THE UNIVERSITY

\title{
OF ILLINOIS
}

\author{
LIBRARY \\ 630 \\ Un 3 an
}

no.1-5

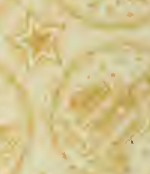

int 
The person charging this material is responsible for its return to the library from which it was withdrawn on or before the Latest Date stamped below.

Theft, mutilation, and underlining of books are reasons for disciplinary action and may result in dismissal from the University.

To renew call Telephone Center, 333-8400

UNIVERSITY OF ILLINOIS LIBRARY AT URBANA-CHAMPAIGN

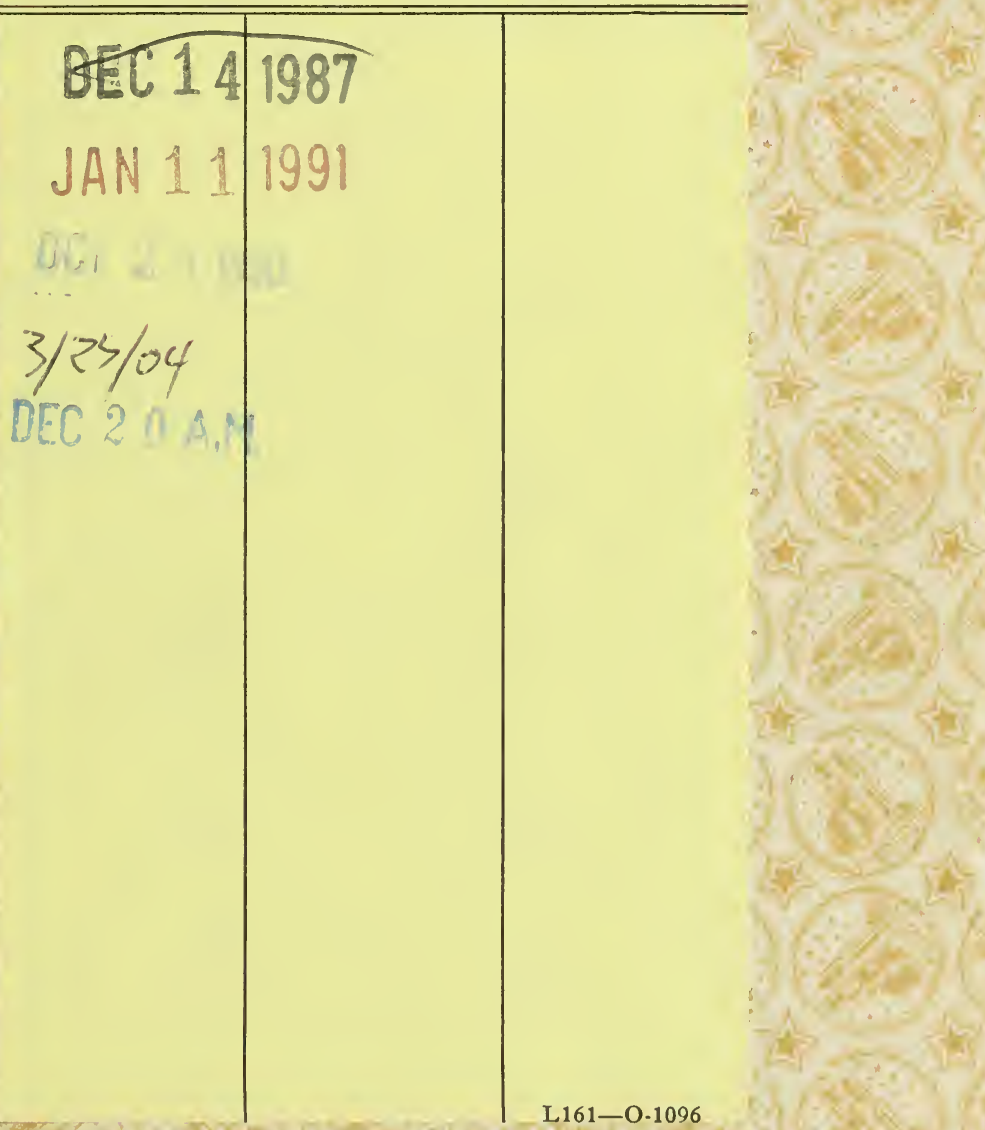




Digitized by the Internet Archive in 2016 with funding from

University of Illinois Urbana-Champaign Alternates

https://archive.org/details/investigationsin1518smit 

U.S. JEPARTMENT OF AGRICULTURE.

BUREAU OF ANIMAL INDUSTRY.

BULLETIN NO. 1.

IN V EST I GATIO NS

INTO THE

\section{NATURE, CAUSATION, AND PREVENTION}

\section{$\mathrm{OH}$ \\ TEXAS OR SOUTIIERN CATTLE FEVER.}

MADE UNDER THE DIRECTION OF DR. D. E. SALMON, CHIEF OF THE BUREAU OF ANIMAL INDUSTRY,

BY

THeOBALI) SMITH, Рн. B., M. D.,

AND

F. L. KILBORNE, B. Agr., B. V. S.

PUBLISHED BY AUTHORITY OF THE SECRE'TARY OF AGRICULTURE.

WASHINGTON:

GOVERNMENT PRINTING OFFICE.

1893. 


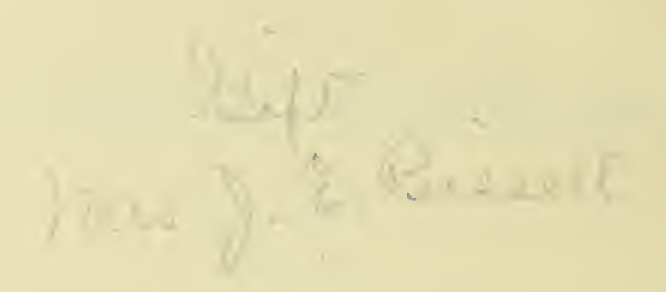




\section{TABLE OF CONTENTS.}

Page.

Letter of transmittal........................................... 7

Letter of submittal ............................................. 9

Historical review ................................................ 11

The nature of Texas cattle fever .................................... 15

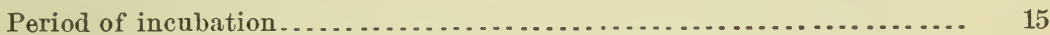

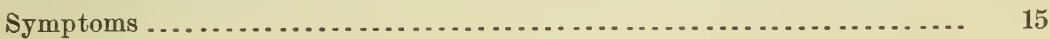

Acute type ................................................. 15

Mild, non-fatal or chronic type ............................. 22

The relation between the acute and the mild type of Texas fever-re-

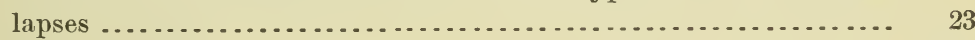

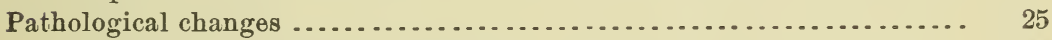

Changes in the organs and tissues.......................... 25

Changes in the corpuscular elements of the bloorl............... 35

The destruction of red blood corpuscles ................... $\quad 36$

The regeneration of red blood corpuscles .................. 41

The causation or etiology of Texas fever........................... 51

Texas fever is not cansed by bacteria........................... 51

The microörganism of Texas fever (Pyrosoma bigeminum n. sp.)......... 55

Peculiar bodies found in the red blood corpuscles of healthy eattle... $\quad 56$

The microörganism in the acute type of Texas fever ............ 58

The microörganism in the mild type of Texas fever.............. 65

The probable life history of the microörganism in the body of cattle.. 68

The nature of the Texas-fever microörganism and its relation to the parasites of the red corpuscles of other animals and of man ........

Probable action of the microörganism in the body of susceptible

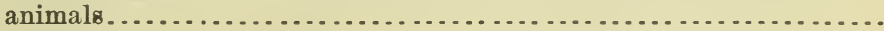

Outbreaks in which the Texas-fever parasite has been demonstrated.

The production of Texas fever in cattle by the inoculation of blood

from cases of this disease .................................

The inoculation of animals other than cattle with Texas fever blood..

The transmission of Texas fever by means of the cattle tick, Boöphilus bovis

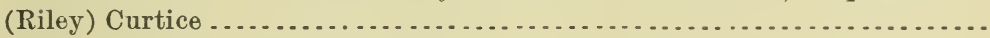

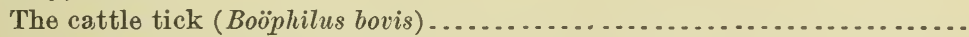

Field experiments to determine the precise relation between the cattle

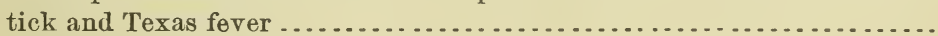

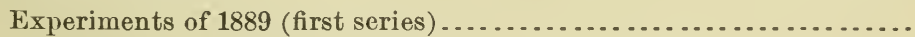

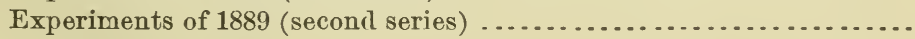

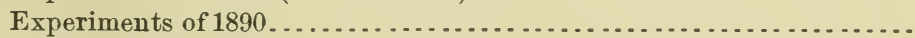

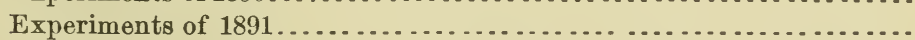

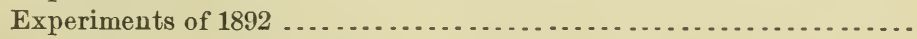

Miscellaneous experiments ..................................

General summary of the field experiments relating to the cattle tick..

The relation of the cattle tick to the "period of incubation" of Texas fever and to the infectiousness of Southern cattle.................. 
'The transmission of Texas fever by means of the cattle tick, etc.-Continued.

The relation of the cattle tick to the micrörganism of 'Texas fever ..... 117

The relation of Southern cattle to the 'Texas fever infection.......... 118

Sick natives as sources of infection when the cattle tick is present...... 122

Sick natives are harmless when the cattle tick is absent ............. 125

May Texas fever be communicated by agencies other than the cattle tick?. 126

Immunity and protective inoculation.-Distribution of diseases resembling

Texas fever on other continents ............................. ${ }^{130}$

Immunity in Southern cattle............................... ${ }^{130}$

Natural immunity of Northern cattle........................ 131

Acquired immunity of Northern cattle ......................... 132

Problems concerning preventive inoculation .................... 135

Is Texas cattle fever restricted to the American continent?............ 137

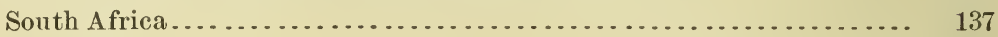

Roumania ............................................... 140

Practical observations and conclusions ........................... 145

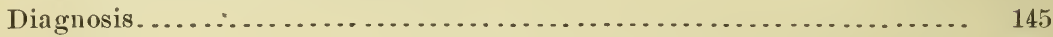

Prevention ................................................... 147

Treatment.................................................. 150

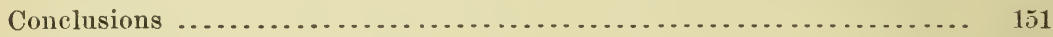

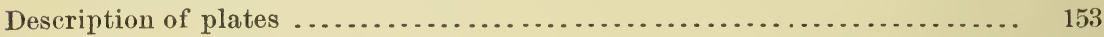

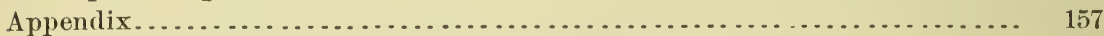

Explanatory introduction to notes and tables.................. 157

Some preliminary bacteriological observations................... 159

Cases of Texas fever examined at the experiment station and the pathological laboratory $(1889-1892$, inclusive) .................... 165

Temperature record of the various cases of 'Texas fever investigated..... 269 


\section{LIST OF ILLUSTRATIONS.}

Page.

Fig. 1. Diagram of the temperature curve in four cases of Texas fever...... 17

2. Method of preparing dry blood films on cover-glasses..............

3. Diagrammatic illustration of the stages of growth of the Texas fever microörganism.................................... 70

4. Plat showing arrangement of experimental field inclosures for 1889.. 94

5. Plat showing arrangement of experimental field inclosures for 1890 .. 99

6. Plat showing supplementary fields for $1890 \ldots \ldots \ldots \ldots \ldots \ldots \ldots \ldots . . .6102$

7. Plat showing arrangement of experimental field inclosures for 1891.. 108

Plate I. Diseased and healthy spleen........................... 156

II. Diseased and healthy liver........................... 156

III. Bile stasis and hæmoglobinuria ....................... 156

IV. Microörganism of Texas fever within the red blood corpuscles . $\quad 156$

V. Microörganism of Texas fever within the red blood corpuseles . $\quad 156$

VI. Microörganism of Texas fever .......................... 156

VII. Capillaries filled with infected blood corpuscles.............. 156

VIII. Microörganism of Texas fever within the blood corpuscles, Amoboid changes ................................... 156

IX. Modified (embryonic) red blood corpuscles after severe hemorrhage and after Texas fever ......................... 156

X. The cattle tick - the carrier of Texas fever................ 156 



\section{LETTER OF TRANSMITTAL.}

\section{U. S. Departiment of Agriculture, Bureau of Animal Industry, Washington, D. C., February 6, 1893.}

SIR: I have the honor to submit herewith a report covering "Investigations into the Nature, Causation, and Prevention of Texas or Southern Cattle Fever," which have been conducted under my direction by Drs. Theobald Smith and F. L. Kilborne, of this Bureau. These investigations have extended over a period of several years, and it is gratifying to be able to state that they have been successful in discovering the cause of the disease and the means by which it is transmitted.

In the whole list of diseases affecting the domesticated animals, there is none so peculiar in its character or so mysterious in its phenomena as was this one previous to these researches. The dissemination of the deadly contagion by apparently healthy cattle, and the harmlessness in general of the really sick animals were inexplicable by any facts which were furnished by the study of other diseases. Veterinarians who had not had an opportunity to observe this disease were skeptical in regard to the correctness of such conclusions, and some spoke of them as a "romance in pathology." These early observations have not only been confirmed, but the phenomena have been explained, and our knowledge placed upon a scientific basis.

It had long been believed by the eattle-raisers of the West that Texas fever was caused by the ticks which were carried and scattered everywhere by the Southern eattle; but scientists were incredulous, because they could not understand how the bite of these insects could produce such an acute disease, with destruction of the blood corpuscles and lesions of internal organs. It was not until the protozoal microörganism was discovered in the blood corpuscles, and its destructive effects were revealed, that the action of the ticks could be explained.

When the writer investigated the extent of the infected district he was strongly impressed with the fact, which then first became apparent, that this district almost exactly corresponded with the habitat of this suspected tick. This led to the experiments which demonstrated that ticks carried the infection, introduced it into the tissues of the susceptible cattle, and in that way produced the disease. We have to 
deal, therefore, with a complicated infection, in which two very different kinds of parasites play an important part.

Another significant discovery, not less marvelous, is that the microorganism which constitutes the contagion of the disease is transmitted through the egg to the young tick, and it is this, and not the adult tick carried by the Southern cattle, which finds its way upon susceptible animals and causes the disease. In the absence of the tick, the disease is probably not communicable except by artificial inoculation.

The accompanying report gives all the details of the investigations referred to, and will be found of the greatest interest both to the cattleowner desirous of preserving his animals from this dangerous malady, and to the scientist who wishes to know the various methods by which contagion may be distributed. The researches have been made with great care and thoroughness, and the gentlemen who have conducted them have shown perseverance and scientific ability of the highest order: Their work, as described in these pages, has for its foundation accurate scientific experiments, and, however unexpected may be the results, these may be relied upon as furnishing the basis for the true exposition of the disease.

Very respectfully,

Hon. J. M. RusK,

D. E. SALMON,

Chief of Bureau.

Secretary. 


\section{LETTER OF SUBMITTAL.}

\section{U. S. Department of Agriculture,}

Bureav of Antmal Industry,

Washington, I). C., November 15, 1892.

SIR: I have the honor to submit herewith a report on the nature, causation, and prevention of Texas cattle fever. The investigations upon which it is based have been carried on more or less continuonsly since 1888. That part of the work dealing with the intimate nature of the disease, its pathology and etiology, and the microscopical and bacteriological work involved in their elucidation was carried on by myself, at the experiment station and at the laboratory of the Division of Animal Pathology, while the field work at the experiment station, embracing the planning and arranging of the field experiments in general and those relating to the cattle ticks in particular, was carried on by F. L. Kilborne, B. V. S. Much assistance has been rendered by E. C. Schroeder, M. D. V., who, under my direction, did the major part of the work of estimating the blood corpuscles in the various cases of Texas fever, and who assisted at the post-mortem examinations from the latter half of the season of 1890 up to the present. Latterly S. D. Maynard, B. s., has also rendered some assistance in this work.

Much difficulty has been experienced in the proper arrangement of the subject-matter, owing to the large number of experiments which have been carried out and the various directions which the investigations har to take. The large amount of material which has accumulated had to be condensed and brought into a compact form. The plan adopted relegated the detailed history of the various cases into an appendix. By this means the report itself could be brought into a form which includes all the important results of the yrork fully discussed. The verification of these results must be sought for in the appendix under the individual cases. Such an arrangement occasionally deinanded repetitions and cross references, since individudl experiments have frequently borne fruit of several kinds which had to be brought into relation with several different subjects.

In the present report the old name, "Texas fever," has been used, since it is the name first given to the disease in the earlier publications and probably the one by which it is most readily recognized. The reten- 
tion of this name does not, of course, imply any restriction of the disease to the State which bears its name, for it is now well known, largely owing to your investigations, that the entire southern belt of this country is equally responsible for it. The term "Southern cattle fever" is not much better, since it is now highly probable that this disease is not peculiar to our country, but exists in other countries situated as we are with reference to latitude. In fact it is hardly to be doubted that this malady exists along the Danube River, in the Balkan provinces, and in the southern territories of Africa. The time has not yet arrived, therefore, to give this disease a more scientific name worthy of its cosmopolitan character, and for this reason the old, familiar, and shorter appellation of "Texas fever" has been temporarily retained.

Theobald Smith, Chief of the Division of Animal Pathology.

Dr. D E. Salmon,

Chief of the Bureau of Animal Industry. 


\section{NATURE, CAUSATION, AND PREVENTION OF TEXAS FEVER.}

\section{HISTORICAL REVIEW.}

Up to the time at which these investigations were begun, a certain number of very important facts had already been ascertained and repeatedly confirmed concerning the nature of Texas or Southern cattle fever. There were also a number of theories in the field concerning the causation or etiology of this disease, based in part on investigation, in part on speculation.

Of those definitely ascertained facts, we may mention as the most important the one which traced the distribution of the infection to cattle brought from a large but well-defined territory, including most of the Southern States, into more northerly regions. The Southern cattle bearing the infection were, as a rule, free from any signs of disease. It was likewise settled that this infection was carried only during the warmer season of the year, and that in the depth of winter Southern cattle were harmless. It was also known that the infection was not communicated directly from Southern to Northern cattle, but that the ground over which the former passed was infected by them, and that the infection was transmitted thence to susceptible cattle. All that was necessary for the production of disease was the passage of Southern cattle over a given territory and the grazing of Northern cattle over the same or a portion of the same territory during the same season.

It was also discovered that Southern cattle, after remaining for a short time on Northern pastures, lost, in some mysterious way, the power to infect other pastures and were, for the remainder of their stay North, harmless. Again, cattle driven over a considerable distance lost, after a time on their way, the power to infect pastures. When pastures and trails had been passed over by Southern cattle, it was observed that the disease did not appear at once in the Northern cattle grazing on them, but that a certain period of not less than thirty days elapsed before the native cattle began to die. More curious even than these 
facts, was the quite unanimous testimony of stock-owners who had had more or less experience with this disease, that native susceptible animals who had become diseased did not transmit the disease to other natives, and that they were harmless. We shall discuss this statement in detail, in connection with experiments made to test its accuracy.

If we turn our attention to the opposite aspect of this interesting series of facts which deals with the introduction of Northern cattle into Southern territory, we learn that such cattle may contract Southern cattle fever, and that it is only under considerable risks that Northern cattle can be introduced into what has been called the permanently infected territory.

These interesting facts about a mysterious disease were largely reasoned out by farmers and stock owners in their trying experience with it and were well known before 1868, when the disease began to arouse the attention of the Government, owing to its widespread devastations in the Northern States in that year. The historical record of the development of these ideas is therefore very meager. That Southern cattle in a state of health might bring destruction to Northern herds was observed late in the last century by Dr. Pease. A herd of cattle was driven in $\mathbf{1 7 9 6}$ from South Carolina into Pennsylvania, where disease broke out in Lancaster county and other places. This disease was directly traced to the Southern herd by Dr. Pease, who made it the subject of an interesting communication and dwelt particularly upon the fact that the cattle bringing the disease were themselves in good health.

In 1868, Texan cattle shipped up the Mississippi River to Cairo and thence by rail into Illinois and Indiana early in June caused during the summer of that year enormous losses of cattle in these States. Moreover the East began to be aroused becanse Western cattle infected with the disease had been shipped eastward for beef and were dying of Texas fever on the way, in the New York stock yards and elsewhere. The question as to the effect of such diseased flesh upon human health was at that time entirely new and caused much uneasiness. The cattle commissioners of New York State and the board of health of New York City made a vigorous effort to check the importation of diseased cattle from the West, and to their efforts we owe much valuable information of this disease. During that year it was investigated by Dr. R. C. Stiles for the Metropolitan Board of Health and by John Gamgee and Drs. John S. Billings and Curtis for the National Govermment. Since then investigations have been made and published by Drs. D. E. Salmon and Detmers for the United States Department of Agriculture, and by Drs. Frank S. Billings, Paquin, Dinwiddie, and Francis for the experiment stations of certain States. 'These various reports will be again referred to under the special subjects to which their authors have given more or less attention. 
Perhaps the most important and special contribution to the subject since the earlier investigations of 1868 is the determination of the boundary line of the permanently infected district by Dr. D. E. Salmon $(\tilde{5}, 6,7)$.* From what has already been stated it will be readily understood that this line marks the northern limit of the territory from which cattle may carry the infection into the territory north of this line. On the other hand to cross this line from north to south obviously places cattle in the position to contract Texas fever under favorable conditions. The investigations of Dr. Salmon have shown that this permanently infected area does not extend north of the 37 th parallel of latitude excepting along the eastern slope of the country, where it extends halfway between the 38th and 39th parallel. The order of the Secretary of Agriculture issued February 26, 1892, puts the following States and Territories entirely within the permanently infected area: South Carolina, Georgia, Florida, Alabama, Mississippi, Arkansas, Louisiana, and Indian Territory. The following are crossed by the boundary line and are therefore not entirely within the infected area: Virginia, North Carolina, Tennessee, Oklahoma, and Texas. The line as at present defined begins at the Atlantic coast, passing westward on the 38th parallel, and follows the lower boundary of Maryland to the Potomac. It then passes westward across Virginia as far as the eastern slope of the Blue Ridge, which it follows in a southwesterly direction through North Carolina, thus exempting the cooler mountainous regions of these two States from permanent infection. It continues in a nearly westerly direction across the sonthern strip of western North Carolina and the southern portion of Tennessee. Across the Mississippi it follows the northern boundary of Arkansas and that of the Indian and Oklahoma Territories and finally passes southward through Texas on or near the 100th meridian.

In addition to this work of accurately defining the territorial distribution of the infection nothing has been done to add materially to the permanently valuable knowledge concerning this malady. Although attempts have been made to discover the cause they were not successful, as we shall be able to show. In 1889 the first systematic experiments were made by the Bureau of Animal Industry and these were at once fruitful in the discovery by one of us of a peculiar micro-organism in the red blood corpuscles which corresponds in every respect with what we should expect as the true cause. At the same time the other showed by field experiments that the cattle tick was somehow necessary to the transmission of the disease. These observations were fully confirmed in 1890. In the fall of the same year it was observed that when young ticks hatched artificially are placed on cattle there is a sudden extensive loss of red blood corpuseles accompanied by fever which could in no way be explained by the simple abstraction of blood. This discovery, at once followed up by additional experiments, brought to light the remarkable fact that Texas fever is caused by putting recently

*These numbers refer to the publications on this subject, p. 14. 
hatched cattle ticks on susceptible cattle. All these results were reconfirmed in the summers of 1891 and 1892.

These investigations have thus far brought to light two important facts: (1) The constant presence of a blood corpuscle-destroying microorganism in Texas fever, and (2) the transmission of the disease from cattle to cattle by the cattle tick. The various experiments and observations which have led to these results are embodied in the following report and appendix.

The subject of Texas cattle fever has been treated of in the following publications, which are referred to in the text by the number prefixed to each title:

(1) Transactions of the New York State Agricultural Society, 1867, part 2.

(2) Report of the Commissioner of Agriculture on the Diseases of Cattle in the United States. Washington, 1871. (Reports by Mr. John Gamgee, J. R. Dodge, and Drs. J. S. Billings and Curtis.)

(3) Contagious Diseases of Animals. Special report No. 22. (Report by Dr. D.

E. Salmon, pp. 98-142.)

(4) Report of the Coumissioner of Agricultúre for 1881-'82. (Report by Dr. D. E. Salmon, pp. 300-306.)

(5) Contagious Diseases of Animals. Washington, 1883. (Report by Dr. D. E. Salmon, pp. 13-44. Report by Dr. J. H. Detmers, pp. 103-145.)

(6) First Annual Report of the Bureau of Animal Industry, 1884. (Report by Dr.

D. E. Salmon, Chief of the Bureau, pp. 214-221.)

(7) Second Annual Report of the Bureau of Animal Industry, 1885. (Report by Dr. D. E. Salmon, Chief of the Bureau, pp. 247-274.)

(8) Bulletin of the Agricultural Experiment Station of Nebraska, II, No. 3. (Southern Cattle Plague and Yellow Fever from the Etiological and Prophylactic Standpoints. By Frank S. Billings.)

(9) Texas Fever. By Paul Paquin. (Missouri Agricultural College Experiment Station, Bulletin No. 11, May, 1890.)

(10) Third Annual Report of the Arkansas Experiment Station, 1890. (Report by R. R. Dinwiddie, Veterinarian, pp. 98-122.) 


\section{THE NATURE OF TEXAS CATTLE FEVER.}

\section{PERIOD OF INCUBATION.}

This term has no very definite significance in this disease, for it is used to designate different things. Thus it has been employed to indicate the period elapsing between the exposure of susceptible cattle to Southern cattle, or upon fields infected by them and the appearance of the disease. If taken in this sense it may vary from ten to ninety days. The great variation here observed is readily explained by the life-history of the cattle-tick, with which this period is intimately associated. A discussion is therefore postponed until the life-history of this parasite has been described (p. 114), and we content ourselves here by simply mentioning the facts as observed.

This term may also be used to signify the time elapsing between the introduction of the infectious agent into the tissues and fluids of the body and the first appearance of disease. This period is ascertainable by inoculation. In the case of subcutaneous and intravenous injection of blood from cattle suffering with Texas fever, the fever temperature appeared within a few days of the inoculation and outward signs of illness were manifest on or even before the sixth day. It is probable, therefore, that multiplication begius at once after the micro-parasite has been introduced into the body, and when it has attained a sufficient momentum the external symptoms of disease appear. This may be in from six to ten days, depending on the number of micro-parasites originally introduced, the predisposition and age of the animals, and the season of the year.

\section{SYMPTOMS.}

ACUTE T'YPE.

In our experiments two types of disease have manifested themselves, the acute fatal type and a mild, rather prolonged, usually nonfatal type. As they differ in many respects, they will be considered separately.

The acute disease is the disease of the hot summer months. It appears suddenly and as a rule at the same time in all animals of a herd 
which have been exposed to the same infection together. The fever usually precedes the outward symptoms by several days, and animals apparently quite well will show a high rectal temperature ranging from $105^{\circ}$ to $108^{\circ} \mathrm{F}$. The value of the elinical thermometer in thus detecting disease was pointed out by Gamgee and by the Metropolitan Board of Health in 1868. The latter have recorded a temperature of $109^{\circ} \mathrm{F}$. (1, p. 1098.) A temperature above $108^{\circ} \mathrm{F}$. has not been noted in our experiments. A glance at the tables in the appendix will show that the normal morning temperature of the cattle in our experiments ranges from $100.5^{\circ} \mathrm{F}$. to $102.5^{\circ} \mathrm{F}$., being in general somewhat lower in autumn than in midsummer. The temperature of calves and young animals may rise to $103^{\circ} \mathrm{F}$. without being accompanied by any sigus of disease.*

If the temperature of exposed animals be taken once daily, say in the morning, it will be found that at the onset of the disease it will rise within twenty-four hours from the normal to $104^{\circ} \mathrm{F}$. or even higher. In the following twenty-four hours it may rise to $105^{\circ}$ or $107^{\circ} \mathrm{F}$. The continued daily record will then show a high temperature until the disease terminates fatally or in recovery. In the former case it may fall from $2^{\circ}$ to $4^{\circ}$ below the normal just before death. When recovery ensues it falls as quickly to or even below the normal as it rose in the beginning of the attack. If the temperature be taken twice daily, in the morning and the evening, a new set of phenomena appear. The temperature at the outset rises during the day, is highest in the evening, and may be low again in the morning. This oscillation, partly a normal occurrence, may be noticed for three or four days in some cases, the morning temperature gradually rising until it is as high as the evening temperature. The nigh temperature then remains continuous until the end of the fever. These facts are well exemplified in the following diagram:

*'These figures agree fairly well with those of other observers. "The temperature of healthy cattle ranges from $37.6^{\circ}$ to $39.6^{\circ} \mathrm{C}$. (99.7 $7^{\circ}$ to $103.3^{\circ} \mathrm{F}$.). In some cases it may be even a trifle higher or lower. In the morning it is usually, but not invariably, $0.2^{\circ}$ to $0.4^{\circ} \mathrm{C}$. $\left(0.4^{\circ}\right.$ to $0.7^{\circ} \mathrm{F}$.) lower than at night. In calves and heifers it is usually somewhat higher than in old cows. *** The general average from the results obtained by numerous observers is $38.8^{\circ} \mathrm{C}$. (101.8 $8^{\circ}$ F.)." -Dieckerhoff (Lehrbuch $d$. spec. Pathologie und Therapie f. Thierärzte, $I I$.) During very hot weather the evening temperature of cattle more or less exposed to the sun in the pastures of the station has been found to rise to $104^{\circ}$ and even $105^{\circ} \mathrm{F}$., although the animals were, so far as could be determined, in good health. 
over a herd of cattle and select those having a high temperature by simply placing the hand on the anus.

The pulse and respiration rise with the fever. There is considerable variation in the number of the pulse-beats and of the respirations during health, and there is also in some cases the excitement incident to being canght, which prevent our giving any very accurate figures. In health the number of respirations of the eattle used in our experiments may be put down as between 20 and 40 per minute, according to the temperature of the air and the age of the animal, while the pulse seems to range between (i0 and 80 beats per minute.* In animals in the acute stage of Texas fever the respiration may rise to between 60 and 100 and the pulse to between 90 and 110. As the fever subsides and recovery begins the great weakness of the animal still keeps the pulse very high for a time, especially when the animal is moverl about or excited in any way. The respirations, on the other hand, are apt to fall below the normal in this same period. When death approaches the heartbeats increase in number as they grow feebler, and the respirations fall with the body temperature below the normal. These statements are fully illustrated in the appendix by the individual cases.

Next to the high temperature the condition of the urine demands our attention. The one sign regarded as peculiar and pathognomonic in this disease is the discharge of urine having the color of blood. This color is not due to a discharge of blood from the kidneys and subsequent breaking up of the red corpuscles, but to a filtration of the coloring matter of broken-down red corpuseles (hamoglobin) already in solution in the eirculation into the urine in the excretory structures of the kidneys. This fact was first pointed out in 1868 by R. Cresson Stiles. In using the term hamolobinuria this is all that is meant in this report. The precise state or condition of this coloring matter in the urine does not call for consideration.

IIrmoglobinuria may be said to be present in most acute fatal cases of Texas fever. Out of 46 fatal cases in which urine was in the bladder after death, hemoglobin was present in 33 cases.t $A$ careful exanination of the notes will show that in 13 negative easest the animals were killed in the earliest stages of the fever, or clse they died or were killed after the number of blood corpuscles had been greatly reduced

* The respiration in our cattle seems to have been higher than the normal of othex observers. "Respiration in healthy cattle varies from 16 to 25 per minute, and may fall as low as 14 or rise as ligh as 30. According to the observations of riirstenbere it is 21 per minute for cows and 24 for bulls."

The "pulse, like the respiration, varies greatly. According to Hering aud Fiirs. tenlserg it is from 90 to 130 per minnte during the first two weeks of lifo; from 70 to 80 from two weeks to two years. The average for a full-grown animal is 60 , for old eows 50 to 5.5."-Lor. cil.

$\dagger$ Nos. 5, 6, 7, 8, 9, 11, 17, 50, 54, 66, 69, 74, 76, 90, 10:2 $2_{\mathrm{n}}, 103,106,108,128,129,130$, 139), 110, 141, 162, 163, 180, 186, 197, 198, 200, 203, 223.

$\ddagger$ Nos. $4,10,44,48,51,71,95,134,137,169,181,181,227$. 
and the acute stage of the disease was over. In the former cases the hamoglobin had not yet been set free from the corpuscles; in the latter cases it had probably been eliminated one or more days before death. How frequently "red water" is passed before death we can not state with any degree of certainty, since its discharge may wholly escape observation. We have a record of hamoglobinuria in but four cases: in No. 43 on the third day before death, in No. 44 on the fourth day before death, in No. 80 before it was killed (probably twelve to twenty-four hours before death), and in No. 198 twenty-four hours before death. In some of these cases it so happened that the urine was passed while the animal was undergoing examination. It is interesting to note in comnection with the statements made that in No. 44 no "red water" was found in the bladder after death, although it had been passed four days previously.

Whether hæmoglobinuria is always present in acute cases of Texas fever it is impossible to state definitely. As it seems to depend upon the rapidity with which the red blood corpuscles are infected and destroyed, a slower destruction may allow other organs to take charge of the debris and thus forestall the diseharge of hemoglobin in the urive. In the notes will be found the record of hemoglobinuria in but one acute case which recovered (No. 49), while in a number of cases in which the urine was collected, sometimes in the height of the fever, sometimes after it had departed, no hremoglobinuria was detected.* In this solitary case the high temperature first appeared August 18. On August 23 the temperature being still above $105^{\circ}$, the urine was free from hromoglobin, but contained a small quantity (.05 per cent) of albumen. On August 27 the temperature had become normal, but a second paroxysm followed soon after, and on September 4 and 5 the urine was of a port-wine color. Urine collected September 6 was again of normal color.

The urine during the fever, when free from hamoglobin, contains in many instances a small quantity of albumen. The specifie gravity may at first be high (1030-1040), and it may be strongly alkaline and effervesce with acids as in health, but, as the disease progresses and when the animal eats but little, its specific gravity will fall to 1010-1020; it fails to effervesce with acids and is faintly alkaline or even slightly acid. When the fever has subsided the urine has been observed to be in a few cases very watery, $i$. $e$, of very low specific gravity and feeble in color. Within one or two weeks, however, the normal condition is restored.

The urine which contains the coloring matter of the blood varies, as might be expected, very much in depth of color, according to the concentration of the hremoglobin. It may have a very light claret color, or it may be so deeply tinted as to appear opaque and blackish. In a

*Nos. $95,105,135,137,138,182,185$, and many others in which the urine was observed to be of the normal color, but was not examined more closely. 
test tube when viewed by transmitted light it may barely permit the light to pass unless diluted with water. (See Plate III, Fig. 4.) Such urine is, as a rule, entirely free from suspended matter and blood corpuscles. The latter may sometimes be found in small numbers when the urine is permitted to stand, and they may be derived from small hemorrhages in the pelvis of the kidney, quite regularly observed at autopsies. The coloring matter, as has been stated above, is derived from corpuseles broken up within the circulation, and not outside in the bladder. When such urine is treated with a little acetic acid a brownish floceulent precipitate, probably of the derivatives of hæmoglobin, appears. When boiled, a brownish flaky precipitate forms, which rises to the surface as a scum. As might be expected, such urine always reacts in presence of the usual tests for albumen. How much of the precipitate formed is the ordinary serum-albumen found in various forms of kidney disease and how much belongs to hæmoglobin does not come up for consideration here. Suffice it to say that in very opaque urines the precipitate is quite abundant and corresponds when Esbach's test is applied, to from one to three per cent of albumen.*

The subject of hæmoglobinuria is more fully discussed here under the symptoms because it is occasionally observed during life and probably with the aid of a catheter may be seen much more frequently. The causes of its occurrence and the way in which it is brought about will be discussed in connection with the microörganism. Very little need be said of the other characters of "red water." When found in the bladder after or collected shortly before death its specific gravity is usually low (1010-1020) and it is feebly alkaline or acid. There is no effervescence with acids. After standing a few granular casts and rarely urates are found in the very slight sediment. The greater the number of days before death that it is collected the more nearly it approaches normal urine as regards specific gravity and alkalinity.

The bowels are as a rule constipated during the high fever, and on post-mortem examination the large bowels (cxeum and colon) are found in some cases compactly filled with small, very firm, hard balls of dung. As the fever subsides the frecs again become softer and are then found more or less deeply tinged with bile.

Loss of appetite always, and cessation of rumination usually, accompany the high fever after the third or the fifth day. These, together with the enormous destruction of the red blood corpuscles and the temporary disorganization of some of the vital organs, lead to a rapid loss of blood during the fever and even to extreme emaciation during the period following the fever. Some observers have recorder the surlden and partial cessation of milk secretion. We have had no opportunity to observe this symptom.

* If a few drops of acetic acid be added to nrine holding much coloring matter in solution and allowol to stand over night, a clear yellowish liquid may be filtered off which gives the ordinary reactions for albumen. 
Symptoms referable to disturbances of the brain and the spinal cord were rarely noted. They usually manifested themselves in partial loss of vision, delirium, staggering gait, and swaying of the hind quarters. These latter may in part he referred to the great weakness which eattle manifest after some days of fever and perhaps to the æedema around the kidneys. This weakness may become so great that they will be unable to rise even when urged. When standing there may be noted a trembling of the muscles especially of the hind quarters and limbs. Icterus or jaundice has not been noted in any case during life.

Another character of this disease, the most constant and valuable of all and of which the hæmoglobinuria or "red water" is but a part, is the thinness of the blood. A more thorough discussion of its condition will be given further on. In this connection we only mention those phenomena which can be witnessed by the naked eye. Soon after the high temperature sets in, the blood begins to grow thin and after some days of fever it has become very pale and watery. An incision into the skin readily shows this to be the case. The difference between the drop of rich red blood issuing from a slight cut of the skin in healthy cattle and the thin, pale drop oozing from such a cut in Texas fever is very marked. This difference is due to the loss of red corpuscles which give the blood its characteristic color. Associated with this there may be in some cases a marked bloodlessness of the skin in the later stages. A number of small incisions are often required to obtain a few drops of blood. In some cases shortly before death the blood slowly trickles from a slight incision for some time before it is checked by the natural process of coagulation.

When freshly-drawn blood is allowed to stand the serum forced out of the clot has in the acute stage a very dark-red color, indicating the presence of much coloring matter in solution. As regards the coagulability, which some observers have regarded as feeble, we have no facts pointing in one dfrection. In a few cases the coagulation appeared retarded; in others it appeared to be normal in rapidity and effectiveness. As will be seen further on, the condition of the blood must vary considerably from time to time. At one time it may contain the débris of destroyed corpuscles equal in number to one-tenth, or even one-fifth, of all circulating in the body. That under such circumstances its coagulability may be affected is evident. Frequently, however, the blood comes under observation when the destruction of red corpuscles has ceased and the products have either been excreted or metamorphosed. In this way conflicting observations may perhaps be harmonized. In general, we may say that the coagulability of the blood is not much altered.

We have thus briefly sketched the symptoms of an acute attack of Texas fever and noted three important diagnostic features, high temperature (or fever), hæmoglobinuria (or red water), and thinness of the 
blood (or destruction of red corpuscles). The last is the most constant, and. in fact, the one essential character of Texas fever Among the other less important symptoms and appearances, many of which are always associated with one or the other of those mentioned, are dry, hot skin, high rate of pulse and respiration, loss of appetite, cessation of rumination and of milk secretion, constipation, hypersemia followed by bloodlessness of the skin and mucous membranes.

The course and the duration of the disease are subject to variations. We have seen that it begins somewhat abruptly with a high temperature, runs its course in a few days, and terminates fatally, or else it disappear's as quickly. In the latter case the disease is followed by a period of great debility, owing to the impoverished condition of the blood and the degenerative processes set up in the various vital organs, and not infrequently by relapses. Some animals never fully recover; in others recovery takes place after weeks and months.

The duration of the disease varies more or less, but the continuous high temperature rarely lasts longer than eight to ten days. The fatal termination may take place in the height of the fever-that is to say, four or five days after the appearance of a high morning temperature, and may be the direct result of the derangement of the vital functions, due to the rapud destruction of red corpuscles by the microörganism, or it may take place after the fever has subsided, when the animal fails to rally from the shock imparted to the system and from the drain of its blood-furming resources. If we take as our starting point of the fever the first high morning temperature death may ensue from four to fourteen days thereafter, or it may be delayed still longer, when the animal dies slowly of exhaustion. The period of disease for such as recover is practically the same. A fever period of eight to ten days is followed by a period of normal or subnormal temperature. The falling of the temperature marks the end of the destruction of red blood corpuscles and the disappearance of the parasite from the blood. The subject of relapses and secondary attacks during the season by which the period of disease as a whole may be prolonged into months is discussed on page 23 .

The mortality fiom southern cattle fever varies greatly, as will be seen in the following pages on the mild chronic form of the disease. The time of the outbreak will largely decide whether practically all the attacked animals die or all survive. A midsummer ontbreak, when acute in its nature, is the most fatal. From this there may be all gradations towards the mild nonfatal form of late autumn.

MILD, NONFATAL OR CHRONIC TYPE.

This type of sonthern cattle fever has hitherto remained unobserved. The reason for this is quite simple. It can be recognized only by an exannination of the blood, which must determine the presence of the 
micro-parasite in the red (orpuseles and their approximate rate of destruction.

The mild form of 'Texas ferer is largely a disease of antumn when the heat of smmmer has passed away. In the latitude of Washington, D. C., October and November, rarely the first week of December, are favorable to it. It is not, howerer, strictly limited to this period, as it may be observed fom early Ingust on through the entire season. Its occurence during this latter period, which is commonly characterized by acute disease, is limited largely to the less susceptible calres and to a very few of the exposed arlults. Cattle which have passed through the acute discase may have a relapse in the form of the mild type in alutumu.

The essential difference between this and the acute type rests on the fact that a stage of the parasite circulates in the bloor of the mild eases, which is different from the one observed in acute cases. This difference will be made clear in the chapter on the Texas fever parasite. When we come to the various symptoms there is only a difference of degree. The fever temperature is low and fluctuating, rarely rising above $105^{\circ} \mathrm{F}$. in the evening. In the morning the temperature is usually normal or very slightly elevated. The destruction of red blood corpuscles by the micro-parasite goos on as in the acute form, but much more slowly and deliberately, and hence the period of disease itself-that is, the time during which the parasite is present in the blood in considerable numbers-is much prolonged. The hemoglobinuria is probably never present. The various symptoms which accompany the ferer are only present when the temperature is above $103 \circ \mathrm{F}$. There is loss of appetite and dullness, especially when the number of red corpuscles has reaclied its lowest limit, followed by a slight falling away in the condition of the animal.

It will thus be seen that there are no symptoms manifest to the unaided eye which we might put down as characteristic of Texas fever in its mild type. It might be confused with a variety of disorders incirlent to the bovine species or else be entirely overlooked. From an economic point of view it is of not rery great consequence, since it is not fatal, and the loss in weight, thongh quite considerable in some cases, is soon made $u$ ) in the winter months.

THE RELATION BETWEEN THE ACUTE AND THE MILD TYPE OF TEXAS FEVER-RELAPSES.

In certain cases it was noticed that after the animal had recovered from the acute attack and the number of blood corpuscles had nearly reached the normal maintained before the attack it would again fall, and in the blood many corpuscles conld be found containing the small stage of the Texas fever parasite which is always associated with the mild type. In other words, the acute attark would be followed after a 
certain interval of time by a mild attack. This interval may vary considerably. Thus in No. 105 the acute attack began early in August, and the secondary, or mild attack, about one month later. In No. 160 the acute attack began early in September, the mild attack about three weeks later. In No. 49 the acute attack began near the middle of August, the mild attack was observed in the second week of October. Two cases of Texas fever (Nos. 206 and 219) induced by the intravenous injection of blood are particularly noteworthy in this respect. In No. 206 the disease began July 13, in No. 219 July 23. In both the mild attack was detected at the same time at the end of August, although it may have begun some days before.

The question naturally presented itself whether these secondary attacks are simply relapses or whether they are reinfections from without. As will be fully discussed further on, the young cattle-tick induces the disease in natives as soon as it has attached itself to their skin. Since the cattle-ticks are present on the infected field during the entire summer and autumn it is reasonable to suppose that the secondary or mild attacks may be cansed by ticks which have remained on the field or by the second generation, since the usual time for mild attacks, late September and October, is the time for the appearance of a second generation of young ticks. Whether these mild attacks are always due to an invasion of the animal by such a second generation or whether they may be due in some cases to the recrudescence of the microörganism not yet entirely eliminated from the system was settled by the cases Nos. 206 and 219 above referred to. Since they were inoculated but once and there was no opportunity for reinfection these cases prove that a mild attack may follow an acute attack without a fresh importation of the microörganism from without; in other words, that the mild attack may be considered as a relapse. This conclusion does not operate against the probability that some mild attacks may be due to secondary infection from without.

In addition to the true relapses just referred to we may observe more or less oscillation in the course of the disease as expressed by the destruction of red corpuscles. This oscillation is probably dependent on the periodical multiplication of the micro-parasite and in this respect may be regarded essentially identical with the relapse. The latter follows the primary disease at long intervals, while the oscillations are but one and one-half to two weeks apart as a rule.* Neither the relapses nor the oscillations have been marked by any distinetive clinical signs excepting a slight rise of temperature in some instances. Such undonbtedly do exist and may perhaps be detected by more frequent daily measurements of the temperature and a closer and more frequent examination of the animals and their excretions than our time has permitted us to make.

* See also p. 40. 


\section{PATHOLOGICAL CHANGES.}

In dwelling upon the pathologieal ehanges caused by Texas fever we have had two objects in view; first, to add as much as possible to the information already on hand, and, secondly, to furnish such a complete record of the cases in the various experiments that there would be $n$ room for doubt in the reader's mind that we were dealing with Texas fever wherever this is claimed to be so. Inasmuch as the causation or etiology was the essential object of the researches, everything else had to be subordinated to lines of investigations which endeavored to get at the causes and the modes of transmission of the malady before us. The following account, though somewhat meager for this reason, is given as a description of the nature of the disease we were dealing with. We may note here that the lesions induced by North Carolina cattle and those induced by Texas cattle at the same season were identical in every respect. (See Nos. $74,80,82,107,128,129,139$. )

\section{CHANGES IN THE ORGANS AND TISSUES.}

Cattle which have succumbed to Texas fever undergo post-mortem changes very rapidly. This may be largely due to the fact that the disease occurs in the greatest heat of summer. Hence facts relating to the appearance of organs and tissues are of doubtful value if the animal has not been examined soon after death. This oceurs as a rule during the night, and post-mortem changes are begun when the morning arrives. It is frequently desirable therefore to kill animals in different stages of the disease to obtain trustworthy facts.

The skin presents nothing abnormal to the unaided eye and, as will be pointed ont later on, certain regions are beset with ticks. In one case (No. 197) which came to our notice very recently the hair on the abdomen and the inner aspect of the thighs was matted into little tufts by dried blood. The skin showed at such places a bluish elevated spot and when incised a little blood was found in the subcutis. This may be what has been called blood sweating.

The subcutaneous tissue and fat in our cases were free from any changes except in three (Nos. 7, 102 a, and 227), in which they were of a decidedly yellowish tinge. Jaundice was thus of rare oceurrence. In the report of the Metropolitan Board it is stated that "the fat has a deep or high colored greenish appearance and has not the firm resistance of health. The lean meat is of a brownish mahogany color and on being cut into has a peculiar sickening odor." The muscular tissue in our eases was normal in color or perhaps a trifle paler. Edema of the subcutaneous tissue of the ventral aspect of the body was occasionally present (No. 69) and referable to the debilitated condition of the animal.

The brain was removed in a smail number of cases (Nos. 198, 200, 203, and 223) and carefully examined, but no lesions which can be re. 
garled in any sense as peculiar to or characteristic of the disease were observed. It may be said, in general, that the brain shared the general tendency towards the injection of the capillany system. The vessels of the pia and the plexuses were engorged, and over the frontal lobes and near the great transverse fissure it was more or less pigmented--a condition also nnet with in other diseases. The gray matter of the cerebrum and especially of the cerebellum appeared of a more pinkish color. The white substance was normal in color, the ventricles free from fluid.

Lungs._-The luugs are, as a rule, healthy. There is, in many cases, pulmonary oedema, with or without emphysema, noticeable after death. In a few instances foci of dark red hepatization were observed in one of the principal lobes, which involved one or several lobules.

Heart.-At the antopsy the right ventricle is always distended with blood, fluid or clotted, according to the time elapsing between death and the examination. The left ventricle is usually firmiy contracted, and may contain a small quantity of fluid or elotted blood. The clots are quite firm and very rarely mixed with firmer, pale yellowish clots. A very constant lesion is the extravasation of blood beneath the epiand endocardium. This is mainly restricted to the icft ventricle, although peterhice are not infiequently met with on the right ventricle. On the external surface of the heart the petechire are usually grouper along the interventricular groove and near the base, although cases occur in which the whole ventricular surface is sprinkled over with them. The inner surface of the left ventricle shows larger patches of extravasation usually on, or at the base of, the papillary muscles. On the large vessels at the base of the heart, within the pericardial sae, there are frequently very delicate shreds of tissue or patches in a hyperxmic condition. The heart muscle, on closer inspection, is observerl to have its minute vessels markedly injected, and in fresh sections the capillary network is found densely packed with red eorjuscles. In cases which have sucemmed after the subsidence of the fever the heart musele is quite pale. Clondy and fatty changes of the fibers are in some cases quite marked; in otliers absent or restricted to a small number of fibers.**

Lesions of the abdominal avity are not infrequent. CErlematons conditions are quite common arouml the kidneys and will be refered to again. Gelatinous ardemas are sometines encountered in the portal

* An interesting appearance, which may now and then deceive observers, is the presence, nuder the endocardium, of minute, whitish, fusiform bodies, perhapes one millimeter long, which seem to follow tho rourse of the smperficial veins and rest upon or near their walls. 'They are sareosporidia (psolosprermia, eysts lilled with falciform hodies. These eysts are likew ise present in tho rejoths of the lieart musclo and in the skeletal muscles. Vnder the endocarlium their mumbrr is greatest in old cows. They are in some cases so numerons that fifty may be included in an area of a square centimeter. They are casily removed rintire by rareful teasing. 
region between duodenum and liver. The omentum firequently dis. plays peculiar hyperemic patches consisting of delicate shreds of vascular tissue made visible to the naked eye by the injected condition of the blood-vessels.

Spleen.-That this organ in Texas fever is very much enlarged was commented upon by the earlier observers, and the name "splenic fever" took its origin from this condition. Gamgee, in 1868, caused to be weighed the spleens of a large number of native Western cattle, of Cherokee cattle (supposed to have come from the Indian Territory) and of Texan cattle. These animals were considered healthy and fit for human consumption.

The average weight of the native Western spleen was 1.45 pounds; of the Cherokee spleen, 1.94 pounds; of the Texan spleen, 2.5 pounds.

A number of spleens were weighed at one of the Washington abattoirs to determine the normal weight. The result is given in the following table:

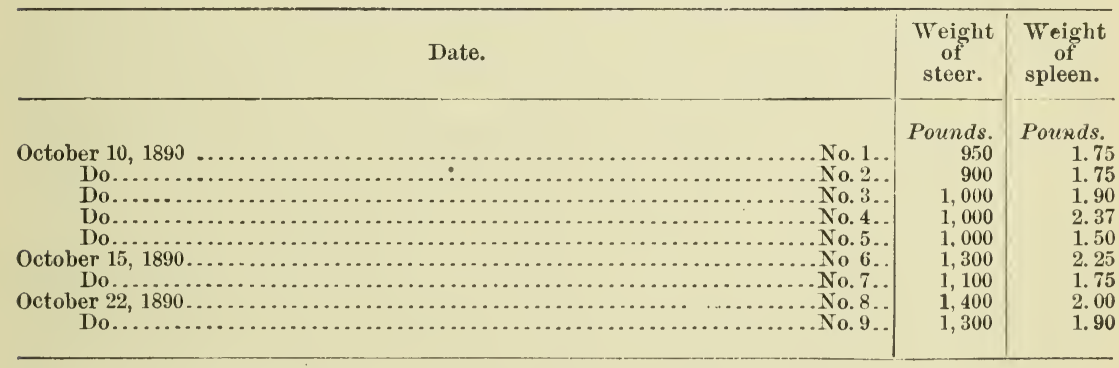

The source of these animals was not determinable. It will be noted that the weight varies considerably, although in all the appearance of the organ, both as to its capsule and pulp, was the same. The weight of each animal was estimated by the butcher in charge. 'These examinations were made at a time when any destructive influences of the summer on the blood may not have been entirely neutralized. How far the bleeding of the animals at the time of slaughter may lave affected the weight of the spleen in comparison with that of cattle which succumbed to the fever with the blood in their system it is of course impossible to estimate. By taking the average of the above nine cases, the weight of the spleen of a steer weighing 1,000 pounds would be 1.72 pounds.

The weight of the spleen in Texas fever varies considerably, according to the stage of the disease in which the animal succumbs. Animals which die after some days of high fever have usually the largest spleens, although this is not an invariable rule. If we compare the weights of spleens as given in the appendix under many of the cases it will be seen that in acute cases the spleen is generally from two to four times its weight in health.

If we turn for a moment to examine its appearance we find its general shape unaltered, but the ordinarily rather thick whitish capsule very much distended and attenuated so that the dark pulp shows 
throngh it very distinctly. (Plate I.) The veins of the capsule are distinct, the minute vessels markedly injected and occasionally accompanied by extravasations of blood. The organ is firm to the touch owing to its distended condition. When it is incised, the pulp appears as a dark brownish-red, glistening, homogeneous mass, which has been compared to "blackberry jam" in its appearance. The usual markings of the parenchyma of the normal spleen are effaced. The grayish Malpighian bodies and the whitish trabeculæ have all disappeared from view within the distended pulp. (The differences between the cut surface of the spleen in health and in Texas fever are well brought out on Plate I.) The pulp may be still firm, or it may be partly diffluent, welling out as a semi-fluid mass from the incised retracting capsule. It has occasionally been reported as ruptured, but this may be a combination of post-mortem softening with carelessness in its extraction. In some cases the spleen may be much heavier than in health, but its markings still visible on section.

A microscopic examination shows that the enlargement and peculiar color of the spleen tissue is due to an engorgement with red blood corpuscles. With this engorgement there may be associated a variable number of large cells containing coarse granules and from two to twelve red corpuscles, or else the remains of these corpuseles in the form of irregular clumps of yellowish pigment. The pigment is also free in masses of variable size. Examination of fresh pulp from spleens of healthy cattle showed that the presence of large quantities of free pigment of the form described is not uncommon.

Of all the organs the liver is the most seriously involved. (See Plates II and III.) The enlargement, congestion, bile-injection, and fatty degeneration were pointed out by R. C. Stiles, in 1868. Gamgee limited himself to matters of weight and evidently did not observe the extensive changes which the parenchyma underwent. In our own observations of healthy and diseased livers, the latter were probably from 3 to 5 pounds heavier than the former. The edges were well rounded off. The color of the surface was usually paler than in normal livers and in most eases of a peculiar mottled appearance. The mottling was due to minute irregular grayish-yellow patches usually 1 millimeter or less in diameter. When incised the parenchyma was remarkably bloodless in most cases, and a lac colored, thick blood poured from the cut ends of the larger hepatic veins. The color of the cut surface was either a uniformly brownish yellow or else mottled as on the surface. (Plate II, Fig. 1.) The mottling, on closer scrutiny with the naked eye or hand lens, was found to be due to a paler-yellowish discoloration of the zone bordering the intralobular veins. (Plate II, Fig. 2.) This zone of discoloration was the wider the more prolonged the disease, and in a few cases involved the entire lobule. Parallel to this degenerative process the consistency of the organ became less resistant, more doughy, and brittle. 
In thin sections of fresh tissue* the most striking phenomenon was the filling up of the ultimate bile canaliculi so that the hepatic cells were inclosed in polygons of yellow lines forming a beantiful network. (Plate III, Figs. 1 and 2.) When the liver is teased and crushed the contents of these bile canaliculi may be found floating free in the form of rods, sometimes with $\mathrm{Y}$.shaped ends. (Plate III, Fig. 3.) This stasis or filling up of the ultimate bile capillaries was present in nearly all animals examined. It was most pronounced in those whose death followed quickly after a high fever. In one case purposely killed in the early clays of the fever the liver was the seat of marked congestion, the bile-stasis not having taken place yet. The extent of this stasis varies considerably. It may be seen in small isolated areas or else it may involve a large continuous territory. Owing to absence of connective tissue between the lobules it is quite impossible in fresh sections to make out accurately its distribution. It seems to be most frequently met with in the innermost or hepatic zone of the lobule (Plate IIr, Fig. 1; Plate II, Fig. 4), but it may also be found involving the entire lobule. Small bile ducts between the lobules are often found injected, and rarely lines of yellow injection may be visible to the unaided eye.

Associated with the occlusion of the biliary canaliculi and ducts is a more or less extensive fatty degeneration of the hepatic cells. This is most advanced in prolonged cases of disease. In several which came under our observation the fatty changes were so extensive that cells free from large quantities of fat could not be seen. Among other abnormal appearances may be mentioned the presence of irregular yellow clumps of pigment in the hepatic cells, and of stellate masses or bloodred needle-like erystals (Plate III, Fig. 2) of very minute size (hæmoglobin?). In one case (No.139) large branched thrombi were found in some of the hepatic veins.

The pathological changes observed in sections and teased preparations of fresh liver tissue are more accurately interpreted in sections of tissue hardened in Miiller's fluid and in alcohol. The material was imbedded in paraffin after having been passed through chloroform par. affin. Sections cut in this way were far more serviceable than thoses cut in alcohol. The injection of the bile canaliculi is seen only in Müller's fluid preparations or in alcoholic material cut directly without imbedding. The extent and location of the injection are variable. It may appear over an entire lobule or only a small portion of it. The fatty degeneration so regularly seen in fresh material shows itself in sections of hardened material in a peculiar vacuolated appearance of the cell protoplasm, the fat having been dissolved out. Tbe vacuolation may be more pronounced near the center of the lobule, where the individual vacuoles may be as large as red corpuscles. Of these there may be

* These were usually made with a razor and examined in iodized serum. The freezing microtome was not generally used because it was desirable in this examination to preserve the red corpuscles. 
several in a single cell, very little of the protoplasm remaining. The cell protoplasm of the peripheral zone of the lobule is uniformty vacuolated, the vacuoles being very small.

Another change that is of considerable importance in estimating the pathological effect of the disease is a tendency toward necrosis of the inner zone of the lobule. This process, which shows itself to the naked eye as a faint paler mottling of the liver tissue limited to the inmer zone of the acini, seens to begin around the central vein and extend toward the periphery.* It is characterized by a degeneration and loss of the nuclei of the parenchyma rells. These changes are observable with various stains, such as hamatoxylin, alum carmine, and the anilines (alkaline methylene blue, aniline water-methyl violet, etc.). Ehrlich's acid hrematoxylin, with or without eosin, is a very satisfactory dye, owing to the intensity of the nuclear staining. The changes undergone by the nuclei are at first manifested by a feebler stain. The margin, which may be irregular, is stainerl, but the body of the nucleus is pale and usually contains several deeply stained round bodies simulating nucleoli. Later on these bodies are all that is left. They shrink together or even unite into a small irregular deeply stained mass. The cell protoplasm is much more feebly stained than in normal areas and its outlines are indistinct. This nuclear degeneration may appear in a compact area uniformly or we may find all grades of degeneration intermingled. $\dagger$ In tissue undergoing such changes the central portions of each lobule may appear much paler than the peripheral. The trabecular arrangement of the cells may be exaggerated by a widening of the lumen of the capillaries in the periphery and made indistinct or become obliterated in the central portions. The destructive changes in central regious may go on to a complete loss of the nuclei. This appears very well in methylene-blue stains. The necrotic portion refuses to stain at all and the result is a mottled section with the isolated unstained areas inclosed in an irregular network of stained material very striking even to the naked eye. Such mottling will, of course, appear with other stains, but not so distinctly. The extent of the necrosis may be as much as one-third or one-half of the entire volume of the lobule.f

In endeavoring to account for the fatty and necrotic changes of the parenchyma we think it probable that the bile stasis, by plugging up with solid bile the ultimate bile canals, may interfere in some way with

* One may be at a loss to determine the limits of the lobules in the ox, owing to the absence of any complete connective tissue boundary. In stained sections they are readily made out by taking as a guide the connoctive tissue with its numerous stained nuclei in the spaces in which the interlobular vessels and duets pass.

†.J. H. Detmers (5, p. 137) observerl in 1883 the disappearance of the nuclei and the reticulated appearance of the cell protoplasm. Babes, in 1889, described a similar condition in Rommanian cattle affected with infections hemoghohinuria. (See page 140.)

$\ddagger$ See the appendix under Nos. 3, 6, 9, 47, 69, 71, 95, 106, 112, 139, and 198. 
the nutrition of the parenchyma or exereise upon it some deleterious influence through the stagnating bile and thus set the degenerative processes in motion. The bile stasis is undoubtedly due to the breaking up in the eapillaries of the liver of immense numbers of infected corpuscles. A large amount of débris is thus brought to the cells for transformation into bile. The result is an abuormal fluid containing a superabundance of solids (pigment) which is unable to flow in the bile channels. How far the degenerative process may be aided by any plugging of the capillaries with infected corpuscles it is impossible to state. In fact, the relation of the disintegration of the red corpuseles and of the bile stasis to the fatty degeneration and the necrosis around the central vein should be made the object of special pathological study.

Bile is found in the gall bladder in considerable quantity (one-half pint to a quart) after death. As might be anticipated from the deseription of the changes in the liver, this fluirl is greatly altered. The usual limpid greenish fluid is replaceỏ by an almost semi-solid mass. As it flow's from the incised bladder it has been aptly compared to chewed grass. The presence of mucus makes it cohesive enough to be drawn out into long flat bands as it flows. When it is allowed to stand quietly in a cylindrical vessel a layer of tlakes settles down which occupies not infrequently one-half of the entire columm. The supernatant fluid is much darker than normal bile. The suspended matter appears to be marle up chiefly of small yellowish floceuli or Hakes. A deep yellow tinge is imparted to all vessels aud to the hands coming in contact with it. When examined under the microscope, the suspended particles are resolved into amorphous yellowish masses mingled with bright golden points barely visible at 500 diameters. The common bile duct has always been found pervious and in many cases an abundance of bile is found in the small intestine.

The kidneys.--We have in a preceding chapter referred to the condition of the mine in this disease and have found it altered by the presence of certain abnormal products, hamoglobin and albumin. We might therefore anticipate more or less alteration in the secreting organ, the kidneys. In a considerable number of cases a sero-sanguinolent condition of the connective tissue and fat about the kidneys is observed. In a few cases the ventral surface of the organs appeared like two large blood blotches. The portion of the abdominal wall upon which the dorsal surface of the kidneys rest is free from these effusions.

The kidneys themselves, like the other organs affected by this disease, vary more or less in color, according to the severity and stage of the disease. In those cases which suceumb early in the fever and in which the bladder is filled with port-wine colored urine, the kidneys are enlarged and of a uniform dark brownish-red color tinronghout. The usual markings are pretty well effacer. When fresh sections are examined from different regions, the vascular system is found quite 
uniformly engorged and distended with red corpuscles. The section is likewise sprinkled over with very minute pigment particles. Sometimes irregular masses of red corpuscles, run together as it were, are met with in the vessels of the pyramids. I Lesions of the secreting structures are not discoverable. Hemorrhages are uncommon. In those cases which succumb after the hæmoglobinuria and the fever have passed away, the kidneys are paler than usual and the texture is quite flabby. Sections of the fresh tissue show in the cortex a considerable amount of pigment. In some cases the convoluted tubules are the elected seat of pigment deposit, and the epithelium of these tubes may be so filled with yellowish red pigment that they are easily traceable in their windings by their decided color. Fatty changes are occasionally met with in the epithelium, and the straight tubules of the pyramids may be filled with fat globules. Degenerative or necrotic changes of the epithelium were not noticed in sections of hardened tissue from a few cases stained in various ways. In those cases in which the capillaries were filled with red corpuscles, the latter were usually all infected with Texas fever parasites.

The pelvis and its ramifications were usually found beset with blood extravasations. It has already been remarked under the head of symptoms that in most cases the bladder is found containing from one to four quarts of urine holding more or less hæmoglobin in solution. Under the same head will be found a full discussion of this phenomenon so that it need not be touched upon here. The bladder itself may show a few ecchymoses on its inner surface.

Digestive organs.-The upper portion of the digestive tract, including the paunch and reticulum, is generally free from morbid changes. The third stomach or manyplies was, in a few cases, somewhat "impacted;" that is, the contents were firm and rather dry and the superficial layer of epithelium of the lining membrane tended to peel off. In most cases it was normal. The fourth or true stomach (abomasum) shows not infrequently a hyperæmic condition. In some cases the laminated portion was of a uniformly bluish-pink color. Both Gamgee and the Metropolitan Board of Health of New York City have laid much stress upon the lesions observed in this organ. Gamgeo describes in addition to the general hyperiemia three kinds of lesions of the laminated, cardiac portion. He finds in sone cases petechiæ, "resembling flea-bites" in some respects, whose "center is dark and sometimes softened and perforated." In others "the reddened folds are studded with miuute yellowish-gray granulations due to a change in the epithelium, which becomes swollen and has a tendency to drop off. Each granulation does not usually exceed the size of a pin's hearl. This appearance is most marked where the folds are most congested and in some cases where the congestion is slight it requires a somewhat careful inspection to recognize the presence of this change." The third lesion is described as follows : "Scattered throughout the folds, 
especially near their free edges, we find * * * marked erosions, as if the epithelium had been peeled off with a shary linger-nail."

The lesion described as the second was also present in many of the cases recorded in the appendix. Its constant appearance was very puzzling and might readily lead one to suspect some relation to the disease. Many of the granulations had their center perforated so that they suggested the presence of enlarged glands with hyperplasia of the tissue surrounding the mouth. It was not until the fall of 1890 that, the nature of these little elevations was solved. In an animal killed for some purpose, though free from the disease, marked lesions of the mucous membrane of the fourth stomach were found. These consisted of yellowish-white exudations about as large as split peas, viscid and composed of round cells mixed with mucus and associated with the mouth of these elevated spots. A careful microscopic examination of this exudate showed the presence of a very minute nematode, a strongyle, imbedded in the exudate. That this was the cause of these lesions was soon determined. Some sections of the fourth stomach of a case of Texas fever in which these lesions were present had been prepared sometime ago, but had not been studied, for want of time. These were now examined, and in the minute pits corresponding to the perforation in the center of these granulations the worm was seen coiled up at the base of the epithelial layer. It was also recognized as the worm found and described a few months before by Ostertag in cattle slaughtered in Berlin, Prussia. The worm described by him was larger, but the fact that it produced the same lesions made it highly probable that the two strongyli are of the same species.* This disposes of the second lesion seen by Gamgee. As regards the first, it is not unlikely that it represents the earliest stage of the invasion of the mucous membrane by the worm, but we will not be dogmatic on this point.

As regards the erosions, it may be said that in a small proportion of our animals, irregular, very shallow, flattish excavations of the mucous membrane were found which harl a blackish base. They varied much in size, some being quite small. They were most numerous on the laminæ. Some were occasionally encountered in the pyloric portion. After finding these same erosions even quite abundantly in some healthy stomachs from an abattoir we interpreter them simply as traumatic erosions due to the accidental presence of some foreign body.

In the investigations of the Metropolitan Board in 1868, the pyloric portion of the fourth stomach was found in many cases to contain deep, ragged excavations with hemorrhagic base. It is not improbable that at least some of these may have been the result of vascular occlusion, since in the animals examined at that time there seems to have been so far as the descriptions and illustrations go, much more congestion

\footnotetext{
*Ostertag named the worm Strongylus convolutus, but this was changed by Dr. C. W. Stiles, of this Division, who gave some attention to the worm subsequently, to Strongylus Ostertagi. Jonrn. Comp. Med., 1892, p. 147.
}

$10320-$ No. $1-3$ 
of the fourth stomach and intestines than in our own cases. These aosions were extensive in but one case (No. 198) of ours. Their constancy led Morean Morris in his report to the Board to consider them as a more certain indication of Texas fever than the other lesions commonly present. Witin this we can not agree. In fact we regard the digestive lesions as perhaps the least pathognomonic of the disease.

In a few cases affected with a more or less chronic after-disease, there was much redema of the coats of the fourth stomach, extending also to the mesentery.

The lesions of the intestines are limited to hyperæmia and pigmentation. Beginning with the duodenum, there is found generally an abundance of bile and more or less injection and pigmentation of the villi appearing in the form of closely set points and fine lines. The remainder of the small intestine may show with the stomach more or less marked congestion, or there may be patches marked by the injection of minute vessels. In many of the cases examined the mucosa was pale and concealed by a thin layer of a grayish pasty consistency made up largely of desquamated epithelium. The walls of the lower half of the small intestine contained quite invariably small worm tubercles. These appeared from the serous surface as dark bluish, slightly elevated nodules. In passing the opened intestine between the fingers the mucosa was found intact while the tubercles gave one the sensation of small shot in the walls. They harbor a parasitic worm and have nothing to do with the disease.

In the large intestine we find more or less hyperæmia and pigmentation in longitudinal lines corresponding to the summits of the folds of the mucous membrane. This condition is more marked in the cxcum and rectum than in the colon and seems to be associated with the constipated condition. Thus the cæcum is in some cases distended with very hard, dry, fecal balls and some may be found in the rectum. In some cases no abnormal condition of the large bowel is discoverable.

Differences in the pathological changes of our cases and those studied by former observers.-We have already called attention to the fact that, while jaundice was rare in our cases, it has been rather common according to other investigators. In fact it has been named "the yellow fever of cattle" on the strength of this symptom. Attention has also been called to the lesions of the fourth stomach in this respect. The causes for these differences may perhaps be looked for in the different condition of the animals examined. The Metropolitan Board in 1868 examined cattle which had been traveling and had undergone much hardship both by rail and on foot. They were all western animals which succumbed soon after their arrival in New York. How far the deprivation of food and water, the crowding, the constant motion, and the marching may have contributed to a more active circulation and to an absorption of the obstructed bile from the liver into the blood must remain a conjecture. In our cases the animals were simply 
pastured and the frequent blood exmininations as well as the taking of the temperature were carried out with the least possible disturbance to the animals. Again the animals used by us weighed between 500 and 800 pounds. They were not more than average animals in an average condition of flesh. It may be that the large fat animals in a plethoric condition would develop the peculiar condition of the muscular system, the jaundice and the more marked hyperæmia (and sloughing?) of the fourth stomach and intestines observed in 1868. The essential lesions, however, are precisely the same. The disease first studied by Gamgee and the Metropolitan Board of Health in 1868 is the same as that now occupying our attention. The changes going on in the blood, the liver, spleen, and kidneys are so striking and peculiar that they could not very well belong to two different maladies.

\section{CHANGES IN THE CORPUSCULAR ELEMENTS OF THE BLOOD.}

The condition of the blood, so far as determinable by the naked eye, has already been referred to. It grows very thin and watery as the disease progresses. This fact was emphasized by the earliest students of this disease, the investigators of the Metropolitan Board in 1868. Its prime significance seems to have escaped them and subsequent ones. In the preliminary pathological examination of four cases in 1888 (Nos. 3 to 6 inclusive) the destruction of red corpuscles explained best of all the conditions observed. Hence the importance of concentrating the attention on the blood and its cellular elements was at once recognized. In 1889 arrangements were made by which cases of the disease could be studied during life at the experiment station, and within easy reach of the laboratory, in the District of Columbia. In order to measure in some accurate manner the changes going on in the blood, the red corpuscles were counted as soon as living cases were accessible. The result proved surprising in the extreme. It was found that there is a destruction of red corpuseles going on from day to day quite enormous in acute cases. Going parallel with this diminution in the number of corpuscles a change in their size and appearance became manifest which demanded a careful study in order that a distinction between the stages of the intraglobular parasite and the altered corpuscles which might be confounded with them could be made. As the investigations proceeded an accurate knowledge of these changes proved very valuable as a means of diagnosis. In a number of cases the recent existence of Texas fever could be at once determined by their presence, even though the Texas fever parasite was no longer to be detected in the blood. These changes must now be considered as next in importance to the parasite itself in the diagnosis of Texas fever in all its forms. The present chapter is therefore a consideration of the changes, both quantitative and qualitative, affecting the red corpuscles without reference to the micro-parasite accompanying them. This will be described in another chapter. 
THE DESTRUCTION OF REI BLOOI) CORPISCIES.

The red corpuscles were comted with the aplaratus of Thoma, constructed by Zeiss. In the direction for use accompanying the apparatus it is suggested that 200 spaces should be connted in orler to reduce the errors to a minimum. Owing to the large quantity of work that had to be done in connection with the various field experiments to be described, the counting could not be carried to the point of accuracy indicated. Moreover, the quantitative changes in this disease are so gross that a slight error will not affect the comparative results. The method adopted was to count 40 spaces. Two parallel rows of squares through the ruled field were counted. Such rows were chosen throngh which an addi. tional line was drawn in order to guide easily the eyes. Hence these rows were always four squares apart. By counting the red corpuscles in a row of squares any differences in their distribution from one side of the cell to the other were thus averaged. A comparison of the results of counts in the case of healthy controls, or of animals before the onset of the disease, shows a remarkable uniformity of results. Moreover, successive counts either from the same dilution or from separate dilutions of blood from the same animal collected at the same time, showed that the greatest margin of error was one to two hundred thousand, a comparatively insignificant figure in the work before us.

It was necessary also to make a modification in the collection of blood. The uneasiness of many animals, the presence of flies, the heat and wind on the fields made it neressary to act with great rapidity. Hence the complete filling of the capillary tube was dispensed withOnly a fraction of the length was filled with blool, usually from 0.6 to 0.9. The quantity aspirated was at once noted and the 3 per cent salt solution or 'Toison's fluid was drawn up to the mark indicated. In those cases in which the blood was very thin and the various squares contained only from 0 to 3 corpuscles abont 80 squares were counted.

If, in the collection of the blood, the dilution with the salt solution or Toison's fluid, its proper mixing with the blood, and especially the placing of the drop in the cell, be properly carried out the necessity for counting a large number of squares is made nugatory. Special care should be devoted to the cleaning of the glass cell and cover and the keeping away of all dust. When the coverslip has once been laid on the cell it should not be slid or moved about, so that the miformity of distribution is not disturbed. 'The process of collecting the blood for' counting is as follows:

In most cases the animals could not be removed from the field and the examination of the blood had to be proceeded with on the field itself. The various appliances necessary for the securing of fresh and dried preparations of blood and the counting of the blood corpuscles, were carried in boxes or trays. The animal was secured by its head and, in rare cases, one hind foot was tied, to forestall any injury to the 
one collecting the blood, whose entire attention had to be given to this work. If desiced, a rectangular box or stall may be placed in each field into which the animal may be led and secured. Or such a box may be placed under cover and then rainy weather will not interfere with the work.*

In collecting the blood the hair is clipped and shaved away over an area 2 or 3 inches square on that region of the rump overlyiug the Haring hip bones (ilium), where the animal is most accessible for this work. The shaven skin is washed and rinsed with clear water and dried with absorbent cotton. To make the incision a spring lancet is used resembling those advertised and figured in most catalogues of medical and veterinary instrument makers. The incision must pass through the depth of the skin in order that a sufficient flow of blood be secured. The depth to which the blade of the lancet penetrates may be regulated by a screw in the forked guard attached to the lancet. The lancet should be tlamed in passing from one animal to another. The soap and razor should not be used on sick and healthy alike, for, although we have no positive evidence that the disease may be transmitted, either by these things or even by the lancet, such transmission is within the range of possibility. $\dagger$

*Such a box is best constructed as follows: Place three pieces of 2 by 4 studding, $4 \frac{1}{2}$ feet long, on the floor parallel to each other and 3 feet apart. Erect uprights also of ' 2 by 4 studding, and 3 feet high, 15 inches from the ends of each horizontal piece, and brace securely from the outside. Within this framework build, by boarding up on the inside, a rectangular box 6 feet long, 3 feet high, and 2 feet wide, open at the top and one end. The frout closed end of the box is loollowed out to a depth of 7 to 8 inches to receive the neck of the animal in the standing position, so that the head may extend over the end and be secured to a framework extending $1 \frac{1}{2}$ feet beyond the box and attached to the box 2 feet from the floor.

The whole framework must be very securely put together. The projection of the studding at the base with the loraces on the outside serves to strengthen the box and to prevent its npsetting by the strnggling of the animal. A bar can be slipperl in behind the animal to keep it from backing out, and a rope or strap over the withers fastened to the sides of the box will prevent the animal rearing forward.

t We give a specimen page of the figures obtained from counting the blood corpuseles as deseribed above.

No. 218 (healthy control.)-August 6, 1892, 10 a. m.: Temperature, 101.8; repiratiou, 64 ; pulse, 56; blood collected, 7.3 divisions.

('Toison's fluid was user in this estimation. It consists of distilled water $160 \mathrm{ec.}$, neutral glycerine $30 \mathrm{ec}$. (at $30^{\circ}$ ), sodium sulphate 8 grams, sodium chloride 1 gram, methyl violet .025 gram. It staius the white corpuscles so that both red and white may be conited in the same preparation.)

First row of squares:

\begin{tabular}{rrrr}
8 & 12 & 9 & 13 \\
13 & 11 & 10 & 14 \\
12 & 13 & 13 & 10 \\
14 & 19 & 8 & 13 \\
12 & 7 & 10 & 10 \\
\hline $61+62+50+60$ & $=233$
\end{tabular}$\quad \frac{463 \times 100 \times 4000 \times 10}{40 \times 7.3}=6,342,465$ red corpuscles.


The number of red corpuscles in cattle (obtained from the counties around the I) istrict of Columbia) during heaith fluctuates more or less, as might be expected, but may be put down as six millions in a cubic millimeter. Seven millions in winter, and five millions in late summer and early autumn seems to be not uncommon. The number may be said to fluctuate, however, between four and one-half and eight millious, since these extremes are occasionally met with. The following counts from healthy animals will serve as illustrations:

No. 109.

September $18,1890 \ldots \ldots \ldots .5,726,000$

October 3, $1890 \ldots \ldots \ldots . . .6,190,000$

October $14,1890 \ldots \ldots \ldots \ldots .5,807,000$
No. 91 . October 1, $1890 \ldots 4,672,000\left\{\begin{array}{l}\text { Placed in } \\ \text { infected } \\ \text { field. }\end{array}\right.$

October 7, $1890 \ldots 4,833,000$

October 30,1890 . . 4, 670,000

No. 143 (control animal).

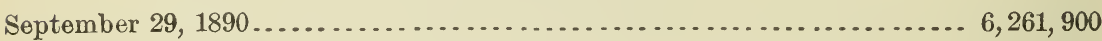

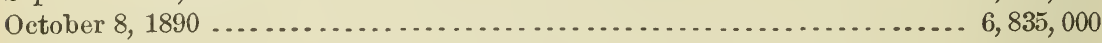

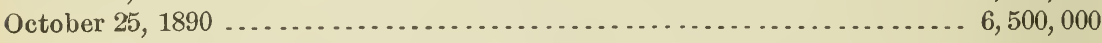

In addition to these illustrations there may be found in the appendix a large number of figures relating to the number of red blood corpuscles of cattle in infected fields, but not yet diseased. The examination of the blood in 1891 was extended to many, and in 1892 to all, animals at the beginning of experiments, in order to get at the approximate normal for each animal, and also to make sure that the animals were in good health. Among the many cases which came under observation only one anæmic cow was found; that is, only one whose red corpuscles fell below four and a half millions. This animal (No. 136) was affected with some catarrhal discharge from the vagina. Her record was:

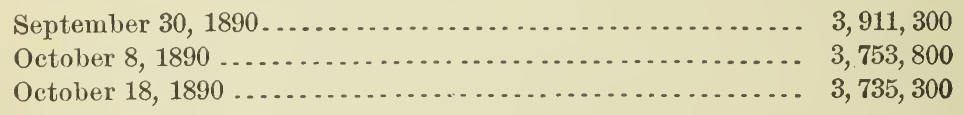

Second row:

$\begin{array}{rrrr}8 & 14 & 7 & 13 \\ 11 & 9 & 10 & 8 \\ 15 & 13 & 13 & 13 \\ 8 & 8 & 15 & 17 \\ 12 & 13 & 9 & 14\end{array}$

$54+57+54+65=\frac{230}{463}$

Seveu white corpuseles in 400 squares.

$\frac{7 \times 100 \times 4000 \times 10}{400 \times 7.3}=9,589$ white corpuscles.

In counting 40 squares the varions factors in the fraction above balance each other in such a manner that it is only necessary to divide the number of corpuscles (463) by the quantity of blood collected (7.3 divisions of melangeur instead of 10 , the quantity usually collected). The first figure of the quotient gives millions. A similar simplification of the formula for the white corpuscles may bo used. 
In one case (No.51) tuberculosis, limited ehiefly to the lymphatics, was discovered at the autopsy. Even in this animal, after three days of high temperature from Texas fever, the corpuscles still numbered $5,125,000$. There could have been no ancmia, therefore, in spite of the tuberculosis.

The destruction of red corpuscles is the essential phenomenon of Texas fever from which all the various pathological processes take their origin. A glance at the figures in the appendix will show that this is a constant occurrence and present in every case in proportion to the acuteness and severity of the attack. Some illustrations will demonstrate these statements.

No. 80 .

[July 5, 1890.-Beginning of exposure in infected field (Texas cattle).]

\begin{tabular}{|c|c|c|}
\hline Date. & $\begin{array}{l}\text { Number of } \\
\text { corpuscles. }\end{array} \mid$ & Remarks. \\
\hline 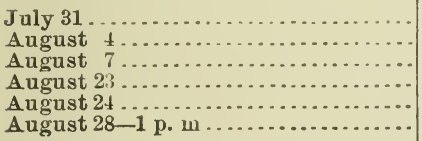 & $\begin{array}{l}6,290,000 \\
5,052,000 \\
5,631,000 \\
5,422,000 \\
5,434,000 \\
2,025,000\end{array}$ & $\begin{array}{l}\text { First high morning temperature on Aug. } 24 . \\
\text { To all appearances in dying condition; killed. }\end{array}$ \\
\hline
\end{tabular}

No. 129.

[July 5, 1890.-Beginning of exposure in infected field (Texas cattle.)]

\begin{tabular}{|c|c|c|}
\hline 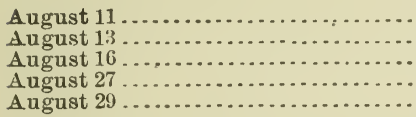 & $\begin{array}{l}6,123,000 \\
7,171,000 \\
5,370,000 \\
3,210,000 \\
1,675,000\end{array}$ & $\begin{array}{l}\text { First high morning temperature A } 1 \text { g. } 24 \text {. } \\
\text { Died at } 8 \text { p. m. }\end{array}$ \\
\hline
\end{tabular}

No. 163.

[July 2, 1891.-Beginning of exposure in infected field (North Carolina cattle).]

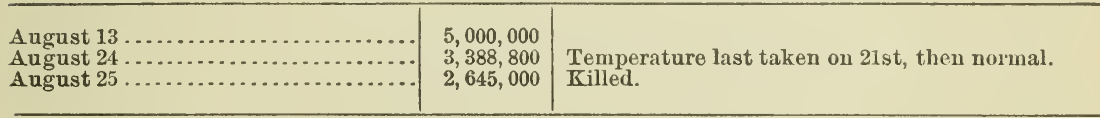

These few examples will suffice to illustrate the rapid disappearance of red corpuscles from the circulating blood. They are by no means extreme cases, but stand for the average rate of disappearance in acute cases. This would be for No. 80 at the rate of about 1,000,000 corpuscles per cubic millimeter a day during the last three days; for No. 129 at the rate of 800,000 , and for No. $163,700,000$. That this rate of destruction is very high becomes evident when we bear in mind that in No. 80 it represents the loss in twenty-four hours of one-sixth of all the red corpuscles usually circulating in the body. In the other cases it represents from one-seventh to one-eighth of the whole number.

In the mild non-fatal type the rate of lestruction is lower. 
No. 56 .

[September 8, 1890.-Beginning of exposure in infected field (North Carolina cattle).]

\begin{tabular}{|c|c|c|}
\hline Date. & $\begin{array}{l}\text { Number of } \\
\text { corpuscles. }\end{array}$ & Remarks. \\
\hline 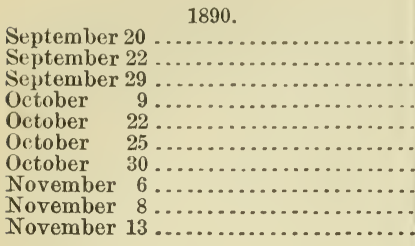 & $\begin{array}{l}6,844,000 \\
5,640,000 \\
5,307,000 \\
5,436,000 \\
4,666,000 \\
2,754,000 \\
2,720,000 \\
2,344,000 \\
1,984,000 \\
1,183,000\end{array}$ & 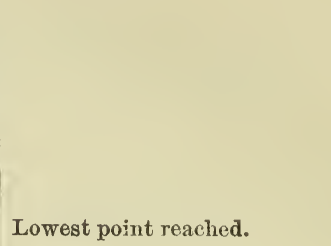 \\
\hline
\end{tabular}

In this animal, the loss which in an acute case would have taken place in four or five days occupied from seven to eight weeks. In these cases, however, other elements enter, such as the constant active production of new corpuscles which masks to a great degree the actual rate of disappearance. Moreover, the destruction seems to go on not regularly but in jumps or paroxysms. Thus in the case before us there was a decrease of 1,912,000 from October 22 to October 25, but practically a standstill from October 25 to October 30 , and so on.

Another fact of considerable interest brought out by the periodic estimates of the red corpuscles is the oscillation of the number up and down during the disease in some cases. It seems as if a period of destruction were followed by a period of regeneration, and this again by a period of destruction. This oscillation is occasionally traceable to the reappearance of the inicro-parasite in the blood, as in No. 160, in which three different downward movements in the number of red corpuscles are associated with the reappearance of infected corpuseles. In other cases the microscope did not, during the downward movement, demonstrate the presence of the parasite, probably because such observations were often one or two weeks apart. The supposition at the time was that such cases were getting well, and the tardy examination of the blood showed instead of the expected return to the normal another downward movement. Very good illustrations of this oscillation are afforded by Nos. 111 and 142, both the result of the intravenous injection of blood from sick natives. In No. 142 the figures were as follows:

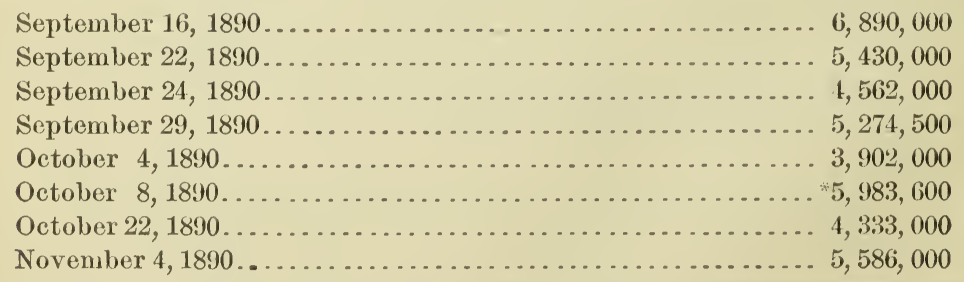

*This number is evidently too high as compared with the preceding, and must bo explained by assuning other forces at work in concentrating the blood beside the mere regeneration. 
It has been assumed above that the disappearance of the red corpuscles is chiefly due to their destruction. We have already seen that in the cases under observation there were very few hemorihagic lesions which might for the time being reduce the number. The ticks can not be regarded at all as abstractors of blood in this stage.* That they are largely destroyed within the body is shown (1) by the loss of hemoglobin through the kidneys, (2) by the overproduction of bile which is abnormal in the abundanes of pigment flakes, and (3) by the actual observation of this destruction by the micro-parasite under the microscope.

THE REGENERATION OF RED BLOOD CORPUSClES.

As determined by actual enumeration.-Passing by, for the present, any further discussion of this interesting subject, let us turn to the regeneration of the red corpuscles. This, of course, varies in accordance with the vigor of the animal, its food, and the season of the year. It is, even under adverse circumstances, remarkably rapid and well adapted to occasion surprise.

The regeneration of corpuscles as indicated by the microscope is not in all cases indicated by the counting apparatus. That is to say, the regeneration may begin before the destruction has ceased, and if the latter process is the more active the count will show a loss, although the microscope may demonstrate the presence of a large number of new corpuscles. This actual regeneration, as indicated by abnormal forms, will be discussed farther on; here we will simply refer to the increase of the corpuscles as indicated by actual counting. A few illustrations will serve our purpose:

No. 64 .

September $9,1890 \ldots \ldots \ldots . . .3,154,000$

September 16, $1890 \ldots \ldots \ldots .4,575,000$

September $29,1890 \ldots \ldots . . .4,869,000$

$$
\text { No. } 102 .
$$

September 18, $1890 \ldots \ldots \ldots . . .1,950,000$ October $4,1890 \ldots \ldots \ldots \ldots . .2,682,700$ October 17, $1890 \ldots \ldots \ldots \ldots . . .3,894,700$ November $6,1890 \ldots \ldots \ldots . .5,120,000$

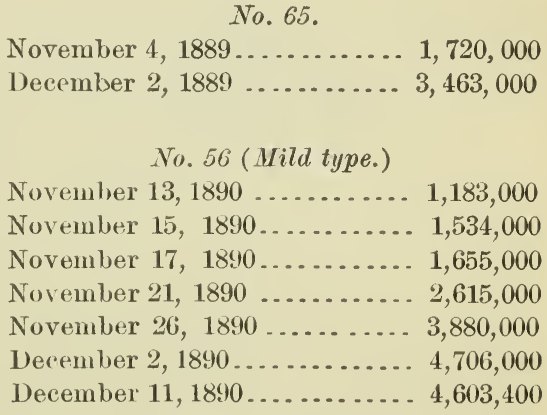

* The enlarged spleen, it is true, absorbs from $1 \frac{1}{2}$ to 3 pounds of red corpuscles roughly speaking, since its enlargement is mainly due to an engorgement with them. If we regard the red corpuscles as constituting one-third of the weight of the blood, this quantity would correspond to $4 \frac{1}{2}$ to 9 pounds of blood. If we take the blood in cattle as one-thirteenth of the borly weight ( $\mathrm{v}$. Limbeck, Klinische Pathologie des Blutes. S. 49) an animal weighing 800 pounds would carry 61.5 pounds of blood. The spleen would thus absorb the corpuscles of one-fifteenth to oneseventh of the entire blood and reduce the number of corpuscles in a cmm. onethird to one million. The capillary engorgement of the kidney, heart muscle, and perhaps other organs may account for some losses, but this is mainly due to infected corpuscles which may be regarded as destroyed. The above calculation is, of course, very approximate. 
The activity of the regeneration is well brought out in No. 56, a large, vigorous ox. From November 17 to November 26 the red corpuseles appeared in the circulation at the rate of 250,000 per cubic nillimeter per day. From November 26 to December 2 the rate of increase was about 140,000 a day. It is furthermore remarkable that in the case of a few calves under observation the corpuscles rose rapidly in number, although the animals did not thrive after the fever departed. This was likewise observed in some adults. The blood forming function seems to go ou independently of downward processes of other functions.

No.82.

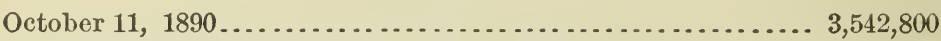

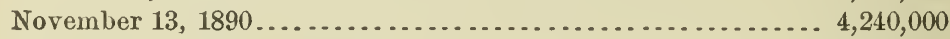

December 2, $1890 \ldots \ldots \ldots \ldots \ldots \ldots \ldots \ldots \ldots \ldots \ldots \ldots \ldots \ldots . . .5,643,000$

In this calf, the number of corpuscles steadily rose after the disease had passed away, in spite of growing weakness and diarrhea. On December 4 it was unable to get on its feet, so that it had to be killed December 6. Opposed to these cases in which the blood-forming function asserts itself under difficulties, there are others in which the strain upon this function has been so severe that several phenomena appear. The corpuscles may increase in number but not reach the full tide of the number present before disease until the following season. Or there may be a temporary standstill in the production of corpuscles when the number is still very low. In none of the cases in which the convalescence was followed with the corpuscle counter did the number remain below four millions after the end of three or four months,

As determined by microscopical examination.-The reproduction of new corpuscles as witnessed by microscopical examination presents a number of important phenomena. Taking it for granted for the present that we are able to detect newly formed corpuscles by certain peculiarities of form and staining which they possess during the more advanced stages of anæmia, we may lay down a few general propositions concerning this production. In the acute type of Texas fever, when the daily loss of corpuscles amounts to from one-sixth to oneeighth of the normal number, there is observed little or no production of new corpuscles, until the number has fallen to one million or two millions, and the normal temperature has returned. Then an abundant crop of new forms is seen, even when the animal succumbs in the end. In the mild, non-fatal type, in which the destruction of red corpuscles goes on much more slowly, and in intervals, and in which there is but little fever and general disturbance of health, the production of new corpuscles begins at once and continues parallel with the destruction of older ones throughout the course of the disease. It is in such slowly progressive cases that the changes in the corpuscles accompanying their regeneration is best studied. Before proceeding to a description of these forms, so valuable in the diagnosis of Texas fever, a brief description of the methods employed is called for, since they are the 
same as those used in the important study of the micro-organism or blood-parasite of this disease.

The blood was examined in the fresh and in the dried condition. The drop of blood as it oozed from the incision was received at once on a flamed platinum loop soldered into a glass rod like the ordinary bacteriological loops used for inoculating, etc. The platinum loop is simply brought in contact with the blood, and the drop placed on a clean glass slide and immediately covered with a cover glass and sealed with paraffin if the preparation is to be kept under observation for some time. The sealing is best done with a camel's hair brush dipped into melted paraffin. It is always desirable to have only a single layer of corpuscles in the preparation. To insure this there should be no speck of dust on slide or cover, and the quantity of blood taken must be small. This can be regulated by adjusting the size of the platinum loop. In the usual method of touching the drop of blood with the cover directly the quantity of blood can not be limited, and many preparations are subsequently found to contain too many corpuscles.

The preparation of dried blood requires much care. Without going into an extended discussion of the relative merits of different methods we give the one found most satisfactory and adopted in these investigations. It is most readily understood by referring to the annexed figure:

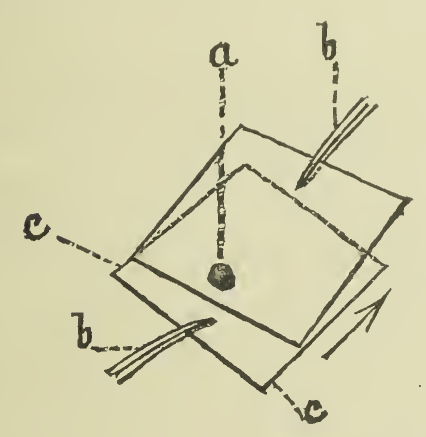

A

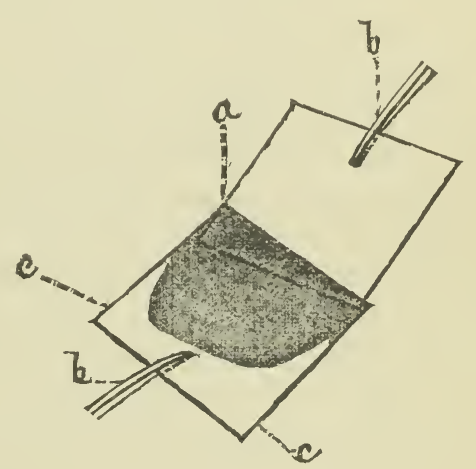

B

Fic. 2.-Method of preparing dry blood films on cover glasses.

In $\mathbf{A}$, a cover glass $c c$ is held by a pair of forceps $b$ and has on its upper surface a drop of blood $a$ placed there by a platinum loop. A second cover glass or "scraper" held by forceps is resting on the first with one edge and is held at an angle of $15^{\circ}$ to $20^{\circ}$ to it. As shown in B the upper cover is drawn over the lower, and as its edge sweeps over it it spreads out the drop of blood in a thin layer. In rapid work the forceps may be dispensed with. The lower cover is held between thumb and forefinger of the left hand at $c c$ and steadied below with the tip of the middle finger, and the upper is firmly seized between thumb and forefinger of the right where the blades of the forceps would rest. 
By using a pair of forceps which may be adjusted by a clamp, the scraper may be fastened between the blades, and by rotating it its edges may serve to spread out three or four separate preparations. The scraper should be thick and its edges smooth in order to insure uniform distribution of the blood. It is needless to say that this simple process is much superior to the barbarous one of placing two cover glasses together face to face in order to allow the blood to spread in a film between them, and then drawing them apart. Each corpuscle is thereby subjected to a long crushing process, whereas in the method before us this is entirely avoided. The thinness of the blood film depends upon several things, such as the condition of the cover as regards freedom from all grease, the size of the drop of blood, the regularity of the edge of the second cover or "scraper," and the angle at which it is held during the operation. The layer is not of even thickness over the entire cover glass, but is thinnest where the scraper has begun its work, and densest where it has left off at the edge of the cover, as shown in the figure. This is no disadvantage, however, but rather an advantage, as it furnishes us with a layer of varying thickness which is of service, as will be pointed out farther on. The place where the scraper began and where the layer is composed of isolated corpuscles or groups of contiguous ones, they have dried so rapidly that they are in a state of perfect preservation. Every preparation has thus some spots where the corpuscles are thoroughly "fixed," even if as a whole it may have been a failure.

The essential condition of success in dry preparations of blood is to get the corpuscles in to a dried state as soon as possible after the blood is shed. For this reason it might seem desirable to eliminate the use of the loop and touch the exuding blood directly with the cover glass, as is frequently done in the study of human blood when the finger tip is pricked. But the circumstances are different in cattle. The prick is useless and an incision must be made. The surface of the skin is that and a cover glass touched to the oozing blood may bring with it epithelial scales and other objectionable things from the skin accidentally touched, however much the latter may have been cleansed beforehand. Still, in rapid work, it is now and then of advantage to touch the oozing blood directly with the edge of the scraper. Not infrequently the quantity of blood is small and does not well out of the incision. A loop then becomes indispensable in lifting it out.*

The dried filns of blood, kept labeled in small pill boxes until used, are exposed in a dry-air oven to a temperature of $110^{\circ}-120^{\circ} \mathrm{C}$. for one and one-half to two hours. Drawing the covers through the Bunsen flame as for bacteriological preparations is liable to fail at any time from overheating or underheating, and is not to be recommended.

* More recent observations during the fall of 1892 have shown quite conclusively that cold rapidly destroys the form of red corpuseles. In fact it was impossible to prepare films out of doors in a temperature below $50^{\circ} \mathrm{F}$. 
When, for rapid work, this method must bo used the cover glass should be drawn through the Hame four times, each movement to occupy a second. Three movements are usually insufficient, for when the stain is applied the coloring matter of the corpuscles is dissolved out and the preparation is spoilt. When overheated the red corpuscles are apt to stain so deeply that any granules or parasites within them are hidden from view.*

The staining process used for the dried and heated cover-glass preparations is very simple. The cover glass is either allowed to float on a filtered solution of Löffler's alkaline methylene blue or else the staining fluid is dropped upon the cover glass and allowed to remain from one and one-half to two minutes. It is thereupon washed in distilled water and dipped into a one-third per cent solution of acetic acid for an instant to remove any diffuse stain in the red corpuscles. Lastly the acid is washed away in distilled water. $t$ It is then ready for examination in water or for drying and mounting in xylol balsam. Care must be taken to make the action of the acetic acid solution momentary, otherwise the decolorization may go too far.

If we examine the blood of a mild autumnal type of fever every one, two, or three days, as described, certain phenomena appear regularly at certain stages of the aniemia. When the number of corpuscles has fallen to three millions a variable number of enlarged corpuscles appear. While the normal ones measure about 5 or $6 \mu$ in diameter, the enlarged forms will be from 6 to $8 \mu$ in diameter. This is the first change observable, and it appears only when, as stated, the number has fallen to onehalf the normal. As the destruction goes on and the number sinks lower, the large cells become more numerous, but they at the same time grow thinner and more delicate. When the number is below two millions, hæmatoblasts or nucleated red corpuscles begin to appear, and their number may be as high as 5 per cent of all corpuscles still in the circulation. It may alsu be noted at this stage that some of the large corpuscles show one or more small vacuoles in a cluster in the center of the corpuscle. These contain sometimes a barely visible $(\times 1000)$ particle in rapid dancing motion. These phenomena are all the result of the anrmia, as will be shown later, and have nothing to do with the micro-parasite. The variation in size of the red corpuscles is illustrated in Plate IV, Fig. 3, Plate V, Fig. 3, and on Plate Ix.

* In place of a hot-air oven kept at the proper temperature by a thermo-regulator the device of Ehrlich may be used. This consists of a Bunsen burner or a small ker osene stove and a strip of sheet copper laid over it. It is evident that at different distances from the source of heat the copper will be of different temperatures. By placing drops of water on it the place where the temperature is $100^{\circ} \mathrm{C}$. can be approximately ascertained by the behavior of the water. The cover glasses are laid upon the sheet of copper for a certain length of time at a point corresponding roughly to $120^{\circ} \mathrm{C}$.

t If the film has been properly heated (not overheated), decolorizing is quite unnecessary. 
When preparations of blood are dried and stained, another set of changes are observed which were hidden in the fresh preparations. These changes are limited to the enlarged corpuscles. When the number falls below 3,000,000, a few corpuscles are now seen among large numbers of others, whose dise is sprinkled over with a variable number of granules which stain deeply in the alkaline methylene blue.* These granules vary in size. In some preparations of blood at this stage, they may be as large as $0.5 \mu$ in diameter, and there may be from 15 to 30 in a corpuscle. A prolonged observation of these granules has suggested the theory that their size depends largely on the rapidity with which the film of blood has been dried. In those portions of the layer which are thinnest and fixed instantly, only small granules are seen; that is, such as are, perhaps, not more than $0.1 \mu$ in diameter. But in those portions of the layer in which the corpuscles are massed two or three deep, the large granules are found, if present at all. The immediate inference is, that the stainable matter diffused through the corpuscle collects into larger nuclei if there is any time elapsing between the sherdling and the drying of the blood. This time is longest in the dense portions of the film. (See Plate IV, Fig. 3, Plate v, Fig. 3, and Plate Ix.) The granules in a cell are not all of the same size, although there is not much variation in this respect in the same corpuscle. There are cells with very fine granules, and cells with very coarse granules. Cells with granules of intermediate size are also found. The large granules are usually round, and resemble very closely micrococci, but the slight irregularity in form and size disposes one to reject at once the view that they may be micrococci. The large granules closely resemble one of the stages of the micro-parasite of Texas fever, as will be pointed out later on. The small granules do not appear round, but more angular, and even slightly rod-shaped. They are distributed quite uniformly over the disc, excepting in a few cases in which there was a central space free from them. These bodies stain, as nuclei and bacteria do, with basic aniline dyes, and they are not readily decolorized with acids. They are stained by hæmatoxylin and refuse to stain with Ehrlich's acid or neutrophile dyes.

The granular forms are characteristic of that stage of the anæmia in which the number of corpuscles stands between two and three millions. When it falls below 2,000,000 other peculiar forms appear. The enlarged corpuscles grow thinner and larger, more easily distorted when the drying is retarded, and, when stained as above indicated, many of them show a diffuse, rather pale bluish coloration not easily removable by acetic acid. (Plato V, Fig. 1; Plate Ix.) Some show instead of this diffuse coloration an aggregation of exceedingly minute granules which might easily give the impression of a diffuse stain. These types are

* It should be borne in mind that under the conditions formulated above the normal red corpuscle does not retain the stain, either in the form of granules or diffusely. 
not infrequently accompanied by hematoblasts. It must not be understood that the different stages of the ancmia are characterized by the exclusive presence of one or the other of these changed forms. The granular or "punctate" cells may be met with in the various stages of the anæmia. Likewise the diffusely stained forms are in a few cases encountered with the punctate forms before the anæmia has become advanced. But as a rule we meet first with the simply enlarged corpuscles, next with the "punctate" forms, and lastly with the diffusely stained or "tinted"* forms and the hæmatoblasts.

It is not desirable to go into any details concerning the nature of these corpuscles, as this has already been done from the standpoint of general pathology in another publication where the literature is also taken into consideration.t A few remarks are, however, in order as bearing upon an understanding of the disease before us. The various modified forms of red corpuscles, which we have been considering, are perhaps all embryonic or immature forms. They have been hastened into the circulation from their place of manufacture, the red marrow of the bones, to supply an urgent demand created by the destruction of vast numbers of red corpuscles by the Texas fever parasite. This demand grows more and more pressing as the number of corpuseles continues to go down, and consequently more and more immature forms are sent until the hæmatoblasts themselves, the progenitors of the red corpuscles, appear. The reasons for considering them embryonic or immature red corpuscles can not be entered into here. It must suffice to state that a comparative study of the embryonic cells in the red marrow and of these modified corpuscles in the circulation shows them to be the same.

The stainable material in these new corpuseles may be some form of protoplasm imperfectly converted into the discoplasm of the adult red corpuscle. We have already presented the theory that the granules may be derived from the diffusely stained material by a condensation in the shed blood. This, of course, will demand special study. It is enticing to interpret, as has been done, the larger granules as fragments of the nucleus of the hæmatoblasts, but there are no observations directly supporting this view. $\ddagger$

The same modified or embryonic forms of corpuscles appear in the acute type of Texas fever after the high temperature has disappeared and the stage of convalescence has begun. They disappear speedily from the circulation when the number of corpuscles again begins to rise. In fact they seem to disappear when the number has risen to 2.5

* These terms are used in the appendix to designate these modified corpuscles.

†Theobald Smith: On changes in the red blood corpuscles in the pernicious anæmia of Texas cattle fever. Trans. Assoc. Amer. Physicians for 1891.

$\ddagger$ It is a curious fact that the granular, or " punctate" cells have not been seen in the parenchyma of the various organs (spleen, liver, kidneys), although the diffusely stained or "tinted" cells are present. 
millions. Even when the regeneration roes not go on quickly and the aniemia remains stationary for a time the punctate and tinted cells speedily disappear while the simply enlarged corpuscles or macrocytes remain in the circulation or rather are produced as such until the number is above 3 millions and they do not regularly disappear until the number is over 4 millions.

While there could be no reasonable doubt that the forms rescribed as abnormal are immature red corpuscles, there was enough resemblance between the larger granules and the smaller stages of the parasite to make a crucial experiment necessary. It might likewise be claimed that the body to be presently described as the micro-organism of Texas fever is nothing more than a phenomenon of embryonic or perhaps degenerated red corpuscles caused by some still unknown agency, which itself is the direct cause of the disease. It became therefore necessary to show that the parasites are not the result of the disease and that in artificial anxemia they do not appear. To prove this, bleeding was resorted to, first upon a sheep then upon a cow. The artificial anæmia brought about caused the various modifications described above to appear in the blood of both sheep and cow, but the various forms of the parasite did not show themselves at any time. These experiments are of sufficient importance to warrant their publication here.

Male lamb, 5 months old, still nursing-gross weight, 65 pounds.

\begin{tabular}{|c|c|c|c|}
\hline Date. & $\begin{array}{l}\text { Number of } \\
\text { red corpus- } \\
\text { cles in a } \\
\text { cmm.* }\end{array}$ & $\begin{array}{l}\text { Quantity } \\
\text { of blood } \\
\text { withdrawn } \\
\text { from jug. } \\
\text { ulars. }\end{array}$ & Remarks. \\
\hline 1890 & & $\underset{336}{\text { Grams. }}$ & - \\
\hline June 18 & $\begin{array}{l}11,500,000 \\
10,500,000\end{array}$ & & Blood corpuscles not visibly altered. \\
\hline June 19 & $9,200,000$ & 406 & Do: \\
\hline June 20 & $6,500,000$ & & Do. \\
\hline $\begin{array}{l}\text { June } 23 \\
\text { June } 25\end{array}$ & $8,000,000$ & 330 & $\begin{array}{l}\text { Many corpuscles enlarged (inacrocytes). A few punctate cells. } \\
20-30 \text { per cent corpuscles enlarged, about } 10 \text { per cent punctate. }\end{array}$ \\
\hline June 27 & $8,200,000$ & 160 & $\begin{array}{l}\text { The per cent corpuscles enlarged, about } 10 \text { per cent punctate. } \\
\text { The same number of macrocytes as before. Punetate cells have }\end{array}$ \\
\hline $\begin{array}{ll}\text { July } & 1 \\
\text { July } & 3\end{array}$ & $\begin{array}{l}7,500,000 \\
6,500,000\end{array}$ & $\begin{array}{l}546 \\
441\end{array}$ & $\begin{array}{l}\text { Macrocytes as before. No punctate cells. } \\
\text { Do. }\end{array}$ \\
\hline July 5 & $6,600,000$ & 650 & $\begin{array}{l}30-40 \text { per cent macrocytes. } 5-10 \text { per cent punctate and tinted } \\
\text { cellst }\end{array}$ \\
\hline July 7 & $5,600,000$ & n............. & $\begin{array}{l}20-30 \text { per cent punctate and tinter cells, the latter relatively in } \\
\text { creased. }\end{array}$ \\
\hline July 10 & $7,100,000$ & & Macrocytes diminishing. Punctate aud tinted cells absent. \\
\hline $\begin{array}{l}\text { July } 15 \\
\text { July } 25\end{array}$ & $\begin{array}{l}8,900,000 \\
8,900,000\end{array}$ & n.......... & $\begin{array}{l}\text { Corpuscles normal. } \\
\text { Do. }\end{array}$ \\
\hline & & & \\
\hline
\end{tabular}

* This number was obtained before the bleeding in every case.

t See Pl. Ix, Figs. 1 and 2. 
Cow No. 168.

\begin{tabular}{|c|c|c|c|}
\hline Date. & $\begin{array}{l}\text { Number of } \\
\text { rod corpus- } \\
\text { cles in a } \\
\text { cmm. }\end{array}$ & $\begin{array}{c}\text { Quantity } \\
\text { of blood } \\
\text { withirawn } \\
\text { from jug- } \\
\text { ular. }\end{array}$ & Remarks. \\
\hline $\begin{array}{l}\text { 1891. } \\
\text { Aug. } 3 \\
\text { Aug. } 4 \\
\text { Ang. } 5 \\
\text { Ang. } 6 \\
\text { Aug. } 7 \\
\text { Aug. } 8 \\
\text { Aug. } 10 \\
\text { Ang, } 11 \\
\text { Ang } 12 \\
\text { Aug. } 14 \\
\text { Aug. } 17 \\
\text { Aug. } 22 \\
\text { Aug. } 29 \\
\text { Sept. } 8\end{array}$ & $\begin{array}{l}6,762,500 \\
4,988,700 \\
4,652 ; 700 \\
5,227,800 \\
3,820,000 \\
3,094,600 \\
2,253,700 \\
2,143,000 \\
2,114,750 \\
2,538,400 \\
3,202,000 \\
3,200,000 \\
4,325,000 \\
4,784,000\end{array}$ & $\begin{array}{r}\text { Grams. } \\
2,268 \\
2,325 \\
3,827 \\
4,251 \\
4,989 \\
\cdots \\
\cdots\end{array}$ & $\begin{array}{l}\text { Blood elements not visibly changed. } \\
\text { Do. } \\
\text { Do. } \\
\text { Do. } \\
\text { Do. } \\
10 \text { per cent macrocytes, } 2-3 \text { per cent punctate corpuscles. } \\
20 \text { per cent macrocy tes, } 15 \text { per cent punctate corpuscles, } \\
\text { Same as yesterday. } \\
\text { Same as yesterday (Plate Ix, Figs. } 3,4 \text { ). } \\
\text { Numerous macrocytes. } 5 \text { per cent punctate corpuscles. } \\
\text { Macrocytes as before. A few punctate corpuscles. } \\
\text { Macrocytes as before. No punctate corpuscles. } \\
\text { Only a few macrocytes. } \\
\text { Do. }\end{array}$ \\
\hline
\end{tabular}

1 This number was obtained before the bleeding in every case.

These two experiments show that the various changes which the red blood corpuscles undergo in Texas fever are solely the result of the rapid and enormous loss of red corpuscles. The enlargement of the corpuscles, the presence of stainable matter in them in the form of large and small granules, and uniformly diffused, are phenomena accompanying severe loss of blood by whatever means this may have been brought about, and are indicative of an active regeneration of the blood elements. We are therefore justified in drawing a sharp line between these phenomena and those to be subsequently described as the Texas fever parasite.

The white corpuscles of the blood did not obtrude themselves so far as fluctuation in numbers is concerned during the various stages of the acute and mild types of the disease. Hence quantitative determinations were not attempted until the latter part of the season of 1891 , when Toison's fluid was used.* Both red and white corpuscles were then estimated with little extra labor in the same preparation of blood. In all cases the 400 squares of the ruled cell of the Zeiss apparatus were counted. The number of leucocytes in these spaces is, however, so small (from 3 to 15 ) that we might anticipate only an approximate accuracy, unless we take the average estimate of 3 or 4 preparations. For this no time could be taken. Hence the figures as given in the appendix based upon the method here described can not be regarded as of much value. $\dagger$

\section{* See page 37 .}

† The inefficiency of the method used has been commented upon recently by other investigators who have had occasion to use it. A melangeur is now specially prepared by Zeiss for the estimation of the white corpuscles, and constructed to give a onetenth dilution of a considerably larger quantity of blood, in order to concentrate the white corpuscles. This was latterly tried but found useless, since the capillary tube is so wide that it no longer retains the column of fluid by capillarity and the blood drops away before it can be mixed with the diluting fluid. We trust that this defect may be speedily remedied, 
As far as they go, they indicate not very much fluctuation. Any unusual increase in numbers was not noted in the stained preparations of any case which came under observation. In some cases an abnormal crowding together of leucocytes was observed in dried preparations, which crowding must be regarded as having existed within the blood vessels, for there was no time for any massing together after the blood had left the vessels.

Whether the disease affects the different kinds of leucocytes either qualitatively or quantitatively has not entered into the scope of this investigation. It should be said, however, that in the stages of advanced anæmia when hæmatoblasts are occasionally detected in the circulating blood, peculiar round bodies, which stain deeply and solidly with methylene blue, and which are a trifle smaller than red corpuscles, are frequently detected. A careful comparison of these with the nuclei of the hæmatoblasts makes it safe to regard the former as such nuclei which have been set free in the circulating blood. 


\section{THE CAUSATION OR ETIOLOGY OF TEXAS FEVER.}

\section{TEXAS FEVER IS NOT CAUSED BY BACTERIA.}

We have thus far considered only the changes caused by the disease in the blood and the organs of infected cattle and the manifestation of these changes during the life of the animal and after death. They are the concomitants and the resultants of certain causes at work in the body of the animal and are to us interesting and important only in so far as they shed light upon the nature of these causes. And what are the causes at work in producing Texas fever? This problem has occupied the attention of a number of investigators since 1868. The general belief that Texas fever could be nothing else than an infectious disease due to the multiplication of some minute organism entering the borly from without, led to a search for this microörganism by most of those who made this disease a special study. Dr. R. C. Stiles of the Metropolitan Board found in 1868 in the bile of Texas fever "preserved for analysis" minute vegetable organisins "in the form of spherical or irregular aggregations of micrococcus." From bile sent to Prof. Ernst Hallier, of Jena, Germany, this savant cultivated a mold (1, p. 1141-1150). It is needless to go into the details of this investigation, for its methods are exploded and its results fantastical to say the least. Gamgee examined the blood of Texas fever with high powers, but found nothing unusual. Drs. John S. Billings and E. Curtis of the army studied the blood with reference to the presence of cryptogamic growths at about the same time, but their efforts were fruitless.

Dr. D. E. Salmon, in 1883, described a diplococcus obtained from the spleen in cultures, but left its relation to the disease undecided $(5, \mathrm{p} .13)$.

Dr. J. H. Detmers (5, p.134) mentions the presence of bacilli and micrococei in the liver just after death, but none in the blood.

In a report published in 1888 Dr. Frank S. Billings claimed, somewhat pompously, to have discovered the "true germ" of Texas fever.*

* The announcement of this supposed discovery is entitled to quotation:

"Hence the germ of the southern cattle plague has been discovered, and I think that I may be pardoned the egotism of claiming this to be the first occasion in American medicine that not only one but two germ diseases of animal life have been traced out and their origin placed upon an impregnable basis.

"The order of events seems to be reversing itself! The sun of original research, in disease, seems to be rising in the West instead of the East, so far as America is concerned. This honor does not belong to me alone," etc. (8, p. 72). 
This germ is said to be like the germ of Billings's swine plague (hog cholera). It "has been found in the blood, the gall, the urine, the liver, spleen, and kidneys" of every diseased animal that was exanined. It produces Texas fever in cattle when inoculated in "unquestionably pure cultivations."

This seems to be sufficient proof. In scientific research, however, especially when an important discovery is involver, it is incumbent upou the investigator to give at least to some extent the details of his experiments, so that others may form an opinion of their own as to whether the work was properly done and the conclusions or inferences warranted. Instead of a conscientious report of work done we find in this bulletin of 138 pages the same padding used in the swine-plague report of the same author. Quotations, criticisms, and discussions, mostly foreign to the object of the report, together with an unwarranted dragging in of yellow fever, constitute the bulk of the text.

The germ of Texas fever as found by Billings stains at the ends. It grows on potato with a delicate straw color, which finally becomes a brick-red yellow. In the beef infusion gelatine tube it does not liquefy gelatine. These meager facts are not sufficient to distinguish this organism from a large group of bacteria living especially in the intestines of all domesticated animals. In fact the few characters apply very well to the bacillus coli communis, a universal saprophyte in decomposing organic matter of intestinal origin, and one that has pathogenic properties with reference to smaller animals. This supposition is strengthened by the fact that Billings found in fresh and old manure bacteria not to be distinguished from the supposed Texas-fevor germ.

As to the crucial test-the production of Texas fever by the inoculation of eattle with cultures of this germ-one case is reported. A black steer ealf five months old presented four days after inoculation a temperature of $42.5^{\circ} \mathrm{C}$. (106.5 $\mathrm{F}$.). The temperature remained high for two days, when the animal was killed for examination. A glance at the autopsy notes shows that there is nothing to prove that the disease was Texas fever. In the liver "each acinus was most beautifully denarcated by delicate lines of a bright yellow eolor, which represented the interacinus and distended gall ducts." In Texas fever the bile injection is, as a rule, limited to the ultimate bile canalienli within the acinus and rarely extends into the interlobular bile ducts. Was the bile stasis in the intralobular tissue actually seen under the microscope in this case? The important pathognomonic sign of Texas fever-hamoglobinuriawas absent. The evidence that this steer was suffering from Texas fever is therefore not sufficient by any means, although we do not wish to claim that it was not Texas fever. This latter disease may have been induced by contact with ticks, or by the presence of the Texas-fever parasite in the eultures originally introduced with blood or bits of tissue. The total absence of any experimental details as to what culture was used, how it was injecterl, where the animals came from, ete, leaves us wholly in the dark as to the aceuracy of the experiment. 
These are all the facts of importance communieated by lillings in his report on the supposed bacterium of 'Texas fever." Hen if the evidence to be adduced farther on were not diametrically opposed to them, the meagerness and vagueness of the statements made by him would prevent any candid unbiased observer from accepting them without great reservation. As to some other theories presented by Billings in his report, we shall recur to them farther on.

In May, 1890, a bulletin on Texas fever was publisherl by Dr. Paul Paquin, of the Missouri Experiment Station (9), which describes investigations conducted between September, 1888, and March, 1890. Paquin claims to have found a germ, but it is impossible to discover from the descriptions anything concerning the nature of this germ, excepting perhaps that it does not exist, and that a variety of microscopic things were seen in the débris of the blood, bile, and liver, which were considered by the author without any supporting proof as the Protean forms of a single organism. This kind of logic may suit disappointed observers, but it does not contribute anything to our knowledge of the subject. Moreover, in departing from established methods $\dagger$ and in describing forms presumably existing which are wholly unlike any already recognized, the burden of proof rests upon the author and the work must be unusually well done to merit any attention. The conclusions reached by the author and presented on page 43 of the bulle. tin have none of them received even the shadow of a proof in the text. 'That bacteria may be found in cases of Texas fever is unquestioned, but that they have anything whatever to do in producing the disease demands rigorous proof. It would be difficult therefore to analyze a report of experiments, however conscientiously pursued, in which the fundamental elements of scientific research-a careful record of such experiments and their details and somnd logic in drawing conclusions from such experiments are at fault. We refer here only to the work involved in the study of the cause of the disease. The vaccination theory will be discussed farther on.

In the third annual report of the Arkansas Experiment Station (1890), Dr. R. R. Dinwiddie reports that in a large number of cultures on different media from three cases of Texas fever no bacteria de-

\footnotetext{
* After the above was written an article by Billings on the etiology of southern cattle plague ( Fexas fever) appeared in the Journal of Comparative Medicine for 1892, beginning with the July number. The remarks in the July and August numbers may be passed over without comment. In the September and October numbers is contained practically what has been stated above, largely drawn from his report. There is quoted in addition an experiment with cultures from ticks with which he claims to have produced Texas fever. While we are pleased to see that, since reading preliminary articles in the reports of the Secretary of Agriculture, Billings is now paying some attention to ticks, we must wholly dissent from his conclusions, which are practically the same as those reviewed in the text. The conclusions which we have drawn and those presented by Billings in these articles may be safely left to the judgment of future workers in this field.
}

t See p.8, which describes the method of preserving tissues. 
veloped. He also isolated bacteria from the intestines which proved negative when inoculated.

In our own work the first problem which naturally presented itself was to determine whether bacteria could be regarded as the cause of the disease. Hence the very first and some of the later cases were utilized for this purpose. As to the first postulate necessary to be fulfilled in demonstrating the cause of any infectious disease-to find with the microscope the bacterium or other organism in the body of the diseased animal-this failed utterly in all the cases examined. The thousands of cover-glass preparations of the blood, spleen, liver, kid. neys, etc., examined fresh and stained never showed any bacteria excepting when the animal had been dead for a number of hours. Animals killed in a dying condition were almost invariably free from bacteria. Those which succumbed in the night in midsummer contained usually large bacilli which are denominated post-mortem bacilli in the appendix, and which are familiar to every worker in bacteriology. They are specially abundant in the carcasses of large animals, from the pig up, which have been dead some time; probably because a large carcass remains warmer and more thoroughly deoxidized than a small one, and thus becomes a good medium for this anaërobic bacillus to flourish in. This bacillus does not grow in ordinary culture tubes, excepting perhaps very feebly in the bottom of bouillon tubes and in impure cultures on agar-agar. These bacilli are mentioned as being present in Nos. 5, 6, 65, 106, 129, 144, 164, 169, 180, 227. They are somewhat broader than anthrax bacilli, have rounded extremities and usually occur single. They stain readily and deeply in all aniline dyes. The mention of this familiar intercurrent bacillus might have been passed over were it not that observers who have described bacteria in connection with Texas fever may have occasionally mistaken this as the canse $(9, \mathrm{p} .43$, third conclusion).

As to the cultivation of any bacteria from the blood and tissues in Texas fever, the results are equally negative. Special attention was paid to this phase of the problem in 18s8. In the appendix will be found a brief statement of this work under the six first eases and after the sixth a summary of the results obtained with a bacterium which appeared a number of times in the cultures. 'That it was the ordinary bacillus coli communis of the intestines which had found its way into the liver and thence into other organs there can be no doubt, for subsequent comparisons with bacillus coli from the intestines of healthy cattle proved them to be identical. It should also be remembered that this bacillus was present in exceedingly small numbers, as will be seen from the quantity of tissue or fluid used for inoculating the various culture media and from the fact that they were never seen in coverglass preparations. A large series of cultures were also made from several eases of the disease in 1889 and 1890 with equally negative results. In most cases the cultures remained absolutely storile. In 
some a few "ultures developed, the contents of which were explainable either as contaminations or as coming from an animal in which frequent skin incisions in the last stages of the disease may have led to the introduction of a few bacteria into the circulation. The results obtained from cattle infected by Texan animals were as negative as those from North Carolina cattle. (See No. 128.) Cultures have thus far been inade from four different outbreaks, and the blood and the tissues have been examined microscopically from as many more. (See Nos. $1,2,3,4,5,6,43,44,48,54,70,128$.)

We are, therefore, ready to admit that there are no bacteria in the blood and tissues of animals suffering with Texas fever, excepting occasional individuals which probably enter the circulation from the intestines by way of the disintegrated liver. But may there not be bacteria living only in the intestinal tract which send their toxic products into the circulation and thus cause disease? This hypothesis might be attractive to those who will insist on bacteria as the cause of Texas fever, but there are no facts to support it, and in view of the more definite results obtained by us its discussion is useless.

\section{THE MICROÖRGANISM OF TEXAS FEVER.}

(Pyrosoma bigeminum, $n . s p .^{*}$ )

Although Texas fever is essentially a blood disease, and only secondarily affects the spleen, liver, and kidneys, most observers have failed to recognize this fact. R. C. Stiles (1) was the earliest and the only observer who laid any stress upon the changed condition of the blood corpuscles. He says: "The red blood corpuscles when examined immediately after removal from the body were shriveled and crenated without artificial provocation. *** In one case many of the disks appeared to have lost a portion of their substance, as if a circular piece had been punched out, the addition of water failing to restore the disk to completeness." There can be little doubt that Stiles saw at that time the microörganism of Texas fever, without, of course, recognizing it, since this description applies very closely to the appearance of red corpuscles infected by this micro-parasite when the blood and the parenchyma of liver, spleen, and kidneys are examined tresh soon after death. Other observers have examined the blood, but have seen nothing unusual.

In 1888 during the examination of portions of the organs of cases Nos. 3 to 6 inclusive the destruction of the red corpuscles seemed to be the one prime phenomenon of the disease. The large quantity of hæmoglobin in the urine, and the peculiar condition of the liver and

${ }^{*}$ For the preliminary announcement of the discovery of this microörganism see the Annual Report of the Secretary of Agriculture for 1889, the Medical News for December 4, 1889, or the Proceedings of the American Public Health Association for 1889 . 
the bile indicative of hyper-secretion could not but lead to the hypothesis that there was some destructive agency at work in the blood. $R$. C. Stiles in 1868 assumed the liver to be the primary focus of the disease and believed that the alteration of the blood elements was due to the absorption of bile from the liver into the circulation. This inference from the observed pathological phenomena is erroneous, for the liver is doing too much work rather than not enough, and the destruction of blood corpuscles goes on very early in the disease. The outcome of the work in 1888 was the formulation of several theories as to how the blood corpuscles came to their destruction:

(1) There may be organisms in the blood which by the production of toxic products act directly on the corpuscles.

(2) There may be some toxic substance in the digestive tract which is absorbed into the blood and causes a dissolution of the red corpuscies. This substance may be the product of specific bacteria multiplying only in the digestive tract.

(3) There may be micro-parasites which invade the red corpuscles in a manner similar to those of malaria, and which by their growth disiutegrate the containing corpuscle.

The first hypothesis was soon made improbable by the absence of any demonstrable organisins in the parenchyma of the various organs which are abundantly supplied with blood, such as the liver, spleen, and kidneys. To test the second the contents of the digestive tract, more particularly the small intestine, were carefully examined microscopically in 1888 and many plates and rolls of gelatine were made with the intestinal contents without bringing to light any other than the ordinary intestinal bacteria. It is true that this method was merely preliminary and would have been followed by more exhaustive bacteriological studies of the digestive tract had not the third hypothesis furnished the clue. This, however, could not be tested in 1888, since no living animals were accessible, and the results of the study of the blood elements could not be considered reliable when obtained only from the organs of animals dead twenty-four hours or even longer. In the very first case which succumbed on the experiment station at Washington, in 1889, certain microörganisms were found within the red corpuscles which will now claim our attention. It should be said, however, that these bodies were noticed in the spleen of a case as early as 1886, as will be seen from the notes of No. 2, of which only this organ was brought to the laboratory.

PECULIAR BODIES HOUND IN THE RED CORPUSCLES OF HEALTHY CAT'TLE.

In endeavoring to prove the existence of specifie parasites in the blood as causes of disease it becomes necessary to prove their absence during health. A large series of microscopic observations have been made upon the blood of eattle which were not infected as well as upon 
those which were infected before the disease had appeared and after it had passed away. In a preceding chapter we have treated of the number of red corpuscles in health and in Texas fever, also the changes which they undergo in this disease and the methods to be used in studying them. These methods apply in the study of the microörganism and the reader is referred to them (p. 43). The red corpuseles of cattle retain their form pretty well when examined in the fresh condition. After a time small conical protrusions form on them as they shrink and shrivel, and the stramonium forms begin to appear.

In 1890 certain minute bodies were first observed within red corpuscles of eattle in health. They are present in variable numbers. In some cases they are not found even after prolonged examination of cover-glass preparations (apochrom. $2 \mathrm{~mm}$ oc. 4 or 8 ). In some a few may be seen in a single field. In several cases as many as 10 per cent of the corpuscles contained them. They may appear as barely visible points with a bright luster. Whether this brightness is a resultant of the color of the body itself and that of the corpuscle within which it is lodged it is impossible to find out. Suffice it to state that as we look into the microscope at a corpuscle containing one of these bodies it appears as a bright, almost golden, speck. These bodies are not all of the same size and form, although their minuteness makes it impossible to express differences in figures. They range in size from mere specks to quite appreciable coccus-like bodies. Frequently a rod-like form with a central constriction, reminding one of diplo-bacteria, appear's. It may be that the rod-like forms are observed as round bodies when standing on end within the corpuscle. In general they are rarely 0.5 $\mu$ large, usually much smaller. In the table of the appendix they are indicated provisionally as bright bodies. Plate VI, Fig. 9, gives an approximate idea of the relative size of these bodies. The third and the fourth corpuscle contain bodies which are much too thick, however.

Another interesting phenomenon of these bodies is their occasional motility. Many change their place within the corpuscle. When first detected the speck is usually situated at the periphery of the corpuscle. When watched closely for a few minutes it may be seen to move toward the center of the corpuscle, then back again toward the periphery. Then the movement may be along the periphery for a distance, succeeded perhaps by a movement across the entire corpuscle. The smallness of these bodies does not allow us to state whether this movement is passive and due to currents within the corpuscle or whether it is the active, spontaneous movement of a living organism. There are, however, cases in which it is difficult not to accept the view that the movement belongs to a living body. The warm stage seems to accelerate these movements, but since heat is also likely to cause disturbance of the fluid within the corpuscle, this acceleration does not add to the proof that we have organisms before us. Fig. 10 on Plate VI shows the path of one of these motile bodies. They do not 
reappear in dried and stained preparations, which means that they do not stain.

It has already been stated that these bright specks are present in the red corpuscles of healthy cattle. They are found in all seasons of the year and in most animals examined, in southern (North Carolina and Texas) as well as native cattle. Besides these bright bodies, many of which are constantly changing their places within the corpuscle, there are occasionally seen in the fresh blood, both in health and during the fever period of this disease, bright rod-like bodies within corpuscles, which do not changetheirplace. They lie usually at the edge of a paler area within the corpuscle, and the impression is conveyed that they are crystals derived from the hæmoglobin of the adjacent pale spot. There are from two to four of these minute rods in the affected corpuscle.

In addition to these intraglobular bodies present in healthy blood, certain forms are now and then seen in dried preparations stained in methylene blue which might be mistaken for Texas fever parasites. They are round, deeply stained coccus-like bodies situated quite near the periphery of the corpuscle and about one to two $\mu$ in diameter. There is never more than one in a cell. They differ from the intraglobular parasite by a deep blue stain and by the compact round form. They are probably remnants of the nucleus of the ancestor of the corpuscle-the hæmatoblast.**

\section{THE MICROÖRGANISM IN THE ACUTE TYPE OF TEXAS FEVER.}

In describing the micro-parasite of Texas fever we shall describe the various forms and stages as they are met with in actual examinations first and then construct its life history as far as that is possible from the recorded facts.

\section{In fresh blood of the acute disease during life.-When blood is drawn}

* The interpretation of appearances in the field of the microscope is frequently beset with difficulties, and certain foreign bodies are likely to intrude and give rise to false impressions. To those accustomed to the examination of the blood elements this is not likely to happen, but to the bcginner in this work certain suggestions will not be superfluous. In preparations of fiesh blood from cattle a large number of very minute refracting spherical bodies abont as large as the earlier stages of the Texas fever parasite are frequently found in all parts of the preparations. They may be attached to the disks of many corpuscles and appear like intraglobular bodies. Their presence in other parts of the field free from corpuseles as well as careful focusing shows them to be foreigu bodies. Prolonged observation has led to the inference that they are derived from the fat in the sebaceous follicles of the skin, because they have bcen occasionally encountercd in masses on the slide. The incision perhaps dislocates such masses and the blood carries them ont.

In stained preparations bluish spots are not infrequently found on red corpuscles which might be mistaken for parasites. They are nothing more than blood plates which have attached thenselves during the drying of the film to the corpuscles. In general it may bo said that such misinterpretations will not occur after tho varions stages of the micro-parasite have been once recognized. 
from the skin during the fever and examined at once with high powors (500 to 1,000 diameter's, Keiss apochnom., 2mm., oculars 4 and S) certain corpuscles will be found containing two pale bodies of a pyriform outline. One end of each body is round and the body tapers gradually to a point at the other. They vary somewhat in size in different cases, but the two bodies in the same corpuscle are as a rule of the same size. They are from 2 to $4 \mu$ in length and 1.5 to $2 \mu$ in width at the widest portion. (Plate vr, Figs. 4, 5, 6.) Their tapering ends are directed toward each other and usually close together; their rounded broad ends may occupy various positions with reference to each other. They may be seen together with the axes of the bodies nearly parallel or they may be far apart, the axes forming a straight line. (Plate V, Fig. 2.) The bodies themselves have a homogeneous, pale appearance, contrasting markedly with the inclosing red corpuscles from which they are sharply outlined. There is no differentiation into peripheral and central zone, no granular appearance of the body. Several slight variations in the appearance of these bodies at different times have been noted. The smaller forms are as a rule homogeneous; the larger forms are very frequently observed to be provided, in the rounded end of the pyriform body, with a very minute spherical body probably not more than 0.1 to $0.2 \mu$ in diameter, which contrasts dark with the body itself. In several cases it manifested a brilliant luster with very high powers. (Plate VI, Figs. 4, 5; Plate VIII, Figs. 4, 5.) In the largest pyriform bodies there was seen in the center of the enlarged end a somewhat larger round or oval body which seemed to take the place of the smaller body or else be associated with it. This second body was from 0.5 to $1 \mu$ in diameter. It changed its appearance with the focus. At a low position of the objective the parasite appeared dark with a light round spot in the enlarged end. At a higher position of the objective the inner body appeared dark, inclosed in the lighter pyriform outline. One or both of these bodies were observed in some of those forms undergoing amoboid changes.

A question of considerable interest to be discussed farther on is the relation of these two pyriform bodies to each other in the same corpuscle. Any direct mutual connection of their tapering ends is not demonstrable in the fresh preparation.

When exposed to a temperature of $35^{\circ} \mathrm{C}$ to $42^{\circ} \mathrm{C}$ on the warm stage* some of these bodies, by no means all, exhibited changes of outline.

\footnotetext{
*Pfeiffer's warm stage as constructed by C. Zeiss was used. The entire microscope is inclosed in the box (with exception of ocular and adjustment screws). The heat is communicated to the heavy iron bottom of the box and thence to the air and the microscope stand which rests upon it. The drawback to this apparatus is the large amount of heat which is stored in the iron base and which may cause the temperature of the stand to rise faster than that of the surrounding air. As these observations upon the fresh blood had to be made mainly at the experiment station with no gas at hand, the heat was applied with an alcohol lamp and the thermometer carefully watched.
} 
These may go on contimmonsly in some bodies, in other's quite slowly. The motion most frequeutly exhibited consists not so mueh of a thrusting ont and withdrawing of psendopodia as of a continual recasting of the general outline of the body as we find it for example in the leucocytes of mammalian blood. (Plate viIr, Figs. 1, 2,3.) The changes of form may go on so continuously and so rapidly that it is not possible to sketch them all, as some escape observation during the sketching. The motion described does not of necessity require the stimulus of heat. During the past summer the same continuous rapid changes were observed in preparations of blood, sealed with paraffin, at $75 \circ$ and at $85^{\circ} \mathrm{F}$. In the former case the slide had been prepared at 10:45 a. m. The motion was still noticeable at 3:10 p. m., when the observation was discontinued. In the latter case the observation was discontinued six hours after the drawing of the blood, although the motion had not yet ceased. The sparseness of the micro-parasite in the blood makes it impossible to state definitely whether this amœeboid motion belongs to a certain stage of its life. On the whole the observations tend toward the inference that the pyriform bodies do not change their form and that the motion belongs to a younger stage. It should likewise be stated that the amoboid bodies observed were apparently single within the corpuscle.

If dried cover-glass films, heated, stained in alkaline methylene blue and decolorized as described on p. 44 be examined in water or balsam-preferably the former-it will be found that the forms described have become stained. The staining, however, is more feeble than in those micro-parasites found in the internal organs after death. It is limited usually to a zone on the periphery of the body, the center being feebly blue or entirely free from coloring matter. (Plate v, Fig. 2, 3d; Plate vi, Fig. 7.) In the latter condition it has been observed that these circulating forms have a peculiar luster, as if they possessed (in the dried and stained condition) feebly refracting powers. Other basic aniline dyes, such as methyl violet and gentian violet, are equally applicable. Fuchsine stains the organism, but also affects the containing corpuscle, so that the pictures obtained with it are not satisfactory. Hæmatoxylin likewise stains the organism fairly well. In general the clearest, most distinct pictures lıave been obtained with Löffler's alkaline methylene blue.

The intraglobular parasites found in the acute stage are not all pyriform and paired. In fuct a considerable number as seen in stained preparations are somewhat irregular in outline and single. These are probably the bodies which were undergoing amoboid changes when they were dried in the film on the cover glass. Some of these irregular forms are shown on Plate v, Fig. 3.

The corpuscle which contains such a pair of microparasites has fully one-fourth of its area occupied by them. That this invasion is detrimental to the corpuscle is easily understood. In preparations of 
fresh blood the corpuscle has a peculiar appearance. Its margin is irregularly notched and creased, the border may be beset with projecting spine-like processes and its color may be darker than that of the normal corpuscle. It has, to use a fitting expression, a wrecked appearance. This change is more marked in some cases than in others. Such corpuscles have lost their characteristic flexibility. They retain their disc-like form, even after normal corpuscies have become shriveled and folded in preparations kept under observation for some time.

The number of infected corpuscles circulating in the blood during the high fever is usually quite small. It is difficult to make an approximate estimate without careful counting. Probably one or two in a single field of the 2-mm. objective, or from half to 1 per cent is near the truth in most cases. In some, however, a long search is necessary before one is brought into view. When the number grows larger, death is not far distant and nay be expected within twenty-four hours. Toward the fatal termination, there may be from 5 to 10 per cent of the corpuscles with the pyriform parasites present. Fig. 2 on Plate $v$ is an illustration of a group of such infected corpuscles taken from the blood on the last day. Very rarely large numbers of parasites may be present and yet the animal recover. The only case of this kind is No. 49, in which hæmoglobinuria appeared at the same time. When present in considerable numbers in the blood the infected corpuscles usually appear in groups in the field of the microscope, as is shown in the figure referred to, and not uniformly distributed.

When the fever has subsided and the number of red corpuscles has been greatly diminished, the parasites disappear quite rapidly from the blood. In fact, the reduction of temperature usually coincides with the more or less complete disappearance of the infected corpuscles, and their place is then taken by the large number of embryonic corpuscles which begin to replace the losses. An occasional infected corpuscle may be detected for some days or even a week after recovery has set in. But they are so scarce that their detection is more of an accident. After the subsidence of the fever, when there is a general sinking of the vital powers, leading to death, the parasites may linger on in the blood in small numbers or they may disappear as in recovering cases.

Parasites in internal organs.-With only 1 or 2 per cent, or even 10 per cent, of infected corpuscles in the circulating fluid, it would be difficult to account for the enormous daily losses of blood corpuscles in the acute fever. The difficulty is cleared up by sacrificing an animal in the earlier days of the fever and examining the internal organs for infected corpuscles. Large numbers of parasites are found within corpuscles in the capillary blood of congested areas, such as those of the heart muscle and of the omentum. In the latter membrane there are delicate fringes containing capillaries which may be placed entire on a slide and examined with the highest objectives. In such capillaries in the fresh condition, with perhaps a little iodized serum added, the pale 
intraglobular parasite may be seen quite distinctly. When such fringes are torn and crushed on cover glasses and dried films prepared and stained, the large number of parasites is at once revealed. (Plate VI, Fig. 1.) The same may be said of the muscular walls of the heart. In these the smaller vessels are seen by the unaided eye to be engorged, and in sections the capillary network is found in the same condition. (Plate VI, Fig. 2; Plate VII, Fig. 1.) If a piece of such muscular tissue be compressed and dried films made from the blood squeezed out, an unusually large number of infected corpuscles will be found.* These statements are best illustrated by a case:

No. 163 was killed August 25, 1891, when her temperature was 107. On the morning of August 21 her temperature was still normal (101.6). It was not taken until August 24, when it was 106.8. If we assume that the first high morning temperature occurred August.22, she was killed at the end of the third day of continued fever. Even at this time there had been great losses in blood corpuscles.

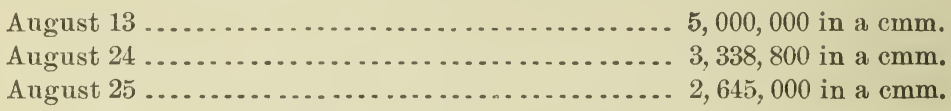

Before she was killed there were 2 to 3 per cent of infected corpuscles in the circulating blood. In the internal organs there were found in cover-glass preparations made at the autopsy-

In blood from skeletal muscles very few infected corpuscles.

In blood from the right heart very few infected corpuscles.

In blood from marrow of sixth rib very few infected corpuscles.

In blood from the left heart 2 to 3 per cent infected corpuscles.

In blood from lnng tissue 2 to 3 per cent infected corpuscles.

In spleen pulp 5 per cent infected corpuscles.

In liver tissue 10 to 20 per cent infected corpuscles.

In kidney tissue 10 to 20 per cent infecterl corpuseles.

In hyperæmic fringes of omentum 50 per cent infected corpuscles.

In heart muscle 50 per cent and many free parasites.

This distribution of the infected corpuseles and their localization in the capillaries will receive more attention later (Plate VII). Meanwhile we simply wish to point out that, while only a few parasites may circulate in the blood, the infection may reach 50 and even more per cent in the internal organs. The parasites as they appear in the capillaries differ somewhat in form from those in the circulating blood. Their form may be best seen in dried and stained preparations of the capillaryblood of the heart muscle. (Plate IV, Fig.5.) They appear slightly smaller than in the circulating blood and the outline of many is spindleshaped or fusiform, i. e., tapering at both ends. (Plate VI, Fig. 1.) In this stage, which is probably one of active growth, they stain very well. The stain is deeper in that half of the body directed towards its mate in the same corpuscle. Distiuctly pyriform bodies are also present,

* In such preparations, the falciform bodies of sareosporidia cysts are frequentiy present, especially whon tho preparation is from a cow ovor 5 years old. 
and these as a rule take the stain quite uniformly. In preparations of fresh blood from the same source no differences are observed except an absence of the minute nuclear (?) body in this stage. It may be that we have to deal with forms younger than those which circulate in the blood (see Fig. 3, p. 70).

Changes of form of an amœboid nature have already been referred to. If the organs of an animal which has been dead for five or six hours be examined it will be found that all the intraglobular parasites have a roundish form and that distinctly fusiform or pyriform bodies are to be seen only occasionally in preparations from the heart muscle. (Plate v, Fig. 1.) The inference is that the microörganisms have assumed the spherical form under the adverse conditions presented by the death of the host. Similar changes are observed after a time on the warm stage. The pyriform and spindle-shaped bodies which have been thus far described may therefore escape the attention of those who study the blood and the organs after death only. The blood is rarely in a condition to be examined after death, because the corpuscles lose their dise-like form very speedily. In the various organs they are preserved fairly well even for hours after death.

- The relative number of infected corpuscles in the internal organs demands some attention. This was estimated approximately in dried and stained cover-glass preparations after examining a large number of fields. The cover-glass films were made like those from the blood. A smooth, fresh incision was made into the organ, the cover glass gently drawn over the cut surface, and the film allowed to dry. This gave thin and uniform films. The very soft and partly disintegrated spleen pulp required some other procedure. A little of the pulp was scraped up with the edge of one cover glass and then quickly drawn over another, as in the preparation of blood films. This usually insured layers thin enough for microscopic examination.

A comparison of the various cases in the appendix will show that there is a considerable variation in the number of infected corpuscles found in the body after death, according as the animal succumbed in the fever stage or after the number of red corpuscles had been greatly reduced and the fever had passed away. In the former case the infection is very extensive, as the following illustrations show:

\section{No. 128 (Texas infection).}

Blood from skin and heart, 10 to 20 per cent corpuscles contain parasites.

Blood from spleen, 10 to 20 per cent corpuscles contain parasites.

Blood from liver, 40 to 50 per cent corpuscles contain parasites.

Blood from kidneys, 80 to 90 per cent corpuscles contain parasites.

\section{No. 130 (North Carolina infection).}

(Number of corpuscles two days before death, 3,922,000.)

Marrow of rib, 5 per cent of corpuscles contain parasites.

Blood from skin and heart, 10 to 15 per cent corpuscles contain parasites. 
Blood from spleen, 10 to 20 per cent of corpuscles contain parasites.

Blood from liver, 20 to 30 per cent of corpuscles contain parasites.

Blood from kidneys, 60 to 80 per cent of corpuscles contain parasites.

Capillary blood from heart muscle, and omentum, 50 per cent of corpuscles contain parasites.

In those cases in which the number of corpuscles has fallen quite low, $i$. e., below $2,000,000$ before death, the number of such as are infected must necessarily be low because there are so few corpuscles remaining. Of these the majority may be embryonic or new forms.

No. 184. (Temperature on the last day, 103.2; number of corpuscles, 1,822,500.)

Blood (subcutaneous and from heart cavities) contains $\frac{1}{2}$ to 1 per cent infected corpuscles.

Spleen, 2 to 3 per cent infected corpuscles.

Kidney and liver, 20 to 30 per cent infected corpuscles.

Heart muscle, 10 to 15 per cent infected corpuscles.

No. 95. (Chronic case. Infection first detected August 7; killed in dying condition August 25. Blood corpuscles 1,858,800.)

Blood before death contains 5 per cent infected corpuscles.

Spleen contains 2 per cent infected corpuseles.

Kidney and liver contain 5 per cent infected corpuscles. The former organ also contains many freed parasites.

These illustrations may suffice here. Many more may be found in. the appendix to demonstrate the variable number of infected corpuscles found at the autopsy. As to their distribution over the body, something has already been stated. They are very abundant, as determined thus far, in the capillary blood of the heart muscle, but quite rare in that of the skeletal muscles. Of the internal organs the kidueys usually contain the largest numbers; not infrequently from 50 to 80 per cent of all the corpuscles are infected. (Plate IV, Fig. 4; Plate VII, Fig. 2.) Next comes the liver, then the spleen. In spite of the fact that this latter organ is loaded by several times its own weight with red corpuseles, rarely more than one-tenth contain parasites. Infected corpuscles have been found in great abundance in the capillaries of the choroid plexus of the lateral ventricles of the brain and in the ressels of the pia and the brain substance. They have also been detected in the capillaries of the intestinal mucosa.

Freed parasites. - In view of such enormous destruction of red corpuscles the question naturally arises whether freed forms of the parasite are not regularly observed. In the circulating blood none have been seen. In the preparations from the heart muscle of various cases there are seen a large number of free bodies in pairs as they are found in the corpuscle. Unstained, they float in pairs in the blood under the cover glass, sometimes as pyriform, sometimes as round bodies. (Pl. vI, Fig. 8.) They have a homogeneous grayish appearance. Whether there is at this time any organic connection between the pair by their tapering ends or simply by invisible remmants of the once enveloping corpusele has not yet been made out. In some instances the shadowy outline of the corpusele may still be seen around them. Motion has not 
been observed. The only other organs in which free bodies are found are the kidneys. (Plate vi, Fig. 3, and notes on Nos.47, 50, 66, 69, 74, $95,128,134,139$.) These organs are generally filled with infected corpuscles. In the fever stage we may find in dried films and in stained sections very few corpuscles which do not contain a pair of parasites. When the number of corpuscles has fallen quite low before death and the destruction has practically ceased there may still be found, in dried and stained films of the parenchyma, immense numbers of fiee parasites. They appear as roundish coccus-like bodies grouped in pairs and varying slightly in size, never as pyriform or fusiform bodies. To a casual observer they might appear as coarse granulations of brokendown cells and cell nuclei, but a little study and comparison of different cases soon dispels this view.

\section{THE MICROÖRGANISM IN THE MILD TYPE OF TEXAS FEVER.}

We have thus far considered only those forms of the parasite found in the acute type of the fever. This type will now be understood to be one in which there is a very rapid multiplication of the micro-parasite in the blood vessels corresponding to an equally rapid disappearance of the red corpuscles. The forms of the micro-parasite are pyriform and fusiform bodies chiefly intraglobular, occasionally free. The post-mortem forms are roundish. In size the pyriform bodies are quite large, and the question arises: are there any smaller forms to be found? For these we must turn to the mild (usually autumnal) eases of the disease. It is an interesting fact that these cases are characterized by the presence of the smaller stages of the parasite. While the pyriform bodies are not entirely absent they are very rare. In the acute type only the latter and not the former are seen.

In the mild type (see p. 22) we have from 5 to 50 per cent of the red corpuscles in the circulating blood infected for a period of from one to five weeks. In the acute type, on the other hand, the circulating blood contains usually from one-half to 2 per cent of infected corpuscles; 10 to 15 per cent is a rare occurrence, usually just before death. In the fresh preparations of blood this small stage of the parasite is as a rule invisible. Rarely we may observe it on the very border of the corpuscle as a round pale spot about $0.5 \mu$ iu diameter, which does not change its place. When dried films of blood are stained in alkaline methylene blue the parasites appear as round coccus-like bodies from 0.2 to $0.5 \mu$ in diameter and situated within the corpuscle on its border. They sometimes appear as if situated on the border but outside of the corpuscle. As a rule only one is found in a corpuscle. (Plate IV, Fig. 1, 2, 3.) In many cases a division of the coccus-like body into two could be clearly made out. The separation was noticeable as a paler line and a constriction at either end similar to the division of certain micrococci. This division usually appeared in all bodies of a preparation fiom one $10320-N_{0}, 1-5$ 
case, but could not be noticed in any preparation of perhaps the next rase.

These bodies stain as well as the larger pyriform bodies in basic aniline dyes and in hamatoxylin. They do not stain in acid dyes such as eosin or in Ehrlich's dye for nentrophile granules. When this stain is employed the corpuscles, beantifully tinted, show a small round unstained spot where the parasite is sitnated. When the dried films are treated with dilute acetic acid the corpuscles fade out, while these coccuslike bodies remain behind and stand out prominently.

It has already been stated that these bodies are characteristic of the mild, autumnal type of the disease. . glance at the appendix will show how numerous these cases may be. This stage of the parasite is there indicated provisionally as "peripheral bodies" or "peripheral coccus-like bodies." A more careful examination of these cases will reveal three groups:

(1) Animals exposed to Texas fever late in the season (October and November).

(2) Animals which have passed through an acute attack earlier in the summer (second attack or relapse in October and November).

(3) Animals which contract a mild disease during or previous to the season of the acute disease.

In the first group (see Nos. 47, 48, 51, 52, 53, 56, 64, 65, 66, 69, s2, $85,86,89,93,95,100$ ), the disease is mild and may pass unnoticed. The corpuseles with peripheral bodies appear in the blood as the number of corpuscles begins to fall, and disappear when it again begins to rise. Rarely a corpuscle with a pair of large pyriform bodies is detecter.

In the second group the phenomena are the same. (See Nos. 49, 64, $104,105,107,160,206,219,222,230$.)

To the third group belong a few cases which showed a blood infection screral weeks before the fever appeared among all the susceptible animals in the infected field. (See Nos. 66, 69, 95, 129.) In No. 66 the infection was at first by peripheral coccus-like bodies. This, after a week's time, developed into an acute fatal infection, in which only the large forms were found after death. The same is trine of No. 69. In No. 95 the infection by peripheral cocei was noticed as early as August 7. From 10 to 20 per cent of infected corpuseles circulated in the bloor until August 19, when some large pyriform bodies made their appear. ance. The blood contained both small and large parasites until August 25, when the animal was killed in a dying condition.

In the foregoing it has been tacitly assumed that these intraglobular cocens-like bodies are living organisms. This position withont finther proof would undoubtedly be open to objection, and hence the reason for cousidering them parasites will be discussed somewhat in detail. In a foregoing chapter (p. 42) all those changes which the red corpuscles undergo as the result of anxemic conditions have been described. Certain corpuscles when dried and stained presented numerous gramules 
which varied more or less in size, the largest rarely exceeding $0.5 \mu$. The eocens-like bodies resemble the larger gramules very closely, and it might be argued that they are of the same origin. This is not so, however, for the following reasons: The coccus-like bodies appear with or immediately before the destruction of red corpuscles. The granules (or punctate cells) appear after the number has fallen below one-half the normal, and when the destruction ceases the punctate ceils still persist or increase and the coccus-like bodies disappear. The coccus-like bodies are with rare exceptions included in normal corpuscles; the granules belong to the large new cells (macrocytes). As to the bodies themselves, they are all of the same size in the same preparation of blood, while the granules vary considerably in this respect. Again, the gran. ules are present in considerable numbers in the same corpuscle, while the coccus-like bodies are present singly or in a state of division; rarely two are found in the same cell.

When dried films are treated with one-half to one per cent acetic acid the coccus-like bodies come out distinctly as the cell fades. The granules can not be made to appear in this way. Finally the punctate cells can be produced by artificial venesection, but the coccus-like bodies do not appear in the blood under this condition. The coccuslike bodies are thus of a character entirely different from that of the granules, although they take the same stain and appear together in the blood. (Plate IV, Fig. 3; Plate IX, Fig. 5.) Many of the same reasons will also apply in refuting the possible objection that they may be the result of disturbances of the blood other than those of a loss of corpuscles. Heinz* found certain bodies stainable in methyl violet appear in the red corpuscles of rabbits twenty-four hours after the subcutaneous injection of phenyl-hydrazin and its derivatives. These bodies are described as "strongly refracting spheres which are attached, button-like, to the red corpuscle. Often they are connected with it by a pedicle, or they may be entirely free in the plasma surrounded by a shred of protoplasm." In Texas fever the corpuscles containing the coccuslike bodies are always of normal form and appearance. It would be diffieult to find reasons for believing them to be the result of somo chemical action on the blood corpuscles. The ticks, which might be regarded as secreting a poison in their parasitic life, are very searce on the animals during the autumm and early winter, when the mild type of disease prevails. When they are most abundant, during the period of the acute disease, the coccus-like bodies give way to the pyriform bodies,

If we admit their parasitic nature as highly probable we have still the question before us whether they are stages of the Texas fever parasite or of another parasite transmitted with it. This question can not be positively answered until, by methods akin to those of bacteriology, we shall be enabled to isolate the Texas fever organism and ob- 
serve the transformation of one stage into the other, either in cultures or in the blood of inoculated animals. In the absence of such rigorous proof the presumption is nevertheless strongly in favor of the unity of this and the larger forms already described. We observe in the first place the appearance of both types of the disease in all outbreaks studied at the experiment station since 1889, though at different periods of the same season, the coccus-like bodies being associated chiefly with cool weather. An outbreak produced after the middle of September in 1889 developed cases containing the coccus-like bodies only (Nos. 47, 48, 51, 52, 53, 64, 65). In one of these cases (No. 48), killed in a dying condition, the spleen and liver were affected as in acute cases, but hæmoglobinuria was absent. There are a few (Nos. 66, 69, 95), already referred to, in which there is a transformation of the mild into the acute type with a corresponding change in the form of the parasite.

Perhaps the strongest proof that the coccus-like bodies and the pyriform, amoboid bodies are stages of the same parasite was furnished recently in an unexpected manner. Two cows (Nos. 206 and 219) inoculated with blood from healthy North Carolina cattle early in July, 1892, developed the acute type of Texas fever with the appearance of pyriform parasites within the red corpuscles. Both recovered, and the number of corpuscles was rising toward the normal, when, at the end of August, a relapse was detected in both animals. The number of corpuscles was rapidly falling again and many were infecter with the coccus-like bodies. Reinfection from withont can hardly be considered in these cases, as there were no ticks in the field and two control animals had normal blood throughout the season.*

I'HE PROBABLE LIFE HISTORY OF THE MICROÖRGANISM IN THE BODY OF CATTLE. .

We have thus far presented in a somewhat fragmentary manner the observations bearing upon this microörganism. It now remains to put them together in a way which will illustrate its probable develop.ment.

In the early stages of the high fever in a few acute cases, before the destruction of red corpuscles had gone far, very minute bodies were seen in fresh blood. Their form, so far as determinable (apochrom $2 \mathrm{~mm}$., ocular 8) appeared as an elongated figure of eight or two short rods attached end to end. They had a very active Brownian motion in addition to a movement which carried them from one place to another in the field. This latter movement may have been due to currents in the liquid. They could not be detected in preparations stained with methylene blue. That this is the free form which precedes the parasitic stage must remain at present a mere conjecture.

The (hypothetical) swarming or motile stage (intraglobular).-We have

* Since writing this two other cases (Nos. 222 and 230) inoculated in the same manner have passed through a relapse. 
already (on p. 56) referred to certain very minute, well defined, bright, frequently motile, bodies seen within the red corpuscles of healthy eattle at various seasons of the year. As might have been expected, these bodies were found in Texas-fever blood as well. It has also been stated that they vary more or less both in size and form. The question has frequently presented itself; whether some of these bright motile bodies were the progenitor's of the coccus-like and the pyriform bodies of the Texas-fever parasite. Inasmuch as they are present both in health and in disease, only a most trying examination of the blood in many cases could decide whether certain forms only appeared in disease or not. These bodies are so minute and so inacressible that it is by $u$ means certain whether such a prolonged study would bear fruit. In the course of these investigations such a study was impossible, and we have simply to present the facts that these bodies are present in health and disease and that they vary in size and form. In one case it was difficult not to accept the hypothesis that some of the bodies are a stage of the micro-parasite. In No. 56, affected with the mild autumnal disease, these bodies grew in number with the peripheral coccus-like bodies and disappeared at about the same time. This view is presented simply to serve as a working hypothesis for such as are inclined to follow this phase of the subject more minutely. There is nothing in this hypothesis not in harmony with the positive observations concerning the Texas-fever microbe. Such a motile, swarming stage is one which can readily be conceived of as finding its way into the red corpuscle constantly in motion in the vessels of the body. Why it is not seen in every case may be explained by the same hypothesis which accounts for the presence of the peripheral coccus-like stage in the milder type of Texas fever. This hypothesis assumes a retardation in the intraglobular development of the micro-parasite by which the smaller stages remain long enough in the blood to be detecter. If the retardation is still more pronounced, it is easy to conceive of the motile or swarming stage as circulating in the blood long euough to be detected.

The stage of the peripheral coccus-like bodies.-After the (hypothetical) swarm-spore has penetrated into the corpuscle it comes to rest, loses its bright, refrangent appearance, and attaches itself near the periphery of the corpuscle as a pale body which is only detected with difficulty in the unstained corpuscle. This body next undergoes division which is probably incomplete, for in the more advanced stages the two resulting bodies are as a rule still attached to each other. These remain close together while the infected corpuscle is circulating in the blood. This stage of the coccus-like body, like the preceding hypothetical stage, must be regarded as recognizable because of a retarded development of the micro-parasite. It is probable that this retardation of development in susceptible animals is due to meteorological conditions, such as low temperature of the air, and to partial immunity. In acute attacks the 
enormous multiplication of the parasite in the blood shows how rapid in such cases its development and how ephemeral these intermediate stages must be. The period of retardation may vary in length, but it serms probable that this stage may remain in the cirenlation at least several days.

The stage of the larger forms (pyriform and spindle-shaped bodies).The two cocens-like borlies resulting from division begin to grow and assume fisiform outlines. It is probable that they remain attacher to each other at least for some time, for in stained preparations a very delicate stained line may occasionally be traced passing from one to the other. In this stage they stain very well in hamatoxylin and basic aniline dyes. As they continue to enlarge, the two members of the pair remaining always of the same size, a more elongaterl, pear-shaped ontline is assumed, and in the unstained condition a minute dark particle is observed in the broad end of each body. Under conditions not definable a larger or smaller number of the red corpuscles contain but one body. These unpaired forms are found most abundantly in the circulating blood, where they may manifest amoboid changes.

The larger forms circulating in the blood do not stain so well as the somewhat smaller bodies fonnd in the rapillaries after death. This may be due to degenerative processes or to a transformation into some unknown reproductive state. The annexed figures illustrate, diagrammatically, the intraglobular stages of the Texas-fever parasite, $i$. e., those forms which have only been found in the blood during Texas fever, and very rarely in sonthern cattle.
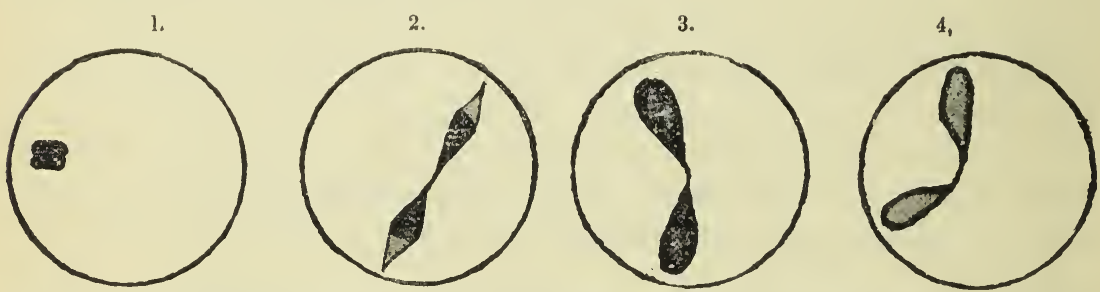

Fig. 3.-Intraglobular forms of the Texas-fever parasite. 'The shaling shows the relative staining capacity with methylene blue. 1. 'The peripheral coccus-like body (0.t $\mu$ long) in process of division (from the mild type). 2. Larger spindle shaped forms from the capillary blood of the heart musch. 'T'he free cuds are but feebly stained. 3. Larger pyriform bolies from the same source staining entirely. 4. Fyriforn bodies ( $2 \mu$ loug, $1.5 \mu$ wide at the widest portion) staining but feebly; from thes (circulating blood. (The last three forms from the acute type.)

Free bodies.-These are set free after they have reached the preceding stage by the disintegration of the infected corpuseles. They may be found in capillary blood of the heart mnscle in abundance. Tlopir most rommon location is in the kidneys, however.

$\mathrm{No}_{0}$ forms which might be interpreted as reproductive stages have been reeognized at any time in the many cases which have been studied. 'That the organism multiplies very rapidly in the blood of susceptible "attle is demonstrated by the fact that the injection of a small quantity of infected blood gives rise to the disease. How does this multiplica- 
tion take place? There are two possibilities in view. Either the large pyriform body, while within the corpuscle or after it is set free, may enter the reproductive stage and produce a generation of very minute borlies akin to the motile, bright intraglobular bodies seen in fresh blood, or there may be a fiee reproductive phase, distinct from the intraglobular forms, taking place in the blood.

TIE NATURE OF THE TEXAS-FEVER MICROÖRGANISM AND ITS RE-

LA'TION TO THE PARASITES OF THE RED CORPUSCLES OF OTHER ANIMALS AND OF MAN.

It has been known since 1881 that the various types of malarial fever in man were accompanied by minute organisms living within the red corpuscles. This discovery by Laveran has been followed by confirmations in various parts of the world, and it is generally accepted that these intraglobular organisms are the cause of malaria. Stimulated by this important discovery, various observers have studied tile blood of many animals (frog, turtle, and various birds) and have found therein certain minute parasites which likewise pass their life chiefly within red corpuscles. Much has been written upon the interrelation of these forms and their bearing on malaria in man. Nothing positive, however, has come of it, although there is a close resemblance between some of the parasites found in birds and those found in mall. They all have in common the peculiar habit of living in the red corpuscles. Those of cold-blooded animals (frog and turtle) do not contain any pigment granules. On the other hand, those of birds and man do, as a rule, contain. granules of dark pigment which is derived from that portion of the red corpuscle destroyed by them. These pigment granules are by some regarded as the more abundant the more retarded the growth of the parasite, and consequently the slower the destruction of the containing corpuscle. The parasites found in the blood of birds and man first appear as minute, slowly enlarging amoboid bodies in the red corpuscles. Soon pigment granules appear. When of a certain size these bodies break up within the corpuscles into a variable number of spores. These are set free and begin life as a new generation by entering other red corpuscles and undergoing the same development. These cell parasites are not associater with a visibly diseased state of the animals in which they are found. In man it is well known that certain kinds of fevers known as malarial are produced by them.

The Texas-fever parasite differs in many important respects from all those thus far described. Its morphology is quite unique. It contains 110 pigment. It probably runs through its whole development in a short time, otherwise it would be difficult to account for the rapid destruction of red corpuscles. Nevertheless, no distinctiy reproductive phase has heen seen during four years of observation of a great variety of cases.

It is hardly within the scope of this report to go into any details con- 
cerning the parasites of the red corpuscles of other animals and of man. The literature of this subject has now grown quite voluminous, although the progress made is not very great, owing to the limitations of methods. None of these cell parasites have thus far been cultivated according to bacteriological methods, and it is not likely that they ever will be. Inoculations of blood containing them seem to succeed only when they are made on the same varieties of the same species of animals, according to Celli and Sanfelice, ${ }^{*}$ and even then success is limited to a small number of the cases inoculated. Observations are thus of necessity statistical and comparative rather than experimental, and must extend over a large number of cases before the significance of the various facts observed can be even formulated. Even then it is difficult to impart to others the conviction which comes from prolonged observation, while the desultory observations of many individuals lead to wide differences of opinion.

Classification of the parasites of red corpuscles has been attempted by Kruse and others and the various forms indicated under the following scheme: $\dagger$

\begin{tabular}{|c|c|c|}
\hline Genus. & Species. & Tariety. \\
\hline 1. Hoenogregarina (Danilewsky) .......... & 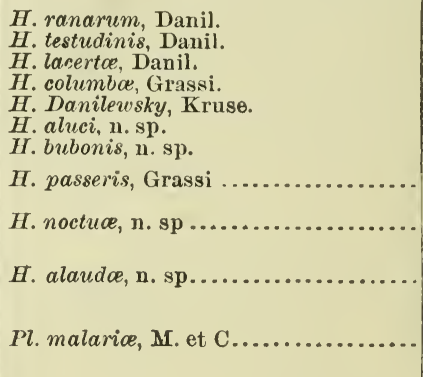 & $\left\{\begin{array}{l}\text { a.' } \\
c . \\
a . \\
c . \\
a . \\
b . \\
c .\end{array}\right.$ \\
\hline
\end{tabular}

1 The letters refer to the relative rapidity with which the development goes on; $a$ signifies slow; $b$, accelerated; $c$, rapid.

It seems improbable that the Texas-fever parasite will ever be ranged under any of these genera, and therefore a new genus has been created for it (Pyrosoma). The specific name (bigeminum) is derived from the peculiar character which this organism has of appearing in pairs within the red corpuseles. This name does not commit it to any special group of protozoan parasites, although it is not improbable that it may belong to the sporozoa to which most cell parasites belong. The peculiar pair of pyriform bodies within the red corpuseles might be homologized with the falciform bodies or crescents of the sporozoa, which in this case are without a cyst of their own and make use of the cell wall for this purpose.

* Fortschritte der Medicin, 1891. Nos. 12, 13, and 14. †Loc. cit., No. 14. 
The great rapidity with which this parasite multiplies in the system of susceptible cattle may perlaps be explained along lines suggested by R. Pfeiffer* in his observations on Coccidium oviforme and $C$. perforans in the rabbit. These two species of sporozoa (the former inhabiting the bile ducts of the adult rabbit, the latter the intestinal epithelium of the young rabbits), Pfeiffer maintains, are one and the same which runs through a rapid entogenic development, with the formation of an immense number of individuals in the intestines of young rabbits, and thereby causes a severe (mostly fatal) disease, while in the adult it remains largely restricted to the liver, where it rums through a slow partial development, and the spore produced requires conditions only found externally for its further development. Applying these observations to the Texas-fever organism, we may assume some dual development of the parasite, one taking place in susceptible, the other in insusceptible animals. Or the conditions may be much simpler in the latter, and depend largely on a simple repression of the multiplication of the parasite in the red corpuscles, owing to some unknown modification of these bodies.

PROBABLE ACTION OF THE MICROÖRGANISM IN THE BODY OF SUSCEP'TIBLE ANIMALS.

This topic has been touched upon in the foregoing chapters, but only in a desultory manner, and the known facts are brought together here in a more compact form. The demonstration of the microörganism in the organs does not present any special difficulties. The organs were examined fresh or after being in the ice-chest for twenty-four hours, both in teased preparations and in sections. The teased preparations as well as the sections were examined in iodized serum to avoid any injury to the blood corpuscles. For the same reason the sections were prepared with the razor and not on the freezing microtome. In such thin sections or in teased preparations some of the capillaries frequently remained intact and the microörgauisms could be made out as pale, roundish dots within the red corpuscles. Plate vir, Fig. 3, is drawn from an unstained teased preparation of the fresh spleen. Tissues were hardened both in strong alcohol and in Müller's fluid and alcohol, according to the usual procedure. The staining presented some difficulties, and in general the aniline dyes did not give satisfactory results. Hæmatoxylin in the form of Ehrlich's acid hæmatoxylin has proved very satisfactory in bringing out the intraglobular parasites. In tissue hardened in Muiller's fluid the outer layers showed a peculiar modification of the parasites. Instead of appearing as blue bodies, they assumed a brownish-red color. This was especially noticeable in tissues which are very delicate, such as the choroid plexus. In these all the parasites appeared brownish red instead of blue. This may be due to the action of the

* Beiträge zur Protozoen-Forschung. I. Heft. 
Miiller's Huid. 'Tissue hardened in alcohol, while it demonstrates the intraglobular bodies very distinctly, does not preserve the corpuscles so well.

The destruction of red corpuscles by the micro-parasite within them is the main fact in the pathology of Texas fever. There are, however, some secondary phenomena associated with this destruction which acconnt in part, at least, for the peculiar lesions of this disease. The infected corpuscle remains in the circulation as long as the contained parasite is below a certain size. 'Thus, in the mild autumnal form of the disease, the number of circulating corpuscles invaded by the cocrus. like bodies is rarely below 5 per cent, commonly from 10 to 30 per cent, and at times near 50 per cent. The corpuscles are not changed in form or size, and in fact the parasites are very small when compared with the size of the corpuscle and only brought out distinctly by staining. When the parasite has reached a certain size we may assume that the rorpuscles lose their flexibility and adaptability to the minute channels or capillaries and become lodged in them, forming emboli, so to speak. The capillary becomes obstructed and red corpuscles are wedged in behind the infected ones. It is highly probable that at this time a favorable opportunity is offered to the free, earliest stage of the microorganism to attack the remaining corpuscles, otherwise it would be difficult to understand why capillaries are frequently found which contain, to all appearances, only infected corpuscles. It may be that other fiactors come into play, such as the changed specific gravity of the infected corpuscles, by which their relation to the blood plasma becomes changed and in virtue of which they act as foreign bodies in the vessels. Whatever may be the reason, the fact remains that the paucity of infected corpuscles in the blood taken from the large vessels and the heart is counterbalauced by a very extensive infection of the corpuscles found in the capillaries. The smaller the capillary, the higher the percentage of infected corpuscles. In the white substance of the brain, in No. 198, for instance, the capillaries in a section were found conpletely filled with red corpuscles and every corpuscle was infected. Similarly, capillaries have been observed both in sections of hardened tissue and in teased preparations of fresh tissue from the spleen, kidneys, intestinal mucosa, omentum, plexuses of the brain, heart mus. cle, and red marrow of the ribs, which were either completely or partly filled with infected corpuscles alone.

This capillary plugging or embolism may account, to some extent at least, for the enormous distention of the spleen and for the degenerative changes of the parenchyma of the liver. The perinephritic sanguinolent redema so frequently observed on post-mortem examination is probably due to the complete filling up of the vascular system of the kidneys with infected and uninfected corpuseles. Similarly the ecchymoses in the calyces of the pelvis may be accounted tor by this condition. 
Another phenomenon of interest is the remarkable injection of all pathological growths of a vascular character, such as old fibrous arlLesions and pleuritic fringes. Similarly vascular fringes found on the omentum covering the paunch, and on the origin of the large vessels at the base of the lieart appear as dark-red spots. Here microscopic examination shows the same abundant infection. The intense injection of the vascular plexuses of the brain cavities is associated with extensive infection within the apillary network. This injection of the entire capillary system of the body may be largely aided by the presumable rise of blood pressure which must take place after the enlargement of the liver and the engorgement of the spleen and kidneys practically shut these organs out of the general circulation.

While the skeletal muscles show but slight infection, the heart muscle is severely involverl. Probably owing to the smaller caliber of its capillaries and the constant contraction of the muscular walls, a favorable opportunity for the lodgment of infected corpuscles is afforded. In the capillaries of the heart muscle the infection of corpuscles is always very great and freed forms of the parasite are abundant. The apillary plugging will account for the almost constant ecchymoses of the external and internal surfaces of the ventricular walls.

The breaking up of the infected corpuscles probably takes place in the capillaries over the entire body, for, as stated above, freed parasites are found in the capillaries of the myocardium after a certain perior of fever. This leads to the presence of free hamoglobin in the blood (hæmoglobinæmia). This condition was strikingly demonstrated in the case of a foetus about three months old taken from a cow which had suceumbed in the acute stage. The amniotic fluid had a beantiful wine-red color. It is probable that many infected corpuscles which break away from the capillary plugs are carried into the kidneys, where the final dissolution takes place. Otherwise it would be difficult to account for the usually enormous numbers of free as well as intrag lobular parasites which are found in the kidneys toward the end of the fever.

The hæmoglobinuria observed in nearly all acute cases may be due in part to a transudation of the hæmoglobin dissolved in the blood, in part to the destruction of red corpuscles in the kidney itself. The same may perhaps be true of the material transformed into bile by the liver. This organ is as a rule heavily infected, though not to the extent observed in the kidneys. The bile contains to all appearances a very large quantity of bile pigment. This may be derived in part from the dissolved hæmoglobin in the general circulation and in part from the corpuscles undergoing destruction in the capillaries of the liver itself.

The cause of the high temperature in the later stages of the fever, when the pathological changes are well under way and when the blood is loaded with the débris of corpuscles and free parasites, may not be open to discussion. In the earliest stages, however, the explanation of 
waste products in the blood does not seem to follow in every case. In a few the fever was high, although there was no apparent reduction of red corpuscles. (See Nos. 166, 180, 198, 206, 219.) It must be stated that in view of the fluctuations to which the number of corpuscles is subject the counts in these cases may be somewhat misleading. Yet on the whole the initial fever seems to be caused by something other than the destruction of the red corpuscles, and we may invoke two possible causes, leaving their determination to more accurate continued observations on single cases. These are the multiplication of the parasite in. the blood, perhaps independent of the corpuscles and the thrombosis of capillaries in the nerve centers.

The question of a cyclical destruction of red corpuscles corresponding to the different generations of parasites is an interesting one, but the observations put on record in the appendix do not give us any definite information. In acute cases after the first few days the fever is continnous or nearly so and does not indicate any intermission or remission of the acting cause. Whether the individual generations follow one another so rapidly or whether there are a number of generations intermingled has not been determined. To the eye there is more or less uniformity in the size of the parasites observed in any given case throughout the body. They may be all minute in the stage of the coccus-like bodies or they may all be unusually large (No.66) or they may all be in a stage intermediate between these extremes. It should be stated, however, that in a few cases the fluctuation in the destruction of the red corpuscles was regular enough to suggest a period of from one and a half to two weeks in such cases. (Page 40.)

What becomes of the micro-parasites in those cases which recover? We have already signalized the setting free of the parasites and their accumulation in large numbers in the kidneys. Further than this the observations do not go. The parasites are perhaps destroyed by a combination of circumstances, one of which is the small number of red corpuscles finally left for infection. Thus in blood containing only one and a half to two million red corpucles fully one-half are enlarged, embryonic forms which may not be so well fitted for the growth of the parasite. Another eircumstance may be the unfit condition of the blood due to the presence of the very débris which the parasites have aided in producing.

OUTBREAKS IN WHICH THE TEXAS-FEVER PARASITE HAS BEEN DEMONSTRATED.

The parasite of Texas fever, or more particularly the coccus-like and the larger pyriform stage of this microörganism, have been demonstrated in the following outbreaks:

(1) In the splecn of a case from an outbreak in Virginia, September, 1886.

(2) In the organs of cases from an outbreak in Maryland, September, 1888.

(3) In the blood and the organs of eases from an outbroak on the experiment sta. tion (North Carolina infection), August to October, 1889. 
(4) In the blood and the organs of cases from an outbreak on the experiment station (North Carolina infection), September to November, 1889.

(5) In the blood and the organs of cases from an outbreak on the experiment station (North Caroliua infectiou), August to November, 1890.

(6) In the blood and the organs of cases from an outbreak on the experiment station ('Texas infection), August to October, 1890.

(7) In the spleen of a case which died in North Carolina, June 29, 1891.

(8) In the blood and the organs of cases from an outbreak on the experiment station (North Carolina infection), August to November, 1891.

(9) In the blood of a case from an outbreak in Penusylvania, November, 1891. (Specimens of organs and urine sent by George Jobson, jr., v. s.)

(10) In the organs of a case at Fort Smith, Ark., March, 1892. (Preparations sent by R. R. Dinwiddie, v. s.)

(11) In the organs of a case produced by the intravenous inoculation of blood from North Carolina cattle, July, 1892.

(12) In the organs of cases from an outbreak produced at the experiment station in the usual way by North Carolina cattle, August and September, 1892.

(13) In the organs of cases from an outbreak in New Jersey, August, 1892.

(14) In the spleen and blood of cases from an outbreak at Camden, N. J., August. 1892. (Specimens sent by Drs. Miller and Sellers.)

In Nos. 7 and 13 there were the usual lesions (hæmoglobinuria, etc.,) observed by Dr. F. L. Kilborne at the autopsies. In No. 9 there were the usual fatty degeneration and bile injection of the liver and hæmo. globinuria. The diagnosis of Texas fever was thus assured in all the outbreaks mentioned.

THE PRODUCTION OF TEXAS FEVER IN CATTLE BY THE INOCULATION OF BLOOD FROM CASES OF THIS DISEASE.

The demonstration that Texas fever is caused by a certain microörganism is not absolutely made by showing that it is always associated with this disease and not observed in health. It may be argued that such bodies are the concomitant rather than the cause of the fever. Nevertheless it may be said that no microörganism constantly associated with a given infectious disease has yet been found which is not demonstrably or presumptively the cause of the disease. Hence the probability that the micro-parasite described is the canse of Texas fever is very high, although the demonstration can not be made until such organism can be cultivated in some manner outside of the animal body and inoculations made with pure cultures. There is nothing today to encourage us in the hope that parasites so highly adapted as the one under consideration will ever submit to the crude culture methods successful with many bacteria.

The high probability that we have the cause of Texas fever before us is increased by the fact that when blood from cases of this disease is injected into the circulation of healthy susceptible cattle, the disease is produced and the micro-parasite appears in the blood under the same conditions under which it becomes manifest in the natural disease. There is still the possibility before us that the microparasite is transmitted in the diseased blood and that some unknown agent has been 
transmitted with it which is the true cause of the infection. It is useless to discuss this further, and each reader must form his own opinion of the value of the experimental evirlence adduced in this report.

Before quoting our experiments in the production of the disease, a few observations on the attempt of others to produce it are in order.

Dr. J). E. Salmon in 1880 (4, p. 303), made a number of inoculations with tissues and fluids taken from cases of 'Texas fever, some of which were successful:

(1) November 7, 1879. Calf 6 to 8 months old inoculated subcutaneously with bile and blood kept ten days in a sealed pipette. No result.

(2) September 14, 1881. Yearling inoculated subcutaneously with $5 \mathrm{~cm}^{3}$ blood from a case dead three or four hours. No result.

(3) September 29 . Yearling bull inoculated subcutaneously with $5 \mathrm{~cm}^{3}$ blood containing some spleen pulp, which had been kept twenty-two hours in sealed pipette.

(4) Red cow inoculated as No. 3; also drenched with a mixture of blood, urine, and bile.

(5) Heifer received a subcutaneous injection of $5 \mathrm{~cm}^{3}$ of bile.

(6) Bull 3 years old drenched with one ounce of urine.

(7) Steer 2 years old drenched with one ounce of bile.

(8) Cow received $5 \mathrm{~cm}^{3}$ of urine under the skin.

Of the cases from Nos. 3 to 6 , inclusive, No. 3 and No. 4 reacted with a high temperature and No. 4 became very weak and emaciated. In $1883(5$, p. 34) three additional experiments are reported. A steer and a heifer, $2 \frac{1}{2}$ years old, received August 7 subcutaneous injections of spleen pulp suspended in water. The spleen pulp had been kept in a sealed tube for seven days. Neither animal became aftected. A third animal, a cow which had been moculated subcutaneously with fresh splenic pulp, October 3 , was taken sick in ten days and died three days thereatter. There were evidences of hæmoglobinuria. Two young animals drenched with the same splenic pulp did not become seriously affected.

A number of additional inoculations were made with cultures of a micrococcus cultivated from the spleen of a case of Texas fever with negative results in all cases.

Dr. Billings gives the notes of a case inoculated with eultures of what he regards as the Texas fever bacterium $(8, \mathrm{p}$. 100). We have already commented on this rase on p. 5:. In this commection it is sufficient to saly that the proof of T'exas fever has not been brought in this rase althongh it shonld have been above reproach since it is supposed to establish the etiology of Texas fever. The observations in this report show that there is no Texas fever without a marked reduction in the number of red corpuseles. This is the essential sign of Texas fever. Secondary to this are lesions of liver, spleen, and kidneys, and hemo. globinuria and the presence of embryonic corpuseles in the bloot. There is nothing in the antopsy notes as published by Billings to demonstrate the presence of 'Texas fever in the absence of red water. It is also cmious that in his experiment the young animal of 5 months should take the disease more severely than the "large red cow," since calves are proverbially resistant. 
In the report of Panl Paquin (9, p. 46) we find the following statements:

Texas ferer is transmissible not only from Sonthern stock to susceptible Northern cattle, but under favorable circumstances is inoculable between Northern natives, although in the ordinary conrse of things in our climate transmission does not ocenr. We have inoculated native Missouri eattle with spleen and liver pulp from other diseased natives and produced typical cases of Texas fever, but it took large doses of virus. The rapidity of the course of the malady depends much on the origin aud age of the virus. It was more rapid from old pulp kept in warmth and properly preserved than it was from virus of fresh matter, and it seems impossible to ranse severe Texas fever with fiesh urine, whilst the same exposed to warmth awhile becomes dangerous.

There are no experiments reported to convince the reader of the truth of these statements, though the direct transmission of disease from Southern eattle and sick natives to susceptible cattle by inoculation has been confirmed by us. Why old spleen pulp and old urine should be more dangerous, excepting as producers of septic conditions, is by no means clear. We should believe the contrary. We have no information at all as to how the inoculation was made or any to show that the inoculated animals did contract Texas fever excepting the bare statement that the inoculations were successful.

R. R. Dinwiddie (10) made subcutaneous inoculationsupon four different animals with fluids and tissues from cases of 'Texas fever. We are glad to see the experiments reported so that they may be estimated at their true value. The inoculations were made with fresh urine, spleen pulp kept over night, with bile kept in a sealed pipette, and with a culture of a micrococens from the liver of a case of Texas fever. These inoculations proved negative. A fifth animal which received spleen pulp kept over night as a drench remained well. We have no reason to donbt the accurary of these results. The negative outcome may have been due to the fact that only young animals were used and that the season was perhaps too advanced for experimental cases to snceed.

In all of these experiments the uncertainty of the couchusions reached as regards the negative results must be evident to all who have read the foregoing part of this report. Many of the cases, which to all ap. pearances were not affected, may have passed through a mild attark, recoguizable only by the microscopic examination of the blood corpuscles and a determination of their number.

Our own experiments were made mainly with fresh material, and this; was injected under the skin and into the blood directly. Nine inoculations were made in all.* We shall in this place only refer to the important points in each case, and leave the reader to consult the appendix for fuller details.

(1) On September 1, 1890, No. 111, a heifer about 21 months old, received into one of the jugular veins 13 cc. of whipped blood. This was

* Five additional cases of Texas fever were produced with the blood of healthy North Carolina cattle (page 119). 
obtained from No. 128, which had just died, and in whose blood there was a large number of infected corpuscles. The defibrinated blood was kept in a warm chamber at $35^{\circ} \mathrm{C}$. for three hours before the injection. An examination of the table in the appendix shows a decided fall in the number of red corpuscles ou the thirteenth day, and several days thereafter a cousiderable number of new red corpuscles (macrocytes) were found in the blood. There can be no doubt that this was a mild case of Texas fever. The subsequent gradual weakening of this animal and death 3 months after the inoculation could not be accounted for.

(2) On September 16, 1890, a similar injection with defibrinated blood was made on No. 142. The blood was taken from the heart of No. 90 about one-half hour after death, and, after defibrinating, it was kept at $35^{\circ}-40^{\circ} \mathrm{C}$. for one and one-half hours before it was injected. In the table we observe a marked fall in the number of red corpuscles at three different times from two to three weeks apart*. The animal fully recovered subsequently. Neither of these cases would probably be considered conclusive evidence that the disease can be reproduced in this way. The seven following cases will dispel any doubt on this point.

(3) On September 19, 1891, a portion of the heart muscle of No. 181, just dead, was pounded in a mortar with sterile normal salt solution. The resulting reddish fluid was filtered and injected into the jugular vein of No. 182, after standing in a warm chamber for about one hour. The table in the appendix with this case leaves no doubt as to the nature of the disease. The temperature (see appendix) rose on the sixth day in the evening and a high evening temperature was observed for ten days thereafter. A high morning temperature was first noted on the eighth day and the fever remained continuously high for at least four days thereafter. The number of blood corpuseles had fallen from $6,000,000$ to $2,000,000$ eleven days after the inoculation. The Texas-fever parasites were found in the blood. The animal fully recovered subsequently.

(4) On the same day blood was withdrawn from the jugular vein of No. 181, then still alive, and injected at once into the jugular of No. 185. The whole operation lasted one or two minutes. Of this blood, which contained at the time perhaps one-half to one per cent infected corpuscles, two syringefuls, or $28 \mathrm{cc}$., were injecter. The disease produced in this animal was severe enough to leave no doubt as to its nature. The evening temperature was high on the third day and was low again on the ninth day. The continuous high temperature lasted four days. The number of blood corpuscles had fallen from 5,000,000 to $2,000,000$ on the tenth day. The Texas-fever parasites were foumd in the blood. The animal fully recovered subsequently.

(5) No. 186 was treated precisely as No. 185 at the same time. A

*See appendix for record of control animal No, 143 in the same inclosure, 
rery severe case of Texas fever was the result. The temperature and the loss of red blood corpuseles were the same as in No. 185 (see page 17 for curve). On the ninth day she could scarcely stand and was trembling and quivering over the whole body. A syringeful of blood was withdrawn at the time from a jugular vein for other inoculatious, and the operation was followed at once by convulsions and death. The very advanced lesions of the liver and spleen, the dark red, portwine-colored urine, and the immense number of infected corpuscles in the various organs, made this case one of the most severe of the seasoll.

In 1892 four cows were inoculated with blood obtained firom a case of the disease. All became affected within a week and three diel. The more important facts in connection with these inoculations are reproduced here.

On August 27 blood was withdrawn from the left jugular vein of No. 222, then suffering with the disease. In the blood a small number of large intraglobular parasites were found. The skin over the jugular was shaved and washed with .1 per cent mercuric chloride and the vein opened with a scalpel. The blood was caught in sterilized bottles, containing glass beads, and defibrinated by shaking vigorously for ten minutes. The bottles were kept in a water bath at $40^{\circ}-42^{\circ} \mathrm{C}$. The injections were performed not longer than fifteen to twenty minminutes after the withdrawal of the blood from No. 222 .

(6) No. 197, a cow 6 years old, received into the left jugular 14 ec. (one syringeful) of this blood.

(7) No. 227, a cow 11 years old, received under the skin of the neck $\frac{1}{2}$ cc. of the same blood in four different places, $i$. e., 2 ce. in all.

(8) No. 228, a cow 7 years old, received subcutaneously $\frac{1}{2}$ cc. in two places, i. e., 1 cc. in all.

No. 197 died quite unexpectedly September 4, eight days after the inoculation. The temperature had been high since August 31. The autopsy left no doubt as to the nature of the disease.

No. 227 died September 9, thirteen days after the inoculation. The temperature had risen and other symptoms of disease had appeared September 2. On the day of death the red corpuscles had fallen to 1.5 million. The autopsy revealed the usual lesions of Texas fever in a very marked degree. The urine was visibly free from hrmoglobin. The small number of red corpuscles just before death indicated that the period of hæmoglobinuria was past.

No. 228, which had received the smallest dose, reacted as promptly as the foregoing with a high temperature. The usual symptoms appeared, but more tardily, and the animal finally recovered. On September 14 the red corpuscles numbered 1,5 million. From this time there was slow improvement in the condition of the blood.

(9) One bottle of the defibrinated blood with which the preceding animals had been inoculated was placed in a refrigerator at an average 10320-No, 1—6 
temperature of $50 \circ$ F. $(10 \circ$ C. ) from August 27 until August 30. On this day $14 \mathrm{ce}$. (one syringeful) was injected into the left jugular vein of No. 200, a cow 8 years old. After five days of elevated evening temperature and two of continuous high temperature, this animal succumbed September 8 . The organs presented the usual lesions of Texas fever. The urine had a dark port-wine color. In the various orgaus and the blood many infected corpuscles were detected.

With these positive results before us we need not hesitate to make the statement that there is something in the blood of cattle during 'Texas fever which, introduced into the body of healthy susceptible cattle, gives rise to the disease. This something is capable of repro. ducing itself indefinitely in the blood of susceptible animals. In all cases there had been multiplication of the Texas-fever parasite, and these inoculations furnish additional proof that this parasite may be regarded as the cause. These inoculations show, also, that a comparatively small quantity of blood from diseased cattle placed under the skin is capable of causing a severe and even fatal infection. In this respect the microörganism seems to have as powerful an effect as the bacteria which produce acute fatal forms of septicæmia and seems to be capable of almost equally rapid multiplication. The sojourn of three days in a refrigerator did not destroy the vitality of the microörganism as it exists in the blood. The very severe inoculation disease produced in 1891 and 1892, as compared with 1890, is partly to be accounted for by the fact that only old animals were used latterly, while in 1890 the animals were young. The observations made in the field experiments and by former observers, that the susceptibility seems to increase with age, provided there has been no exposure to the disease at any time in life before, is thus indirectly confirmed by inoculation. The very striking susceptibility of cattle to this disease was furthermore demon strated by the intravenous inoculation of three guinea-pigs at the same time with three of the cases eited above (Nos. 6-8, inclusive). These animals remained perfectly well, though they had received relatively to their body weight a very mueh larger quantity of the defibrinated blood. (Page 84.)

THE INOCLLATION OF ANIMALS OTHER 'IHAN CAT'TLE WITH TEXASFEVER BLOOD.

The inoeulation of animals other than cattle had a twofold purpose: first, to determine whether other domesticated animals are likely to become infected with the micro-parasite and perhaps canse the dissemination of 'Texas fever, and, second, to find some small animal to take the place of the much more costly cattle in the study of the parasite and the disease. This was especially desirable since this parasite can not be cultivated ontside of the animal body.

Sheep.-CSince sheep and cattle are so closely related it was thought that the disease might perhaps be induced in them. For this purpose 
a lamb was used. A syringeful (7 ce.) of blood was drawn from the right jugular of cow No. 184, which was very sick at the time and whose blood contained the micro-parasite in small numbers, and injected at once into the left jugular vein of the lamb. The operation was performed October 1, 1891; the blood contained 10,442,000 red corpuscles in a cubic millimeter. No parasites of any kind were detected in thein.

October 13.- Red corpuscles 8,282,000. Nothing abnormal detected.

October 2\%.- Red corpuscles 11,538,000. Several bright intraglobular bodies seen in the fresh preparation, but no parasites.

From October 1 to October 27 the temperature was taken twice daily. It fluctuated between 101 and 103 .

Though the inoculation was made somewhat late in the season the outcome plainly indicates no susceptibility of sheep to this disease.

Rabbits.-September 20, 1889, immediately after cow No. 51 had been killed a quantity of spleen pulp containing many corpuscles infected with large paired parasites was mixed with sterile salt solution. The reddish liquid was injected into the ear vein of three rabbits. No rise of temperature and no symptoms of disease were noticed. One rabbit was killed on the seventh day and the blood and organs carefully examined for infected corpuscles with negative result. The others were watched for several months, bnt nothing abnormal detected in their action. The second rabbit, which had become scabby, was killed Janu. ary 18,1892 . The various organs and the blood were examined micro. scopically with negative result. The following may also be eiter:

October 1, 1891.-With the blood of cow No. 184 two rabbits (Nos. 140, 141) were inoculated at the same time with the lamb. Each received 1 cubic centimeter into the ear vein.

No. 140 (black rabbit) showed no external symptoms of disease. The blood was examined twice and no infected corpuscles found.

October 19, 1891, 6,537,000 red corpuscles in a cmim.

December 3, 1891, 7,134,613 red corpuscles in a cmm.

No. 141 (white rabbit) remained equally well. The following blood examinations were made:

October 19, 1891, 5,268,000 red corpuscles in a $\mathrm{cmm}$.

December 3, 1891, 4,533,000 red corpuscles in a cmm. Infected corpuscles absent.

Pigeons.-September 28, 1891. Blood containing infected corpuscles is drawn from the jugular vein of cow No. 186 and injected at once into the wing vein of three pigeons (Nos. 2, 3, and 4). In a foruth pigeon (No. 1) the blood failed to enter the vein and was deposited in the surrounding connective tissne. Each received about 1 ce.

No. 1 died October 13, though not from the inoculation, as its feather's were ruffled at the time of the operation and it was probably not well at that time. Examination of the blood and organs negative. The other pigeons remained well. On October 5 the blood of No. 3 contained 3,926,800, that of $\mathrm{No}_{0} 4,4,094,300$, red corpuscles, in a cubio millimeter. They were killed Jannary 22, 1892. The blood of No. 3 was searched in vain for parasites. 
Guinea-pigs.-August 27, 1892. Blood was drawn from the left jugular of cow No. 222 affected with 'Texas fever into sterile wide-mouthed bottles containing glass bearls and defibrinated by slaking vigorously. Three guinea-pigs were inoculated: No. 1 received into the exposed jugular 1 cr. of defibrinaterl blood; No. 2 receiverl into an ear vein $\frac{1}{2}$ a.; No. 3 received into an ear vein $1 \mathrm{cc}$.

The injections were completed fifty to seventy minutes after the blood had been drawn from No. 222. The injection into the ear vein was a perfect success in the two cases on which it was tried. These guinea-pigs remained entirely well. The blood was examined from time to time both in fresh and in dried and stained preparations, but the corpuscles were not counted, owing to the pressure of other work. There was no evidence, however, from the microscopic examination of any change from the normal-condition or of any infection. The guineapigs were watched for more than a month after the inoculation.

Strongly contrasting with the result on guinea-pigs is that obtained with the same blood on cows. (See page 82 and Nos. 197, 200, 227, and 228.) The largest quantity injected into the eirenlation of the guinea-pigs was relatively to the body weight not less than twentyfive times greater than the largest dose and three hundred times greater than the smallest dose injected into the cattle. Yet all four cows contracted Texas fever and three died.

Of other observers who have tried to produce Texas fever in other animals we find Paquin (9, p. 46) making the following statement: "We have succeeded also, though with great difficulty, to induce the disease in sheep, guinea-pigs, white mice, white rats, aud very rarely rabhits, kittens, and swine. The germs may be reproduced by inoculation of liver and spleen pulp in any of these subjects, but the quantity must be large and the gross typical spleen lesions are not always to be found." Inasmuch as spleen lesions are associated with a variety of infectious and septic diseases in animals, and as there is no record of other lesions peculiar to Texas fever in these inoculated animals, we are compelled to eall in question the accuracy of the diagnosis in these cases.

The inoculations made by us demonstrate that sheep, pigeons, rabbits, and guinea-pigs are to all appearances insusceptible to this disease, whereas in cattle the disease may be invariably produced by the injection of infected blood. It is to be hoped that opportunity will be presented the comiug summer to try other species of animals. 


\section{THE TRANSUISSION OF TEXAS FEVER BY MEAIS OF THE CATTLE TICK.}

Boöphilus boris (Riley) Curtice.

It has been a more or less prevalent theory of cattle-owners in the districts occasionally invaded by Texas fever from the South that ticks are the canse of the disease. Mr. J. R. Dodge, (2) in his historical report of this plague, mentions the fact that in 1869 an outbreak in Chester County, Pa., was believed to be caused by ticks. Gamgee in 1868 (2) states: "The tick theory lias acquired quite a renown during the past summer, but a little thought should have satisfied any one of the absurdity of the idea." The officer's of the Metropolitan Board (1, p. 1084) and most subsequent observer's seem to have entertained the same view of the harmlessness of the cattle tick as a carrier of the infertion. In fact few observers have given it any thought. In the entire report of $\mathrm{F}$. S. Billings we find no reference whatever to these pests. Paquin $(9, \mathrm{p} .45)$ states that he has "found the parasites also in ticks bloated with blood of infections southern cuttle. So this must be added to the list of sources." But the ubiquity of this "germ " rather predisposes one against any belief in its existence if" we did not have sufficient positive evidence that bacteria have nothing to do with the disease. 'The statement thus depends simply upon the tinding of a "ger'm" in adult ticks resembling that found in diseased cattle and in fact everywhere else (waters, soil, manures from the South, urine, bile, liver, spleen, kidneys, etc., of infectious Northern stock). Experiments to demonstrate the relation which ticks bear to Texas fever were not made.

Nothing positive was thus contributed to the elucidation of the action of ticks in carrying the disease until the subject was taken up at the Experiment Station of the Bureau near Washington, in 1889. Here it was found by experiments to be detailed in the remainder of this report that the disease can be produced by ticks hatcherl artificially in the laboratory, without the presence of sonthern cattle. Before giving in detail the experiments which led up to the final determination of this important discovery a few facts concerning the cattle tick which have come under our observation are necessary for the information of the general reader. We do not propose to give anything more than a general account of the tick, leaving problems of biology and mor. phology to those pursuing special lines of work in this tield. 


\section{'HE CA'T'TLE 'T'IC (Bö̈philus bovis).}

Plate $\mathrm{X}$.

'The first description of this parasite was made by Prof C. V. Riley, in 1868, under the name Ixodes bovis (2, p. 118):

Ixodes bovis Riley. - A reddish, coriaceous flattened species with the body oblongoval, contracted just behind the middle, and with two longitudinal impressions above this contraction, and three below it more especially visible in the dried specimen. Head short and broad, not spined behind, with two deep, round pits. Palpi and beak together unusually short, the palpi being slender. Labium short and broar, densely spined beneath. Mandibles smooth above with terminal hooks. Thoracic shield distinct, one-third longer than wide, smooth and polished; convex, with the lyrate medial convexity very distinct. Legs long and slender, pale testaceons red; coxæ notspined. Length of body 0.15 of an inch ; width 0.09 of an inch.*

The generally accepted idea as to the harmlessness of this parasite causer it to be neglected as an object of study until 1889, when our preliminary experiments seemed to indicate that ticks must be present to convey the infection from southern to northern stock. Hence, Dr. Cooper Curtice, at that time in charge of the investigation of animal parasites, began the study of the life history of this species.t It was discovered quite accidentally that adult females kept confined in bottles or other glass receptacles always lay their eggs. Such a stock of eggs furnished the starting point of Dr. Curtice's investigations. The eggs were placed in covered glass dishes containing a little soil and kept in a warm place. After a period of three to four weeks the young ticks appeared. These were placed on a calf kept in an artificially heated stable, as the season was already advanced (November 15). 'The earliest or larval stage as it emerged from the ovum had three pairs of legs. After one week's sojourn on the calf it was ready to moult. The emerging nymphal stage was provided with an additional pair of legs. After another week's life on the calf the tick was ready to moult a second time and become sexually mature. Curtice thus showed that in this particular species there are two periods of moulting before the parasite becames matured. He likewise created for it a new genus (Boöphilus). Dr. George Marx has given more or less attention to the classification of ticks, and places the species under consideration as followst: Cliss, Arachnida; order, Acari; suborder, Cynorhosta; family, Rhipistomida; genus, Bö̈philus; species, bovis.

In our experiments with this cattle tick we have confirmed and extended the observations recorded above chiefly in the direction of the life history, since this is the most important aspect in its relation to 'Texas fever.

\footnotetext{
* We sinply quote this description here as a matter of historical interest, withont romment as to its acenracy. We may state, however, that the color of adult females is not reddish. The back is olive brown, the belly slate colored. 'The dinensions given in this diagnosis probably belong to an adult male. For the dimensions of the parasite in its different stages, see this chapter.

t'The biology of the cattlotick. Jomrn. of Comp. Medicine and Veteriuary Ar(chives. July, 1891, aud Januiny, 1892.

†Proc. Hntomological Society of Washington, II, p. 292.
} 
The laying of the eggs may be observed by anyone by simply placing full-grown ticks in some vessel from which they can not escape. The tick remains quiet for from two to four and one-half days, according to our observations; then a few eggss will be observed on the mouth parts, which gradually increase in number. The period of oviposition varies somewhat. Confined in bottles, for instance, at a temperature of $68^{\circ}$ $78^{\circ} \mathrm{F}$. the laying was observer to continue from eight to fifteen days in a lot of 23 mature ticks, each one of which was kept in a separate bottle. The number of eggs varies in general with the length of the egg-laying' period. 'Those which took the longest time laid the largest number. Of four large ticks laying from twelve to sixteen d.uys, each averaged $118 \mathrm{mgr}$. ( $1 \frac{13}{1} \frac{3}{6}$ grains) of eggs. Carefiul counting gave an average of $1,300 \mathrm{eggs}$ per grain. If we take the actual weight of all the eggs laid by the twenty three ticks, which is 2.41 grams (371 grains), a sirigle full-grown tick averages about 2,100 eggs. Ticks do not need to be fully gorged with blood before they are capable of laying eggs. Even such as are half-grown will begin to lay after a few days, but the number is much less than that laid by the large, gorged individuals. Tests showed that 40 half-grown ticks laid no more egg's than would have been laid by seven or eight full-grown individuals. During the process of oviposition the female slowly shrinks in size and when it iscompleted she appear's shriveled and not more than one-half or one-third her former size. The eggs appear as dark, brownish-red masses of oval bodies. The color varies somewhat and its depth appears to be connected somehow with the quantity of blood with which the female is gorged be. fore oviposition. Measurements of freshly laid ova in 1889 made the long diameter $0.519 \mathrm{~mm}$., the transverse $0.38 \mathrm{~mm}$. Measurements in 1892 gave nearly the same figures, 0.496 and $0.384 \mathrm{~mm}$. They are thus, roughly speaking, one-fiftieth of an inch long and one-sixty-sixth of an inch broad at their widest portion.

When masses of ova are placed in glass dishes with a little soil or some leaves and a few drops of water, and the dishes kept closed with glass covers so that the emerging young may not escape, the incubation goes on without any difficulty. The period required for the young to emerge from the shell varies very markedly with the surrounding temperature. In Curtice's first experiment it required from three to four weeks. The temperature of the bacteriological culture room where they were kept could not have been lower than $70^{\circ}$ to $80^{\circ} \mathrm{F}$. at that time. This relation to temperature is well exemplified in the following' experinents:

(1) Ticks sent from North Carolina and received here July 29, 1890, have laid a considerable number of eggs on the way. These are placed in glass dishes and kept in the laboratory. Many young ticks moving about on August 13. Here the period of incubation was from fifteen to eighteen days. The weather during this time was very hot.

(2) Eggs two to three days old placed in glass dishes August 8 . Young ticks ap. peared August 29. Period about twenty-four days. 
(3) Egges several days old placed in dishes August 13, 1890. Young ticks appear in large numbers September 4 and 5. Period approximately twenty-five days.

(4) On September 17, 1890, eggs two to three days old placed in glass dishes. Yomng ticks first appear Octoler 24, and their number increases until October 28. Period about forty days.

(5) Eggs one to three days old are plaeed in glass dishes September 20, 1890. Young ticks present in abundance November 1. Period abont forty-three days.

(6) Eggs one to three days old are placed in glass dishes September 23, 1890. Young ticks begin to appear November 1. Period abont forty days. The eggs from experiments 4,5, and 6 were taken successively from the same adnlt ticks. The temperature of the laboratory at this time was $75^{\circ}-80^{\circ} \mathrm{F}$. during tle day, but fell $5^{\circ}$ or $10^{\circ}$ at night.

(7) Eggs one to two days old plaeed in dishes October 6 and 9, respectively. The dishes were kept on shelves several feet above a steam heater. On November 9 all eggs were found batched out. Period about thirty days or less.

(8) Eggs two to fonr days old placed in dishes October 9, 1890. Orer steam register only a part of the time. A few young ticks appeared November 15 . Hatching completed November 17. Period about thirty-eight to forty days.

From these recorded dates it will be seen how essential a high temperature is for the rapid development of the embryo in the egg. The period of development may vary from fifteen days to six or seven weeks, and may perhaps be prolonged still more by lower temperature. It is evident, however, that a certain temperature level exists below which no development takes place. In the experiments above described there was considerable daily fluctuation in the temperature, and hence they can be made to show only the general relation subsisting between heat and development. To find the lowest temperature at which development may go on would require thermostats in which a certain low temperature could be constantly maintained. It is probable that the shortest period of incubation might be shortened still more by placing the eggs in continuous high temperatures. We have considered this matter more in detail owing to the intimate relation between the period of development of the young tick and the so-called "period of incubation" of Texas fever.

There are some changes which the ova undergo during development which are visible to the naked eye. After a variable number of clays each ovum presents a white spot. Under the microscope this corresponds to the position of the cloacal opening, and is nothing else than a mass of white powder composed of very minute spherical crystals. It is an excretory product (urates?) of the young tick, the outlines of whose body and limbs are now visible through the shell under a low power of the miscroseope. The color of the egg itself becomes lighter, and of a inore opaque, milky eharacter. Towards the end of the period of development it assumes a peculiar metallic luster. These changes are all causerl by the changes going on within the shell.

The minute six-legged ticks (Plate $x$, Fig. 3) after emerging fiom the shell are at first of a pale brownish, translucent, waxy color, which soon changes into an opaque brownish hue. They are about $0.67 \mathrm{~mm}$. 
(.0268 inch) in length, including the mouth parts. 'They move actively about, earrying in their cloacal opening the chalky mass of urates(?) mentioned above. They collect along the edge between dish and cover, and scatter as soon as the cover is removed. When confined for some time in the dish, this becomes soiled with a large number of white dots discharged by the ticks.

These minute creatures are very tenacious of life when kept confined in glass dishes containing a little loam or some leaves. Young ticks hatched about the middle of December, 1890, were confined in the same glass dish in the laboratory during the winter. On May 1, 1891, four and a half months after hatching, they were still active. On May 19 a few were still active; some were inert, but not yet deard. Young ticks hatched about July 20, 1891, were still active August 29. The parasitic habit of the tick is probably so complete that no growth and no further development takes place unless the larvæ gain access to cattle. When they have once attached themselves to the host and begin to get nourishment in the form of blood their growth is assured.

We have already referred to the larval and nymphal stage, so-called, as observed by Curtice. In his observations each stage oceupied about a week, so that at the end of two weeks the female tick is sexually mature, prepared to become fertilized, swell up and drop off to lay her eggs as the beginning of another generation. When young ticks hatched within a few days of one another are placed on cattle they do not necessarily mature at the same time. The dropping off of ripe ticks may go on some days before the animal is completely freed. In general, the time required for the tick to mature and drop off is from twenty-one to twenty-three days. These figures are the result of numercus observations made in the experiment fields at the station. The date being known when the larve were placed on the cattle, this period was easily determined.

The life history of the tick after it has attached itself to cattle is thus easily told. Taking two weeks for the tick to become sexually mature, the fertilization takes place as described by Curtice. An examination of the skin of cattle at this time shows each female provided with a male. After fertilization the female enlarges very slowly until from the nineteenth to the twenty-second day, when she swells up very rapidly, a day or two prodncing great change in size. When the proper stage is reached she loosens her hold upon the skin and drops to the ground, where the laying of eggs begins in a few rlays. The length of time elapsing between successive generations of ticks may be tabulated approximately as follows:

From oviposition to the larval stage (period of incubation) ........ 20 to 45 days From larval to adult state (parasitic stage) .................. 21 to 23 days

Age of one generation 
It should be borne in mind that the young, after emerging from the egg, may perhaps live on the fields an indefinite length of time before they gain access to cattle. We have kept them alive for several months. How they would fare under the varying conditions of moisture and dryness and of a fluctuating temperature we are unable to state, from lack of observation. This free-living period must be adderl to the total given above to obtain a more accurate iclea of the life of a single generation. Yet it is of little importance and without doubt very brief, for when cattle are within accessible distance the young ticks soon find their way to their host.

The problem how the tick passes the winter is an jinportant one which needs special investigation. In the warmer climates ticks are found on cattle during the winter season, and hence the development from the egg goes on during the entire year. It is highly probable that in those regions where the temperature falls too low for the tick to live on cattle the species is carried through the winter in the ovum. The great vitality of the ova is illustrated in the following experiment:

A number of dishes containing eggs were placed in a cold storeroom in the attic of the Department building during November and December, 1890, and January, 1891. The eggs were placed on the bottom of the dishes, which, otherwise empty, were covered with glass covers. They were occasionally inspected in the course of the winter and early spring, but not thereafter until July 15, 1891. It was tinen found that in three out of nine dishes (one placed there in November, one in December, and one in January) the embryos had developed and hatched at the approach of hot weather, but were now dead. The young ticks had left behind the little chalky masses of urates (?) over the inner surface of the dishes. In the other dishes the eggs were shriveled. Signs of development were absent. This shows that the ova lived through the winter under unfavorable conditions of moisture, since the air of the room was quite dry. The capacity of the tick to survive occasionally the winter in regions north of its natural habitat was demonstrated in an unexpected manner on the station grounds in 1891. In September of 1890 ticks hatched in the laboratory were placed on two cows (Nos. 137, 144) in a piece of woodland belonging to the station, but some distance remover from it (page 105). These contracterl the disease in dne time. One died during the acute attack, the other succumbed after it. The ticks, matured from this case, wintered over, probably, among the leaves under the trees, and on September 1, 1891, one young animal in this inclosure was found with many ticks attached to it, and the examination of the blood demonstrated 'i'exas fever. The other animals in the inclosme were insusceptible Southern animals, kept over from previous years, but likewise infested with ticks. Since it is quite impossible that any ticks conld have been taken to this inclosure during the summer of 1891, the explanation given above is the only admissible one. The ticks did not reappear in 1892. 
Aside from the relation which the tick bears to Texas fever as the ("arrier of the microörganism of this disease, it is pertinent to inquire in this place what other injury this parasite might inflict on cattle. That it abstracts a certain quantity of blood during the later days of its parasitic existence is evident. The intestine is distended with a dark-red, tarry, viscid mass, from which an abundant crop of hæmin crystals may be obtained according to the well-known method of adding: a crystal of common salt and some glacial acetic acid to some dried contents of the tick's body cavity on a glass slide and heating the latter until bubbles of gas are given off. These crystals show that there is much concentrated coloring matter of the blood corpuscles in the body of the tick. Yet it is doubtful whether in the aggregate very much blood is abstracted by the ticks, and the various cases under ob. servation did not warrant the conclusion that any marked impression was made on the number of red corpuscles of insusceptible or recovered cases.

The tick produces more or less inflammation of the true skin and subcutis where it is attached. Sections of skin examined under the mi croscope show a very intense cell infiltration at the place of attachment, and for several millimeters around it. This infiltration is not noticer by the unaided eye. It is probable that it is due to the irritation caused by certain unknown secretions of the tick, which aid it in working its way throngh the upper layers of the skin and in obtaining blood in an uncoagulated state from the blood vessels attacked by it. After having attached themselves, ticks are in communication with blood vessels, for in removing them a drop of blood oozes from the place of attachment.

The young ticks attach themselves by preference to the more tender regions of the hide, such as the inner aspect of the thighs, the pubic region (escutcheon), and around and on the udder. When numerous they may attach themselves to the neck, the sides of the thorax, the ears, and even the back. In searching for them the regions first mentioned must be thoroughly examined. It must also be borne in mind that when the disease appears the ticks are still quite small and may be overlooked. Even at the time of death only a small number may have passed beyond the second moult. The ticks still within the second skin are only $3.2 \mathrm{~mm}$. (about one-eighth inch) long. Those just emerged are of the same length. The more active males of the same stage are only $2 \mathrm{~mm}$. (one-twelfth inch) long. The largest ticks found on animals which die during the acute attack are not more than 5 to $8 \mathrm{~mm}$. (one-fifth to one-third inch) in length. When ready to drop off from insusceptible or recovered cases, they are about balf an inch long (12 $\mathrm{mm}$. long, $7 \mathrm{~mm}$. broad, and $4 \mathrm{~mm}$. thick).

In the foregoing it has been stated that the female tick remains until maturity upon the same animal to which it attacherd itself after emerging from the egg. Each tick, in other words, is parasitic upon but one 
animal. What beeomes of the ticks not yet matured, which are at. tached to the skin of natives when the latter succunb to Texas fever, we can not state definitely. It is certain that they do not at once leave the dead body, for in the case of eattle which die early in the night the ticks are still found attached next morning. In the case of a calf kept in a large refrigerator several were found attached forty-eight hours after death. If ticks are removed by the hand from the dead body it will be found that the males as well as those females which have passed through the second moult move about with some show of activity, while those individuals which have not yet cast off the moulted skin are motionless. Taking these facts into consideration, we must regard the movement of ticks from one animal to another as an unnatural process which, so far as we know, may take place, but which from general observations does not appear to be of much importance. Still, it is nevertheless desirable that experiments be made to determine positively whether ticks may be transplanted after the last moult, and whether at this advanced stage in their life history they are still capable of producing Texas fever.

Paquin states $(9, \mathrm{p} .45)$ that full grown ticks contain the "bacteria" of Texas fever. In order to see whether ticks contain bacteria, and what kind, the following inoculation experiments were made:

(1) July 7, 1890.-A large North Carolina tick was taken and its back scoreher throngh with a red hot platinum spatula, thus exposing the body cavity. A loop of the black tarry contents transferred to peptone bouillon. A coccus, arranged in the form of tetrads, produces a faint cloudiness and a deposit in the inoculated tube.

(2) A second tick from the same source treated in the same way. The culture contains a coccus of the same form.

(3) $J u l_{y}$ 10, 1890.-From a large tick from 'l'exas cattle a peptone bouillon tube inoculated. Remains sterile.

(4) Another large Texas tick used. The resulting peptone bouillon ('ulture contains a flocculent growth of large bacilli in chains.

(5) July 18, 1890.-An agar tube inoculated from a large tick, as before. Remains permanently sterile.

(6) Another agar tube inoculated from another tick. A considerable number of small colonies develop, having an opaque center and trauslucent periphery. 'They are made up of short bacilli.

(7) A peptone bouillon tube inoculated from a large tick becomes clouded with large motile spore-bearing bacilli.

These experiments show that the tirk may harbor a varicty of bacteria or none at all.

FIELD EXPERIMENTS TO DETERMINE THE PRECISE RELATION BLTWEEN 'THE CATTLE TICK AND 'TEXAS FEVER.

These experiments were begun in the summer of 1889 and have been continued up to the present. 'They have been carried on in threedif' ferent directions:

(1) Ticks were carefully picked from Southern animals, so that none 
conld mature and infeet the ground. The object of this group of experiments was to find out if the disease could be conveyed from Southern to Norther'n stock on the same inclosure without the intervention of ticks.

(2) Fields were infected by matured ticks and susceptible cattle placed on them to determine whether Texas fever could be produced without the presence of southern cattle.

(3) Susceptible northern cattle were infected by placing on them young ticks hatched artificially, i. e.; in closed dishes in the laboratory.

These three lines were not followed simultaneously because, for instance, the fact that the disease can be produced by placing young ticks on eattle was discovered in 1890 , and hence only tried then and thereafter. In giving the details of the various experiments we shall adhere not to the classification given above, but rather to the chronological order in which the experiments were performed. This is necessary in order to describe successively the experiments of the same year which were more or less connected with one another and also to show the process by which the various facts eoncerning the cattle tick came to our knowlerlge.

The disease was introduced into one field each year by North Carolina cattle brought here for this purpose. In 1890 a field was infected by cattle from Texas.

The field experiments were all conducted on the experiment station of the Bureau of Animal Iudustry within half a mile of the limits of the city of Washington. The arrangement of the various experimental fields is shown for each year on a plat of the station grounds. The isolated condition of the field in use in any given season may be seen by an inspection of these plats. They are either separated from one another by a piece of ground remaining permanently free from infection or by a lane or by*a strip of ground purposely fenced off between them. No two fields in use are thus separated merely by a fence. In every case, with the exception to be noted, a strip of ground intervenes which is at least 36 feet wide. A small brook passes through a portion of the gromuds as is shown in the various plats, and the space between the fields along this brook is about 20 feet wide.

EXPERIMENTS OF 1889 (HIRST SERIES).

To carry on the experiments in the early part of the season of 1889 , seven head of cattle were collected in Craven County, N. C., which is a portion of the permanently infecter territory. On June 25 they were shipped by steamer from New Berne, N. C., aud they arrived at the station near Washington June 27. They had thus been two days on the way. These animals were rather thin and a large number of eattle ticks (Boöphilus bovis) in various stages of development were attached to them. Only a few were full grown. 
Experiment 1 (exposure to Sonthern cuttle with ticks).-Of these seven head four were placed in field I (see Fig. 4) on the day of arrival, June 27. The field contains about nine-sixteenths of an acre. The soil is a dry, gravelly loam. A small stream passes through it, from which the cattle obtain their drinking water.

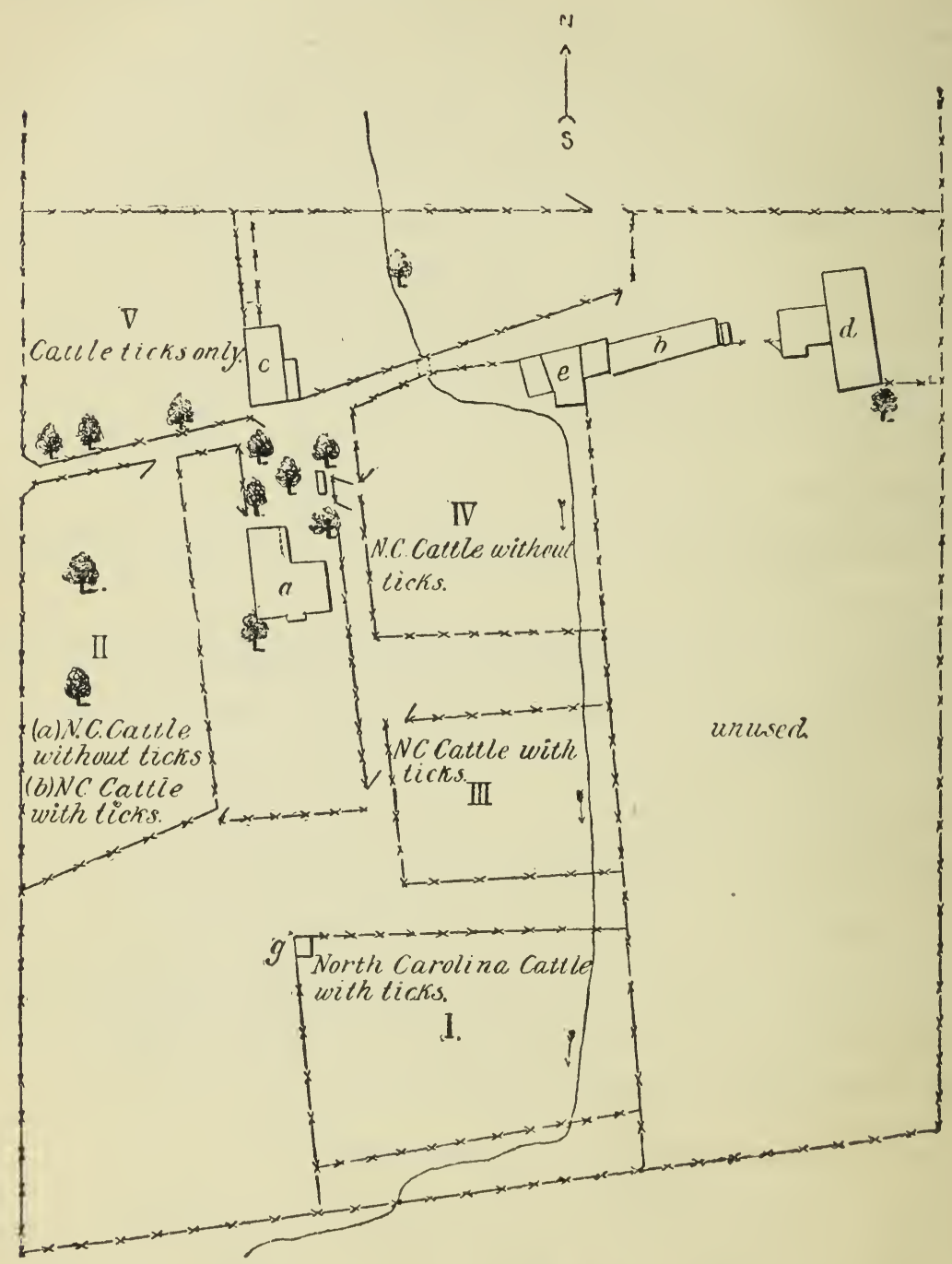

Fif. 4.-Field inclosures for 1889 (scale, $\frac{1}{4}$ inch $=33$ feet).

$a$, dwelling house; $b$, station laboratory; $c$, lorse stable; $d$, cow stable; $e$, breeding pons; $f$. tool house; $g$. slied in fielil.

The history of the native eattle placed in this field may be briefly summarized. Notes concerning symptoms of disease, post-mortem examination, microscopical and bacteriological data, may be found in the appendix under each case. 
(a) North Carolina cattle with ticks:

No. 12, placed in this field June 27, removed August 17.

No. 40, placed in this field June 27, removed August 17.

No. 42, placed in this field June 27, removed August 17.

No. 45, placed in this field June 27, removed August 17.

(b) Native cattle:

June 27, No. 7 (cow, 6 years) placed in this field. Dead* Angust 23.

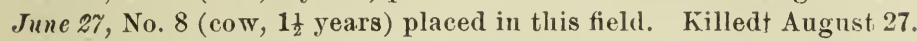

June 27, No. 75 (calf of No. 8, 4 months) placed in this field. Recovered.

June $2 \%$, No. 9 (bull, $1 \frac{1}{2}$ years) placed in this field. Died August 31.

June 2\%, No. 10 (calf of No. 7, 4 months) placed in this field. Died August 31.

June 27, No. 11 (calf of No. 7, 4 months) placed in this field. Killed September 10. August 20, No. 46 (heifer, 1\% years) placcd in this field. Killed September 10. August 24, No. 43 (steer, 3 years) placed in this field. Dead September 13. August 24, No. 44 (steer, 4 years) placed in this field. Dead September 17. September 6, No. 53 (heifer, $1 \frac{1}{2}$ years) placed in this field. Recovered.

September 6, No. 54 (heifer, 2 years) placed in this field. Killed September 20.

September 14, No. 57 (cow, 9 years) placed in this field. No result.

September 30 , No. 70 (steer, $2 \frac{1}{2}$ years) placed in this field. Died October 19.

October 19, No. 71 (heifer, $3 \frac{1}{2}$ years) placed in this field. Probably no disease.

The disease in this field was designed to furnish material for general investigation as well as to serve as a control for experiment 2 below. It illustrates admirably a number of important characters of this remarkable disease and demonstrates once again the frequently observed fact that cattle, to all appearances healthy, may become the cause of an extensive fatal disease when transferred in the warmer seasons of the year from a certain permanently infected area to territories north of this area.

The first high morning temperature appeared August 15, or thirtynine days after the native and Southern cattle were placed on this field together. The first death occurred August 23, or forty-seven days after this same date. In other words, the cattle exposed at this time died not less than forty-seven days after the beginning of the exposure. After a certain time, however, death follows more speedily after exposure, as may be seen when we consult those cases exposed August 20 and thereafter, for which this period was only fourteen to twentythree days. The field remained infected so as to cause death as late as October 19. The later the exposure the less likely is the disease to end fatally.

Omitting the last case, No. 71, as having been exposed too late, we have ten deaths from thirteen cases exposed, or 76.8 per cent. It should be noted that, although the Southern cattle were removed from the field August 17, the infection on the field remained unimpaired.

Experiment 2 (exposure to Southern cattle without ticks).-For this experiment, field II was selected (see Fig. 4). The soil is the same as

* Unless otherwise stated the cause of sickness and death is Texas fever.

+With one exception (No. 163) all native animals reported killed in this report were in a dying condition at the time. 
that in field I, but there is no running or standing water in it. It contains one-third of an acre.

This experiment differed from the first in that the ticks were carefully picked from the three North Carolina cattle left after stocking field $I$. The picking was done by hand. On July 6 and 17 , the eattle were again carefully inspected, and any ticks which had thus far escaped attention were carefully removed. On July 2:3 no more ticks could be detected. In this way it was expected that no ticks would mature and infect the field. 'The following cattle were placed in this field June 27, 1889:

(a) North Carolina cattle without ticks, Nos. 28, 29, and 30.

(b) Native cattle: No. 51 (eow, 3 years); No 52 (calf of No. 51, 4 months); No. 53 (heifer, $1 \frac{1}{2}$ years); No. 54 (heifer, "2 years).

On September 6, no ticks and no disease having appeared in this field, Nos. 53 and 54 were transferrerl to field I. Their further history is given under experiment 1 . It would have been more satisfactory to have left theseanimals on this field until the close of the season of 1889. But the evidence is decidedly in favor of the assumption that there was no infection of these animals when they were transferred to the infected field. This evidence is twofold: (1.) The three adult animals and one calf in control field I were dead by August 31 and the remaining ealf was killed in a dying condition September 10. Hence all five animals exposed at the same timein the field containing ticks were either dead or very siek on the date of the removal of these two to field I. They on the other hand were at this time to all appearances healthy. (2.) One transferred case (No.54) was dying of an acute attack September 20, as the autopsy notes and microseopical observations demonstrate. If this animal had been affected september 6 , at the time of transfer, the blood corpuseles would have shown later on enlarged and stained forms (macrocytes) always associated with prolonged disease. No. 53 first showed external signs of disease in the last week of September, at which time it lost much flesh and was very weak. In October it was passing through a mild or secondary attack. Both transferred animals, therefore, must be regarded as having contracted Texas fever after September 6 on field I. The same arguments apply to Nos. 51 and 52, which were reëxposed later on in the season (experiment 4).

EXPERTMETS OF 1889 (SECOND SERIES).

In September of 1889 a second series of experiments were carried on in order to repeat the observations on the relation of ticks to Texas fever. Nine head of eattle were collected in Oraven County, N. U. Three were taken from each of three farms located several miles from New Berne and in opposite directions fiom that eity. The three cattle from one farm were shipped from New Berne September 10 and reached the station September 14. The remaining six were shipped september 12 and arrived September 15. The three of the lot to arrive first wero 
placed in their respective fields a day earlier than the remaining six. All eattle were well loaded with cattle ticks, many of which were nearly matured.

Experiment 3 (exposure to Southern cattle with ticks).-For this experiment field III was chosen (see Fig. 4). It resembled field $I$ in having a running stream and contained about three-eighths of an acre. It was separated from field I by a lane 36 feet wide. The experiment was designed as a control to the others below, and in order to insure the same conditions in every respect one of each of the three lots of cattle was placed in it. The following animals were in this field:

(a) North Carolina cattle with ticks:

No. 113, placed in field III September 14.

No. 60, placed in field III September 15 .

No. 62, placed in field III September 15.

(b) Native cattle:

No. 35 (heifer, 2 years), placed in field III September 14.

No. 47 (cow, $3 \frac{1}{3}$ years), placed in field III September 14 .

No. 49 (heifer, 3 years), placed in field III September 14.

Of these three natives only No. 47 passed through a severe attack of the disease, as the notes in the appendix demonstrate. The season was somewhat too far advanced when the exposure began, and of the new generation of ticks only very few appeared on the native cattle afterward.

Experiment 4 (exposure to Southern cattle with ticks).-This experiment is the counterpart of experiment 3 , excepting that it was conducted in field II, which is without running water. Field II, moreover, was occupied by Southern cattle without ticks in July and August, as will be seen by referring to experiment 2. From this experiment there remained in the field natives No. 51 and 52. On September 14 and 15, three North Carolina animals, one from each of the three lots, were placed in this field, and one native, No. 56, was added September 14. There were, ti. :efore, in this field on September 15-

(a) North Carolina cattle with ticks, Nos. 32, 61, and 67.

(b) Native cattle:

No. 51 (from experiment 2). Passed through the disease and recovered.

No. 52 (from experiment 2). Passed through the disease and recovered.

No. 56 (steer, $2 \frac{1}{2}$ years). Probably not affected.

On October 9, ticks had almost entirely disappeared from the Southern cattle, and very few young ticks subsequently appeared on the natives. There can be no doubt of the nature of the disease in Nos. 51 and 52 , as will be seen from the notes in the appendix.

Experiment 5 (exposure to Southern cattle without ticks).-For this experiment field IV was chosen (see Fig. 4). It covers about threeeighths of an acre, is situated above field III, and separated from it by a lane 36 feet wide. The stream passes through it on the east. Three Southern cows, one from each lot, were placed in this field after the 10320-No.1—7 
ticks had been carefully picked off so far as they could be seen. In this field there were the following animals:

(a) North Carolina cattle without ticks:

No. 55,59 , and 63 , the first put on the field September 14, the others September 15 .

(b) Native cattle:

No. 41 (heifer, 4 years) placed in this field September 14.

No. 50 (cow, 3 years) placed in this field September 14.

No. 97 (calf of No. 50, 2 months old) placed in this field September 14.

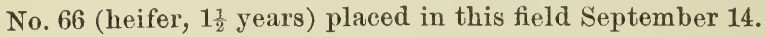

The Sonthern cows were reëxamined September 18, 26, October 1 and 9, and some remaining ticks removed. On October 9 only two or three were found. Up to November 27 no ticks were detected on the native cattle, and no symptoms of disease were noticed.

Experiment 6 (exposure to cattle ticks only).-This experiment was carried on in field $V$, an inclosure consisting of about three-eighths of an acre. The soil is a heavy clay loam, and contains neither running nor standing water. On September 13 several thousand, mostly fullgrown ticks, were scattered over the ground in this field. These ticks had been collected from cattle near New Berne, N. C., September 9 and 10. There were placed in this field, September 14, four natives:

No. 48 (cow, $2 \frac{1}{2}$ years).

No. 83 (calf of No. 48,2 months).

No. 64 (steer, $2 \frac{1}{2}$ years).

No. 65 (heifer, $2 \frac{1}{2}$ years).

Of these. Nos. 48, 64, and 65 contracted Texas fever. No. 83 was not examined as to its blood, but it showed no external symptoms of disease. No. 48 was killed in a dying condition, October 21. The autopsy, as well as the examination of the blood before death, demonstrated Texas fever. Nos. 64 and 65 recovered.

\section{Summary of the Experiments for 1889.}

The first series (Nos. 1 and 2) go far toward demonstrating that a field must be infected with ticks before Texas fever ean appear among natives. The second series confirms the first as far as it goes. The arvanced season gave rise only to what has been called the mild or autumnal type of the disease, characterized by the presence in the blood corpuscles of the peripheral coccus-like stage of the Texas-fever parasite. If we bring together the results of the four experiments we find that in the field containing the ticks only, and in which Southern cattle at no time entered, all three exposed adult natives took the disease. In the field containing Southern cattle from which the ticks had been picked no disease appeared. Finally, in the two fields which contained Southern cattle and ticks together three out of six natives became diseased. In these experiments the great importance of the method of blood examinations as described in the first part of this volume is plain. To rely upon external symptoms in mild attacks is 
out of the question. The counting of the red corpuscles, the changes going on in the latter, and the presence of the Texas-fever parasite as determined by microscopical examination are indispensable in determining whether Texas fever is present or not.

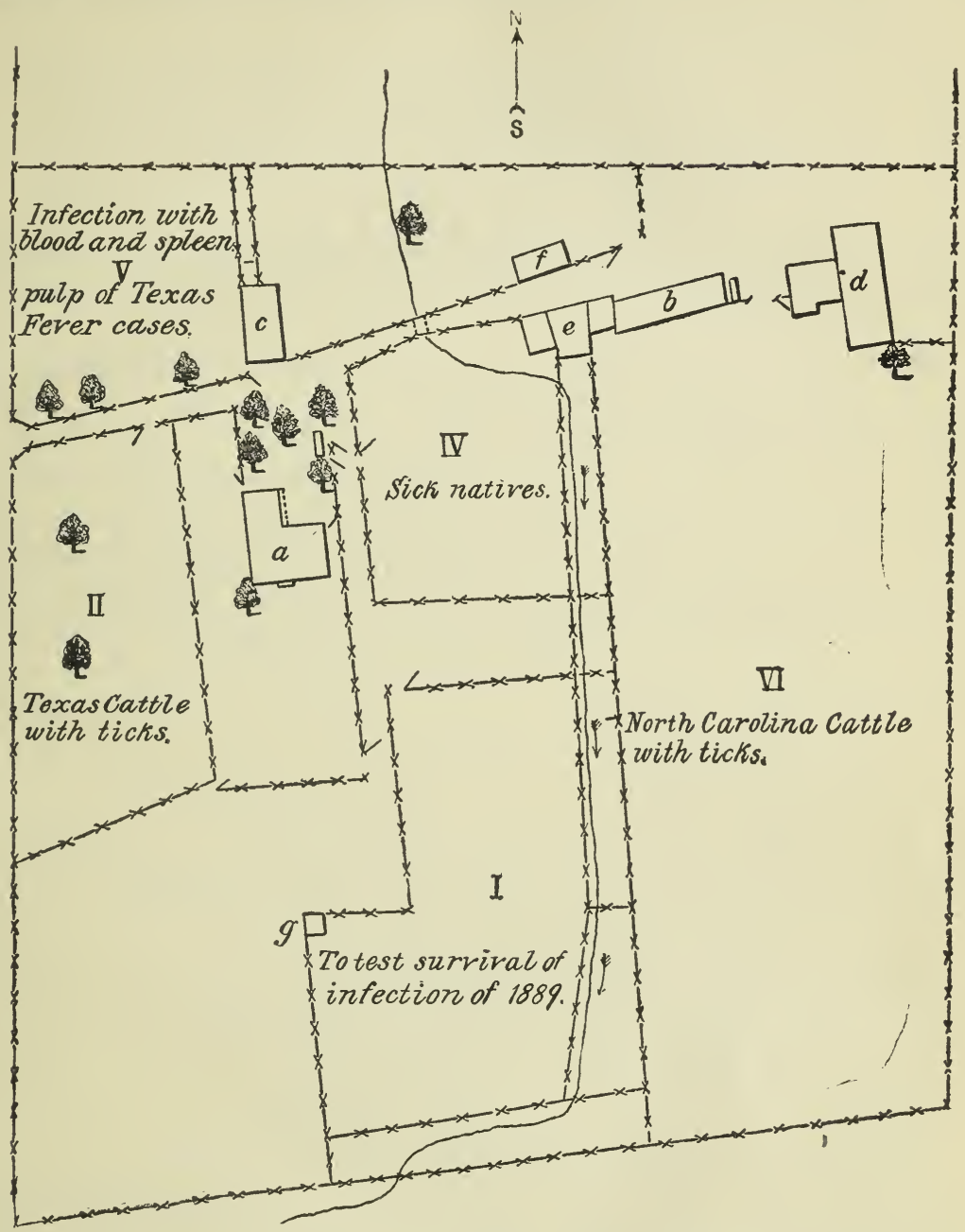

Fia. 5.-Field inclosures for 1890 (scale $\frac{1}{4}$ inch $=33$ fcet). For an explanation of theletters see fig. 4 on page 94 .

EXPERIMENTS OF 1890.

The experiments of this year were chiefly occupied with the relation of ticks to Texas fever. The experiments of last year were repeated and in addition ticks were hatched artificially and placed on cattle with the result that Texas fever appeared in every case (experiments 12 and 13). Sonthern cattle were obtained as before from Noith Carolina (Experiment 9) and also from Texas (Experiment 8). 
Experiment $y$ (to ascertain whether the infection of 1889 survived the winter).-For this purpose fields I and III of 1889 were thrown together by removing the intervening fences and the whole designated field I (see Fig. 5). The little stream was likewise fenced off in July to prevent any infection from field VI reaching it. A number of animals were pastured on this field.

May 26, 1890.-No. 74 (heifer, 2 years.) Transferred to field II September 25.

May 26, 1890.-No. 91 (heifer, 3 years.) Transferred to field VI October1.

July 4, 1890.-(Stream fenced off, as field VI is now used for the first time.)

July 9, 1890.-No. 130 (cow, 5 years).

August 25, 1890.-No. 97 (bull, 1 year).

During the summer no ticks appeared in this field, so that it was evident that they had not survived the winter. No disease appeared in any of the animals exposed.

Experiment \& (to ascertain whether the disease introduced by Texas and that introduced by North Carolina cattle are the same).-Four heifers were removed from their pasture near Houston, Tex., June 30, and sent by express to the station, whore they arrived July 4. Hence, they were but four days off the Southern pasture before they were placed in field II (see Fig. 5). The heifers were in rather poor condition and all well supplied with cattle ticks of all stages. The field corresponded with field II, of 1889 , but it was slightly enlarged so as to include about $\frac{9}{16}$ of an acre. The field thus contained-

(a) T'exas cattle with ticks:

No. 124 (heifer, 2 years).

No. 125 (heifer, 3 years).

No. 126 (heifer, 4 years).

No. 127 (heifer, 5 years). All placed in field II, July 4.

(b) The native cattle were exposed in the following order:

July 4, No. 128 (cow, 12 to 14 years). Dies September 1.

Ju7y 5, No. 80 (cow, 7 years). Killed August 28.

July 5 , No. 82 (calf, 5 months). Diseased, but recovered.

July 5, No. 107 (heifer, 1 year). Diseaserl, but recovered.

July 5, No. 129 (heifer, 2 years). Dies August 29.

August 30, No. 139 (cow, 6 years). Dead September 13.

September 25, No. 74 (heifer from field I). Dies October 16.

September 25, No. 62 (N. C. heifer of 1889). Exposure negative.

As regards the cattle ticks, the following observations were made. On July 30, only a few arlults were still attached to the Texas cattle, the rest having disappeared. On October 20, only very few young" ticks were still found on the surviving cattle, and eight days later they had all disappeared.

Any differences between the disease in this and the North Carolina fields could not be found.

Experiment 9 (cxposure to North Carolina cattle with ticks. General control field for 1890).- Field VT was chosen for this purpose. It covers $1 \frac{7}{8}$ acres, and is fenced off from the stream. Between it and the other fields (I, IV) is a strip of land containing the stream berl. 
'To carry on the various experiments of the year, and to infect this field, cattle were taken from North Carolina tields as in 1889. The cattle were collected July 1, shipped by steamer from New Berne, N. C., July 2, and received at the station July 4. Of those received, the following were placed in field VI on July 4:

No. 114 (heifer, 2 years), from North Carolina; farm 1.

No. 112 (old cow), from North Carolina; farm 4.

No. 120 (cow, 7 years), from North Carolina; farm 2.

No. 119 (calf of No. 120, 3 months), from North Carolina; farm 4.

No. 121 (cow, 3 years) from North Carolina; farm 4.

No. 122 (heifer, 2 years), from North Carolina; farm 3.

These Southern animals were in fair condition, excepting No. 112, which was very thin and weak. All excepting No. 114 were well supplied with cattle ticks.

The following Northern animals were placed in field VI:

July 4.-No. 49 (cow, 4 years), exposed in 1889, but probably not affected at that time.

July 4.-No. 85 (calf of No. 49, 3 months).

July 4.-No. 50 (cow, 4 years), in field IV in 1889, but not affected.

July 4.-No. 57 (cow, 10 years), exposed in field I in 1889, but probably not affected.

July 4.-No. 79 (calf of No. 50, 3 months).

July 4.-No. 66 (heifer, 2 years), exposed in field IV in 1889, but not aftected.

July 4.-No. 69 (cow, 3 years).

July 4.-No. 100 (calf of No. 69, 2 months).

July 4.-No. 95 (cow, 4 years).

July 4.-No. 93 (calf of No. 95, $1 \frac{1}{2}$ months).

August 13.-No. 71 (heifer, $4 \frac{1}{2}$ years), transferred from field I.

August 13.-No. 134 (heifer, 2 years).

August 25.-No. 90 (bull, 1 year).

September 8.-No. 56 (steer, $3 \frac{1}{2}$ years), exposed in field II in September, 1889, but probably not affected.

These various animals (excepting Nos. 49, 56 and 57) may be regarded as unexposed natives, although some of them had been used the year previous and early in this season in fields presumably free from infection. The result of the exposure may be tabulated as follows:

No. 49 has a severe attack but recovers.

No. 85 has a mild attack.

No. 50 dies September 6, sixty-four days after the beginning of the exposure.

No. 57 is not affected.

No. 79 has a very mild attack and recovers.

No. 66 dead September 1, fifty-nine days after the beginning of the exposure.

No. 69 dies September 3, sixty-one days after the beginning of the exposure.

No. 100 has a mild attack, but succumbs in December.

No. 95 killed in dying condition, A ugust 25, fifty-two days after exposure.

No. 93 has a mild attack.

No. 71 dead September 6, twenty-four days after beginning of exposure.

No. 134 killed in dying condition August 28, fifteen days after exposure.

No. 90 dies September 16, twenty-two days after exposure.

No. 56 has a prolonged but mild attack and recovers.

It will be seen from this table that all animals exposec in inicisummer (July and August) died or were killed in a dying conratios, excepting 
the calves. These were all affected; one dierl late in the fall and one was killed, but in every ease the attack was mild. The mortality among those over 1 year old was 80 per cent. It will be noticed also that animals exposed in August died with those exposed a month earlier. Those exposed on July 4, when the field was first infected, died from fifty-two to sixty-four days thereafter. Those exposed in August died in fifteen to twenty-four days after the first day of exposure.*

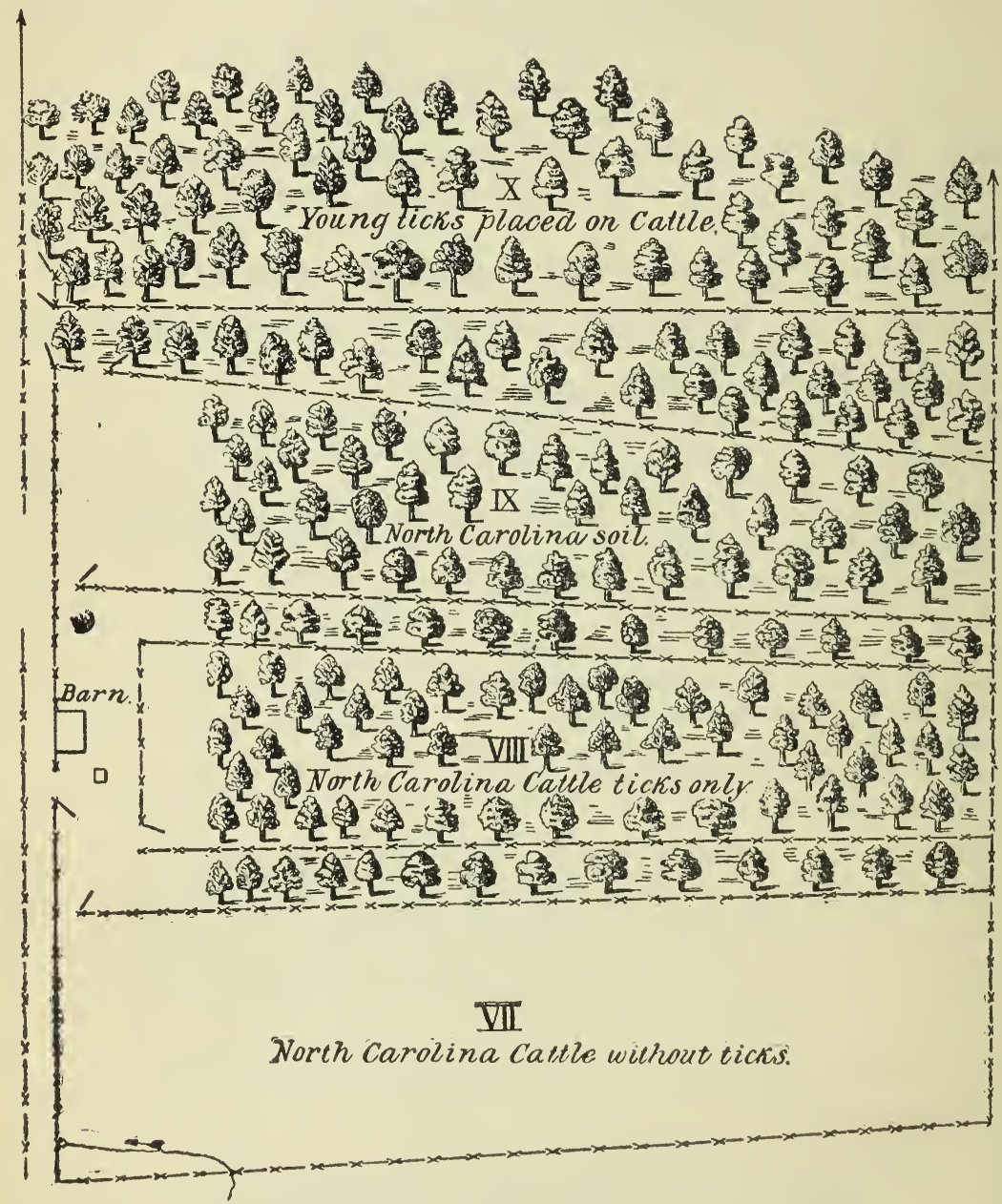

Fra. 6.-Supplementary inclosures for 1890 (seale $\frac{1}{4}$ ineh $=33$ feet.)

On July 30 only a few full-grown ticks were left on the Southern stock. August 13, two weeks later, young ticks had appeared on all the cattle, native and Southern alike. August 25 some of these had become full grown. In the middle of October only a few young ticks could be seen, and byr.the end of the month they har practically disappeared.

* In this figle' there were placed during the summer some animals which had passed through one attack of the disease and some Southern animals kept on the station from the year previous. 'These cases will be reviewerl under another subject (p. 130). 
Experiment 10 (exposure to ratlle ticks only).-Field VIII was used in this experiment. (See Fig. (6.) It is a fenced-off portion of a piece of ground situated about one-fourth mile north of the station grounds upon which the experiments thus far recorded were carried on. This particular field covers about $1 \frac{3}{8}$ acres, and is separated from the adjoining fields VII and IX by strips of ground as shown in the plat. The ground is covered largely with trees (oak and chestnut) and may be regarded as sparsely wooded. It slopes towards field VII at an angle of $20^{\circ}-30^{\circ}$. On July 4 about 4,000 matured and 1,000 half-grown ticks were scattered over the ground in this inclosure. The ticks had been collected between June 28 and July 2 about New Berne, N. C., and placed in a large can containing grass from the Washington station. At the time they were scattered over the field many had already laid a portion of their eggs.

In this field were placed the following native cattle:

July 4.-No. 76 (heifer, 1 year).

July 4.-No. 102 (cow, 6 years). No. $102_{\text {a }}$ (calf of No. 102, born on this field September 1).

July 4.-No. 105 (heifer, 2 years).

August 21.-No 47 (cow, $4 \frac{1}{2}$ years, recovered case of 1889).

August 21.-No. 135 (heifer, two years).

The result of the exposure is briefly as follows:

No. 76 killed in dying condition August 18.

No. 102 very sick with Texas fever, but recovered.

No. $102_{\mathrm{a}}$ died of Texas fever thirtcen days after birth.

No. $105^{*}$ very sick, but recovered.

No. $47^{*}$ died September 12 .

No. $135^{*}$ very sick but recovered.

Of the six cases exposed to the ticks only, all were unmistakably affected with Texas fever, as the notes in the appendix show. Three died and the autopsy confirmed the diagnosis. The reason why the mortality was not so high here as in the preceding experiment is probably to be sought for in the fact that under natural conditions the young ticks appear more successively and cause a more prolonged infection, while in this experiment they probably appeared nearly all at the same time.

The young ticks were seen in this field August 8, and on August 23 full grown specimens were tound on No. 102.

Experiment 11 (exposure to Southern cattle without ticks).-This experiment was conducted on field VII adjoining the field of the preceding experiment. (See Fig. 6.) It covers about $1 \frac{3}{4}$ acres and contains both ruming and standing water from a spring. It is not wooded. 'The following Southern eattle broaght from North Carolina (with those

* These cases were transferred subsequently to an uninfected field in order to determine whether they could communicate the disease to other natives. (See appen. dix under each case and p. 122.) 
placed in field VI of this year) were put into this inclosure after all ticks that could be found were carefully removed:

July 4.-No. 115 (cow, 6 years) from farm 4.

July 4.-No. 116 (heifer, 2 years) from farm 2.

July 4.-No. 117 (heifer, 2 years) from farm 3.

July 4.-No. 118 (cow, 10 years) from farm 4.

July 4.-No. 123 (heifer, 3 years) from farm 1.

Into this inclosure were placed on the same day the following native cattle: No. 103 (heifer, 3 years); No. 106 (heifer, 2 years); No. 108 (heifer, 2 years).

The Sonthern cattle were reëxamined three times a week between July 7 and July 28, to remove any ticks which on account of their small size had escaped detection. On a final examination July 30 no more could be found.

Nevertheless, on August 15, a few young ticks were found on the natives, and three days later a large number had attached themselves. The outeome was the death of the three natives. No.103 died August 28; No. 106 died September 6; No. 108 died September 9.

The experiment had thus failed in so far as ticks had not been kept out of the field. Although it is not to be denied that some may have escaped attention and fallen to the ground, yet it is highly probable that most if not all the ticks or their eggs were washed in from the adjoining field VIII, which is considerably higher and slopes toward VII. There had been exceptionally heavy showers August 1 and August 8, which had carried much soil, and even stones as large as a fist, into this field. At all events, this difficulty might have been avoided by reversing the location of the two experiments and placing the animals fiee from ticks on the higher ground.

Experiment 12 (production of Texas fever by placing on native cattle young ticks artificially hatched in the laboratory).-Witherto we had supposed that the cattle tick acts as a carrier of the disease between the Southern eattle and the soil of the Northern pastures. It was believed that the tick obtained the parasite from the blood of its host and in its dissolution on the pasture a certain resistant spore form was set free, which produced the disease when taken in with the food. The feeding of one animal (No. 145) for some time with grass from field VI, the most abuudantly infected of all, without any appearance of disease made this hypothesis untenable. But even before this feeding experiment was undertaken other facts were noticed which militated against this hypothesis and which proved that the young tick ealls forth the disease. In the first place, animals exposed when the field was first infected did not die until fifty to sixty days after the beginning of the exposure, while those exposed thirty or more days later on the same ground died in fifteen to twenty-five days thereafter. In the second place, all animals which succumbed had young ticks on them. In other words, the appearance of the disease was in some manner associated with the appearance of the new generation of ticks. Even 
with this fact fairly well determiner the true explanation that the young ticks were directly responsible for the disease seemed too farfetched to deserve attention until it was demonstrated in the following manner. A yearling heifer (No. 138) had been placed in a box stall and a number of young ticks, hatcher artificially in glass dishes, had been placed on this animal at intervals, beginning August 14, in order to determine whether ticks in the eapacity of blood-sucking parasites made any impression on the number of blood corpuscles. It was found by a periodical estimation of the number of red corpuscles that after a certain time this number fell so quickly and so markedly as to be wholly incommensurate with the small amount of blood abstracted by the ticks. At the same time other symptoms of Texas fever appeared and the parasite was detected in the blood.

The experiment was repeated on several other animals as soon as young ticks could be obtained.

No. 140 (heifer, 2 years old) kept in a box stall on a neighboring farm. The young ticks 3 to 4 days old were placed on it September 9. It was found dead October 2. Both blood examination during life and the autopsy demonstrated Texas fever.

No. 137 (heifer, 1 year old) was placed in field $\mathrm{X}$, a wooded lot to which no infected cattle had been admitted, and on September 9 young ticks were placed on it. It passed through a severe attack of Texas fever and was killed in a dying condition November 6 .

No. 144 (cow, 8 years old) was also kept in field X. The young ticks were placed on it September 17 and it was found dead October 3. In this case also the nature of the disease was beyond question.*

Experiment 13 (production of Texas fever in the winter season by placing young ticks on cattle kept in an artificially-heated stable).-The result of experiment 12 was so important that it was deemed best to repeat it in an artificially-heated stable, as the season was too far advanced for ticks to thrive in the open air. The stable was warmed by means of a coal stove. The temperature fluctuated between $65^{\circ}$ and $80^{\circ} \mathrm{F}$. A complete history of all the cases experimented on in this way is given in the appendix.

The following animals were exposed and infected with young ticks:

\begin{tabular}{|c|c|c|c|c|c|}
\hline No. & Age, etc. & $\begin{array}{l}\text { Placed in } \\
\text { stable. }\end{array}$ & $\begin{array}{c}\text { Infection with } \\
\text { ticks. }\end{array}$ & Number of infections. & Result. \\
\hline 143 & Heifer, $1 \frac{1}{2}$ years... & Oct. 27,1890 & Oet. 28 -Nov. 8 & 2 (200-300 each time). & Slight, if any effect. \\
\hline 145 & 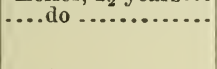 & ....do...... & Nov. 21-Dec. 3 & 7 (200-300 each time). & $\begin{array}{l}\text { Prolonged case of Texas } \\
\text { f } \mathrm{v} \text { ver. Recovered } \\
\text { Mar. 18, } 1891 \text {. }\end{array}$ \\
\hline 149 & ....do .............. & ....do........ & Oct. 28 -Nov. 21 & 6 (15 each time). & Slight, if any effect. \\
\hline $117^{1}$ & $\begin{array}{l}\text { Southern heifer, } 2 \\
\text { ytars. }\end{array}$ & Nov. 19,1890 & Nov. 21-Dec. 3 & $7(200-300$ ea & Do. \\
\hline 130 & Cow, 2 years...... & Dec. 12,1890 & Dec. 13-Dec. 29 & 9 (200-300 each time) & $\begin{array}{l}\text { Marked case of Texas } \\
\text { fever. Ricoverel } \\
\text { Feb. 18, 1891. }\end{array}$ \\
\hline 152 & Cow, $4 \frac{1}{2}$ years. & .... rlo & $\ldots$. do .... & 9 (200-300 each time) . & $\begin{array}{l}\text { Mild case of Texas } \\
\text { fever. Recovered } \\
\text { Jan. } 20,1891 \text {. }\end{array}$ \\
\hline
\end{tabular}

1 In stall with No. 145.

The young ticks placed on Nos. 117, 143, 145, and 149 were descended from adults picked from dis. eased natives (Nos. 137, 138, and 140). Those placed on Nos. 130 and 152 were descended from adults received directly from North Carolina.

* For fulldetails concerning the ticks and the course of the disease in these cases seo appendix. 
'This experiment demonstrates that Texas fever may be produced at any season of the year if the conditions are fairly favorable; if, in other words, the temperature of the air is sufficiently elevated to permit the cattle tick to carry on its parasitic existence. Of five presumably susceptible animals infected with ticks three showed well-marked symptoms of Texas fever, and the remaining two reacted with a high temperature for a few lays. In these latter cases there may have been a reduction in the number of red corpuscles also, but we can not regard such reduction demonstrated until the number falls below 5,000,000. The temperatures taken twice a day will be found in the appendix, and we limit ourselves here to the following extracts:

No. 143. Temperature 104 to 105.3 on November 21 to 23 , inclusive.

No. 145. Temperature 104 to 106 on December 4 and 5.

No. 145. Temperature 104 to 105 from January 19 to 27.

No. 149. Temperature 104 to 105 on November 12, 13, and 25.

No. 117. Temperature 103.8 on November 26, and 103.4 on December 16 and 17.

No. 130. Temperature 104 to 106.4 from December 26 to January 1, and 103 to 104 on January 9.

No. 152. Temperature 103.8 to 106.3 on December 27 to 31 .

In these casses the high temperature appeared generally in fifteen days after the first lot of young ticks had been put on the animal. In No. 145 the period of marked destruction of red corpuscles was associated with high fever.

This experiment does not definitely prove that the progeny of ticks collected from susceptible Northern animals which have passed through the disease may produce as severe an attack as those descended from ticks picked directly from Southern animals. The positive result in No. 145, infected with "Northeru" ticks, is vitiated by the fact that this animal was in the same stall with a Sonthern cow, No.117. The severe secondary attack appeared in No. 145 in the midlle of January. This would allow time for the ticks to mature on No. 117, and the next generation to attack No. 145. Hence No. 145 may actually have received the severe secondary infection from "Southern" ticks, in so far as they were descended from those matured on No. 117. This interpretation may be wrong and the secondary infection in No. 145 may have been a true relapse resulting from the primary infection with "Northern" ticks. The experiment as it stands, however, can only be interpreted as showing that ticks produce welk marked ciisease in artificially-heated stables in winter, and the other question, whether "Northern" ticks may do this, must be left open.

Summary of the experiments of 1890.

The discovery of 1889 that ticks alone are sufficient to infect a field was confirmed this year. The experiment designed to test the theory that Southern cattle are infections only throngh the ticks they carry failed this year for the field became infected with ticks after all. Lastly, the demonstration of the important fact that the infection is 
conveyed by the young tick, and is probably introduced by it into the blood, was a very great stride in advance in our understanding of the external characters of the infection.

In field IX (see Fig. 6) several natives were exposed to North Carolina soil without becoming diseased.

On the station grounds field V (see Fig. 5) was infected with the blood and spleen pulp of cattle which had succumbed to Texas fever. The exposed natives did not become infected.

In field IV (see Fig. 5) during this same year a number of sick natives were brought together and some healthy natives added. The latter had a mild attack late in the season, only detected by the microscopic examination of the blood.

These three experiments will be fully discussed farther on, and we simply refer to them here to show that the animals not exposed under certain conditions did not become infected although pastured not far from Texas-fever cases during the summer.

Experiments of 1891.

The arrangement of the fields for this year and the uses to which they were put are indicated on the accompanying plat. A tract of land adjoining the station grounds on the north was added to the territory in use. On this tract were situated a dwelling house and a number of unused sheds. For the purpose of carrying on the various experiments, cattle were collected near New Berne, N. C., as in previous years, and shipped by steamer from New Berne, June 30. They arrived at the station July 2, having been but two days on the way.

Experiment 14 (exposure to North Carolina cattle with ticks).-The general control experiment of producing the disease in the natural way was conducted, as before, by exposing natives to Southern animals on the same field. For this purpose inclosure VI was again selected (see Fig. 7). In this experimen $\lrcorner$ not only unexposed natives but also recovered natives were reëxposed to test any acquired immunity (p. 133). Similarly Southern animals, kept for one or two years on the station, were reëxposed to determine any loss of immunity (p. 131). These collateral experiments will be discussed in dealing with these subjects. In this place we simply summarize the results of the exposure of fresh natives.

The animals placed in this field comprised the following:

(a) North Carolina cattle:*

July 2-No. 172 (cow, 6 years), from farm No. 6.

Juty 2-No. 174 (cow, 3 years), from farm No. 5.

July 2-No. 177 (cow, 5 years), from farm No. 3.

July 2-No. 178 (cow, 4 years), from farm No. 2.

* Eight animals were brought North, two from each farm, and divided equally between this and the following experiment. 
TEXAS OR SOUTHERN CATTLE FEVER.

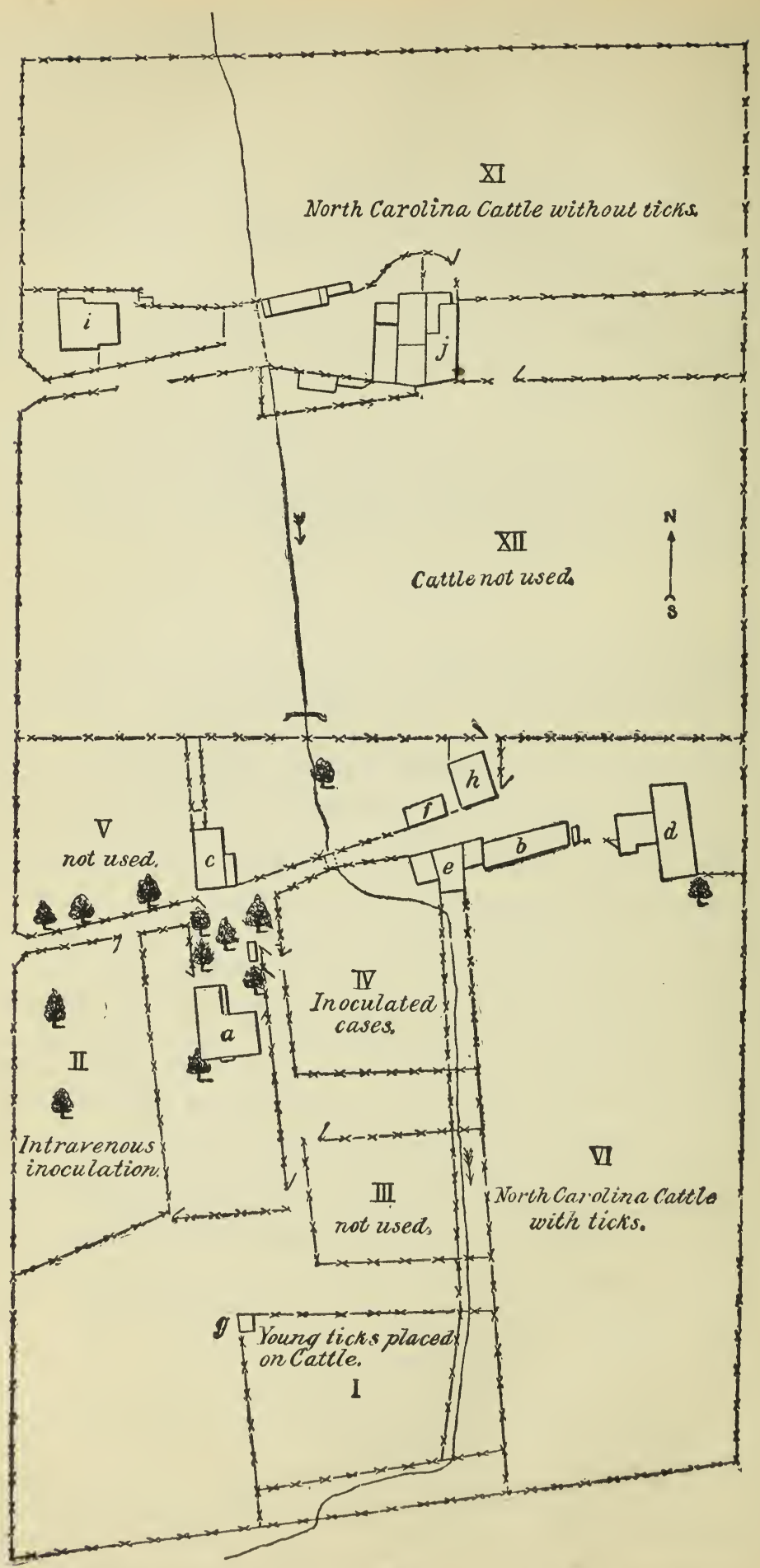
Fir. 7.- Field iuclosures for 1891 (scale: $\frac{1}{4}$ inch $=36$ feet), a to $g$, as in lig. $4 ; h, 8 w i n e$ pen; $i$, dwoll.
ing houso; $j$, cow stalls. 
(b) Natives:

July 2-No. 104 (cow, 4 years). Very sick; recovered.

July 2 -No. 159 (heifer, 2 years). Not very sick; recovered.

Juiy 2-No. 163 (cow, 6 years). Very sick; killed August 25.

September 1-No. 169 (cow, 8 years). Died September 14.

September 1-No. 181 (cow, 21 years). Killed in dying condition September 19.

Septemiber 15-No. 184 (heifer, 2 years). Died October 2.

September 21-No. 160 (cow, $2 \frac{1}{2}$ years). Prolonged attack; recovered.

September 21-No. 187 (calf of No. 160, 4 months). Not affected.

All animals excepting the calf were attacked by Texas fever. No. 163 was killed at the height of the fever and probably would have died. The mortality among these animals was lower than in the preceding year, the conditions being precisely the same. Among those exposed later on, the mortality was higher than among those exposed early. The ticks in this field had entirely disappeared from the Southern stock by the end of July. The young ticks had appeared in considerable numbers on all the cattle August 10 and continued to increase in number during August on all cattle alike. They had all disappeared by the last of October.

Experiment 15 (exposure to North Carolina cattle without ticks).The great importance of determining whether or not the ticks are the only carriers of the infection from the permanently infected regions of the South imposed upon us the necessity of trying this experiment until it could be stated with certainty that no ticks had passed from Southern to native cattle. The experiment of 1890 (Exp. 11) had failed because young ticks had appeared on the natives in course of the summer. For the repetition of this experiment the hitherto unused in. closure XI (see Fig. 7), covering over 2 acres, was chosen. The following North Carolina cattle were put into it July 2, after the ticks had been carefully picked off: No. 173 (cow, 3 years), from farm 6 ; No. 175 (cow, 4 years), from farm 5; No. 176 (cow, 6 years), from farm 3; No. 179 (cow, 5 years), from farm 2.

The following natives were added at the same time: No. 161 (cow, 6 years); No. 164 (cow, 7 years); No. 167 (heifer, 3 years).

The Southern animals were reëxamined daily and a few small ticks found each day until July 22. Thereafter very few were found and after July 29 none. Nevertheless a few adults must have escaped and dropped on the ground, for young ticks made their appearance, though in small numbers, in August. From August 22 to September 3 several hundred were removed from the three natives. No. 164 was most abundantly infected. Next came 167 in this respect. No. 164 suffered a severe attack and was found dead September 3. No. 167 likewise passed through a severe attack, but recovered, while in No. 161 signs of infection were not detected, as will be seen by an examination of the notes in the appendix. The severity of the disease was thus in general proportional to the number of ticks found on these animals. 
Experiment 16 (production of disease with young ticks hatched artificially).-This is a repetition of experiments 12 and 13 of last year, but is made much earlier in the summer. A large number of adult ticks were collected June 25-27 from cattle near New Berne, N. C. An abundance of eggs had been laid by July 7 , which were placed in glass dishes containing a few fresh leaves and a few drops of water and covered snugly with a piece of glass. The young ticks began to appear July 23.

July 25, Nos. 166 and 180, both 2-year old heifers, were placed in field I (see Fig. 7). To the rump and neck young ticks were applied daily for 10 days until August 4. No. 166 received from 200 to 300 daily, No. 180 only 20 to 30 daily. After each application the heifers were held quiet for a few minutes until the young ticks had crawled away through the hairs.

July 29, No. 158, a 2-year old steer, was placed on the same field and several thousand ticks from the same lot applied at one time.

August 20, No. 117, a North Carolina heifer of 1890, was placed in the same inclosure and several thousand ticks of the same lot applied at one time.

The following table summarizes the experiment. The full notes of each case are reserved for the appendix.

\begin{tabular}{|c|c|c|c|c|}
\hline No. & Age, etc. & $\begin{array}{l}\text { Young ticks } \\
\text { applied. }\end{array}$ & $\begin{array}{l}\text { Number of } \\
\text { applications. }\end{array}$ & Result of exposure. \\
\hline $\begin{array}{l}166 \\
180 \\
158 \\
117\end{array}$ & $\begin{array}{l}\text { Native heifer, } 2 \text { years..... } \\
\text { Nado .................... } \\
\text { Sative steer, } 2 \text { years...... } \\
\text { Southern heifer.......... }\end{array}$ & $\begin{array}{l}\text { July } 25 \text {-Aug. } 4 . \\
\text { Judo . . . . . . . } \\
\text { August } 30 \ldots \ldots\end{array}$ & $\begin{array}{l}10(200-300 \text { each time) } \\
10 \text { (20-30 each time). } \\
1 \text { (several thousand). } \\
1 \text { (several thousand). }\end{array}$ & $\begin{array}{l}\text { Severe attack; recovered. } \\
\text { Severe attack; died Aug. } 12 . \\
\text { Severe attack; recovered. } \\
\text { No result. }\end{array}$ \\
\hline
\end{tabular}

In all natives a marked case of Texas fever was produced, which proved fatal in that animal to which the smallest number of young ticks had been applied. It is also interesting to note that in this experiment Texas fever appeared much earlier than in field VI (experiment 14), when the infection took its natural course. Thus in the latter field the disease was observed first August 18, while among the artificially infected it appeared as early as August 7.

\section{EXPERIMENTS OF 1892.}

The field work of this year was mainly directed to wards determining whether or not Texas fever is transmitted withont ticks. The experiments of the two preceding years had failed, because young ticks appeared on the native eattle though they had been very carefully removed from the Southern animals. This year the experiment was tried over again, and with entire success. The young ticks did not appear on the native cattle, and the latter remained free from discase in 
spite of the presence of North Carolina eattle. Another very important fact was demonstrated. Texas fever was produced in natives by the intravenous injection of blooel from healthy North Carolina cattle, and natives exposed to these sick natives did not contract the disease because ticks were absent.

The Southern animals used in the field work of the summer were six in number, gathered together from two farms near New Berne, N. C., three being taken from each farm. They left New Berne by steamer June 29, and reached the station July 1, being off their native fields about four days before they were placed in the fields at the station.

The field inclosures for this year are numbered as they were last year. Hence the plat of 1891 (Fig. 7) will serve to illustrate the experiments to be described.

Experiment 17 (exposure to North Carolina cattle with ticks).-This experiment was to serve as a control upon the following experiments. Field VI was again used for this purpose. Two Southern animals, one from each North Carolina farm, were introduced as sources of the infection. The contents of the field and the general results of the exposure may be tabulated as follows:

June 30, 189:.-No. 201 (cow, 5 years). Removed to uninfected field July 20; not diseased.

June 30, 1892.-No. 203 (cow, 6 years). Died of Texas fever August 22.

July 1, 1892.-No. 216 (North Carolina cow, 6 years).

July 1, 1892.-No. 217 (North Carolina cow, 6 years).

July 20, 1892.-No. 220 (steer, $2 \frac{1}{2}$ years). Very sick; recovered.

July 20, 189?.-No. 223 (heifer, 6 years). Died of Texas fever August 23.

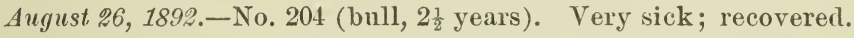

On July 20 only a few half-grown ticks were found on the Southern cows. On August 5 the young ticks were first noticed on the natives; at this time they had probably been on the cattle only two or three days. The outcome of the exposure does not differ from that of preceding summers. All exposed animals contracted Texas fever, and two of the three early exposures died.

It is interesting to note that No. 201, though it was pastured on the infected field for twenty days, remained perfectly healthy, because it was removed to an unused field (IV) before the young ticks had appeared in the infected field. It is likewise worthy of notice that No. 223, though exposed twenty days later than No. 203, became infected at about the same time and died only a single day later. The reason for this has already been pointed out, but it deserves repetition. The infection of the field is established when the young ticks have hatched, and not before. Hence a field is not dangerous until twenty or twenty-five days (according to the average temperature) after Southern cattle have pastured on it.

Experiment 18 (exposure to North Carolina cattle without ticks).Field I (see Fig. 7) was used for this test. Two North Carolina cows (Nos. 212 and 213), one from each farm, were chosen, so as to make the 
conditions as nearly like those in the control field as possible, and the ticks carefully picked off before they were placed in this ficld. They were subsequently examined daily, as will be seen from the notes in the appendix under each case, for any ticks too small to be seen originally. Two native cows (Nos. 208 and 209) were placed in the inclosure with them. These remained perfectly well throughout the summer.

Experiment 19 (exposure to North Carolina cattle without ticks).-This is an exact duplicate of the preceding experiment, to insure the success of one in case the other failed, by reason of the appearance of young ticks. Two North Carolina animals (Nos. 214 and 215), one from each farm, were carefully picked over and all ticks removed so far as they could be detected. They were placed in field No. II, July 2. Two native cows (Nos. 205 and 210) had been placed in the same field two days before. The Southern animals were carefully reëxamined for ticks, as in the experiment preceding, for several weeks. In this field no disease appeared during the entire summer. The results of the examination of the blood of these cases at different intervals are to be found in the appendix.

The cases in these fields may be tabulated as follows:

\section{Field $I$.}

July 2-No. 212 (North Carolina cow, 3 years).

July 2-No. 213 (North Carolina cow, 5 years).

Jnne 30-No. 208 (cow, 5 years). Exposure negative (October 1).

June 30-No. 209 (cow, 9 years). Exposure negative (October 1).

Field 11 .

July 2-No. 214 (North Carolina cow, 4 years).

July 2-No. 215 (North Carolina cow, 4 years).

June 30-No. 205 (cow, 5 years.) Exposure negative (Octıber 1).

June 30-No. 210 (heifer, 2 years.) Exposure negative (October 1).

MISCELLANEOUS EXPERIMENTS.

Experiment 20 (to test infection by way of the digestive tract).-No. 131, a heifer, 2 years old, was placed in a box stall July 29, 1890, and fed at three intervals about 2,000 adult live ticks in all. The animal remained well. No blood examinations were made.

No. 110, a heifer, one year old, was placed in a box stall August 14, 1890, and fed several thousand young ticks and egg cases. The result was to all appearances negative. Unfortunately the blood was not examined.

No. 145, a heifer, 18 months old, was placed in a box stall September 17,1890 , and fed once daily with one-fourth bushel of grass cut from the infected field VI together with hay and mill-feed. The experiment, was closed October 12. No indications of disease during the feeding or until November 21, when it was used in another experiment (see appendix). 
1.xperiment 21 (to test presence of infection in young ticks).-August 29, 1891, a large number of youm ticks nearly $1 \frac{1}{2}$ months old, and still confined in the glass dish in which they were hatched, were crushed in a mortar in sterile distilled water. The turbid brownish liquid was filtered through two thicknesses of filter paper. A portion of this filtrate was passer through a Pasteur filter to remove organisms of every kind.

No. 165 (heifer, $2 \frac{1}{2}$ years) receives into the right jugular vein 10 cubic centimeters of the fluid passed through the Pasteur filter.

No. 183 (heifer, $2 \frac{1}{2}$ years) receives into the right jugular vein 5 cubic centimeters of the turbid fluid not passed through a Pasteur filter. Repeated examination of the bloor in both cases failed to show any disease.

In 1892 a similar experiment was tried with equally negative outcome. Nos. 202 and 207 were used for this purpose and their history together with the blood examinations may be found in the appendix.

The result of these two experiments is at present inexplicable. The crushed ticks introduced into the blood fail to produce any infection whatever, while ticks from the same lots when placed on the skin (see No. 224) produce Texas fever. The experiment simply demonstrates our incomplete knowledge of the life history of this parasite.

GENERAL SUMMARY OF THE FIELD EXPERIMENTS RELATING TO THE CATTLE TICK.

We are now in a position to review the results of the field work of the past four summers and determine how far they enable us to draw definite conclusions. In addition to the general control experiments (Experiments No. 1, 3, 4, 8, 9, 14, and 17) by which Texas fever was produced in the natural way in natives which pastured on the same ground with Southern (North Carolina and Texas) cattle, experiments have been carried on in the three directions outlined on p. 92.

(1) Experiments with Suuthern cattle from which the ticks were picked off were made every year. (Experiments No. 2, 5, 11, 15, 18, and 19.) Those made in 1889 and 1892 were successful. Those made in 1890 and 1891 failed because young ticks appeared subsequently. The conclusiou from these experiments that the tick is necessary to cause infection in Northern cattle may be regarded as demonstrated.

(2) Experiments to show that fields may be infected by cattle-ticks alone were made in 1889 and 1890. (Experiments No. 6, 10.) In both Texas fever was produced.

(3) Experiments to show that young ticks artificially hatched produce Texas fever when placed on susceptible cattle were made in 1890, 1891, and 1892. (Experiments No. 12, 13, 16, and Nos. 224 and 225.) These were uniformly successful in the summer and fall months.

It was observed, however, that the disease induced by such ticks is less fatal than that produced in the fields in the natural way. We are 10320 -No. $1-8$ 
not prepared to account for this difference, unless it be the mode of incubation. The artificial condition of heat and moisture under which the eggs are kept may iead to a speedy destruction of the microparasites which are in some unknown way associated with then.

THE RELATION OF THE CATTLE TICK TO THE "PERIOD OF INCUBATION" OF TEXAS FEVER AND TO THE INHECIIOUSNESS OF SOUTHERN CATTLE.

In the foregoing experiment everything points to the cattle tick as the natural transmitter of the disease. It has been definitely demonstrated by our experiments that not only fields may be infected by simply scattering matured ticks over them, but that cattle themselves may be infected in stables away from all infected ground by placing on them young ticks artificially hatched.

We are now in a position to understand the peculiar variability in the period of incubation. We have seen from the experiments related that when native cattle are exposed to Southern cattle on a given field the period elapsing before the disease appears is generally over fortyfive days, and the first deaths usually occur one or two weeks later, as is illustrated by the following table:

\begin{tabular}{|c|c|c|c|c|c|}
\hline Year. & $\begin{array}{l}\text { Experi- } \\
\text { ment. }\end{array}$ & $\begin{array}{l}\text { Date of ex- } \\
\text { posure. }\end{array}$ & $\begin{array}{l}\text { First high } \\
\text { morning } \\
\text { tempera- } \\
\text { ture. }\end{array}$ & First death. & $\begin{array}{l}\text { Number of } \\
\text { days after } \\
\text { exposure. }\end{array}$ \\
\hline $\begin{array}{l}1889 \\
1890 \\
1890 \\
1891 \\
1892 \ldots \ldots\end{array}$ & $\begin{array}{r}1 \\
9 \\
8 \\
14 \\
17\end{array}$ & $\begin{array}{l}\text { June } 27 \ldots \ldots \\
\text { July } 4 \ldots \ldots \\
\text { July } 4 \text { and } 5 . \\
\text { July } 2 \ldots \ldots \\
\text { July } 1 \ldots \ldots\end{array}$ & $\begin{array}{l}\text { Aug. } 15 \ldots \\
\text { Aug. } 18 \ldots \\
\text { Aug. } 23 \ldots \\
\text { Aug. } 18 \ldots \\
\text { Aug. } .6^{1} .\end{array}$ & $\begin{array}{l}\text { Aug. } 23 \ldots \\
\text { Aug. } 27 \ldots \\
\text { Aug. } 29 \ldots \\
\text { Aug. } 29 \ldots \\
\text { Aug. } 22 \ldots\end{array}$ & $\begin{array}{l}57 \\
54 \\
55 \\
58 \\
52\end{array}$ \\
\hline
\end{tabular}

1 There was a period of high evening temperature in this field $J u l y 20-25$, the significance of which is not clear.

This long period coincides with the time necessary to produce a new generation of ticks. When Southern cattle graze on a certain pasture in early summer, say for a day only, a few ripe ticks drop off. They lay their eggs in about seven days. These are hatcher in about twenty days and are at once ready to crawl on cattle. Ten days thereafter the first high temperature usually appears. If we add these figures together we find that the disease may appear about thirty-seven days after the field was infected.* 'To be sure these figures are liable to fluctuations, which may make this period much longer or perhaps a little shorter at times.

When Southern cattle are placed on a certain field and kept there, as in our experiments, the field becomes much more abundantly infeeted with ticks, for the reason that all the tieks in their various stages ripen and fall on the same ground. Hence there is a continuous infec-

* In 1890 the Texas-fever parasite was first detected in the blood (No. 95) 34 days after the first day of field infection. 
tion of the field going on for several weeks, until all the ticks originally attached to the Southern cattle have disappeared. 'This may increase the severity of the disease.

But how may we account for the fact that cattle placed on infected pastures later may become diseased at the same time and may die in less than fifteen days after the first day of exposure? Simply by taking into account the fact that cattle exposed late are at once attacked by the young ticks already present on the field. Hence, if we allow ten days for the fever to appear after the ticks have crawled on the cattle the mystery is at once explained.

The explanation of unusually prolonged periods of incubation is equally simple. They are associated with very early importations of cattle and the low temperature retards the development of the young in the egg. We have already shown that this development may be greatly retarded by cold, and we have observed periods of incubation ranging from twenty to forty-five days and have kept eggs over winter which developerl when the temperature became warm enough the following spring.*

Billings has compiled a table $(8, p .47)$ embracing ten outbreaks of Texas fever which have occurred in various Westeru States since 1868. The period elapsing from the date of exposure to that of the first death ranges from thirty-three to ninety days. Leaving out of account these two extreme periods, the remaining ones range between forty-six and sixty-five days. The short period of thirty-three days is probably due to the fact that the native cattle were exposed on a field which had been infected some time before and on which the tick eggs had consequently undergone more or less development. This is made highly probable by putting this outbreak with the one after it in the table.

(6) Tolono, Ill., June 2.5, 1868 (date of arrival of 'Texans)..... July 28 (first death). Period 33 days.

(7) Sodorus, Ill., June 1, 1868 (date of arrival of 'Texans)......July 28 (first death). Period 56 days.

Tolono and Sodorus are on the same railroad and but 5 miles apart. The Sodorus fields were infected June 1 and the period of incubation is fifty-six days, the usual time. The Tolono fields were most likely infected at the same time, since the first death occurred at both places on the same day. That they were not also infected twenty-five days later we do not pretend to gainsay. At this time of the year, i.e., June and July, the period of thirty-three days is somewhat short for the appearance of a new generation of ticks, and the explanation given will clear up this difficulty. Billings has furnished a very good illustration $(8, p .47)$ of the prolongation of the period between infection of pastures and the appearance of the disease in an outbreak at Tekamah, Nebr., studied by him in 1887. The Texans infected the field as early as April 1 or thereabouts, but the disease did not appear until ninety days thereafter. 
There is general unanimity on this point, that a long period elapses between the date of infection of a given pasture and the appearance of the disease, so that further illustrations may be dispensed with. In searching over all the various publications on this subject we have not yet encountered any authentic statement which gave dates to support its claims that Texas fever ever appeared on a field within thirty days of the time that it was entered by tick-bearing cattle. If there are any such outbreaks, they may have been produced either by ticks wintering over in the egg or by an infection of the field earlier than that actually noted. It is not improbable that Southern animals may accidentally carry some eggs of ticks nearly hatched on their feet or other parts of their body. In such a case disease might appear several weeks earlier. The same would be true if ticks which have once attached themselves to cattle may, after being accidentally torn or brushed off, crawl upon natives, provided they are still infectious.

The relation of young ticks to Texas fever explains why natives placed in an infected inclosure at various intervals before the appearance of the young ticks will all contract the disease at the same time. They may mingle with freshly arrived Southern stock for twenty or twenty-five days before becoming infected. If removed at the end of this period before the appearance of young ticks they remain healthy. (See Expt. 17, page 111.) We now understand why natives placed on an infected field after the young ticks have appeared will contract Texas fever in ten to fifteen days. The life history of the tick likewise explains the frequently observed fact that Southern cattlelose the power of infecting Northern pastures after a certain number of days. We have already stated that the ticks on Southern cattle gradually disappear as they become matured. It is evident that when all have dropped off, the power of the cattle to infect fields is lost. It is possible to give the exact period of time required, provided we know the time which has elapsed since Southern cattle left their pastures, where they are being continually infected with young ticks. On the station pastures the time required for all the ticks to disappear was twenty-five to thirty days. Very soon thereafter the young ticks, descended from the ticks which matured first, appeared on all cattle, and the Southern animals again became infectious. The maturation of the second generation may push the period of disease into the fall and thereby rob it of its fatality.

We now likewise understand how cattle driven slowly northward lose their infection after a time. As soon as they have left the territory where ticks abound they receive no more accessions of young ticks and they are continually dropping mature ones. After twenty-five to thirty days, or perhaps sooner, they have parted with all and are henceforth harmless to Northern stock.

Let us now review briefly what oecurs when Southern tick-bearing cattle are placed on the sane inclosure with matives. If the animals be brought together early in July, as in our experiments, nothing un- 
usuah will be noticed for weeks. The ticks on the Southern an imals slowly mature, swell up, and drop off, one by one, so that after three or four weeks all have practically disappeared. If during the second week in Angust the cattle be carefully examined, young ticks will be found attached to the skin and buried within the coat of hair. They may be overlooked if the examination is superficial and hasty. A week later, generally in the third week in August, the temperatures of all exposed native cattle suddenly rise to $105^{\circ}$ or $107^{\circ} \mathrm{F}$. within a few days of one another. The ticks at this time are still quite small, and have not yet passed through the second molt. Even at the post-mortem examination of many cases only small, immature ticks are found. If the natives survive the attack, the ticks mature, swell up, and drop off, ready to give birth to a second generation if the season permits.

\section{THE RELA'TION OF THE CATTLE TICK TO THE MICROÖRGANISM OF} TEXAS FEVER.

The hypothesis which seemed most plausible after the experiments of 1889 was that the tick, while withdrawing the blood from Southern cattle, drew out in it the Texas-fever parasite, which, entering into some more resistant state, perhaps some spore state, was disseminated over the pastures when the body of the mother tick became disintegrated. These spores were then supposed to enter the alimentary tract with the food and infect the body from this direction. The later experiments, however, completely demolished this conception. Neither the feeding of adult ticks and tick eggs* nor the feeding of grass* from infected pastures gave any positive results. On the other hand, the unmistakable outcome of the experiments was that the young tick introduced the infection into the borly. This fact implies two possibilities. Either the tick is a necessary or a merely accidental bearer of the micro-parasite. If a necessary bearer of the infection, we must assume that the latter undergoes certain migrations and perhaps certain changes of state in the body of the adult tick and finally becomes lodged in the ovum. Subsequently it may become localized in certain glands of the young tick and discharge thence into the blood of cattle. This hypothesis assumes a complex symbiosis between the tick and the parasite on the one hand and the cattle and the tick on the other. According to another, simpler hypothesis the tick would be merely an accidental bearer of the infection. The parasite entering the body of the tick with the blood of cattle may be already in the spore state or about to enter upon such a state. The young ticks, as they are hatched near the dead body of the female, may become infected from this. This infection, elinging to their mouth parts, is introduced into the blood of the cattle to which they subsequently attach themselves. Further investigations are necessary before the probable truth of one or the 
other of these hypotheses can be predicated with any degree of certainty.

It should be stated that the contents of the bodies of ticks in various stages of growth have been examined microscopically with considerable care. The abundant particles resulting from the breaking up of the ingested blood corpuscles obscured the search so that nothing definite has thus far resulted from it. The very minute size of the microörganism renders its identification well-nigh impossible, and any attempts will be fraught with great difficulties.

A question of much interest, but one upon which we have no information, is the relation of the cattle tick to the enzoötic Texas fever area. Is the distribution of the tick coextensive with that of the Texas fever micro-parasite, or does their distribution obey different laws? This question could be solved by a thorough investigation of a small portion of the border line of the enzoötic territory. This border line probably depends on the mean annual temperature, and hence we can not expect to find it very sharply defined. Ticks may extend farther north during some seasons than others, and hence there may be a belt or strip on which cattle are partially insusceptible because of former repeated attacks, although for the time being ticks may be absent. The entire subject is at present speculative, and is simply referred to liere to arouse the attention of those who are in a position to record observations concerning it.

THE RELATION OF SOUTHERN CATTLE TO THE TEXAS-FEVER INFECTION.

What has already been said concerning the tick makes it certain that all Southern cattle are dangerous when they bear the cattle tick, whether they are sick or healthy. On the experiment-station fields, the North Carolina and Texan cattle which called forth Texas fever during the four years of the investigation were, in general, healthy. Two cows (Nos. 63 and 112) were killed. One of these had impoverished blood, although positive signs of Texas fever were not detected. Another died of peritonitis. The remainder were healthy, improved on the pastures, and were sold at the beginning of winter or before.

In the foregoing pages it has been assumed that the tick obtains the micro-parasite from Southern cattle. Withont demonstration it might be claimed with equal propriety that the micro-parasite belongs essentially to the cattle tick, and that its multiplication in the body of susceptible cattle is perhaps an accidental phenomenon against which southern cattle have been amply protected by frequent infection. Lixperiments made latterly, as well as the microscopic examination of the blood, prove that the micro-parasite is harbored by Sonthern cattle in a state of health. These interesting experiments, as far as they have been carried up to the present, are briefly summarized here. The complete record will be found in the appendix under each case. 
Inoeulation with blood of healthy North Carolina cows soon after the lutter had left the Southern pasture.*-On July 6, 1892, a native cow, No. 19s, received into a jugular vein 28 ce. of blood drawn from a jugular vein of a North Carolina eow, No. 217. The quantity of blood injected was large, becanse it was supposed that if the microörganisms were present at all in the Southern cattle they would be very scarce. The blood was drawn fiom the Southern cow by piercing the wall of the vein with the needle of a previously sterilized and warmed hypodermic syringe holding $14 \mathrm{cc}$., and injected immediately after into the exposed jugular of the native cow by simply piercing the wall of the vein. The entire operation lasted about two minutes. The high temperature became continuous on the seventh day after inoculation, and the number of red blood corpuscles had begun to fall on this same day. The various symptoms of Texas fever became gradually intensified, and the animal died July 19, thirteen days after inoculation. On the day before death the urine was claret-colored. The autopsy revealer the usual lesions of Texas fever.

On the same day No. 206, another native cow, was inoculated in the same way with blood drawn from a jugular vein of North Carolina cow No. 216. The same quantity, 28 cc., was injected as before, $14 \mathrm{cc}$. being injected at a time. The high temperature and the destruction of red blood corpuscles set in at the same time with those of the preceding case. This animal did not die, however. After passing through a prolonged fever period the animal slowly recovered, to suffer a relapse, which kept the number of red corpuscles below 2,000,000 during the whole of september.

On July 16, ten days after the preceding inoculations, a third cow, No. 219, received the same quantity of blood into a jugular vein. The blood was drawn from North Carolina cow No. 216. The continuous high temperature began July 24 , at which date the destruction of corpuscles had set in. The further history of this case is very similar to that of the preceding, No. 206. The blood corpuscles continued to decrease in number until August 6. After this there was a slow rise. At the end of August a relapse was detected, which continued throughout September.

Inoculation with blood of healthy North Carolina cattle sometime after the latter had left the Southern pasture.-On August 15, a steer, No. 222, received 28 cc. of blood drawn from North Carolina cow No.214. The same procedure as above was adopted. The disease had become established by August 25, as is shown by the high temperature and the marked reduction in the number of red corpuscles on this day. The movement of the disease was markedly slower, so far as this latter phenomenon is concerned. In the middle of September a relapse was detected, which was still in progress at the beginning of the second week in October.

\footnotetext{
* For the examination of the blood of the Southern cattle see appendix under each case.
} 
On September 9, a cow, No. 230, received the same quantity of blood from No. 214 into a jugular vein. In this case the first high temperature appeared September 14, and by October 1 the number of red corpuscles had fallen to 2.2 million.

These positive results demonstrate that the Texas fever microörganism was present in the blood of North Carolina eattle as long as seventyfour days after they had left the permanently infected territory. One cow, No. 217, was tested once on the ninth day; another, No. 216, was tested on the ninth and the twentieth day; a third was tested on the forty-ninth and the seventy-fourth day. That the microörganism was the one found in natives infected in the ordinary way on pastures was demonstrated in every one of the five cases by a large number of microscopic examinations. No difference whatever could be detected. Moreover, three out of the five inoculated cases passed through relapses or mild secondary attacks, in which the stage of the peripheral coccus-like body appeared constantly in the blood, as in the ordinary mild type of the disease. There cau be no doubt, therefore, that the micro-parasite transmitted in the blood of Southern eattle was the same as the one introduced into the blood of natives by the cattle tick.

It might be claimed that the Southern cattle harbored this microparasite because they are being constantly reinfected by the cattle tick. This might be true of Nos. 216 and 217, but it does not hold for No. 214 . This animal was one of the four from which the ticks had been carefully picked in July, so that at the date of the last inoculation with blood from this animal it must have been entirely free from ticks for at least fifty days, and it had received no fresh accession of ticks since leaving its native pasture, June 27 (seventy-four days).

Whether the Texas fever parasite resides permanently in the bodies of Southern cattle or whether its presence, after all, depends on that of the cattle tick, these experiments do not permit us to decide definitely at present.* From an economic standpoint this is of little importance, since in many parts of the permanently infected territory of the South ticks are present during the entire year.

The presence of the parasite in Sonthern cattle does not seem to materially affect their health, although it may maintain a more or less constant breaking up of the red corpuscles on a small scale, which would necessarily tax certain vital organs. The parasite is present in the circulating blood in such small numbers, however, that ouly after a most

* The production of disease in 1891 by ticks which wintered over on one of the fields of the station would at first sight suggest the inference that the cattle tick carried the parasite throngh the winter in the egg. (See 1. 90 and No. 154.) 'This does not follow from the circumstances, however, for there were in the same inclosure Southern eattle which had been kept over one or two years for purposes of rë̈xposuro. Ticks hatched in spring may have invaded all the cattle in the inclosure, matured and dropped off, and given rise to mother generation, which produced the disease late in Angust. This second generation may have obtained the micro-parasite from the Southern cattle. 
tedious microscopic examination is it occasionally encomtered. The fact that Southern cattle rid themselves of infectious properties on Northern pastures after twenty-five to thirty days does not, therefore, imply that their blood is no longer infectious. It simply signifies that they have rid themselves of the means by which this parasite is transmitted, namely, the cattle tick.

In this connection the reader may be referred to the various Southern animals whose blood was examined at one time or another during these investigations. Thus, in the Appendix, estimates will be found of the number of red blood corpuscles of Nos. 121, 123, 124, 126, 216, and 217 , obtained at intervals after therr removal from southeru pastures varying from two to ten weeks. The number was, in general, fully up to the level maintained by the natives used in the experiments. There are, also, records of a few southern animals kept on the station grounds for longer periods, and subsequently exposed to Texas fever infection fresh from the South. Thus, No. 117 exposed, in winter, five months after arrival from the South, Nos. 32, 62, and 59 exposed one year thereafter, and Nos. 55 and 60 two years thereafter, show the normal number of red corpuscles.

The discussion which has raged so persistently about the health of Southern eattle has outlived its usefulness or suggestiveness, for it does not matter in what condition they are. So far as our evidence goes-and this is very strong-they are quite harmless, provided they do not carry the cattle tick. Hence there is no necessity for going into a review of the statements of Gamgee, the Metropolitan Board, and of F. S. Billings on this point. It is not claimed that Southern cattle may not and do not contract Texas fever. It is highly probable that every Southern calf has to go through the process of natural inocnlation and reinoculation to a greater or less extent, and we have the records of several calves of Southern parents (Nos. 86 and 87) which passed through a mild form of the disease. It is likewise probable that a certain percentage of Southern animals which have not been sufficiently exposed while young may contract Texas fever in adult life under abnormal conditions. It is not impossible that under the influence of prolonged marches, crowding in cattle cars and on vessels, with insufficient air and food, the natural resistance of the body may break down and the mild or unobserved infection break out into an acute disease. These are possibilities as yet unproved, but they are by no means ignored when we state that Southern cattle, to all appearances healthy, do transmit Texas fever, and it is not necessary that they have any symptoms of disease, recognizable by clinical methods, to make them dangerous. We do not now wish to enter into any philosophical discussion as to what constitutes disease. From a practical economic standpoint we must maintain that Southern cattle may be healthy and yet be the cause of Texas fever.

The various hypotheses which observers have framed concerning the 
infectious character of the excreta of cattle, the saliva hypothesis of Detmers, the manure hypothesis of Billings, or the urine hypothesis of others must now be considered as unfounded so far as these excreta are claimed to be the direct source of the disease, since the excreta of Southern cattle on Northern fields can not produce Texas fever. In those experiments (p.111) which demonstrate that Southern cattle may pasture with susceptible Northern cattle throughout the summer without imparting disease, provided all ticks be removed, we have all the necessary proof for refuting these hypotheses, since the excreta and secretions of all kinds are left on the field. The only object missing is the cattle tick.

SICK NATIVES AS SOURCES OF INFECTION WHEN THE CATTLE TICK IS PRESENT.

This matter has called forth much discussion by scientific observers as well as by cattle-owners. It is a question of considerable importance to determine whether cattle which have contracted Texas fever in the ordinary way may transmit it to other natives coming in contact with them. That such transmission inust be very rare is evident, otherwise there would have been no discussion and no divergent opinions. It is certainly a very curious fact that animals, which are affected with an infectious disease contracted indirectly through the presence of certain presumably healthy cattle, should not also transmit the same disease to other susceptible cattle. Theoretically, there is nothing opposed to the view that sick natives may infect other natives, and we shall show that they actually do so; but the conditions under which this infection takes place are rarely realized, and hence very little disease due to natives comes under observation. The fact that the disease may be transmitted from sick to healthy natives directly by injections of blood into the veins does not help us in solving the problem before us, since the disease is not conveyed in this way. If we turn, however, to the life history of the tick we shall find the explanation sought.

Sick natives have ticks on them. But only those which survive the disease or die after a prolonged attack ripen the tick on their bodies. Those which die of an acute attack in a short time after infection have only immature ticks on them. If the fever has occurred early enongh in the season to permit a second generation of ticks to appear before the cold weather arrives, we may expect Texas fever on fields on which sick natives only have pastured. Usually the first ontbreak occurs in August, and the second, to be looked for in late September or early October, is so mild as to pass mobserved. If, however, the first outbreak occurs in July, the second may appear in September and perhaps be of greater virulence. 
In order to test this problem the following experiment was carried out:

In 1890 Field IV (see Fig. 5, p. 99) was set aside to be infected with sick natives only. The following sick animals were introduced:

August 21.-No. 49 and calf No. 85 exposed in Field VI since July 4; elevated temperature since August 19.

August 21.-No. 105, exposed in Field VIII (ticks only) July 4; elevated temperature since August 13.

September 3.-No. 50, exposed in Field VI July 4; elevated temperature since August 27.

September 5.-No 47, exposed in Field VIII (ticks only) August 21; elevated temperature since September 1.

September 8.-No. 135, exposed in Field VIII (ticks only) August 21; elevated temperature since August 30.

From the notes of the appendix it will be seen that all these cases excepting the calf went through a severe attack of Texas fever, to which No. 47 and No. 50 succumbed. The field was therefore infected, so far as this was possible, by sick natives.

In this field were placed two natives (Nos. 132, 133) on August 21. These animals passed through a mild but undoubted attack of Texas fever. In both, blood parasites were observed early in October, and the number of red corpuscles shows evidence of infection after the middle of September. Though this experiment is sufficient to demonstrate the ability of sick natives to infect pastures, a much more obvious and striking result might be obtained by an early infection of the fields.*

There are several instances reported of the transmission of disease by sick natives. The Metropolitan Board of New York City reported an outbreak of Texas fever among cattle at Hamptonburg, Orange County, N. Y. (1, p. 954), due to the importation of native cows from Painesville, Ohio, on the Lake Shore Railroad, over which a large number of Texas cattle had been passing. The disease, supposed to have been introduced by the Ohio natives, broke out in October, 1868, and deaths occurred as late as October 24 and 27. The cows brought from Ohio were received Angust 25, and deaths occurred among them on that day and up to September 10. It is also stated that several native cows died of Texas fever fourteen, sixteen, and nineteen days after exposure to these infected natives. This last statement is open to question, for if our deductions be correct and the general experience of those who have observed Texas fever be trustworthy, it would take from one and a half to two months for such infection to take place.

A very good illustration of the infecting power of diseased natives is that given by F. S. Billings ( 8, p. 41). According to his statement 1,100 Texan cattle reached Tekamah, Nebr., March 30-31, 1887.

* A similar experiment (p. 105) in the artificially heated stable with the progeny of ticks matured on sick natives is not conclusive on this point. 
Twenty-one native cows put into one of the inferted pastures May 1 began to die early in July. On June 19, twenty-four native steers broke into a pasture infected April 1-15 by the Texan cattle. They were returned next day to a pasture containing 114 natives. The twenty-four steers began to show signs of disease July 9, and only two recovered. Curiously enough Texas fever broke out among the 114 natives, and several were found dead September 21. These circumstances are all perfectly intelligible, if we apply the facts which we have worked out concerning the life history of the cattle tick and its relation to Texas fever. It is to be regretted that Billings did not make any observations on the ticks present on the infected cattle.

We will take it for granted that the Texan cattle brought cattle ticks with them, and that ripe females dropped on the pastures about Tekamah, Nebr., from April 1 to April 15. We have received such from North Carolina in midwinter which, confined in a paper boxin the laboratory, promptly laid a large number of eggs. It might be claimed that at this date the low temperature would destroy the ticks entirely. It is true that low temperatures interfere with the growth of ticks on cattle and with the development of the young tick in the egg, but the embryo is not destroyed and simply lies dormant until the warmer season approaches. Thus on the experiment station ticks (probably in the egg) actually wintered over on a wooded pasture in 1890-91. We are indebted to the Weather Bureau for the daily maximum and minimum temperature of De Soto, Nebr., about 25 miles south of Tekamah, from March 30 to May 15, 1887. From this table we learn that the thermometer fell at night below $32 \circ \mathrm{F}$. only seven times after March 30 , and that on April 8 the maximum temperature was $92{ }^{\circ} \mathrm{F}$. There was nothing in the weather, therefore, to prevent the ripe ticks laying their eggs. The young ticks probably did not hatch before the middle of June, because the twenty-one native cows which were put on an infected pasture did not begin to die until early in July. The twenty-four steers which broke into an infected pasture Jume 19 began to show signs of disease at about the same time (July 9). This short period of twenty days indicates that the ticks were probably just hatehed when these steers broke in.

The time of infection of the large lot of natives by these steers may be easily calculated. They returned from the infected pastures June 20 with young ticks on them. If we allow twenty to twenty-five days for maturing, seven to ten days for egg-laying, twenty days for hatching, ten to fifteen days for the appearance of the fever, and seven to fourteen days for the first fatal case, we have in all sixty-four to eighty-four days from June 20 for the first death among the natives infected by natives. This could bring us to August 23 or September 12 as the probable date of the appearance of the disease originating from ticks which matured on native cattle. 'The actual date was September 21. Or we 
may calculate it in another way. When Southern cattle infect the ground by simply passing over it, they do so by dropping ripe ticks ready to lay their eggs. In such a case we usually find a period of fifty-five to sixty days elapse before the first death. In the case before us the twenty-four steers which broke into the infected pasture June 19 brought only young ticks with them. Hence to the usual period of fifty-five to sixty days we must add twenty to twenty-five days to allow the ticks on the native steer's to ripen. This would make the period seventy-five to eighty-five days and the first death might occur between September 3 and September 13, provided the case were acute and rapidly fatal, as is the case in midsummer.

The mortality of such secondary outbreaks due to sick natives is probably very low. In the case before us we are not told definitely by Billings how many of the 114 head exposed to sick natives succumbed, excepting that several were dead on a certain date. It has already been stated that only those natives which survive or die after pro. longed illness can mature ticks on their bodies. Hence where the mortality is very high, the ticks may mature in but small numbers, so that the secondary outbreak due to sick natives may be mild for this reason as well as on account of the advanced season; for there seems to be, up to a certain point, a more or less direct relation between the number of ticks which attack cattle and the severity of the disease.

In regard to the infectious character of sick natives it may be concluded that the infection really exists and it may be transmitted to other natives by the cattle tick. The severity of the secondary disease will depend upon the time of the first outbreak among the natives and upon the number of ticks matured. It is as a whole not a very serious element and the losses result mainly from the impoverished condition of the animals which pass through such attacks.

SICK NATIVES ARE HARMLESS WHEN THE CATTLE TICK IS ABSENT.

This investigation is largely of theoretical interest in confirming the experiments which demonstrate that Texas fever is not transmitted from Southern to Northern stock without the intermediation of the cattle tick. Natives are not supposed to be sick excepting as they are infected by the cattle tick, hence the existence of sick natives withont ticks must be of such rare occurrence that it is of no practical importance. In the following experiment the disease was produced in native cattle by the intravenous injection of blood drawn fiom the jugular vein of healthy Southern cattle. For a more complete discussion of these inoculations the reader is referred to page 119 and to the history of the individual cases mentioned below in the appendix. Here we simply mention the fact that the disease was actually produced and 
that two natives, placed with such cases as controls, remained well, as is indicated in the annexed table:

\begin{tabular}{|c|c|c|c|c|}
\hline Date. & No. & $\begin{array}{l}\text { Quantity } \\
\text { of blood in- } \\
\text { jected into } \\
\text { vein. }\end{array}$ & $\begin{array}{l}\text { Source of } \\
\text { blood. }\end{array}$ & Remarks. \\
\hline \begin{tabular}{lr}
\multicolumn{2}{c}{1892.} \\
July & 6 \\
July & 6 \\
July & 16 \\
& \\
Aug. & 15 \\
Sept. & 9
\end{tabular} & $\begin{array}{l}198 \\
206 \\
219 \\
222 \\
230\end{array}$ & 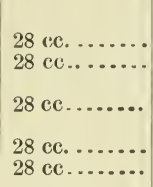 & $\begin{array}{l}\text { N. C. cow, } 217 \text {. } \\
\text { N. C. cow, } 216 \text {. } \\
\text { N. C. cow, } 216 \text {. } \\
\text { N. C. cow, } 214 \text {. } \\
\text { N. C. cow, } 214 \text {. }\end{array}$ & $\begin{array}{l}\text { Disease begins July } 13 \text {. Cow dead July } 19 . \\
\text { Disease begins July 13. Acute attack followed by re- } \\
\text { lapse in Soptember. Recovery. } \\
\text { Disease begins July 13. Acute attack followed by re- } \\
\text { lapse in September. Recovery. } \\
\text { Disease begins August } 18 \text {. Recovery. } \\
\text { Disease begins September } 14 \text {. Recovery. }\end{array}$ \\
\hline $\begin{array}{l}\text { July } 16 \\
\text { Aug. } 15\end{array}$ & $\begin{array}{l}218 \\
221\end{array}$ & …....... & n................ & $\begin{array}{l}\text { October } 1 \text {, exposure negative. } \\
\text { October } 1 \text {, exposure negative. }\end{array}$ \\
\hline
\end{tabular}

The disease began in this field as early as July 13. Five animals had passed through the disease and one had died on it. The control No. 218 had been in it from July 16, i. e., 77 days up to October 1 without manifesting the slightest signs of infection. The second control spent 46 days in this inclos ure up to October 1, with the same negative outcome.

\section{MAY TEXAS FEVER BE COMMUNICA'TED BY AGENCIES OTHER THAN THE CATTLE TICK?}

We have seen in the foregoing pages that the transmission of Texas cattle fever may be prevented entirely by removing the ticks from Southern cattle in such a way that a new generation is suppressed. We have likewise seen that sick natives may remain in the same inclosure with health y natives for months without transmitting the disease to them, provided the sick natives have no ticks on them, or, in other words, provided the disease has been produced by direct inoculation. These facts go far toward bringing us to the conclusion that no outbreaks of Texas fever are produced withont ticks. Yet we can not deny the possibility of a conveyance of the disease by other agencies, for this possibility is demonstrated by the fact that by a direct transferrence of blood from sick natives, and even from healthy Southern animals, the disease may be reproduced with all its characteristic virulence. We know as yet so little of the ectogenic life of the Texas fever parasite that whatever hypotheses may be made must remain such until our knowledge has become more defined. Meanwhile we may formulate certain possibilities of transmission without the aid of the cattle tick to call the attention of future observers to them.

It is possible that the disease may be conveyed by insects, which pierce the skin and draw blood. Such pests, when moving fiom sick to healthy animals very rapirly, may carry enough blood on their mouth parts to inoculate healthy animals. But under such circum- 
stances several factors come into play, such as the probable destruction of the micro-parasite by drying and other unknown agencies, and the probability that the quantity of blood is too small to contain any parasites. Moreover, a single parasite, or even a few parasites, may not produce anything more than a mild, unnoticed affection. The possibility of direct inoculation by insects may depend on the distribution of insects which draw blood. In the District of Columbia Texas fever was not carried by insects, with the possible exception of a single instance, to be described below, during the four summers of work from 1889 to 1892, inclusive, although the very best opportunities were offered them to carry on direct inoculation, especially during the present summer. There may be parts of our country, however, where such direct inoculation from sick to healthy natives in midsummer is favored by the presence of certain insects not to be found near Washington.

If we consider for a moment the probability of an infection of native from Southern cattle directly by means of flies, etc., we shall find it very slight. Though we now know that Texas fever parasites exist in the blood of presumably healthy Southern animals, we must regard these parasites so scarce in number, if we are to be guided by the microscopic examinations of the blood, that insects can not draw enough blood to become dangerous. The infection of natives by Southern animals in this way must be considered probable only when authentic cases of this disease are on record which appeared ten or fifteen days after contact with Southern cattle, provided the ground has not been previously infected with ticks from other Southern herds. There seems to be no earefully investigated outbreak of Texas fever on record which occurred within thirty days of the ground infection or of contact with Southern animals.

In ease Southeru droves of cattle contain animals actually diseased with Texas fever, their blood would contain more parasites that that of the healthy, and hence might serve more readily as an inoculating fluid for insects, but Southern animals and natives are not allowed to mingle so as to bring this about. The disease is produced, in most cases, where Southern and native cattle do not come in contact at all. If insects distribute Texas fever they could only do it accidentally, and hence the result would be a few isolated cases. But Texas fever attacks 90 to 95 per cent of all natives.

Texas fever, as elucidated in the foregoing pages, is essentially a disease of the blood. The parasite producing it must be transferred in some manner from the blood of one animal to that of another. There is no evidence to support the view that it may gain entrance by way of the digestive tract, and hence several channels by which the microparasite might get into the body are necessarily cut off. Though the parasite is very likely present in the discharges and the urine of the sick, and perhaps in smaller numbers in the excretions of Southeru 
animals, yet pastures infected by such excretions are not infectious. In 1890 the following experiment was made :

Blood and spleen pulp from several natives which had succumbed to Texas fever was scattered over the ground in Inclosure V, and two natives (Nos. 109 and 136) pastured in it from August 25 to November. There was no trace of Texas fever discovered in either case, althongh the blood was examined at three different times. The number of blood corpuscles in one of these animals (No. 136) was below normal, but as it remained at this low point throughout the season, and as the animal had some vaginal discharge, the low number must have been due to disturbances of the generative organs. In the other animal the red corpuscles remained above 5.7 millions.

Perhaps the best evidence which can be adduced that the excretions do not have anything to do in transmitting the disease is the experiment in which healthy natives were exposed to sick natives free from ticks for months without any result.

The only exception, and this a doubtful one, to the general result of our experiments and experiences at the station, that Texas fever appears only with ticks on native cattle, occurred in 1891. Field XII (see Fig. 7, p. 108) was used only for the storing of unused healthy cattle during the course of the experiments of that summer. In this field a cow was kept (No. 168) upon which bleeding had been performed a number of times for the study of changes going on in the blood in anæmic conditions. The examination of the blood in this case began August 3 and was continued at intervals to September 8 . The auimal was led out of the field during each examination to a box, into which she was fastened during the venesection and the collection of the blood. On September 1 No. 162 was received and placed in this field. On September 26 she was observed to be dull and to refuse to eat. The temperature on September 28 was over $104^{\circ} \mathrm{F}$. On the following day the animal was found dead. The autopsy revealed an acute case of Texas fever, with an enormous infection of the red corpuscles of the blood with the Texas-fever parasite. An examination of the other animals in this field showed that only one other was diseased. This was the case upon which venesection had been practiced and whose blood had been examined last on September 8. How was this infection brought about? In a preliminary report* the probability of direct inoculation by flies was emphasized. The disease had appeared on the station as early as August 8 in those cases artificially infected with young ticks, and it appeared subsequently in the generalcontrol Field VI. Hence the opportunity was afforded for the conveyance of the virus by insects from sick animals. Instead of this channel of infection there is one other possible one. Though no ticks could be found on the animals in this field, a few may have been earried there in the course of the season, or they may have crawled there. A few

* Report of the Secretary of $\Lambda$ griculture for 1891, p. 134. 
ticks on an animal may have been overlooked, since they are still quite small when animals succumb in the acute stage. Moreover, they may have attached themselves in places not regularly selected by the young ticks (inner aspect of thighs and escutcheon), in which case they would have been quite certainly overlooked. On the whole we must confess that the infection of these two animals is a matter the obscurity of which can not be cleared up. They are the only cases of Texas fever which have occurred on the station fields during the four summers of experimentation which are not directly traceable to Southern cattle carrying ticks, to ticks alone, or to direct transferrence of blood from sick native or healthy Southern animals to susceptible natives by inoculation.

10320-No. $1-9$ 


\section{IMMUNITY AND PROTECTIVE INOCULATION-DISTRIBUTION OF IDISEASES RESEMBLING TEXAS FEVER ON OTHER CONTI- NENTS.}

\section{IMMUNITY IN SOUTHERN CATTLE.}

It has been stated by some observers that Southern cattle soon lose their immunity against Texas ferer after they have been taken to Northern pastures, and that they are liable to be attacked by this disease after having been away from the permanently infected territory for a year or longer. These statements, so far as we know, are not based upon experimental evidence, but upon observation of natural outbreaks, and hence the evidence is likely to be weak in one or more points. As we were more or less favorably situated to test these statements, some of the Southern animals were kept on the station grounds for one or two winters and then reëxposed to freshly imported Southern animals together with Northern stock. These experiments are a part of those already described in connection with ticks, and the following numbers, therefore, belong to the original experiments.

Experiment 8 (exposure of native to Texan cattle, page 100).-In addition to the natives, a Southern animal (No. 62), brought from North Carolina in 1889, was exposed on this field September 25, 1890, with a native (No. 74). The latter died October 16, while the Southern animal appeared not to be affected. The blood was examined three times. The corpuscles did not fall below five and one-half millions. All adult natives exposed in this inclosure during the summer succumbed to Texas fever.

Experiment 9 (exposure of natives to North Carolina cattle, page 100).In addition to the natives placed in this inclosure, the following North Carolina cattle of the previous year were introduced July 4, 1890 :

\footnotetext{
No. 32 (heifer, 3 years). Exposure negative.

No. 59 (cow, 5 years). Exposure negative.

No. 87 (calf of No. 59, $3 \frac{1}{2}$ months). Slight infection.

No. 61 (steer, 2 years). Exposure negative.

No. 67 (cow, 5 years). Exposure negative.

No. 86 (calf of No. 67, 2 months). Slight infection.
}

From this table it will be seen in the first place that none of the cattle died or became visibly diseased. In Nos. 32 and 59 the blood was examined September 3, when all exposed natives were either sick or dead, and found normal. Nos. 61 and 67 were not examined in this way. In case of the two Southern calves descended from Southern 
parents, but born on the station, there was a slight infection characterized by the presence of the intraglobular coccus-like stage of the Texas-fever parasite. In No. 87 these were detected September 20, but the blood corpuscles did not fall below five millions, as far as the few examinations are evidence. In No. 86, the younger calf, there was a decided fall in the number of red corpuscles associated with the presence of the parasite in the same stage as in No. 87. The corpuscles numbered but three and one-half millions October 30 .

Experiment 14 (exposure of natives to North Carolina cattle, page 107).In addition to the natives placed in this field the following Southern animals were introduced July 2, 1891:

No. 55 (cow, 5 years old, from North Carolina in 1889). Exposure negatize.

No. 62 (heifer, 3 years old, from North Carolina in 1889, exposed in 1890). Exposure negative.

No. 121 (cow, 4 years old, from Texas in 1890). Exposure negative.

No. 126 (cow, 6 years old, from Texas in 1890). Exposure negative.

These four exposures proved negative (so far as any outward signs of disease are concerned). No. 55 had been away from Southern pas. tures and not reëxposed for two years. Her blood August 29 was normal. The blood of Nos. 62, 121, and 126 was not examined. In these experiments the examination of the blood was not carried on systematically, and hence no very definite conclusions can be drawn as to the presence or absence of all disease. However, the examination of the blood of adults when made was negative. It is evident that the immunity of Southern cattle is not lost in one year or in two years. And by this we mean insusceptibility so far as a severe attack is concerned, for none of the adults showed any signs of disease, while none of the exposed natives resisted.

It is especially interesting to note that the two Southern calves exposed for the first time were not entirely insusceptible. A mild form of the disease was detected late in the season, and it is not beyond probability to assume that they may have been slightly affected through the entire summer. This seems to make it probable that Southern animals acquire at least some of their immunity by mild attacks very early in life.

\section{NATURAL IMMUNITY OF NORTHERN CATTLE:}

Natural immunity of cattle more than 1 year old.-This we know is very slight, for the mortality in many outbreaks has been found to be nearly 100 per cent. Still, there are animals which have more or less immunity, though never exposed to the virus of this disease. By compiling the cases exposed on the station in the ordinary way to Southern cattle in 1889, 1890, and 1891 (experiments 1, 9, and 14), and rejecting all those exposed after September 15, we may obtain approximate percentage of insusceptible cattle. There were exposed in all during these three years 24 head over 1 year old. Of these only one animal 
remained unaffected, though exposed twice. This was an old cow (No. 57). The remainder passed through more or less severe attacks and five (Nos. 49, 53, 56, 104, and 159) recovered. Some animals, it is true, were killed, but in a dying condition, and these are included with those that died. It may be said, therefore, that about 95 per cent of adult Northern animals are susceptible to Texas fever. When we examine the record of the animals under 1 year of age we obtain a somewhat different result.

There were exposed in the general fields in 1889 and 1890 in all eight calves. Of these two (Nos.10, 11) died in 1889 from an acute attack of Txas fever and two (Nos. 52 and 75) recovered. The remaining four (Nos. 79, 85, 93, and 100) exposed in 1890 were all affected, but none died of an acute attack. The disease was of the more or less mild, prolonged type, with the intraglobular coccus-like stage of the parasite in the blood.* Some succumbed at the beginning of winter from exhaustion, but not so far as could be discovered from the after effects of Texas fever. In general, calves are not insusceptible to Texas fever, but the disease is milder and the mortality is lower than with those more than 1 year old. Attention is here called to the case of No. $102_{\text {a }}$, a calf, which was found dead thirteen days after birth in a field infected with ticks only (experiment 10). The lesions were unmistakably those of Texas fever.

\section{ACQUIRED IMMUNITY OF NORTHERN CATTLE.}

This is a problem of far more economic importance than those just discussed, since it affords us some insight into the possibilities of producing immunity artificially. The various field experiments of 1889 , 1890 , and 1891 furnished a number of animals, some of which had passed through a mild attack, others through an acute attack. Many of these were reëxposed the following year to freshly introduced North Carolina cattle in company with fresh native animals. The following summary includes all such exposures. The number of the experiment corresponds in every case to that alleady described, of which the one under consideration forms a part.

Experiment 9 (see page 100).-The following recovered cases of 1889 were exposed with fresh natives to North Carolina cattle:

July 4, 1890.-No. 51 (cow, 4 years) passed through a mild attack in the fall of 1889.

July 4, 1890.-No. 53 (cow, $2 \frac{1}{2}$ years) passed through a fairly severe attack in the fall of 1889 .

July 4, 1890.-No. 64 (steer, 3 years) passed through a mild attack in the fall of 1889.

September 20, 1890.-No. 65 (cow, $3 \frac{1}{2}$ years) passed through a mild attack in the fall of 1889 .

July 4, 1890.-No. 75 (heifer, 16 months) passed through a prolonged attack in the summer of 1889 .

${ }^{*}$ It is probable that in all of these cases a short acnte attack preceder the ruild attack. 
In these animals the severity of the first attack is best measured by the intensity of the destruction of red corpuscles. The number of corpuscles in No. 51 had fallen below 1.5 millions on November 4,1889 . In No. 53 they fell to 2.5 millious. In No. 64 they had fallen to $2.7 \mathrm{mil}$ lions on November 7 , but there were still many infected corpuscles in the circulation. In No. 65, they numbered only 1.7 millions November 4. No. 75 was not examined. The result of the second exposure in 1890 is, briefly, as follows:

No. 51 dies of an acute attack August 26.

No. 53 probably not affected.

No. 64 passes through a prolonged, but rather mild attack.

No. 65 passes through a mild attack (exposure late) and dies some time after.

No. 75 probably not affected.

Experiment 10 (see page 103).-No. 47 (cow, $4 \frac{1}{2}$ years) had passed through a rather severe attack in the fall of 1889. The red corpuscles at that time fell below one million. July 4, 1890, she was placed in Field VIII, into which only adult eattle ticks had been thrown. She died of an acute attack September 12.

Experiment 14 (see page 107).-The following recovered cases of 1890 were exposed with fresh natives to North Carolina cattle July 2, 1891:

No. 56 (steer, 4 years) passed through a prolonged attack in fall of 1890 .

No. 102 (cow, 7 years) passed through an acute attack (due to ticks only) in summer of 1890 .

No. 130 (cow, 6 years) passed through a rather severe attack (Experiment 13) in artificially heated stable (1890-'91),

No. 143 (heifer $2 \frac{1}{3}$ years) passed through a doubtful attack (Experiment 13) in artificially heated stable (1890-'91).

The result of this exposure is, in brief, as follows:

No. 56 passed through a mild attack.

No. 102 was slightly; if at all, affected.

No. 130 died from an acute attack August 27.

No. 143 passed through an acute attack and recovered.

Experiment 17 (see page 111).-In addition to the natives, not hitherto exposed, which were placed into Inclosure VI, in 1892, together with fresh North Carolina cattle and ticks, the following recovered cases were included:

No. 135 reëxposed July 20. This animal had passed throngh an attack in Field VIII (ticks only) in 1890 . This summer it was again attacked, and the red corpuscles at one time were found as low as $2,000,000$.

No. 167 was reëxposed on the same day with the preceding. It had passed through an acute attack last year, and was very low for a time. This summer there was probably a slight attack, as the blood corpuscles, though they did not fall below 5,000,000, showed signs of regeneration.

The following eases were reëxposed together on August 26, in the same field, and a hitherto unexposed native, a bull (No. 204), $2 \frac{1}{2}$ years old, was put in with them as a control, since the season was now somewhat advanced:

No. 56 had passed through a prolonged but mild attack in the fall of 1890 . In 1891 it was again exposed and passed through a short, acute attack. This summer there was probably a very short attack, as is indicated by the record of the red corpuscles. 
No. 105 had passed through an acute and prolonged fever in Field VIII (ticks only) in 1890, followed by a relapse later on in the same season. A second exposure in September of 1891 was apparently negative. This summer, however, the disease reappeared on exposure, the loss of red corpuscles being fairly severe.

No. 160 was exposed late in 1891 and passed through a short but acute attack, followed by a relapse which lasted into December. This summer the exposure was evidently negative.

No. 166 was infected with artificially hatched ticks in 1891, and passed through an acute and rather prolonged attack. This summer the exposure was negative.

No. 182 passed throngh an acute attack late last year as the result of the intravenous injection of infected blood. This year the exposure resulted in a slight attack.

No. 185 at the same time passed through a similar attack, due to inoculation. This summer the exposure was negative.

No. 204. The control exposed at the same time in this field passed through a very acute attack, and was probably saved by its age. No. 225, which had just passed through the disease due to an infection with artificially hatched ticks, and whose blood corpuscles had nearly reached the normal, was transferred to this field August 30 , four days later than the preceding lot. A second attack was the result, with a rapid and extensive loss of corpuscles. This case is merely introduced to show the intensity of the infection still existing in this field, but it can not be wholly regarded in the light of a control.

The following table gives a brief résumé of these exposures:

Nature of attack.

\begin{tabular}{|c|c|c|c|}
\hline No. & 1890. & 1891. & 1892. \\
\hline $\begin{array}{r}56 \ldots \ldots \ldots \\
105 \ldots \ldots \ldots \\
135 \ldots \ldots \ldots \\
160 \ldots \ldots \\
166 \ldots \ldots \\
167 \ldots \ldots \\
182 \ldots \ldots \\
185 \ldots \ldots \\
204 \ldots \ldots \\
\text { (Control.) }\end{array}$ & 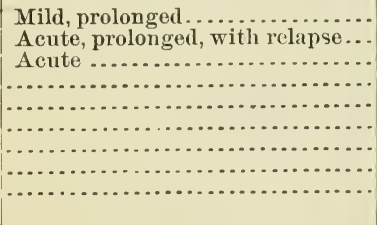 & 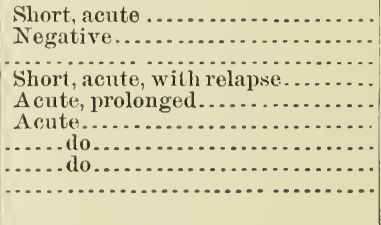 & $\begin{array}{l}\text { Slight(?). } \\
\text { Fairly severe. } \\
\text { Do. } \\
\text { Negative. } \\
\text { Do. } \\
\text { Slight(?). } \\
\text { Mild, short. } \\
\text { Negative. } \\
\text { Very acute. }\end{array}$ \\
\hline
\end{tabular}

These experiments demonstrate the important fact that one attack of Texas fever does not necessarily protect the animal from a second attack. Of the eighteen cases seven may be said to have remained practically unaffected during the second exposure. Of the remaining eleven three died during the second exposure. It is impossible to determine in such cases how much natural immunity existed before the first attack. Thus No. 53 survived the first attack while another cow oxposed at the same time and nearly of the same age succumbed to an acute attack. No. 75, the heifer which passed through the first exposure as a walt 4 months old, is hardly to be regarled as a fair case. INence we must be cautious in giving even in these cases too much crelit to the first attack in warding off the following one. It is not to be lenied that in the case of animals not more than $2 \frac{1}{2}$ or 3 years old a first mild at tack may prevent a second fatal attack in many cases, and a first acute attack may be followed by a very mild infection, but it may be laid down as a ren. eral proposition that a single attack is not sufficient to produce complete immunity. 
PROBLEMS CONCERNING PREVEN'IIVE INOCULA'ION.

If a single attack of the disease itself does not afford complete protection it is not likely that any process or method of artificial inoculation will be successful in this respect. The profound effect which is necessarily produced in the body of an animal by a destruction of red corpuscles equal in amount to all those circulating in the body at any given time should make much more impression than any method of inoculation is likely to do. And yet such an attack not only does not prevent a second attack but may not prevent death during a second attack. Aside from the difficulties attending the production of insusceptibility under any circumstances the difficulties of preparing a "vaccine" according to the method hitherto practiced are at present insurmountable. The mieroörganism which we have deseribed as the presumable cause can not be cultivated. Hence the method first practiced by Pasteur of using an attenuated form of the virus itself is not within reach and other means must be sought. Before suggesting any lines of experimentation in this field let us examine briefly unter what conditions it is desirable to have an animal insusceptible to Texas fever.

On pastures north of the permanently infected area Texas fever can be kept away by properly applied preventive measures. Hence the protection of Northern cattle by some artificial process is unnecessary and practically out of the question. It is, however, of great importance to be able to protect from a fatal attack valuable animals which are to be taken South into permanently infected territories. It is probable that if ealves be taken they may, without treatment of any kind, survive the infection upon Southern pastures and become gradually insusceptible. But in case of animals more than 12 to 18 months old the first attack might be fatal, and if a preliminary mild attack could be induced by artificial means the fatal effect of a second attack might be averted.

Perhaps the simplest manner of producing a mild, usually nonfatal attack is to expose cattle on pastures which have been infected with ripe, egg-laying ticks at some specified time in the fall. This time must depend on the climate of the locality where the infection is to be practiced. In the latitude of Washington we found in 1889 the middle of September a convenient time for the infection. In more northerly latitudes the exposure should be correspondingly earlier. Cattle exposed in this way take Texas fever invariably, but the mortality is practically zero. Such animals may die of a second attack during the succeeding summer, but a second mild exposure during the following autumn may furnish a sufficient protection. Inasmuch as the recovery from even severe attacks of Texas fever is usually complete and not followed by any permanent debility, such mild attacks would not be likely to cause any permanent injury to the exposed animals.

Another method of inducing Texas fever is the injection of blood 
from cases of Texas fever. Such inoculations are apt to result in a mild attack if practiced after the hot weather of midsummer. The blood of Southern cattle will serve the same purpose, as our experiments carried on this year (1892) have shown. If practiced in early summer the injection of such blood induces a prolonged attack, and may cause death. (See Nos. 198, 206, 219, 222, and 230.) These latter methods of inoculation require either the presence of Texas fever or of freshly imported Southeru cattle.* The former method of exposure to ticks is on the whole simpler, since it requires no operation, and since ticks are easily procurable from the permanently infected Southern territory. Mild attacks of this kind should be watched with care and the blood examined from time to time to obtain positive information concerning the severity of the induced attack. The temperature should likewise be taken morning and evening.

Efforts to protect Northern cattle by inoculation were made by Dr. Paul Paquin (9, p. 14). We can not review these experiments in detail. While we must commend the faithful work, we must dissent from the method, and hence can not regard it as applicable in practice. The wholly different outcome of our experiments concerning the microörganism and the cattle tick as probably the only transmitter prevents us from accepting any results based upon hypotheses which are now shown to be unfounded. But if we look at the results obtained by Paquin's vacci nation we will be convinced that they are far from being satisfactory. Thus Dr. Dinwiddie (9, p. 23) reported a mortality of 100 per cent among nonvaccinated animals, and of 75 per cent among vaccinated animals exposed in Arkansas. Of vaccinated and nonvaccinated cattle sent to Texas $66_{3}^{2}$ per cent of the former and $88_{3}^{2}$ of the latter died. These percentages show so little difference between the mortality of vaccinated and nonvaceinated cattle that, bearing in mind the various unknown factors which come into play in such experiments, we may regard the effect of this mode of vaccination as negative. What is meant by the author when he speaks of the virus used in these inoculations as doubtful it is difficult to understand. A culture always contains a definite kind of bacteria and they are either of the wrong kind or the right kind, and no vaceination experiments should be attempted or, if attempted, reported without an exact description of the underlying conditions, so that they can be repeated if necessary by others. The real difficulty, however, with these experiments lies deeper. Vaccination experiments were tried before anything definite was known concerning the nature and causation of the disease, and hence were built on hypotheses of a vague character in place of demonstrated facts. Any reader of the foregoing pages of this report will be satisfied that the diagnosis of Texas fever must now require a careful

\footnotetext{
* Since the above was written we have determinol that the Texas fover parasite was carried in the blood of a North Carolina animal 3 yoars after loaving tho pormanently infected territory.
} 
periodical examination of the blood, and that unless this is carried ont the disease may escape observation. Again all test exposures must he made under precisely the same conditions and not in different inclosures with a doubtful or a variable infection, since we now know that the infection is carried by the newly hatched tick.

The statements made above concerning the possible uses of mild infections as means of subsequent protection must be regarded as mere suggestions which may or may not prove of practical utility on a large scale. They are carried out so easily, however, that they may be tried by anyone exercising a certain amount of care.

IS TEXAS CATTLE FEVER RESTRICTED TO THE AMERICAN CONTINENT?

Among the diseases carried from their natural habitat by intercourse Texas fever occupies a very prominent position. Existing chiefly as a mild, rarely recognizable, malarial infection in certain regions of our country, it becomes a highly fatal infectious disease when transported beyond its natural confines. The movement of cattle is entirely responsible for the phenomenon. The question naturally presents itself whether such a disease is not to be found in other countries situated as we are. Only an active movement of cattle, such as took place in our country in 1867 and 1868, in the hot months of the year, together with their dissemination over Northeru pastures, would demonstrate the presence or absence of such a plague on other continents. But there is evidence even now that a disease resembling Texas fever very closely, if not actually identical with it, exists in southern Africa and in Europe along the Danube.

\section{SOUTH AFRICA.}

In 1883 a i'eport was presented to the English Parliament by a commission of inquiry concerning a disease among cattle in the colony of the Cape of Good Hope known as "redwater." This disease is defined by the commission as-

an infective and malignant fever in horned cattle, characterized by the passing of urine of a color varying from blood-red to purplish-red, and holding the hæmatin or coloring matter of the blood in solution. One ox can not give auother red-water as a smallpox patient can give his disease to his neighbor. Redwater is not contagious in that way. The poison of redwater passes from a suffering animal on to pasturage. What, if anything, happens to the poison at this stage is not fully determined. Another ox feeds over the pasturage thus contaminated and becomes in his turn the sufferer.*

The disease was first observed in 1870. Since then it has been introduced from time to time by oxen used as carriers which keep up communication with the territory north of the colony. Of the precise manner in which the virus is communicated by the cattle only this was known, that while they did not infect other cattle directly they did infect the ground over which they passed. 


\section{'The symptoms of this disease are summarized by the commission as follows :}

The beast, when first observed, appears dull and sluggish, with a tendency to leave the rest of the herd; the hair stands erect, like that of an animal on a cold day (a staring coat); the ears liang, and the eyes have a dull, lusterless appearance. In some eases the beasts will cease feeding; in other cases they continue to nibble at the herbage until nearly the last, but in an indifferent manner, indicating that they have no relish for their food. There is generally a dribbling of saliva from the mouth, the nose or muzzle may appear quite moist during the early stages of the disease, but it invariably becomes dry and erusty as the disease advances. Later on, the animal will manifest a disinclination to move, and when compelled to do so, will walk with a dragging, straddling gait, as if weak across the loins. In some cases where the sick beast is left undisturbed, it will remain almost constantly in one place, and while standing with head depressed, and ears hanging, in a drowsy, semi-comatose condition, look the very picture of complete nervons prostration. Some such cases will lie down the greater part of the time and scarcely move, and when found dead the head and limbs will be resting in their natural position, as if the beast was asleep. In one very marked case of this kind the colonial veterinary surgeon, on making a post-mortem examination, found the carcass pale and almost bloodless, as if the animal had been bled to death. In other severe cases a twitching and quivering of the muscles will be observed, especially of those situated in the flank and behind the shoulder, while the animal will stand and grind its teeth and curl up its upper lip. The beast's dung during the early stages of the disease is very often soft, with a tendency to diarrhœa in some cases; but it almost invariably becomes hard as the disease advances. In some very severe cases, where recovery has taken place, the favorable crisis appeared to be ushered in by a salutary diarrhøa. But whether the dung is hard or soft it is generally of a brownish tinge, and mixed with blood and mucus. In milch eows the very first symptom observable, is the sudden cessation of milk; and in many mild eases, of which there is generally a considerable percentage in a lierd, the oniy symptoms discernible are a dull, dejected appearance, staring coat, and a slight stiffness for a day or two, after which these symptoms disappear, and the animals resume their usual appearance. Of course, the most prominent and diagnostic symptom in this disease is the color and the character of the urine, which varies generally as the disease advances from a pale yellow to a dark port-wine color, in many very typical eases, however, even amongst those which terminate fatally, the urine does not aequire that deep tinge. In many cases, also, even when the attack has been very severe, when the crisis is passed, recovery is very rapid, and it is very remarkable, in such eases, how soon the urine reassumes its normal color and density with the disappearance of the albumen. In other cases, again, where the liver fails to resume its healthy function, the beast will become hidchound and unthrifty-looking, while a thick scurf will form on the skin.

The lesions observed on post-mortem examinations were reported by the veterinary surgeon of the colony as follows:

On eutting throngh the skin the flesh is seen to be pale and bloodless, and oceasionally of a yellowish tinge; sometimes there is subcutaneous emplyysema and infiltration of jellowish-colored serum.

Liver.-This organ is nearly always more or less affected, being angmented in volume, aud in many instances altered in texture, and so softened as to beeasily broken up with the finger. On eutting into it the ducts are often found filled with bile, and sometimes from the cut surfaces large quantities of black blool escapes. The gall bladder is nsually full of thick bile, and many have thought that this distmiled condition of it was in some way the canse of the disease, which is annite a mistaken notion, as this state may be olsserved in other diseases, and in any case whore the 
process of digestion is arrested, as the bile continnes to be secreted and simply collects in its natural receptacle till wanted for use.

Spleen.-This viscus I have invariably found affected, it being generally enlarged to three or four times its natural size, and filled with black blood, giving to its exterual surface a livid blue or black color. On holding it up by oue end, it will be found that the blood will gravitate to the most dependent part, showing that the splenic tissue is disintegrated. On cutting into it, black incoagnlable blood escapes from the incision.

Kidneys.-In a few instances I have found these organs looking quite natural, but, as a rule, they are much congested, dark in color, and augmented in volume, and sometimes easily broken down.

During the outbreak of this disease in 1871-'72, many persons noticed engorgement or discoloration of the tissues surrounding the kidneys, while the capsules of the kidneys contained a fluid more or less dark colored.

Bladder.-This usually contains urine of a high color, often quite black, but sometimes not much altered in appearance. If left to stand it deposits a sediment, which on examination is found to consist of mucus corpuseles, hippurates, etc., while the urine itself contains a variable quantity of albumen.

The color I find due to the escape of the hematin of the blood.

Mouth.-On the tongue I have sometimes seen dark-colored spots or patches, but this condition is by no means constant, as in many instances the mouth looks quite healthy.

Rumen.-In this stomach I have found the inner coat much discolored after the animal has been dead some hours, the epithelium peeling off readily. In other cases when I have opened animals immediately after death, I liave not found this condition, though in a few instances I have noticed a slight redness.

Reticulum.-This stomach has not exhibited any symptoms of disease in any animal that I have examined.

Omasum (third stomach). - In most cases I have found this organ healthy; sometimes I have noticed the leaves slightly reddened, and the vessels radiating from their attached border injected, but I have never observed the sloughing which occurs in cases of rinderpest, and some other diseases, nor ecchymosis either. When I have found the tissues discolored and the epithelium'peeling off readily, it has been after the animal has been dead some time, and the same oceurs in cattle which have died from other causes. In a few instances I found the contents hard and dry, in others quite soft.

This dry, impacted state is not peculiar to this disease, nor has it anything to do with the cause of it, as some have supposed.

Abomasum (fourth stomach).-I have noticed intense congestion of this organ in all cases, with more or less ulceration penetrating to the inuscular coat. In some instances there were superficial erosions not extending to the submucous tissue.

The mucous membrane was covered with mucus generally tinged with blood.

Intestines.-The small intestines are invariably congested, and in other respects present the same appearance as the fourth stomach. Peyer's glands I have found enlarged and dark in color, but I have not detected ulceration of them.

The large intestines present a similar appearance to the small, but in a lesser degree.

In a few instances a kind of crompous exudation has been seen, and casts of portions of the intestines have passed with the feces.

Chest.-In this cavity I have not observed any particular indication of disease, with the exception of patches of eechymosis in the lining membrane of the heart.

Brain.-In the few instances where $I$ have examined the brain $I$ have found the membranes covering it much injected and yellowisl colored serum in the ventricles.

Enough of the report has been quoted to illustrate the striking sim- 
ilarity of this South $A$ frican disease and Texas cattle fever as regards the symptoms and lesions and the noncontagious character of both maladies. Both are carried by cattle from warmer, permanently infected territories, and in both the pastures become infecterl. In fact, the Commission reports that a certain line exists which represents the boundary of the infected district. This is deducible from the minutes of the proceedings, where the following passage occurs:

The Commission recommend that the southern Redwater line, at present drawn at the Umtata River, be strictly respected, and that no cattle, either loose or in yoke, be allowed to cross that line except from a portion of East Pondoland, where no Redwater is known to exist, and from such portion of East Pondoland only by certificate.

It is to be hoped that this peculiar disease will be soon made the subject of investigation to determine whether or not it also is transmitted by some specific parasite like the cattle tick, and whether or not it is really the same as Texas fever.

\section{ROUMANIA.}

Still better evidence of the existence of Texas cattle fever outside of our own country is furnished by certain investigations made by Prof. Victor Babes,* of Bucharest, in 1888, conceruing epizoötic hæmoglobinuria among cattle in Roumania. According to Babes-

A peculiar disease devastates, since olden times, the herds of Roumania. Native veterinarians have given it the name gastro-entero-nephritis. Nowhere are references to be found in publications concerning this plague, which formerly was regarded the same as rinderpest. It is not less fearful than the latter in the persistence with which it demands annually thousands of victims from among the most powerful draft oxen, especially in the swampy lowlands of the Danube River. * * * Government commissions harl endeavored to determine the nature of the plague in former epizoötics, but ueither the infectious nor the contagious character could be determined. The disease was looked upon as a kind of malarial disease.

Its dissemination seems to be largely due to draft oxen. Babes is inclined to consider it as spreading from public drinking places, and that the infection starts from such fountains as centers and extends over a restricted area therefrom. Babes also makes the curious statement that "the disease moves from one end of the village to the other, reaches after a few days a certain place in the village from which it does not spread farther, while those animals in the infected part of the village hitherto spared from the disease may succumb later." It is evident that the way in which the virus is disseminated is not known, and that the above statements are more or less contradictory and need elucidation. It should be borne in mind that the conditions as described by Babes must be very complicated, owing to the employment of draft oxen moving from place to place. No mention is made in these investigations of any ecto-parasites.

*Die Aetiologie der senchonhaften Hïmoglobinurio des Rinles. Archiv. für pathol. Anatomic und Physiologie, CVx, (Jan., 1889), p. 81. 
Symptoms.-The most powerful dralt oxen are the chief victions. Cows are rarely attacked; calves never. An animal affected with the disease appears weak, the head and ears droop and the back is arched. The temperature is elevated, the pulse and respiration rapid. After two days some recover, others begin to pass dark-red urine. In such cases emaciation becomes marked, muscular tremors appear, and the temperature rises to $40-41{ }^{\circ} \mathrm{C}$. $\left(104^{\circ}-105.8^{\circ} \mathrm{F}\right.$.). The animal now sways and drags its hind limbs after it. The bowels may be constipated or pass liquid, reddish-brown, sometimes bloody stools. The disease may terminate fatally in four to seven days. In rare cases fatal relapses occur after apparent recovery. The urine contains but rarely red corpuscles. Usually albumin and coloring matter of the blood are present.

Pathological changes.-The lesions found by Babes are intermingled with those produced by Pentastomum, so that it is difficult to determine which are due to the specific fever and which to the parasites. Of the more important we may extract the following brief statements:

The lungs may be the seat of emphysema and hyperæmia. The heart muscle is pale red, friable. The fluid blood and the clots in the heart cavities are quite pale, indicating a marked loss of coloring matter. The liver is enlarged, in some cases yellowish brown, in others dark brown and rich in blood. Babes dwells upon the peculiar mottled appearance of stained liver sections due to the fact that the central zone of each acinus is in a necrotic condition; $i$. e., the nuclei have wholly or partly disappeared from the parenchyma cells. This condition is precisely similar to that observed in Texas fever. The gall ducts are not obstructed. The gall bladder contains fluid, orange-yellow bile.

The spleen is always enlarged, black or blackish red, the capsule tense. The enlargement resides in the pulp, which is blackish, disin, tegrated.

The third stomach is impacted. The fourth or true stomach is always hyperæunic. As a rule, hæmorrhagic erosions are found in the pyloric portion, still more frequently flat or deep excavations are observed along the mucous folds, with hæmorrhagic base and covered with a slightly elevated greenish-brown slough more or less easily removable. The hæmorrhagic and œedematous changes along the digestive tract and its mesenteries seem to be much more pronounced than in Texas fever, but, as stated above, Pentastomum may have something to do with these.

The kidneys are surrounded by hæmorrhagic, œdematous tissue. Frequently the site of a kidney is indicated by a large blackish, hrmorrhagic area. The kidneys are enlarged, the cortical portion dark red. In the pelvis more or less extravasation of blood. The blatider contains much dark-red urine.

The musculature of the body is in parts pale and friable. The membranes of the brain and spinal cord are injected, the nervous tissue rich in blood, sometimes softened and cedematous. 
That portion of the work most interesting to us is the description of bodies within the red corpuscles, which are strikingly like the parasite of Texas fever in its intermediate stages. Babes finds peculiar microorganisms, which he calls bacteria within the red corpuscles in the capillaries of the mucosa of the stomach and intestines, in the mesenteric glands, in the liver, spleen, and kidneys. In the mesenteric glands they were found free in masses. In the kidneys they were exceedingly abundant, both free and within red corpuscles. He also detected them in the musculature of the body, sometimes in the marrow of the bones. In the brain and spinal cord they were not found.

Babes describes these peculiar micro-organisms when stained in Löffler's methylene blue or methyl violet as squarish bodies, each divided by a light line so as to form a body like a diplococcus. The description is vague, but an examination of the illustrations shows that the corpuscles may contain two such diplococci hanging together at one corner and making an angle with each other. Babes finds also that these organisms can not be stained in sections by the current bacteriological methods. He resorts to the following procedure to avoid the decolorizing action of the alcohol: The sections are stained in Löfler's methylene blue for one hour, then dehydrated in an alcoholic solution of methylene blue. Thence they are transferred to an alcoholic solution of eosin and lastly to aniline oil and xylol.

The author believes that owing to the massing together of the "diplococci" in the mesenteric glands, the capillaries of the mucosa of the stomach and the odemas surrounding these organs that they enter by way of the ulcers of the fourth stomach, become disseminated in the blood, and then attack the red corpuscles. The probable truth of the matter is, however, that the bodies which he saw have already been in corpuscles and have been set free by their breaking down.

Bacteriological observations were made on a certain number of cases, of which some are reported. The whole work is regarded as preliminary, however, for the results are by no means conclusive. Several kinds of bacteria were isolated from oxen which had succumbed to the disease. One of these was cultivated with great difficulty and is fatal to rabbits in about two weeks. Its relation to the disease, though assumed by the author, is not yet proven, since no inoculations are reported which show that it is capable of reproducing the disease in cattle.

In a more recent communication* Babes gives some additional facts bearing on the micrö̈rganism of this disease.

The parasites are quite polymorphous. The characteristic form is that of a diplococcus in the interior of the red corpuscle. In other cases there are two or even three pairs of the microörganism in a red corpuscle. The size of these bodies varies. Some individuals are $2 \mu$, others $0.5 \mu$ in dianeter. In the fresh condition they are recognizable within the red corpuscles by their moderate refrangibility and

*Verhandlungen des X. internationalen medizinischen Congresses (1890). II. Dritte Abtheilung., S. 104-108. 
their colorlessuess. They do not move within the corpuscles. Stained with methyl violet their interior shows a peculiar line of division. The microbes are more tubular (tubisch), with ends rounded off, and they often hang together by means of a fine thread. Stained blue, the bodies are spherical and the chromatic substance is found more on the periphery. The parasites are colored brown in chromate of potash.

It will be observed that this description accords much more closely than the one given in his first communication with that given in this report of the Texas-fever micrö̈rganism. Babes has detected no movement or changes of form of the microörganism within the corpuscles. This may be due to the failure to examine the blood during the life of the animal. In fact, his description of these bodies indicates that he has thus far seen them only post-mortem. He also adds the following information concerning the transmissibility of the disease from one animal to another.

Blood of sick or dead cattle 2 or 3 days old may still produce the disease, but frequently a considerable quantity of fresh blood does not infect them. Undoubtedly this depends upon other conditions of development of the parasite in the animal body. Thus the parasite can only be transmitted once from cow to cow and inoculations from rabbit to rabbit can only be carried through two to three generations with success. Of twelve inoculated beeves only four contracted the disease.

In our experiments the infectious agent has been transmitted from a Southern cow (No. 214) to a Northern cow (No. 222) and from this subsequently to four other Northern cows (Nos. 197, 200, 227, 228) without any diminution of virulence. In fact, three of the four died. As the evidence for the above statement of Babes that the transmission of the disease can be effected but once from cow to cow is not presented we can not examine into it more closely.

As to the cultivation of the microörganism, Babes is more cantious in his statements in this second communication. Of 200 inoculated tubes only twelve showed a feeble growth of diplococci of various sizes. These are said to produce the characteristic disease in rabbits with a hemorrhagic odematous exudate of the peritoneum and great masses of parasites in the same, exceptionally in blood corpuscles. This sounds more like the more chronic forms of the ordinary septicamia hemorrhagica in rabbit. As to the nature of the microörganism Babes now hesitates to express an opinion, and inclines to the view that it may stand between the bacteria and the protozoa.

It is difficult not to come to the conclusion that this disease is identical with Texas fever. The pathological changes are almost precisely the same, and any minor differences are explainable by the assumption that Babes may have largely examined animals after the acute attack had passed away. The microörganisms of both diseases, their general appearance, their habitat are strikingly alike. The fact that Babes cultivated his organism and produced disease in rabbits is not a strong argument against their identity, for it seems very probable that he may have had under observation one of those not very uncommon bacteria accidentally associated with various disease processes whose form is 
too small to resemble anything in particular and the cultivation of which is attended with many failures. Such forms are familiar to most working bacteriologists. It is nevertheless impossible to come to any positive conclusion that the Roumanian and the American diseases are the same until the investigations concerning the former are carried beyond the preliminary stage in which Babes has left them.

In the Caucasus* there prevails a disease during the hot season which is called "Tschichir," a name also applied to a kind of red wine, because the urine of affected cattle is red in color. The disease is said to kill thousands of the best cows and oxen annually, and peasants lose the major part of their stock in a few days without being able to do anything to check the disease. The details concerning the disease are very meager. "At first the animal is dull, with drooping head and ears. It champs its teeth, moans, and discharges from its mouth a viscid, foulsmelling mucus. The bowels may be loose or costive and the urine is bright red." From observations of the disease the following conclusions are drawn:

The "Tschichir" has no infectious properties. The disease attacks mainly working oxen, more rarely mileh cows, and never young animals. It is more severe and acute in spring than in fall. It does not last more than three weeks in any one locality. In the first week it begins to show itself, in the second it is at its height, and in the third it disappears completely. The flesh of animals which have died of this disease is consumed without any ili effects by the nomadic tribes of the Caucasus.

* T. Praktische Bemerkungen iiber die im Kankasus Tschichir (Hæmaturia) genannte Krankheit des Hornviehs. Med. Ztg. Russlands, St. Petersburg, 1853, x, 209. 


\section{PRACTICAL OBSERVATIONS AND CONCLUSIONS.}

It will undonbtedly be conceded by all impartial readers of the foregoing pages that the economic value of the results derived from these investigations is very promising. As yet they are undeveloped, however, and their true importance can not be estimated. Experiments must be built upon them in various directions. These we have thus far been unable to undertake, owing to the large amount of labor involved in determining the relation of ticks to the disease. In the following pages, in addition to deductions immediately available in the control of this disease, a few suggestions are made in regard to the objects to be attained by further investigations and the manner in which they should be conducted. Those readers technically interested in carrying on such investigations will undoubtedly have read between the lines of the foregoing chapters all that can be suggested here.

\section{DIAGNOSIS.}

One of the immerliate results of the work is the simplicity and ease with which an outbreak of Texas fever can be positively determined. Iost veterinarians and pathologists are able to recognize Texas fever when an acute case presents itself for post-mortem examination. The greatly enlarged spleen, the peculiar coloration of the liver, the thick bile, and especially the hæmoglobinuria are so obvious that no one trained to a knowledge of the appearance of the healthy organs and excretions in cattle can make a mistake. But all cases are not in the acute stage at the time of death, and one or several of these important pathological changes may be missing or barely recognizable when present. In fact, there may be no animals which can be sacrificed, and all may be on the road to recovery. In such cases even the clinical signs, such as the high temperature, may be missing.

Among the diagnostic characters to be added to the list are the examination of the blood and the presence or absence of the cattle tick (Bö̈philus bovis). We may now consider it demonstrated that Texas fever outbreaks in the North are not possible without the cattle tick. Isolated cases may occur through other agencies perhaps, but no general infection of fields or pastures is possible without the cattle tick. Hence, in any doubtful disease where Texas fever is suspected, ticks should be looked for, and in doing so all those facts concerning the size of the ticks on animals in the acute stage and during recovery and their location on the body must be borne in mind. On animals which have

$10320-$ No. 1 
passed through the disease the ticks are nearly or quite full grown and therefore easily detected. But even when great care is exercised the ticks may be overlooker or in a late fall infection they may have speedily disappeared. In such cases the examination of the blood will give the necessary information. This requires some skill, and a good microscope with objectives and oculars giving a magnification of not less than 500 diameters is necessary. The method of examination as well as the pitfalls to be avoided in interpreting appearances under the microscope have been discussed at length, and need not be again referred to here.* While the presence of the micro-parasite within the red blood corpuscles and the changed size and appearance of many of the corpuscles themselves are usually of sufficient diagnostic value, it is always desirable that the number of red corpuscles be estimated at the same time.

In the microscopic examination of the blood attention should be paid, first of all, to the presence of the various stages of the micro-parasite. In the mild type, the minute coccus-like body will be found within the corpuscle, near its periphery. As it is rarely seen in fresh preparations, stained preparations should invariably be examined. In the acute type of midsummer, associated with high fever, the larger, paired, pyriform bodies are always present, but usually in very small numbers. They may be detected as readily in fresh blood earefully mounted as in dried and stained preparations. Next in importance to the micro-parasite of the disease are the changes induced in the blood corpuseles by the anamia. In fresh blood the variation in size of the individual corpuseles and the very large size of many (from one and one-half to one and three-quarters times the diameter of the normal red corpuscles) is at once apparent. In properly stained preparations the peculiar granulations and the diffusely stained appearance of a greater or smaller number of the large corpuscles as depicted on Plate IX and other plates is quite characteristic. These changes may, of course, be the result of very severe, repeated hemorrhages, and these must be excluded first before the former ean be considered as due to Texas fever. The changes in the blood corpuscles may be directly associated with the parasite in the mild type, but they usually follow the parasite in the acute type. Hence they may be the only indication of disease recognizable under the microseope in some cases.

A rednetion in the number of corpuscles is a very reliable sign of Texas fever. If we except the occurrence of severe hemorrhages and the feeding of ehemical poisons, their number is but slightly, if at all, influenced by diseases of various kinds. In several eases of advanced tuberculosis no reduction was noticed. In fact there seems to be but little specific action of bacterial poisons on the red corpuscles, while the Texas fever microbe limits its destructive action entirely to them. Anamia in cattle seems to be rare, as we found it but once among the 
many cases under observation. Hence the counting apparatus is of great service in detecting Texas fever in all its phases and should be used whenever possible.

A summary of the diagnostic characters to be looked for when this disease is suspected would include among others the following salient ones:

(1) Cattle ticks.

(2) Gross pathological changes: Hamoglobinuria; enlarged spleen; enlarged, yellowish liver; thick, flaky bile; ecchymoses on the external and internal surfaces of the heart.

(3) The micro-parasite within the red corpuscles.

(4) Modified or changed corpuscles (enlargement, the presence of stainable granules, etc.).

(5) The reduction in the number of red corpuscles.

\section{PREVENTION.}

Texas fever in the territory outside of the enzoötic region is the result of the distribution of ripe egg-laying ticks by cattle from the enzoötic region. Hence such cattle should not be allowed on uninfected territory during the warmer half of the year. It is also evident that all cars carrying Southern cattle contain a larger or smaller number of ticks which have dropped off during the journey and which are ready to lay their eggs. The sweepings of such cars, wherever depositerl, may give rise to a crop of young ticks and these, when they have access to cattle, will produce the disease. Wherever Southern tick-bearing cattle are kept within twenty-five to thirty days after their departure from their native fields they are liable to infect such places, since it requires the period mentioned for the smaller ticks to ripen and drop off: But under special conditions even this period is too short and the Southern cattle may remain dangerous a longer time. This would oceur when such cattle remain in any one inclosure long enough (four to five weeks) for the progeny of the first ticks which drop off to appear on the same cattle.

The above points are covered in the regulations of the Department of Agriculture concerning cattle transportation. These regulations insist on the complete isolation of cattle coming from the permanently infected territory between March 1 and December 1 of each year and on the proper disinfection of the litter and manure from such cattle during transportation. Furthermore such cattle can only be transported into uninfected territory for immediate slanghter during the prescribed period. These regulations if properly carried out would prevent the appearance of Texas fever at any time in those areas north of the enzoötic territory. The only question which now presents itself with reference to them is the efficiency of the prescribed disinfection. It has been shown that the infection resides only in the cattle ticks and their eggs; hence the destruction of these is absolutely essential 
to make the disinfection of any value. In the present report this question has not been touched upon; therefore, pending the trial of various disinfectants, which is now going on, any discussion or any suggestions are of little value.

The harmlessness of Southern eattle after being deprived of the cattle tick brings up the very important question whether such cattle can not by some means be freed from ticks so that their transportation may go on without any restriction during the entire year. There are several ways in which experiments might be undertaken. Cattle might be subjected to disinfecting washes of various kinds, or else they might be run throngh disinfecting baths which expose the whole body to the action of the liquid used. Such processes would require careful attention. The survival of a very few ticks might lead to serious consequences, since a single ripe tick averages about 2,000 eggs.

Cattle may be deprived of ticks on a large scale without the use of any disinfection if the following plan be adopted: Two large fields in a territory naturally free from cattle ticks are inclosed. The tick-bearing cattle are put into the first inclosure and kept there about fifteen days. They are then transferred to the second inclosure for the same length of time. Thirty days after the beginning of their confinement they may be considered free from infection. The reason for this procedure is simple enough. The eattle drop the ticks as they ripen in the inclosures. By being transferred to a second (or even a third) inclosure they are removed from the possible danger of a reinfection by the progeny of the ticks which dropped off first. It is evident that such inclosures can only be used once a season, since the young ticks subsequently hatched remain alive for an indefinite length of time on the ground. Such inclosures must not be located where there is a possibility that the ticks might survive the winter.

For cattle which are introduced into the enzoötic territory two modes of prevention may be adopted. Either they are kept entirely free from ticks by confinement in stables or upon pastures known to be free from ticks, or else they are exposed to the infection in such a way as to become insusceptible to it after a time. The first method is open to the objection that ticks may at some time accidentally gain access to such cattle and produce a fatal disease. The second method seems the more rational, provided it can be successfully carried out. We know that Southern cattle are insusceptible to the disease, and the way in which this insusceptibility has been acquired has been already discussed (p. 130). Young animals seem to be largely proof against a fatal infection, although they are by no means insusceptible. The repeated mild attacks to which they are subjected finally makes the system indifferent to the virus. The introduction of young animals in to the permanently infected territory, though not without danger, is far safer than the introduction of animals older than one year. 'The danger of a fatal infection increases with the age of the animal and is very great in cows over 5 or 
6 years old, as is distinctly shown by the experiments recorded in this report.

The subject of preventive inoculation has already been discussed and experiments cited on page 132. It has been shown that while in general two mild attacks may not prevent a third attack, this will not be fatal. One very acute attack will usually prevent a second severe attack. Hence it is possible to prevent cattle, even when fairly along in years, from succumbing to a fatal attack by several preliminary carefully guarded exposures to a mild infection. This infection may be produced by scattering ripe ticks in an enclosure, or by placing young ticks on cattle in the fall of the year (page 135). Protective inoculation of this kind should be carried on at some locality outside of the enzoötic territory carefully chosen for the purpose. A few years of careful experimentation would probably lead to an efficient method which, when definitely formulated in all its details, could be applied in different parts of the country. Such experimentation should, of course, pay special attention to the relative susceptibility of the various higher grades of cattle, a matter which we have been unable to touch upon thus far.

What can the individual farmer or stock-owner do in the event that Texas fever has been introduced into his pastures? From what has been said thus far pastures which have been infected by Southern cattle or ticks from the litter and manure of infected cattle cars should be avoided during the entire summer season. While we know that young ticks may remain alive in jars for two or three months without food, it would be premature to conclude that such is the case on pastures, as the conditions are quite different. Yet everything seems to point to a long sojourn of young ticks on infected fields, and pending the carrying out of experiments to test this question we would recommend that native cattle be not allowed to graze on infected fields until after the first frosts, for even a mild attack in fall before the ticks have been destroyed by frosts is debilitating to cattle. The period of time during which infected localities remain dangerous varies, of course, with the latitude, and would be shorter the colder the climate.

The infection of stables, stalls, and other structures with the ticks should be counteracted by thorough disinfection. The adult ticks and the eggs must be destroyed. As stated above, we know as yet very little concerning the agents which will destroy the vitality of the eggs of ticks, but the use of water near the boiling point may be sufficient, if liberally applied, to destroy the life of the embryos. In the case of litter and manure heaps the thorough saturation with some strong mineral acid in dilution may accomplish the purpose. Ordinary lime, slaked or unslaked, densely sprinkled over infected places so as to form a continuous layer may be recommended. The slow incrustation of the egg masses with carbonate of lime may be expected, provided the manure is under cover. Otherwise it will be washed away and may 
leave the eggs unharmed. In regions outside of the enzoötic territory the absence of ticksmay be accounted for by the severity of the winter; hence in unprotecter localities disinfection is unnecessary after the winter has set in. But it inay occur that in sheltered places the eggs will winter over and the ticks reappear the following spring. Whether such ticks are likely to produce any serious trouble in the absence of Southern cattle we are mable to state definitely. All that we know is that disease may break out when Southern cattle of the preceding year are in the pasture, as was demonstrated accidentally in our investigations during 1891. (See p. 120 and No. 154.) Hence all infected material should be freely exposed to the frost, even though treated with disinfectants beforehand.

\section{TREATMENT.}

If the disease is suspected in a herd, the animals should be searched thoroughly for the presence of small ticks and the temperature of every animal taken with a clinical thermometer with which every stock-owner should be provided. This, which should be 5 inches long, is inserted well into the rectum and held there 3 to 5 minutes. If the temperature is $104^{\circ}$ to $107^{\circ} \mathrm{F}$., fever is present. The combination of ticks and fever, or the presence of the former in a locality where they do not naturally exist, may be considered a sure sign of the imminence of Texas fever. Though there are at least two species of ticks regularly infesting cattle in the permanently infected territory, these remarks can apply only to the species described in this report, since we know nothing as yet of the fever-producing capacity of the other species (Amblyomma unipunctata).

In case the ticks are found on the cattle they should be carefully removed and the cattle transferred at once to uninfected grounds. The cattle should be repeatedly examined for ticks and all found destroyed. While the change of pasture and the removal of ticks may not prevent the attack nor cut short the disease after it has once shown itself, we feel certain that fewer animals will succumb to it. A single infection is sufficient to cause severe and prolonged disease, as is shown by the injection of infected blood; but the mortality seems to be lower than in natural exposures, where the infection is intensified with every additional tick.

We are unable to recommend any specific remedies to be applied after the disease has appeared, because none have been tried as yet. Quinine and its various preparations fed or injected under the skin may prove of value in destroying the parasite, or perhaps methylene blue, recently recommended for malaria, may be of some service. We hesitate, hower, to do more than suggest these remedies, since their efficiency should first be carefully tested by well-planned experiments, which should only be undertaken on a large seale with a sufficient number of control animals and guided by a repeated examination of the blood. 
The general indications to be followed in attempting to save diseased animals are perfect rest in a sheltered place. Sick animals should not be driven or excited, for the condition of the circulation is such that any effort may bring about rupture of blood vessels and lead to speedy death. The heart, moreover, is always seriously involved and should not be strained in any way. Again, the exposure of sick cattle in the sun's heat withont shelter is liable to increase the ahready abnormally high temperature. We have, in fact, observed on unsheltered fields during very hot days a rise of from 2 to 3 degrees F. in presumably healthy eattle during the day, which we must attribute to the effect of the sun's heat. A sheltered place, preferably in the open air, in which the sick animal remains free from the annoyances of other animals, is therefore best suited to its condition. An abundance of pure water should be supplied to aid the overtaxed liver and kidneys to excrete their abnormal products in a more diluted condition. The food given should be readily digestible. It may be on the whole better to withhold food entirely until the high temperature begins to subside, since the various digestive organs are in a congested state and not in a condition to do any work.

The disinfection of infected pastures is out of the question, and must be left to nature in winter. They may, however, be used for sheep, since we have found these animals unharmed after grazing on them during an entire summer. It is highly probable that all other domesticated animals may run over such pastures with impunity, since Texas fever outside of the bovine species has not yet been observed.

\section{CONCLUSIONS.}

(1) Texas cattle fever is a disease of the blood, characterized by a destruction of red corpuscles. The symptoms are partly due to the anæmia produced; partly to the large amount of débris in the blood, which is excreted with difficulty, and which causes derangement of the organs occupied with its removal.

(2) The destruction of the red corpuscles is due to a microörganism or micro-parasite which lives within them. It belongs to the protozoa and passes through several distinct phases in the blood.

(3) Cattle from the permanently infected territory, though otherwise healthy, carry the micro-parasite of Texas fever in their blood.

(4) Texas fever may be produced in susceptible cattle by the direct inoculation of blood containing the micro-parasite.

(5) Texas fever in nature is transmitted from cattle which come from the perman ently infected territory to cattle outside of this territory by the cattle tick (Boöphilus bovis).

(6) The infection is carried by the progeny of the ticks which matured on infected cattle, and is inoculated by them directly into the blood of susceptible cattle. 
(7) Sick natives may be a source of infection (when ticks are present).

(8) Texas fever is more fatal to adult than to young cattle.

(9) Two mild attacks or one severe attack will probably prevent a subsequent fatal attack in every case.

(10) Sheep, rabbits, guinea-pigs, and pigeons are insusceptible to directinoculation. (Other animals have not been tested.)

(11) In the diagnosis of Texas fever in the living animal the blood should always be examined microscopically if possible. 


\section{DESCRIPTION OF PLATES.}

Every figure illustrative of red blood corpuscles containing the parasites or modified by the disease, was made from one field of the microscope, and this only, unless especially mentioned to the contrary in the descriptions below. The drawings are therefore equivalent to photographic reproductions, in so far as nothing was omitted from the field or inserted from other fields (with exceptions to be given). The slight differences in the coloring is due to the different intensity of the stain in the preparations, and was copied as accurately as possible by the artist.

'I'he preparations illustrating blood corpuscles were all drawn with the aid of a Zeiss apochromatic objective, $2 \mathrm{~mm}, 1.30 \mathrm{n}$. a. and the measurements made with the compensating micrometer ocular No. 6. The occasional variation in the magnification is due to the fact that the earlier drawings were made with the draw tube in, the later ones with the tube length so adjusted that each division of the ocular micrometer was exactly equivalent to $2 \mu$. The history of the cases from which the drawings were made may be found recorded in detail in the appendix.

Plate I. Diseased and healthy spleen.

Fig. 1. Spleen of No. 130. The smaller ventral end is here represented. Weight of entire spleen $6 \frac{7}{8}$ pounds

Fig. 2. Spleen of healthy steer killed for beef. The same region selected as in the diseased spleen. Weight $2 \frac{8}{8}$ pounds.

Note the enormous enlargement of the diseased spleen, the almost blackish appearance of the pulp, and the concealment of the trabeculæ and Malpighian bodies as compared with the healthy spleen. The ratio of the weight of the diseased to that of the healthy spleen is in this case as $2 \frac{1}{2}$ to 1 , while the weights of the animals is as 2 to 3 (800 to 1,200).

Plate II. Diseased and healthy liver.

Fig. 1. Liver of No. 50. The figure shows the cut surface at right angles to the peritoneal surface.

Fig. 2. Cut surface of the same liver enlarged two diameters to show the distribution of the yellowish zones along the course of the hepatic vessels.

Fig. 3. Cut surface of healthy liver. The coloring is not properly reproduced in this figure.

Fig. 4. Section parallel to the peritoneal surface of fresh liver from No. 106, in iodized serum. Slightly magnified. The yellow regions correspond to the regions in which the bile capillaries are distended with bile.

Plate III. Bile stasis and hæmoglobinuria.

Fig. 1. Section from liver of No. 130. Cut on freezing microtome, stained in alum carmine, floated upon the slide, dried in thermostat and mounted in xylol balsam. Drawn with Zeiss apochr. $4 \mathrm{~mm}$. and compens. ocular 4. Outlined with camera lucida. ( $\times 250$.) The hepatic cells are shown to be inclosed in a network of bile canaliculi distended with rods of solid bile. The space to the left represents the intralobular vein. 
Plate III-Continued.

Fig. 2. From a fresh section of liver of No. 144, showing the notwork of injected bile canaliculi and the needle-like, red crystals.

Fig. 3. Rods of solid bile obtained from teased preparations of the liver of No. 184. $(\times 1,000$. $)$

Fig. 4. Urine from No. 80.

Plate IV. Microörganism of Texas fever.

Fig. 1. Blood from a skin incision of No.74, taken September 30, 1890. The blood was spread in a thin layer on a cover-glass as described in the text, dried in the air. Subsequently heated for one to two hours in a dry hot-air oven at $110^{\circ}-120^{\circ} \mathrm{C}$. Stained for two to three minutes in Löffler's alkaline methylene blue, washed in water, then dipped for a moment in a one-third per cent solution of acetic acid, washed again, dried in the air, and finally mounted in xylol balsam. The micro-parasite is represented by the exceedingly minute blue points within the red corpuscles.

In this preparation the infection is shown to be very extensive. This is the only case in which the parasite was detected in this exceedingly minnte stage. $(\times 1,000$. $)$

Fig. 2. Covel-glass preparation of spleen pulp from No. 70. Stained as described in Fig. 1 above. The intraglobular bodies are slightly larger than those of Fig. 1. $(\times 900$.

Fig. 3. Blood from a skin incision of No. 160. Prepared November 7, 1891. Method as described. The small bodies are situated within the red corpuscles near the periphery. The large red corpuscle in the center containing a number of stained particles of different sizes is a result of the loss of corpuscles, or anæmia. $(\times 1,000$.

Fig. 4. Cover-glass preparation from kidney of No. 130. Method of fixing and staining as described above. The large, blue body in the center of the group is one of the cellular elements of the kidney. The parasites are usually in pairs, and roundish. This form is generally assumed in the dead body. $(\times 1,000$.

Fig. 5. Preparation made by rubbing a piece of the heart muscle of No. 186 on a cover-glass, drying and staining as before. In this way the blood corpuscles from the smaller vessels and capillaries are obtained. The large blue borly represents a leucocyte. The parasites are mostly in pairs and pearshaped. $(\times 1,000$.

Plate V.

Fig. 1. Cover-glass preparation of spleen pulp from No. 66. The two large blue bodies represent cell elements of the spleen pulp, and the uniformly bluishpink body represents an "anzemic" red corpuscle. The red corpuscles are mostly larger than normal, owing to the anæmic condition induced in the animal before death. Many parasites are in pairs and have assumed the spherical form. The animal had died in the night. $(\times 1,000$.

Fig. 2. Preparation made as before from blood taken from a skin incision of No. 106, August 27, 1890, less than twenty hours before death. The appearance of the infected corpuscles in groups, as shown in the figure, was especially marked in this animal. The parasites are nuinly in pairs, and pyriform. The stained body on the right is a white corpuscle. The largo parasite in a corpusele to the left was drawn in from an adjacent field. The reddish color of the stained bodies is due to the fact that the preparation was diawn while still mounted in water, which partly dissolver out the coloring matter. $(\times 1,000$.

Fig. 3. Cover-glass preparation of blood taken from a skin incision of No. 185, (Oetober 9, 1891. (Case of intravenous injection of T'exas-fever blood.) On this date only about 1,000,000 red corpuseles in a cmm. All objects within the dotted line are in one field of the microseope. The rest are drawn in 
Puate V-Continued.

from other fields in the same preparation. a represents modified red corpuseles, $b$ a leucocyte, $c$ a liematoblast, and $d$ the parasites. Note the variation in the size of the red corpuseles. The parasites are mainly in pairs. They vary in size and form, and perhaps represent stages of degeneration. $(\times 1,000$. $)$

Plate VI. Mieroörganism of Texas fever.

Fig. 1. Vascular fringes on omentum of No. 130, crushed on cover-glass. Dried and stained as before $(\times 1,000)$. Shows the extensive uffection of blood corpuscles in the eapillaries. The large blue boty represents a cellular element. One corpuscle shows a double infection. The parasites are mainly pyriform.

Fig. 2. Heart muscle of No. 130, crushed on cover-glass, dried and stained to show presence of parasites freed by the disintegration of the infected corpuscles. One corpuscle in the lower part of the figure to the right shows faintly. The two pairs of free parasites above are drawn in from another field.

Fig. 3. Preparation from kiduey of No. 74, showing the iarge number of freed parasites in addition to an extensive infection of the red corpuscles. The free bodlies largely in pairs.

Fig. 4. Infected corpuscle, unstained, from cutaneous blood of No. 56, collected November 28.1890. Each pyriform body is provided with a minute dark body not seen in stained preparations. $(\times 1,000$.

Fig. 5. A similar pair of parasites, unstained, from No. 130. Taken December $30,1890 . \quad(\times 1,000$. $)$

Fig. 6. A spherical form from the same case at the same time.

Fig. 7. Stainerl corpuscle from cutaneons blood of No. 137, prepared November 6,1890 , shortly before it was killed, showing how large these bodies may occasionally become with reference to the enveloping corpuscle. Note also the peripheral stain.

Fig. 8. Free parasites not infrequently observed in crushed, fresh, and unstained preparations from heart muscle of various cases. They are seen usually in pairs.

Fig. 9. A series of corpuscles containing bright motile bodies, observed both in health and disease. Somewliat coarsely outlined.

Fig. 10. Path of one of the bright notile bodies within a red corpuscle, as observed during a period of 15 minutes. Sketched from a fresh preparation of cutaneous blood from No. 107, August 25, 1890.

\section{Plate ViI.}

Fig. 1. Capiliary from heart muscle of No. 181. From tissue hardened in Miiller's fluid and alcohol. Sections cut after imbedding in paraffin and fastened to the slide with a few drops of 70 per cent alcohol. Stained for an honr in Ehrlich's acid henıatoxylin and eosin, dehydrated in alcolol containing eosin, cleared in clove oil, and mounted in xylol balsam. There are a considerable number of parasites in pairs within the red corpuscles, the majority of which show only in outline, since they have lost their coloring matter, probably as a result of disintegration. $(\times 500$. $)$

Fig. 2. Capillary from the medullary portion of kidney of No. 186. Nearly every corpuscle contains a pair of parasites. Those drawn in shadow below the optical section in focus are also infected. The section was prepared in the same manner as detailed above, with the exception that it was not fastencd to the cover glass and not passed through eosin alcohol. $(\times 500$.)

Fig. 3. Capillary containing infected corpuscles almost exclusively. From a teased preparation of fresh spleen pulp of No. 131, in iodized serum. The unstainel parasites appear as minute round white spots in the corpuscles. $(\times 500$. 
Plate VIII. Amoboid changes of the microörganism of Texas fever.

Fig. 1. Fresh preparation of blood from No. 69, five hours post-mortem. Prep. aration sealed with paraffin and kept in a warm chamber with microscope at $35^{\circ}-40^{\circ} \mathrm{C}$. Showing changes of form in an intraglobular parasite.

Nos. 1 to 6, changes going on as fast as could be sketched.

Nos. 7 to 15 , sketched twenty minutes later.

Fig. 2. An intraglobular parasite in subentaneous blood of No. 95 a few hours before death, showing changes of ontline.

Fig. 3. Another parasite from the same source, showing similar changes.

Fig. 4. Parasites showing a nuclear (?) body from the same source.

Fig. 5. Similar parasites sketched from the fresh cutaneous blood of No. 90, shortly before death. Note the different forms and relative positions occupied by the intraglobular parasites as well as the presence of the nuclear (q) body.

Plate IX. Modified or embryonic red corpuscles after severe hemorrhage and after 'Texas fever.

Fig. 1. Blood from sheep No. 160, upon which venesection had been practiced. Prepared July 7, 1890, a fter the number of red corpuscles had been reduced from 11 to $5 \frac{1}{2}$ millions. Dried and stained as described for Fig. 1 on Plate IV. Note variation in the size of the normal corpuscles and the presence of large corpuscles containing a large number of stained particles or granules of variable size. $(\times 1,000$.

Fig. 2. Another field from the same preparation, showing also a tinted form without granules. $(\times 1,000$.

Fig. 3. Cutaneous blood from cow No. 168, drawn Angust 12, 1891, after the red corpuscles had been reduced by venesection from $6 \frac{1}{2}$ to 2 millions. Stained as indicated in Fig. 1. Note the presence of large and small granules in the corpuscles; also a uniformly stained corpuscle. $(\times 1,000$. $)$

Fig. 4. From another field of the same preparation, showing two large corpuscles containing stained granules. $(\times 1,000$.)

Fig. 5. Cutaneous blood from No. 160 (case of Texas fever). Preparation made November 12, 1891. A corpuscle on the left contains a Texas-fever parasite. $(\times 1,000$.

Fig. 6. Another field of the same preparation, showing uniformly stained as well as granular red corpuscles. Note also the great variation in size of the corpuscles having a normal appearance. $(\times 1,000$. $)$

Plate X. The cattle tick-the carrier of Texas fever.

(Figs. 1, 3, 4, and 5 were drawn under the direction of Dr. Curtice. Nos. 4 and 5 were slightly modified before insertion.)

Fig. 1. A series of ticks from the smallest, just liatehed from the egg to the matured female ready to lay eggs.

Fig. 2. Eggs magnified 5 diameters.

Fig. 3. The young tick just hatched $(\times 40)$.

Fig. 4. The sexually mature male after the last moult. Dorsal view. ( $\times 10$.

Fig. 5. The sexually mature female after the last moult. Dorsal view. $(\times 10$.

Fig. 6. A portion of the skin of the udder of No. 140. (Ticks artificially hatched and put on when small.)

Fig. 7. A portion of the ear of the same animal, showing adults ready to drop off and lay their eggs. 


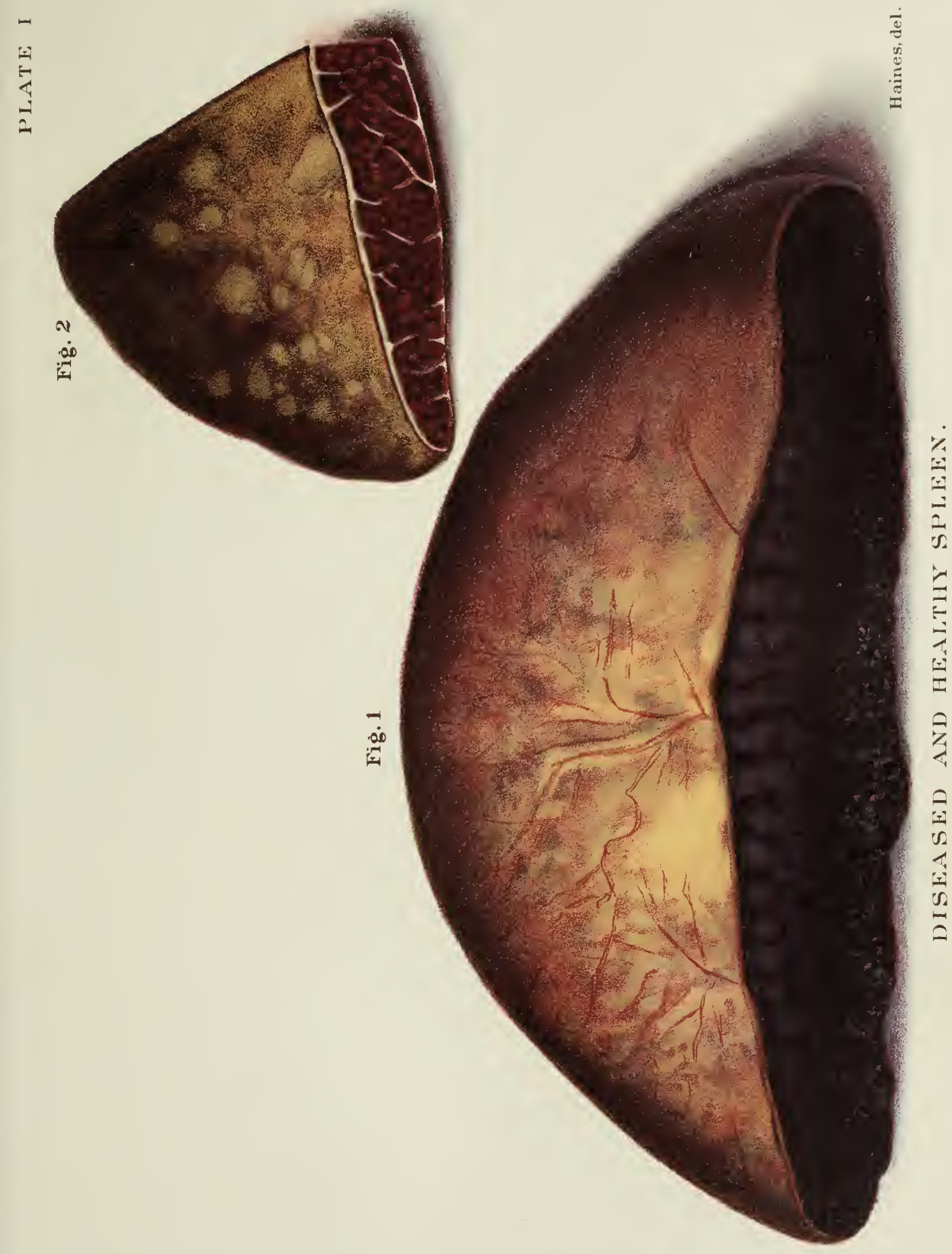



Fig. 1

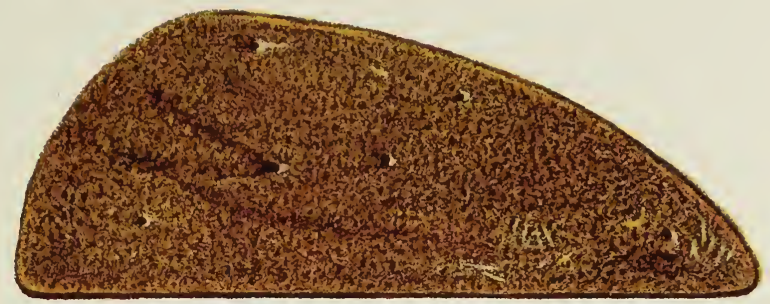

Fig. 3

Fig. 2

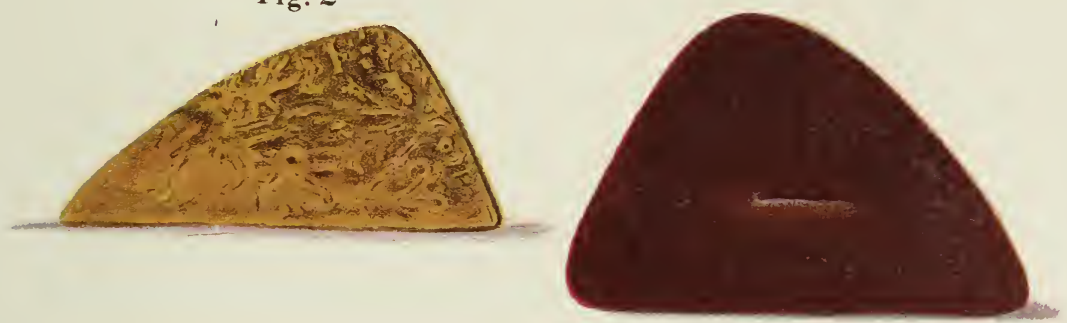

Fig. 4

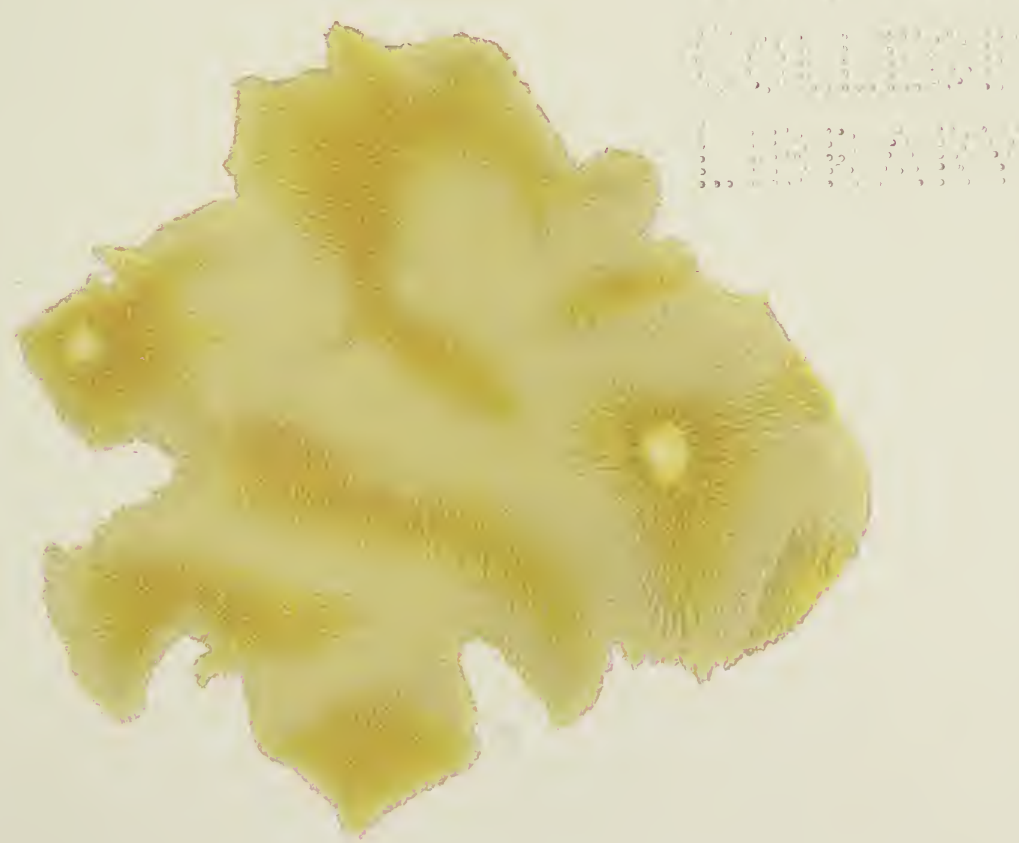

Haines, del 

Fig. 2

Fig. 1
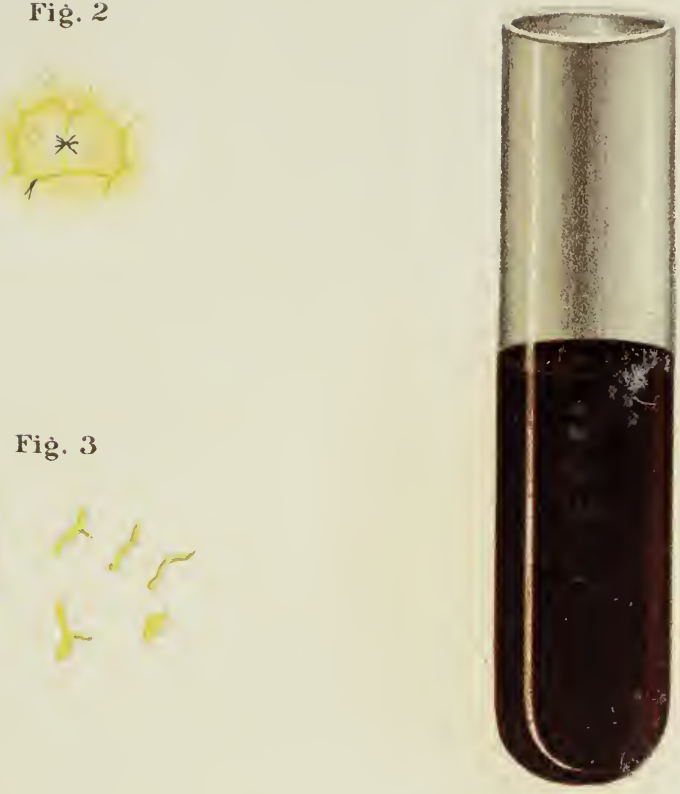

Fig. 3

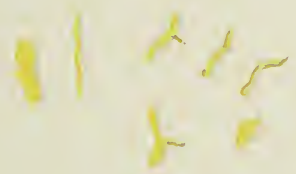

Fig. 1

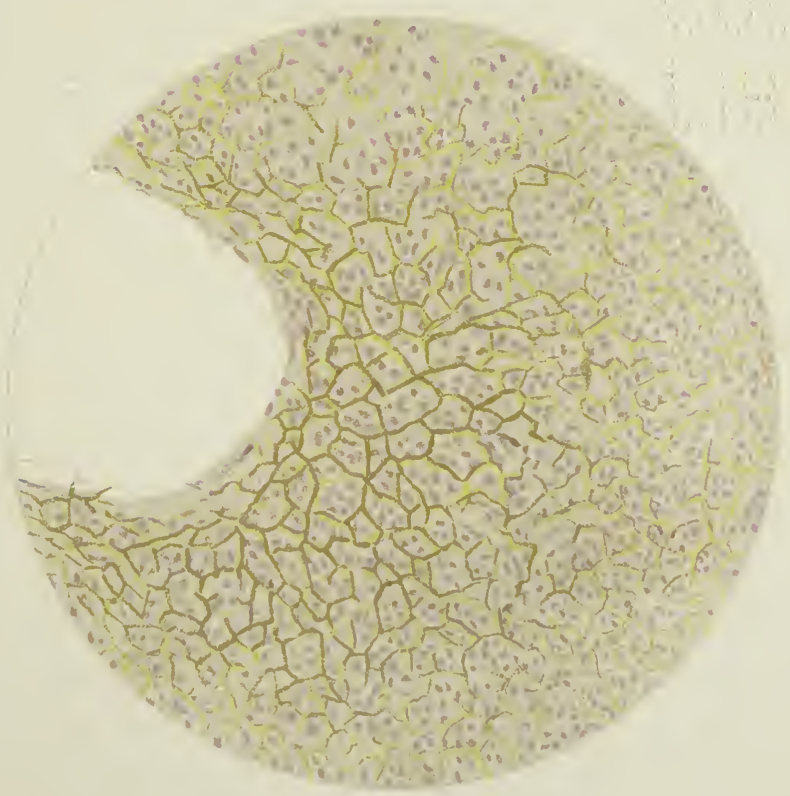



Fig. 1

Fig. 2

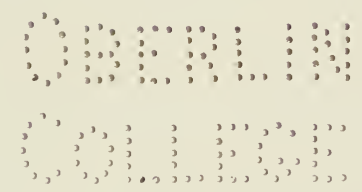

Fig. 3

Fig. 4

Fig. 5
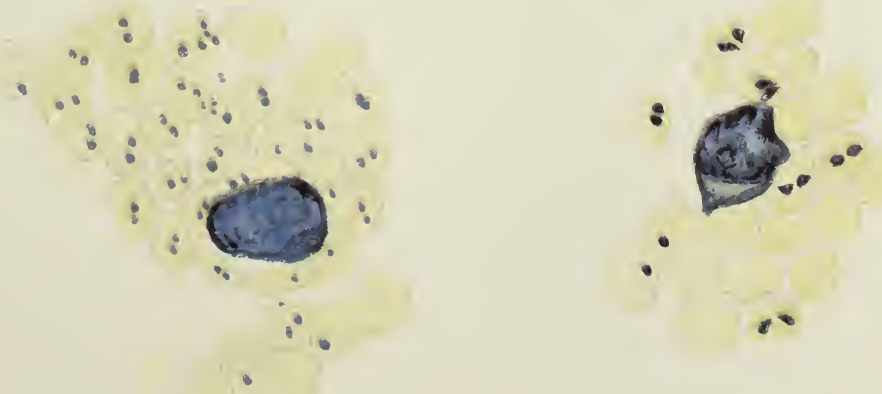

Haines, del.

MIGRO-ORGANISM WITHIN THE RED BLOOD GORPUSGLES. 

Fig. 1

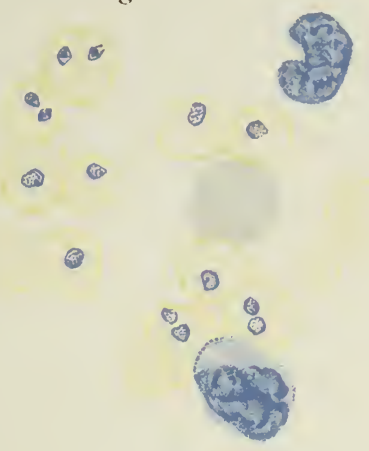

$c$

$d$

$\therefore$

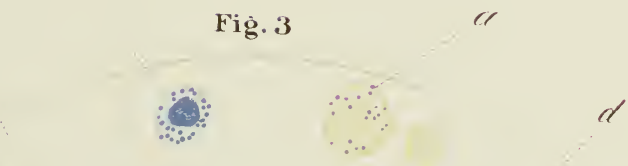

2.

is
Fig. ?

$$
\begin{aligned}
& 7 \text {, } \\
& \text { a d o n }
\end{aligned}
$$

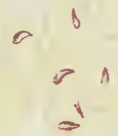

$a^{1}$
8 $\checkmark$

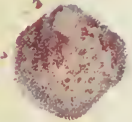

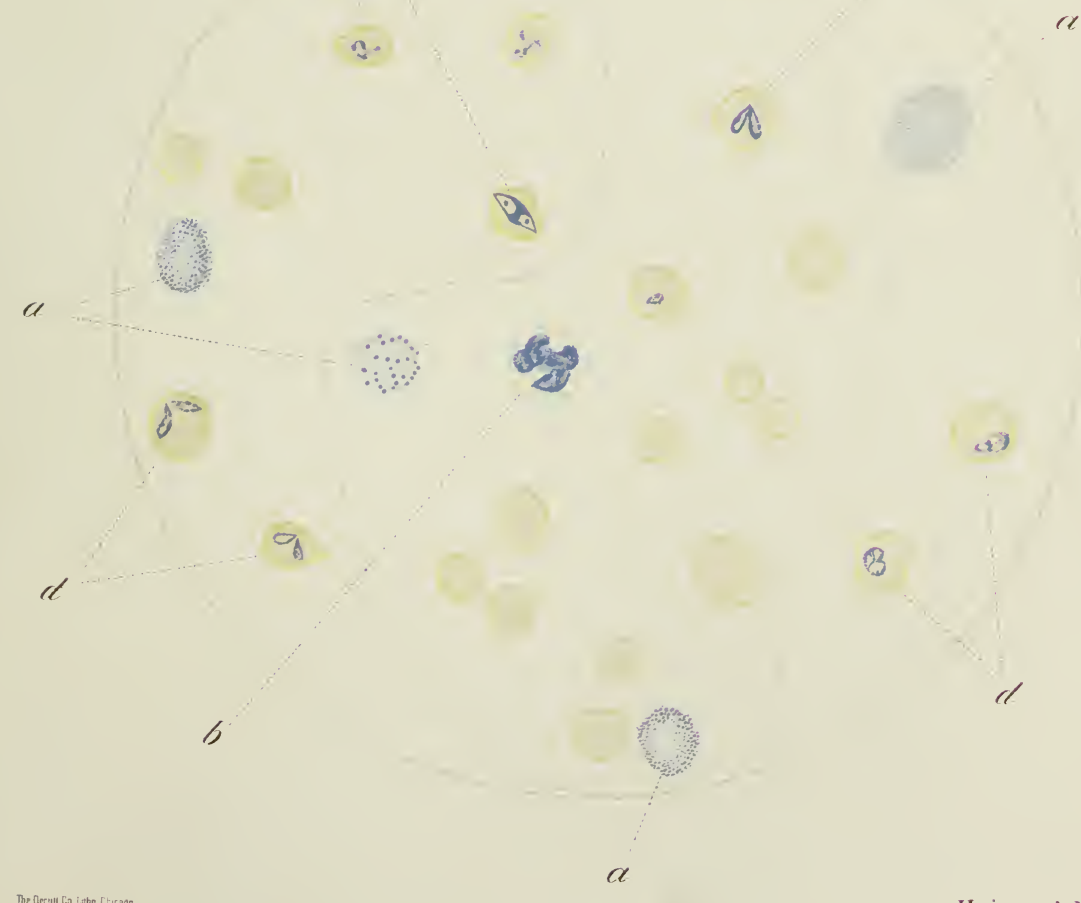

Haines,del. 
Henthes

+4a $x$ 
: Fig. 2

Fig. 1
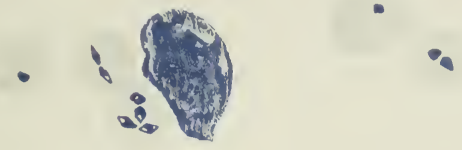

Fig. 4

Fig. 5

Fig. 3

$:$

Fig. 6

Fig. 7

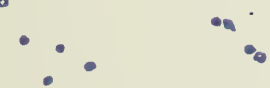

Fig. 8

Fig. 9 

Fig. 1

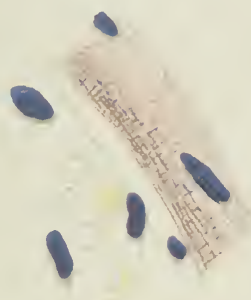

Fig. 2

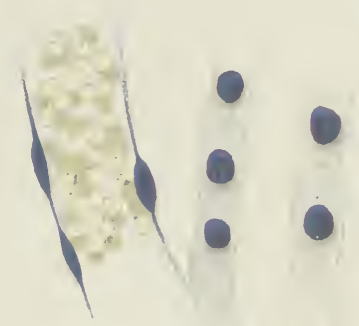

Fig. 3

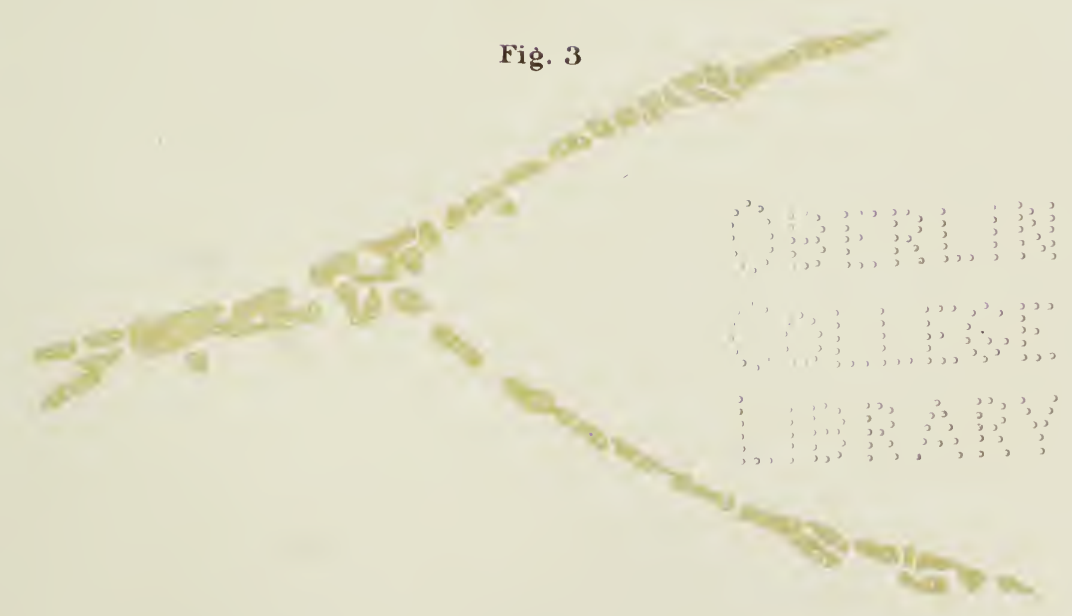



Fig. 1

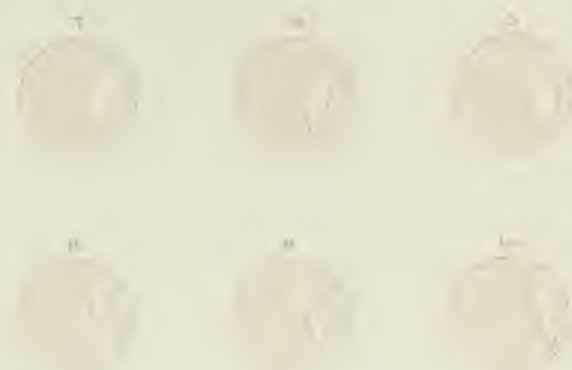

Fig. 2

Fig. 3

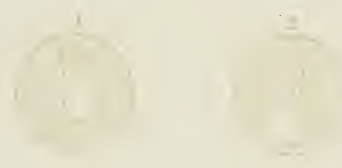

Fig. 4

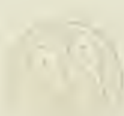

Fig. 5

PARASITE IN THE BLOOD GORPUSCLES.

Figs.1,2 and 3 show amoeboid changes. 


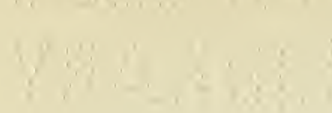


Fig. 1

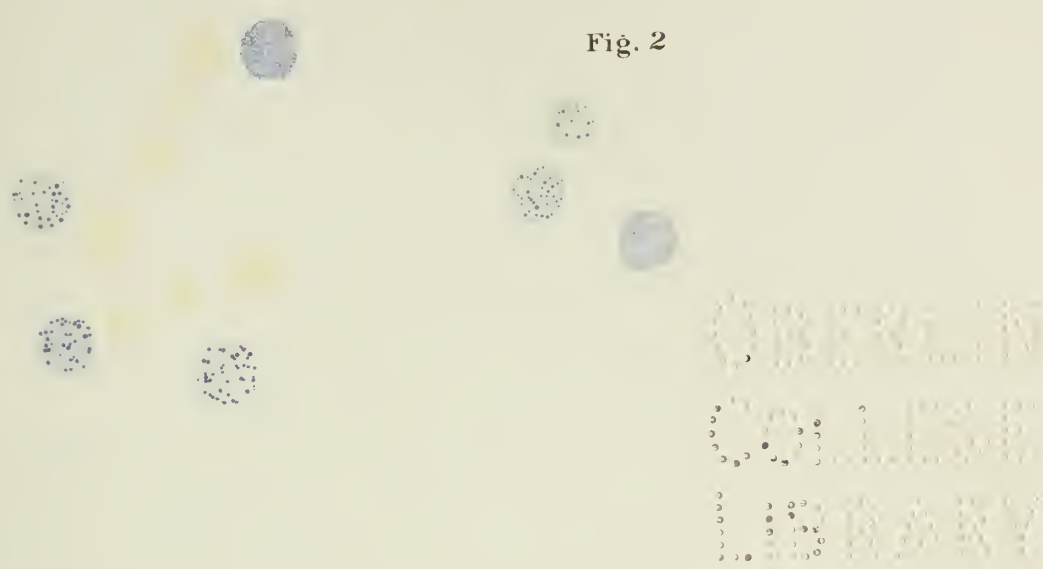

Fig. 4

Fig. 3
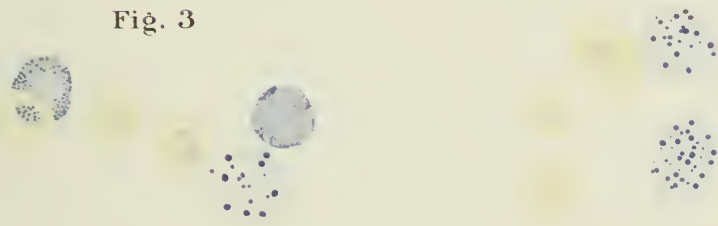

Fig. 5

\section{Fig. 6}
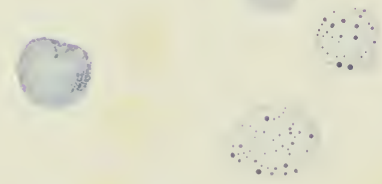

MODIFIED (Embryonic) RED BLOOD CORPUSGLES AFTER SEVERE HEMORRHAGE AND AFTER TEXAS FEVER. 

Fig. 1

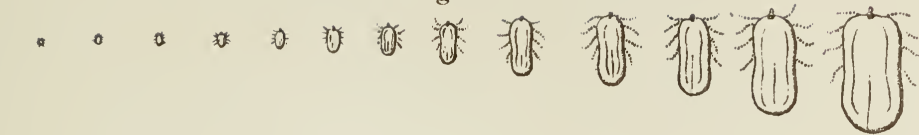

Fig. 2
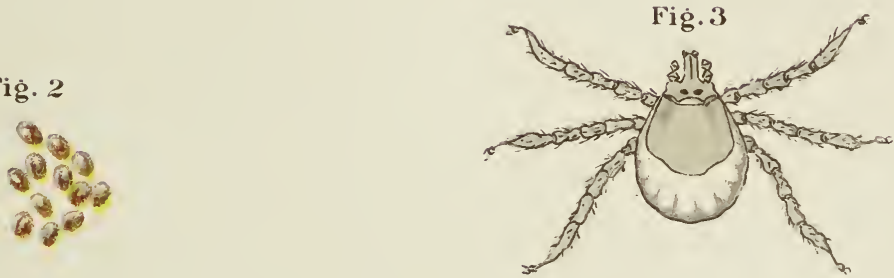

Fig. +
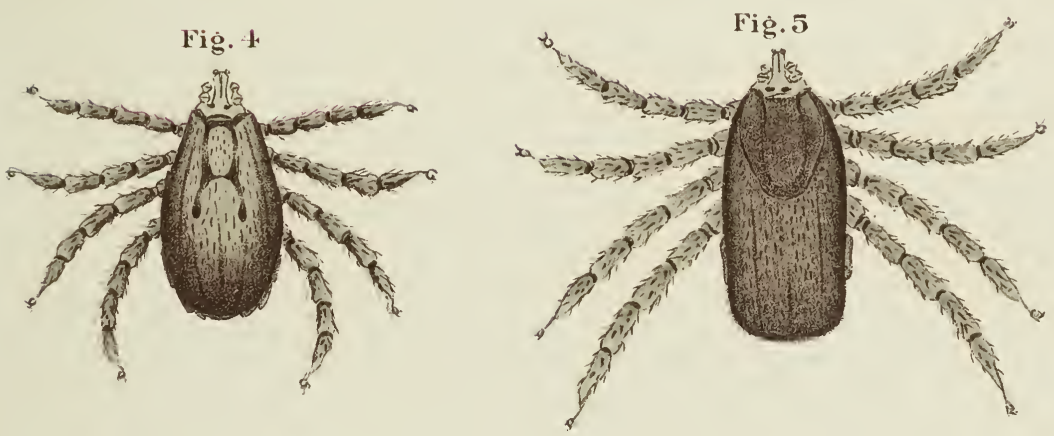

Fig. 7

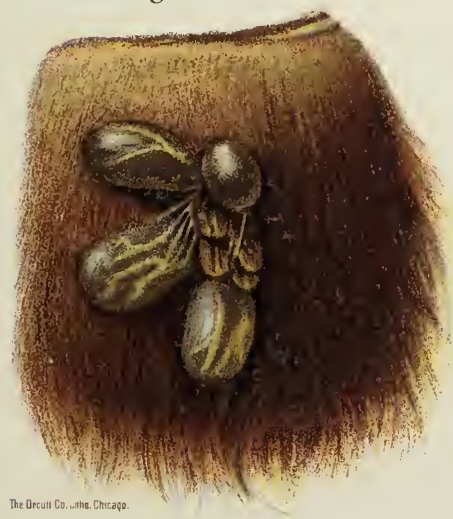

Fig. (
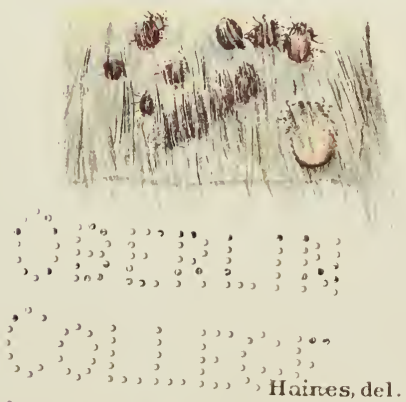

THE GATTLE TIGK- THE GARRIER CWE, TSXAS WVER. 



\section{APPENDIX.}

\section{EXPLANATORY INTRODUCTION TO NOTES AND TABLES.}

In the following pages will be found the various observations upon the cases of Texas fever investigated upon which the deductions in the foregoing report are based. They consist of clinical, pathological, bacteriological, and microscopical observations, and are given as fully as is possible without going into frequently i epeated details. The terms used will not in general be intelligible without the text and the notes as a whole are not intended to replace this. They may in fact be entirely passed over by those not especially interested in the methods and details of the work.

The cases are arranged with regard to the numbers which the animals accidentally bore and not with regard to the experiments of which they formed a part. This method was adopted to facilitate reference while reading the text in which the general plan of all experiments may be found. Frequent reference is made to special cases by the numbers of the animals, and the appendix may thus be regarded as a kind of index to the text. This plan of transferring the greater part of the experimental detail to a compact appendix is tried with the hope that it will make the text less bulky and hence more readable, while including everything that may be necessary to an impartial judgment on the part of the reader. The arrangement therefore throws cases together which perhaps belong to different experiments in different fields. But the material has grown so bulky that any other arrangement would be more confusing than this seems to be. As the appendix has been entirely subordinated to the text proper the serial arrangement by numbers remained the only altermative. It was thought best to include the temperature records of all animals in their entirety, and these will be found at the end arranged, not numerically, but with reference to the experiment to which each animal belongerd. Under each case the page where such record can be found is given.

In order to facilitate the reading of the notes the observations upon many of the cases have been thrown into a tabular form. This necessitated very brief statements concerning the condition of the blood and the parasite, which in some respects is to be regretted. This tabular form is, however, so much superior in permitting a ready comparison of the condition of the blood, etc., from time to time, that it was 
nevertheless used in all cases in which the examination of the blood was frequently marle.

In the interpretation of the tables the following things should be borne in mind: In quoting the number of red blood corpuscles the three last figures have in general been replaced by ciphers to facilitate and simplify the reading.

The sign of equality indicates the same condition as in the column immediately precerling.

The third and fourth columns always refer, unless it is otherwise stated, to bodies within the red blood corpuseles. A record has been made of the oceurrence of the so-called bright bodies described on page 56 because of a possible connection of some of them with the earliest stages of the Texas-fever parasite as suggested on page 68 .

The estimated percentage of infection is not from actual counting of the corpuscles in the dried and stained films (as this would have consumed an amount of time not warranted by the results), but is a simple approximation or guess from the examination of a large number of fields. In those cases in which the infection is very slight and indicated by actual figures (as "one bright body," "three pairs pyriform bodies," etc.) these are based on the more or less prolonged examination of a single cover-glass preparation. This does not mean, however, that only one preparation was examined. Not infrequently three or four in doubtful cases were examined at different intervals. The same may be said of those preparations in which no intraglobular bodies were found. In some cases one, in others two or three, were carefully scrutinized. It is of course evident that the absence of all parasites from the blood can not be predicated when the evidence is based on the examination of a very small quantity of blood. It may be said that at least several thousand red corpuscles were passed in review in every preparation.

Attention is likewise called to the fact that the two columns giving the result of the examination of the blood in the fresh and in the dried and stained condition are not necessarily identical, but supplement one another as to the information given. Thus the minute bright, intraglobular bodies are visible only in fresh blood. The reverse is true of the earlier coccus-like forms of the parasite, which are rarely visible in the fresh blood.

The fifth and sixth columns of the tables refer to the condition of the red corpuscles at the time of the examination. The importance of a recognition of their condition in the study and diagnosis of 'Texas fever has been fully discussed on pages 46 and 146.

The record of the pulse and respiration is not reliable in many cases, owing to the excitability of the animals on being caught. This unreliability disappears more or less during the fever, since they are then easily canght and manifest little if any excitement.

In the notes on the individual eases, facts obtained by subsequent microscopical and bacteriological examnination of tissues have been 
placed with the gross post-mortem notes to simplify the arrangement. For instance, the result of the examination of hardened tissues, which in a few cases was delayed a year or even longer, is quoted in the postmortem notes, thereby bringing all the observations upon any one organ together. In some cases discoveries have been anticipated in rewriting the notes. Though the eanse of the minute worm pits in the fourth stomach was not recognized until the fall of 1890 (see page 33 ) the lesions in some earlier cases have been referred to the strongyle in writing up the notes for publication.

In general, the notes of the later cases are fuller with reference to the microörganism, and perhaps more exact, and the reader is referred to them more particularly. It was thought best, however, to include the notes of all the cases which have entered into the field experiments in order to preclude any doubts in the mind of the reader as to the correctness of the inferences drawn from these field experiments. Many of the notes are, therefore, merely of diagnostic value, and their meagerness in other respects is excusable on the ground that it was a physical impossibility during the prevalence of the acute disease in summer to do justice to all cases which came under observation.

For the diagrams of the field inclosures see pp. 94, 99, 102, and 108.

\section{SOME PRELIMINARY BACTERIOLOGICAL OBSERVATIONS.}

The following observations were made on material brought to the laboratory in Washington, D. C. Cases No. 1 aurl 2 belonged to an outbreak near Hamilton, Va., in 1886. Cases No. 3-6, inclusive, belonged to an outbreak in Carroll County, Md., in 1888. Dr. Cooper Curtice reported the field notes of this outbreak in the volume comprising the fourth and fifth annual reports of the Bureau of Animal Industry (p. 429):

Case 1-(August 30, 1886). Spleen from a case of Texas fever which had died during the night at Hamilton, Va. About four times the normal size. Wrapped in cloths saturated with a one-tenth per cent solution of mercuric chloride, brought to the laboratory and kept on ice nntil next morning. Cultures were made from the spleen pulp which was very soft, dark-colored, by making several successive incisions with sterile knives and withdrawing bits of pulp with loops.

September 1.-Three tubes of neutralized beef infusion peptone, into which small bits of the pulp had been dropped, were faintly clouded to-day. One contained two forms of bacilli, a slender and a plump form; the second, a large bacillus and a micrococcus; the third, a large spore-bearing bacillus and a slender one. Two tubes of the same culture liquid, into which the platinum loop previously forced into the spleen pulp was dipped, remained sterile. Of two tubes of blood serum, inoculated with bits of spleen tissue, one remained free from growtl, the other contained a plump bacillus. Several gelatin-plate cultures nade at the same time remained free from growth.

A portion of the same spleen was kept in a warm room for one day wrapped in sublimated cloths. The pulp then examined microscopically contained two forıns of bacilli, a plump and a slender form, the former in chains, the latter predominating in numbers. Three tubes of gelatin inoculated therefrom were liquefied in a few days and contained the same organism found in the liquid cultures.

The various forms thus brought to light no doubt found their way into the spleen soon after the death of the animal, and the want of uniformity in the results of the cultivations indicated that nothing could be expected from them. 
Case 2.-September 1, 1886. Another cow died this morning at Hamilton, Va. The spleen was immediately removed, wrapped in sublimated cloths, and kept on ice until it could be examined next morning.

This organ is likewise very large, the tissue disintegrated and very dark. No bacteria could be seen in cover-glass preparations. In or on many red blood-corpuscles there are small round bodies perhaps $1 \mu$ in diameter, centrally or slightly eccentrically situated, which stain poorly in an aqueous solution of methyl vivlet, very well in methyl violet to which aniline water has been added (Koch-Ehrlich tubercle stain). They then resemble micrococci in size and form. Only a few are found outside of the corpuscles. Unstained they can be seen as mere transparent spaces in the corpuscles.

Five tubes containing bouillon peptone were inoculated from the spleen, three with the platinum loop simply, two with bits of tissue. Several agar and one blood serum (beef) culture were also made. All remained sterile. The method of inoculation consisted in thoroughly scorching the uninjured capsule, making an incision through this scorched area with a flamed scalpel, a second at right angles to this with a fresh knife. From the latter incision bits of pulp were torn away with flamed forceps and transferred on platinum loops to the various culture tubes.

Case 3.-Cow died at 9 a. m., August 28, 1888, in Carroll County, Md. The autopsy was made by Dr. Farrington at $10 \mathrm{a}$. $\mathrm{m}$. some of the organs were placed in different compartments of a refrigerator pail specially constructed for this purpose, in which they were surrounded by a jacket of ice. The temperature of the air in the inner compartments varied from $32^{\circ}$ to $40^{\circ} \mathrm{F}$. No decomposition could thus begin, or continue if already begun, while the organs were kept in the pail packed with ice. Cultures were made from the various organs as soon as they reached the laboratory. The unbroken surface was thoroughly scorched and bits of tissue, etc., cut out from within this scorched area with sterile instruments.

The splecn is very large, pulp dark and disintegrated. Red blood-corpuscles do not appear altered. When stained with alkaline methylene blue on cover glasses the splcen pulp. reveals no bacteria. Four tubes were inoculated with bits of the pulp, two containing bouillon peptone, two agar. After a week all sterile.

The liver is of a mahogany color, dne to the accumulation of bile. When a bit of the liver tissue is crushed in salt solution and examined unstained under the microscope the liver cclls themselves show their nucleus with five or six fat granules around it. Each cell is encircled by a narrow band of reddish-yellow material, forming a polygonal network and representing the bile canaliculi filled with inspissated bile. This peculiar injection is even visible on stained cover-glass preparations. It breaks up into straight and Y-shaped rods, indicating that it is solid in consistency. The rods are about $1.5 \mu$ thick.

Sections of liver tissue (hardened in Mïller's fluid and alcohol imbedded in chloroform paraffin and) stained in alum carmine, show a marked distention of the capillaries of the middle zone of the acini with red corpuscles. The bile canaliculi in the innermost and outermost zones appear as a yellow network. The nuclei of the cells of the innermost zone have become disintegrated, each appearing as a group of roundish granules. The middle zone shows degenerated and normal nuclei mingled together.

Blood corpuscles begin to crenate rapidly; in other respects no changes are manifest.

Cultures from the liver, three in bonillon peptone and two in agar, contain active growths ou the following day. Nearly all contain the same motile bacillus. It is short, with rouncied ends. In gulatin-roll cultures its colonies appear in the form of very thin, iridescent patches on the surface of the gelatin.

A portion of the large intestine contained lumps of hand fisces. 'The numeosil was considerably reddened from the injection of minute vessels, especially on the summit of the longitudinal folds. Numerous roll cultures in gelatin were mado from unterial scraped from the surface of the membrane. In most of theso tubes the samo 
peculiar iridescent patches appeared, which consisted of short motile bacilli. 'Two mice and a rabbit were inocnlated with the seraped naterial suspended in water. One monse died in less than twenty-four hours. 'The spleen was enlarged to twice its nommal size. A sero-sanguinolent oedema in the subeutis of thigh and abdomen. In eultures from the spleen, liver, and blood the same iridescent colonies developed as those obtained from the intestine directly in enltures. The second mouse dicd "few hours later. Local lesion as in the first monse; spleen small. The same bacteria observed in the local orlema, but apparently absent from the spleen. An agar culture from the blood develops a rather thick, whitish, glistening growth of the same bacteria. The rabbit showed no ill effects after the inoculation.

From the kidney of the cow bits of tissue were transferred to bouillon peptone, agar, aud gelatine. Of these five tubes one (agar) contains a feeble grow th of small motile rods. 'The remaining four permanently sterile.

At the autopsy the bladder was empty, hence the condition of the urine could not be determined.

Material from this cow was bronght to the laboratory in the refrigerator pail September 3, 1888. The spleen is abont 8 inches wide in the middle and $1 \frac{1}{2}$ inches thick. The capsnle is so tense that the softened pnlp wells ont wherever an incision is made and is so soft as to be readily broken up with a stout platinum loop. A bit of the pulp placed in salt solution, under the microscope shows immense numbers of apparently normal red corpuseles. No bacteria seen either in the unstained or the stained preparations. Four eultures made by adding bits of spleen pulp as large as split peas remain sterile. A gelatine roll culture contains but one liquefying colony.

Only a small mass of liver tissue bronght for exanination. It has a peculiar sickening but not putrefactive odor. The eut surface is yellowish. In salt solution the hepatic cells appear finely gramlar with nuelens distinct. Some contain fat globules and irregular masses of yellow pigment. The intercellular bile canaliculi are distended irregularly with yellow masses. The injeetion is not so uniform as in the preceding case. On stained cover-glass preparations no bacteria are visible.

Roll cultures of gelatine show that small bits of liver contain but few gerus, as only two or three colonies appear in each tube. The germs of which they are made up are probably identieal with those fomd in two liquid and one agar eultnre.

In the fourth stomach no lesions are perceptible. The dnodenum is filled with brownislı food-particles suspended in a thick liquid. 'The mucosa of the upper small intestine is covered with a yellowish white layer resembling in its eonsistency flour paste, and made 11p ahnost entirely of desquamated epithelial eells. The mucosa itself is injected in patches. Cover-glass preparations as well as roll cultures reveal the presence of the same germ found in previous eases. In fact it seems to be the only germ present in very large numbers. Two mice inoculated with some sterile water in which the pasty intestinal contents were suspended remained well. The gall bladder is moderately distended with a thick liquid containing in suspension flakes from 1 to $6 \mathrm{~mm}$ across, whieh are very numerous. This abnormal mass is free from bacteria according to microseopie examination and roll eultures. The urinary bladder liad been ligatured at the autopsy. Specifie gravity of the nrine was 1020 . It was yellowish red, slightly alkaline. The bladder contained a large deposit of anorphous salts of a pinkish hue, dissolving when heated. Tests showed the al)sence of bile pigments, sugar, and albumen. When the urine was removerl the mncosa of the bladder near the neck showed a spot abont 1 ineh square covered with eechymoses as large as pin's heads. In order to obtain cultures from the urine the wall of the hladder was burned through witl a platiuum spatula and the uriue withdrawn witl a flamed pipette. About $\frac{1}{4}$ cubic eentimeter was added to a peptone bouillon culture, and a gelatine roll culture. One liquid culture became turbid, and a roll cultnre made from this revealed the fact that the germ was identieal with the intestinal germ. The roll and the other liqnid cultures remained sterile.

$10320-$ No. $1-11$ 
Case 1 (stcer).-Dr. Curtice's notes are as follows: "This animal died September 2, alout noon. 'The examination was undertaken about two hours later. Its temperature about one hour and a half before dying was $106^{\circ}$, taken at two separate trials. Its eyes wore inflamed and whitened; after death their interior appeared opaque and yellowish. When first seen the animal was standing in the pasture; it had previously been lying down and had paroxysmal attacks, from one of which it afterwards died. The animal, though the flanks were tucked up, was moderately fat. The fat had an orange yellow tinge. 'The spleen was enlarged and tumid with blood of black-red color, and of blackberry jam consistency. The liver had a heavy waxy feel, and a decidedly yellowish color. The gall-bladder was normal in size and full of bile. The kidneys were overlooked. The urine was very high colored, butnot bloody or wine-colored. The abomasum had some slightly reddened patches on the mucous coat; no ulcerations were seen. The rumen was impactcd. 'The small intestine contained occasioual dark spots about as large as the head of a lead pencil. The prominency of some portions of the villous surfacc was injected with blood, so that a redicned appearance was presented. The patches of inflammation were decidedly more abundant in the ileum than in the jejunum. The duodenum was blackened on its villous surface, and slight decomposition had set in. The odor was fetid. The lungs contained some blood spots, which appeared to have been made about the time of death. Some scattered lobules were collapsed. One side was emphysematous. The thoracic walls near the diaphragm were roughened as if from inflammation. The heart showed blood-red spots, which followed the direction of the muscles beneath the pericardium. These were more abundant on the left side toward the apex. Washed clots stained with yellow were found in each half. The surface of the lungs was stained yellow. The fauces were dark and inflamed, but this inflammation did not extend into the trachea."

Case 5 (heifer).-Deptember 4. This animal died during the night. "The oxamination took place about 11 a. m., but the morning had been rainy and cold so that decomposition had not set in to any great extent. She carried a large number of ticks.

"The abdominal cavity contained rather more than a normal amount of serous fluid. This fluid was rather dark. One portion of the mesentery appeared as though there were some peritonitis present. The fat was of an orange yollow color. The spleen was enlarged, but the color was nearly normal. The liver liad a yellowish cast; it was beginning to decompose on its concave surface, and more especially toward that part which was next the duodenum. The gall bladder was tumid and somewhat larger than normal. The left kidney was very dark colored (from decomposition (?)) on the outside, but apparently sound in the central portion. The bladder contained abont a gallon of claret-colored urine. The omasum or manifolds was inpacted. The abomasum or fourth stomach was normal. The left lung was normally contracted, but the right, was full and spougy. It presented a ycllowisli tinge on section. The heart prescnted bright red spots underneath the epicardial serous membrane. These spots bad their longest dimension in the direction of the inuscles, and were more abundant in the left portion near the apex. There were clots in each side of the heart, and that on the left side was slightly washed. The perieardial sac contained an excess of reddish tiuged fluid. The fauces presented but little inflaumation."

The organs reached the laboratory Seplomber 5. The spleen shows the same enlargement as the former ones. The pulp is so soft that it wells out as a tarry black mass when the capsule is incised. No bacteria were detected on cover-glass preparations aud three bouillon peptone tubes inoculated with spleen pulp remained stcrile.

The liver was pale, emitting a slightly disagreeable odor. The acini appeared distinct and there was no injection of the bile analiculi observable. In portions of the parenchyma a large bacillus liad multiplied. Cultures remained sterile. The gall-bladder was distended with a very thick viscid bile scarcely flowing from the opened bladder. It holds in suspension rather coarse granules. 
The duodenum is distended with brownish semi-solid food débris. The mucosa is swollen, shining, and discolored, but nothing is found pointing to severe inflammatory or hemorrhagic lesions. Roll cultures from its walls contain the same germ found in preceding cases. A loop of the small intestine had a slightly putrefactivo odor; its contents were like those of the preceding case. The mucosa is swollen, glistening, deeply reddened in patches. The roll cultures reveal the same germ found in the duodenum.

The kidney presents no abnormal appearance excepting that the cortex has a dull washer-out appearance. One out of three (2 liquid, 1 agar) cultures inoculated with bits of tissue becomes turbid.

The urine brought in the ligated bladder has a dark wine-red color, but is clear. The specific gravity 1010, sediment slight; no red blood corpuseles could be seen. On boiling, a brownish scum rises to the surface and a flaky deposit settles to the bottom. Of three liquid cultures receiving in all 1 cubic centimeter of urine all remain clear.

Case 6 (cow).--"This animal dicd on the night of September 4. Her temperature, taken the forenoon before, was $103^{\circ} \mathrm{F}$, but in the afternoon it was $106^{\circ} \mathrm{F}^{\mathrm{r}}$. This cow, like the other two, had the habit, during the last days of sickness, of pressing the head against the wall at times; she was very weak and showed some pain when lyung down. The examination was made about 9 a. m. The carcass was but little if any decomposed. The spleen was enlarged, and had the blackberry jam appearance. This spleen showed a marbled appearance of the surface with the inottling, varying between the normal gray appearance and that due to the blood showing through the serous membrane. The liver seemed slightly enlarged and with a yellowish tinge, but this wits not as marked as in No. 5. The gall-bladder and surrounding tissues were stainer with bile. The fat was of the orange yellow tinge. The kidneys were enlarged and had acute congestion. The bladder was very full, over one gallon of dark claret-colored fluid being present. The fourth stomach harl all of its mucous coat congested. The animal had been drenched the day before with Glauber salts, and this may have given rise to this appearance. The third stomach or manifolds was slightly impacted. The dnodenum was black in the mucous coat. The small intestine had numerous small black spots along its length. The villous coat was reddened and in some places, more especially the ileum, blackened by inflammation. There had been some peritonitis indicated by the presence of considerable serum in the abdominal cavity. The lungs were normal; the extreme tip of the right was affected with worms (Strongylus micrurus)."

In this case the spleen was likewise exceedingly large, the pulp disintegrated so as to be readily scooped out with a platinum loop. It contains an immense number of apparently normal red corpuseles. Bacteria seemingly absent. Two liquid cultures made with pulp remain sterile.

The surface of the liver is smooth, the bile canaliculi not injected. The orlor is disagreeable but not putrefactive. 'Throughout the parenchyma are spots of a more yellowish tinge from $\frac{1}{8}$ to $\frac{1}{4}$ inch in diameter. The discoloration is dne to the active multiplication of a large anaërobic bacillus. Roll and liquid cultures from bits of tissue remain sterile. There is about $300 \mathrm{cc}$. of bile in the distender gall bladder. It is thick, of a dark amber color, holding in suspension small flakes. These flakes are covered with masses of bacteria and in roll cultures are found later on to be the same organisms obtained from intestines of preceding cases.

Sections of liver tissue hardened in absolute alcohol, imbedded in paraffin and stained in alkaline methylene blue (Löffler's) are peculiar in appearance. To the naked eye they appear mottled. Patches of unstained tissue are inclosed in an irregular meshwork of stained tissue, both summing up about equal in extent. Under a low power the unstained areas are quite variable in size and irregnlar in outline. They border upon the central vein of the lobule and evidently begin to form 
there. Two or three such patches radiate from the center into different directions, some joining a patch form a neighboring lobule, others extending only a short distance. No lobule is entirely intact. Under a high power (Zeiss $2^{\mathrm{mm}}$. obj. ocular 4) the unstained areas show the cells faintly as yellowish masses of a finely granular character with a faint indieation of the nucleus. The peripheral, stained areas show the cells with protoplasm very finely vacuolated (effect of the fatty degeneration?), the nuclei still distinct. In the capillaries are occasional clumps of yellow pigment evidently inelosed in cells.

In sections from another portion of the liver, which are stained in alum carmine, and in aniline water methyl violet, the necrotie areas are present only in a shallow zone under the capsule. The remainder of the section shows the same faintly vacnolated condition already referred to. In the alum carmine section the cell protoplasm is seen sprinkled thoroughly with very minute pigment grauules. In the capillaries of all regions the T'exas fever parasites are distinctly (methyl violet stain) seen within the red corpuscles which distend the lumen of the vessel. Each corpusele contains apparently but one round coccus-like body stained blue (1892).

In 1889 several of the permanent cover-glass preparations of the liver were carefully reëxamined. In one preparation stained with gentian violet and decolorized with acetic acid, intraglobular bodies are distinctly visible, though present in very small numbers. In some corpuscles there were two bodies, in others but one. They appeared as round eocci stained blue in the uncolored corpuscle.

The mucosa of the fourth stomach is rather dark in color and contains very many small pits. The duodenum is filled with a dark-red thick liquid with putrefactive odor, probably bile. The membrane is swollen, glistening, dark red, owing to punctate and linear pigment spots closely set in the membrane. Portions of the small intestine were brought with ends tied. The mucosa covered by a dirty creamcolored pasty layer consisting of desquamated epithelium and molecular débris. Portions of mucosa have a deep wine-red color. Roll cultures made from the scrapings of the wall in these various situations contain the same germ found in o the intestines and in the liver.

Kidney, dark red; the surface presents minute blood-red points. All but the lip of the papillie deeply congested. Ecchymoses in the pelvis. Of three cultures made from bits of tissue one becomes faintly clouded; the others remain clear. From the former a roll culture fails to develop. The ligated bladder contains about 1 quart of deep brownish red, slightly opaque urine; specific gravity, 1030. Sediment very slight. In it red blood corpuseles are still distinetly visible. The mucosa of the bladder has a pale-red appearance, probably due to the imbibition of hæmoglobin. of three cultures made from the urine all remain sterile.

'The microbe, isolated from the intestines of the four cases examined, and from tho liver in two cases, was a saprophyte so far as its appearance and growth in culture media are concerned. It produces considerable turbidity in neutralized bouillon, with or without peptone, but there is no distinct membrane formerl. The bacteria are motile, but their motility may be overlooked. In the hanging drop they appear quiet for a time, then one or more in the field of the microscope suddenly begin to move rapidly across the field, at the same time executing revolutions about that point as an axis where two are attached to each other. The peculiar bluish expansive colonies on gelatin, taken in comnection with the other biological characters and the presence of these bacteria in the intestines of healthy cattle and other domesticated animals, identify them with the very common bacillus $\mathrm{B}$. coli commums.

Inoculated into the small experimental animals this bacillus seems to exert no bad effect if the dose be limited. Large doses have not been tried, for the reason that some putrefactive bacteria which are know $n$ to be harmless may produce death when large doses are injected subcutaneously more by a kind of poisoning than hy the actual infeetion of the organism with the injected microhes.

From an agrar culture originally inoculated from a colony in a roll eulture pre- 
pared from the duodenum of No. 6, material was stirred up in sterile beef infusion until a clondy suspension was obtainer. From this-

Two mice received subcutaneonsly $\frac{1}{16}$ and $\frac{1}{8} \mathrm{ce}$.

Two rabbits receiver subentaneously $\frac{1}{8}$ and $\frac{1}{4} \mathrm{ce}$.

One guinea-pig received subcutaneonsly $\frac{1}{4}$ ce.

All remained well.

The same negative results were obtained when cattle were fed with enltures of this germ.

September 5, 1888. - A flask containing $600 \mathrm{cc}$ of sterilized slightly alkaline bonillon was inoculated from a culture obtained from case No. 3 and placed in the incubator. On the second day the liquid was very turbid, of a pale straw color, with no perceptible inembrane on the surface. On the bottom a small quantity of a flaky deposit. No putrefactive odor. On this same day a black heifer aud a calf were drenched with the cnlture liquid, the former receiving $400 \mathrm{cc}$, the latter $200 \mathrm{cc}$. There was a slight elevation of temperature in both animals, which may have been dne to other canses, however. Five days after feeding, the calf had quite a liquid diarrhea, but this disappeared in a few days. Both animals remained well.

October 15 five roll cultures in gelatin of the same germ were dried in watch glasses until the gelatin was very brittle. After drying eleven days a 2-year-old heifer was drenched with the dried gelatin enltures suspended in water. The feeding was followed by no symptoms of disease.

CASES OF TEXAS FEVER EXAMINED AT THE EXPERIMENT STATION AND THE PATHOLOG-

ICAL, LABORATORY OF THE BUREAU OF ANIMAL, INIUSTKY, 1889-1892, INCLUSIVE.

No. 7 (native).-Black cow, 6 years old, has been on the station 2-3 years. Placed in field I* June 27, 1889, containing North Carolina cattle with ticks.

No symptoms of disease noticed until the midlle of Angnst, when she began to lose flesh rapidly, becane dull, and refused to eat. Rumination ceased Angust 21. At this time she wandered aimlessly abont or stood with head low and back arched. On the $22 d$ very weak and scarcely able to walk. Disinclined to move except when urgerl, and standing most of the day in the bed of the stream passing throngh the yard. Dung passed in small quantities, which has a yellowish tinge and is coated with mucus. Color of urine not noticed. 'The mucous membrane of vagina dotted with hemorrhagic streaks and patches. Rectum not redilened.

August 23. - Found dead this morning. Ticks found on escuteheon (pubic region) and on hiud limbs; few on fore limbs. Body greatly emaciated. The subcutaneous tissne of an abnormally deep orange color (janndice).

Spleen very large, pulp dark and soft. Among the red corpnscles a considerable number have each a single round body within them readily brought out by aniline colors.

Kidneys deeply congested throughout the parenchyma. In the bladder about one quart $\theta^{\circ}$ blood-red urine. When poured into a beaker it is entirely opaque and of a dark port-wine color. Specific gravity, 1022. When diluted with two parts of water in a test tube and held up to the light, it appears trauslncent and of a beantiful wine-red color. When boiled a slight quantity of a dirty brownish flocculent precipitate is formed.

The liver is enlarged, discolored, and on mieroscopic examination the bile canaliculi surromding the hepatic cells appear as a yellow retwork; the cells themselves contain irregular masses of pigment. Many red-blood corpnscles contain the round stainable bodies. Bile, so thick that it scarcely flowed from the exciserl bladder, die to the presence of small yellowish flakes and plates.

Slight extravasation beneath endocardinm and ejicardium of heart. Lungs with a few old arhesions, otherwise healthy. 
In the digestive tract considerable congestion and pigmentation, chiefly on the summits of the longitudinal folds, extending from the duodenum with variable intensity as far as the upper portion of the colon.

The blood of this case was not studied during life excepting to count the number of red corpuseles July 11 and August 20. They numbered at the former date about $6,000,000$ in a cubic millimeter, at the latter date about $4,000,000$.

The temperature of this case rose on August 16-17 from 101.2 to 106.9 and remained high until death, August 23. (For the complete record, see p. 270.)

No. 8 (native).-Black heifer, aged 18 months, raised on the station. Placed in field I (containing North Carolina cattle with ticks) June 27, 1889.

August 26.-Animal has become gaunt and thin, walks as if hind limbs were weak. Vision seems impaired. The animal raises its forefeet quite high in walking and stumbles on inequalities of the ground. Refuses to eat to-day, although it ate yesterday. Wanders aimlessly abont, preferring the bed of the stream. When standing the head droops and the back is arched.

Angust 27.-Animal lies down this morning and refuses to rise when urged to do so. At $8 \mathrm{~A}$. M. stretched ont at full length, lying quietly, apparently nuable to raise the head. At 10 A. M. temperature $99.8^{\circ}$ F. Pulse 80, irregular. Killed at 11.30 by a blow on the head.

Autopsy notes: Moderately emaciated. A few ticks attachei to the udder and to the skin on pubic region. Janndice absent. Spleen 18 inches long, 6 inches wide, and $1 \frac{1}{2}$ inches thick; pulp very soft and dark. Kidneys and urine very nearly like No 7 ; the urine perhaps even darker in color. The same condition of the liver and bile as in No. 7. Heart very flabby, left ventricle not firmly contracted. Small blood extravasations beneath epicardium of left ventricle and along interventricnlar grooves.

Digestive tract: Contents of third stomach rather dry and firm. In the fourth stomach a small quantity of very dry food. The mucosa considerably reddened. On the laminæ small erosions from the size of a pin's head to $\frac{1}{4}$ inch across, the base covered by a black (hemorrhagic?) scab. (These lesions were found in healthy cattle slanghtered for beef, and are probably wonnds due to foreign borlies taken in with the food.) In the small intestine Peyer's patches are considerably infiltrated and raised above the general mucous surface. In the cacum are lines of pigment abont $\frac{1}{8}$ inch in diameter. They continue about 12 inches below the valve. The remainder of the large intestine normal with the exception of a small hyperæmie patch in rectum.

The blood taken from a skin incision on the day and in the morning before the animal was killed showed a very small number of round intraglobular bodies, as a rule one in each corpuscle; in one corpuscle three were observed. The number of blood corpuscles before death was somewhat more than 5,000,000. In stained coverglass preparations of the spleen pulp a considerable number of red globnles contain the small round bodies, one in each cell usually between the centre and the erlge of the corpuscle. Although various stains were nsed snch as a watery solution of methylene blue, methyl violet and gentian violet, the alkaline methylene blue seems best. These bodies conld be seen in the fresh pulp, spreal in a thin layer between slide and cover-glass, as round, pale, sharply defined spots between centre and periphery of the corpuscles. After the spleen had been in a refrigerator thirteen days they were still visible.

The temperature of this case rose on August 23 to 103.4 and remained at this point 111til shortly before deatl, August 27. (For the complete record, see p. 270.)

No. 9 (native).-Bull, eighteen months old, raised on the station. Exposed iu field I (North Carolina cattle with ticks), June 27, 1889.

Angust 26.-Blood, withdrawn from an incision through the skin, was dried and stained in an arneous solution of methylene blne. In many red corpuseles, which are paler and larger than normal ones, from fifteen to twenty very minuto stained 
bodies of slightly variable dimensions aro present, some not so large as the smallest cocei. Some corpuseles appear as if sprinkled over with these colored points (modified, embryonic red corpuscles).

August 30.-Blood witlidrawn, and stained on cover-glass preparations in alkaline methylene blue slows a large number of red corpuscles containing five to teu stained coccus-like points. In a few red corpuscles are seen peculiar oval or pearshaped bodies in pairs, lying parallel to each other. One end of the body is romder, the other drawn out into a short terminal point.

Angnst 31.-The animal died this morning. Mucous nembranes and tissnes quite pale; fat alsent; no janndice. Spleen 22 inches long, 6 wide, and $2 \frac{1}{2}$ thick in the middle. The pulp so soft that it almost flows out of the incised, tense capsule. Cover-glasses placed against the eut surface and then directly on the slide furnish a thin film of spleen blood in which about one out of every twenty-five corpuscles ( 4 per cent.) contains one or more parasites. They appear as circular, sharply defined pale spots, either centrally situated or nearer the periphery of the corpuscle. In a small number two are present in one corpuscle. They are about $2 \mu$ in diameter whell observed in the fresh state.

Kidneys resembling those of No. 8. In the blood therefrom the intraglobnlar bodies observed. The urine taken from the bladder is almost black when viewed in bulk. In a small test tube, viewed by transmitted light, it is of a deep port-wine color. Specific gravity, 1018. Reaction acid. A dirty brownish, flocculent precipitate, formed after boiling, occupies abont one-third of the liquid colnmn after settling.

The liver presents the same appearance as in the preceding cases. Each liver cell is snrrounded by a polygonal line of yellow eylindrical rods (bile pigment) representing the plugged bile capillaries. Nucleus of hepatic cells distinct. Among the blood corpuscles in the liver tissue nearly one-half contain the intraglobular bodies, resembling those found in the spleen.

Sections of liver tissue hardened in Miiller's fluid and in alcohol and stained in various ways show no necrosis of tissue. The cells, however, contain much pigment in the form of minute granulcs. Large lnmps are rare.

Bile very thick, containing the minute yellowish flakes already described.

Considerable extravasation on the epicardium of the ventral surface of the heart. The blood itself of a rather pale color clots very firmly. In the blood taken from the right ventricle perhaps one hour after death a very large number of intraglobular bodies could be observed. These are nearly round and quite invariably occur in pairs, being close together in the corpuscle. There are few eorpuscles which have not thus been invaded by the parasites.

In the digestive tract the mu cosa of the fourth stomach is normal with the exception of a few small hemorrhagic erosions. Feces in upper colon dry and hard. No other abnormal appearances noticed.

The tempcrature of this case rose to 106.7 August 15, fell to 102.2 Angust 19, rose again on August 22, and remained high until death. (For the complete record, see p. 270.)

No. 10 (native).-Calf, four months old, raised on the station. Exposed, June 27, 1889, in field I (North Carolina cattle with ticks).

August 31. - The animal died to-day, and was examined immediately after death. No emaciation; no jaundice. Spleen as usual very large, soft, and dark $\left(13 \frac{1}{2} \times 4 \times\right.$ $1 \frac{1}{2}$ inches). Urine contains no hæmoglobin; of norinal color. Liver, however, very much enlarged, of a peculiar "mahogany" color and a rather sickening odor. The fluid which exudes from the cut surfaces bas the color of bile. The bile canaliculi are shown completely occluded by an injection of yellowish bile pigment forming a meshwork enclosing the hepatic cells. Bile very thick, due to the presence of yellow floceuli.

The mucosa of the fourth stomach dotted with minute opaqne white elevations, which, on closer observation, represent the swollen periphery of minute worm-pits 
(Strongylus Ostertugi). A few erosions with hemorrhagic base near pylorus. In the duodenum the mucosa is quite deeply stained with bile. No lesions in the intestine.

In a preparation of heart's blood, taken one-half hour after death, a considerable number of intraglobular parasites are found. They are more or less pyriform in ontline; very few round forms seen.

The temperature of this animal fluctuated more or less, but did not rise above 104.5 at any time. (For the temperature record, see p. 270.)

No. 11 (native).-Red calf, 4 months old, raised on the station. Exposed June 27, 1889, in field I (North Carolina cattle with ticks).

September 10.-Found at 2 p. m., lying down, unable to move. Respirations irregular, hardly perceptible. It was killed by a blow on the head and examined immediately.

Animal very much emaciated. Several ticks on inner surface of thighs. Spleen moderately engorger; pulp soft and dark. In preparations a very large number of corpuscles were seen with parasites within them. Probably not less than $\frac{1}{5}$ of the corpuseles invaded. Sometimes 2 bodies, very rarely 3 in the same corpuscle. They were the largest seen thus far. In form they are circular, and two, side by side, stretched almost across the diameter of the corpnsele. Kidneys but slightly congested in appcarance. The urine has a port-wine color, which is not so dense as in most preceding cases. In a test tube it is translncent without requiring dilution with water. Reaction, alkaline. A dirty scmm forms on boiling.

The liver is rather firm, nearly normal in eolor. Little or no injection of bile capillaries observed. The bile is somewhat more viseid than normally, but of normal color and containing very little floceulent matter in suspension.

The blood corpuscles in the liver are invaded by parasites in the same degree as those in the spleen. They are very distinct in fresh, unstained preparations. Occasionally a large body 2 to $2.5 \mu$ in diameter is associated with a very small one in the same corpuscle.

Nothing abnormal in the stomachs and intestines. Duodenum bile-stained.

Temperature fluctuating between 103 and 104.5 mnch of the time from August 16 until a few days before death. (For complete record, see pp. 270 and 271.)

No. 12 (Southern).-Heifer, 2 years old; received Jnne 27, 1889, from near New Berne, N. C., and placed in field I.

August 17.-Removed and sold.

No. 28 (Southern).-Cow, 5 years old; received June 27, 1889, from near New Berne, N. C., and placed in field II after the ticks had been picked off.

Auginst 17.-Removed and sold.

No. 29 (Southern).-Cow, 7 years old; received.June 27, 1889, from near New Berne, N. C., and placed in field II after the ticks had been picked off.

Angust 17.-Removed and sold.

No. 30 (Southern).-Heifer, 3 years old; received June 27, 1889, from near Now Berne, N. C., and placed in ficld II after the ticks had been picked off:

Angust 17.--Removed and sold.

No. 32 (Southern).-Heifer, 21 years old; received September 14, 1889, from Now Berne, N. C. Placed in field II for remainder of season.

July 4, 1890.-Exposed in field VI (Southern cattle with ticks).

The hlood was examined september 3. The animal became greatly excited while being canght. 'Temperature, $101^{\circ} \mathrm{F}$; respiration, 60; pulse, 90. 5,875,000 red corpuscles in a cubic millimeter. In the blood no ahormal eloments dotected.

Urine collected at the same time. Color, light yellow; specific gravity, 1035; strongly alkaline. No albumen.

Animal remained in this field until November 3. Exposure without result. Only slight fluctuations of temperature (seo 1$) .277$ and 278 ).

No. 35 (natine).-Gray and white heifer, 2 years old; received Angust 16, 1884, 
from St. Marys Connty, Md., and kept in field IV until September 14. On this day transferred to field III (Southern cattle with ticks).

The animal manifested no symptoms of disease during exposure. On November 5 the blood was examined and no abnormal elements observed. The corpuscles numbered $6,000,000$. The animal was in rery good condition. (For temperature record, see p. 272.)

No. 40 (Southern).--Steer, 3 years old; received June 27, 1889, from near New Berne, N. C., and placed in field I. Removed and sold August 17.

No. 41 (native).-Roau cow, 4 years old; received aud kept with No. 35 until September 14, 1889. On this day exposed in field IV (Southern cattle without ticks).

On November 6 the blood was examined. No abnormal forms observed. The corpuscles numbered 6,520,000. (For temperature record, see p. 273.)

No. 42 (Southern).-Cow, 6 years old; received June 27, 1889, from near New Berne, N. C., and placed in field I (North Carolina cattle with ticks).

August 17-Removed and sold.

No. 43 (native).- Rerl steer, about 3 years old, from Charles County, Md. Placed in field I (North Carolina eattle with ticks) Angust 24, 1889.

September 10.-Urine collected to-day has a port-wine color. Reaction alkaline. Liberation of large quantities of gas when acidified. When boiled it remains clear until an acid is added, when a brownish flaky precipitate forms, which, after settling over night, is equivalent to about one-sixth of the column of liquid in test tube.

September 11.-Animal very weak towards evening, lying down most of the time. Continues in this condition for another day, and is found dead on the morning of September 13. Examined immediately.

Autopsy: On the surface of the heart somesmall blood extravasations. Small, firm, dark-red clots in both cavities of the heart.

Slight hypostatic congestion of the right lung; no lesions observable.

Spleen very large $\left(21 \times 7 \frac{1}{2} \times 1 \frac{1}{2}\right.$ inches $)$, soft, dark-colored. In fresh and stained preparations of spleen pulp a moderate number of round, intraglobular bodies.

Liver of a yellowish color; the bile-canaliculi more or less occluded with cyliudrical masses of yellow bile, as in preccding cases. There is also a considerable amount of fat in the hepatic cells. A preparation from the blood in the liver showed probably 10 per cent of the corpuscles infected with parasites. Bile contains a dense suspension of minute yellow flakes.

The bladder contains a liter of clear wine-colored urine without sediment; slightly acid; specific gravity, 1013. A precipitate forms with picric acid as well as on boiling.

The digestive tract normal, with exception of the duodeunm, the mucosa of which is considerabiy pigmented and bile-stained. In the rectum the feces covered with mucus.

Cultures were made from this animal as follows:

A minute bit of spleen pulp was placed in four tubes containing respectively bonillon-peptone, blood serum (cattle), glycerin agar, simple agar. These tubes, kept under observation in a thermostat for several weeks, remained sterile.

Blood taken from the lieart with pipette was added to tubes containing the same media, $\frac{1}{8}$ ec. being used for each tube. These also remained sterile.

From the kidney bits of tissue were added to the same media. Two tubes contained subsequently a large bacillus with terminal spore; one contained a colony of yellow eoccus, and one a colony of a large coceus. These were probably contaminations. (For the temperature record, see pp. 270 and 271.)

No. 44 (native).-Black and white steer, 4 years old, from Charles Connty, Md., placed in field I (North Carolina cattle with ticks), Angust 24, 1889.

September 13.-Urine observed to be tinged with hamoglobin to-day (red water). It behaves towarls reagents precisely like that from the preceding case, No. 43. 
September 14.--Blood examined to-day, but nothing definite observed.

September 16.-Lies down most of the time and is slow to rise when urged to do so. Temperature gradually falling, $103^{\circ} \mathrm{F}$. at 3 p. m. Cover-glass preparations of blood show a great decrease of red corpuseles (1,063,333 in 1 cubic millimeter). When stained in alkaline methylene blue and decolorized in 1 per cent acetic acid, a moderate number of red corpuscles slightly larger than normal forms are seen, containing from 5 to 10 stained granules.

September 17.-Animal died during the night and was examined this morning. Autopsy: Several large and small ticks on fore and hind limbs and escutcheon (pubic regiou). Mucous membranes and subcutaneous tissue very pale. No janndice observed.

In right ventricle of heart a large dark clot; in left, a small one; both are very firm, alınost like liver tissue. Lungs more or less cdematous. In the trachea a considerable quantity of pinkish-white foam.

Spleen is very large $\left(20 \times 6 \frac{1}{2} \times 1 \frac{8}{4}\right.$ inclies $)$. The pulp more disintegrated than any thus far observed. It oozes ont from the incision through the capsule as a semiliquid dark-red mass. Intraglobular bodies in only a few blood corpuscles.

Liver: Color, yellowish. The bile canalieuli injected with solid bile. Large oil globules in liver cells. Bile, almost solid, owing to presence of mucus and flaky particles. It can be drawn out into long bands. No intraglobular bodies detected in several preparations from blood in the liver. Urine in blalder free from hæmoglobin, yellowish-red in color; reaction, acid; specific gravity, 1013. A flocculent albuminous precipitate appears on boiling equivalent to about one-tenth of the volume of liquid.

Digestive tract: The mucosa of fourth stomach near cardiac orifice is reddened in a punctiform manner. The small intestine contains a large quantity of turbid liquid. The duodenum and upper jejunum dotted with numcrous pigment points. In the remainder of the small intestine, points and patehes of ecchymosis, especially marked in the ileum. Contents of large intestine quite firm; in the rectum the feces in balls covered with mucus. The minute vessels of the mucosa injected.

A number of culture tubes were inoculated with blood from the heart in order to determine whether any one species of bacteria were present. From $\frac{1}{4}$ to $\frac{1}{2}$ cc. of blood was added to each of three tubes of bouillon peptone, two of blood serum, and three of agar. In each of the agar tubes a large bacillus growing in long chains and producing gas was present. The same bacillus and a coccus present in one bouillon peptone tube, the remaining two clear. One blood serum tube remained sterile, the other contained a pigment coccus. Bits of spleen were placed in three tubes, two containing glycerin agar, the third bouillon peptone. One agar tube contained subsequently one colony of a coccus, the others remained clear.

The temperature in this case rose September 6, and remained high (104-107.2) until death. (For complete record, see pp. 270 and 271.)

No. 45 (Southern).-Cow 7 years old. Received June27, 1889, from near Now Berne, N. C., and placed in field $\mathbf{I}$.

Angust 17.-Removed and sold.

No. 46 (native).-Roan and white heifer 20 months old. Received August 20, 1889, from the District of Columbia and placert in field I (North Carolina eattle with ticks).

September 5.-Blood examined after the temperature had been high for four or five days showed a very few intraglobular bodies.

September 10.-Animal found lying down, unable to rise. 'Temperature $96.2^{\circ} \mathrm{w}$, killed at 10 a. m. Blood taken immediately from some superficial veins showed very few intraglobular parasites.

Animal in good condition. On the pubic region a fow small ticks. Fat of normal yellow color. Spleen very large ( $22 \times 7 \times 2$ inches), pulp soft and dark. In preparations of the pulp, examined fresh and stained, abont 2 per cent of the corpuselos contain one or two round bodies each. Liver weighs 12 pounds, affected as in previous 
cases. Quite friable to the touch. 'The same injection of the bile canaliculi. Considerable fat in the hepatic colls. Very fow blood corpuseles containing parasites. Bile very thick and viseid, resembles finely chewed grass.

Urine of a deep port-wine color, faintly translucent in a layer several inches deep.

Digestive tract: Greater portion of laminated part of the fourth stomach is of a bluish-pink color. On the lamina a large number of dark depressed points, also small erosions with reddened periphery and larger slightly depressed areas to which are attached shreddy masses of a diphtheritic character. Pyloric portion of stomach and duodenum normal. In the remainder of the small intestine a considerable quantity of turbid watery liquid. No appearance of catarrhal inflammation. Peyer's patches slightly infiltrated. In cecum and for 2 feet below valve the longitudinal ridges of the mueous membrane extensively pigmented. Near rectum, more or less hyperæmia.

Blood from the heart, examined soon after death, failed to show any intraglobular bodies.

The temperature of this case rose Angust 31 to 105 and remained high (105-107.3) until before death. (For complete record, see pp. 270 and 271.)

No. 47 (native).-Cow, aged $3 \frac{1}{2}$ years. Received September 4, 1889, from Prince George County, Md., and placed in field IV until September 14, 1889, when it was exposed in field III (Southeru cattle with ticks).

November 11-15.-Has been growing thin quite rapidly, and very weak, especially in hind quarters. Dull and lying quietly most of the time.

November 15. - The blood was examined to-day and found very anæmic. Only 865,000 corpuscles in a cubic millimeter. Fully one-third are abnormal. They are, as a rule, larger than in health, from 7.5 to $8.5 \mu$ in diameter. When dried and stained with methylene blue, the following forms are noted:

(1) Corpuscles showing on their disk a variable number of stained particles, from 0.3-0.4 $\mu$ in diameter. They number from 5 to 20 in a corpuscle.

(2) Corpuscles which have retained a diffuse stain.

(3) Corpuscles intermediate between these which have not only retained a feeble bluish tint but are beset with a large number of very minute stained points.

Besides these modified transitional forms of red corpuseles, about 5 per cent of the total number have on their periphery a very minute well-stained body from 0.3 to $0.5 \mu$ in diameter (Texas fever parasite).

November 16-30.-Its condition lias been slowly improving but it is still emaciated.

December 2.-Blood again examined. Now 3,126,000 corpuscles in a cubic millimeter. The various transitional forms and the parasites have disappeared. The only abnormal condition still prevailing is a variation in the size of the red corpuscles. Some are still over $8.5 \mu$ in diameter.

August 21, 1890.-Continued to improve slowly during the winter and is now in only fair condition. Exposed to-day in field VIII (tieks only).

September 5.-Temperature 106; respiration 42; pulse 60. Red corpuscles $3,916,666$ in a cubic millimeter.

September 8. Very sick and emaciated. Transferred to field IV (sick natives only).

September 12.-Died quite suddenly at 9 a. m. The blood had not been examined since September 5 .

Autopsy notes: Animal in rather poor condition. Weight about 600 pounds. $\Lambda$ considerable number of half-grown ticks on the inner side of thighs and on escutcheon. In abdominal cavity nothing abnormal. Heart large, considerable fat on pericardium and around base. Ecchymoses under the epicardium of the left ventricle, fewer on right ventricle. In right heart a large, dark clot extending into the venous trunks. A large clot of the same character in the left side. Groups of fibers of the heart muscle show fatty degeneration. Interlobular emphysema in left veutral and principal lobes. Slight vesicular emphysema of right cephalic lobe. 
Spleen weighs 28 pounds. Pulp dark-brownish in color; no disintegration. Mal. pighian bodies still visible on section. Engorgement slight.

Liver wcighs about 13 pounds, of a uniform yellowish brown color on section. In thin sections of the fresh organ, complete yellow injection of the bile canaliculi and of some of the sinallest dnets observed. Considerable yellowish pigment within the hepatic cells. Very little blood in capillaries. Fatty degeneration slight.

Sections of liver tissue (hardened in Miiller's fluid, embedded in chloroform paraffine and stained in Ehrlich's hæmatoxylin) show the same intermingling of stained and nearly unstained areas mentioned under No. 6. The unstained areas equal together perhaps one-quarter of the whole area of the section, and are isolated. They radiate from the central vein. Within them neither the cells nor the cell nuclei are any longer visible as such. The connective tissue nuclei not increased in number. On the border of these patches are zones of bile injection. Red corpuseles are not seen.

In the gall bladder about 6 ounces $(180$ cc.) of dark green bile containing a large quantity of flakes, and hence very thick.

Kidneys embedded in much fat; not surrounded with any œdematons tissue. On section, the cortex brownish-red; congestion slight. Pigment in epithelium of the convoluted tubes in small masses. Bladder contains about $2 \frac{1}{2}$ quarts of claret-colored urine. The hæmoglobin slight in quantity. Reaction barely alkaline. Specific gravity 1015. Abundant floceulent precipitate after acidifying and boiling.

In the rumen, contents dry and in lumps. Third stomach normal. In fourth stomach contents as in the rumen. On the laminated portion, a small number of irregular scars or erosions with hemorrhagic base. Injection of the minute vessels in spots. Dnorlenum with mucosa pale, not bile-stained. In the ileum mucosa normal, contents of a greenish-yellow color, with a fecal odor, and undergoing fermentation. Cæcum empty, normal. In colon and rectum, large, firm balls of fecal inatter. Mucosa, normal.

Cover-glass preparations of the various organs, dried and strained, were examined with the following results :

In blood from the right heart infected corpuscles very rare. In the spleen only one-half to 1 per cent of the corpnscles contain the parasite. Each infected corpuscle contains two round or slightly elongated bodies 1 to $1.5 \mu$ in diameter, situated near the center.

In the liver, between 5 and 10 per cent of the corpuscles infecter. In one, four parasites observed. Diffnsely stained corpuscles, indicating a beginning regeneration of the blood corpuscles, present in small numbers; also some hiematoblasts.

In the kidney, not less than 20 per cent of the corpuscles contain twin parasites. There are present a very large number of free bodies which, from their form, size, and staining, are evidently parasites, the corpuscles once containing them having heen broken up. (For the temperature record, see pl. 272, 281, and 282.)

No. 48 (native).-Red and white heifer, aged 3 years. Received August 14, 1889, from Montgomery County, Md.

September 14.-Placed in field V (cattle ticks only).

October 20.-Loss of appetite first discovered to-day. Animal in a dumpish condition.

October 21.-Very weak, can scarcely walk. Blood taken from a skin incision contained only 1,285,000 red corpuscles in a cubie millimeter. It clots quickly and firmly. It was thought best to kill the animal, as it did not seem likely that it would live until next day. Killed by a blow on the head.

Spleen large, engorged (22 inches $10 n g, 6 \frac{1}{2}$ wide at widest portion). Only a fow infected red corpuseles detected in fresh preparations. A large number of masses of yellowish pigment in irregular chmps from 2 to $8 \mu$ in cliameter. When spleon pulpdried on cover.glasses and stained in alkaline mebylene blue about 10 per cent of all the red corpuseles in the field eoutain each a minutes spherical stained body, 
not more than $\frac{1}{2} \mu$ in diameter. Rarely two are present in one corpusele, and then so near together that they ean scarcely be distinguished. They are situated near the periphery, a few on the very edge of the corpuscle and seemingly attached to, not within, the corpuscles. The pulp contains also a large number of large corpuscles and a small number of hismatoblasts.

Liver enlarged, edges rounded. Surface every where mottled with minute irregular yellowish patches. This appearance also on section. The parenchyma examined microseopically in fresh sections in several places does not show any biliary injection. The hepatic cells contain variable numbers of oil globules. A small number of intraglobular parasites observed after staining.

In the blood of kidneys which appear normal, very few intraglobular parasites. Urine of a pale yellow color, clear. Reaction acid. Specific gravity 1018. Albumen absent.

In the fourth stomach over cardiac half, many little pits due to Strongylus Osterfayi. Mucosa otherwise normal. 'The mucosa of duodenum and upper jejumum covered with a semiliquid layer, which has a deep yellow eolor. Hucosa of ileum more or less bile-stained. Peyer's patches infiltrated. Large intestine normal. Uterus contains a foetus four months oldi.

Cultures were inade at the antopsy by adding a trace of blood and spleen pulp to tubes of agar and agar containing glycerin, both aeid and alkaline. No growth in any tube. (For the temperature record, see 1. 273.) 


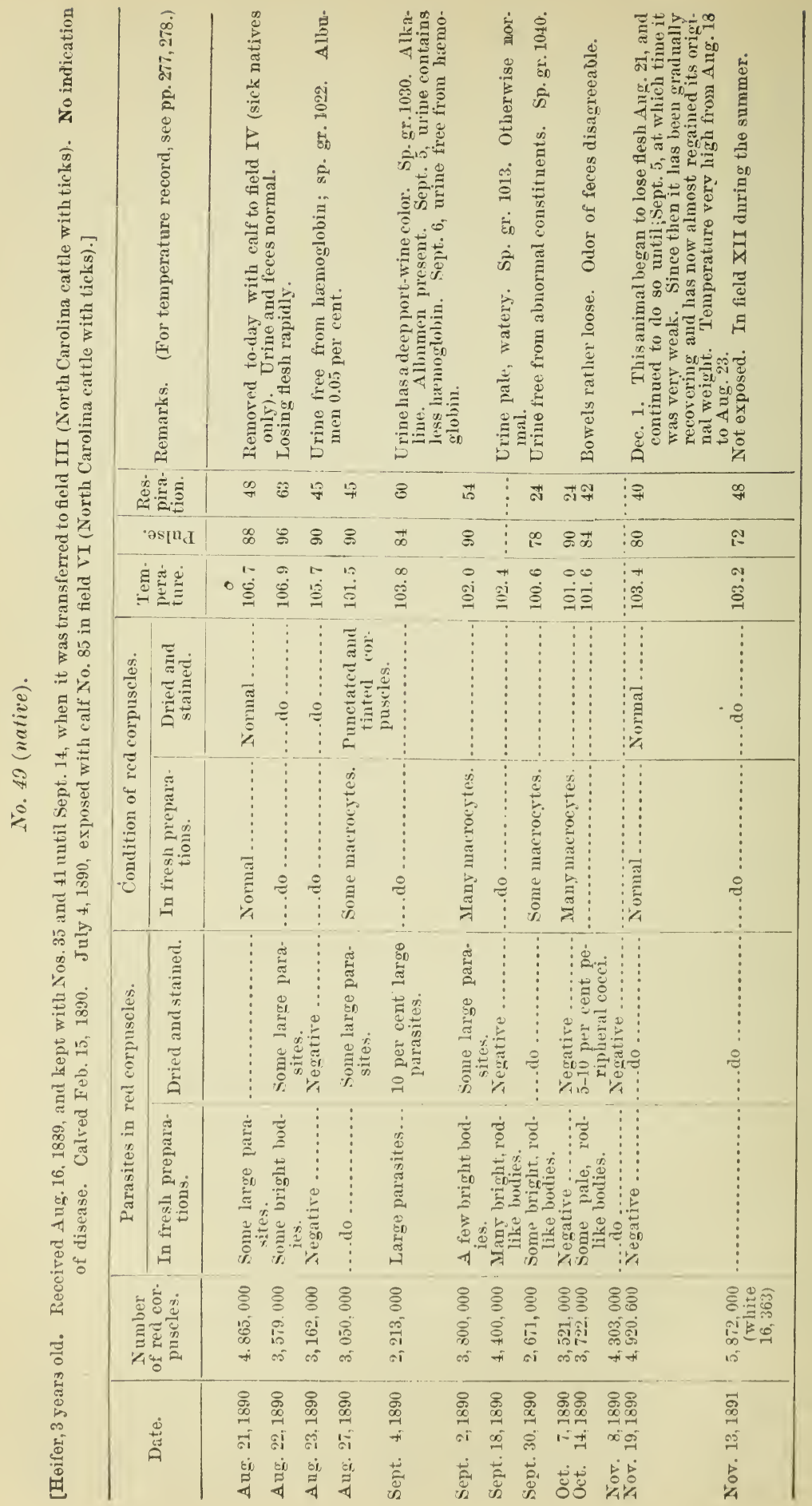


No. 50 (native).-Red and white cow, Jersey grade, aged 3 years. Received with calf No. 97 August 20, 1889, from Montgomery County, Mr., and placed in field V. Transferred, with calf, September 14, to field IV (Southern cattle without ticks). No elevation of temperature was noted. The blood was examined twice:

November 7.-Red corpuscles, 6,220,000. No abnormal forms detected.

November 15.-Red corpuscles, 6,044,000. No abnormal forms detected.

April 9, 1890.-Calved to-day.

July 4, 1890.-Exposed, with calf 79, in field VI (Southern cattle with ticks).

September 3.-Animal very sick. Has growu very thin lately. A large number of ticks, including adults, attached to skin. Transferred to field IV. Blood examination showed only 1,127,000 corpuscles in a cubic millimeter. A moderate number of corpuscles are infected, the parasites being either single or in pairs. A few corpuscles contain the minute bright bodies. The extreme anæmia is manifested by the presence of large corpuscles which stain diffusely or contain stained granulations, also a small number of hæmatoblasts. These are quite small compared with the hrematoblasts of other observed cases. The nucleus is either partially extruded or else within the corpuscle near the periphery.

September 6,9 a. m.-The animal is exceedingly weak. Temperature, 99 ; respiration, 24 ; pulse, 84 . It is unable to rise and groans slightly with each expiration. Dies at $1.30 \mathrm{p.} \mathrm{m}$.

Before death the blood was carefully examined. The hæmatoblasts were increased in number. A small number of intraglobular parasites, some of which manifest changes in form on the warm stage at $35^{\circ}$ to $40^{\circ} \mathrm{C}$. The changes follow each other so rapidly that it is impossible to sketch all consecutive stages. Some corpuscles also contain the bright bodies. In stained preparations very few corpuscles appear normal. The larger number are either diffusely staincd or dotted with stained granules or else represented by infected corpuseles and hæmatoblasts.

Autopsy notes: Body emaciated. To the skin adhere a large number of ticks of various stages, mature forms predominating.

Lungs normal. Emphysema on the right side of the right principal lobe. Right side of heart greatly distended with still fluid blood. 'The left side also distenderl. The usual subepicardial ecchymoses over the entire surface of both ventricles and on fatty tissue around the base.

Spleen weighs $4 \frac{1}{2}$ pounds. Quite firm to the tonch, the Malpighian bodies distinctly visible on section. The organ is but slightly changed. In it a considerable number of hematoblasts and hæmatoblast nuclei. Intraglobular parasites rare.

Liver weighs $12 \frac{1}{2}$ pounds. It is manifestly enlarged, the edges rounded, the whole firm to the touch. The surface mottled with minute yellowish patches. On section, the color is yellowish brown, and on closer inspection the minute patent vessels are seen sheathed with brighter yellow zones. (Plate II, Figs. 1, 2.) The bile capillarics are quite generally injected with bile; the hepatic cells themselves have undergone fatty degeneration. Many hrematoblasts present. In the gall bladder about 16 ounces of thick, dark bile loaded with a large quantity of solids.

The kidneys are paler than normal, the cortex brownish, the pyramids near the base whitish. Under the microscope the epithelium generally is found filled with pigment granules. The tubes of the pyramids filled with fat globules. In sections from material hardened in alcolol and stained in hrmatoxylin and alum carmine no pathological changes detected.

The urine taken from the bladder (about 8 ounces) slightly tinged with hæmoglobin. It is faintly alkaline, and contains a small quantity of albumen. Specific gravity 1012. On standing, slight deposit of urates.

The digestive tract is normal as a whole. The rumen fairly well filled, the manifolds with contents dry and firm. The mucosa of the fourth stomach normal, with the exception of minute nodular swellings containing a central perforation (Strongylus Ostertagi). 
In the small intestines nothing abnormal. The large intestine also iutact. In the cacum the feces yellowish brown; in the rectum a few hard balls.

In the spleen, liver, kidneys, and marrow the intraglobular parasites correspond hoth in number and character with those found in the blood before death. In the kidueys there are in addition an immense number of free forms.

T'he temperature of this case was high from Augnst 25 to September 1, inclusive. (For the complete record, see pp. 273, 277, and 278.)

No. 51 (native).-Brindle cow, 3 years old, received June 27, 1889, from Maryland. Placed in field II (North Carolina cattle withont ticks). No result up to September 14. On this day fresh North Carolina cattle with ticks were put into this field, No. 51 remaining there.

October 28.-Quite weak and refuses to eat. Losing flesh rapidly.

October 29, 30.-Weakness and emaciation much more pronounced, so that it seemed as if she were going to die.

October 31.-Slight improvement. Appetite begins to return.

November 4.-Animal in very poor condition. Blood examined to-day; corpuscles down to $1,330,000$. There are present a large proportion of red corpuscles which stain diffusely and which contain stained gramnles. White corpuscles evidently increased in number, some of them probably identical with free hæmatoblast nuclei. A sinall number of corpuscles infected with parasites. These appear in the form of peripheral coccus-like bodies.

Throughout November improvement gradnal. Her lost flesh not entirely regained.

The blood of this animal was not examined again until June 18, 1890. The corpuscles at this date were normal and numbered over 6,000,000 in a cubic centimeter.

July 4, 1890.--Reëxposed in field VI (North Carolina cattle with ticks).

Angust 21.-Temperature 107; respiration 60; pulse 78. Number of corpuscles $5,125,000$, When examined unstained, a small number of corpuscles contain each one, rarely_two bright bodies, very minnte. In staiued preparations nothing abnormal observed.

Augnst 26.-Animal found unable to rise this morning; expiration accompanied by a slight groall. Temperature 95.5 ; respiration 30 ; pulse 42, very feeble. Blood corpuscles 2,730,000 in a cubic millimeter. A small number contain large parasites; a cousiderable number contain the bright points, many in active motion. In stained preparations nothing else observed.

Dies at 11 a. m.

Autopsy notes: Weight about 500 ponnds. A moderate number of ticks on inner surface of thighs, escutcheon, and neck. Muscular tissue normal. Patches of extravasation in subcutis over sternum probably dne to ante mortem position. On serons covering of rumen a sulall number of caleified tubercles. Similar ones on costal pleura.

Lungs normal. Caleified tuberculous nodules in bronchial aud mediastinal glands. Considerable ecehymosis under epicardimm of the left ventricle and on the right near interventricular groove. Blood clots readily and firmly. Sarcosporidia cysts very abundant under endocardinm of both venticies. They are 11 to $\frac{1}{2} \mathrm{~mm}$ loug and from $\frac{1}{2}$ to $1^{\mathrm{mm}}$ apart. Under endocurdium of left ventricle near septum, and of right auricle some ecchymotic patches. Heart muscle in state of fatty degeneration.

Spleen weighs $5 \frac{3}{4}$ ponnds. On the convex surfare about six partly ralcified tuberculons nodules $\frac{1}{4}$ inch in diameter. Spleen very mueh engorged. On soctinn the pulp is of the nsual dark brownish red (blacklocry jaun) color, very soft and almost. wells ont; trabecula and Malpighian bodies not visible. A large number of the large granular cells $(22 \mu)$ contain from 1 to 12 red corpuseles.

Liver greatly enlarged, weighing 19 pounds. Weight in part made up by large tuberculons glands abont the portal fissure. Suall tubereular masses disseminated throughout the liver tissue, especially abundant in small lobe. Parenchyma has a 
yellowish-brown color. Under the microscope this color is found due partly to the advanced fatty degeneration of the cells, partly to the yellowish masses of pigment within the cells.

Bile so thick from armixture of amorphous solids that it scarcely flows from the incised bladder.

The fatty tissue around both kidueys, especially the left, in a condition of sanguinolent œedema. More or less congestion of the entire parenchyma. Minute petechir in cortical substance. In sections of fresh tissue the capillaries are seen filled with dense masses of blood corpuscles, in some of which the parasites may be distinguished. Urine pale, free from hæmoglobin. Specific gravity 1015. Neutral. Albumen present in small quantity.

In stained preparations of kidney, liver, and spleen a small number of red corpuscles are found invaded by large paired parasites.

The digestive tract normal with following exceptions: Contents of manifolds somewhat dry. On mucosa of fourth stomach a few slightly depressed ecchymoses. In duodenum Dochmius present. In the ileum worm-nodules of various sizes under mucosa. Very little dry feces in large intestine. Mucosa shows occasional ecchymoses. (For the temperature record, consult pp. 272 and 277.)

No. 52 (native).-Bull calf of No. 51, aged 4 months. On June 27, 1889, placed in field II (North Carolina cattle without ticks) with No.51. No result up to September 14. On this day North Carolina cattle with ticks were put in field II, in which No. 52 remained.

October 29-November 11.-Calf during this period very sick, as manifested by marked emaciation, weakness, and loss of appetite.

November 7.-Blood contains 2,219,000 corpuscles in a cubic millimeter. There is considerable variation in their size, some having a diameter of $9 \mu$. When stained, a small number (perhaps 1 to 2 per cent) are observed with the peripheral coccus-like bodies.

November 11.-After this date slow improvement. Unthrifty and stunted condition of calf through the winter. (For the temperature record, see p. 272.)

No. 53 (native).-Heifer, 18 months old, raised on the station. Placed in field II (North Carolina cattle without ticks) June 27, 1889.

September 6.-There being no result up to date, the animal was transferred to field I (North Carolina cattle with ticks).

October 4.-Has been losing flesh,during the past week and is now very weak and thin. Can not walk without staggering. Blood examined and found quite thin. Only 2,766,666 corpuscles in a cubic millimeter. In stained coverglass preparations the red corpuscles vary in size; some are quite large and show a large number of stained points on the dise, as if dusted over with granules of coloring matter.

October 10.-Slight improvement in the general condition since October 4. Blood examined to day. Only one intraglobular coccus-form seen, while there are a small number of the punctate corpuscles, described above, present. In one cubic millimeter of blood 2, 626,666 corpuscles present.

November 7.-Blood examined. About 3,560,000 corpuseles in one cubic millimeter. In preparations stained in alkaline methylene blue there are in perhaps 10 per cent of the red corpuscles barely visible coccus-like bodies, quite invariably on the periphery of the corpuscle, often on the very edge, one in each corpuscle.

November 11.-General condition has remained unchanged during the past mouth. Still weak and thin. Gives birth to a $6 \frac{1}{2}$ months' foetus.

December 2.-Blood examined, but no abnormal forms detected. In one cubic millimeter about 3,080,000 red corpuscles. Slight general improvement. Animal still very thin. Appetite has returned.

April 1, 1890.--Improvement slow during the winter. Now in fairly good condition,

July 4.-Now in fine condition. Exposed in field VI (North Carolina cattle with ticks).

10320-No. $1-12$ 
Octoher 15.-Temperature, 101; pulse, 78; respiration, 33. A few ticks on animal. To-day is the only time the blood was examined. Corpuscles normal; 4,836,000 in a cullie millinneter. Urine passed during the exannation was pale yellow. Specific gravity, 1040. Reaetion strongly alkaline. No albumen.

(For temperature record, see pp. 271, 272, 277, and 278.)

No. 54 (native).-Red heifer, 2 years old, from Maryland. Placed, June 27, in field II (North Carolina cattle without ticks).

September 6.-Apparently no result up to late. Transferred with No. 53 to field I (North Carolina eattle with ticks).

September 20.-The heifer is lying down and rises only when urged. Led with difficulty to the laboratory. The temperature has fallen to $101.2 \mathrm{~F}$. Animal killed hy a blow on the head at $1 \mathrm{p}$. m., when it seemed quite certain that she wonld not live throigh the night. Shortly before death the blood was examined fresh, with and without salt solution, as well as stained in alkaline methylene blue. A sinall number of red corpuscles were found eontaining the miero-parasite. At the same time the corpuseles were counted and found to number 1,075,000.

Autopsy notes: On udder and pubic region a moderate number of ticks.

Spleen very large and engorged; 22 inches loug and 7 inches across the widest portion. The pulp is very soft, thongh not yet disintegrated. Many of the red eorpuscles eontain the parasites, chiefly in pairs and laving the form of an apple-seed. The two bodies are, as a rule, close together, parallel, slightly diverging, or end to end.

On the epicardium of the heart, ecchymosis limited chiefly to the left ventricle. Respiratory organs normal.

Fourth stomaeh shows a few superficial erosions of the mueosi, with hemorrhagic base. Duodenum and upper jejunum bile-stained. Mucosa of small and large intestines normal.

The liver but slightly discolored, and scrapings examined fresh under the microscope show very little injection of the bile capillaries. Bile holds no solids in suspension although the eolor is very much deepened. In blood from the liver a large number of intraglobular bodies seen, both iin fresh and stained preparations. They also occur in pairs, some beiug round, some having the apple-seed form. The individuals of each pair are, as a rule, of the same size, althongh this is not invariably true.

Kidneys on section are of a dark red color. 'The wrine is of a dark wine color and quite opaque, although it holds no solids in suspeusion; reaetion arid. On boiling, a brownish flocenlent semm rises to the surface. In blood from the kidheys a very large number of red corpuseles contain the parasites, guite invariably in pairs, as in spleen and liver.

From the organs a number of enltnres were mate in different media. Three inoeulated with blood, and four with bits of spleen pulp, remained sterile. In a honillon tube of the blood a small streptococeus appeared. In a bouillon peptowe tube inoculated with a lit of liver tissue a diplococens appeared. In a glyeerin agar tube no development took place, while in a blood serum tube a surall oval coecus grew. In cultures made from the kidney various germs appeared, such as a streptococcus, a vibrio, and a small coccus. These were probably mainly contaminations from the air, as the cultures were prepared at the station, whereopportunity for contamination is favorable. (For tho temperature record, see ply. 271 and 272.)

No. 55 (Southern).-lleifer; age, $3 \frac{1}{2}$ years; from near New berme, N. C., roceived September 14, 1889, and placed in dield IV after ticks had been picked off.

July 2, 1891.-Kept in stork since 1889 , bnt not exposed to 'Texas fever in 1890.On this day exposed in field VI (North Carolina cattle with ticks).

August 29, 1891.-Temperature, 102.1; pulse, 72; respiration 51. Red corpuscles, $5,103,000$. Nothing abnormal in fresh or stained preparations.

October 20.-No result firom this exposme. (For temperature record, soo p. 286i). 


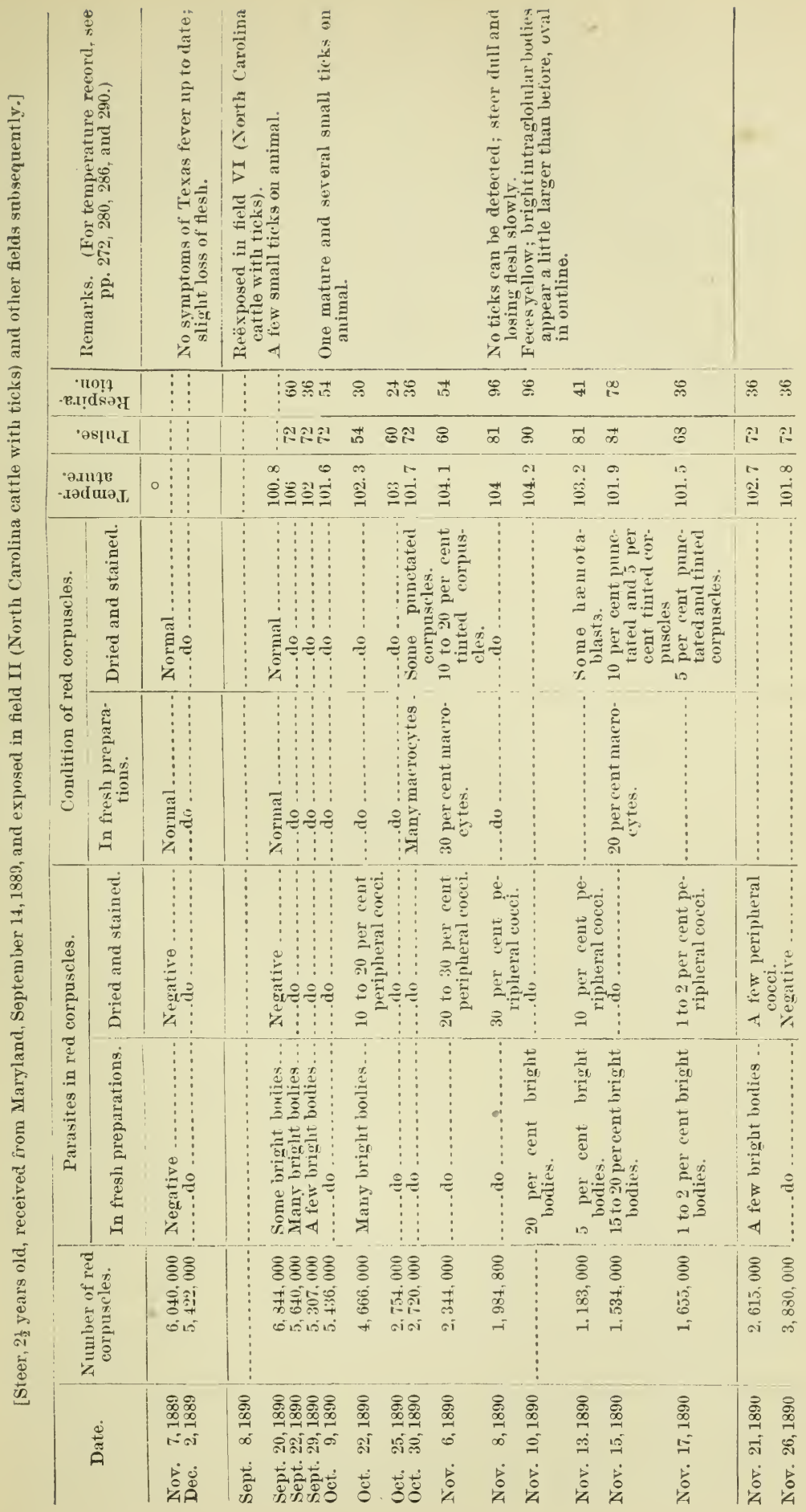




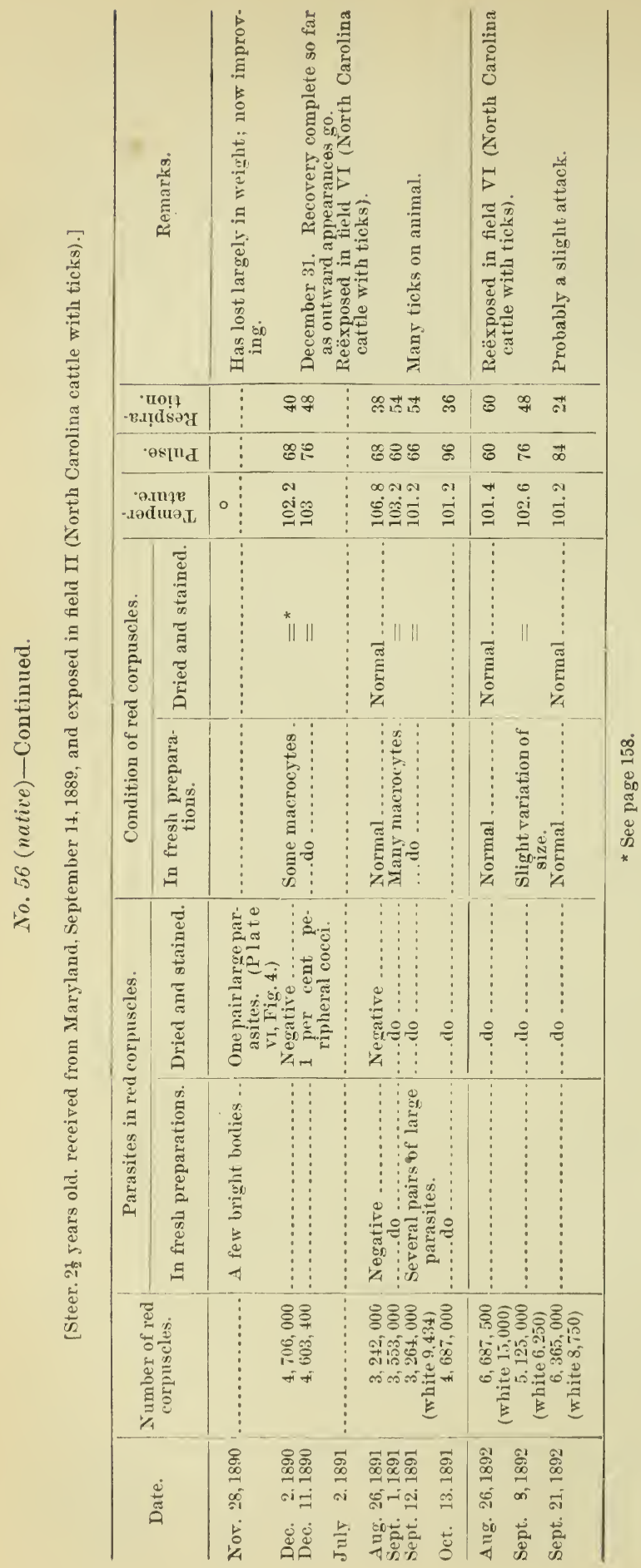


No. 57 (native).-Cow, 9 years old, from the District of Colmmbia, receiver Augnst 31, 1889. September 14, placed in field I (North Carolina eattle with ticks).

November 5.-The blood is normal. Corpuseles, 6,089,000 in a cubic millimeter. No abnormal or enlarged forms detected.

May 16, 1890.-Cow in good condition. Blood corpuseles normal; 6,662,000 in a enbic nillimeter.

July 4.-Reëxposed in field VI (North Carolina cattle with ticks).

September 20.-A small number of ticks on animal. Some matured. Blood corpuscles normal; 5,362,000 in a cubic millimeter. In one corpuscle a motile bright body detected. (The only native which has resisted midsummer exposure.) For temperature record, see pp. 271, 277, and 278.

No. 59 (Southern).-Cow, age 4 years, from near New Berne, N. C, placed in field IV, September 15, 1889, after ticks picked off.

July 4, 1890.-Placed in field VI (North Carolina cattle with ticks) to test insusceptibility.

September 3.-Temperature, 103; pulse, 80 ; respirations, 42 . Corpuscles, 6,313,000. In fresh preparations a small number of corpuscles, several in a single ficld, contain the bright bodies.

No result from this exposure. (For temperature record, see pp. 277 and 278.)

No. 60 (Southern).-Cow, aged 4 years, from New Berne, N. C. Received September 15,1889 , and placed in field III.

September 1, 1891. - This cow has been kept in stock since 1889, but not exposed to Texas fever. On this date exposed in field VI (North Carolina cattle with ticks).

September 1.-Temperature, 102 ; pulse, 81; respiration, 48 . A few ticks on animal. Corpuscles, 5,470,000. Nothing abnormal in fresh and stained preparations.

October 9.-Temperature, 101.5 ; pulse, 60 ; respiration, 40 . Red corpuscles, 4,984,000 ; white, 15,625, or 1 to 319 red. No abnormal forms observed.

Oetober 20.-No result from this exposure although ticks just as abundant on this animal as on natives. (For temperature record, see p. 286.)

No. 61 (Southern).--Red steer, 18 months old; received September 15, 1889, from near New Berne, N. C., and placed in field II.

July 4, 1890.- Exposed in field VI (North Carolina cattle with ticks).

November 3.-No result. (For temperature record, see pp. 277 and 278.)

No. 62 (Southern).-Heifer, 18 months old, received from near New Berne, N. C., September 15, 1889, and placed in field III.

September 25, 1890.-Exposed in field II ('Texas cattle with ticks).

October 15.-Temperature, 101.4. Corpuscles, 5,444,000. No abnormal forms.

October 22.-Temperature, 102.2; pulse, 72; respirations, 24 . Corpuscles, $6,333,000$. Several bright intraglobular bodies.

October 30.-Temperature, 102.5 ; pulse, 66 ; respiration, 18 . Corpuscles, $5,695,000$. A considerable number of bright intraglobular bodies from such as are barely visiible $(\mathrm{x} 500)$ to those $\frac{1}{2} \mu$ in diameter; all sizes motile.

On this date she was placed in a barn artificially heated and kept there until December 12.

July 2, 1891.-Placed in field VI (North Carolina cattle with ticks.)

October 20.-No result from exposure. (For temperature record, see pp. 275, 284, and 286.)

No. 63 (Southern).-Cow, $3 \frac{1}{2}$ years old, received September 15, 1889, from near New Berne, N. C., and placed in field IV after the ticks had been picked off.

November 5.-General condition very poor. Corpuscles, 5,540,000.

December 9.-This animal was killed to-day owing to its unthrifty condition. The antopsy revealed a small number of firm yellowish masses from $\frac{1}{8}$ to $\frac{1}{4}$ inch diameter, with cheesy contents, in the liver. There are a considerable number of entozoa in this animal, in the small intestine worm tubercles, in the oesophagus a filaria. In the fourth stomach the mucosa is dark pink in color and beset with uumerous 
slight elevations having a central fine hole. Thesc were subsequently found to be tue to parasites (Strongylus Ostertagi), which are encysted under the mucosa for a time.

No. 64 (native.) - White steer, aged $2 \frac{1}{2}$ years, received from Maryland September 10, 1889. Exposed in field V (cattle ticks only) on Scptember 14.

November 7.-Blood corpuscles, 2,780,000. Fully 20 per cent of the corpuseles contain the coccus-like peripheral body. During the first half of November this animal was dnll, bnt continued to eat fairly well. No appreciable loss of flesh during this time. The disease wonld have passed unnoticed except for the examination of the blood.

Jnly 4, 1890.-Reëxposed in fielı VI (North Carolina cattle with ticks).

August 19.-Temperatnre, 105.8; pulse, 66; respiration, 80. Blood corpuscles, $3,740,000$. In two stained preparations, after a long seareh, one pair of intraglobular pyriform parasites detected. Urine normal. Feces soft.

August 20.-Temperature, 105.4; pulse, 64; respiration, 42 . Corpuscles, 3,710,000. Fresh and stained preparations negative.

September 9.-Temperature, 102.7; pulse, 80 ; respiration, 96. blood corpuscles, $3,154,000$. In blood preparations no parasites seen. From 20 to 30 per cent of all corpuscles art enlarged.

September 16.-Temperature, 101. Blood corpuscles, 4,575,000. Enlarged corpuscles as before. A few ticks, one full grown, on animal.

September 29.-Temperature, 100.8. Blood corpuscles, 4,869,000. Macrocytes diminished in number. One red corpuscle detected containing a pair of pyriform parasites. Several corpuseles with peripheral coccus-like bodies.

October 7.-Temperature, 101.2; pulse, 39; respiration, 21 . Corpuscles, 4,431,000. Blood examination negative.

Oetober 14.-Temperature, 102; pulse, 96; respiration, 33. Corpuseles, 4,490,000. Blood examination negative.

November 1.-Temperature, 102.3 ; pulse, 51 ; respiratiou, 34 . Corpuscles, 5,916,000. In fresh preparation nothing observed. In staired, one eorpuscle with a pair of pyriform bodies.

November 3.-No marked loss of thesh from this exposure. (For temperatme record, see pp. 273, 277, and 278.)

No. 65 (wative).-Spotted heifer; age, $2 \frac{1}{2}$ years; received with No. 64 from Marylaud, September 10, 1889.

Soptember 14.-Exposed in field V (cattle tieks only).

November 4.-Animal apparently in good condition, but has been dnll during the past ten days. Blood corpuscles have fillen to $1,720,000$. There are present nmmerons corpuscles containing large stained gramnles, and an equal number of snch as stain diffusely. A small number are infeeted with the small peripheral cocei.

December 2.-Blood contains to-day 3,463,000 corpuscles in a enbie millimeter. There has been no appreciable loss of Hesh dinring this exposure. Disease only positively recognized by blood exanination.

March 27, 1890.- Gave birth to a healthy calf at full term (No. 89).

September 20.-Along with calf rë̈xposed in field VI (North Carolina cattle with ticks).

September 30.-Temperature, 100.8; pulse, 72 ; respiration, 24. No ticks detecterl on animal. Blood rorpuseles number $1,213,000$. Nothing abnotmal in fresh and stained preparations.

Octoluer 1.-Temperature, 100.4; pulse, 66; respiration, 66. Blood eorpuscles, $3,896,000$. No abourmal forms in stained preparations.

Oetoler 9.-Temperatme, 101.t; pulse, 81; respination, 54. Bloot corpuseles, $3,738,000$. In one fresh preparation of blood several large parasites seen in red corpuscles. Stained preparation negative.

October 18.--Temperature, 101.6; pulse, 60; respiration, 24. Blood corpusules, 
3,609,000. A considerable number of large corpuscles now present. No parasites detected.

November 2.- - Lying down all day muble to rise and dies about $5 \mathrm{p}$. m. Had beo come greatly emaciated and weak during the first lialf of October and continued so during the second half withont change. A ehronie fotid diarrea accompanied the entaciation during Ortober, and np to the time of death.

Autopsy at noon November 3 : Body in poor condition. Weight about 650 pounds. several adnlt ticks on escnteheon. (Edenatons condition of the musenlar tissne. In the abdominal cavity 3 to 4 liters of elear sermu. Mesentery cedematous.

Heart: Eechymoses very abmulant over the apex and walls of the left ventricle. Few on the right. In both ventrieles small, partly white, elots. Under the endocardimm of the left, eechymoses on the papillary muscles. Muscular tissue pale but nor'mal; minute vessels injecterl.

Iungs normal with exception of slight entphysema of the cephalic lobes of right lnug. Mncosa of trachea and bronchi coated with delicate white froth.

Spleeu wcighs but 1 pound. Not enlarged or congested. Several areas of extravasation nnder capsule.

Liver weighs about $7 \frac{1}{2}$ pounds. Not enlarged. Tissue quite tlabhy, probably due largely to post-mortem changes. Bile normal. Kidneys markedly pale. Post-1nortem changes lave begm. No lesions determinable. Urine quite yellow in color, barely alkaline; specific gravity, 1016. No albumen.

(Edlematons condition of submucons tissue of fourth stomach. Slight arborescent injection of mucosa. In the small intestine a large number of worm nodules showing as bluish spots on the serons surface, and from $\frac{1}{4}$ to $\frac{1}{2}$ inch in diameter. The remainder of digestive tract normal. Mucosa generally quite pale.

In blood from the subentis, spleen, kidneys, and liver a considerable number of large (post-mortem) bacilli. No intraglobular parasites present. (For the temperature record, see pp. 273 and 280.)

No. 66 (natice).-Spotted heifer, age 18 mouths, reeeived September 10, 1889, from Maryland, and exposed in field IV (North Carolina eattle withont ticks) September 14.

November 15.-Blood corpuseles abont six millions.

November 27.-No result from this exposure.

Jnly 4, 1890.-Reëxposed in field VI (North Carolina eattle with ticks).

Angust 22.-Temperature, 104.3; pulse, 80 ; respiration, 72. Corpuseles, 3,953,000. In fresh preparatious a few corpuscles contain the bright bodies. In stained preparations 25 to 30 per cent contain the minnte peripheral coceus-like bodies.

September 1.-Found dead, but still warm, early this morning. Except for the elevated temperature and slight dullness it had not appeared serionsly ill up to this time.

Autopsy: Body in good condition, weighs about 500 pounds. A considerable number of ticks from the mature size down. Considerable snbentaneons fat.

Heart with some ecchymoses on ventricles along interventrienlar grooves and base. Both sides distended with large, rather soft dark elots. Heart miscle rather pale; cloudy and finely gramlar condition of the fibers. Heart's blood contains from 10 to 20 per cent of corpuscles infected with parasites.

(Edema of the lungs with two dark red hepatized masses, one in the left principal lobe, the other in one of the smaller lobes of the same lung.

Spleen weighs $4 \frac{1}{2}$ pounds; considerably engorged, but the pulp still quite firm and the Malpighian bodies partly visible. In cover-glass preparations of fresh pulp perliaps one-third of all the corpuscles contain large, romnd parasites. When stained it appeared that not less than 50 per cent of all the red corpuscles were infected with parasites abont $2 \mu$ in diameter. Many of the corpuscles contained donble parasites; not infrequently fonr were present.

Liver weighs 10 ponnds, enlarged. The parenchyma on section is yellowish brown. Minute bile ducts are visible to the naked eye as banched yellow lines. In sections 
of fresh tissue there is seen an almost universal plugging of the ultimate bile cana. liculi with solid bile associated with fatty degencration. Rarely places are found with very little of such injection in which the fatty rlegeneration is most advanced. Numerous infected corpuscles observed in fresh preparations. In stained preparations fully one-half the red corpuscles are shown to be infected as in the spleen.

The gall bladder contains about 400 grams (1 pint) of very thick bile. Sp. gr., 1022. When allowed to stand, the lower half of the liquid represents a dense stratum of flakes and granules.

No extravasation or codema about kidneys. Cortical portion sprinkled with petechiæ as large as pin heads. The color in general somewhat brownish. In fresh sections nothing abnormal. In stained preparations 10 to 20 per cent of the corpuscles infected. The bladder contains about 1 liter (1 quart) of urine of a dark, port-wine color, barely translucent in a test tube $\frac{3}{4}$ inch in diameter.

The contents of third stomach dryer and firmer than usual. Both second and third contain much gravel. In the fourth stomach also considerable sand and gravel. Mucosa normal. Mucosa and contents of duodenum bile-stained. In the lower ileum mucosa pinkish. Contents of large intestine dry and coated with mucus. (For temperature record, see pp. 273 and 277.)

No. 67 (Southern).-Cow, 4 years old, received September 15, 1889, from near New Berne, N. C., and placed in field II.

April 29, 1890, gives birth to a calf, No. 86.

July 4.-Exposed in field VI (North Carolina cattle with ticks).

November 3.-No result. (For teniperature record, see pp. 277 and 278.)

No. 69 (native).-Heifer, $2 \frac{1}{2}$ years old, received from Maryland, September, 1889, used during fall and winter in another experiment with No. 74 (exposure to skiu disease of No. 73, which see) and not exposed to Texas fever until

July 4, 1890.-On this day, with her calf (No. 100), 8 weeks old, exposed in field VI (North Carolina cattle with ticks).

July 25.-Temperature 101.8. Corpuscles 5,341.000. Blood examination negative. July 29.-Corpuscles 5,536,000. Blood examination negative.

August 4.-Corpuscles 6,050,000. Blood examination negative.

August 11.-Corpuscles 4,698,000. A few corpuscles containing intraglobular coccus-like bodies.

August 13.-Corpuscles 6,090,000. Condition of blood the same as at preceding examination.

August 15.-Temperature, 100.9 ; pulse, 66 ; respiration, 30 . Corpuscles, 4,640,000. About 10 per cent contain the peripheral cocei.

August 23.-Temperature, 105.8; pulse, 76; respiration, 28 . Corpuscles, 2,855,000. In stained preparation very few coccus-like bodies detected.

September 3.-Dies at, 7 a. m. Exanined several hours later. Body generally emaciated. Weighs about 400 pounds. Many ticks of all stages on body.

Heart muscle pale, with some ecchymoses beneath epicardium. Parenchyma in condition of fatty degeneration. Blood taken from the heart which appears quite thin and watery, was cxamined fresh in the warm chamber betweeu $35^{\circ}$ and $43^{\circ} \mathrm{C}$. Many of the corpuscles are quite large, some containing minute vacuoles in gronps. A considerable number of hrematoblasts are present, some with protruding nuclens. The blood probably did not contain more than a nillion red corpuseles in a cubie millimeter at the time of death. A considerable number are invaded by large parasites; some contain two or thrie individuals. Some of the parasites have already assumed the spherical form. $\Lambda$ few still show distinct and rapid changes of form in the warm chamber five honrs after death (Plate vm, Fig. 1). In many corpuscles the bright bodies present. In stained preparations some diffusely stained corpuscles are seen. (These are present in spleen and liver also.)

Lungs, normal. 
Spleen weighs 25 ponuds. Only moderately engorged. 'Trabecula and Malpighian bodies visible on section. Moderate number of rod corpuscles within large cells and considerable free pigment in irregular masses. In stained preparations 10 to 20 per cent of the corptiscles are seen infected with from one to four parasites.

Liver weighs abont 8 pounds. Somewhat enlargerl. At the hilus some oedema of the connective tissue. Parenchyma yellowish brown. The intralobular veins appear distínctly to naked eyo as patent vessels surromuled by a yellowish zone. In the peripheral portion of the acinus the bile injection is complete. Numerons intraglobular parasites detected in fresh preparations. In stained cover-glass preparations a rough estimate makes the infection as extensive as in the spleen.

In sections of liver tissue (hardened in alcohol imbedded in chloroform paraffin and) stained in hæmatoxylin and cosin or in alum carmine, the protoplasm of the cells is in a peculiar reticulated or vacuolated condition. In some places, the vacuoles are as large as or cven larger than the nuclei and from one to three in a cell (fatty degeneration). Nuclei show signs of degeneration and there are some nissing, but there are no compact necrotic areas in the sections examined. Bile injection and red corpuscles containing parasites not traceable. Bile in the gall bladder extremely loaded with suspended flakes; semiliquid.

Kidneys have a uniformly dark-brownish appearance on section. Noperinephritic vedema or extravasation. In fresh sections the epithelium of the convoluted tubes of the cortex contains much pigment. In the capillaries some of the corpuscles show the pale intraglobular bodies quite distinctly in such sections. In stained preparations the corpisscles are infected as in spleen and liver. There are also a very large number of stained bodies abont the size of the parasites free. In the bladder are 2 to 3 quarts of urine having a deep port-wine color. In layers $\frac{8}{4} \mathrm{inch}$ deep it is translucent. Abundant precipitate is formed when acetic acid is added and it is boiled.

In fourth stomach a considerable quantity of sand and gravel. In the lamellar portion some erosions of irregular shape with hemorrhagic base. Mucosa otherwise normal. In the lower small intestine numerous worm tubercles. In the large intestine very dry balls of feces coated with mucus. (For temperature recort, see p. 277.)

No. 70 (native).-Yellow steer, $2 \frac{1}{2}$ years old. Received September 30, 1889, from Maryland, and exposed in field I (North Carolina cattle with ticks). Died about noon October 19.

Autopsy: Steer in fairly good condition. A very few small ticks on the body.

The surface of the heart, especially on the left ventricle and interventricular grooves, sprinkled with small ecchymoses.

Lungs normal.

Spleen 17 inches long, $5 \frac{1}{2}$ inches across the widest portion. Pulp soft, dark, concealing the Malpighian bodies and trabeculae completely. In stained preparations many infected corpuseles (Plate IV, Fig. 2).

Liver enlarged, firm, cdges rounded. The surface has a mottled yellowish appearance. On section small areas of a yellowish color throughout. Blood flows freely from the eut surface. The biliary injection was not observed in a few preparations of fresh tissue. The hepatic cells contain large and small fat globules. Blood from the liver, when stained, shows a large number of red corpuscles invaded each by a pair of parasites elongated in form, usually close together.

Bile of a dark-green color and containing as yet only a slight amount of solisls.

Under the fat enveloping the kidneys, there is considerable redema of a sanguinolent character. The parenchyma of the kidneys uniformly of a dark brownish-red color. In the blood from the kirlneys the intraglobular bodies are as numerons as in the blood from the liver.

Gastrointestinal tract appears normal with exception of caecum and upper colon, the mucosa of which contaius bluish spots $\frac{1}{2}$ inch across, the result of blood extravar sation. 
Cultures were made from the various organs, but in place of taking large quantities of blood or tissnes for inoculation only loops of blood were nsed, and in inoculating from the tissues a straight platinum needle was used to pierce the organ and the quantity thus adhering nsed to inoculate the culture media. In this way cultures were prepared from the blood (heart), spleen, liver, and kidneys, three or four tubes being inocnlated from each organ. The media nsed were simple agar, agar with glycerin, neutralized and slightly acid, and blood sernm. Of the 14 thbes thus prepared, only one showed signs of growth after ten days. 'This contained one colony made up of micrococei. (For the temperature record, see p. 271.)

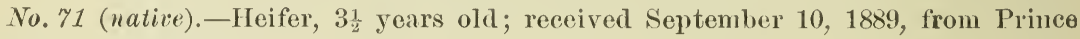
George County, Ma.

October 19.--Exposed in field I (North Carolina cattle with ticks).

December 5.-No result from exposure. Blood was not examined at any timo.

August 13, 1890.--Reёxposed in field VI (North Carolina cattle with ticks).

September t.-Temperatnre, 105.3; pulse, 108; respiration, 60; corpuseles, 1,655,000. One infecterl corpnsele detected. Enlarged forms with feebly tinted dise and several hæmatoblasts.

September 6.-Animal found dead this morning. Had been very weak for several days. Yesterday she was taken with convulsions, falling down several times within fifteen minntes. She appeared blind and ran against various obstacles.

Autopsy: Considerable loss of flesh. Weight abont 600 pounds. On the skin nmmerons smiall and a few large ticks.

Lungs emphysematons and cedematous. Very extensive ecchymoses of the ventricular surface of the heart under epicardinm. Both sides distended with large, lark, soft clots. In the blood very few parasites. Corpuscles shriveled. Hrmatohlasts in small numbers. Capillaries and larger vessels of myocardinm filled with red corpuscles. No fatty degeneration.

Spleen weighs $3 \frac{3}{4}$ pounds. The pulp is of a mniform dark brow nish red appearance, quite soft and wells out from the incision. Very few corpuseles with parasites. Some hæuntoblasts and feebly stained corpuscles.

Liver weighs 14 pounds, mnch enlarged. Parenchyna yellowislı brown. Bileinjection and fatty degeneration as in No. 69. Much blood flows from the larger hepatic vessels when the organ is cnt into. Gronps of very minute ruby-red needlelike crystals scattered over fresh sections. In stained preparations very few corpuscles containing parasites. A small number of faintly stained macrocytes. Gallblarler contains about one-half pint of bile, so thick that it scarcely flows, having become nearly solid with suspended flakes and particles. Kitheys with eortical portion very pale. In fresh secticus nothing abnormal observed. Blood corpuseles and parasites as in liver. Bladder contains abont 2 quarts of nrine free from hamoglobin, pale yellow. Specific gravity, 1014, neutral. $\Lambda$ slight flocenlent precipitate of albumen obtained. In marrow of ribs many diffusely stained macrocytes but no infected corpuscles.

In digestive tract the fomth stomach shows on the mucosa of lamellar portion a very large number of dark-red dots from $\frac{1}{2}$ to $1^{\mathrm{mm}}$ in dianeter and abont $3^{\mathrm{mm}}$ apart. The center of these dots is paler than periphery. The same appearance in npper duodenum which, in addition, is much pigmented. In ilemu a considerable number of worm tubereles of which a few are foumd seattered throngh the large intestine. Pigmented eondition of the snmmit of the mucons folds in eiecmur and upper eolon. In rectum small dry fecal balls covered with unens. (For the temperatme record, see pp. 271, 279, and 280.)

No. 73 (native).-Cow, 6 years old, received November 13, 1889, from (Yark Commy, Va., alfected with neerotic skin disease prevalent during that season.

May 23, 1890 - Several of the larger patches, from which the skin had slonghed away, still mulealed and covered with seabs.

July 1.-Exposed in field IX (North (Aarolina soil). 
Soptember 2.--Temperature, 101.2; pulse, 58; respiration, 24. Corpuscles, 7,516,000. In fresh preparations corpuscles aro normal. A fow contain the minnte bright bodies. In stained preparatıon no intraglobnlar bodies detected.

September 18.-Temperature, 101. Corpuseles, 6,389,000.

October 8.- Temperature, 101. Corpuseles, 5,327,000. In fresh preparations corpuscles are normal. A few contain the motile bright bodies. Some of these have a short, rodlike form. In some eorpmscles a larger, paler, romul body, abont $0.7 \mu$ in diameter, also elianging place within the corpuscle. In stained preparations no intraglobular bodies seen.

November 3.-Exposure without result. (For the temperature recold, see p. 281.)

No. 74 (native). - Heifer, 15 months old, received September. 1889, from Maryland. (For its nse mutil exposnre to Texas fever, see No. 69.)

May 26, 1890.-Placed (with No. 91) in field I to determine whether the infections agent of Texas fever had snrvived the winter.

September 25.-No result from this exposure. Trausferred to field II (Texas cattle with ticks).

September 30.-Temperature, 101.4 ; pnlse, 66 ; respiration, 30. Corpuscles, 6,662,000. In fiesli preparations, blood-corpnscles normal in appearance. A very large number contain each a very minnte bright body. One of these observed more closely, appears as a short rod, and has a restricted movenent within the globnle. It goes toward the center and back, occasionally disappeariug from view.

In stained preparations nearly every corpuscle contains a very minnte, not well ontlined, stained body nsmally half way between center and border of the globule. In some, two are seen near each other (Plate IV, Fig. 1).

October 8.-Temperature, 102.2 ; pulse, 72 ; respiration, 30 . Corpuscles, 5, 783,000. In fresh preparations only a small number of corpuscles containing bright bodies. In stained preparations the infection appears as extensive as at preceding examination.

October 12.-Qnite weak and losing flesh.

October 15. - Temperature, 106.5; pulse, 120 ; respiration, 48. Corpnscles, 2,185,000. Blood corpuseles in fresh preparations largely erenated and shriveled. Several bright bodies within corpuscles; one in motion. Also several pyriforn intraglobnlar borlies. In stained preparation a few of the latter forms detected, also a small $\mathrm{mm}$ ber of red corpuscles sprinkled over with very minnte stained particles.

October 16.-Cow found dead early this morning.

Antopsy: Condition of boly still fair, althongh there has been considerable emaciation since the exposnre. Weight, 450 ponnds. Three ticks in last monlt fomnd on the body.

Thoracic orgaus. Faint ecchymosis on the left ventricle of heart. Considerable extravasation under endocardium of the same ventricle, especially on papillary muscles. Contents of right ventricle still fluid; left, empty. Capillary injection of the nyocardinm and finely grannlar condition of the fibers.

The blood coagulates slowly and imperfectly when placed in beakers. After several hours it has assumed a soft, gelatinous condition and may be poured from beaker in lumps mingled with thick liquid hloor. In fresh preṕarations of blood at 10:30 a. m., both from heart and jngular, a moderate number of corpuscles contain ronnd parasites either single or in pairs, $1.5 \mu$ in diameter, and very few of the bright bodies. In stained preparations the parasites appear both as round and pyriform bodies.

Lings imperfectly collapsed. In the trachea and bronchi mnch eream-colored frotl. In the right ventral lobe, dorsal portion, a dark-red hepatized mass under pleura abont 1 inch in diameter.

Ablomen: The omentum covered with hyperemic spots, cousisting of delicate vascular fringes. When they are placed nuder the microscope the capillaries are seen filled with red globnles, some of which show the pale parasites within. 
Spleen weighs $4 \frac{1}{4}$ pounds; very large and soft to the tonch. 'The distended cap)sule retracts from the incision. Parenchyma has a dark, glisteuing appearance, resembling blackberry jam and is partly disintegrated. In teased preparations much phagocytosis (involving red corpuscles) and very little free or intra-cellular pigment. About 10 per cent of the corpuscles contain parasites $1 \mu$ in diameter, fully one-half of which are double forms.

Liver weighs $10 \frac{8}{4}$ pounds. Considerably enlarged. Color yellowish brown with paler mottling over the surface more especially in the middle portion. The parenchyma on section shows the same yellowish color. From the hepatic vessels blood flows abundantly. The smallest bile duets visible to the naked eye distencled with bile. Thin sections have a decided yellowish color. The bile injection appears in spots under the microscope and the smallest interlobnlar bule ducts are filled with yellow bile. Fatty degeneration slight. In preparations not less than 30 per rent of the blood corpuscles are invaded by pairs of parasites.

Portions of the liver were hardened in alcohol, imbedided in chloroform paraffine ind the sections stained in hrematoxylin and eosin. In sucl sections under a low power only a narrow peripheral zone remains in which the acini appear normal. There is even here an exaggerated size of the capillary network. Under a high power the difference between the central and peripheral regions is observed to bedne to a partial disintegration of the cell nuclei. These instead of staining almost uniformly consist of a pale round body containing a variable number of larger grannles, or the nuclei may be represented merely by a group of such deeply stained granules. The protoplasm of cells with sueh nuclei is not distinctly ontlined. The capillaries of the acini are well filled with red corpuscles and in many of them every corpuscle contains one or two parasites. These appear at 500 diameters as munte as coeci stained blue. In some capillaries no infection is noticeable and in general the infection includes every corpuscle when present at all.

The gall bladder contains about 8 ounces of bile so thickly impregnated with flakes and mucus that it scarcely flows. The flakes of yellow pigment are up to $2^{\mathrm{mm}}$ in diameter.

Around and in the fat of both kidneys considerable oedema associated with the blood extravasation. The parenchyma of both has a uniform dark brownish-red appearance, even to tips of papillie. In fresh sections the entire eapillary system is found gorged with red bloor corpuseles. In some the latter have broken down into gramular masses. Pigment granules diffused throngh the parenchyma more or less densely everywhere. In stained cover-glass preparations nearly every corpusele is found infected. There are in addition large numbers free (Plate vI, Fig. 3). In sections of the kidney (hardened in alcohol) stained in Ehrlich's hematoxylin no structural changes of the parenchyma were recognized. The capillaries are distended with red corpuseles within which the parasites are readily distinguished.

The bladder contains abont 3 quarts of urine which has an opaque, dark red appearance. It is barely translucent in layers, $\frac{3}{4}$ inch leep. Reaction slightly alkaline. Sp. gr. 1012. An abundant floceulent brownish precipitate with acetic acid. According to Esbach 1.2 per cent albumen.

Digestive tract. Fourth stomach contains some turbid liquid. Mueosa uniformly bright pink in color. In pyloric portion a few erosions with blackish base. Mucosa of duodenum covered with a layer of bile-stained mucus; pinkish in color. Mucosa of ilenm similarly injected.

Ciecum and upper colon distended with firm masses of feces. Pigmentation and injection appear in occasional patches. Worm nodnles 2 to $3^{\text {mm }}$ in diameter. $\Lambda$ few specinens of (Esophagostoma and numerous specimens of l)ochmins imbedded in a soft coaguhm mixed with mucus. In lower colon and rectum many fine foral balls. Mueosa slightly injected in patches. (For the temperature, see 11p. 27., 275.)

No. 75 (nutive). - Calf, age 4 montlis, hom on the Station, of eow No. 8. Exposed in field I (Southem eattle with ticks), Jume 27, 188\%. Very sick fiom August 22 to 
September 10. At the latter date it was very weak and thin and its abdomen enlarged (pot-bellied). It remained in this condition up to October, at which time it hegan to improve. In December it was still thin and stunted. It did not recover until the following sprung.

December 2, 1889.-Corpuscles 5,620,000. Stained preparations show nothing abnormal.

July 4, 1890.-Placed in field VI (Southern cattle with ticks). Up to November 1 there were no noticcable symptoms of Texas fever. For the temperature record, see pp. $270,271,277$, and 278 .

No. 76 (native).-Heifer 1 year old, when received May 20, 1890, from the District of Columbia.

July 4.-It was exposed in field VIII (cattle ticks only).

August 14.-Temperature, 104.1. Blood corpuscles, 4,966,000. In fresh preparations nothing abnormal detected. Stained preparations equally negative.

August 16-18.-Heifer growing thin and weak quite rapidly.

August 18.-7 a. m. Anımal walking about, but very weak and unsteady in its movements. At 9 a. m. lying down, unable to rise. Temperature, 99. 7; pulse, 124; respiration, 16. The skin almost bloodless. Blood obtained with difficulty from skin incisions. Corpuscles, $3,475,000$. In fresh preparations a few double pyriform intraglobular parasites observed. The same detected in stained preparations. As the temperature of the anımal was falling rapidly and the animal now unable to rise and evidently dying, it was killed at 11 a. m., by a blow on the head.

Autopsy: On the skin of thighs, escutcheon, and belly a large number of ticks just completing the last moult. Lungs normal. Some adult specimens of Strongylus micrurus (both sexes) in terminal bronchi. Heart fibers have undergone cloudy swelling. In blood from the right ventricle scarcely any parasites present. Splcen weighs $1 \frac{8}{4}$ pounds. Enlarged; capsule tense, under it a few hemorrhagic patches. On section, pulp dark, still consistent, however. In teased preparations some large cells containing from one to four red corpuscles. No free pigment. In stained preparations not more than 1 per cent of the red corpuscles contain the parasite.

Liver weighs $5 \frac{1}{2}$ pounds. Evidently enlarged. Yellowish brown. In fresh sections small areas of the lobules show bile injection, while the fatty degeneration is more or less uniform over the lobule. Occasional interlobular bile ducts appear as yellow streaks. In teased preparations made some hours after death fully 10 per cent of the corpuscles contain apparently round or oval pale bodies from 1.5 to 2 $\mu$ in diameter. These are usually in pars situated a variable distance apart. In some only one, in others four bodies are seen. In stained preparations made at autopsy the parasites are all pyriform in shape.

About 8 ounces of bile in gall bladder. Spccific gravity, 1022. Holds in suspension a small amount of flaky yellow material.

Kidneys are of a uniformly dark-brownish red color throughout. Fresh sections magnified appear dusted over with minute reddish pigment granules. The capillaries everywhere distended with blood corpuscles. In those of the medulla it is easy to see with high powers in fresh sections each corpuscle containing one to four parasites. When cover-glass preparations are stained few corpuscles are present; but everyone contains a pair of parasites. Besides these there are numerous free bodies identical with those in the corpuscles.

In the bladder about 3 pints of urine containing a large amount of hremoglobin so that it is barely translucent in layers an inch deep. Specific gravity, 1017. Slightly acid. On boiling, a brownish flocculent precipitate is formed. In the slight, amorphous deposit a few short granular casts.

Digestive tract: In fourth stomach the mucosa of laminæ pinkish and beset with small elevations having a central hole (worm pits). Numerous specimens of Strongylus contortus actively moving. In duodenum specimens of Dochmius. Mucosa bile-stained. Worm nodules in ileum. In the upper colon masses of clotted blood 
in which are imbedded round worms (Esophagostoma). The origin of the blood not traceable. (For the temperature record, see p. 281.)

No. 79 (native).-Born April 9, 1890, of cow No. 50.

July 4, 1890.-Exposed in ficld VI with No. 50 (North Carolina cattle with ticks). October 9.-Temperature, 103.4 ; pulse, 96 ; respiration, 72 (probably elevated by excitcment in being eaught). Corpuscles, $6,767,800$. In fresh and stained preparations corpuscles appear normal. About 1 per cent contain peripheral cocci. These are $0.5-0.6$ in diameter.

October 25.-Temperature, 104 ; pulse, 72 ; respiration, 30 . Corpuscles, 5,707,000. In fresh preparation several corpuscles containing each a bright motile body. In stained preparations from 1 to 2 per cent of peripheral cocei.

No noticeable symptoms of Texas fever excepting the slightly elevated temperature (for record see pp. 279, 280) and the blood parasites as observed above.

No. 80 (native).-Cow, age 7 years when received May 26, 1890, from St. Mary County, Maryland. Several adult specimens of a speeies of tick (Amblyomma unipunctata), differing from the cattle tick proper, found on this animal when received.

July 5, 1890.-Exposed in field II (Texas cattle with ticks). (See No. 82.)

July 31.-Corpuscles, $6,290,000$. In fresh and stained preparations nothing abnormal.

August 4.-Corpuscles, 5,052,000. In fresh and stained preparations nothing abnormal.

August 7.-Corpuseles, 5,631,000. In fresh and stained preparations nothing abnormal.

Animal is losing flesh slowly.

August 23.-Temperature, 101.6; pulse, 42; respiration, 15 . Corpuscles, 5,422,000. Botd fresh and stained preparations negative.

Angnst 25.-Temperature, 106.2; pulse, 56 ; respiration, 27. Corpuscles, 5,434,000. In fresh preparations a moderate number of corpuscles contain bright motile points. In stained preparations no parasites were detected.

Angust 28.-1 p. m. Tempcrature, 101.8; pulse, 100; respiration, 48. Corpuscles, $2,025,000$. Perhaps 2 per cent contain sinall round parasites $0.8-1 \mu$ in diameter near the center of the corpusele. Emaciation verymarked. Animal very weak and sways with its hind quarters when it attempts to stand or walk. This morning urine contained much hemoglobin. The feces are thin and streaked with blood. As the cow would presumably not survive the night, she was killed by a blow on the head.

Autopsy: $\Lambda$ few small ticks on inside of thighs and around ndder.

Thoracic cavity. Lungs normal with exception of several small areas of collapse in both principal lobes. Eechymoses on both auricles and along interventricular groove of heart. Infection of one to two per cent of corpuscles in blood from the right ventricle. Some of the intraglobular bodies pyriform, others roundish ; most in pairs.

Abdoinen: Spleen weighs 3 pounds. Moderately firm to the tonch. Capsule retracts when incised, displaying an engorged, uniformly, dark-red pulp. In teaser pulp much pigment in lumps, either free or intracellular. About 10 per cent of the corpuseles contain one or two parasites each. When in pairs the parasites usually elongated, pyriform.

Liver weighs 127 pounds. Cousiderably enlarged and congestod. In fresle sec. tions slight fat infiltration observed. Nnelei of hepatic cells distinct. The latter are in some places surrounderl by a network of injecterl bile eapillaries. There are also some spherical reddish masses of a pecnliar luster, slightly larger than red corpuscles, scattered over the section. Parasites easily rletected within the corpuscles. In stained preparations from 20 to 30 per cent of all corpuscles contain oach one or two parasites, nsually pyriform.

In the gall bladder nearly a pint of bile of a deep greenish color and containing much tlocculent matter in suspension. When allowerl to subside only the upper fifth of the layer free from solids. The remainder scarcely flows from the bottle. 
Slight sanguinolent adema around pelves of both kidlueys. Parenchyma more or less congested and considerable extravasation in pelvis. In fresh sections, inchding base of pyramids, the bloot vessels found distended with blood corpuscles, in ahmost everyone of wheh a pair of pale parasites conld be readily distinguished. 'The tissuesprinkled over with very minnte pigment particles. In stained preparations the infection of corpuscles ahnost as extensive as in liver. There are also numerous free parasites. Bladder contains nearly 3 pints of urine, whieh has a leep portwine color in layrrs three-quarters inch deep (Plate III, Fig. 4). Faintly alkaline. Speeifiegravity, 1016. Abundant preeipitate when simply aeidified with acetic acid. In the amorphous deposit formed after some time a few straight granular casts.

In the marrow of ribs very few hematoblasts. One capillary observed which had been preservel entire in a eover-glass preparation. This was packed with eorpuscles, in many of which parasites are present.

Digestive traet. Mucosa of fourth stomach of a uniformly bluish-red color. In duodenmm much bile. Mucosa with minnte vessels injeeted. Mucosa of the entire small intestine hyperimic; in ileum punetiform hemorrhages. Similar petechix and small hemorrhagie patches in large intestine. (For the temperature roeord, seo pp. 274 and 276.)

No. $\delta 2$ (native).-One week old when received, June 5, 1890, and placed with and adopted by eow No. 80 , which was still giving milk.

July 5.-Placed with No. 80 in field II (Texas eattle with ticks).

Angust 20-September 11.-Has shown slight symptoms of Texas fever, as indicated by elevated temperature and emaciation.

September 11.-Teinperature, 101.4 ; pulse, 72 ; respiration, 48. Corpuscles, 5,630,700. In fresh preparations eorpuscles crenated and examination imperfect. In stained preparations nothingabnormal. A small number of ticks of varions sizes on animal.

Oetober 11.-Temperature, 102.5 ; pulse, 84 ; respiration, 33 . Corpuscles, $3,542,800$. In fresh preparations nothing abnormal. In stained preparations a few corpuseles containing peripheral coeei observed. A few ticks observed.

November 13.-Temperatnre, 100.5; pulse, 75 ; respiration, 18. Corpuseles, 4,240,000. In preparations of blood, enlarged eorpuseles hut vo parasites observed. Animal very weak and emaciated and eovered with lice brought by Texas eattle. Blood obtained from skin with difficulty.

Deeeuber2.-Temperature, 100.4 ; pulse, 104 ; respiration, $26 . \quad$ Corpuscles, 5, 643,000. Examination of fresh and stained preparations negative. Animal very weak and scareely ahle to stand. A liquid foetid diarrhea for several days past.

December 6.-Animal has heen nuable to get np since December 4. Diarrhea persists.

Killed by a blow on the hear. General eondition very poor, weighs 93 pounds. Heart and lungs normal with the exception of a f'ew lung worms in one bronchus. Spleen weighs $3 \frac{8}{8}$ ounees, liver $1 \frac{8}{4}$ pounds. Both normal in appearance. Gall-bladkler contains viseid bile with more or less flocenlent inatter in suspension. Kidneys congested. Trine in bladder free from hemoglobin. Edematons rondition of coats of fourth stomaeh and slight hyperemia of mneosa of small intestine. The organs were not examined microseopically. (For the temperature record, seo pp. 274, 276.)

No. 83 (native).-Calf of cow No. 48; 8 weeks old when exposed, September 14, 1889, in field V (tieks ouly). (For the temperature during this period, see p. 273.)

The blood was not examined. Lxterual evidences of disease not ohserved.

No. 85 (native).-Calf, 4 weeks old, May 7, 1890. At this date placed on and adopted by eow No. 49.

July 4.-Exposed in field VI (North Carolina cattle with tieks).

Augnst 21.--Removed with No. 49 to field IV (field for exposure of healthy to sick natives). Temperature elevated from Angust 23 to September 1 indicating the pres ence of Texas fever.

September 4.-Temperature, 103.1; pulse, 72; respiration, 72. Corpnseles, 5,040,000. In stained preparations a considerable number of large red corpuscles (maerocytes). 
September24.-Temperature, 102.7; pulse, 87 ; respiration, 48. Corpuseles, 6,113,000. In fresh preparations nothing abnormal. A very few peripheral cocci in stained preparation.

October 6.-Temperature, 102.4; pulse, 84; respiration, 27. Corpuscles, 5,138,000. Fully 10 per cent of corpuscles infected with peripheral cocci.

November 11.-'Temperature, 102.2; pulse, 78; respiration, 45 . Corpuscles, 5,843,000. About 1 to 2 per cent of infected corpuscles still in the circulation. Animal in fairly good condition. (For the temperature record, see pp. 279 and 280.)

No. 86.-April 29, 1890. Born of eow No. 67 (North Carolina cow brought from the South last summer).

July 4.-Exposed to North Carolina cattle with ticks in field VI.

October 9.-Temperature, 103.2; pulse, 180; respiration, 120. (Caught after much chasing.) Corpuscles, 5,240,000. Four bright bodies within corpuscles detected in fresh preparation; two are rod-like. In stained preparation 2 to 3 per cent of corpuscles containing peripheral cocei. One nearly adult tick on animal.

October 22.-Temperature, 104.3; pulse, 108; respiration, 30. (Rapidity of pulse probably due to chasing.) Corpuseles, 4,308.000. They vary more or less in size. In a fresh preparation one minute, pale, peripheral body seen. In stained preparations about 5 per cent of the corpuscles infected with peripheral cocei.

October 30.-Temperature, 103.5 ; pulse, 96 ; respiration, 30 . Corpuseles, 3,579,000. Fresh preparations negative. In stained preparations about 5 per cent of infected corpuscles.

November 4.-Temperature, 103 ; pulse, 84 ; respiration, 24. Corpuseles, 4,312,500. Some enlarged. In several, bright motile bodies and pale peripheral bodies. In stained preparations still 2 to 3 per cent of infected corpuscles. (For the temperature record, see pp. 279 and 280.)

No. 87-March 21, 1890. Born of eow No. 59 (North Carolina cow, brought from North Carolina last summer).

July 4.-Exposed in field VI (North Carolina eattle with ticks).

August 21.-Temperature, 107. Corpuscles, 6,687,500. A few corpuseles contain exceedingly minute bright bodies. Stained preparations negative.

September 20.-Temperature, 102.1. Corpuscles, 5,896,000. Fresh preparatious negative. In stained preparation not less than 5 per cent of corpuscles containing peripheral cocci about $0.6 \mu$ in diameter. A small number of half-grown ticks on thighs.

October 7.-Animal excited in being caught. Temperature, 102.7; pulse, 96 ; respiration, 40. Corpuscles, 5,084,000. In fresh preparation numerous corpuscles containing bright bodies, chiefly near periphery of cell. In stained preparation about 1 to 2 per cent of infected corpuseles.

October 22.-Animal excited. Temperature, 104 ; pulse, 96 ; respiration, 12 . Corpuscles, 6,270,000. Fresh preparations negative. In stained preparations 1 to 2 por cent of corpuscles containing peripheral cocei.

October 25.-Temperature, 104.1; pulse, 87; respiration, 27. Corpuseles, 5,596,774. One intraglobular motile bright body in one fresh preparation. In stained preparations 1 to 2 per cent peripheral cocei. (For the temperature, see pl. 279 and 280.)

No. 89 (native).-Born of cow No. 65, March 27, 1890.

September 20.-Placed with No, 65 in field VI (North Carolina eatt?e with ticks).

October 6.-Temperature, 104.5 ; pulse, 120 ; respiration, 60 . Corpuseles, 3,936,500. In fresh preparation slight tendency of corpuseles to crenate. One corpuscle containing a large pyriform parasite detected. Stained preparations lost.

October 11.-Temperature, 103.8; pulse, 108; respiration, 54. Corpuscles, 3, 027,000. No parasites detected in fresh and stained proparations. Corpuseles tend to crenate. Some macrocytes present. Several small ticks found.

October 25.-Temperature, 103.4; pulse, 96; respiration, 30. Corpuscles, 3,606,000). Both fresh and stained preparations negative.

Oetober 30.-Temperature, 104.2; pulse, 78 ;respiration, 27. Corpuseles, 4,254,500. 
Several very minute bright and pale bodies within corpuscles. Stained preparations negative.

November, 11.-Temperature, 103.6; pulse, 78; respiration, 42. Corpuscles, 3,218,800. Corpuscles somewhat enlarged. No parasites detected.

November 24.-Temperature, 103.8; pulse, 92 ; respiration, 52 . Corpuscles, 3,300,000. In stained preparatious 2 to 3 per cent of corpuscles contain peripheral cocci. Animal in fair condition. (For temperature record, see p. 280.)

No. 90 (native).-Young bull, one year old when received, May 26, 1890, from Prince George County, Md.

Augnst 25.-Exposed in field VI to North Carolina cattle with ticks.

September 12.-A large number of small ticks on animal. Temperature, 103.4; pulse, 96 ; respiration, 36 . Blood-corpuscles, 1,974,000. Several bright bodies but no parasites detected in corpuscles. In stained preparations corpuscles more or less crenated. About 10 per cent contain each from 5 to 10 unusually large granules. There are in addition some diffusely stained corpuscles and some with many very fine stained particles.

Sept. 16, 11 a. m.-Animal lying down unable to rise. Temperature, 100.8. Blood exceedingly thin; only 1,000,000 red corpuscles. From 20 to 30 per cent contain large parasites in pairs, the majority pyriform and provided each with a dark round body barely visible at 500 diameters (Plate viII, Fig 5 ).

1.30 p. m. - In same preparation most of the pyriform bodies have assumed the round form. Animal killed by a blow on the head. Temperature at this time 101.4. Heart's blood examined at $2 \mathrm{p} . \mathrm{m}$. and $3.15 \mathrm{p} . \mathrm{m}$. The same appearances as in blood from the skin. Hæmatoblasts present. A few bright motile bodies within corpuscles detected. At 3.15 p. m. many of the parasites had become spherical.

Autopsy immediately after death.--Weight of animal about 450 lbs. Many ticks on thighs and abdomen. Nothing abnormal in thoracic cavity excepting slight clouding of heart fibers when examined microscopically. Spleen weighs $2 \frac{8}{8}$ pounds. Slightly engorged and quite firm. In both fresh and stained coverglass preparations about one-third of all corpuscles contain round parasites about $2 \mu$ in dianeter. Fully one-half are double. Liver weighs about $7 \frac{1}{4}$ pounds. Parenchyma yellowish brown. In fresh sections the bile injection is observed in spots within the lobules. Fatty degeneration marked throughout. In fresh and stained preparations the same infection of red corpuscles as in spleen. Many bodies are pyriform. Gall bladder contains a little bile holding much solid matter in suspension.

Kidneys are somewhat paler than normal. In fresh sections little blood in capillaries. The convoluted tubules are plainly marked as yellowish brown bands owing to the pigment contained in the epithelium. Considerable pigment generally diffused in very minute particles. The infection of corpuscles as in liver. Urine contains considerable hæmoglobin. Specific gravity, 1013. Neutral. Digestive tract with its mucosa rather pale. Marrow of ribs shows nearly the same infection of corpuscles as that observed in liver, spleen, kidneys, and blood preparations. (For temperature record, see pp. 279 and 280.)

No. 91 (native).-Heifer, 3 years old, received May 26, 1890, from Prince George County, Md. Placed in field I on this day to test survival of infection of 1889 . (See also No. 74.)

September 16. - Temperature, 101.8. Corpuscles, 5,540,000. No abnormal condition of the blood detected in fresh and stained preparation.

October 1.-There being only a negative result from this exposure, the animal was transferred to field VI (North Carolina cattle with ticks). Temperature, 101.8; pulse, 60 ; respiration, 22 . Corpuscles, 4,672,000. One corpuscle containing a bright motile body detected. Examination of stained preparations negative.

October 7.-Temperature, 101; pulse, 96; respiration, 18 . Corpuscles, 4,833,000. Blood examination as before. 
October 14.-Temperature, 101.8 ; pnlse, 66 ; respiration, 30 . Corpuscles, 4,837,500. Blood examination as before.

October 30.-Corpuscles, 4,670,000. Examination of blood negative. (For temperature record, see pp. 274, 275, and 280.)

No. 93 (native).-May 23, 1890. Born to-day of cow No. 95.

July 4.-Placed with No. 95 in field VI (North Carolina cattle with ticks).

Angust 16.-'Temperature, 104.4. Corpuscles, 4,775,000. They tend to become crenated. In stained preparations not less than 20 pcr cent contain each one or more peripheral cocei.

August 19.-Temperature, 103.8; pulse, 96; respiration, 40 . Corpuscles, 3,460,000. In stained preparation many corpuscles are enlarged. Not less than 10 per cent contain peripheral cocci.

August 23.-Temperature, 102.6 ; pulse, 88; respiration, 18 . Corpuscles, 3,160,000. Enlarged corpuscles as before; a few are punctated. In stained preparations from 1 to 2 per cent of corpuscles infected with peripheral bodies.

September 22.-Temperature, 103.7; pulse, 96; respiration, 80. Corpuscles, $3,940,000$. Enlarged corpuscles very few. Only an occasional infected corpuscle detected.

This calf has been sick and in very poor condition since August 16. The cornea of left eye is ulcerated. Similarly the left ear in which the metal tag was inserted.

A considerable number of ticks of various stages, including such as are fully matured on the animal.

Killed by a blow on the head.

Autopsy.-Weight, 150 pounds; heart and lungs normal; spleen, of normal consistency, weighs $\frac{3}{4}$ pound; liver weighs about 2 pounds, normal in appearance; cells contain exceedingly minute pigment granules; bile holds in suspension a small number of large flakes; kıdneys rather pale, otherwise normal; small amount of pigment in cells; urine pale yellow, alkaline. Specific gravity, 1037. No albmmen.

Digestive tract not markedly affected. In the fourth stomach minnte worm-pits (Strongylus Ostertagi). Strongylus contortus present in large numbers. In ileum many worm-nodules under mucosa.

In stained preparations of spleen, liver, and kidneys, no parasites detected. In heart's blood there were the same number of corpuscles infected as in blood from the skin.

Marrow of rib contains hæmatoblasts with 2 to 4 nuclei each. (For temperature record, see pp. 279 and 280.) 
(1)
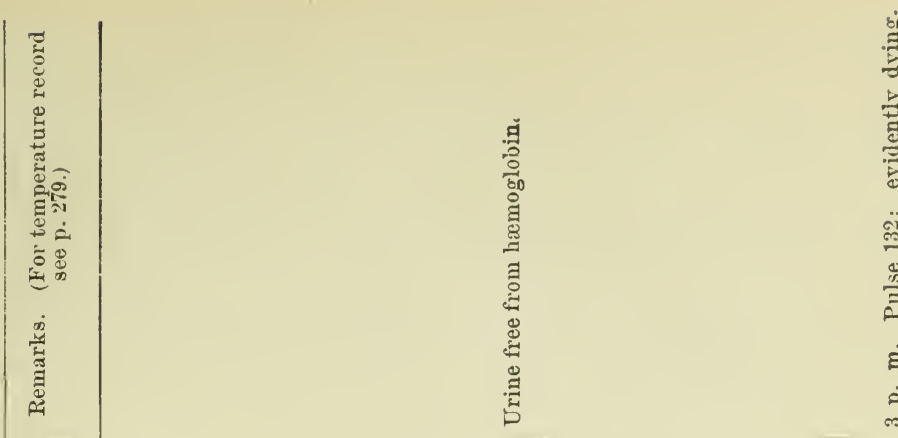

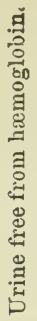

宅

害

ำ

กิ่

is

है

घ่

六莯

- notper

ㄱำ

คำ ติ ต

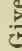

ค่ำ

字

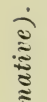

-!dsey

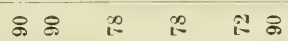

8 $\stackrel{\infty}{\cong} \stackrel{\circ}{\circ}$

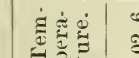

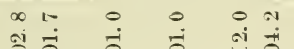

?

:

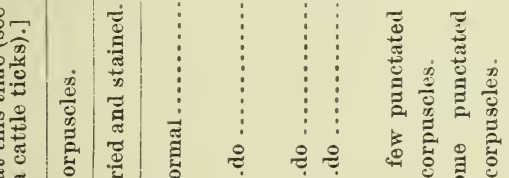

范苟

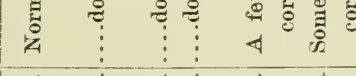

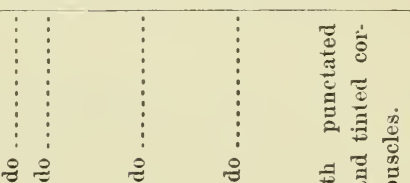

$\begin{array}{ccc}\vdots & \vdots & \vdots \\ \vdots & \vdots & \vdots\end{array}$

苛

4

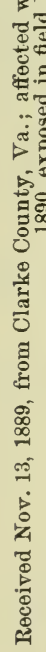

항

离

$\begin{array}{cccc}1 & \vdots & \vdots & \vdots \\ 1 & \vdots & 0\end{array}$

$\vdots$
$\vdots$
$\vdots$
$\vdots$
$\vdots$
$\vdots$

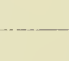

交

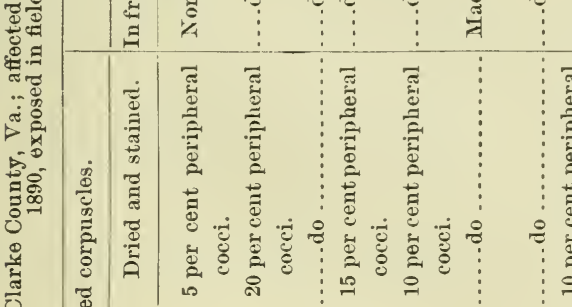

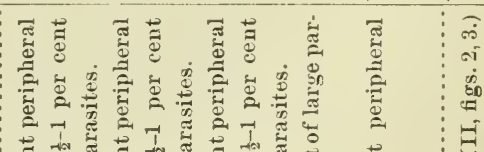

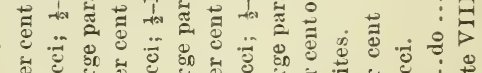

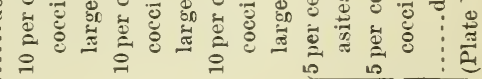

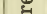

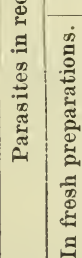

$\vdots$

\begin{tabular}{|c|c|c|c|c|c|c|}
\hline 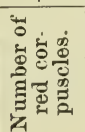 & 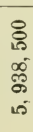 & $\begin{array}{l}8 \\
8 \\
0 \\
\text { 帘 } \\
+1\end{array}$ & 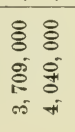 & 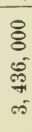 & 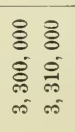 & $\begin{array}{l}8 \\
\varnothing \\
0 \\
\text { को }\end{array}$ \\
\hline
\end{tabular}

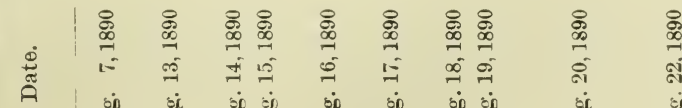

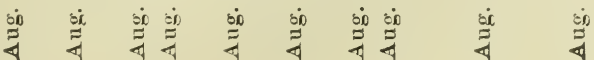

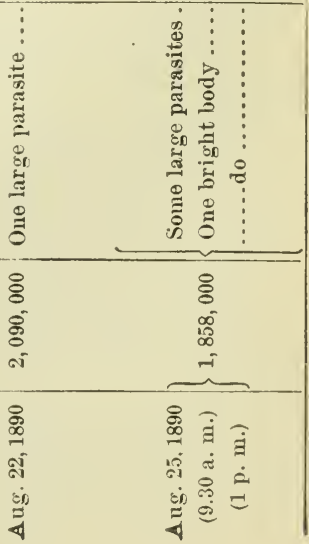


Autopsy: Subcutaneous fatty tissue of ventral aspect of body from sternum to udder infiltrated with straw-colored serum. The infiltration several inehes thick near udder. Blood clots quickly and firmly as it flows from the subeutaneous veins.

Slight eechymosis of heart muscle. No degeneration of fibers. Numerous sarcosporidia cysts under endocardium. In blood from the right ventricle a few intraglobular parasites present. In principal lobe of right lung on convex surface six lobules in state of dark-red hepatization. Lung worms in bronchi.

Spleen weighs 3 pounds, moderately engorged. About 2 per eent of the red corpuscles eontain the large paired parasites, some roundish, others pyriform.

Liver weighs $13 \frac{1}{4}$ pounds. The eut surface presents a mottled (nutmeg) appearance. In fresh sections under the mieroscope large fat globules uniformly sprinkled over the section. No bile injection.

Sections of liver tissue (hardened in aleohol, imbedded in ehloroform paraffin) stained in hæmatoxylin show more or less of pathologieal ehanges. The trabecular arrangement of the outermost zone of the lobule is exaggerated, the capillaries wide. The eells appear large and the protoplasm uniformly vaeuolated. The nuclei are no longer normal. They appear as pale bodies with a stained periphery. The interior is disintegrated into a variable number of deeply stained roundish granules. In the eentral half of lobule the trabeeular arrangement more or less effaced. The protoplasm is markedly vaeuolated and the nuclei represented by three or more deeply stained gravules which have shrunken together, in some cases coaleseed into a single irregular mass. Capillaries do not show any red eorpuseles.

Gall-bladder contains $1 \frac{1}{2}$ pounds of bile, very thick, dark colored and viseid. Holds in suspension an abundanee of yellow amorphous flakes. In stained preparations of liver pulp about 5 per cent of the corpuscles eontain one or more parasites. Kidneys somewhat pale (left weighs $1 \frac{1}{8}$ pounds). No anatomieal changes observable in fresh sections. In the capillaries and larger vessels of pyramids numerous corpuscles may be seen $(\times 500)$ with the pale parasites within them. Occasional bunches of red needle-like crystals deteeted. In stained preparations few parasites within corpuscles, but a very large number of free bodies. In sections of tissue hardened in aleohol and stained in hæmatoxylin no pathologieal changes detected. Much fine pigment in convoluted tubules. Urine free from hæmoglobin; specific gravity, 1020 ; aeid. No reaction for albumen, bile pigments, or bile salts.

Digestive tract normal with exception of fourth stomaeh, of which the mucosa has a pale bluish-red color. Mueh bile in duodenum. In red marrow from ribs perhaps 1 per eent of eorpuseles contain one or two parasites.

No. 97 (native).-Bull calf of cow No. 50, born July 20, 1889.

September 14.-Placed with eow No. 50 in field IV (North Carolina cattle without ticks).

November 27.-No symptoms of disease observed up to date.

August 25, 1890.-Plaeed in field I to test presenee or absence of infeetion of 1889. (See 74, 91.)

November 3.-No result to date. Animal removed. (For temperature reeord, see pp. 273,274 , and 275 .)

No. 100.-May 10, 1890. Born to-day of eow No. 69.

July 4.-Exposed with No. 69 in field VI (North Carolina eattle with ticks).

October 11.-One tick found on animal. Temperature, 102.6 ; pulse, 72 ; respiration, 36. Red corpuscles, 4,393,000. In fresh preparations many corpuscles appear enlarged. Stained, not less than 10 per eent eontain the small peripheral eoccus-like bodies.

October 15. - A few small tieks on animal. Temperature, 104; pulse, 90; respiration, 42. Red eorpuscles, 3,685,000. In fresh preparations a fow corpuscles olsserved with a minute, pale, round body within each, near periphery. (Nuch bodies had also been observed in the proceding examination.) In stained preparations proba- 
bly 15 per cent of all corpuscles contain the peripheral coccus-like bodies, scen in the fresh preparations as pale round bodies.

October 22.-T'emperature, 103.8; pulse, 68; respiration, 22 . Corpuscles, 3,254,000. In fresh preparations a bright and a pale intraglobular body detected. In stained preparations about 5 per cent of the corpuscles contain peripheral cocci.

November 13.-Temperature, 100.8; pulse, 64 ; respiration, 12 . Animal emaciated and weak. A few lice observed. Corpuscles, 3,379,000. Some enlarged. Only about 1 per cent of corpuscles carry parasites.

November 24.-Temperature, 103.4 ; pulse, 84 ; respiration, 9. Corpuscles, 2,760,000, In stained preparations infected corpuscles rare. Animal very weak.

December 2.-Animal has been lying down, unable to move, since November 30 . Probably dying. Temperature, 88; pulse, 42; respiration, 8. Corpuscles, 3,057,000. After long search several infected corpuscles detected in stained preparations.

December 3.-Animal killed by a blow on head. At the autopsy no lesions were observed excepting the presence of numerous lung worms in the bronchi and an cdematous condition on the submucous tissue of the fourth stomach. (For temperature record, see pp. 279 and 280.)

No. 101 (native).-Heifer, one year old, received May 31, 1890, from the District of Columbia.

July 4.-Placed in field IX (North Carolina soil only).

August 14.-Termperature, 101.6; pulse, 68; respiration, 30, Corpuscles, 5,600,000. September 2.-Temperature 101.7; pulse, 60; respiration, 30. Corpuscles, 8,475,000. A few bright bodies detected within red corpuscles. Blood elements normal.

September 18.-Urine collected and examined. Normal. Blood also examined. After a long search one motile bright body detected within a red corpuscle.

November 3.-No symptoms of disease noticed up to date. (For the temperature record, seo pp. 281 and 282. 


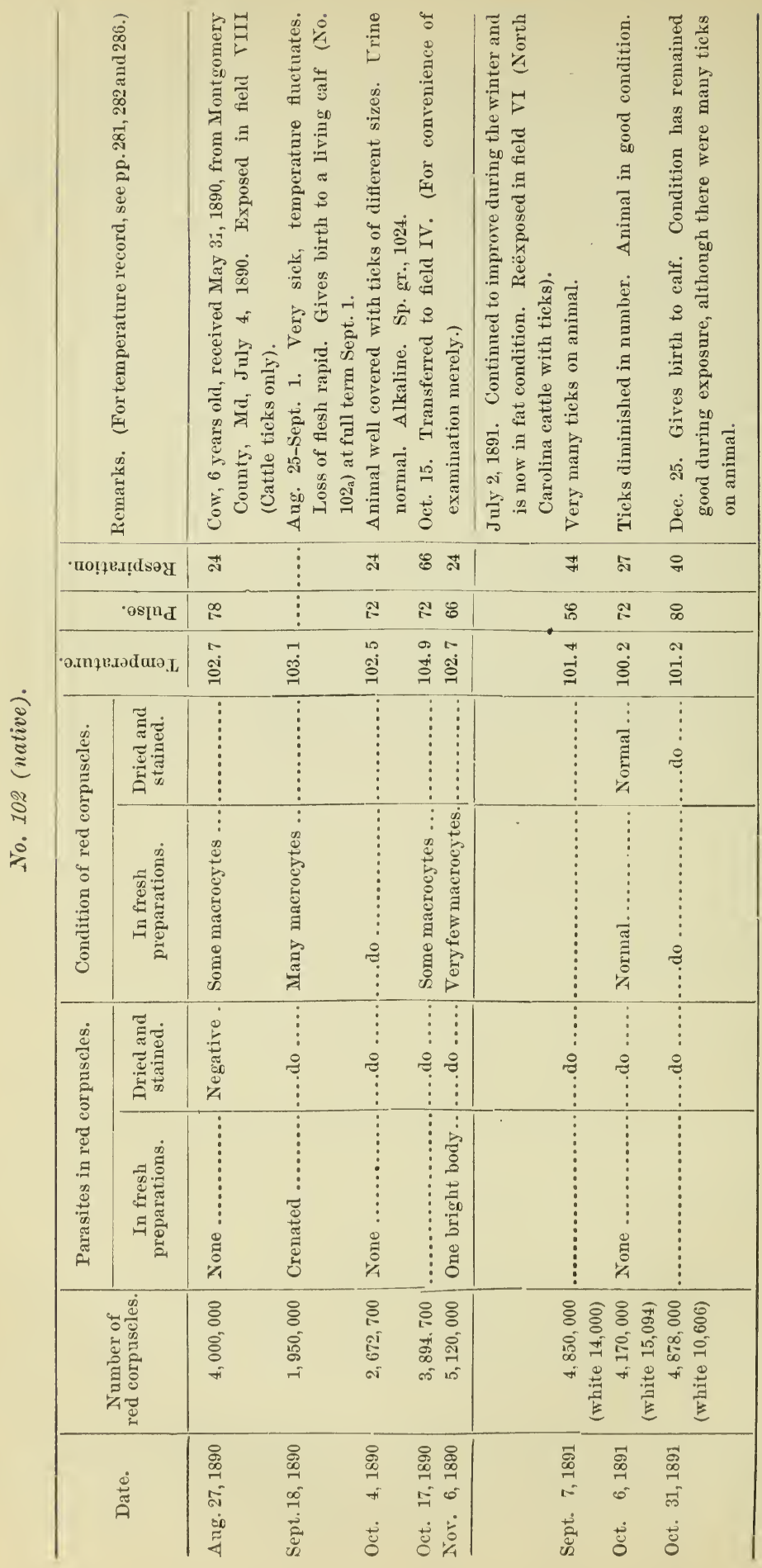


No. 102 a (native),-Calf of No. 102. Born September 1, died September 13, kapt in refrigerator until september 15. The examination was delayed because of other work. It was presmmed that the calf had sucenmbed to other causes, but to our surprise the autopsy denionstrated a marked casc of Texas fever as the following notes will show.

Autopsy: Several small ticks found on skin of thighs. The subeutaneous as well as viseeral fat over the whole body has a decided yellow tinge.

Heart empty, contraeted. Fatty degeneration of fibers. Spleen (weight 13 ounces) was dark, enlarged and softened. Liver (1 $\frac{8}{4}$ ponnds), firm, brownish red. In fresh sections and teased preparations fatty degeneration of the hepatic eells moderate; nuclei distinct. Occasional spots showing bile injection. Considerable number of minute golden needle-like crystals scattered over the section. In stained preparations about 10 per cent. of the red eorpuscles in the liver contain eaeh a pair of parasites. Bile thick and full of flaky sediment. Not so dark in eolor as with adults. Kidneys very hyperæmie, of a dark brownish-red color. In fresh sections eapillaries distended with red corpuseles. About 20 per cent. of corpuscles invaded by parasites.

About 500 ee. (1 pint) of urine in bladder deeply eolored with hremoglobin, not translueent in layer $\frac{8}{4}$ inel deep. Specifie gravity, 1022. Reaction, aeid. 1.4 per per cent. albumen (Esbaeh.)

No. 103 (native).-Heifer, three years old, received June 4, 1890, from Prince George County, Md.

Jnly 4.-Plaeed in field VII (North Carolina cattle without ticks. Ticks washed in later from adjoining field VIII.)

Augnst 16.-Temperature, 102.8. Corpuscles, 5,500,000. Nothing abnormal in fresh and stained preparations.

August 25.-Temperature, 103; pulse, 60; respiration, 25. Corpuseles, 5, 133,000. In fresh preparation 5 eorpuseles observed eontaining each a motile, bright body. In stained preparations nothing abnormal.

September 6.-Found dead this morning quite unexpeetedly.

Autopsy atnoon: A large number of ticks of all sizes on the animal.

Heart: Slight ecchymosis ou pulmonary artery, near root. Small quantity of fluid blood in both ventricles. Considerable extravasation under endocardium of left ventriele. In fresh preparations of heart's blood a considerable number of bright bodies within corpuseles, in motion and of pale round forms, equal to about one-third or less of the diameter of the eontaining corpuseles. In stained preparations eorpuscles eontaining one or two parasites, distributed more or less in groups.

Lungs cedematous.

Spleen weighs $3 \frac{1}{2}$ pounds. Pulp very dark, partly disintegrated. In stained preparations from 5 to 10 per cent. of eorpuseles infected with parasites.

Liver weighs 12 pounds. Color more yellowish than nornal, slightly mottled in appearanee. Slight fat infiltration, with general engorgement of capillaries with corpuseles, but no bile injection. In stained preparations, from 15 to 20 per cent. of the corpuseles eontain each one or two parasites. Bile in bladder very thick, and flows like sirup, leaving a golden brownish coating on vessels. Large quantity of suspended solids.

Kidneys have a uniform brownish-red eolor on section. In fresh sections capillaries in general distended with corpuscles. In stained preparations not less than 50 per cent. of those contain one or two parasites. Urine of a decp port-wine color. Speeific gravity 1017; faintly alkaline. Albumen present in slight amount.

Digestive traet normal, with exception of fourth stomaeh, the mueosa of which is dark pink in eolor. In the duodenal portion there are erosions, with blackish base $\frac{1}{8}$ to $\frac{1}{2}$ ineh long.

In uterus a fœtus seven to eight months old.

In marrow from the ribs a small number of parasites detected. (For the temperature reeord, see p. 281.) 


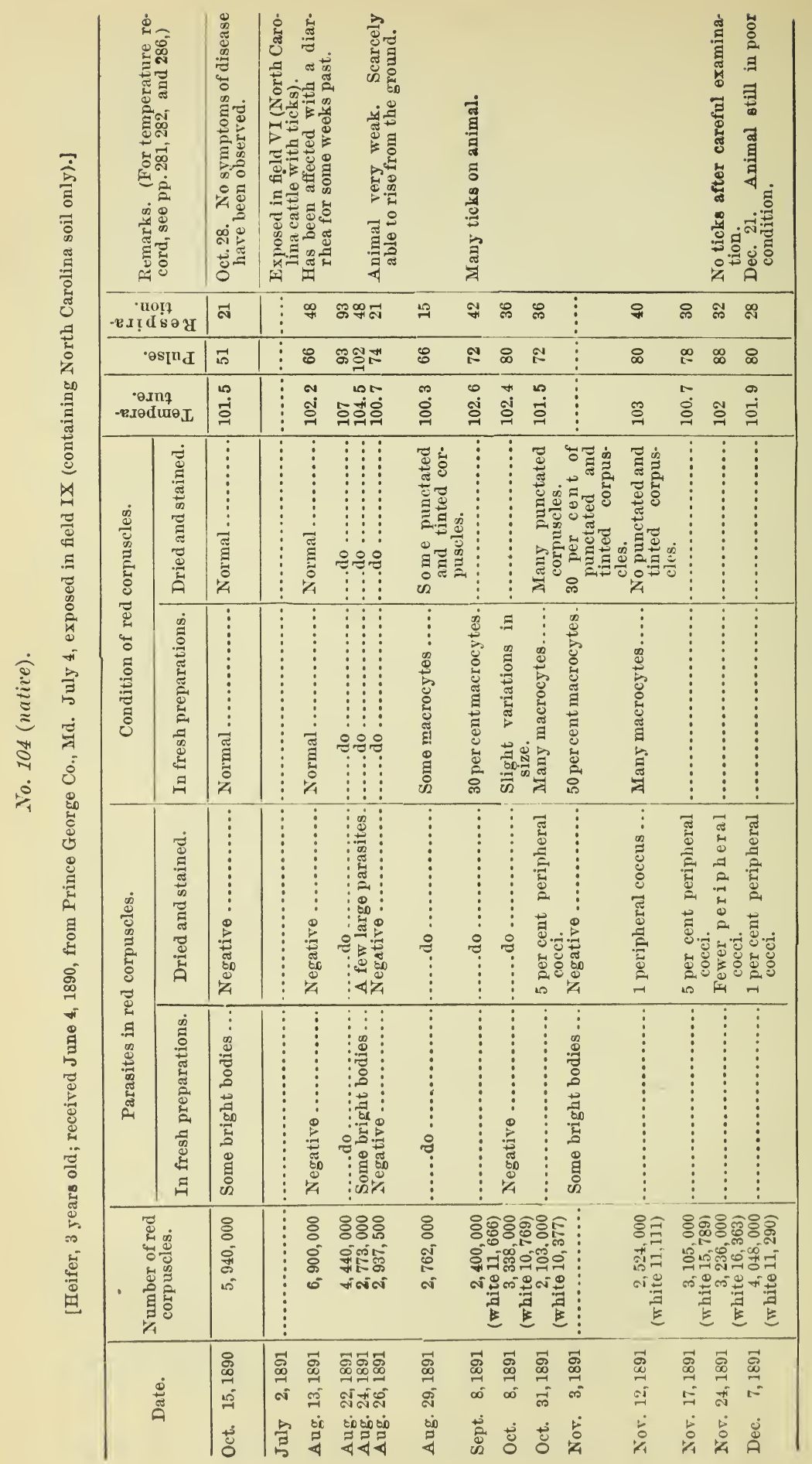


APPENDIX.

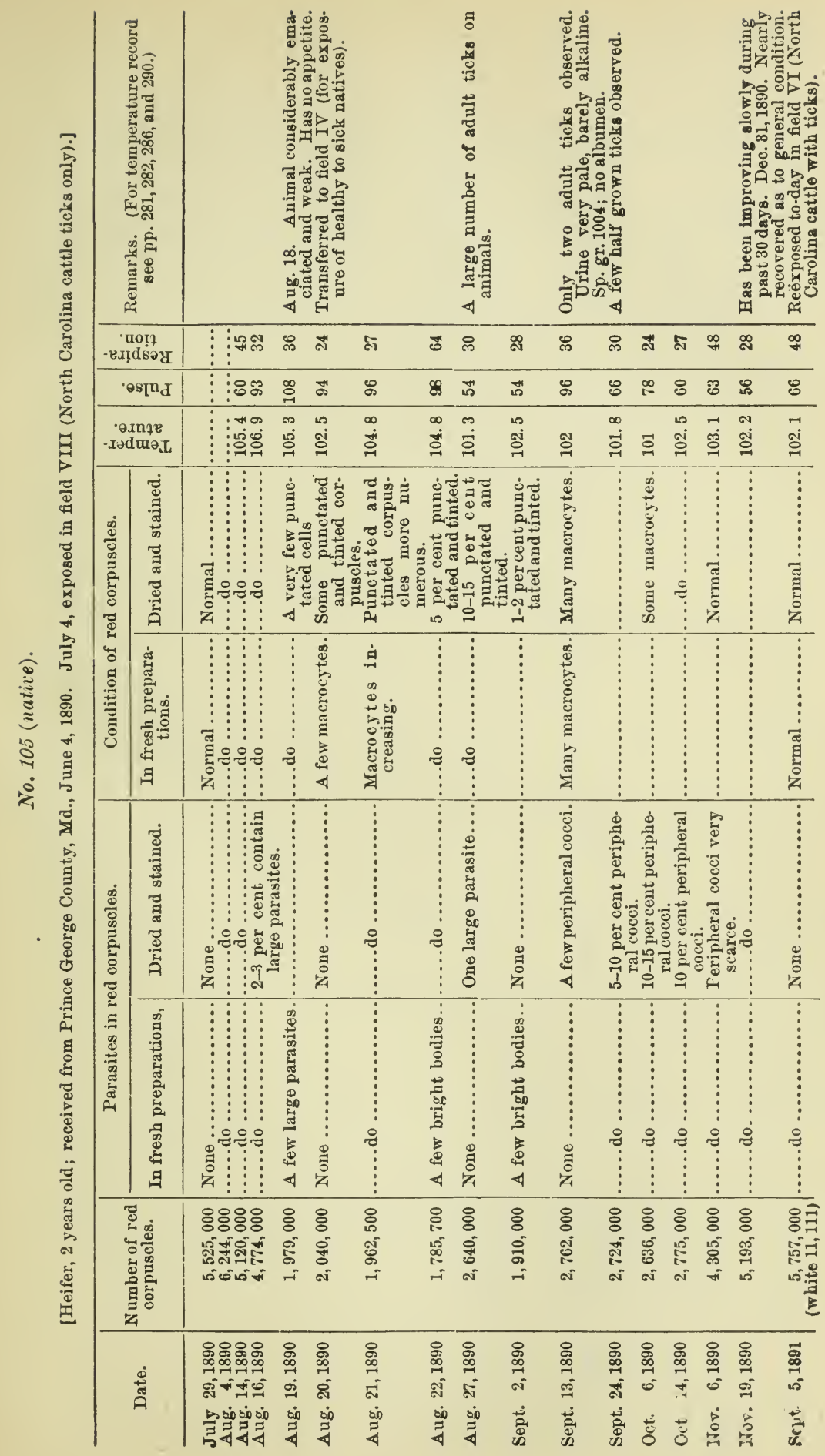




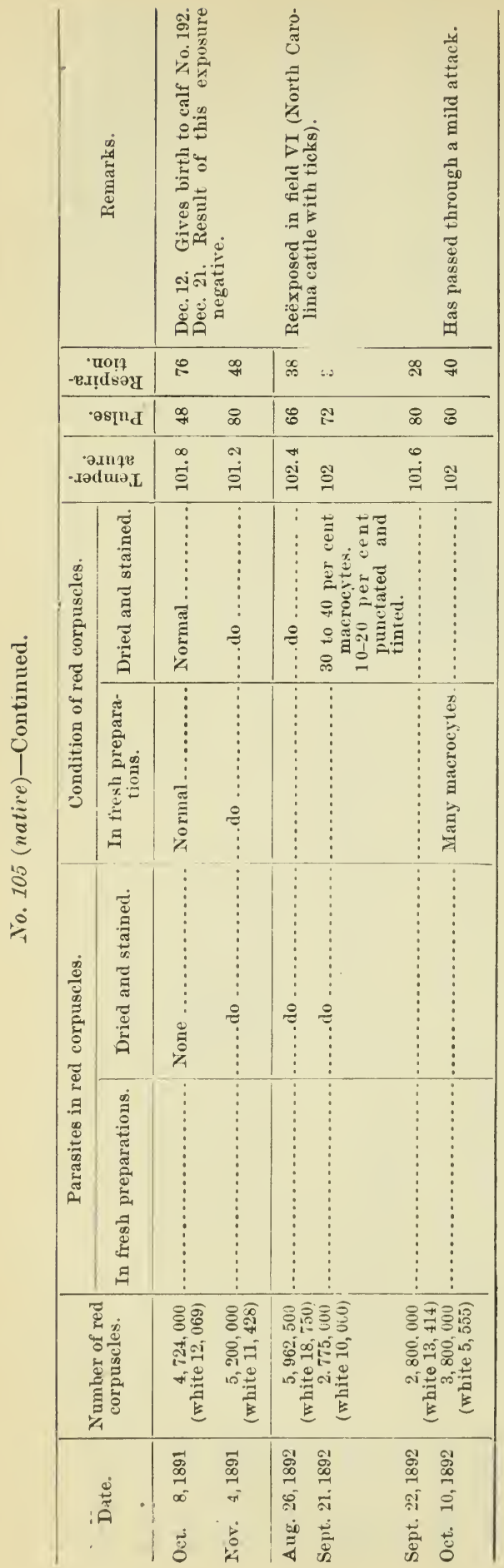


No. 106 (native).-Heifer, 2 years old, received June 4, 1890, from Prines Georges County, Mal.

July 4.-Exposed in field VII (North Carolina cattle without ticks. Ticks washed in from arljoining field VIII. See No. 103).

August 25.-Temperatnre, 105.9; pulse, 72; respiration, 48. Red corpuseles 6,175,000. In preparations of fresh and stained blood a very few corpuscles observed with intraglobular bodies rather irregular in ontline.

Angust 27.-Temperature, 105.8; pulse, 72; respiration, 48. Corpuscles 3,400,000. Corpuscles in fresh preparations mostly crenated. In stained preparations from 5 to 10 per cent contain large pyriform parasites in pairs or else smaller ronndish bodies singly or in pairs. (Plate v, Fig. 2.) Skin almost bloodless. Blood obtained from incisions with difficulty and in very small quantities.

Angust 28.-Found dead this morning. Seen alive at $6: 30$ the preceding evening. Autopsy at 9:30 a. m.: Animal in good condition. Emaciation very slight. To skin of abdomen, neck, and inner snrface of thighs a large nmmber of small ticks are attached. On opening the abdomen a distinct odor of decomposition noticeable.

Heart surface sprinkled over with minute petechix, most numesons near the base. In both ventricles small clots and some liquid blood which contains about the same number and kind of parasites as those observed yesterday in blood firom the skin. Some large bacilli present. A few patches of extravasation beneath the endocardium of left ventricle and one on mitral valve. A similar patch on one of tricuspid valves. Lings slightly emphysematous; lungworms absent.

Spleen weighs $3 \frac{1}{2}$ pounds. Pulp dark, soft, very much engorged with blood corpuscles. Much pigment within large cells and free. In stained preparations from 10 to 20 per cent of all corpuscles contain single or paired parasites irregularly roundish and from $\frac{8}{4}$ to $1 \mu$ in diameter. Bacilli present.

Liver weighs $11 \frac{1}{2}$ pounds. Enlarged, yellowish brown on surface and section. Yellowish zones visible to naked eye borlering the minnte vessels. In fiesh sections the bile capillaries are injected with bile in the central portion of lobule. (Plate II, Fig. 4.) Fatty degeneration slight. In stained preparations fully 50 per cent of all corpuscles contain single or paired parasites. Some large bacilli likewise detected. In sections of tissue hardened in Miiller's fluid and alcohol and cut in alcohol without imbedding no structnral changes are observed. The bile injection is well bronght out over the whole area of some of the lobules.

About $\frac{8}{4}$ ponnd of bile in gall bladder, which holds a large quantity of solids in suspension.

Kidneys deeply congested throughout. Occasional petechia in cortex. In fresh sections all capillaries distended with red corpnseles in which very few parasites can be distinctly seen in this way. Nevertheless in stained preparations fully 50 per cent of all corpuscles are fonnd to contain parasites. Bacilli present.

About one quart of nrine in the bladder. This has a deep port-wine color, barely translucent in layers an inch deep. When dilnted with 2 parts of water it assumes a beautiful wine-red color in the test tube with transmitted light. Specific gravity 1022; feebly acid. 1.4 per cent albumen present.

Digestive tract not materially affected. Much bile in duodenum and parasite nodules in walls of ileum. Some patches of extravasation on mucosa of rectum.

In the marrow of ribs many hæmatoblasts with 2 to 5 nuclei. (For the temperature record, see p. 281.) 


\begin{tabular}{|c|c|c|c|c|c|c|c|c|c|}
\hline 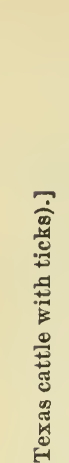 & 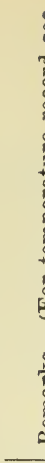 & & & 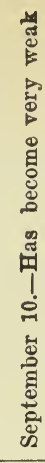 & 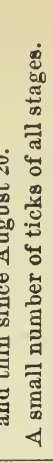 & & 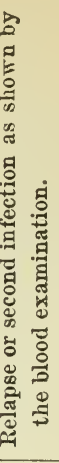 & & \\
\hline$\Xi$ & & (3) & ฉั ลั & ลี & 8 & तี & In & $\stackrel{\sim}{\sim}$ & ह్ల \\
\hline $\overrightarrow{\breve{u}}$ & & ${ }^{8} \mid n_{d}$ & 옹 & 8 & 8 & ละ & & $\mathfrak{1}$ & கే \\
\hline 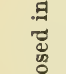 & & 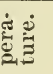 & 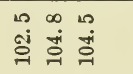 & $\begin{array}{l}\text { N } \\
\text { sं }\end{array}$ & s. & 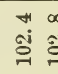 & & 点 & $\stackrel{\circ}{\circ}$ \\
\hline 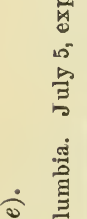 & 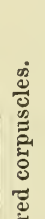 & 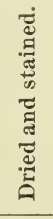 & 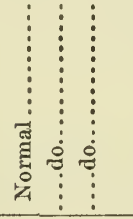 & $\begin{array}{c}\vdots \\
\vdots \\
\vdots \\
\vdots \\
\vdots \\
\vdots \\
\vdots\end{array}$ & 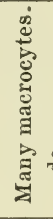 & $\begin{array}{c}\vdots \\
\vdots \\
\vdots \\
\vdots \\
\vdots \\
\vdots \\
\vdots \\
\vdots\end{array}$ & & $\begin{array}{c}\vdots \\
\vdots \\
\vdots \\
\vdots\end{array}$ & $\begin{array}{c}\vdots \\
\vdots \\
\vdots \\
\vdots\end{array}$ \\
\hline 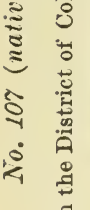 & 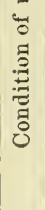 & 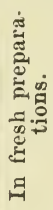 & 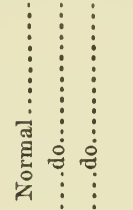 & $\begin{array}{c}\vdots \\
\vdots \\
\vdots \\
\vdots \\
\vdots \\
\vdots \\
\vdots \\
\vdots\end{array}$ & 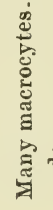 & $\begin{array}{c}\vdots \\
\vdots \\
\vdots \\
\vdots \\
\vdots \\
\vdots \\
\vdots \\
\overbrace{2}\end{array}$ & 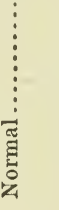 & 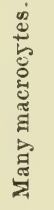 & $\begin{array}{l}\vdots \\
\vdots \\
\vdots \\
\vdots\end{array}$ \\
\hline 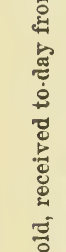 & 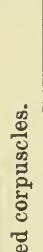 & 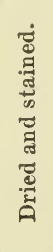 & 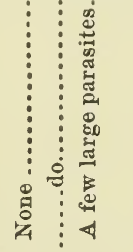 & 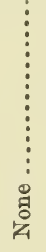 & $\begin{array}{c}\vdots \\
\vdots \\
\vdots \\
\vdots \\
\vdots \\
\vdots \\
\vdots\end{array}$ & $\begin{array}{cc}\vdots \\
\vdots \\
\vdots \\
\vdots \\
\vdots \\
\vdots \\
\vdots \\
0 \\
\vdots \\
\vdots \\
\vdots \\
\vdots\end{array}$ & 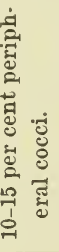 & 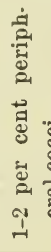 & $\begin{array}{c}\vdots \\
\vdots \\
\vdots \\
\vdots \\
\vdots \\
\vdots \\
\vdots\end{array}$ \\
\hline 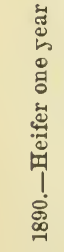 & 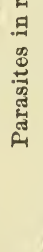 & 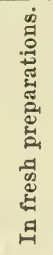 & 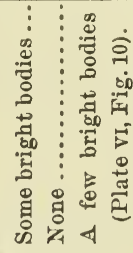 & & $\begin{array}{c}\vdots \\
\vdots \\
\vdots \\
\vdots \\
\vdots \\
\vdots \\
\text { \% } \\
4\end{array}$ & 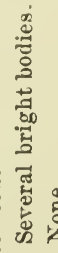 & & $\begin{array}{c}\vdots \\
\vdots \\
\vdots \\
\text { ọ }\end{array}$ & $\begin{array}{r}\vdots \\
\vdots \\
\vdots \\
\vdots \\
\vdots \\
\vdots\end{array}$ \\
\hline $\begin{array}{l}0 \\
\text { : } \\
\text { 号 }\end{array}$ & & 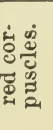 & 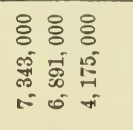 & $\begin{array}{l}8 \\
\text { in } \\
\text { ती } \\
\text { का }\end{array}$ & 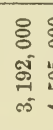 & $\begin{array}{l}8 \\
0 \\
5 \\
5 \\
0 \\
\pi\end{array}$ & & 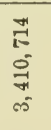 & $\begin{array}{l}8 \\
8 \\
18 \\
20 \\
7\end{array}$ \\
\hline & & ค็ & 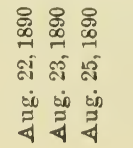 & 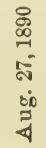 & 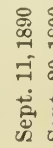 & 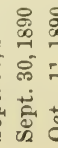 & & 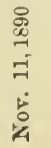 & 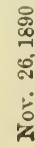 \\
\hline
\end{tabular}


No. $10 S$ (native).-Heifer, 2 jcars old when reccived, June 7, 1890, from the District of Columbia.

The blood of this animal was examincl June 18, 1890. The corpuscles number $7,808,000$.

July 4.-Exposed in field VII (North Carolina cattle without ticks. Ticks washed in from adjoining field VIII. See Nos. 103, 106.)

Scptember 9, 1890.-Died during the night. In fair condition as to flesh. Weighs between 500 and 550 pounds. A large number of ticks of all stages attached to body.

Heart: Muscular tissue rather pale. Faintly granular appearance of fibers under microscope. In right ventricle and auricle a rather firm dark clot extending iuto the large vessels. The left side contracted. The blood, examined fresh, contains many macrocytes and a few hæmatoblasts. A certain number of corpuscles contain rather large parasites. In stained preparations 5 to 10 per cent of the corpuscles are infected. A small number of the corpuscles are quite large and uniformly tinted. No punctated cells detected.

Lungs œdematous, only partly collapsed.

Spleen weighs $3 \frac{5}{8}$ pounds. Enlarged, but still firm. Malpighian bodies visible. In teased preparations many large corpuscles, but no hæmatoblasts detected. Very little pigment.

In stained preparations 10 to 20 per cent of corpuscles contain one or two rather large, roundish parasites $2 \mu$ in diameter.

Liver weighs about twelve pounds and has a uniformly brownish yellow appearance, due to the extensive injection of the bile-capillaries with bile. Much fatty degeneration associated with this injection. Ten to 20 per cent of the corpuscles contain parasites. Bile very thick and flaky.

In stained preparations from the kidueys about 30 per cent of all corpuscles contain one or two parasites. A few large bacilli present and some hæmatoblasts.

Urine in bladder (about 2 quarts) is of a port-wine color. Specific gravity 1013. Slight precipitate when acidified and boiled.

Digestive tract: Mucosa of fourth stomach has a few erosions, with a partly yellowish, partly blackish base. Some sand and gravel mixed with the food. In pyloric portion some round worms. Mucosa of duodenum pigmented in points and striæ. Worm tubercles in wall of ileum. In cæcum many firm fæcal balls, covered with mucus.

The red corpuscles of the marrow infected as in spleen and liver. (For temperature record, see pp. 281 and 282.)

No. 109 (native).-Cow, 10 years old when received, June 7, 1890, from the District of Columbia.

August 25.-Placed in field V to be exposed to blood and splenic pulp from natives which had died of Texas fever. This had been scattered over the ground.

September 18. - Temperature 101.6. Red corpuscles 5,726,000. In fresh preparations they appear normal. Within, several minute, bright, short rods present. In stained preparations corpuscles are normal and parasites absent.

October 3.-Temperature, 102.2 ; pulse, 54 ; respiration, 48 . Red corpuscles, 6,190,000. In fresh preparations corpuscles normal. In every five or six fields one containing a motile bright body detected. Stained preparation as above.

October 14.-Temperature, 100.5; pulse, 48; respiration, 24. Red corpuscles, 5,807,000. Condition of corpuscles in fresh preparation as before. Motile bright bodies perhaps more numerous. Stained preparation as above.

November 3.-Result of exposure negative. (For temperature record, see p. 283.)

No. 110 (native).-Heifer, 1 year old when received, June 9, 1890, from Charles County, Md.

August 14.-Placed in a box-stall and fed several thousand young ticks and egg cases, weighing in all about $4 \frac{2}{8}$ grams. Feeding negative. (See temperature record on p. 283.) 


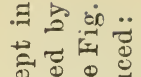
$\pm \&$ 둥

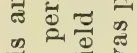
क 口

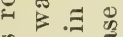

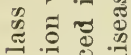
कo 웡 5 के

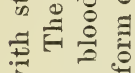
푤

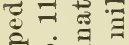
完

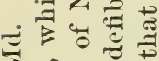

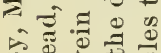
政

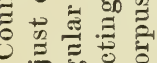

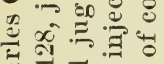

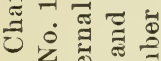
छ $\exists \quad$ 3 $0 . \Xi 0$

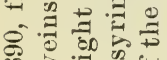
$0>-1000$

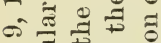

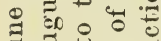

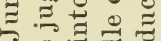
政 政 $\frac{1}{0} 000$ 仓 -0 क त 교

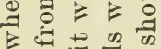

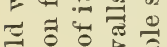

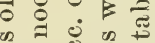

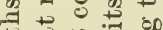
ह ㅁำ

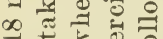
年

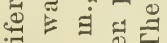
a T. का है

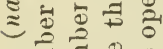

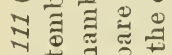
4 을

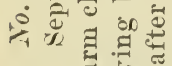
政

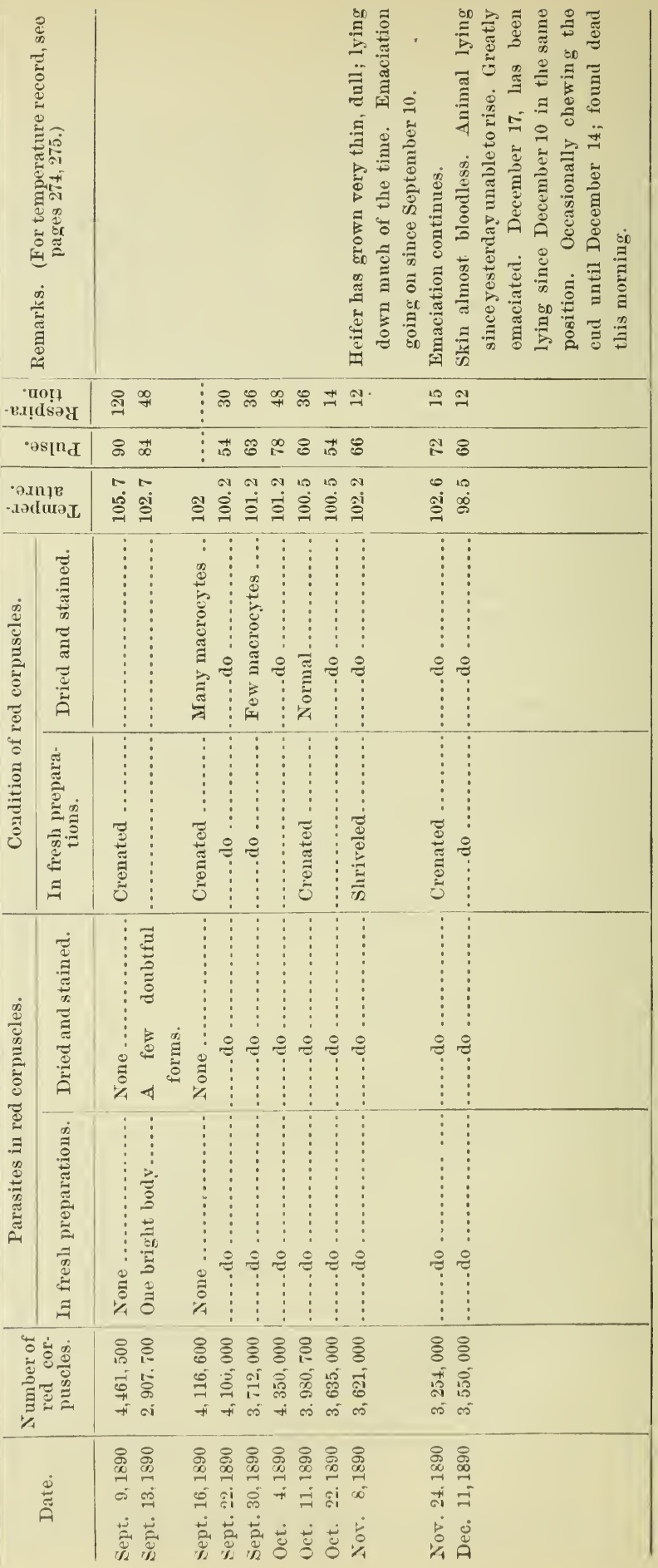


Autopsy: Animal very thin and the tissues quite anxmic in appearanee.

In abdominal eavity eousiderable colema of mesentery and eonneetive tissue around kidneys.

Lungs nelematous. A few small areas are emphysematous. In both sides of the heart large firm clots (larger in right), partly pale, extending in to the large vessels.

spleen small, unehanged. No pigmeut in pulp. Liver weighs $4 \frac{1}{8}$ pounds, with gall-bladiler. In general normal in appearance. Abont 6 onnees viscid bile with much floeculent matter. Kidneys normal. Urine in bladder clouderl, free from hiemoglobin and albumen.

Digestive tract. Mueh redema of submucous coat of fourth stomaeh espeeially in the laminated portion. The mueosa has a markedly pink color in patches. Otler portions of the traet normal. In the blood and organs no traee of the Texas fever parasite.

No. 112 (Southern).-Cow from near New Berne, N. C., age 12 to 15 years. Received July 4, 1890. Placed in field VI. This cow is very thin and weak. Neck and inner aspect of thighs loaded with ticks.

July 8.-Temperature 105.2. Corpuscles 2,786,000. In preparations of fresh blood, a slight tendency towards distortion and increase in size noticeable. No eorpuseles detected in stained preparations indieative of a reeent aente attaek of Texas fever. No parasites present.

July 15.-Corpuscles $3,095,000$. Owing to the enfeebled condition of this animal it was killed. It was likewise loped that some information might be obtained by a post-mortem examination of a Soutlern animal at this time.

Autopsy: At apex of heart the wall of left ventricle thinned out to a translucent membrane which can be pushed out with the finger and withdrawn. Under endocardium a large number of sareosporidia cysts. Lungs contain a few small firm subpleural nodules; otherwise normal.

Spleen weighs $\frac{8}{4}$ pound, small and firm; microscopically nothing abnormal in teased preparations. No parasites detected in stained preparations.

Liver weighs, without gall bladder, 6 pounds. Minute, ocher-yellow dots and lines, noticed on the cut surface and associated with the central vein of the lobule, are found to be due to advaneed fatty changes of the hepatic eells in this situation. Among the fat déhris are round, reddish spheres slightly larger than red corpuseles. In seetions from alcoholie material stained in carmine and in hrmatoxylin, small pale areas are seen around the central vein of some of the lobules in which cells contain very large vacuoles. The nucleus is contracted and pushed to periphery of cell. In the carmine section considerable pignent is observed in granules.

Digestive tract: In the fourth stomach mucosa is more or less pitted by Strongylus Ostertagi. The minute holes surrounded by a slightly thiekened border. In the lower small intestine and caeum the walls studded with numerons worm tubereles.

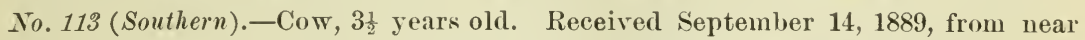
New Berne, N. C., and placed in Fielıl III (North Carolina cattle with ticks).

November 27.-Removed from field.

No. 114 (Southern).-Heifer 2 years old. Reeived July 4, 1890, from near New Berne, N. C., and placed iu Field VI (North Carolina cattle with ticks).

November 3.-Removed from field and sold.

No. 115 (Southern).-Cow, 6 years oll. Received July 4, 1890, from near New Berne, N. C., and placed in Field VII (North Carolina cattle without ticks) after the ticks had been carefully picked off.

July 13.-Gives birth to calf.

October 20.--Removed and sold.

No. 116 (Southern).-Heifer, 2 years old. Received July 4, 1890, from near New Berne, N. C., and placed in Field VII (North Carolina cattle witlont tieks) after the ticks had been carefully picked off.

November 3.-Removed trom field and sold. 
TEXAS OR SOUTHERN CATTLE FEVER.

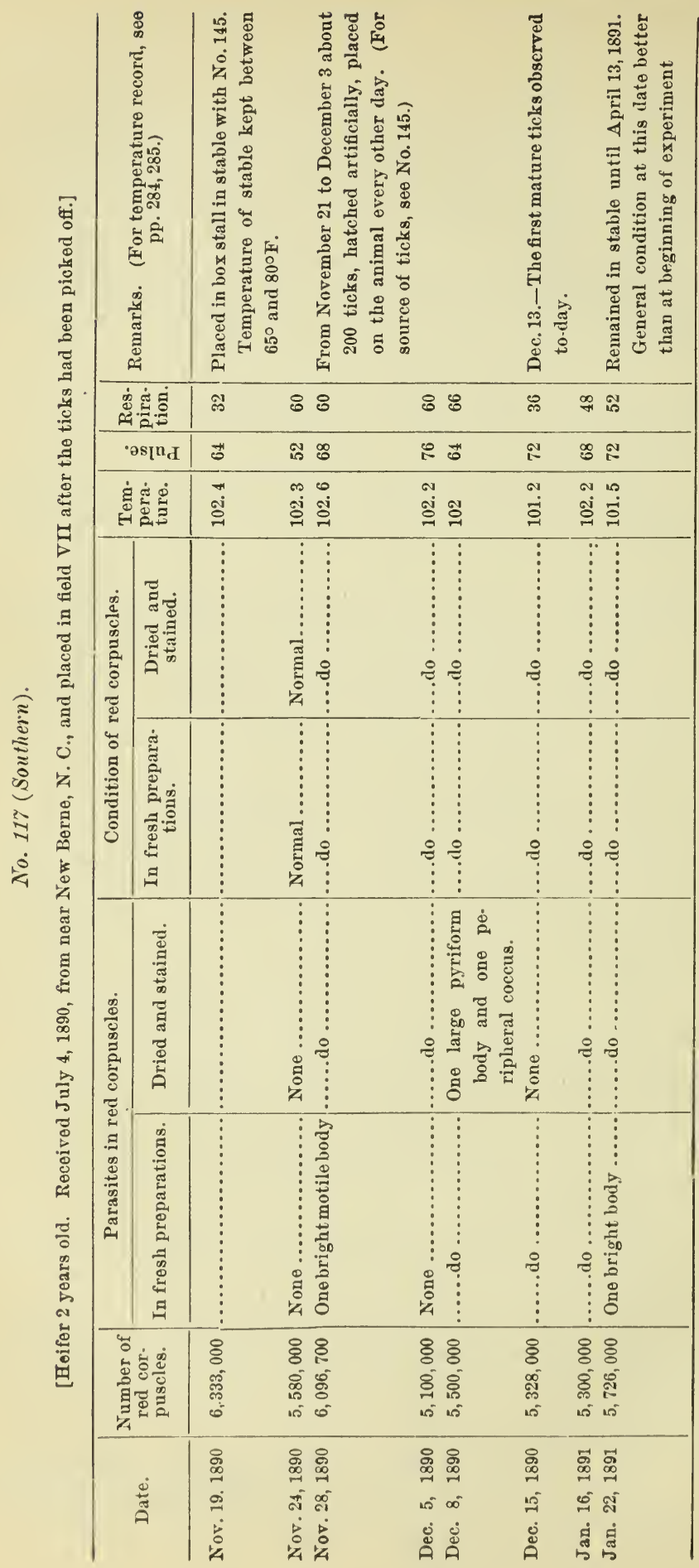


No. 118 (Southern).-Cow, 10 years old; received July 4, 1890, from near New Berne, N. C., and placed in field VII (North Carolina cattle without ticks) after the ticks had been carefully picked oft.

November 3.-Removed from field and sold.

No. 119 (Southern).-Calf, 3 months old; received July 4, 1890, from near New Berne, N. C., and with its dam, cow No. 120 placed in field VI (North Carolinacattle with ticks).

November 2.-Removed from field and sold.

No. 120 (Southern).-Cow, 7 years old; received July 4, 1890, from near New Berne, N. C. Placed in field VI on that day.

September 21.-Dies about 3 p. m. to-day. Had been bloated for several days. Examination postponed till next morning, at which time decomposition was already advanced. There was found extensive exudative peritonitis.

No. 121 (Southern).-Cow, 3 years old; received July 4, 1890, from near New Berne, N. C. Placed in field VI on this day.

September 2, 1890.-Temperature, 102.6; pulse, 106; respiration, 42. (Greatly excited on being caught). Corpuscles, 6,683,000. Nothing abnormal detected in fresh preparations of blood. In one stained preparation about 12 peripheral coccus-like bodies detected. Urine passed at the time normal.

September 28.-Gives birth to calf, No. 148.

July 2, 1891.-Exposed in field VI to Southern cattle with ticks.

October 20.-No evidence of infection up to date. (For temperature record, see p. 286.)

No. 122 (Southern).-Heifer, 2 years old, received July 4, 1890, from near New Berne, N. C., placed in field VI.

September 2, 1890.-Temperature, 103.9; pulse, 88; respiration, 56. (Considerably excited on being caught.) Corpuscles, 5,737,000. Nothing abnormal detected in fresh preparations of blood. In two stained preparations a marked variation in the size of the red corpuscles noted ( 5 to $8 \mu$.) Two peripheral coccus-like bodies and one large parasite found after a long seareh. Urine passed at the time normal.

No.123 (Southern).-Heifer, 3 years old. Received July 4, 1890, from near New Berue, N. C., and placed in field VII (North Carolina cattle without ticks) after ticks had been carefully removed.

November 3.-Removed from field and sold.

No. 124 (Southern).-Cow, 4 years old, received July 4, 1890, from near Houston, Tex., and placed on this day in field II.

September 11, 1890.-Temperature 103. Corpuscles 7,482,000. In fresh preparations of blood several bright motile bodies observed within corpuseles. Two appeared as mere refrangent points and two had a rodlike form. In a stained preparation no parasites detected after a long search. Red corpuscles vary slightly in size. A small number of ticks of various sizes on this animal.

No. 125 (Southern).-Heifer, 3 years old. Received July 4, 1890, from near Houston, Tex., and placed in field II (Texas cattle with ticks).

November 3.-Removed from field and sold.

No. 126 (Southern).-Cow, 5 years old. Receiver July 4, 1890, from near Houston, Tex. Placed in field II on this day.

July 10.-Temperature 100.8. Corpuscles 5,947,000. No abnormal forms in stained preparations.

September 11.-Corpuscles 6,383,000. Somewhat variable in size. In fresh preparations one doubtful bright body observed. In several preparations no abnormal forms detected. Ticks of various stages on animals.

July 2, 1891.-Exposed in field VI to southern cattle with ticks.

October 20.--No indication of infection up to date. (For tenuperature record, see p. 286.)

$10320-$ No. $1-14$. 
No. 127 (Southern).--Heifer, 2 years old. Received July 4, 1890, from near Houston, Tex., and placed in field II (Texas cattle with ticks).

November 3.-Removed.

No. 128 (native).-Cow, 12 to 14 years old. Received July 4, 1890, from the District of Columbia, and exposed in field II, to Texas cattle with ticks.

July 25.-Corpuscles 6,360,000. They appcar normal in fresh and stained preparations.

July 29.-Corpuscles 5,673,000. Normal in fresh and stained preparations.

July 31.-Corpuscles 5,820,000. Normal in fresh and stained preparations.

September 1.-Dies at noon and examined at once.

Animal in fairly good condition. Weighs about 650 pounds. A small number of ticks of various stages attached to skin.

Blood from a skin incision examined a few minutes after death. In a considerable number of red corpuscles parasites singly or in pairs. The forms are mostly round, rarely spindle-shaped or pyriform, and their diameter is about one-third the diameter of the corpuscle. In several slight changes of outline observable. Besides these some corpuscles likewise contain each one bright body changing its position rapidly. In the fresh preparations are also noticed very minute bacteria-like bodies moving or dancing about frec in the plasma. Whether these are mere débris particles in Brownian motion is not determinable. In stained preparations from 20 to 30 per cent of all corpuscles contain the parasite in its large stage. The majority of the infected corpuscles contain each but one body which is usually roundish rarely pyriform in outline. Occasionally, however, a group of corpuscles is encountered which contain each a pair of pyriform bodies. All parasites stain feebly and show more or less refrangence when examined in water.

Blood from the external jugular and the heart shows the same features.

Heart: Slight, mottled discoloration of muscular tissue of left ventricle. Considerable extravasation of blood under endocardium. Many sarcosporidia cysts in this situation. Muscular fibers in state of cloudy swelling.

Lungs: Considerable interlobular œdema in both ventral and adjacent portion of principal lobes. In the right principal lobc, near the lateral edge, a mass of tissue $1 \frac{1}{2}$ to 2 inches in diameter partially hepatized with interlobular cffusion of serum.

Spleen large, weighs $4 \frac{8}{4}$ pounds. Pulp dark, almost disintegrated. A few intraglobular parasites and much pigment in lumps, either free or intraccllular observed in teased preparations.

Liver wcighs about 12 pounds. Very much congested, parcnchymatous swelling. Bile injection observed in restricted places and fatty changes absent. Numcrous infected corpuscles detccted in tcased preparations.

Kidneys also intensely congested, the whole parenchyma has a uniformly dark brownish-red color. In sections all capillaries gorged with corpuscles, in some of which parasites are noticed. The epithelium of the convoluted tubules contains much pigment in granules. Bladder contains about 3 quarts of urine, having a very dark port-wine color. In a test tube having a diameter of $\frac{8}{4}$ inch this urine is practically opaque. When acidificd with acetic acid and boiled an abundant precipitate is formed.

Digestive tract. Mucosa of fourth stomach dark pink. Pits due to Strongylus Ostertagi present and numerous erosions with a depressed hemorrhagic base from $\frac{1}{8}$ to $\frac{1}{4}$ inch in diameter. Considerable hyperæmia of the mucosa of the entire small intestine. Pigment patches in cæcum and hyperæmia in the rectum on the longitudinal folds.

This being a very favorable ease, examined immediately after death, the follow. ing cultures were made:

Blood: Peptone-bouillon, peptone agar with and without glycerine.

Splcen: The same modia. 
Liver: Agar with and without glycerine.

Kidney: The same.

Bile: The same.

The tubes were noculated from the blood and the bile with a looped platinum wire, from the organs with a straight platinum wire.

These various tubes were kept in the thermostat several weeks, but all remained sterile.

On the other hand, stained preparations of the various tissues show the following results as to the presence of the intraglobular parasite approximately stated:

Blood from the skin contains about 20 per cent of infected corpuscles. The parasites are largely in pairs and pyriform. Blood from the right heart contains about the same number. Blood from the jugular contains fewer (about 10 per cent).

Spleen contains from 10 to 20 per cent. of infected corpuscles. The parasites roundish, chiefly in pairs.

Liver contains from 40 to 50 per cent of infected corpuscles. These are also mostly in pairs and many are pyriform.

Kidneys contain between 80 and 90 per cent of infected corpuscles. These parasites are nearly all double. Some corpuscles contain three and four parasites. There are also an immense number of bodies set free from disintegrated corpuscles in this organ. (For tempcrature record, see pp. 274, 275, and 276.)

No. 129 (native).-Heifer, two years old. Received July 5, 1890, from the District of Columbia, and placed in field II (Texas cattle with ticks).

August 11.-Corpuscles 6,193,000. Nothing abnormal in fresh and stained prepations.

August 13.-Corpuscles 7,171,000. Microscopic examination as before.

August 16.-Temperature, 100.6; pulse, 60; respiration, 30. Corpuscles 5,370,000. Nothing abnormal detected in fresh and stained preparations.

August 27.-Temperatnre 106.6; pulse 96; respiration 30. Corpuscles 3,210,000. About 5 per cent of the red corpuscles contain peripheral cocci.

Heifer very weak. Barely able to move.

August 29.-Continues in the same condition. Temperature 101.2; pulse 100 ; respiration 30. Corpuscles $1,675,000$. In fresh preparations on the warm stage a considerable number of corpuscles found to contain bright, motile bodies. One corpuscle found containing a large amœboid form. In stained preparations are found both peripheral cocci and slightly larger round bodies ncarer the center of the corpuscle. About 5 per cent of corpuscles infected in this way.

August 30.-Heifer died at 8 p. m. yesterday. Weighs about 500 pounds. Considerably emaciatcd. Only a few ticks on body. Marked odor from the abdominal cavity when opened. Blood in subcutaneous veins liquid.

Heart: More or less extravasation on the left ventricle near base. In both sides clots of blood extending through both auricle and ventricle. Under endocardium of left ventricle much extravasation of blood, especially around and on papillary muscles. Cloudy swelling of fibers. Lungs collapse but slightly. More or less emphysema and velema.

Spleen weighs $3 \frac{1}{8}$ pounds. Pulp dark, disintegrated. Wells out when capsule is incised. In teased preparations very few parasites detected. There are a considerable number of large coarsely granular cells containing red corpuscles or pigment.

Liver weighs about 11 pounds. Doughy to the touch. On section the parenchyma presents a markedly yellowish-brown appearance. Very extensive fatty degeneration of hepatic cells, which also contain more or less pigment. Bile canaliculi and small bile ducts largely plugged with solid bile.

Bile in gall bladder scarcely flows owing to the large quantity of flocculent material suspended in it.

Both kidneys congested uniformly throughout. In fresh sections capillaries found densely filled with corpuscles. Very fine pigment particles abundant. 
Urine, about two quarts in bladder; has a deep wine-red color. Specific gravity 1013. Considerable brownish floceulent precipitate on boiling.

Digestive tract normal, with exception of upper small intestine, in which some patches of extravasation are found in mucosa.

In stained coverglass preparations the infection of the red corpuscles ran approximately as follows:

In heart's blood corpuscles are all shriveled. Parasites detected with difficulty. Probably 5 per cent infected.

In splecn about 10 per sent infected.

In liver about 20 per cent infected.

In kidneys over 50 per cent infected.

The parasites are all roundish, about $1 \mu$ in diameter, many of them double.

In liver, kidney, and blood preparations there are also large bacilli with rounded ends. Each rod is $3-4 \mu$ long and $1.5 \mu$ broad. The post-mortem changes fully account for the presence of these bacilli. (For temperature record see pp. 274, 276.)

No. 130 (native).-Cow, 5 years old, received July 8, 1890, from Prince George County, Md.

July 9.-Placed in Feld I (see Fig. 5) to test survival of last year's infection.

October 28.-No evidence of infection up to date.

November 6.-Gives birth to a calf.

The following table gives the result of placing young ticks upon this animal in midwinter in an artificially heated stable. The experiment was begun December 12, and the table shows an attack of Texas fever beginning December 30. 


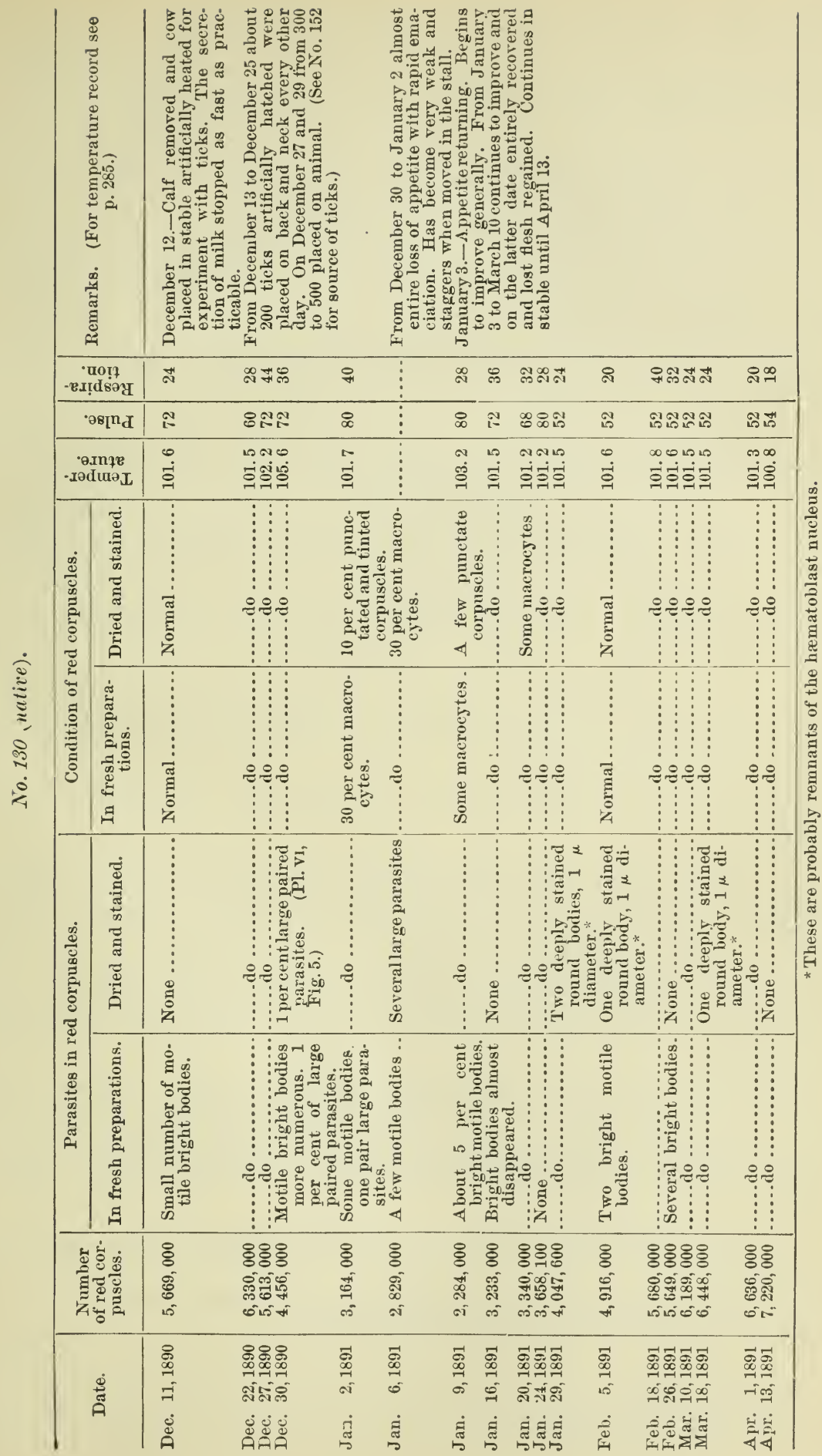


July 2, 1891.-Reёxposed in field VI to Southern cattle with ticks.

August 25.-Temperature 107; pulse, 96 ; respiration, 54 . Red corpuscles 3,922,000. In the fresh blood several corpuscles in every field of the microscope (estimated afterwards in stained preparations as 10 to 15 per cent of the whole) contain large parasites. These are either round or pyriform and some in pairs. The infected corpuscles have a dark-red color, the edges are irregular, and in general they have a wrecked appearance quite different from the corpuscles when crenated or wrinkled from external causes. Minute motile bodies not observed.

August 27.-Has been sick three days; failing very rapidly since yesterday. Has been lying down all the forenoon, excepting to change her position a few times, and then she moved 10 or 15 yards before settling down. At 1:15 p. m. she rose and staggered along 20 or 30 feet, then fell on her side and died soon after. Temperature shortly before death, $100.8^{\circ} \mathrm{F}$.

Autopsy: Weight of animal 750 to 800 pounds. In fairly good condition. A considerable number of small ticks on escutcheon and thighs (froin 8 to 12 to a square inch). A few on udder and neck.

Lungs slightly hyperæmic; trachea contains whitish foam. Heart: Considerable ecchymoses under endocardium of left ventricle, especially on and around papillary muscles. Heart muscle shows slight fatty changes.

Spleen weighs $6 \frac{7}{8}$ pounds, enormously enlarged. The pulp is of a blackberry-jam color. (See Pl. I.) In teased preparations no pigment or phagocytosis of red corpuscles observed. Occasionally a roundish parasite with a dark point (nucleus?) within its substanee seen in red corpuscles.

Liver much enlarged, weighing 20 pounds. Color from the surface rather pale; texture somewhat flabby. On section a small quantity of thick blood flows from the hepatic veins. The parenchyma has a peculiar mottled appearance. The minute hepatic vessels are surrounded and sheathed by zones of a pale yellowish color. The intervening territory of a brownish-red color corresponds to areas containing much blood. Much bile injection observed (Pl. Ir, Fig. 1). Fatty degeneration every where noticeable with variable intensity.

About one pint of very thick, flaky bile in the gall bladder. Extensive deposit on standing.

Kidneys weigh each about $2 \frac{1}{2}$ pounds. The parenchyma has a uniform dark, brownish-red color throughout. The glomeruli appear as blood-red points. In fresh sections of cortex and medulla, the entire capillary system found gorged with blood corpuscles. Very few parasites detected by this method. In the medulla the straight tubules contain granular reddish pigment. In the bladder fully three quarts of dark-red urine, barely translucent in layers $\frac{8}{4}$ inch deep. Reaction alkaline. Sp. gravity 1020. Precipitate with acetic acid. About 1 per cent of albumen according to Esbach's test. Slight deposit of urates on standing in refrigerator, disappearing when heated.

Digestive tract. Mucosa of fourth stomach uniformly reddened. Scattered over it are small papular elevations, $1.5 \mathrm{~mm}$. diameter, with central perforations. (Pits made by Strongylus Ostertagi.) Marked hyperæmia of jejunum and ileum. In the large intestine, the summits of the longitudinal folds are either hyperæmic or pigmented.

In uterus a foetus 3 months old.

In the marrow from the sixth rib most hrmatoblasts have two nuclei, a few thre $\theta$ and even four.

In stained preparations from the various organs and tissues the following percentage of infected corpuscles represents a rough estimate:

Per cent.

Marrow from rib......................................... 5

Blood from subcutaneous veins............................ 10-15

Blood from right heart................................. 10-15 
Spleen ........................................... 10-20

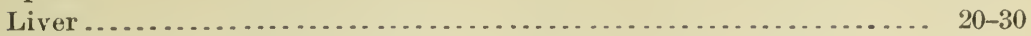

Capillary blood from heart muscle and omentum (Pl. vI, Figs. 1, 2)... 50

Kidney (Pl. Iv, Fig. 4) ................................. $60-80$

Blood of fotus, umbilical vein, none.

The parasites were either round or pyriform, from 1 to $2 \mu$ in diameter. Many were paired. In capillary blood the pyriform bodies more abundant than in the parenchyma of the organs. (For temperature record, see pp. 274, 275, 285, and 286.)

No. 131 (native).-Heifer, 2 years old, received July 8, 1890, from Prince George County, Md.

July 29.-Placed in box stall and fed 600 adult live ticks collected July 21-26 near New Berne, N. C. A large mass of eggs had been laid meanwhile. Of this mass a quantity weighing 70 grains was removed for hatching. A still larger quantity was fed with the ticks. The whole was fed after being mixed with lawn grass and mill feed.

August 12.-Fed 900 mostly adult live cattle ticks, collected August 4-7 inclusive, near New Berne, N. C. A mass of eggs weighing 330 grains was removed. A large mass remained which was fed with the ticks as before.

August 18.-Fed 500 adult ticks, mostly alive, with a mass of eggs weighing 200 grains. These had been collected at the same time with preceding lot.

September 30.-Removed from stable to field I (free from infection).

November 3.-No result to date. (For temperature record, see pp. 274, 275, and 283.) 


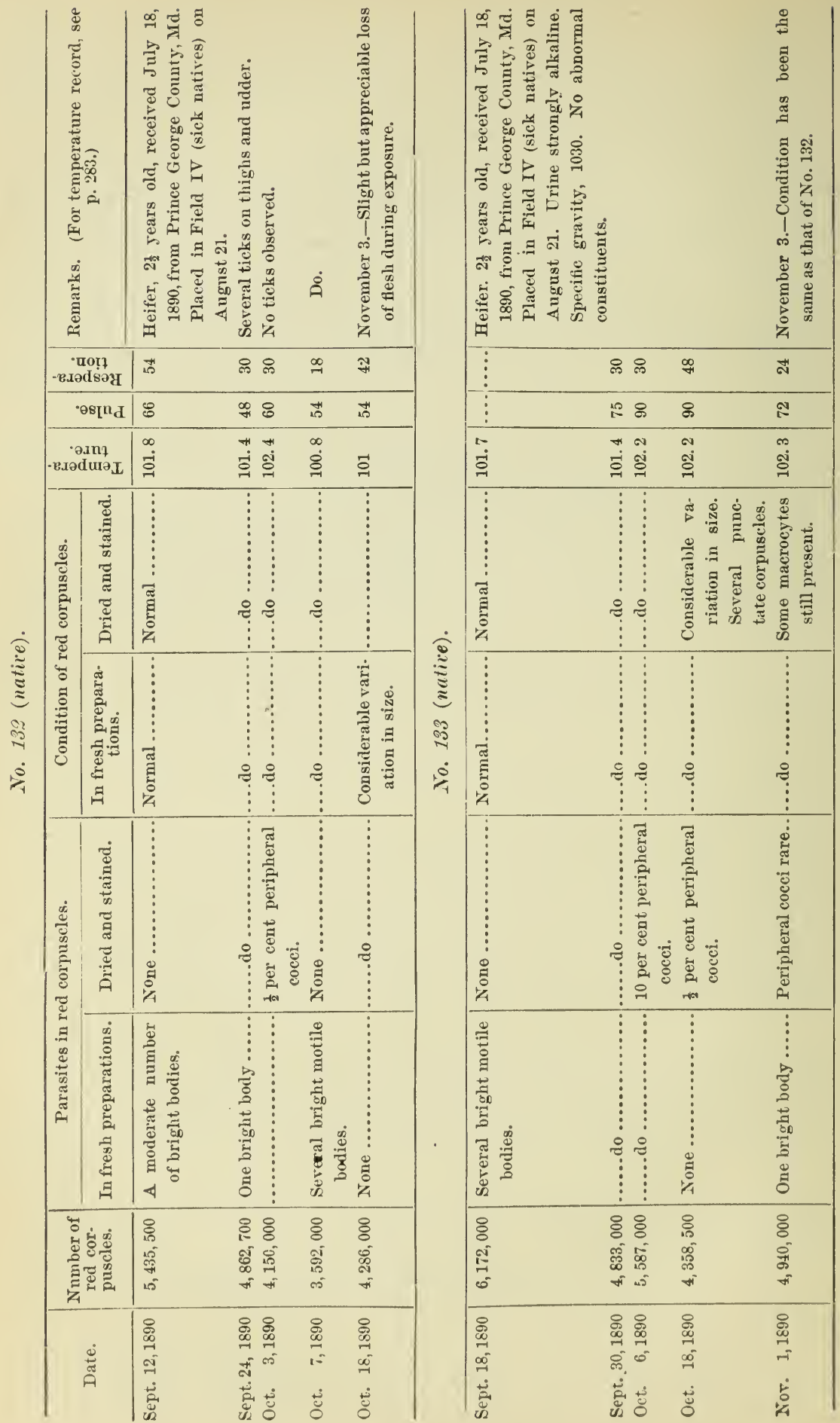


No. 134 (native).-Heifer, 2 years old, received July 18, 1890, from Prince George County, Md.

August 13.-Exposed in Field vi (Southern cattle with ticks); kept up to this date in an uninfected field.

August 27.-Temperature, 106.4; pulse, 96; respiration, 36 . Corpuscles, 2,213,000. In stained preparations of blood one-half to 1 per cent of red corpuscles contain large parasites.

The animal is at this date very much emaciated and weak. Has been sick for two days. A large number of ticks about one-eighth inch long attached to its body.

August 29.-Temperature, 97.4; pulse, 92; respiration, 28 . Corpuscles, 1,840,000. Parasites are scarce in stained preparations, but punctate and tinted corpuscles have appeared; in fresh preparations a small number of corpuscles containing motile bright bodies and amœboid parasites.

Inasmuch as the animal was in a dying condition, it was killed by a blow on the head and the autopsy held at once.

Animal weighs about 450 pounds. Many small ticks on thighs and udder.

Heart: Diffuse extravasations beneath endocardium of left ventricle, especially marked near apex. In right ventricle extravasation slight. Myocardium not affected. Condition of blood the same as that from skin incisions.

Lungs normal.

Spleen weighs $2 \frac{1}{4}$ pounds. Pulp dark brownish red and sonewhat friable. Malpighian bodies still visible on the cut surface. (See Plate VII, Fig. 3.)

Liver weighs about 9 pounds. Appears enlarged. The surface reddish brown, with paler mottling along the course of minute veins. In fresh sections very extensive fatty degeneration observed. Each cell contains from 1 to 5 fat globules as large as red corpuscles. Bile injection observed in small, restricted areas. In fresh teased preparations corpuscles with large parasites readily detected.

About one pint of bile in gall bladder. It contains an abundance of flakes and mucus, so that it pours like very thick molasses, cohering in long flat bands as it leaves the vessel.

Kidneys on surface and section have a uniformly brownish-red color, with exception of tips of papillæ, which are somewhat paler. In fresh microscopic sections much granular pigment observed, some in the form of easts in the straight tubules.

In the straight vessels of cortex and medulla masses or plugs of red corpuscles may be seen, every one containing one or two large, roundish parasites. In a teased preparation in one field out of 11 corpuscles 4 contained pairs of pyriform parasites.

Urine reddish yellow. A slight flocculent precipitate forms on standing. Acid. Sp. gr., 1013. A small quantity of albumen present.

Digestive tract: Mucosa of fourth stomach has a faintly bluish color. The characteristic pits due to Strongylus Ostertagi present. Mucus in small intestine slightly inereased in quantity. Cæcum thoroughly impacted with very dry fecal balls. The same condition of colon and rectum.

In stained preparations of spleen about 2 per cent of the red corpuscles coutain single or paired pyriform bodies. Liver as many as 5 per eent.

Kidneys: An immense number of free parasites. Of the small number of corpuscles present fully 30 per cent contain pairs of pyriform bodies. (For temperature record, see p. 279.) 


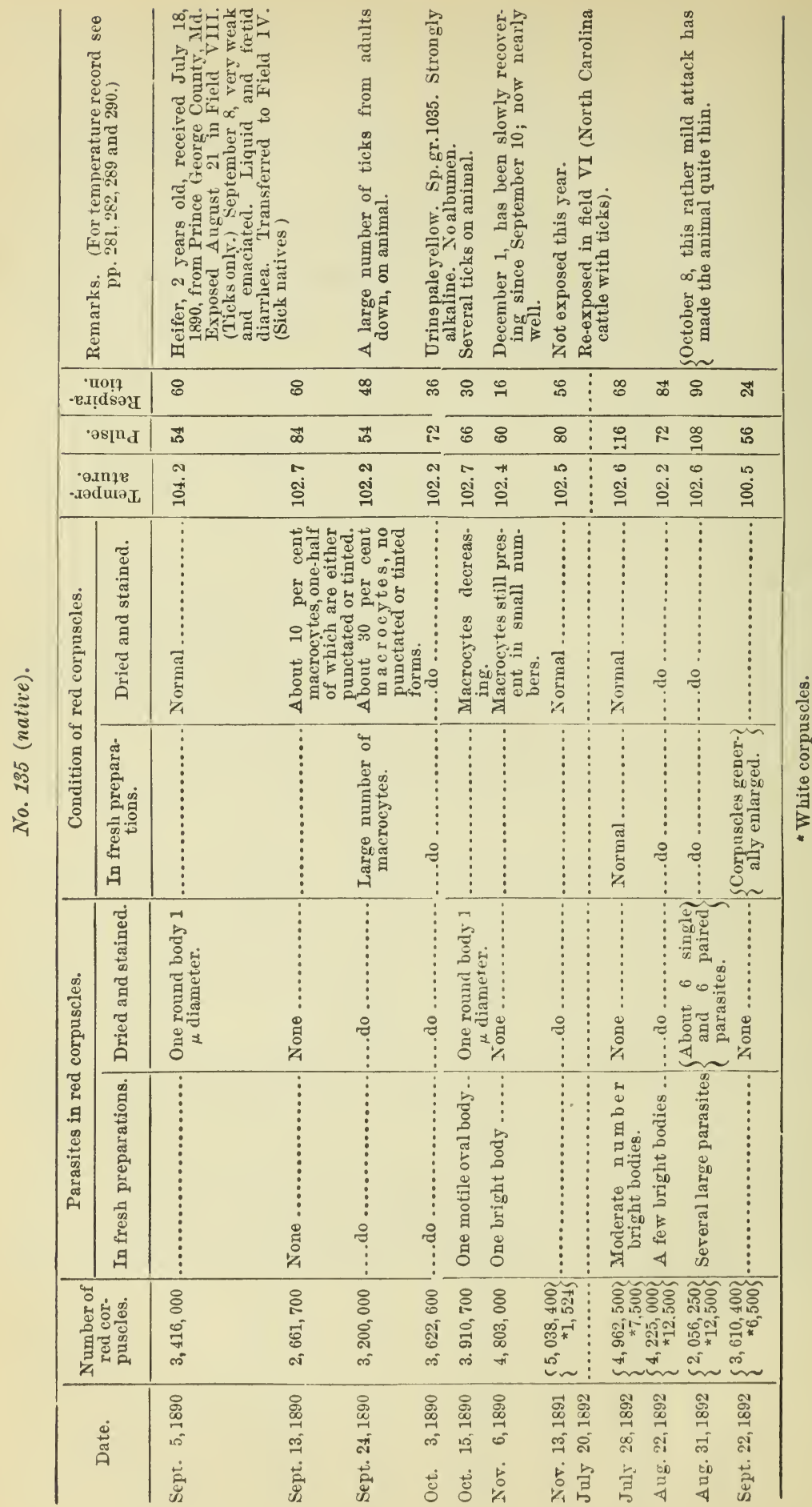




\begin{tabular}{|c|c|c|c|c|}
\hline \multicolumn{2}{|c|}{ 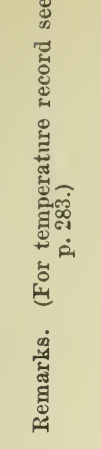 } & \multicolumn{3}{|c|}{ 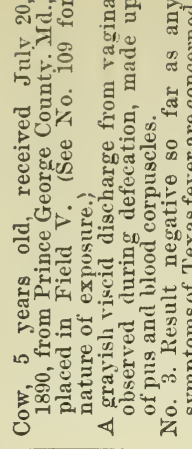 } \\
\hline \multicolumn{2}{|c|}{$\begin{array}{c}\text { •uot7 } \\
\text {-xxịdsay } \\
\end{array}$} & $\vec{N}$ & థి & নే \\
\hline \multicolumn{2}{|c|}{$\cdot \theta s\left[u_{d}\right.$} & ळ & $\stackrel{\infty}{\rightarrow}$ & $\stackrel{\infty}{\sharp}$ \\
\hline \multicolumn{2}{|c|}{ 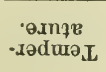 } & ๙ั. & 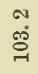 & 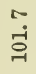 \\
\hline \multirow{2}{*}{ 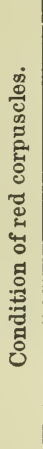 } & 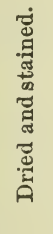 & 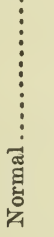 & ج̊ & $\begin{array}{c}\vdots \\
\vdots \\
\vdots \\
\vdots \\
\vdots \\
\vdots \\
\vdots\end{array}$ \\
\hline & 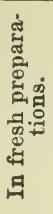 & 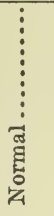 & ְุ & $\begin{array}{c} \\
\vdots \\
\vdots \\
\vdots \\
\vdots \\
\stackrel{\rho}{0}\end{array}$ \\
\hline \multirow{2}{*}{ 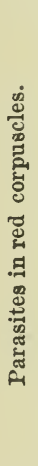 } & 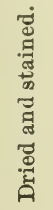 & 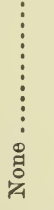 & ㅇํ & $\begin{array}{c}\vdots \\
\vdots \\
\vdots \\
\vdots \\
\vdots \\
\vdots\end{array}$ \\
\hline & 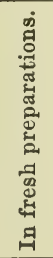 & 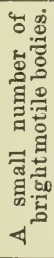 & \begin{tabular}{l}
$\vdots$ \\
$\vdots$ \\
$\vdots$ \\
$\vdots$ \\
\hdashline
\end{tabular} & 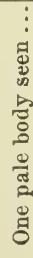 \\
\hline \multicolumn{2}{|c|}{ 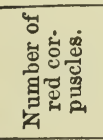 } & $\begin{array}{l}8 \\
\text { के } \\
\overline{5} \\
\infty^{-1}\end{array}$ & $\begin{array}{l}8 \\
\infty \\
10 \\
\text { का } \\
\text { is }\end{array}$ & $\begin{array}{l}\text { के } \\
\text { फ़ } \\
\text { c5 }\end{array}$ \\
\hline \multicolumn{2}{|c|}{ ब̊ँ } & 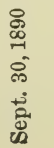 & $\begin{array}{l}\stackrel{8}{8} \\
\stackrel{\infty}{\sim} \\
\infty\end{array}$ & 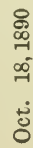 \\
\hline
\end{tabular}

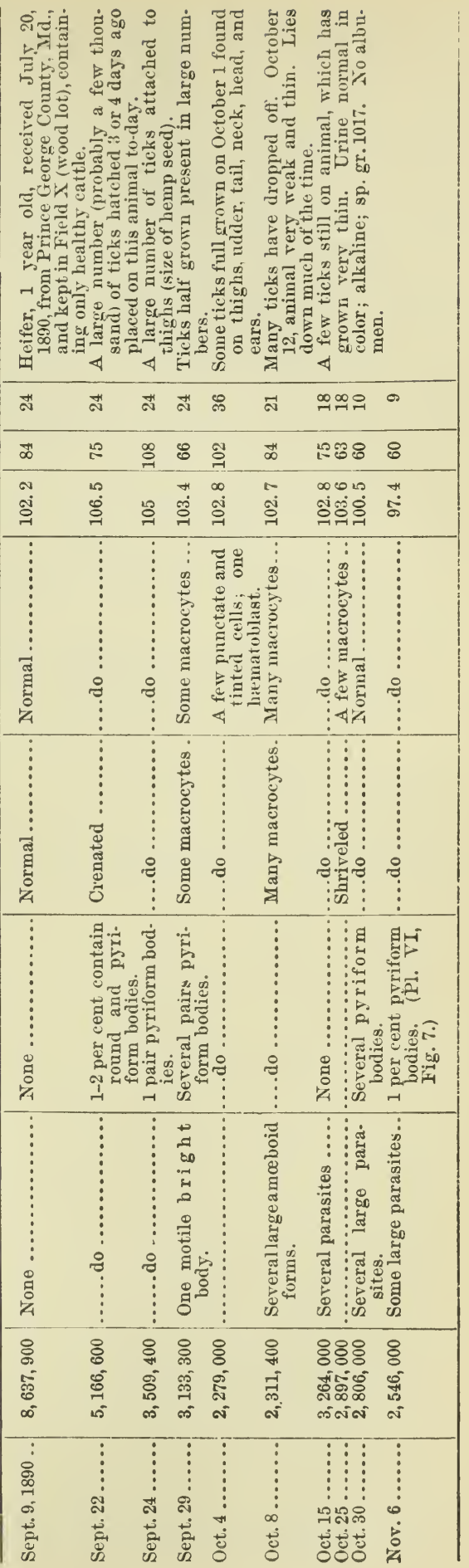


From October 25 the emaciation was extreme. It finally became so weak that it was unable to move. On October 30 it had been lying in one position for thirty-six hours past, chewing the eud incessantly, eating a little nay and drinking water freely. On November 2 it passes into a partly comatose condition. Eating and rumination have ceased. On November 6 it was killed by a blow on the head.

Autopsy: Extreme emaciation. Heifer weighs 200 pounds. Original weight about 350-400 pounds. A few small, and one nearly full grown tick still on animal. Edema of the subentis over the region of the sternum. Indications of fat absorption everywhere in the large cavities. Blood clots quickly and firmly.

Lungs normal. Heart somewhat flabby ; fatty, and cloudy changes of fibers of left ventricle. Spleen weighs $\frac{1}{2}$ pound, small, flabby. Malpighian bodies prominent. Much pigment in lumps found in teased preparations, both free and intracellular.

Liver, small; weighs about 4 pounds. In general pale and bloodless. Cells contain much pigment in form of minute granules. There are also a considerable number of cells observed in teased preparations up to $15 \mu$ in diameter completely filled with lumps of brownish pigment. These are found quite uniformly distributed when sections are made.

Gall bladder contains nearly a pint ( $375 \mathrm{cc}$.) of viscid, dark bile, holding in suspension a moderate quantity of flakes.

Kidneys pale. No gross lesions observable. In sections of fresh tissue considerable pigment in lumps in glomeruli; in the epithelium generally much pigment in minute granules.

Urine from bladder yellowish, faintly alkaline. Sp. gravity, 1028. No albumen.

Digestive tract: Contents of fourth stomach smell badly. Considerable mucus on the membrane, which is beset with worm pits. Many worm modules in walls of ileum. In large intestine a considerable quantity of turbid liquid. Many specimens of Esophagostoma in the rectum and upper colon imbedded in a mass of partly coagulated blood mixed with mucus.

In stained preparations of blood from the right heart, of spleen, liver, and kidney, one red corpuscle in every one or two fields of the microscope contains a pair of pyriform bodies. (For the temperature record, see page 283.) 


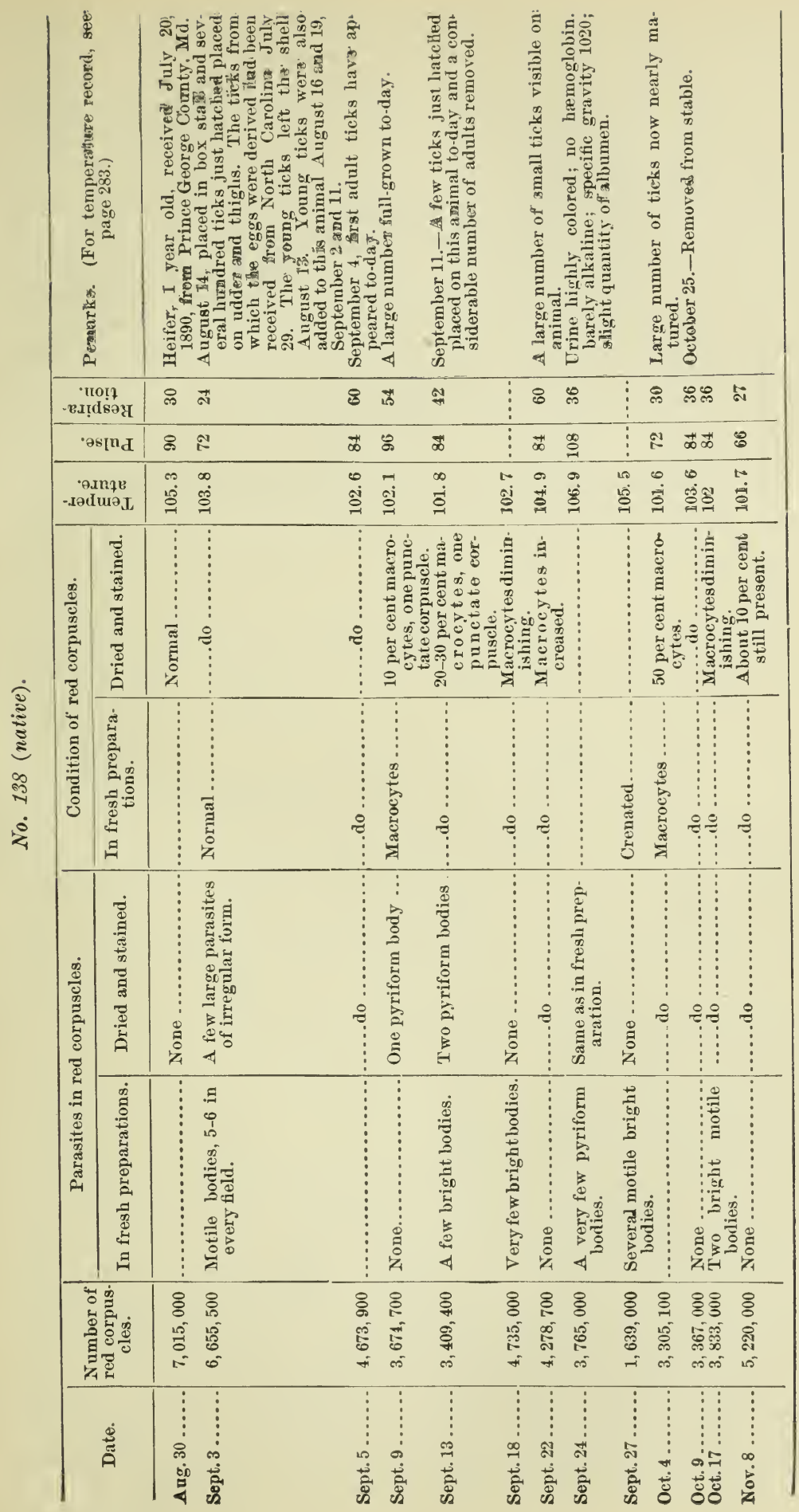


No. 133 (native).-Cow, 6 years old, received August 30, 1890, from Prince George County, Md., and exposed in field II ('Texas cattle with ticks).

September 11.-Temperature, 105.2; pulse, 84; respiration, $72 . \quad$ Red corpuseles $5,285,000$. In fresh preparations of blood a comparatively large number of eorpuscles contain bright motile points, perhaps 10 or more in each field. In stained preparations no abnormal elements detected.

September 13.--Cow dead this morning. Some animal heat still present in the body. Quite sick yesterday evening, but still on her fect.

Autopsy: Animal weighs about 700 pounds. A small number of quite small ticks attaehed to body. Blood from a subcutaneous vein was examined at 9:50 a. $\mathrm{m}$. In fresh preparations a considerable number of eorpuscles contain the bright bodies. A small number contain each one or two roundish pale bodies 1 to $2 \mu$ in diameter.

In stained preparations about 10 per cent of the corpuscles arc infected. The parasites are not all of the same age. Some are round bodics 0.7 to $0.8 \mu$ in diameter, situated near or at the periphery of the corpuscle. Usually but one in a corpusele. In a small pereentage the parasites are in pairs, from 1 to $2 \mu$ in diameter, slightly pear shaped and more centrally situated.

In blood from the heart the fresh preparations show numerous corpuscles containing the bright bodies as well as the larger pale parasites. The stained preparations do not differ from those obtained from the blood of the subcutaneous veins.

Lungs not fully collapsed. The small anterior lobes somewhat hyperæmic and emphysematous.

Heart shows more or less ecchymosis under epicardium of left ventricle, with petechiæ on the fat at the base. In both ventricles a small clot with some fluid blood. Extensive extravasation under endocardium of the left ventricle. One small area in the right. Myocardium normal.

Spleen weighs $4 \frac{1}{8}$ pounds; engorged, very friable. The pulp has a dark-red glistening appearance, obscuring Malpighian bodies entirely.

Liver weighs 16 pounds; enlarged, edges rounded. Much blood flows from the liepatic veins on section. In the larger ones are oceluding thrombi $6-7 \mathrm{~mm}$ in diameter, some branched. They are mottled grayish and dark red and appear to be composed chiefly of blood plates. In teased preparations and fiesh sections of liver tissue the bile injection is found generalized and the fatty degeneration coextcnsive with it. Occasionally minute red needle-like erystals observed. In sections of liver tissue (hardened in alcohol and embedded in chloroform paraffin) stained in hamatoxylin, the trabecular armugement of the parenchyma has largely disappearcd. The cell protoplasm is finely vacuolited in some places. In others the vacnoles are quite large. There is more or less nuclear disintegration over the inner zones of the lobule. Cells with normal and partly degenerated nuclei and some without nuclei are intermingled.

In the gall bladder about one pint of bile, which loolds in suspension an abundąnee of yellowish flocculi, but still flows readily.

Kidneys show intense congestion throughout. All eapillaries gorged with blood. In tubules lumps of yellowish pigment. In sections of kidney tissue (hardened in Miiller's fluid and embedded in elıloroform paraftin) stained in lianiatoxylin, the parenchyma appears undistmbed by pathological changes. In all the capillaries of the glomeruli and the tubules which are disteuded with red corpuseles, the latier quite invariably contain each one or two parasites. Urine in bladder (2 to 3 quarts) has a decp port-wine color and is quite opaque. $\Lambda$ faint light transmitter through a layer $\frac{8}{4}$ inch deep. Specifie gravity 1027. Alkaline. Acetic aeid gives an abundant flocenlent precipitate. With kshach test 2.1 per cent albumen present.

Digestive tract: Contents of third stomach dry and hard. Mucosa of fonth stomach is of a dark, bluish-pink color. Worm pits abundant. In the pylorie portion a considerable number of erosions with blackish base and puekered margins. 
The mucosa of the small intestine hyperæmic and covered with a thin layer of mucus.

Cæcum contains soft feces. Mucosa hyperæmic. In the rectum considerable pigmontation along summits of longitudinal ridges. Feces soft.

In stained proparations the following approximate figures of infected corpuscles were obtained:

In blood from subcutis and right heart, 10 per cent; in spleen, 20 to 30 per cent; in liver, 20 to 30 per cent; in kidneys, 50 per cent; in addition a very large number of free parasites coming from disintegrated corpuscles. Perhaps one-half of the infected corpuscles contain each a pair of parasites. They are 1 to $2 \mu$ in diameter. (For the temperature record, see pp. 274, 275, and 276.)

No. 140 (native).-Heifer, 2 years old, from Prince George County, Md. Received September 9, 1890, and placed in box stall on a neighboring farm on which Texas fever had never existed. The animal, therefore, has not been on the station grounds. Blood corpuscles, 6,047,000. In several preparations of fresh blood a small number (perhaps a dozen found in a preparation after a little searching) of bright bodies observed within corpuscles, some in motion.

Several thousand young ticks, hatched three to four days ago, were placed on the thighs, udder, and flank of this heifer, which was thrown for this purpose. (See also No. 137.)

September 22.-Temperature, 105.5; pulse and respiration, 96. Corpuscles, $5,790,000$. The number of bright intraglobular bodies increased. Several small parasites observed within corpuscles in dried and stained preparations. Animal well covered with young ticks the size of hemp seeds.

September 27.-Temperature, 104.4; pulse, 84; respiration, 96. Corpuscles, $2,800,000$. Tend to crenate and shrivel. Bright bodies diminished in number. No parasites detected. The urine examined to-day is normal. The amount of carbonates diminished.

September 30.-Temperature, 102.5; pulse, 96; respiration, 78. Corpuscles, $2,628,500$. A considerable number of macrocytes now present. In general the corpuscles show a tendency to crenate. In fresh preparations a moderate number of intraglobular, rather large, paired parasites are detected. In stained preparations about $\frac{1}{2}$ to 1 per cent of the corpuscles are infected with large parasites. There are also about 2 per cent of the cells punctate, and a smaller number are diffusely stained. Brought to experiment station to-day and placed in a stall in the baru.

October 2.-Found dead in her stall, with head drawn back and to one side. Considerable heat still in body.

Autopsy at 10 a. m.: Animal more or less wasted by the disease; weighs about 500 pounds. On thighs, udder, neck, and shoulders a large number of ticks, some fullgrown and ready to drop off; others just completing the last molt.

Lungs retain expanded form when thorax is opened. One of smaller lobes on the right slightly hyperæmic. In both bronchi are firm, dark blood clots, extending into branches. There is no blood in the mouth or upper air passages nor in the œsophagus. The source of the blood not discoverable.

Heart: Ecchymoses on the left ventricle, with marked injection of the smallest vessels visible to the naked eye. 'The right heart contains a firm, dark clot extending through auricle into large veins. Left contains a small clot, quite firmly contracted. Fatty degeneration of fibers pronounced.

Spleen weighs $3 \frac{1}{2}$ pounds. Parenchyma soft and yielding; congested. Malpighian bodies prominent. Very little pigment observed in teased preparations.

Liver weighs about 11 pounds. More or less enlarged. Parenchyma of a pale brownish-red color; texture still firm. In the hepatic veins a considerable quantity of fluid blood. In sections of fresh tissue the bile injection appears only in small patches, accompanied by extensive fatty changes. In the remainder of the parenchyma the hepatic cells contain much pigment in the form of granules barely visible 
About 400 cubic centimeters of very thick, turbid bile in the gall bladder, holding a large quautity of yellowish flocculi in suspension.

The kidneys are very pale throughout, and quite flabby to the touch. In fresh sections no marked changes in the epithelium observed.

The bladder contains $\frac{3}{4}$ liter of light claret-colored urine. Faintly alkaline; specific gravity, 1012. Slight flocculent precipitate after boiling.

Digestive tract: Contents of third stomach somewhat firm. In fourth stomach from 1 to 2 liters of a turbid liquid. Mucosa quite pale and free from anatomical changes. In duodenum, bile-staining marked. Occasional patches of vascular injection. In the walls of the ileum worm tubercles. The mucosa normal. Soft feces present.

In the cæcum a large quantity of soft feces; colon and rectum empty. Mucosa throughout unaffected.

In the various organs infected corpuscles are present, but in small numbers. The parasites are roundish, about $1.5 \mu$ in diameter. Fully one-half of the infected corpuscles contain two, the rest one parasite. Their distribution is as follows: Heart's blood very few ; spleen 2 to 3 per cent; liver and kidneys 5 to 10 per cent. (For temperature record, see p. 283.)

No. 142 (native).-Heifer, 1 year old when received, September 16, 1890, from Prince George County, Md.

Blood was taken on this day from heart of No. 90 about one-half hour after death. This was beaten in a sterile bottle with sterile glass rods and kept at $35^{\circ}$ to $40^{\circ} \mathrm{C}$. in a warm chamber from 2 to $3: 30 \mathrm{p}$. m.

The left external jugular vein of No. 142 was exposed by making an incision through the skin $1 \frac{1}{4}$ inches long. The needle of a syringe was made to puncture the wall of the vein made tense at the root of the neck by pressure, and 7 cubic centimeters of blood injected slowly. Three stitches were used to bring together the edges of the skin incision. The animal was placed in the same inclosure with No. 111 (field I) and a healthy control animal, No. 143, from the same source, was placed with it. The wound healed quickly and normally. The following table gives the result of the examinations of the blood. The complete temperature record may be found on page 275 :

No. 142 (native).

\begin{tabular}{|c|c|c|c|c|c|c|}
\hline \multirow[b]{2}{*}{ Date. } & \multirow{2}{*}{$\begin{array}{l}\text { Number } \\
\text { of red cor- } \\
\text { puscles. }\end{array}$} & \multicolumn{2}{|c|}{ Parasites in red corpuscles. } & \multicolumn{2}{|c|}{ Condition of red corpuscles. } & \multirow{2}{*}{ 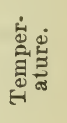 } \\
\hline & & $\begin{array}{l}\text { In fresh } \\
\text { prepara. } \\
\text { tions. }\end{array}$ & Dried and stained. & $\begin{array}{c}\text { In fresh prepara- } \\
\text { tions. }\end{array}$ & Dried and stained. & \\
\hline 1890 & & & & & & \\
\hline Sept. 16 & $6,890,000$ & & None - & & & 102. 2 \\
\hline Sept. 22 & $5,430,000$ & None... & Two doubtful forms & Normal ......... & Normal ................ & 100.8 \\
\hline Sept. 24 & $4,562,500$ & & Two doubtful forms & $\ldots .00 . . . . . . .$. & A few macrocytes.. & 101.8 \\
\hline Sept. 29 & 5,274500 & None... & None ................... & ....do........... & Normal............ & 102.6 \\
\hline Oet. 4 & $3,902,400$ & n........ & $\begin{array}{l}\text { Several parasites of } \\
\text { i) regular form. }\end{array}$ & .....do.... & .... dlo ..... & 100.2 \\
\hline $\begin{array}{lr}\text { Oet. } & 8 \\
\text { Oct. } & 22\end{array}$ & $\begin{array}{l}5,983,606 \\
4,333,000\end{array}$ & $\begin{array}{l}\text { None... } \\
\text {.. do .... }\end{array}$ & None & $\begin{array}{l}\text { A fow maerocytes } \\
\text { flo....... }\end{array}$ & $\begin{array}{l}\text { A fow macrocytes } \\
\text { do }\end{array}$ & 102.5 \\
\hline Nov. 4 & $5,586,000$ & ...do .... & $\ldots$. do $\ldots . . . . . . . . .$. & ...do................ & ....do ............... & 102 \\
\hline
\end{tabular}




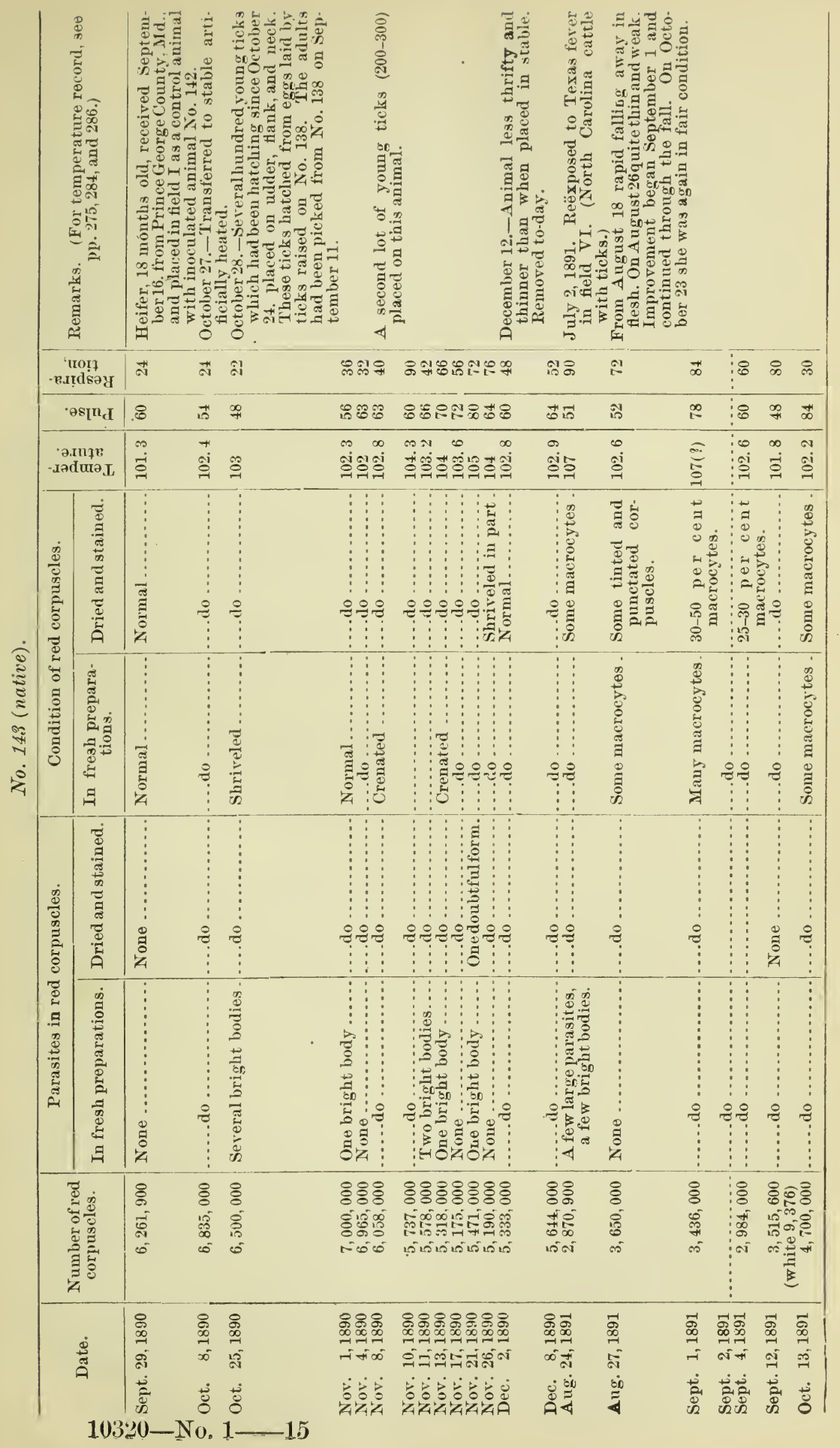


No. 144 (native).--Cow, 8 years old, received September 17, 1890, from Maryland, and placed in wooded Field $X$ (Fig. 6, p. 102). On this day a considerable number of ticks hatcher in the laboratory were placed on this animal. The hatehing began September 4 and continued several days. (See also Nos. 137 and 140, to which ticks from the same lot were applied.)

September 18, $11: 30$ a. m.-Temperature, 101.2. Red corpuscles, 6,299,000; normal. In fresh blood a few corpuscles contain bright motile bodies. Nothing detected in stained preparations.

September 25.-A second lot of young ticks placed on this animal.

September 29.-Temperature, 106.2; pulse, 54; respiration, 27. Rerl corpuscles, $4,934,000$. In fresh preparations a few corpuscles contain bright motile bodies. In one corpuscle a large parasite. In stained preparations about one infected corpusele in every two or three fields. The contained parasite is irregularly roundish and about $1 \mu$ in diameter. A large number of small ticks visible between thighs and on escutcheon.

October 3.-Cow found dead this morning; seen alive yesterday at $5 \mathrm{p} . \mathrm{m}$.

Autopsy notes.-Body in fairly good condition, weighing abont 550 pounds. A large number of ticks on the inside of the thighs down to hocks, on the escutcheon, and udder; a few on neck, shoulders, and in the axilla.

Heart.-A considerable number of punctiform hentorhages nuder the epicardium of both ventricles. In the right ventricle a small dark clot and some fluid blood. In the left, which is quite firmly contracted, a little clotted blood. Much extravasation under the endocardium of this ventricle; especially marked on the papillary muscles.

In blood from the heart examined fresh a snall number of corpuscles contain motile and nonmotile bright bodies. Several contain the large stage of the Texasfever parasite. Examination made unsatisfactory, owing to post-mortem shriveling and distortion of the red corpuseles.

Lungs only partially collapsed, slightly congested, and oedematous. Trachea and bronchi eoated with fine froth; mucosa of trachea reddened.

Spleen weighs $4 \frac{1}{8}$ pounds. Uuder the capsule several large patches of extravasation. Pulp very soft, dark. Usual markings no longer visible on section. Very little pigment detected under mirroscope.

Liver weighs about $12 \frac{1}{2}$ ponnds. Pale yellowish brown. On section, the yellowish color very marked. The cut ends of the hepatic vessels discharge thick bloor. On the various surfaces are seen irregular yellow spots about $\frac{1}{2}$ inch in diamoter, which eorrespond to yellow foci extending into the liver tissue for $\frac{1}{4}$ to $\frac{1}{2}$ inch. In fresh sections under the microscope abont $\frac{a}{4}$ of each lobule las its bile eapillaries distended with bile, accompanied by extensive fatty degeneration. The yellow spots above mentioned represent masses of tissue completely degenerated. Numerous microscopic blood-red needle-like erystals in the fresh tissue. (Plate 1II, Fig. 2.)

In the gall blarlder the bile is very thick with solids and mueus, so that it scarcely flows when poured.

Kidneys miformly and deeply congested. In the convoluted tubes of cortex a large quantity of fine yellowish pigment nay be seen in fresh seetions. In the bladder over three pints of uriue, having a deep port-wine color. It is barely translucent in a $\frac{3}{4}$-inch test tube. Feebly alkaline. Specific gravity, 1015. Heavy flocculent preeipitate, with aretic acicl in the rols. Abont 0.6 per cent albunen present. In the various organs and tissues examined stained, the intraglobular parasites appear as round cocens-like bodies, either one or two in a corpuscle from 1 to $1.5 \mu$ in diameter. 'The infected corpuscles ne scarce in blood from heart; in the spleen there are ahout 2 per cent. They are slightly more abundant in the liver. In the kirlney there are from 20 to:30 per cent. Besirles theso parasites there are present large post-mortem bacilli, in small mumbers in the blood and splecil, abundant in liver and kidney. (For temperatmre record, sec p. 283.) 


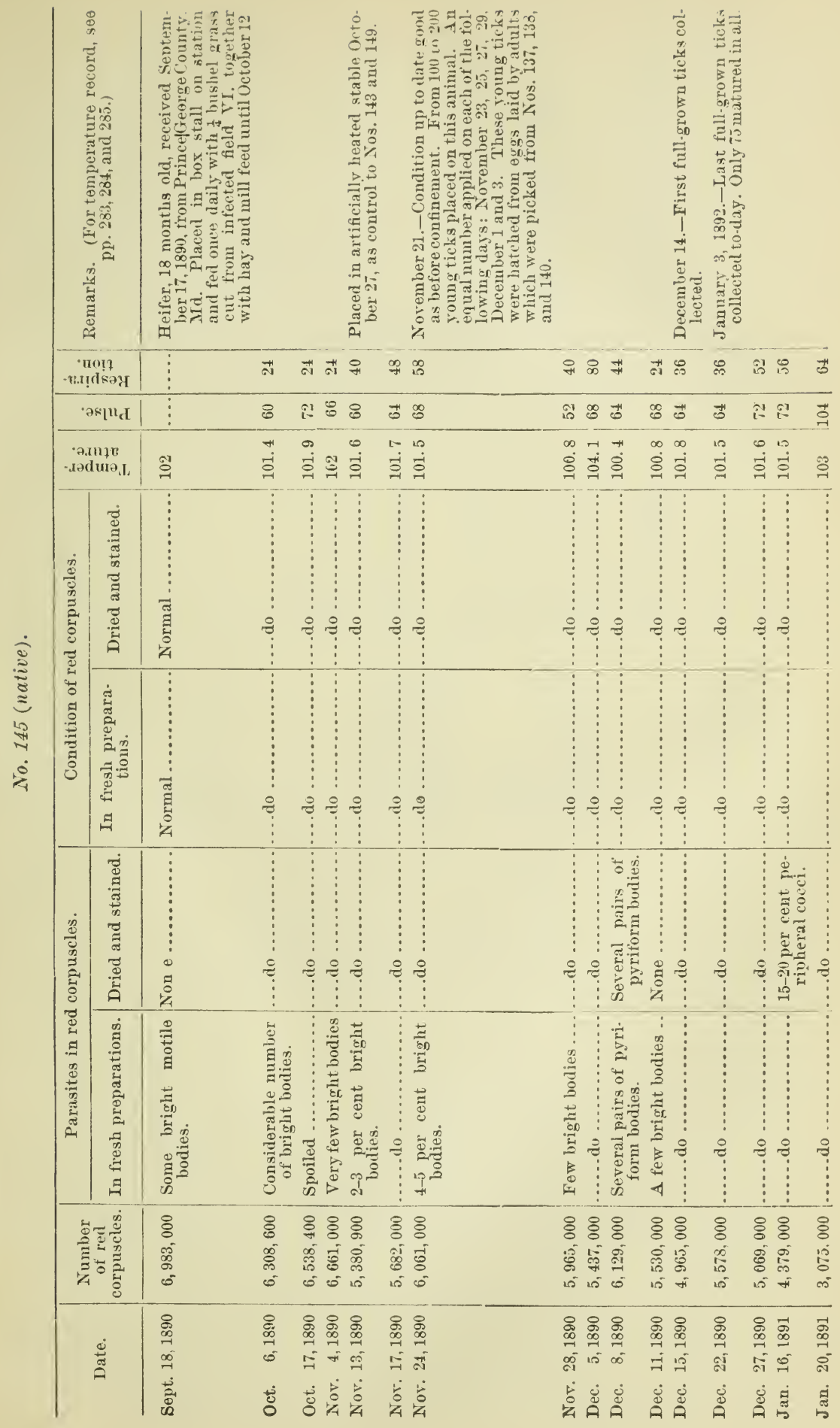




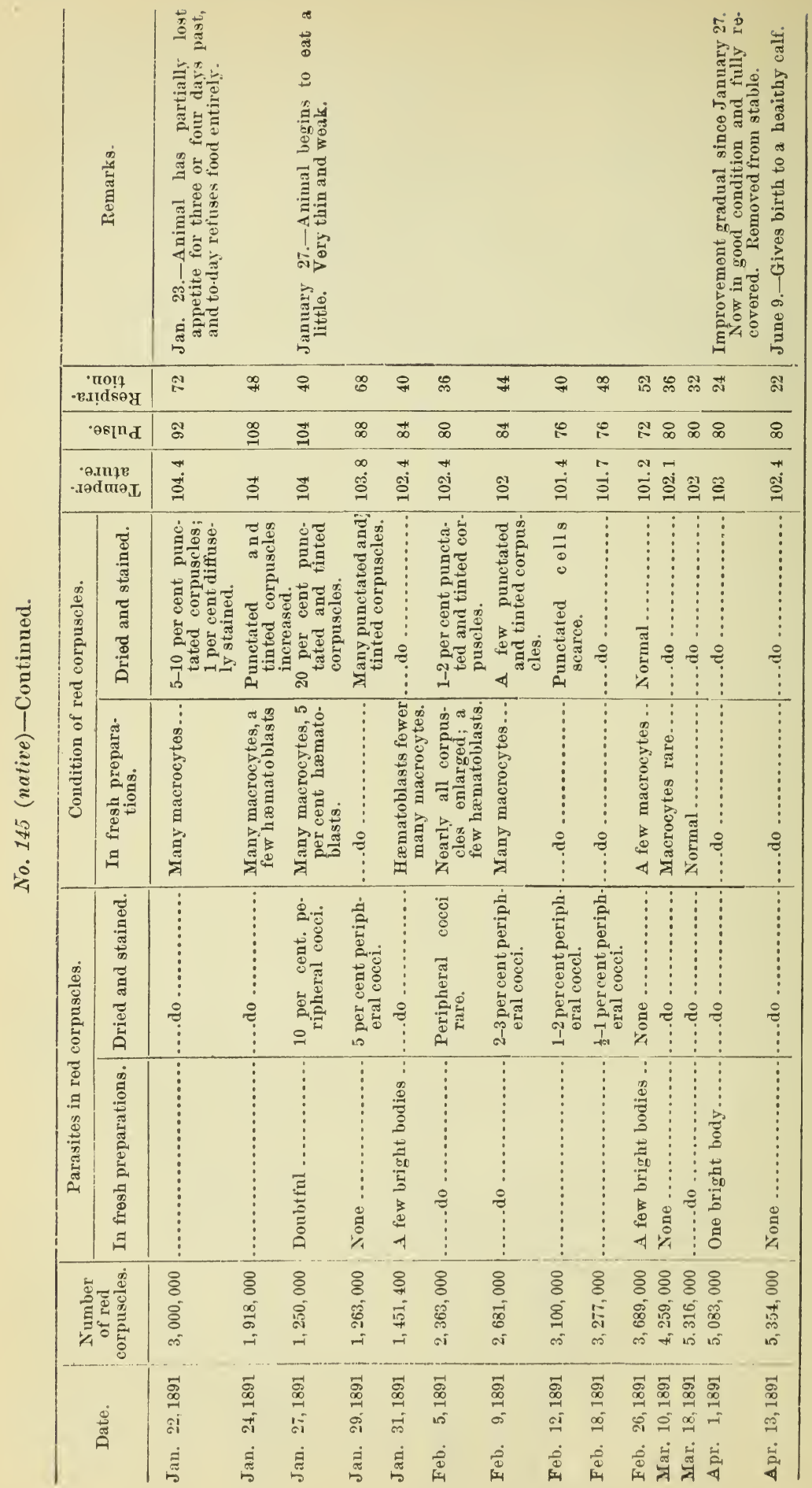




\begin{tabular}{|c|c|c|c|c|}
\hline 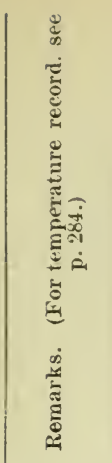 & 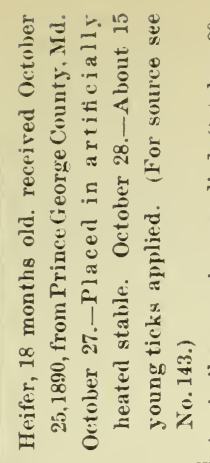 & 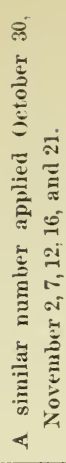 & 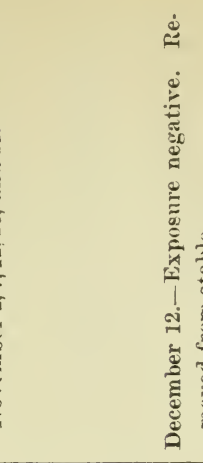 & 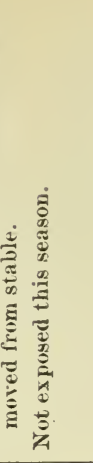 \\
\hline $\begin{array}{c}\text { uolf } \\
\text { rexidsey }\end{array}$ & o & 㐫 & F 공 & $\pi$ \\
\hline$\cdot \partial s\left[n_{d}\right.$ & 8 & 8 & 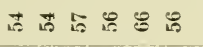 & 8 \\
\hline 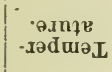 & 量 & $\begin{array}{l}0 \\
\text { sij } \\
\stackrel{5}{0}\end{array}$ & 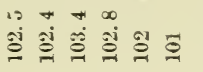 & 离 \\
\hline 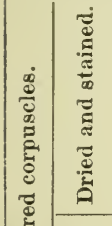 & 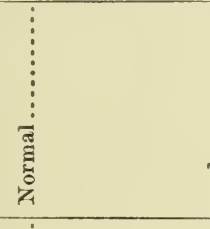 & $\begin{array}{c}\vdots \\
\vdots \\
\vdots \\
\vdots \\
\vdots \\
\vdots \\
\vdots \\
\end{array}$ & 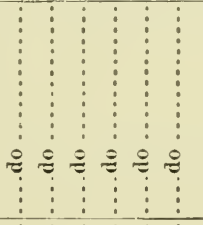 & $\begin{array}{c}\vdots \\
\vdots \\
\vdots \\
\vdots \\
\vdots \\
\vdots \\
\vdots\end{array}$ \\
\hline 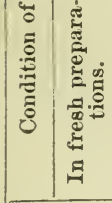 & 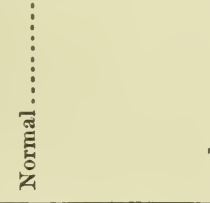 & \begin{tabular}{c}
$\vdots$ \\
$\vdots$ \\
$\vdots$ \\
$\vdots$ \\
\hdashline \\
$\vdots$ \\
$\vdots$
\end{tabular} & 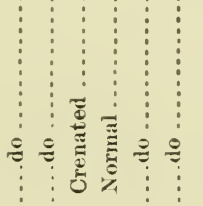 & $\begin{array}{c}\vdots \\
\vdots \\
\vdots \\
\vdots \\
\vdots \\
\vdots \\
\vdots\end{array}$ \\
\hline 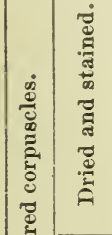 & 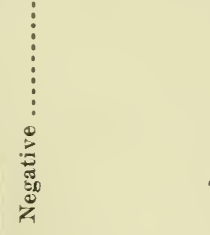 & $\begin{array}{c}\vdots \\
\vdots \\
\vdots \\
\vdots \\
\vdots \\
\vdots \\
\vdots \\
\vdots\end{array}$ & 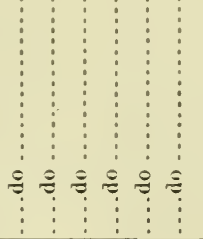 & $\begin{array}{c}\vdots \\
\vdots \\
\vdots \\
\vdots \\
\vdots \\
\vdots \\
\vdots\end{array}$ \\
\hline 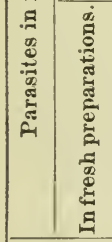 & 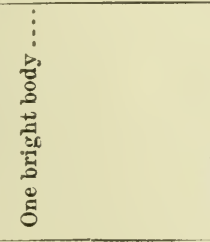 & 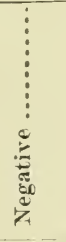 & 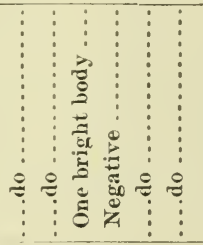 & 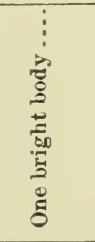 \\
\hline 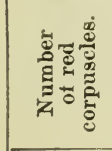 & 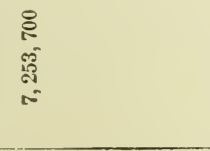 & 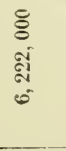 & 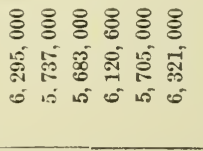 & 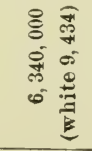 \\
\hline 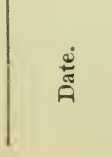 & 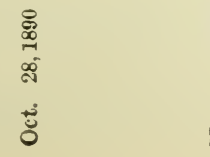 & 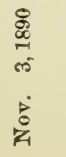 & 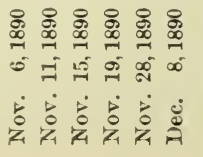 & $\begin{array}{l}\vec{D} \\
\underset{0}{\Delta} \\
\dot{\tilde{\sigma}} \\
\dot{\delta}\end{array}$ \\
\hline
\end{tabular}




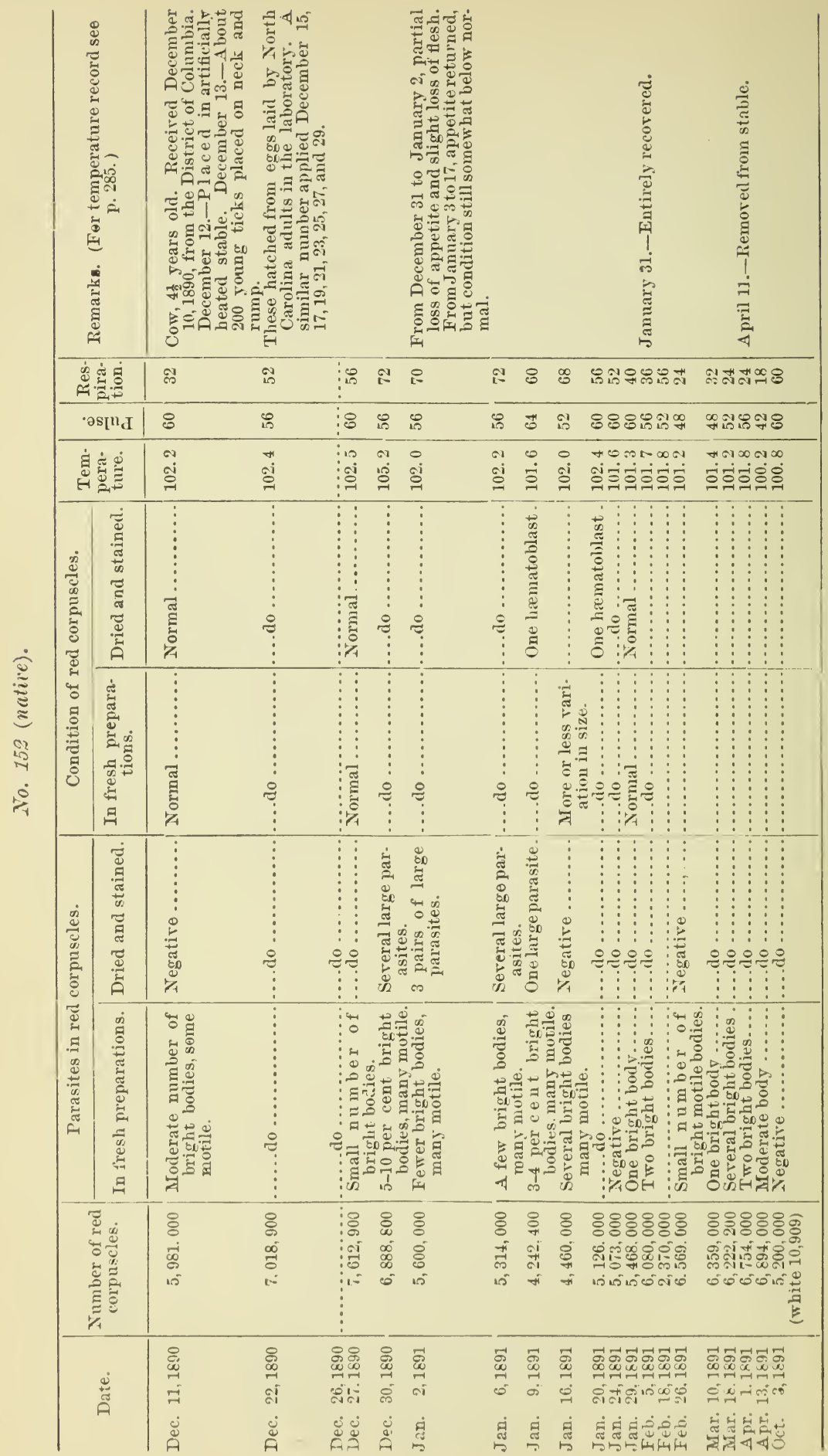


No. 15.4 (natire).-Heifer, 1 year old, received Mareh 16, 1831, from the District of Columbia. In May, placed in wooded field No. X, with several North Carolina cattle of 1889 and 1890 . (The disease was inluced in this animal by ticks which wintered over from the fall of 1890 .)

September 1, 1891.-Temperature, 103.7; pulse, 132 (due to (hasing); respiration, 5.1. The animal to-day is thin and weak. A consiclerable number of ticks of varions stages on the thighs and udder. Condition of the blood: Red corpuscles 2,075,400. In the fresh preparation of blood from skin probably 20 per cent of the corpuscles are eularged (macrocytes). One intraglobular bright body and one hematoblast detected in one fresh preparation. In several stained preparations a large percentage of the macrocytes are either uniformly tinted or else punctated. After a long search, several large pyriform parasites deterted within corpuscles.

September 7.-Temperature, 103.2; pulse, 96; respiration, 52. Red corpuscles, $3,433,300$; white corpuseles, 10,000 . Fully 30 per cent are larger than normal. Tinted and punctated forms have disappeared.

October 6.-A few large ticks on animal. Temperature, 100 ; pulse, 85 ; respiration, 42. Red corpuscles, 4,161,800; white, 10,909. A considerable number are macrocy tes. In stained preparations nothing abnormal besides the variation in size.

November 4.-Temperature, 100.6; pulse, 72; respiration, 24. Rerl corpuscles, $6,984,000$; white corpuscles, 18,781 . Nothing abnormal in stained and fresh preparations. 
TEXAS OR SOUTHERN CATTLE FEVER.

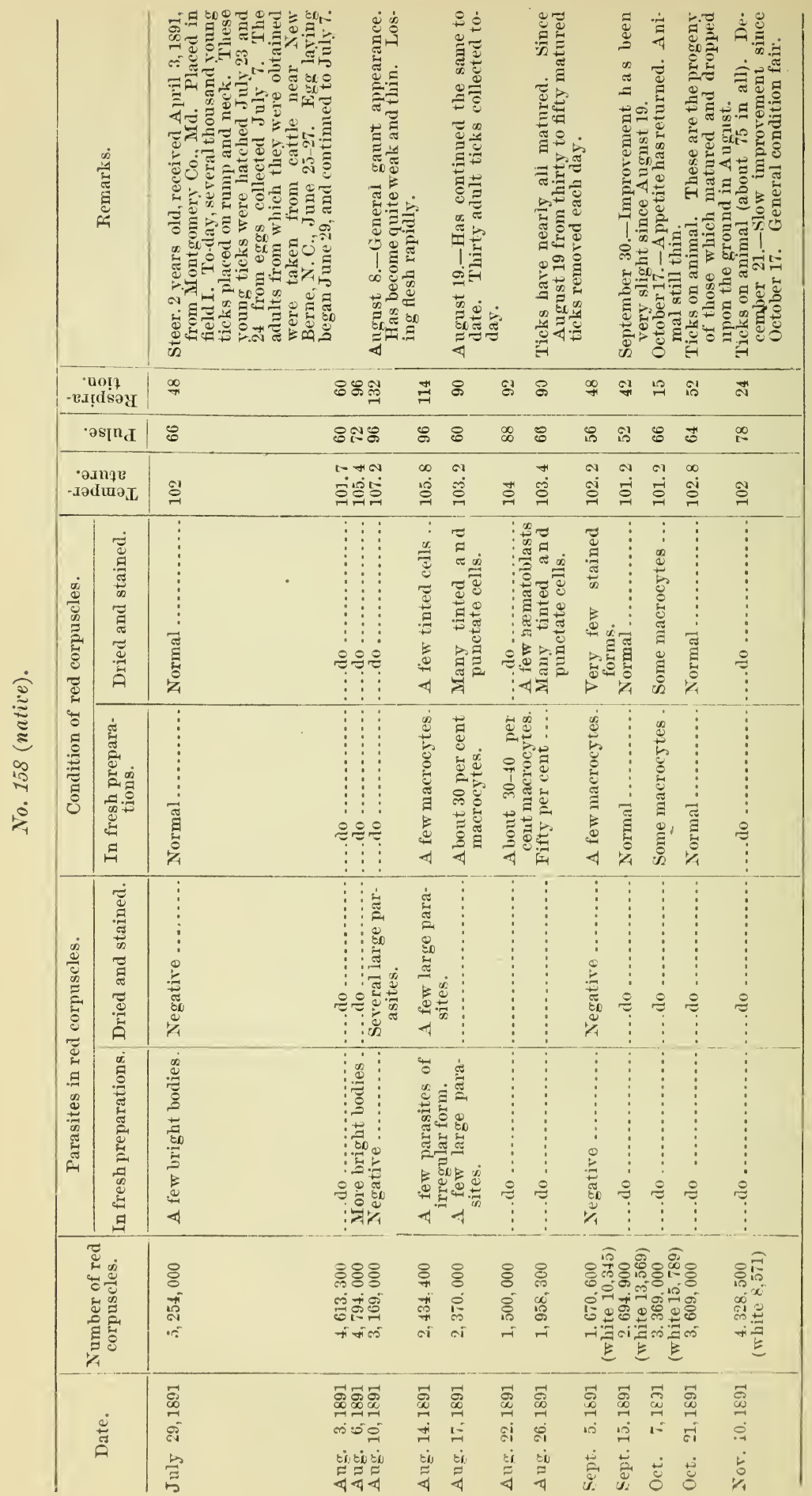


APPENDIX.

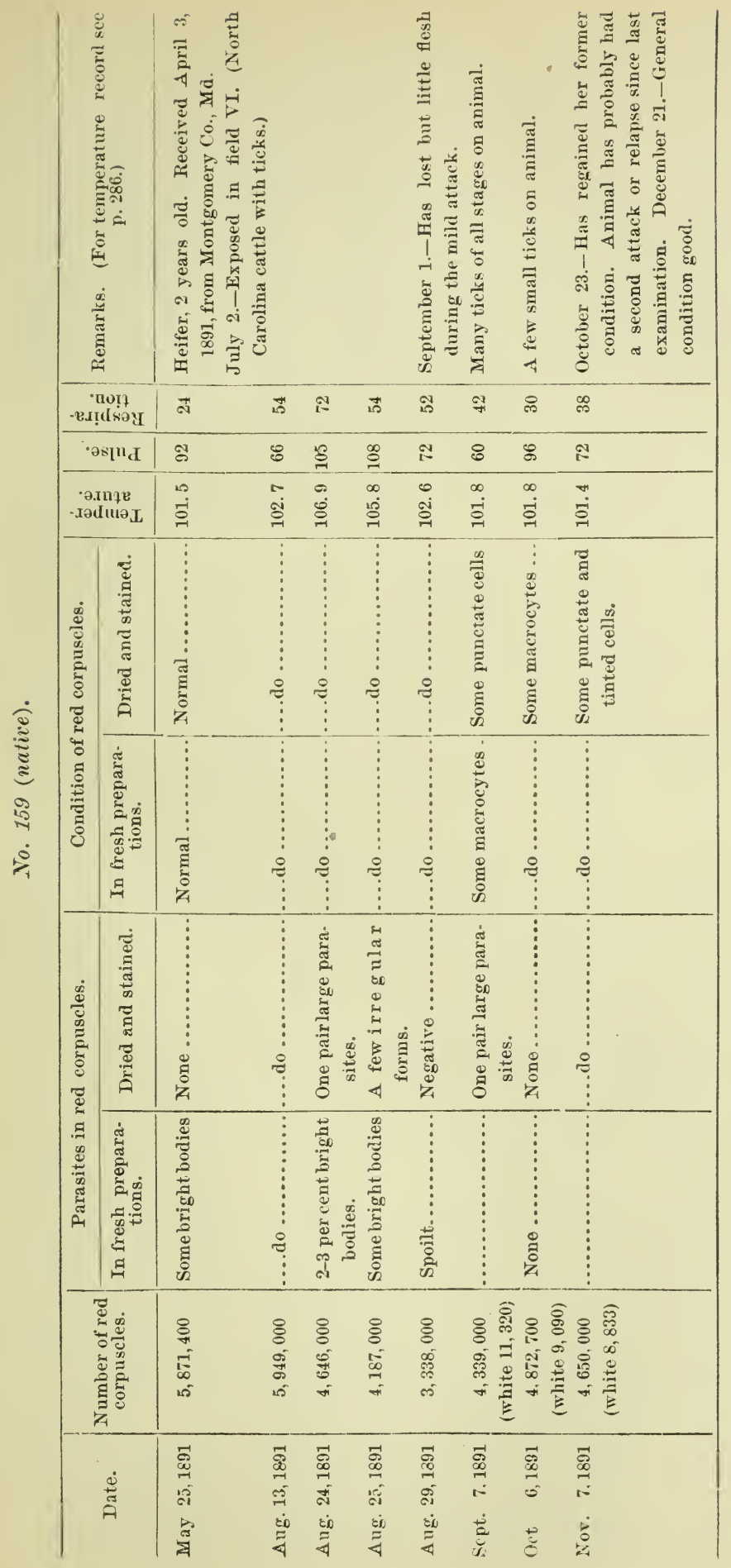




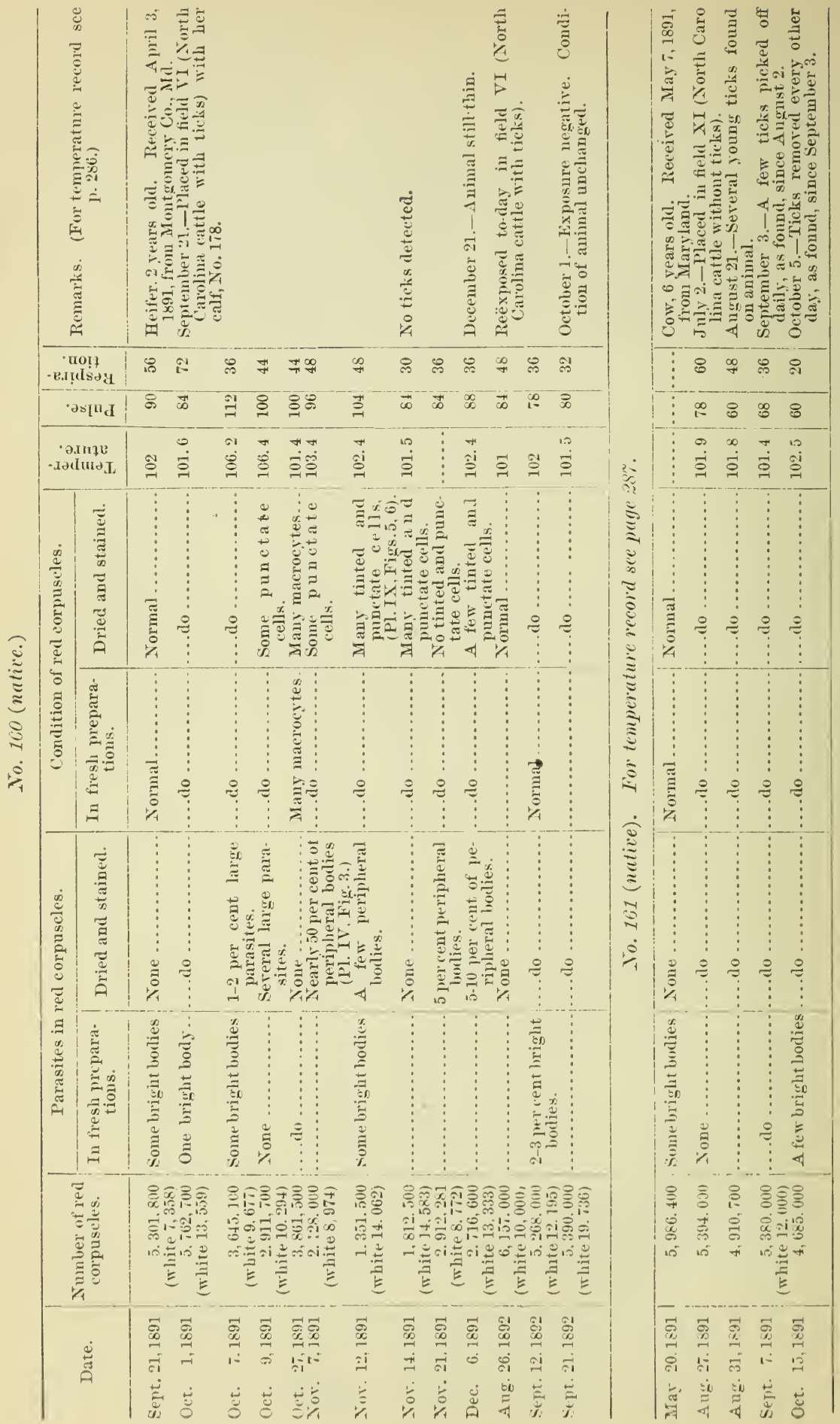


No. 162 (nutive).-Cow, ago 6 years; received September 1, 1891, from Maryland. Placed in uninfected field XII.

September 26.-Animal first observed dull and refusing to eat.

September 28.-Temperature above $104^{\circ} \mathrm{F}$. The animal is very weak, disinclined to move, and remains in shed. Feces passed in small quantities at short intervals; stained yellow and quite liquid.

September 29.-Found dead this morning.

Autopsy: Body fairly well nourished; weighs about 650 pounds. No ticks observed, though very carefully looked for.

In abdomen the omentum shows injected patches. Under the serosa of fourth stomach a patch of extravasated blood 10 inches long and $2 \frac{1}{2}$ to 3 inches wide.

In thorax, lungs somewhat oedematous. In both ventricles of heart considerable fluid blood and a small clot. Capillaries of heart muscle gorged with red corpuscles. Fatty degeneration of fibers.

spleen weighs 4 pounds. Pulp blackish, softened. Usual markings on section no longer visible.

Liver weighs about 16 pounds. On surface and on section the tissue has a diffuse yellowish hue. Fatty degeneration and bile injection as yet slight. In the gall bladder about 6 ounces of yellowish green bile, holding an abundance of flakes in suspensiou.

Kidneys uniformly congested throughout. All capillaries choked with red corpuscles. In the bladder about 1 quart of urine, having a deep port-wine color. Specific gravity, 1022; reaction, neutral. Abundant precipitate, with acetic acid in the cold. Esbach's test gives 3 per cent albumen. In uterus a foetus about 6 months old.

Stained cover-glass preparations from the blood and tissues show an extensive infection of red corpuscles with Texas-fever parasites. The parasites are mainly roundish, either single or in pairs, and not yet full grown. Their approximate ummbers may be given as follows:

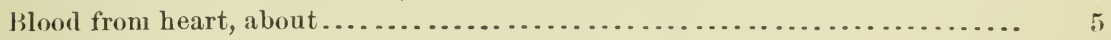

Blood from marrow of rib, about....................................

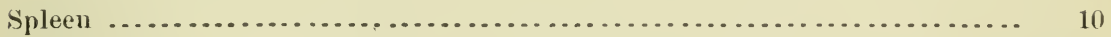

Liver tissue. ................................................. 40-50

Kidneys ...................................................... $70-80$

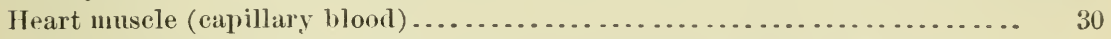

No. 163 (native).-Cow, 6 years old, received May 7 , 1891, from Maryland.

July 2.-Placed in field VI (North Carolina cattle with ticks).

August 13.-Temperature, 102.3; pulse, 54; respiration, 48. Red corpuscles, $5,000,000$. A small number of corpuscles contain briglit bodies. Stained preparations negative.

August 24.-Temperature, 106.8; pulse, 66 ; respiration, 57 . Corpuscles, 3,388,800. In fresh preparations a moderate number of corpuscles containing bright bodies. They vary in size from barely visible points to $\frac{1}{2} \mu$ in diameter $(\times 680)$. The smallest are most active, although all sizes are observed to change their place within the corpuscles. In stained preparations a very few corpuscles contain each a pearshaped parasite.

August 25.-Animal is dull, quite weak, and losing flesh rapidly. Temperature, 107 ; pulse, 80 ; respiration, 24 . Corpuseles, 2,645,000. The blood from the skin, examined fresh, contains corpuseles with bright bodies and some which contain the pale, pear-shaped parasites. Those containing the latter have a degenerated appearance. Amoboid changes deterted on the warm stage.

Killed by a blow on the head at 11.45 a. m., and the autopsy made immediately.

Weight of animal abont 500 poumls. A large number of ticks on the esenteheon and inner surfice of thighs; they are especially abundant on the escutcheon, where 
they are crowded close together over an area 6 inches long by 2 inches wide: on the abdonien and thighs they average abont 4 to the square inch. A considerable numlier are attached to the neck, in front, from the larynx to the sterumm.

In abdomen the omentum over the pannch shows numerous hyperiemic patches.

Heart and lungs normal. Strongyli in the bronchi.

Splern weighs 25 ponnds; engorged with blood-corpuscles as shown on the ent surface, which has a blackberry-jam color. Trabecula and Malpighian bodies barely visible. Several specimens of an unknown filaria in the connective tissne between spleen and paunch. In the pulp, examined fresh, a little pigment in irregular lumps. Liver weiglıs 14 pounds; it appears slightly enlarged and paler than in the normal condition, both on surface and on section. In fresl sections the intralobular capillaries are fomnd filled with corpuscles, the cells somewhat clouded and containing very minnte pigment particles. Bile injection and fatty degeneration absent. The bile, although darker than in healtl, contains no flocculent matter. The mneosa of the fourth stomach quite nniformly reddened thronghout; it is beset with paler spots from 2 to 8 to a square inch (probably the places where Strongylus Ostertagi is encysted). Strongylus contortus abmulant. The villi of the small intestine are injecter, the injection diminishing in intensity from above dowuward. Mucosa of crecum pigmented along the summits of the longitudinal folds. Colon and rectum normal.

Kidneys muiformly and intensely congested. Capillaries distenderl with red corpuscles. The urine in bladder of a dark port-wine color. Specific gravity, 1,020. Alkaline. An abmndant brownish precipitate when acetic acil is added. Witl Esbach's test, 0.3 per cent albnmen.

A large nnmber of dried and stained preparations from blood and the tissnes of various organs were examined to determine the relative abunlance of the infected corpuscles. The parasites were, as a mle, in pairs within the corpuseles, eaclı containing one, very rarely two pairs. In form they were quite invariably pearsliaped. A few were romblish, and of these there was but one in a corpuscle. The relative distribution is approximately as follows:

Marrow of rib, very few infected corpuseles.

Skeletal numscles, very few infected corpuscles.

Blood from skin, very few infected corpuseles.

Blood from left heart, 2 to 3 per cent; blood from right heart fewer than in preceding; blood from lung tissue, 2 to 3 per cent; spleen pulp, 5 per cent; liver tissue, 10 to 20 per cent; kidney tissue, 10 to 20 per cent; lyperiemic spots on onentun, 50 per eent; heart mnscle, 50 per cent and many free forms.

At the antopsy many fresh preparations frou the various regions of the body were likewise serntinized. In achlition to the par-shaped pairs within corpuseles the miunte bright bodies were always detected. (For teniperature record, see p. 286.)

No. 164 (native).-Cow, 7 years old, received May 7, 1891, from Maryland.

July 2.-Placed in field XI (North Carolina cattle withont tieks).

Angust 26.-Temperature, 106.4; pulse, 93; respiration, 100 . Corpuscles 4,142,800. In fresh preparation, a few bright motile intraglobular bodies. No large parasites seen. In stained preparations, after a long searrh, two intraglobular forms of irregnlar ontline seen.

Angust 31.-Temperature, 102 ; pulse, 64 ; respiration, 44 . A large unuber of small tieks on animal. Skin practically bloodless, so that many incisions were made before a drop of blood could be obtained. Red corpuscles, 1,661,000. Both fresh and staiued preparations nogative as regards parasites. Animal quite sick.

September 2.-Animal very weak, lying down aud unable to rise. Has lost flesh rapidly within the past four days. Feces bile-stained. Temperature, 98; pulse, 78; respiration, 20. In two fresh preparatious ouly three infecterl corpuseles detected after a long search. There are present about 10 per ceut of macrocytes, some fully 
twice the diameter of normal red corpuscles. When stained a fer tinted corpuscles and some hematoblasts detected.

September 3.-Found read early this morning.

Autopsy, 3 a.m.: A fow ticks on thighs and urlder. Rather strong odor of decomposition when abdomen opened.

Thorax. Heart appears pale and flabby. In both sides large, dark soft clots. Cloudy swelling of muscular tissue. Lungs adematous. Interlobular empliysema in right principal lobe.

Abdomen. Spleen weighs 4 pounds. Verysoftand flabby. Pulpdifinent. Considerable amonnt of pigment in lumps and gramules.

Liver weighs $15 \frac{1}{2}$ pounds. Enlarged and flabby to the touch. Color markedly paie yellowish, with nccasional spots of deeper yellow sprinkled throughont parenchyma. On closer inspection, the yellowish color is limited to the inner zones of the acini. The cut surface has thus a mottled appearance. In fresh sections much fatty degeneration of the hepatic cells, many of which contains lumps of pigment. Very little bile injection.

Gall bladder contains over a pint of very thick, viscid bile, holding in suspension a large anount of flocculent matter.

Kidneys pale and flabby. Pelvis ecchymosed. No distinct pathological changes observed in fresh sections. Very little pigment in the epithelium. Bladiler empty.

Paunch nearly empty. Contents of third stomach more or less dry, impacted.

Microscopic examination of dried and stained cover-glass preparations gave the following result:

Blood from the heart contained large post-mortem bacilli, but no parasites. Blood from the subcutis equally negative.

In the spleen no parasites but bacilli present. In the kidney and liver very few blood corpuscles; many bacilli but no parasites.

In the nterus a foetus 7 months old was found. Skin stained bright yellow over the entire body. In blood from the heart and in spleen pulp no parasites were detected. (For temperature record, see p. 287.)

No. 165 (native).-Heifer, two and one-half years old; receiver June 22, 1891 , from Maryland. On August 29 it received into the right jugular vein ten cubic centimeters of fluid made by crushing ticks one and one-lialf months old in sterile water and passing the flnid through a Pasteur filter. (See also No. 183.) Up to October 20 no signs of disease could be discovered, as is shown by the following table of the blood examination. The temperature record will be found on page 287 .

\begin{tabular}{|c|c|c|c|c|c|c|c|c|}
\hline \multirow{2}{*}{ Date. } & \multirow{2}{*}{$\begin{array}{l}\text { Number of red } \\
\text { corpuscles. }\end{array}$} & \multicolumn{2}{|c|}{ Parasites in red corpuscles. } & \multicolumn{2}{|c|}{$\begin{array}{c}\text { Condition of red eor- } \\
\text { puscles. }\end{array}$} & \multirow{2}{*}{$\begin{array}{l}\text { Tem- } \\
\text { pera- } \\
\text { ture. }\end{array}$} & \multirow[b]{2}{*}{$\frac{\dot{D}}{3}$} & \multirow{2}{*}{$\begin{array}{l}\text { Res } \\
\text { pira } \\
\text { tion. }\end{array}$} \\
\hline & & $\begin{array}{l}\text { In fresh prepara- } \\
\text { tions. }\end{array}$ & $\begin{array}{l}\text { Dried and } \\
\text { stained. }\end{array}$ & $\begin{array}{l}\text { In fresh } \\
\text { preparations. }\end{array}$ & $\begin{array}{l}\text { Dried and } \\
\text { stained. }\end{array}$ & & & \\
\hline 1891. & & & & & & & & \\
\hline July 25 & $5,233,700$ & Some bright bodies. & Negative & Normal.... & Normal.. & 103.4 & 72 & $\begin{array}{l}72 \\
36\end{array}$ \\
\hline $\begin{array}{l}\text { Aug. } 29 \\
\text { Sept. } 5\end{array}$ & $\begin{array}{l}5,854,000 \\
5,545,000\end{array}$ & 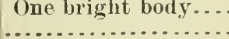 & 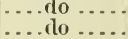 & ....do & $\begin{array}{l}. \text {. } \\
\text {. do } \ldots \ldots . . . . .\end{array}$ & $\begin{array}{l}101.0 \\
101.0\end{array}$ & $\begin{array}{l}54 \\
78\end{array}$ & $\begin{array}{l}36 \\
42\end{array}$ \\
\hline Sept. 10 & $\begin{array}{r}\text { (white 12, 727) } \\
5,673,000\end{array}$ & One bright borly... & ....dlo & Normal. & . . dlo . & 101.6 & 68 & To \\
\hline Sent 18 & $\begin{array}{r}\text { (white } 9,615 \text { ) } \\
5,384,600\end{array}$ & & & & & & & \\
\hline Deper 10 & (white 15, 384) & & & & & 102,6 & 81 & 54 \\
\hline Oct. 15 & $5,188,400$ & One bright body... & ....do. .. & Normal.... & .. dlo ........ & 101.5 & 72 & 36 \\
\hline
\end{tabular}




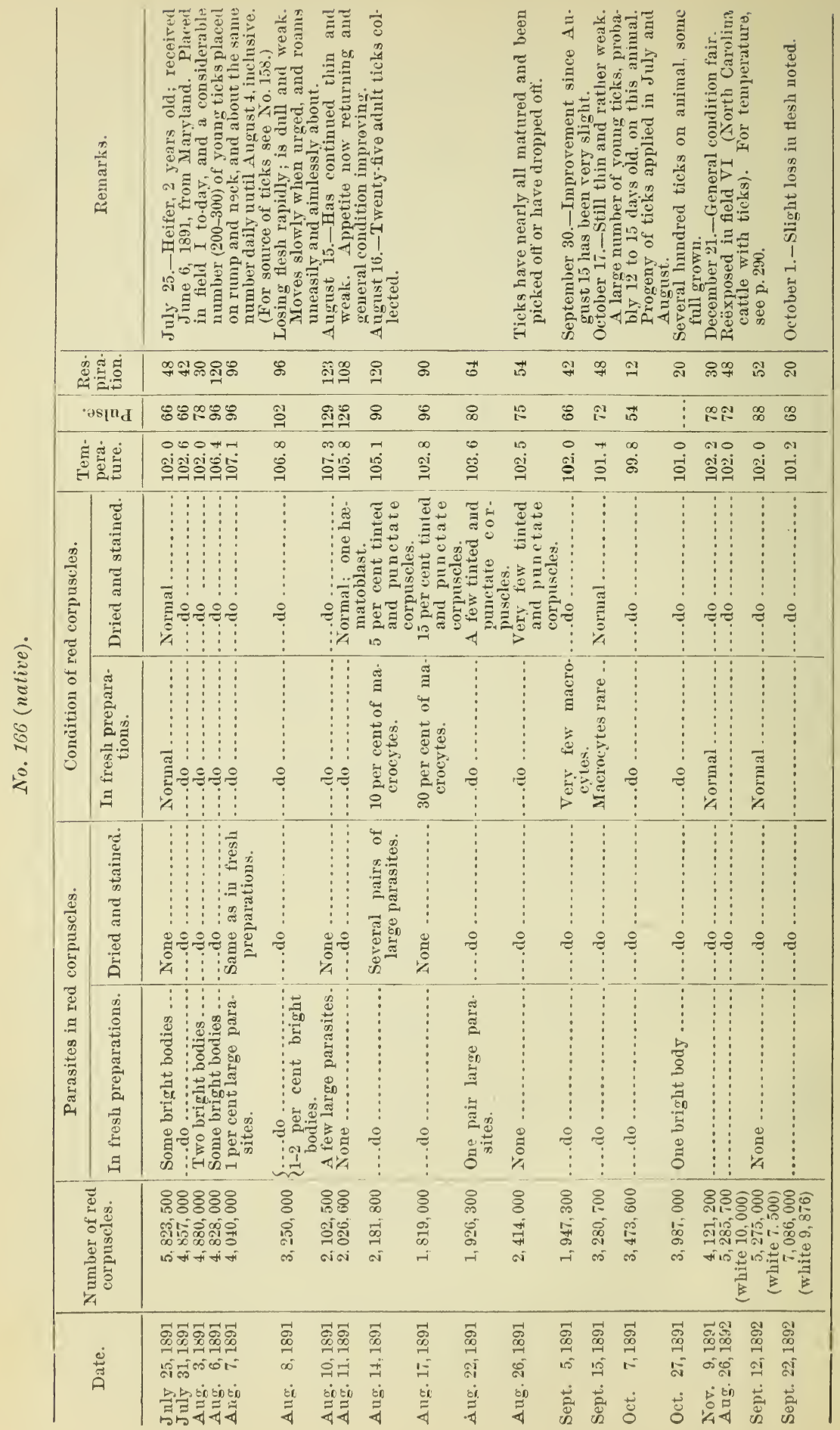




\begin{tabular}{|c|c|c|c|c|c|c|c|c|}
\hline 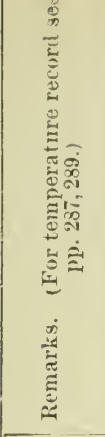 & & 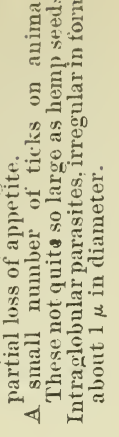 & 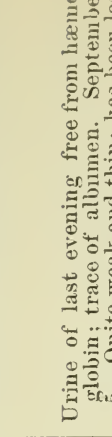 & 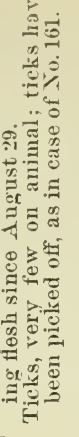 & 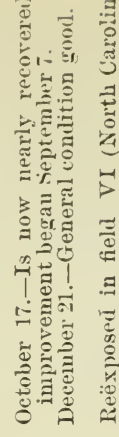 & 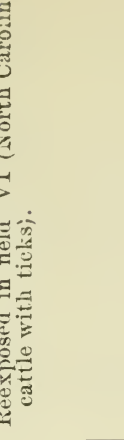 & & 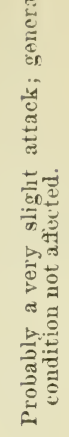 \\
\hline 新新 & 8 & $\& \&$ & $\infty \varnothing$ & $\because$ & $\&$ \& & $\& \vec{\infty}$ & 8 & $\stackrel{\infty}{\infty}$ \\
\hline$\cdot{ }^{\prime}={ }^{\prime}\left[u_{C} I\right.$ & 13 & î & $\stackrel{\infty}{\stackrel{\infty}{\Xi}}$ & 옷 & ใี้ & : & 일 & $\vec{\infty}$ \\
\hline 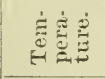 & $\stackrel{\infty}{\Xi}$ & 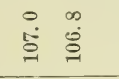 & & $\stackrel{0}{\dot{\Xi}}$ & $\begin{array}{lll}0 & 13 \\
\stackrel{8}{g} & \stackrel{a}{\Xi}\end{array}$ & 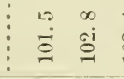 & $\stackrel{\overrightarrow{g i}}{\mathrm{gi}}$ & \\
\hline 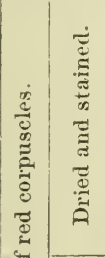 & 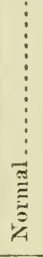 & \begin{tabular}{cc} 
& $\vdots$ \\
$\vdots$ & $\vdots$ \\
$\vdots$ & $\vdots$ \\
$\vdots$ & $\vdots$ \\
$\vdots$ & $\vdots$ \\
\hdashline & $\vdots$ \\
$\vdots$ & $\vdots$ \\
$\vdots$ & $\vdots$
\end{tabular} & 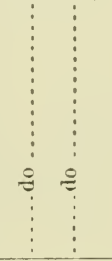 & 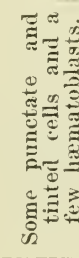 & 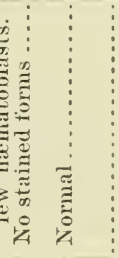 & 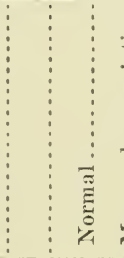 & 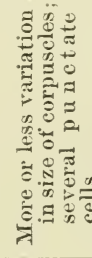 & 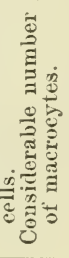 \\
\hline 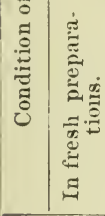 & & 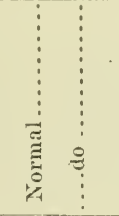 & \begin{tabular}{cc}
$\vdots$ & $\vdots$ \\
$\vdots$ & $\vdots$ \\
$\vdots$ & $\vdots$ \\
$\vdots$ & $\vdots$ \\
\hdashline & $\wp$ \\
$\vdots$ & $\vdots$
\end{tabular} & 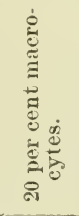 & 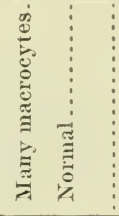 & 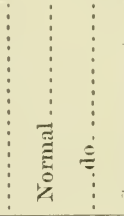 & 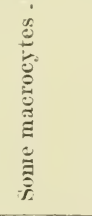 & \begin{tabular}{c} 
\\
$\vdots$ \\
\hdashline
\end{tabular} \\
\hline 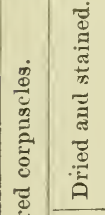 & 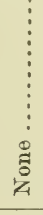 & 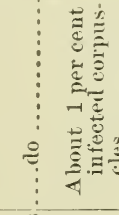 & 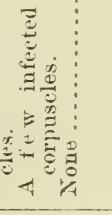 & 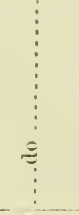 & $\begin{array}{cc}\vdots & \vdots \\
\vdots & \vdots \\
\vdots & \vdots \\
\vdots & \vdots \\
\ddots & \ddots \\
\vdots & \vdots\end{array}$ & $\begin{array}{c}\vdots \\
\vdots \\
\vdots \\
\vdots \\
\vdots \\
\vdots \\
\vdots \\
\vdots \\
\vdots\end{array}$ & $\begin{array}{c}\vdots \\
\vdots \\
\vdots \\
\vdots \\
\vdots \\
\vdots\end{array}$ & : \\
\hline 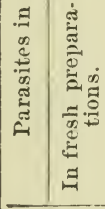 & : & 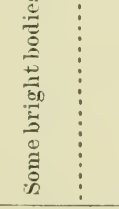 & 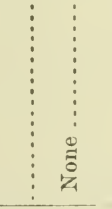 & 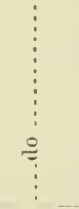 & $\begin{array}{cc}\vdots & \vdots \\
\vdots & \vdots \\
\vdots & \vdots \\
\vdots & \vdots \\
\ddots & \vdots\end{array}$ & 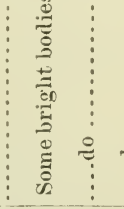 & $\begin{array}{c}\vdots \\
\vdots \\
\vdots \\
\vdots \\
\vdots \\
\vdots\end{array}$ & 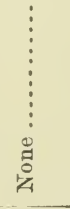 \\
\hline 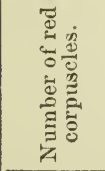 & 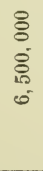 & 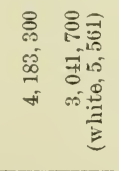 & 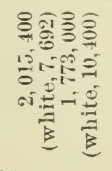 & 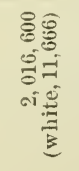 & 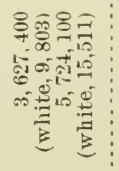 & 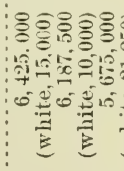 & 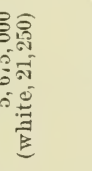 & 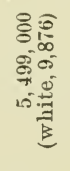 \\
\hline 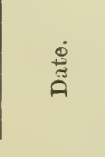 & 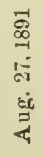 & 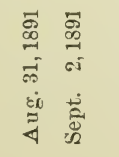 & 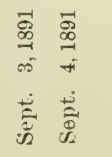 & 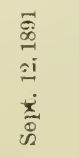 & 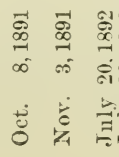 & 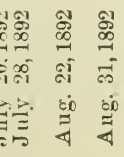 & & 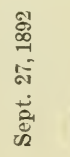 \\
\hline
\end{tabular}




\begin{tabular}{|c|c|c|c|c|c|c|c|c|c|c|c|c|}
\hline \multicolumn{2}{|c|}{ 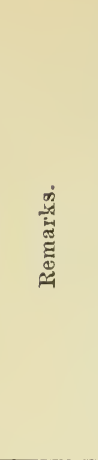 } & 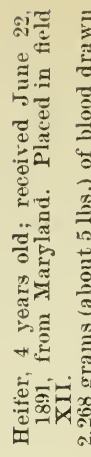 & 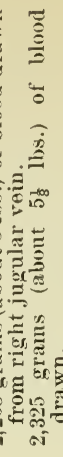 & 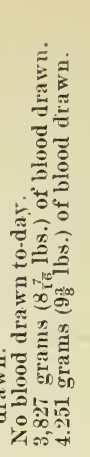 & 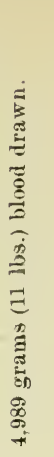 & & 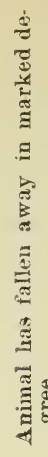 & & \multicolumn{4}{|c|}{ 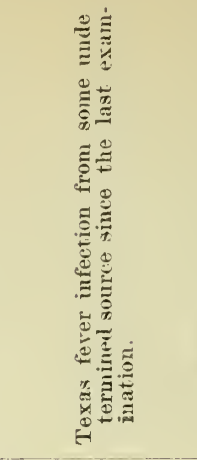 } \\
\hline \multicolumn{2}{|c|}{ 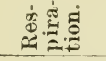 } & దे & สี & $\stackrel{\infty}{\infty} \underset{+\infty}{\infty}$ & 8 & $i_{i=1}^{\infty}$ & 落 & in & ํำ & & त & ถి \\
\hline \multicolumn{2}{|c|}{$\cdot \operatorname{es}\left[{ }^{u} d\right.$} & 8 & 8 & ஜ ๑ : & $\hat{\imath}$ & $\hat{i} \hat{i}$ & $\because$ & 8 & 8 \& & & 8 & 8 \\
\hline \multicolumn{2}{|c|}{ 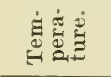 } & 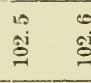 & $\stackrel{\infty}{\dot{\Phi}}$ & 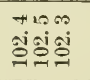 & $\begin{array}{l}0 \\
\text { si } \\
\stackrel{-}{a}\end{array}$ & $\begin{array}{l}00 \\
90 \\
9 \\
g\end{array}$ & 힘 & $\begin{array}{l}0 \\
\dot{\Phi}\end{array}$ & 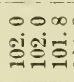 & & $\stackrel{+}{\dot{\sigma}}$ & ن. \\
\hline \multirow{2}{*}{ 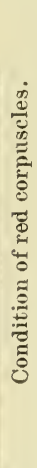 } & 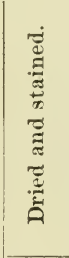 & 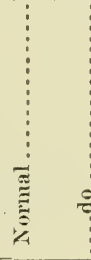 & $\begin{array}{c}\vdots \\
\vdots \\
\vdots \\
\vdots \\
\vdots \\
\vdots \\
\vdots \\
\vdots \\
\vdots \\
\vdots \\
\vdots\end{array}$ & 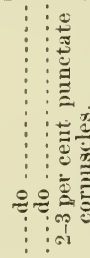 & 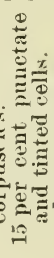 & 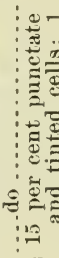 & & & 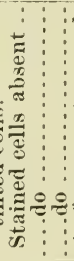 & 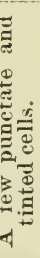 & $\begin{array}{c}\vdots \\
\vdots \\
\vdots \\
\vdots \\
\vdots \\
\vdots\end{array}$ & $\begin{array}{l}\vdots \\
\vdots \\
\vdots\end{array}$ \\
\hline & 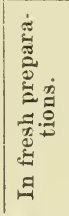 & $\begin{array}{c}\vdots \\
\vdots \\
\vdots \\
\vdots \\
\tilde{\Xi} \\
\tilde{\sigma} \\
\frac{\pi}{4}\end{array}$ & ! & 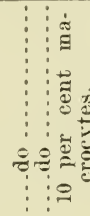 & 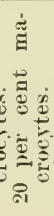 & 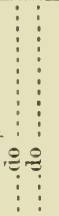 & 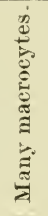 & $\begin{array}{l}\vdots \\
\vdots \\
\vdots \\
\vdots\end{array}$ & 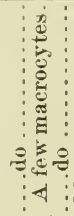 & & $\begin{array}{c}\vdots \\
\vdots \\
\vdots \\
\vdots \\
\vdots\end{array}$ & 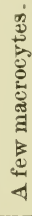 \\
\hline \multirow{2}{*}{ 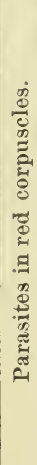 } & 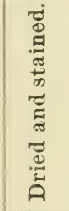 & 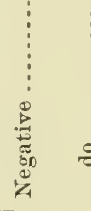 & \% & 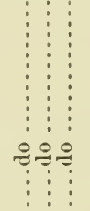 & 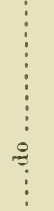 & ध⿻ & \begin{tabular}{l}
$\vdots$ \\
$\vdots$ \\
\hdashline
\end{tabular} & $\stackrel{0}{\dddot{\gamma}}$ & $\begin{array}{c}\vdots \\
\vdots \\
\vdots \\
\vdots \\
\vdots \\
\text { केष } \\
\vdots \\
\vdots\end{array}$ & & $\begin{array}{c}\vdots \\
\vdots \\
\vdots \\
\vdots \\
\vdots\end{array}$ & $\begin{array}{c}\vdots \\
\vdots \\
\vdots \\
\vdots \\
\vdots\end{array}$ \\
\hline & 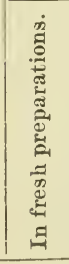 & 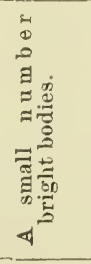 & 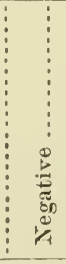 & 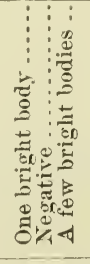 & 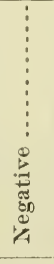 & 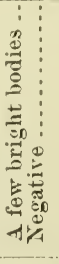 & \begin{tabular}{l}
$\vdots$ \\
$\vdots$ \\
\hdashline
\end{tabular} & \begin{tabular}{c}
$\vdots$ \\
$\vdots$ \\
$\vdots$ \\
$\vdots$ \\
\hdashline
\end{tabular} & 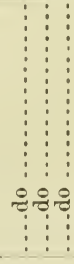 & & 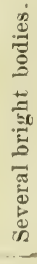 & \\
\hline \multicolumn{2}{|c|}{ 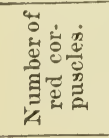 } & $\begin{array}{l}8 \\
0 \\
01 \\
0 \\
0 \\
0\end{array}$ & 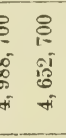 & 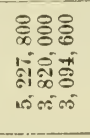 & 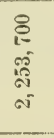 & 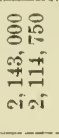 & $\begin{array}{l}8 \\
\& \\
\infty \\
0 \\
0 \\
\text { oi }\end{array}$ & $\begin{array}{l}8 \\
\delta \\
\text { cิi } \\
\text { ô } \\
\text { ri }\end{array}$ & 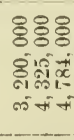 & & $\begin{array}{l}8 \\
8 \\
00 \\
\text { N. } \\
\text { co }\end{array}$ & $\begin{array}{l}\text { षे } \\
\text { \&ิ } \\
\text { + }\end{array}$ \\
\hline \multicolumn{2}{|r|}{$\dot{\stackrel{\Phi}{*}}$} & 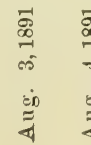 & 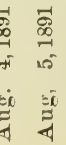 & 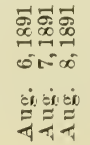 & 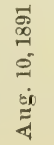 & 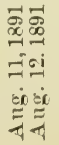 & 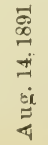 & 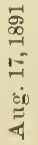 & 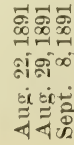 & & $\begin{array}{l}\overrightarrow{8} \\
\stackrel{0}{7} \\
\stackrel{10}{2} \\
\dot{0} \\
\dot{0}\end{array}$ & $\begin{array}{l}\overrightarrow{8} \\
\stackrel{0}{0} \\
-1 \\
\text { m } \\
\dot{8} \\
\dot{0}\end{array}$ \\
\hline
\end{tabular}


No. 169 (native).-Cow, 8 years old, received May 28, 1891, from Maryland.

Septcmber 1.-Placed in field VI (North Carolina cattle with ticks). Temperatnre, 101.6; pulse, 66; respiration, 12. Red corpnseles, 5,423,000. Fresh and stainerl preparations slow nothing almormal.

September 7.-Temperature, 101.2 ; pulse, 60 ; respiration, 60 . Red corpuscles, $4,966,600$; white, 10,000 . A few ticks and many lice on this animal.

September 12.-Temperature, 103.4 ; pulse, 72 ; respiration, 48. Red corpuscles, $4,335,400$; white, 12,903. Nothing abnormal in fresh and stained preparations.

September 14.-Found dead early this morning; abdomen distended with gas. Autopsy a few liours later.

Animal weighs about 900 pounds. A large number of small ticks on thighs, escutcheon, udder, neck, and axilla. On removing the skin a small tumor observed in the left flank, which proves to be a hernia of a coil of the ileum. Slight extravaation surrounding the opening in abdominal muscles. The peritonenm covering sac very hyperamic, similarly the mucous membrane of the involved coil. Nostrangulation. The protrusion probably occurred a short time before death.

Heart shows punctiform ecchymoses over left ventricle. A small white clot in this cavity. The right ventricle contains a large dark clot. Limgs cedenatous.

Spleen weighs $3 \frac{1}{8}$ pounds. Pulp soft; not so dark as in the average case of this disease.

Liver weighs 13 pounds. Advanced post-nortem changes. In the gall bladder some turbid bile.

Kidneys apparently unchanged. Bladder contains a small quantity of turbid urine free from hamoglobin. It is alkaline and contains much albumen.

Digestive tract. Contents of third stomachdry, and somewhat impacted. Fourth stomach normal. Mucosa of upper jejunum and of a portion of cæcum markedly injected. The other portions normal, with exception of the prolapsed portion of ileum.

The various tissues show advanced post-mortem chauges, and contain large postmortem bacilli. Intraglobular parasites are very scarce in all the organs. Those present have the usual roundish form as found in the dead animal, and occur singly or in pairs. (For temperature, see p. 286.)

No. 170 (native).-Heifer, 1 year old received June 6, 1891. Kept in field XII.

September 30.-Temperature, 101.8; pulse, 60; respiration, 40. Red corpuscles, $4,300,000$; white, 12,000. Nothing abnormal in fresh and stained preparations. This animal was not used in any experiment, but its blood was examined on this date owing to the unexpected appearance of Texas fever in this field at this time.

No. 172 (Southern).-Cow, 6 to 7 years old, received July 2, 1891, from near New Berne, N. C., and placed in field VI (North Carolina cattle with ticks).

December 21.--Removed and sold.

No. 173 (Southern).-Cow, $3 \frac{1}{2}$ years old, received July 2, 1891, from near New Berne, N. C., and placed in field XI after the ticks had been carefully picked off by hand. (North Carolina cattle without ticks.)

December 21.- Removed and sold.

No. 174 (Southern).-Heifer, $3 \frac{1}{2}$ years old, received July 2, 1891, from near New Berne, N. C., and placed in field VI (North Carolina cattle with ticks).

December 21.-Removed and sold.

No. 175 (Southern).-Cow, 4 years old, received July 2, 1891, from near New Berne, N. C., and placed in field XI, after the ticks had been carefully picked off by hand (North Carolina cattle without ticks).

December 21.-Removed and sold.

No. 176 (Southern).-Cow, 6 years old, received July 2, 1891, from near New Berue, N. C., and placed in field XI after tho ticks had been carefully picked off by hand. December 21.-Removed and sold. 
No. $17 y$ (Southern).—('ow, 5 rears old, received Iuly 2, 1891, from near New Berme, N. C., and placed in field VI (North Carolina cattle with ticks).

October 20.--Removed and sold.

No. 178 (Southern).-Cow, 4 years old, reccived July 2, 1891, from near New Berne, N. C., and placed in field VI (North Carolina cattle with ticks).

December 21.-Removed and sold.

No. 179 (Southern).-Cow, 5 years old, receiverl July 2, 1891, from near New Berne, N. C., and placed in field XI, after the ticks had been carefully picked off by haud (North Carolina cattle without ticks).

December 21.--Removed and sold.

No. 180 (native).-Heifer, age 2 years, received July 25, 1891, from Maryland. Placed in Field I. From this date to Angust 4, inclusive, abont 20 to 30 young ticks were placed on this animal daily. (See No. 158 for source of ticks.)

July 25.-Temperature, 102.4; pulse, 80 ; respiration, 48 . Red corpuseles, 5,396,800. In fresh preparations a few corpuseles containing bright bodies. Stained preparations negative.

July 31.-'Tenperature, 104; pulse, 70 ; respiration, 30. Corpuseles, 4,462,700. Fresh and stained preparations negative.

August 3.-Temperature, 102.1; pulse, 60; respiration, 30. Corpuscles, 4,560,000. Fresh and stained preparations negative.

August 6.-Temperatnre, 106.7; pulse, 72; respiration, 48. Corpuscles, 4,636,300. Blood examination negative.

August 8.-Losiug flesh aud becoming weak.

August 10.-Animal very weak and thin. Temperature, 107.7 ; pulse, 120 ; respiration, 87. Corpuscles, $1,86+, 900$. In fresh and stained preparations a very small number of corpuscles detected, containing parasites of medium size.

August 12.-Died between 6 and 7 a. m.

Autopsy (9 a. m.). Animal has lost considerable fleslı since the beginning of the fever. Weighs now about 400 pounds.

Ticks on the inner surface of thighs, on abdomen, and neck. On the average about one to a square inch. They are still small, about $\frac{1}{8}$ inch long.

Heart surface well sprinkled with ecchymoses. These are most numerons on the left ventricle. Both sides of the heart contain rather large, dark, firm elots, that in the right being the larger. Considerable blood extravasation under endocardium of left ventricle, especially marked on septum.

Lungs somewhat œdematous.

Spleen weighs 2 pounds. Small blood tnmors along the course of the ressels under capsule.

Liver weighs 10 pounds; enlarged, edges ronuded off. The parenchyma has at yellowish hue. When examined with a lens the yellow coloration is foumd limited to the tissue around the hepatic vessels. In fresh sections bile injection appears restricted to small areas. Fatty degeneration well advanced. The hepatie cells contain lumps of pigment, and red needle-like erystals are sprinkled over the section. Bile in gall bladder very viscid, and holds in suspension a large quantity of flocculent matter.

Kidneys deeply congested thronghont. In hladder 3 guarts of nrine, which has a light elaret color. Specific gravity, 1018. Acid reaction. Albumen, according to Esbach, 0.2 per cent. On standing, urates are deposited.

Digestive tract: A fow hemorrhages on lamellae of fourth stomach. In the large: intestine more or less pigmentation of mucosa. Contents dry and massed into fine halls. Intestines otherwise normal.

In preparations of leart's blood, parasites are rare. They are in general romdish in ontline and but one within a corpusele. In the liver there are about 1 to 2 per cent; in the spleen still less. In one preparation of the spleen pulp a capillary is 
seèn filled with infected corpuseles only. Each contains two parasites. In the kidney not less than 10 por cent of the corpuseles contain either one or two parasites. The large post-mortem bacilns is likewise present in small nmmbers.

No. $1 S 1$ (native).-Cow, $2 \frac{1}{2}$ years old, received August '28, 1891.

September 5.-Placed in field VI (North Carolina eattle with ticks). Temperaturc, 101.6; pulse, 54; respiration, 90. Red corpuseles, 5,673,400. White, 12,245. Nothing abnormal in stained preparations.

September 12.-Temperature, 101; pnlse, 72 ; respiration, 60. Red eorpuscles, 5,707,000. White, 6,900. Botlu stained and fresh preparations negative.

September 18.--Temperature, 106.4; pulse, 66; respiration, 102. Red corjuseles, 4,134,600. White, 7,692. In stained preparations two eorpuseles found infected with lirge parasites.

September 19.-Temperature, 106.2; pulse, 76; respiration, 100. Red corpuseles, $2,970,000$. White, 4,177. In stained preparations from one-half to 1 per cent infeeted corpuscles. From this animal blood was withdrawn to-day from the left jugnlar and injected into the jugular of No. 185 and 186 . After the withdrawal of blood the animal refuses to rise. It was killed by a blow on the head and examined immediately.

Autopsy: Animal weighs abont 750 ponnds; in fair eondition. Ticks on the thighs, udder, pubie region, abdomen, and axilla in moderate numbers.

Firm attaehment of omentum and slight adhesions of spleen to wall of abdomen.

Heart and lungs normal. Blood withdrawn before death and placed in beaker; chots readily and firmly. In two homs much serum liad been presserl ont.

Spleen weighs 4 pomnds. Considerably enlarged. Pulp, dark and soft, concealing Malpighian bodies and trabeenlie.

Liver weighs 15 pounds. Enlarged, but nearly normal in appearance. The intra. lobular capillaries are distended with blood. No fatty degeneration and bile injeetion as yet noticeable in fresh seetions. The hepatie cells contain minnte pigment granules. Gall bladder contains abont one-half pint of bile which is eonsiderably darker than in the normal state, but holds only a small quantity of floceulent matter in suspension.

Kidneys more or less eongested. In fresh seetions eapillaries densely packed with red corpuseles. The urine in the bladder is still free from coloring matter of the blood. Specifie gravity, 1024. Strongly alkaline. Trace of albumen.

Digestive tract normal.

The distribntion of the infected corpuscles examined both in fiesh and stained preparations. In the tissnes the following approximate numbers were observed:

Blood of heart and skin, $\frac{1}{2}$ to 1 per cent; spleen, liver, and marrow, $\frac{1}{2}$ to 1 per cent; kidney 20 to 30 per cent; lieart muscle (eapillary blood), 50 per cent or more (Plate VII, Fig. 1). The parasites were likewise readily found in unstained preparations. In all of these corpuscles the bright bodies frecunently referred to were present. The parasites were all pear-shaped, nsually a pair in a corpusele. In the tluid expressed from the heart muscle many free pairs were seen. (For temperature reeord, see 1. 286.)

No. 18: (native).--September 19.-A portion of the heart muscle of No. 181, just dead, was ent up into small pieces and pounded in a sterile mortar with normal sterilized salt solution. The reddish fluid was passed throngh filter paper at $1 \mathrm{p}$. $\mathrm{m}$. and kept at $95-105 \cup \mathrm{F}$. 1mtil 2 p. m., when 14 cc. (one syringe finll) was injected into the left jngnlar of No. 182 (see, also, Nos. 185 and 186). The subsequent history of this case is comprised in the following table: 


\begin{tabular}{|c|c|c|c|c|c|c|c|c|c|}
\hline \multirow{10}{*}{ 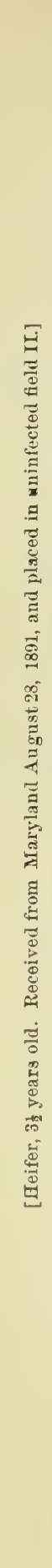 } & \multirow[t]{2}{*}{ 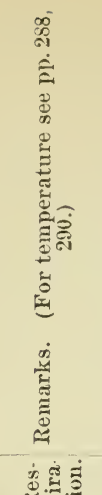 } & \multicolumn{2}{|c|}{ 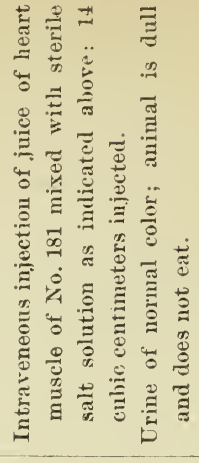 } & \multicolumn{3}{|c|}{ 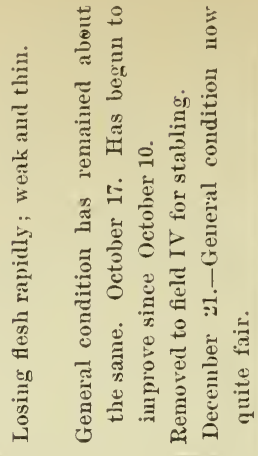 } & \multicolumn{2}{|l|}{ 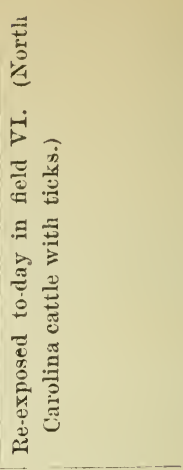 } & 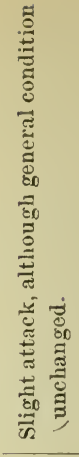 \\
\hline & & I & $\stackrel{\infty}{\stackrel{-1}{-1}}$ & $\stackrel{\infty}{\longleftarrow}$ & $\underline{\varphi}$ & $\infty$ & $8 \%$ & $\stackrel{\infty}{\text { N }}$ & \& \\
\hline & 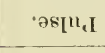 & 8 & 온 & 8 & $\stackrel{\infty}{\infty}$ & 8 i & $\vec{\infty} \quad \infty$ & $\vec{\infty}$ & $\infty$ \\
\hline & 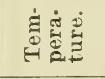 & 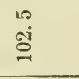 & $\triangleq$ & 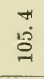 & @i & $\stackrel{10}{\circ}$ & 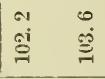 & $\stackrel{H}{\Xi}$ & $\stackrel{\cong}{\leftrightarrows}$ \\
\hline & 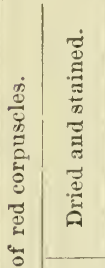 & 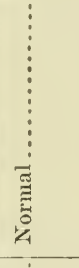 & $\begin{array}{c}\vdots \\
\vdots \\
\vdots \\
\vdots \\
\vdots \\
\vdots\end{array}$ & ؛ & 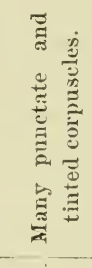 & 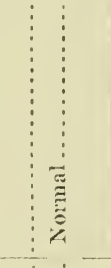 & 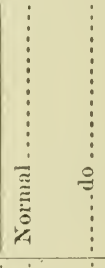 & 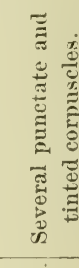 & 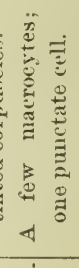 \\
\hline & 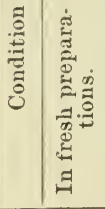 & $\begin{array}{c}\vdots \\
\vdots \\
\vdots \\
\vdots \\
\vdots \\
\vdots \\
\vdots \\
\vdots\end{array}$ & $\begin{array}{c}\vdots \\
\vdots \\
\vdots \\
\vdots \\
\vdots \\
\vdots \\
\vdots\end{array}$ & $\dddot{\ddots}$ & 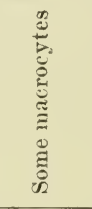 & 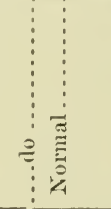 & & 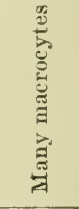 & \\
\hline & 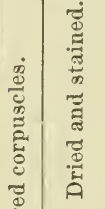 & 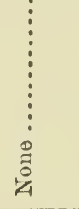 & & 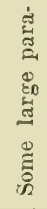 & & $\stackrel{3}{\vdots}$ & 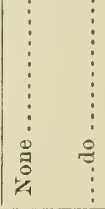 & ְे & శి \\
\hline & 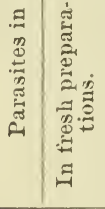 & 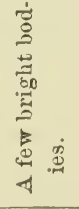 & 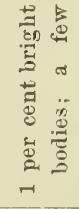 & & ְִ & 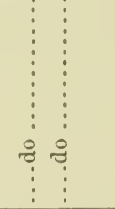 & & & \\
\hline & 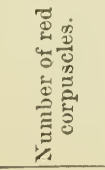 & 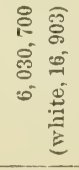 & 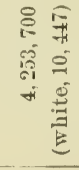 & 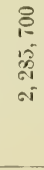 & 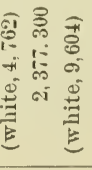 & 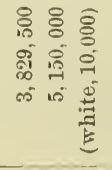 & 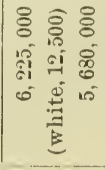 & 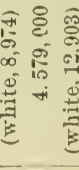 & 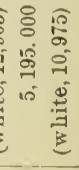 \\
\hline & مُ & 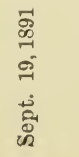 & 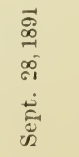 & 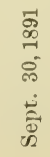 & $\begin{array}{l}\vec{D} \\
\stackrel{8}{0} \\
0 \\
\dot{0} \\
\dot{0}\end{array}$ & 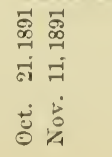 & 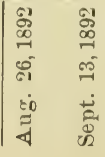 & 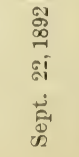 & $\begin{array}{l}\stackrel{1}{\circ} \\
\stackrel{0}{0} \\
\stackrel{-}{0} \\
\dot{0} \\
\dot{0}\end{array}$ \\
\hline
\end{tabular}


No. 183 (native).-Heifer, $2 \frac{1}{2}$ years old, received Angust 28, 1891, from Maryland. August 29.-Receives into right jugnlar vein $5 \mathrm{cc}$. of fluid made by crushing young ticks, $1 \frac{1}{2}$ months old, in sterile water and filtering throngh filter paper. (See No. 165.) Placed in field IV.

The following fignres were obtained before the injection: Temperature, 101.2; pulse, 72) respiration, 54. Red corpuseles, 5,770,000. Fresh and stained preparations of blood normal.

September 5.-Temperature, 101.2; pulse, 84; respiration, 42. Red corpuseles, $5,583,300$; white, 11,666. Microscopic examination, negative.

September 10.-Temperature, 101; pulse, 60; respiration, 36. Red corpuseles, $6,557,000$; white, 9,615. Examination of blood, as before.

September 18.-Temperature, 102.7; pulse, 72 ; respiration, 60. Red corpuscles, $6,000,000$; white, 14,035. Exanination of lood, as before.

October 15.-Temperature, 101.8; pnlse, 80; respiration, 36. Red corpuscles, $6,206,800$. In fresh preparations, a few bright bodies; stained preparations, negative.

October 20. -No result to date. Animal sold. (For temperature record, see p. 287.)

No. 184 (native).-Heifer, 2 years old, received Angust 28, 1891. Kept in uninfected field II.

August 29.-Temperature, 102 ; pulse, 96 ; respiration, 54. Red corpnscles, 7,530,000. Fresli and stained preparations, normal.

September 15.-Placed in field VI (North Carolina cattle with ticks). Temperature, 101.6; pulse, 78; respiration, 40. Red corpuscles, 5,826,900; white, 5,769. Fresl and stained preparatious, normal.

September 29.-Temperature, 106.2; pulse, 90; respiration, 84. Rerl corpuscles, $4,350,000$; white, 10,000 . In both fresh and stained preparations a few corpuscles found infected with large parasites.

October 1.-Temperature, 103.2; pulse, 108; respiration, 60. Red corpuscles, 1,822,500; white, 6,451. Parasites as on September 29.

October 2.-Found dead at 6 a. m. Animal heat not entirely dissipated.

Autopsy: Weight of animal, about 500 pounds. Condition still good. Vew few ticks on thighs.

In the abdomen the omentum is injected in patches.

Lungs slightly œdematous. Several small foci of red hepatization. In both heart cavities a considerable amount of fluid blood.

Spleen weighs $3 \frac{1}{2}$ pounds; enlarged; pulp dark and softened. Liver weighs $11 \frac{1}{2}$ pounds, of a markedly yellowish color and donghy to the tonch. In fresh sections the bile injection (Plate III, Fig. 3) and the fatty legeneration of the parenchyma very extensive. Large bundles of minnte acicular red erystals scattered over the section. Gall bladder contains abont 10 ounces of very thick bile, holding mnch floccnlent matter in suspension and scarcely flowing from the bladier.

Kidneys miformly congested. Pelvis dotted with ecchymoses. In fresh sections capillaries observed gorged with red corpuscles. Bladder contains 2 quarts of urine liaving a wine-red color. Aeid. sp. gr. 1,018. Aluundant flocenlent precipitate, with acetic acid in the cold. Albumen, according to Esbach, 0.2 per cent. On stauding a slight deposit of urates.

Digestive tract normal.

Stained preparations of the blood and organs contained infected corpuseles approximately as follows:

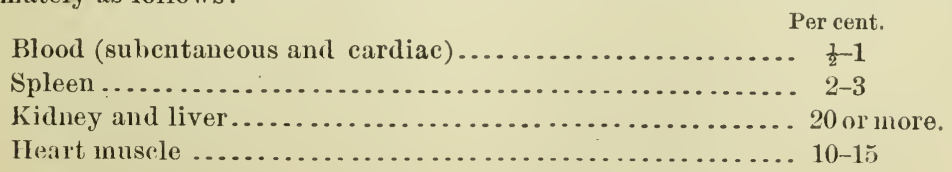

The parasites are roundish, abont $1 \mu$ in diameter. Some corpuscles contain two, others but one. (For temperature record, see 1.286.) 


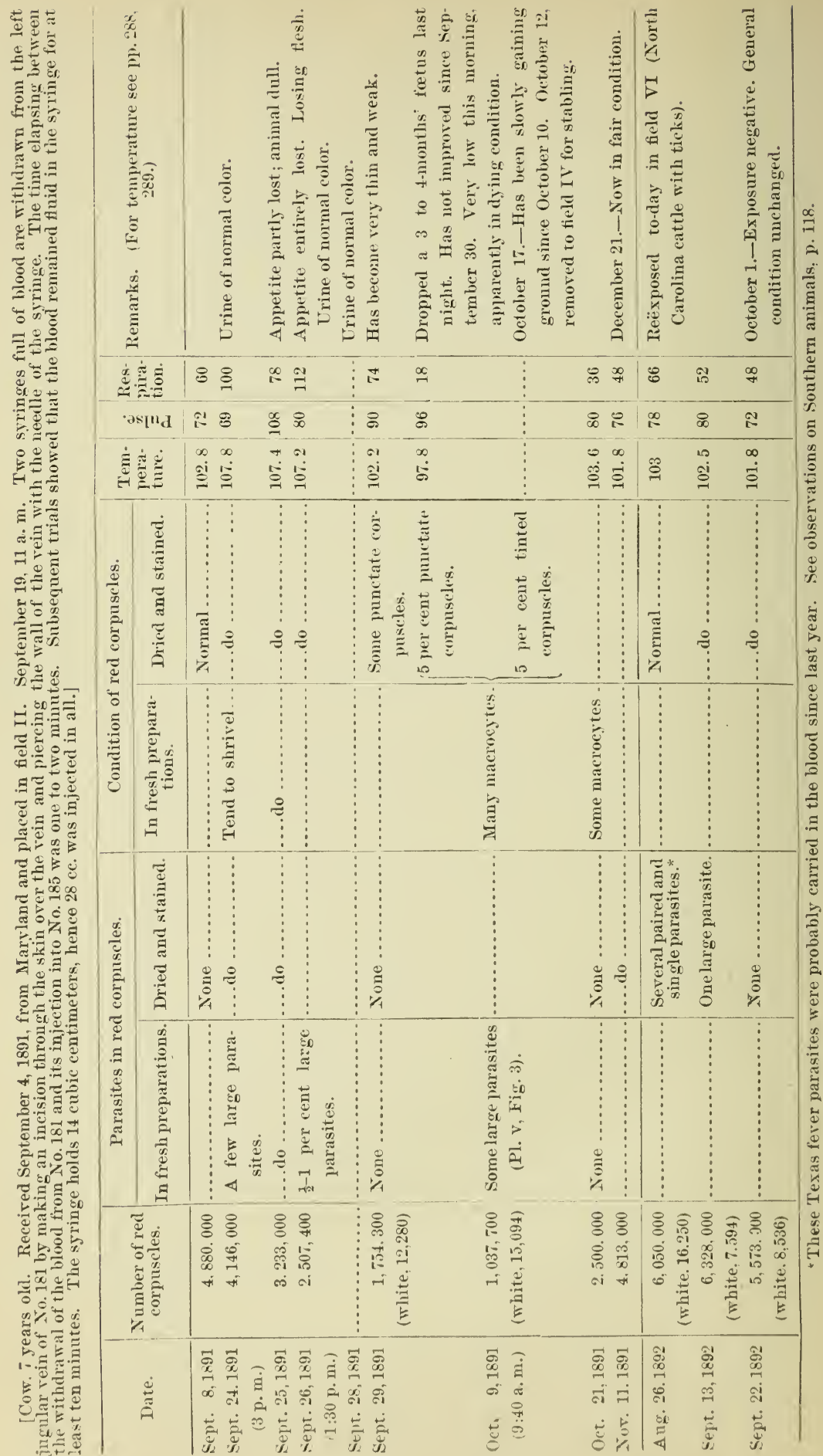


Yo. 1S6 (native).---Red cow, from 10 to 12 years old, received from Maryland September 4,1891 , and placed in nninfecterl tield II.

september 8.-Temperature, 102.2; pulse, 72: respiration, 48. Rerl corpuscles, $4,980,700$; white, 13,461 .

September 19.- From the jugnlar vein of No. 181, sick with Texas fever, a syringe finll of blood (14 ece) was withdrawn and injected directly into the right jugnlar of No. 186. (For details see the preceding case.) The blood of No. 186 was carefinly examined microscopically before the injection. In a fresh preparation one ninnte round body, $1 \mu$ in diameter, seen in a corpuscle, slightly rlunging place.

September 25.-Temperature, 106; pulse, 72; respiration. 78. Rerl corpuseles, $4,761,905$. In preparations of fresh blood several corpnseles detected witl large pyriform parasites, each with a dark point (molens?). In stained preparations none observerl.

September 26, 2:30 p. m.-Temperature, 107; pulse, 96 ; respiration, $10 x$. Red enrpuscles, 4,333,300. In fresh and stained preparations a small number of corpuseles containing large parasites.

September $28,2: 15$ p. m.-Temperature, 101.2; pulse, 108 ; respiration, $60 . \quad$ Red eorpuscles. 2,123,077; white, 4,615. From 1 to 2 per cent of corpuscles contain amal,iform parasites.

The animal stands trembling and quivering, swaying with her hind quarters, and scarcely able to remain on her feet while a few drops of hlood are being collected from a skin ineision for examination. Soon after sle falls down and remains on the ground.

At $3: 30 \mathrm{p} . \mathrm{m}$. a syringeful of blood $(7 \mathrm{ce}$.) is withdrawn from a jugular vein to inoculate several pigeons. The blood was withdrawn as deseribed under No.185 above. After this insignificant operation the row goes into convulsions and dies.

Autopsy notes: Animal has lost much flesh. Weighs now abont 700 pounds.

Lungs normal. Heart firmly contracted. Considerable extravasation under the epieardium of left ventricle, much less on the right ventricle. Many eechymoses and small hematomata under endocardinm of both ventricles. Heart muscle shows slight fatty degeneration. In the sermm expressed from the heart muscle a large number of large parasites hoth free and within corpuscles. In stained preparations from 30 to 40 per cent of all corpnscles are infected. Many parasites in pairs. (Plate IV, Fig. 5.)

Spleen weighs $4 \frac{9}{16}$ pounds. Very mmel enlarged. 'Tortuons injected ressels on capsule with hemorrhages along their course. The pulp is rery dark and very soft. Malpighian bodies invisible. In teased preparations examined fresh there are many large eells eontaining from two to eight red corpuseles. Two eapillaries seen, in which nearly every corpuscle is infected. Pigment present in small quantities. In stained preparations about 8 to 10 per cent eorpuscles contain large parasites.

Liver weighs 14 pounds. Some old adhesions on the right between it and diaphragm. Tissue rather firm, color departing slightly from the normal. Yellowish dots and lines are seen on section eorresponding to the zone around hepatic veins (intra aud sub lobnlar). In several larger branches of the hepatie vein are thrombi. In fresh sections and teased preparations, bile injection localized to small areas aronnd intralobular viens. In stained preparations from 20 to 30 per cent of the eorpuscles infected.

Bile very thiek and flaky, holding a large amount of amorphous matter in snspension.

Considerable odema in the fatty tissue around kirlneys. The organs are in a condition of general congestion; all normal markings effaced or indistinct. Glomernli prominent. Cut surfiee gramlar. Many eechymoses in pelvis. In fresh seetions all capillaries choked with red eorpuscles. In stained preparations nearly all corpuscles contain parasites. There are also many free forms. In sections of tissue (hardened in Miiller's thuid and alcohol and embedded in chloroform paratine) 
stained in hematoxylin the engorged capillaries contain only infected red corpus. cles (Pl. vir, lig. 2). Structural changes not noticeable.

In bladder, 2 quarts of urine of a dark reddish color. No sediment on standing. Specific gravity, 1015. Frebly acid. About 0.05 per cent albumen (Esbach). Precipitate with acetic acid withont heat.

The mucosa of fonrth stomach has a blnish red to bright red color. Digestive tract otherwise not affected. (For temperature record, see 1. 288.)

No. 187 (native).-Calf of No. 160. Abont 4 montlis old (Sept. 21), bnt no larger than a calf 4 weeks old (first ealf of a small heifer).

September 21.-Canght after much chasing and placed in lield VI (North Carolina cattle with ticks) with No. 160. 'Temperature, 105; pnlse, 117; respiration, 52. Red corpuseles, 5,870,000; white, 22,222. Nothing abnormal in fresh and stained blood preparations.

October 13.-Exeited on being chased and canght. Temperature, 103.5; pulse, 132; respiration, 30. Red corpuscles, 5,774,000. Nothing abnormal in fresh and stained preparations.

November 12.-Temperature, 102.4; pulse, 92; respiration, 60. Red corpuscles, 7,224,000; white, 15,517. Corpuscles normal. Result of exposure, uegative.

No. 188 (native).-Cow, 6 years old, received September 25, 1891, from Marylaud, and placed in field XII.

October 1.-Temperatnre, 100.8; pulse, 60; respiration, 36. Red corpuscles, $5,596,400$; white, 8,772 . In fresh preparations a considerable number of corpuscles with bright bodies. In stained preparations nothing abnormal.

Oetober 20.-Sold.

No. 189 (native).-Cow, 7 years old, received September 25. 1891, from Maryland, and placed in field XII.

October 1.-Temperature, 101 ; pulse, 66 ; respiration, 30 . Red corjuscles, 5, 706,800; white, 10,344. Some bright intraglobnlar hodies; eorpuscles otherwise normal.

October 20.-Sold.

No. 190 (native).-Cow, 6 years old, received September 25, 1891, from Maryland, and placed in field XII.

October 1.-Temperatnre, 100.2; pulse, 65; respirationi, 60. Reil corpnseles, $5,966,000$; white, 10,169. Some bright intraglobular borlies deterted in fresh preparations.

October 20.-Sold.

No. 197 (native).-..Grade Jersey, 6 years old, received Junc 3, 1892, from District of Colmubia. Placed in field $X$ to test smrvival of ticks amd Texas fever infection of 1891. Gives birth to calf No. 226, June 20.

Angnst 27.-Up to this date no ticks had appeared on animals in this field. No evidence of infection as shown by the condition of the animal and the following results of the blood exanination. Red corpuscles, 5,012,500. White, 6,250. In preparations of fresh blood many red eorpuseles contain bright bodies. Stained preparations negative. Injection into lelt jugnlar 14 ec. (one syringefinl) of blow from sick native No. 222 prepared in the following mamer:

No. 222 was secured in a rectangnar box and the heal raised and finstened in such a way as to be immovable and to stretch the left side of the neck. This was shaven over the jugular vein and the skin disinfected with 0.1 per cent merenric chloride. With a flamed sealpel the skin and wall of the jugnlar vein, kept tenso by pressnre applied at the root of the neck, was incised. The blood llowing freely was canght in sterilized bottles containing glass beads and defibrinated by shaking for ten minntes. The bothles were then placed in a water bath at $40^{\circ}-42^{\circ} \mathrm{C}$. until used for inocnlation. (For the condition of the blood of $\mathrm{No} .222$ on this day see this appendix muler No. 222.) The blood drawn about $11: 05$ :1. m. was injected into this animal at $11: 15$ a. 111.

Placed in a fenced off portion of field XII $\left(\mathrm{XII}_{n}\right)$. 
September 4.-Dies suddenly abont $9: 30$ i. m., after having shown :u elevated temperature 4 days.

Autopsy notes: The animal having died on Sunday, only such examination was made on the spot at $2: 30 \mathrm{l}$ m. as wonld determine the canse of deatl. The following notes amply demonstrate 'Texas fever:

Spleen very large, blackish, normal markings effaced on the cut surface. The liver has a bluish slate color mottled with minute paler dots. On sertion this mottling is yellowish and confined to the tissne aromnd the intralobular veins, the remainder of the cut surface being a brownish color. In fresh sections much fatty degeneration, also ronndish bodies (crystals?) larger than red corpuseles which are refrangent like fat globules and have a pecnliar yellowish red color. Bile injection not observed in such sections.

Kidneys pale brownish red with a granular cut surface and usnal markings efiaced. Epithelium of convolnted tnbules markedly grannlar. In pelvis extensive patehes crowded with small ecchymotic spots. Urine, claret colored. Specific gravity, 1017. Fine precipitate with acetic acid cold. Albnmen (Eshach), 0.35 per cent. In the deposit collected by standing in refrigerator, a few red eorpuseles, gramlar masses, and mueus.

The skin over abdomen shows (5 hours after death) dried blood crusts $\frac{1}{4}$ to $\frac{1}{2}$ inch apart, matting the hair together into little tufts. Theskin under these placessliows a bluish spot, and when incised a small collection of blood is found under the trne skin.

In the spleen and liver very few corpuscles infected with paired romdish para. sites 1 to $1.5 \mu$ in diameter. In the kidneys abont 10 per cent are infected. (For temperature record, see p. 294.)

No. 198 (native).-Cow, 7 years old, received Jnne 3, 1892, from Prince George Connty, Md., and kept on an adjoining farm until Jnne 30, then transferred to field III.

Jnly 6.-Injected into left jugular vein $28 \mathrm{cc}$. (two syringefuls) of blood drawn from the jugular vein of North Carolina cow No. 217. The transfer of blood from the vein of one animal to that of the other was made in the same syringe ant occupied not more than two minutes. The syringe had been warmed previonsly to $105^{\circ}$ F., after being thoroughly disinfected in 5 per cent carbolic acid and boiling water. (See No. 206 for a similar injection.)

The following table gives in brief the clinical history of the animal 1 p to the time of death: 


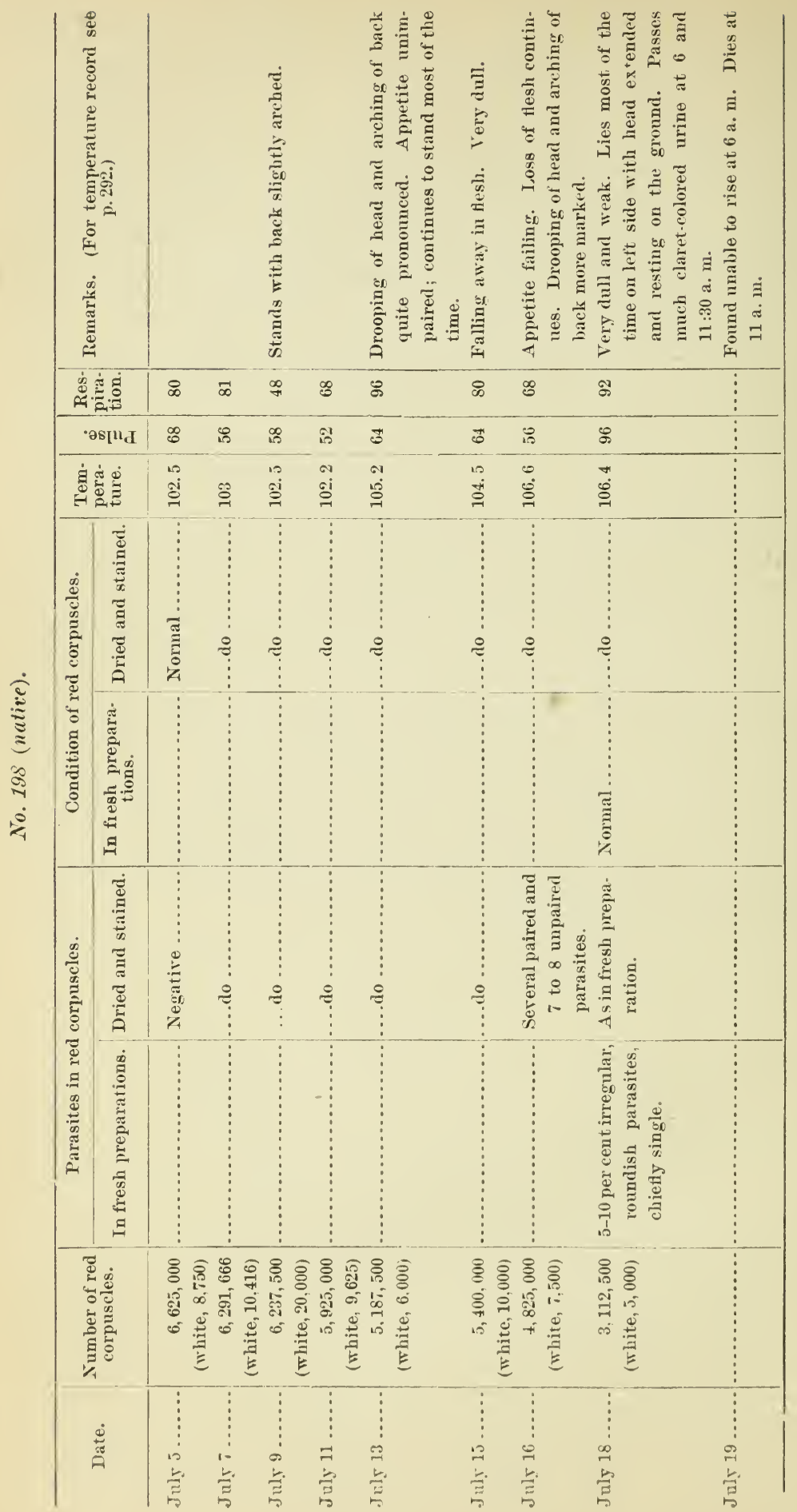


Autopsy abont $1 \frac{1}{2}$ hours after death. Animal thin; weighs about 600 pounds; has lost about 200 pounds during illness.

Several small areas of intralobular and subpleural empliysema in the principal lobe of both lungs and in the right ventral lobe. The connective tissue of old plenral alliesions forms a fringe along lateral borders of principal lobes which is in a dark-red hyperemic condition. A dark red, airless lobule in the right cephalic and the left principal lobe.

Slightly odematous condition of the fat aronnd heart ease. Very marked ecchymosis of the ventricular surfaces of the lieart, the discoloration extending in some places from $\frac{1}{8}$ to $\frac{3}{16}$ inch into the heart muscle. Extensive extravasation beneatl endocardium of left ventricle; slight extravasation in right ventricle. Blood from the lieart coagulates promptly in beakers. The serum is much more deeply colored than that from healthy attle.

Spleen werghs $5 \frac{1}{2}$ pounds. Capsule very much distended. Pulp dark brownish, still consistent in texture. Malpighian bodies barely visible on section.

Liver weighs $13 \frac{3}{4}$ pounds (without gall bladder). It is paler than normal and shows a peculiar mottling with irregular yellowislı-gray patches, each less than $1 \mathrm{~mm}$. in diameter. On section the organ appears yellowish brown and the course of the intralobular veins is marked by yellowish-gray borders. In fresh sections fatty degeneration of the parenchyma and the bile stasis quite extensive. Sections were also examined from tissne liardened in Miiller's fluid and alcohol. In these after staining with acid hiematoxylin or alkaline methylene blue, only a narrow region of the acini bordering on the intralobular tissue was found free from necrotic changes. These were manifested hy partial or total loss of the nncleus. The eapillaries of these areas were in places very much distended and filled with red corpuscles, many of which contained parasites.

In the gall bladder abont $\frac{1}{2}$ liter of very thick, flaky bile.

The fat aronnd kidneys contains a moderate amount of serous effusion. Kidneys eularged (left, $1 \frac{7}{8}$ pounds; right, 2 pounds); capsule readily removable. Parenchyma of a uniform dark brownish red. Murle serum Hows from the "ut surface. The glomeruli stand out as minute blood-red points. 'The tips of papillie hyperemic and the calices of the pelvis surrounding them ecchymosed. Microscopic examination of fresh sections shows extreme engorgement of all blood vessels.

Urinary bladder contains $1 \frac{1}{2}$ liters of urine having a dark port-wine color and barely translucent in a layer 훈 inch deep. No sediment on standing.

The second stomach adherent to the diaphragm by means of old connective tissue over an area 6 inches square. This tissne dark red, very hyperæmic. The mucosa of fourth stomach in the laminated portion is of nniformly pink color. The pyloric portion is beset with a number of irregular shallow erosions with hemorrhagic base. They vary from $\frac{1}{2}$ to 2 inches in length, and are elongated in shape. The mesentery of duodenum (near portal fissure) is infiltrated with pale reddislı serum. Mucosa of smiall intestine coated with a pasty bile-stained substance representing desquamated epithelinm. Macosa of rectum congested in patches and containing fecal balls.

The pia covering the hemispheres of the brain injected and pigmented. The plexuses are considerably engorged with blood. No fluid in the ventricles and no abnormal appearance of the brain substance itself. In sections of the cerebral tissue hardened in Miiller's fluid and alcohol and stained in hamatoxylin capillaries conld be traced for some distance in the white substance underlying the gray, which were filled completely with infected corpuscles. From one of the puncta vasculosa on the cut surface of the white substance of the cerebrum, while still fresh, a bit of tissue was crushed uniler a cover gliss. In it a capillary was traced for some distance containing only infected corpuscles. In the choroid plexus of lateral veutricles many of the gorged capillaries are observed containing infected corpuscles only. 
Stained cover-glass preparations from various tissues and organs were examined for infected corpuseles with the following resnlts:

\begin{tabular}{|c|c|}
\hline \multicolumn{2}{|l|}{ Per cent. } \\
\hline 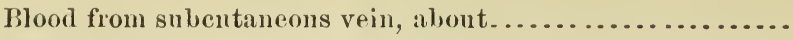 & 10 \\
\hline 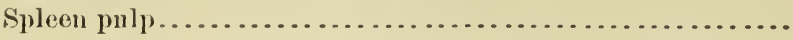 & 10 \\
\hline 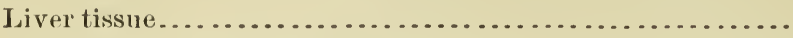 & 10 \\
\hline 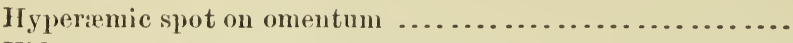 & 10 \\
\hline 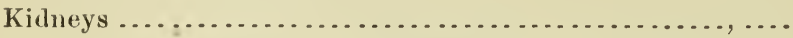 & 50 \\
\hline (excluding free parasites) .. & 30 \\
\hline . & $2-3$ \\
\hline (1) & $10-20$ \\
\hline second stomach............... & 10 \\
\hline
\end{tabular}

The parasites appeared in the red corpuscles, both single and in pairs. The numerical relation, in the different preparations, of single and double bodies varied more or less, the former being in some regions in the majority, in others in the minority. The single bodies were always roundish, the double bodies ronndish or pyriform. The best pyriform bodies appeared in preparations of corpuscles from the heart muscle, next in the circnlating blood, and in the capillaries of organs excepting spleen, liver, and kidneys.

No. 199 (native).-Cow, 8 years old, received June 3, 1892, from the District of Colnmbia, and placed in field $\mathrm{X}$ to test survival of ticks and Texas fever infection of 1891.

September 12.-No ticks, or evidence of Texas fever infection $n p$ to date. Calf due in October. Temperature, 102.4. Red corpnseles, 4,820,000. Nothing abnormal in fresh and stained preparations exeepting a slight variation in size.

No. 200 (native).-Cow, 8 years old, received from Charles County, Md., June 20, 1892, and kept on an adjoining place nntil Augnst 30. Injected into left jugnlar vein 14 cc. (one syringeful) of defibrinated blood from sick native No. 222, being a portion of the same blood injected into Nos.197, 227, and 228, and kept at a temperature of $50^{\circ} \mathrm{F}$. in a refrigerator since Angust 27. (See No. 197 for details.)

Placed with above-mentioned cases in a fenced-off portion of field XII ( XII $\left._{n}\right)$.

No. 200, before the operation, had a temperature of $102.3^{\circ}$ F'. Red corpuscles, $5,400,000$. In fresh preparations of blood a considerable nmmber contain the bright bodies. Stained preparation negative.

September 6.-Temperature, 107; pulse, 75; respiration, 72. Red corpuscles, $3,950,000$; white, 7,500 . In fresh preparations of blood, probably 2 per cent of corpuscles contain large parasites of irregular outline. 'The corpuseles themselves are darker in color and wrinkled and notched along border. Some contain bright bodies. In stained preparation some of the intraglobnlar parasites are in pairs and pyriform, others single and irregular in outline.

September 8.-Dies about 8:30 a. m.; autopsy threo hours later. Animal thiu. No skin lesions. In abdominal cavity hyper:enic patehes on omentun. Gelatinons adema between spleen and pannch.

Lungs, normal. Marked ecchymosis of the epicardium and endocardium of the ventricular surfaces of the heart.

Spleen weighs $4 \frac{3}{8}$ pounds. Pulp has a blackish homogeneons appearance. Markings of cut surface effaced.

Liver weighs 17 pounds. Has a brownish color, mottled with paler dots. On the cut snrface, which is more or less nniformly brownish, the paler yellowish lines and dots corresponding to the tissue immediately arome the intralobular veins appear only in localized patches. In sections of fresh tissue the intralobnlar capillaries markedly distended with red corpuscles; bilo injection detected in small patehes. latty degeneration very slight. Some hepatic cells contain pigment particles.

(iall bladder contains 1,200 ce. (3 pints) of very dark bile fiee from suspended matter. 
Kinmeys show the usual dak brownish red appearance. The right weighes 2 pounds; the left $1 \frac{7}{8}$ pounds. In fresh sections, the usual ougorged eondition of all the eapillaries.

Urine has a dark port-wine color. Specific gravity, 1020. A fow drops of acetic acid give a precipitate. Next dily the filtrate is free from the blood-coloring matter. When tested for albumcu hy lieat an abundant precipitate forns. Total alloumen of the uriue (ksbach) 1.2 pre eent.

The walls of the small intestine show marked injection of the minnte vessels. Contents fecal and bile stained. Mucosin uniformly pinkish red, due to injection of the capillaries.

Iterus contains a foetus 4 to 5 mouths old.

Brain. Marked pigmentation of the pia of the frontal lobes and of the lateral aspect extending upon the base at the optie commissure. Similar pigmentation at the great transverse fissure. The minnte vessels of pia injected. The injection most marked within the sulei as seen on cross section.

The Texas fever parasites were found in the various organs examined. They aro either single or in pairs, mostly romulish, and nearly $1 \mu$ in diameter. According to stained eover-glass preparations, the approximate infection is as follows:

Blood from subeutis contains 3 to 5 per cent infected corpuseles.

Spleen contains 5 per cent of infected corpuseles (parasites chiefly single).

Liver contains 30 per cent of infected corpuseles (parasites single and paired).

Kidneys contain 30 per cent of in fected corpuseles (parasites single and paired).

Heart muscle contains 50 per cent of in fected corpuscles (parasites chiefly in pairs).

In the choroid plexus where it enters the transverse fissure of the brain the parasites were observed within the capillaries in a fresh preparation. (For temperature record, see p. 294.)

No. 201 (native).-Cow, 5 years old, received June 20, 1892, from the District of Columbia. Kept in field between inclosures I and II until June 30, then placed in field VI for exposure to North Carolina cattle with ticks. (North Carolina cattle added July 1.) This animal is handled with considerable difficulty, hence the highpulse rate as given below.

July 7.-Temperature, 102 ; pulse, 92 ; respiration, 44 . Red corpuscles, $6,300,000$. White, 8,750 . In stained preparation several maerocytes and an occasional punctate corpusele.

July 12.-Temperature, 102.6; pulse, 80; respiration, 44. Red corpuscles, 5, 762,500; white, 9,555. Blood examination as before.

July 18.-Temperature, 102.8; pulse, 68 ; respiration, 72. Rerl corpuscles, $5,887,500$; white, 10,000. More or less variation in the size of the corpuseles; examination otherwise negative.

July 20.-Transferred to uunsed field IV.

Angust 24.-Temperature, 101.8; pulse, 75; respiration, 54. Rerl corpuseles, $5,950,000$; white, 10,000 . Slight variation in size of the red corpuscles. A few bright intraglobular bodies in fresh preparatiens of blood. In stained preparations one deeply stained round intraglobular body, $2 \mu$ dianeter (unclens of hiematoblast.)

September 3.-Temperature, 102.4. Red corpuseles, 6,187,500; white, 7,500. Condition of blood corpuseles normal both in fresh and stained preparations.

September 27.-Temperature, 103 (animal excited by chasing). Red eorpuseles, $6,875,000$; white, 7,500. $\Lambda$ moderate number of bright bodies detected in fresh preparations of blood. Stained preparations negative. (For temperature seo pp. 289, 290.)

No. 202 (native).-Cow, 7 years old, received June 20, 1892, from Charles Connty, Md., and placed in field between inclosures I and II.

Juue 30.-Transferred to Field XI.

August 31.-Transferred to Field XIII. (See also No. 207.)

Injected into the left jugnlar vein 14 ce. of a fluid prepared by grinding up in a mortar a large number of young ticks in a small quantity of distilled water and filtering throngh filter paper. The ticks were hatched artificially from adults received 
July 9, 12, and 19, from near New Berne, N. C. The hatching began August 9, 11, and 15 , respectively. The injected thid was turbid and grayish in color and passed through the filter with sone difficulty.

('Ticks from lots received July 9 and 19 placed on the skin of No. 224 August 30 and September 3, produced Texas fever (see No. 224), showing that the ticks were capable of producing infection in the ordinary way.)

The condition of the blood on the the day of the injection was as follows: Red corpuscles, 6,228,500; white, 7,142. In fresh preparations a slight variation in size of the former and a few bright intraglobular bodies observed. Stained preparation negative.

September 9.-Temperature, 101.5; pulse, 72; respiration, 39. Red corpuscles, $6,050,000$; white, 10,000. Nothing abnormal detected in preparations of fresh and stained blood.

September 21.--Temperature, 102.2. Red corpuscles, 5,666,000; white, 4,938. Nothing abnormal in dried and stained preparations of blood. (For temperature record, see p. 294.)

No. 203 (native.)-Cow, 6 , years old, received June 20, from Charles County, Mrd., and placed in field between inclosures I and II until June 30. Then transferred to field VI for exposure to North Carolina cattle with ticks (North Carolina Cattleadded July 1 ).

July 7.-Temperature, 102.5 ; pulse, 84 ; respiration, 48 . Red corpuscles, $6,375,000$; white, 10,000. In stained tilms slight variation in size of the red corpuscles. One corpuscle contains what appears to be a peripheral cocens.

July 12.-Temperature, 102.8 ; pulse, 80 ; respiration, $52 . \quad$ Red corpuscles, $5,800,000$; white, 12,500. In stained preparation a slight variation in size of corpuscles. One intraglobular parasite (?) of irregular outline.

July 18.-Temperature, 102.4 ; pulse, 80 ; respiration, 52. Red corpuseles, 5,875,000; white, 13,750 . In stained preparation a slight variation in the size of the red corpuscles.

August 22.-Temperature, 101.1; pulse, 108; respiration, 72. Red corpuscles, $1,659,000$; white, 6,813 . In the fresh blood about 2 to 3 per cent of the corpisscles appear infected with single parasites chiefly. There are besides a few bright intraglobular bodies, and a large number of very minute free diplococcus-like bodies not more than 0.2 to $0.3 \mu$ in diameter in violent (Brownian?) motion. In stained preparation the irregular intraglolular parasites stain on the periphery only. Cow has continuer eating until this morning; dies suddenly at $2 \mathrm{p} . \mathrm{m}$. The very brief autopsy notes are appended to confirm the diagnosis.

Animals weighs about 50 n pounds; in quite poor condition, A large number of ticks on udder and escutcheon, perliaps six to the square incli. Few on abdomen and dewlap. The ticks were of several sizes:

(1) Ticks within the last moult and abont ready to emerge, $3.211 \mathrm{~m}$ ( $\frac{1}{8}$ inch) long.

(2) Ticks just emerged from the last moult and quite active. Size as before.

(3) Adult ticks from 4 to $7 \mathrm{~mm}$. $\left(\frac{1}{6}-\frac{7}{2}\right.$ inch) long.

(4) Males about $2 \mathrm{~mm}$. ( 12 inch) long.

Brain: Marked pignentation and injection of the minute vessels of the pia on the hemispheres. $\Lambda$ small hemorrhagie spot 3 mm. in diameter in the third ventricle on left thalamus. The gray matter of eerehrum and cerebellum appears slightly reddeneil. Other changes absent.

The heart shows the usnal epicardial and endocardial extravasations. "Heart fibers show many fat gramules.

The spleen weights 35 pounds. Large and softened. Markings no longer visible on section in the blackish pulp.

The liver weighs $13{ }_{1}^{1}$ pounds. Paler than normal and mottlod on the surfaco. The cut surface is brownisl yellow. Bile in gall bladder (1 $\frac{1}{2}$ pounds) very thick and flaky. The kidneys weigh each $1 \frac{1}{4}$ pounds, of the usual dark loownish red appearance. In the hladiler 2 quarts of very dark port-wine colored urine barely translucent in a layer $\frac{8}{4}$ inch deop. 
The mucosa of small intestine injected and coated with a pasty layer of desquamated epithelium. The cernm contains soft feces and is extensively pigmented along the summits of the longitudinal folds.

In the serum expressed from the heart muscle a large number of pairs of parasites inclosed in barely visible bodies representing the outlines of former red corpuscles. In a teased preparation of spleen pulp several capillaries seen, plugged with infected corpmscles. In stained preparations the infection was roughly estimated as follows:

Spleen and liver about 10 per eent infected corpuscles.

Kidneys about 50 per cent infected corpuscles and many free forms.

Heart muscle about 30 per cent infected corpuscles and many apparently firee forms. (For temperature record, see pp. 289, 290.)

No. 204 (native).-Bull, $2 \frac{1}{2}$ years old; received from Prince George County, Md., June 24, 1892, and placed in field between inclosures I and II until June 30. Then transferred to field $X I$.

August 26.-Temperature, 102.8. Red corpuscles, 5,540,000; white, 7936. Stained preparations show nothing abnormal. Transferred from field XI to field VI (North Carolina cattle with ticks) as control to reëxposed animals Nos. 56, 105, 135, 160, $166,167,182,185$.

September 8.-Temperature, 107.4 ; pulse, 72 ; respiration, $44 . \quad$ Red corpuseles, $4,225,000$; white, 5,000 . In the fresh blood a considerable number, perhaps 10 per cent, of very minute intraglobular bright bodies, of variable size, situated mainly near the periphery. A few are in motion. In stained preparations a few irregular intraglobular bodies of doubtful nature.

September 13.-Temperature, 103.5; pulse, 76 ; respiration, 28. Red corpuscles, $1,963,000$; white, 1.219. Stained preparations slow macrocytes.

September 27.--Temperature, 102; pulse, 60; respiration, 42. Red corpuscles, $3,753,000$; white, 7,407. In fresh blood 30 to 40 per cent of the rorpuscles are enlarged. Several bright bodies. In stained preparations no parasites detected.

October 10.-Temperature, 103.5; pulse, 64; respiration, 32. Red corpuscles, $5,012,000$; white, 7,564 . In stained preparations about 30 per cent macrocytes.

From September 10 to 15 the animal appeared decidedly sick. Appetite partly lost. It lay down much of the time and appeared gaunt and weak. Lately it has been improving rapidly. (For temperature record, see p. 290.)

No. 205 (natice).-Grade Jersey cow, five years old; received June 30, 1892, from District of Columbia. Placed in Field II (North Carolina cattle without ticks). (North Carolina cattle added July 2.)

July 5.-Temperature, 103.8 ; pulse, 60 ; respiration, 64 . Red corpuscles, 5,975,000; white, 6,250 .

July 18.-Temperature, 102.8 ; pulse, 56 ; respiration, 48 . Red corpuscles, $5,400,000$; white, 6,250. Stained preparatious show nothing abuormal.

August 25.-Temperature, 101; pulse, 60 ; respiration, 54. Red corpuseles, 5,962,500; white, 10,000. Slight variation in size of red corpuscles. A small number contain the bright bodies. Stained preparation negative.

September 2.-Temperature, 101.2; pulse, 54: respiration, 30. Red corpuscles, $5,887,500$; white, 5,000. A small number of red corpuscles contain bright boclies. Stained preparation shows nothing abnormal.

September 16.-Temperature, 101.8 ; pulse, 48; respiration, 40. Red corpuscles, $5,370,000$; white, 5,714. Nothing abnormal in stained preparation. (See p. 291.)

No. 206 (native).-Cow, seven years oid; received June 30, 1892, from the District of Columbia and placed in Field III.

July 6, 9 a. m.-Received into left jugular vein 28 ce. (2 syringefuls) of blood drawn from left jugular of North Carolina cow No.216. (See No. 198 for details of operation.) The following table gives the essential facts of the history of the disease induced by the injection. 


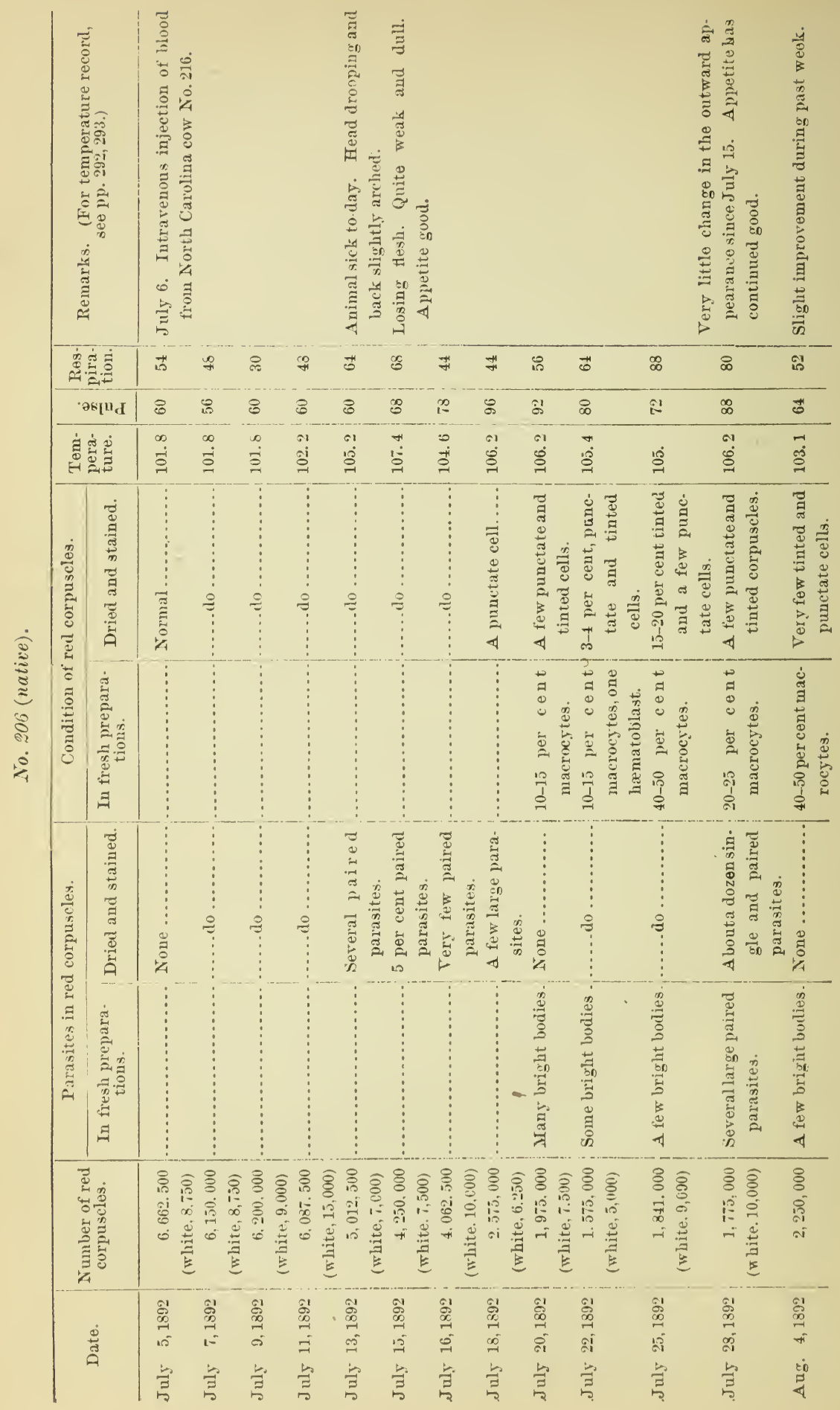




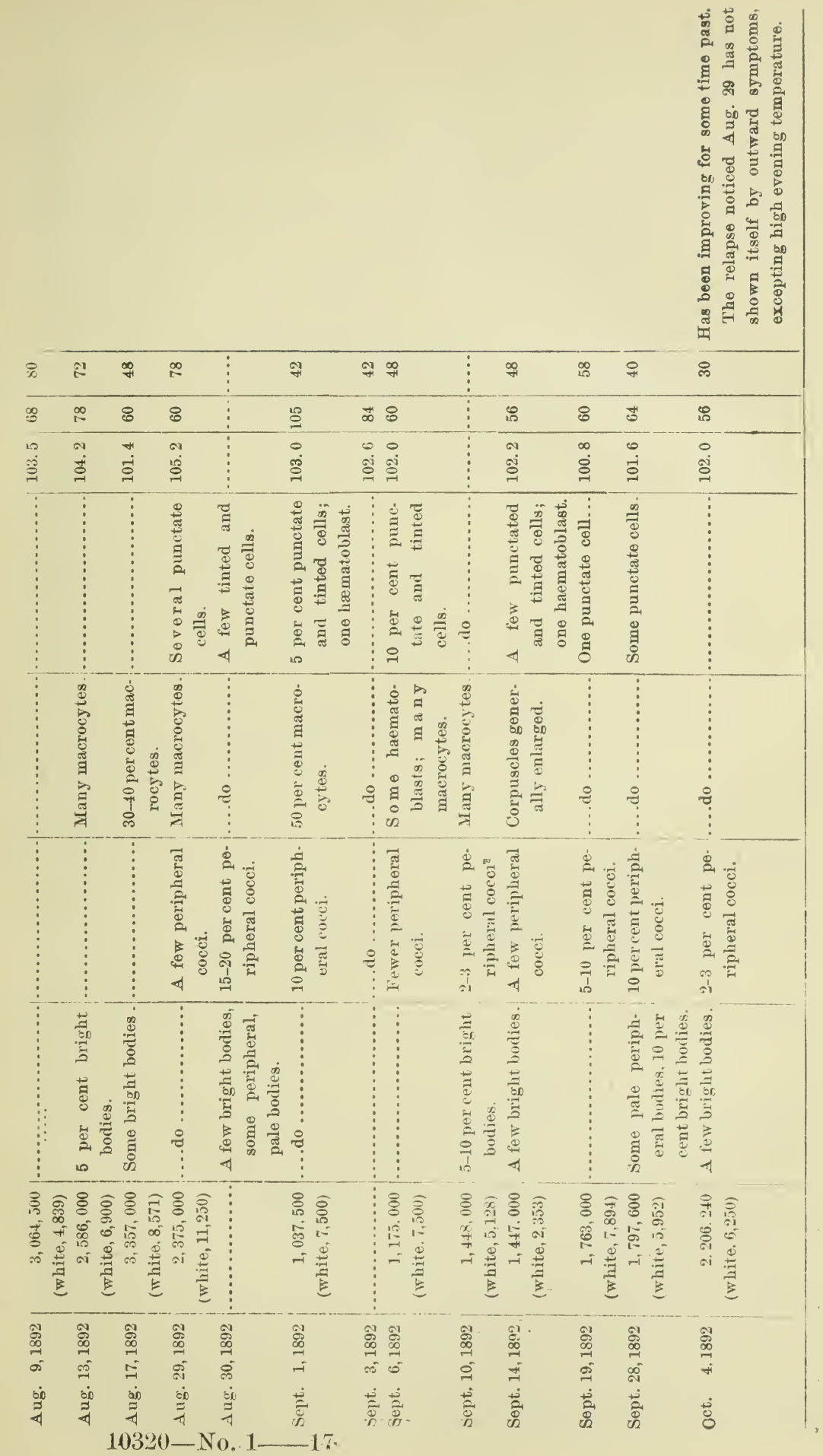


No. 207 (native). - Steer, ? years old, received June 30, 1892, from Prince George County, Md, and placed in field XI.

Angust 31.--Transferred to field XIII.

Temperature, 103.2; pulse, 98; respiration, 78. (Animal much excited on being eaught.) Red corpuseles, $5,985,700$; white, 10,000. In preparations of fresh blood a moderate number of bright bodies detected. Nothing abnormal in stained preparation. The red corpuscles vary slightly in size.

Injected into left jugntar vein 14 ' '. of the same fluid used upon No. 202 (flnid from young ticks), with the exception that in this case the fluid was filtered through muslin instead of filter paper. 'The turbid filtered Huid held in snspension a considerable quantity of minute particles rapidly settling when the fluid was allowed to stand. (See also No. 224.)

September 9.-Temperature, 102.2. Red corpuseles, 5,275,000; white, 7,500.

In fresh and stained preparations nothing abnormal.

September 21.-Temperature, 102.4. Red corpuscles, $6,570,000$; white, 6,024. In a stained preparation nothing abnormal. (For temperature record, see p. 294.)

No. 208 (native).-Cow, 5 years old, received .June 30, 1892, from District of Colun. bia and plaeed in field I (North Carolina cattle without ticks. North Carolina eattle added July 2$)$.

July 6.-Temperature, 102. Red corpuscles, 6,800,000; white, 7,500. Nothing abnormal in stained preparation.

July 15.-Temperature, 101.8. Red corpuseles, 5,811,000; white, 10,000. Stained preparation as before.

August 25.-Temperature, 101.2. Red corpuscles, 6,350,000; white, 11,250. Slight variation in size of red corpuseles. A fow eontain bright bodies. In stained preparation nothing abnormal.

September 2.-Temperature, 101.6. Red corpuscles, 5,500,000; white, 10,000. Slight variation in size of the former. Stained preparation as before.

September 16.-Temperature, 101. Red corpuscles, 6,080,000; white, 12,345. In stained preparation slight variation in size of the former. Otherwise nothing abnormal. (For temperature record, see p. 291.)

No. 209 (native).-Cow, 9 years old, received June 30, 1892, from the District of Columbia. Placed in field I (North Carolina cattle without ticks. North Carolina cattle added July 2).

July 6.-Temperature, 102.6. Red eorpuseles, 6,787,500; white, 10,875.

July 15.-Temperature, 103 . Red corpuseles, 6,000,000; white, 13,750.

July 25.-Temperature, 105.5. Red eorpuseles, 5,675,000; white, 11,250. In a few red eorpuseles bright hodies.

Augnst 25.-Temperature, 102.8. Red corpuscles, 5,687,500; white, 10,000. Slight variation in size of the former. A fow contain bright bodies.

September 2.-Temperature, 102. Red eorpuseles, 5,225,000; white, $15,000 . \quad \Lambda$ few of the former eontain bright bodies.

September 16.-Temperature, 100.6. Red eorpuscles, 5,309,000; white, 8,333.

Stained preparations of blood eolleeted on all of the above dates show nothing abnornal. In the last there is a very slight variation in size. (See p. 291.)

No. 210 (native).-Heifer, 2 years old, reeeived June 30, 1892, from Prince Goorge's County, Md.; plaeed in field I (North Caroliua cattle without ticks. North Carolina eattle added July 2 ).

July 6.-Temperature, 102.2. Rod corpuseles, 6,875,000; white, 16,625.

Jnly 18.-Temperature, 102. Red corpuseles, 6,425,000; white, 10,000 .

Augnst 25.-Temperature, 102. Red corpuscles, 6,025,000; white, 12,500. Slight variation in size of the former. A few eontain bright bodies.

September 2.-Temperature, 101.2. Red eorpuseles, 5,650,000; white, 5,000. A few red eorpuscles eoutain bright hodies.

September 16.-Temperature, 101.8. Red corpuscles, 6,076,000. 
Stained coverglass preparations of blood eollected at every one of the ahove dates show nothing abnormal alter areful nirroscopic examination. (See p. 291.)

No. 21: (Southern).-Cow, 3 years old, received from near Now Berne, N. C., July $1,189 \%$.

July 2.-Kept in a fencerl-off portion of field VI over night. To-day tieks picherl oft, after which process the animal was placed in field I (Nerth Carolina cattle without ticks).

July 3-27.-Animal examined daily and any ticks found carefinly pickerl off. No ticks found after July 21.

No. 213 (Southern).-Cow, 5 ycars old. For source, removal of ticks, and field, see No. 212.

No. 214 (Southern).-Cow, 4 years old. For source of animal and removal of ticks sec No. 212. Placed in field II July 2.

Angust 15. - Blood withdrawn from jugular to inoculate No. 222. Slight variation in the size of the red corpuscles $(5$ to $7 \mu$.) No parasites detected in two stained preparations after prolonged search.

September 9.-Blood withdrawn to inoculate No. 230. No parasites detected in a stained preparation even after a long search.

No. 215 (Southern).-Cow, 4 years old. For source of animal and removal of ticks see No. 212. Placed in field II July 2.

No. 216 (Southern).-Cow, 6 years old, received from near New Berne, N. C., July 1, 1892, and placed in field VI (North Carolina cattle with ticks).

July 6.-Blood withdrawn from jugular vein to inoculate native No. 206.

July 14.-Red corpuscles, $6,050,000$. In two stained preparations of blood, one peripheral coccus-like body detected after a long search.

July 16.-Blood withdrawn to inoculate native No. 219.

September 3.-Temperature, 102.8. Red corpuscles, 6,112,500; white, 15,000. In fresh preparations nothing abnormal. In a stained preparation nothing abnormal detected aiter a long search.

No. 217 (Southern).-Cow, 6 years old, received from near New Berne, N. C., July 1, 1892, and placed in field VI (North Carolina cattle with ticks).

July 6.-Bloood withdrawn from jugular vein to inoculate native No. 198.

July 8.- Red corpuscles, 6,012,500. In two stained preparations of blood, after a long search, a peripheral coccus detected in process of division.

September 3.-Temperature, 102.6. Red corpuscles, 6,600,000. Fresh preparations show nothing abnormal. In a stained preparation a pair of intraglobular pyriform bodies detected after a long search.

No. 218 (native).-Cow, 6 years olđ, reccived July 15, 1892, from Prince George County, Md., and placed in unused field XI.

July 16.-Tempcrature, 103. Red corpuscles, 6,450,000); white, 10,000. 'Transferred to field III, as control to inoculated cow No. 219.

Angust 4.-Temperature, 102.2. Red corpuscles, 5,437,500. In preparations of fresh blood about 10 per cent of all corpuseles contain bright motile bodies. August 6.-Temperature, 101.8. Red corpuseles, 6,342,000; white, 9,589. Condition of red corpuscles as on August 4. Angust 15.-Temperatire, 102. Red corpuscles, 5,973,000; white, 10,666. Condition of corpuscles as before. August 29.-Temperature, 102.2. Red corpuscles, $6,587,500$; white, 10,000. In fresh preparations 3 to 5 per cent of the former contain bright bodies. September 1.-Temperature, 101.8. Red corpuscles, 6,787,500; white, 8,750. Condition of the former as on Angust 29. September 17.Temperature, 101.8. Red corpuscles, 5,820,000; white, 4,494. September 29.-Temperature, 101.5. Red corpuscles, 5,650,000; white, 10,000. From 2 to 3 per cent of the former contain bright bodies, varying in size.

Stained films of blood, prepared at every one of the above dates. show nothing abnormal after careful examination. (For tcmperature record, see pp. 292, 293.) 
TEXAS OR SOUTHERN CATTLE FEVER.

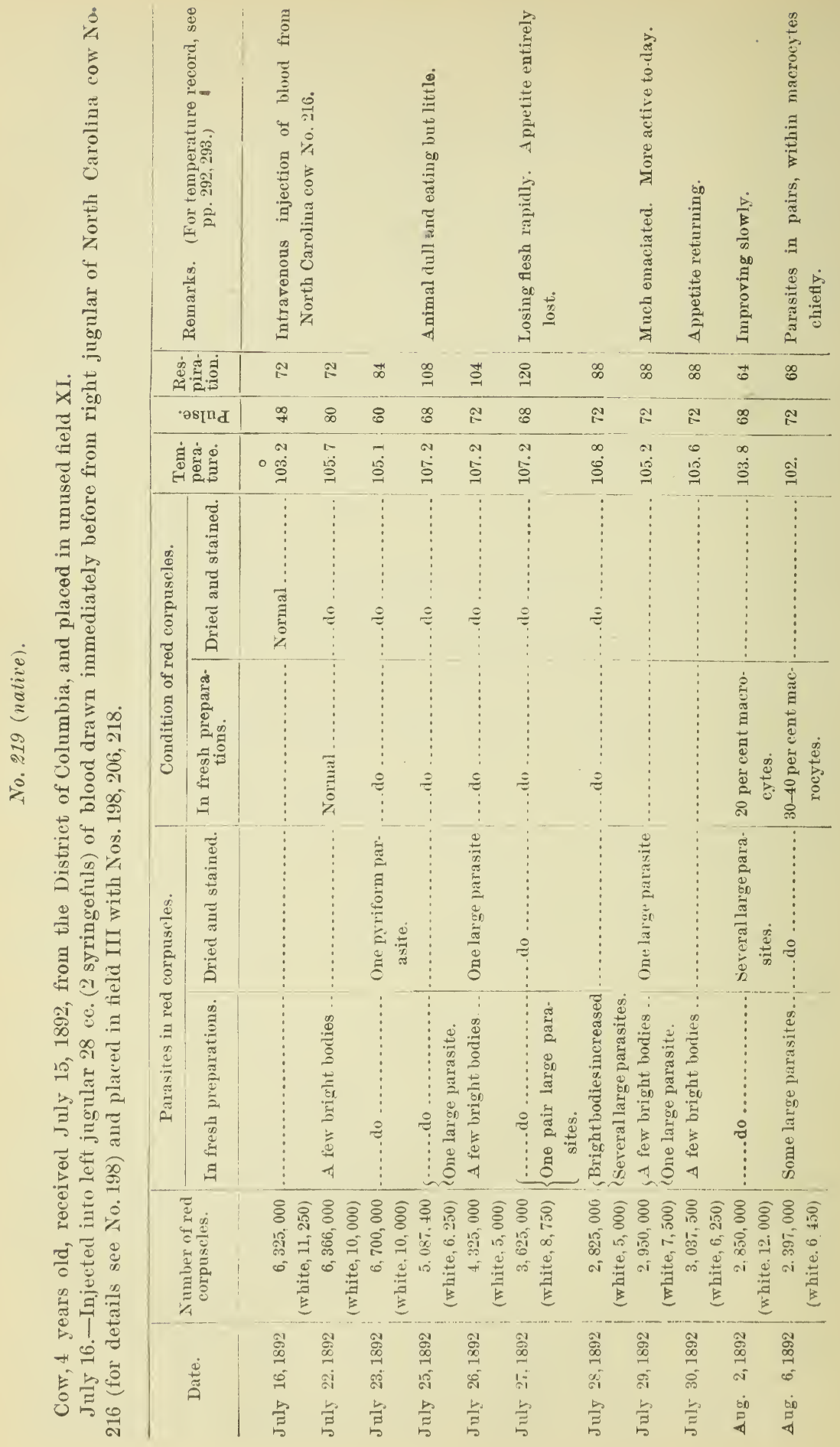




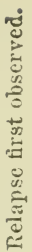

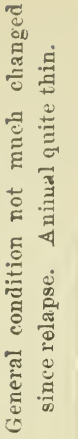

\begin{tabular}{|c|c|c|c|c|c|c|c|c|c|c|c|}
\hline த & 8 & 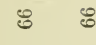 & 8 & 5 & & స్లి & 8 & 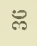 & $\&$ & ํํํ & है \\
\hline 8 & $\because$ & $\stackrel{\infty}{i}$ & $\infty$ & $\bar{\infty}$ & & 88 & 8 & 8 & $\&$ & $\&$ & DD \\
\hline $\begin{array}{l}\infty \\
\text { i } \\
\vec{c} \\
\overrightarrow{1}\end{array}$ & $\ddot{n}$ & 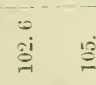 & $\stackrel{8}{8}$ & $\stackrel{\text { ஜ் }}{g}$ & & 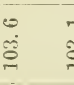 & $\ddot{8}$ & gi & में & 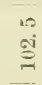 & बे \\
\hline 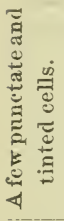 & $\begin{array}{c}\vdots \\
\vdots \\
\vdots \\
\vdots \\
\vdots\end{array}$ & 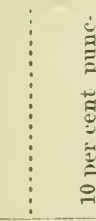 & 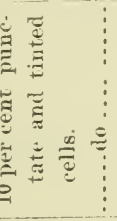 & 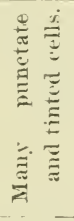 & $\begin{array}{c}\vdots \\
\vdots \\
\vdots \\
\vdots \\
\vdots \\
\vdots\end{array}$ & 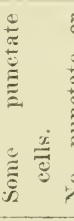 & 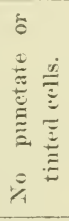 & & 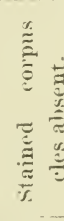 & $\vdots$ & $\begin{array}{l}0 \\
\vdots \\
\vdots \\
0\end{array}$ \\
\hline 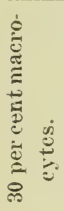 & 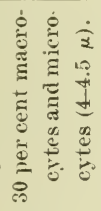 & 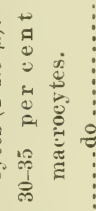 & $\begin{array}{c}\vdots \\
\vdots \\
\vdots \\
\vdots \\
\vdots \\
\vdots \\
\vdots\end{array}$ & 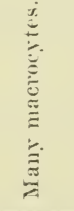 & & & $\begin{array}{c}\vdots \\
\vdots \\
气 \\
\vdots \\
\vdots\end{array}$ & & 苞 & $\stackrel{\leftrightarrow}{ٍ}$ & $\begin{array}{c} \\
\vdots \\
\vdots \\
\vdots \\
\vdots\end{array}$ \\
\hline
\end{tabular}

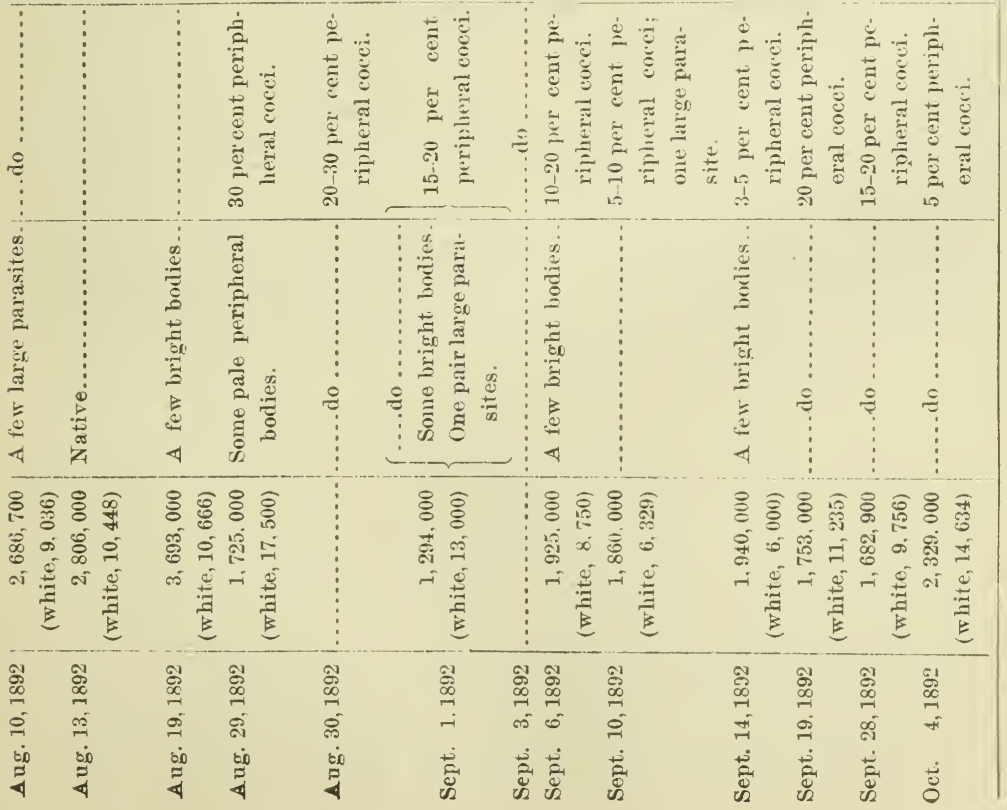


No. $2 \% 0$ (native).-Steer, $2 \frac{1}{2}$ years old, received July 1.5, 1×92, from Montgomery ('omnty, Md., and placed in an unused field Xl.

July 20.--Transferred to field VI (North Carolina eattle with ticks). Temperature, 105.6 (elevated by prolonged chasing). Red corpuseles, 6,425,000; white, 12,500. In fresh prepurations a few corpuseles containing bright hodies.

Augnst '2.--Animal sick, cating but lit tle.

Angust 21.-Temperature, 106.6; pulse, 105; respiration, 93. Rerl corpuscles, $2,727,000$; white, 8,080 . In fresh preparations one pair of large parasites and a fow niacrocytes.

August 25.-General condition slightly improved. Appetite returning.

Angust 27.-Temperature, 103.6; pnlse, 84; respiration, 60. Rerl corpuscles, $2,550,000$; white, 10,000 . In fresh preparations parasites not detected. Abont i to 10 per cent macrocytes. In stained preparations a small number of marrorytes are feebly tinted or finely punctated.

September 1.-Temperature, 103; pulse, 108; respiration, 79 (animal excited). Red corpuscles, $3,662,500$, white; 15,000 . About 30 per cent marrocytes and a few bright bodies seen in fresh preparation. Macrocytes very large. A few feebly tinted and punctated corpuscles in stained preparation.

October 1.-Temperature, 101.5; pulse, 68; respiration, 36. Red corpuscles. 1,425,000; white, 12,500. Over 50 per cent macrocytes. This animal lost considerable flesh during the disease. Has been slowly gaining since the middle of september. (For temperature record, see pp. 289, 290.)

No. 221 (native).-Cow, 9 years old, received Jnly 18, 1892, from Montgomery C'ounty, Md., and placed in nnused field XI.

August 15.-Temperature, 102.4. Red corpuscles, 6,550,000. A moderate number contain bright bodies. Transferred to field III as control to inocnlated case No. 222.

August 29.-Temperature, 102.4. Red corpuscles, 5,487,500; white, 10,000. Condition of blood as before.

September 1.-Temperature, 101.6. Red corpuscles, 5,912,500; white, 20,000. $\Lambda$ few of the former contain bright bodies.

September 17.-Temperature, 102.5. Red corpuscles, 5,375,000; white. 10,000.

September 29.-Temperature, 101.8. Red corpuscles, 5,912,000; white, 9,876. A very few bright bodies in fresh preparation.

Stained films of blood prepared on each of the above dates show nothing abuormal. (For temperatnre record, see p. 293.)

No. 222 (native).-Steer, 5 years old, received Jnly 20, 1892, from Montgomery Connty, Md., and placed in unused field XI.

Augnst, 15.-Received into left jugular vein 2 syringefuls, or 28 cubic centimeters, of blood drawn immediately before from jugular of North Carolina cow No. 214 (see No. 198) and placed in field III. The following table gives the subsequent history of this case and indicates a relapse lirst detected september 28. 


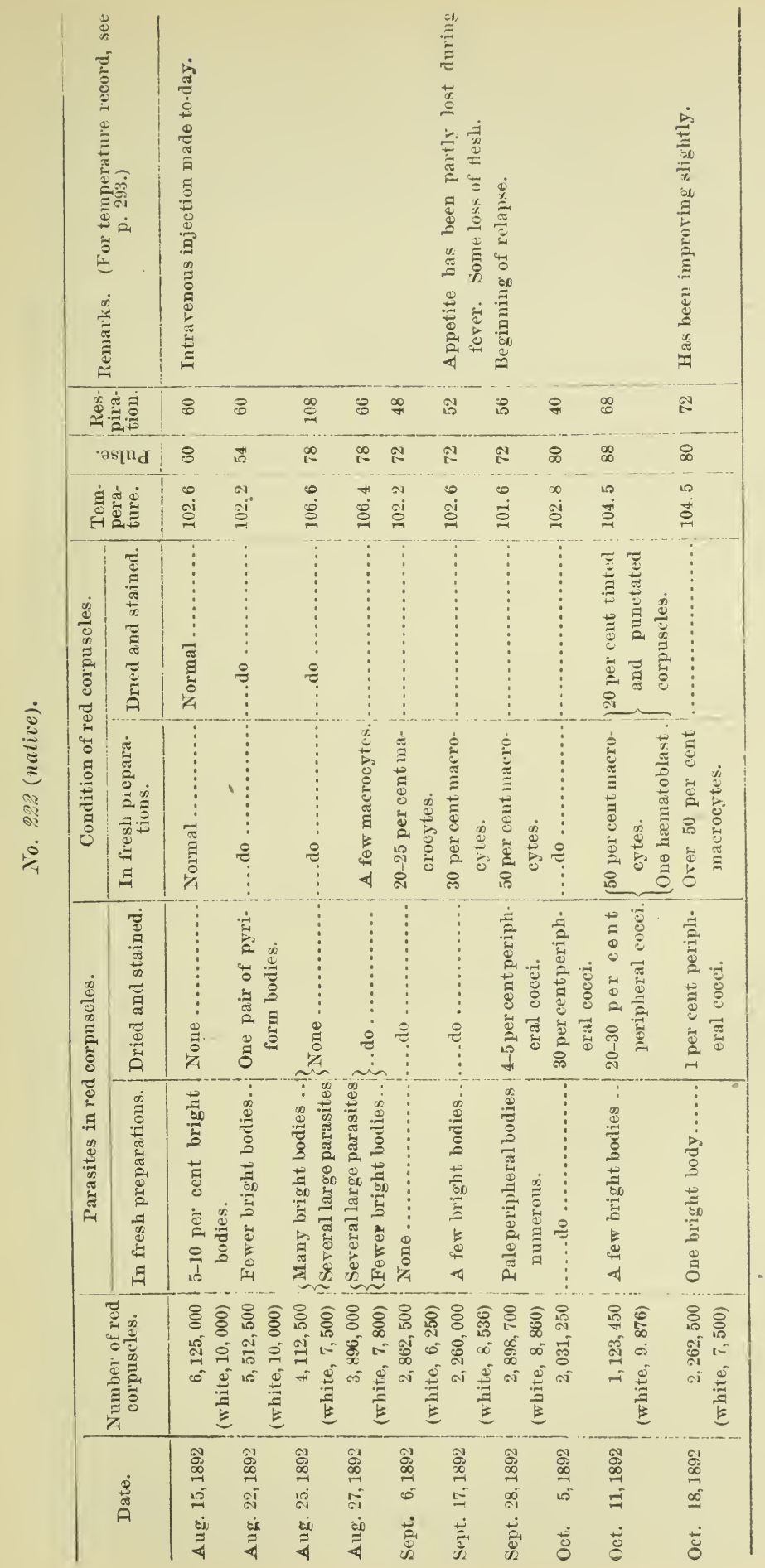


No. 223 (native).-Heifer, 6 years old, received July 20, 1892, from Montgomery ('ounty, Md., and placed in field VI (North Carolini cattle with ticks). Temperature, 102.5. Red corpuscles, 6,500,000; white, 10,000. In fresh preparations of blood a few corpuscles contained the bright bodies. Stained prejarations negative.

August 19.-Temperature, 106.8; pulse, 72; respiration, 84. Red corpuscles, $5,087,500$; white, 5,000. In fresh preparatious of blood about 1 per cent of the corpuscles have a dark wrecked appearance, and contain the Texas fever parasite.

August 20.-Auimal sick. Eating but little.

August 22.-Animal refuses food entirely.

August 23.-Animal died during the night.

Autopsy at 9 a. m.: Body in good condition as regards flesh. Weight about 600 pounds. A moderate number of ticks on inner aspert of thighs, escutcheon, and udder. The sizes are the same as those found on No. 203.

Both lungs quite full of blood. The left principal adherent to chest wall and the left ventral to the former by old connective tissue. Very marked extravasation of blood on the base of the heart, extending about 2 inches each side of the interventricular groove. Slight ecchymosis beneath endocardium. Injection of heart muscle. Cloudy and fatty changes of fibers.

Spleen weighs $4 \frac{1}{4}$ pounds. Capsule much distended. Pulp blackish, but still firm and markings effaced.

Liver weighs $14 \frac{2}{8}$ pounds. It is of a uniform yellowish-brown color, as viewed from the exterior, excepting near the left border, where there is more or less grayish mottling. More or less bile injection in circumscribed areas detected in sections of fresh tissue. The gall bladder contains 4 ounces of bile, having a dark yellowishgreen color, and holding a very large quantity of flaky material in suspension.

The fat surrounding kidneys infiltrated with reddish serum. With capsule removed each weighs $1 \frac{8}{8}$ pounds. The parenchyma has the usual dark brownisli-red granular appearance of the eut surface. Slight eechymosis of the calices of the pelvis. In sections of fresh tissue marked injection of all capillaries. In the bladder about 2 quarts of port-wine colored urine slightly more translucent than that of No. 203.

Digestive tract: In the fourth stomach the mucosa of laminated portion shows a large number of round whitish spots, not elevated above the general surface, 2 to 5 mm. in diameter. (Strongylus Ostertagi?) The epithelium over these spots intact. In pyloric portion two or three small blackish depressed scars. In the suall intestine the mucosa is injected as usual, and covered with a thin grayish pasty layer composed entirely of desquanated epithelium. There is much bile in the intestine. In sections of the fresh mucosa, the parasites of 'Texas fever can be seen distiuctly in every corpuscle of some of the distended capillaries.

Uterus contains a fœtus 7 to 8 inches long. Amniotic fluid free from hæmoglobin.

The pia covering the hemispheres of the braiu injected and pigmented. The brain substance and the ventricles show no lesions excepting a more pinkish appearance of the gray matter of the cerebellum.

In the organs the number of infecterl corpuscles, estimated from stained coverglass preparations, is approximately as follows:

Spleen, 10 per cent, parasites single and in pairs.

Liver, 30 per cent, parasites chiefly in pairs.

Kidney, 30 per cent, together with a very large number of free forms.

Heart muscle, 30 per cent, and many free pairs. (For temperature, see pp. 289, 290.)

No. 224 (native).-Cow, 11 years old, received August 1, 1892, from Prince George County, Md., and placed in field XI.

August4.--Temperature, 103.8; pulse, 68; respiration, 76 (excited in being canght). Red corpuscles, 6,194,000. No abnormal appearances in fresh and stained prepara. tions. 
Augnst 9.-Placed in box stall to-day, and the artificially hat.hed young tieks of Amblyomma unipunctata placed on neck and rump.

Angust 30.- The ticks have not developed. A large number of artificially liatched ticks of Bö̈philus bovis placed on rmup and neck. Adults of these ticks received from near Now Berne, N. C., July 19, 18:92. The young hegan to hatch August 15.

The condition of the blood to-day is as follows: Red corpuscles, $6,112,500$; white, 7,500. A few corpuscles contain bright bodies. In stained preparation nothing abnormal.

September 3.-A second lot of yonng ticks (B. bovis) placed on auimal. Adults were received July 9. The young began to hatch August 9. (See also Nos. 202 and 207.)

September 10.-Temperature, 106.8; pulse, 72; respiration, 24. Red corpuscles, $3,925,000$; white, 5,000. In a stained preparation two corpuscles found containiug limge parasites.

September 15-20.-Animal has lost considerable flesh. Dull.

September 22.-First matured ticks fonnd.

September 26.-Temperatine, 102 ; pulse, 80 ; respiration, 24. Red corpuscles, $2,062,500$; white, 5,000. From 25 to 30 per cent macrocytes and a very few punctate corpuscles. A large number of ticks almost ready to drop off on this animal.

September 29.-Very lit tle improvement; animal thin and weak, probably in part due to the abstraction of blood by the many maturing ticks.

No. 225 (native).-Cow, 8 years old, received August 1, 1892, from Prince George County, Md. Placed in field XI.

Angust 2.-Transferred to box stall in stable. A few young recently hatched ticks placed on rump and neck. Temperature, 102.2. Red corpuscles, 6,405,000; white, 9,459. A few bright bodies in corpuscles. Stained preparation negative.

August 10.-This process of adding young ticks repeated daily from August 2 until to-rlay. The adult ticks were collected near New Berne, N. C., June 27, from Hyde Connty cattle. Eggs laid June 29-July 11. The young began to appear July 30.

August 15.-Refuses food.

August 17.-Losing flesh rapidly. Temperature, 105.8; pulse, 72 ; respiration, 42. Red corpuscles, 4,800,000; white, 10,750. One large parasite detected in fresh blood after some searching. In stained preparation 10 to 12 corpuscles detected containing pyriform bodies.

August 18.-Feces passed in small quantity and deeply bile-stained. Animal weak and lying down much of the time. Urine appears free from coloring matter of the blood.

August 19.- Very weak. Urine as yesterday. Feces soft. Drinking much water, but refusing solid food.

August 24.-Has been slowly improving since Augnst 21. Appetite returned. Ticks beginning to mature. Temperature, 102 ; pulse, 75 ; respiration, 51 . Red corpuscles, 2,987,000; white, 8,860. Macrocytes 10 to 15 per cent. One hæmatoblast seen. A few punctate macrocytes in stained preparation.

August 30.-A large number of ticks have matured since August 24 and many are still maturing. Animal improving. Transferred from stable to field VI (North Carolina cattle with ticks).

September 5.-Temperature, 101.7; pulse, 63; respiration, 39. Red corpuscles, 4,600,000; white, 10,000. A few bright bodies seen in fresh blood. Twenty per cent macrocytes.

September 23.-Temperature, 102.7; pulse, 80; respiration, 52. Red corpuscles, $1,888,000$; white, 7,407. A few pairs of large parasites detected in fresh and stained blood. Ten to 15 per cent macrocy tes up to $11 \mu$ in diameter. Some punctated and tainted. Several hæmatoblasts.

October 1.-Temperature, 102.2; pulse, 60; respiration, 24. Red corpuscles, $3,300,000$; white, 1,111. Macrocytes over 50 per cent ( 8 to $10 \mu$ in diamoter). 
October 10.-Temperature, 102.7; pulse, f8; respiratiou, 36. Red corpuscles, 3,623,000; white, 4,706. Macrocytes still ummerous. Animal still thin. Slowly improving. (For the temperature record, see p. 290.)

No. 297 (native).-Cow, 11 years old, received August 27, 1892, from the District of Columbia. Received, on this day, under the skin of the neck, with a hypodermie syringe, 2 cc. of defibrinated blood from sick native (No. 222). The injection was made in four places, $\frac{1}{2} \mathrm{ec}$. being injected in each place. Animal placed in fenced-ofi portion of field XII $\left(\mathrm{XII}_{\mathrm{n}}\right.$ ) (For the preparation of the blood and other details, see No. 197.)

Angust 30.-Temperature, 102.2; pulse, 60; respiration, 72. Red corpuscles. $5,312,500$; white, 5,000. A few corpuscles contain bright bodies.

September 5.--Temperature, 105.4; pulse, 78; respiration, 66. Rel corpuscles, $3,887,500$; white, 3,750 . A few corpuscles contain large parasites.

September 6.-Animal weak and eating but little.

September 8.--Animal very weak; stands with head drooping; hind quarters sway in walking; refuses food entirely.

September 9.-Temperature, 101.5; pulse, 84; respiration, 12. Reil corpuscles, $1,555,000$; white, 6,666. A few enlarged corpuscles detected.

Dies about $6 \mathrm{p}$. m. to-day.

September 10, 10 a. m.-Autopsy: Cow now weighs about 500 pounds; weight before disease, 750 to 800 pounds. Fat over the entire body of a deeper yellow than is found in cows of this age. Slight post-mortem decomposition.

Extensive ecchymoses beneath epicardinm and endocardium of ventricular surfaces of heart. In both cavities small firm clots, having on the surface a bright golden color in patches.

Lungs full of blood, collapsed only in part. Old fibrous adhesions to chest wall. Slight in terlobular emphysema.

Spleen very large and of the usual blackish appearance on sertion. Much pigment in large lumps detected in teased preparations. Also many large red corpuscles and some hrematoblasts. Liver shows a uniform yellowish color throughout. In fresh sections, the parenchyma found in state of complete fatty degeneration. Individual cells contain 2 to 4 oil globules larger than red corpuscles. Occasional patches of bile injection. Bile viscid and loaded with flocculent matter, which stains the hands intensely yellow.

Kidneys surrounded by reddish œelematous tissue. Parenchyma pale. On section there is a swollen odematous appearance with absence of the usual markings. The base of the pyramids marked with pale strix. In fresh sections these correspond to tubules completely filled with fat globules. Clumps of anorphous yellow pigment are scattered through the epithelium of the straight tubules and bundles of needle-like yellowish crystals 4 to $8 \mu$ long are also present. Urine free from hæmoglobin, rather yellowish in color. Albumen present in very small quantity.

In stained preparations of the various organs many large bacilli (post-mortem) detected. There are present perhaps ten per cent of quite large macrocytes which have retained a very fecble stain. The intraglobular parasites are quite scarce. In the preparations of liver and the heart muscle, about one-half to one per cent of the red corpuscles contain single or paired pyriform bodies. In the spleen and the kidney preparations none are found. (For the temperature record, see p. 294.)

No. 228 (native).-Cow, 7 years old, received August 27, 1892, from the District of Columbia. Received under the skin of the neck 1 ec. of defibriuated blood from sick native No. 222. The injection was made in two places, $\frac{1}{2}$ ec. being injected at a time (see No. 197 for preparation of blood and other details). Placed in fenced-off portion of field XII. The blood examined before the inoculation gave the following figures: Red corpuscles, 5, 948, 700; white, 9,874. In preparations of fresh blood 2 or 
3 per ecnt of all corpuseles contain bright hodies varying in size from mere points to $\frac{1}{2} \mu$. in diameter. In stained preparation nothing abnormal.

September 5.-Temperature, 106. Rer corpuscles, 4,586,900; white, 5434. In fresh preparations of blood 2 to 3 per cent of corpuscles found with large 'Texas fever parasites and a few with bright bodies. One stained preparation negative.

September 6.-Eating very little.

September 7.-Appetite entirely gone.

September 9.-Temperature, 105.8. Red corpuscles, 3, 185, 700; white, 10,000. In fresh preparations nothing abnormal detected. In stained preparation several corpuscles containing large pyriform parasites.

September 10.--Animal weak. Stands with head drooping. Hind quarters sway in walking. Has grown thin.

September 14.-Temperature, 102.1. Red corpuscles, 1,512,200; white, 1087. In fiesh preparations a few corpuseles with bright bodies. Fully 10 per cent are macroeytes. Of these a very few are found punctate and tinted in stained preparation.

September 26.---Temperature, 101.2; pulse, 48; respiration, 12. Red corpuscles, $2,757,000$; white, 11,428. Fully 60 per cent macrocytes present, of which 10 per cent are finely punctated or tinted in stained preparation. The animal is improving slowly.

October 8.-Still very thin and weak, although appetite good.

October 27.-Temperature, 102.6; pulse, 60; respiration, 24. Red corpuscles, $3,300,000$; white, 11,250 . In fresh preparations 1 to 2 per cent bright bodies within corpuscles. In stained preparation 5 to 10 per cent of the red corpuscles are infected with the peripheral coccus-like bodies. (For the temperature record, see p. 294.)

No. 230 (native).-Cow, 9 years old, received September 7, 1892, from the District of Columbia, and placed in unused field XI.

September 9.-After the examination of the blood, this animal received into the left jugular vein 28 cubic centimeters (two syringefuls) of hlood, drawn inmediately before from the left jugular vein of North Carolina cow No. 214. Transferred to field III, now containing Nos. 206, 218, 219, 221, and 222 (see No. 198 for details of the operation). The following table indicates an acute attack followed br a relapse: 


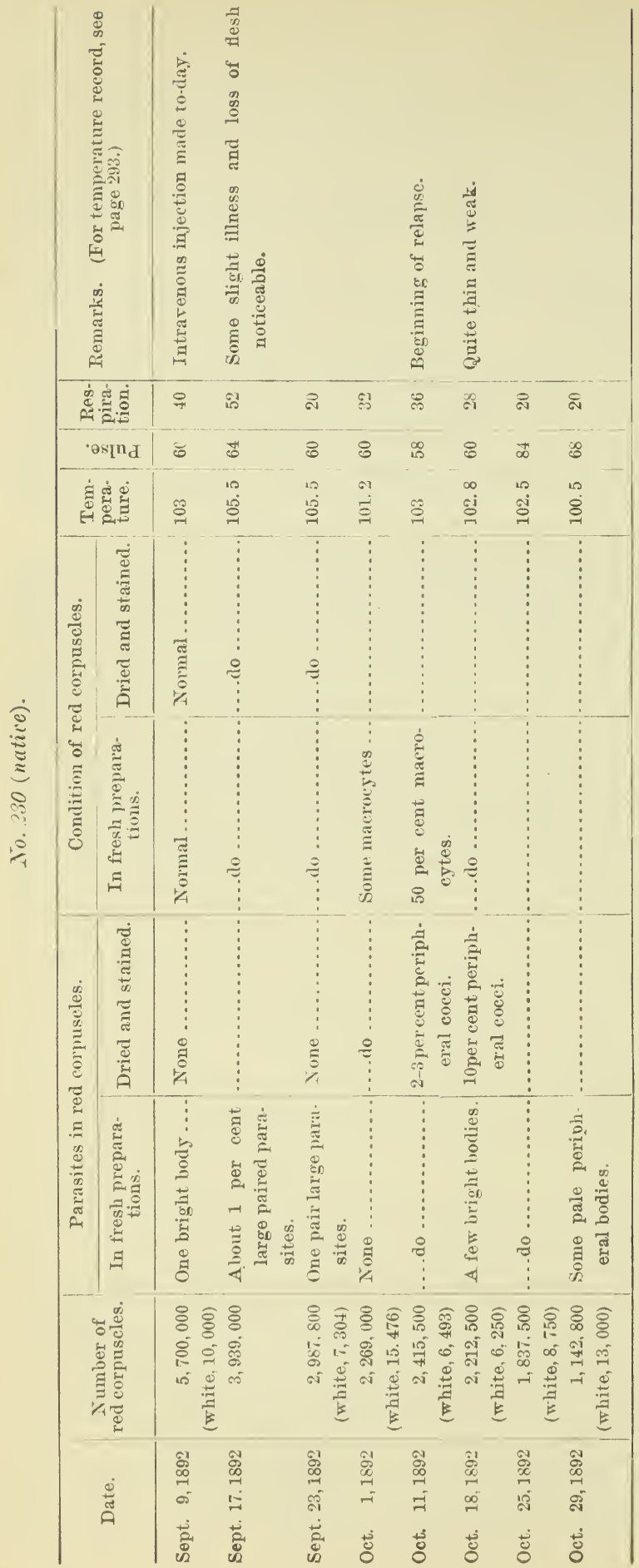


TEMPERATURE RECORD OF THE VARIOUS ANIMALS USED IN THE EXPERIMENTS FROM I889 TO I892, INCLUSIVE.

The following tables include with but three exceptions (Nos. 158, 166, and 180) the temperature record, in degrees and tenths of degrees Fahrenheit, of all cattle used in the field experiments at the Washing ton station. The temperature of the three cases noter as exceptions was not taken regularly, and hence the only record forthcoming is that printed with the notes under each case.

In consulting these tables the reader may find what appear to be discrepances between the temperature as given here and that already printed with the notes under each case. This apparent discrepancy is due to the fact that the temperature as given in the tables and as tabulated with the blood examination was, as a rule, taken at different hours of the same day or on different days. Hence, in case of considerable daily fluctuation, such as is usually the rule, at least in hot weather, in mild attacks and relapses and at the beginning and at the end of the acute attacks, these two records may not agree closely.

The hour in which the temperature was taken is given in the tables. Slight fluctuations in healthy animals may here and there be explaned by taking into account the time of day since there is a slight rise of temperature towards evening.* The hour of the blood examination is not given in the notes of the individual cases, but it was made, with few exceptions, before noon.

The record of the same animal exposed for several successive seasons is given in a separate table for each fresh exposure. The pages on which such records may be found are given at the end of the notes for each case or with the remarks, if the notes have been tabulated.

The daily fluctuations associated with the beginning of the fever, and quite marked in some cases may be studied in tables on pages 276,284 , $285,288,291,292,293,294$, and 295 , where the morning and evening temperature is recorded.

The temperature of Southern animals used as sources of infection in the various inclosures was not taken. Whenever such animals were exposed ou subsequent occasions, for the purpose of determining any loss of immunity, their temperature was recorded. The record of such cases and of a few natives which remained healthy during the period of exposure (page 291, and elsewhere) furnishes a valuable basis of comparison with the record of the artually diseased animals. 


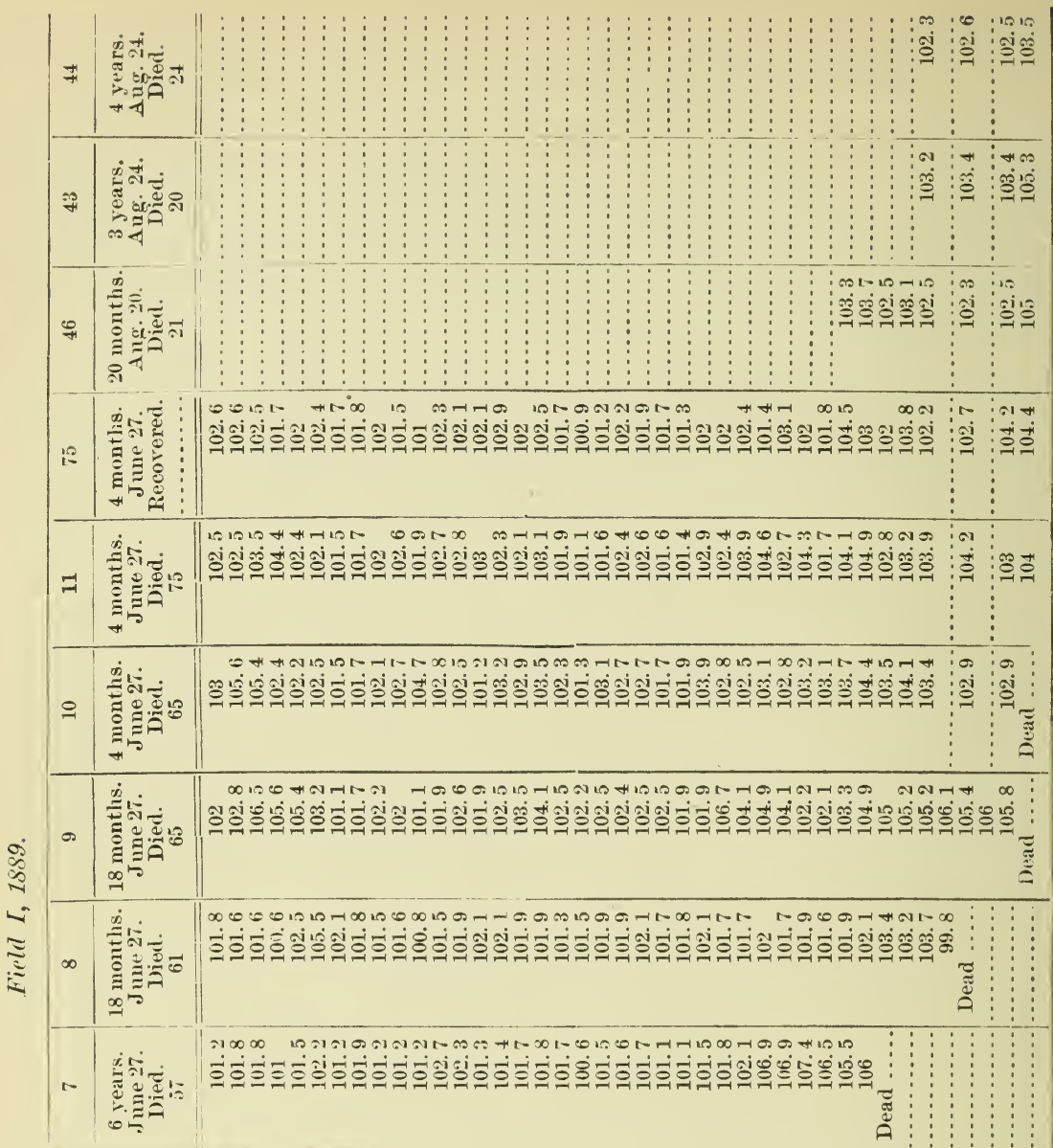




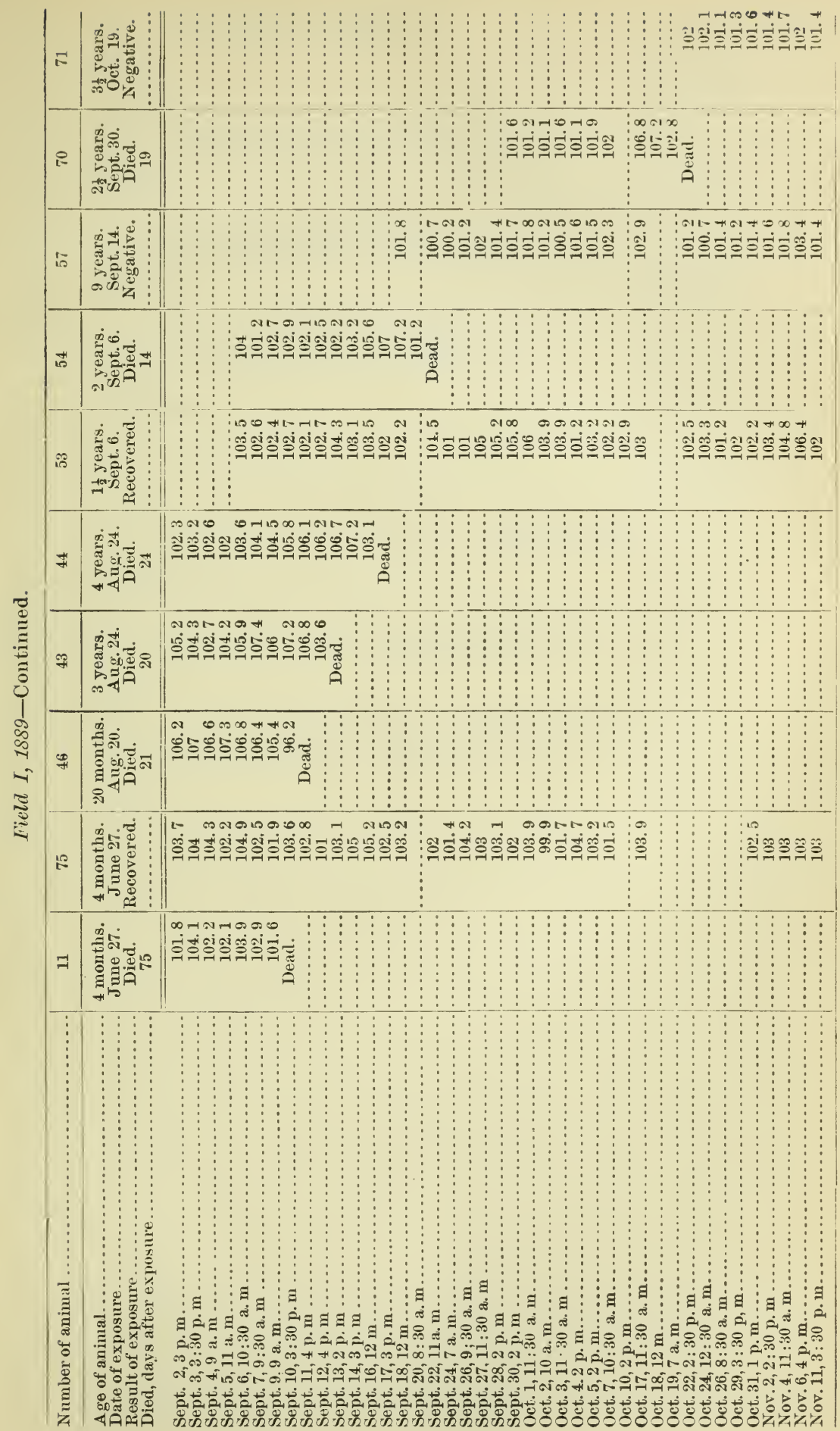




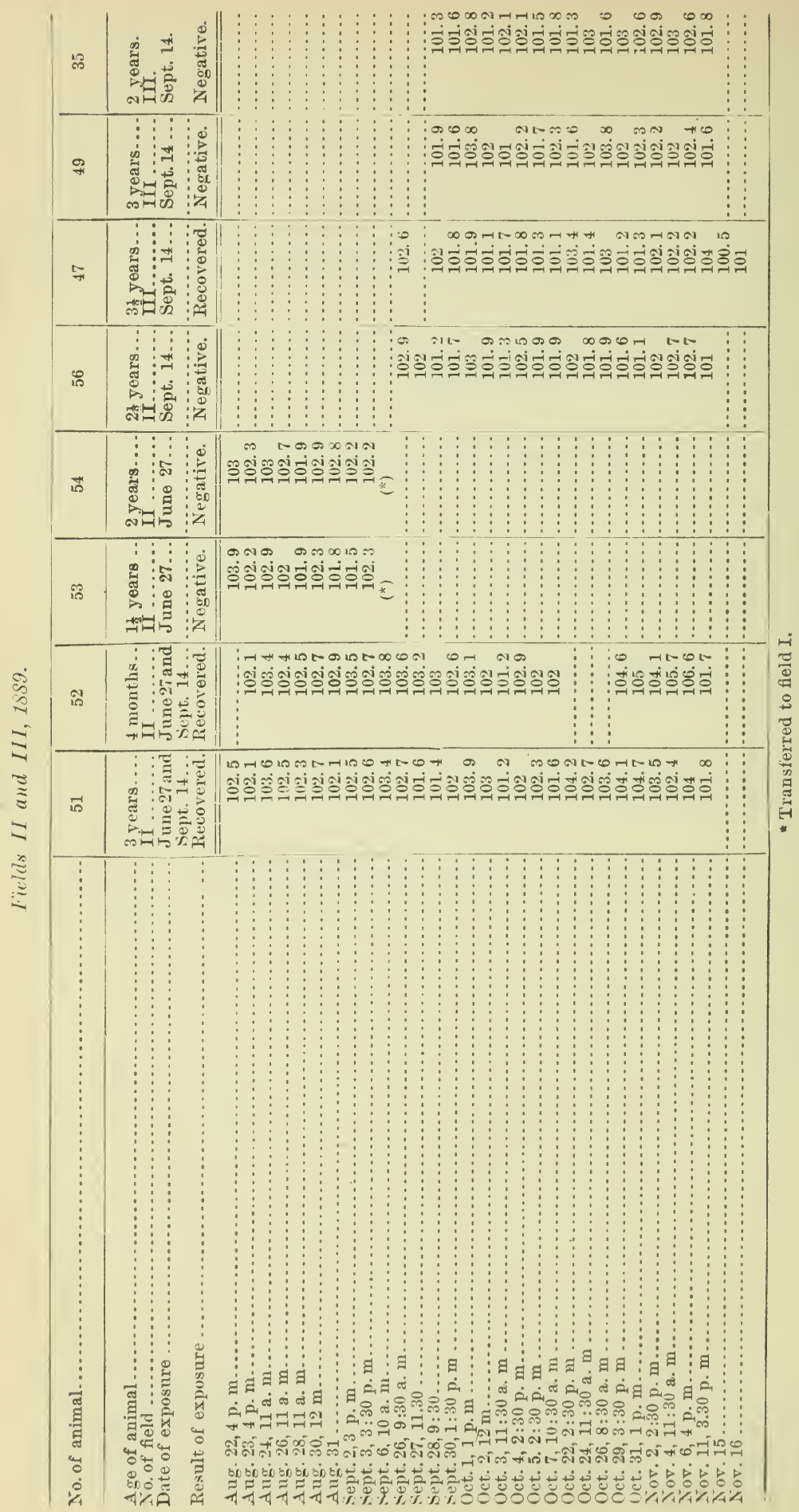




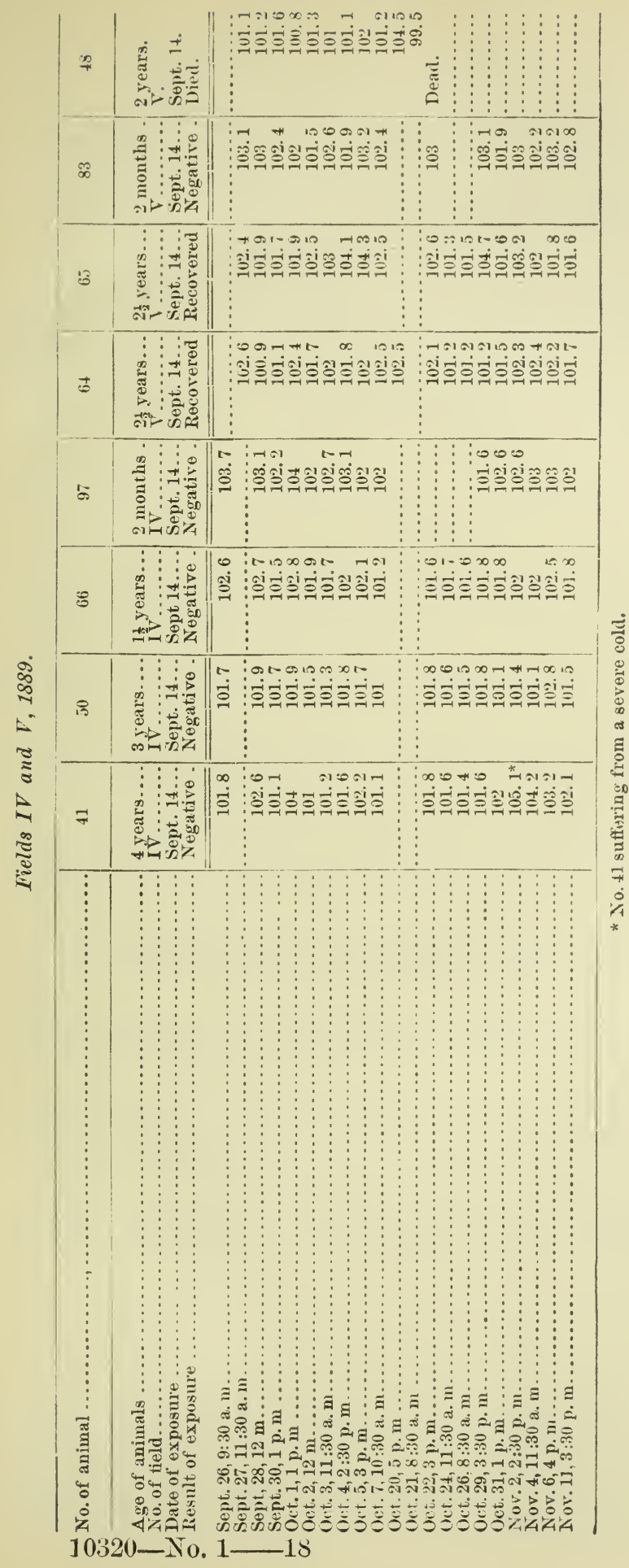




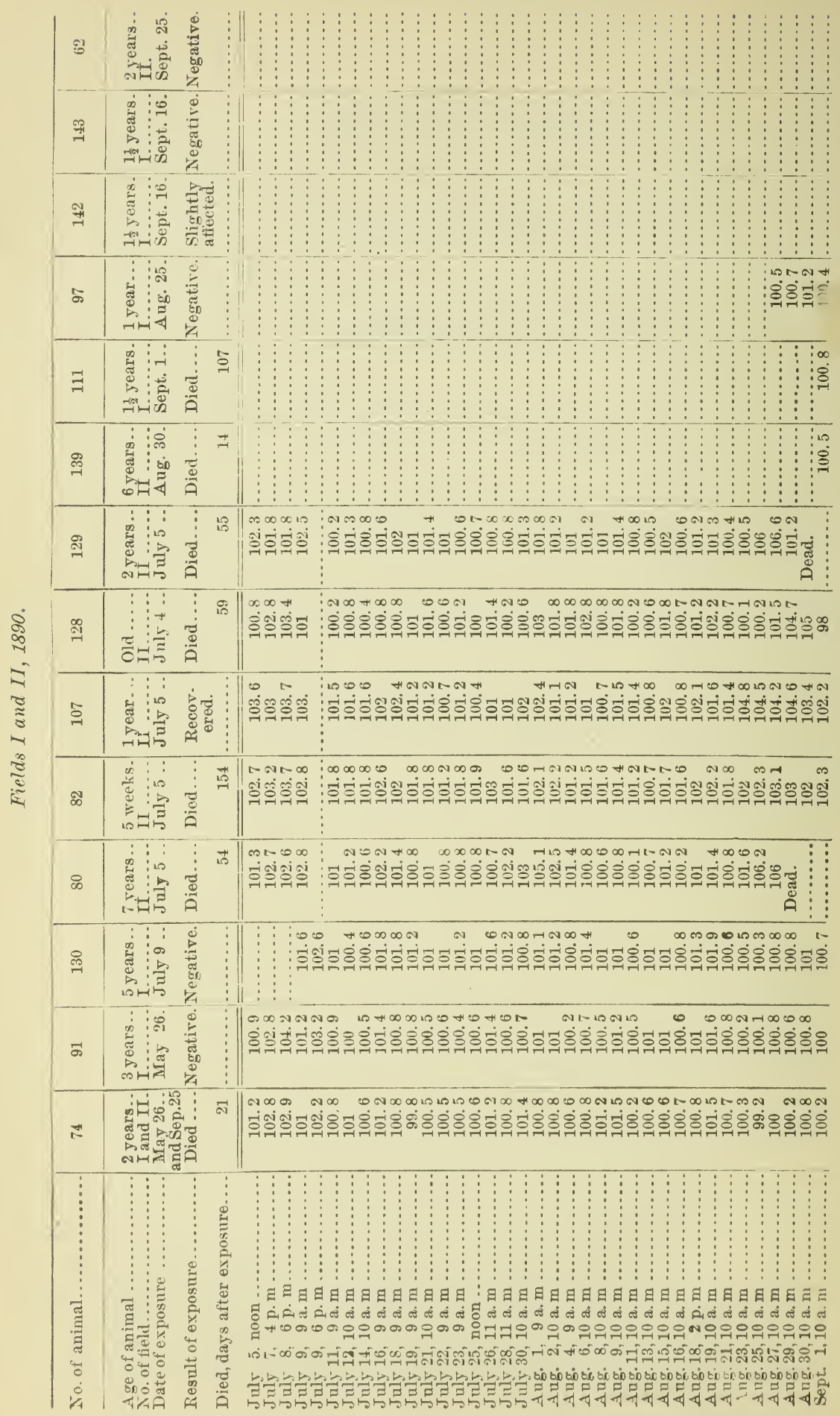


APPENDIX.

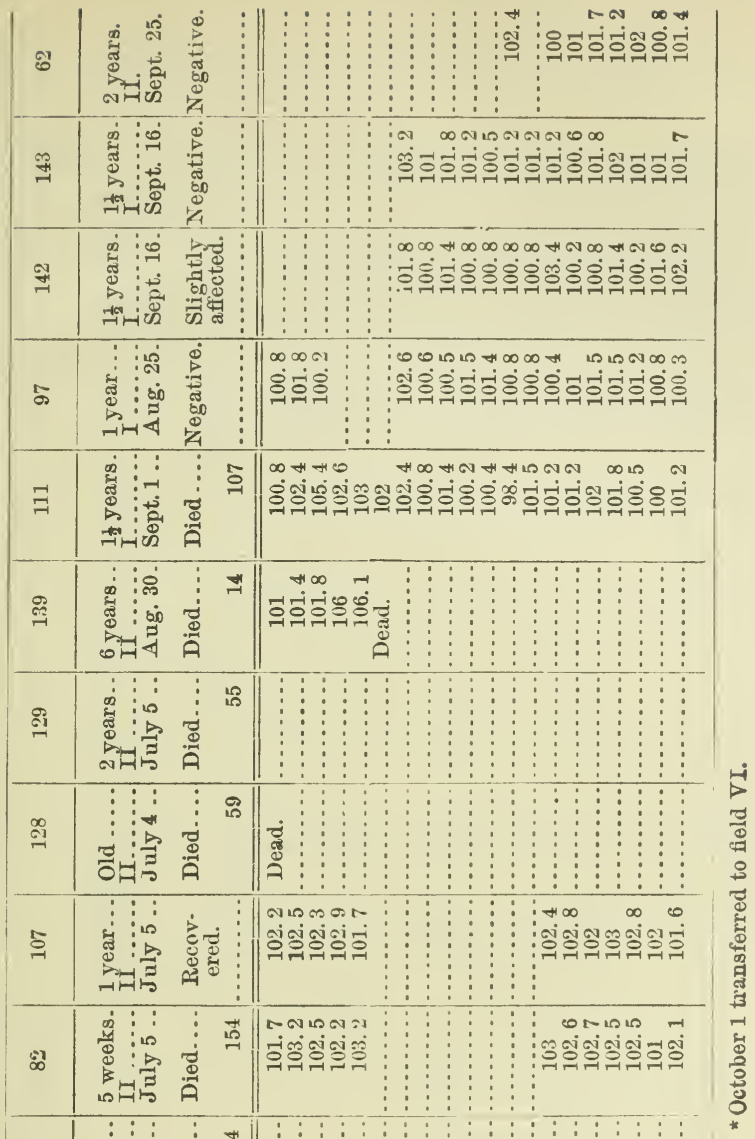

\begin{tabular}{|c|c|c|c|}
\hline$\varnothing$ & 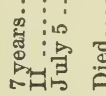 & & \\
\hline$\stackrel{m}{-1}$ & 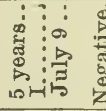 & $\vdots$ & 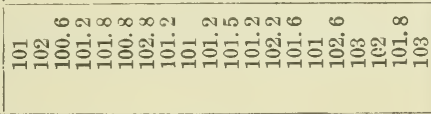 \\
\hline 5 & 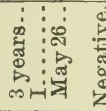 & $\vdots$ & 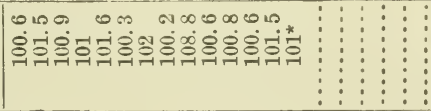 \\
\hline$\vec{Z}$ & 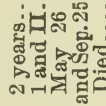 & ने & 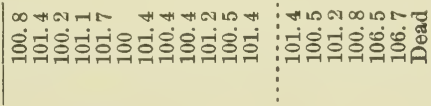 \\
\hline 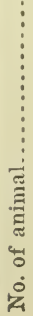 & 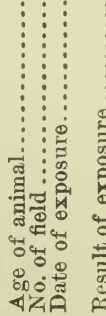 & 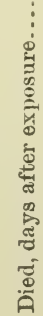 & 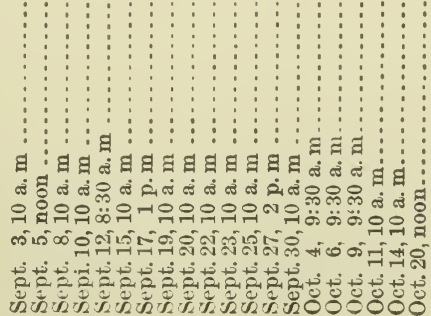 \\
\hline
\end{tabular}


Field II, 1890 .

\begin{tabular}{|c|c|c|c|c|c|c|c|c|c|c|c|c|}
\hline \multirow{2}{*}{$\begin{array}{l}\text { No. of amimal. } \\
\text { Temperature } \\
\text { taken. }\end{array}$} & \multicolumn{2}{|c|}{80.} & \multicolumn{2}{|c|}{82.} & \multicolumn{2}{|c|}{107.} & \multicolumn{2}{|c|}{128.} & \multicolumn{2}{|c|}{129.} & \multicolumn{2}{|c|}{139.} \\
\hline & Morn. & Eve. & Morn. & Eve. & Morn. & Eve. & Morn. & Eve. & Morn. & Eve. & Morn. & Eve. \\
\hline July 24...... & 102.2 & 101.4 & 101.5 & 102.2 & 101.8 & 102 & 102.2 & 104.7 & 100.8 & 101.4 & & \\
\hline July $25 \ldots . .$. & 100.8 & 102.9 & 101.9 & 102.5 & 100.4 & 103 & 100 & 103.9 & 100.7 & 101.8 & & \\
\hline July $26 \ldots . .$. & 100.7 & 102.8 & 103 & 102.8 & 101 & 102 & 101.4 & 103.2 & 100.8 & 101.6 & $\ldots$ & . \\
\hline uly $27 \ldots \ldots$ & 102 & 103.6 & 101.5 & 102.8 & 101.9 & 103.1 & 101.4 & 102 & 101.2 & 101.8 & & $\ldots$ \\
\hline July $28 \ldots .$. & 102.2 & 101.8 & 101.6 & 102.5 & 101 & 101.8 & 100.2 & 101.5 & 100.8 & 102 & ..... & $\ldots$. \\
\hline J 11 प $29 \ldots \ldots$ & 101.4 & 104.2 & 101.4 & 102.6 & 101.3 & 102.3 & 100.2 & 103.2 & 101 & 102.4 & ...... & .... \\
\hline July $30 \ldots \ldots$ & 103 & 103.8 & 101.6 & 102.5 & 102 & 103.7 & 100.6 & $10: 3.2$ & 101.3 & 101.8 & ..... & \\
\hline July $31 \ldots \ldots$ & 101.5 & 103.6 & 101.7 & 102.4 & 101. 6 & 102.6 & 101.1 & 103.8 & 101.4 & 101.8 & $\ldots$ & \\
\hline Aug. $1 \ldots .$. & $10 \% .1$ & 102.8 & 102.1 & 102.2 & 102.4 & 102.6 & 103 & 102.5 & 101.8 & 102 & & \\
\hline A 1 g. $2 . . .$. & 102.5 & 104.2 & 102.2 & 102.8 & 101.1 & 103 & 101.8 & 104 & 101. 2 & 10 & . & $\ldots$ \\
\hline Aug. 3..... & $10 \div .5$ & 103.6 & 102 & 102.7 & 102.8 & 102.8 & 103.2 & 103.6 & 101.2 & 101.6 & $\ldots$ & .... \\
\hline A॥g. $4 \ldots . .$. & 101.4 & 103.6 & 101. 2 & 102.2 & 101.2 & 102.5 & 101.8 & 104 & 101 & 102 & $\ldots$ & \\
\hline Aug. $5 \ldots . .$. & 101.2 & 103.8 & 101.2 & $10^{\circ}$ & 100.5 & 102.7 & 102.5 & 104 & 100.8 & 101.6 & ... & $\ldots$. \\
\hline Аाँ. $6 \ldots . .$. & $10 \% .8$ & 103.2 & $1 \mathrm{C1.} 5$ & 101.8 & 01 & 103 & 102.8 & 104 & 101.2 & .5 & $\ldots .$. & $\ldots$. \\
\hline Ang. $7 \ldots . .$. & 101 & 101.8 & 101.4 & 101.8 & 101.3 & 102.2 & 102.4 & 16 & 101 & .6 & & \\
\hline Aug. 8...... & 100.6 & 101.6 & 101.6 & 102.8 & 100.7 & 101 & 100.8 & 2.8 & & 102.2 & & \\
\hline AıI $9 \ldots . .$. & 100.8 & 102.5 & 101.4 & 102.4 & 101.5 & 103.2 & 101.8 & 10 & 104.1 & 102.3 & $\ldots$ & $\ldots$ \\
\hline A $11 \% .10 \ldots .$. & 101.2 & 101.4 & 101.9 & 102.6 & 102.6 & 103 & 103 & 102.8 & 101.5 & 101.8 & & \\
\hline Aıg. 11..... & 100.1 & 101.2 & 101. 2 & 102.3 & 101.4 & 10 & 100.2 & 103.2 & 100.8 & 102.2 & $\ldots$ & . \\
\hline Aug. 12..... & 100.8 & 101. 6 & 102 & 102.8 & 101.6 & 102.7 & 101.5 & 1026 & 101.2 & 103.2 & & \\
\hline A ug. $13 \ldots . .$. & 100.7 & 101.6 & 100.7 & 102.6 & 100.8 & 10 & 100 & 10 & 00.5 & .8 & & \\
\hline A $1 \mathrm{~g} .14 \ldots . .$. & 100.2 & 102.5 & & 10.3 & & & & & 0.3 & .8 & & \\
\hline Aıg. $15 . . .$. & 101.2 & 101.8 & 101. 7 & 1027 & 102 & 103.5 & 101.8 & 103.4 & 102 & 101.6 & $\ldots$ & $\ldots$ \\
\hline A $11.16 . . .$. & 100.2 & 102.3 & 101.6 & 102.2 & 100.8 & 103.2 & 100.7 & 103.2 & 100.6 & 101.8 & & $\ldots$ \\
\hline Aug. 17...... & 101.2 & 102.4 & 101.5 & 102.9 & 101.8 & .2 & 101.4 & 103 & 100.8 & 101.5 & & .. \\
\hline Alig. 18..... & 101 & 101.4 & 102 & 102 & 102.1 & 101.2 & 101.2 & .3 & 101.2 & .5 & & \\
\hline Aıg. $19 \ldots . .$. & 101.4 & 101.6 & 102.2 & 102.7 & 101.6 & 103.8 & 102.2 & 102.4 & 101.3 & 101.6 & & . \\
\hline Aug. $20 \ldots . .$. & 100.8 & 102 & 102.1 & 103 & 101.5 & 104 & 101.2 & $10^{\circ} .8$ & 101.4 & 1. 6 & & .. \\
\hline A119. $21 . . .$. & 100.8 & 104.8 & 101.8 & 103 & 101.4 & 104. 6 & 103.7 & 102.2 & 4 & 10 & & \\
\hline A $11 \mathrm{~g} .22 \ldots .$. & $10^{6} .6$ & 104.8 & 101. 7 & 103.6 & 102.5 & 10 & 100.7 & & & & & \\
\hline Aug. 23...... & 101.6 & 104.8 & $100^{\circ}$ & 104 & 104.8 & 105. 4 & 100.1 & 102.5 & 100.5 & 102 & & $\ldots .$. \\
\hline A $11 \mathrm{~g} .24 \ldots . .$. & 104.7 & 105.7 & 102.2 & 103.2 & 105.8 & 105.5 & 100.2 & 101.5 & 104 & 104.8 & & \\
\hline A $119.25 \ldots . .$. & 106.2 & 106.8 & 102.3 & 104.5 & 104.5 & 105.6 & 100.2 & 102.2 & 106 & 106.4 & & \\
\hline A $119.26 \ldots . .$. & 105.8 & 106.6 & $10 \% .8$ & 103.8 & 104.5 & 103.8 & 100.7 & 102.6 & 106.4 & 106.3 & & \\
\hline Ang. 27..... . & 106 & 104.8 & 103.1 & 103.8 & 104.2 & 105.4 & 101.5 & 105.6 & 106.6 & 106.2 & & \\
\hline A 1 g. 28..... & 102.7 & Dead. & 102.5 & 104.4 & 103.4 & 105 & 103 & 104.5 & 105.6 & 104.6 & & \\
\hline $29 \ldots \ldots$ & ...... & $\ldots \ldots$ & 103 & 104 & 104.6 & 105 & 104.7 & 107.1 & 101.2 & 100.1 & & \\
\hline Aug. $30 \ldots . .$. & ...... & ....... & 102 & 103 & 103.4 & 104.7 & 105. & 107 & Deart. & ...... & & 101.8 \\
\hline Aug. 31. & & & 101.2 & & 104.7 & & 106. & & - & & 100.5 & \\
\hline Sept. $1 \ldots .$. & & & 102.3 & 103 & 102.2 & 101.3 & 98 & Dead. & & & 100.8 & 101. 2 \\
\hline Sept. & & & 100.8 & 102.4 & 101.2 & 101.4 & $\ldots$ & ....... & & 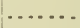 & 100.6 & 101.3 \\
\hline Sept. & & & 101.7 & 102.9 & 102.2 & 103.8 & & & & $\ldots$ & 101 & 102 \\
\hline Sept. & & & 102 & 102.5 & 101.4 & 102.8 & & & & & 101 & 101.6 \\
\hline Sept. & & & 103.2 & 103.4 & 102.5 & 103. & & & & & 101.4 & 101.7 \\
\hline Sept. & & & 102.5 & 102.6 & 102 & 104. & & & & & 101.2 & 102 \\
\hline Sept. $7 \ldots . .$. & & & 102 & 103.9 & 102.5 & 103.8 & & & & & 101.4 & 102.6 \\
\hline Sept. $8 \ldots .$. & & & 102.5 & 103.2 & 102.3 & 103.8 & & & & & 101.8 & 103.4 \\
\hline Sept. 10. & & & 102.2 & 103.7 & 102.9 & 103.4 & & & & - & 106 & 105.2 \\
\hline & & & 101.4 & 101. 2 & 101.2 & 101.8 & & & & ..... & 105.2 & 106 \\
\hline Sept. 12. & & ..... & 103.2 & 103 & 101.7 & 102.6 & & & & 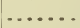 & 106.1 & 102.4 \\
\hline Sept. 13. & & & & & & & & & . & $\ldots \ldots$ & Dead. & $\ldots \ldots$ \\
\hline
\end{tabular}




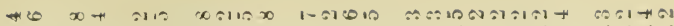

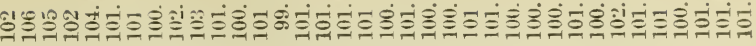

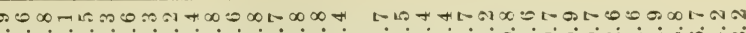
Бं

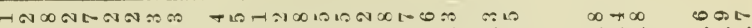

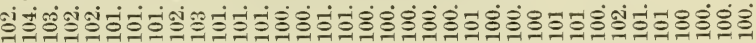

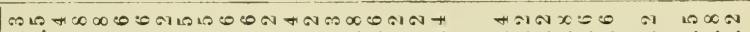

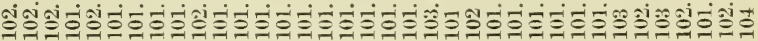

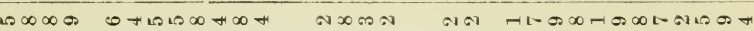
க்

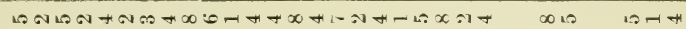

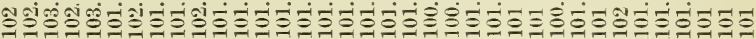

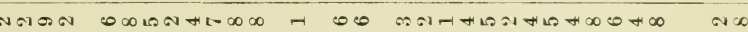

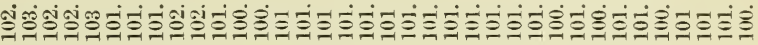

RाM த்த் فंڤ

hen एका க் gi

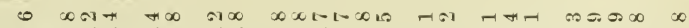

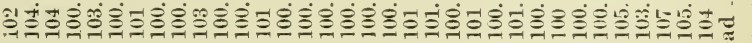

Henthatis

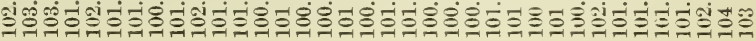

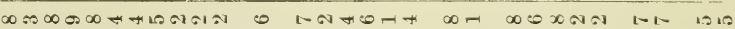

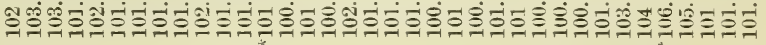

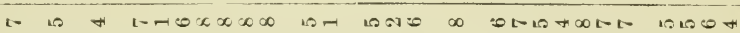

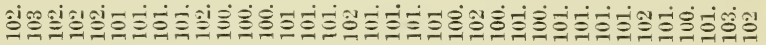
ॠ б 궁 เ

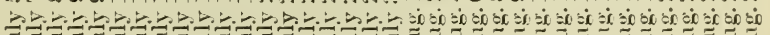

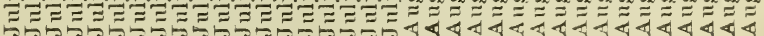




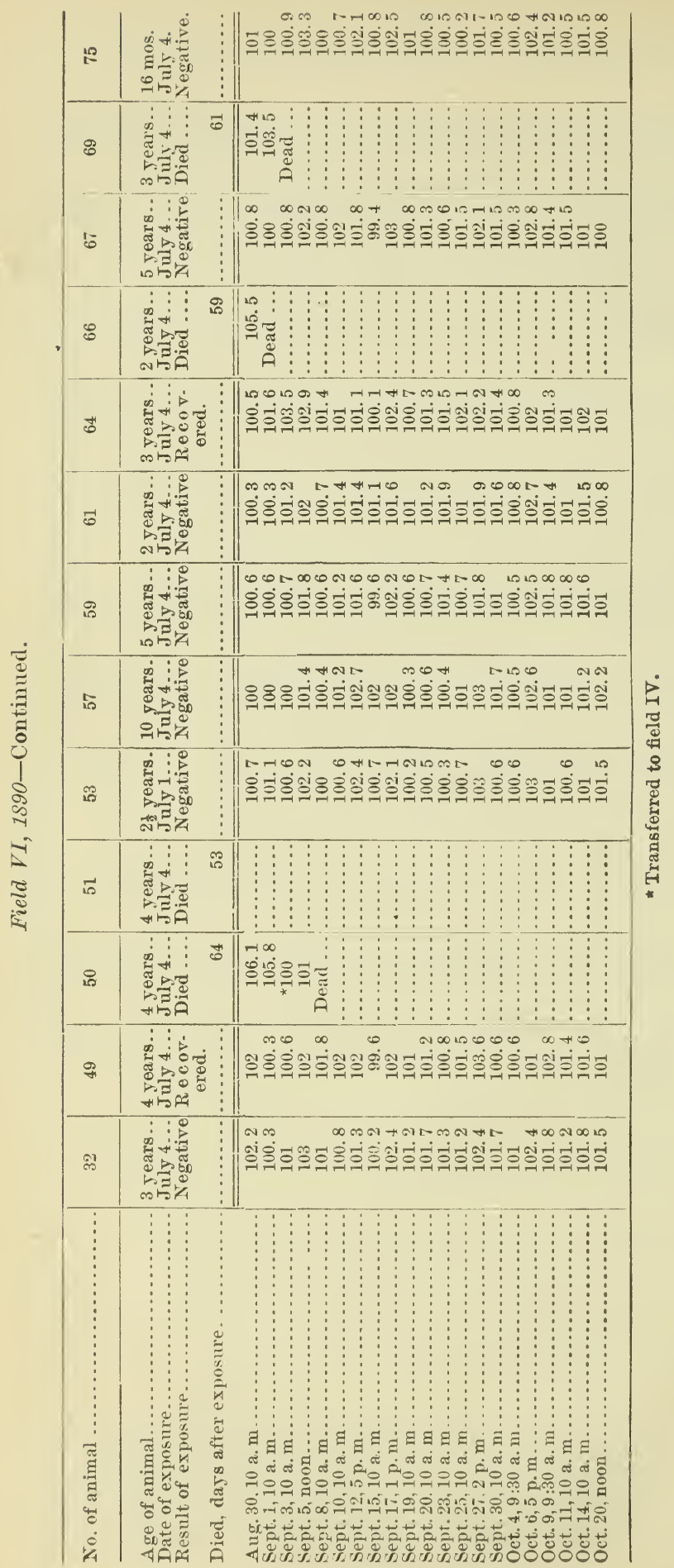




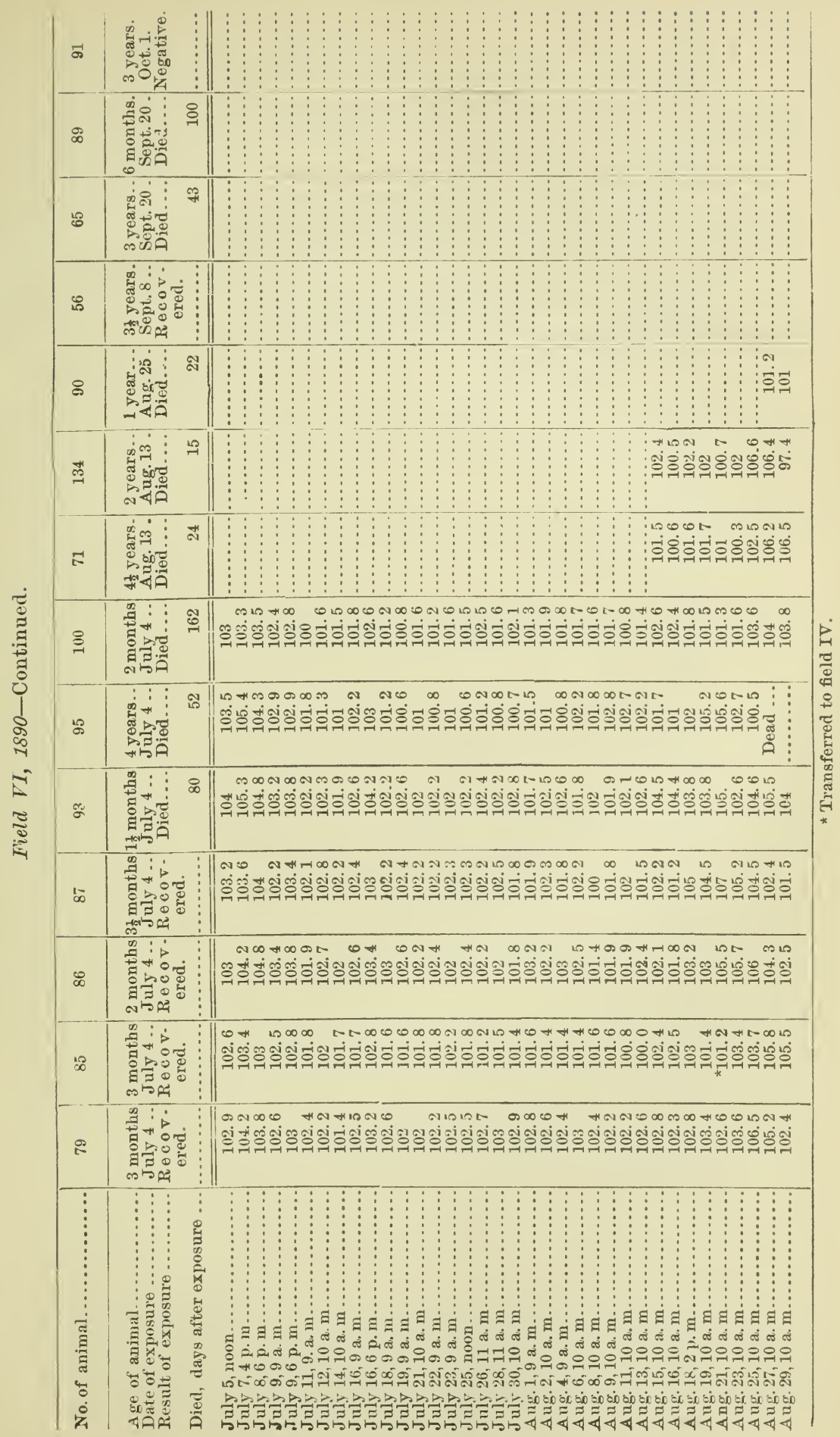




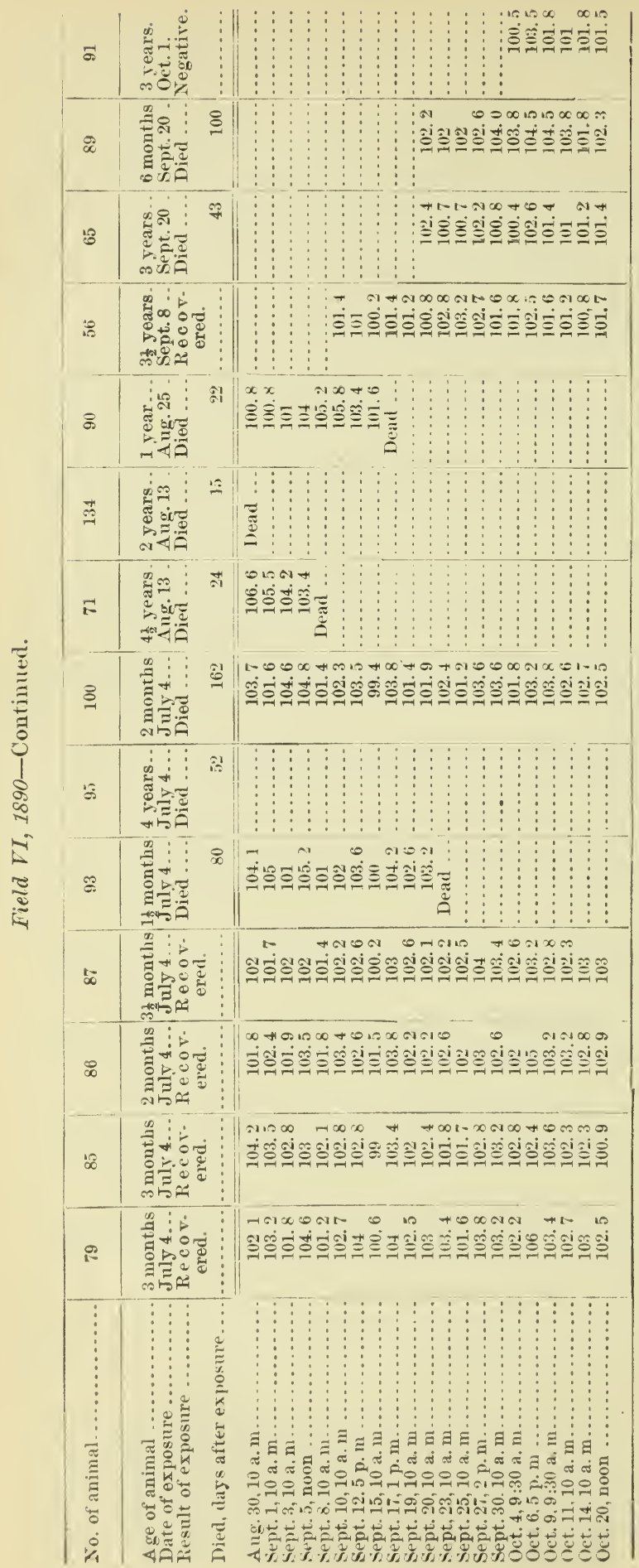




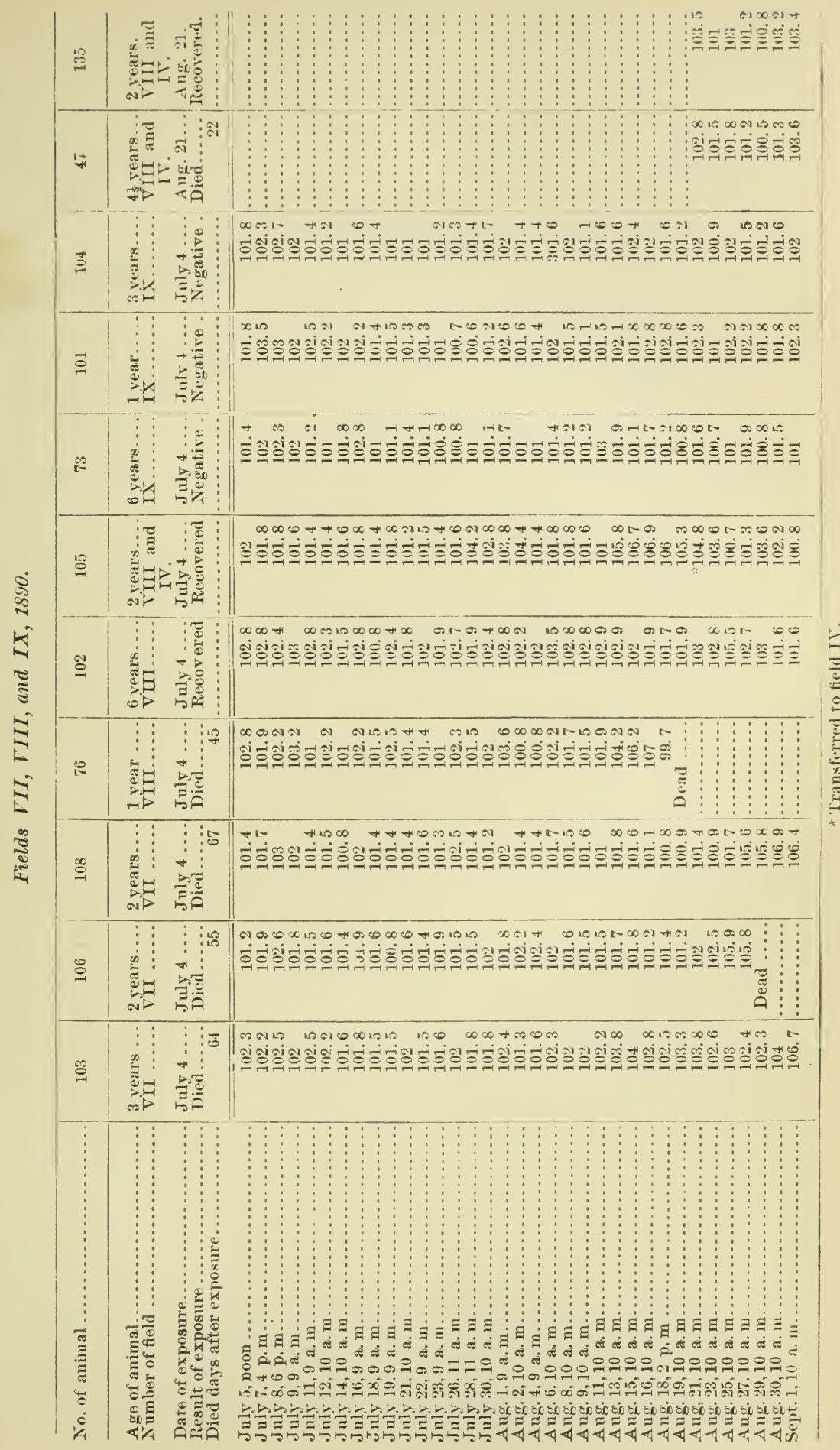




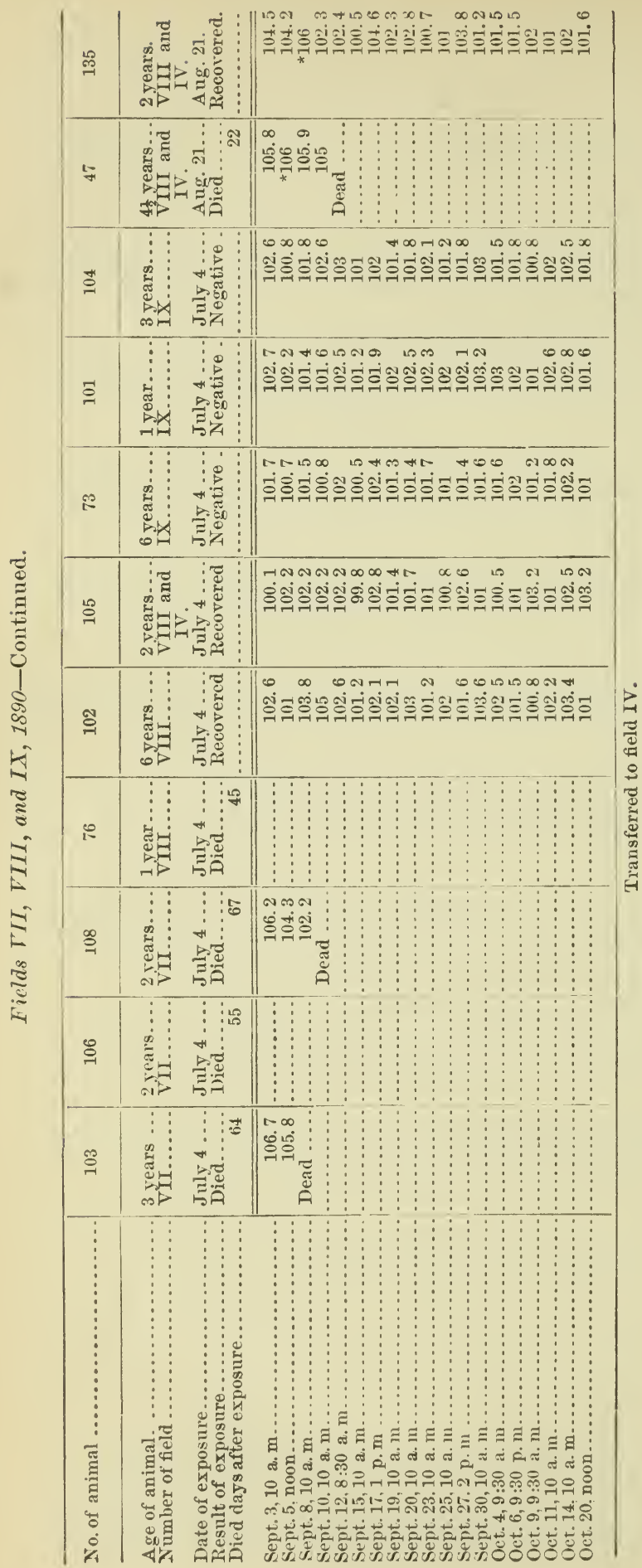




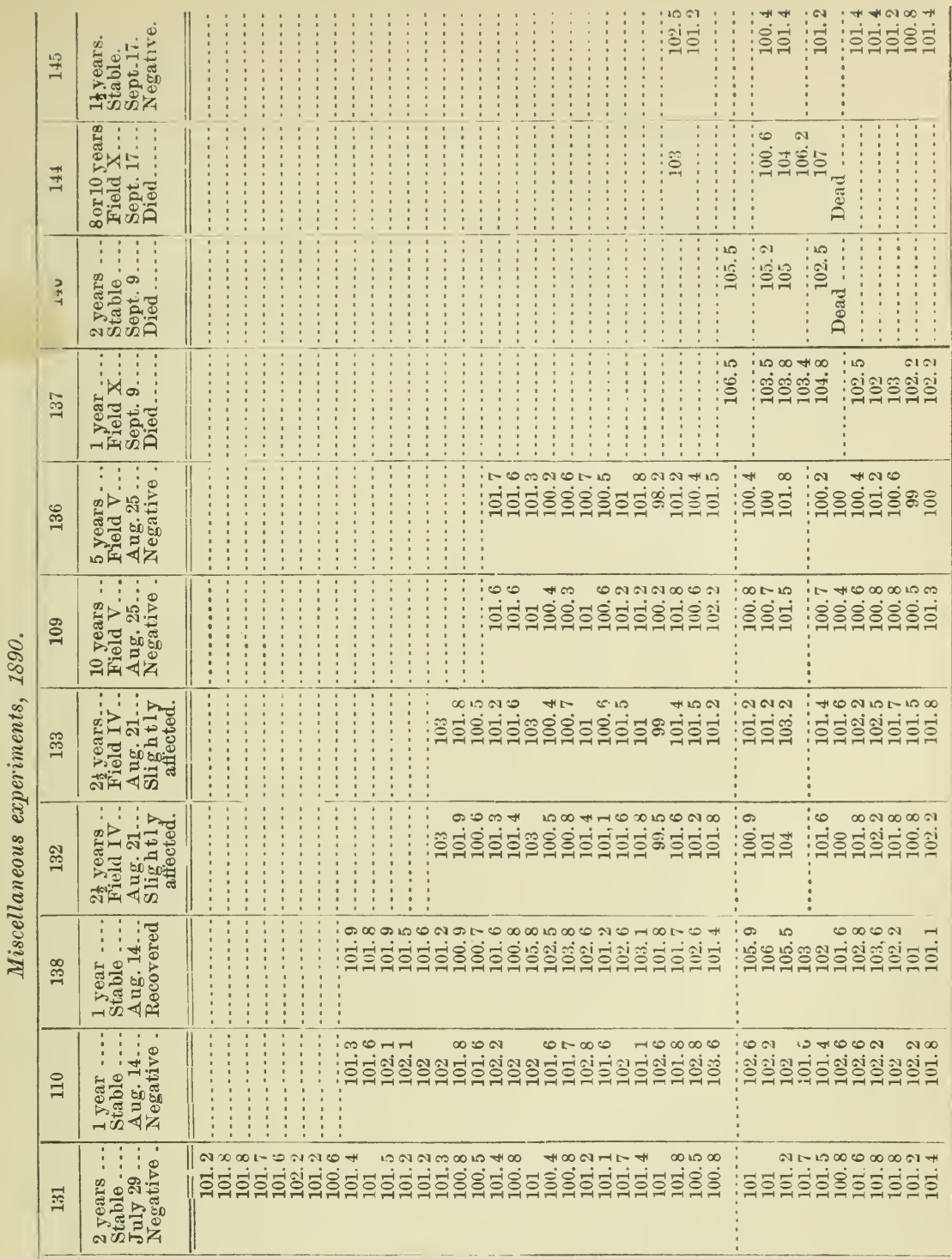


Animals in stable arlificially heated, 1890.

\begin{tabular}{|c|c|c|c|c|c|c|c|c|c|c|c|c|}
\hline \multirow{2}{*}{ Date. } & \multicolumn{2}{|c|}{$\begin{array}{l}\text { Teinperature } \\
\text { of stable. }\end{array}$} & \multicolumn{2}{|c|}{62.} & \multicolumn{2}{|c|}{143.} & \multicolumn{2}{|c|}{145.} & \multicolumn{2}{|c|}{149.} & \multicolumn{2}{|c|}{117.} \\
\hline & $\begin{array}{l}\text { Morn- } \\
\text { ing. }\end{array}$ & $\begin{array}{l}\text { Even- } \\
\text { ing. }\end{array}$ & $\begin{array}{l}\text { Morn- } \\
\text { ing. }\end{array}$ & $\begin{array}{l}\text { Even- } \\
\text { ing. }\end{array}$ & $\begin{array}{l}\text { Morn- } \\
\text { ing. }\end{array}$ & $\begin{array}{l}\text { Even- } \\
\text { ing. }\end{array}$ & $\begin{array}{l}\text { Morn- } \\
\text { ing. }\end{array}$ & $\begin{array}{l}\text { Even- } \\
\text { ing. }\end{array}$ & $\begin{array}{l}\text { Morn- } \\
\text { ing. }\end{array}$ & $\begin{array}{l}\text { Even- } \\
\text { ing. }\end{array}$ & $\begin{array}{l}\text { Morn. } \\
\text { ing. }\end{array}$ & $\begin{array}{l}\text { Even. } \\
\text { ing. }\end{array}$ \\
\hline Oct. 30 & 70 & & 102.5 & & 101.9 & & 101.9 & & 102.3 & & & \\
\hline Nov. 1 & 74 & & 101.8 & & 102.3 & & 101.4 & & 101.8 & & & \\
\hline Nov. 2 & 70 & 78 & 101.7 & 102.2 & 102.8 & 103.3 & 101 & 101.9 & 102 & 100.2 & & \\
\hline Nov. 3 & 68 & 67 & 101.6 & 102 & 102.4 & 102.8 & 101.8 & 101.7 & 102.6 & 102.2 & & \\
\hline Nov. 4 & 70 & & 101.6 & & 102 & & 102 & & 101.6 & & & \\
\hline Nov. 5 & 67 & & 101.7 & & 103.1 & & 101.4 & & 102.3 & & & \\
\hline Nov. 6 & 65 & 80 & 101.6 & 101.4 & 103 & 103.2 & 101.3 & 101.8 & 102.5 & 102.7 & & \\
\hline Nov. 7 & 67 & & 102 & & 103.1 & & 101.3 & & 101.5 & & & \\
\hline Nov. 8 & 70 & 84 & 101.9 & 101.4 & 102.8 & 103.8 & 101.4 & 101.4 & 102 & 103 & & \\
\hline Nov, ! & 7 & 80 & 101.2 & 102.2 & 103.8 & 105.8 & 101.2 & 101 & 101.8 & 103.5 & & \\
\hline Nov. 10 & 76 & $\ldots$ & 101.5 & & 104.3 & ....... & 101. 2 & & 102.2 & & & \\
\hline Nov. 11 & 7 & & 101.6 & & 103.2 & & 101. 2 & & 102.4 & & & \\
\hline Nov. 12 & 72 & 74 & 101.7 & 101.9 & 103.3 & 103.5 & 101.3 & 101.4 & 104.5 & 104.1 & & \\
\hline Nov. 13 & 73 & 80 & 102. 2 & 101. & 103. 3 & 104.3 & 101.5 & 101 & 103.8 & 104.7 & & \\
\hline Nov. 14 & 7 & 80 & i01.5 & 102. & 103. 3 & 103. 8 & 101.3 & 102.7 & 103 & 103.4 & & \\
\hline Nov. 15 & 80 & 83 & 102.4 & 102.1 & 103.2 & 104.4 & 102 & 102 & 103.4 & 103.7 & & \\
\hline Nov, 16 & 74 & 72 & 101.5 & 102 & 103.2 & 103.2 & 100.9 & 102.1 & 102.3 & 102.7 & & \\
\hline Nov. 17 & 72 & 76 & 102.2 & 102.2 & 103.6 & 103.9 & 101.7 & 102 & 102.1 & 103.5 & & \\
\hline Nov. 18 & 72 & 81 & 102 & 101.8 & 103.8 & 103.4 & 101.5 & 101 & 102.4 & 102.8 & & \\
\hline Nov. 19 & 78 & 70 & 102 & 102 & 103.2 & 103.5 & 101.2 & 101 & 102.8 & 163 & & 102.4 \\
\hline Nov. 20 & 70 & 78 & 102 & 101.8 & 102.7 & 103. 2 & 101 & 101 & 102.1 & 102.1 & 102.1 & 103 \\
\hline Nov. 21 & 74 & 80 & 101.6 & $10^{\circ}$ & 105 & 104.2 & 101.4 & 101 & 103. 1 & 102 & & \\
\hline Nov. 22 & 79 & 78 & 102 & 101.8 & 104 & 104 & 102.5 & 102 & 103.6 & 103. 2 & 102.4 & 103.2 \\
\hline Nov. 23 & 7 & 82 & 101.9 & 102. & 105. 3 & 10 & 101.7 & 102.7 & 103.2 & & 02.5 & 103.6 \\
\hline Nov. 24 & 76 & 78 & 102 & 102.1 & 102 & 1.03 & 101.5 & 101. & 103.4 & 103.2 & 102.3 & 103.2 \\
\hline Nov. 25 & 7 & 82 & 102 & 102.2 & 103 & 103.8 & 101.6 & 102.5 & 104 & 103.6 & & $10 t$ \\
\hline Nov. 26 & 80 & 77 & 101.9 & 102 & 104 & 104.6 & 101.7 & 101.2 & 102.8 & 102.6 & 103.7 & 103.8 \\
\hline Nov. 27 & 75 & & 101.6 & & 103 & & 101.8 & & 102.2 & & 102.7 & \\
\hline Nov. 28 & 72 & 80 & 101.7 & 101.7 & 103.5 & 104 & 100.8 & 101.8 & 102 & 101.7 & 102.6 & 102.7 \\
\hline Nov. 29 & 72 & 80 & 101.5 & 101.9 & 102.5 & 103.5 & 101 & 102 & 102.1 & 102.5 & & \\
\hline Der. 1 & & 78 & 101.7 & 102.5 & 102.5 & 103 & 101.6 & $10^{2} .4$ & 102 & 103 & 102.1 & 103. 2 \\
\hline Dec. 2 & 7 & 74 & 101.7 & 101.7 & 102.8 & 103 & 101.5 & 102.4 & 101.5 & 102.5 & $10 \% .2$ & 102.8 \\
\hline Dee: 3 & & $7 x$ & 101.8 & 102.2 & 103.4 & 103 & 102 & 103.2 & 103 & 102.5 & 102.4 & 103.4 \\
\hline leee. 4 & 7 & 77 & 101.4 & 101.5 & 102.2 & $10^{\circ} .8$ & 103 & 105.2 & 101.6 & 101.9 & 101.5 & 102.6 \\
\hline 1)ec. 5 & & 77 & 101.8 & 102 & 102.9 & 104.2 & 104.1 & 106 & 102.6 & 102.8 & $10 \div .2$ & 103 \\
\hline Dee. 6 & 76 & 80 & 101. (; & 103 & 102.2 & 103.4 & 103.4 & 103.2 & 101.8 & 102.7 & 102.6 & 103 \\
\hline Her. 7 & & 74 & 101.5 & 101.5 & 103.2 & 103.2 & 100.2 & 101 & 102.4 & 102.2 & $10: 3$ & $10^{2} .9$ \\
\hline Dee. 8 & 71 & 74 & 101.5 & 102.5 & 102.9 & 102.6 & 100.4 & 101.2 & 101 & 102.2 & 102 & 101.5 \\
\hline Hec. 9 & 76 & 76 & 101.8 & 101.8 & 102.5 & 102.5 & 101.2 & 101.5 & 167 & 107 & 102 & 102.6 \\
\hline Dee. 10 & 75 & 78 & 101.7 & 102.3 & 102.2 & 103.5 & 101. 2 & 102.2 & 101.2 & 102 & $10^{2}$ & 103 \\
\hline 1)ee. 11 & 70 & 70 & 101.8 & 101.8 & 102.5 & 103.5 & 100.8 & 101.6 & 101.7 & 101. 7 & 102 & 101.8 \\
\hline Hee. 12 & 62 & & 102 & & 102.2 & & 101.5 & & 102.1 & & 101.8 & \\
\hline
\end{tabular}


Animals in stable arlificially heated, 1890-Contimust.

\begin{tabular}{|c|c|c|c|c|c|c|c|c|c|c|}
\hline \multirow{2}{*}{ loate. } & \multicolumn{2}{|c|}{$\begin{array}{l}\text { Temperature } \\
\text { of stable. }\end{array}$} & \multicolumn{2}{|c|}{117.} & 130 & & 145 & & 159 & \\
\hline & $\begin{array}{l}\text { Morn. } \\
\text { ing. }\end{array}$ & $\begin{array}{l}\text { Even- } \\
\text { ing. }\end{array}$ & Morning. & $\begin{array}{l}\text { Evell- } \\
\text { ing. }\end{array}$ & Morning. & $\begin{array}{l}\text { Fven- } \\
\text { ing. }\end{array}$ & Morning. & $\begin{array}{l}\text { Even. } \\
\text { ing. }\end{array}$ & Morning. & $\begin{array}{l}\text { Hven- } \\
\text { ing. }\end{array}$ \\
\hline Hece 12 & & 64 & & 101. & & 102. 3 & & 103.4 & & 102.2 \\
\hline Ileer. 13 & 68 & $1 i 7$ & 101.7 & 101.8 & 10.3 .8 & $10: 3 .+4$ & 101.4 & 102.4 & 102.4 & $10: 3.3$ \\
\hline loee il & 71 & & 101.9 & ...... & 1003 & $\ldots . .$. & 101.5 & (2) & 102.5 & \\
\hline T)er 1 , & 72 & 76 & 101.2 & 101.9 & 1012.5 & 10). 6 & 111.8 & 101.8 & $10)^{2}$ & $10: 4$ \\
\hline Jeer if & (i)? & 74 & 101.8 & $10: 3$ & 101.9 & 10.2 .4 & 101.7 & 102.2 & 10.26 & 103.2 \\
\hline 1) 17 & 74 & it & 1003.4 & 1023 & $101 . ?$ & 1011.8 & 102.2 & 101.8 & $10: 3.2$ & i(1):?. 23 \\
\hline Ines in & (ii) & 71 & 101 & $10 \% 2.4$ & 101.5 & $10 \%$ & 101.3 & $10^{*}$ & 101.6 & 102.7 \\
\hline There: 19 & 69 & 72 & 101.6 & 102 & 102 & 101.8 & 101.5 & 101.7 & 101.7 & 101.8 \\
\hline I)ece 20 & 68 & 71 & 101.6 & 102 & 101.4 & 101.5 & 101.8 & 101. j & 101.5 & 101.8 \\
\hline Hea. $=3$ & 72 & 78 & 101.6 & 102,5 & 101.5 & 101.8 & 101.5 & $10^{2}$ & 10.2 .4 & 102 \\
\hline 1)ee. 23 & 71 & 74 & 102.5 & 102.2 & 101.4 & 101.6 & 101.2 & 101.6 & 102.6 & 102.6 \\
\hline Here. 24 & 68 & 70 & 101.7 & 102.1 & 101.5 & 101.5 & 101.8 & 102 & 101.4 & $10 * 2$ \\
\hline 1$)$ ec. 25 & 72 & $\cdots$ & 10:2. 4 & $\ldots$. & 101.8 & $\ldots \ldots$ & 101.8 & $\ldots .$. & 102.2 & \\
\hline Deee 26 & 72 & 134 & 102.2 & 102.3 & 104.7 & 104.4 & 101.6 & $10^{2}$ & 102.2 & 101.8 \\
\hline Dee. 27 & 70 & 72 & 102 & 103.1 & 102.2 & 105 & 101.6 & 101.5 & 102.5 & 103.8 \\
\hline 1)ee. $2 x$ & 76 & 76 & 102.2 & 102.8 & 104.5 & 106.4 & $101 .+$ & $10: 3.1$ & $10: 3.7$ & $10.5,4$ \\
\hline Dec. 29 & 74 & 77 & 101.7 & $10 \pm .5$ & 106.3 & 105.8 & 101.2 & 102 & 105.4 & 106.3 \\
\hline Dec. 30 & 70 & $8: 3$ & 102.2 & 103.6 & 105.6 & 105.7 & 102.2 & 101.8 & 105.2 & 104.8 \\
\hline Dees. 31 & 72 & 80 & $10 \% .4$ & $\ldots \ldots$ & 105.3 & 105.6 & 101.5 & $\ldots . .$. & 101.2 & 105.4 \\
\hline JaII. 1 & 74 & 80 & 102.2 & $\ldots \ldots$ & $10: 3.8$ & 104.2 & 101 & $\ldots \ldots \ldots$ & 101.4 & 104.5 \\
\hline Jan. 2 & 81 & 84 & 102.8 & 103 & 101.7 & 102.6 & 101.6 & 101.8 & 102 & 102.5 \\
\hline Jan. & 67 & 76 & 101 & 101 & 101.2 & 101. & 100.8 & 101 & 103. 2 & 102.7 \\
\hline Jan. & 74 & 76 & 101.5 & $10 \div .4$ & 101.8 & $10^{\circ}=2$ & 101.6 & 101.7 & 1112.4 & 103.2 \\
\hline Jan. & 70 & 70 & 101.2 & 102 & 101 & 102.2 & 101.2 & 10 i. 6 & 103 & 104.3 \\
\hline Jall. & 70 & 75 & 101 & 102 & 1017 & 102.5 & 101.5 & 101.8 & 102.2 & 1013 \\
\hline Jan. & (j) & 73 & 101.4 & 101.8 & 101.2 & 10) 2 & 101.4 & 101.7 & 102.2 & 102. 2 \\
\hline Jan. & 72 & 78 & 101.2 & $10 \div .3$ & 102.5 & $10: 3$ & 101.4 & 102 & $101 .(j$ & 102. \\
\hline Jan. 9) & $7: 2$ & 78 & 101.5 & 102.6 & :03. 2 & 104 & 101.6 & $1(12.2$ & 101.6 & 101. $x$ \\
\hline Jan. 10 & 74 & $81)$ & 101.5 & 103 & 102.5 & 103 & 101.4 & 102.6 & 101. 8 & 102.5 \\
\hline Jaı. 11 & 78 & $\ldots$ & $10: 3.2$ & $\ldots \ldots$ & 102.8 & $\ldots \ldots$ & 102.4 & $\ldots \ldots$. & $10: 2.2$ & $\ldots . .$. \\
\hline Ja11. 12 & 80 & $\ldots$ & 102 & $\ldots \ldots$ & 101.7 & ........ & $102.1 ;$ & $\ldots \ldots \ldots$ & 101.6 & ...... \\
\hline Ja11. 13 & 72 & 74 & 101. 4 & 102 & 101.6 & $10: 3$ & 101.2 & 101.8 & $101 . \overline{5}$ & 101.7 \\
\hline Jan. 14 & 73 & 81 & 101.5 & 102.3 & 101.8 & 102.5 & 102.8 & $10: 3.5$ & 101.6 & $10: 2$ \\
\hline Jan. 15 & 80 & 81 & 101. 6 & 102.2 & 101.5 & 101.8 & 101.2 & 102 & 10)2.5 & 101.8 \\
\hline Јан. 16 & 78 & 82 & 102.2 & 102.3 & 101.5 & 101.8 & 101.5 & $10: 3$ & 102 & $102.1 \mathrm{i}$ \\
\hline . Тan. 17 & 76 & 78 & 102.2 & 103.2 & 101.4 & 102 & 102 & 103.4 & 102 & 101.8 \\
\hline Jau. 19 & 76 & 74 & 101.3 & 102.6 & 101 & 101.6 & 103.2 & 104 & 101.8 & 101. 7 \\
\hline Jan. 20 & 79 & 78 & 101.2 & 101.8 & 101.2 & 102 & 103 & 104.5 & 102.4 & 102.6 \\
\hline Jan. 21 & 72 & 76 & 100.7 & 102 & 101.4 & 101.8 & 103.9 & 105.2 & $10 * 2.2$ & 102 \\
\hline Jan. 22 & 78 & 78 & 101.5 & 102 & $10 \div 2.2$ & 102.6 & 104.4 & 105 & 101.3 & 302 \\
\hline Tan. 23 & 74 & 80 & 101.4 & $10^{\circ} .6$ & 101.7 & 101.8 & 104 & 104.5 & 101.6 & 102.2 \\
\hline Jan. 24 & 72 & 70 & 101.8 & 102 & 101. 2 & 101,6 & 104 & 105 & 101.6 & 102.3 \\
\hline Ja11. 25 & 58 & 68 & 101.4 & 102.5 & 101.5 & 101.4 & 104 & 104.8 & 100.6 & 101.6 \\
\hline Jan. 26 & 67 & 70 & 101 & 101.4 & 101.2 & 101.5 & 103.7 & 104 & 101.5 & 101.4 \\
\hline Ja11. 27 & 62 & 72 & 101.7 & 102.5 & 101.4 & 102.2 & 104 & 104.5 & 101 & 101.8 \\
\hline Ja11. 28 & 66 & 76 & 101.6 & 101.8 & 101.8 & 101. 6 & 103.8 & 104.3 & $i 01$ & 101.5 \\
\hline Jan. 29 & 72 & 75 & 101.2 & 101.6 & 101.5 & 101.8 & 103.8 & 104 & 101.3 & 101. 4 \\
\hline Jan. 30 & 71 & 72 & 101.2 & 102.2 & 101.4 & 101.7 & 103 & 103.6 & 101.5 & 101.5 \\
\hline Jan. 31 & 65 & 74 & 101.8 & 102.5 & 101.3 & 101.7 & 102.4 & 103.8 & 103.4 & 101.6 \\
\hline Feb. 2 & 72 & $\ldots$ & 101.4 & $\ldots \ldots$ & 101.3 & $\ldots \ldots \ldots$ & 1()$^{2}, 4$ & $\ldots . .$. & 101.5 & 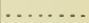 \\
\hline Feb. 3 & 72 & 76 & 101.2 & 102 & 101 & 101.4 & 102.2 & 102.8 & 101.4 & 101.5 \\
\hline Feb. & 60 & 70 & 101. 2 & $10^{\circ}$ & 100.8 & 101.5 & 101.8 & 103 & 100.5 & 101.6 \\
\hline Feb. & 68 & 70 & 101.7 & 102 & 101.6 & 101.6 & 102.4 & 102.6 & 101.7 & 101. $\frac{1}{4}$ \\
\hline Feb. & 75 & 70 & 102.2 & 101.8 & 101.5 & 101.8 & 102 & 102.6 & 101.7 & $10^{2} 2$ \\
\hline Feb. & 64 & 70 & 101.4 & 102.4 & 101 & 101.6 & 101.8 & 102.2 & 101.6 & 101.5 \\
\hline Feb, 9 & 68 & 70 & 101.8 & 102 & 101 & 101.4 & 102 & $10^{\circ} .8$ & 101.8 & 101.5 \\
\hline Feb. 10 & 65 & 73 & 100.8 & 102.4 & 101.4 & 101.3 & 101.4 & 102.2 & 101 & 101.8 \\
\hline Fel. 11 & 68 & 76 & 101.6 & $102.1 j$ & 101 & 101.6 & 101.7 & 102.4 & 102 & 101,8 \\
\hline Feb. 12 & 70 & 68 & 101.2 & 102 & 101.2 & 101.6 & 101.4 & 101.4 & 101.5 & 101.8 \\
\hline Feb. 13 & 71 & 72 & 101. 4 & 102 & 101.8 & 101.7 & 102.4 & 102.6 & 101.6 & 101.6 \\
\hline Feb. 14 & 69 & 72 & 101.5 & 102.2 & 101.5 & 101.8 & 102 & 102.6 & 101.7 & 102 \\
\hline Feb. 16 & 71 & 72 & 101.8 & 106.2 & 101.4 & 101,6 & 101.2 & 101.5 & 101.6 & 102 \\
\hline Feb. 18 & 73 & $\ldots$ & 101.5 & & 101.8 & & 101.7 & 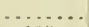 & 101.8 & \\
\hline Feb. 19 & 60 & 70 & 100.6 & 102 & 101.4 & 101.8 & 101.3 & 102 & 101. 7 & 102 \\
\hline Feb. 21 & 74 & 75 & 101.6 & 102. 2 & 101.8 & 101.6 & 101.8 & 101.7 & 101.2 & 101.4 \\
\hline Feb. 23 & 68 & $\ldots \ldots \ldots$ & 101.6 & & 101.4 & $\ldots \ldots$. & 101.8 & & 101.3 & \\
\hline Feb. 24 & 72 & 78 & 101. 4 & 102 & 101.5 & 102 & 101.7 & 102 & 101.3 & 101.6 \\
\hline Feb. 26 & 70 & 74 & 101.5 & 102.2 & 101.6 & 101.6 & 101.2 & 101.8 & 101.2 & 101.5 \\
\hline Mar. 2 & 62 & 65 & 102 & $10: 3$ & 102.4 & 102.8 & 101.8 & 102.2 & 101.7 & 101.8 \\
\hline Mar. 5 & 60 & 66 & 102 & 101.6 & 101.4 & 101.2 & 101.5 & 101.6 & 101.4 & 101.5 \\
\hline Mar. 9 & 68 & 70 & $10^{2}$ & $10^{-3}$ & 101.6 & 101.3 & 102.2 & 103.1 & 101. 6 & 101.7 \\
\hline Mar. 12 & $\ddot{n} 9$ & 68 & 101.2 & 101.6 & 101.5 & 102 & 101.6 & 102.6 & 101.8 & $10^{\circ} .1$ \\
\hline Mas. 16 & 65 & & 101.2 & & 101. & & 102.2 & & 102.4 & \\
\hline Mlar. 21 & (i5) & 70 & 101.6 & 101.5 & ion & 101.6 & 101.5 & 101.6 & 101.8 & 102 \\
\hline Mar. 24 & 73 & 72 & 101.2 & 1111.8 & 101.2 & $101 .+$ & 102 & 101.2 & $10^{\circ} 3$ & 102 \\
\hline Mar. 28 & (ii) & 70 & 101.2 & 101.4 & 1011.4 & 101.1 & 102.4 & 101.8 & 101.8 & 101.8 \\
\hline$A_{1} \mu . \quad 1$ & (i) & 68 & 101.2 & 101.6 & 1111.3 & $101.1 i$ & $10: 3$ & $10: 3.7$ & 101.8 & 101.8 \\
\hline Apr. 4 & (i:) & 64 & 101.4 & $10: 2$ & $101 . x$ & 101.4 & 102 & 101.8 & 101.7 & $10: 3$ \\
\hline A pr. 7 & 60 & 72 & 101.6 & 102.2 & 101.4 & 101.8 & $10 \% .2$ & 101.8 & 101.5 & 101.7 \\
\hline
\end{tabular}




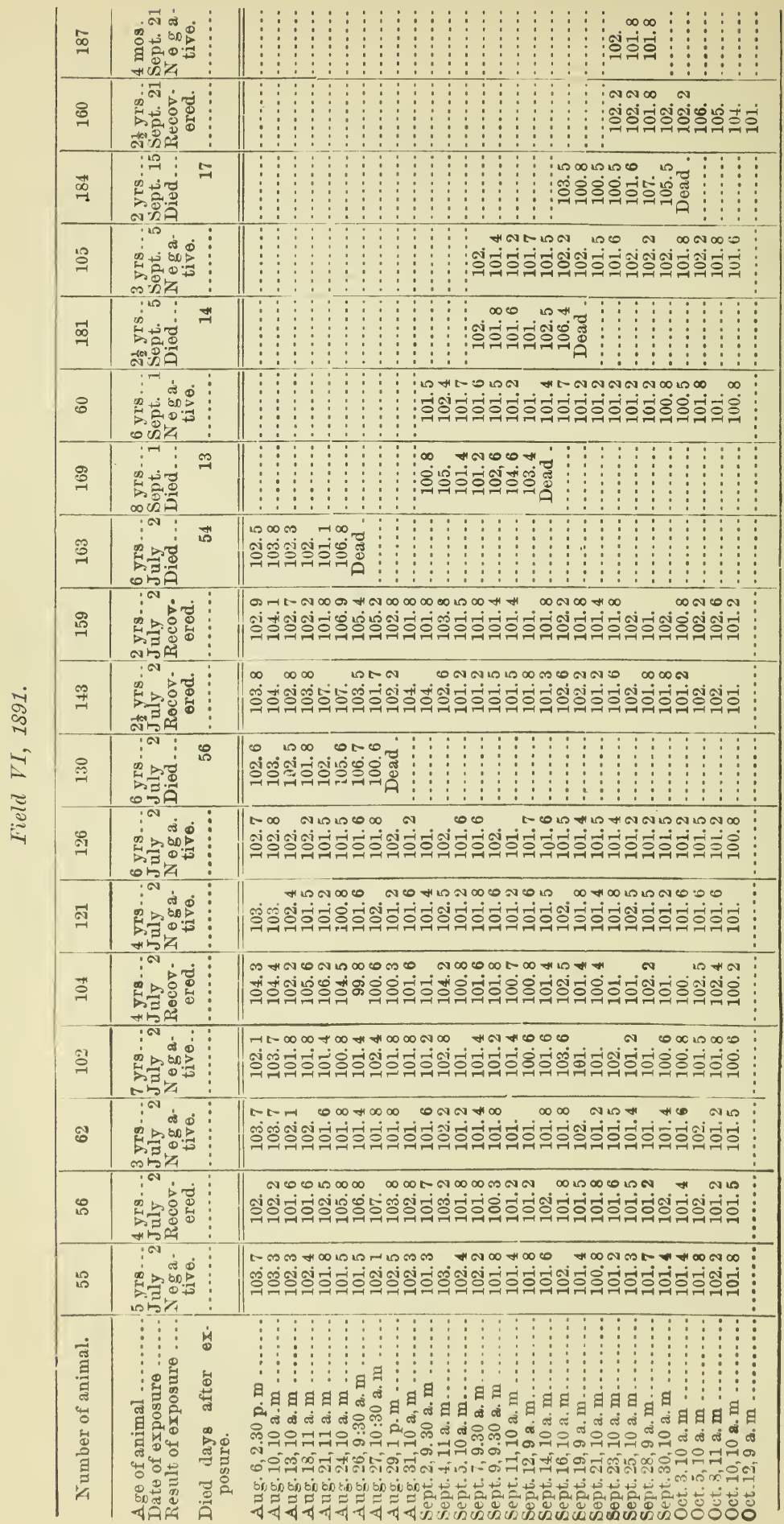


Field XI, 1891.

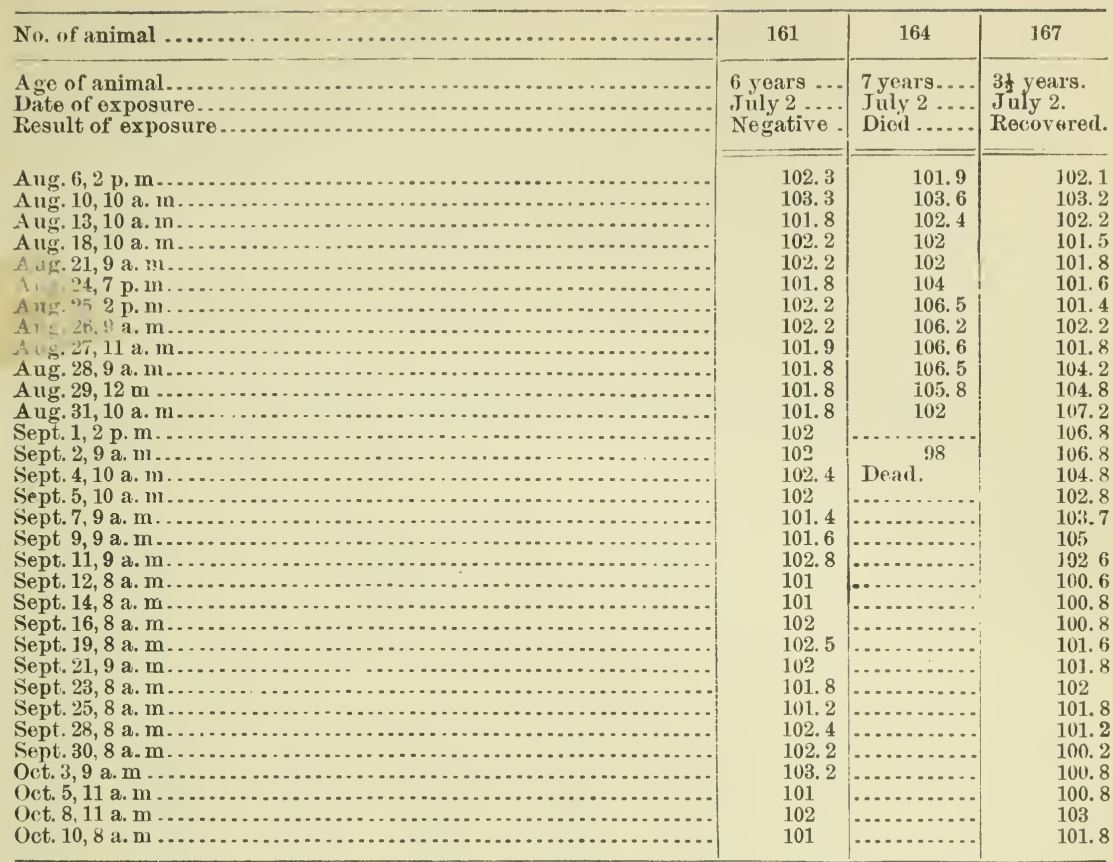

Intravenous injection of infusion of ticks, 1891.

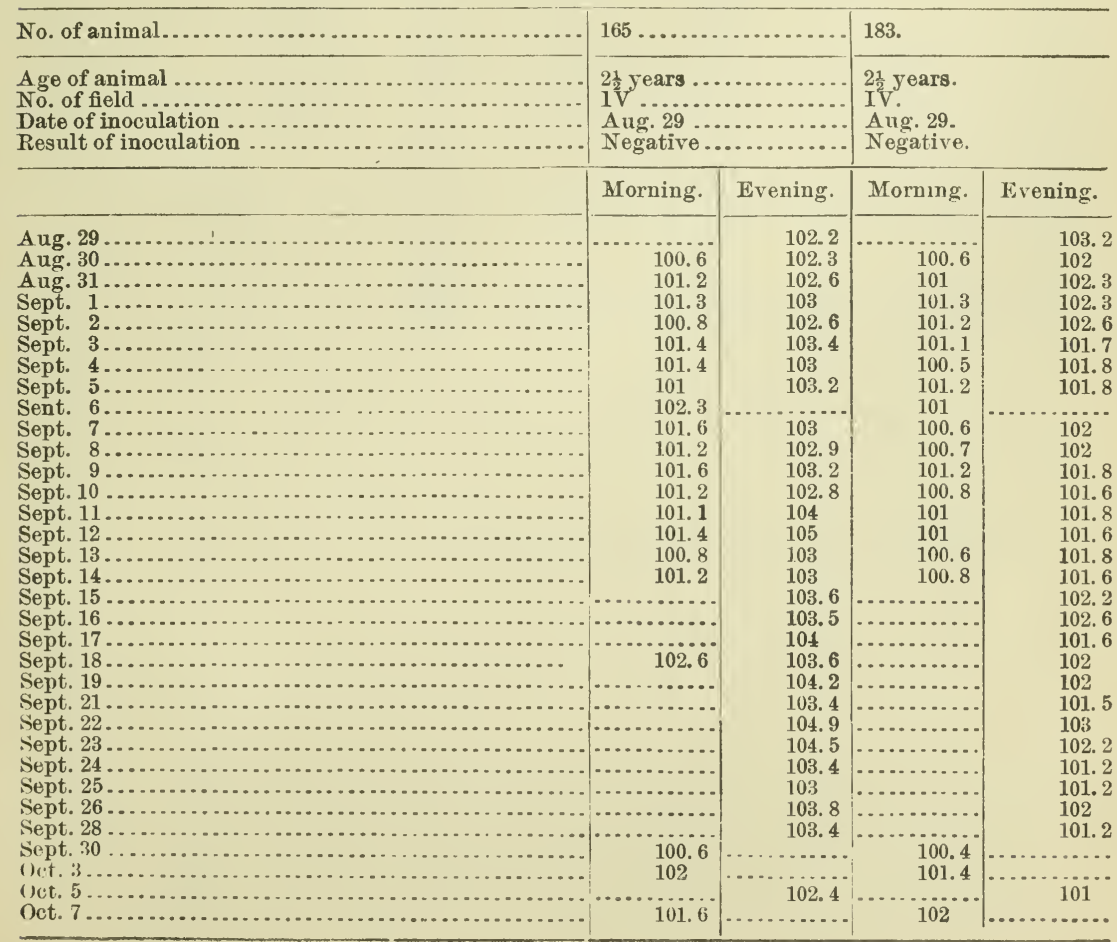


Intravenous injection of Texas fever hlood. Field II. 1 S31.

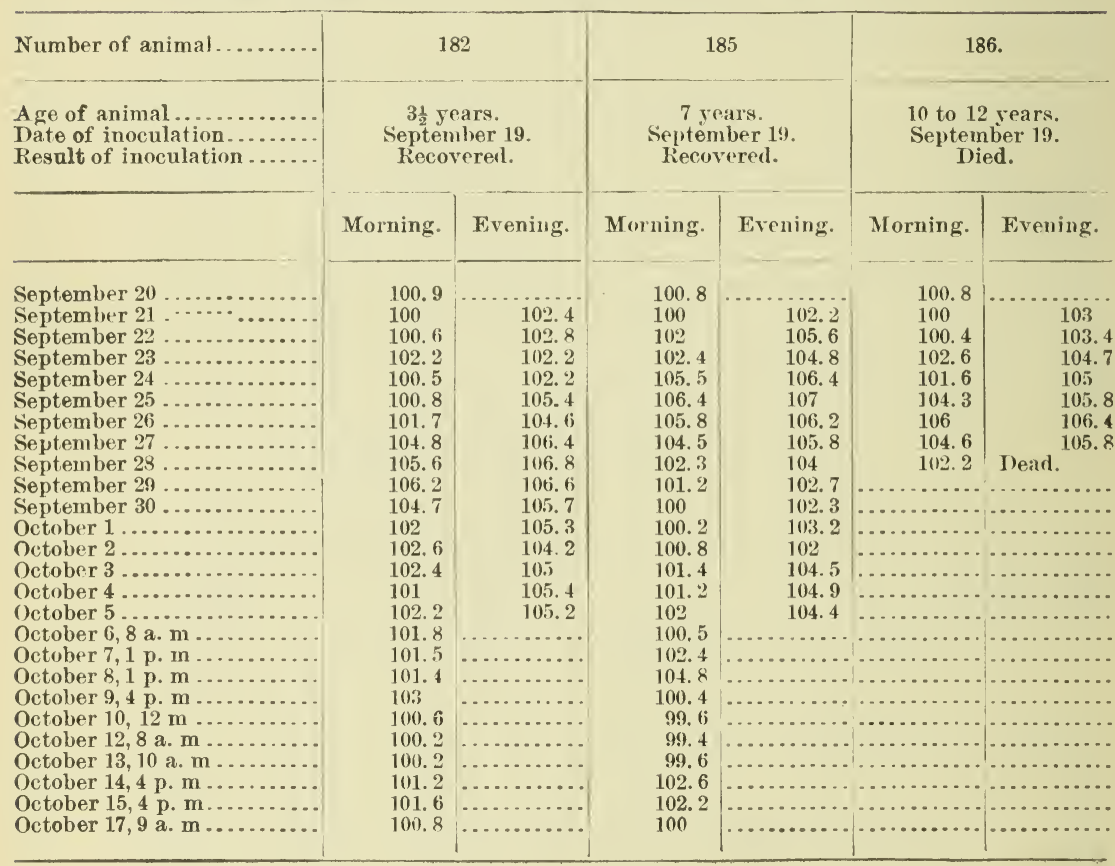




\begin{tabular}{|c|c|c|}
\hline Бे & 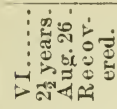 & \\
\hline$\stackrel{\infty}{\infty}$ & 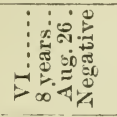 & $\begin{array}{l}\vdots \vdots \\
\vdots\end{array}$ \\
\hline$\stackrel{\substack{\infty \\
-\infty}}{ }$ & 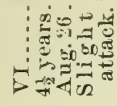 & $\begin{array}{cc:}\vdots & \vdots \\
\vdots & \vdots \\
\vdots & \vdots \\
\vdots & \vdots\end{array}$ \\
\hline 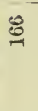 & 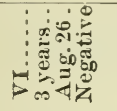 & $\begin{array}{cccccc}0 & 0 & 0 & 0 & 0\end{array}$ \\
\hline : & 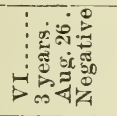 & : : : : : : \\
\hline 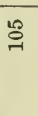 & 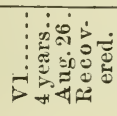 & : : : : \\
\hline : & 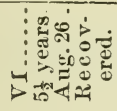 & 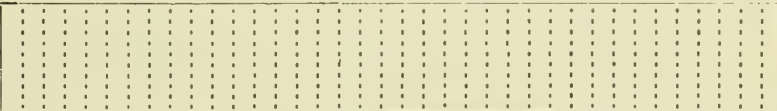 \\
\hline 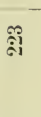 & 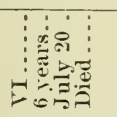 & 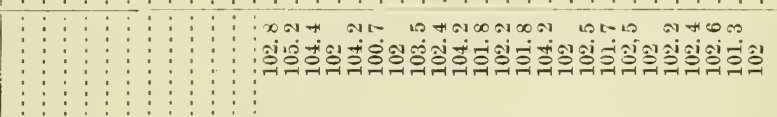 \\
\hline สิ & 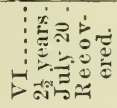 & 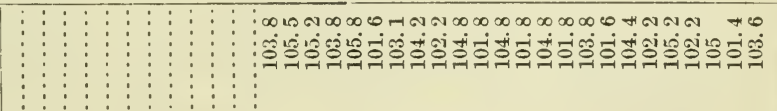 \\
\hline$\stackrel{\bullet}{\bullet}$ & 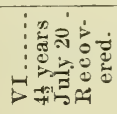 & 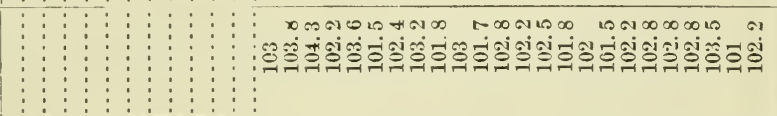 \\
\hline స్తి & 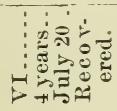 & 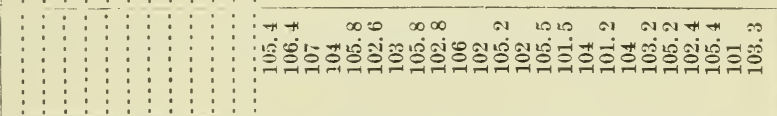 \\
\hline ڤึ & 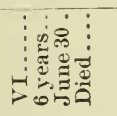 & 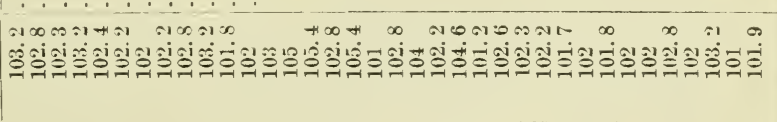 \\
\hline కి & 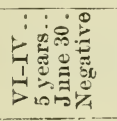 & 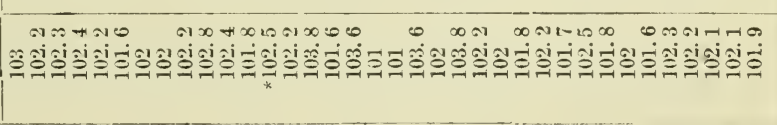 \\
\hline 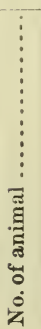 & 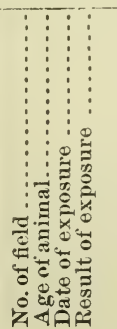 & 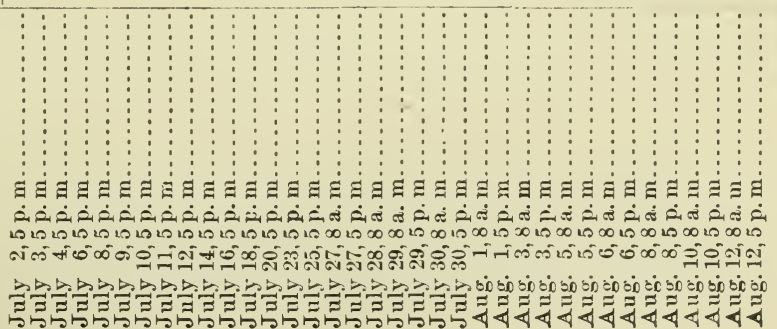 \\
\hline
\end{tabular}


Fields $I$ and II, 1892.

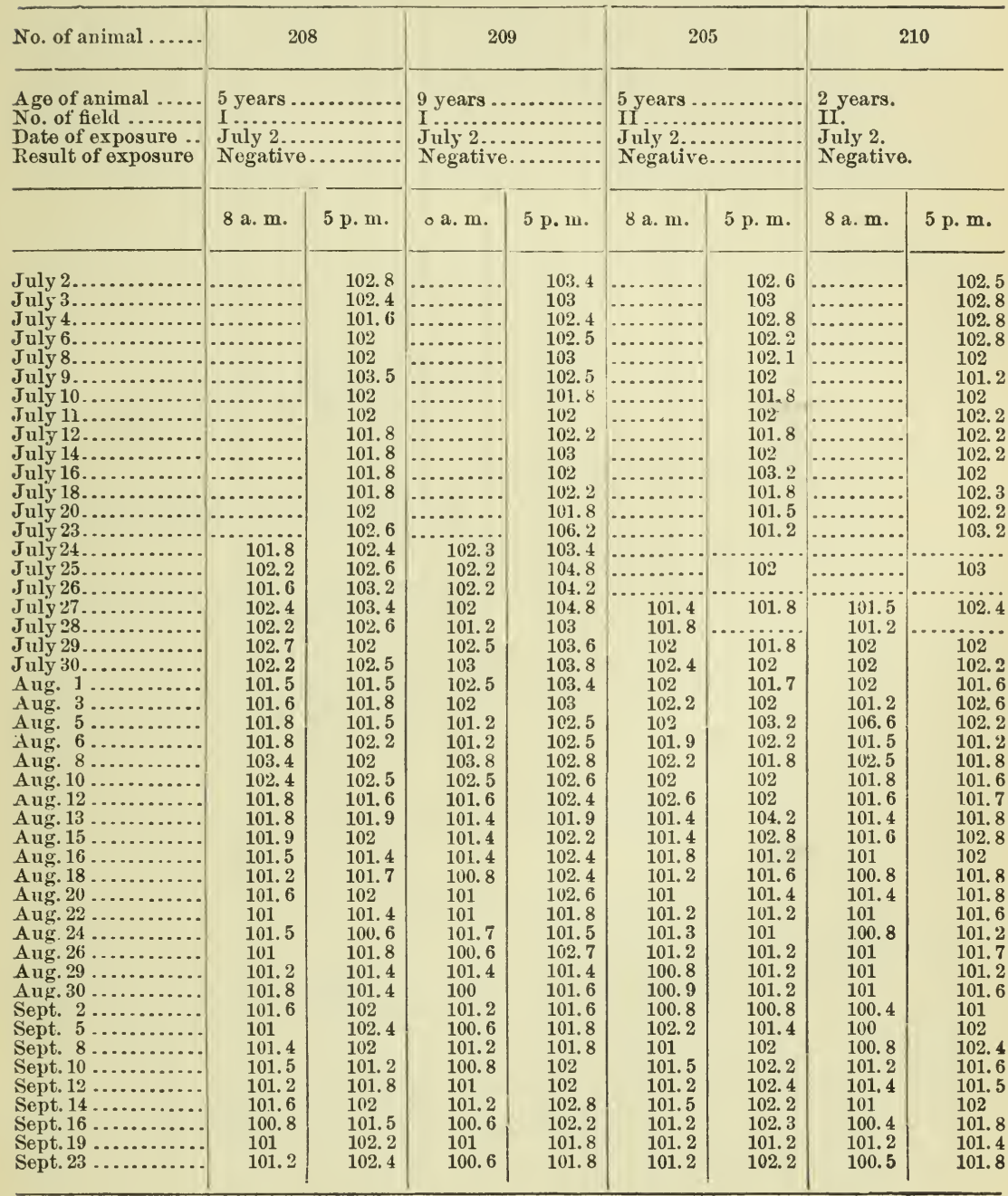




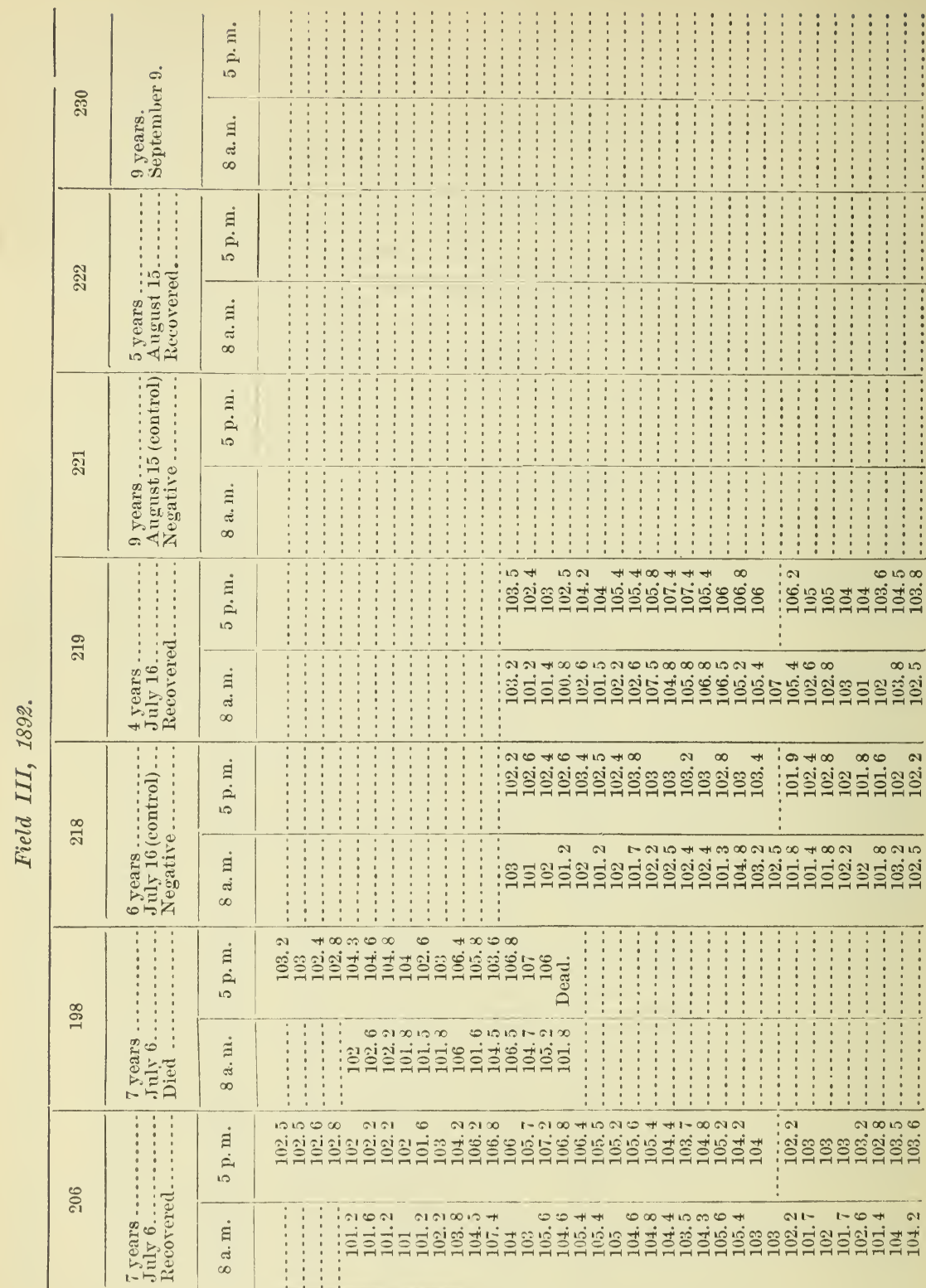




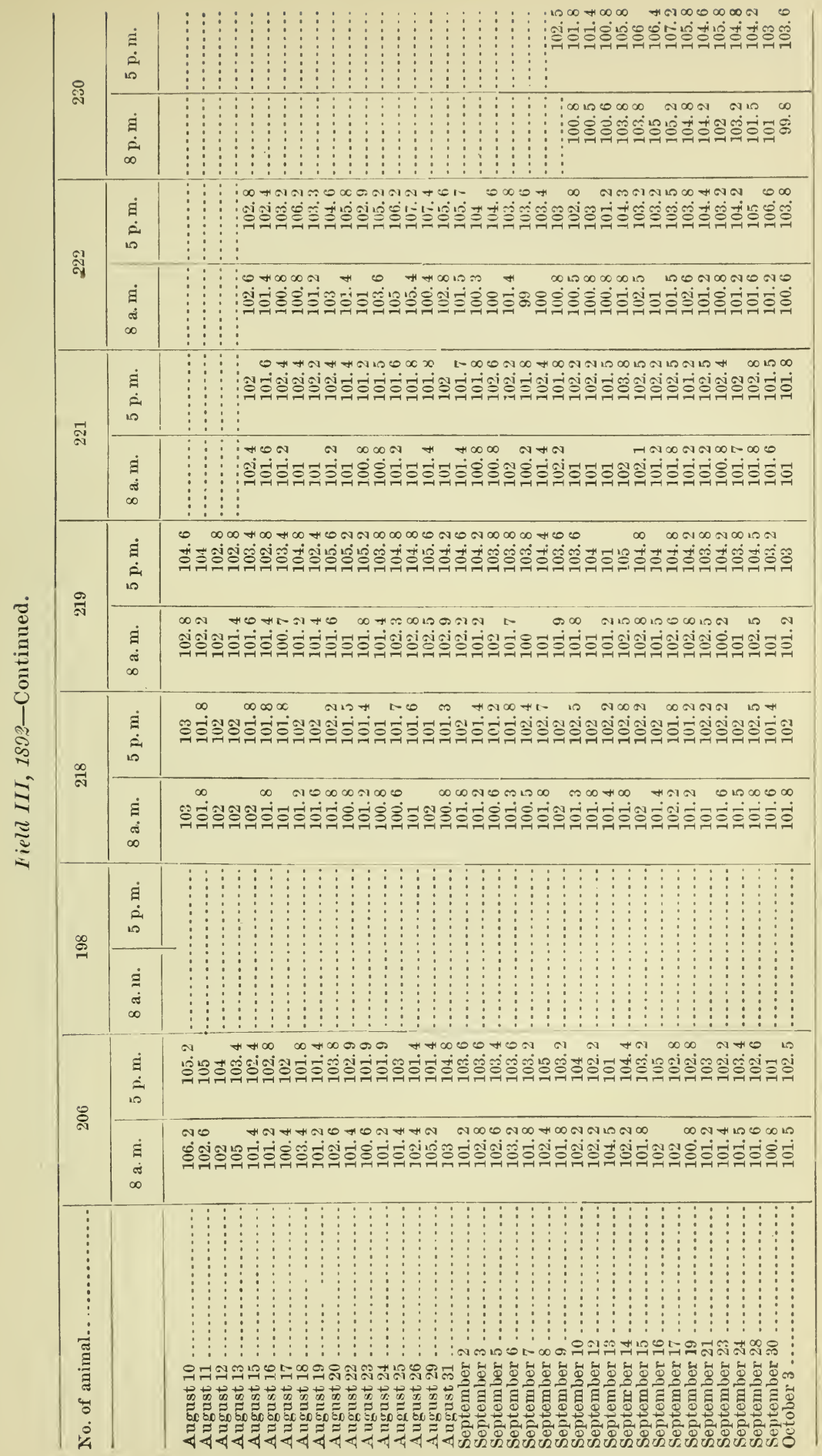


Miscellaneous experiments, 1892.

\begin{tabular}{|c|c|c|c|c|c|c|c|c|c|c|c|c|}
\hline No. of animal. & \multicolumn{2}{|c|}{197} & \multicolumn{2}{|c|}{227} & \multicolumn{2}{|c|}{228} & \multicolumn{2}{|c|}{200} & \multicolumn{2}{|c|}{202} & \multicolumn{2}{|c|}{$20 \%$} \\
\hline $\begin{array}{l}\text { Age of ani- } \\
\text { mals. } \\
\text { Number of } \\
\text { field. } \\
\text { Date of inoc- } \\
\text { ulation. } \\
\text { Result of in. } \\
\text { oculation. } \\
\text { Died days } \\
\text { after inocu. }\end{array}$ & \multicolumn{2}{|c|}{$\begin{array}{l}6 \text { years ...... } \\
12 \mathrm{~A} \ldots . . . . \\
\text { A } \ldots . . \\
\text { Died ......... } \\
8 \ldots \ldots \ldots\end{array}$} & \multicolumn{2}{|c|}{$\begin{array}{l}11 \text { years .... } \\
12 \mathrm{~A} . . . . . \\
\text { A ug. } 27 . . . \\
\text { Died ........ } \\
13 \ldots . . . .\end{array}$} & \multicolumn{2}{|c|}{$\begin{array}{l}7 \text { years ...... } \\
12 \mathrm{~A} . . . . . \\
\text { Aug. } 27 . . . \\
\text { Recovered .. } \\
\ldots . . . . . . . .\end{array}$} & \multicolumn{2}{|c|}{$\begin{array}{l}8 \text { y ears ...... } \\
12 \mathrm{~A} \ldots \ldots . . \\
\text { A ug. } 30 \ldots . \\
\text { Died ........ } \\
9 \ldots \ldots \ldots\end{array}$} & \multicolumn{2}{|c|}{ 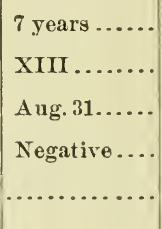 } & \multicolumn{2}{|c|}{ 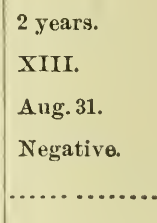 } \\
\hline & 8 a. m. & 5 p. m. & 8 a. m. & 5 p. m. & 8 a.m. & 5 p. m. & 8 a. m. & 5 p. $\mathrm{m}$. & 8 a.m. & 5 p. $\mathrm{m}$. & 8 a. m. & 5 p. m. \\
\hline $\begin{array}{l}\text { Aug. } 29 \ldots \ldots \\
\text { Aug. } 31 \ldots \ldots \\
\text { Sept. } 2 \ldots \ldots \\
\text { Sept. } 3 \ldots \ldots \\
\text { Sept. } 5 \\
\text { Sept. } 6 \ldots \ldots \\
\text { Sept. } 7 \ldots \ldots \\
\text { Sept. } 8 \ldots \ldots \\
\text { Sept. } 9 \\
\text { Sept. } 10 \\
\text { Sept. } 12 \\
\text { Sept. } 13 \\
\text { Sept. } 14 \\
\text { Sept. } 15 \\
\text { Sept. } 16 \\
\text { Sept. } 17 \\
\text { Sept. } 19 \\
\text { Sept. } 21 \\
\text { Sept. } 23 \\
\text { Sept. } 28 \\
\text { Sept. } 30 \\
\text { Oet. } 3 \\
\text { Oet. } \\
\end{array}$ & \begin{tabular}{l}
101 \\
103.2 \\
105.4 \\
106.7 \\
Dead \\
$\cdots \ldots .$. \\
$\cdots \ldots .$. \\
\hdashline$\ldots .$. \\
$\ldots \ldots$.
\end{tabular} & $\begin{array}{l}101.6 \\
105 \\
106.6 \\
107.4 \\
\text { Dead } \\
\cdots . . . \\
\ldots . . .\end{array}$ & \begin{tabular}{|l|}
100.8 \\
100.4 \\
100.6 \\
102.4 \\
103.8 \\
103 \\
103 \\
98.5 \\
97.5 \\
Dead
\end{tabular} & 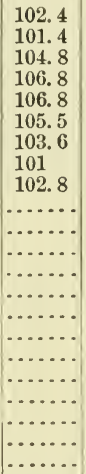 & $\begin{array}{l}100.8 \\
101.2 \\
100.8 \\
102.8 \\
103.6 \\
105.8 \\
106 \\
106 \\
104.2 \\
103.6 \\
102.8 \\
102.2 \\
102.1 \\
101.6 \\
101.6 \\
100.8 \\
101.7 \\
101.5 \\
100.8 \\
101 \\
100.6 \\
100.4\end{array}$ & $\begin{array}{l}101.6 \\
102 \\
106.6 \\
107 \\
106 \\
107.2 \\
108.1 \\
106.2 \\
105.8 \\
103.6 \\
105.8 \\
102 \\
105 \\
105 \\
104.5 \\
104.4 \\
105.2 \\
104.4 \\
103.5 \\
105 \\
103 \\
102.8\end{array}$ & $\begin{array}{l}100.8 \\
100.6 \\
100.6 \\
101.2 \\
106 \\
105.2 \\
\text { 1ead. } \\
\cdots \ldots . . . \\
\cdots \ldots . .\end{array}$ & $\begin{array}{l}101.4 \\
100.2 \\
104.8 \\
105.4 \\
107.4 \\
107.5 \\
\cdots \ldots \ldots \\
\cdots \ldots \ldots \\
\cdots \ldots \ldots\end{array}$ & $\begin{array}{l}104 \\
100.8 \\
100.8 \\
101 \\
101 \\
100.8 \\
101.8 \\
101.8 \\
101.4 \\
101.6 \\
101.8 \\
101.5 \\
102.2 \\
101.4 \\
101.5 \\
102.4 \\
101.5 \\
101.2 \\
102\end{array}$ & $\begin{array}{l}105 \\
101.8 \\
101.9 \\
101.4 \\
101.2 \\
102 \\
101.7 \\
101.7 \\
101.8 \\
102 \\
102.4 \\
101.8 \\
102 \\
102.4 \\
102 \\
101.4 \\
101.6 \\
101.8 \\
\cdots \cdots . .\end{array}$ & $\begin{array}{l}103.2 \\
101 \\
101.8 \\
101.4 \\
101.4 \\
100.4 \\
101.7 \\
101.2 \\
101.6 \\
101 \\
101.4 \\
101.8 \\
101.8 \\
101.2 \\
101.9 \\
101.6 \\
100.5 \\
100.8 \\
101.8 \\
\ldots \ldots \ldots\end{array}$ & $\begin{array}{c}105 \\
101.3 \\
102 \\
102 \\
101.4 \\
102.4 \\
101.8 \\
102.2 \\
101.5 \\
161.8 \\
102 \\
101.8 \\
102 \\
101.8 \\
102.5 \\
101 \\
101.5 \\
101 \\
\ldots \ldots \ldots \\
\ldots \ldots \ldots \\
\ldots \ldots \ldots\end{array}$ \\
\hline
\end{tabular}


Lieiscellaneous expcriments, 159 ?.

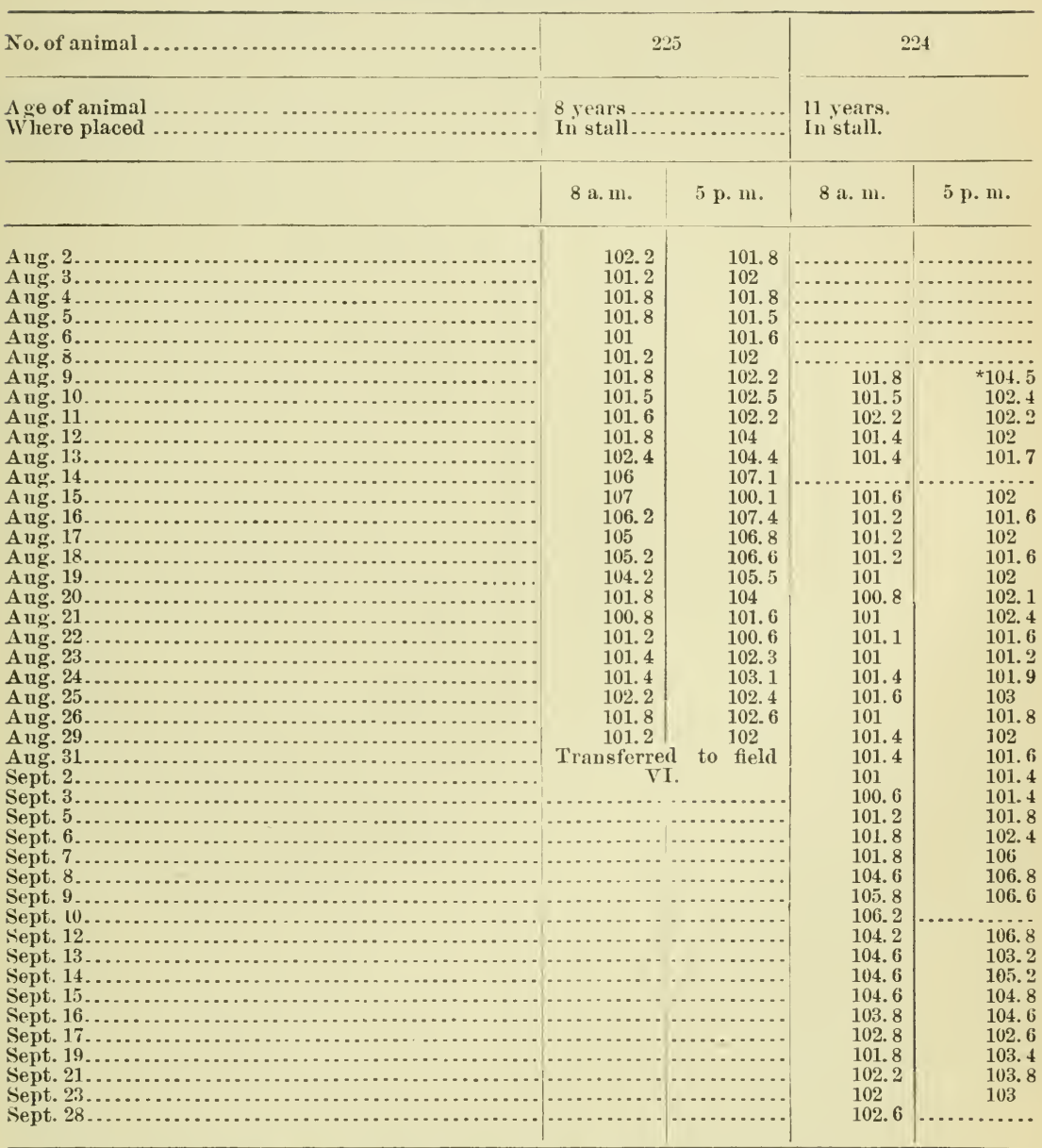

* High temperature perbaps due to the struggle necessary to get animal in stall. 



\section{N D E X.}

$\begin{array}{lll}\text { Page } & \end{array}$

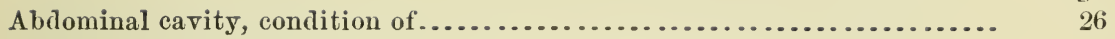

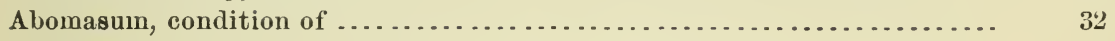

Acute and mild type of Texas fever, relation between ................ 23

type of Texas fever ................................... 15

microürganisms in ........................ 58

Anæmia in Texas fever....................................... 36,45

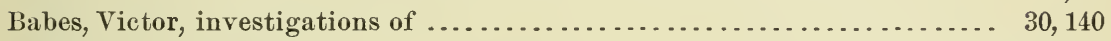

Bacillus coli communis..................................... $52,54,164$

Bacteria in cattle ticks....................................... 92

Bacteriological investigations in Texas fever ..................... 51, 159

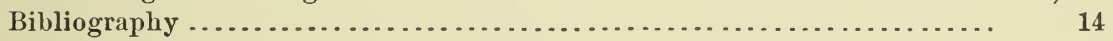

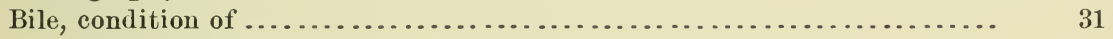

stasis in bile capillaries .................................. 29

Billings, Frank S., investigations of ......................... $51,78,115,123$

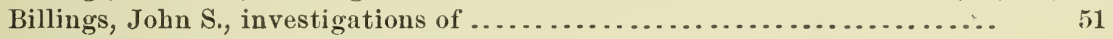

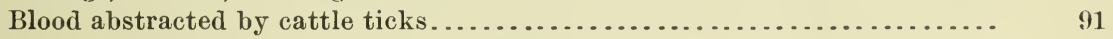

changes in the corpuscular elements of .................... 35

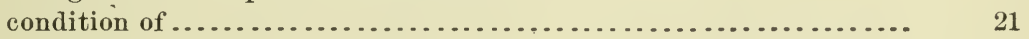

corpuscles. (See Red blood corpuscles.)

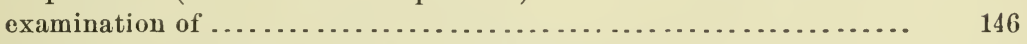

films on cover glasses, method of preparing .................. 43

method of examining fresh ............................. 43

method of obtaining preparations of ........................ 36

of Sonthern cattle will produce Texas fever .................. 119

Texas ferer is infectious ............................ 77

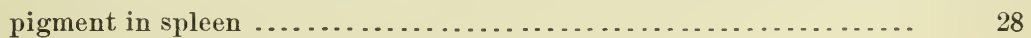

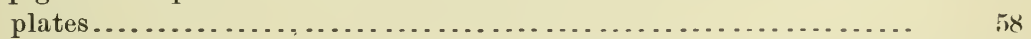

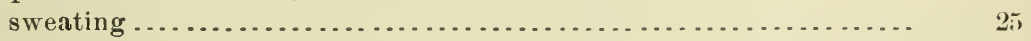

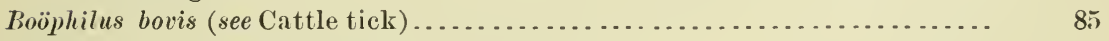

Boundary of permanently infected territory ...................... 13

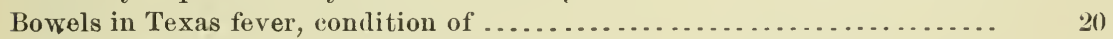

Box for confining cattle during examination............................. 37

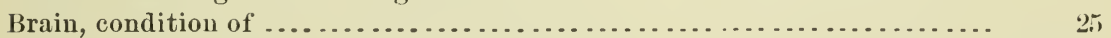

Calves less susceptible to Texas fever than adults .................. 13:2, 148

Capillaries filled with infected corpuscles ........................ 74

Cars conveying Southern cattle as sources of infection ................ $\quad 147$

Cattle, location of ticks on ................................... 91

number of red blood corpuscles in health ................... 38

peculiar bodies in red corpuscles of during health............ 56

Texas fever produced in, by inoculation of infected blood ......... $\quad 77$

ticks and Texas fever, experiments to determine relation between... 92 are they necessary in transmission of Texas fever.......... 126

as abstractors of blood ............................. 91

aids to preventive inoculation.................... 135 
Cattle ticks containing bacteria

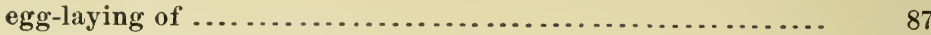

feeding experiments with ............................ 112

hatched artificially will produce Texas fever....104, 105, 110, 264, 265

how long do they survive on field.................. 90, 89, 149

infection of sick natives due to ...................... 122, 125

inflammation of the skin produced by .................. 91

intravenous injection of extract of .................... 113

larval stage of .................................... 88

period of incubation of in ovum ...................... 87

places of attachment of on cattle ..................... 91

producing Texas fever without the presence of Southern cat-

tle ...........................98, 103, 104, 105, 110, 264, 265

relation of to period of incubation of Texas fever........... 114

thrown on field when ripe produce Texas fever ............ 98, 103

transmitting Texas fever............................ 85

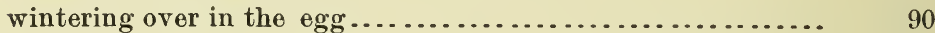

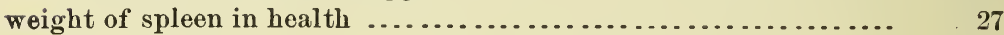

Causation of Texas fever ....................................... 51

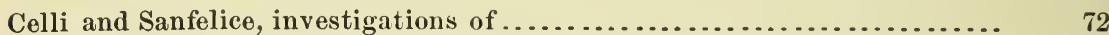

Chronic type of Texas fever..................................... 22

Circulating blood in Texas fever, micrö̈rganisms in............... 58,65

Classification of parasites of red blood corpuscles.................... 72

Cold, action on red blood corpuscles............................ 44

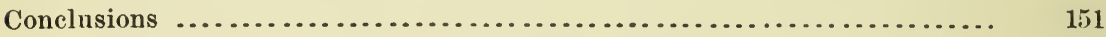

Curtice, Cooper, investigations of Texas fever outbreak.............. 159

investigations concerning cattl e ticks................ 86

Curtis, E., investigations of .................................... 51

Detmers, J. H., investigations of.......................... 30,51

Diagnosis of Texas fever ..................................... $\quad 145$

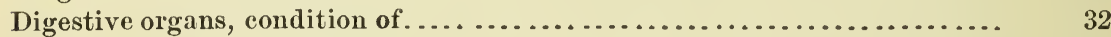

Dinwiddie, R. R., investigations of ........................... 53,79

Disinfection .............................................. $\quad 149$

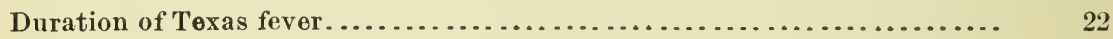

Ehrlich's method of heating dried blood films..................... 45

Embryonic red corpuscles in Texas fever......................... 47

produced in healthy cattle and sheep by with.

drawing blood........................ 48

Field infection, when does it begin............................ 111

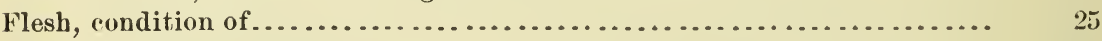

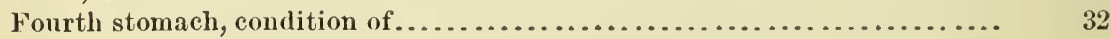

worm pits in ..................................... 33

Frost, action on cattle ticks................................. 149

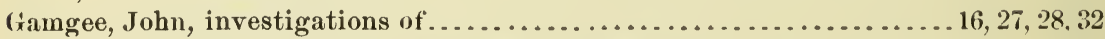

(xrass from infected field does not produce disease................... 112

Guinea-pigs not susceptible to inoculation with Texas-fever blood .......... 84

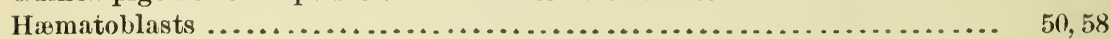

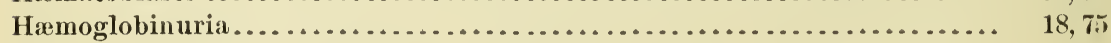

Hallier, Ernst, in vestigations of . .............................. 51

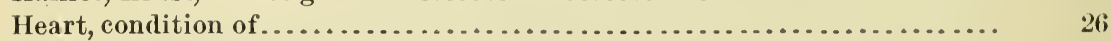

Heinz, investigations of ........................................ 67

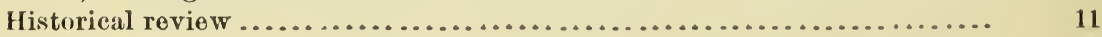

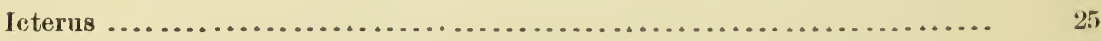

Jmmunity acquired by repeated infection....................... 132 
Page.

Immunity of Northern cattle, experiments

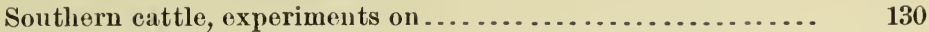

Incubation of Texas fever, period of. ........................... 15

relation to life history of cattle ticks ......... 114

Inoculations with Texas fever blood in cattle and other animals ......... 77,82

Intestines, condition of ...................................... 34

Intestines of cattle, worm tubercles in ............................. 34

Ixodes bovis (see Boöphilus bovis) ............................... 86

Jaundice ............................................. 25

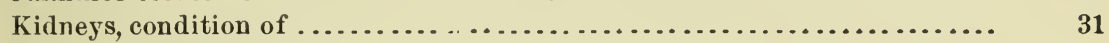

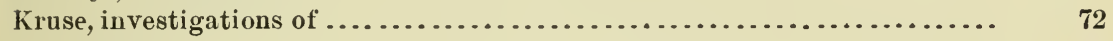

Leucocytes ............................................. 49

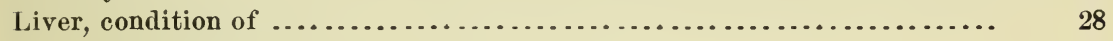

fatty degeneration in .................................. 29

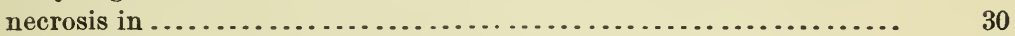

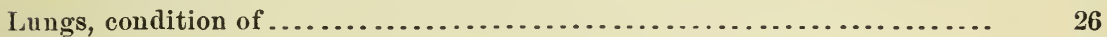

Macrocytes .......................................... 45

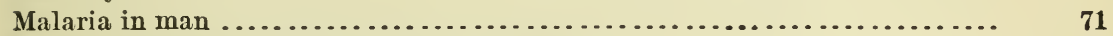

Marx, George, on classification of cattle ticks . . . . . . . . . . . . . . . . . 86

Method of counting blood corpuscles ............................... 36

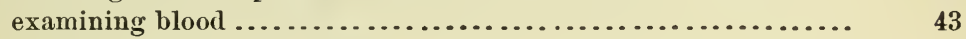

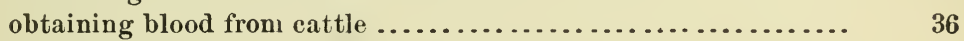

staining blood ................................... 44

Microörganism of Texas fever, amœboid motion of................... 59

distribution of, in the body............. 62

found in fourteen outbreaks............. $\quad 77$

in acute type ........................ 58

blood of Southern cattle.............. 118

mild type ........................ 65

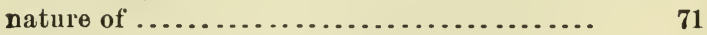

probable action of in the body ............ 73

life history of ............... 68,69

relation to other parasites of red blood corpuscles.................... $\quad 71$

the cattle tick............... $\quad 117$

set free from red blood corpuscles ........ 64,70

staining of .......................... 60

Micro-parasite of Texas fever (see Microürganism) $\ldots \ldots \ldots \ldots \ldots \ldots \ldots \ldots \ldots \ldots \ldots$

Mild type of Texas fever. ........................................ 22

the microörganisın in the .................. 65

Milk secretion in Texas fever.................................... 20

Mortality from Texas fever...................................... 22

Native cattle infectious when cattle ticks present................... 122, 125

Nervous symptoms in Texas fever ................................. 21

North Carolina cattle producing Texas fever.................93, 96, 99, 107, 111

Ontbreaks of Texas fever in which micro-parasites have been demonstrated. 76

Paquin, Paul, investigations of ........................53, 79, 84, 85, 92, 136

Parasites of red blood corpuscles ............................... 72

Pathological changes in Texas fever. . . . . . . . . . . . . . . . . . . . . . . . 25

Pease, Dr., investigations of ................................... 12

Period of incubation ......................................... 114

Peripheral coccus-like bodies................................... 66

Pigeons not susceptible to inoculation with Texas-fever blood .......... 83

Plates, description of ...................................... 153 
Post-mortem bacilli . Page.

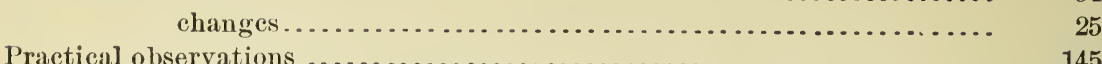

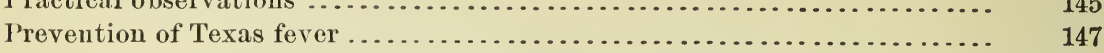

Preventive inoculation...................................... 135, 149

Pulse in Texas fever.......................................... 18

of cattle in health ......................................... 18

"Punctate" corpuscles ...................................... 46, 47, 48

Pyriform stage of the microörganism of Texas fever .................. $\quad 70$

Pyrosoma bigeminum (see Microörganism of Texas fever) ................. 55

Rabbits not susceptible to inoculation with Texas.fever blood............ 83

Red blood corpuscles, changes, in during Texas fever ................ $\quad 45$

containing stainable granules.................. 46

destruction of, in Texas fever ............... 36, 39, 62, 75

enlarged in the course of Texas fever ............ 45

method of counting ......................... $\quad 36$

number of, in health ........................ 38

of healthy cattle containing peculiar bodies ......... $\quad 56$

other animals and of man containing parasites.... $\quad 71$

oscillation in the destruction of ............... 24, 40

rate of destruction in acute type............... $\quad 39$

mild type.................. 40,76

regeneration of, as determined by countiug......... 41

the microscope.... 42

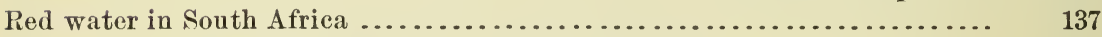

Texas fever......................................... 18

the Caucasus ......................................... 142

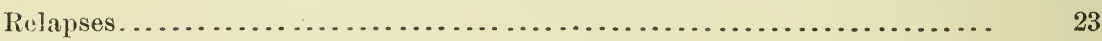

Respiration in Texas fever .................................... 18

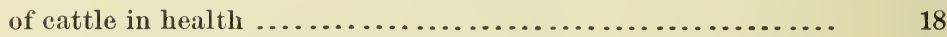

Riley, C. V., description of Ixodes bovis........................ 86

Roumania infected by a disease resembling Texas fever............... 140

Salmon, D. E., investigations of ............................. 51,78

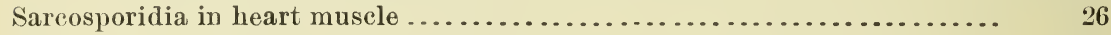

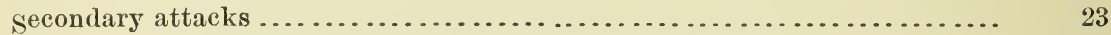

Sheep not susceptible to inoculation with Texas-fever blood ............ 82

Skin in Texas fever ........................................... $\quad 25$

inflammation of, due to cattle ticks ............... 91

temperature of ................................ $\quad 17$

South Africa infected by a disease resembling Texas fever ............. 137

Southern cattle carry the micrö̈rganisin of Texas fever in the blood....... $11^{8}$

how deprived of ticks ........................... 148

infectious when cattle ticks are present..........94,97, 100, 107

loss of power to infect natives .................... 116

not infectious when cattle ticks are absent...........95,97, 112

Spleen absorbing red blood corpuscles in Texas fever ................. 41

in Texas fever, condition of . ........................... 27

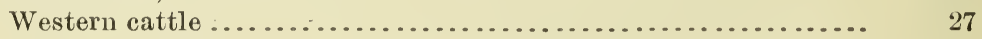

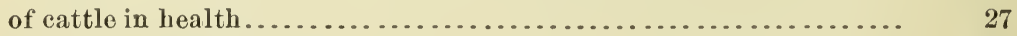

Staining of blood preparations .................................. 44

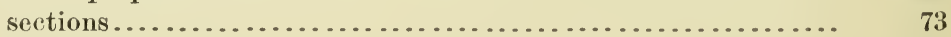

Stiles, C. W., on Strongylus Osterlagi............................. 33

Stiles, R. C., investigations of................................ 28,55

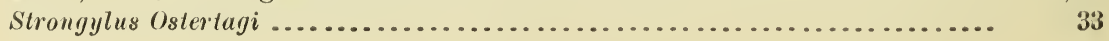


Swarming stage of the microörganism of 'T'exas fever ........................... 69

Symptoms of 'Texas fever...................................... $\quad 15$

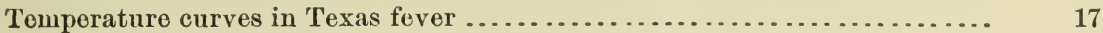

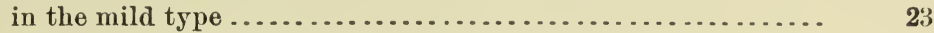

Texas fever, causes of .............................. 75

range in Texas fever ................................ 16

of cattle in health ............................. 16

record of various cases of Texas fever ................... 269

Texas cattle, diseases produced by, not dne to bacteria ............... 55

producing Texas fever ............................ 100

fe

and cattle ticks, experiments to determine relation between ... 92

etiology of ........................................ 51

infection, hypotheses conceruing .................... 121

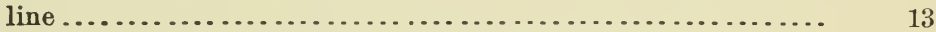

may it be communicated withont the cattle tick ............ 126

microörganism of ................................... 55

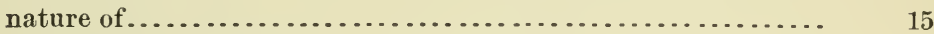

not caused by bacteria ............................... 51

produced without ticks .......................... $95,97,112$

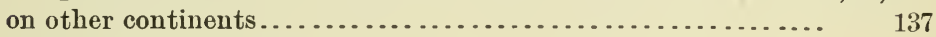

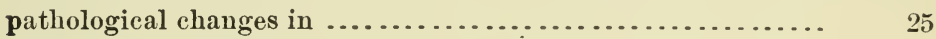

period of incubation in . ............................ 114

produced by cattle ticks alone........... 98, 103, 104, 105, 110, 261, 265

North Carolina cattle...............93, 96, 99, 107, 111

Texas cattle .......................... 100

the inoculation of blood from sick animals...... 77

reports on, by various investigators ................... 14

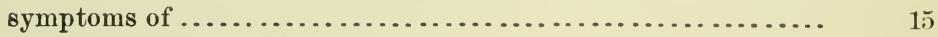

transmitted by the cattle tick ......................... 85

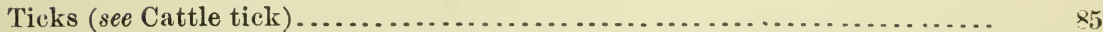

"Tinted" corpuscles......................................... $46,47,48$

Toison's fluid ................................................. 37,49

Treatment of 'Texas fever...................................... $\quad 150$

United States, permanently infected territory of..................... 13

Urine in Texas fever, condition of . .............................. 18

White blood corpuscles, counting of ............................. 49

Yellow fover of cattlo........................................... 34 

U.S. DEPARTMENT OF AGRICULTURE.

BUREAU OF ANIMAL INDUSTRY'

BULLETIN No. 2.

\author{
REPORT UPON INVESTIGATILNS
}

IELATLNG TO THE

\title{
'TREATIMEN'T' OF LUMPY-JAW,
}

or

ACTINOMYCOSIS, IN CATTLE.

BY

Dr. D. E SALMON, CIIEF OF THE BUREAU OF ANIMAL INDUSTRY.

PUBLISIIED BY AUTHORITY OF THE SECRETARY OF AGRICULTURE.

WASHINGTON:

GOVERNMENT PRINTING OFFICE.

1893. 



\section{TABLE OF CONTENTS.}

Report npon investigations relating to the treatment of lumpy-jaw in cattle..

Review of the work

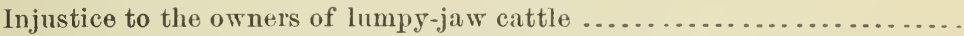

The jurisdiction of the Illinois Board of Live-Stock Commissioners over animals affected with lumpy-jaw............................ 24

Experiment to test the contagionsness of actinomycosis ............. 30

Does the iodide treatment drive the disease to the internal organs? ..... 31

The cost of treatment.......................................... 34

Successes obtained by individuals with the iodide treatment.......... 35

Report of Dr. V. A. Norgaard, inspector in charge.................... 39

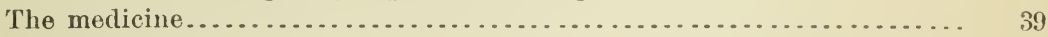

Effect of iodide of potassium on the different actinomycotic new forma-

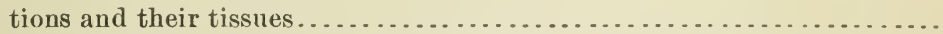

Individuals which show the most pronounced symptoms of iorlism will improve the fastest........................................ 43

Post-mortem examination ...................................... 46

Appendix................................................ 17 



\title{
LETTER OF TRANSMITTAL.
}

\author{
U. S. Departuent of Agricinture, \\ Bureau of Animat Indistry, \\ Washington, D. C., February 10, 1893.
}

SIR: I have the honor to transmit for publication the following report upon investigations relating to the treatment of lumpy-jaw, or actinomycosis, in cattle, together with a detailed report upon the experiments conducted at Chicago by Dr. V. A. Norgaard, to whom was assigned the personal supervision of the work.

Very respectfully,

Dr. D. E. SALMON, Chief' of Bureau of A nimal Industry.

Hon. J. M. Rusk,

Secretary of Agriculture. 

REPORT UPON INVESTIGATIONS RELA'TING TO THE TREATHEAT' OF LUNPY-JAW, OR AC'T'LNONYC'OSIS. IN CA'T'I'LE.

\section{RFVIEW OF 'IHE WORK.}

Since 1889 the disease of cattle known scientifically as actinomycosis, and popularly as "lumpy-jaw," has attracted much attention in the United States on account of sensational publications alleging its great prevalence and dangerously contagious character. The Illinois board of live-stock commissioners appears to have beeu the first to accept this view and the most active in promulgating it. Since 18s' that board has seized and slaughtered a large number of animals both at the Union Stock Yards at Chicago, and at the National Stock Yarks at East St. Louis. As there were included among the animals seized many in the best of condition and but slightly affected, and as these were turned over to parties for rendering purposes who allowed to the owners for a long time less than one-third of what the carcasses were worth even for that purpose, there was naturally much dissatisfaction created. It was claimed that, in addition to the umnecessary and unjust loss on affected animals which the owners were compelled to suffer, the inspection was not uniform; that many affected animals were not siezed; that those badly affected were in some cases passed by the inspectors as fit for food, while in other cases those but slightly affected and in good condition were sent tu the rendering tanks. More than this, the contagiousness of the disease among animals and the danger of its transmission to man, as well as the jurisdiction of the State board of livestock commissioners in assuming the duty of meat inspectors were seriously called in question.

The Bureau of Animal Industry was confronted with this condition of affairs when it began the inspection of meat at Chicago under the act of Congress approved March 3, 1891. The shippers of cattle at once made numerous complaints of the arbitrary, arrogant, and unjust manner in which the State board was acting, and, on the other hand, the board attempted to dictate to the Department as to what carcasses should be condemned by its inspectors and what passed as fit for fool. It became necessary, therefore, that this Bureau should make an investigation as to the facts and take such action in its own work as would secure justice to the cattle owners, and at the same time pro- 
tect the consumers of meat from any carcasises that were in the slightest degree dangerous to the public health.

The matter has been a difficult one to adjust because there existed, on the one hand, a board which has not hesitated to make the most sensational assertions, alarming to the consumers and damaging to the meat trade of the country, and on the other, a body of consumers exacting as to the quality of their food, sensitive to any aspersions upon it, and disposed to ask the destruction of meat though perfectly wholesome, if a breath of suspicion had been directed against it. This disposition of consumers, and the desirability of protecting the public health at any cost, no doubt accounts for the ability of the board to maintain its seizures for so long a tim e, though they were generally rerognized as unjust, and suspected to be without legal authority.

In the spring of the year 1892, the attention of the Department was called to a remedy which appeared to have extraordinary effects in the treatment of this disease. An experiment was made on a single steer, which was badly affected, by Dr. Norgaard, an inspector of the Bureau of Animal Industry, and this steer was completely cured.

The following announcement was then made for the information of those interested in this subject:

The interest which has been shown by the stockmen of the United States in regard to the disease kuown as "lumpy-jaw," or that form of actinomycosis which appears as external swellings on the head, renders it desirable that a preliminary statement should be made concerning the treatment of this disease. Until recently it has been the opinion of the veterinary profession that a cure could only be obtained by a sirrgical operation, and that this should be performed in the early stages of the disease in order to insure success.

In March last an important contribution to our knowledge of this subject was made by M. Nocard, of the Alfort Veterinary School, in a communication to the French Central Society of Veterinary Medicine. Ho showed clearly that the actinonycosis of the tongue, a disease which appears to be quite common in Germany and is there known as "wooden tongue," conld be quickly and permanently cured by the administration of iodide of potassium. M. Nocard calls attention to the success of M. Thomassen, of Utrecht, who recommended this treatment as long ago as 1885, and who has since treated more than eighty cases, all of which have been cnred. A French veterinarian, M. Godbille, has treated a number of cases with the sane remedy, all of which have been cured. M. Noeard also gives details of a case which was cured by hinself.

All of the cases reforred to were actinomy cosis of the tongne, and no one appears to have attempted the cure of actinomycosis of the jaw until this was undertaken hy Dr. Norgaard, veteriuary inspector of the Burean of Animal Indnstry. Heselecterl a young steer in April last, in fair condition, which had a tumor on the jaw measnring $15 \frac{3}{2}$ inches in circumference, and from which a discharge had already been established. This animal was treated with iodide of potassium, and the result was a complete cure, as stated in the reports which were recently given to the pross at the time-the animal was slanghtered in Chisago, If Inmpy-jaw can be eured so easily and cheaply as this experiment wonld load one to smppose, the treatment will prove of great value to the cattle-raisers of the comntry. As is well known, there is a considerable number of steers weekly coming to onr markets which are condemued hecanse they are diseased to such an extent that the general condition of the animal is affected. If these conld be cheaply and radily anred hy thr owners, it would 
prevent the loss of the carcass and solve all the tromblesome questions which have been raised in regard to the condemnation of such animals.

The curability of the disease does not affect the principles which have been adopter in insperting and condemning animals affected with it. This Department has never considered it necessary to condemn animals affected with actinomycosis on account of the contagiousness or the incurability of the disease. Such condemnations have bern made when the disease was so far advanced as to affect the general condition of the animal, and all such carcasses would be condemned whether the disease from which the animal suffered was contagious or not, or whether it was curable or incurable.

The treatment with iodide of potassium consists in giving full doses of this medicine once or twice a day until improvement is noticed, when the dose may be reduced or given less frequently. The size of the dose should depend somewhat upon the weight of the animal. M. 'Thomassen gives $1 \frac{1}{2}$ drams of iodide of potassium daily in one dose dissolved in a pint of water until improvement is noticed, which, he states, is always within eight days. Then he decreases the dose to 1 dram. The animals do well under this treatment, showing only the ordinary symptoms which follow the use of iodine, the principal ones being discharge from the nose; weeping of the eyes, and peeling off of the outer laycr of the skin. These symptoms need cause no uneasiness, as they never result in any serious disturbance of the health.

M. Godbille has given as much as 4 drams (half an ounce) in one day to a steer, decreasing the dose half a dram each day until the dose was $1 \frac{1}{4}$ drams, which was maintained until the twelfth day of treatment, when the steer appeared entirely cirred.

M. Nocard gave, the first day, $1 \frac{1}{2}$ drams in one dose to a cow; the second and succeeding days a dose of 1 dram in the morning and evening, in each case before feeding. This treatment was continued for ten days, when the animal was cured.

Dr. Norgaard gave $2 \frac{1}{2}$ drams, dissolved in water, once a day for three days. He then omitted the medicine for a day or two, and continued it according tosymptoms. These examples of the treatment as it has been successfilly administered by others will serve as a sufficient indication for those who wish to test it.

Experiments are now being conducted on a large scale by the Bureau of Animal industry in the treatment of lumpy-jaw with this remedy, and the results will be published as soon as possible. In the meantime it would be well for all who have animals affected with this disease to treat them according to this method, and report results to the U.S. Department of Agriculture, Washington, D. C.

Arrangements were made at as early a clate as possible to test the remedy on a large scale with the affecterl animals as they came to the Union Stock Yards at Chicago. As the State board was still exercising its assumed power to seize, quarantine, and slaughter affected animals, they were requested to release from quarantine all such animals arriving during the time necessary to collect the number which was desired for the experiment, and allow them to be taken withont interference to the stables where they were to be fed and treated. This they agreed to do.

No sooner, however, was an attempt marle to collect affecterl animals for the experiment than it was reported by the inspector of the department at Chicago that the State veterinarian was passing all these animals as fit for food except those in the very worst stages of the disease. This change in the policy of the State board made it difficult to obtaii the necessary cattle for treatment, but some were collected, and these the board put in quarantine, so that they could not be taken out of the 
stock yards. This action made it necessary for the Secretary of Agriculture to call upon the governor of Illinois for his interference, which was done in the following telegram:

Gov. J. W. FrFer,

Viroqua, Wis., August 23.

Springfield, Ill. :

Have received following telegram from Dr. Melvin, in charge of meat inspection at Chicago: "Stock Yards Company refuse to allow cattle to go ont of yards on orders issued by you or myself. Baldridge will have carload by Wednesday. They will only recognize orders of the State." Will you please instruct your live stock buard to allow us to ship out all cattle for experiments in lumpy-jaw I am anxious to have cattle in all stages of disease for experiment by Department's remedy. I am now two weeks behind in this work on acconnt of not being able to procure cattle, and if I am to be subjected to further delay I will have to go to Kansas City to make experiments. Answer here.

J. M. Rusk, Secretary of Agriculture.

To this request Governor Fifer replied in two telegrams, as follows:

SpringField, Ill., August 23.

Hon.J. M. Rusk, Secretary of Agriculture,

Viroqua, Wis.:

I have directed the Illinois live-stock commissioners to allow your agents to ship cattle diseased with lumpy-jaw for experiments by your Department, as requested by you.

JOSEPH W. FIFER.

Hon. J. M. Rusk, Secretary of Agriculture,

Springfield, Ill., August $\$ 9$. Tiroqua, Wis.:

I am advised that the United States officials have full charge of the cattle in question, for the purpose of experiment, and are only held responsible for their saftkeeping. The ofticials of this State are seeking to exercise no control over them beyoud this. Is not this satisfactory?

Joserit W, Funer.

After the governor's assistance had been secured, the animals purchased were allowed to leave the yards for treatment, and a better class was obtained, but the influence of the State veterinarian was still exerted to prevent the Department fiom collecting the milder cases. Although animals so affected had been freely condemned before, they were now said to be either free fiom the disease or perfectly fit for fond. Under such circumstances the owners naturally preferred to sell their affected animals on the market inchuded in the lots with which they had been shipped.

Notwithstanding the obstacles put in the way of the experinent, 1s() animals, in various stages of the disease, were fiually collecterl and put, under treatment. No sooner had the first lots arrived at the stables than they were placed in quarantine by order of the Illimois bomal of live-stock commissioners. This hostile action was also laid betore tho 
governor by the Secretary of Agrieulture, and on September 14 the quarantine was withdrawn by order of the governor. The following is the correspoudence in the case:

\section{U. S. Department of Agriculture, OfFicli of the ShCRetary, Washington, D. C., September 6, 189 .}

SrR: Allow me to thank you for your assistance in securing for me animals affected with actinomycosis for the purpose of experimenting with the same with a view to enring this disease.

As I telegraphed to you, your live-stock commissioners, immediately after I obtained these animals, placed upon them an order of quarantine, and I now write replying to your telegram asking whether said quarantine would in any way interfere with my experiments in this direction. I desire to call your attention in this connection to one or two facts. One of the purposes for which the Bureau of Animal Industry of the Department of Agriculture was organized was to investigate the diseases of domestic animals and to endeavor to ascertain the causes of the same and "means for the prevention and the cure" of animal diseases. At the same time, the Bureau was, by its organic act, empowered to extirpate all contagious and communicable diseases among the domestic animals of the United States and to enforce all necessary measures to prevent their spread. These being some of the purposes for which the Bureau was organized, it would seem that when it undertakes to carry on an experiment with a view to discovering a cure for some certain disease, that all necessary measures to prevent any danger of the spread of such disease from animals being experimented on would be provided by the officers of the Bureau. For a State board, therefore, to place in quarantine animals being experimented upon by the Department of Agriculture is not only unnecessary, but has the appearance of being discourteons. It is a reflection upon the honesty and ability of the officers of the Department. It is not to-day believed by any competent person that actinomycosis, the disease with which the animals that are now being treated at Chicago are affected, is a contagious disease, or that there is any danger of its being communicated from one herd of cattle to another or to people. There is consequently no danger to be apprehended from the animals now in the possession of the Department at Chicago undergoing treatment. The great public benefit sought to be obtained by the experiment is snch that it should not be interfered with or impeded by hostile acts of State officers. It would seem as if State authorities should coöperate in every way possible towards the successful accomplishment of such a purpose, and not, from jealonsy or other cause, seek to prevent its success. The quarantine placed upon these animals, which the National Government has bought and paid for and which are now its property, is to me exceedingly irksome, and I consider it almost a personal insult; not only that, but, in my judgment, it is an illegal quarantine, so far as it applies to the work which is being carried on by the Department in the State of Illinois. 'The Department of Agricultnre is operating in Illinois under the provisions of the act of Congress of May 29, 1881, and of the act of the State of Illinois of June 28, 1887, and under these acts it is beyond the control w interference of State officials.

I wonld further say that in conversation with Mr. C. P. Johnson, secretary of the board of live-stock commissioners of the State of Illinois, I was informed by him, in a very gentlemanly manner, that the reason of this quarantine was that there "was some liability on the part of the State in case any disease should be spread from these animals. I have to assure y.ou that there is no such liability, and whatever liability there might possibly be is assumed by the National Goverument, and that the law of Illinois above referred to expressly provides in section 3 that the state shall not be liable for damages or expenses of any kind under the provisions of the 
same, and that this Department will hold itself responsible for any damages that may be inflicted by reason of the experiment which is now being carried on.

This experiment is heing watched with a great deal of interest, not only by the stock-owners of the State of Illinois, but by the stock-owners of the entire United States, for, in case it proves successful, it will be of great benefit to all owners of cattle. I feel assured that I will have your best efforts to prevent any impediments being placed in my way in carrying the experiment to a snccessful end, and I would therefore request that you will kindly have the quarantine order made by the live stock commissioners of your State at once rescinded.

Trusting that this request will meet with your approval, I an, Very respectfinly,

His Exellency Joseph W. Frere,

J. M. Rusk,

Secretary.

Gocemor of the State of Illinois.

Hon. J. M. RUsK,

Springateld, Ili.., September 14.

Secretary dgricultural Department, Washington, D. C.:

The live-stock commissioners of this State will remove quarantine from the diseased cattle your Department is experimenting on in Chicago, as requested in your letter to me.

JOSEPII W. FIFER.

On November 29, 1892, eighty head of the cattle which had been under treatment were shipped to Chicago and slanghtered. There were two lots of forty each. The first lot was supposed to be nearly or quite all cured, while the second lot was made up of the worst cases, many still having large swellings upon their heads, which were believed to be incurable. By direction of the Secretary of Agriculture, the following notice was mailed to the governor:

\section{U. S. DEPAR'TMENT OF AgRiculture, BUREaU OF ANmal INDUSTRY, Chicago, IIl., November $26,1899$.}

DEAR SIR: I am directed by IOn. J. M. Rusk, Secretary of Agriculture, to inform you that a portion of the eattle which have been under treatment for actinomycosis or "hmmpy-jaw," will he slaughtered at the slaughterhouse of Jacob Hess, Inion Stock Yards, Chicago, on Tueselay, the 29th instant. The Secretary will be pleased to extend every fucility for examination to any iuspectors or other's whom you may desire to have present on that oceasion.

Very respectfinlly,

1). K. S.MIMUN, Chirg of liurean.

Hon. JosEPII W. WHER, Gorernor of Illimois,

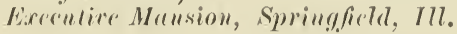

There were present on the part of tha state Mr. H. MrChesnoy, of the Illinois board of live-stock commissioners; Mr. Johnson, the sereretary of the hoard; Dr. Casewell, State veterinarian, and Drs. A. H. Baker and Joseph Hughes, assistant State veterinarians. 'There wero 
also present on the part of the eity, Mr. Pieree and an assistant. The inspectors representing the Bureau of $A$ nimal Industry who were present were Dr. A. D. Melvin, inspector in charge at Chieago, and I)rs. Lew is li. Baker and Victor $A$. Norgaard, all being under the direction of the Chief of the Bureau of Animal Industry. Dr. Melvin was instructed to make the inspection carefully, and condemn all carcasses about which there could be any doubt as to their fitness for human food.

When the slaughter began, the State veterinarian assumed to be in 'harge of the inspection; he put his assistants on the floor, and with them examined the various organs of the animals, cutting them open, and in some cases removing them before they were seen by the inspectors of the Bureat, and even, in some instances, directing the removal of such organs by the workmen of the slaughterhonse before they had been seen by I)r. Melvin, and withont any consultation with the Chice of the Bureau, who was constantly present. Several times it was necessary to go to Mr. Hess, at whose slaughterhouse the animals were killed, and ask him to instruct his men not to remove the organs of any animals from the killing floor or from places where they could be identified, until Dr. Melvin had given his consent to such removal. A number of students of the Chicago Veterinary College, at which institution the two assistant State veterinarians mentioned are professors, had been brought there to witness the slaughter without the knowledge or consent of the Chief of the Bureau. A number of newspaper correspondents were also present, and as they did not get their information of the killing from anyone connected with the burean, it is assumed that they were informed by some one conmected with the State board. The result of so many persons being present who were unexpected was that the floor was crowded; the inspectors were obliged to work under difficulties, and it was only by exercising constant vigilance that the organs of the animals were examined before they were removed from the position where they could be identified with the carcass from which they came.

As long as these animals belonged to the Department of Agriculture, and were being slanghtered under its direction, it certainly was no more than courtesy demanded that those in charge should have been consulted before information was given to so many persons not officially connected with the State, in order that it might be determined whether or not their presence would interfere with the proper performance of the work in hand. In addition to this, those present on the part of the State did not hesitate to speak disparagingly of the experiment, freely stating that they were condemning as large a proportion of these carcasses as were condemned from the "lumpy-jaw" cattle which come to the stockyards and are slaughtered without treatment.

At the close of the day's inspection the State veterinarian, without consulting with the Chief of the Bureau or with Dr. Melvin, or with- 
out informing the representatives of the Burean what he had done, gave a certificate to the inspector present on the part of the city to the effect that eighteen carcasses which had been passed by the inspectors of the Bureau of Animal Industry, and tagged with the ineatinspection tag of this Department, were affected with actinomycosis. As a result of this the Chief of the Bureau was notified by the city inspector that these carcasses could not be sold for consumption in the city of Chicago.

It should be stated that, during the whole day, in spite of the offensive action above mentioned on the part of those representing the State, these gentlemen were treated with every courtesy and given every facility for making their examinations, and no disagreeable word was spoken in their presence by any one representing this Department.

The inspectors of the Bureau of Animal Industry condemned every carcass in which a lesion was found which was of sufficient importance to justify the least suspicion that the carcass would be affected by it. None of the eighteen carcasses passed by the United States inspectors and condemned on the certificate of the State veterinarian were affected in such a way as to cause any suspicion in the minds of any of the representatives of this Department as to the perfect wholesomeness of the carcass. The lesions in all were very small, in one case not being larger than a grain of wheat, and in every case being insignificant. In a number of the cases these lesions, when examined microscopically, were found to be free from the actinomyces fungus, and, consequently, in these cases, there was an error of diagnosis on the part of the State veterinarian. I have been informed by Dr. Melvin that cases with much larger lesions than existed in any of the eighteen cases mentioned have frequently been passed by the State veterinarian in his inspections at the stock yards and allowed to go on the market as fit for food. If the statement is correct, which was freely made, that no larger proportion of these carcasses were passed than were ordinarily passed anong the lumpy-jaw animals which come to the stock yards, that of itself is sufficient evidence that the carcasses about which this unfortunate difference of opinion exists should have passed, because no animal without greater lesions than many of these had would have been held for examination.

From the statements made above it will be seen that the inspection made by the inspectors of this Department was entirely ignored, and that the State representatives, who were present by the courtesy of the Department and who have no responsibility as meat inspectors, assumed anthority to certify as diseased the carcasses of animals belonging to the Department which the Govermment inspector's declared to be entirely fit for food. Considering all these cireumstances, and particularly that Governnent property was at stake which these gentlemen had shown their determination to ause to be lestroyed by misiepresentation, I decided that it was no longer my duty, either on the 
grounds of courtesy or by any previous arrangement which had been made, to invite their presence when other animals belonging to the Goverument were to be slaughtered.

It may not be out of place to mention in this connection the fact, already referred to, that at the time these animals were being purchased the representatives of the State board apparently did everything in their power to prevent the collection of a sufficient number of animals for this experiment, and that in order to accomplish this, affected ani mals, such as had previously been condemned, were freely passed as fit for human food.

I am free to state that the disease with which these animals were affected when they were purchased for experiment-that is to say, actinomycosis or "lumpy-jaw"-can not properly be considered as a contagious disease, requiring any such action to prevent its spread as is being taken by the Illinois board of live stock commissioners. If the disease is contagious at all, which is doubtful, it certainly is not contagious to the degree which requires measures to prevent its dissemination by animals in transit for slanghter. There is no satisfactory evidence that it can be disseminated by animals mingling with each other in cars or yards, and it is even doubted by recognized authorities if it can be transmitted by inoculation, on account of the many failures which have been recorded.

In the International Congress of Hygiene and Demography, held at London a year ago, there was unanimity of opinion to the effect that there was no danger of this disease being transmitted to persons who used the flesh of animals so affected for food, and, so far as I remember, I was the only member of that congress who sanctioned the condemnation of carcasses of animals affected with this disease, except when it was disseminated throughout the body, and my position was that this was only justified when the malady had made such progress as to have affected the general health of the animal, as shown by loss of flesh or by large suppurating abscesses. In no other country than the United States, so far as our information extends, are carcasses condemued as unfit for food when locally affected with the actinomyces fungus, provided the animal was in good health and in good condition of flesh when slaughtered. The action of the Illinois State live stock commission in deciding this to be a dangerously contagious disease and in forcing affected animals with even slight lesions to be destroyed as unfit for food is, in my opinion, unjustified as a measure to prevent the spread of disease, and is a great wrong to the cattle producers of this country. There is no doubt that many thousands of dollars' worth of property have been unjustly taken from the cattle owners by such action. This would be the case, even if animals slightly affected were passed, as it is understood they have been in many cases. If, on the other hand, animals are to be coudemned for the same reasons for which the eighteen carcasses above mentioned belonging to the Govern- 
ment were condemued, then a much greater number of animals would necessarily be sacrificed. If a great department of the National Govermment can not secure justice in the inspection of animals belonging to it, when it las the muloubter anthority to make such inspection with its own inspectors, then the individual shipper certainly has a very small chance to secure justice in the handling of his prorluct.

In conclusion, I would ask attention to the fact that the Union Stock Yards of Chicago is one of the clanmels of interstate commerce; that the cattle received there do not come from the State of Illinois alone, but from nearly every State of the Union; that this is not their final restination, but that many of the animals and most of the products are shipped from there to other States. From these reasons it would appear that the United States Department of Agriculture should have jurisdiction in such yards, both to prevent the spread of eontaginus diseases and to insme a just and miform inspection of animals and meats.

The experience in the slaughter of the animals belonging to the Department, and the action taken by the State veterinarian, evidently under the direction of the board of live stock commissioners, convinces me that there is much truth in the charges of arbitrary and inconsistent condemnation, and that something should be done by the United States Government to secure justice to the many cattle owners in various parts of the country, who are under the necessity of shipping their cattle to the Union Stock Yards of Chicago for market.

Of the eighty eattle slaughtered on November 29, forty-three were pronounced practically cured and fit for food by the inspectors of the Bureau of Animal Industry, and twenty-five were pronounced to be completely cured and fit for food by the State veterinarian. Considering that this number included forty of the very worst cases taken for experiment, the result ean not but be considered as extremely satis. factory. If the conclusions of the Bureau inspectors are taken, the result is a cure of 53 per cent of the affected animals; while should we accept the extraordinarily biased report of the State veterinarian, we still find that over 31 per cent of the animals were completely cured.

The following report given to the press by the State board explains itself:

NO UURES (IF LIMIY-JA W EFFECTED.

REPORT OF STATE VETERINARIANS ON FXPEIRMENTS MAIE IN CHICAG().

SPRINGFIEL, ILL., l)ecember 7 (special).

The State board of live-stock commissionors to-diug gave out for publication tho following:

To the Slate Board of Live-Stock Commissiomers, Springfield, III.:

Gentemwe: Tuestay, the 29th of November, at the request of Mr. Me('hesmey, of your board, wo, the undersigued, assisted in making post.mortom eximimations of 80 head of attle that wero slaughterod in Hess Brothers' slanghferlouso in this 
city. We beg to report on the same as follows: Fifty-five of these cattle were found to be affected with actinomycosis (lumpy-jaw) and 25 were healthy.

Respectfully submitted.

\author{
John Casewell, M. R. C. V. S., \\ State Veterinarian. \\ Joserh Hughes, M. R. C. V. S. \\ A. H. BAKER, D. V. S., \\ Assistant State Veterinarian.
}

Forty of the cattle found diseased were, after fully two months' treatment, pronounced by the Burean officials incurable; the other 40 were composed of the class of cases that the State veterinarian does not pronounce diseased on ante-mortem inspection, but holds for post-mortem inspection before deciding them affected with actinomycosis. After being treated for over two months, 15 of these 40 questionable cases were found, upon post-mortem examination, to be affected with actinomycosis. For months the percentage of this class of cattle pronounced free from disease, upon post-mortem examination each week by the State veterinarian, exceeds that of these 40 experiment cattle, so that so far as the experiment cattle already killed are concerned there is no evidence that the treatment used has effected any cures.

The statement that 40 of the cattle belonged to the "class of cases that the State veterinarian does not pronounce diseased on ante-mortem inspection, but holds for post-mortem inspection before deciding them affected with actinomycosis" is misleading, as there was not an animal among them that was not plainly affected with that disease when it was taken for treatment.

The other statement that "for months the percentage of this class of cattle pronounced free from disease upon post-mortem examination each week by the State veterinarian exceeds that of the 40 experiment cattle" is absolutely incorrect, unless it refers to the months during which the affected animals were being collected for this experiment. During these months it is freely admitted that the State veterinarian passed a large proportion of such cases as fit for food, even when they were badly diseased. This fact, almitted in the above press dispatch given out by the board, shows the insincerity and lack of principle governing their inspection. When they inspected the carcasses belonging to the Government they condemned every carcass which showed a tumor the size of a grain of wheat or an abscess the size of a hazelnut. When they inspected in the yards they passed cattle as sound which had tumors and abcesses larger than a man's clenched hand. In the latter case they were trying to prevent the Department from obtaining cattle for experiment, while in the former case they were trying to disparage the results of the treatment. Admitting, then, this statement of the board that "for months the percentage of this class of eattle pronounced free from disease upon post-mortem examination each week by the State veterinarian exceeds that of these 40 experiment cattle," it can be shown from their own reports that this has not always been the case. Of the 40 experinent cattle referred to, 25 were pronounced free from the discase by the State veterinarian; that is to say, 62.5 per cent.

$14411-\mathrm{No}_{2} 2-2$ 
Referring to the reports of the board of live stock eommissioners for the State of Illinois for the years ending October 31, 1889, 1890, and 18:1, we find the figmes given for the number isolated and the number condemued as follows:

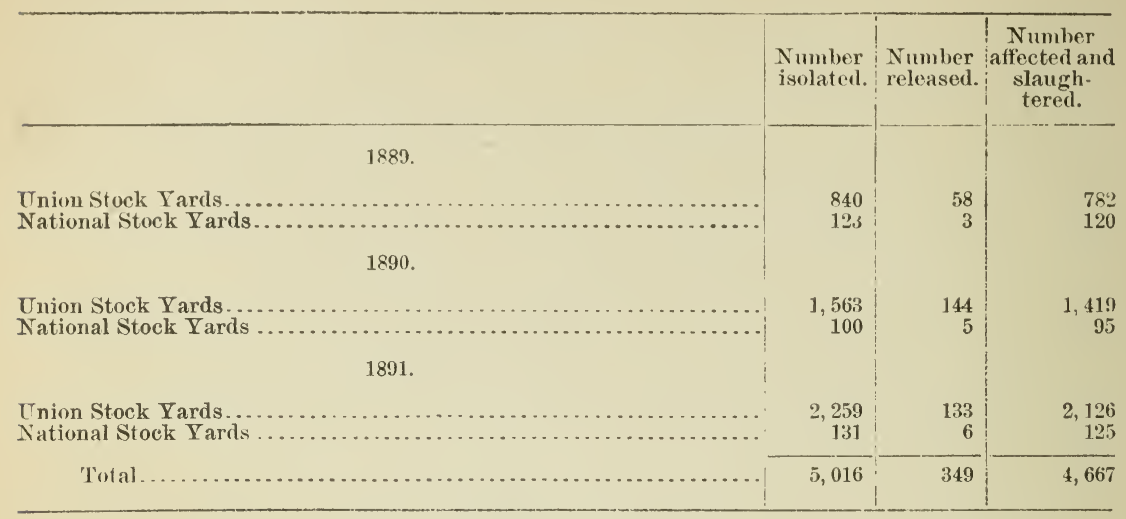

This table shows that for three years there was less than 7 per cent of the isolated animals which were pronouncer free from the disease. To explain even this number, considered by the board to be large, the following statement is made in their report:

The large number of animals released, as shown by the first table, is largely composed of those that were returned to the yards by the purchasers after they had reached the slanghterhouses, because they had a small lump, due to injuries, or other causes than actinomycosis, fearing that this wonld be made an excuse by the city health inspectors in the slaughter-house to eut down the carcasses, in which ease the purchaser would have to bear the loss. All such cases turned back were inspected by our veterinarian, and we are advised that not a single one of these 'ases proved to be actinomycosis. (Anmual Report of the Board of Live Stock Comnissioners for the State of Illinois, 1890, p. 52.)

If during the years 1889,1890 , and 1891 the State veterinarian was so expert in determining actinomycosis m the living animal that less than 7 per cent of those isolated were found free from disease, and even these were mostly animals with small lumps due to injuries, turned back by purchasers and not by the veterinarian, how can we explain the sudden loss of this expertuess in 1892 and the isolation of over 60 per cent of cattle which are afterwards pronounced free from the disease? This question is a pressing one at this time, for the statements of the board are inexplicable and inconsistent, and it is plain either that these statements are incorrect or that there has been an extraordinary change in the principles governing the inspection.

The animals taken for the experiment of the Department did not include any turned back by the purchasers on accomt of injuries, but every one was pronounced by the veterinary inspectors to be affected with actinomycosis. How, under such circmmstances, the board can state that they have for months pronounced over 62 per reut of such cases to be free fiom the disease is a mystery and shows conclusively that 
their action either in 1889, 1890 , and 1891 , or in 1892 w was unskillful and lacking in uniformity or that they purposely modified their principles of inspection in order to disparage the results of this experiment. Whatever may be the final conclusion as to the cause of their action, the open hostility of the board has been apparent from the time the first animals were selected, and it has continued with increasing intensity as the success of the treatment became more and more apparent.

As the officionsness of the State inspectors made a proper inspection by the Bureau inspectors a difficult matter, and as the former assumed jurisdiction as meat inspectors which is not given them by law, and as finally they had shown a determination to cause the destruction of carcusses of eattle belonging to the Government, which carcasses were not diseased, I decided not to invite them to be present when the next lot of cattle was slaughtered.

On December 2 a lot of twenty head, all of which had been cured, were slaughtered at the same place, in presence of the Bureau inspectors above named and also of the inspector of the city department of health. Every one of these animals was free from disease, as was slown by the most careful post-mortem examination.

The examination of the one hundred head of cattle first slaughtered shows, according to the reports of the Bureau inspectors, who were unbiased and had no interest in the experiment beyond a desire to get accurate results, that sixty-three of these animals had been cured by the treatment. This is a cure of nearly two-thirds of the animals treated, and certainly can not be surpassed by the results of treatment with any of the internal diseases of a progressive nature which affect animal life. This is the more gratifying as some of the animals which were pronounced healthy by the State veterinarian had been affecter with enormous tumors, which entirely disappeared during the course of the treatment.

The following correspondence in regard to the slaughter of twenty animals without notifying the State board explains itself:

State of Illinois, Executive Office, Springfield, December 3, 1892.

DeAR SIR: Complaint is marle to me by the live-stock commissioners of Illinois that on yesterday the second consignment of cattle delivered to your agent for experiment were slanghtered in Chicago without notice to the live stock commissioners. of this State. I am also informed that there was an agreement previously entered into, when these cattle were taken for experiment purposes, that the live stock commissioners would be notified when said cattle were slaughtered, and afforded an opportunity to make a post-mortem inspection.

Permit me to call your attention to this complaint, believing that you will direct youragents in Chicago to earry out the agreement above named, and that, before slaughtering the remaining cattle under experiment, the commissioners will be duly notified and given an opportunity to make an inspection.

Yours, very truly,

Joseph W. Fifer.

Hon. J. M. Rusk,

Secretary of Agriculture, Washington, D.C. 


\section{U. S. Department of Agriculture, OFFICH OF THE SECRETARY, Washington, D. C., December 6, 1892.}

DEAR SIR: I am in receipt of your favor of the 3 instant, in which you state that complaint is made to you by the live-stock commissioners of Illinois that on December 2 the second consignment of eattle from the animals under experiment by this Department was slaughtered in Chicago without notice to such commissioners.

In reply I wonld say that this is correct and that, in order to give you a full state ment of the reasons for such action, I inclose with this, for your information, a copy of the report made to me by Dr. D. E. Salmon, chief of the Bureau of Animal Industry, under whose direction the slaughter was made.

It appears from this report that the State veterinarian attempted to take charge of the inspection of the animals, that the inspection made by the inspectors of this Department was ignored, and that a certificate was given to the inspectors of the city department of health that eighteen carcasses were unfit for food which had been passed by the United States inspectors.

The invitation was extended to you by my direction, according to agrcement, as an act of courtesy, to enable your representatives to witness the results of the experiment as revealed by the post-mortem examination, and every facility was given them for this purpose. Being there by invitation, their attempt to assume control and to enforce their unjust decision as to the disposition of the meat was an interference with the jurisdiction of this Department which conld not be tolerater.

The Department inspectors were acting under the acts of Congress authorizing the inspection of meats, as well as the investigation and prevention of disease, and the test which they were conducting was to discover a cure in the interest of the cattleraisers of the whole country. I am, therefore surprised to learn that the live-stock rommissioners of Illinois and their assistauts have gone, not only to the full extent of their authority, but beyond such anthority, to obstruct and delay this experiment and to disparage its results.

I regret that the action of the commissioners, or those acting for them on the day mentioned, was such as to lead Dr. Salmon to the couchision that he could mot conscientiously invite them to be present at the slaughter on Friday, the $2 d$ instant.

I think you will agree with mo that if his statement of facts is correct, and I have no donbt it is as he understood them, that he could not properly have done otherwise.

The stand which is taken by the live-stock commissioners of Illinois in regard to the condemnation of these eareasses is a matter of great consecynence to the stork owners of the United States, and it also involves property belongiug to the Inited States fovernment. The inspection of animals for food should be miform and just, and the meat inspection of this Departinent, which is anthorized by act of Congress, and which is carried on by competent men, should be reeognized. As the responsible officer in charge, I can not permit it to be ignored or ealled in question by State officials who are not even given jurisdiction as meat inspectors by the laws of the State.

Very respectinlly,

J. M. Risk, Secrelary.

Hon. JOSEPUI W. FIFER,

Governor of Illinois, Exccutive Mansion, Springfeld, 111.

Soon after this the chairman of the Illinois board of live-stock commissioners addressed a comanuication to the Secretary of Agriculture, 
which was of so semrilous and discourteous a chatacter that it was returned to Gov. Fifer with the following letter:

\section{U. S. Department of Agriculture, OHHCE OF THE SECRETARY, Washiugton, I). C., December 19, 18??.}

SIR: I inclose herewith a communication addressed to me by the chairman of the lllineis State board of live-stock conmissioners, which not only refers to a number of matters with which he has nothing to do officially, but which is so discourteous in its tone that I must decline to receive it. If the board mentioned has any thing to bring to the attention of this Department which you deem proper to transmit, I shall be glad to give it carefnl consideration, but I can not have any direct communication with a man in that position whose ideas of ofticial courtesy are exemplified by such a letter as the one I return.

'The letter in question is incontestable evideuce of Mr. McChesney's unfitness for the office which he holds.

Very respectfully,

Нов. JOSEPH W. FIEER,

J. M. Risk, Seretary.

Governor of Illinois, Lixecutive Mansion, Springfirld, Ill.

To this the goveruor replied as follows:

State of Iulinots, Executive Oriati, springfield, Jamuary 3. 1893.

MÝ DEAR SIR: I am in receipt of your favor of December 19, and contents noterl. When I received your letter of December 6 I immediately called upon the hourd of live-stock commissioners of this State for a full and complete statement concerning their action in the matter of diseased cattle turned over to your Department for experimenting in the city of Chicago, and I inclose you a copy of their report to me on that subject, dated December 16.

I regret exceedingly the conflict that has arisen. I had no knowledge whatever, directly or indirectly, that Mr. McChesney would write yon as he did under date of December 16, and I regret exceedingly that he did so. My administration closes now in a few days. The general assembly of this State convened to-day. I an very busy with official business that must be closed out before I give up the office to my sucessor. Were it not that my term of office expires in a few days I would give this controversy between the live-stock commissioners and your representatives further consideration.

Assuring you of my high regard and esteem, I remain, yours very respectfully, JOSEIII W. FIFER.

Hon. J. M. Rusk, Secretary of Agriculture, Washington, D.C.

The Secretary of Agriculture replied to the above letter and asked for the removal of the quarantine which the board had again imposed upon the cattle. His letter is given below:

\section{U. S. Department of Agriculture, OFFice of the SeCretary, Washington, D. C., January $\%, 1893$.}

My Dear Sir: I am in receipt of your very satisfactory letter of the 3 d instant, for which I wish to express my thanks. The report of the board of live-stock commissioners, which you state was inclosed, has not reached me, and for that reason I do not know what position they have taken in regard to the cattle belonging to this Department. 
The ehairman of the hoad, after writing his very discourteons lefter to me, had the quarantine again put upon the cattle. I feel sure that yon were not informed of this, or you wonld have taken action to cause its revocation. Un to the present it has caused me no embarrissment, because I was not ready to slanghter the cattle, but I wish no:v to have them slanghtered at once. I would request you, therefore, to have this quarantine removed, so that the cattle can be shipped to Chicago for slanghter the first of next week.

I an sure that you have been desirous of giving every facllity for making this experiment, which is of so much interest and value to the farmers of your State and of the whole country, and that you are willing to do everything in your power to prevent delay in completing it, and giving the results to the cattle owners, to whoin it is of vital importance.

There was no reason whatever for the board of live-stock commissioners to put a quarantine upon these cattle, because they knew very well that I would ship them to Chirago and have them slaughtered. Cattle affected with actinomycosis are daily allowed to be shipped to the Union Stock Yards for slaughter, and, therefore, in case any of these are still affected, this Department is asking nothing beyond what is allowed without restriction to every cattle owner in the country.

I hole that the board of live-stock commissioners will no longer be allowed in milintain their position for the purpose of obstructing an investigation marte as munc. $\mathrm{I}_{1}$ in the interest of the people of your State as for the people of any other section of the comitry.

Assuring yon of my appreciation of your conrtesy in this matier, I an, very respectiully,

J. M. Risk, Secretary.

Hon. JOSEPH WV. FIFER, (iocernor of Illinois, Springfield. Il1.

\section{IN.ITSTICE TO OWNERS OF LITMPY-JAW CAT'TLE.}

Although the Illinois board of live-stock commissioners began quarantining and condemning cattle affected with actinomycosis in 1888 , it was not until their meeting of February 21, 1890, that they discovered that the owners of such cattle were only receiving from $\$ 2.50$ to $\$ 4$ per head for then. 'This was less than the value of their hides. Fven at that late late the board did not make the discovery itself, but the lacts were presented to it by a committee of the Live-Stock Exchange.

It is usually admitted that when the property of citizens is taken and restroyed for the good of the community, the citizen who suffers the joss should receive a just compensation, and the law of Inlinois under which the board has acted provides for the appraisement and compensation for cattle lestroyed to prevent the spread of disease. Althonlin the cattle affected with actinomycosis were ostensibly condemmed to prevent the spreal of that disease, it appears that no appraisement or compensation has been made as provider in the law. On the contrary, the destruction of these animals was enforeed without any compensation from the State, and with no effort on the part of the board to sece that the owners received even a just compensation fiom the parties who took possession of the carcasses. The owners were powerless. The cattle were seized and destroyed by order of the state board, and. 
the arreasses were turned over to the Union Rendering Company, which gave for them what it saw fit, and no more. The result was the owners were robbed of two-thirds or three-fourths of the value of their cattle even for rendering purposes.

According to the inspection rules of every country which carries on a meat inspection, and also according to the teachings of the most eminent scientists who have investigated the question, actinomycosis is generally considered a local affection, and in its early stages does not affect the general health of the animal or the wholesomeness of the meat. There is no good reason, consequently, for condemning animals in good condition which present only a small swelling on the jaw cansed by the actinomyces fungus, and from which there is no discharge. In this case the part of the animal involved is not used for food, and when that is removed it is the general opinion of scientists that the remainder of the carcass is not affecter. To condemn and destroy such carcasses as this is, therefore, unjustifiable from any point of view. It is especially uncalled for as an act to prevent the spread of the disease, as the most extreme believers in the contagion theory can not explain how the disease could be transmitted from an animal which had no discharge from the tumor by which the fungus could be disseminated.

It has not been uncommon to see steers in fine condition, weighing from 1,600 to 1,800 pounds, and worth 5 cents a pound, condemned because of a small swelling on the jaw which was scarcely more than visible. Such a steer would bring on the market for beef $\$ 80$ or $\$ 90$, but when condemned and delivered to the Union Rendering Company, the owner received, according to the board's own statement, not to exceed $\$ 4$. And this wholesale appropriation of the farmer's property was allowed to proceed for nearly two years before the members of the board became officially aware of what an outrage they were assisting to perpetrate.

Since that time a more satisfactory arrangement has been made, through the exertions of the Live-Stock Exchange. Under the new plan the carcasses are turnerl over to a rendering establishment in the stock yards, and the owner receives 1 cent a pound, less $\$ 3$ a head. That is to say, the owner of a steer weighing 1,600 pounds would now receive $\$ 13$ for the careass. Even this is less than its real value for rendering purposes. In disposing of the cattle purchased by the Department for the experiment, no difficulty was found in getting offers of 1 cent a pound without any discount. An offer of this kind was received from the very firm which obtains the other cattle condemned at $\$ 3$ per head less. The conclusion is unavoidable, therefore, that the shippers of these cattle do not receive as much by $\$ 3$ a head as they are entitled to and as the carcasses are really worth.

If we should admit, which we do not, that the condemnation of the board is in all cases legal and justifiable, there would still remain this great wrong of forcing the owners of these cattle to take $\$ 3$ per head 
less than they are actually worth when sold with an opportunity for free competition in the purchase. One would suppose that a board assuming the authority and the responsiblity of making such seizures, would feel it incumbent upon itself to see that the owners were fairly treated in the disposal of their property, but apparently this is not the case.

THE JURISDICTION OF THE ILLINOIS BOARD OF LIVE-STOCK COMMISSIONERS OVER ANIMALS AFFECTED WITH LUMPY-JAW.

The Illinois board of live-stock commissioners is given no authority or duties by law in the inspection of meat. It was ereated to suppress and prevent the spread of contagious diseases among animals in the State of Illinois. Its powers to quarantine and cause the slaughter of animals is expressly limited by the statute under which it acts to animals affected with "dangerously contagious or dangerously infectious disease." Lumpy-jaw is, in the opinion of the State veterinarian, a dangerously contagious disease, and consequently the board assumes that it is fully empowered to quarantine and cause the destruction of animals so affected.

As many of the carcasses destroyed were plainly unfit for human food, being emaciated and in some cases affected with a more or less generalized form of the disease, there were many who were inclined to sustain the action of the board on this account, even if it should be shown that the disease was not "dangerously contagious" and consequently not within the intent of the law. However, the board did not confine itself to such carcasses, but seized animals in good condition, with only small lumps which were not discharging, and in general their actions were so arbitrary, dictatorial, and burdensome, that the cattle-owners of a large section of the country tributary to Chicago have been loud in their protests and vigorous in their demands for relief.

This situation raises the question as to whether the board has, under the Illinois law, any jurisdiction over animals affected with this disease-in other words, whether actinomycosis is a dangerously contagious disease, as stated in the opinion of the State veterinarian. To sustain itself the board has collected a number of reports of experiments in which it is alleged that the disease was communicated by inoculation with the tissue and discharge from the diseased parts of affected animals.

The reasoning appears to be that if a disease is inoculable it must be contagious, and if it tends to progress until the life of the animal is destroyed it must be dangerously contagious (Report, 1890, p. 20). 'This reasoning may be satisfactory to the board, but it will hardly be considered so by any unbiased scientist.

In the first place, a disease is not necessarily contagious because it is inoculable. Pyæmia, septicæmia, malignant redema, black quarter, and tetanus (lockjaw) are all inoculable diseases, much more so than is 
actinomyeosis, and yet it is well known that these diseases do not spread by contagion from animal to animal, and they can not properly be regarded as contagious diseases. All of these diseases, with one exception, affect mankind, but it would be absurd to claim that people are infected from diseased animals because of the coincidence that man and animals both suffer from the germs of these diseases. It is well known that these germs exist nearly everywhere and that animals and men get them from the same source in nature. The actinomyces fungus is also widely distributed, and grows upon the straw and beards of grain, and probably upon grasses, and for that reason it must be almost universally present. Leading scientists have, therefore, concluded that, with this disease, as with lockjaw, the germs of the disease are obtained by man from the same sources as by animals-that is, from the sources in nature where they abound.

The next question which suggests itself is, what is meant by " a dangerously contagious or dangerously infectious disease?" The word "dangerously" is evidently an adverb qualifying the words "contagious" and "infectious," and, as all words in a statute are supposed to have been put there for a definite purpose, we can only conclude that not all contagious and infectious diseases are meant, but only the dangerously contagious and dangerously infectious diseases, as stated. The meaning of "dangerously contagious" or "dangerously infectious" in this connection can only be taken as highly contagious or highly infectious, or at least having such a degree of contagiousness or infectiousness as to make the affected animals dangerous to the stock interests of the State.

The board says, however, that "no one will claim that actinonycosis is a highly contagious disease" (Rep. 1890, p. 20). They go on to say that "when the actinomyces have invaded the vital organs of the body, the disease, in both man and animals, is pronounced, by physicians and veterinarians, as fatal as tuberculosis, hence it must be listed in the catalogue of dangerously contagious diseases."

This reasoning would make the word "dangerously," which is an adverb, qualify the word "disease," which is a noun. That is, it is assumed because this disease is dangerous and fatal, when not properly treated, that it is a "dangerously contagious disease." But adverbs do not qualify nouns, and hence the word "dangerously" can only refer" to the degree of contagiousness or infectiousness, and not to the fatal character of the malady. A disease may be extremely fatal, but not in the least degree contagious, and it may be very fatal and only contagious when purposely inoculated, as in the case of tetanus, or lockjaw, but it would be perverting the use of language to speak of either of these classes of disease as "dangerously contagious."

It has been quite generally held by scientists who have investigated actinomycosis that it should be classed with those diseases which, like tetanus, or lockjaw, are produced by vegetable parasites inoculated by 
hasd bodies of rarious kinds which are infected, and which accidentally wound the skin, the mucous membrane, or other tissues, and thus gain entrance to the body.

There have been a number of cases recorded in which actinomycosis has been experimentally transmitted by inoculation from diseased to healchy animals. A large proportion of the successful inoculations were made with rabbits and guinea-pigs_animals which are very susceptible to most kinds of infection. In some cases large animals have been used, and with these the disease has been sometimes transmitted, and in other cases no result has followed the inoculation. The methods employed have been to deposit infectious matter in the abdominal cavity or in the connective tissue, or to inject it directly into the blood vessels. The results in most of the cases are of theoretical rather than practical value, because it is not likely that animals would be accidentally inoculated into the abdominal cavity or into the blood vessels. It is also an admitted fact with other diseases that those which require wounds of the skin and mucous membrane for the parasites to gain entrance to the tissues only in exceptional instances spread from animal to animal under natural conditions. So with actinomycosis, it is theoretically possible, but not probable, that an accidental inoculation might oceur by which the disease would be transmitted from a diseased to a healthy animal. This contingency is too remote, however, according to our present knowledge, to give any warrant for considering the disease as dangerously contagious.

The Illinois board of live-stock commissioners admits that before suppuration sets in, and there is a discharge, the disease is probably not transmissable, naturally, from one animal to another. (Rep. 1890, p. 20.) This conclusion is incontestable, but in spite of it many animals, which did not have a discharge, have been seized by order of the board, taken from the owners and condemned to the rendering tanks. How the board can justify such action under a law which only permits it to quarantine and slaughter animals affected with a "dangerously contagious" or "dangerously infectious" disease, it is difficult to understand.

If this board actually believes that actinomycosis is a dangerouslycontagious disease, its action has not been cousistent with that opinion. Affected animals have been allowed to come freely into the Union Stock Yards to the number of 100 or 200 a month. They go into all of the pens, mix with thousands of cattle, and the action of the board, instead of hastening their slaughter, actually delays it and gives additional opportunity for contagion.

'The diseased animals, instead of being slaughtered as soon as discovered, are placed in open pens, surrounded on all sides by healthy cattle, and are held in that condition until the end of the week, when the State veterinarian comes and decides as to whether they shall he condemned or not. There is no effort to clean and disinfect the pens 
where the distased animals have heen, and I have, myself, seen the State veterinarian open abcesses of affected animals and allow them to drip pus by the pint into the public alleyways of the yards, where thousands of cattle were driven, without any thought of having this infectious material cleaned up or disinfected.

Instead of lessening the chances of infection in the Union stockyards, the action of the State board actually increases it. Indeed, it is difficult to see loow any one believing in the contagiousness of the disease would cause the affected animals to be held in the interior of such great stock yards from the beginning to the end of the week before they are disposed of, and equally difficult to understand how constant and thorough disinfection could be dispensed with. It is impossible to find any measures adopted by the board which are calculated to prevent the spread of the disease should it be contagious, but on the contrary, the measures enforced, as already stated, are of such a nature as to assist the dissemination of contagion.

The board has devoted considerable space in its reports to arguments by which it attempts to show the danger to consumers of the meat of animals affected with actinomycosis. Their extravagant statements have excited the wonder and ridicule of sanitarians the world over. It would appear from the attention which the board has given to this aspect of the question, from the condemnations by it of some carcasses of affected animals and the passing of others as fit for food, from the neglect to disinfect cars, yards, and alleys where diseased cattle have been, and from the holding of diseased cattle in the stock yards a whole week for the visit of the State veterinarian, that its action in regard to lumpy jaw is a meat inspection measure and not a measure for the purpose of preventing the spread of a contagious disease among smimals.

The board, lowever, has no authority to inspect meat. but was created for the purpose of "the suppression and prevention of the spread of contagious and infectious diseases among domestic animals." When it goes beyond this purpose, it would appear to be acting ontside of the law, and it certainly causes great and unnecessary hardship to cattle owners in holding their animals a whole week for inspection. If there is to be a State meat inspection applied to cattle in the interstate trade, that inspection should be promptly made by competent inspectors, and there should be an end to this ridiculous methor by which suspicious cattle are collected for a whole week by employés ignorant of the nature of cattle diseases, and then passed upon by the State veterinarian by a cursory examination on Saturday. An inspection of value to the consumers or satisfactory to the producers can not be conducted in yards where from 50,000 to 75,000 head of cattle arrive weekly, by a single veterinarian, who devotes but one day in the week to this duty.

The important question in this connection is as to whether the board 
las jurisdiction for the inspection of meat. If it has not, then its acts in condemning animals and meats for the purpose of preventing certain carcasses from going on the market for food can only be considered as illegal. In that case there would be no need of considering whether or not actinomycosis might be communicated to the consumers of the flesh of affected animals, as the question at issue would turn on the jurisdiction of the board, and the cattle owners would thus secure relief.

The danger of the transmission of this disease to people from affected animals has been insisted upon by the board in such unequivocal terms, however, that a public interest has been aroused which makes it desirable to quote the opinions of some of those unbiased scientists who have given most attention to the subject. Actinomycosis in people is a very rale disease, and but few cases are on record as having occurred in this country. If there were any great danger of its being communicated by the flesh of affected animals we should certainly expect that it would be more frequent. On the other hand tuberculosis is one of the most common diseases of mankind, and is known to be communicable among: animals by taking tuberculous germs with the food, and yet, as appears from Dr. Norgaard's report attached hereto, the inspectors of the State board and of the city health department pay no attention to meat that is covered with tubercles even when it is exposed to their view in the slaughterhouses. Tuberculous meat is incomparably more dangerons. than that of animals affected with actinomycosis, and, hence, it is difiicult to understand this inconsistency in the action of inspectors who claim to be so solicitous for the public health.

Prof. Crookshank, in the course of an exhaustive review of our knowledge of actinomycosis, published in the annual report of the agricultural department, privy comcil office, on the contagious diseases, inspection, and transit of animals for the year 1888, says, by way of conclusion :

The successful transmission of actinomycosis from man to bovines suggests intercommunicability, but the negative evidence as to infection of man from bovines supports the view that the disease is derived from some sonrce which is common to both species. (S. C., p.115.)

In summing up the evidence as to the source of the disease in man, he says:

Many interesting observations have been made upon the origin of this disease in man. Two cases have been recorded in support of the theory of direct infection from the cow. Stelzner described a case of the actinomyeosis in a man who had had the care of animals some of which had suppurating glands. Hacker had a case of the tongue in a man who had charge of cows, one of which had a tumor of the jaw, which he had opened. On the other hand, Moosbrugger found that ont of 75 cases 54 were in men and 21 in women, including 2 children. In 11 of these the occupation was not stated. In 33 their occupation did not bring them into contact with liseased animals. They were, for example, millers, glaziers, tailors, shop people, and students. Only 10 cases oceurred anong farmers, peasants, and farm laborers. In 1 case only out of the 10 had the patient been in contact with diseased auimals. 
Out of the 21 women there were only 4 peasants, and none of them had been associated with diseased cattle.

Infection by the flesh of diseaser animals has also been discussed, but there is no evidence of the prevalence of the disease among slaughterers and butchers, who would be particularly liable to it if tlesh wore a source of infection. The chances of infection by ingestion are minimized by the flesh being alnost always cooked. Actinomycosis occurs also in pigs, and pork is often eaten in an uncooked state, but Israël has pointed out that this may probably be excluded, as many of the cases occurred among strict Jews.

The evidence points to the disease originating in man and lower animals from the same source, and there is a very strong suspicion attached to cereals. This view is supported by important observations with reference to the part played by cereals in inducing the disease in cattle, which have already been mentioned, and it gailis additional support from a case described by Soltmann, where the disease resulted from swallowing an awn of barley. A boy, aged 11, accidentally swallowed an awn of Hordeum murinum. He became very ill and suffered great pain behind the sternum, extending over six intercostal spaces, and when opened the awn of barley was found in the evacuated pus. The pain, however, continued and fresh deposits occurred, and when the boy was taken to the hospital the ray-fungus was detected. Possibly the spores of the fungus can be conveyed both by air and water.

Friedberger and Fröhner, in the last edition of their excellent Treatise on the Special Pathology and Therapeutics of the Domesticated Animals, published in 1892, give the following unbiased opinions, which they form from the scientific investigations made up to the present time:

Experiments to transfer the same to other animals have been made by several parties, but were always unsuccessful (Rivolta, Bollinger, Seidamgrotzky, Perroncito, Johne, Ullmann, Bodemer, Boström). Cattle, calves, goats, sheep, wethers, hogs, dogs, cats, rabbits, guinea-pigs, did not suffer after inoculation; only in some cases a granulation, outlined by inflammation, developed as the reaction of the organism against the inoculated foreign bodies. The alleged positive results of inoculation of Ponfik, Israël, Rotter, and Hanau refer, according to Boström, only to inoculation material which remained and was encysted (encapsuled). It appears that the actinomyces fungus produces a pathogenic effect only in that stage of development reached in connection with the beards of grain; but when once introduced into the animal body it is no longer transferable, because it immediately assumes involution forms (calcification) after inoculation. 'The negative results of the inoculation experiments are of great importance for the solution of the question whether or not a transmission of actinomycosis occurs from animal to animal or from animal to man. All are of the opinion that an infection of this kind does not take place (p. 585).

Inspection of meat.-As already mentioned in the article on "Pathogenesis," it must be considered as very improbable that the actinomycosis is a contagions or an infectious disease in the sense of its transmission from animal to animal or firom animal to manl. A case of infection of man from cattle affected with actinomycosis has never been observed which was free from all objections. At the most, such a transmission may be considered perhaps as possible from a theoretical standpoint, but not as probable. Expericnce is positive against the occurrence of a direct transmission. The actinomycosis, therefore, in relation to the inspection of meat is by no means so important as tuberculosis, even setting aside the fact that the former usually represents only a local affection. The meat of cattle affected with actinomycosis may be commercially disposed of without hesitation after the affected parts have been removed; it is objected to only in the rare cases of a generalized acținomycosis (p. 592). 
Ostertag, in his haudbook of meat inspection, published in 1892 , says, atter reviewing the evidence at hand:

Accorling to this, we are hardly justified in assuming that the use of actinomycoticorgans as food is injurious to health. At any rate, we must deny the possibility of this for the meat of actinomycotic animals. The activity of the sanitary police should therefore be restricted to the detection of all organs affected with actinomycosis, and the withdrawal of them from the market as highly spoiled food products in proportion as the specifically changed portions can not be certainly removed. In isolated lesions in the tongue this will usnally be possible, and is firther recommended, as the tongue is a valuable organ. In cases of generalization the whole animal is to be withdrawn from the market, becanse the generalization in actinomycosis seems to have an entirely atypical canse, and the detection of the total lesions (in the bone, in the depth of the muscles) is much less easy thin in tnberculosis, in which the regular lymph gland affection answers as an excellent guide for the discovery of the diseased areas.

Prof. Thomas Walley, in the second edition of his very thorough Practical Guide to Meat Inspection, published in 1891, says (p). 135-387):

The malady affects man, and is in him known as actinomycosis hominis, and while it has been transmitted by inoculation from man to the calf (Crookshank), so far as I am aware there is no direct evidence of the transmission of the disease from :nimals to man. Nor indeed is such a contingency ever likely to arise, seeing that the vitality of the spores of the hyphomycetes is much inferior to those of the pathogenetic fungi,'and that the changes produced by it in the organs attacked are so marked as to attract the immediate attention of even ordinary persons; and what is of more importance, the lesions of the disease are seldom localized, except in the pix, in the muscular tissue of the body. * * * Although certain organs, such as the tongur, may be largely involved, there is not much accompanying fever or interference with the normal functions or with the nutrition of the blood, and any systemic changes that arise are dne mainly to annihilation of the functions of the tougue, or of the particular organ involved, and as a result of this a state of poverty is indnced. See. ing that the disease is rarely of a systemie nature, and that there is an absence of fever, the nutritive changes induced in the muscles are so slight as to do away, in the great majority of eases, with the question of nocuity of the flesh; but, notwithstanding this, if there is any evidence of malnutrition of the blood or of the flesl, the carcass should be condemned for human food and in every instance the affected organ or organs should be effectually destroyed.

\section{EXPERIMEN'T TO TEST THE CONTAGIOUSNESS OF ACTINOMYGOSIS.}

When the cattle affecter with actinomycosis were placed under treatment 21 healthy eattle were placed among them in the stables in such a way that they would be most exposed to the infection of the disease. The healthy animals were tied between diseased ones, where they were obligerl to eat food soiled with the discharges from the tumors, and where they could not but inhale the breath warm fiom the lungs. There would appear to be no way possible of making a more severe test, and yet after being in such proximity with diseased animals for four months not a single one showed signs of being afrected either while alive or on post-mortem examination after they were slaughtered. 
DOES TIIE IODIDE TREATMENT DRIVE THE DISEASE TO TIIE INTELNAL ORGANS?

In a weekly agricultural journal printed in Chicago appeared a statement, under date of December 7, 1892, of the results of the post mortem examinations made of the eighty cattle slanghtered on November 29. This statement was evidently inspired by the State veterinarian or one of his assistants, as the post mortem notes of each case as taken by these officials were given in full. In this statement occurs the following language:

From the experiment just concluded we learn conclusively that iodide of potash has an effect upon cattle afflicted with actinomycosis, and possibly that it may cure simple cases characterized by glandular swellings or abscesses not attached to the bone. The general effect, however, is more of a dangerous than beneficial character as regards severe cases of lumpy jaw, where suppurating tumors implicating the bones are noticeable abont the head. Used in such cases iodide of potash apparently causes a superficial drying up of the suppurating sores which might lead the owner to believe that a cure had been accomplished, when, on the contrary, such external effects are no safe criterion of internal conditions. The post-mortem examination of animals so healed externally developed serious tronble within, trouble that had evidently been carried by the blood and possibly driven inwards by the drug employed.

The question now is, was there anything in the results of the examination to justify the conclusion that there were more internal lesions than are usually found in animals affected with actinomycosis? So tar from this being the case, there was a very much smaller proportion of the animals affected internally than was to be expected. The State veterinarian and his assistants are certainly the last people who would be expected to give the impression that a larger proportion of these animals were affected internally than is usually found.

The following table is compiled from the reports of the Illinois board of live-stock commissioners for 1890 . It shows the proportion of untreated animals which were found affected in the lungs or abdominal organs:

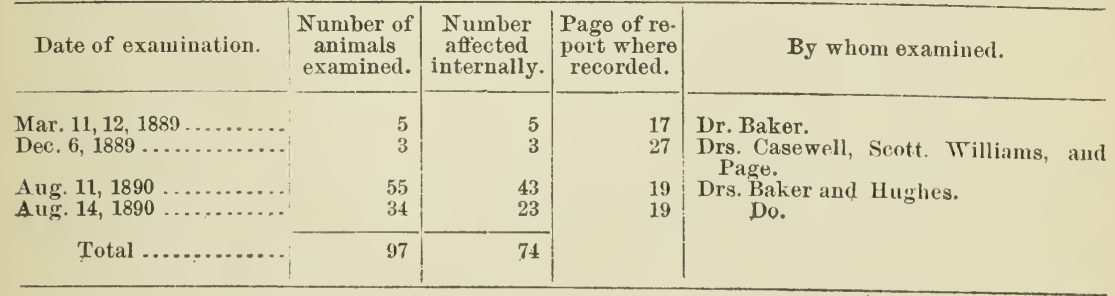

Fully 76 per cent of these animals were, therefore, affected in the lungs or abdominal organs. Now, taking the notes of the State veterinarian as published in the paper above mentioned, we find that they admit fifty-five of the animals had actinomycosis at the time of slaugh ter, while they found lesions of the lungs and abdominal organs in only twenty-three; that is, turning all the facts so that they will bear as 
much as possible towards this view that the iodide treatment tends to develop the disease internally, we find that, of the fifty-five affected animals which had been treated, but 42 per cent were affected internally, as against 76 per cent found with untreated animals by the State veterinarian and his assistants.

This, however, is not a fair statement of the case, because eighty animals, every one of which had the disease before treatment, were examined, and of these only $28 \frac{3}{4}$ per cent were found affected internally at the time of slanghter. And this is taking the statements of men who were doing everything in their power to disparage the results of the experiment and make it appear valueless.

As a matter of fact, the statement referred to which was printed from the notes of the State veterinarian does not correspond with the records of the bureau inspectors. Dr. Norgaard dictated a record of the condition of each animal as examined to a stenographer as the examination was made, and this record is the only accurate one that exists. From this official record it appears that seventeen of the animals reported by the State veterinarian to have internal lesions of actinomycosis did not have such lesions. Some of them had small nodules along the intestinal walls, which are common in healthy cattle, but when these were examined microscopically they were shown not to be affected with the actinomyces. There are several other cases reported with internal lesions, the numbers of which do not correspond with the numbers of any of the cattle slaughtered. There was evidently an error in printing the numbers of these animals, and I am unable for that reason to compare the notes with those made by Dr. Norgaard. Taking the examination made by Dr. Norgaard, therefore, as a basis for calculation, we find that among the eighty cattle killed on November 29) there were but four which showed internal lesions cansed by the actinomyces fungus. As twenty additional animals were killed and examined on December 2, in none of which any trace of internal lesions was found, the result was that in the 100 animals first slaughtered but 4 per cent showed evidence of the disease having affected the internal organs located in the thorax and abdomen.

The proportion of animals affected internally was very much less than was expected, and it is conclusively demonstrated by this fact that the iodide of potassium reduces the proportion of internal lesions and that it has fully as wonderful an effect on these as it has on the external lesions. This is precisely what we should expect from internal and constitutional treatment. If the remedy had been applied externally and caused the tumors to heal from the surface, there might have been some reason for anticipating a change of the seat of disease from external to internal organs. Being, on the contrary, administered internally, the remedy penetrated the whole organism, acting upon the internal organs at the same time as upon the external tumor, and the effect was not, only to prevent the extension of the disease, but to cure any manifestations of it which may have existed in the interior of the body. 
It can not be stated too emplatically nor repeated too often that the State veterinatian and his assistants carried out their examination with the determination to disparage the results of the experiment. Every inch of the intestines and every portion of the internal viscera were scrutinized in the most deliberate and anxions manner. The least abnormal sign, though it affected tissue of no greater magnitude than the heal of a pin, was set down as a serious internal lesion cansed by the actinomyces. Inspection of Dr. Norgaard's notes will show that in a number of cases these lesions were examined microscopically and were proved to be free from the actinomyces germs. Anyone who examines the intestines of healthy cattle will find in their walls, in a considerable proportion of cases, such small tumors, which have not the slightest effect upon the health, and which in many cases are caused by animal parasites. To set these down as internal lesions of lumpy jaw is to show either deplorable ignorance of the subject or deliberate falsification of the facts.

On January 23, 1893, the Secretary of A griculture informed Governor Altgeld that the cattle remaining in the experiment were ready for slaughter, and requested the removal of the quarantine for that purpose. The governor immediately replied by telegraph that he had directed the board of live stock commissioners to permit the removal of the animals.

The eighty-five animals which had been under treatment were slaugh. tered on January 27 and 28 at Hess Brothers' slanghterhouse. There were present, besides the Chief of the Bureau of Animal Industry, the following members of the Bureau force: Dr. A. D. Melvin, chief meat inspector at Chicago; Dr. W. S. Devoe, chief inspector for New Jersey; Drs. V. A. Norgaard and L. R. Baker, meat inspectors at Chicago; Dr. II. R. Trumbower, State veterinarian of Illinois, and the inspectors of the Chicago department of health.

The number found on post-mortem examination to be cured was sixtyeight, or 80 per cent of the whole number. Of the seventeen condemned as not cured there were internal lesions of actinomycosis in the lungs of three. About $4 \frac{1}{2}$ per cent of this lot of animals, therefore, showed internal lesions.

Of the whole number under treatment, which were killed and examined, viz, 185, there were found to be cured 131, or about 71 per cent. The number showing internal lesions was 7 , or 3.8 per cent of the animals in the experiment.

This result is extremely gratifying, and proves that a large preportion of the advanced cases of actinomycosis are curable by the internal administration of iodide of potassinm. If taken in the early stages of the disease, there is no doubt that 85 or 90 per cent would yield to this treatment.

14411-No. $2-3$ 
THE COS'T OF TREATMEN'T.

It has been repeatedly stated by those who have opposed the Department in its effort to relieve the eattle owners from the injustice which has been practiced upon them at Chicago, by giving them a specific treatment for this disease, that farmers can not afford to use a medicine for this purpose which costs $\$ 3$ a pound. Let us examine this statement in the light of the facts.

The result of the experiment shows that about two-thirds of the lumpy-jaw steers which come to the stock yards can be cured. If they were taken in an earlier stage of the disease the proportion would be much larger. Taking, however. two-thirds as a basis of calculation, we will see what would be the result on twelve animals averaging 1,200 pounds in weight. If shipped without treatment and condemned they would bring $\$ 9$ per head, or $\$ 108$ in all. If treated with the same success as the animals in the Department's experiment, there would be eight cured ones, which would bing not less than 4 cents per pound in good flesh, or $\$ 48$ per head. This would amount to $\$ 3 \$ 4$ for eight head, to which should be added $\$ 36$ for the four not cured, making a total of $\$ 420$ receipts as against $\$ 108$ if not treated. The medicine does not cost over 7 cents for each day it is administered, and no animal should receive over one ponnd in all. Taking the largest figures and the medicine for treating twelve steers would cost \$36. Deducting this from $\$ 420$, we have remaining $\$ 384$, a gain of $\$ 276$ in all, or $\$ 23$ per head over what would have been received if the cattle were not treated. It must be plain to every experieneed cattle-feeder that there is no other known medicine, nor indeed any kind of food, which ean be given to his animals with a prospect of such returus for the money invester.

It is difficult to understand the motive which will lead men to make such absurd objections and such incorrect statements in regard to a discovery which must prove of such incalculable advantage to the farmer without in the leastinjuring any other class of our citizens. It appears, however, that certain parties have conmitted themselves so irrevocably to the position that actinomyeosis is a dangerously contagions and incurable disease that they are willing to go to almost any length to prevent the overthrow of this theory. The action of the Illinois live stock commissioners, for instance, made it difficult to obtain eattle for experiment. They quarantined the cattle in the stock yards so that until the governor interfered they could not be taken to the stable for treatment. After the cattle were taken to the stables they were again put in quarantine until the governor ordered them released for a second time. Incorrect reports were sent ont apparently on anthority of the boarl, giving the inpression that the results were less favorable than was actually the ease. Other statements were made as to the proportion of isolated cattle condemned by the board, and as to the proportion affected intermally, which are at variance with the rec- 
ords given in the printed reports; and after the quarantine was removed from the cattle under experiment it was again imposed on December 31 , when the results of the first killing became known. If these several actions were not taken in the first place with a view to preventing the experiment being made, and afterwards to disparage the results obtained, and to delay the completion of the test, it is impossible for me to explain them on any other hypothesis.

\section{SUCCESSES OBTAINED BY INDIVIDUALS WITH THE IOIDID TREAT-} MEN'T.

A considerable number of cattle-feeders have tested the treatment of actinomycosis with iodide of potassium, and their results, so far as reported, have been remarkably successful. Below are given some of the letters received:

$$
\text { Bradfold, Stakk County, Ill., November 21, } 1892 .
$$

\section{U. S. Department of Agriculture:}

In October I received a circular giving treatment for actinomycosis. I tried it on three head of cattle, and it worked to a charm. I told other cattlemen, and several have tried the treatment. All are well satisfied but one, who informs me that he could see no change. His steer was very barl. The three I treated had not fully developed, and the veterinarian could not operate at the time. I therefore commenced treatment, and all swelling of the throat disappeared.

Yours, truly,

R. W. Phelis.

VAN Bures, IL.., Norember 22, 1892.

Burfau of Axmat. Industry,

IIashington, D. C. :

I treated three 3-year-old feeding steers according to the advice contained in your circular of October 10, 1892, for actinomycosis, or lumpy-jaw, with iodide of potassium. One had a lump under the ear; one on the jaw, and one under and between the jaws. The first was 3 inches in diameter; the second about $3 \frac{1}{2}$ by 6 inches long, and the third abont 5 inches in diameter. I commenced with 2 drams daily, and increased to 3 drams for twelve days, when the first named was entirely cured. I then gradually decreased to $1 \frac{1}{2}$ drams, and at the end of sixteen days the second was cured, the third being cured on the twenty-first day. The three neat were all slaughtered at Chicago thirty-five days from commencement of treatment.

Yours, very respectfully,

L. A. KEENE.

Dr. D. E. SAlimon,

Weltox, Cunton County, Iowa, November 26, 1892.

Chief of the Bureau of Animal Industry :

I received a circular from your Burean on October 18, with instructions for the treatment of lumpy-jaw in cattle with iodide of potassium. The cow I treated is 4 years old, and was bought at a sale for a trifle on account of her jaw. I thought I could cure it with corrosive sublimate, but failed. The jaw had been affected for a year before I began the iodide of potassium treatment. I had it lanced several times, 
aud for two months it had been running almost eonstantly. I began the treatment on Oetober 22, and gave $1 \frac{1}{2}$ drants every morning for ten days, then every alternate morning for a week, and the lumps are gone and the sore healed up. The eow now appears to be beginning to thrive. In this case, and it was a radieal one, the treatment has been a positive sueeess.

Respeetfully yours,

JohN Howat.

King Center, Mo., December 2, 1892.

DEAR SIR: I have been reading of your experiments with iodide of potassium. I would like to tell you of my experience with it. I have been treating "big-jaw" with it for more than a year, and have eured more than twenty eases.

A. J. Wheatley.

Hon. J. M. Rusk,

Secretary of Agriculture, Washington, D. C.

Creston, Iowa, December 5, 1892.

DeAr Sir: I tried your remedy for lumpy-jawed eattle on five steers out of niy herd, and it has proved sueeessful in eaeh case.

Daniet, D. Dorn.

Hon. J. M. Rusk,

Secretary of Agriculture.

Warsaw, INd., December 19, 1892.

DeAr Sir: Some two months ago I asked the Department for a bulletin on "lumpyjaw." I had a 2-year old Durham heifer with a lump on her jaw the size of a small hen's egg. I purehased 1 ounee of the prescribed remedy, iodide of potassium, divided it into eight portions, and gave as direeted, in bran. I saw no ehange exeept the softening of the tumor. I bought another quantity, and after a rest of a week gave it in the same manner. The lump has disappeared, exeept a small callous. 'The animal's eyes beeame weak, which is the only eliange I eould see. The last ounee of the remedy I gave in seven doses. I think the heifer is cured of the ailment.

Yours,

Dr. D. E. SALMON

A. T. Cоoк.

Burcau of Animal Industry, Washington, D.C.

Algona, Iowa, December 18, 1892.

Dear Sir: I cured two lumpy-jaw heifers, a 3-year old and a 2-year old, giving each 1 onnee of iodide of potash, in six doses, two months ago, and as yet there is no sign of a return of the discase.

Very respectfully,

Hon. J. M. RUsk,

R. W. BARIRTT,

Secretary of Agricullure. 
Kinntone, Chautauqua County, N. Y., January 2, 1893.

DEAR Sir: Some time ago wo wrote you, asking if the Department had ever made an investigation of the lumpy-jaw in cattle, and we reccived in reply a circular containing the iodide of potassium remedy, and asked us to rejort results. We had two cases.

No. 1, a cow in milk, was taken in July. Had four different sores that discharged matter previons to treating her with this remedy. A local veterinarian had advised killing her. One of the sores has cntirely healcd; the other three are greatly diminished in size. Her flesh has dropped off until she is getting quite thin, and is at the present time and has been for the last threc weeks, very stitf. Her eyes discharge very badly; nose but little, and the outer skin looks as if some one had poured her hair full of the coarsest bran. Her appetite is as good as any cow we have.

No. 2, a cow also in milk when taken, but dried off since. She was taken about middle of November, one bunch only. It did not discharge until broken by halter pulling on it. Had treated her ten days. The matter discharged was white, with a yellowish cast, and was very thick and sticky. She discharged from the nose very badly, and also from the eyes, but the outer skin cleaving off was hardly noticable in this case. She did not drop off in flesh, nor did she show any stiffness. The sore has greatly diminished in size, and think we will have to give her no more medicine, as all that remains is apparently dead flesh, and does not seem sore. We are highly pleased with the remedy so far.

Yours, truly,

Hon. J. M. Rusk,

S. SPENCER \& SON.

Secretary of Agriculture, Washington, D. C.

Vine Hill, Conn., January 27, 1893.

Patient, thoroughbred Gnernsey; age, 8 years; calved July 11, 1892. Observed bunch on left upper jaw November 1, 1892. At this time enlargement was about size of two fists, and very firm. Herdsman then reported to me he had observed swelling in August, 1892. For four days after November 12, administered 2 drams iodide of potassium twice daily. Onc week later repeated same doses for same period of timc. From November 30, for seven days following, gave 2 drams once daily. About one week thereaftcr administered 1 dram once daily for ten days. From November 12 the swelling rapidly increased in size until November 27, when at about the size of a man's head, it broke. In a few days indications of resolution set in, and for the past six weeks she has, to all intent and purpose, appeared to be perfectly recovered. At this date, the seat of attack is barely visible. The cow's appetite improved shortly after receiving first dose of the iodide, and milk sccretion also increased.

F. H. Stadtmueller, One of the managers of Vine Hill and Ridge farms, West Hartford, Conn.

Fontana, Miami County, Kans., February 1, 1893.

DEAR Sir: I have tried iodide of potassium for the cure of lumpy-jarw in cattle on 12 head, as prescribed under the treatment made by your direction at Chicago. Nine head, are entircly well; three need further treatment. Five head, with large, lumpy jaws, would have been worthless without the iodide cure. The balance might have got well without it, as they had only bad lumps. I have full 400 hcad of cattle this winter, and will not have a worthless steer amongst them. The experiment made by your direction has saved me $\$ 200$ or $\$ 300$. John Shean lost 10 out of his herd of 100 head from lumpy-jaw. He then tried the iodide of potassium remedy on two 
very bad cases, and cured them. I know hmupy-jaw can be successfully treated by farmers and feeders with iodide of potassium. Accept my thanks for the interest you take in the welfare of farmers.

Respectfully, yours,

Hon. J. M. Rusk,

Fred. Mathews.

Secretary of Agriculture, Washington, D. C.

Mr. J. W. Wampler, a stock-breeder of Brazilton, Kans., in a letter recently publisherl in the Kansas Farmer, says:

I will tell yoll all I know about curing lumpy-jaw in cattle. Last summer 1 saw an account in some paper that Seeretary Rusk wanted 300 head of lumpy-jawed cattle to experiment on, and he thought he could cure them. I wrote him to send me his remedy and I would experiment, too, as I had three of my own, badly diseased. In a few days I received his answer, and also the renedy, and low different cattle had bcen treated.

The remedy is iodide of potassium. It costs 30 cents per onnce here in Girard, and 1 ounce will cure one animal if the disease las not gone too long. The longer the disease has run, the more of the iodide of potassinm it will take. I use 1 dram in 1 pint of fresh water. It dissolves readily if well pulverized. This much I give to each animal every morning. Don't mix any more than you use each day, as it is better while fresh. Place a good leather halter on the animal and draw its head up by placing the strap or rope over sonething above. Place a cow horn in its mouth, then pour the medicine through. Repeat every morning unless the animal gets to ruming too much at the nose, so much that it interferes with its brcatling, then stop a day or two, and commence again, and so on, until the animal is well, which, if the disease is not too old, will be in ten or fifteen days.

I have a fine heifer that was badly affected for over a year. 'The lnmp on the left side of her lead was half as large as her head, and had become honeycombed and running. I fed her 5 ounces and then quit, thinking the disease had gone too far, and left her run, thinking I would kill her and drag her away. We were busy sowing wheat, and did not notice lrer for some two weeks. I then went to look at and arrange to kill her, as I did not want her to be with the other cattle, but was surprised to seo the lump half gone and the remainder soft, and it has been going away ever since, and now she is well.

I cured two steers, also, that liad not run so long. One I fed 1 onnce and the other about 2 omnces. I told a neighbor that had three badly discased. He cured them all. Another neighbor eured three. Another had one that was fat, but had a lump on jaw. He tried to sell it to a shipper, but he wonld not take it for a gift. He tried the remedy, and in two weeks she was well. He sold her and she went to Kansas City, passed the inspection, went on the market, and he got a good price for her.

Don't get scared if they run at the cyes and nose and the outer coat of skin peck off, like the hair was full of bran, for this is characteristic of the effects of the iodide. The cattlo will fatten while under treatment, and so much better and faster afterward, that I am convinced that it would pay to treat all cattle to about 1 ounce to purify the blood and system, especially those put up to feed.

I have given to others that I have not heard from yet, except one that had two stecrs diseased. I saw him the other day, and asked him how he snceeded in enring the lunıy-jaw? Ite saicl he had bonght the medicine and laid it "1) in the kitchen cupboard, and it was there yet. So you can see his cattle have the hmpy-jaw yet.

Tell the people not to buy the medicine muless they intend to use it, and use it right and regular. I will give anyone all I know about curing lumpy-jaw if they will write me, inclosing stamp, and not charge any thing, feeling that what is good for me is good for my neighbors.

The report of Dr. V. A. Norgaard is hereto appended. 


\section{REPORT OF DR. V. A. NORGAARD, INSPECTOR IN CHARGE.}

Cincago, It..., February 6, 1893.

SIR: I have the houol to sulumit herewith a peport upon the results of experiments in the treatment with iolide of potassium of "attle affected with actinnmyeosis.

Very respectfully,

Dr. D. E. Salmon,

VICTOR A. NORisaARD, Velerinary inspector.

Chief Bureau of Inimal Industin.

THE MEDICINE.

It has already been mentioned that the medicine used in treating actinomycosis is iodide of potassium. This is a common drug and may be obtained at any drug store, the price being about $\$ 3$ per pound.

All the iodine salts have a faint antiseptic power, which is produced by the iodine getting free under a prolonged influence of varions organic substances. A 5-per cent solution of iodide of potassium will render tuberculous sputum inactive in about twenty-four hours.

Iodide of potassium has a strong absorbing effect when given internally, especially on all pathological accumulations of cells. In connection with protoplasm, earbonic acid, and water the iodine will become fiee and combine with the cells in the pathological prodnct, which will decrease their vital power and accelerate their absorption. It still remains to be explained in what that specific effect consists, which the iodine salts has on the actinomyces fungus. Several prominent anthorities have investigated the subject, but all have given a different explanation. Nocard succeeded in cultivating actinomyces in gelatine containing 1 per cent of iodide of potassium, but this proves nothing, as the salt in this case will remain unchanged, and in that form has not even a slight antiseptic power. It is not necessary, however, that the iodine should have a specific influence on the fungus to produce that remarkable effect, which the drug evidently exercises on all artiuomycotic new formations. This effect may simply be attributed to the strong absorbing power which iodicle of potassimm exercises on all pathological neoplasms and waste products. The colonies of artinomyees, when living and possessed with regenerative power, are always imbedded in a small amount of liquified tissue surrombled by a wide zone of inflammation. When an actinomycoma consists exclusively of granulomatous tissue and comnective tissue, the fungus is dead, or at least dying (Bostroem).

It will therefore be miderstood that the liquified tissue surromuling the parasite under the influence of the administered iodide of potassinm will be absorberl, the soft granulomatous tissue when partly deprived of the necessary pabulum by absorbtion will undergo a degenerative met- 
amorphosis, and the round cells be converted into spindle cells, and the whole actinomycoma in this way will be turned into an inert mass of fibrous tissue, inclosing the dead calcified actinomyces tufts.

The yellow pus-like substance in which the living actinomyces is always imbedded is not true pus, though it has that appearance, but is merely the product of a degenerative metamorphosis (necrobiosis). True pus must contain pus corpuscles and specific pus-producing micro-organisms (pyogenic cocei and bacilli) (Bostroem).

Where iodide of potassium is administered in medium doses for any length of time it will affect not only the diseased parts, but the liberated iodine will combine with the normal cells in the body and produce a series of symptoms called iodism. This consists of a general enaciation, slırinking of the fat, atropliy of lymph glands, and of mammary glands, in connection with a chronic catarrh of nose and conjunctiva, and desquamation of the epidermis. When given in over doses, it will produce a catarrh of the stomach and intestines, and even cause hemorrhages. The manure will get dry and hard and coated with thick mucous, or eventually with blood. All these inconveniences, however, are easily avoided when the medicine is given in proper doses, care being taken that the animal gets plenty of nutritious and easily digestible food. It is not, however, advisable to ad minister iodide of potassium to milch cows, as it will reduce the milk secretion considerably or stop it altogether. Furthermore a great part of the drug is excreted through the milk, making it unfit for any use. When administered internally the drug permeates all the tissues in the body, even the muscles and bones, but it is, however, rapidly excreted again, especially through the urine and inilk.

In treating actinomycosis in cattle with iodide of potassium the dose should never exceed one gram ( $\frac{1}{4}$ dram) for every lunndred pounds live weight, the proper dose being from 8 to 12 grams ( 2 to 3 drams) according to the size of the animal and the extent of the lesion. This dose may be given from five to six days, when the animal will show slight symptoms of iodism, viz: discharge of thick mucus from the nose and excretion of tears. The manure will become rather dry, but that is easily repaired by giving a dose of glauber salts and some bran mash. This will restore the appetite, and two days after the last dose was given the animal will be ready for another week's treatment, and so on until a cure is affected. If these precantions are taken, no ill effect will result from the treatment, and if properly fed the animal will gain in condition uninfluenced by the medicine. There is, however, a great difference as to the individual effect of the medicine on animals, but any farmer who takes an interest in seeing his stock doing well will easily perceive when it is time for him to stop and give the animal rest for two or three days.

The medicine is best administered dissolved in water and given by means of a slender, long-necked bottle, for example, a common Rhine- 
wine bottle. One dose of medicine is dissolved in about a pint of water, the steer is seized by the nose to hold up the hearl, and the contents of the bottle is emptied into the mouth without fixing or securing. the tongue in any way.

Where a man has several head of humpy-jawed cattle to treat, they may not be of the same size and therefore not require the same dose of medicine. As the farmer or breeder is not usually in possession of a pair of scales sufficiently fine to weigh off such small doses as required, the most convenient way is to have the medicine, which is: easily dissolved, prepared in a concentrated solution of the strength 1 to 2 (2 drams of the solution to contain 1 dram of iodide of potassium). The drug must be dissolved in distilled or rain water, as otherwise a precipitate will form from the salts present in common water. With such a concentrated solution and a measuring glass, it is easy to measure out the exact dose for every animal and pour it into the winebottle, half filled with common water.

EFFECT OF IODIDE OF POTASSIUM ON THE DIFFERENT AC'INOMYCOTIC NEW FORMATIONS AND THEIR TISSUES.

To give a detailed description of the various forms under which actinomycosis appears in cattle is not necessary here, as there is an abundance of literature on this subject. It shall only be mentioned that, from a therapeutic standpoint, we may divide them all into two classes: (a) those where the bones are involved and $(b)$ those where only the soft tissue has become affected.

Of the 150 head experimented on, about sixty had lesions where the bone was affected. These may again be divided into two classes: first, where the lesion is located on the lower jaw, which is by far the most common, and, second, those where the facial bones and upper maxilla have become affected. In both cases, the lesion has been produced by an invasion of the germ, by means of a spikelet of barley or some other stiff vegetable particle, on which the germ has regetated in nature. No other satisfactory explanation of the infection has been given so far, whether the invasion occurred through a decayed tooth or through some abrasion in the mucous membrane in the mouth, on the tongue, or in the pharynx. Bostroem has examined thirty-two cases of actinomycosis in the jaw bones, minutely, and in every case he found some vegetable particle located in the socket of the decayed molar, or in the gums, or even as deep as in the center of the swollen rarefied bone. Where the tongue is affected these foreign bodies may be found in almost every one of the nodules, and a microscopical examination will show them to be closely covered with actinomyces. In five cases of actinomycosis in human beings, this anthority has traced the infection back to the same origin, and thereupon advises people who may have that habit not to chew a piece of straw or to put grain, especially those kinds which are armed with a spikelet, in their mouths, as he 
feels satisfied that this is the only way in which an infertion can take place.

Where the lesion has only affecterl the soft tissues, the most common appearance is in form of a hard, round or egg-shaped tumor, lying loose in the connecting tissue under the skin, usually in the submaxillary, sublaryngeal, or parotid region. The tumor may vary in size and character, ranging from a walnut to a child's head and from being hard and solid to soft and fluctuating. In almost all cases, bony or soft, the skin that covers the tumor or swelling is more or less indurated and may on the large bony swellings obtain a thickness of 2 or 3 inches. These fibrous indurations, however, show a pronounced inclination to shrink where iodide of potassium has been administererl for some time, and will, in most of the cases, disappear altogether, leaving the skin in its natural condition.

The first step toward a medical treatment of actinomycosis was when M. Thomassen, of Utrecht, in 1885, announced that he had successfully treated actinomycosis in the tongue of eattle by giving iodide of potassinm internally and applying tincture of iodide to the ulcers on the tongue, which he first scraped. Since then this experiment has been repeated all over Europe by many different authorities and practitioners, with similar success. This lesion, however, is very seldom seen in this country, but seems to be rather common in Germany and certain parts of England. Of all the cattle in this experiment, not one had actinomyeosis in the tongue, and the inspectors in the stock-yards have only seen it two or three times. The reason for this may be that actinomycosis in the tongue has a comparatively acute progress, and that an animal so affected rapidly loses in flesh, if medical treatment is not adopted. Now we know, howerer, that we can always master a case like this, even if the tongue is swollen to such a size that it protrudes

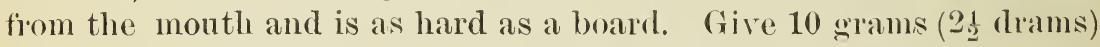
of iodide of potassium a day, in the above-describerl way, are being taken to give the animal time to swallow the medicine, as deglutition is impaired considerably by the hard and swollen condition of the tongue. Feed the animal gruel and other fluid food, with a bottle if necessary. In ten to twenty days the tongue will regain its natural size and condition and the animal be enabled to eat hay.

Though none of the cattle in this experiment had the lesion in the tongue, several of them had it in the mueous membrane in the mouth, especially on the inside of the lips, but not to any great extent. It ap. pears as small, flat tumor-formations, the size of a dime to that of a fiftycent piece, of a reddish-gray color and only protruding a little over the surrounding parts. They will shrink and disappear completely in about two weeks when medieine is administered regularly.

When a medium dose of iodide of potassium has been administered from three days to a week, symptoms of iodism will appear, and at the lapse of two to three weeks a descyumation of the epidermis will take 
place, especially on the neck, shonlders, and rump. But there are many exceptions. Some animals will show only a slight eatarih of the nose and none of the conjunctiva, while the desquamation will not take place before a month or six weeks or may not oceur at all. Several of the steers experimented upon showed under the whole treatment not a single epidermic seale on their body, even if the dose of medicine was increased up to 15 grams a day. They would get a catarrh of the intestines, with constipation, but show no external symptoms of iodism. Such an insusceptibility of the constitution usually extends to the actinomycotic lesions, which wonld remain almost unaffected or only inprove slightly. There are, however, also exceptions here, as one steer with a large tumor in the sublaryngeal space was almost completely cured without showing any epirlermic desquanations.

INDIVIDUALS WHICH SHOW THE MOST PRONOUNCED SYMPTOMS OF IODISM WILL IMPROVE THE FASTEST.

The effect of the medicine is rarely alike in two individuals, even if they are affected exactly the same way and to the same extent. The hard fibrous tumors lying loose in the connective tissue under the skin will require a treatment of from three to six weeks with proper intervals, according to their size. But in many cases a tumor the size of a child's head will shrink and disappear faster than a similar one located on the same place in an animal of the size and condition, but with the tumor only as large as a goose egg; the former wili show a pronounced iodism, while the latter will show little or none at all.

When one of those loose fibrous tumors become fluctuating and is left alone, it will break open after a while and a comparatively small amount of pus will be evacuated. The pus cavity will rapislly fill up with the soft granulomatous tissue characteristic of actinomycosis, and when it is full it will grow out of the opening like a mushroom and form a granuloma, which will increase in size very fast as long as the supply of nutrition is abundant, after which the surface will begin to ulcerate.

If the treatment is commenced at this time, say with a daily dose of 10 grams of iodile of potassium, the rule is that the ulcerating surface of the granuloma will dry up and the granuloma itself will gradually shrink, apparently as if it were drawn in through the same hole it had grown ont of. Then the fibrous parts of the tumor will commence contracting until the whole tumor has disappeared, leaving a smail white cicatrix where the granuloma was. But here there are also exceptions. In some cases the granuloma may remain unaffected, while the fibrous parts begin to shrink first, and after a while the granuloma will become dry, shrinking slowly, but not disappearing completely, although the actinomyces are killed or rendered ineffective by the treatment.

When the disease affects the bony parts, the periosteum will become covered with thick layers of connective tissue inclosing centers of soft granulomatous tissue, and when it penetrates into the internal parts 
the bone will increase in bulk in two different ways, either by a rarefying granulating ostitis or by an ossifying periostitis. The invading germ will cause an irritation, which will be followed by a formation of granulomatous tissue in the cancelli of the spongiosa and in the Haversian canals. The bony plates of the former and the walls of the latter will be destroyed and replaced by large pockets or alveoli filled with soft granulomatous tissue and with a creamy, viscid pus-like mass, containing numerous actinomyces.

The proliferating granulomatous mass will finally penetrate the surface of the bone and infiltrate the covering tissues, and at last break throngh the skin, which will become thick and indurated and form a bulky fibrous wall around the fast-growing granuloma. The picture here described is that frequently seen on the lower maxilla. These tumefactions may attain enormous dimensions, and even reach the size of a half-peck measure, with granulomas 6 to Sinches in diameter. Several specimens of this kind were included in the experiment, thongh such extreme cases practically never will be submitted to treatment.

Nevertheless, the effect of the medicine by far exceeded the expectations. With a few exceptions, they all showed wonderful improvement. Under the effect of a 12 to 15-gram daily dose, those large fetid ulcerating granulomas would dry up and shrink, most of them disappearing altogether, only leaving a white contracted cicatrix, while the fibrous parts of the tumefaction would become thinner every day and finally leave the swollen bone directly covered by the muscles and the skin. The post-mortem examination of these bony tumors revealed similar inprovements. An incision through them showed the internal parts to be spongy, but the alveoli and pockets only contained very little granulomatous tissue, and this was not soft, but of a more elastic and dense character, and only contained calcified actinomyces. In many of them no pus was found at all, while others contained small quantities. This remarkable inprovement was seen in all those cases where the iodide of potassium had caused a well-prononnced iodism, while in those eases where the medicine had a less pronounced effect the improvement was equally small and quantities of pus and soft tissue could be found in the alveoli. Similar to the loose-lying tumors, the medicine may in these cases have a stronger effect on either the grannloma or the fibrous parts of the tumefaction. The first is the most common, but in a smaller number of cases the medicine takes effect exclusively on the fibrous parts, making them shrink so rapidly that the thick fibrous wall surrounding the granuloma contracts to such an extent that the whole granulona is expelled, partly by force and partly on account of obstructed blood circulation.

There is no doubt that these results, obtained by a treatment which in no case lasted more than seven weeks and as an average notexeceding five weeks, permit us to say that in iodide of potassium we have found a valuable remedy in treating actinomycosis. 
It now remains to decide which cases will prove of financial value for the farmer and breeder to treat, and this partly depends upon the facility with which the administration of the medicine can be performed. The amount of medicine used in a single case ought never to exceed 1 pound, equal to an expense of $\$ 3$. We suppose any steer will be worth that much. If the affected animals are being kept in a stable, as, for instance, in distilleries, the trouble about giving the medicine amounts to nothing. But where the animals are running loose in a pasture and there is no convenient place to tie them up it is no easy matter to catch them and secure them every day while the medicine is given. On ranges and similar places, where several hundred head of cattle are kept, the easiest way is to keep the affected cattle in a large pen, in the one side of which is built a narrow chute, into which the cattle can be driven one by one and treated. Such a chute may be constructed out of a few solid boards without much expense. To administer the medicine in the drinking water is not advisable, as it is then rather difficult to control the right dose. The eattle will not drink a pailful of water with a dose of iodide of potassium, except when they are very thirsty.

As to the different lesions, it has already been said that when the tumor is not connected with the bony tissue, but is lying loose in the connective tissue under the skin, a favorable result may be expected in from two to five weeks, according to the size of the tumor and to the susceptibility of the individual toward the effect of the medicine.

It is not necessary to continue the treatment until the tumor has disappeared completely, but it may be stopped when it has shrunk to about one-third of its original size, and the remainder will usualy disappear without further treatment. When a small tumor about the size of a hen's egg is located on the cheek or on the outside of the lower jaw, it is usually very hard and can only be moved very little, so it is easily mistaken for a bony tumor. A careful examination, however, will soon prove that it is not bony, and when treatment is commenced it will rapidly decrease in size.

When the bone is affected, treatment is only advisable when the swelling does not exceed a goose egg in size on the lower jaw or a walnut on the upper jaw, and has not penetrated the skin; but in any case the medicine will avert a further development of the disease if the animal is at all susceptible to its effect.

Two cases as above described, and with a small bony tumor on the lower maxilla, were killed on October 29 , 1892, after a six weeks' treatment. The bony swellings were then almost solid, contained no large cavities and no traces of pus or soft tissue. In the center of them was a bunch of white connective tissue, and from this extended a cord of the same material to the socket of the first molar. This was an obliterated fistzla. The molar was not decayed and was firmly embedded in the socket. This is a clear proof that actinomycotic lesions in the bony tissue can be successfully treated when they are taken in time. 


\section{POST'MORTEM LXAMINA'IION.}

November 29, 189:, two lots of cattle experimented npon were killed. Each lot contained forty head, the one consisting of cattle that were supposed to be completely cured, while the second lot included all the old chronic cases, where a successful result could not be expected in the time limited for the experiment.

In the special report of each animal the description of the post-mortem examination will be found. This is necessarily very brief.

The forty cattle which were supposed to be cured proved to be so with the exception of two, which had small actinomycotic tumors in the lung containing living actinomyces. In nine other cases traces of the disease were found at the place where the tumors had been located, but these traces were so insignificant, ranging in size from a pin's head to a bean, that they did not amount to anything.

The first lot were ail in good condition, some of them very fat, and the greater part of the second lot also were in a very satisfactory condition, only a few of them being really poor. A noteworthy fact is that only two out of each lot had actinomycotic lesions in the internal organs, all cases in the lungs. This would hardly have been the case if the cattle had not been treated. In some cases a few small nodules, in size from a millet seed to a pea, containing a cheesy, greenish deiritus mass, were found in the wall of the small intestines, but a microscopical examination of these lesions gave negative results with regard to actinomycosis. The final result of the investigation was that out of the first lot thirty-eight were passed as fit for human food, while the two cases affected with actinomycosis in the lung were condemned. Of the other forty only five were passed and thirty-five condemned, though many of them were big fat steers with the lesion located on the jaws and no internal lesions of any kind. The tumors on the greater part of these did not contain any pus, and the granulomatous tissue had undergone a fibrous metamorphosis, and subsequently did not contain any actinomyces possessed with regenerative power.

December ¿ twenty more head of cattle were killed. These had been stabled in the same barn as the rest and had been treated from three to six weeks each with proper intervals. They were all used to the distillery slops before the treatment began and continued to grow in Hesh while they were treated. They were all pronounced eases, but only the soft tissues were involved. The lesions were about the same as those in the first lot, hard, fibrous tumors, the size from a goose egg to a child's head, loeated in the loose eommective tissue under the skin in the submaxillary or sublaryngeal space. They all recovered completely, and at the post-morten examination no traess of the disease were found except in two eases, a hard, fibrous induration in the skin, where the tumors had been. They were all passed as fit for human food.

The notes in regard to the treatment and the post-mortem examination of the individual cases are given in the form of an appendix to this report. 


\section{APPENDIX.}

No. 533796.

Dark-red hornless steer; received August 22.

In the submaxillary space is located an oblong tumor extending from the chin to the neck, with a dependence of $t$ inches. The tumor was of a fibrous condition, but not very hard. In the parotid region on the right side is located a hard, fibrous tumor the size of a goose egg.

Treatment was commenced August 24 with a daily dose of 12 grams and was continued until September 13, at which time the swelling underneath had disappeared and the tumor in the parotid region had shrunk considerably. October 20 the tumor in the parotid region was swelled a little and fluctuating. It was opened with a knife, and a small amount of pus discharged. Medicine was again administered for two weeks, and by November 12 the steer was regarded as cured.

Post-mortem, Norember 29.-Skin under submaxillary space indurated. Fibrous scar in parotid region. No traces of actinomycosis. No internal lesions. Carcass passed as sound.

\section{No. 4598\%.}

Roan steer, white sirles, horuless; received September 5.

In sublaryugeal space on left side is located a hard fibrous tumor the size of a double clinched fist, extending up in parotid region toward the ear. The lower part of the tumor is lying loose under the skin, but the part in the parotid region is in close connection with it.

Treatment was commencerl September 7 with a daily dose of 12 grams and was continued until October 12 , by which time the tmmor had shrunk to the size of a walnut and only left a fibrous induration in the skin in parotid region. The steer was considered cured about October 15.

Post-mortem, Nocember 29.-No traces of the tumor left. No internal lesions. Carcass passed as sound.

\section{No. 39976}

Dark-red steer, white face and rump, horns; received Angust 28.

In sublaryngeal space in left side is locaterl a fibrous tumor the size of a child's lread. The tumor is not quite as hard as is generally the cise, but is closely counected with the skin.

Treatment was commenced August 30, with a daily dose of 10 grams, aud was contimued until October 1 . Two weeks later no traces of the tunor conld be felt, hut in the skin was still left a thick fibrous induration. Medicine was again atministered until November 1 , by which time the skin had almost regained its natural coudition. Abont November 10 the steer was considered cured.

Post-mortem, November 29. - Slight thickening of the skin in sublaryngeal space. No traces of the tumor left. No internal lesions. Carcass passed as sound. 
No. 39963.

White roan steer, hornless; received August 28.

In parotid region on the left side is located a tumor the size of a large goose egg, which at the lower end extends into a hard fibrous swelling, filling the intermaxillary and sublaryngeal space, depending about 5 inches below the throat.

The treatment was commenced August 30, with a daily dose of 10 grams. The medicine had a good effect, causing a strong catarrh of nose, mouth, and conjunctiva and made the tumor and swelling shrink rapidly. After four weeks the treatment was stopped and the steer regarded as cured about October 10.

Post-mortem, November 29.-The skin that covered the tumor a little indurated. No traces of the disease left here nor internally. Carcass passed as sound.

No. $49385 \%$.

Red steer, white star, rudiments of horus; received August 16.

In the sublaryngeal space on the right side is located a tumor the size of a clincherl fist. The tumor is hard and fibrous with a smooth surface and lying loose under the skin.

The treatment was commenced August 18 and continued for three weeks with daily dose of 10 grams. The medicine had a strong effect on the steer, and already, September 12, the tumor had completely disappeared. In spite of a well-defined iodism the steer did not lose flesh under the treatment, but his condition improved considerably.

Post-mortem, November 29.-No traces of the tumor left. No internal lesions. Carcass passed as sound.

\section{No 545801 .}

Speckled dehorned steer, white face; received August 22.

In sublaryngeai space on right side is seen a tumor the size of a clinched fist located in loose connective tissue under the skin. The tumor is hard and fibrous with no evidence of having been opened.

Treatment was commenced August 24; daily dose, 10 grams. The tumor began to shrink from the fourth day, and in less than three weeks it had gone down to the size of a walnut. The steer was then covered all over with epidermic scales, and was consillered cured about October 7.

Post-mortem, November 29.-No traces of the disease were found either on neck or internally. Carcass was passed as sound.

No. 545593.

Brindle steer, hornless; receiverl August 16.

On the ontside of the right clieek, near the lower edge of the inferior maxilla, is located a hard fibrous tumor the size of a goose egg. The tumor is situated in tie short connective tissue under the skin. In submaxillary space there is a similar tumor the size of a double clincherl fist.

Treatment was commenced August 18, with a daily dose of 10 grams, and was continned until September 12. The steer showed a pronounced iodism, aud the tumors shrunk rapidly. When the treatment was stopped the tumor in submaxillary space had disappeared, and the other one had shrunk to the size of a waluut. Octolyer 1 the steer was considered cured.

Post-mortem, November 29.-No traces of the tumors left, except.a slight iuduration in the skin. No introinal lesions. Carcass passed as sound. 


\section{No. 5340:4.}

Brindlo steer, horns; received August 22.

On left cheek outside second molar is located a hard fibrons tumor the size of a walnut. It is situated in the short comnective tissue unter the skin and can only be inoved very little. In the intermaxillary space there are two tumors, the foremost one a little larger than the above mentioned, and the other one about the size of a double clinched fist.

The treatment was commenced Angust 24 with a daily dose of 10 grams. Two weeks later the two smaller tumors had disappeared, and by September 20, when the treatment was stopped, there was only a small part of the largest one left.

Post-mortem, November 29.-In intermaxillary space was found a small fibrous induration the size of a robin's egg. The surrounding lymph glands were a little swollen, but sound. The carcass was passed as sound.

\section{No. $54.551 \%$.}

Red dehorned steer, white triangular spot on left side; receiverl Angust 16.

On the left cheek are located four hard fibrons tumors from the size of a walnut to a hen's egg. They form a line from the angle of the month toward the back edge of the jaw, the last one being located a little below the ear. They are very hard and can only be moved slightly. In sublaryngeal space on left side is located a tumor the size of a double clinched fist.

Treatment was commenced August 18 with a daily dose of 10 grams, and was continued until September 10, at which time the four tumors on the cheek had disappeared and the one on the throat had shrunk to the size of a hen's egg. September 25 the steer was regarded as cured.

Post-mortem, Nocember 29.-One of the sublaryngeal lymph glands contained a small actinomycoma the size of a bean. No traces left of the tumors, excepting a small induration in the skin covering the throat. No interual lesions. Carcass passed as sound.

\section{No. 39964.}

Red spotted steer, white star; received Augnst 28.

The right lower maxilla is swollen to the size of a goose egg, between the incisor and the first molar. The bony swelling is covered with a layer of fibrous tissue, which makes the swelling appear the size of a donble clinched fist. In the submaxillary space are a couple of small soft swellings the size of walnuts.

Treatment was commenced September 1, with a daily dose of 12 grams and was continued until October 30, with proper intervals. By this time the sivollen glands in the submaxillary space had regained their normal size and condition, while the tumor on the jaw had only shrunk very little, but lad become considerably harder. Only on the lower part of it was left some of the fibrous covering. The bony swelling seemed to have increased a little in size, while the fibrous tissue was absorbed.

Post-mortem, November 29.- Small fibrons infaret in sublingual lymph gland. The bony swelling was spongy, but did not contain any pus cavities. It was covered by a thin layer of fibrous tissue, which on the lower side of the swelling inclosed a small soft granuloma. In the wall of the small intestines were located a few small round hard nodules. * One of the mesenteric glands was a little enlarged. By the microscopic exanination no actinomyces was found in any of these. No other internal lesions. Carcass passed as sound.

*These nodules, which are frequently mentioned in the post-mortem notes, have nothing to do with actinomycosis or lumpy-jaw. They are very common in healthy cattle and are caused by animal par. asites.-D.E. S. 


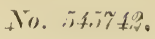

Brown steer, horms; received Angust 22.

On the right cheek is locater a hard fibrous tmmor the size of a hen's egg outside the third molar. In the submaxillary space there is another tmonor the size of a cocoannt. The lower part of this tumor was fluetuating, and couseguently was opened with a knife. It only container aloont one ounce of pus. The rest of the tumor was hard and fihous.

Treatment was commenced August 24, with a daily dose of 10 grams, and was contimued until September 1, with proper intervals. The medirine did not have any strong effect, the tnmors only shrinking slowly. Oetober 7 there was still left a tumor the size of a goose egy under the jaw, while there only remained a small fibrous bunch from the tumor on the cheek. Medicine was then again administered for three weeks, and by November 12 the steer was considered enrer.

Post-mortem, Norember 29. - No traces of the disease left except a small fibrous cicatrix on the cheek and a tibrous induration in the skin in snbmaxillary space. No internal lesions. Carcass passed as sound.

\section{No. 545559.}

Light brown Montani-Texas steer; reeeived August 16.

In right parotid region is loeated a hard fibrous tumor the size of a goose egg. In the submaxillary space on right side there is a similar tumor the size of a donble elinched fist lying loose in the eonnective tissne moler the skin.

Treatment was commenced Angust 18 with a daily dose of 10 grams, and was continued nutil owtober 1, with proper intervals. The steer showed no symptoms of iodism, the tumors shrinking only slowly, "sprecially the one in the parotid region. From November 1 to November 15 medieine was again administered, and soon afterwards both tumors had disappeared, the upper one leaving a small fibrons induration on the angle of the lower maxilla.

Post-mortem, Norember 29.-No traces of the tmmors left. No internal lesions. Carcass passed is soumd.

\section{No. .540.519.}

Black steer, dellorued; received August 16.

On the right side of the face, between rye and nose, is located a bouyswelling the size of a hen's egg, with a sear on the top, indicating that it has been suppurating. On the right cheek, ontside the third molar, is located a lard fibronstmmor, the size of a goose egg, lying loose in the comedive tissnemder the skin. In sublaryugeal space there is located another tumor the size of a donble clinched list.

The treatment commeneed Angust 18, with a daily dose of 10 grams of iodide of potassimm, and was kept up for four weeks, with the exception of one or two days a werk. The tmmor on the cheek and under the throat soon commenced to shrink, while the bony one brokr open and discharged a little pus, after whieh it healed "1p again. At the end of the fourth wrek the two fibroms tumors had gone down to alont one-third the original size, and the treatment was stopped. 'Three weeks later they had almost disappeared, while the bony one was exactly the same size as before the treatment. From Or.tober 15 to October 24 medieine was again administered daily, but the bouy tmmor remained mehanged.

Post-mortem, Norcmber 29.- - T'he bony swelling on right upper maxilla consisted of solid hone, and containod neither pos nor soft tissne. No traces were left of the other tumors, No internal lesions, Carcass passed as sound. 
No. $545 \% 9 \%$.

Red hornless bull; received August 22.

On the right lower nilxilla, ontside the first molar, is located a hard fibrous bony tumor the size of a clinched fist. In submaxillary space on left side is seen another tumor, the size of a child's head, lyiug loose in the connective tissue uncler the skin. 'The skin covering the lower part of the tumor is fibrous indurated.

Treatinent was commenced August 24, with a daily dose of 10 grams of iodide of potassium, and was continned until October 15 , with proper intervals. The tumor uucler the throat had then gone down to the size of a goose egg, while the fibrous parts of the bony swelling on the lower jaw had disappeared, leaving it bony, hard, and the size of a hen's egg. November 15 the tumor under the throat had disappeared completely, only leaving a fibrous thickening in the skin.

Post-mortem, November 29.-Fibrous induration in skin under throat. An incision throngh the bony swelling shows this hard and solid, with no cavities, but with a suall fibrous mass in the center, from which the obliterated remainders of a fistula goes up to the socket of the first molar. No lesions internally. The carcass was passed as sound.

\section{No. 59141.}

Dark red steer, hornless, roan face; received August 28.

The entire intermaxillary and sublaryngeal space is occupied by an oblong and rather soft tumor. The skin covering the tumefaction is inclurated, and closely attached to the underlying tissue. The tumor depends about 5 inches below the lower erlge of the under jaw.

Treatment was commenced about August 30, with a daily dose of 12 grains, and was continued until October 5, with proper intervals. The steer showed a pronounced iodism, and the tumor shrunk rapidly, but for a long while the skin remained very thick and indurated. This, however, disappeared slowly after the treatment was stopped. About October 20 the steer was regarded as curerl.

Post-mortem, November 29.--In intermaxillary space is located a flat fibrous induration, about 2 inches long and 1 inch thick, inclosing a small abscess the size of a bean, containing thin yellow pus. No actinomyces was found by microscopical cxamination. No internal lesions. Carcass passed as sound.

\section{No. 45983.}

Red steer, hornless, white star; received September $\mathbf{5}$.

On the left side of the face, about 3 inches below the eye, is located a hard bony swelling the size of a clincherl fist.

Treatment was commenced September 7 with a daily dose of 12 grams, and was continued until November 1 . The steer showed a pronounced iodism, but the size of the tumor remained almost unchangerl.

Post-morlem, November 29.- The thin fibrons covering on the bouy swelling contained a fow small pockets the size of peas. The contents of these pockets had originally been soft granulomatous tissue, but consisted now of a fibrous lomogeneous mass of a light yellow color, but still bearing resemblance in structure to the former tissue, which during the treatment had mndergone a fibrons metamorphosis. On the cut surface these centers protruled a little over the indurated tissue in which they were embedderl, showing that they had been under a pressure. The internal parts of the bony swelling were spongy, but contained neither soft tissue nor pus. The lymph glands on the head were sound. No internal lesion. Carcass was passed as sound. 
No. 40001.

Roan heifer, horns; received August 22.

The left cheek, from the eye down to the lower edge of the jaw, is covered by a hard fibrous tumor, which is tluctuating on the most prominent part. It was opened with a knife and a few ounces of good yellow pus evacuated.

Treatment was eommcneed August 24, with a daily dose of 10 grams, and was continued until October 10, with proper intervals. The heifer showed only slight symptoms of iodism, and the tumor only shrunk slowly. When the treatment was stopped another abseess formed in the same plaee, which was also opened. From October 20 to November 10 medicine was again administered, and the tumor was considerably redueed in size.

Post-mortem, November 29.-On the right cheek is located a fibrous induration about 1 inch thick and 3 inehes in diameter, containing slight traces of soft tissue centers. The lymph glands on the head were sound. No internal lesions. Carcass passed as sound.

No. $399 \% 4$.

Spotted roan steer, horns; reccived August 28.

On the right lower maxilla, below the first molar, islocated a fibrous bouy swelling the size of a clinched fist. In the intermaxillary space there is a hard fibrous tumor about the same sizc, lying loose in the conneetive tissue under the skin.

Treatment was commeneed August 30, with a daily dose of 12 grans, a:ld was continued until October 20, with proper intervals. The steer showed a pronounced iodism, and the tumor in the intermaxillary space disappeared eompletely, while the swelling on the lower jaw was reduced to the size of a goose egg by the shrinking of the fibrous covering.

Post-mortem, November 29.-No traces of the fibrous tumor left, exeept a small induration in the skin. Surrounding lymph glands sound. An incision through the bony swelling showed this to be solid bone throughout, with the exception of a small bunch of white connective tissue in the center, from which a cord of the same material leads to the socket of the second molar. No internal lesions. Carcass passed as sound.

No. 45594.

Dark red steer, horns; received September 12.

In the loose connective tissue in the submaxillary space is located a tumor the size of a double clinehed fist.

Treatment was commenced September 14, with a daily dose of 12 grams, and was continued until October 10, by which time the tumor had shrunk to the size of a walnut. About October 25 the steer was considered eured.

Post-mortem, November 29.-Small, fibrous induration in the skin in submaxillary space. No traces of the tumor left. No internal lesions. Carcass passed as sound.

\section{No. 25873.}

Dark red steer, hornless, white star aud white spot on left hip; received September 12.

The intermaxillary space is eompletely filled from the chin to the noek with a fibrous swelling, which, on the left side, extends up into the parotid region toward the ear. The swelling is rather soft, and is elosely connected witl the indurated skin.

The treatment was commenced September 14, with a daily dose of 10 grams, and was continued until October 25 , by which time the swolling liad disappeared, but 
the skin was still hard and very thick. This, however, improved rapidly, and by November 12 the steer was considered cured.

Post-mortem, November 29.-No traces of the swelling left. No internal lesions. Carcass passed as sound.

\section{No. 546042.}

Blue roan steer, hornless; received August 22.

In the sublaryngeal space on the left side is located a hard fibrous tumor the size of a cocoanut, lying loose in the connective tissne under the skin.

Treatment was commenced August 24, with a daily dose of 12 grams, and was continued until Oetober 7, with proper intervals. The steer showed no symptoms of iodism whatever, and when treatment was stopped the tumor had only shrunk to about one-half its original size. Medicine was again administered from October 20 to October 31, during which timo the tumor improved rapidly. About November 12 the tumor had disappeared, only leaving a thick induration in the skin.

Post-mortem, November 29.-No traces of the tumor left. No internal lesions. Carcass passed as sound.

\section{No. 48040 .}

Red steer, hornless; received August 30 from Kansas City.

In the sublaryngeal space on left side is located a bard fibrous tumor the size of a child's head, lying loose in the connective tissue under the skin. In right parotid region there is a hard, flat, fibrous swelling, which is closely connected with the skin.

Treatment was commenced September 1, with a daily dose of 12 grams, and was continued until October 1, with proper intervals. By this time the tumor under the throat had shrunk to the size of a hen's egg, while the swelling in the parotid region had become considerably reduced. About October 20 the steer was regarded as eured.

Post-mortem, November 29.-No traces of the tumors left, except a fibrous thickening in the skin in the parotid region. No internal lesions. Carcass passed as sound.

\section{No. 39985.}

Red steer, white face, white rump and legs; received August 28.

In the loose connective tissue in the submaxillary space are located three hard fibrous tumors the size of goose eggs. The skin covering them is considerably indurated.

Treatment was commenced August 30, with a daily dose of 12 grams, and was continued until September 20, by which time the tumors had shrunk to the size of a walnut, and the induration in the skin had become thinner. October 18, the steer was considered cured.

Post-mortem, November 29.-Slight induration of skin in submaxillary space. No traces of the tumors left. No internal lesions. Carcass passed as sound.

No. 45953 .

Red steer, white star, hornless; received September 5.

In left parotid region is located a hard fibrous tumor the size of a goose egg. In the sublaryngeal space on left side there is a similar one the size of a double clinched fist. The first one is closely connected with the underlying tissue, but the other one is located in the loose connective tissue under the skin.

Treatment was commenced September 7, with a daily dose of 12 grams, and was continued until Oetober 7, with proper intervals. By this time both tumors had shrunk considerably, and about October 28 the steer was regarded as cured, though there was still left a small deeply situated induration in the intermaxillary space. 
Post-mortem, Norember 29.-In the intermaxillary space is located a small, flat, fibrous induration the size of a walnut, inclosing three granulomas the size of beans. The skin in left parotid region a little indurated. No internal lesions. Carcass passed as sound.

$$
\text { No. } 9.48 \% \text {. }
$$

Red steer, white face, horns; received August 30 from Kansas City.

In the submaxillary space are located three hard fibrous tumors the size of goose eggs, and in the right parotid region there are two similar tumors, the lower one the same size and the upper one the size of a heu's egg. The skin covering the tumors in submaxillary space is hard and thickened.

Treatment was commenced September 1, with a daily dose of 12 grams, and continued until Oetober 15 with proper intervals. The steer only showed slight symptoms of iodisin, and the tumors shrunk rather slowly. When treatment was stopped none of the tumors were larger than a walnut. About November 10 the steer was considered cured.

Post-mortem, November 29.-In the intermaxillary space were found two small fibrous indurations, each containing a couple of small soft granulomas the size of a pea. No internal lesions. Carcass passed as sound.

\section{No. 39994.}

Red steer, horus; received August 28.

The intermaxillary space is completely filled, from the chin to the neck, with a fibrous swelling of rather soft conlition. The skin is closely connected with the nnderlying swelling.

Treatment was commenced September 1, with a daily dose of 12 grams, and was eontinued until September 30, by which time the swelling had shrunk considerably. October 12 the steer was regarded as cured.

Post-mortem, November 29.-The skin that covered the swelling is fibrous indurated. No traces of actinomyces to be found. No internal lesions. Carcass passed as sound.

$$
\text { No. } 44531 .
$$

Red steer, horns; received Scptember 12.

In sublaryugeal region on left side is located a hard, fibrous tumor the size of a double-clinched fist, lying loose in the conuective tissue under the skin.

Treatment was commenced October 14, with a daily dose of 12 grams, and was continued until October 15, by which time the tumor had shrunk to the size of a hen's egg. November 10, the steer was considered cured.

Post-mortem, Norember 99.-One of the submaxillary lyuph gland's was swollen to the size of a walnut, and contained an abscess the size of a bean, filled with pus containing antinomyces. Sublingual lymph gland in right side indurated. No internal lesions. Carcass passed as sound.

$$
\text { No. } 25386 .
$$

Red steer, white star, roan spot on right side and left thigh, hornless; received September 12, 1892 .

In the loose connective tissue in the submaxillary space is located a hard fibrous tumor the size of a child's head.

Treatment was commenced September 14, with a daily dose of 15 grams, and was continued until October 12, by which time the tumor had shrumk to the size of a hen's egg. Oetober 25 , the steer was regarded as cured.

Post-mortem, November 29.-No traces of the tumor left. No internal lesious. Carcass passed as sound. 
No。, 5.,311.11.

Dark red, Hereford-crossed steer, white on hips; received $\Lambda$ ugust 22.

In the left parotid region is located a hard, flat, fibrous swelling, which at the lower endextends into a tumor the size of a child's head, located in the sublaryngeal space. Two inches in front of this tumor, in the submaxillary space, there is another one the size of a goose $\operatorname{egg}$.

Treatment was commenced September 24, witl a daily dose of 12 grams, and contimued until Oetober 1 . The steer showed no symptoms of iodism, and no desquamation of the epidermis appenred under the whole treatment. Nevertheless the tumors shrunk rapidly, especially the largest one in the sublaryugeal space. Octobet 16 , the steer was regarded as cured.

Post-mortem, November 29.- In one of the submaxillary lympli glands was located a granuloma the size of a bean. The skin in the parotid region a little indurated. No internal lesions. Carrass passed as somul.

\section{No. $4000 \%$.}

Black speckled heifer, hornless; received August 22.

In the loose connective tissue in the intermaxillary space is located a hard fibrons tumor the size of a donble clinched fist.

Treatment was commenced August 25, with a daily dose of 10 grams, and was continned until September 20. By this time the tumor had almost disappeared, and by Octolser 1 the steer was considered cured.

Post-mortem, November 29.-No traces of the tumor left. No internal lesions. Carcass passed as sound.

\section{No. 131056.}

Red steer, horns, white on left flank; received September 5.

In the sublaryngeal space on left side is located a hard fibrous tmmor the size of a double clinched fist, lying loose in the connertıve tissue under the skin.

Treatment was commenced September 7 , with a daily dose of $10 \mathrm{grams}$, and was continued nntil October 7, with proper intervals. By this time the tumor liad shrnnk to the size of a walnut. October 20 the steer was regarded as cured.

Post-mortem, November 29.-No trace of the tmmor left. No iuternal lesions. Carcass passed as somud.

$$
\text { No. } 25150 \text {. }
$$

Red steer, horns; received September 5.

In the sublaryngeal space on the right side is located a hard fibrous tumor the size of a cocoanut, lying loose in the connective tissue nuder the skin.

Treatment was commenced September 7 , with a daily dose of 12 grams, and was continued October 1 . The steer showed a pronounced iodism, and the tumor shrmik rapidly. October 15 the steer was considered cured.

Post-mortem, Norember 29.-No traces of the tumor left. No internal lesions. Careass passed as sound.

$$
\text { No. ..5 11,is. }
$$

Red steer, white star, horns; received September 12.

In the sublaryngeal space on the left side is located a tumor the size of a ehild's head. On the lower end of it, the skin is in close conncetion with the tumor, and out through the skin extends a round soft granuloma, about 4 inches in dianeter and 1 inch thick. The surface of the gramuloma is nueven and covered with pus crusts.

Treatment was conmenced september 14 , with a daily dose of 14 grams, and was 
continued until Oetober 20. with proper intervals. The granulona began to dry up right away, and in the third week it had shrunk to a level with the surrounding parts and was covered with cpidermis. When the treatment was stopped the tumor had gone down to the size of a hen's egg, with a sinall white scar at the place where the gramloma was, surrounded by heavy wrinkles of skin. Abont November 12 the steer was regarded as cured.

Post-nortem, Norember 29.-Small fibrous thickening in the skin where the tumor was. No internal lesions. Carcass passed as sound.

\section{No. 131035.}

Red steer, horns, white star and spot on shoulder; received September 5.

In the sublaryngeal space on the left side is located a fibrous tumor the size of a child's head. It is lying loose in the comnective tissue under the skin.

Treatment commenced Septenber 7, with a daily dose of 12 grams, and was continued until Oetober 10, witl proper intervals. By this time the tumor had sunk to the size of a goose egg, and the rest disappeared without further treatment. October 25 the stecr was considered cured.

I'ost-mortem, November 29.-One of the submaxillary lymph glands showed a fibroid degeneration, having evidently contained an actinomycoma, which under the treatment had undergone a fibrous metamorphosis. No trace of the tunor was left except a fibrous induration in the skin. No internal lesions. Carcass passed as sound.

$$
\text { No. } 25870 \text {. }
$$

Dark red steer, hornless; received September 12.

In the left parotid region is located an extensive, flat, hard, fibrous swelling, which bclow extends into a tumor filling the intermaxillary space.

Treatment was conmenced September 14, with a daily dose of 15 grams, and was continued until October 15. By this time the tumor below liad shrunk to about onethird its original size, while the swelling in the parotid region remained almost nnchanged, though not quite as prominent as before. November 1 the treatment was commenced again, and the tumors now began to improve rapidly. November 10 treatment was discontinued, and shortly afterwards the steer was considered cured.

Post-mortem, November 29.-One of the sublaryngeal lympl glands contained an abscess the size of a hazclnut, in which was fornd yellow pus with actinomyces. No internal lesions. Carcass passed as sound.

\section{No. 39984.}

Dark red, hornless steer; reecived August 28.

In the sublaryngeal space on the right side is located a tumor the size of a child's head. It is lying loose in the connective tissue unler the skin.

Treatment was commenced September 1 with a daily dose of 10 grams, and contimued until October 10. The steer showed marked symptoms of iodism and the medicine had a good effect on the tumor. When the treatment was stopped there still remained a tumor the size of a hen's egg, covered by a rather extensive hard induration in the skin. November 1 the tumor had completely disappeared, and even the induration in the skin had grown thinner.

Post-mortem, November 39.-Fibrous thickening in skin under larynx. One of the sublingnal lymph glands contained a very small actinomycoma the size of a large pin head. No internal lesions. Carcass passed as sound.

\section{No. 48056 .}

Red steer, hornless, speckled body, white belly; received August 30, from Kansas City.

The intermaxillary space is completely filled with an oblong tumor, extending 
from the chin to the neck, and depending abont 3 inches below the lower edge of the jaw. The tumor is hard and n̂brous and in elose connection with the skin. On the inside of the npper lip are located two soft red actinomyeomas about the size of a nickel.

Treatment was commenced September 1st, with a daily dose of 12 grams, and was contimed mintil October 7 , by which time ther: only remained a fibrons induration in the skin under the jaw. The actinomyeomas inside the lip lad disappeared during the third week of the treatment. The steer was considered cured abont October 15.

Post-mortem, November 99.-No traces of the disease left under the jaw or in the mouth. No internal lesions. Careass passed as sound.

No. $4503 \%$.

Red steer, hornless, received Angust 30, from Kansas City. On both cleeks outside the third molar is located a hord fibrous tumor the size of a hen's egg. They are sitnated in the short connective tissue under the skin, and as they are hard and can only be moved slightly, give the impression that they are bony. In the loose connective tissue in submaxillary space there is another tnmor the size of a clinched fist.

The treatment was conmeneed September 1st with a daily dose of 12 grains, and was continued until October 1 , at which time there was only a small trace left of the tumors. October 12 the steer was regarded as cured.

Post-mortem, November 29.-The sublaryngeal lymph glands were a little swollen, but no traces of the tumors were left. No interual lesions. Carcass passed as sound.

$$
\text { No. 48,020: }
$$

Red steer, horuless, brockled face, white on shoulder and hip; received August 20 from Kansas City.

In right parotid region is located a hard, fibrous tumor the size of a goose egg, and another one, the size of a child's head, is seen in the loose connective tissue in the sublaryngeal space.

Treatment was commenced September 1, with a daily dose of 12 grams iodide of potassium, and was continued until October 10 . The steer showed very pronounced iodism, and the tumors shrunk fast. October 28 they had disappeared.

Post-mortem, November 29.-No traces of the disease left except a small callous in the skin where the tumors had becn. No internal lesions. Carcass passed as sound.

\section{No. 533950.}

Red steer, white face, shonlder and rump; received August 22.

On the back edge of the right lowc jaw is located a fluctuating tumor the size of a goose egg, and two hard fibrous tumors of the same size in submaxillary space.

Treatment was commenced August 24, with a daily dose of 10 grams. The abscess was opened with a knife, and a few onnces of thick yellow pus evacuated. The pus did not contain actinomyces. The treatment was continued until September 20. The tumors shrunk rapidly and had disappeared about October 2.

Post-mortem, November 29, 1892. - In the submaxillary space was left a small, fibrous induration containing two abscesses the size of beans. The contents of these was a thin yellow pus. The microscopic examination proved actinomyces to be present. Treatment was stopped a little too soon. Carcass passed as sound.

$$
\text { No. } 25854 \text {. }
$$

Red hornless steer; received September 12.

In right parotid region is located a fibrous tumor the size of a goose egg, and 
another the size of a child's heal is soen in sulmaxillary space. Both are hard, round, and with a smooth surfare lying loose under the skin.

Treatment was commenced Septenber 14, with daily dose of 12 grams. Both tumors shrunk very fist, and one month later had gone down to one-third their original size. No nore medicine was given and about November 12. the steer was ronsidered curec'.

I'ost-mortem, Norember 29.-Fibrous incluration of skin underneath the throat. No traces of actino. In the liver there were two small abresses the size of hazelnuts. Microscopir cxamination proved they were not caused by actinomycosis. The carrass was passed as sound.

\section{No. 45943.}

Brown horuless steer, white star; received September 5.

In left parotid region is located a hard fibrous tumor the size of a donble clincherl tist, and another one the same size in submaxillary space.

Treatment was commenced September 7 with a daily dose of 12 grams, and was continued until October 15 . The tumors shrunk fast and by November 12 no trace of them was left, not even in the skin.

I'ost-mortem, Nocember 29.-Neither on the hear nor in the internal organs was any of the disease found. The carcass was passed as sound.

\section{No. 39988.}

Dark red steer, hornless, received Augnst 28.

The right lower maxilla below the first molar is swollen to "e size of a donble clinched fist. It is bony, hard, but the covering skin is thick and indurated, and closely attached to the underlying tissue.

Treatment was commenced, August 30, with a daily dose of 12 grams, and was continued until October 20, with proper intervals. The steer showed a prononnced odis $m$, and the tumor shrunk a little and became bony hard.

Post-mortem Norember 29.-In the indurated skin on the swelling was located a few small centers of soft tissues the size of peas. The interual tissue of the bone was rather solid, only a trifle spongy with no traces of the disease. The lymph glands on liead were sound. No internal lesions. Carciss passed as sound.

\section{No. $.9999 . ?$.}

Black hornless steer, August 28, 1892.

Bony swelling of right lower maxilla, similar to above No. 39988. Treatment the same.

Post-mortem, Norember 29.-The internal parts of the swelling were spongy, and contained several centr.rs of soft tissue the size of hazelunts. No pus was fonud. Lymph glands sound. No intermal lesious. Carcass was condemned.

No. 13015 .

Red white-speckled steer; white star and horns; received August 30.

All the facial bones on the right side of the bead are immensely swollen from the edge of the lower eyclid down to the nose. 'There is hardly any fibrous avering on the bony parts, except below, where the tmmol gradually goes over on the lower maxilla. On the most prominent part of the swelling is an ulerethe sizo of the palu of a hand, located abont 4 inches below the eye. The lachrymal duct isobliterated so that the tears run down over the tumor.

Treatment was commenced September 1st, with a daily dose of 15 grams and contimed to Octoher 25 , with proper intervals. The mediciue caused woll-marked 
symptoms of iodism, but the tumor remained almost nnclianged, the fibrons swelling on the lower jaw shrinking a little. The ulcer dried up and left a small sear 1 by 2 inches. As no further improvement could be experter the treatment was stopper.

I'ost-mortem, November 39.-An incision throngh the bony swelling shows this completely filled with numerous eavities the size of peas, filled with pus and soft tissue, hoth containing actinomyces. No lesions in intermal organs. Careass condemmed.

$$
\text { No. 5455:24. }
$$

Red dehorned steer, white star, white on loft fore knee, side, and hip; received Angust 16, 189:.

The left lower maxilla is inmensely swollen from the mouth and backwards. On the lower part of the swelling, which occupies the whole intermaxillary space, and has a dependence of 5 to 6 inches below the original edge of the jaw, is located an nlcerating granuloma about 7 inches in diameter, protruding about 3 inches over the surroundings. The deeper parts of the swelling are hard like bone, covered with a thick layer of fibrous tissne, which around the granuloma forms a heavy swollen wall 2 to 3 inches thick. Upwards the fibrous part of the tumor extends over the upper jaw and the parotid region, gradnally growing thinner up against the eye and ear. The skin covering the tumor is closely connected with it all over. The surface of the granuloma is uneven, covered with crusts, and small drops of pus constantly ooze out at many different places. The base of the granuloma is a little sunaller than the circumference of it. A 2-inch deep fissure separates the edges of the granuloma from the epidermis covered parts. The steer is constantly grinding his teeth and slobbering from the mouth. Condition ratler poor.

The treatment was commenced August 18 with a daily dose of 12 grams, and was continued until October 25 , with proper intervals. The tumor apparently did not shrink much, though the fibrous parts of it, especially on the upper jaw, were reduced considerably. The granuloma dried np, but shruuk only very slowly. By November 12 there was only a scar about 5 inches in diameter left at the place where the base of the granuloma was, and the fibrous wall which surrounded it had also grown considerably thimmer, but is now the most prominent part, as it has not shrunk as fast as the granuloma.

Post-nortem, November 29.-An incision through the bony tumor shows numerous alveoli filled with soft granulomatous tissue containing very little pus. Directly above the scar on the lower surface is a large granuloma the size of a goose egg. The consistence of this is not soft as usual, and contains less pus. In this actinomyces were found.

In the wall of the small intestines was located a few hard small nodules the size of millet seeds, containing a dry mass, consisting mostly of limesalts. No actinomyces were found. Other interual organs sound. Carcass condemned.

No. 534819.

Red steer, white star, white left shoulder and rump, dehorned; received Angust 16.

The right lower maxilla is immensely swollen from the ramus to the angle. The swelling is bony and covered with a thick fibrons layer, which extends up over the cheek towards the eye. Underneath_it occupies the whole intermaxillary space and has a dependence of 4 inches.

The treatment commenced August 18 with a daily dose of 12 grams and was contimued until September 25. The fibrous parts of the swelling had by this time shrunk considerably, leaving the tumor hard as bone. The swelling on the upper jaw had almost disappeared, only in the submaxillary space there remained a good deal of fibrous covering The treatment was then contiuued for three weeks more withont much improvement. 
Post-mortem, November 29. - The fibrous tissne eovering the lower part of the bony swelling contained a few deposits of soft tissne, while the bony swelling contained several cavities filled with the same and a few filled with pus. No internal lesions. Carcass eondemned.

\section{No. $4808 \%$.}

Red steer, white star and shoulder, hornless; received August 30 from Kansas City.

The right ramus of the lower maxilla is swollen to an enormous size. The swelling fills the entire intermaxillary space from the ehin to the neck and extends up over the right cheek toward the eye. The deeper parts of the swelling are hard, covered with a thiek layer of fibrons tissue, which on the lower part of the swelling is 4 or 5 inches thick. There is locater at this point an nleerating granuloma 6 to 8 inches in diameter and protruding 3 to 4 inches over the swollen margin of the surroundings. The surface of the granuloma is uneven, partly raw and bleeding, and partly eovered with crusts of pus, which is constantly diseharged through mumerous minute openings on the surface. The ulcer has a very fetid odor. The skin eovering the tumefaction is elosely attached to the underlying tissue. The entire swelling was fully the size of a gallon jug.

Treatment was eommenced September 1 with a daily dose of 15 grans and was eontinued until November 1, with proper intervals, two of which extended over a week each. The steer showed a pronounced iodism, and, though the manure constantly was rather hard, the steer did not lose in eondition. The nlceratiug surface of the granuloma soon began to dry up and no more pus was discharged. The swollen indurated margins shrunk and contracted considerably, and by this contraction the whole granuloma, which then had shrunk to the size of a clinched fist, was expelled, leaving a dry scar. The softer parts of the swelling, especially in the submaxillary space and the parts extending up over the eheek, disappeared almost entirely, leaving the bony part elosely exvered by the indurated skin,

Post-mortem, November 29.- The skin covering swelling is indurated and about threc-fourths of an inch thick. On the lower part of the tuitefuction is seen a white contracted cicatrix 3 to 4 inehes in diameter, and in the thin fibrons covering of the bony swelling were a few small centers of reddish granulomatoms tissme. A seetion through the bone shows this spongy and filled with numerous centers and pockets containing yellow pus of a rather slimy eharacter, and several cavities filled with soft red tissue intermpted by the characteristie small yellow spots. All the surronnding lymph glands were perfectly sound and no lesions were found in the interual organs. 'The carcass, though it was in splendid condition, was eondenned.

\section{No. $4824 \%$.}

White roan steer, horns; received Angust 30 from Kansas City.

Immense swelling of right lower maxilla with ulcerating gramuloma. Case similar to above number (48087).

Post-mortem, Norember 29.-The grannloma had not disappeared altogether. It shrumk to the size of a hen's egg and was rather hard and dry. The fibrons tissue eovering the bony swelling contained several small eenters of soft tissue. An ineision through the bony swelling shows this to be spongy, with numerous pockets and eavities filled with soft red tissue and pus. The lymph glands on head wero sonnd. Carcass eondemned.

\section{No. 534031 .}

White steer, dehorned; roeeiver Angnst 22.

The left side of the faee from the eye down to the nose, is immensely swollen. The swelling is of a bony consisteney, tho covering skin only a littlo indurated. On the 
most prominent part, 3 inches below the eye, there is an ulcor 4 by 5 inches in diameter and only protruding a little over the surrounding parts. On the surface of the ulcer, which is covered by dry pus crusts, 3 fistulas open out, constantly discliarging a little yellow inodorous pus.

Treatment was commenced August 24 with a daily dose of 15 grams, and was continued until Oetober 7 , with proper intervals. By this time the fibrous part of the swelliug had shrunk considerably, leaving the rest hard like bone. The ulcer had healed up, but one of the fistulas was still discharging a little pus. Medicine was again administered for two weeks without any noticeable improvement as to the size of the swelling, but no more pus was discharged from the fistula, which shortly afterward healed up. The steer showed no symptoms of iodism during the entire treatment.

Post-mortem, November 29.-The bony swelling was spongy, but contained no pus and only a few centers of soft tissue. Two cords of white connective tissue extended from the contracted cicatrices on the surface toward the deeper parts. The lymph glands on the head were sound. No internal lesions. Carcass condemned.

\section{No. 39989.}

Red hornless steer, white star, white spot on right hip and left flank; received August 28.

The right side of the lower maxilla is enormonsly swollen. The deeper parts of the tumefaction are of a bony consistency, but are covered with a thick layer of fibrous tissue, which on the lower part is about three inches thick and is closely attacherl to the skin. At this place there is an ulcerating granuloma, 5 to 7 inches in diameter, protruding 3 to 4 inches over the thick swollen margins of the surrounding parts. (See picture of No. 545524.)

Treatment was commenced August 30 with a daily dose of 15 grams and was contimued until October 20, with proper intervals. The steer showed a pronounced iodism and the granuloma dried up and shrunk rapidly. When the treatment was stopped there was only left a contracted scar, the fibrous parts of the swelling had shrunk considerably, and afterward continued to shrink.

Post-mortem, November 29.-The bony swelling is covered by a thin layer of fibrous tissue, which on the lower part contains a few centers of soft red tissue, ranging in size from a pea to a bean. The iuternal tissues of the bone are spongy, with several pockets filled with soft tissue, but none filled with pus. The surrounding lymph glands are all sound. No internal lesions. Carcass was condemned.

\section{No. 48159.}

Black steer, white star, hornless ; received August 30 from Kansas City.

Immense fibrous bony swelling of left lower maxilla, with ulcerating granuloma 6 to 8 inches in diameter. (See No. 545525.)

Treatment was commenced September 1 with a daily dose of 15 grams and was continued until Norember 1, with proper intervals. Though the steer showed a pronounced iodism, the tumor showed no tendency to heal up or shrink; in fact, the large and frequent doses of medicine had very little effect on it. The granuloma remained unclianged in size and continued to discharge drops of pus through minute openings in the surface. The fibrous parts of the tumefiation shrunk almost entirely, nuaking the gramloma appear about twice as large as it was originally.

Post-mortem, November 29.-An incision through the granuloma showed this to consist of very soft red tissue, with ummerous pus foci, ranging in size from a pin head to a pea. The internal part of the bony swelling was spongy, containing numerous centers of soft red tissue, the largest being about the size of a waluut. The lymph glands on the head were sound. No internal lesion. Carcass was condemued. 
No. 130988.

lirrl steer, hornless, white spot on right shonlder; received September 5, 1892.

In sulhlaryugeal space on left side is located a fibrous tumor the size of a cocoaunt. The tumor was slightly flnctuating, and was opened with a knife. It inclosed a deeply sitnated abscess, which contained from 1 to 3 ounces of good yellow pus.

Treatment was commenced September 7 with a daily dose of 12 grams, and was rontinued until October 10, by which time the tumor had disappeared, and only leaving a fibrons thickening in the skin. About October 20 the steer was considered cilred.

Post-mortem, Norember 29. - No traces of the tumor left, except a slight induration of the skin. In the upper part of the right lung was located a tumor the size of a clinched fist. In the left lnng in the same place was a tumor the size of a walnut. Both tumors consisted of fibrous tissue, and contained several cavities filled with pus and granulomatous tissue. The microscopic examination proved them to be actinomycomas. 'The carcass was condemued.

\section{No. $4706 \%$}

Red dehorned steer; received August 16.

On the left cheek are located seven hard fibrons tumors, ranging in size from a hazelnut to a walunt. They are situated in the short connective tissue under the skin, and can only be moved slightly. In the submaxillary space is located another one the size of a double clinched fist.

Treatment was commenced August 18, with a daily dose of 10 grains, and was continned until October 1 with proper intervals. By this time the small tumors in the cheek had disappeared, each leaving a small fibrous thickening in the skin. The tumor underneath had shrunk to the size of a hen's egg. Abont October 20 the steer was considered cured.

Post-mortem, November 29.-No traces of the tumors left, except a sliglit induration in theskin mider the throat. In the upper part of the left lung were found three tumors about the size of a walnut. In the right lung a similar one the size of a hazelnut. All of them contained soft granulomatous tissne and pus. The microscopic examination proved the pus to contain actinomyces. The carcass was condemned.

\section{No. 130916 .}

Red steer, white face, horns; received August 30 from Kansas City.

Immense fibrous bony swelling of left lower maxilla, with nlcerating granuloma 6 to 8 inches in diameter. (See No. 545524.)

Treatment was commenced September 1 with a daily dose of 12 grams, and was continued until October 25 , with proper intervals. The steer showed symptoms of iodism. The grannloma slirunk considerably, bnt did not dry 11 ). The fibrons parts of the swelling were rednced.

Post-mortem, Norember 29.-The surface of the external granuloma is ulcerated. An incision throngl it slows that it consists of soft red tissne, with nmmerous jus foci the size of small peas. The fibrons covering on the bony swelling eontainer traces of soft tissne. The internal parts of the bone were spongy, with a few centers of soft tissne the size of liazelnuts. In the wall of the small intestines were located a few small hard nodules, containiug a green colored detritus mass, consisting mostly of limesalts. The microseopic exammation proved them not to bo actinomycotic lesions. No other lesions internally. Careass was condemned.

\section{No. $4.410 \%$,}

Red roan dohorned steer, white on left shonlder and brisket; recoived August 16. Immense fibrous, bony swelling of right lower maxilla, with nlcorating granuloma 6 to 7 inches in diameter. (Seo No. 515524.) 
Treatment was commenced August 18 with a daily dose of 12 grams and was eontinued until October 10, with proper intervals. The steer showed pronouneed ioclism. The granuloma dried up, but only shrmk a little. The fibrous parts of the swelling were rednced sontewhat in size. Medicine was again administered from October 20 to November 1, by which time the gramuloma had shrunk to the size of a hen's egg, and the fibrous parts had disappeared almost entirely.

Post-morlem, November 29.-The external granuloma, which is about the size of a walnut, is rather hard, having a dry surfare. It contains no pusfoci, but extends up into a cavity in the bony swolling the size of a goose egg. Under the peritoncal covering on the small intestines were located a few small hari nodules, containing a grecnish detritus mass with eonsiderable lime salts in it. No actinomyces was fonnd by the microscopical examination. On the surface of the liver was located an abscess the size of a hazelnut, filled with ycllow pus. This also was free from the actinomyces. The carcass was condemned.

$$
\text { No. 45\%2. }
$$

Red steer, deliorued; received September 5, 1892.

The right ramus of the lower maxilla below the first molar is swollen to the size of a double clinched fist. The skin covering it is thickened and indurated, being about 1 inch thick on the lower part.

The treatment was commenced September 7 , with a daily dose of 12 grams, and was continued nutil November 1 . The steer showed a pronounced iodism, but the tumor shrunk only very little.

Post-mortem, November 29.-The induration covering the bouy swelling contains a few centers of soft granulomatous tissue the size of hazeluuts. The internal parts of the bone were a little spongy, but contained no pus eavities. No lesious in the lymphatic glauds and no internal lesions. The carcass was conclemned.

\section{No. 1804 ?}

Red stecr, partly dehorned; received August 30 from Kansas City.

On the right side of the face, 1 ineh below the eye, is located a bony hard swelling the size of a clinched fist. The skin that covers the sweling is thick and indurated.

Treatment was commenced september 1 with a daily dose of 12 grans and continned until October 30, with proper intervals. The tumor remained almost unchanged, only the induration in the skin becoming thinmer.

Post-mortem, November 29.-The internal parts of the bony swelling were very spongy, with several pockets filled with soft granulomatous tissne, but without any pus. A cord of white connective tissue extended from the ecnter of the swelling down to the socket of the third molar in the upper jaw, which was loose. The lymph glands on the head were ail sound. No internal lesions. Careass condemned.

\section{No. 39978 .}

Dark red steer, horns; received August 28.

( $)$ the right side of the face a little below the eye there is a flat bony swelling as large as the palm of a hand, and protruding about one inch. The skin covering the swelling is a little indurated. In the submaxillary space is located a hard fibrous tunor the size of a double clinched fist lying loose in the connective tissue under the skin.

Treatment was commenced August 30 with a daily dose of 10 grams, and was continued until October 15 with proper intervals. The bony swelling remaincel unchanged, while the tumor in submaxillary space completely disappeared. 
Post-morlem, November 29.-The bony swelling contained a few centers with soft tissue, but 110 pus. No traces of the other tumor left. Lymph glands on head sound. In the left lung was a fibrous induration the size of a hen's egg, containing several small pockets filled with pus and soft tissue. Microscopical examination proved them to be actinomycomas. Carcass was condemned.

No. 48510 .

Red steer, hornless, white star; received September 5 .

Immense bony swelling of lower maxilla on right side with large ulcerating granuloma on lower end. Case similar to No. 48087.

Treatment was commenced September 7 with a daily dose of 15 grams and was continued until November 1 with proper intcrvals. The granuloma dried $\mathrm{np}$ and disappeared altogether, only leaving a large contracted eicatrix. The thick indurations which covered the bony swelling were reduced to a thin layer of connective tissue half an inch thick.

Post-mortem, November 29.-An incision througl the bony part of the swelling shows this to bespongy. In the lower part of it, just inside the scar left by the granuloma, there was a large sinus the size of a hen's egg, and filled with the characteristic soft red tissue with the small yellow spots in. No pus could be squeezed out of the cut surface. The rest of theswelling contained neither pus nor soft tissue. No internal lesions. Lymph glands on head sound. Carcass was condemned.

No. 48155.

Black steer, horuless, spotted, white star; received August 30 from Kansas City. All the left side of the face from the eye down to the nose is immensely swollen. The swelling is hard as bolle and the covering skin is thick and indurated. Two or three fistulas open out on the surface, discharging yellow pus containing actinomyces.

Treatment was commenced September 1 with a daily dose of 15 grams, and was continued until October 20 with proper intervals. By this time the tumor had shrunk to its capacity the fistulas had healed up, each leaving a small contracted scar which remained closed until the steer was killed six weeks later.

Post-mortem, November 29. - The skin is closely attached to the bony swelling. An incision through the bony swelling slows the internal tissue spongy, bnt containing neither pus nor soft tissue. The lymph glands on the head wcre sound and no internal lesions found. The carcass was condemned.

\section{No. 39972.}

Red steer, horns, received August 28, 1892; symptoms and treatment exactly like above number (48155).

Post-mortem, November 29.-The internal spongy part of the bony swelling eontained a pocket the size of a robiu's egr filled with pus. In the fibrous covering of the bone there werc a few centers of soft tissue the size of beaus. Lymph glands on head sound. No internal lcsions. Carcass condemned.

\section{No. 39953.}

Roan stecr, horns; received August 28.-On the right sirle of the face, 2 inches below the eye there is a hard bony swelling the size of a goose egg. In the loose conncctive tissue in the submaxillary space is located a hard fibrous tumor as largo as a clinched fist.

'Treatment was commenced August 30 with a daily dose of 10 grams, aud was continued until October 7, with proper intervals, By this time the tumor in the sub. 
maxillary space lad completely disappeared, while the bony swelling remained almost unchanged.

Post-mortem, Ninember 29.-No traces of the fibrons tumor left. Surrouuding lymph glands all sound. An incision through the bony swelling proved it to be spongy with numerous centers of soft tissue. No internal lesions. Carcass was condemned.

\section{No. 39955.}

White steer, rudiments of horns; received Angust 28.

Large fibrous, bony swelling of left lower jaw, with ulcerating grannloma 4 to 5 inches in diameter. (See No. 545524.) On the right side of the face a couple of inches below the eye there is a small, flat, bouy swelling.

Treatment was commenced August 30 with a daily dose of 12 grams and was continued until Octooer 25 , with proper intervals. The fibrous part of the tumefaction shrunk alınost entirely and the granuloma dried up and disappeared, leaving a contracted scar not larger than a silver dollar. The steer showed pronounced iodism.

Post-mortem, November 29.-No traces of actino in the thin fibrous cover of the bony swelling. 'Ihis one, however, contained several cavities filled with soft tissue and pus. The small bony swelling on the upper jaw was solid and contained no traces of the disease. The lymph glands on the head were sound. No internal lesions. Carcass was condemned.

\section{No. 531593.}

Spotted brown steer, dehorned, white star; received Angust 22.

Immense fibrous bony swelling of left lower maxilla, with ulcerating granuloma, 5 to 7 inches in diameter. (Case similar to No. 545524.)

Treatment was commenced August 24, with a daily dose of 12 grams, and was continued until October 25 , with proper intervals. The steer showed plain symptoms of iodism, and the fibrous parts of the tumor shrunk considerably. The granuloma did not dry up, but a piece of it, about one-third, was expelled by the shrinking of the fibrous surroundiugs. Medicine was again administered for two weeks, but without any noticeable improvement.

Post-mortem, November 29.-The thick induration in the skin on the bony swelling contained several centers of soft tissue the size of hazelnuts. The bony part of the swelling was spongy, with numerous pockets, filled with pus and soft tissue. The inside of the right lower maxilla was slightly affected in the sane way. The lymph glands on the head were sound. No internal lesions. Carcass contemned.

\section{No. 545543.}

Red steer, horuless; received August 16.

Immense fibrous bony swelling of right lower maxilla, with ulcerating granuloma 5 to 6 inches in diameter. (Case similar to No. 545524.)

Treatment was commenced Angust 18, with a daily dose of 12 grams, and was continued until October 25, with proper intervals. By this time the granuloma had dried up and disappeared, leaving a white contracted cicatrix. The fibrous parts of the swelling had also shrunk considerably. The steer showed pronounced rodism.

Post-mortem, November 29.-The bony swelling is covered by a thin layer of fibrous tissne. The internal tissue of the bone is spongy, with one large cavity the size of a clinched fist filled with soft tissue which, on the lower part, is covered by the scar left from the external granuloma. No pus can be squeezed out of the cut surface. The lymph glands on the head were all sound. No internal lesions. Carcass was condemned.

$14411-$ No. $2-5$ 
No. 45591 .

Red steer, rudiments of horns; receiver Angust 30 from Kansas City.

Swelling of facial hones, left sicle from eye to nose, with ulcer size of the palm of a hand (see No. 130915).

Treatment was commencer September 1 with a daily dose of 12 grams and was continued until Oetober 25 with proper intervals. The steer showed pronounced iodism. The ulcer dried up, only leaving a small cicatrix. The fibrous parts of the swelling shrunk almost completely, leaving the bony part covered by the slightly indurated skin.

Post-mortem, November 29.-The bony swelling was spongy and container several centers of soft tissue, but no pus. The lymph glands on head were sound. Small actinomycotic tumor in the lung. Carcass was condemned.

\section{No. 39951.}

Red steer, large horns, white stripe across forehead; received August 28, 1892.

Immense fibrous bony swelling of right lower maxilla. (See No. 545524.) Ulcerating granuloma, 4 to 5 inches in dianeter.

Treatment was conmenced Angust 30 with a daily dose of 15 grams and was continued until October 25 with proper intervals. The steer showed pronounced iodism, and the fibrous parts of the tunor shrunk considerable. The granuloma dried up and shrunk to the size of a walnut.

Post-mortem, November 29.-The fibrous part of the swelling contained several centers of soft tissue, especially in the lower part around the scar left from the granuloma. The remaining part of this was rather hard and dry. The internal tissue of the bone dyas spongy with a few centers of soft tissue and pockets filled with pus. Just inside the granuloma was a large cavity filled with soft tissue. The lymph glands on head were sonnd. No internal lesions. Carcass condemned.

\section{No. 39981.}

Speckled steer, dehorned; received Angnist 28.

Immense fibrous bony swelling of right lower jaw, with ulcerating grauuloma. (Case similar to No. 545524.)

Treatment was commenced August 30, with a daily dose of 15 grams, and was continued until November 1 with proper intervals. Thongh the steer showed a fair degree of iodism, the effect of the medicine on the tumefaction was scarcely noticeable. The granuloma only dried up around the edges, but continued to ulcerate in the central part of the surface, with a very fetid odor. The surrounding fibrous swelling shrunk somewhat, which made the granuloma appear more prominent than it did originally. In spite of the large and frequeut doses of medicine no further improvement was obtained.

Post-mortem, November 29. - The head had been removed by the butchers before examination. No internal lesions. Carcass was condemned.

\section{No. 39966.}

Light red steer, hornless; received $\Lambda$ ugust 28.

On the right lower maxilla, below the third molar, is loeated a hard bony swolling the size of a goose egg. The skin covering the swelling is thick aud indurated, and closely attached to the underlying tissue.

Treatment was commenced August 30, with a daily dose of 10 grans, and was continued until Oetober 15 with proper intervals. The first two wooks the swolling increased considerably in size, and becane a trifle larger than a clinehed fist. Tho dose was then increased to 12 grams, and later on to 15 grams, but in spito of this the 
steer, which weighed about 1,100 pounds only showed obscure symptoms of iodism. The growth of the tumor was ehecked, but it did not shrink any.

Post-mortem, November 29. - The head of the steer had bcen removed by the butchers before it was examined. No internal lesions. Carcass condemued.

No. 59805 .

Brindle steer, lorns; received August 30, from Kansas City.

Extensive swelling of facial bones on right side. (Casesimilar to No. 130915, but without granuloma).

Treatment was commenced September 1, with a daily dose of 15 grams, and was continued until November 1, with proper intervals. Steer showed a prononnced iodism, but the tumor only shrunk very little.

Post-mortem, November 29. - The skin covering the bony swelling was thick and in. durated. The internal tissue of the tumefaction spongy with numerous centers of soft tissue and pockets filled with pus. Lymph glands on head sound. No internal lesions. Carcass condemned.

No. 39959.

Red stcer, hornless, brindle head and neck; received August 28.

Immense fibrous bony swelling of left lower jaw. Ulcerating granuloma 6 to 7 inches in diameter, and 2 to 3 inches thick. (Case similar to No. 545524.)

Treatment was commonced August 30 with a daily dose of 15 grams, and was continued until October 25, with proper intervals. The steer showed a pronounced iodism. The fibrous part of the tumor shrunk almost entirely. Granuloma dried up and was drawn in. By November 12 it had disappeared, only leaving a large white contracted cicatrix.

Post-mortem, November 29.-The fibrous parts of the swelling around the cicatrix contained a few centers of soft tissuc the size of hazel nuts. The bony swelling was spongy with a few pockets containing yellow inodorous pus. Surroundiug lymph glands were sound. No internal lesions. The carcass was condemned.

No. 48043.

Red steer, white star, hornless; receiverl August 30, from Kansas City.

Bony swelling of the left side of the face, from the eye to the nose. (Case similar to No. 130915.)

Treatment was commenced September 1, with a daily dose of 12 grams, and was continued until Oetober 25th, with proper intervals. The steer showed the usual symptoms of iodism. The tumor remained unchanged in size, only the thick indurated skin covering it became a trifle thinner.

Post-mortem November 29.-No traces of actino in the indurated skin. 'The internal tissue of the bony swelling was spongy, and contained a few centers of soft tisswe the size of hazel nuts. The lymph glands on the head were sound. No internal lesions. Carcass was condemued.

No. $4588 \%$.

Red steer, hornless, white star; received September 5.

Immense fibrous bony swelling of left lower maxilla, with ulcerating granuloma 6 to 7 inches in diameter. (See 545524.)

Treatment was commenced September 7 with a daily dose of 12 grams, and was continned nntil October 25 with proper intervals. The steer showed a prononnced iodism and the granuloma soon began to dry up and shrink, and finally disappeared entirely. The thick fibrous induration in the skin was reduced considerably. 
Post-mortem, November 29.-The fibrous tissue on the lower part of the tumefaction inclosed a few small eenters of soft tissue. The internal parts of the bony swelling were spongy, with several pockets and eenters of pus and soft tissue. One of these just inside the scar left by the external granuloma was the size of a hen's egg. 'The lymph glands on the head were sound. No internal lesions. Careass was eondemned.

\section{No. 545544 .}

Dark red steer, white faee and rump; reeeived Angust 16.

Large fibrous bony swelling on left lower maxilla with ulcerating granuloma 4 to 5 inches in diameter. (See No. 545524.)

Treatment was eommeneed Angust 18, with a daily dose of 12 grams, and was eontinued until Oetober 1 with proper intervals. The steer showed no symptoms of iodism and the tumor improved only slowly. The granuloma dried up and shrunk slightly, and so did the fibrous parts of the tumefaetion. Medicine was again administered from Oetober 15 to October 30 , by which time the granuloma had disappeared and the swelling had been reduced considerably.

Post-mortem, November 29.-The internal parts of the swelling were spongy and contained several eavities filled with soft tissue, one of which, just inside the eieatrix left by the external granuloma, was the size of a walnut. The lymph glands on the head were sound. No interual lesions. Careass condemned.

\section{No, 39983.}

Red steer, white star, hornless, white spot on shoulder; received August 28, 1892.

Large fibrous bony swelling of right lower maxilla with ulcerating granuloma 4 to 5 inches in diameter. (See 545524.)

Treatment was commeneed August 30 with a daily dose of 12 grams and was continued until Oetober 25 , with proper intervals. The steer showed a pronouneed iodism, the granuloma dried up and disappeared, and the fibrous part of the swelling was redueed considerably.

Post-mortem, November 29.-The dense fibrous tissue surrounding the sear left by the granuloma contained a few centers of soft tissue, the internal tissues of the bony swelling were spongy with several pockets filled with soft tissue, one of them corresponding to the eieatrix, being the size of a hen's egg. No internal lesions. Carcass was condemned.

\section{No. 131026.}

Light roan steer, horns; received September 5.

\section{No. 131030}

Speekled steer, red head and neek; received September 5.

Large, fibrous, bony swelling of right lower maxilla with uleorating grannloma 2 to 3 inches in diameter. Treatment was eommenced September 7 with a daily dose of 12 grams, and was continued until October 28, with proper intervals. Both steers showed a pronouneed iodism. The granuloma dried up and disappeared. and the fibrons parts shrunk eonsidcrably.

I'ost-mortem November 29.-No. 131026. The fibrous eovering on the bony swelling showed no traces of actinomycosis. The interual tissue of the bone was spongy, and contained only a few small centers filled with soft tissue. The lymph glands on the head were sound. No interual lesions. As the steer was in a very poor eondition the careass was eondemned.

No. 131030. The fibrous part of the swelling contained several eenters of soft tissue. The internal parts of the bone were spongy, and eontained several large pock- 
ets with soft tissue, especially just inside the sear left by the external granuloma. No internal lesions. Carcass was condemned.

Though both steers showed exactly same external lesions, and received exactly the same treatment as to dose of medicine and length of time administered, and though they both apparently improved equally, the post-mortem examination slowed that the medicine had had a much stronger effect on the internal parts of the tumefaction on the first one than on the other.

\section{No. 130905.}

Red speckled steer, horus; received August 30 from Kansas City.

Fibrous bony swelling of the left side of the face from eye to nose. (See No. 130915.)

Treatment commenced September 1 with a daily dose of 12 grams and was contiuued until October 25, with proper intervals.

The steer showed a pronounced iodism and the fibrous parts of the tumefaction shrunk considerably, leaving the bony swelling covered by the slightly indurated skin.

Post-mortem, November 29.-The thin fibrous covering of the bony swelling contained a few small centers of soft tissue. The internal tissue of the bone was spongy and contained several pockets filled with soft tissue and pus. The lymph glands on the head were sound. No internal lesions. Carcass was condemned.

\section{No. 48089.}

Red steer, hornless; received August 30 from Kansas City.

Immense swelling of the facial bones on right side from eye to nose. The covering skin is very thick and indurated. On the most prominent part, 3 inches below the eye, is located an ulcer, 4 to 5 inches in diameter. (Case simil ar to 130915.)

Treatment was commenced September 1 with a daily dose of 15 grams and was continued until October 25 , with proper intervals. The steer showed a pronounced iodism and the fibrous parts of the swelling were reduced considerably, while the ulcer dried up and became covered with epidermis, leaving three small fistulous openings, which soon afterwards closed up.

Post-mortem, November 29. - Slight traces of soft tissue centers left in the indurated skin. The internal tissues of the bony swelling were spongy, but contained only a very few cavities, filled with soft tissue. The lymph glands on head were sound. No internal lesions. Carcass was coudemned.

\section{No. $545 \% 64$.}

Red steer, white star, small horns; received August 16, 1892.

The right lower maxilla is swollen from the mouth to the neck. The swelling is hard as bone near the mouth, while the posterior parts are soft.

Treatment was commenced August 18 with a daily dose of 10 grams, and was continued until September 12, when the soft parts of the swelling had disappeared, leaving a hard, bony tumor the size of a clinched fist on the lower edge of the under jaw near the chin.

The treatment was again commenced October 10 and continued until November 15 , with proper intervals, but apparently without further improvement in the size of the tumor. The steer being in a rather poor condition was not killed with the first lot slaughtered on November 29.

Post-mortem, January 27, 1893.-In right lower maxilla is a bony swelling the size of a goose egg. A section through this shows it to consist of compact bone, which around the dental canal is spongy, and contained a little pus. The fifth molar was decayed. No internal lesions. Carcass passed as sound. 
No. 130926.

White speckled cow, short horns; received September 12, 1892.

In the sublaryngeal space, on the right side, is located a hard, fibrous tumor the size of a double clinched fist lying loose in the connective tissue under the skin.

Treatment was commenced September 14 with a daily dose of 10 grams, and was continued until October 20, when the tumor had shrunk to the size of a hen's egg.

Medicine was again administered from November 13 till January 25 with one dose twice a week, which removed the last traces of the tumor.

Post-mortem, January 27, 1893.-No traces of the tumor left. A few small hard nodules in the wall of the small intestines. Lungs and liver sound. Carcass passed as sound.

\section{No. 545750 .}

White speckled steer, with horns; received August 16, 1892.

The right upper maxilla a little below the eye is swollen considerably. The covering layer of the swelling is fibrous, the deeper parts bony. In sublingual space is located a hard, fibrous tumor the size of a clinched fist.

Treatment was commenced August 18 with a daily dose of 10 grams, and was continued until October 2 at proper intervals. By this time the tumor in sublingual space had disappeared, while the other one had grown considerally smaller. From November 1 until January 25 medicine was given about twice a week, only reducing the swelling a little.

Post-mortem, January 27, 1893.-No traces of actino in the fibrous covering on the bony swelling. This, however, is completely filled with actinomycotic new formations which extend into the maxillary sinus, and from here into the nasal fossa, where it may be felt by inserting a finger through the nostril.

Pleuritic adhesions in right lung. Internal organs sound. Carcass condemned.

$$
\text { No. } 480 \% 1 .
$$

Red-spotted steer, hornless; received Angust 30, 1892, from Kansas City.

In right parotid region is located a hard, fibrous tumor the size of a goose egg, and a similar one is seen in sublaryngeal region on same side.

Treatment was commenced September 1, with a daily dose of $10 \mathrm{grams}$, and was continued until October 10, with proper intervals, by which time the steer was regarded as cured.

Post-mortem, January 27, 1893.-Notraces of the tumors loft. A few small hard nodules in the wall of the small intestines. Anterior lobes of both lungs adherent to pericardium. Carcass passed as sound.

\section{To. 480496 .}

Dark red steer, dehorned; received August 16, 1892.

On both cheeks are located four to five small hard tumors the size of walnuts, and in sublaryngeal space one the size of a double clinched fist.

Treatment was commenced August 18 with a daily dose of 10 grams, and was continueit until October 7, with proper intervals, by which time all the smaller tumors had disappeared and the largest one had shrunk to the size of a hen's egg. Medicine was again administered from November 1 to November 20, and soon afterwards the steer was curcd.

Post-mortem, January 2\%, 1893. - No traces of the tumors left. A fow small nodules in the wall of the small intestines. Other internal organs sound. Carcass passed as sound. 
No. 6 .

No tag. Dark red steer with horns, white bclly, white spot on flank.

The steer has been under treatment for the last three months, though not steadily.

It had a fibrous tumor under the throat as big as a child's head.

At the post-mortem examination on January 27, 1893, no traces of the disease were found, and the carcass was passed as sound.

\section{No. 25875 .}

White steer, hornless; received September 12, 1892.

In left parotid and sublaryngeal region is located a fibrous tumor the size of a child's head. The covering skin is connected with the tumor.

Treatment was commenced September 14 with a daily dose of 12 grams, and was continued until October 20, when the tumor had shrunk to the size of a goose egg.

From November 20 to January 25 medicine was again administered. The steer showed a pronounced iodism under the whole treatment.

Post-mortem, January 27, 1893.-No traces of the tumor left, except a fibrous induration in the skin where the tumor had been located. No interual lesions. Carcass passed as sound.

No. $5913 \%$.

Red Hereford steer, white head and shoulder; received August 28, 1892.

In left parotid region are located two tumors, the size of a hen's egg. Under the throat, a similar one, the size of a double-clinched fist.

Treatment was commenced August 30, with a daily dose of 12 grams, and was continued until October 25 , when all three tumors disappeared, with the exception of a little induration in the skin.

Post-mortem, January 27, 1893.-Submaxillary glands a little swollen, but sound. The skin in parotid region and under the throat fibrous and indurated. No interual lesions. Carcass passed as sound.

\section{No. $4595 \%$.}

Red steer, white breast and back; received September 5, 1892.

In sublaryngeal space on right side is located a fibrous tumor, the size of a child's head.

Treatment was commenced Scptember 7, with a daily dose of 12 grams, and was continued until Oetober 10, with proper intervals. By this time an abscess had formed, which was opened, and four to five ounces of good yellow pus evacuated. The tumor had shrunk considerably in size. Medicine was again administered from November 15 till January 20. The steer showed pronounced iodism, and the tumor shrunk rapidly.

Post-mortem, January 27, 1893.-No traces of the tumor left except a 1-inch thick fibrous induration in the skin. No internal lesions. Carcass passed as sound.

\section{No. 130914.}

Red steer, white face and belly, with horns; received Angust 30, from Kansas City.

In sublaryngeal space is located a hard, fibrous tumor, the size of a child's head. Inside the lips are located several small granulomas.

Treatment was commenced September 1, with a daily dose of 12 grams, and was continued until October 15 , with proper intervals. The granulomas in the mouth disappeared after two week's treatment, and the tumor nuder the throat shrunk rap- 
idly, the steer showing a pronounced lodism. Medicine was again administered from November 1 until November 20, when the steer was regarded as cured, though it was in a rather poor condition.

Post-mortem, January 27, 1893.-Postpharyngeal glands indurated. No internal lesions. Carcass passed as sound.

No. $5337 \% 0$.

Red steer, white forehead and jaws, hornless; received August 22, 1892.

In right parotid region, just below the ear, is located a fibrous tumor the size of a clinched fist. In sublaryngeal space a similar tumor, the size of a child's head.

Treatment was commenced August 24, with a daily dose of 12 grams, and was continued until October 20, with proper intervals. By this time the tumor in parotid region had disappeared, and the other one slirunk to the size of a goose egg. Medicine was again administered from November 9 until January 10, when this tumor had almost disappeared.

Post-mortem, January 27, 1893.-No traces of the tumor left except a fibrous induration in the skin. No internal lesions. Carcass passed as sound.

No. 39995.

Roan steer, hornless; received August 28, 1892.

In sublaryngeal region is located a hard, fibrous tumor the size of a cocoanut.

Treatment was commenced on August 30, with a daily dose of 12 grams, and continued until October 20. The steer showed no symptoms of iodism, and the tumor remained almost unchanged. Treatment was continued from November 10 until January 20, but the tumor only shrunk very little.

Post-mortem, January 27, 1893.-Under the throat is located a fibrous tumor, the size of a child's head, containing several large centers of soft tissue and pus. The lymph glands on head sound. In the wall of the small intestines were located a few small nodules. Carcass condenined.

\section{No. 39969.}

Strawberry roan steer, dehorned; received August 28, 1892.

In left sublaryngeal space is located a fibrous tumor the size of a donble clincher fist, and on the middle of the left cheek is a similar one the size of a goose egg.

Treatment was commenced August 30, with a daily dose of 10 grams, and was continued until October 15, with proper intervals. By this time the tumor on the cheek had almost disappeared, and the other one grown considerably smaller. Medicine was again administered from November 12 till Jannary 15, when the steer was regarded as cured. The steer showed ouly slight symptoms of iodism.

Post-mortem, January 27, 1893.-No traces of the tumor's left. No internal lesions. Carcass passed as sound.

No. 545511 .

Light red steer, dehorned; received Angist 16, 1892.

In sublaryngeal space, on left side, is located a tumor the size of a child's head. On the lower part of the tmmor extends through the skin an ulcerating granuloma, the size of a hen's egg.

'Treatment was commenced $\Lambda$ ugust 18 , with a daily dose of 12 grams, and was contimned mntil October 7, with proper intervals. The steer showed no symptoms of iodism, and the tmnor shrmnk only slowly, while the grannloma showed no inclination to dry up. From November 9 tho treatment was again commenced, and this time with better effect. 'The dose was increased to 15 grams. The tumor shrmk 
completely, and the granuloma dried up. January 5 the treatment was discontinued, and $t$ wo wecks later the steer was regarded as cured.

Post-mortem, January 27, 1893.-No traces of tumor left, except a white cicatrix in the skin. Left postpharyngeal lymph gland considerably swollen, containing sevcral small pus centers. No internal lesions. Carcass passed as sound.

\section{No. 130921.}

Red steer, hornless; received September 5, 1892.

In right parotid region is located a fibrous tumor the size of a double-clinched fist, a similar one under the throat, the size of a child's head, and another in left parotid region, the size of a goose egg.

Treatment was commenced September 7, with a daily dose of 12 grams, and was continued until November 10, with proper intervals. The steer showed a pronounced iodism, and the tumors shrunk rapidly. When the treatment was discontinued, there only remained slight traces of the tumors, which disappeared soon afterwards.

Post-mortem, January 27, 1893.-No traces of tumors left. No internal lesions. Carcass passed as sound.

\section{No. 39965.}

Red steer, white face and stomach; received August 28, 1892.

In right parotid region is located a hard, fibrous tumor, the size of a double-clinched fist, lying loose in the connective tissue under the skin.

Treatment was commenced August 30, with a daily dose of 10 grams, and was contimued until October 20, with proper intervals. By this time the tumor had almost disappeared, the steer showing a pronounced iodism. November 17 a new tumor began to form on the opposite side, and medicine was again administered until December 20 , when the steer was regarded as cured.

Post-mortem, January 27, 1893.-No traces of the tumor left. No internal lesions. Carcass passed as sound.

\section{No. 25364.}

Dark red steer, horns; received September 12, 1892.

Under the throat on left side is located a liard, fibrous tumor the size of a big rhild's head, lying loose in the connective tissue under the skin.

Treatment was commenced September 14 with a daily dose of 12 grams and was continued until October 20. The steer showed only slight symptoms of iodism and the tumor shrank slowly. November 10 the tumor had the size of a clinched fist, and medicine was again administered until December 12, when only a small bunch was left under the skin, which disappeared soon afterwards.

Post-mortem, January 27, 1893.-No traces of the tumor left except a thick fibrous induration in the skin. No internal lesions. Carcass passed as sound.

\section{No. 131004.}

Dark-red steer, roan face, hornless; received September 5, 1892.

In sublaryngeal space is located a tumor the size of a child's head. On the lower end of this there is a granuloma about 2 inches in diameter.

Treatment was commenced on September 7 with a daily dose of 12 grams and was continued until October 7, when the granuloma had dried up and the tumor shrunk to the size of a goose egg. November 5 the granuloma began to grow again, and medicine was arministered until Dccember 12, when the steer was regarded as cured. The animal showed a pronounced iodism under the treatment. 
P'ost-mortem, January 27, 1893. - No traces of tumor left, except a white contracted cicatrix on the slightly indurated skin. No internal lesions. Carcass passed as sound.

No. 19.

No tag. Red steer, horns.

On post-mortem examination, January 27, 1893, there was found no trace of actinomycosis, except a fibrous induration in the skin under the throat.

NTo. 20.

No tag. Same as above.

Both carcasses passed as sound.

No. 534838 .

Dark-red steer, white forehead, hornless; received Angust 16, 1892.

In left parotid region, jnst below the ear, is located a hard, fibrous tumor the size of a hen's egg, and below this one, in the sublaryngeal region, another the size of a child's head.

Treatment commenced August 18 with a daily dose of ten grams and was continned until October 23, with proper intervals. The steer showed a slight iodism, and when the treatment was stopped the smaller tumor had disappeared and the larger one shrunk to the size of a walnut, but covered by a very thick, fibrons induration in the skin. Medicine was therefore commenced again on November 10 and continued until December 20, with two doses per week. By this time the steer was regarded as cured.

Post-mortem, January 27, 1893.-The skin in the parotid region slightly indurated. No traces of the tumors left. No internal lesions. Carcass passed as sound.

No. 47988.

Red steer, hornless; received Angust 30 from Kansas City.

Flat, fibrous, bony swelling on left npper maxilla, with a granulating ulcer 3 inches in diameter.

Treatment was commenced September 1 with a daily dose of 12 grams and was continued until October 20, when the fibrous parts of the swelling had disappeared and the ulcer dried up, leaving only a small white cicatrix. November 10 this broke open again, and medicine was again administered for four weeks. The steer showed a pronounced iodism under the treatment.

Post-mortem, January 27, 1893.-The superior maxilla slightly swollen. The maxillary sinus contains a little granulomatous tissue of a rather solid character, and not containing any pus. The lymph glands on head sound. No internal lesions. Carcass passed as sound.

\section{No. 68000 .}

Red steer, white face, horns; received Angust 22, 1892.

In left parotid region, just below the ear, is located a hard, fibrous tumor the size of a double clinched fist. In submaxillary space is a similar one thesize of a child's head.

Treatment was commenced August 24 with a daily dose of 12 grams and was continued until October 7, with proper intervals. By this time an abseess had formed in the tumor below the ear, which was opened, and a few ounces of pus evacnated, after which the tumor rapidly disappearerl. As the steer got sore fcet from standing on the hard floor, he was turned out, and not taken in again before October 25. By this time the tumor under the jaw had shrunk to the size of a hen's egg and medi- 
rine was again administered two weeks. Shortly afterwards the steer was regarded as cured.

I'ost-mortem, January 27, 1893.-No traces of the tumors left. Nointernal lesions. Carcass passed as sound.

\section{No. 39958.}

Red steer, white star, hornless; received August 28, 1892.

In sublaryngeal space on left side is located a fibrous tumor the size of a large child's head. The superior maxilla on same side is a little swollen.

Treatment was commenced on August 30 with a daily dose of 12 grams and was continued until October 28, with proper intervals. The steer showed only slight symptoms of iodism, but the tumor had by this time shrunk to the size of a goose egg, while the swelling on the upper jaw remained unchanged. From November 15 until December 29 medicine was again administered in doses, two or three times a week, and the remaining part of the tumor disappeared slowly.

Post-mortem, January 27, 1:393. - The superior maxilla above the third molar slightly swollen. By opening the maxillary sinus is seen a bony new formation, the form and size of a cherry, extending from the socket of the molar up into the sinus. It contained granulomatous tissue, but no pus. No traces left of the tumor under the jaw. No interual lesions. Carcass passed as sound.

\section{No. 25.}

No tag. Red steer, white star, horns.

Left lower maxilla swollen to the size of a double clincherl fist.

At the post-mortem examination on January 27 the swelling prover to be spongy, filled with granulomatous tissue and pus. In the wall of the small intestines were a few small nudules. The carcass was condemned.

No. 39968.

Black steer, white star, hornless; received August 28, 1892.

In left sublaryngeal region is located a hard, fibrous tumor the size of a child's head.

Treatment was commenced on August 30 with a daily dose of 12 grams and was continued until October 15, with proper intervals The steer showed a pronounced iodism, and the tumor shrunk until about one-third of its original size. From November 10 until January 15 medicine was given about twice a week, and the tumor disappeared completely.

Post-mortem, January 27, 1893.-No traces of the tumor left. A few small nodules in the wall of the small intestines. The carcass was passed as sound.

\section{No. 39973.}

Red speckled steer, horns; received August 28, 1892.

In left parotid and sublaryngeal region is located a large, rather soft tumor the size of a cocoanut. On the left cheek there is a very hard, fibrous tumor the size of a hen's egg.

Treatment was commenced on August 30 with a daily dose of 12 grams and was continued until October 20, with proper intervals. The little, hard tumor on the cheek disappeared remarkably fast, in about two or three weeks, and the larger one also shrunk considerably, the steer showing a well-pronounced iodism. From December 5 until January 20 medicine was again administered and the tumor shrunk to the size of a hen's egg.

Post-mortem, January 27, 1893.-In left parotid region is found a fibrous induration the size of a hen's egg. It contained traces of soft tissue. No internal lesions. Carcass passed as sound. 
No. $13101 \%$.

Dark red steer, horns, received September 5, 1892.

In sublaryugeal region, on the left side is located a hard, fibrous tumor, the size of a double clinched fist. On the lower end of it there is a white contracted scar, 3 to 4 inches in diameter.

Treatment was commenced September 7, with a daily dose of ten grams, and continued until October 20. The tumor shrunk to about half its original size, but the thick indurations in the skin did not improve very much. From December 5, to January 15, medicine was given every second day, and the tumor shrunk constantly but slowly.

Post-mortem, January 27, 1893.-The skin under the throat indurated and about 1 inch thick. No traces of the tumor left, no internal lesions. Carcass passed as sound.

\section{No. 59146.}

Red steer, white brisket, hornless; received August 28, 1892.

In sublaryngeal region on right side is located a fibrous tumor the size of a child's head.

Treatment was commenced August 30, with a daily dose of 12 grams, and was continued until October 15. The steer showed very little iodism, and the tumor only shrunk to about half the original size. From November 10 until January 15 medicine was given about every second day, the tumor going down slowly.

Post-mortem, January 27, 1893.-In connective tissue under the throat is located a fibrous tumor the size of a small hen's egg. It contains a few centers of soft tissue of a rather dense character. The left sublaryngeal lymph-gland swollen to about double its size, containing several small pus foci. A few small nodules in the wall of the small intestines. No other internal lesions. Carcass passed as sound.

No. 25886 .

Red steer, white spot on each shoulder, horns; received September 12, 1892.

In sublaryngeal region, on right side, is located a hard, fibrous tumor the size of a cocoanut. From its lower surface depends a cylindrical granuloma, 3 inches thick, covered with thin epidermis, and 5 to 6 inches long.

Treatment was commenced on September 14, with a daily dose of 12 grams. The fibrous tumor shrunk very rapidly, but the granuloma remained almost unchanged. On November 20 the large fibrous tumor had disappeared completely, leaving the granuloma hanging loose from the skin, with no connection with the underlying tissue. It had by this time shrunk about 3 inches, and had the form and size of a goose egg, attached with the broad cnd to the skin, and was now of an almost fibrous character.

Post-mortem, January 27, 1893.-No notes taken about the granuloma, which has been left in the skin and overlooked. Lymph gland on head sound. No internal lesions. Carcass passed as sound.

No. 131033.

Red steer, white star, and spot on shoulder and hip, horus; received September 5,1892 .

In sublaryngeral space on right side, is located a tumor the size of a double clinched fist.

Treatment was commenced on September 7, with a dose of $10 \mathrm{grams}$, and was continued until October 15, when the tumor had disinpeared. About Oetober 25; the respiration became accompanied by a snoring sound. The breathing was oxtremely diffienlt, the steer's appetite failed, and it lost rapilly in Hesh, and stood 
with the head stretched forward, with staring ejes. Medicine was at once administered in doses of 15 grams a day, and with good success. In less than a week the respiration became almost normal, the steer regained its appetite, and soon picked up in flesh again. Above the pharynx could be felt a tumor the size of a goose egg. Medicine was then given in doses of 10 grams about every second day, and December 10 th the steer was regarded as cured.

Post-mortem, January 27, 1893.--Right postpharyngeal lymph gland swollen to alout three times its original size, containing a few small pus foci. No internal lesions. Carcass passed as sound.

No. 399S2.

Roan steer, white brisket, hornless; received August 28, 1892.

In the loose connective tissue in the submaxillary space, are located four hard, fibrous tumors, the size of goose eggs. Two similar ones of the same size are located in the left parotid region.

Treatment was commenced on August 30, with a daily dose of 12 grams, and was continued until October 20. The steer showed no symptoms of iodism and the tumors had only shrunk a little. From November 12 the medicine was given again, and the tumors now improved faster. January 15 , the steer was regarded as cured.

Post-mortem, January 27, 1893.-No traces of the tumors left. No internal lesions. Carcass passed as sound.

No. 33.

No tag, N. M. Spotted dehorned steer.

Post-mortem, January 27, 1893.-In the left parotid'region was located a fibrous tumor the size of a double clinched fist. It was closely covered by the skin and contained several centers of soft granulomatous tissue, out of which pus drops might be squeezed.

No internal lesions. Carcass condemned.

No. 39970.

Dark red steer, horns; received August 28, 1892.

In sublaryngeal space, on right side, is located a fibrous tumor the size of a child's head, on the lower surface of which is located a granuloma 3 to 4 inches in diameter. In parotid region on same side there is a similar one, the size of a goose egg.

Treatment was commenced on August 30 with a daily dose of 12 grams and was continued until October 28 , but with little success. The steer showed no symptoms of iodism, and the tumors remained almost unchanged, and the granuloma showed no tendency to dry up. Medicine was again administered from November 10 until January 15, but the tumors only shrunk to about half their original size and the granuloma became a little smaller.

Post-mortem, January 27, 1893.-In sublaryngeal region is located a hard, fibrous tumor the size of a double clinched fist, containing numerous pockets and centers filled with soft granulomatous tissue and pus. In parotid region a fibrous induration the size of a walnut. Lymph glands on head sound. No internal lesions. Carcass condemned.

\section{No. 35.}

No tag, N.M. Red dehorned steer, white star in forehead, stripes on shoulder, white belly.

Post-mortem, January 2\%, 1893.-In the sublaryngeal region is located a fibrous induration the size of a clinched fist, containing a few centers of granulomatous tissue, of a rather solid character, not containing pus. No internal lesions. Carcass passed as sound. 


\section{No. 39954 .}

Red steer, hornless; received August 28, 1892.

In left parotid region is located a hard, fibrous tumor the size of a clinched fist. It is open on the most prominent point and pus flows out. Under the throat, on the right side, is a similar tumor, the same size.

'Treatment was commenced on August 30 with a daily dose of 10 grams, and was continned until Oetober 20, when the steer was regarded as cured. The animal showed a pronouneed iodism.

Post-mortem, January 2\%, 1893.-No traces of the tumor left. No internal lesions. Carcass passed as sound.

\section{No. $3996 \%$.}

Red steer, white star, white belly, hornless; received Angust 28, 1892.

In the submaxillary space are loeated five tumors, the largest the size of a goose ega, the smallest the size of a walnut.

Treatment was eommeneed on August 30, with a daily dose of 12 grams, and was continned until Oetober 20, when the tnmors had slirunk to half their original size.

Erom November 12 until Deeember 30 medicine was given twice a week, and the tumors disappeared completely.

Post-mortem, January 2\%, 1893.-No traces of the tumors left. No internal lesions. Carcass passed as sound.

\section{No. 39971.}

Brindle steer, white forehead, horns; received August 28, 1892.

Left superior maxilla is slightly swollen and eovered by an ulcer 3 inches in diameter.

Treatment was commenced on August 30, with a daily loss of 10 grams, and was continued until October 15 with proper intervals. By this time the ulcer had dried up, and only left a little eontraeted scar. From November 12 until Jamuary 15, medicine was given twice a week, apparently without further improvement.

Post-mortem, January gr, 1893.-The-skin covering the upper maxilla a little indurated. The maxillary sinus contained an aetinomycotic new formation the size of a hen's egg, but of a rather fibrous character, and not containin $\tilde{\sigma}_{6}$ any pus. No internal lesions. Carcass passed as sound.

No. 40.

No tag. N. M. Red-roan, dehorned steer.

Post-mortem, January 2\%, 1893.-Left upper maxilla slightly swollen and corerel with a layer of fibrous tissue. Small new formations in maxillary sinus. A few small nodules in wall of small intestines. Careass passed as sound.

\section{No. 39986 .}

Red steer, white face and back, hornless; received $\Lambda$ ugnst 28, 1892.

In the intermaxillary space are located three hard, fibrous tumols the size of goose eggs.

Treatment was eommenced $A$ unust 30 , and was eontinued until Oetober 14, when all three tumors had disappeared. 'The steer showed a pronouneed iodism and lost in condition under the treatment.

I'ost-mortem, January 2\%, 1893.-The skin covering the submaxillary space slightly induraterl. Lymph glands on hearlsound. No internal lesions. Careass passed as sound. 
No. 25855 .

Red-speckled steer, hornless; recoived September 12, 1892.

The sub and intermaxillary region is filled with an oblong fibrous tumor formation, closely attached to the covering skin.

Treatment was commenced September 14, with a laily dose of 12 grams, and was continued until October 25, wlien the swelling had almost disappeared. One of the sublingual glands could then be felt swollen to the size of a hen's egg. Medicine was given from November 10 to November 25, when the steer was regarded as cured.

Post-mortem, January 27, 1893.-No traces of the swelling left. No interual lesions. Carcass passed as sound.

\section{No. 45879.}

Red steer, hornless, white shoulders and hips; received September 5, 1892.

In sublaryngeal region are locateil threc distinet fibrous tumors in close conncction, together the size of a cocoanut. On outside of the largest one is an ulcerating granuloma 3 inches in diameter.

Treatment was commenced on September 7 with a daily dose of 12 grams, and was continued until October 27. The steer only showed slight symptoms of iodism, but the tumors shrunk slowly, and the ulcer dried up. From November 12 until January 17 medicine was administered, and the tumors disappeared, only leaving the covering skin hard and indurated with a white contracted cicatrix thereon.

Post-mortem, January $27,1893 .-$ No traces of the tumors left except the induration in the skin. No internal lesions. Carcass passed as sound.

No. 44 .

No tag. Red steer with white sloulders and belly.

Post-mortem, January 2\%, 1893.-In left parotid region is a fibrous tumor the size of a double clinched fist, almost entirely consisting of white fibrons tissue, with a few deposits of soft tissue. On the ontsicle of it is located a clried-up granulona, the size of a hen's egg.

The carcass was passed as sound.

No. 533794 .

Red steer, white star, and white on hips; received Angust 22, 1892.

In left parotid region is located a hard, fibrous tumor the size of a clinched fist. From this extends a softer swelling filling the whole sublaryngeal space, ascending on the right side up into the parotid region toward the ear.

Treatment was commenced on August 24, with a daily dose of 12 grams, and was continued until October 12. By this time the soft swelling in the sublaryngeal space had almost disappeared, while the tumor on the left side still remained, thongh it had shrunk a little. From November 9 until December 20 medicine was given regularly, and from then until Jannary 20 every second day. The tumor beneath the left ear did not disappear entirely.

Post-mortem, January 2\%, 1893. - In left parotid region is a fibrons tumor the size of a walnut, containing a small amount of pus. In the surrounding tissue were located a few small centers of soft tissue. In the walls of the sinall intestines were located a few small, hard nodules. The carcass was passed as sound.

No. 534105.

Dark-red steer, lehorned; received August 22, 1892.

In the submaxillary and sublaryngeal space is located an immonse, soft, undulating tumefaction the size of a half-peck measure. On the right cheek, outside the third molar, is a hard, fibrous tumor the size of a clinched fist, 
Treatment was commenced August 24 and continued until October 25, with a daily dose of 12 grams. The steer showed a pronounced iodism and the tumefaction inproved fast. By this time the tumor on the cheek had almost disappeared, while the swelling underneath was not one-third its original size. From November 11 until January 20 the medicine was given two to three times a week, and the head of the steer almost regained its normal appearance.

Post-mortem, January 27, 1893.-In the sublaryngeal space is a soft, fibrous induration containing a number of small fibrous nodules, varying in size from a pea to a bean, containing no pus, but a small amount of soft tissue. Lymph glands on head sound. No internal lesions. Carcass passed as sound.

No. 131038 .

Brown steer, horns; received Sepiember 5, 1892.

In right parotid region is located a hard, fibrous tumor, the size of a double clinched fist.

Treatment was commenced on September 7, with a daily dose of 10 grams, and continued until October 25, with proper intervals. The steer showed a pronounced iodism and the tumor disappeared al together.

Post-mortem, January 27, 1893.-No traces of the tumor left. Lymph glands on head sound. No internal lesions. Carcass passed as sound.

\section{No. 45973.}

Black steer, speckled back and breast, dehorned; received September 5, 1892.

In left parotid region is located a hard, fibrous tmmor the size of a goose egg. A similar one the size of a child's head is located in the submaxillary space.

Treatment was commenced on September 7, with a daily dose of 12 grams, and continued until October 22 . The steer showed a pronounced iodisin, and the tumors shrunk fast. From November 9 until Deccmber 20 medicine was again administered, and both tumors disappeared completely.

Post-mortem, Jamuary 97,1893 . - No traces of the tumors left except a slight induration in the skin. Lymph glands on head sound. No internal lesions. Carcass passed as sound.

\section{No. 45909.}

Dark-red steer, white star and spot on shoulders, ears cut; received September 5 , 1892.

In sublaryngeal and submaxillary space is located a large, fibrous tumor, on the lower surface of which is located an nlcerating granuloma, 6 to 7 inches in diameter.

Treatment was commenced on September 7, with a daily dose of 12 grants, and continued until October 20. The steer showed a pronounced iodism, and the fibrons parts of the tumor shrunk and contracted so fist that a large piece of the granuloma was expelled, and the rest dried up and disappeared, leaving a white contracted scar. Medicine was again administered from November 10 until January 20, when the tumefaction had disappeared, only leaving the skin hard and indurated.

Post-mortem, January 27, 1893.-No traces of the tumor left, except a fihrons induration in the skin showing traces of soft tissue. No internal lesions. Carcass passed as sound.

\section{No. 40733.}

Brown bull, horns; received $\Lambda$ ngust, 30, 1892, from Kansas City.

In the submaxillary space on the right side is located a hard, fibrous tumor the size of a child's head. On the right check is a similar one the size of a goose ogg. 
Treatment was commenced September 1 by a daily dose of 12 grams, and continued until December 25, with proper intervals. The steer showed a pronounced iodism, and the tumor on the chcek disappeared fast, while the larger one shrunk considerably. From November 10 until Deccmber 28 medicine was again administered, and the remaining tumor disappeared slowly, leaving the skin indurated.

Post-mortem, January 2\%, 1893.-No traces of the tumors left. Lymph glands on head sound. In the wall of the small intestines were found located a few. small, hard nodules. Carcass passed as sound.

No. 131063.

Black steer, horns, ears cut; received September 5, 1892.

In sublaryngeal space is located a hard, fibrous tumor the size of a child's head.

Treatment was commenced on September 7, with a daily dose of 10 grams, and continued until October 22, when the tumor had almost disappeared. The steer showed a pronounced iodism.

Post-mortem, January 2\%, 1893.-No traces of the tumor left, except a fibrous induration in the skin. Lymph glands on head sound. No internal lesions. Carcass passed as sound.

\section{No. 45932.}

Roan bull, hornless; received September 5, 1892.

In the loose connective tissue under the skin covering the sublaryngeal region on the left side is located a tumor the size of a double clinched fist. It is hard and fibrous, with a smooth surface. On the lower end there is a scar, and here the skin is in close connection with the tumor.

The treatment was commenced September 6 by giving a dose of 15 grams iodide of potassium daily, with proper intervals of about one or two days a week. The medicine did not have any marked effect on the bull, which only showed vague symptoms of iodism, the tumor shrinking but slowly. At the end of the fourth week the treatment was stopped, the tumor having then shrunk to about one-third its original size. In less than three weeks the rest disappeared without any further treatment, leaving only a hard fibrous thickening in the skin, the size of the palm of a hand, which made it look as if there was still a tumor there, but by manipulation no traces of the original lump can be detected.

Post-mortem, January 27, 1893.-The skin covering the left parotid region a little indurated, and covered by a white scar. Lymph glands on head sound. On surface of liver a small nodule the size of a bean. Carcass passed as sound.

No. 53.

No tag; red hornless steer, white belly, white spot on left side.

Post-mortem, January 27, 1893.-No lesions of any kind found. Carcass passed as sound.

No. 39990.

Roan dehorned heifer; received Angust 28, 1892.

In the submaxillary space is located a hard, fibrous tumor the size of a large cocoanut.

Treatment was commenced August 30, with a daily dose of 10 grams, and was continued until October 25, with proper intervals. The heifer showed no symptoms of iodism, and the medicine had hardly any effect on the tumor. The treatment was continued from 9 th of November nutil January 25, but without success. The tumor only shrunk a little.

$14411-$ No. $2-6$ 
Post-mortem, January 27, 1893.-In left side of the submaxillary space was located a hard, fibrous tumor the size of a child's head, containing several centers of soft granulomatous tissue and pus. No internal lesions. The carcass was condemned.

No. 531549 .

Red steer, hornless; received August 22, 1892.

In the sublaryngeal space on the left side is located a hard, fibrous tumor the size of a double clinched fist.

Treatment was commenced on August 24, with a daily dose of 12 grams, and was continued until October 15, when the tumor had disappeared. The steer showed a pronounced iodism.

Post-mortem, January 2\%, 1893.-No traces of the tumor left. No internal lesions. The carcass was passed as sound.

\section{No. 45930 .}

Red bull, hornless; received September 5, 1892.

In sublaryngeal space on left side is located a hard, fibrous tumor the size of a child's head, lying loose in the connective tissue under the skin.

Treatment was commenced on September 7, with a daily dose of 12 grams, and was continued until October 20, when the tumor had shrunk to the size of a clinched fist. From November 10 until December 20 medicine was again administered and the rest of the tumor disappeared.

Post-mortem, January 2\%, 1893.-No traces of the lump left. No internal lesions. Carcass was passed as sound.

No. 59131.

White steer, hornless; received August 30, 1892, from Kansas City.

The left upper maxilla is slightly swollen. In sublaryngeal space on left side is located a hard, fibrous tumor the size of a cocoanut.

Treatment was commenced September 1, with a daily dose of 12 grams, and was continued until October 20, when the soft tumor had almost disappeared, while the bony swelling remained the same. The treatment was continued from November 10 to January 20. The steer showed a pronounced iodism, and the tumor underneath disappeared completely.

Post-mortem, January 27, 1833.-The left upper maxilla is swollen slightly. The maxillary sinus was completely filled with actinomycotic new formations extending into the nasal fossa. The three first molar's were pushed slightly outward. Lymph glands on head sound. No internal lesions. Carcass condemned.

No. 39962.

Red heifer, white belly; received August 28, 1892.

In left sublaryngeal space is located a tumor the size of a double clinched fist.

Treatment was commenced on August 30, with a daily dose of 10 grams and continued until October 10, when the heifer was cured.

Post-mortem, January 27, 1893.-No traces of the tumor left, no internal lesions. Carcass was passed as sound.

\section{No. 40000 .}

Red cow, hornless; received Augnst 22, 1892.

In left parotid region is located a large, rather soft tumor, extending from the ear down into the sublaryngeal region. 
Treatment was commenced on Angust 24, with a daily dose of 10 grams and continued until October 27 , when the whole tumor had disappeared, except a fibrous induration in the skin.

Post-mortem, January 2\%, 1893.-No traces of the tumor left except a fibrous induration in the skin. No internal lesions. Carcass passed as sound.

$$
\text { No. } 4592 \% \text {. }
$$

Brindle steer, white star, hornless; received September 5, 1892.

In sublaryngeal space is located a fibrous tumor the size of a cocoanut, and a similar one the size of a goose egg in the intermaxillary space

Treatment was commenced on September 7, with a daily dose of 10 grams, and continned until October 10. The steer showed a pronounced iodism, and the tumors shrunk considerably. From November 12 until December 20, medicine was again administered, and the tumors disappeared completely.

Post-mortem, January 27, 1893. - No traces of the tumors left except an induration in the skin No internal lesions. Carcass was passed as sound.

\section{No. 39996.}

Red bull, dehorned; received August 28, 1892.

In sublaryngeal space is located a fibrous tumor the size of a child's head. On the inside of the lips are located a few small granulomas.

Treatment was commenced on August 30, with a daily dose of 12 grams, and continued until October 25. The bull showed hardly any symptoms of iodism, and the tuınor shrunk but slowly. The granulomas on the lips disappeared after two weeks' treatment. Medicine was given from November 10 until December 20, when most of the tumor had gone.

Post-mortem, January 27, 1893.-No traces of tumors left, except a fibrous induration in the skin. No interual lesions. The carcass was passed as sound.

\section{No. 62.}

No tag. Red steer, hornless, white face and neck.

Post-mortem, January 27, 1893.-No traces of actinomycosis found. The carcass was passed as sound.

No. 45918 .

Speckled steer, red head and neck, hornless; received September 5, 1892.

In sublaryngeal space is located a fibrous tumor the size of a cocoannt. On the lower surface is located an ulcer, from 5 to 6 inches in diameter.

Treatment was commenced September 7 with a daily dose of 12 grams and continued until October 20, with proper intervals. The steer showed a well-pronounced iodism, and the ulcer soon dried up and shrunk. From November 10 until Jauuary 20 medicine was again administered and the tumor disappeared entirely, leaving a hard, fibrous induration in the skin, covered by a white scar.

Post-mortem, January 27, 1893.-No traces of the tumor left, except the induration in the skin. In sublingual gland a granuloma the size of a walnut, not containing any pus. No interual lesions. Carcass passed as sound.

No. 64 .

No tag. Black, dehorned steer.

Post-mortem, January 27, 1893.-In submaxillary space a fibrous induration 2 inches thick, showing traces of soft tissue. Lymph glands on head sound. No internal lesions. Carcass passed as sound. 
No. 65 .

No tag. Red steer, horns, white face.

Post-mortem, Jumuary 2\%, 1893.-On left lower maxilla was a bony tumor the size of a double clinched fist. The internal parts of the tumor were spongy, and contained numerous cavities filled with soft granulomatous tissue and pus. No internal lesions. Carcass condemned.

\section{No. 48140.}

Red steer, white star, hornless; received August 30, 1892, from Kansas City.

In right upper maxilla is a fibrous enlargement the size of a clinched fist. On the most prominent point is located a small ulcer 1 to 2 inches in diameter.

Treatment was commenced on September 1, and continued until October 20, when the fibrous points of the tumor had shrunk and the ulcer dried up. The treatment was continued from November 10 until January 20, with doses twice a week.

Post-mortem, January 27, 1893.- The swelling on the right upper maxilla is covered by a thin layer of fibrous tissue, inclosing a few small centers of soft tissue containing pus. The inaxillary sinus is completely filled by actinomycotic new formations, extending into the nasal fossa. Lymph glands on head sound. No internal lesions. Carcass condemned.

\section{No. 44534.}

Red steer, large horns; received September 12, 1892.

In right parotid region are located three tumors the size of goose eggs, a similar one the size of a cocoannt in sublaryngeal region, and two the size of goose eggs in left parotid region.

Treatment was commenced on September 14 with a daily dose of 12 grams, and continued until October 25. October 2 two abscesses were formed in right parotid region. The steer showed a pronounced iodism and all the tumors shrunk considerably.

From November 12 medicine was again administered, and the tumors disappeared, leaving only a fibrous induration in the skin.

Post-mortem, January 27, 1893,-No traces of tumors left. Lymph glands on head sound. Mesenteric glands swollen to the size of hen's eggs containing pus (probably not actinomycotic). Numerous small abscesses in both lungs. Carcass condemned.

\section{No. 45969.}

Red steer, white star and rump; delorned; received September 5, 1892.

In left parotid region were located two tumors the size of hen's eggs. Three similar ones the size of goose eggs in intermaxillary space.

Treatment was commenced ou September 7 with a daily dose of 10 grams, and continued until September 29, when the steer was almost curer. It showed a pronounced iodism.

I'ost-mortem, January $27,1893$. - No traces of tumors left. In liver an abscess the size of a hazeluut. Carcass passed as sound.

\section{No. 69}

No tag. Speckled steer, horns.

P'ost-mortem, January 27, 1893.-All the left side of the face inmensely swollen from the eye down to the nose. The hony swelling covered by a thin layer of fibrous tis. sue. The maxillary simus is filled with grannlomatons tissne, and the bordering bones are rarefied. The actinomycotic new formations extend into the nasal fossa almost down to the nostril. The carcass was coudemued. 
No. 541268.

Strawberry roan steer, dehorned; received August 16, 1892.

On the right cheek are located three hard tumors the size of walnuts. In submaxillary space is a tumor the size of a child's head.

Treatment was commenced August 17, with a daily dose of 10 grans. On August 22, there appeared suddenly a granulomatous growth in the middle of the forehead, and another one behind the crest of the occiput. They were both round, 2 inches in diameter, red, and blood-filled, and looked like a large strawberry. The dose of medicine was increased to 12 grams a day, and in less than a week they had both completely disappeared. The treatment was continued until October 12, when all the tumors had disappeared.

Post-mortem, January 27, 1893.-No traces of timors left. No internal lesions. Carcass passed as sound.

\section{No. 45896 .}

Red steer, speckled side and back; received September 5, 1892.

In left parotid region and sublaryngeal space is located a rather soft tumor the size of a child's head.

Treatment was commenced on September 7 and continued until October 22, with a daily dose of 12 grams. The steer showed a pronounced iodism, and the tumor shrunk fast. From November 10 until December 15 medicine was again arlministererl, and soon afterwarts the tumor had disappeared completely.

Post-mortem, January 27, 1893.-No traces of tumor left. Sublingual lymph glands indurated and swollen to the size of a walnut, containing a few drops of pus. No internal lesions. Carcass passed as sound.

\section{No. 45942.}

Brown steer, hornlcss; received September 5, 1892.

In sublaryngeal region on left side is located a hard, fibrous tumor, the size of a large cocoanut. On lower surface there is an ulcerating granuloma 4 to 5 inches in diameter.

Treatment was commenced on September 7, with a daily dose of 12 grams, and continued until October 25 . The steer showed hardly any symptoms of iodism, and the tumefaction only improved slowly. Medicine was again arministered from November 10 until January 20, but the tumor only shrunk to about half the original size, and the granuloma grew a good deal smaller, without showing any tendency to dry up.

Post-mortem, January 27, 1893.-The parotid glands on both sides swollen and containing several pus-foci. In sublingual space is located a fibrous tumor, the size of a child's head, containing one big pus cavity, and several small centers of soft tissue and pus. In both lungs were numerous small abscesses.

The carcass was condemned.

No. 73.

No tag. Dark red steer, horns.

Post-mortem, January 27, 1893.-On right lower maxilla is a bony swelling the size of a clinched fist. The internal parts of the tumor were spongy, and contained a quantity of soft tissue and pus.

The carcass was condemned.

No. 74.

No tag. Dark red bull, horns.

Post-mortem, January 27, 1893.-In submaxillary space is a small fibrous induration in the skin. No traces of soft tissue. No internal lesions. Carcass passed as sound. 
No. 75 .

No tag. Red steer, hornless, white star, belly and flank.

Post-mortem, January $27,1893 .-O n$ right lower maxilla is a bony enlargement the size of a hen's egg. The internal parts were rather compact and only spongy in the center, where a small amount of granulomatous tissue was present. No internal lesions. Carcass passed as sound.

\section{No. 39956.}

Speckled steer, horuless; received August 28, 1892.

In submaxillary space are located three hard fibrous tumors, the size of a goose egg, loose in connective tissue under the skin. On right cheek a similar one, the size of a hen's egg.

Treatment was commenced on August 30, with a daily dose of 12 grams, and continned until October 20. The steer showed a pronounced iodism, and the tumors disappeared completely.

Post-mortem, January 2\%, 1893.-Small fibrous indurations in the skin in submaxillary space. Large contracted scar on anterior surface of liver. Carcass passed as sound.

$$
\text { No. } 7 \% \text {. }
$$

No tag. Spotted steer, horns.

Dost-mortem, January 2\%, 1893.-On left lower maxilla is a bony tumor the size of a double clinched fist. It is covered by a thick layer of fibrous tissue, containing several centers of soft tissue. The internal parts of the tumor were spongy, the cavities filled with soft tissue and pus. No internal lesions. Carcass condemned.

\section{No. $545 \% 89$.}

Red steer, speckled on head, brisket, belly, and legs; received August 16, 1892.

All the left side of the face from eye to nostril considerably swollen. Three inches below the eye is located an ulcer 4 to 5 inches in diameter. The swelling is hard and supposed to be bony in the deeper parts.

Treatment was commenced on August 18 with a daily dose of 10 grams, and continued until October 7, when the ulcer had healed up completely and the swelling had been reduced to the size of a goose egg. No more medicine was givelu until November 10, and the swelling improved slowly, and it was therefore concluded that it did not extend into the maxillary sinus. From November 10 until January 20 medicino was given about twice a week and the swelling shrunk so that it was hardly noticeable.

P'ost-mortem, January 27, 1893.--The left upper maxilla a little swollen, covered by a thin layer of fibrous tissue, not containing any soft tissue. The bone itself is a little rarefied, but the lesion does not extend into the sinus. The infection has probably taken place through some external abrasion in the skiu. The lymph glands on head sound. No internal lesions. The carcass was passed as sound.

\section{No. 59092.}

Brindle heifer, white face and back, dehorned; received Angust 28, 1892.

In submaxillary space are located three fibrous tumors, the size from a child's head to a goose egg, closely comected with each other.

Treatment was commenced on Angust 30 with a daily dose of 10 grams and continued until October 25, when there only remained one tumor the size of a goose ogg. The heifer showed a pronounced iodism. From November 10 mtil December 15 medicine was again administered, when the heifer was regarded as cured. 
Post-mortem, Jamuary $27,1823$. - No traces of the tumors left. Lymph glands on head sound. No internal lesions except a cysticircus bovis in the liver. Carcass passed as sound.

No. 252388.

Rerl steer, white on left flank and belly, white forehead; received July 25, 1892.

Condition very poor. The left lower maxilla is immensely swollen from the angle of the jaw to the chin. 'The swelling extends up over the cheek towards the eye and is of a very dense, fibrous character, on some places almost bony. On the lower surface are located two ulcerating granulomas, one smaller, about 2 inches in diameter, and a larger one, 5 or 6 inches in diameter. The surface of the granulomas is raw, covered with pus crusts. In the parotid region on the same side are located two round, hard tumors, one just below the ear the size of a hen's egg, and below this another the size of a clinched fist. The deformation of the lower maxilla has interfered with the mastication in a considerable degree, so the steer is only able to eat soft or ground feed, but no hay. When not eating he is grinding his teeth and the saliva flows incessantly from his mouth. The steer is very weak, lies down almost all day, and looks as if he were not able to live until the next day. The excretion from the ulcers is exceedingly fetid.

Treatment.-Fron .July 27 until August 1, 5 grams of iodide of potassium dissolved in 15 grams of water were injected into the jugular vein. It had to be stopped, as phlebitis resulted. The three following days the same amount was injected hypodermically on the neck, but this caused mortification of the tissue at the place of injection. Nevertheless, it had a good effect, and the granulomas began to dry up at the elge. From August 4 the medicine was given internally in doses of 10 grams daily, which was continued until October 25, when the steer was cured. The little granuloma had disappeared completely, while the large one had been reduced to a scar in the skin the size of a silver dollar. The fibrous swelling was reduced to a small induration in the skin on the cheek. The steer ate and drank all he was given, and became fat. The two tumors in the parotid region became fluctuating, were opened with a knife, and a few ounces of good pus evacuated, after after which they disappeared completely. During the treatment the ulcers had been washed with a 2 per cent creolin solution to prevent the smell and dusted with equal parts of iodoform and boric acid. When they had dried up they were painted with tincture of iodide.

Post-mortem, January 27,1893 .- Under the skin of the left cheek is a small fibrous induration, and outside a white scar the size of a silver half dollar. Of the tumors in parotid region no traces are left. No internal lesions. Carcass passed as sound.

\section{No. $15968 \%$.}

Light red steer, dehorned; received July 25, 1892.

The right upper maxilla swollen to the size of a clinched fist. The swelling is bony and closely covered by the skin, and on the lower end of it is a small scar where the skin has been broken. On the inside of the upper lips are located two flat granulomas, one to two inches in diameter.

Treatment was commenced July 27 with a daily dose of 10 grams. The swelling began to grow right away, so it was evirlent that the maxillary sinus was filled with new formations. August 1, the scar on the point of the swelling broke open and a few drops of the pus were discharged. The tumor kept on growing until August 15, when it began to decrease. The scar healed up and about the middle of September the tumor had regained its original size. By this time the maxilla on the opposite sidle began to swell. It had from the beginning been slightly swollen, but so little that it was hardly noticeable. In spite of increased and frequent doses of merlicine, it increased rapidly in size. This is the only case where the medicine did not at least stop the progress of the disease. The treatment was continued until the ani- 
mal was killed, by which time the tumor on the right side had its original size, and the one on the left side was about the size of a small hen's egg. An oxternal treatment with unguentum iodi had no effect.

Post-mortem, January 27, 1893.-The maxillary sinus on both sides fiiled with actinomycotic new formations. The bony swelling is on the outside covered by a thin layer of fibrous tissue containing a few centers of soft tissue. No internal lesions. The carcass was condemned.

No. 252388.

Red steer, white on left flank and belly, white forehead; received July 25, 1892.

The left lower maxilla is immensely swollen from the chin to the angle of the jaw. The swelling is of a fibrous character, but seems to be bony in the deeper parts. The surface is covered by two ulcerating granulomas, the smaller one $1 \frac{1}{2}$ inches in diameter, and the larger one almost circular, 5 to 6 inches in diameter. The granulomas are partly covered with dried pus, and are partly raw and bleeding. In the parotid region on the same side is located two tumors, one just below the ear and the size of a hen's egg, and below this anotlier the size of a clinched fist. Both of these tumors were slightly fluctuating. The deformation of the lower maxilla has interfered considerably with the mastication, so the steer is only able to eat soft or ground feed, but no hay. When not eating he is constantly grinding his teeth, and the saliva flows incessantly from his mouth. The steer is in very poor condition, lies down most of the time, and looks as if he had not many more days to live.

The treatment was commenced July 27 with a dose of 10 grams of iodide of potassium. The three following days a solution of 5 grams of iodide of potassium in 15 grams of distilled water was injected into the jugular vein, but had to be stopped, as there resulted phlebitis. The same treatment was then tried hypodermically, the solution being made a little weaker (1-4), but this resulted in a necrosis of the tissue at the place of injection. The treatment was therefore continued by giving the medicine internally until the 1st of September. The steer showed pronounced iodism, the granulomas dried up and shrunk, and the whole tumefaction was reduced to less than one-third its original size. The ulcers, which in the beginning were exceedingly fetid, were washed in a 2-per cent solution of creolin and dusted with iodoform and boracic acid, equal parts. The two tumors in the parotid region had been opened with a knife and a small anount of pus evacuated. From September 15 till October 15 medicine was again administered and the tumor on the jaw was reduced to a small fibrous induration in the skin, covered by a pink cicatrix, the size of a silver dollar. The two tumors in the parotid region disappeared completely. The lesion did not extend into the bony tissues of the lower maxilla as was at first supposed. About November 1 the stcer was regarded as cured and was in splendid condition.

Post-mortem, January 29, 1893.-On the left cheek and in left parotid region is a slight fibrous induration of the skin. No traces of soft tissue left. No internal lesions. Carcass passed as sound.

\section{No. $15968 \%$.}

Light red stcer, hornless; white forehead, white marks on belly, flank, and legs; received July $25,1892$.

On the right upper maxilla is located a swelling the size of a large goose egg, about 5 inches long and $3 \frac{1}{2}$ inches wide. The swelling is bony, hard, and closely covered by the skin. On the lower end of it is located a small scar, where the skin has been broken, but now is healed np. The left upper maxilla is slightly swollen, hardly noticeable. On the inside of the upper lip is located two small flat actinomycomas $1 \frac{1}{2}$ to 2 inches in diameter. The steer is in fairly good condition.

The treatment was commenced July 27, 1892, with a daily dose of 10 grams. August 1 the swelling had apparently increased a little, and the scar on the lower 
end broke open and a fow drops of pus was discharged. For the first two wecks the tumor increased in size slowly, in spite of the increase in the dose to 12 to 15 grams a day. Then it commenced to shrink again, until it had reached the original size. About September 1 the upper maxilla on the felt side began to swell, regardless of the large doses of medicine. This is the only case out of 192 animals affected with actinomycosis where the medicine did not at least arrest the progress of the disease, but the steer showed a perfect insusceptibility to the effect of the treatment. The administration of medicine was continned with proper intervals until the steer was slaughtered, by which time both tumors were about the same size, the one on the right side a little larger than originally. The two granulomas inside the upper lip disappeared after three to four wceks' treatment. An external application of iodine ointment to the tumors had no effect.

Post-mortem, January 29, 1893.-The maxillary sinuses on both sides completely filled with soft actinomycotic new formations, extending into the nasal fossa. Infection has evidently taken place through the dental alveola of the third molar on both sides, as they were pushed slightly out of their places. The lymph glands on the head were sound. No internal lesions. Carcass condemned.

\section{No. 396938.}

Red steer, white forehead, rudiments of horns; received July 30, 1892.

In the sublaryngeal region is located a hard, fibrous tumor, the size of a double clinclied fist, lying loose in the connective tissue under the skin. From the lower surface of the tumor projects a round, red granuloma about 4 inches in diameter.

Treatment was commenced August 1 with a daily dose of 12 grams and was continued until January 20, 1893. The steer showed no symptoms of iodism and the tumor improved only slowly. About October 1 the tumor had shrunk to half its original size and the granuloma had dried up and shrunk to the size of a silver dollar. By this time an abscess formed in the left parotid region just below the ear, which broke open, and a small amount of pus was evacuated. 'The tumor kept on shrinking slowly and had almost disappeared when the animal was slaughtered.

Post-mortem, January 29, 1893.-In the sublaryngeal region is a fibrous induration in the skin about 1 inch thick, on the outside of which is located a white contracted cicatrix. In the left parotid region is a small induration in the skin. The lymph glands on the head were sound. In the upper part of the left lung was located an actinomycotic tumor the size of a child's head. The carcass was condemned.

\section{No. 373406.}

Red stag, horns; received July 30, 1892.

In the right parotid region is located a flat, hard, fibrous tumor, the size of the palm of the hand. It is closely connected with the covering skin. In the center of the swelling is an open scar, ont of which flow a few drops of yellow pus. In the sublaryngeal space, on the right side, is located a hard, fibrous tumor, the size of a goose egg, lying loose in the connective tissue under the skin. On the lower end of it is a small scar where it has been broken, and here the skin is in close connection with the tumor. On the middle of the right cheek, outside the third molar, is located a red granuloma the size of a silver half dollar. The surface is ulcerating and covered with pus crusts. The surrounding skin is hard and indurated. On the inside of the upper lip is located a small oblong granuloma one inch long and half an inch wide.

Treatment was commenced August 1 with a daily dose of fifteen grams and was continued until October 15 , with proper intervals. The stag showed a prononncerl iodism and the tumors improved rapidly. The granuloma in the mouth disappeared after two weeks' treatment. The one on the cheek dried up and disappeared after four weeks' treatment, and the hard swelling in the parotid region became soft and 
disappeared. From Novomber 1 until November 15 medicine was again adm1nis. tered, and shortly afterwards the stag was regarded as cured.

I'ost-mortem, Jannary 29, 1893. - No traces of the tumor's left. No internal lesions. Carcass passed as sound.

$$
\text { No. } 294924 .
$$

White steer, horns; received Jnly $30,1832$.

In the sublaryngeal space on the right side is located a hard, fibrous tumor, the size of a double clincherl tist, lying loose in the connective tissue under the skin.

On the left shoulder is located a red, soft granuloma the size of a large hen's egg. The surromuling skin is thick and indurated, but is not connected with the underJying tissue.

Treatment was commenced Angust 1 with a daily dose of 10 grams and was continned nutil september 12 , when the tumor in the sublaryngeal space had disappeared aud the granuloma on the shoulder had shrunk to the size of a hazelnut. From September 20 to september 30 medicine was again administered and the steer was regarled as cured.

Post-mortem, January 29, 1893.-No traces of the tumors left; no interual lesions. Carcass passed as soumd.

$$
\text { No. } 322415 .
$$

Dark-red steer, horns; received July 25, 1892.

Both the lower maxillas, especially the left one, are immensely swollen from the chin to the neck. The swelliug exteuds up over the left side of the face toward the eyc. On the lower surface is located an ulcerating granuloma, 6 inches in diameter, and protruding 4 to 5 inches over the swollen edges of the surrounding tissne. The whole tumefaction is the size of a half-peck measure. The leeth in the left lower maxilla are pushed out of their sockets, so that inastication is considerably impaired.

'Treatment was commenced July 27 with a daily dose of 10 grams and was continued until January 25, 1893, with proper intervals, some of these extending over two to three weeks. The steer showed no symptoms of iodism whatever, even if the doses were increased to 15 grams per day and contiuued for two weeks without stopping; consequently the effect on the tumor was very little. 'The granuloma did not dry np after the treatment had been continued for three months, and neither this nor the fibrous tumor becume smaller. Injections into the granuloma of a concentrated solution of iodide of potassium or of tincture of iodine only produced a small abseess at the place of injection, which would break opeu and a small amount of pus be evacuated. In spite of the daily doses of medicine the steer, which was rather poor at first, put ou flesh and became fat.

Post-mortem, Jonuary 29, 1893.-The granuloma was covered with a thin layer of epidermis, and was not soft, but had become of a more fibrous character. A section through it proved it to consist of a stroma of fibrous tissue in which were embedded the larger and smaller centers of gramlomatous tissue. This is of a dense character and does not contain pus. 'The surrounding parts of the tumefaction consisted of soft, white, fibrous tissue, with no traces of soft tissue. The lower maxilla was considerably swollen, the inner parts being spongy aud containing numerous centers of soft tissue, but no pus. The lymph glands on the head were sound. No internal lesions. Carcass condemned. 


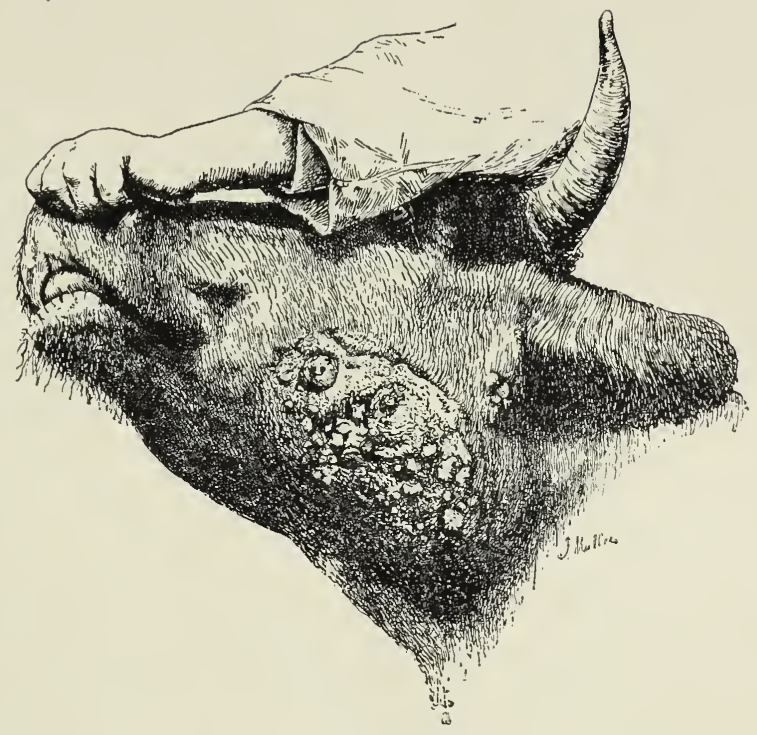

252388

Before treatment-afterward Cured.

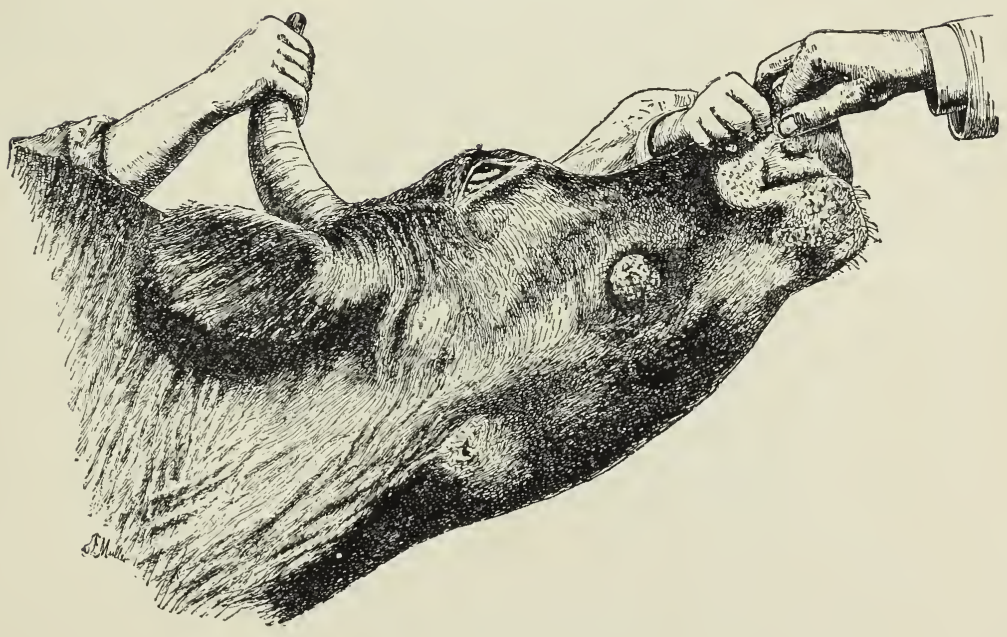

373406

Before treatment-afterward Cured. 



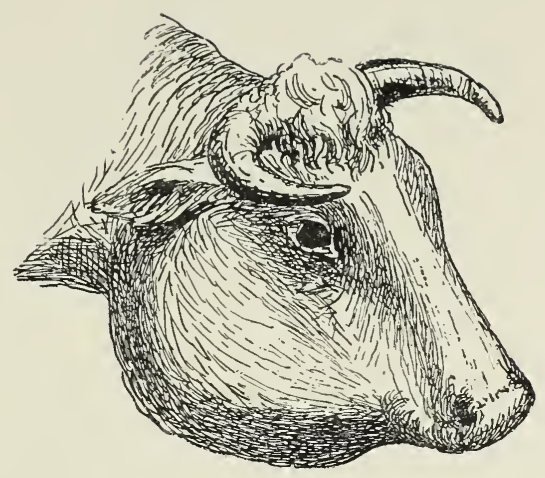

545764

Before Treatment-afterward Cured.

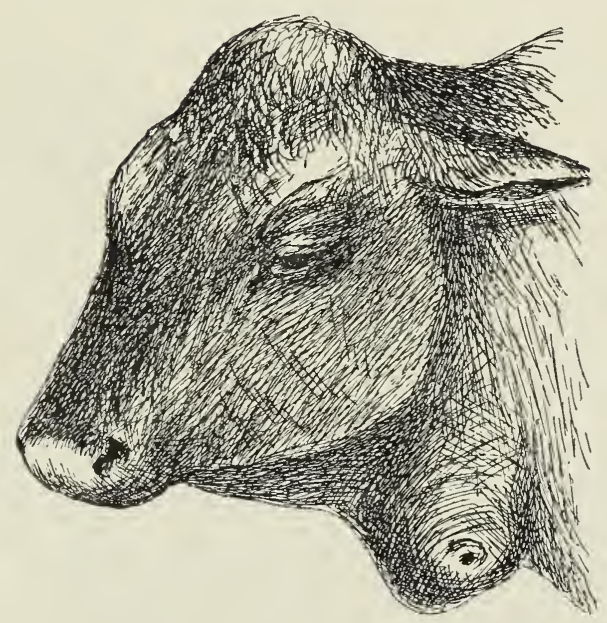

45932

BEFore TREATMENT-AFTERWARD CURED. 



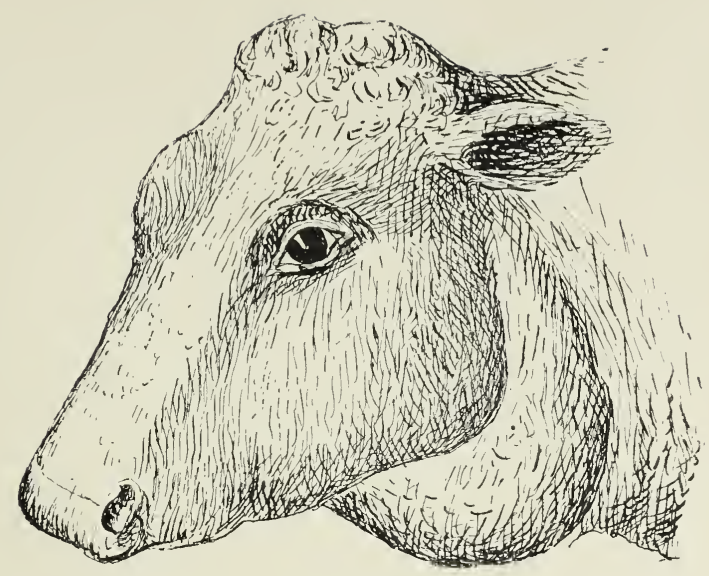

45982

Before TREATMENT-AFterward CuREd.

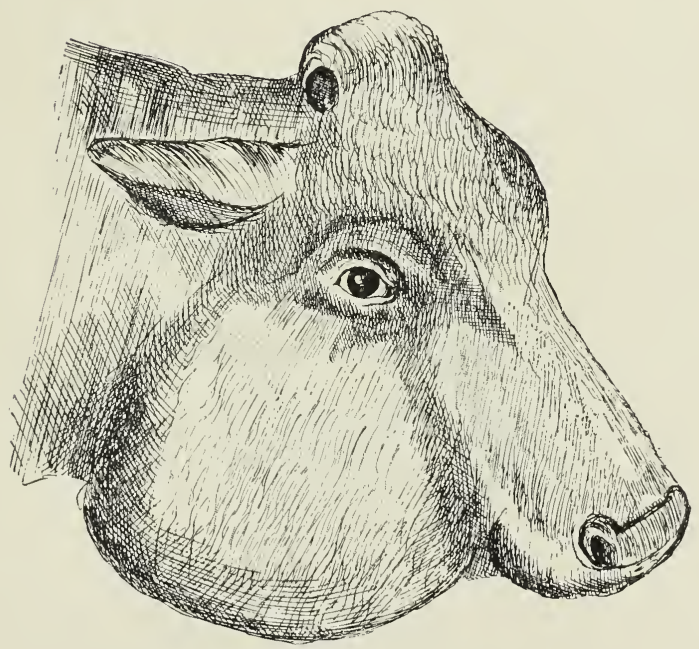

534819

Animal Treated, But not Cured. 



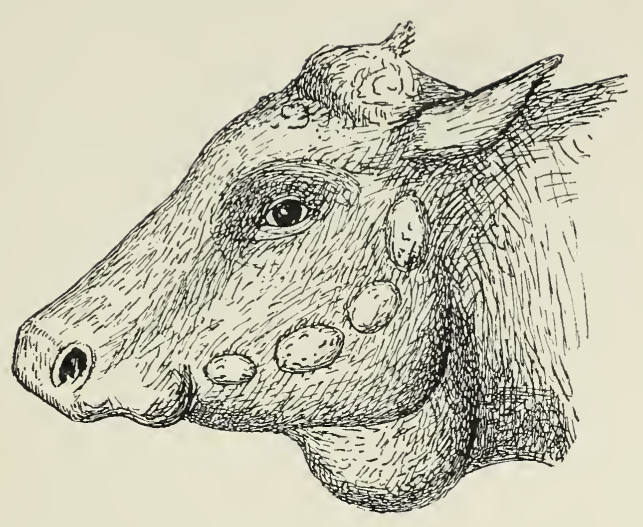

545512

Before treatment-afterward Cured.

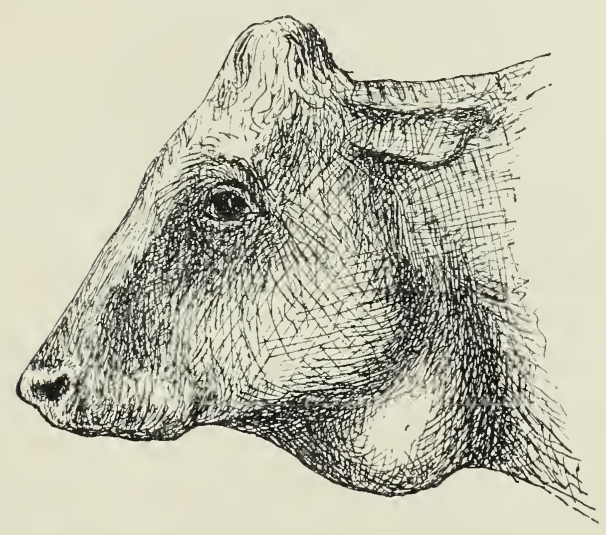

39963

Before Treatment-afterward Cured. 



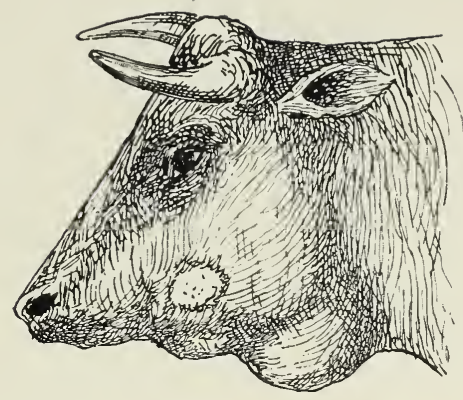

534024

Before Treatment-afterward CuREd.

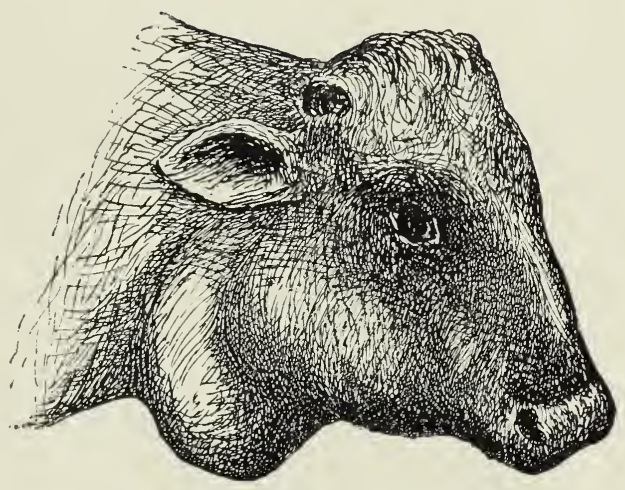

545801 .

Before TREatMent-AFterward Cured. 



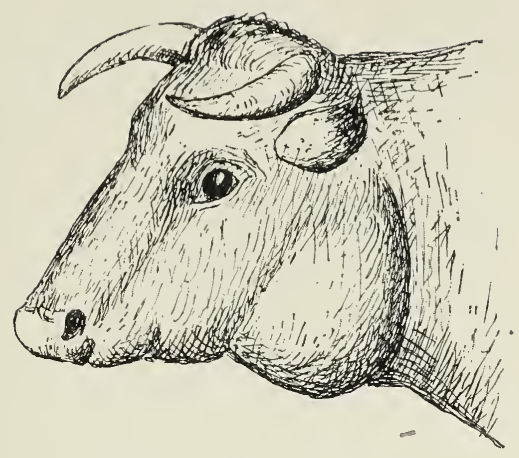

\section{6}

Before Treatment-afterward Cured.

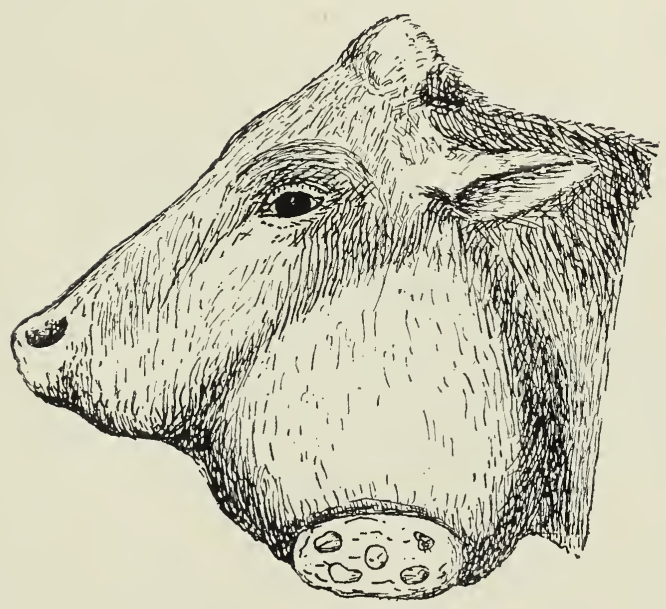

545524

ANimal Treated, But not Cured. 



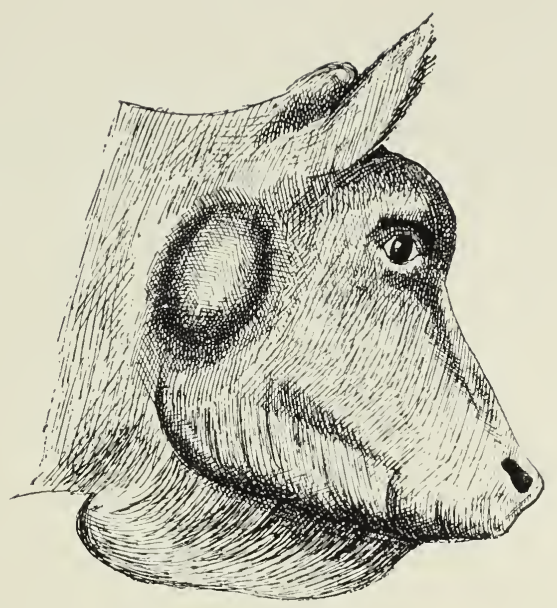

533796

Before Treatment-afterward Cured.

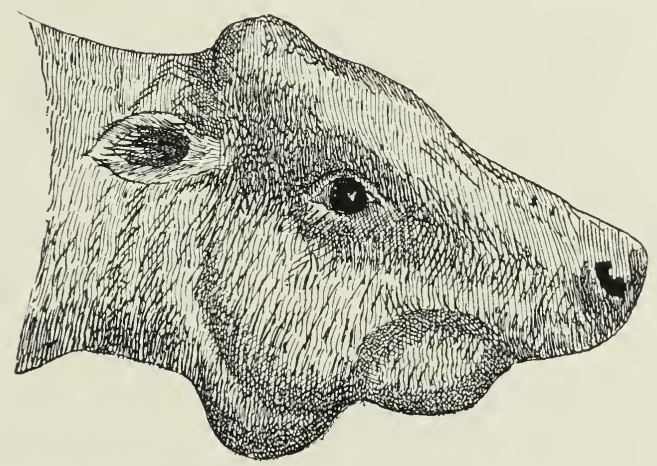

545523

Befcre Treatment-afterward Cured. 



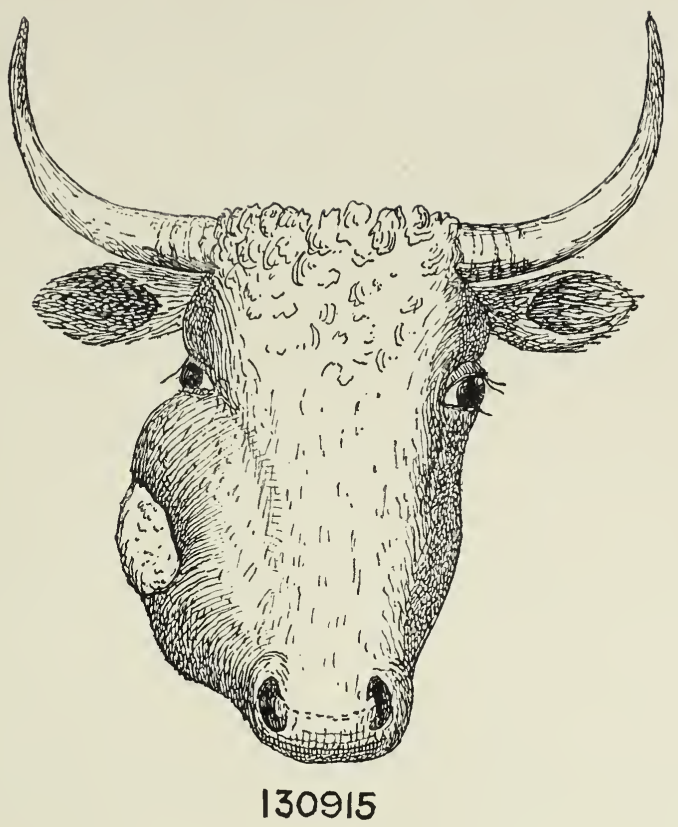

animal Treated, But not Cured.

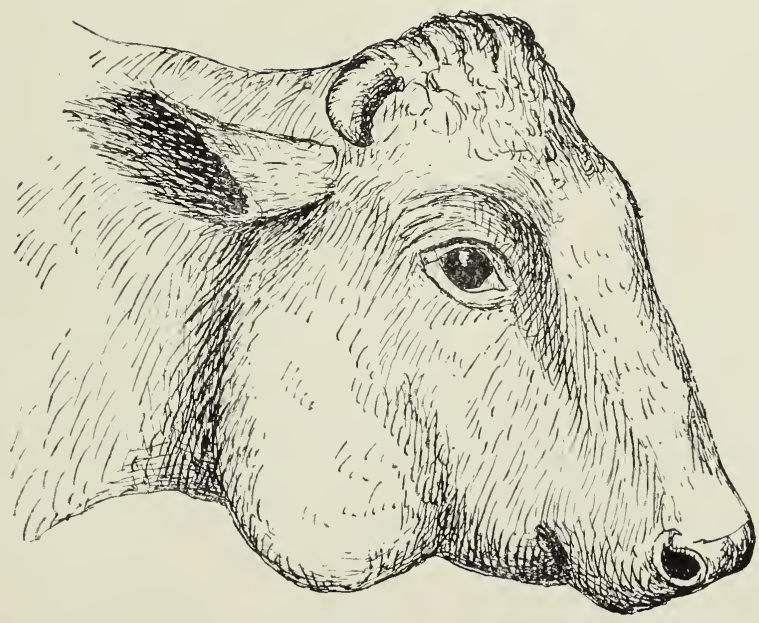

499857

Before Treatment-afterward Cured. 



\section{U.S. DEPARTMENT OF AGRICULTURE.}

BLREAU OF ANIMAL INDUSTRY.

BULLETIN No. 3.

MISCELLANEOUS INVESITIGATIONS

CONCERNING

\section{INFECTIOUS AND PARASITIC DISEASES}

OF

\section{DOMESTICATEI) ANIMALS.}

CONDUCTED UNDER THE DIRECTION OF DR. D. E. SALMON, CHIEF OF THE BUREAU OF ANIMAL INDUSTRY,

F. L. KILBORNE, VERANUS A. MOORE, E C. SCHROEDER, THEOBALD SHITH, AND C. W. STILLS.

PUBLISHED BY AUTHORITY OF THE SECRETARY OF AGRICULTURE.

WASHINGTON:

GOVERNMENT PRINTING OFFICE.

1893. 



\section{TABLE OF CONTENTS.}

Observations on the morphology, biology, and pathogenie properties of twentyeight streptococei found in the investigation of animal diseases. By Vk-

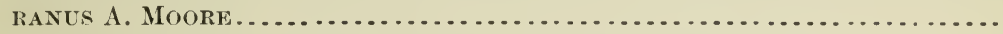

A non-motile pathogenic bacillus, closely resembling the bacillus of hog cholera, found in the lung and spleen of a pig. By Veranus A. Moore...........

Pathogenic and toxicogenic bacteria in the upper air passages of domesticated

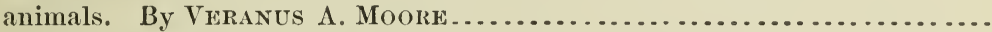

An outbreak of abortion in mares. By F. L. KrLborNe .................... On a pathogenic bacillus from the vagina of a mare after abortion. By Trivo-

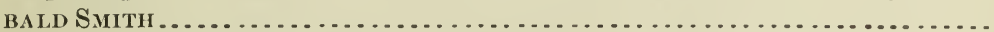

Some experimental observations on the presence of tubercle bacilli in the milk of tuberculous cows when the udder is not visibly diseased. By Theoratil

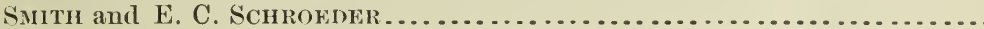

Adrlitional observations on Texas cattle fever. By Theobald Sirti, F. L.

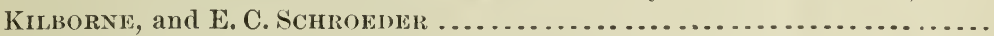

Preliminary notes on a sporozoön in the intestinal villi of cattle. By TneoBALD SMITH .

........

Notes on parasites.-18: On the presence of Sarcosporidia in birds. By C. W. STILES

\section{ILLUSTRATIONS.}

Plate I. Sporozoön in the intestinal villi of eattle .................... 78

II. Balbiania Rileyi sp. n.................................... 86

III. Balbiania falcatula sp. n. and sarcocystis falcatula sp. n........... 88

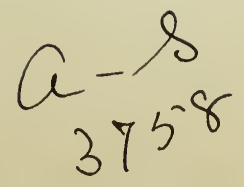





\section{LETTER OF TRANSMITTAL.}

U. S. Departient of Agriculture, Bureau of Antmal Industry,

Washington, D. C., August 29, 1893.

SIR: I have the honor to herewith transmit the results of some im. portant investigations concerning infectious and parasitic diseases, as revealed by researches made by my assistants in the laboratory and at the Experimental Station of the Bureau while engaged in efforts to determine the causes of some of the more destructive communicable and contagious diseases of domesticated animals.

Very respectfully,

Hon. J. Sterling Mor'Ton,

D. E. SALmon, Chief of Burean.

Secretary of Agriculture. 



\title{
LETTER OF SUBMITTAL.
}

\author{
U. S. Depar'tMent of Agriculture, \\ Bureau of Animal Industry, \\ Washington, D. C., April 25, 1893.
}

SIR: I have the honor to submit herewith some investigations concerning infectious and parasitic diseases of domesticated animals, which are either the outgrowth of more important researches already published or else fragments of such work under way.s. The slow progress made, largely due to the want of suitable material for investigation, makes it very desirable that integral portions of our work be not witlheld too long from publication, provided premature conclusions are not drawn therefrom. The minor investigations herewith presented are all of them important in aiding our understanding of infectious animal diseases, and in paving the way for their control or suppression.

Very respectfully,

THeOBald Shíth, Chief of the Division of Animal Puthology.

Dr. D. E. Salmon,

Chief of the Bureau of Animal Industry. 

MISCELLLANEOUS INVESTIGATIONS CONCERNING IN: FEC'IIOUS AND PARASITIC DISEASES OF DOMESTICATED ANIMALS.

OBSERVATIONS ON THE MORPHOLOGY, BIOLOGY, AND PATHOGENIC PROPERTIES OF T'WENTY-ELGHT STREPTOCOCCI FOUND IN THE INVESTIGATION OF ANIMAL DISEASES.

By Veranus A. Moore.

In the bacteriological investigation of diseased animal tissues which has been carried on in this laboratory, streptococci have frequently been found, either alone or associated with other bacteria. Their occasional appearance in greater or less numbers in the variously affected organs, more especially when associated with those morbid conditions which could not be attributed to any specific cause, suggested the desirability of determining as far as possible the relations existing between the streptococci and the lesions in which they were found. The results that had been obtained, and the observations that had been made by a few investigators with reference to the etiological value of certain streptococci, gave hope that with a better knowledge of this group of bacteria the cause of a greater or less number of morbid conditions might be explained. Since the summer of 1858 , therefore, the streptococei that have appeared in the regular examinations in this laboratory have for the greater part been isolated and subjected to a somewhat thorough investigation with reference to their morphology, biology, and especially their pathogenic properties. It should be stated that several of the streptococei that had appeared prior to this time had been quite carefully studied, but no attempt had been marle to isolate and compare all of these forms. My work upon this group of bacteria has been done under the direction of Dr. Theobald Smith, Director of the Laboratory, to whom I am indebted for valuable suggestions and assistance in the isolation of many of these streptococci. For the inoculation of pigs and the careful watching of the same subsequently, I am indebted to Dr. F. L. Kilborne. Other and more important work has necessarily confined this study within quite narrow limits, and prevented the carrying out of extended experiments in any of the many directions suggested by these preliminary observations. 
Since Billroth ealled attention to this group of bacteria, much has been written concerning the streptococci that have been found to be associated with lesions in a large number of diseases in both man and the lower animals. A few of these streptococci have been found by careful study and investigation to be of specific etiological value, but a considerable number of them, on account of their frequent appearance and their pathogenic effect on certain of the experimental animals, have been considered as bearing a more or less causal relation to the lesions with which they were associated. While it is my purpose to present simply the results of the observations made on the streptococci examined, a brief mention of the more important work that has been done on this subject, and from which many suggestions have been obtained, should not be omitted.

Koch found in putrid fluids a streptococcus which, when inoculated into the ear of a mouse, produced necrosis of the surrounding tissue, destruction of the cartilage cells, and death of the animal in about three days.

In the earlier investigations of swine diseases carried on by the U. S. Department of Agriculture, Dr. Salmon isolated micrococci from the diseased organs of pigs. The micrococei grew in shorter or longer chains, and in stained cover-glass preparations they exhibited a light center. These forms were so constantly present that they were supposed to have a more or less causal relation to the diseases with which they were associated.

Klein, in his investigation of a cattle disease known as "cow scarlatina," or the "Hendon cow disease," found a streptococeus in the sores on the udders of the diseased cows. He isolated and carefully studied this streptococcus. When it was inoculated into calves and mice it produced a definite disease, which he considered to be identical with the disease that was produced by the scarlatina streptococcus. In another outbreak, which manifested itself by a contagious, ulcerative process on the teats and udders of certain cows which furnished milk to the Ediuburgh dairy, other streptococei were obtained, one of which Klein considered as standing in some "necessary relation to the disease."

The same author, in his investigations of "foot-and-mouth disease" in sheep, concludes that the cause of the disease is a micrococens, which grew in chains. He obtained the germ from cultures made from the lymph of a sheep which was affected with the disease. Inoculation experiments with this germ were negative, but by feeding sheep with cultures of the twentieth generation the typical disease was produced. From the vesicles of the artificially-produced disease pure cultures of the micrococcus were obtained.

Sand and Jensen in 1887 investigated the cause of strangles in horses. In 29 cases they invariably found in the pus from the suppurating glands and in the nasal discharge a characteristic streptococcus. 
It grew on all of the ordinary culture merlia. It destroyed mice, and two of the three rabbits that were inoculated with it in the veins died. Guinea-pigs were immune. Horses were not affeeted, but colts developed typical strangles after spraying the nasal mucous membrane with a bouillon culture of the streptococeus. One of these died. Two colts that were inoculated intravenonsly suffered from phlebitis and abscesses, after which they were immune.

Schiitz found a streptococcus in the pus from the glands of horses suffering from strangles. It was fatal to mice, but rabbits, guinea-pigs, and pigeons were immune. The nasal cavities of a colt, 6 to 8 weeks old, were sprayed several times with a pure bouillon culture of this germ. It died of strangles on the 23d day. Schiitz examined the pus from the suppurating glands of a considerable number of horses suffering from strangles, in all of which he found the streptococcus. Zschokke, in 1886, found a streptococcus in horses suffering from strangles, but supposed it, prior to Schuitz's investigations, to be identical with Streptococcus pyogenes.

In addition to these observations it is a fact worthy of note that streptococci are frequently found associated with a considerable number of morbid conditions which thus far have not been satisfactorily attributed to any specific germ.

In human pathology the presence of streptococei is not unusual. The etiological value of Streptococcus pyogenes Ogston, and Streptococcus erysipelatos Fehleisen, is very generally conceded. Such affections as erysipelas, pyamia, empyema, septicemia, diphtheria, progressive gangrene, scarlatina, Impetigo contagiosa, pericarditis, ostemyelitis, and influenza have been attributed with more or less positive evidence to the invasion of certain streptococei. The investigations of the etiology of la grippe has revealed the fact that streptococci are very frequently associated with the inflammatory condition of the air passages in that disease. Prudden has made the observation that streptococci are found associated, apparently as the etiological factor, with those inflammations, particularly of the serous membranes, in which the formation of fibrin is a most marked feature.

Investigators are not unanimous in the belief that streptococei which have been found to be associated with the diseases mentioned belong to different species. It is a well known fact that their common morphological and biological properties are much more numerous than their differences, and that it is exceedingly difficult to point out a distinguishing trait of any one of these streptococci that is sufficiently characteristic to differentiate it from the others.

The description by Fliigge of Streptococcus pyogenes applies equally as well to Streptococcus erysipelatos, and also to a large number of other streptococei which have no pathogenic properties by which they can be differentiated. Hajek could not find any morphological or cultural difference between Streptococcus erysipelatos and Streptococcus pyogenes, 
but by inoculation experiments he found that they possessed marked differences.

Prudden compared very carefully Streptococcus pyogenes, Streptocoo cus erysipelatos, and Streptococcus diphtherice, but found that there was not a single constant feature of difference between thein that could be detected by cultural characters (parallel cultures), or by inoculations into the smaller experimental animals.

Fraenkel isolated from a case of purulent peritonitis a streptococcus which had apparently played the part of a pyogenic agent, and succeeded in producing erysipelas on the ear of a rabbit with a pure culture of this streptococeus. When it was inoculated beneath the skin on the back of mice and rabbits it produced suppuration.

Prof. Welch was unable to determine any decisive differences between Streptococcus erysipelatos and Streptococcus pyogenes. He adds that "the efforts to differentiate into distinct species the pathogenic streptococei have thus far met with little success, so that the weight of opinion favors the view that the streptococci of erysipelas, phlegmonous inflammations, septicæmia, puerperal fever, and of the various forms of angina belong to one and the same species."

Klein, however, in his report on "The Morphology and Biology of the Streptococcus," in which he discusses the properties of nine selected streptococci, concludes that they are separate and belong to distinct species or varieties. He also believes that a large number of streptococei that have the appearance, on a superficial examination, of belonging to the same species, may, by careful study, be differentiated into separate species or varieties.

Von Lingelshein has recently divided the streptococei into two groups; one non-pathogenic, called Streptococcus brevis, and the other pathogenic, called Streptococcus longus. The streptococci which he studied were from various sources, principally, however, from the normal human saliva and diphtheritie membranes. He made some interesting investigations upon the effect of disinfectants on the various streptococei which he stud ied. He lays much stress upon the importance of blood serum from $\mathrm{v}$ arious animals as a culture medium for these germs. His streptococei grew very vigorously on the serum from rabbit's blood, while they failed, with one exception, to grow on that from pig's blood.

Kurth, in his extensive work upon the differentiation of streptococei and their appearance, especially streptococcus conglomeratus in scarlatina, makes the following provisional classification. Streptococci rigidi, which grow in short chains in bouillon, the sediment loose, the chains not hanging together. Streptococci flexuosi, which grow in long chains in bouillon, the sediment hanging together.

Pasquale, in an exhaustive discussion of the greater number of the streptococci that have heretofore been described, has tabulated the varions properties of 33 different species, making note of their effects on milk, acid formations, pigment-producing properties, growths npon 
various kinds of blood serum, and their pathogenic effects. Their morphology and cultural characters on the ordinary media are also carefully considered. The large variety of species with which he has worked has enabled him to take a nore comprehensive view of the entire group of streptococei than had heretofore been done. The result of his study of the pathogenic powers of the different species of streptococci differs in many respects from those obtained by other authors. He has, as a result of his observations, formulated a new classification of this group of bacteria based upon their morphological and pathogenic properties. He does not, however, draw sharp lines between the different streptococci, but on the contrary groups them within the natural boundaries which he finds to exist between them.

Pasquale's classification of the streptococei is as follows:

I. Short Saprophytic Streptococei.

(1) Develop in a low temperature (Feces and external surroundings).

(2) Develop in a higher temperature (Mouth and bronchial mucosa).

II. Long, Not Virulent, Streptococcr.

(1) Feces. (Example, Streptococcus coli gracilis.)

(2) Mucous membrane of the mouth (Strepococcus of Kruse u. Pansini).

III. Long Pathogrenic Streptococei.

(1) Streptococci of erysipelas, suppuration, pneumonia, diphtheria, scarlatina, etc.

(2) Sputum of pueumonia (Streptococcus of Kruse u. Pansini).

IV. Short, Highly Infectious, Streptococci.

(1) From tuberculosis, etc. (Diplococcus pyogenes).

(2) From pneumonia (Diplococcus pncumonia).

It is believed that the investigations eited are sufficient to show the general scope of the work that has been done and the more important opinions entertained concerning this very interesting class of the shizomycetes, although a very loug list of minor observations could be added. Without entering into detail, it may be stated that subsequent investigations have shown that in some cases, especially in human pathology, the streptococcus which was first supposed to be the etiological factor in the disease gave way to other bacteria* and in a few other cases the question is still an open one. With this brief review I will pass to the consideration of the streptococei that have come under my observation. It should be stated at the outset that the streptococei which are here considered were obtained exclusively from the organs and secretions of domesticated animals, and that no attempt is made to identify them with the streptococci that have hereto. fore been described. This is omitted on account of the great similarity,

\footnotetext{
* Streptococcus diphtherice is a marked example of this fact. Prudden found this streptococcus almost iuvariably in the false membrane and occasionally in the spleen, liver, and kidneys in a series of twenty-four cases in children who perished with the disease. In none of these did he find the Klebs-Loeffler bacillus. In a later series of investigations, however, he found the Klebs-Loefler bacillus in every case.
} 
generally speaking, that exists in the morphological and biological characters between the streptococei isolated from different sources and the variability of their cultural characters when grown under different conditions.

The first streptococcus to which my attention was given was discovered by Dr. Theobald Smith, in the spring of 1857, while he was investigating an outbreak of swine plague.* Since tlat time several other outbreaks of swine disease have been investigated, in each of which one or more streptococci have been isolated from the diseased organs of one or more of the pigs that were examined. In addition to these, streptococci have been isolated from diseased organs taken from animals that died from varied and widely separated diseases. They have also been found to to be quite numerous in the secretions covering the different mucuous membranes of the animal body as well as in various extraneous material. This varied, and we may almost say, universal distribution of these organisms, renders it of much importance in view of the great similarity that is known to exist between them, that differential biological or morphological characters should be determined, if they exist, between the purely saprophytic streptococei and those that have a greater or less etiological value.

In all we have isolated during the past four years more than fifty streptococci, many of which perished before they were sufficiently studied to be considered here. In a few of the earlier cases streptococci, which appeared upon a somewhat superficial examiuation to be identical, were found in several organs of the same animal, and consequently only one culture was retained for further study and comparison. This was unfortunate, as subsequently it was found in a few cases that the streptococei that were present in different parts of the body could be differentiated by one or more distinct characteristics. $\mathrm{A}$ few of the streptococci were obtained in pure culture from the diseased organs of the animals that were examined, while others were isolated from impure original cultures by means of agar plates. The germs thus obtained have been carefully studied and compared microscopically and in their growth upon certain culture merlia. Various experimental animals have been inoculated with pure cultures of these germs, and subsequently very carefully watched in order to detect any effect that might have been produced by the inoculation. The greater number of the streptococci considered here were isolated from morbid tissues, but for the purpose of comparison a few that were obtained from the mucous membranes of certain animals are inserted.

The cultivation of these streptococci has shown them to bo very sensitive to any change in the conditions under which they were grown, especially to any variation in the composition or chemical reaction of the eulture media. On account of this extreme delicacy in the great majority of their differential biological characters, it seems best at 
this time to limit the descriptions of these characters to those more hardy properties which repeaterl cultures have shown to be constant, and eonsequently recognizable under slightly varying conditions as having a more or less differential value. It will be observed subsequently that the greater number of constant differences that exist between these streptucocei were manifested in their physiological effect, and it is believed by the writer that when our methods for the determination of these properties are sufficiently elaborated the facts necessary for a specific differentiation of the varions classes of the schizomycetes will be forthcoming.

Although several classifications of the streptococci have been made, there appears to be no system of subdivision which is applicable to all of the series of streptococei which have been described. My observations have shown that there are streptococei which possess biological characters that give them an intermediate position between the forms which have been designated as belonging to subgroups. On this account I have not adopted any of the classifications which have been formulated. The streptorocei which I have studied, as the subsequent descriptions will show, exhibit a more or less marked variation in their various properties, but, with one exception, they were as sensitive to changing conditions of growth as streptococcus erysipelatous and other recognized pathogenic forms. It would seem, therefore, that many of these forms have become parasitic in their habits. A classification based on the various morphological, biological, or pathogenic properties of a limited number of streptococei must continue to suffer modifications as more extended observations are made, until the saprophytic, parasitic, and pathngenic forms are more clearly defined, and the range of their biological variations more accurately determined.

Before considering the special qualities of the streptococci about to be described, a few of the more important properties which have been found to be common to them all may be mentioned:

1. They are readily stained with the ordinary aniline dyes.

2. They grow in gelatine, but do not liquefy it.

3. The growth on the different media is not viscid.

4. The growth at the ordinary temperature is less vigorous than at the temperature of the body (excepting streptococcus $\mathrm{O}$ ).

5. In simple beef broth the growth is more feeble than in peptonized bouillon.

6. The growth is most vigorous in peptonized bouillon containing glucose.

7. With one exception (streptococens $\mathrm{O}$ ) the time during which cultures will remain alive, is very variable and uncertain. (It is due to this fact that several forms were lost before their characteristics were (determined.)

In order to present, in a form most convenient for comparison, a description of the more important properties of the streptococci studied the following table has been constructed: 
Description of the

\begin{tabular}{|c|c|c|c|c|c|c|c|}
\hline \multirow{2}{*}{ 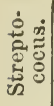 } & \multirow{2}{*}{ Source and date. } & \multirow{2}{*}{$\begin{array}{c}\text { Accompanying } \\
\text { disease. }\end{array}$} & \multirow{2}{*}{$\begin{array}{l}\text { Length of } \\
\text { chains. }\end{array}$} & \multirow{2}{*}{$\begin{array}{l}\text { Dianneter } \\
\text { of coeci. }\end{array}$} & \multicolumn{3}{|c|}{ Growtl on solid media. } \\
\hline & & & & & A.gor. & Gelatine. & Potato. \\
\hline$\frac{\mathrm{A}}{\mathrm{B}}$ & $\begin{array}{l}\text { Lung, pig, } 1887 \ldots \ldots \\
\text { Spleen, pig, } 1888 \ldots\end{array}$ & $\begin{array}{l}\text { Swine plague. } \\
\text { Disease (?) ... }\end{array}$ & $\begin{array}{l}\text { Very long. } \\
\text { Long ..... }\end{array}$ & $\begin{array}{l}0.8 \mu \text { oval . } \\
0.6-0.9 \mu \ldots\end{array}$ & Nucleart.. & $\begin{array}{l}\text { Fecble... } \\
\text {. do } \ldots . . .\end{array}$ & $\begin{array}{l}\text { No growth. } \\
\text { Grayish ... }\end{array}$ \\
\hline $\mathrm{C}$ & Lung, pig, 1889... & Hog cholera .. & Short and & $0.7-0.9 \mu \ldots$ & ...do.. & ...do & \\
\hline D & ... do ...... & ....do & $\ldots d$ & $0.7-0.9 \mu \ldots$ & ...do. & ... do & \\
\hline $\mathbf{E}$ & Spleen, pig, 1889 .. & ....do & $\begin{array}{l}\text { Long and } \\
\text { very long. }\end{array}$ & $0.5-0.7 \mu \ldots$ & ...do. & & No growth. \\
\hline G & $\begin{array}{l}\text {... do do ... } \\
\text {.... do ... }\end{array}$ & $\begin{array}{l}\text { Morlified hog } \\
\text { cholera. } \\
\text { Disease (?) ... }\end{array}$ & Very long. & $\begin{array}{l}0.7-0.8 \mu \ldots \\
0.8-1.0 \mu \ldots\end{array}$ & ...do + & ... do & $\begin{array}{l}\text { Thin, gray. } \\
\text { ish. } \\
\text { Feoble..... }\end{array}$ \\
\hline $\mathrm{H}$ & Lung, pig, 1889.... & ....do ........... & Short. & $0.7-0.9 \mu \ldots$ & ... do . & ...do & \\
\hline I & ...do . ............. & Swine plague. & Long & $0.6-0.7 \mu \ldots$ & ...do. & ... do & No growth. \\
\hline$J$ & Blood, pig. $1889 . .$. & $\begin{array}{l}\text { Modified hog } \\
\text { cholera. }\end{array}$ & ...do .. & $0.6-0.7 \mu \ldots$ & ... do. & ...do & Feeble.. \\
\hline K & $\begin{array}{l}\text { Peritoneal exu- } \\
\quad \text { date, pig, } 1890 .\end{array}$ & Swine plague. & ...do. & $0.6-0.8 \mu \ldots$ & $\ldots d o \S$ & ... do . & \\
\hline $\mathbf{L}$ & Liver, pig, $1890 \ldots$ & ....do ........ & ...do . & $0.8-1.8 \mu \ldots$ & ... do . & ...do & \\
\hline M & Spleen, pig, 1890 .. & Discase (?) & ...do. & $1.0-1.5 \mu \ldots$ & $\ldots d o t$ & ...do. & No growth. \\
\hline$N$ & $\begin{array}{l}\text { Kidney, cow, } 1890 \\
\text { Feces, cow, } 1890\end{array}$ & Texas fever... & Short. & $0.8-1.0 \mu \ldots$ & ..do ...... & $\ldots$ do ... & \\
\hline & Feces & & $\cdots d$ & & Vige & $\sqrt{i s}$ & \\
\hline $\mathbf{Q}$ & $\begin{array}{l}\text { Trachea, pig. } 1890 . \\
\text { Lung, cow, } 1891 \ldots\end{array}$ & Pneumonia... & ...do ....... & $\begin{array}{l}0.8-1.0 \mu \\
1.2-2.0 \mu . .\end{array}$ & $\begin{array}{l}\text { Nuclear.. } \\
\text { Moderate. }\end{array}$ & $\begin{array}{l}\text { Moderate } \\
\text { Fecble... }\end{array}$ & \\
\hline R & $\begin{array}{l}\text { Absecs s, knee, } \\
\text { pig, } 1891 \text {. }\end{array}$ & $\begin{array}{l}\text { Chronic hog } \\
\text { cholera. }\end{array}$ & & & ...do & ...do & \\
\hline $\mathrm{S}$ & $\begin{array}{l}\text { Abscess, elbow, } \\
\text { pig, } 1891 \text {. }\end{array}$ & & ...do & $0.7-0.8 \mu \ldots$ & ... do & ...do & \\
\hline $\mathbf{T}$ & Lung, cow, $1891 \ldots$ & $\begin{array}{l}\text { Pleuro-pneu- } \\
\text { monia. }\end{array}$ & ...do .. & $1.0-2.0 \mu \ldots$ & $\therefore$ do & ... do . & No growth. \\
\hline U & Trachea, h or s e, & & & $0.9-1.0 \mu \ldots$ & $\begin{array}{l}\text { Translu- } \\
\text { cest. }\end{array}$ & ...do. & Feeble... \\
\hline $\begin{array}{l}V \\
W\end{array}$ & $\begin{array}{l}\text { Luing, pig. 1891... } \\
\text { Liver, pig, 1892. . }\end{array}$ & $\begin{array}{l}\text { Swine plague. } \\
\text { Disease (?) ... }\end{array}$ & 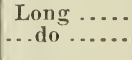 & $\begin{array}{l}0.7-0.8 \mu \ldots \\
0.8-0.9 \mu \ldots\end{array}$ & $\begin{array}{l}\text { Moderato. } \\
\text { Nuclear... }\end{array}$ & $\begin{array}{l}\ldots \text { do } \\
\ldots \text { do }\end{array}$ & $\begin{array}{l}\text { No growth. } \\
\text { ‥do } . . . . . . . .\end{array}$ \\
\hline $\mathrm{X}$ & Blood & do & Shor & $0.7-0.9$ & ... do & $\ldots d$ & ...de \\
\hline$Z$ & $\begin{array}{l}g, 1892 \ldots \\
, 1892 \ldots\end{array}$ & 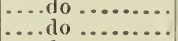 & $\begin{array}{l}\text { Long } \ldots . . . \\
\text { Short .... }\end{array}$ & $\begin{array}{l}0.7-0.8 u . . \\
0.8-1.3 \mu . .\end{array}$ & Moderate. & $\begin{array}{l}\ldots \text { do } \\
\ldots \text {. do }\end{array}$ & 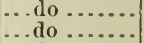 \\
\hline$a$ & Blood, pig, $1892 \ldots$ & .... do ........... & Long ..... & $0.7-0.9 \mu \ldots$ & ... do ....... & ... do ..... & ... do ......... \\
\hline$\beta$ & $\begin{array}{l}\text { Nas a l } \operatorname{mn}_{\text {a }} \mathrm{cus} \text {, } \\
\text { horse, } 1892 \text {. }\end{array}$ & Glanders.. & Short... & $0.9-1.0 \mu \ldots$ & Nuclear .. & ... do .... & $\begin{array}{c}\text {...do .... } \\
\text { - }\end{array}$ \\
\hline
\end{tabular}

*The streptococci from $Q$ to $\beta$ inclusive were cultivated in peptonized boullin containing 2 per cent glucose in the fomentation tube. Streptococci Q, R, and S, did not develop in the closed portion of the tube. The growth in the open bulb was exceedingly vigorons. The others developed in both sicles of the tube. 'The growth was more vigorons but otherwises similar to that in simple bouillon. 'The alkaline reaction of the bouillon was changed in every case to an acid me fluring their multiplication.

t'There was no formation of pigment in the growth on the surface of agar.

An unusually vigorous growth.

$\$$ An unnsually fueble growth.

ilSeveral weckis. 
twenty-eighth streptococci.

\begin{tabular}{|c|c|c|c|c|c|c|}
\hline \multirow{2}{*}{$\begin{array}{l}\text { Growth in } \\
\text { bonillon.* }\end{array}$} & \multirow[b]{2}{*}{ Reaction. } & \multirow{2}{*}{$\begin{array}{l}\text { Bonil- } \\
\text { lon } \\
\text { cleared }\end{array}$} & \multirow[b]{2}{*}{ Effect on milk. } & \multicolumn{2}{|c|}{ Stain after- } & \multirow[b]{2}{*}{ Pathogenesis. } \\
\hline & & & & $\begin{array}{l}\text { Gram's } \\
\text { method. }\end{array}$ & $\begin{array}{l}\text { Weigert- } \\
\text { Grammethor. }\end{array}$ & \\
\hline Flakes....... & & $\begin{array}{l}\text { Days. } \\
\text { D....... }\end{array}$ & Not changed.. & Nicely. & & Destroys mice. \\
\hline $\begin{array}{l}\text { F'ntly cl'd'd, } \\
\text { tlakes. }\end{array}$ & & 10 & $\begin{array}{l}\text { Thickened in } \\
30 \text { days. }\end{array}$ & Not stained. & Not stained. & $\begin{array}{l}\text { Local swelling in rab } \\
\text { bits. }\end{array}$ \\
\hline $\begin{array}{l}\text { Uniformly } \\
\text { cloulded. }\end{array}$ & & 26 & No change.... & Nicely . & Feebly... & No effect. \\
\hline $\begin{array}{l}\text { Fiakes, faint- } \\
\text { ly clouded. }\end{array}$ & & $\begin{array}{l}19 \\
12\end{array}$ & $\begin{array}{l}\text { Casein coag- } \\
\text { ulated. }\end{array}$ & Feebly... & ... do $\ldots$ do............ & $\begin{array}{l}\text { No. } \\
\text { Destroys mice and } \\
\text { rablits. }\end{array}$ \\
\hline $\begin{array}{l}\text { Uniform } \\
\text { clonded. }\end{array}$ & & 25 & No change... & Nicely & Nieely .... & No effect. \\
\hline $\begin{array}{l}\text { Flakes in } \\
\text { suspension. }\end{array}$ & & 12 & ....do do & Fcebly. & ... do & Do. \\
\hline $\begin{array}{l}\text { Unif orm ly } \\
\text { clouded. }\end{array}$ & & 10 & ....do ..... & ... do ... & ... do ....... & Do. \\
\hline ....do ........ & & 3 & $\begin{array}{l}\text { Casein coag- } \\
\text { nlaterl. }\end{array}$ & Nicely ...... & ... do .......... & \\
\hline .... do ........ & & 40 & Thiekened... & ...do. & Feebly...... & Do. \\
\hline $\begin{array}{l}\text { Flaky sedi- } \\
\text { ment. }\end{array}$ & & & No change... & Not stained. & Very feebly. & $\begin{array}{l}\text { In rabbit eleration of } \\
\text { temperature. L } \\
\text { cal swelling. }\end{array}$ \\
\hline .... do ........ & & & Casein coag. & ... do .... & Feebly...... & No effect. \\
\hline $\begin{array}{l}\text { Heavily cl'd. } \\
\text { ed, flakes in } \\
\text { suspensiou. }\end{array}$ & & 6 & No change... & Nicely .... & Nieely.... & Do. \\
\hline Turbid...... & & (II) & & $\ldots$ do ....... & Frebly. & Do. \\
\hline .... & & 15 & $\begin{array}{l}\text { Casein coag- } \\
\text { nlated. }\end{array}$ & Not stained & Not stained. & Do. \\
\hline F'tlyclor & & 8 & . & Feel & Nicely ... & Do. \\
\hline $\begin{array}{l}\text { Floce ulent } \\
\text { sediment. }\end{array}$ & & & 1ango.... & & Fcebly..... & $\begin{array}{l}\text { Slight local reaction } \\
\text { in rabbits. }\end{array}$ \\
\hline Flakes ...... & .. do & & Casein coag- & ...do & Nicely..... & No effect. \\
\hline ....do & ...do & & No change... & Nicely... & Not stained. & Do. \\
\hline $\begin{array}{l}\text { Uniformly } \\
\text { clouded. }\end{array}$ & Alkaline.. & 8 & Casein coar- & ...do. & Nicely...... & Destroys mice and \\
\hline 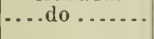 & Acid...... & 4 & & Feebly & Not stained. & Do. \\
\hline$\because d 0=. .$. & Alkaline.. & 7 & .... do & Nicely ...... & Nieely & No effect. \\
\hline $\begin{array}{l}\text { Ft'ly cloud- } \\
\text { ed, flakes. }\end{array}$ & Acid...... & 5 & & Feebly ...... & Not stained. & Do. \\
\hline 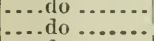 & $\begin{array}{l}\ldots \text { do } \\
\ldots \text { do }\end{array}$ & $\begin{array}{l}2 \\
4\end{array}$ & ...do ..... & $\begin{array}{l}\text { Nieely ....... } \\
\ldots \text {... do ........ }\end{array}$ & $\begin{array}{l}\text { Nicely....... } \\
\text {...do......... }\end{array}$ & $\begin{array}{l}\text { Do. } \\
\text { Do. }\end{array}$ \\
\hline$\ldots$ & ... do & 2 & ....d & ...do ... & Not stained. & Destroys mice. \\
\hline Very fa & Alkaline.. & $6-8$ & 0 & ...do & ...do ... & $\begin{array}{l}\text { bits, and pigs. } \\
\text { No effect. }\end{array}$ \\
\hline & & & & & & \\
\hline
\end{tabular}

* According to observations recently made br Dr. Theobald Smith, the initial acidity of the cultures of many bacteria is demonstrably die to the presence of muscle-glneose in the bouillon. As this varies in quantity and is occasionally absent, the inconstant and conflicting results on the acid and alkaline reaction of cultures is readily explained.

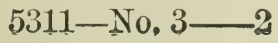


A few explanations seem necessary, however, for an understanding of the terms employed in tabulating these facts:

1. In discussing these streptococci it was necessary to assign to each some name by which it could be designated. For this purpose the let. ters of the alphabet were chosen.

2. In the beginning of our work several of the tests were not employed that have been used in studying the forms more recently isolated.

3. The streptococei are given in the order of their discovery.

4. The words short, long, etc., used in designating the length of the chains, have been assigned the following arbitrary definite meaning: Very short chains are composed of from 3 to 4 cocci or segments; short chains of from 4 to 10 cocci; long chains of from 10 to 40 cocci; very long chains of more than 40 cocci. In the table these terms have reference to the growth in bouillon only. In cultures containing chains ranging from 3 to 50 or more cocei, the term has been employed that applies to the greatest ummber of individuals.

5. The media used were faintly alkaline in reaction. The agar, gelatine, and bouillon container one-quarter per cent peptone and onehalf per cent sodium chloride. Bouillon used in the fermentation tubes contained, in addition, 2 per cent glucose. The word "nuclear" is used to designate colonies from 1 to 2 millimeters in diameter (on the inclined surface of agar) which have a convex, grayish, opaque center, with a spreading translucent border. The word "moderate" represents a corresponding homogenous non-nuclear growth. In the growth in gelatine "feeble" indicates the development of colonies varying from one-eighth to three-fourths millimetre in diameter.

The fermentation tube* enables us to determine approximately the aërobic and anaërobic properties of the bacteria; as the liquid in the closed bulb is deprived of its air during the sterilization, all bacteria that grow in it can be considered as more or less anaërobic. The glucose was added in order to detect the power of the various streptococei to cause the fermentation of sugar with or without the production of gas.

\section{BIOLOGY.}

In addition to the media inentioned, glycerine agar, blood serum, agar-gelatine, glycerinated bouillon, acid bouillon, and potato broth have been employed in the cultivation of these streptococci. Although von Lingelsheim found blood serum very advantageous, my experience with the streptococci in question showed this medium to be quite unsatisfactory. (I lave used the serum from cattle only.) The growth was variable on the various other solid media and in the boullon con-

*Dr. Theobald Sinith ealled attention to the value of the formentation tube in bacteriological work in 1890. Centralblatt f. Bacteriologie, Bd. vII, p. 502. It is figured and briefly described hy the same anthor in his Report on the Cause and Prevention of Swine Plagne.

U. S. lepartment of A griculture, 1891, p. 81 . 
taining glycerine. Like the swine-plague germ, the great majority of these streptococei would not grow in acid bouillon, although many of them would change the reaction of the alkaline medinm to an acid one during their multiplication.

The character of the growth in bouillon is interesting. With few exceptions it will be observed that the long-chained streptococci grew in flakes, while those composed of short chains imparted a uniform cloudiness to the liquid. This property was constant. Streptococcus $\mathrm{Q}$ was carried through a series of 20 subcultures made at short intervals with no alteration in the character of the growth. Shorter series were made with several of the other streptococci with the same result. In a few cases, there was a faintly clouded appearance of the liquid in which the streptococci grew very largely in flaky masses. The flakes are due to the interlacing of the chains, presumably during the process of multiplication. The time required for the liquid to become clear was very constant (as tested by several cultures), although it varied very much with the different species (?). The settling of the streptococci did not indicate their death, but the limit of their growth in the liquid. The sediment was not viscid.

Although the growth on agar and gelatine varied slightly, the constant characters were very similar, and, with few exceptions, these cultures could not be considered of any differential value. Their growth on potato, their ærobic and anaerobic powers, and their effect on the reaction of bouillon and the casein of milk show more marked differences. Although with every germ tested the reaction of the alkaline bouillon containing glucose was changed to a very acid one, no gas was produced. These facts are significant in showing the differential importance of the physiological properties of these bacteria.

In considering the minor differences that exist between these streptococci, especially those that were isolated from animal tissues, it should be remembered that they were obtained presumably at different periods of time after their invasion, and that there is no positive information by which we can determine the changes produced in their various properties by the influence of their new environments and the variable time in which they were subjected to them.

\section{MORPHOLOGY.}

When examined in a fresh condition, (hanging drop) with a magnification of 500 diameters, the chains appear, as a rule, to be made up of micrococei 0.7 to $1.8 \mu$ in diameter. When stained with methylene blue, or fuchsin, they appear as deeply stained oval or spherical bodies. With a magnification of 1,000 diameters, the coeci, in the majority of the forms, appear to be more or less elongated. Many of these exhibit the polar stain or belted appearance quite characteristic of the swine plague group of bacteria, while others show simply a light unstained center. The spherical forms usually appear as deeply stained bodies. 
In many species (?) there seems to be a tendency of the segments to be closely united in pairs. This is believed to be due to the incomplete division of the cocei. The Gram and Weigert-Gram staining methods can not be considered of much importance as means of differentiation, on account of the variable degree to which the stain is retained by the different species. The individual deviations from the typical streptococcus (micrococci united in chains) are as follows:

In streptococci $\mathrm{A}, \mathrm{F}, \mathrm{G}, \mathrm{O}, \mathrm{Q}$, and $\mathrm{W}$, the individual segments were oval, elongated, and resembled somewhat, in a stained specimen, the appearance of the swine plague organism. In $G$, the long diameter appeared to be transverse to the long axis of the chain. An examination of a properly stained specimen, magnified 1,000 diameters, revealed the fact that a single segment was composed of two of the supposed cocci separated by an unstained band. The ends were nearly square, giving them the appearance of minute rectangular parallelograms. In streptococei B, E, M, and $\mathrm{U}$, the terminal cocci, and frequently one or two within the chains, were much larger than the other segments. When only two were present they were invariably at the extremities. They were never observed in the other streptococci, and their siguificance is still somewhat speculative. Streptococcus L presented a great variation in the size of the cocei and length of the chains. So varied was the size that it was thought for a considerable time that it was an impure culture. Repeated plate cultures, however, developed only one form of colonies which were composed of streptococci of a similar character. The shorter chains were composed of the larger cocci and the longer ones of the smaller cocci. This variation* was also present, but to a less degree, in streptococcus $\mathrm{Z}$.

Streptococcus O varied very considerably when grown in different media. In bouillon the segments were spherical, while on agar and gelatine they were oval. The morphological as well as biological characters of this streptococcus indicated its close relationship to the ordinary saprophytic micrococci. The length of the chains in nearly all of the species varied with the media; usually they were longer in bouillon than on solid media, and longest in bouillion containing glucose.

Although considerable variation was found in the length of the chains, and in a few cases in the size of the individual cocci, there was no appreciable change produced by artificial cultivation in the morphology of any of these forms. Streptococeus $\mathbf{Q}$ was carried through a long series of cultures in a liquid containing very little nutriment ( 0.5 cc. of bouillon and 9.5 ce. of sterile distilled water). The number of chains was greatly diminished, but no appreciable change was produced in the morphology of the individual chains or cocci. Cultures in bouillon containing 1 per cent peptone gave similar results. It is

* Koplik and Van Arsdale have recently noted the variation in the diameter of the cocei, in the different chains of the streptococens found by thom in diseased joints (osteomyelitis). 
interesting to note in this comnection that in the pathogenic forms the chains were very short, and often single cocei appeared in cover-glass preparations from the organs of the dead animals. The few forms that were fatal to experimental animals possessed no morphological or biologieal peculiarities that would suggest their pathogenic properties, or separate them as a class or group from the others. The parasitic tendency in the cultural characters of all the streptococci (excepting O) was very marked.

It is observable that, while there are many minor differences, there is still a strong similarity existing between the different streptococei mentioned (excluding streptococeus $\mathrm{O}$ ), and that the difference in the species of the host animal or the disease with which they were associated was not marked by any distinct characteristic of the invading streptococci. It will be observed from the table that in the differential tests no two of the streptococei that were studied gave precisely the same reaction throughout, but each streptococcus differed in some respect from all of the others. This fact appears to support Klein's statement that there is a specific or at least variatal difference existing between the streptococci (isolated from different sources) which appear in a superficial examination to be identical. In many cases, however, the variation was so slight that it seems possible to find its cause in external conditions rather than in the inherent properties of the germ.

The statements at hand concerning the variations in the morphology, biology, and pathogenic properties of certain bacteria as found in nature, and the power to modify to a greater or less degree these properties in certain species by cultivating them under different conditions, would warrant the supposition that many of the minor differences in these streptococci could have been brought about by the different influences to which they had been subjected prior to their isolation. The close resemblance of the streptococcus from the tracheal mucus of a pig (streptococcus P), to those found within the organs of diseased swine is at least suggestive in tracing the source of these organisms. The fact that intestinal and tracheal bacteria invade the inuer organs and cavities of the body is so well established that it is not difficult to believe that streptococci, known to be more or less numerous in the flora of the mucous inembranes of the healthy animal, should occasionally, under favorable conditions, make their way into the various organs of their host.

A few very interesting differences in the reaction of streptococci isolated from different organs of the same animal have been observed. Streptococci $\mathrm{G}$ and $\mathrm{H}$ were isolated from the liver and lung respectively of a pig that presented the lesions on post-mortem examination of a somewhat modified case of swine plague. In sections of the lung streptococcus $I I$ was seen winding in and out among the cells in the alveoli. As there was considerable lung disease but no swine plague 
or hog cholera bacteria discovered, the pathogenesis of this germ was thoroughly tested on mice and rabbits, but with negative results. Streptococcus G grew without exception in the longest chains of any form studied, but like streptucoceus $\Pi$ it prodnced no effect on animals, the difference between them existing in their morphology and biology.

Streptococci $\mathrm{R}$ and $\mathrm{S}$ were isolated from the pus in the joint abscesses (knee and elbow) of a pig that died of chronic hog cholera. (The hog-cholera bacilli were obtained from the same abscesses). As they were isolated from the abscesses, produced presumably by the same cause in the same animal, it is evident that they were subjected to practically the same conditions after their invasion. They were not rlis. tinguishable excepting in their reaction to Gram's and Weigert-Gram's staining processes and in their effect upon milk, where repeated experiments proved conclusively that streptococcus $R$ wiould invariably coagulate the casein, while streptococcus $\mathrm{S}$ produced no ajpreciable change. The constancy in their reaction to the staining reagents was likewise determined. A third and similar illustration of this peculiar variation of streptococci inhabiting the same host and apparently identical in the majority of their characteristics is foumd in streptococei $\mathrm{Z}$ and $\alpha$. These were obtained from the same animal and differed principally in their virulence, the one being fatal to mice only, the other destroying mice, rabbits and swine.

Pasquale makes the remark that streptococei differing in their characters were isolated from different organs of the same animal.

\section{OTHER STREPTOCOCCI.}

The streptococei that perished before they were studied sufficiently to be considered here did not, as far as they were observed, present any characteristics that were markedly different from those here described. A large number of them were inocnlated into mice or rabbits, or both, but always with negative results. There are, however, several streptococei that have been isolated and studied more or less thoroughly, which have not been included in the preceding series, but which appear to be of sufficient importance in throwing light upon the distribution of the delicate and more saprophytic forms of streptococci to be briefly considered. A glance at the table will show that streptococcus $\mathbf{O}$ (isolated from the feces of a cow) differs very deciledly from the other forms in its biological characters, and that in every way it was more saprophytic in its tendencies. Several apparently similar streptococci have been isolated, which, upon subsequent cultivation, lost their power to grow in chains and appeared as ordinary saprophytic micrococci or diplococci. Some of these only softened gelatine, while others liquefied it. I will describe briefly two of these supposed streptococei :

(1) April 4, 1830.--T examined a rabbit which had a large and apparently elosed alosecss $1 n$ one of its nasal cavities. From the pus in this abscess I obtained a strep- 
tococcus. It grew in short chains, and was composed of micrococci 1.2 to $1.5 \mu$ in diameter. It stained nicely after Gram's method, and grew very vigorously on the ordinary media. After about four generations, however, it ceased to grow in chains, aud appeared subsequently as simply micrococci.

(2) From the tracheal mucus of a sheep a streptococcus was isolated by means of gelatine plates. It consisted of micrococi 1.2 to $1.8 \mu$ in diameter, united in short (hains. It took the Gram stain and grew very vigorously on all of the ordinary media. After a few generations very few ehains were observed, but diplococci and single germs appeared in large numbers. It liquefied gelatine. The variations in the size of the cocci is especially worthy of note.

During an investigation of an outbreak of abortion in mares, cultures were made from the vaginal secretion of five healthy animals, from four of which a streptococcus which grew in long chains was obtained, which, in the minuteness of its structure and delicacy of its cultural characters, surpassed any of those described in this article. The streptococei from the different animals appeared to be identical in their microscopic appearance and growth on certain media. They were cultivated only with difficulty. In the examination of Potomac river water and a series of soil examinations varions streptococci were found, some of which, upon superficial examination, presented characteristics of strongly saprophytic bacteria, while others were parasitic in their tendencies. Dr. Smith gave me a culture of a streptococcus isolated by him from drinking water that was biologically as parasitic in its tendencies as any of those obtained from the organs of diseased animals.

Although these observations on streptococci from extraneous material could be multiplied, the facts stated are sufficient to show the wide variations found to exist in this group of bacteria, and that those forms which we have isolated from the organs of diseased animals do not possess, biologically, parasitic tendencies that are not equally as well marked in some of the streptococei found apparently in their normal habitat in nature. These facts are of much importance iu considering the economic value of this group of bacteria.

\section{PATHOGENIC PROPER'TIES.}

It will be observed from the table that fifteen of the streptococci were obtained from the organs of animals that had perished from a known disease, while nine of them were isolated from animals that were either healthy or that had died from a disease the specific cause of which was not positively determined. These facts are important in their bearing upon the economic value of these forms. Of the twentyeight streptococci described, six were fatal to some one or more of the experimental animals. The source of these destructive forms shows that two of them were associated with specific disease germs; two were found in a pig in which no disease germ was discovered, although the pig was from a herd in which several of the animals had succumbed to hog cholera: one from a pleuro-pneumonia lung, and the other was 
firm the tracheal inucus of a lame, but otherwise healthy horse. In addition to these, three of the forms produced more or less local reaction in rabbits, but apparently had no effect on mice. Of these, two were associated with specific pathogenic bacteria, and the third was found in the organs of an animal that perished from an undetermined disease. The other nineteen forms exhibited no pathogenic properties whatever. In addition to the usual subeutaneous and intravenous inoculations several of them were injected in large quantities into the pleural and abdominal cavities of rabbits with no appreciable effect. It should be observed that six of the nine more or less pathogenic forms grew in long chains, while in the other three the chains were short.

Besides the fact that 79 per cent of these streptococei, and all of a considerable number of other forms isolated from similar sources, failed to destroy experimental animals, we should note a few other general facts which are important in considering the pro and con of their etiological value.

1. The streptococci were isolated from a very large variety of lesions, from many of which the specific germ was obtained.

2. Comparatively speaking, a small minority of apparently similarly affected organs contained streptococci.

3. The streptococci isolated, even those from undoubtedly the same kind of lesions, were not identical as indicated by the various differential tests applied.

In these respects my observations have differed from those of Fehleisen, Klein, Schuitz, and others who have attributed specific etiological value to certain of the streptococei which they have studied. It will be remembered that in their investigations the same streptococei were found to be nearly or quite unversally present in a greater or less number of similarly diseased conditions.

As my observations and results do not conform with the necessary requirements to establish a specific pathogenesis for a micro-organism, it would seem that the streptococei here described (that were obtained from animal tissues) must be considered as having accidentally* invader the organs from which they were isolated. Their etiological effect is therefore reduced to a secondary place, which assures them of no greater economic value than that possessed by a large number of other bacteria that are frequently found in diseased animal tissues. $\Lambda$ more detailed account of the inoculation experiments with the nine path. ogenic forms is appended:

Streptococcus A.-For the pathogenic effect of this streptococcus I quote from the Report of the Burean of $\Lambda$ nimal Industry for 1887 : "Two rabbits and two mice wero inoenlated subentancously with one-twelfth ce. each of a pure bouillon culturo. Both

*By the term "acciclentally" is meant a passive invasion, brought about by conditions produced by other eanses in contradistinction to the iuvasion of virnlent pathogenic bacteria. 
mice were found dead on the morning of the second day. One, on acconnt of decomposition, was not examined; the other presented at the point of inoculation a slight reddish serons infiltration containing numerous streptococci. There was a moderate number in the spleen and blood from the heart. The two rabbits remained well. Cultures of this microbe were injected beneath the skin into the thorax and trachea of pigs withont causing any distnrbance."

Two series of inoculations in mice were male with this streptococcus for the purpose of testing the effect of repeated inoculations upon the virulence of the germ. The first monse was inoculated with a pure culture, the second from the blood of the first and so on. The appended table is self-explanatory:

\section{Inoculation experiments.}

\begin{tabular}{|c|c|c|c|c|c|}
\hline $\begin{array}{c}\text { Mouse } \\
\text { No. }\end{array}$ & $\begin{array}{c}\text { Date } \\
\text { of inocu- } \\
\text { lation. }\end{array}$ & $\begin{array}{l}\text { Inoculated subcu- } \\
\text { taneously with- }\end{array}$ & $\begin{array}{l}\text { Date of } \\
\text { cieath. }\end{array}$ & $\begin{array}{l}\text { Time re- } \\
\text { quired to de- } \\
\text { stroy life. }\end{array}$ & Remarks. \\
\hline $\begin{array}{l}2 \\
3 \\
4 \\
5\end{array}$ & $\begin{array}{c}\text { 1887. } \\
\text { Aug. } 15 \\
\\
\text { Aug. } 17 \\
\text { Aug. 20 } \\
\text { Aug. } 25 \\
\text { Aug. } 29\end{array}$ & $\begin{array}{l}2 \text { to } 3 \text { drops bouillon } \\
\text { culture. } \\
\text { Loop blood mouse } 1 . . \\
\text { Loop blood mouse } 2 . . \\
\text { Loop blood mouse } 3 . \\
\text { Loup blood mouse } 4 .\end{array}$ & $\begin{array}{l}\text { 1887. } \\
\text { Aug. } 17 \\
\\
\text { Aug. } 20 \\
\text { Aug. } 25 \\
\text { Aug. } 29 \\
\text { ……... }\end{array}$ & $\begin{array}{l}2 \text { days } \ldots . . . \\
3 \text { days } \ldots . . . \\
5 \text { days } \ldots . . \\
4 \text { days } \ldots . . \\
\ldots \ldots\end{array}$ & $\begin{array}{l}\text { Streptococei in blood and spleen not } \\
\text { found in cover-glass preparations } \\
\text { from liver. } \\
\text { Do. } \\
\text { Do. } \\
\text { Do. } \\
\text { Remained alive and well. }\end{array}$ \\
\hline
\end{tabular}

A second series gave the same result, showing that the streptococcus lost its virulence very rapidly.

Streptococcus B.-This streptococcus was obtained in pure cultures (bonillon and agar) from the spleen of a pig that died at the Experiment Statiou apparently from a disease of the liver. Mice inoculated subcutaneously with a pure culture of this microbe remained apparently perfectly well. A rabbit inoculated in a similar manner with $0.25 \mathrm{cc}$. of a turbid suspension in bouillon of the growth from an agar culture exhibited on the following day considerable reddening and slight swelling at the point of inoculation. No elevation of temperature. 'These symptoms soon disappeared.

Streptococcus E.-This streptococcus was obtained from the spleen of a pig. The animal was one among others that perished in an outbreak of modified hog cholera near Washington, D. C., in 1889. Streptococci C and D were obtained from pigs in the same ontbreak.

Mice inocnlated subcutaneously with from 1 to 3 drops of a bouillon culture died in from 2 to 14 days. The livers and spleens hyperiemic. Coagulation necrosis in the liver when the animals lived several days after inoculation. Stained cover-glass preparations from the organs showed the cocci single, in small clnmps, and short chains, never in long chains. Pure cultures of the strcptococcus were obtained from the organs in every case. A rabbit inoculated witl one-eighth cc. of a bouillon culture in the ear vein died within 24 hours. Slight enlargement of spleen, liver hyperamic, blood-stained serum in pericardial sac. In stained cover-glass preparations of the blood a few micrococci, usually in pairs, were discovered. No bacteria werc observed in similar preparations from the spleen. Pure cultures of the streptococcus were obtained from both spleen and blood. A rabbit inoculated subentaneonsly with one-fourth cc. of a bouillon culture was found dead on the seventh day. Surrounding the point of inoculation a slight injection of the blood vessels. Over the coils of the intestines, liver, and splecn a thin, semi-membranous exudate. Spleen somewhat enlarged, liver hyperæmic. The exudate contained a large number of single microcoeci, also short chains. No bacteria were observed in stained cover- 
gliss preparations from the spleen, liver, and blood. A pure enlture was ohtained from the splcen. 'This streptococeus lost its virulence after having been eultivated for about one your, so that it wonld not destroy either mice or rabits even when inoculated with very large quantities of a pure culture.

Streptococcus $\mathrm{K}$.--This germ was associated with swine plagne bacteria in a bonillon culture which was marle from the peritoneal exulate of a pig that died of swine plagne in an outbreak of that disease in New Jersey in 1890. (Streptococens L was obtained from the liver of another pig in the same outbreak.) It produced no effect on mice. A rabbit was inoenlated subcutaneonsly with 0.25 ce. of a turbid suspension of agar growth in bonillon. On the following day there was considerable reddening and slight swelling at the point of inocnlation, temperatnre $104.4^{\circ} \mathrm{F}$. (Normal temperature $103^{\circ} \mathrm{F}$.) On the second lay the rabbit appeared unwell; the fur was ruffled and it ate sparingly. Temperature $103.6^{\circ} \mathrm{F}$. The next day it appeared well. Temperature normal and the local reaction had subsided.

Streptococens Q.- This germ was found in large numbers in the lung of a cow affected with pneumonia (bronchial?). It produced no appreciable effect on mice. A rabbit inoculated in the ear vein with 0.25 ce. of a bouillon culture appeared nnwell on the following day. Temperature $102.8^{\circ} \mathrm{F}$. A rabbit inoculated subcutaneously in the ear with a loop of the surface growth from an agar cnlture developed a suall abscess at the point of inoculation, with slight reddening of the surrounding tissue. No other symptoms.

Streptococcus T.-This was obtained from a pleuro-pneumonia lung in 1891. Mice inoculated subentancously with a few drops of a bonillon culture died in from 3 to 4 days. No local reaction. Abdominal organs more or less hyperamic. In coverglass preparations from the spleen and liver were a large number of oval bacteria, surrounded apparently by a capsule. Pure cultures of the streptococcus were obtained from the blood. A rabbit inoculated in the ear vein with $0.50 \mathrm{cc}$. of a bouillon culture was found deal on the fifth day. 'The leart, nuscles, and abdominal organs pale. About the right knee joint were several small abcesses in the inter-muscular tissue containing grayish pus. No other abcesses were foumd. No bacteria were observed in stained cover-ghlass preparations from the blood, liver, and spleen. In a similar preparation from the abcesses about the joint a large number of micrococci single, in pairs, and in short chains, were found. A pure culture was obtained from the blood.

Streptococeus $U$.- On June 24, 1891, two rabbits were inoculated with the mucus from the trachea of a horse which was killed on account of lamencss. One of the rabbits died on June 28, from the organs of which this streptococcus was obtained. No other bacteria were discovered. Mice inoculated subcutaneously with a pure culture died on the fifth day. At the place of inocnlation there was a purulent thickening of the skin and subentis over an area one-half inch in diameter. Liver, spleen, and kidney swollen aud discolored. No bacteria found in stained preparations made from the local lesions. Pure cultures were obtained from the spleen and blood.

A rabbit, which was injecterl subcutaneously with $0.10 \mathrm{ec}$. of a bouillon culturo died on the second day. At the proint of inoculation there was a slight injection of blood vessels. Liver pale, spleen enlargerl, dark, and friable. Kidnoys liyperamic. Punctiform hemorrlages in lower colon. Lung emphysematons. Blood-stained sernm in pericardial sac. Many cocci, usually in short chains, in cover-glass preparations from the organs. A pure culture from the blood. $\Lambda$ fter four months this germ had lost its virulence to such a degree that a rabbit inoculated subentancously witl $0.2 \mathrm{ce}$ of a bonillon culture remained well. It was reinocnlated with $0.5 \mathrm{ce}$. five days later, and died on the second day following the inoculation. At point of inoculation a slight purulent iufiltration into the subcutis, liver reddened, swollen, and sprinkled with minute romd grayish aroas (coagulation necrosis?). Spleen much onlarged, dark-colored, and friablo. Considerable bloot-stained liquid in 
perieadial sac. Cover-glass preparations showed namy micrococci, but no chains or eapsules were observed. After five months (nine months in all) $0.75 \mathrm{ce}$ of a buillon enlture destroyed a rabbit in five days. A slight local reaction; exulative peritonitis; exudate eontained coeci. Spleen dark. Liver enlarged, hyperemic, kidlneys redlened. Many bacteria appeared in preparations made from the liver, but few in those made from the blood. Pure cultures were obtaincd from the organs.

Streptococens $Z$. - This germ was found in the liver of a pig that died in an outbreak of swine disease in Illinois in 1892. No hog-cholera or swine-plague bacteria were found in this animal, although the hog-cholera germ was found in several of the animals that perished in the same outbreak. Two mice inoenlated subentanconsly with this streptococeus died, one on the fourth and the otlicr on the fifth day. At point of inocnlation a purnlent thickening of the skin and subcutis extended over an area one-half incl in diameter. Livers pale (fatty), spleens and kidneys eongested. Stained eover-glass preparations from the internal organs showed no bacteria, while those from local lesions exhibited a very large number of micrococci. Agar tubes inoculated with the blood of the first mouse that died, and with the spleen of the second one, developed pure enltures of the streptococens. A rabbit inoculated subcutaneously with $0.75 \mathrm{cc}$. of a bonillon culture remaincel perfectly well.

Streptococcus $\alpha$.-This organism was obtaincd from the blood of the same pig from which strcptococeus $\mathrm{Z}$ was isolated. Micc inoculated with this germ died on the third day, and presented lesions similar to those produced by streptocoecus Z. The local lesion was more extended in this case.

A rabbit that received 0.25 cc. of a boullon enlture in the ear vein was found dear on the following morning. Blood-stained liquid was found in the peritoneal cavity and pericardial sac. Heart muscle (right ventricle) hemorrhagic. Enormons numbers of oval bacteria, many of which exhibited a light central band, were found in cover-glass preparations from the various organs. They were usually mited in short chains. A rabbit inoculated subcutaneously with 0.75 ce. of a bouillon culture remained well.

March 1, 1892, two pigs (Nos. 91 and 92) were each inoculated intravenonsly with 5 cc. of a bouillon culture. On the following day both animals were apparently unwell, ate sparingly, and moved about very little. March 4, inprovement in appetite and general appearance, but both animals used the right fore leg very little. March 6, appetite fairly good, but pigs have no use of their right fore limbs. Mareh 7, no apparent chauge in their eondition when seen in the morning. At 1 p. m. pig 91 was found dead; examination made at once..

Pig No. 91 (female), Essex grarle, aged 3 montlıs, weight 65 pounds, eondition good. The capsnlar ligancnt of the right shoulder joint thickened, deeply reddened areas on the inner surface, otherwise the eapsule pale and apparently necrosed. Articular cartilage normal in appearance. An infiltration of a thick yellowish purulent substance in the intermuscular tissue surrounding the joint. Other joints normal.

In the abdominal cavity abont $300 \mathrm{ec}$ of light amber-colored liquid, which coagulated on exposure to the atmosphere. A few elastic shreds adhered to the abdominal parietes. Spleen very much enlarged, discolored, and on the lesser surface scveral blood tumors. Liver swollen, hyperæmic. Kidneys swollen, surface mottled. A microseopical examination showed parenchymatous degeneration of both liver and kidneys. Stomach containerl a small quantity of undigested food; mucosa of fimdus slightly congested and pigmented. Upper portion of the dnodenal mucosa sprinkled with stellate areas of blood injection; mucosa and contents deeply bilc-stained. This was true of the small intestine throughout. Large intestive normal. Lungs hyper:emic and slightly emphysematous; a eonsiderable area of the right lung agghtinated to eostal surface hy a fibro-gelatinous substance. An area 4 by $2 \mathrm{~cm}$., of right lung, was deeply congested and partially hepatized. Heart well 
filled with post-nortem slots; several punetiform hemorrhages on right auricle and base of right ventricle.

Stained cover-glass preparations from the various organs showed many micrococci, often in short chains. In the liver, the chains were from 4 to 8 cocei long, surrounded by an unstained border which appeared to be a capsule. From the peritoneal and pleural exudates, and from the organs pure cultures of the streptococcus were obtained.

Pig No. 95 remained apparently quite well, excepting the loss of the use of the right fore limb. Killed for examination March 23. Female, Essex, weight 90 pounds, in good condition. Right elbow joint considerably enlarged, articular surfaces normal, capsule very much thickened, eartilaginous and sprinkled with centers of ossification. Spleen consirlerably enlarged, engorged and on section shows a few hemorrhagic infarets. Otherwise the tissues appeared to be normal. No bacteria found in cover-glass preparations made from the difterent organs, and media inoculated from the organs remained sterile.

The appended table contains a summary of the inoculation experiments with the pathogenic forms:

Summary of inoculation experiments with the pathogenic streptococci.

\begin{tabular}{|c|c|c|c|}
\hline $\begin{array}{l}\text { Strepto- } \\
\text { coccus. }\end{array}$ & Fatal to- & $\begin{array}{l}\text { Time required } \\
\text { in destroy- } \\
\text { ing life. }\end{array}$ & liemarks. \\
\hline $\begin{array}{l}\mathbf{E} \ldots \\
\mathrm{K} . . \\
\mathrm{Q} . . . \\
\mathrm{T} \ldots \\
\mathrm{U} \ldots \\
\mathrm{Z} . . .\end{array}$ & 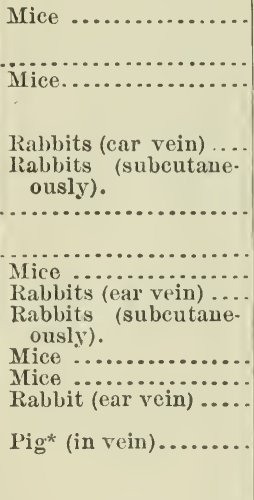 & 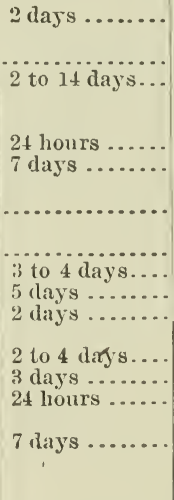 & 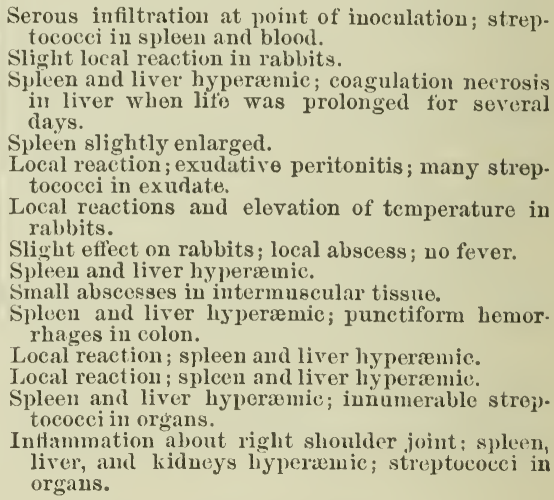 \\
\hline
\end{tabular}

* Streptococci A $a$ and are the only ones that were inorulated into pigs.

Unfortunately streptococei $Z$ and $\alpha$ perished before a further study could be made of their pathogenic properties.

Although the effect produced on animals by the nine pathogenic streptococei varied to a greater or less degree, in general the lesions produced were more characteristic of septicamia and pyamia than of any other general or local disease. The localization of the lesions in rabbits appeared only when the animals lived for several days-a condition comparable to that produced by attemuted rabbit-septicamia bacteria. The comparatively rapid attenuation of the more virulent streptococci (excepting $\alpha$, which perished before this point was determined) is also observable. This is important, as it points to a temporary virulence lather than to distinctive specific pathogenic species. Tho temporary virulence may, in some cases at least, be explained on the 
ground suggested by Lesage and Macargne,* who disenvered that Bacillus coli comunis acquired more or less virulence when exposed in diseased animal tissues. $\dagger$ Althongh we are not able to show that streptococei were in any case primarily the cause of the lesions in which they were found, or that they are capable of producing any disease in the experimental animals manifested by characteristic lesions, $\ddagger$ their multiplication within the organs of the body undoubtedly had a more or less secondary influence in the production of the lesions from which they were obtained. The similarity that exists between the description of the streptococcus, supposed to be the canse of strangles and streptococcus $U$ of this series, should be noted. The toxic effect of filtered or sterilized cultures of these streptococci was not tested.

The organs from which these streptococci were originally obtained presented such a variety of appearances that it was impossible to predict with any degree of certainty the presence of streptococei in any of the lesions examined. This is further evidence of their accidental invasion and non-specific etiological value. The results obtained and recorded in the preceding pages-limited and variable as they areappear to be sufficiently clear to warrant the following conclusions:

\section{CONCLUSIONS.}

1. Streptococci are very generally distributed in nature, and especially on the mucous membranes of healthy animals. They fall very naturally into two classes: (1) Those that are strongly saproplytic in all of their properties; and (2) those that lave decirledly parasitic tendencies in their morphological and cultural characters. The second class only has been found (by the writer) in diseased animal tissues.

2. Under certain conditious, presumably a weakened condition of the body with lesions of the mucosa in the air passages or intestinal tract, the streptococei of the mucous membranes invade the inner cavities and organs of the body. The large number of streptococei frequently found in the tissues indicate their invasion and subsequent multiplication prior to the death of the animal.

3. The streptococei that have been isolated in my investigations are capable of being differentiated by one or more distinct and apparently constant characteristics. In several instances, however, the differences are very slight, and may be due to previous conditions of life or unobserved irregularities in their cultivation.

4. Streptococei, quite as delicate and parasitic in their cultural characters as those obtained from diseased organs, are found in the flora of water and the mucosa of healthy animals, although no two of these

*Archiv. de Med., Exper. No. 2, 1892.

tA series of experiments is now in progress which it is hoped will throw some light upon this question with reference to certain streptococci.

$\ddagger$ Excepting more or less purulent infiltration of the subcutaneous tissue at the point of inoculation and general changes indicative of septicæmia or pyæmia. 
contrasted species (?) have been found that possess precisely the same properties.

5. The grouping of the pathogenic and non-pathogenic streptococei by von Lingelsheim into Streptococcus longus and Streptococcus brevis does not hold true with all the forms that I have studied. The pathogenic effect of at least the majority of the destructive forms is septic in its character. Their virulence is soon lost.

6. Like other bacteria, streptococei are frequently found in the organs of animals that have perished from different and widely separated diseases. A few of these streptococci, as well as a small per cent of those from other sources, have been found to be fatal to certain of the experimental animals, while a large majority of them possess (when isolated) no pathogenic powers whatever as indicated by animal inoculations.

\section{LIST OF AUTHORS REFERRED TO IN THE TEXT.}

BiLlRoth._-Untersuchungen iiber die Vegetationsformen von Coccobacteria septica 11. s. w. Berlin, 1874.

Kocr.-Untersuchungen iiber die Aetiologie der Wund-infectionskrankheiten. Leipzig, 1878, p. 47.

SAlmoN.-Reports of the Department of Agriculture, 1880, 1881-1882.

KLers.-Sixteenth Annual Report of the Local Government Board. Supplement eontaining Report of the Medical Officer. London, 1886-'87.

KLern.-The Veterinarian, 1886, p. 92.

SAND AND JENsen.-Reviewed in Baumgarten's Jahresbericht, 1888, p. 86.

'ZscirokKL.-Schweiz. Archiv f. Thierheilk., Bd. xxx, p. 209. Reviewerl in Baumgarten's Jahresbericht, 1888.

SCHÜT7.-Archiv f. wiss. u. prakt. Thierheilkuude, Bd. xrv, 1888, p. 172.

OGston.-Archiv f. klinisch. Chirurgie, Bd. xxv, 1880; British Med. Journal, 1881, p. 369.

Fentersen.-Aetiologie des Erysipels. Berlin, 1883.

RoskNBACH.-Mikro-Organismen bei den Wumd-Infectionskrankheiten des Menschen. Wiesbaden, 1884.

BARBIEr.-Arehives de médecine expérimentale et d'anatomie pathologique, 1891, No. 3, p. 361 .

BArbier.-Ibid., 1892, No. 6, p. 827.

Prudnen.-The American Journal of the Medical Sciences, xcrir, 1889, p. 229.

FlüGGE.-Die Mikroorganismen, 1886.

HАJEK.-Reviewed in Baumgarten's .Jahresbericht, 1888.

Fraenkei.-Centralblatt f. Bakteriologie u. Parasitenkuude, Bdi. vi, 1889, p. 691.

WeLCH.-The American Journal of the Medical Sciences, CII, 1891, p. 439.

KLfix.- Seventeenth Aunual Report of the Local Government Board. Supplement containing Report of the Medical Officer, 1887, p. 256.

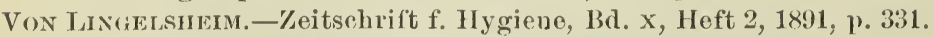

Smitir.-Report of the Burean of Animal Industry, 1887-1888.

Koplik and Van Arspale.-The American Journal of the Medical Seiences, CII, 1892, p. 422.

KURTH.-Arbeiten a. d. kaiserlichen Gesundheitsante, Bd. vir, 1891, p. 389.

Kuntr.-Ibid., Bd. vil, 1892, Heft 2, p. 294.

P'Funh.-Zeitschrift f. Hygiene, Ba, xн, 1892, Heft 4, p. 517.

PAsqualk.-Beiträge zur path. Anat. u. zur allgemeinen Pathologie, Bd. xir, IIeft 3, 1893, p. 433. 
A NON-NOTILE PATHOGENIC BACLLLUS CLOSELY RESENBLING THE BACILLUS ()H' HOG CHOLERA FOUND IN THE LUNG AND THE SPLEEN OF A PIG。

By Veranes A. Moone.

In the bacteriological examination of the spleen and a portion of the lung of a pig early in 1891, a non-motile bacillus was discovered which, on account of its pathogenic properties, has been somewhat carefully studied. Its appearance in a bouillon culture from the spleen and in stained cover-glass preparations from the bronchial secretions of the lung suggested a slightly modified form of the swine-plague germ. Further investigation, however, showed that biologically and in its effect upon animals it resembled very closely the hog-cholera bacillus. In the lung it was associated with attenuated swine-plague bacteria, but it appeared in a pure culture from the spleen. Unfortunately the other organs of this pig were not bacteriologically examined.

The pig from which this bacillus was isolated was of Essex grade, about 10 months old, and weighed 50 pounds. For several weeks prior to its death it had been kept in a pen at the Experiment Station with three other pigs. It had not been exposed to any swine disease. The other animals remained well. From the autopsy notes $I$ take the following:

There was extensive broncho-pneumonia of the cephalic and median lobes, and small areas of hepatization in the principal lobes of both lungs. No pleurisy. Abdominal organs apparent]y normal. A comminuted fracture of the right femur and suppuration, the pus burrowing in all directions, but more especially downward. Both hockjoints much enlarged and deformed. The injury to the femur was probably due to fighting.

The spleen and a small portion of the right lung* were removed with antiseptic precantions, and taken to the laboratory in a sterilized bottle and jar. These tissues were examined at once. No bacteria were found in cover-glass preparations from the spleen. A tube of bouillon inoculated with the spleen pulp developed a pure culture of a

${ }^{*}$ The piece of lung receired at the laboratory was quite firm, pale, the lobules separated by opaque grayish lines, due to thickening of the interlobular tissue. The surface mottled with grayish, more or less elevated nodules, varying from onehalf to two millimeters in diameter. On section the nodules appeared as grayish necrosed tissue. Upon pressure, muco-pus exuded from the larger bronchioles. Near the border there was ecchymosis beneath the pleura, 
non-motile bacillus. Cover-glass preparations from the muco-pus from the bronchioles contained a very large number of oval, elongated bacteria slightly larger than the swiue-plague germ. A smaller number of apparently the same bacteria were found in similar preparations from the consolidated lobules. A rabbit inoculated subcutaneously with the hepatized tissue died of swine plague on the fourth day. The characteristic polar-stained bacteria were found in cover-glass preparations from the organs. Plate cultures in agar developed colonies of swine-plague bacteria only. A second rabbit, inoculated with the muco-pus from the bronchioles, died on the eleventh day with lesions resembling those produced by hog-cholera bacteria. Pure cultures were obtained fro $\mathrm{m}$ the spleen, liver, and blood of a non-motile bacillus slightly larger than that of swine plague, but apparently identical with those obtained from the spleen of the pig.

The marked difference in the character of the lesions and the number of bacteria present in the organs of the two rabbits led to further investigations of the bacteria obtained (1) from the hepatized lung tissue, (2) from the muco-pus of the bronchioles, and (3) from the spleen. The methods employed and the results obtained were as follows:

\section{INOCULATION OF RABBITS.}

The necessary information concerning the inoculations for differential purposes is summarized in the appended table:

\begin{tabular}{|c|c|c|c|c|c|}
\hline $\begin{array}{l}\text { Rabbit } \\
\text { No. }\end{array}$ & $\begin{array}{l}\text { Date of } \\
\text { inocula- } \\
\text { tion. }\end{array}$ & $\begin{array}{l}\text { Tissue and cultures inocu- } \\
\text { lated subcutaneously. }\end{array}$ & $\begin{array}{l}\text { Date of } \\
\text { death. }\end{array}$ & $\begin{array}{l}\text { Time } \\
\text { required to } \\
\text { destroy } \\
\text { rabbits. }\end{array}$ & Romarks. \\
\hline 1 & $\begin{array}{l}\text { 1891. } \\
\text { Jan. } 9 \\
\text { Jan. } 24\end{array}$ & $\begin{array}{l}\text { Hepatized tissue pig's lnng. } \\
0.01 \text { cc. bouillon culture }\end{array}$ & $\begin{array}{l}\text { 1891. } \\
\text { Jan. } 14 \\
\text { Jan. } 28\end{array}$ & $\begin{array}{l}5 \text { days } . . . \\
4 \text { days .... }\end{array}$ & $\begin{array}{l}\text { Severe local reaction, perito- } \\
\text { nitis, swine-plague bacteria. } \\
\text { Do. }\end{array}$ \\
\hline 3 & Jan. 9 & $\begin{array}{l}\text { Muco-pus bronchioles, pig's } \\
\text { lung. }\end{array}$ & Jan. 20 & 11 days. & $\begin{array}{l}\text { Local reaction; no peritouitis; } \\
\text { necrosis in liver; very few }\end{array}$ \\
\hline 4 & Jan. 22 & $\begin{array}{l}0.01 \text { ec. bouillon culture } \\
\text { rabbit No. } 3 \text {. }\end{array}$ & Jan. 26 & 4 days.... & $\begin{array}{l}\text { Slight local reaction; no peri- } \\
\text { tonitis: fow bacteria. }\end{array}$ \\
\hline 5 & Jan. 17 & $\begin{array}{l}0.01 \text { ce. bouillon culture } \\
\text { pig's spleen. }\end{array}$ & Jan. 20 & 3 days.... & $\begin{array}{l}\text { Slight local reaction; nocrosis } \\
\text { in liver; fow bacteria. }\end{array}$ \\
\hline
\end{tabular}

The examination of rabbits Nos. 1 and 2 showed typical lesions of attenuated swine plagne. There was severe local reaction and peritonitis with the polar-stained bacteria in cover-glass preparations. The lesions in rabbits Nos. 3, 4, and 5 were characterized by local reaction, which was slight in Nos. 4 and 5 ; enlarged, discolored spleen; necrosis in the liver; heart muscle pale. In stained cover-glass preparations from the spleen and liver were very few elongated oval bacteria slightly larger than the swine-plague germ and usually united in pairs. They exhibited a light unstained center and deeply stained periphery. In similar preparations from the blood a much smaller number of bacteria was found. 
The fact that the rabbit which was inoculated with the virus from the bronchioles lived a day longer than the one inoculated with the culture from the spleen, is worthy of note, as it points to the supposition that the bacteria after penetrating the spleen had become slightly increased in their virulence.

It should be observed that in the cover-glass preparations from the bronchial secretions and the hepatized lung tissue of the pig there was not the visible difference in the size of these bacteria that was apparent in the preparations made from the organs of rabbits that perished from their inoculation, or from subsequent cultures.*

\section{CULTURE TEST.}

Repeated cultures were made on all of the ordinary media of the bacteria obtained from the organs of the pig. Those made from the hepatized lung tissue and from the germ obtained from the rabbit inoculated with the same could not be.distinguished from cultures of swine-plague bacteria. The cultures made from the bacteria obtained from the spleen and from the muco-pus of the bronchioles, by rabbit inoculation, could not be differentiated. They were identical in every respect, and resembled similar cultures of hog-cholera bacteria as the subsequent description will show.

\section{DESTRIPTION OF THE BACILLUS.}

Morphology.-A non-motile, rod-shaped germ, from 1.0 to $2.0 \mu$ in length and from 0.7 to $1.2 \mu$ in thickness. In stained cover-glass preparations from an agar culture they are about $1.0 \mu$ long and from 0.7 to $0.8_{\mu}$ wide; the ends are rounded, giving them the appearance of elongated ovals. In bouillon they are appreciably larger, usually single, but occasionally united in pairs. In stained preparations from the tissues of rabbits they are much larger (1.5 to $2.0 \%$ long and from 0.8 to $1.2 \%$ wide). The periphery stains more deeply than the central portion; this is also frequently observed in preparations from cultures. It does not retain its coloring matter when treated after the Grain or WeigertGram methods.

Biology.-Agar at $36^{\circ}$ C. On the surface of agar, after 24 hours,

* The non-appearauce of the swine-plagne germ in the organs of the rabbit inoculated with the muco-pus of the bronchioles is explained by the fact that they were very largely localized in the hepatized tissue as shown from the plate cultures. In preparing the material for inoculation the surface of the lung was scorched and an incision made with a sterile knife. The section was then scorehed and the muco-pus obtained by pressure. The fow swine-plagne bacteria that were presumably present in the secretions were, on account of their attenuation, overcome by the great excess of other bacteria. It is probable that the swille-plague germs in this case belonged to the tracheal bacteria not distinguishable from those of swine plague that are normally present in the air passages of many healthy swine. (Sce report on Swine Plagne, U. S. Department of Agriculture, 1891, p. 107.)

$$
\text { 5311-No. } 3 \longrightarrow 3
$$


round, convex, grayish, non-viscid colonies appear, varying from 0.5 to $1.5 \mathrm{~mm}$. in diameter, according to the number present. The edge of the colonies is sharply defined, the surface glistening. Within the agar small grayish colonies develop. When grown on agar plates ("double dishes") a peculiar, somewhat musty, penetrating odor is given off.

Gelatine.-At the ordinary temperature the growth is quite slow. After several days round, convex, glistening colonies are developed. Within the gelatine the colonies are smaller, $0.7 \mathrm{~mm}$. in diameter, which have, under a hand lens, a slightly yellowish color and homogeneous appearance. 'The gelatine is not liquefied.

Potato.-At $36^{\circ}$ C. a thin, brownish growth is formed over the sur. face. The growth continues slowly for several days. In old cultures it is of a yellowish brown color.

Alkaline peptonized bouillon.-After twenty-four hours, at $36^{\circ} \mathrm{C}$., the liquid is uniformly heavily clouded with a very slight quantity of a grayish sediment. No appreciable odor. Acid reaction, a few bubbles at surface. In two weeks the liquid is nearly cleared, with the formation of a grayish, friable sediment. At this time the reaction is alkaline.

Acid peptonized bouillon.-In this medium the growth is quite feeble, imparting a faintly uniform cloudiness to the liquid. A grayish band composed of bacteria is formed on the sides of the tube at the surface of the liquid. The reaction becomes alkaline in about two weeks.

Aikaline peptonized bouillon, plus 2 per cent glucose.-In fermentation tubes containing this liquid the growth is quite vigorous throughout. The liquid becomes clouded and acid after twenty-four hours. Gas is formed, which replaces about one-third of the liquid in the closed bulb. The fermentation is very slow; only a few bubbles of gas appear in the first twenty-four hours. The fermentation is completed in about six days. When treated with a solution of caustic potash about one-third of the gas is absorbed. When the remaining gas is brought in contact with a flame there is a feeble explosion.

Milk.-In this medium, at $36^{\circ} \mathrm{C}$., the growth is vigorous. In stained cover-glass preparations, the bacteria are often observed in clumps. The milk remains unchanged in appearance for about two weeks, when it presents a pearly, semi-translucent appearance. A microseopical exanination shows that the fat globules are destroyed.* It is strongly alkaline in reaction. The casein is precipitated by the addition of a few drops of acetic acid. There is a doubtful peptone reaction. $\dagger$ A few experiments were made to test its power of resisting drying, and to determine its thermal death point.

*'This peculiar appearance of the milk is also produced by the hog-cholera bacteria, but usually a much longer time is required.

t As a few drops of caustic potash or soda solution added to a tubo of milk will produce the same appearance, it is probable that the change is largely due to the alkali which is produced durirg the multiplication of the bactoria. 
Drying.-Cover-glasses were placed on a glass stand and covered with a bell jar, all of which were previously sterilized. A drop of a bonillon culture, one day old, was placed, by means of a flamed pipette, on each cover-glass. A tube of bouillon was inoculated with one of these cover-glasses on each day for seven consecutive days. The tubes inoculated on the fifth, sixth, and seventh days remained sterile. The others developed pure cultures of the bacillus.

Thermal death point.-Five tubes of bouillon were inoculated with about six drops each of a fresh bouillon culture and heated in a water bath for five, ten, fifteen, twenty, and twenty-five minutes at $58 \circ \mathrm{C}$. The tube that was heated for twenty-five minutes remained clear. All of the others, including check, developed pure cultures of this bacillus.

\section{PATHOGENIC PROPERTIES.}

In the detailed account of the isolation and differentiation of this bacillus, its effect upon rabbits was partially described. The germ (the one from the spleen) was preserved by means of subcultures until September (eight months), when a series of inoculations were made which demonstrated more fully the range of its pathogenic effect. Rabbits inoculated subcutaneously with 0.01 cc. of a bouillon culture died in from four to six days. The lesions were marked necrosis in the liver, enlarged and discolored spleen, heart muscle pale. In some cases Peyer's patches were pigmented and the kidneys more or less hyperæmic. Occasionally a few shreds of exudate oxer the intestines, but no bacteria present as indicated by cover-glass preparations. The organs contained very few elongated oval bacteria which exhibited a light unstained center and deeply stained periphery in cover glass preparations. This was true in similar preparations from other animals. An intravenous inoculation of the same quantity prover fatal in about forty-eight hours. Guinea pigs were destroyed in eight days by a subcutaneous injection of $0.03 \mathrm{ec}$. of a bouillon culture. The lesions were similar to those produced by hog eholera bacteria. Mice perished in six days after a subcutaneous injection of from one to two drops of a bouillon culture.

A pig weighing 50 pounds, inoculated intravenously with 6 ce. of a four-day bouillon culture, died on the afternoon of the second day. The lesions were not distinguishable from those produced by a similar inoc. ulation of strong hog cholera virus. Pure cultures were obtained from the different organs.

A rabbit that had been inade immune to swine plague was inoculated subcutaneously, together with a check rabbit, with 0.01 cc. of a bouillon culture. The check died in four days. The treated rabbit perished on the sixth day, two days later than the check. This is interesting, as rabbits that have been made immune to swine plague offer no resistance whatever to hog-cholera bacteria.

In June, 1892, this bacillus was as fatal to rabbits as in September, 
1891. This is important in differentiating it from certain so-called toxicogenic bacteria that have been found in water and in diseased animal tissues which were fatal to rabbits when first isolated, but which soon lost their virulence.

From the description of the various properties of this bacillus it will be observed that it resembles the hog-cholera bacillus in (1) its biological characters; (2) the light center and deeply stained periphery in cover-glass preparations;* $(3)$ the appearance of the bacteria in pairs in preparation from animal tissues, and (4) the character of the lesions produced in experimental animals. It differs from it in (1) morphology, (2) its feeble resistance to drying, (3) the small number of bacteria present in the tissues of animals dead from its inoculation, and (4) it is more rapidly fatal to rabbits. Its non-motility, however, is the only specific difference that can be considered between it and the bacillus of hog cholera. Cultures on the various media have been very carefully examined in the very early and advanced stages for the purpose of determining whether or not this germ was motile under any of the ordinary conditions of cultivation, or at any age of the culture, but only negative results were obtained. No flagella could be found, although a very careful examination was made in which the same methods were employed that were satisfactory in demonstrating the flagella on the hog-cholera, coli, and typhoid bacilli. As flagella can be demonstrated on motile but not on non-motile bacteria, it seems fair to presume that after these tests this bacillus can be safely considered a non-motile organism.

Although morphologically it resembles the swine-plague group of bacteria its cultural characters and pathogenic effect on animals differentiate it very distinctly from that group of organisms. The differences between it and the swine-plague and hog-cholera bacteria appear to be sufficiently great to consider it a species rather than a modified form of either of the specific disease germs. Further investigations, however, may reveal intermediate forms which will connect it unquestionably with one or the other of these specific bacteria. The close resemblance that exists between these organisms suggests the importance of a thorough examination of all of the various properties of a germ before it is placed unreservedly in any species, and especially so in eases of pathogenic organisms where a description based on incomplete observations may lead to unexplainable differences in the correlation of our disease-producing bacteria.

* In the earlier investigations of hog cholera (Second Aunual Report Bureau of Animal Iudustry, U. S. Departinent of Agriculture, 1885, p. 212) this reaction of the wacteria to the staining fluids was very constant. It is not, however, so universally observed in the bacteria that have heen isolated from more recent outbreaks, although it is frequently noticed. The difference may bo due to slight variations in the germs themsolves, or to some inappreciable difference in the staining fluids or mode of their application. 
From a baeteriologieal standpoint the natural habitat of this bacil lus is of much interest. The fuct that it has not heretofore been ob. served would indieate that it does not belong to the normal flora of the lungs. As it was the predominating form in the bronchial secretions, it is presumable that it first gained entlance to the air passages, from which it invaded the inner organs of the body. Although it was universally fatal to experimental animals, there is very little if any evidence that it was to any degree responsible for the death of the pig from which it was obtained. This fact reuders it of little economic importance when considered with reference to this case alone, but it seems reasonable to suppose that a germ which possesses such marked pathogenic properties might, under certain conditions, be the cause of a more or less serious outbreak of infectious disease. 


\section{PATHOGENIC AND TOXICOGENTC BACTERTA IN THE UPPER AIR PASSAGES OF DONESTICATED ANINALS.}

By Veranus A. Moore.

In 1888 Dr. Theobald Smith* demonstrated the fact that in the secretions covering the posterior nares, pharynx and larynx of certain numbers of apparently healthy pigs, were bacteria, which could not be distinguished either by their cultural characters or in their pathogenic properties from the swine-plague germs. In an appendix to the same report are the results of a few preliminary examinations of the secretions from the upper air passages of certain other domesticated animals which I made for the purpose of determining the extent of the distribution of these bacteria. In these investigations it was found that similar bacteria were present in the secretions covering the mucosa of the upper air passages in certain numbers of such animals as cattle, dogs, and cats. Since the publication of that report, further examinations have been made, from time to time, from both the same and other species of animals. As a final result of these observations the swine-plague group of bacteria was found to be present, apparently in its natural habitat, in the upper air passages of a certain per cent of healthy pigs, cattle, cats, $\operatorname{logs}$, and horses. In sheep very attenuated forms were occasionally found. Pathogenic bacteria were not discovered in the flora of the mucosa of healthy rabbits, fowls, and guinea pigs. A few examinations were also made from frogs and turtles, but with negative results.

The method that was employed in the latter examinations is the same as that used in the earlier investigations, viz, the subcutaneous inoculation of rabbits with the secretions taken from the mucosa of the upper air passages of the living or freshly killed animals. It is given in detail in the report on swine plague (l.c., p. 110), and consequently need not be repeated here.

The pathogenic bacteria previously reported from the healthy mucosa of the different species of animals could not be distinguished from the swine-plague group, and only slight variations were found to exist between the germs isolated from the various animals, either of the same

\footnotetext{
*Report on the Cause and Prevention of Swine Plagne. U. S. Departmont of $\Lambda$ gri-
} culture, 1891, p. 109. 
or different species. The more recent investigations, however, have revealed the existence of bacteria which vary in a marked degree from the more typical swine-plague germ, and also the presence of toxicogenic bacteria which are widely separated from the swine-plague group of organisms.

The total results, as reported in 1891, are, for the sake of comparison, tabulated here. As previously stated, all the pathogenic bacteria which were obtained could not be distinguished from the swineplague germs. They varied from the rapidly fatal septicæmia forms which destroyed rabbits in sixteen hours when inoculated subcutaneonsly, to those that possessed so little viruleuce that thirteen days were required for the rabbits to die.

Table showing the species of animals from which rabbits had been inoculated and the results reported up to 1891.

\begin{tabular}{|c|c|c|c|c|c|}
\hline Species of animals. & $\begin{array}{l}\text { Number of } \\
\text { aninals } \\
\text { from whicl } \\
\text { rabbits } \\
\text { were inoc. } \\
\text { alated. }\end{array}$ & $\begin{array}{l}\text { Swine- } \\
\text { plague bac- } \\
\text { teria pres. } \\
\text { ent in. }\end{array}$ & $\begin{array}{c}\text { Number } \\
\text { pathogenic } \\
\text { bacterianot } \\
\text { found in. }\end{array}$ & $\begin{array}{l}\text { Per cent } \\
\text { normally } \\
\text { infected. }\end{array}$ & Virulence of the germs. \\
\hline 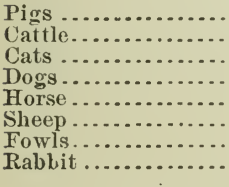 & $\begin{array}{r}19 \\
7 \\
7 \\
6 \\
1 \\
1 \\
2 \\
1\end{array}$ & 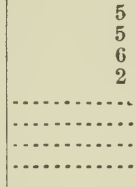 & $\begin{array}{r}14 \\
2 \\
1 \\
4 \\
1 \\
1 \\
1 \\
2 \\
1\end{array}$ & 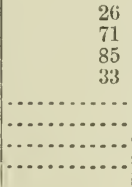 & $\begin{array}{l}\text { Attenuated. } \\
\text { Do. } \\
\text { Very virulent and attenuated. } \\
\text { Virulent. } \\
\text { No patlogenic bacteria. } \\
\text { Do. } \\
\text { Do. } \\
\text { Do. }\end{array}$ \\
\hline
\end{tabular}

In addition to the inoculations from pigs which were reported, another series was made which gave uniformly positive results. The bacteria, however, as in the other cases were attenuated, producing in the rabbit more or less cellular infiltration with the localization of the bacteria on one or more of the serous membranes. The facts necessary for an understanding of these inoculations are given in the appended table:

Inoculation of rabbits with the mucus from the upper air passages of pigs.

\begin{tabular}{|c|c|c|c|c|c|}
\hline $\begin{array}{l}\text { Pig } \\
\text { So. }\end{array}$ & $\begin{array}{l}\text { Mucus } \\
\text { from- }\end{array}$ & $\begin{array}{l}\text { Rab- } \\
\text { bit } \\
\text { inocu } \\
\text { lated. }\end{array}$ & $\begin{array}{l}\text { Date of } \\
\text { inoculation. }\end{array}$ & $\begin{array}{l}\text { Rabbit } \\
\text { died } \\
\text { in- }\end{array}$ & Remarks. \\
\hline 181 & Larynx... & No. & Sept. 23,1889 & $\begin{array}{r}\text { Days. } \\
2 \\
2\end{array}$ & Local reaction; peritonitis. \\
\hline 267 & Trachea .. & 3 & Jan. 2,1890 & 5 & $\begin{array}{l}10 . \\
\text { Do. }\end{array}$ \\
\hline 303 & Pharynx.. & $\begin{array}{l}4 \\
5\end{array}$ & Jan. 28,1890 & $\begin{array}{r}6 \\
3 \\
4\end{array}$ & $\begin{array}{l}\text { Local reaction; hemorrhages in cæcum. } \\
\text { Local reaction; peritonitis. }\end{array}$ \\
\hline 305 & Larynx... & 7 & 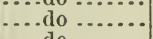 & $\begin{array}{l}4 \\
4 \\
5\end{array}$ & $\begin{array}{l}\text { Do. } \\
\text { Do. }\end{array}$ \\
\hline 308 & Pharynx.. & $\begin{array}{r}8 \\
9 \\
10\end{array}$ & Feb. 11,1890 & $\begin{array}{l}5 \\
4 \\
5\end{array}$ & $\begin{array}{l}\text { Local reaction; hemorrhagic areas in cxcum. } \\
\text { Local reaction; peritonitis; pleuritis. } \\
\text { Do. }\end{array}$ \\
\hline 219 & .... do ..... & 11 & Feb. 14,1890 & 3 & $\begin{array}{l}\text { Do. } \\
\text { Remained }\end{array}$ \\
\hline 202 & Larynx... & $\begin{array}{l}12 \\
13 \\
14\end{array}$ & (ndo & 4 & Local reaction; peritonitis; hemorrhages in cæcum. \\
\hline 7 & ....do. & 15 & Apr. 2,1890 & & Local reaction; peritonitis ; hemorrhages iu crecum. \\
\hline
\end{tabular}


The pigs from which these inoculations were marle came from different herds, excepting Nos. 181 and 305. These were secured from the same farm with an interval of nearly nine months between the times of purchase. The inoculations were made in a few cases as soon as the pigs werepurchased, but in other instances they were kept at the Experiment Station for a greater or less period of time prior to the examiuation. The uniform positive results obtained in this series would indicate a more general distribution of the swine-plague group of bacteria in the flora of the upper air passages of healthy pigs than would be supposed from the previously reported results.

In the consideration of the more recent rabbit inoculations from the various animals, a table has been constructed for each of the species examined. Very few additional examinations have been made from those species from which a considerable number of examinations were heretofore reported. It seemed, for obvious reasons, more desirable to obtain the secretions from freshly killed animals, and on this account the work has extended over a considerable length of time in order to obtain the secretions from suitable animals that were killed for other and more important purposes. In a few instances the cattle and sheep were slaughtered at the abattoir for beef and mutton. I am indebted to Dr.F. L. Kilborne, Director of the Experiment Station, for assistance in making many of the inoculations.

\section{INOCULATIONS FROM CATTLE.}

Rabbits have been inoculated with the secretions from the upper air passages of three animals. Two of these (Nos. 8 and 9), were from the Experiment Station, and the other (No. 10) was a steer killed at the abattoir for beef. No. 8 died of Texas fever. The mucus was taken from the amygdaloid cavities very soon after death. This fact does not detract from the justice in using this animal, as the disease from which she suffered was not bacterial in its nature. The subjoined table is selfexplanatory:

Inoculation of rabbits with the mucus from the upper air passages of cattle.

\begin{tabular}{|c|c|c|c|c|c|}
\hline $\begin{array}{l}\text { Ani- } \\
\text { mal } \\
\text { No. }\end{array}$ & Mucus from- & $\begin{array}{l}\text { Rabbit } \\
\text { inocu- } \\
\text { lated. }\end{array}$ & $\begin{array}{c}\text { Date of } \\
\text { inoculation. }\end{array}$ & $\begin{array}{l}\text { Rabbit } \\
\text { died in- }\end{array}$ & Remarks. \\
\hline 8 & Amygdaloid cavities.. & $N_{27}$ & Ang. 27, 1891 & Days. $_{2}$ & $\begin{array}{l}\text { Local roaction, spleen, liver, and } \\
\text { kidneys hyjeranic. }\end{array}$ \\
\hline 9 & 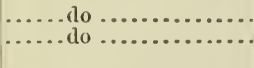 & $\begin{array}{l}28 \\
29\end{array}$ & May 13,1892 & $\begin{array}{r}2 \\
15\end{array}$ & $\begin{array}{l}\text { Local reaction, peritonitis. } \\
\text { Severe local reaction, spleen and } \\
\text { liver hypernemic. }\end{array}$ \\
\hline 10 & Larynx..... & 30 & Juno 2,1892 & 5 & $\begin{array}{l}\text { Severe local reaction, thoracic and } \\
\text { abdoninal organs normal, cultures } \\
\text { remained clear. }\end{array}$ \\
\hline
\end{tabular}

The bacteria * obtained from rabbits inoculated from cow No. 8 could

*The varions morphological, biological, and pathogenic properties of these bacteria will not be mentioned excepting where they differ from the descriptions pul, lished in the provious report (l. c.) or where additioual facts concerning any of these properties have been oltained. 
not be distinguished from the swine-plagie germ in their morphology, biological characters, or pathogenic properties. They gave the phenol reaction, but no trace of indol could be found. They perished after drying on cover-glasses for twenty-four hours, and were destroyed by heating at a temperature of $60^{\circ} \mathrm{C}$. in a water bath for ten minutes.

In order to compare more fully the pathogenic effect of these bacteria with that of the swine-plague germ a pig was inoculated with a culture from rabbit No. 27. The facts concerning the inoculation and its results are as follows:

October 16, 1891.-Pig No. 39, weighing about 25 pounds, was inoculated with 5cc. of a fresh bonillon culture in a subcutaneous vein in the right thigh.

October 17.-Pig barely able to stand.

October 19.-Pig down, unable to rise; ate very little.

October 20.-Pig lies on one side, can not be roused up; refuses food.

October 26. - The pig still refuses food. Metatarsal joints considerably swollen. It breathes with apparent difficulty.

October 2\%. -No appreciable difference in its condition. It was killed by a blow on the head.

Post-mortem examination.- The skin over the ears and above the eyes and nose was of a purplish color; a small amount of subcutaneous fat. Both inetatarsal joints and right elbow joint swollen, fluctuation detected. Subcutis about these joints œdematous. The sections showed the joint capsules to contain a considerable quantity of thick, greenish-yellow, semi-solid substance. About the edges of the joint cartilages were small pockets of pus. The suppuration extended from the capsule between the muscles for from 1 to 2 inches. Spleen slightly enlarged, pulp firm. Liser slightly discolored, fatty, contained several cysts of Tania echinococci. ${ }^{*}$ The gall bladder contained a small quantity of very thick, yellowish-brown bile which could not be forced through the duct. Kidneys, cortex pale (parenchy matous degeneration), pyramids of a glairy, reddish appearance. Capsule easily removed. Stomach contained no food; a small quantity of bile-stained mucus in pyloric end. Mucosa pale. Small iutestines contained bile-stained mucus; mucosa slightly reddened from injected blood vessels. Lymphatics pale and œdematous throughout. Lungs partially collapsed, deeply congested, but no hepatization or hemorrhages. Heart muscle very pale.

Stained cover-glass preparations from the blood, spleen, liver, and pus from elbow joint showed no bacteria. Similar preparations from the kiclneys exhibited a large number of oval bacteria, many of which took the polar stain. Tubes of agar and bouillon inoculated from the blood and spleen remained clear. Those inoculated from the liver, kiducys, and pus from the affected joints developer pure cultures of bacteria which were not distinguishable from the swine-plague germ.

A rabbit was inoculated subcutaneously with a bit of the pus from one of the metatarsal joints. It perished on the third day with lesions characteristic of attenuated swine plague.

The suppurative condition of the joints which formed the most marked lesion is not unlike that found by Dr. Smith in pigs inoculated intraveneously with swine-plague bacteria $(l . c . p .7 \tilde{5})$. The resemblance of this germ to that of bovine pneumonia is worthy of careful attention.

In the rabbit inoculated from cow No. 9 there was a marked difference. It lived for an unusually long period after the inoculation. The

* This appears to be the first time that this parasite has been found in pigs from the District of Columbia. 
subcutaneous and intermuscular tissue was infiltrated with a purulent substance over the entire abdomen and thorax. The abdominal organs were hyperæmic but no exudate. Cover-glass preparations revealed the presence of no bacteria, but culture media inoculated from the spleen and blood developed feeble growths of an elongated non-motile bacillus, which will be briefly described under the provisional name of bacillus No. 1.

BaCIllus No. 1.-Source: From the spleen and blood of a rabbit inoculated with the mucns from the amygdaloid cavities of cow No. 9. Morphology: A non-motile oval bacillus 1.5 to $2.0 \mu$ in length. Stains deeply or with a light center. Polar arrangement of the cellular protoplasm well marked when examined at the edge of a hanging drop preparation. Biology: On the surface of agar it develops a somewhat vigorous gravish, non-viscid growth. Growth very feeble in gelatine. It does not develop on potato. Bouillon becomes very faintly clouded with an acid reaction. In peptonized bouillon containing 2 per cent glucose in a fermentation tube it imparts a uniform cloudiness to the liquid throughont the tube. It ferments the sugar with the production of a large quantity of gas (five-ninths of the contents of the tube) one-third of which was absorbed by potassium hydrate. Milk was thickener in twenty-four hours, not viscid. Pathogenesis: Rabbits inoculated with 0.5 cc. of a fresh bouillon culture exhibited an elevation of temperature which lasted for about five days. Otherwise they appeared to be unaffected.

Rabbit No. 30, inoculated from cow No. 10, exhibited an extensive purulent infiltration and sanguinolent effusion in the subcutaneous tissue over the entire ventral aspect of the body. No bacteria were found in the organs, but cover-glass preparations from the local lesion contained a very large number of oval elongated bacteria, resembling those isolated from rabbit No. 2?. Unfortunately no cultures were made from the local lesion and those made from the spleen and blood remained clear.

\section{INOCULATIONS FROM SHEEP.}

The sheep from which secretions were obtained were killed in part for mutton at the abattoir and in part for various purposes at the Experiment Station. In the examinations that have thus far been made no bacteria have been found which were identical in their varions properties with the swine-plague group of organisms. The facts necessary for an understanding of these inoculations are tabulated below.

Inoculation of rabbits with the mucus from the upper air passages of shecp.

\begin{tabular}{|c|c|c|c|c|c|}
\hline $\begin{array}{l}\text { Sheep } \\
\text { No. }\end{array}$ & Mucus from- & $\begin{array}{l}\text { Rabbit in- } \\
\text { oculated. }\end{array}$ & $\begin{array}{l}\text { Date of in- } \\
\text { oculation. }\end{array}$ & $\begin{array}{l}\text { Rabbit } \\
\text { dierl in- }\end{array}$ & Remarks. \\
\hline 2 & $\begin{array}{l}\text { Larynx an d } \\
\text { pharynx. }\end{array}$ & $\begin{array}{r}\text { No. } \\
\quad 33 \\
34\end{array}$ & Nov. 6,1891 & $\begin{array}{r}\text { Days. } \\
10\end{array}$ & $\begin{array}{l}\text { Sovere local reaction; bacteria from local } \\
\text { lesions only } \\
\text { Killed after thirty-t wo days; no bacteria. }\end{array}$ \\
\hline $\begin{array}{l}3 \\
4\end{array}$ & Month ........ & $\begin{array}{l}35 \\
36\end{array}$ & $\begin{array}{l}\text { May } 26,1892 \\
\text {...do }\end{array}$ & $\begin{array}{l}18 \\
19\end{array}$ & $\begin{array}{l}\text { Loeal abscess; oval bacteria from local } \\
\text { lesion only. } \\
\text { Severo local reaction; no pathogenio } \\
\text { bacteria. }\end{array}$ \\
\hline $\begin{array}{l}5 \\
6\end{array}$ & $\begin{array}{l}\text { Pharynx and } \\
\text { larynx. }\end{array}$ & $\begin{array}{l}27 \\
38 \\
39\end{array}$ & $\begin{array}{l}\text { May } 28,1892 \\
\text {. . do }\end{array}$ & $\ddot{22}$ & $\begin{array}{l}\text { Rabbit remained well. } \\
\text { slight local reaction; many coceidia in } \\
\text { liver, no bacteria. } \\
\text { Rabbit remained woll. }\end{array}$ \\
\hline
\end{tabular}


Rabbit No. 33 exhibited an extensive purulent infiliration in the subentaneous tissue over the ventral aspect of the body, starting from the point of inoculation. The thoracic and abrlominal organs were apparently normal. No bacteria were found in cover-glass preparations made from the spleen and blood. A large number of oval, elongated bacteria were present in similar preparations from the local abscess. Cultures from the spleen and blood remained elear. A tube of bouillon inoculated from the local abscess developed an impure culture of a bacillus resembling morphologically the swine-plague germ. The other rabbit was killed after thirty-two days. No lesions.

A rabbit was inoculated subcutaneously with 0.50 cc. of a bouillon culture of the germ made directly from the local abscess of rabbit No. 33. It perished on the second day. It exhibited slight local reaction; liver large, hyperæmic. Spleen slightly enlarged. No localization of bacteria on serous membranes. Bacteria appeared in cover-glass preparations from the liver as deeply stained bodies. No polar stain observed. Similar preparations from the spleen and blood exhibited no bacteria. Tubes of boullon inoculated with bits of the liver developed pure enltures of a non-motile bacillus 1.5 to $2.5 \mu$ in length; ends rounded. At the edge of a lianging drop preparation the cell protoplasm was observed to be more dense at the poles or extremities. Many of them exhibited the polar stain. The periphery stained more deeply than the center. The cultures from the spleen and blood remained sterile. This bacillus differed very slightly from the swine-plague germ in its biological properties.

The rabbit inoculated from sheep No. 4 presented on post-mortem examination an extensive purulent infiltration into the subcutis. No inflammation of the serous membranes. From the local lesion cultures of an elongated, oval germ were obtained which subsequently destroyed a rabbit in three days when inoculated subcutaneously with 0.75 cc. of a pure bouillon culture. It exhibited a local reaction, but no localization of the bacteria on the serous membranes. This germ differed from the swine-plague organism in (1) not exhibiting a polar stain in cover-glass preparations from the organs of the rabbit; (2) it was slightly larger; (3) the growth on the agar was not viscid, and (4) its localization at the place of inoculation only.

Rabbit No. 36 perished evidently from the effect of the local reaction, which was extremely severe. A micrococcus which grew in clumps was isolated from the infiltrated substance, but it possessed no pathogenic properties, as indicated by an intravenous inoculation in rabbits. Rabbit No. 38 perished presumably from the effect of the coccidia in the liver.

Although the bacteria isolated from the air passages of sheep differed in a marked degree from each other, and from the swine-plague germs in their virulence and pathogenic manifestations, their general morphological and biological characters would indicate that they 
belong to that group of organisms. 'The severe purulent infiltration into the subcutaneone tissue, extending from the point of inoculation, was undonbtedly partially due to other germs that were introduced with the mucus. The non-localization of the bacteria on the serous membranes is the most important variation that was found to exist between these and the attenuated swine-plague bacteria.

\section{INOCULATIONS FROM HORSES. •}

Rabbits were inoculated with the secretions from the upper air passages of five horses. Two of these were the work horses at the Experiment Station. The other three were from Washington, D. C., two of which were killed for lameness. The following table is self-explanatory:

Inoculation of rabbits with the mucus from the upper air passages of horses.

\begin{tabular}{|c|c|c|c|c|c|}
\hline $\begin{array}{l}\text { Horse } \\
\text { No. }\end{array}$ & Mucus from- & $\begin{array}{c}\text { Rab- } \\
\text { bit } \\
\text { inocu- } \\
\text { lated. }\end{array}$ & $\begin{array}{l}\text { Date of in- } \\
\text { oculation. }\end{array}$ & $\begin{array}{c}\text { Rab- } \\
\text { bit } \\
\text { died } \\
\text { in- }\end{array}$ & Remarks. \\
\hline 2 & $\begin{array}{l}\text { Trachea ......... } \\
\text { Larynx ........ }\end{array}$ & $\begin{array}{r}\text { No. } \\
42 \\
43\end{array}$ & $\begin{array}{l}\text { June } 24,1891 \\
\ldots . \text { do } \ldots \ldots . .\end{array}$ & $\begin{array}{r}\text { Days. } \\
5 \\
9\end{array}$ & $\begin{array}{l}\text { Spleen enlarged; strepitococci from organs. } \\
\text { Slight local reaction; peritonitis; polar-stained }\end{array}$ \\
\hline $\begin{array}{l}3 \\
4\end{array}$ & $\begin{array}{l}\text { Root of tongue. } \\
\text { Nares........... }\end{array}$ & $\begin{array}{l}44 \\
45\end{array}$ & $\begin{array}{l}\text { May } 26,1892 \\
\text { Apr. 23, } 1892\end{array}$ & & $\begin{array}{l}\text { bacteria. } \\
\text { Rabbit remained well. } \\
\text { Do. }\end{array}$ \\
\hline 5 & Pharynx....... & $\begin{array}{l}46 \\
47\end{array}$ & Mar. 8,1892 & & $\begin{array}{l}\text { Do. } \\
\text { Do. }\end{array}$ \\
\hline 6 & Root of tongue.. & $\begin{array}{l}48 \\
49 \\
51\end{array}$ & $\begin{array}{l}\text { Apr. } 7,1892 \\
\ldots \text { do } \\
\ldots \ldots \text { do }\end{array}$ & $\begin{array}{r}\cdots \\
26 \\
\cdots \\
\cdots\end{array}$ & $\begin{array}{l}\text { Rabbit emaciated; no bacteria. } \\
\text { Rabbit remained well. } \\
\text { Do. }\end{array}$ \\
\hline
\end{tabular}

The inoculations with the mucus from horses showed the flora of their mouth, nose, and throat to be comparatively free from pathogenic bacteria. No. 2, however, was unusually fertile in these forms. Rabbit No. 42 died evidently from the effect of a streptococcus which was isolated from its organs. This germ was rapidly fatal to rabbits and mice; 0.10 ec. of a boullion culture destroyed a rabbit, when inocnlated subcutaneously, in less than two days. In cover-glass preparations firom the organs of the rabbit it appeared very largely in single forms, frequently, however, in very short chains. The individual segments were elongated and frequently exhibited a polar stain. The resemblance of the individual elements to the swine-plague germ was very marked. This was true of its biological properties. It became much attenuated in about nine months. This germ is described more fully under the name "Streptococcus U," in the article on Streptococci.

Rabbit No. 28 presented lesions typical of attenuated swine plague. Its organs contained bacteria that could not be differentiated from the swine-plague germ.

$\Lambda$ rabbit inoculated subcutaneously with $0.10 \mathrm{cc}$ of a bouillon culture died on the third day with severe local reaction, but no marked localization of the bacteria on the serous membranes. No phenol or indol reaction could be detected in boullon cultures of this germ. Its power 
to resist drying and its thermal death point were not appreciably different firom those of the swine-plague germ.

Rabbit No. 33 perished presumably from the effects of the inoculation, but no bacteria could be found in any of the organs. The other rabbits remained well.

INOCULATION OF GUINEA-PIGS FROM TIIE NASAL DISCHARGE OF A MULE.

September 10, 1892, Dr. F. L. Kilborne inoculated a guinea-pig with mucus from the nose of a mule which was suspected of having glanders. The guinea-pig was found dead September 12. From the organs cultures of bacteria were obtained which were fatal to rabbits in from two to four days. They were not distinguishable from the swineplague germ in their biological and pathogenic properties. In coverglass preparatious from tissues, however, they did not exhibit the polar stain.

September 12 a second guinea-pig was inoculated from the same mule. It died September 14. Tubes of bouillon inoculated from the organs developed pure cultures of the Bacillus fuorescens liquefaciens. A rabbit inoculated subcutaneously with 0.75 cc. of a bouillon culture of this bacillus perished in forty hours. At the point of inoculation there was an infiltration of serum into the subcutaneous tissue. Abdominal organs hyperæmic. Cover-glass preparations from the spleen and liver contained many bacilli. Pure cultures were obtained from the spleen and liver. A rabbit inoculated in the ear vein with $0.10 \mathrm{cc}$. of a fresh bouillon culture perished in seven days. After three weeks cultivation this germ lost its virulence to such an extent that $0.75 \mathrm{cc}$. produced no effect on rabbits. This is the first time, so far as I am aware, that this bacillus has been reported as possessing pathogenic or septic properties.* It did not produce gas. When grown in the fermentation tube it developed in the open bulb only.

\section{INOCULATIONS FROMI CATS.}

Rabbits have been inoculated with the mucus from the upper air passages of three healthy cats, all of which contained bacteria which belong to the swine-plague group of organisms. The facts concerning these inoculations are tabulated below.

* In the summer of 1892 , while making a series of inoculations in pigeons with another germ, the cultures became contaminated with the Bacillus fuorescens liquefa. ciens. It was isolated by means of agar plate cultures and a pigeon inoculated in the pectoral muscle with $0.50 \mathrm{cc}$ of a pure bouillon culture. The pigeon died in forty hours with extensive necrosis of the pectoral muscle. Heart muscle pale. Many bacilli were found in cover-glass preparations from the organs. A second pigeon, inoculated with $0.50 \mathrm{cc}$. of an emulsion of the pectoral muscle from the first pigeon, remained well. 
Inoculation of rabbits with mucus from the upper air passages of cats.

\begin{tabular}{|c|c|c|c|c|c|}
\hline $\begin{array}{l}\text { Cat } \\
\text { No. }\end{array}$ & Mucus from- & $\begin{array}{c}\text { Rabbit } \\
\text { inoculated. }\end{array}$ & $\begin{array}{c}\text { Date of } \\
\text { inoculation. }\end{array}$ & $\begin{array}{l}\text { Rabbit } \\
\text { died in- }\end{array}$ & Renarks. \\
\hline 8 & Larynx...... & No. 63 & Aug. 31, 1890 & $\begin{array}{r}\text { Hours. } \\
21\end{array}$ & Septicamia. \\
\hline 9 & $\begin{array}{l}\text { Trachea and } \\
\text { larvnx. }\end{array}$ & $\begin{array}{l}64 \\
65\end{array}$ & $\begin{array}{l}\text { Aug. 31, } 1890 \\
\text { Sept. 4, } 1892\end{array}$ & $\begin{array}{l}21 \\
20\end{array}$ & $\begin{array}{l}\text { Do. } \\
\text { Do. }\end{array}$ \\
\hline 10 & $\begin{array}{l}\text { Pharynx and } \\
\text { laring. }\end{array}$ & 66 & May 31,1892 & 48 & Local reaction; peritonitis. \\
\hline
\end{tabular}

- The bacteria obtained from cat No. 8 by rabbit inoculation was compared very carefully with the germs of acute swine plague. They gave in bouillon (containing 1 per cent peptone) cultures $2:$ days old a decided phenol reaction, but no trace of indol could be found. Their power to resist drying and their thermal death point were identical with those of the swine-plague germ.

The pathogenic power of these bacteria was tested by a pig, gruineapig, and rabbit inoculations.

October 16, 1891.-A pig weighing about 30 pounds was inoculated in the vein (inside of right thigh) with $5 \mathrm{cc}$. of a fresh bouillon culture. It was found dead on the morning of October 17, twenty-four hours after its inocnlation. The abdominal organs were slightly hyperæmic, lymphatic glands reddened. About 100 ec. of a clear straw-colored serum in the right pleural cavity, a smaller quantity on the left sicle. Beneath the pleura over the lungs were several irregular areas containing petechix. Lungs not collapsed. Slight emphysema. Cover-glass preparatious from the organs contained very many polar-stained, oval bacteria.

A pig inoculated at the same time, and with a similar quantity of a like culture of virulent swine-plague bacteria, perished at the same time and with similar lesions.

A guinea-pig inoculated subcutaneously with 0.001 cc. of a bouillon eulture perished on the tenth day. It exhibited considerable cellular infiltration into the subeutis, involving the superficial layer of the subjacent muscle. The abdominal organs were covered with a cellular, pasty exudate. There was beginning plenritis as exhibited by a large number of bacteria in cover-glass preparations made by drawing a coverglass over the surface of the visceral pleura. A guinea-pig inoculated with a similar quantity of a like culture of a virulent swine-plague germ recovered after some weeks. This fact would indicate a slight increase in the virulence of the tracheal germs over that of the swineplague organism used.

They destroyed rabbits in about sixteen hours. In cover-glass preparations from their organs the bacteria were appreciably larger than the swine-plague germ. They appeared frequently in clumps, and when stained were separated by unstained septa, which sugersted the possibility of a capsule. There were many involution forms, in some of which the cellular protoplasm was condensed near the center of the cells rather than at the ends as indicated by their reaction to the stain. ing fluids. 
The bacteria from cat No. 9 differed from those from cat No. 8 , in that they were larger, did not exhibit the polar stain (excepting involution forms), and were localized more especially in the liver of the rabbit.

Rabbit No. 66 exhibited lesions characteristic of attenuated swine plague.

The almost uniform presence of the swine plague group of bacteria in the normal saliva of cats is of considerable interest; inasmuch as these animals are so largely the living pets of children and the effect of these germs on the human species is not yet demonstrated. Their effect on the smaller experimental animals, however, does not differ, to any marked degree, from those of the germs of pneumonia in man.

Fiocca* has recently described a pathogenic bacillus which he found in the saliva of eats and dogs, and which resembles the germ of rabbit septicæmia, but only one-half its size. The variation which I have found in the size of the bacteria from the saliva of cats would indicate that Fiocea's germ belougs to the same group of bacteria as those which I have found in these animals. It will be observer that there is a considerable variation in both the morphology and virulence of the pathogenic bacteria found in the upper air passages of apparently healthy cats.

\section{OTHER INOCULATIONS.}

Rabbits were inoculated with the secretions from the pharynx, larynx, or trachea of one healthy dog, six rabbits, five guinea-pigs, and four fowls, with negative results.

It is of further interest to note that pathogenic bacteria were not discovered in the flora of the upper air passages of rabbits, guineapigs, and fowls, although it frequently happens that these animals perish from sporadic diseases, the lesions of which resemble those produced by the inoculation of the swine-plague germ, and that bacteria which belong to the swine-plague group can be isolated from the affected organs. On the other hand it should be mentioned that cats are immune to large doses of the virluent swine-plague germ.

From the results recorded in the preceding pages it is found that 48 per cent of pigs, $\dagger 80$ per cent of cattle, 50 per cent of sheep, 16 per cent of hotses, 90 per cent of cats, and nearly 30 per cent of dogs contain among the flora of their upper air passages bacteria which possess more or less pathogenic or septic properties. Of these the greater number belong to the swine-plague group, and can be designated as pathogenic bacteria, while the other forms which possess only temporarily destructive power appear to more properly belong to the toxicogenic bacteria. The extremely attenuated forms from the sheep and the great variation found in the pathogenic properties of those from

${ }^{*}$ Centralblatt f. Bakteriologie u. Parasitenkunde, Bd. xI, 1892, p. 406.

†This is based upon both my own results as recorded in the table and those heretofore published by Dr. Smith. 
the eats are interesting in showing the graduation of the swine-plague gronj of bacteria from the very virulent to the extremely attenuated forms.

The close resemblance of the pathogenic bacteria to the swine-plague germs has been determined by a large number of inoculations and parallel cultures, and by a careful study of their morphology in both fresh and stained preparations. Their feeble power to resist drying and their thermal death point are identical with those of the virulent swineplague bacteria.

Bacillus No. 1 from cow No. 9 is interesting, from the fact that while it resembles in its general morphological characters the swine-plague germ, it possessed the property of fermenting sugar with the formation of gas. These variations are important in the classification of diseaseproducing bacteria. The streptococcus isolated from the nares of a - healthy horse differed very slightly in its more hardy biological characters, as well as in its effect upon certain animals from the description of the streptococcus which has been lescribed as the supposed cause of strangles.

The very general distribution, in the secretions covering the mucosa of the upper air passages of domesticated animals, of bacteria which possess such marked pathogenic and toxicogenic properties, is of much importance, as it explains the cause of sporadic cases of swine-plague, ${ }^{*}$ and, to some extent at least, the possible source and significance of various toxicogenic bacteria which are frequently isolated from variously diseased animal tissues.

* For a discussion of the relation of these bacteria to isolated cases of pneumonia in swine, see Dr. Smitl's Report on Swine Plague, l. c., pp. 109-117. 


\section{AN OUTBREAK OF ABORTION IN MARES.}

By F. L. Kilborne.

During the winter of 1890 -'91 an outbreak of abortion occurred among the mares of a large stud near Franklin, Pa. On visiting the farm February 5, 1891, I found the stud to comprise about one hundred brood mares and several stallions.

The first mare to abort, due to foal March 21, dropped her foal December 2, or 109 days before full term. Between December 2 and February 5 , ten abortions occurred, at periods ranging from 23 days to 195 days, or an average of 100 days, before the completion of the period of gestation. No history of any introduction of the infection from without could be obtained beyond the fact that mares were daily being received at the stock farm from various parts of the State, to be bred to the stallions of the stud. But as far as could be ascertained no mare had been received from any stable in which abortions had previously occurred.

The brood mares were all kept in box stalls, bedded with peat moss, on a dirt floor. The box stalls were arranged in one row around the outside of the barn, and another within. Between the two rows there was a tan-bark track for exercising the animals. The barn was rather damp, but otherwise apparently well suited to the purpose. Each pregnant mare received, on an average, about 6 quarts of oats and a little bran, with a moderate allowance of timothy hay, daily. Mares in poor condition received more, while those in high condition were fed less. The mares were exercised daily, by being ridden on the tan-bark track.

The veterinarian in charge of the stables had taken the usual precaution of removing the aborting mares as soon as discovered, and disinfecting the stall. Such mares were disinfected por vaginum by repeated injections of a diluted solution of corrosive sublimate, 1 part to 10,000 of water, and the external organs, tail and hind limbs sponged over with a stronger solution, 1 part to 1,500 of water. The stablehad also been repeatedly fumigated with burning sulphur. Fluid extract of black haw (Viburmum prunifolium) in half ounce doses once daily, had been given the preguant mares during the month preceding February $\tilde{5}$.

At the time of the above visit there had been no indications of another abortion during the past week, the last abortion having occur$5311-N o .3-4$ 
red January 29. In order to guard against a continuation of the outbreak, a more thorough system of disinfection of the whole stable was advised and carried out. For this purpose a 2 per cent solution (by volume) of commercial sulphuric acid was used. All stalls were to be disinfected once a week, and any stall occupied by a mare aborting was to be immediately vacated, thoroughly cleaned and disinfected.

The practice of exercising the mares by riding was discontinned, and they were exercised by being led at the halter. On account of the extreme cold weather the drinking water was to be given in quantity not to exceed one-half horse-bucketful at one time, and with the chill taken off. All preguant mares were to have the tail and external genital organs sponged over daily with a solution of the mercuric chloride 1 part to 1,000 or 1,500 of water. The fluid extract of black haw was continued throughout February, but apparently without result. The mares aborting February 15 to Mareh 4, were among the number receiving the extract.

From January 29 until February 15, no abortions occurred; but from this date until March 4 six mares aborted. A second visit was made to the farm at this time. The additioilal precautions taken during the past month liaving failed to check the outbreak, it was leemed advisable to remove all the pregnant mares from the large barn to other stables. Accordingly eighteen of the remaining mares were removed to a stable, formerly used only for colts, with high plank floor and roomy box stalls. The otlier mares were to he removed as soon as accommodations could be provided for them. Owing to some delay of the carpenters in completing another stable, these mares were not removed from the large barn as intended.

In the barn to which the eighteen mares were removed, all stalls were to be entirely emptied of bedding, and swept and sprinkled with 2 per cent solution of sulphuric acid, twice a reek. The disinfection of the large barn was continued as before. All preginant mares continued to have the root of tail and vulva washed, once daily, with the mereuric chloride solution. In washing, the lips of the vulva were pressed apart with the sponge in order that the solution might be worked in as far as possible. In attempting to inject a diluted solution, even a short distance into the vagina, it was found to produce nore or less straining, and was therefore discontinued.

Beginning with March 4, one-half of the mares were to receive the fluid extract of black haw, in doses of one-half onnce once daily; the remaining half of the mares to receive one hoaping tablesponnfinl of the following powder in the feed twice daily, omitting the powders for four or five days a fter each two weeks: ('hlorate of potash, phosphate of sorla, of each 1 pound; sulphate of quinine 3 ounces, mix. This treat rent was continuer to the end of the season. No further abortions occurred.

From the last ease to abort, in the evening of March 3 , cultures were made on agar-agar early the following norning. The cultures wero made by separating the lips of the vulva as far as possible, and lightly 
scraping the mucosa of the vulva with a flamed platinum loop and rubbing this upon the inclined agar surface. In these tubes a bacillus appeared, which is described in an article by Dr. Snith, following this account.

Table showing date of abortion and stage of gestation of each mare aborting.

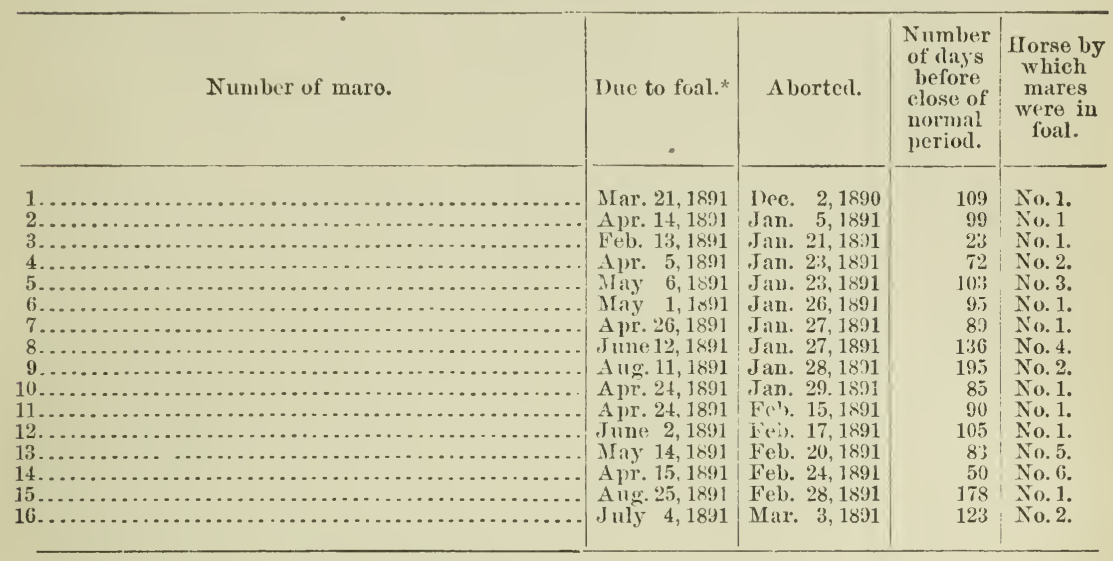

* Eleven months allowed as period of gestation.

Of the sixteen mares nine were in foal by stallion No. 1; three by stallion No. 2, and one each by four other stallions.

Mare No. 5 clied after the abortion. The others all made a good recovery. No. 7 , however, was a chronic aborter, having lost two or three foals previousty.

There still remained, March 4, thirty-six pregnant mares, due to foal March 19 to September 14. Two mares due to foal February 18 and 26, making bag. Live foals at term were dropped February 2 and 11, and March 4. One dead foal dropped at term February 19.

In the fall of 1891, when the mares were first brought in from the pasture, three abortions occurrerl in a small barn where several mares were kept. The aborting mares were at once isolated and the stalls disinfected with the solution of sulphurie acill. The pregnant mares were again given the fluid extract of the black haw in half-ounce doses, once daily, and the vulva and root of tail washed with mercuric chloride solution, as before. No further abortions occurred during the season of 1891-92.

In order to test the pathogenic properties of the bacillus obtained from mare No. 16, and described by Dr. Smith, vaginal injections were made with pure cultures on one pregnant mare and two pregnant cows. Inoculations were also made on two pigs.

Vaginal injection of pregnant mares.-On April 22 a large bay mare, age 7 years, supposed to be in the ninth month of gestation, received by injection into the vagina 10 ce. of a peptone bouillon culture, two days old, of the bacillus. Twenty-four hours after the injection an abundant, purulent discharge of a grayish-white color was 
coming from the vagina. This discharge continued for two days, after which it ceased. The mare developed well-marked symptoms of influenza on the day following the injection, and from April 23 to 30 suffered a severe attack, with a temperature varying from $102.4^{\circ} \mathrm{F}$. to 106.20 F. There were several cases of influenza in the stable from which the mare came, so that it is evident the mare contracted the disease before coming to the station. On May 2 the mare dropped a live foal, probably owing to the attack of influenza. The birth was evidently premature, as shown by the lack of development and weakness of the colt, which lived. but two days.

Vaginal injection of pregnant cows.-On April 11 a two-year-old heifer, No. 160, and a cow, No. 152, five years old, were chosen for the injection. No. 160 was supposed to be in the seventh or eighth month of pregnancy, while No. 152 was in the fifth month, having taken the bull November 29, 1890. Each cow received by injection into the vagina $10 \mathrm{cc}$. of a peptone bonillon culture of the bacillus twenty-four hours old. Twenty-four hours after the injection there was quite an abundant, purulent discharge of a dirty grayish-white color from the vagina of both cases. After two days this discharge gradually diminished and had entirely ceased April 15. On April 22 both cows were given a second vaginal injection of a similar culture of two days' growth. No. 152 received 10 cc. as before, and No. 160 received 25 ce. Twentyfour hours after the injection No. 152 showed a slight vaginal discharge, and No. 160 a much more abundant discharge of the same character as before. The discharge had entirely ceased on the third day.

Below is given the temperature of the two cows taken morning and evening during the period of injection:

\begin{tabular}{|c|c|c|c|c|c|}
\hline & \multirow{2}{*}{ Date. } & \multicolumn{2}{|c|}{ Cow No. $160 . *$} & \multicolumn{2}{|c|}{ Cow No. 152.} \\
\hline & & 9 a. $\mathrm{m}$. & $5 \mathrm{p} . \mathrm{m}$. & $9 \mathrm{a} . \mathrm{m}$. & $5 \mathrm{p} . \mathrm{m}$. \\
\hline & $\begin{array}{l}\circ F . \\
102.8\end{array}$ & $\circ F$. & $\begin{array}{l}\circ F . \\
101.2\end{array}$ & ${ }^{\circ} \mathrm{F}$. \\
\hline \multicolumn{2}{|l|}{ April 13. } & 101.2 & 104.4 & 101.2 & 101.6 \\
\hline \multirow{2}{*}{\multicolumn{2}{|c|}{ A pril 14.. }} & 104 & 104.2 & 101 & 101.4 \\
\hline \multirow{2}{*}{\multicolumn{2}{|c|}{$\begin{array}{l}\text { A pril } 15 . \\
\text { A pril } 16 .\end{array}$}} & 103 & 102.8 & 101 & 102 \\
\hline \multirow{2}{*}{\multicolumn{2}{|c|}{$\begin{array}{l}\text { A pril } 16 . \\
\text { A pril } 17 .\end{array}$}} & 103.2 & 103 & 101.4 & 102 \\
\hline & & 103 & 103.7 & 101.4 & 101.6 \\
\hline \multicolumn{2}{|l|}{ A pril 20.} & 102 & 103.5 & 101.4 & 100.8 \\
\hline \multirow{2}{*}{\multicolumn{2}{|c|}{$\begin{array}{l}\text { A pril } 22 . \\
\text { A pril 23. }\end{array}$}} & 102.2 & 104 & 100.8 & 102.2 \\
\hline & & 102.6 & 103.8 & 101.6 & 102 \\
\hline \multicolumn{2}{|l|}{$\begin{array}{l}\text { A pril 23. } \\
\text { April 24. }\end{array}$} & 102.7 & 103 & 101.6 & 100.8 \\
\hline \multicolumn{2}{|c|}{$\Delta$ pril $25 .}$. & 102.5 & 103.5 & 101.4 & 102 \\
\hline
\end{tabular}

* The continued elevated temperature of No. 160 is dne to the advanced stage of prognancy, per. haps. No. 160 drops a healthy calf May 9. No. 152 calves September 5.

Intravenous inoculation of pigs.-April 11, two pigs, five to six months old, numbers 472 and 473 , received each by inoculation into the vein on the inside of the thigh, 5 cc. of a peptone bouillon culture twenty-four hours old. On A pril 12 and 13 both pigs were quite dull and eating but very little. On April 14 to 16 the pigs were active and appetite returning. On April 17 they had apparently entirely recovered. 


\section{ON A PATHOGENIC BACILLUS FRON THE VAGINA OF A MARE AFTER}

ABOR'IION.

By Theobald Smith.

The two tubes of inclinerl agar inoculated by Dr. F. L. Kilborne, as described in his report, were handed to me the day following the inocnlation. I may repeat here that these tubes were inoculated directly with a platinum loop rubbed over the mucosa of the vulva. They were inoculated in the morning, the abortion having occurred the evening before. In each tube there had appeared, twenty-four hours after inoculation, about a dozen roundish, grayish-white colonies several millimeters in diameter. Some of the growth from each tube seems to have run down the inclined surface of the agar as if semiliquid. Microscopic examination revealed small rods which did not show any motion when stirred up in sterile water and examined in a hanging drop. Both tubes evidently contained but this one species. From a colony in each peptone bouillon, tubes were inoculated and placed in the thermostat. Both were clourled on the following day and contained short motile rods closely resembling in size and motility hog-cholera bacilli. From both cultures gelatine plates were prepared. On the third day colonies appeared on both sets of plates which were all alike. The bacteria composing them were motile and identical with those in the original tubes. From a colony on a gelatine plate a peptone bouillon culture was prepared, and from this two rabbits" were inoculated. The source of the culture may be tabulated thus:

March 5, 1891, agar inoculated from vagina of mare.

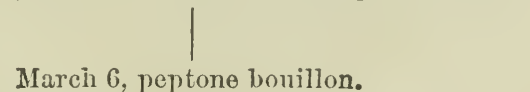

March 6, peptone bovillon.

March 9, gelatine plates.

March 12, peptone bouillon

(from colony).

March 14, two rabbits inoculated.

Rabbit No. 1 received into an ear vein 0.3 ce. of the enlture liquid.

Rabbit No. 2 received under the skin of the abdomen the same quantity. 
The first rabbit appeared ill on the second day; the inoculated ear slightly swollen and turned back on the head. The same condition prevailed on the third day. Seven days after inoculation it was found dead. As the writer was confined to his bed at this time the animal was examined and the cultures prepared by Dr. V. A. Moore. The spleen very much enlarged, dark, friable. The liver sprinkled with minute areas of necrosis as in hog cholera. In pericardial sac blood-stained serum. The left lung oedematous and some serum in left pleural sac. In cover-glass preparations of both spleen and liver stained with alkaline methylene blue are observed groups of short bacilli. In the blood they are not detected. An inclined agar tube inoculated with a loop of blood showed on the following day about ten nearly round, isolater colonies. They are nearly flat, slightly raised disks from $1 \frac{1}{2}$ to 2 millimeters in diameter, of a grayish-white color. An agar tube inoculated with a trace of spleen pulp showed on the following day a large number of isolated and confluent, quite small colonies. The condensation water was covered with a very thin, papery membrane. A peptone bouillon tube inoculated from the spleen at the same time shows, as did the examination of the colonies from the agar tubes, the same motile bacillus which had been injected into the rabbit. The second rabbit remained well until the sixteenth day, when it was unable to move its hind limbs. It was chloroformed at once. No internal lesions were detected. At the place of inoculation slight purulent infiltration of the skin, with enlargement of the neighboring kneefold glands. The spinal cord was not examined.

A second pair of rabbits were subsequently inoculated, the source of the culture used being as follows:

Blood of inoculated rabbit.

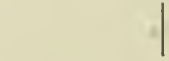

March 21, agar tube.

April 1, peptone bonillon

(from colony).

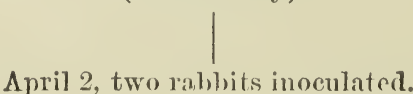

Rabbit No. 3 received 0.3 ce. into abdominal cavity.

Rabbit No. 4 received 0.3 ce into an ear vein.

No. 3 dirl not become ill. Killed on the twellth day. It showed no trace of local or general infection.

No. 4 died suddenly March 6, four days after inoculation.

The spleen very large, dark and quite firm. A portion of dorsal surface converted into a yellowish necrotic layer. Liver pale. Besicles the general parenchymatous changes the tissue was permeated with a network of yellowish necrotic linos. Parenchymatous degeneration of kirlneys. Urine turbid; contains very short fiagments of grauular castis.

Cloudy swelling of myocardium associated with fatty degenoration. Scattered 
over ventricular surfaces are yellowish lines parallel to the conrse of the fibers and abont 1 or $2 \mathrm{~mm}$. long. These are made up of necrosed fibers.

Lungs congested and æedematous. Scattered petechix over surfaces. One-half of the two small right (cephalie and ventral) lobes hepatized. Hepatized foci in left eephalic lobe.

Blackish, hemorrhagie condition of elge of pyloric valve in duodenum. For several inches below this petechise in mucosa. Very many bacteria in preparations from spleen, kidneys, and liver. Few in blood.

From the spleen two gelatine roll eultures prepared. From the blood (right ventricle) agar plates. In all these cultures colonies appearer, to be described more fully below, which were made up of the injected bacteria and these only. At the same time a tube of inclined agar was inoculated with a trace of spleen pulp. On the following day about fifteen colonies of the same bacteria appeared. These inoculations proved the pathogenic character of these bacilli and made it desirable to examine them more carefully.

On gelatine plates or in rolls colonies of these bacilli become distinctly visible to the naked eye about thirty-six to forty-eight hours after sowing. When magnified the deep colonies appear as round, finely granular, brownish disks, which may show later on a more or less distinct peripheral, paler zone. Their size depends on the number of colonies. When about $1 \mathrm{~cm}$. apart they reach a diameter of $0.5 \mathrm{~mm}$. The surface colonies are roundish, slightly convex, whitish, glistening disks with margin slightly wavy or delicately notched. When magnified they show a faintly stippled surface. They become not more than 1 to $1.5 \mathrm{~mm}$. in diameter.

On agar plates the colonies are quite large twenty-four hours after sowing when kept in the thermostat. The deep ones are round or lenticular, opaque with finely knobbed periphery, and $1 \mathrm{~mm}$. in diameter. The surface colonies are flattish, barely translucent, grayishwhite disks. When magnified, finely granular in appearance. They may attain a diameter of 4 to $8 \mathrm{~mm}$. when one to two centimeters apart.

Occasionally the deep colonies may become nearly $2 \mathrm{~mm}$. in diameter and the surface colonies now and then spread out so as to attain a diameter of 1 to $2 \mathrm{~cm}$. They then have a smooth, shining, mucus-like appearance, as if the growth had flowed over the surface. In some gas bubbles appear, and oceasionally some spread out between the agar layer and the glass beneath.

In tubes of inclined agar, the growtl of the isolated colonies is the same as that described for the plates. When confluent a grayish smooth growth is the result. The condensation water becomes turbid.

In tubes of gelatine, the track of the wire appears after two or three days as a thin yellowish-white line, which increases but little in thickness with time. On the surface there is very little growth beyond the point of inoculation.

On the cut surface of boiled potatoes in plugged tubes well supplied with water in the bottom, growth takes place. The potato becomes 
covered with a straw-colored growth, the abundance of which depends upon the state of the surface as regards moisture or dryness as well as on the nature of the potato.

In peptone boullion kept in the thermostat there is at first simple cloudiness withir twenty-four hours of inoculation. After some days the liquid becomes more turbid, and a thin cohesive pellicle forms on the surface when the culture tube remains undisturbed.

In milk at $37 \circ \mathrm{C}$., no change takes place which is visible to the naked eye, even after several weeks' sojourn in the thermostat. Subsequent boiling gives a slightly hrownish, more translucent tint to the milk.

The bacilli have the same power as the hog-cholera bacilli of causing fermentation in peptone bouillon to which some glucose has been added. Thus, in bouillon containing 2 per cent glucose, 48 per cent of the volume of the closed branch of the fermentation tube had been occupied by gas in three days. Of this 34 per cent was absorbed by potassium hydrate as $\mathrm{CO}_{2}$; the rest exploded with air (probably liydrogen). In a recent test with somewhat more alkaline glucose bouillon I obtained the following result:

Fermentation tube inoculated December 30, 1892.

December 31, 10 per cent of closed branch occupied by gas.

January 2, 58 per cent of closed branch occupied by gas.

January 4, 58 per cent of closed branch occupied by gas.

In the temperature of the room the gas occupied only 56 per cent of the closed branch. Of this 37 per cent was absorbed by caustic potash; the rest exploded with air. The culture liquid was markedly acid and free from odor. Gas is not set free in saccharose and lactose bouillon.

In reinoculating this bacillus from time to time into fresh tubes of agar the surface growth assumed a more and more pronounced membranous appearance. The agar was covered with a thin, brittle, somewhat wrinkled layer, which extended down over the condeisation water. The same change was observed in bouillon in which a thin papery membrane became a prominent feature of later cultures. This may be replaced by other membranes when shaken to the bottom.

The bacillus under consideration is a short bacillus with rounded ends, which stains well in alkaline methylene blue. It is actively motile in bouillon cultures. It moves to and fro in a straight or curved line, frequently twirling about its own middle point as an axis at the same time. When traces of growth from colonies on agar or gelatine are suspended in water or bouillon, this property of motility may escape attention, since only a few move under these conditions, and in some preparations no motile forms were observed.

The presence of this bacillus exclusively, so far as the agar eultures indicated, in the vagina of a mare which had just aborted, was of considerable moment, and it became necessary to determine-

(1) May injections of eultures of this bacillus produce abortion in healthy pregnant mares? 
(2) Is this bacillus present in the genital passages of healthy pregnant and noll-pregnant mares?

The first problem was undertaken by Dr. Kilborne, and his notes will be found in the preceding article. They show that an injection of culture fluid into the vagina of one mare and two cows in a state of pregnancy was followed by a discharge, but abortion did not take place.

The second problem was taken up by Dr. V. A. Moore. He failed to find in cultures from one pregnant and four not pregnant mares the pathogenie bacillus just described, as the following brief summary of his observations demonstrates:

"A bacteriological examination was made of the vaginal secretions from five healthy mares. Some of them were on a farm adjacent to the experiment station and some from the stables of a horse car company. One of the mares was pregnant. The method employed was the inoculation of agar tubes with mucus taken directly from the walls of the vagina by means of a sterilized platinum loop. From the colonies of bacteria which developed in these tubes subcultures were made, and from all impure original cultures agar or gelatine plates were made. From mares Nos. 4 and 5 the examination was repeated. The inoculations upon agar directly from the animals were made by Dr. F. L. Kilborne.

The number of cultures that were made and the bacteria that were obtained from the different animals are given in the subjoined table:

\begin{tabular}{|c|c|c|c|c|c|}
\hline $\begin{array}{l}\text { Mare } \\
\text { No. }\end{array}$ & $\begin{array}{l}\text { Number of } \\
\text { cultures } \\
\text { from vagi- } \\
\text { ual secro- } \\
\text { tions. }\end{array}$ & $\begin{array}{l}\text { Date of } \\
\text { cultures. }\end{array}$ & $\begin{array}{l}\text { Number of } \\
\text { tubes that } \\
\text { remained } \\
\text { clear. }\end{array}$ & $\begin{array}{l}\text { Ninmber of } \\
\text { species. }\end{array}$ & Remarks. \\
\hline $\begin{array}{l}1 \\
2 \\
3 \\
4\end{array}$ & $\begin{array}{r}\text { Agar. } \\
2 \\
1 \\
2 \\
2 \\
2 \\
2 \\
2\end{array}$ & 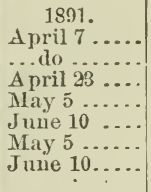 & 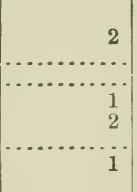 & $\begin{array}{r}\ldots \\
5 \\
\\
2 \\
1 \\
2 \\
2 \\
2\end{array}$ & $\begin{array}{l}2 \text { bacilli, } 2 \text { micrococci, } 1 \text { streptococcus. } \\
2 \text { streptococc1. } \\
1 \text { streptococcus. } \\
1 \text { micrococcus, } 1 \text { streptococcus. } \\
1 \text { micrococcus, } 1 \text { strejtococcus. }\end{array}$ \\
\hline
\end{tabular}

It should be stated that the cultures from mare No. 3 , and the first cultures from No. 5, were pure. The streptococcus from the second set of cultures from No. 5 was not distinguishable from one of the streptococci from mare No. 3. The large number of bacteria from No. 2 and the comparatively small number obtained from the other animals is worthy of note. It will also be observed that streptococci were universally present in the cultures. In all, eleven apparently different species of bacteria were isolated. They were classified as follows: Two non-motile bacilli, four micrococci, and five streptococci. The streptococci were very delicate, and the difterences that were found to exist between them were very slight. The micrococci were more saprophytic in their biological characters, and were readily differentiated. One of bacilli the was club-shaped, and resembled morphologically, and in its 
cultural characters, the pseudo-diphtheria bacillus. The other bacillus was slender and usually united in chains. It was äërobic and did not ferment glucose."

The results tluus far obtained, while they do not demonstrate the causal relation between this pathogenic bacillus and the abortion, nevertheless do not disprove such relation. The negative outcome of the injection experiments should not be rated too high in this connection, for there may be other as yet unknown factors necessary in order that the infectious agent may bring about abortion. It is true that we know nothing as yet concerning the pathological processes involved in. epizoötic abortion, and it would be premature to claim that some living organism like a specific bacterium is responsible for it. There may be other causes at work of which we have no information. Yet, from the standpoint of to-day, the assumption of living organisms, such as bacteria, explains best the occurrence as related above, and the actual presence of a pathogenic bacillus in the vagina of a mare after abortion is a fact worthy of more attention than we have been able to give it thus far.

Another fact of interest in connection with this pathogenic bacillus is its close resemblance to the hog-cholera bacillus. As I shall show by illustrations in a future article, the specific bacillus obtained directly from outbreaks of hog cholera varies slightly in its biological and morphological characters, and if the bacillus in question had been sent to me as having come from swine I should not have hesitated to regard it as a hog-cholera bacillus of rather feeble virulence, and possessing some slight differential characters from the virulent form. In other words, the differences between this form and the virulent hogcholera bacillus are no greater than between the hog-cholera bacilli of widely varying pathogenic power which I have thus far investigated. This fact does not of course prove it to be identical with Bacillus cholera suis, but the presumption is strongly in favor of a close relationship. The points of resemblance include form, motility, growth on the various culture media, including the fermentation tube, staining reactions, and pathogenic action. The only points of difference are fecbler pathogenic action and a tendency to a membranous growth on agar, which tendency, however, is not pronounced.

Granted the close relationslip of this bacillus with the bacillus of hog cholera, it is not safe to infer that abortion in mares stands in a causal relation to hog cholera or the reverse, and yet such relation is by no means disproved by the foregoing investigations. All that we may safely affirm is that there is evidently a wide distribution of bacteria closely related to the hog-cholera bacilus, inchding such bacteria as B. enteriditis Gärtner, B. typhi murium Löffler, and the bacillus under discussion. This possible relationship brings пр the important question whether certain specific infertious diseases of any given spercies of domesticated animals may appear also in other species, but un- 
der a different form, and therefore only identifiable by a study of the bacteria or other microörganisms which produce them. May we, in other words, have diseases among domesticated animals known under different names and yet due to the same canse? Pending the solution of such problems by a thorough investigation of all communicable diseases among domesticated species and species of wild animals coming in contact with them, we would urge owners and breeders of animals not to take it for granter that diseases, even among different species of animals, are necessarily different until such has been demonstrated to be the case by investigation. In other words, the indiscriminate mingling of sick and healthy animals may be really more dangerous than we are in the habit of regarding it.

The results of this investigation may be briefly summarized as follows:

1. The discovery of a pathogenic bacillus in the vagina of a mare soon after abortion.

2. The very close relationship between this bacillus and the hogcholera bacillus.

3. The production of a slight discharge by the injection of cultures into the vagina of a pregnant mare and two pregnant cows.

4. The absence of this bacillus from the genital passages of one pregnant and four non-pregnant mares. 
SOME EXPERTIENTAL OBSERVATIONS ON THE PRFSFNCE OF TUBERCLE BACILLI IN T'HE NILK OF TUBERCULOUS COWS WHEN THE UDDER IS NOT VISIBLY DISEASED.

\section{By Theobald Smith and E. C. Scmroeder.}

The possible dangers to health arising from the consumption of meat and milk from tuberculous cattle were discussed by one of us in a former report,* and the state of the subject summarized from the results of experiments made by various observers up to that time. Since then a small number of milch cows which were affected with this disease have come under observation in the District of Columbia. It was deemed advisable to take up this subject and determine if possible the extent of milk infection and its relation to the general condition of the animal. Only a small number of cases have been experimented on, but the facts are published now, inasmuch as they have accumulated very slowly and are not likely to be collected any more rapidly in the future.

The tuberculous animals were taken to the Veterinary Experiment Station where they were in charge of F. L. Kilborne, B. V. S., to whom we are indebted for some of the clinical notes. The two cases reported first have been already briefly referred to in a former report. $\dagger$ 'To determine the presence or absence of tubercle bacilli a certain quantity of milk, in the later cases centrifugalized, was injected directly into the abdomen of guinea-pigs. These were kept under observation a certain iength of time before being killed for examination.

No. 155, Jersey cow, seven years old, from a large herd in the District of Columbia. Tuberculosis diagnosed, and the animal transferred to the Experiment Station February 28, 1891.

There was at this time some emaciation, which became more marked in the following months. The animal lived until June 24, on which day she was found lying down in an insensible condition, and was dispatched by a blow on the head. At the autopsy advanced caseous masses were found in both lungs as well as in the bronchial and mediastinal glands, which were enormously enlarged. 
Guinea-pigs were inoculated as follows:

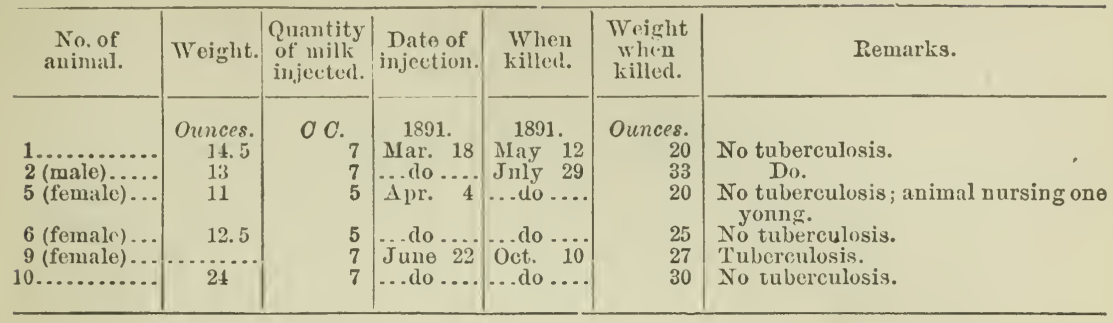

This table shows the absence of tuberculosis in all but one case, which had been inoculated with milk (together with one other) only two days before the cow was in a dying condition, and when the secretion had nearly ceased.

The tuberculous lesions in this guinea-pig are of interest. The anterior mediastinal glands at the root of the thorax were quite large and contained calcareous foci. There were a small number of subpleural tubercles. The spleen, thongh not much eularged, contained bunches of tubercles projecting slightly above the surface, and giving the organ a nodular appearance. A few necrotic foci were found in the liver. The guinea-pig was quite large and fat when killed, and no one would have suspected tubercular infection.

These inoculations show that even though the cow was emaciated the milk did not contain tubercle bacilli until shortly before death, and even then only one out of two inoculated guinea-pigs became infected.

No. 156, Jersey cow 8 or 9 years old, from the same herd as No. 155. Tuberculosis diagnosed February 28, 1891, and the cow transferred to the experiment station on this day. Emaciation had already set in and continued, so that at the time of her death, June 5, 1891, she was a mere skeleton.

There were extensive tuberculous growths on the omentum and in the peritoneum covering the paunch, the diaphragm, the abdominal walls, the spleen, kidneys and liver. The mesenteric, portal, and renal glands were transformed more or less into caseous masses. Of the abdominal organs there were tuberculous foci in the liver and the kidneys. Those in the kidneys were few in number and calcified. The Fallopian tubes and the lining membrane of the uterus contained very extensive cheesy deposits. In the thorax, the lungs were very extensively involved in tuberculosis, similarly all the lymph glands and the costal and pulmonary pleura. There were large deposits on the epicardium. The infection of the glands in the muscular tissue was not specially searched for. It should be mentioned, however, that there were no enlarged glands visible in any portion of the body, and that the axillary glands were normal. The udder was free from all changes visible to the naked eye. 
Only four guinea-pigs were inoculated with the milk of this cow.

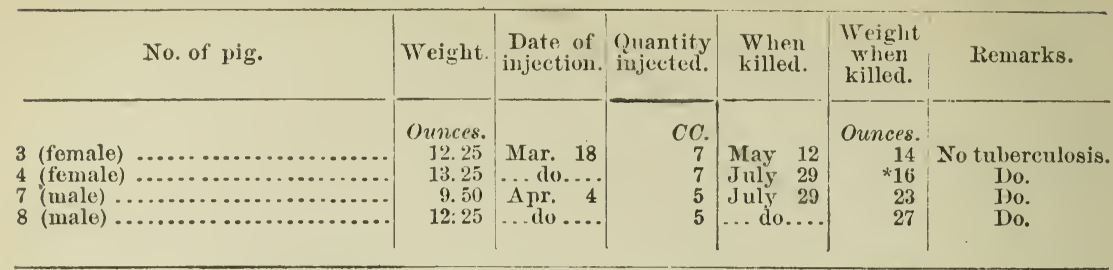

* Nursing three young.

The milk from these cows was used to feed tro pigs as long as it lasted. This was done by Dr. Kilborne, who has furnished the following notes concerning the feeding:

Pig No. 489.-Female, 2 months old on April 16, 1891; weighed 14 pounds.

Pig No. 490.-Female, 2 months old on April 16, 1891; weighed $16 \frac{1}{2}$ pounds.

On April 16 the feeding was begun. Each pig received daily about 1 gallon of milk in two meals until May, when the quantity began to decrease. On June 5, No. 156 died and No. 155 furnished about 1 quart daily until June 20, when the milk gave out.

Both pigs were killed July 29, 1891. At this time No. 489 weighed 63 pounds, and No. 49078 pounds. Both were in excellent condition and the organs free from disease.

No. 233, Jersey cow, 7 years old. Tuberculosis diagnosed, and the animal sent to the Experiment Station August 31, 1892. At this time she was quite thin and coughed more or less. Enlargement of subcutaneous lymphaties not discoverable.

Up to October 5 she was milkerl once a day, and on this day she gave only about a pint. She was failing rapidly, and on October 11 she was found dead.

At the autopsy tuberculous processes were observed in the following organs and localities :

In the cortex of one lymph gland behind udder a few quite small tubercles with yellowish center. The omentum over paunch studded with a large number of confluent tubercles; similarly the abdominal surface of the diaphragm. The capsule of liver and spleen was studded with tubercles, but the parenchyma of both organs was free. The mesenteric and portal glands extensively necrosed. 'Through the parenchyma of kidneys were disseminated cheesy tuberoles from 1 to 2 inches apart, and about one-eighth inch in diameter.

Extensive tuberculous disease of the Fallopian tubes and ovaries, as well as of the mucous membrane of the uterine horns.

In the thorax the anterior and posterior mediastinal glands, as well as the bronchial glands, are greatly enlarged and converted in part into caseous masses. Through the parenchyma of both lungs are disseminated caseous foci of various sizes, while the small bobes of the right lung (cephalic and ventral lobes) are completely transformed into caseous masses. The pleura over these lobes is thickly studded with tubercles. 
The milk was tested a short time before the death of the animal, and although the udder contained, so far as the naked eye could tell, no foci of disease, the secretion contained tubercle bacilli, as the following table demoustrates:

\begin{tabular}{|c|c|c|c|c|c|c|}
\hline $\begin{array}{c}\text { No. of } \\
\text { guinea- } \\
\text { pig. }\end{array}$ & Weight. & Date of injection. & $\begin{array}{l}\text { Quantity } \\
\text { of milk. }\end{array}$ & When killed. & $\begin{array}{c}\text { Weight when } \\
\text { killed. }\end{array}$ & Remarks. \\
\hline $\begin{array}{l}302 \\
303 \\
304 \\
305 \\
306 \\
309 \\
310 \\
311 \\
312 \\
313\end{array}$ & \begin{tabular}{|c|} 
Pounds. \\
1.25 \\
1.18 \\
1.18 \\
1.5 \\
0.95 \\
1.37 \\
1.5 \\
1.18 \\
1.5 \\
1.43
\end{tabular} & 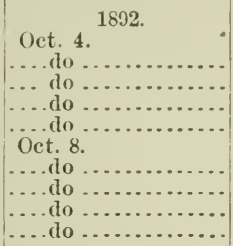 & $\begin{array}{r}C C . \\
5 \\
5 \\
5 \\
5 \\
5 \\
5 \\
5 \\
5 \\
5 \\
5\end{array}$ & 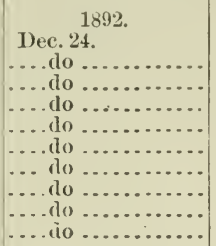 & $\begin{array}{l}\text { Pounds. } \\
1.343 \\
1.343 \\
1.25 \\
1.314 \\
1.047 \\
1.25 \\
1.156 \\
0.953 \\
1.375 \\
1.265\end{array}$ & $\begin{array}{l}\text { No tuberculosis. } \\
\text { Do. } \\
\text { Tuberculosis. } \\
\text { Do. } \\
\text { Do } \\
\text { Do } \\
\text { Do. } \\
\text { Do. } \\
\text { Do. } \\
\text { Do. }\end{array}$ \\
\hline
\end{tabular}

Note.-Nos. 302 to 306 , inclusive, received milk from left forequarter only. Only about 50 ce. could be drawn at the time. Nos. 309 to 313 , inclusive, received milk from the other quarters mixed.

Of the first five cases three were found tuberculous. Of the second lot of five all were tuberculous. The first set had been inoculated with milk drawn from the udder seven days before death; the second set with milk drawn only three days before death. There was, therefore, a distinct increase in the number of bacilli in the milk as death approached. That the animal was perhaps going through the earliest stages of an acute generalized miliary tuberculosis affecting the udiler as well as the other orgaus of the body was suggested in the microscopic examination of a fresh section of liver tissue, in which a group of three giant cells came clearly into view. Sections of hardened tissue have not been examined.

As to the character of the tuberculosis affecting the guinea-pigs the following general remarks will suffice:

1. Marked enlargement of the lymph glands of abdomen, especially the one behind (or dorsad of) the stomach, and of those at root of thorax (anterior mediastinal).

2. Puriform, semi-fluid condition of the contents of these glands excepting in one case in which they were still tirm.

3. In two cases the subcutaneous glands were affected.

4. Marked nodular condition of the spleen owing to agglomerations of tubercles within its substance.

5. Lines of retraction of the convex surface of the liver with very few distinctly tuberculous lesions.

6. Tubercles of the omentum in several cases.

No. 234, Jersey cow from the same herd as No. 233. Sent to the Experiment Station August 31, 1892, with the latter. Tuberculosis suspected from physical signs. Positive reaction with tuberculin at two different times. She was killed February 6, 1893, in a condition of marked emaciation. Of the autopsy notes, the following may be quoted:

Udder, together with lyniph glands, nornal.

In the thorax, the caudal half of both principal lobes firm, nodular to the touch. 
On seetion tubcrculous foci of various dimensions from miliary tubereles up to caseous foei fully three-fourths of an inch in diameter. The bronchi contain an abundance of muco-pus. Scattered masses of tubereles on the pleura of the diseased areas and on eorresponding costal pleura. Bronehial and mediastinal glands very large with easeous contents.

In abdomen the mesenterie glands are very large aud transformed into easeons masses whieh contain calcareous particles. In the eapsule of the liver three cheesy nodules as large as peas. Parenehyma of this organ as well as of spleen and kidney free from tubereulosis, although the gland at the hilus of the latter is enlarged and partly caseous.

The milk from this cow was centrifugalized before injection into guinea-pigs. The small Litten hand-centrifugal machine was used for this purpose. According to Scheurlen* bacteria do not behave alike under the influence of this process. Anthrax bacilli and their spores, the bacilli of typhoid fever and Asiatic cholera, gather in the cream, while tubercle bacilli are thrown down. In the same way tubercle bacilli tend to settle when milk is allowed to stand for some time in the refrigerator. Basing our procedure on these determinations we retained the milk in the bottom of the tubes after centrifigalizing and rejected the rest. The glass tubes which were to hold the milk during centrifugalizing were sterilized dry beforehand. The milk drawn as heretofore directly into cotton-plugged, sterilized 6 -ounce wide-mouthed bottles, was distributed into the four tubes of the centrifugal machine and revolved for forty-five minutes at a rate of 1200 revolutions per minute. The milk and cream at the surface was then carefully withdrawn with a sterile pipette and abont 0.5 ce. in the bottom retained.

The following table gives in bricf the experimental inoculations with the milk from No. 234:

\begin{tabular}{|c|c|c|c|c|c|c|c|}
\hline $\begin{array}{c}\text { No. of } \\
\text { guinea- } \\
\text { pig. }\end{array}$ & Weight. & $\begin{array}{l}\text { Date of } \\
\text { injection. }\end{array}$ & $\begin{array}{l}\text { Quan- } \\
\text { tity in- } \\
\text { jectrel. }\end{array}$ & $\begin{array}{l}\text { Centrifu- } \\
\text { galized } \\
\text { from. }\end{array}$ & $\begin{array}{l}\text { Guinea- } \\
\text { pig } \\
\text { killer. }\end{array}$ & $\begin{array}{c}\text { Weight } \\
\text { when } \\
\text { killed. }\end{array}$ & Remarks. \\
\hline & Pounds. & Dec. 2?, 1892 & $C C$. & $O C$ & $\begin{array}{c}1893 . \\
\text { Mar. } 25\end{array}$ & Pounds. & No tuberculosis. \\
\hline & 1.06 & Dec. 24,1892 & 62 & 20 & Mar. 10 & 1. 11 & $\begin{array}{l}\text { No tinberculosis. } \\
\text { Do. }\end{array}$ \\
\hline & 1.09 & Dec. $28,18 \cap 2$ & $4.5\}$ & & $\ldots$ do .... & 1. 06 & 1). \\
\hline 360 & & Jall. 10, 1893 & $5.5\}$ & & April 13 & 1.5 & Do. \\
\hline 1 & 1.5 & ato do & 65 & & ... do .... & 1. 28 & I)o. \\
\hline & 1. 120 & $\begin{array}{l}\text { Jan. } 11,1893 \\
\text {.... dio ......... }\end{array}$ & $\left.\begin{array}{l}5.0 \\
5,5\end{array}\right\}$ & $100\}$ & ... do .... & 1.28 & $\begin{array}{l}\text { Do. } \\
\text { Found dead of pnoumonia } 5 \text { days }\end{array}$ \\
\hline $\begin{array}{l}272 \ldots \ldots \\
373 \ldots \ldots\end{array}$ & 1.125 & $\begin{array}{l}\text { Jan. } 28,1893 \\
\ldots . . \text { do } \ldots . . . .\end{array}$ & $\left.\begin{array}{l}6 \\
6\end{array}\right\}$ & $60\}$ & 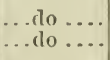 & $\begin{array}{l}1.25 \\
0.93\end{array}$ & $\begin{array}{l}\text { No tuberculosis. } \\
\text { Do. }\end{array}$ \\
\hline
\end{tabular}

In almost every case the milk was allowed to stand over night in the ice chest, and only the lower strata centrifugalized. In this way both sedimentation and centrifugalizing came into play. Granted that the latter process acts as determined by Schemrlen, the milk of this eow, even in the far advanced stage of tuberculosis, was free from tubercle

* Ueber die Wirkung des Centrifugirons auf Bakteriensuspensionen besonders auf die Benrtheilung der Bakterien in der Milch. Arbeiten aus dem Kaiserl. Gesundheitsamte, VII, 1891, 269. 
bacilli. Cover-glass preparations of the serliment, prepared Jauuary 28 , were carefully examined for tubercle bacilli, but only a small number of inultinuclear leucocytes were detected.

Cow No. 303.-The milk from this animal was tested but once. On December S, 1892, 60 ce. was centrifugalized for thirty minutes. In a little of the sediment stained, multinuclear lencocytes were detected, but no tubercle bacilli; 6 ec. of the milk in the bottom of the tubes was injected into the abdominal cavity of guinea-pig No. 356. It was killed with chloroform March 25, 1893, and found healthy.

The cow was killed March 18, 1893, a little more than three months after the milk was tested. Tuberculous foci partly caseous, partly calcareous, were found in the lymphatic glands behind the udder; abont half a dozen tuberculous foci in the lungs, one as large as a hen's egg. The bronchial, mediastinal, mesenteric and portal lymph glands contained tuberculous masses.

Cow No. 314.-The milk of this animal was tested at the same time. The guinea-pig inoculated with 6 cc. of centrifugalized milk was killed with the preceding, three and one-half months after the inoculation, and a little grayish, pneunonic infiltration of one lung discovered. Tuberculosis not detected.

The cow was killed March 18, 1893. The udder and corresponding lymph glands were free from disease. Tuberculosis of the lungs was present in the form of several foci, one 4 by $2 \frac{1}{2}$ inches in size. The bronchial and mediastinal glands were considerably involved ; the portal and mesenterie glands only slightly so.

The facts thus far obtained may be most conveniently put into the form of a table in which the period of injection of the milk is outlined by the last negative and first positive results :

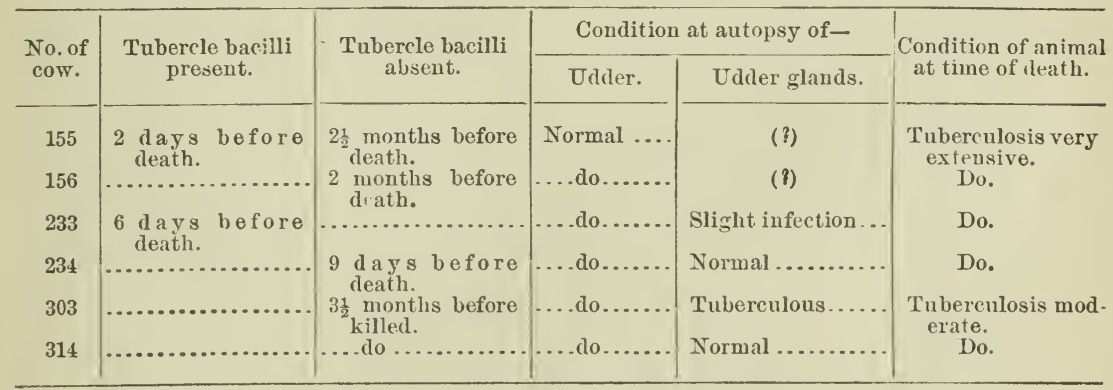

These determinations show, as was suggested in the article referred to above, that if a rigid inspection of all dairy herds be enforced so that all animals showing any affection whatever of the udder, or any emaciation, could be at once removed from the herd and the milk condemned,* perhaps most of the infected milk would be thereby excluded. This inspection does not settie the serious question of bovine tuberculosis in

*'This should be done even if the reaction with tuberculin proves negative. $5311-N_{0} 3-5$ 
its relation to human health. It would, however, be a great stride toward the settlement of this question, pending a more accurate determination of the extent of the disease. 'The use of tuberculin and the more careful examination of the organs at the abattoirs in Europe is revealing a much higher percentage of tuberculous eattle than had been heretofore even suspected, and we shall soon be prepared to publish additional interesting facts and deductions bearing on this important matter. 


\section{ADDITIONAL OBSERVATIONS ON TEXAS CATTLE FEVER.}

By Tireobald Smith, F. L. Kilborne, and E. C. Schroeder.

ON THE INDEFINITE PERSISTENCE OF THE TEXAS-FEVER PARASITE IN THE BLOOD OF SOUTHERN CATTLE.

Since the preparation of Bulletin No. 1 of the Burean of Animal Industry on Texas eattle fever, several additional facts have been discovered concerning the peculiar nature of this disease which we deem of sufficient importance to place on record.

In the bulletin referred to (p. 119) it was shown that, the blood of Sonthern (North Carolina) cattle contained the micro-parasite of the disease some time after such cattle had left the permanently infected territory, and after they had been rid of cattle ticks. In the final experiment recorded, the blood of a Southern cow (No. 214) was still capable of producing disease in natives when injected into a jugular vein seventy-four days after the animal had left the permanently infected territory, and about fifty days after the last ticks had been removed.

In continuation of the same investigations another native (No. 211) was inoculated with the blood of the same Southern cow (No. 214) on October 13, 1892, or one hundred and eight days after the latter had left North Carolina pastures. The native was a tuberculous cow in fairly good condition. An enormously enlarged lymphatic gland was situated behind the angle of the lower jaw. The inoculation was made as in previous cases, the blood being drawn from the jugular of No. 214 with a large hypodermic syringe and injected directly into the jugular vein of No.211. The following table and notes give the subsequent history of this case:

No. 211.

\begin{tabular}{|c|c|c|c|c|}
\hline \multirow[b]{2}{*}{ Date. } & \multirow{2}{*}{$\begin{array}{l}\text { Number of red } \\
\text { corpuseles. }\end{array}$} & \multicolumn{2}{|c|}{ Parasites in red corpuscles. } & \multirow[b]{2}{*}{ Remarks. } \\
\hline & & $\begin{array}{l}\text { In fresh prepa- } \\
\text { rations. }\end{array}$ & $\begin{array}{l}\text { Dried and } \\
\text { stained. }\end{array}$ & \\
\hline Oct. 13,1892 & $\begin{array}{l}5,678,500 \text { (white } \\
7,142 \text { ). }\end{array}$ & $\begin{array}{l}5 \text { percent bright } \\
\text { bodies; s ize } \\
\text { v a r a b b e } \\
\text { some motile. }\end{array}$ & None ... & 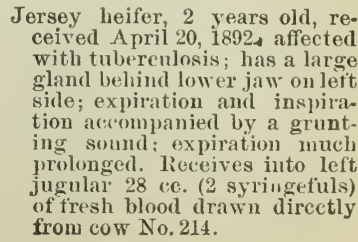 \\
\hline
\end{tabular}


No. 211.-Continned.

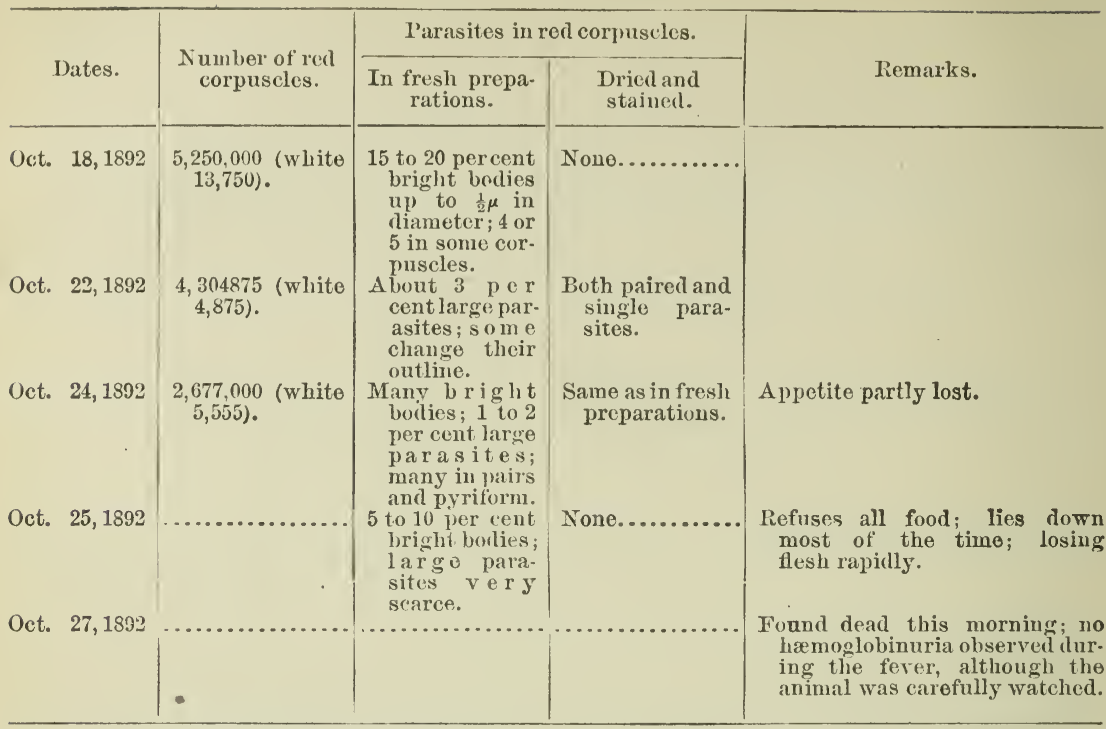

The temperature of this animal began to rise Oetober 14, reaching its highest point (105.8) October 18.

\section{AUTOPSY NOTES.}

Animal in good eondition as regards fat. Weighs about 550 pounds. Hrmorrhagie diseoloration of the left superficial jugnlar vein extending from tho place of injection to the root of the neck and toward the head. On the left side, resting against the larynx, a fluctuating gland fully 4 inches in diameter. It is readily dissected out, and when incised the thickened capsule is fonnd to inelose a turbid fluid holding easeous masses in suspension. Near this gland is a smaller one with eontents eompletely easeous.

Heart.-Usual eeehymosis under epieardium and under endocardium of left ventriele. Both ventricles contain dark coagnla of moderate size. Parenehymatous changes of heart muscle very slight. Lungs not collapsed. Subpleural empliysema over portions of smaller anterior lobes. Red hepatization of a portion of the right ventral, of a eontiguous portion of the right prineipal, and of the entire azygos lobe. The two first-mentioned lobes are interspersed with small neerotic foci. 'The whole einits an offensive odor. The portion of diaphragm nearest the hepatized azygos lobo is sprinkled with hemorrhages. The lung contains some tubereuluus foci as follows: A soft cheesy mass, 1 ineh in diameter, in the left cephalic, a similar one in the right prineipal lobe, and a smaller one in the hepatized region of the right ventral lobe. Bronchi of hepatized regions intensely dark red; oceasionally elmmps of yellowish masses encountered. The pneumonia is probably due to a reeent discharge of a easeous mass into a bronchus, resulting in aspiration pneumonia of a septie character.

Spleen.-Woighs $5 \frac{8}{4}$ pounds, 25 ineles long, 8 wide, and 18 thick. More or less extravasation of blood from vessels of eapsule. Usual markings of pulp effaced by the great ongorgemont with blood corpusclos.

Liver.-Weighs $1 t_{\frac{1}{2}}$ pounds without gall bladder. Color paler than normal. The surface sprinkled with elosely set irregular grayish spots about $\frac{1}{2}$ mm. in diameter. On section the liver presents a decidodly yollowish appearance. Much thick blood flows from hepatic vessels. Tho grayish points not visible on the cut surface. In fresh sections oecasional areas of bilo stasis noticed and much fatty degeneration of the parenchyma. Intracellular pigment abmulant.

Bile,-(8 ounees) is very highly charged with llakes, so that it scarcely flows. 
Kidneys.-Much paler than normal. On the surface asm all number of grayish spots corresponding on section to gray ish eylindrical lines passing through the cortex and indicative of fatty changes. Similar radial lines on pyramidal portion corresponding to tubules filled with fat globules. Much pigment in the form of granules up to $0.5 \mu$ in diameter in the epithelium of cortex and medulla. Papillæ more or less reddened and the inclosing calices are ecchymosed.

Urine.-Pale yellow; contains a trace of albumen.

Digestive tract.-Slight bluish-pink discoloration of mucosa of fourth stomach. Mucosa of small intestines pale and covered with a pasty, bile-stained layer of desquamated epithelium.

Uterus.-Contains a fotus 10 inches long.

The number of infected corpuscles was quite small at this stage of the disease. In the capillary blood from the heart none were seen. In the liver and spleen only a few were detected. In kidney preparations from 1 to 2 per cent were present. They are large, roundish, mainly single. A small number of tinted nacrocytes are present in the various organs.

The following case is of unusual interest, for it demonstrates that the Texas fever parasite may remain an indefinite length of time in the blood of insusceptible animals.

No. 113, a cow brought from North Carolina in September of 1859 , and at that time $3 \frac{1}{2}$ years old, has been kept on the Station since that time. She has not been exposed in fields infected with ticks or Southern cattle or sick native cattle. She has, in other words, not come in contact with the Texas fever infection in any way.

On October 29, 1892, a native cow, No. 235, which had been on the Station during the past two years, was inoculated with the blood of No. 113 in the way indicated in the preceding case. A syringeful of blood (14 ce.) was withdrawn from a jugular vein of No. 113 and injected at once into a jugular of No. 235. This was repeated, hence the latter animal received $28 \mathrm{cc}$. in orler that the experiment might be a precise parallel to similar ones made earlier in the season.* The following tabulated record demonstrates the presence of Texas fever:

No. 235.

\begin{tabular}{|c|c|c|c|c|c|}
\hline \multirow[b]{2}{*}{ Date. } & \multirow{2}{*}{$\begin{array}{l}\text { Number of red } \\
\text { corpuscles. }\end{array}$} & \multicolumn{2}{|c|}{ Parasites in red corpuscles. } & \multicolumn{2}{|c|}{ Condition of red corpuscles. } \\
\hline & & $\begin{array}{l}\text { In fresh prep- } \\
\text { arations. }\end{array}$ & Dried and stained. & $\begin{array}{l}\text { In fresh prep- } \\
\text { arations. }\end{array}$ & Dried and stained. \\
\hline Oct. 29,1892 & $\begin{array}{l}6,375,000 \text { (white } \\
10,000) \text {. }\end{array}$ & $\begin{array}{l}\text { Some bright } \\
\text { bodies. }\end{array}$ & None.............. & Normal... & Slight variation \\
\hline Nov. 9, 1892 & $\begin{array}{l}5,050,000 \text { (white } \\
6,250) \text {. }\end{array}$ & Negative ..... & $\begin{array}{l}\text { Severul irregnlar } \\
\text { and one pair } \bar{y} \text { - }\end{array}$ & .....do .. & Do. \\
\hline Nov 12,1892 & $\begin{array}{l}3,815,555 \text { (white } \\
7,700) \text {. }\end{array}$ & $\begin{array}{l}\text { A few bright } \\
\text { bodies. }\end{array}$ & $\begin{array}{l}\text { liform parasites. } \\
\text { Several parasites } \\
\text { of irregularout- } \\
\text { line. }\end{array}$ & ....do. & Normal. \\
\hline Nov. 14,1892 & $4,2 \overline{0} 7,500$ (white & .... do ... & None............. & ....do ...... & Do. \\
\hline Nov. 19,1892 & $\begin{array}{l}5,575,000 \text { (white } \\
10,000 \text { ). }\end{array}$ & ....do & ....do..... & $\begin{array}{l}5 \text { to } 10 \text { percent } \\
\text { m a c rocytes } \\
\text { up to } 8 \mu \text { in di- }\end{array}$ & $\begin{array}{c}\text { Same as in fresh } \\
\text { preparations. }\end{array}$ \\
\hline Nov. 30,1892 & $\begin{array}{c}4,700,000 \text { (white } \\
18,750) \text {. }\end{array}$ & $\begin{array}{l}\text { (Preparation } \\
\text { poor.) }\end{array}$ & Negative.... & $\begin{array}{l}\text { ameter. } \\
\text { A few macro. } \\
\text { cytes. }\end{array}$ & $\begin{array}{l}\text { A few macro- } \\
\text { eytes. }\end{array}$ \\
\hline
\end{tabular}

NOTE.-The temperature in this case was high from November 6 to November 13.

*Bulletin No. 1, p. 119. 
As a parallel to the precenling inoculation the following may be cited here, although it does not bear directly on the subject under discussion :

On August 27, 1892, defibriuated blood from a case of Texas fever* collected with all precautions necessary in bacteriological work, was drawn up into sterile pipettes and some placed at $36^{\circ} \mathrm{C}$., others in a dark receptacle at the ordinary temperature. These pipettes remained undisturbed until November 3, when the blood was withdrawn from all and mixed together, making about $7 \mathrm{ce}$. It was free from bacteria. This blood was injected into a jugular vein of No. 236. The record below shows that no disease was produced. The blood at the time it was drawn and after a few days' standing in a cool place produced Texas fever in four cases inoculated therewith. $\dagger$

No. 236.-Cow, 5 years old, received October 29, 1892, from Maryland.

\begin{tabular}{|c|c|c|c|}
\hline \multirow[b]{2}{*}{ Dates. } & \multirow[b]{2}{*}{ Number of red corpuscles. } & \multicolumn{2}{|l|}{ Parasites in red corpuscles. } \\
\hline & & In fresh preparations. & $\begin{array}{l}\text { Dried and } \\
\text { stained. }\end{array}$ \\
\hline $\begin{array}{l}\text { Oct. } 29,1892 \\
\text { Nov. } 9,1892 \\
\text { Nov. } 14,1892 \\
\text { Nov. } 19,1892\end{array}$ & $\begin{array}{l}6,609,370 \text { (white } 10,937) \ldots \ldots \ldots \ldots \\
6,737,500 \text { (white } 13,750) \ldots \ldots \ldots . \\
6,093,000 \text { (white } 6,666) \ldots \ldots \ldots \ldots \\
6,475,000 \text { (white } 7,500) \ldots \ldots \ldots \ldots\end{array}$ & 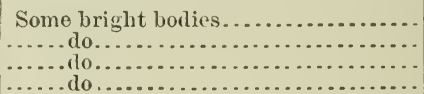 & $\begin{array}{l}\text { None. } \\
\text { Do. } \\
\text { Do. } \\
\text { Do. }\end{array}$ \\
\hline
\end{tabular}

Note.-The temperature of this case at no tine rose above $102.6 \mathrm{~F}$.

The following severe case of Texas fever, followed by relapse, was produced in midwinter by inoculation with blood from a North Carolina cow:

No. 237, a native cow, 5 years old, from Prince George County, Md., was inoeulated January 12, 1893. Twenty-eight ec. of blood, drawn with a hypodermie syringe from a jugular vein of North Carolina cow No. 214, was injected directly into a jugular vein of this cow. The fever appeared in five days, and the destruction of the red blood corpuscles was well under way on the seventh day after inoculation, as the table below shows:

No. 237.

\begin{tabular}{|c|c|c|c|c|}
\hline \multirow{2}{*}{ Dates. } & \multirow{2}{*}{$\begin{array}{l}\text { Number of red } \\
\text { corpuscles. }\end{array}$} & \multicolumn{2}{|c|}{ Parasites in roil corpuscles. } & \multirow{2}{*}{ Remarks. } \\
\hline & & In fresh preparations. & Dried and stained. & \\
\hline Jan. 10, 1893 & $\begin{array}{l}5,962,500 \text { (white } \\
10,000 \text { ). }\end{array}$ & $\begin{array}{l}2 \text { to } 3 \text { per cent louglit } \\
\text { bodies. }\end{array}$ & None .............. & \\
\hline $\begin{array}{l}\text { Jan. } 12,1893 \\
\text { Jau. } 19,1893\end{array}$ & $\begin{array}{l}4,287,500) \text { (white } \\
10,000) .\end{array}$ & $\begin{array}{l}\text { A fow bright bodies; } \\
1 \text { per cent amo bond } \\
\text { and paired parasites. }\end{array}$ & $\begin{array}{l}\text { Ono-hal f per cont } \\
\text { large, single and } \\
\text { paired parasites. }\end{array}$ & Injection maleto-day. \\
\hline $\begin{array}{l}\text { Jan. 21, } 1893 \\
\text { Jan. 25, } 1893\end{array}$ & $\begin{array}{l}3,561,600 \text { (white } \\
42,465) \text {. } \\
2,314,(000 \text { (white } \\
4,271 \text { ). }\end{array}$ & $\begin{array}{l}\text { Parasites very searce. } \\
\text {......do ............... }\end{array}$ & $\begin{array}{l}\text { Soveral large parasites } \\
\text { in each preparation. } \\
\text { Very fow parasites... }\end{array}$ & $\begin{array}{l}\text { Appetito failing. } \\
\text { January } 26 . \text { (i a ve } \\
\text { birtli to a alf last } \\
\text { niglit; has lost con. } \\
\text { siderable llesh and } \\
\text { is very weak. }\end{array}$ \\
\hline
\end{tabular}


No. 237.-Continned.

\begin{tabular}{|c|c|c|c|c|}
\hline \multirow{2}{*}{ Dates. } & \multirow{2}{*}{$\begin{array}{l}\text { Nnmber of red } \\
\text { corpuscles. }\end{array}$} & \multicolumn{2}{|c|}{ Parasites in red corpussles. } & \multirow{2}{*}{ Remarks. } \\
\hline & & In fresh preparations. & Dried and stained. & \\
\hline Feb. 17,1893 & $\begin{array}{c}1,851,300 \text { (white } \\
4,054)\end{array}$ & Nono .................. & Some peripheral cocci. & Relapse beginning. \\
\hline Mar. 1,1893 & $\begin{array}{l}1,033,000 \text { (white } \\
1,515) \text {. }\end{array}$ & $\begin{array}{l}\text { Some pale peripheral } \\
\text { bodies. }\end{array}$ & $\begin{array}{l}5 \text { per cent perıpheral } \\
\text { cocci. }\end{array}$ & \\
\hline Mar. 20, 1893 & $\begin{array}{l}1,246,000 \text { (white } \\
6,350) \text {. }\end{array}$ & $\left\{\begin{array}{l}\text { Some bright bodies... } \\
\text { Some pale peripheral } \\
\text { bodies. }\end{array}\right.$ & Some peripheral cocci. & \\
\hline Mar. 31, 1893 & $\begin{array}{l}2,170,000 \text { (white } \\
6,154) .\end{array}$ & A few bright bodies. & $\begin{array}{l}\text { Very few peripheral } \\
\text { cocci. }\end{array}$ & \\
\hline
\end{tabular}

NoTE-The temperature in this case remained high (103.5-105.5) almost continuously from January 17 to January 25.

ON THE PERSISTENCE OF THE 'TEXAS-FEVER PARASITE IN THE BLOOD OF INOCULA'TED NA'TIVE CAT'ILE.

That the Texas-fever parasite might persist in the blood of susceptible cattle long after recovery from the disease did not suggest itself until this interesting fact was accidentally discovered.

In 1891 several animals which had been inoculated with blood from a case of Texas fever passed through a severe attack of this disease.* Two of these were reëxposed the following summer.

Just before the exposure on the infected pasture the customary blood examination was made, and it was found that in one of these animals the Texas-fever micro-parasite was present, though in very sinall numbers. The peculiar pyriform bodies in pairs within the red corpuscles left no doubt as to their nature. Inasmuch as this animal had not been exposed to the infection since the inoculation, the only conclusion which could be reached was that the micro-parasite had persisted in the blood since last fall. This persistence, astonishing in itself at the time, is now quite in harmony with the long life of the species in the blood of Southern cattle.

Another fact of interest is the insusceptibility on the second exposure of the animal in which the micro-parasite was found. The other animal,whose blood on microscopic examination no longer revealed the micro-parasite, was not insusceptible, for it passed through a wellmarked but mild attack.

\section{ON PREVEN'IIVE INOCULATION.}

The foregoing observations furnish an important basis for a rational method of protective inoculation with reference to Northern cattle about to enter the Southern permanently infected territory. We have shown in the foregoing pages and in the bulletin on Texas fever that the blood of Southern cattle contains the micro-parasite of Texas fever an indefinite length of time, and that every intravenous inoculation with such blood has produced the disease in natives. Moreover, the disease induced by a single inoculation of such blood is by no means so

${ }^{*}$ Bulletin No. 1, p1. 80 and 131; also Nos. 182 and 185 in the appendix. 
fatal as the natural disease. In fact, we have recorded but one death in midsummer due to inoculation. A third important consideration is the production of the inoculation disease at all seasons, even in midwinter.

Putting these facts together we are led to the inference that, inasmuch as a well-marked attack of Texas fever will probably protect from a future fatal attack, the first attack might be induced artificially in fall or winter by direct inoculation. In other words, the protective inoculation would be a real attack of the disease. It is not likely that, even in older cattle, such inoculation would result fatally in late fall or winter. Of inoculation with the blood of sick natives we can not make such positive statements, since we have had a considerable percentage of deaths in September from such inoculations.

In the bulletin on Texas fever it was suggested that native cattle might be exposed to a mild form of the disease by scattering ripe, egglaying ticks on an inclosed pasture upon which the cattle to be exposed are confined. While we have found nothing thus far to invalidate this method, we nevertheless feel that the method of intravenous inoculation can be made more certain by some additional experimenting. It is desirable to determine, also, whether the simple subcutaneous injection of blood from Southern cattle will suffice to induce Texas fever. The subcutaneous injection of defibrinated blood taken from natives during the fever was followed by positive results (in one old cow, by death). The much larger number of micro-parasites in such blood will perhaps account for the very positive results obtained.*

It should be stated that preventive inoculation is of no avail during the prevalence of the disease, owing to the long duration of the disease and the consequent slow acquisition of immunity.

In conclusion, the important facts in this article may be very briefly summarized as follows:

1. The indefinite persistence of the micro-parasite of Texas fever in the blood of Souther'n cattle after removal from the enzoötic territory.

2. The persistence (for nearly a year) of this micro-parasite in the blood of an inoculated native.

3. The production of a severe case of this disease in midwinter by inoculation.

4. The destruction of the micro-parasite in a quantity of defibrinated blood kept sixty-eight days before it was tested by inoculation.

5. The probable development of a simple method of preventive inoculation by the use of blood from Southern cattle.

* Since this was written, subentancous inoculation with blood from Southern catthe has been found equally efficacious. The intravenous injection thus becomes unnecessary. 


\section{PRELIMINARY NOTES ON A SPOROZOÖN IN THE INTESTINAL VILLI OF CAT'ILE.}

By Theobald Smitr.

[Plate I.]

In the summer of 1889 , during the examination of cattle which had succumbed to Texas fever, I observed on the mucous membrane of the small intestine in its lower portion minute white bodies of a spherical outline which were just visible to the naked eye. Several observers, even when informed of their presence in a given portion of the mucous membrane, passed them by at first until they were directly pointed out. They may, therefore, pass unnoticed, unless the scrutiny of the mucosa is sharp. When the membrane is stroked with the back of the scalpel it will be noticed that these bodies change their place slightly without becoming detached, indicating that they are located within the villi near their free end. This supposition is confirmed by carefully removing a number of villi, where such a body is found, with small forceps, and examining them under a low power. It will then be seen that an opaque round or slightly oval body is situated within one of them directly under the epithelial layer. The removal is accomplished best by clasping at their roots the villi in the immediate vicinity of such a borly with delicate forceps and gently pulling upward. The parasite comes away intact, and is easily teased out of the adherent cell mass without rupture of the eyst. These bodies vary considerably in size, and are either spherical or ovoid. Of a number of measurements of bodies of the larger varieties the following may be quoted:

(1) $0.416^{\mathrm{mm}}$ long, $0.364^{\mathrm{mm}}$ broad.

(2) $0.312^{\mathrm{mm}}$ long, $0.208^{\mathrm{mm}}$ broad.

(3) $0.312^{\mathrm{mm}}$ long, $0.312^{\mathrm{mm}}$ broad.

(4) $0.364^{\mathrm{mm}}$ long, $0.312^{\mathrm{mm}}$ broak.

It is probable that the weight of the cover glass may have distorted somewhat the true form, and this may account for the varied relation of length to width in these measurements. We may roughly speak of the larger bodies as from 0.3 to $0.4 \mathrm{~mm}(0.012$ to 0.016 inch) in diameter.

A superficial microscopic examination showed at once that they were sporozoa. At the time of their discovery I had just found the 
intraglobular parasite of Texas fever. Some relation between these intestinal parasites and those of the blood in Texas fever seemed at least a possibility. I therefore gave them as much attention as I could in connection with the somewhat trying microscopic work demanded by the Texas fever investigations. Subsequently I found the same bodies in cattle from a Washington abattoir. Their relation to the Texas-fever micro-parasite could not, therefore, be upheld any longer, even if their morphology warranted such an assumption, which it does not.

The following observations were made exclusively on fresh material. They are incomplete in many ways, since only the reproductive stage has been seen. They will be continued as opportunity presents, since this group of organisms demands the attention not only of the zoölogist, but of the pathologist as well.**

It has already been stated that the lower portion of the small intes. tine is the preferred seat of these sporozoa. A few have been detected in the cxecum. They are especially abundant in young animals, and these animals are the only ones which present a sufficient number of stages of the life history of the parasite for investigation. Older animals generally contain a few eysts in an advanced stage of develop)ment, but they do not repay examination. I am inclined to regard midsummer as the time when the parasite appears most abundantly, but I have not extended the observations sufficiently into other seasons to make any positive statements on this point.

1. Let us first take the largest eysts readily visible to the naked eye. When crushed under a cover glass in some physiological salt solution the eyst wall tears readily and appears as a very delicate membrane of a finely granular appearance, readily thrown into folds by the crushing. A double contour was not observed. The crushing forees ont of the densely packed eyst an immense number of falciform bodies of the sane form and size, showing no connection with one another. These swarm spores are from 10.5 to $12.6 \%$ in length and slightly crescentic in outline (Fig. 2, a). The body is homogeneous in appearance. Any differentiation of the cell contents is not observable in this condition. The majority show distinct movenents like those originally described by Eimer for the coecidia discovered by him in the mouse's intestine. One end of the crescent bends toward the body, the whole appearing in this condition like the figure 6, or like a hook, or even like the letter U. After remaining in this bent, tetanic state for one or two seconds, the crescent suddenly straightens ont, and in some instances it was propelled forward, $i$. e., in the direction of the other extremity, from one half to twice its whole length (Fig. 3, a). Whether only one-half of the erescent is the contractile portion, or whether both share this function, I am not prepared to say. The power to move had not been lost by

*I)r. C. W. Stiles, zoölogist of the Burean, will continue tho study of this organisia. 
a stay in the refrigerator for twenty-four hours ( $55^{\circ}$ to $60^{\circ} \mathrm{F}$.). The path of the crescent is usually a slightly eurved line, corresponding to the curvature of the crescent in its straightened position. In straightening out it may be swung around by this force so as to describe a complete circle. In some eysts forms like $b$, Fig. 3, may be observed moving around this middle point as a pivot withont straightening ont. In one cyst bent forms with a central knob were observed, which, without showing any change of form, moved quite a distance. In one case the path described by the crescent might be compared to a loop. It will be seen from these illustrations that the motion of these bodies, which can be studied to great advantage in this sporozoön, is by no means easily explained, and will repay careful investigation.

The simple crescents retain their form well in normal salt solution, but when this is replaced by distilled water they speedily assume the spherical form and are disintegrated.

2. Cysts containing independent crescents of the simpler form described (Fig. 2, a) are perhaps the most commonly met with. Yet they are not the ouly ones, for after more or less investigation I found a bewildering variety of cyst contents which will require much patient observation to elucidate their relationship to one another. Not infrequently I found large cysts containing, besides the crescents described, peculiar modifications of the latter, as is shown in Fig. 2 . These modifications appear as a central, round finely granular body, about $3_{\psi}$. in diameter attached to the middle point of a thread-Jike body, the whole being as long as the crescent described $(12 \mu)$. These sometimes pre. dominate in a cyst, sometimes they are present in small numbers mingled with the others. I have not observed any motion of the slender filiform bodies. It is safe to assume that they are crescents which have undergone further development within the cyst, and that Fig. 2 represents a related series of forms from $a$ to $e$, inclusive.

3. In the case of calves minute cysts are frequently detected with a hand lens. Such cysts vary much in the morphology of their contents, and give us some idlea of the way in which such a large number of swarm spores may be formed in a single cyst. When such cysts are examined entire under a low power a slight differentiation in the contents may be occasionally detected in the form of miunte darker specks (Fig. 1). When the cyst is crushed the outpouring free crescents are accompanied by peculiar agglomerations of crescents. A small spherical body containing a variable number of refracting granules is everywhere beset with the homogeneous crescents first described. Each crescent is attached with one extremity to the periphery of the sphere, and extends radially or somewhat tangentially outwards. Some of these colonies, as they roll out of the crushed cyst, give the impression of sunflowers, the central sphere representing the disk and the crescents the ray flowers (Fig. 4, $a, b$ ). In several cases I was fortunate enough to find a still younger stage of the same structures, as is shown 
in Fig. 5. The periphery of a rather large splıere loosely filled with refracting granules is beset with quite short conical appendages, the base resting on the sphere.

In some cysts nearly all the crescents are attached in the manner described. The central sphere varies much in size. The largest may be $15 \mu$ in diameter, the smallest but $2 \mu$. The crescents may be so densely packed around the central body as to obscure it almost entirely. In the case of the smaller spheres there may appear but one to three attached crescents.

4. In the contents of the cysts described there are frequently found among the crescents a variable number of round bodies or spheres, from 4 to $9 \mu$ in diameter, which are filled with minute refrangent particles, and to whose periphery no crescents in any stage of development are seen attached.

5. A few cysts, not more than half the diameter of those described, contained only masses of strongly refracting bodies 1 to $2 \mu$ in diameter.

From this cursory description it will be seen that ouly a fragment of the life history of the sporozoön has come under my observation. The youngest stage, its penetration into the tissue of the villus, its true situation there, whether originally as an intracellular or an extracellular parasite, and the form and characters of the adult parasite still need elucidation. That portion of the life history found by me seems to be concerned mainly with the production of a large number of swarm spores. How these are formed in such abundance is shown at least partially by the observations recorded. A large number of centers or foci seem to be present throughout the cyst, from the periphery of which the crescents are produced. The previous history of these foci is not cleared up, but I have seen in various eysts forms roughly reproduced in Fig. 6, which lead me to believe, at least provisionally, that these foci are formed by a simple transformation of some of the crescents into spheres which subsequently produce, peripherally, a second generation of the same elements. In this way we may have several generations of crescents in the same cyst, and the enormous numbers may be the offspring of a few original crescents. As to the correctness of this theory future observations must decide. In several cysts I observed forms resembling $y$, in Fig. 2. The ereseent was bent upon itself and apparently within a eyst of its own. Further observations will be necessary to determine whether such endogenons formation of crescents actually takes place. If so, it may represent crescents of a different functional order from those described, and it may explain the presence of the peculiar bent or U-shaped forms (Fig. 2, f; Fig. 3, b,c).

The functions of the swarm spores probably is to keep up the autoinfection of the intestinal mucosa. The rupture of one of the large cysts sends into the intestine a great number of such bodies, each of which is capable of forming a new focus of infection. The different stages in which these cysts are found lends color to this view. It is probable that 
there exists also a more resistant stage whose function it is to keep up the life of the parasite outside of the animal body. I am inclined to look upon the tailed modifications of the erescents (Fig. s, $c, d, e$ ), found only in the largest and oldest cysts, as such a resistant stage (Dauerform, Dauerzustand). The recent investigations of R. Pfeiffer* on Coccidium oviforme, and of Schubergt on Eimeria falciformis, have shown that for these coccidia two types of reproduction may perpetuate the species, which in the ease of C.oviforme were formerly looked upon as two distinct species. It is not to be denied therefore that the supposition concerning a more resistant stage of the crescents given above may be erroneous, and that future investigations may reveal a quite different type of reproduction.

The evidence thus adduced is not sufficient to bring this sporozoön into any of the known groups, but it at least indicates that it does not belong to the Coccidia, and that it has characters which nnight bring it within the range of the Sarcosporidie.

To give an adequate illea of the varions stages in which the cyst contents are found, I will cite a few illustrations from my notes:

October 11, 1889.- Several cysts, from small intestine of healthy steer slaughtered for beef, contain only the homogeneous crescents (Fig. 2, a), many in motion.

One cyst contains a variety of forms, from the simple erescent to the knobbed forms (Fig. 2, a to $f$, inclusive).

September 20, 1859.-Case of Texas fever. One eyst contains mainly forms shown in Fig. 6.

September 20, 1889.-One cyst, from intestine of healthy steer, contains only such forms as are shown in Fig. 4. All crescents are attached to spherical bodies of various dimensions $(2 \mu$ to $15 \mu)$.

A second cyst from the same animal contains free crescents in motion and a small number of round granular bodies (F'ig. 5, a).

August 20, 1890.-One cyst, from a calf which succumbed to Texas fever, contains an immense number of refracting graumles, $1 \mu$ diameter, and many spherical bodies with small crescents attached (Fig. 5). Whether the free granules were originally contained in small cysts and freed by crushing was not determinable.

A second cyst contains, besides a few sunflower forms (Fig. 4), an immense number of free crescents.

A third very suall cyst contains only refracting granules, 1 to $2 \mu$ in diameter.

September 22, 1890.-One cyst, from a calf killed after a mild attack of Texas fever, contains motile crescents and granular spheres ( 4 to $9 \mu$ in diameter).

A second cyst contains only knobbed crescents in the stage of $b$, Fig. 2 .

A third small cyst contains almost exclusively immature attached crescents (Fig. $5, b, c, d, e)$.

August 11, 1890.-One cyst, from a healthy slaughtered steer, contains mainly tailcd (Fig. 2, $d, e, f$ ) forms and a few crescents.

A second cyst contains (1) homogeneous crescents in motion; (2) tailed or knobbed forms; (3) many crescents bent in form of the letter $U$ (Fig. 2, $f$ ); (4) crescents at. tached to a central spliere; (5) forms like Fig. 2, g.

A third cyst contains erescents only. These are of the usual form or doubled up (U-shaped) or partly extended. The $U$-shaped forms are quite abundant.

*Untersuchungen iiber Protozoen, Heft. I, 1892.

+ Uheber Coccidien des M̈̈usedarmes, Sitzungsber, d. Würzb. phys-mod, Gesellschaft, 1892. 


\section{DESCRIPTION OF PLATE I.}

Illnstrations, somewhat diagrammatic, of the varions observed forms of the sporozoön. Fig. 1 is magnified about 80 diameters, the rest, 1,000 diameters.

Fig. 1. Cyst as seen under a low power of the microscope. The darker spots represent the forms shown in lig. 4. The remainder of the cyst is filled with freo crescents.

Fig. 2. Crescents of various forms.

Fig. 3. Crescents in motion. The dotted lines indicate approxinately the paths.

Fig. 4. Spheres containing grannles, with periphery beset with nearly mature crescents.

Fig. $5 a$. Simple sphere containing granules; $b, c, d$, and $e$, spheres with periphery beset with immature crescents.

Fig. 6. (Hypothetical), trausformation of a crescent into a sphero. 

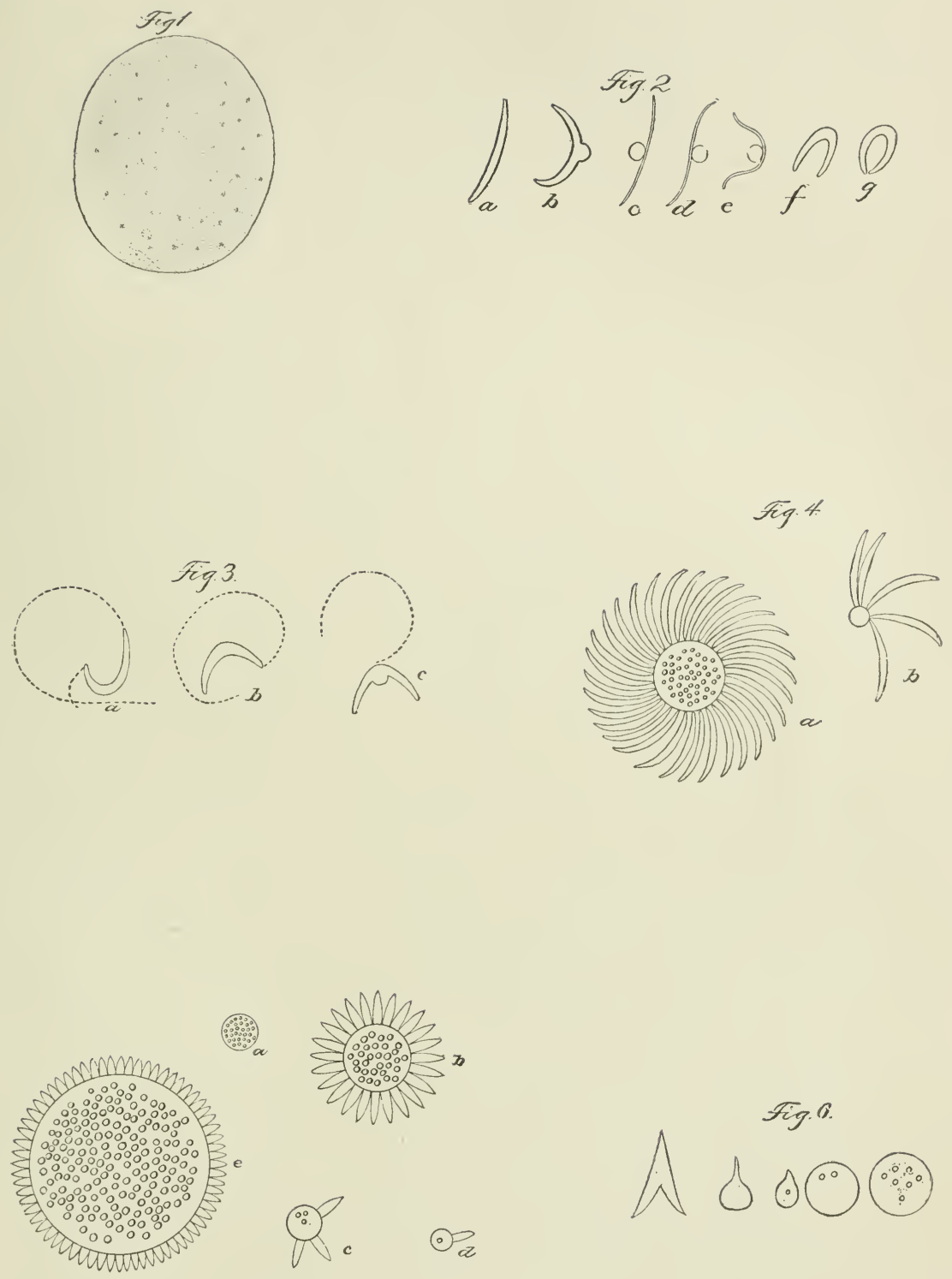

Fig. 5

SPOROZOÖN IN THE INTESTINAL VILLI OF CATTLE. 



\title{
NOTES ON PARASITES-18: ON THE PRESENCE OF SARCOSPORIDIA IN BIRIS.
}

\author{
By C. W. Stiles.
}

[Plates II and III.]

The first reference $\mathrm{I}$ have been able to find in regard to Sarcosporidia in birds is an article by J. Kiihn.* This paper is unfortunately not at my disposal, but it is evident from the statements of later authors that Kiihn found the parasites in chickens.

Rileyt examined a duck whose museles were infested with parasites one-fourth to one-fifth of an inch long; they resembled grains of wheat and contained a viscid yellowish substance. Riley compared them to pork measles (Cysticercus cellulose) and was under the impression that they represented the larval stage of some worm which would reach maturity in the intestine of man. Upon seeing one of the specimens I describe below, Riley stated to me that it was undoubtedly identical with his duck measles.

Bélangerł presented before the Société d'Histoire Naturelle de Quibec a case of parasitism in Spatula clypeata Boie. Small "white worms" were found by thousands in the muscles. They measured $\frac{1}{2}-1$ line long, and appeared to belong "à quelques genres voisius des trichines, bien qu'ils ne fussent pas enroulés, ni renfermés dans des enveloppes." This reference, to which Prof. Riley called my attention, probably refers to the same parasite described below from the same host.

Rivolta§ states:

Gli otricelli psorospermici dei gallinacei, di cui fanno menzione alcuni trattastisti (Röll ecc.) altro non sono che gli otricelli del Miescher o del Renay, che non si devono confondere con i psorospermi, propriamente detti, dei polli.

On page 398, l. c., in discussing Enterite psorospermica, he adds:

Alle pareti poi dell' intestino si scorgono, situati all' incerca nel sottomucoso, dei punti bianchicci sparsi qua et là; quasi tutti della grandezza di un seme di papavero o di un semplice punto. Questi punti bianchicci non sono formati da colonie di psorospermi ma bensì da otricelli del Miescher o del Renay pieni di navicelle.

Rivolta also gives two figures of the parasites.

"Mittheilungen d. landwirthsch. Instit. zu Halle, 1865, p. 68.

tWalsh and Riley. A Measly Wild Duck. The American Entomologist, 1869, I, p. 89.

$\ddagger$ Le Naturaliste Canadien, Tome II, Mai, 1870, p. 188.

$\$$ Dei parassiti vegetali, Torino, 1873, p. 39j. Taf. III, figs. 52, 74 , 
Leidy* describes some eysts found in the interstices of the muscles of Anas boschas L. They were oval, white, 2 to $4 \mathrm{~mm}$. long, $0.7 \mathrm{~mm}$. thick, and contained myriads of "fusiform corpuscles" $17 \mu$ long, resembling minute pseudo-navicellx. He adds that "though the Mallard is not a fish eater, the bird may have become infected by eating diseased fish." Thus it is clear that Leidy was inclined to look upon these parasites as identical with the Myxosporidia.

Leuckart $\nmid$ simply states in regard to Sarcosporidia in relation to birds, "selbst das Haushuhn bleibt davon nicht verschont."

Buitschlił refers to the observations by Kühn and Rivolta as follows:

Kiihn fand sie auch bei Ḧ̈hnern und Rivolta hat auf das Vorkommen ähnlicher Parasiten in der Submucosa des Darmes mehrerer Vögel (der Haushiihner, Schwarzamsel (Turdus merula), des Rabens, etc., aufmerksam gemacht und es scheint auch, dass diesselben mit Recht den parasitischen Schläuchen dor Säugethiere an die Seite gesetzt werden.

Zuirn || states that Sarcosporidia (Psorospermienschläuche) have been reported in the muscles, kidneys, and intestinal walls of various birds. He himself had never found them. The parasites are in some cases microscopic, in others visible to the naked eye; they may be 1 or several mm. long and are surrounded by a resistant but porous membrane. The crescents are $0.01 \mathrm{~mm}$. long and contain one or more vacuoles, together with granules. They are not seattered regularly through the entire cyst, but collected together in balls.

Barrows ๆ found a Parula pitiayumi (Vieill.) whose muscles were parasitized, and upon examining one of my specimens Mr. Barrows said that the parasite appeared identieal with those he found, so far as this could be determined without a microscopical investigation. His description reads:

One specimen (i.e., I'arula pitiayumi) seemed to be abnormal in coloring, showing many white feathers in the forehead, and upon skinning the flesh was found thickly spotted with oval, white lumps about the size of the egg of the common blow-fly. These were most numerous toward the surface of the pectoral muscles, but occurred also deeply imbedded in their substance as well as in the muscles of wings and legs. It was not practicablo to examine their structure with the microscope until the next day, when decomposition was so far advanced that little could be made out. In all probability, however, they were the eneysted larva of some parasitie worm, though whether they had anything to do with the abnormal plumage is an open question.

Barrows' description renders it almost certain that this was a case of sarcosporidiosis, although it is, of course, impossible to determine

* Psorosperms of Mallard Duck. Proc. Acad. of Seience, Phila., 1875, xxvin, p. 125.

† Die Parasiten des Menschen, 2. Aufl., 1879, I, p. 254.

$\ddagger$ Protozoa, I, p. 605. Bronn's Klassen und Ordnungen des 'Thier-Reichs, 1880-82.

§opra i vajuolo dei Columbi e dei Polli. Giornale di Anatomia, Fisiologia o Patalogia degli Animali, 1874, vi, p. 257. (N. B.-The pagination of this journal is incorrect. It should read, p. 321.)

|| Die Krankheiten des Haungefliigels, 1882, p. 146.

IT Walter B. Barrows. Birds of the Lower Uruguay. Bull. of the Nuttall Ornithological Club, viı, 1883 , p. 87. 
whether the parasites were identical with any of the forms described below or not. From the size of the animal ("the size of the eggs of the common blow-fly", Lucilia ccesar) it is probable that the parasites belonged to the genus Balbiania.

R. Blanchard* does not believe that the parasites mentioned by Rivolta in chickens are Sarcosporidia, but rather that they belong to Eimeria.

Mr. Haines, artist, upon seeing my specimens, said that he had noticed this same parasitized condition of the pectoral muscles in chickens upon several occasions, while Prof. C. Hart Merriam, of this Department, tells me that he has not infrequently seen similar bodies in the muscles of various birds.

I have mentioned above all the references I have been able to trace here at Washington of parasitic Sarcosporidia in birds.

While this list may not be entirely complete, it is quite evident that this parasitism, although by no means rare, has attracted very little attention, and that we have as yet no exact description of any one species of Sarcosporidia in birds. On this account I have deemed it advisable to place on record several cases of this kind which have recently come under my observation, and to give a more or less minute description of the animals, so far as the condition of the material at hand will permit.

1. The U. S. National Museum, through Prof. Riley, has recently referred to this Bureau a portion of the pectoral muscles of the Shoveller or Spoon-billed Duck (Spatula clypeata), taken by Walter Brett at Clear Lake, California.

2. From the U. S. Army Medical Museum I have a specimen of parasitized muscle labelled "Cysticerci(?) from the Shovel-bill Duck." (Sp. clypeata).

3. In our own collection in the Bureau of Animal Industry we possess a specimen labelled "From ducks. Prof. Liiger, Minnesota, said that falciform bodies escaped from eysts like Balbiania gigantea."

4. In Leidy's collection of parasites, which the University of Pennsylvania has recently sent to me for revision, I have found a bottle containing a portion of muscle labelled "Oval, smooth bodies, no limbs. In muscles of Mallard. Anas boschas. Dr. H. Coues. Ex. Jan. 26, 1890."

5. Finally, a second bottle referred to me by the U. S. National Museum contains the legs of a Grosbeak (Habia ludoviciana). The label reads "Explorations in Manitoba, Walter Brett. Habia ludoviciana $\hat{~}$ (1166). Riding Mountain, June 16, 1892."

An examination of the muscle in all cases shows that it contains numerous elongated cysts lying parallel to the muscle-fibers and perfectly visible to the naked eye. The parasites found in specimens 1 to

* Note sur les Sarcosporidies et sur un Essai de Classification de ces Sporozoaires. Bull. d. 1. Soc. Zool. d. France, 1885. 
4 are identical, while two different forms of Sarcosporidia are found in specimen 5 .

\section{Balbiania Rileyi sp. n., 1893.}

[Plate II, Figs. 1-5.]

The parasites in specimen 1 are in a tolerably good state of preservation, while specimens 2 to 4 are not so well preserved. They measure $1 \mathrm{~mm}$. to $6 \mathrm{~mm}$. in length, $0.48 \mathrm{~mm}$. in diameter in their middle. They are rather fusiform, but the ends are somewhat blunt. Coloring liquids differentiate the body into two distinct portions-a center core, which has the general form of the entire body and is about $0.16 \mathrm{~mm}$. broad in its greatest diameter, and a peripheral portion having about the same thickness as the center core. In some specimens the peripheral zone is even broader than the central core. The meshes of the central core may either be empty or contain a granular matter which does not take stain; while the meshes of the peripheral layer are filled with numerous lancet or falciform borlies, to the staining of which is due the differentiation of the entire body into the center core and peripheral layer.

The falciform bodies measure 12 to $14 \mu$ long by $2 \mu$ broad. They are slightly more pointed at one end than at the other. They contain a distinct oblong nucleus (about $2 \mu$ ) which stains (Figs. 4,5 ) very clearly with methyl blue or acid carmine. On unstained preparations (Fig. $5, b)$ the nucleus appears as a bright refrangent body. Its position is not constant. The rest of the falciform body is gramular. Occasionally a small but indistinct vacuole is noticed.

The outer covering of the Sarcosporidium is a very thin $(2 \mu)$ cuticular membrane. In this no striation could be discovered. The entire inner portion of the animal is a meshwork or stroma, in the interstices of which lie the numerous falciform bodies. Although there is no regular arrangement or form to the meshes of this stroma, in general they radiate from the center to the periphery and are longer $(i . e$. , in a radial direction) than broad ( $i$. e., in a tangential direction).

The entire stroma can be separated from the cuticle and pressed out, but I was unable to isolate the meshes one from another. Treated with a strong solution of caustic potash the contents of the meshes were destroyed. The walls, however, remained intact. In nearly all cases the wall between any two arjacent meshes appeared to be a simple membrane, common to both lumina, but in one or two cases the walls split longitudinally for a short distance, thus giving the impression that each lumen possessed its own wall and that the adjacent walls of the two meshes were very intimately united. In places where three meshes came together at one point there was frequently a triangular space between the three membranes.

The parasites lie between the muscle-fibers, surromiled by comnective tissue. A very careful examination of a number of microtome sections failed to show any young stages inside of the muscle-fibers.

It is evident from the above that this parasite is very closely allied to 
Balbiania gigantea Railliet, and $B$. mucosa R. Bl. Placing it in the genus Balbiania, I propose the specific name B. Rileyi, dedicating the species to my colleague, P'rof. C. V. Riley, Entomologist of the United States Department of Agriculture, as lie is evidently the first scientist who examined this parasite.

Diagnosis.-B. Rileyi, sp. 11., 1893. 1-6 mm. long by $0.48 \mathrm{~mm}$. broad; rather fusiform, ends not sharply pointed. Cuticle not striated, about $2 \mu$ thick. Center core does not color and does not contain falciform bodies. Peripheral zone as broad as central core $(0.16: 0.16 \mathrm{~mm}$.) or even broader; colors in various liquids (acid carmine or methyl blue), and contains numerons falciform bodies. Form of meshes irregular, but elongated radially. Falciform bodies 12 to $14 \mu$ long, more pointed at one extremity than at the other; contain a very distinct nucleus $(2 \mu)$ which stains clearly in acid carmine or methyl blue, and which contains several chromatophil granules; vacuole quite indistinct.

Habitat.-Intermuscular connective tissue of ducks (the Shoveller or Shovel-bill Duck or Spoon-bill Duck (Spatula clypeata) aud the Mallard Duck or Tame Duck (Anas boschas).

Development unknown.

Geographical distribution.-North America. (?) Philadelphia, Pa. (Coues-Leidy); St. Lonis, Mo. (Riley); Clear Lake, Cal. (Brett); Mirnesota (Liiger); Quebec (Bélanger).

Type material deposited in the U. S. National Museum, in the Bureau of Animal Industry, and in Collection of Stiles, Washington, D. C. Specimens are also to be found in the Army Medical Museum, Washington, D. C., and in Collection of Leidy, University of Pennsylvania, Philadelphia, Pa.

In conclusion, although "measly duck" is not very appetizing in appearance, there are no grounds for believing that it is dangerous to man.

Specimen 5, mentioned above, contained, as already stated, two different forms of Sarcosporidia, which could easily be seen through the skin of the animal. The difference between the two forms, however, could be recognized only in microscopic preparations. One form was thicker, the cuticle unstriated so far as I could discover, a central non-staining core was present, and the parasites were intermuscular. The other form was much thinner, possessed a transversely striated cuticle, contained no central non-coloring core, and the parasites were intramuscular, $i$. e., inside of the muscular fiber's.

Blanchard $(l . c$.$) has proposed the following provisional classification$ of the Sarcosporidia:

I. Family Miescherida :

Situated in the striated muscles, enveloping membrane-

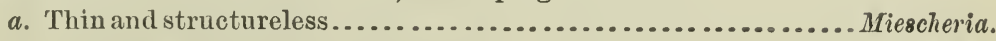

b. Thick and traversed by fine canals..................... Sarcocystis.

II. Family Balbianidce:

Situated in the connective tissue, enveloping membrane thin and

without structure

Balbiania. 
I have already stated * that I agree with those authors who consider that the membrane of Sarcocystis is finely striated, instead of possess. ing pores-there can be absolutely no doubt in regard to this point in S. tenella of sheep-so that according to Blanchard's classification, if we substitute "finely striated" in place of "traversed by fine canals," the thicker specimens mentioned above will belong to the genus Balbiania, while the thinner form must be placed in the genus Sarcocystis.

Finding, as I have, the two forms lying side by side in the same host, there are two alternatives open to us to explain their presence:

(1) Either we have here a case of infection by two Sarcosporidia of different genera; or (2) Sarcocystis is only a younger intramuscular stage of the intermuscular Balbiania.

Although the second explanation is very tempting to us, yet having but a portion of a single animal infested with the two forms now under consideration, it would be too hazardous for me to state that this is the correct explanation, and to attempt to prove it, especially as the following facts can not be considered confirmatory of this view:

(1) In the case of $B$. Rileyi, I have found no intramuscular stage.

(2) In the horse, sheep, and hogs, it is a very common occurrence to find only intramuscular Sarcosporidia.

Thus, for the present, I feel compelled to place the two forms now under consideration in two separate genera, Sarcocystis and Balbiania, respectively, although I will give to both the same specific name, so that they may more easily be united in case they prove to be two stages of the same species.

\section{Balbiania falcatula sp. n., 1893.}

[Plato III, Figs. 1-2.]

This form appears quite distinct from $B$. Rileyi, in that it is longer in proportion to its breadth; the central non-coloring core is much wider in proportion to the peripheral coloring portion; the meshes in the center core are smaller and more regular, while the meshes of the peripheral zone generally have a more regular outline; the falciform bodies are only about half as large $(6 \mu)$ as those of $B$. Rileyi, and generally more curved; they possess a very prominent nucleus, but no vacuole or portion resembling a vacuole could be discovered, except that the middle portion stained more lightly than the ends.

Specific diagnosis.-B. falcatula sp. 11., 1893. The parasites measure $1.3 \mathrm{~mm}$. to $3.2 \mathrm{~mm}$. long by $0.4 \mathrm{~mm}$. to $0.42 \mathrm{~mm}$. broad; fusiform; cuticle not striated, about $2 \mu$ thick. Center core does not coloz, and contains no falciform bodies; meshes quite regular, $8 \mu$ to $12 \mu$. Peripheral zone much narrower than centre core $(0.096 \mathrm{~mm}$. : $0.224 \mathrm{~mm}$.), colors in various liquids (acid carmine, for instance) and contains numerous

* Review of recent pubiications in Medical Zoölogy. The Journal of Compar. ative Medicine and Veterinary Archives, 1891, p. 693. 
falciform bodies measuring $5 \mu$ to $6 \mu$ long by $2 \mu$ broad; the latter curved, containing a distinct nucleus $(1.5 \mu)$ generally situated at one end; no vacuole visible (alcolol material).

Habitat.-Intermuscular connective tissue of the Rose-breasted Grosbeak (Habia ludoviciana).

Development unknown.

Geographical distribution.-North America, Manitoba (Brett).

Type material deposited in the U.S. National Museum, in the Bureau of Animal Industry and in Collection of Stiles, Washington, D. C.

3. Sarcocystis falcatula sp. n., 1893.

[Plate III, Fig. 3.]

These parasites lie within the sarcolemma; they measure on an average $2.4 \mathrm{~mm}$. long by $0.152 \mathrm{~mm}$. broad; the ends are pointed, the membrane ( $3 \mu$ thick) is finely striated; no non-coloring central core could be distinguished. At the extremities the divisions or meshes in which the falciform bodies lie are more rounded and distinct, but near the middle of the body it is almost impossible to recognize these divisions. The falciform bodies I could measure only on microtome sections. They appeared about $6 \mu$ long.

Specific diagnosis.-S. falcatula sp. n., 1893. Body $2.4 \mathrm{~mm}$. long by $0.152 \mathrm{~mm}$. thick; fusiform; cuticle finely striated; meshes at extremities rounded and distinct, in middle portion indistinct; falciform bodies $6 \mu$ long.

Habitat.-Muscle-fibers of Habia ludoviciana.

Life history unknown.

Geographical distribution.-North America, Manitoba (Brett).

Type material deposited in the U.S. National Museum, in the Bureau of Animal Industry, and in Collection of Stiles, Washington, D. C.

Species inquirendæ.

4. Sarcosporidia of chickens found by Kühn (1867), at Halle, Germany, and by Haines (cited above) in Maryland, U. S. A.

5. Sarcosporidia-probably Balbiania sp. found by Barrows in Parula pitiayumi, at Concepcion del Uruguay, province of Entre Rios, Argentine Republic, South America.

6. ? Sarcosporidia described by Rivolta (1874) from chickens, Turdus merula and crows (cited from Bütschli). 


\section{DESCRIPTION OF PLATE II.}

Fig. 1. A portion of the pectoral muscles of a duck (Measly duck).

Fig. 2. Longitudinal section of Balbiania Rileyi (greatly enlarged).

Fig. 3. Transverse section of Balbiania Rileyi (greatly enlarged); ct, eonnective tissue cyst, with numerous nuclei ; cu, cuticle of the parasite; $m$, sections of inuscle.

Fig. 4. Microtome section of meshes containing falciform bodies, greatly enlarged.

Fig. 5. Faleiform bodies of Balbiania Rileyi; a, stained, showing nucleus and vacnole; $b$, unstained.

All drawings by Mr. Haines, artist, detailed to this Bureau, drawn either from nature or after sketches by author.

86 
Bul. 3, Bureau of Animal Industry.

Plate 1 .

sig 2
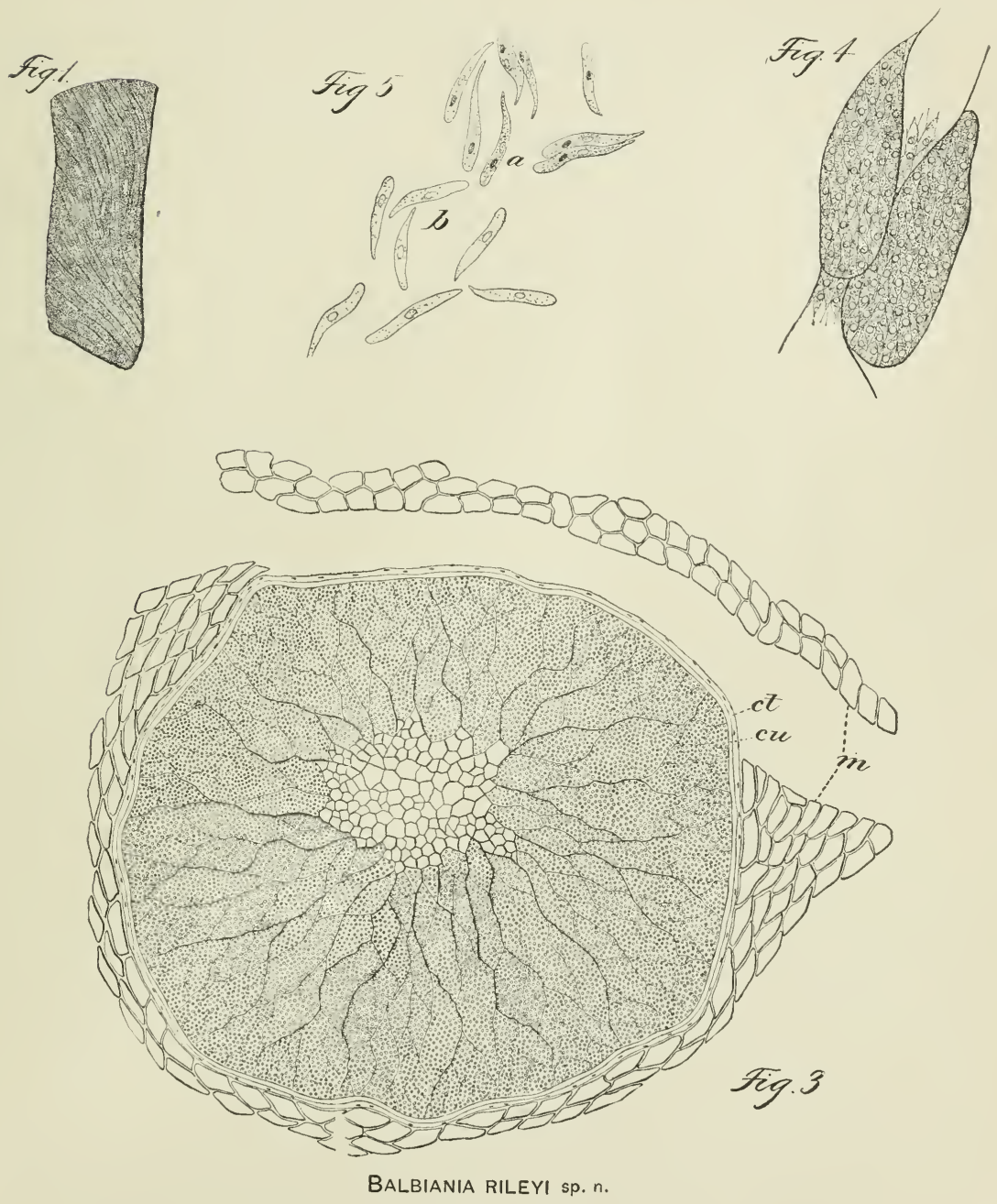



\section{DESCRIPTION OF PLATE III.}

Fig. 1. Falciform bodies of Balbiania falcatula.

Fig. 2. Balbiania falcatula, longitudinal section.

Fig. 3. Sarcocystis falcatula, longitudinal section. It will be noticed that the ends of the parasite are included in a muscle-fiber which has lost part of its transverse striation. In the specimen here drawn the muscle-fiber could not be traced the entire length of the parasite.

Fig. $3 a$. A portion of the striated cuticle, seen on section.

88 

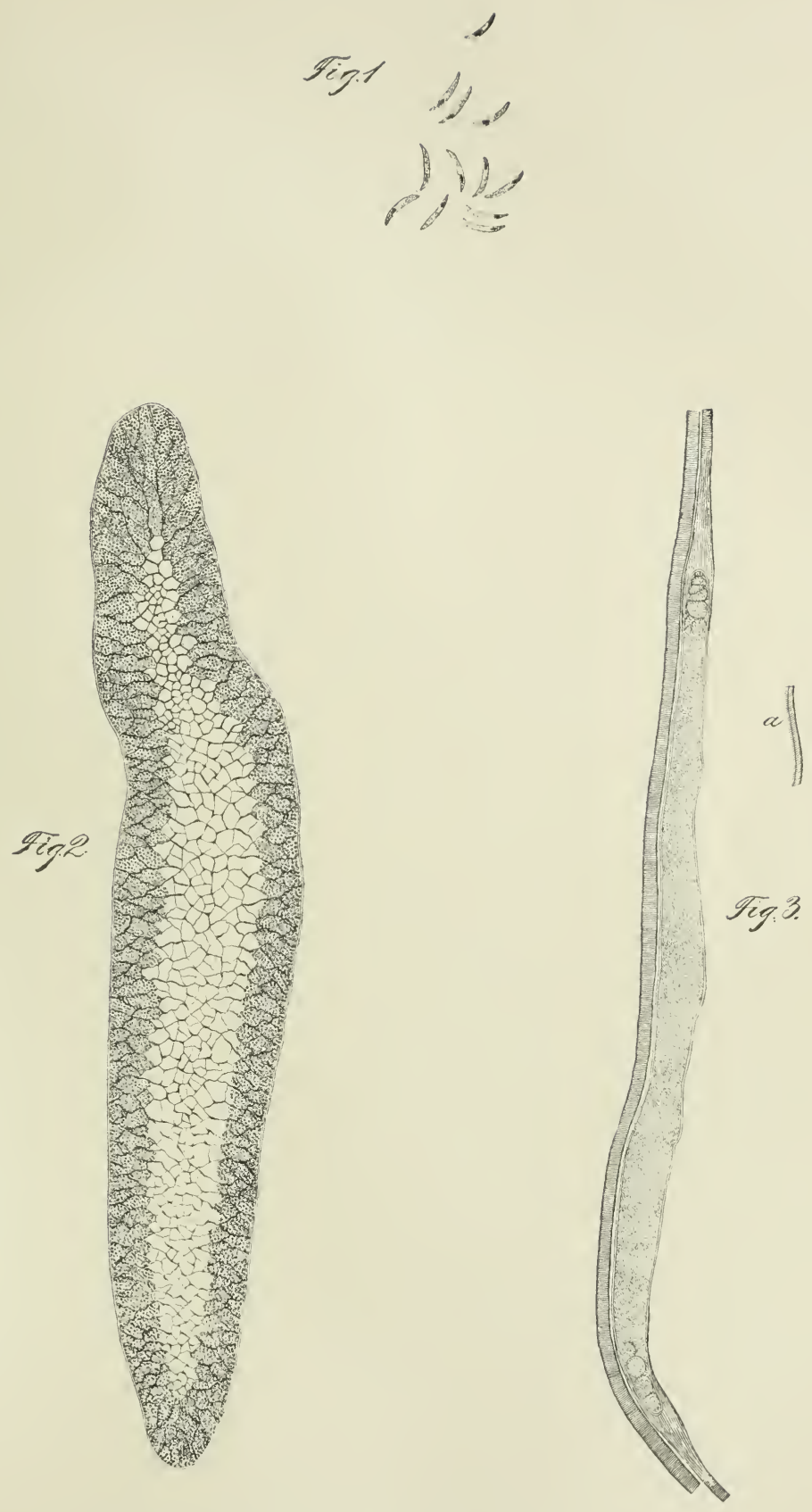

BALBIANIA falcatUla sp. n., and SARcocystis FaLCATULA sp. $n$. 



\section{U.S. DEPARTMENT OF AGRICULTURE.}

BUREAU OF ANIMAL INDUSTRY.

BULLETIN NO. 4.

\section{A REVISION OF THE ADULT CESTODES}

OF

CATTLE, SHEEP, AND ALLIED ANIIALS.

PREPARED UNDFR THE DIRECTION OF DR. D. E. SALMON, CHIEF OF THE BUREAU OF ANIMAL INDUSTRY,

BY

C. W. Stiles, Ph. D., ANd Albert Hassall, M. R. C. V. S.

PUBLISHED BY AUthority OF THE SECRETARY OF AGRICULTURE.

WASHINGTON:

GOVERNMENT PRINTING OFFICE.

1893. 



\section{CONTENTS.}

Letter of transmittal

Letter of submittal.

Part I. Moniezia R. Blanchard, 1891

A. Planissima group ( I. planissima, M. Benedeni, and M. Nenmanni).

(1) Moniezia planissima Stiles and Hassall, 1892.

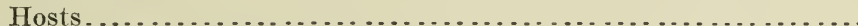

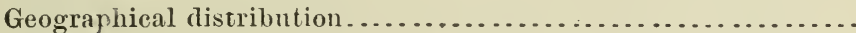

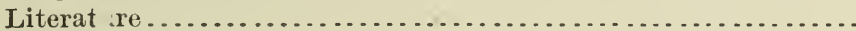

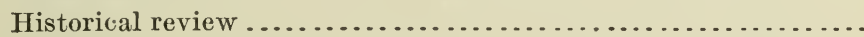

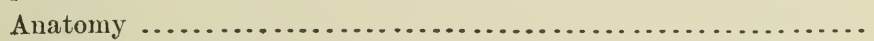

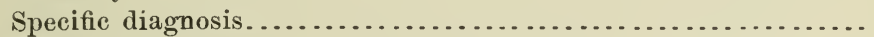

(2) Moniezia Benedeni (Moniez, 1879) R. B1., $1891 \ldots \ldots \ldots \ldots \ldots \ldots . . . . .22-25$

Synonymy....................................... 22

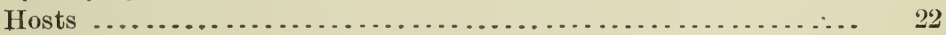

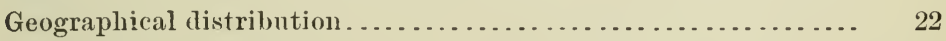

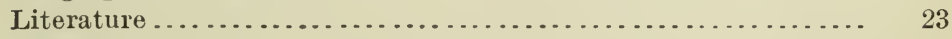

Historical review .................................. 23

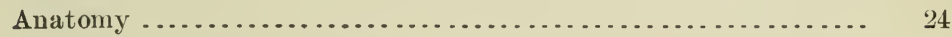

Specific diagnosis................................... 25

(3) Moniezia Neumanni Moniez, $1891 \ldots \ldots \ldots \ldots \ldots \ldots \ldots \ldots \ldots . . .25-26$

Host ............................................... 25

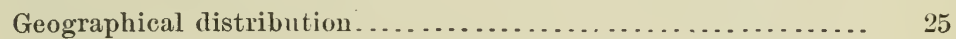

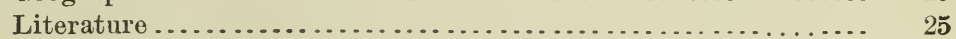

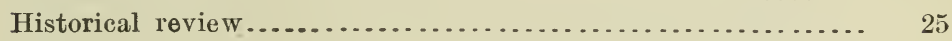

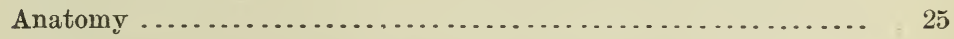

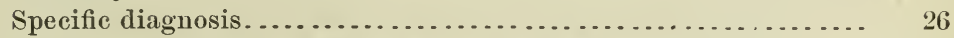

B. Expansa group (M. expansa, M. oblongiceps, and M. trigonophora) . . . 26-42 26-34

(4) Moniezia expansa (R., 1810) R. 131., 1891................... 26-34

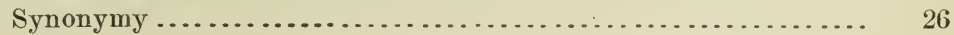

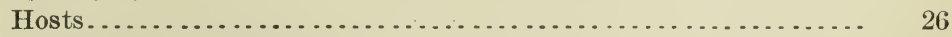

Geographical distribution.......................... 27

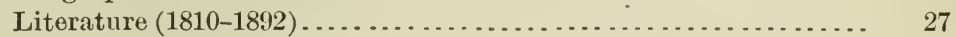

Historical review..................................... 28

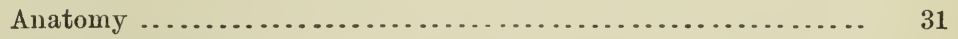

Specific diagnosis................................... 34

(5) Moniezia oblongiceps sp. n., 1893....................... 35-36

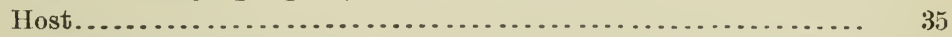

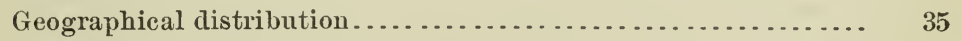

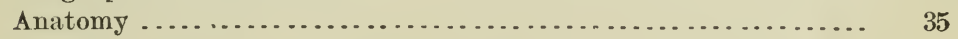

Specific diagnosis .................................... 36

(6) Moniezia trigonophora sp. n., 1893.................... 37-42

Synonymy ............................................ 37 
Part I. Moniezia R. Blanchard, 1891-Continued.

B. Expansa group-Continued.

Host.

Page.

Geographical distribution. ............................ 37

Literature ............................................ 37

Historical review ......................................... 37

Anatomy ................................................. 37

Specific diagnosis ........................................ 42

C. Denticulata group (M. denticulata and M. albu) ................ 42-51

(7) Moniezia denticulata (R., 1810) R. 131., 1891 ... . ............... 42-47

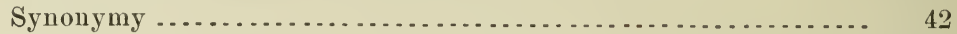

Host................................................. 42

Geographical distribution................................. 42

Literature ............................................ 42

Historical review ........................................ 43

Specific diagnosis .., .................................. 46

(8) Moniezia alba (Perroncito, 1879) R. Bl., 1891 ................. . 47-51

Synonymy ............................................. 47

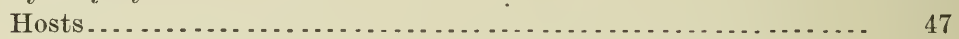

Geographical distribution............................. 47

Literature ............................................ 47

Historical review ....................................... 47

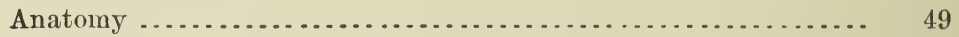

Specific diaguosis ............................................ 51

D. Moniezia. (Undetermined specinens) .......................... 51

General summary of the genus Moniezia ...................... 51-54

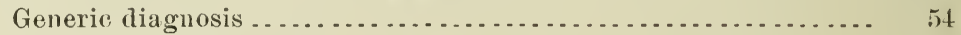

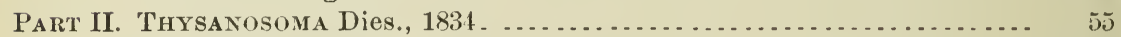

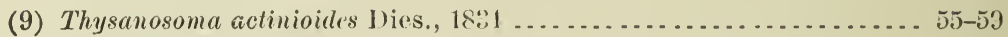

Synonymy ................................................... 55

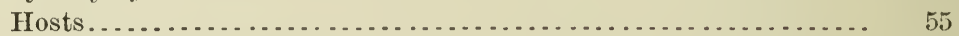

Geographical distribution.............................. 55

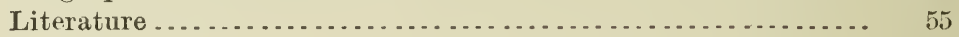

Historical review....................................... 55

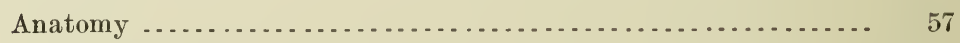

Specific diagnosis .................................... 58

(10) Thysanosoma Giardi (Riv., 1878) Stiles, 1893 ................ 59-69

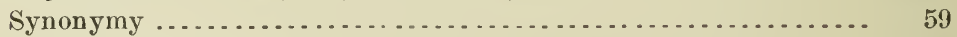

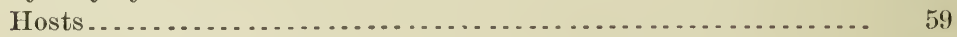

Geographical distribution ................................. 59

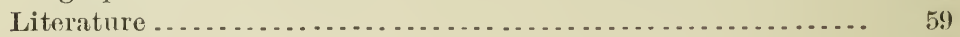

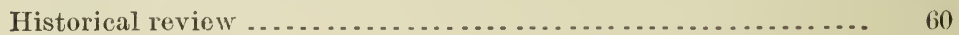

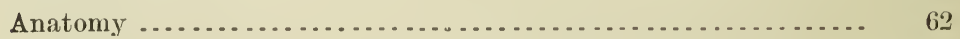

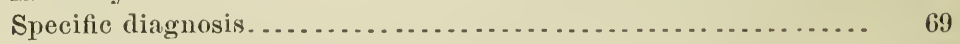

The systematic position of Twuia Giardi................... 69

General consideration in regard to Thysanosoma Giardi and Th.

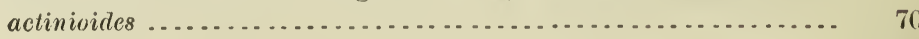

Generic diagnosis .................................... 70

(11) Tania marmote Froelich, 1802 ...................... 71-72

Par't III. Stillesia RaIlliet, 1893 MS . . . . . . . . . . . . . . . . . . . . . $73-82$

(12) Stilesia globipunctata (Riv., 1874) Railliet, 1893 MS.......... 73-79

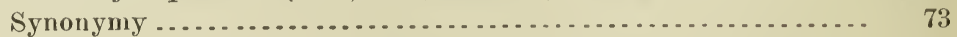

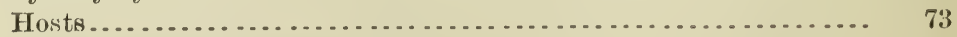

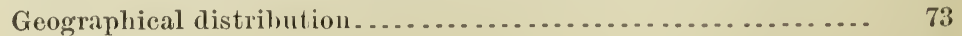

Literature ................................................ 73 
Part III. Stilesia Raillict, 1893-Continued.

Historical roviow ..................................... $7: 3$

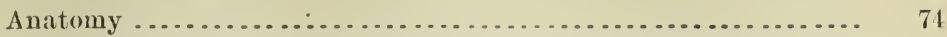

Specific diagnosis.................................. 79

(13) Stilesia centripunctata (Riv., 1874) Railliet, 1893 MS.......... 79-81

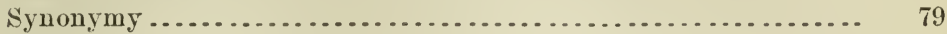

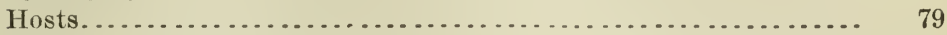

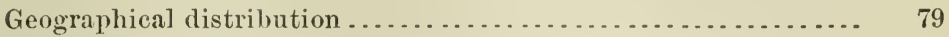

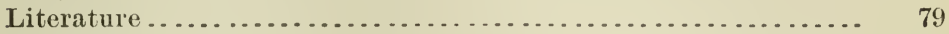

Historical review .................................... 80

Anatomy .................................................. 80

Specific diagnosis........................................ 81

Gencral remarks in regard to stilesin globipunctata and slilesia centripunctata ..................................... 82

Generic diagnosis ....................................... 82

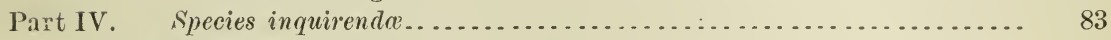

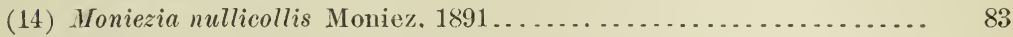

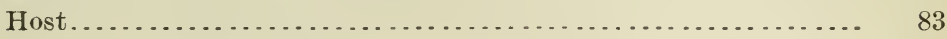

Geographical distribution ............................ 83

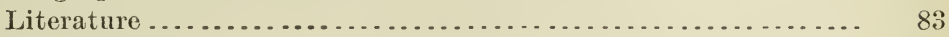

Historical review ................................... 83

Diagnosis............................................ 83

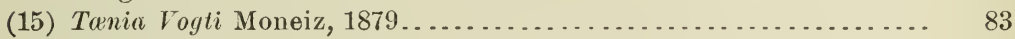

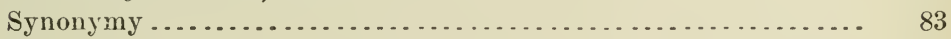

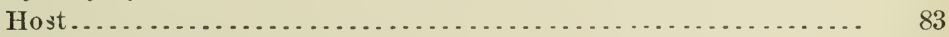

Geographical distribution........................... 83

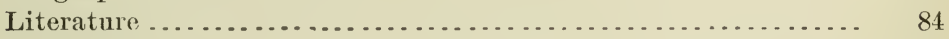

Historical revicw ....................................... 84

Conclusions.......................................... 84

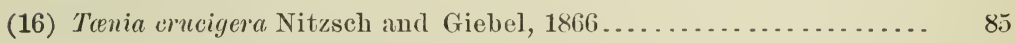

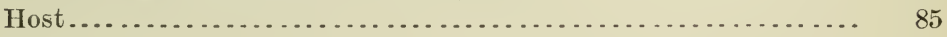

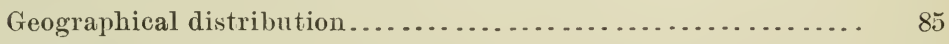

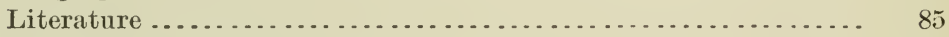

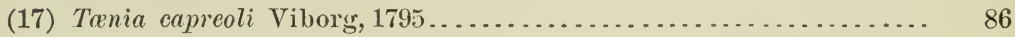

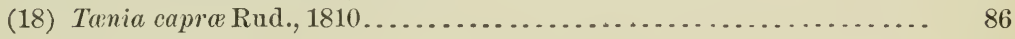

Part V. Life history .......................................... 87

Part VI. Conclusions......................................... 88

Part VII. Compendium of species arranged according to their hosts ....... 90-91

Part VIII. Bibliography of adult Cestodes of cattle and sheep............ 92-96

Addenda ......................................... $97-101$

Index to specific names....................................... 103 



\section{LIST OF ILLUSTRATIOİS.}

Page.

Plate I. Moniezia planissima .................................... 104

II. Moniezia planissima-Yoniezia Benedeni....................... 106

III. Moniezia planissima.................................. 108

IV. Moniezia Neumanni.................................... 110

V. Moniezia expansa-Moniezia denticnlula (Rudolphi's original types) .... 112

VI. Moniezia expansa....................................... 114

VII. Moniezia oblongiceps-Thysanosoma Giardi-Tarnia marmota......... 116

VIII. Moniezia trigonophora............................... 118

IX. Moniezia trigonophora................................. 120

X. Moniezia alba......................................... 122

XI. Thysanosoma actinioides................................. 124

XII. Thysanosoma Giardi..................................... ${ }_{126}$

XIII. Thysanosoma Giardi................................... 128

XIV. Stilesia globipunctata..................................... 130

XV. Stilesia centripunctata................................... 132

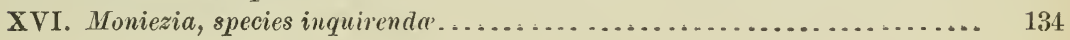





\title{
LETTER OF TRANSIITTAL.
}

\author{
U. S. Department of Agriculture, \\ Burlay of Animal Industry, \\ Washington, D. C., September 15, 1893.
}

SIn: I have the honor to transmit herewith a report covering "A Revision of the Adult Cestodes of Cattle, Sheep, and Allied Animals." It has long been known that herbivorous animals were subject to infection by tapeworms, but it was not known exactly how many different species were to be found, nor has it heretofore been definitely known what particular species were present in America. Since it may be assumed that every separate species of tapeworm found in animals has a separate source of infection, it has been deemed important to obtain definite data for the determination of the various species already described and to give descriptions of the new forms found in this country. With this end in view, the original type specimens of European species were re-studied according to the more modern scientific methods before the American forms were investigated. The manuscript herewith transmitted cover's the results of a more thorough and extensive study of the tapeworms of cattle and sheep than has ever before been attempted, and places the forms mentioned on a scientific foundation.

Very respectfully,

D. E. SALMON, Chief of Bureau.

Hon. J. Sterling Morton, Secretary of Agriculture. 



\section{LETTER OF SUBMITTAL.}

\section{U. S. DFPARTMENT OF AgRiculture, \\ Bureau of Animal Intustry, \\ TI ashing̀ton, 1). C., April 18, 1893.}

SIR: In accordance with instructions received from you to prepare a report on the animal parasites of cattle, I began the work in September, 1891. In the spring of 1892 I took up the tapeworms of cattle, and found that the various diagnoses of the species given by different authors were so contradictory that it was almost impossible to determine a single species with certainty. I was ronvincer that it was useless to write anything on the subject for my report until the entire group of tapeworms found in cattle and allied animals was subjected to a thorough anatomical sturly in order to obtain refinite characters for the differentiation of the various species.

During the conrse of the anatomical sturly then begun I obtained a number of interesting results which are too technical to publish in the biennial report of this Bureau, and yet too valuable to zoiilogists aud veterinarians and too important theoretically to leave unpublished. These results, therefore, have been included in this manuscript, which I offer for publication as a special bulletiu.

In this study I have confinerl myself almost entirely to the microscopic anatomy of those organs which would be of systematic value. No pretensions are made in this publication to treating the worms histologically, although here and there I have mentioned a few histological obervations.

As it was impossible to determine the various species with certainty from their present diagnoses, I obtainer the original type suecimens of a number of the parasites in question, and have made my specific determinations by comparing the worms in the Bureau collection directly with the type specimens. I have further received from various European specialists a number of tapeworms which I have compared with our American forms, and it is a pleasant duty to acknowledge the kindness of these foreign specialists in sending me the specimens referred to. To Geheimrath Prof. Dr. Karl Möbius, Director of the Berlin Museum, I am indebted for segments of the original Tonia expansa and Tania denticulata described by Rudolphi in 1810; to Prof. L. G. Neumann, of the National Veterinary School at Toulouse, France, I am indebted for specimens of Moniezia planissima, M. alba, M. trigonophora, 
T. Giardi, T. centripunctata, and T.globipunctata; to Prof. Moniez, of the Medical Faculty of Lille, France, I an indebted for the original material of the parasites described by him under the names: T. Giardi, I'. Bencdeni, T. Neumanni, and T. nullicollis, as well as for specimens of other French tapeworms; to Prof. Railliet, of the Veterinary School of Alfort, France, I am indebted for specimens of $M$. planissima; to Prof. R. Blanchard, of Paris, France, I am indebted for specimens of Tania marmota, which I wished to compare with the above-mentioned forms found in eattle and sheep; to Jr. von Marenzeller, of Vienna, I am indebted for a specimen of M. Benedeni; to Prof. Perroncito, of Turin, Italy, I am indebted for three strobilæ of his original material from which he described Trnia alba; to Dr. G. M. Giles, of Sanawar, Punjab, India, I am indebted for specimens of T'ania glolipunctata.

Besides the above-mentioned specimens I have had at my disposal the entire Burean collection of tapeworms of cattle and sheep, over 800 in number; collected by Dr. Curtice, Dr. Hassall, and myself ; also a number of specimens frcm Dr. Hassall's private collection, made in England, and from my own collection of parasites, made in France, Germany, Austria, and America.

I wish to acknowledge, also, the services of my assistant, Dr. Hassall, who has made about 2,000 microscopic slides for my use.

For that portion of this bulletin which deals with new species and for the bibliography at the end of this paper, both Dr. Hassall and myself are to be considered respousible, while I alone am responsible for the statements made in regard to the species already known.

According to the rules passed by the International Zoölogical Congress, Paris, 1889, the law of priority must be strictly adhered to in determining the generic and specific names of animals. Most zoölogists have adopted this rule, although it occasionally causes inconvenience for the time being. In a former paper I objected to applying the rule in all cases, stating, as a particular case, that we should speak of Pentastomum proboscideum rather than revert to the specific name crotali. At present, however, I wish to withdraw from that position, for I am thoroughly convinced that we can never obtain a fixed nomenclature until the "law of priority" and the rule "Once a synonym always a synonym" are strictly enforced according to the rules laid down by the International Congress. I am hence in favor of speaking of Linguatula rather than Pentastomum, and if the genus Linguatula is divided we are obliged to accept the genera, Linguatula and Porocephalus-Porocephalus crotali, instead of Pentastonum proboscideum.*

*We should in that case speak of Porocephulus constrictus, Por, annulosus, Por. polyzonus, Por. subuliferus, Por. moniliformis, I'or. megastomus, P'or. oxycephalus, etc. It is not at all improbable that Porocephalus armillntus (Lingualula armillala Wyman, 1845) will prove to be identical with Porocephalus polyzomus, in which case Wyman's specific vame must be accepted. I hope later to compare the type specimens of some of these forms in order to straighten out the synonymy, which seems at present to be very uncertain. 
In the present paper I have held strictly to these rules, and used the terms actinioides and Giordi as specific names, instead of fimbriata and ovilla in speaking of two of the worms found in sheep.

I am well aware of the fact that in following out these rules I am doing that which, although indorsed by most zoölogists, is disapproved of by many helminthologists, who contend that we should accept the specifie name which we find in general use. These anthors, however, lose sight of the fact that many animals have one name in general use in one country, while another name is generally used in another country. Thus Pentastomum is in general use in Germany, while Linguatula is in more general use in France, England, and America. Authors are generally best acquainted with the text-books of their own country, and will naturally assume that the name "in general use" in their own country is the name to be followed. Thus Germans would hold to Pentastomum, while French, English, and American authors would hold to Linguatula.

The only way to establish an international nomenclature in medical zoölogy, as well as in other branches of biology, is to enforce the law of priority.

In the adult tapeworms of cattle and allied animals I recognize several distinct groups, and in the description given below the parasites are classed together in four groups:

(1) Blanchard's genus Moniezia, in which the species naturally fall into three subdivisions:

(a) The Planissima group, characterized by the linear arrangement of the interproglottidal glands (M. planissima, M. Benedeni, Mr. Neumanni).

(b) The Expansa group, characterized by the saceular arrangement of the interproglottidal glands ( $M$. expansa, $M$. oblongiceps, and $M$. trigonophora).

(c) The Denticulata group, which contains forms in which the interproglottidal glands are absent ( $M$. denticulata and $M$. alba).

(2) The genus Thysanosoma Diesing. This genus is characterized by the presence of a single uterus with ascon-spore shaped egg-sics. Further, while in the genus Moniezia the genital canals pass dorsally of the longitudinal canals, in the genus Thysanosoma they pass between the longitudinal canals.

(3) Railliet's new genus Stilesia, based upon Tunia globipunctata. Provisionally the species $T$. centripunctata may be placed in this genus, but I believe that further sturly may result in the formation of a separate genus for that worm.

(4) Owing to our lack of knowledge of the anatomical details of M. nullicollis, $T$. Vogti, T. crucigera, T. capreoli, and $T$. capra, it is impossible as yet to give these species any definite place in the classification.

Besides the above-mentioned species, the Burean possesses three forms of Moniezia, taken from sheep, at least two of which are probably new species.

Unless otherwise expressed the measurements given are in millimeters ( $\left.{ }^{\mathrm{mm}}\right)$.

The cestodes collected by this Bureau for the past eighteen months have been fixed in the following solution: Fifty parts of an aqueous solution of corrosive sublimate +50 parts of alcohol, 70 per cent $+a$ few drops of glacial acetic acid. The worms were placed in this liquid 
which had been heated to $45-53^{\circ} \mathrm{C}$. The liquid was then allowed to cool for 20 to 60 minutes. The parasites were next washed in ruming water from 1 to 24 hours, and passed through 30 per cent, 50 per cent, 70 per cent, 95 per cent, and absolute alcohol.

The preparations have been colored in alcoholic hydrochloric acidcarmine after Grenacher or hæmatoxylin, and finally mounted in Canada balsam. Most of the specimens collected by the Bureau prior to July, 1891, were simply preserved in alcohol, and, with few exceptions, have been of very little use in preparing this bulletin. It is also evident that the specimens we have received from European zoölogists have been killed in alcohol.

Anterior in this paper signifies cephalad.

Posterior signifies caudad.

Proximal refers to the direction toward the head or median line.

Distal refers to the direction from the head or median line.

Ventral refers to the flat surface of the worms, containing the large longitudinal canals and female organs.

Dorsal refers to the opposite surface.

Lateral refers to the direction toward the margins upon which the genital pores are found.

Respectfully submitted.

Charles W. Stilles, Zoölogist, Bureau of Animal Industry.

Dr. D. E. SAlmon, Chief of the Bureau of Animal Industry. 


\section{A REVISION OF THE ADULT CESTODES OF CATTLE, SHEEP, ANI) ALLIED ANIMALS.}

By C. W. Stiles, Ph. D., and Albert Hassall, M. R. C. V. S.

\section{PART I.}

\section{MONIEZIA R. Blanchard, I89I.}

A. Planissima Group. (M.planissima, M.Benedeni, and M.Neumanni.)

Interproglottidal glands linear and not grouped around sacs.

(1) Moniezia planissima Stiles and Hassall, 1892.

[Plates I, II, Figs 1-6; Plate III.]

Synonymy.-Tania expansa, ex parte of various authors.

Hosts. - Sheep (Ovis aries); cattle (Bos taurus): small intestine.

Geographical distribution.-United States of America: Chicago, Ill., and Washington, D. C. (Hassall, Stiles, and Curtice); Fairfax County, Virginia (Norgaard).

- France: Paris (Railliet, of Alfort, Stiles); Toulouse (Neumann).

\section{LITERATURE。}

(Probably all references to Tania expansa 20-25 $\mathrm{mm}$ broad.)

(1) C. Wr. Stiles. Notes sur les Parasites-14: Sur le Tania expansa Rudolphi; Compt. rend. de la Soc. d. Biol. Paris, 1892. Pp. 665-666.

(2) Bemerkungen iiber Parasiten-17: Ueber die topographische Anatomie des Gefasssystems in der Familie Torniade; Centralblatt für Bakteriologie und Parasitenkunde. 1893. Bd. xun, p. 457-165, Fig. 3.

\section{HIS'IORICAL REVIEW.}

Upon studying the tapeworms of cattle and sheep we becane convinced that Moniezia expansa contained more than one species, and diagnosing the proper $M$. expansa by a few segments and the scolex of Rudolphi's original material, we separated from it Moniezia planissima. Stiles found this species at Paris, and Hassall and Stiles found it at Washington, D. C. We have also found several segments of this worm in the material collected by Dr. Curtice, under the label "Tania expansa," and Dr. Norgaard has recently found it in Virginia. Two of the best known medical zoölogists of France (Railliet and Neumanu) have also sent us specimens of this species under the label "Tania expansa."

The only publications on this worm, as a distinct species, are the short preliminary notes by Stiles $(1,2)$. The present account of the anatomy is the paper anticipated by those preliminary papers. 


\section{ANATOMY.}

General appearance.-When fresh the strobila is yellowish, but when preserved it becomes whitish. The worm is remarkably flat and broad much more so than M. expansa. In the best specimens at our disposal the segments are slightly contracted. In a few specimens in the Bureau collection they are not contracted, the segments being slightlylonger and narrower. In the following description, however, we have used the worms which are slightly contracted. Nevertheless, we have given a figure of some uncontracted segments, so that the difference may be readily seen and no confusion arise.

Fig. 1, Plate I, represents a strobila, natural size, slightly contracted. The segments increase in breadth very rapidly. It will be noticed that the segments are very regular, although werged segments, such as have been described in other worms, are occasionally found. The posterior flap of the segment, overlapping the next following segment, is very distinct. The cuticle is very smooth.

Scolex.-The heads of the various preserved specimens in our possession vary greatly in shape and size. They have the appearance presented in Figs. 2, $2 a$ and 2b, Plate r. When viewed from the apex the head is nearly square. The suckers are at the four corners and turned slightly towards the front. In press preparations, viewed from the ventral or dorsal surface, we notice a plane running from the anterior portion backwards and upwards, $i$. e., the posterior elge of this plane is the point of the highest focus, and situated $0.56 \mathrm{~mm}$ from the anterior extremity. A head examined in alcohol measured $0.8^{\mathrm{mm}}$ broad. The suckers were strong, $0.296 \mathrm{~mm}$ in diameter, the muscular wall $0.064^{\mathrm{mm}}$ thick; cavity of the sucker round; the opening somewhat oblong and directed diagonally toward the front. The constriction $\left(0.7^{\mathrm{mm}}\right.$ from the anterior extremity) is $0.664^{\mathrm{mm}}$ broad. Then follows an unsegmented portion (neck) $0.8^{\mathrm{mm}}$ long, at the end of which the first signs of strobilization are visible. In some specimens the neck aplears short and thick, in others very long and thin (contraction). In the neck two longitudinal canals are visible on each side.

In the examination of preserver material too much confidence must not be laid upon the size of the head, as it may contract or shrink in preparation. Press preparations also give more or less artificial pictures. Thus the following measurements were.found in preserved material and balsam preparations:

\begin{tabular}{|c|c|c|c|c|c|c|}
\hline $\begin{array}{c}\text { Dianeter of } \\
\text { head. }\end{array}$ & $\begin{array}{l}\text { Posterior } \\
\text { edge of plane } \\
\text { from ante- } \\
\text { rior point. }\end{array}$ & $\begin{array}{l}\text { Diamoter of } \\
\text { suckers. }\end{array}$ & $\begin{array}{l}\text { Muscular } \\
\text { wall of } \\
\text { suckers. }\end{array}$ & $\begin{array}{l}\text { Distance of } \\
\text { constriction } \\
\text { from ante- } \\
\text { rior ex- } \\
\text { tremity. }\end{array}$ & $\begin{array}{l}\text { Diameter of } \\
\text { comstric- } \\
\text { tion back of } \\
\text { tho head. }\end{array}$ & $\begin{array}{l}\text { Strobiliza- } \\
\text { tion begins } \\
\text { back of } \\
\text { constriction. }\end{array}$ \\
\hline $\begin{array}{l}m m . \\
0.40 \\
0.548 \\
0.448 \\
0.67\end{array}$ & $\begin{array}{l}m m . \\
0.528 \\
0.326 \\
\ldots \ldots \\
\ldots \ldots\end{array}$ & $\begin{array}{l}m m . \\
0.20 \\
0.288 \\
0.192 \\
0.256\end{array}$ & $\begin{array}{l}m m . \\
0 .(172 \\
\ldots \ldots .072 \\
0.06 .1\end{array}$ & $\begin{array}{l}m m . \\
0.8 \\
0.480 \\
0.4 \\
0.56\end{array}$ & $\begin{array}{l}m m . \\
0.312 \\
0.352 \\
0.216 \\
0.496\end{array}$ & $\begin{array}{l}\operatorname{mm} . \\
0.8 \\
0.64 \\
0.8 \\
1.48\end{array}$ \\
\hline
\end{tabular}


Most of these variations are due to contraction. The head of fresh specimens generally measures $0.7-0.9^{\mathrm{mm}}$. The segments a:e at first extremely short and indistinct, but th:y rapidly become much broader, longer, and sharper in their outline. Seven to $12^{\mathrm{mm}}$ from the hear (segment $c a .150$ ) the segments measure $0.062-0.112^{\mathrm{mm}}$ long by $1.5-2^{\mathrm{mm}}$ broad; they are very thin. At this point (Plate I, Fig. 3) we find the first trace of genital organs in the form of round clumps of tissue, $48 \mu$ in diameter, in the lateral portion of the median field (just insile the longitudinal canals); two anlagen* are present in each segment.

Segments $70^{\mathrm{mm}}$ from the head measure $4^{\mathrm{mm}}$ broad by $0.3-0.55^{\mathrm{mm}}$ long: (Plate I, Fig. 4). The genital pores have not yet pierced the edge of the segment. The anlagen of the genital canals are still solid and extend from the lateral margin of the anterior half, a distance of about $0.3^{\text {nim }}$ towards the median field, crossing the longitudinal canals and ending in a double knob. The anterior portion of the kuob belongs to the male genitalia, the posterior to the female genitalia. No testicles are visible.

The distal border of every segment is very slightly wavy and overlaps the proximal border of the next following segment about $0.032^{\mathrm{mm}}$. The juncture of the segments is differentiated in the median field to the extent of showing a linear group of cells each side of the median line; this urgan will be described more fully below.

The longitudinal canals, which were of nearly the same diameter in the head, have changed in position and size. The ventral canal has a lumen of about $0.1^{\mathrm{mm}}$, and is nearer the margin of the segment; the dorsal canal is but $0.05^{\mathrm{mm}}$ broad, and lies on the merlian side of the ventral canal, at the same time slightly dorsal of the latter.

The testicles first appear in segments about $100^{\mathrm{mm}}$ from the head. At first they are arranged in two triangles pointing towards the median line, as in $M$. trigonophora (see p. 38), but they very soon lose this arrangement (see below).

Segments $14^{\mathrm{cm}}$ from the head measure $8^{\mathrm{mm}}$ broad and $0.4^{\mathrm{mm}}$ long. The genital pore is distinct and the male and female canals are very plain. The genital pore is in the anterior half of the edge of the segment. The vas deferens is very slightly convoluted; the testicles have increased in number and they are nearly as numerous in the median as in the submedian fields. None were found in the lateral fields.

Of the female organs one cau distinguish vagina, receptaculum sem. inis, shell-gland, vitellogene gland, ovary, and several small canals which will be considered below. The uteri are not visible. In the

\footnotetext{
*The word "anlage"-plural "anlagen"-is now being adopted by a number of American zoölogists to signify the incipient rudiment of an organ. This is the meaning given to the word in this paper. Prof. E. L. Mark has recently introduced the term "fundament" as equilvalent to the Germañ "Anlage."
}

$7114-\mathrm{No}, 4-2$ 
median field each segment overlaps the one next following by $0.176^{\mathrm{mm}}$; and at the juncture of every two segments there is a differentiation in the median field (about $0.68^{\mathrm{mm}}$ each side of the median line) which colors very dark in acid carmine. Sacs such as we find in $M$. expansa (see p. 32) are, however, nowhere developed. The arrangement of the various organs ean be especially well seen in segments $180-400^{\mathrm{mm}}$ from the head, measuring $9-13.5^{\mathrm{mm}}$ in breadth by $0.67-0.96^{\mathrm{mm}}$ long by $0.17-$ $0.26^{\mathrm{mm}}$ thick.

The segments are quadrate in form (Plate II, Fig. 4). The posterior flap of each segment overlaps the anterior portion of the next following segment by $0.192^{\mathrm{mm}}$ (Plate I, Fig. 5). The first thing which strikes the eye on the stained specimen is the deeply colored line at the juncture of the segments, rumng parallel to the posterior edge and measuring about $1.9^{\mathrm{mm}}$ long by 48 \% wide. Upon examining this in sections with a high power it is found to be distinctly cellular in structure. On frontal sections (Plate II, Fig. 2) the cells are arranger irregularly in groups. This grouping is more or less artificial, and caused by the longitudinal muscles. The cells are drawn out at one end, which inclines toward the nearest muscle-fiber. The round nuclei $(4 \mu)$ are very distinct and the nucleolus $(2 \mu)$ is very sharply defined. Generally only one dark spot is found in the nucleus, but occasionally two are found. In Plate III, Fig. 2, it will be seen that these cells surround the border between two succeeding segments, and that they are directed towards the cuticle. They have the same appearance as the cells surrounding the interproglottidal sacs in $M$. expansa, and probably represent a low stage in the phylogenetic development of those organs. The grouping which, as stated, is in this case irregular and apparently dependent on the position of the longitudinal muscle fibers, reaches its highest development around the sacs in the species of the Expansa group.

The genital pores are in the lateral margin, invariably in the anterior half of the segment (Plate II, Fig. 4, and Plate III, Fig. 3). The male and female openings present the following constant topographical relations: On the right hand side of the segment the vagina is ventral, while the cirrus lies dorsal in the same transverse plane; on the left side the position is reversed so that the vagina is dorsal and the cirrus is ventral in the same transverse plane (Plate II, Fig. 3, 4). This arrangement was found constant in every segment examined.

The vagina leads toward the female glands, which lie just median of the longitudinal canals, while the vas deferens extends from the cirrus across (dorsally) the female glands to the testicles, which are scattered throughout the median field (Plate II, Fig 4).

IFale genitalia.-The cirrus-ponch extends straight towards the median line of the body. It is $0.2 \mathrm{~S}^{\mathrm{mm}} \mathrm{long}, 0.096^{\mathrm{mm}}$ in diameter (Plate II, Figs. 3, 4, and Plate III, Fig 4). 'The inverted cirrus runs throngh its center, forming but comparatively few convolutions. The layers of the cirrus-pouch, beginning at the center, are as follows: (1) 
lumen; (2) viliary projections extending into the lumen. Zschokke states that this is of cellular nature in Tania expansa, but we were unable to convince ourselves of the correctness of this statement as applied to M. planissima. We saw in sections a number of nuclei which were apparently in this layer (Plate III, Fig. 4) but, which upon further study proved to be in the next following layer; (3) a more or less homogenous layer containing numerous nuclei, hence of cellular origin. In (Plate III, Fig 4) some of these nuclei appear as if they were in layer 2 , but the circular muscles prove that the section was not exactly straight; 2 and 3 are probably to be looked upon as a single layer, or more strictly speaking, as arising from a single layer of cells; (4) circular muscles; (5) lougitudinal muscles, $i$. e., longitudinal in reference to the shape of the cirrus-pouch, not in reference to the worm; (6) cellular parenchyma with large nuclei; (7) circular muscles with nuclei which stain very dark. This layer is much thicker in the proximal than in the distal portion of the pouch; (8) longitudinal muscles.

For the study of layers 7 and 8 this species is not very good. In cross sections of the pouch the circular inuscles (Plate III, Fig. 6) are very evident, but it is only with the greatest difficulty that the longitudinal fibers are seen, while in longitudinal sections the reverse is the case, except that the circular fibers of the proximal portion come plainly into view.

Upon emerging from the median end of the cirrus-pouch the male canal immediately increases in diameter, forms a number of convolutions, and crosses into the median field on the dorsal side of the longitudinal canals; most of the convolutions lie anterior to the vagina. Arriving at the ovary it decreases in diameter and crosses the latter dorsally.

Testicles.-At this stage there are 400 to 600 testicles scattered through the entire median field, forming a more or less regular quadrangle. There is generally no portion in the median line where the testicles are absent, such as is seen in $M$. trigonophora. On cross section they are seen to lie in the center portion of the segment, $i$. e., between the transverse muscles.

Female genitalia.-In a depression directly dorsal (left side, ventral right side) of the cirrus lies the vulva (Plate II, Fig. 3), from which the vagina runs almost straight toward the median field of the segment. The layers of the vagina (Plate III, Figs. 4, 5) correspond very nearly to those of the cirrus, $i$. e. (1) lumen; (2) ciliary projections; (3) homogenous layer with nuclei; (4) longitudinal fibers (almost imperceptible); (5) circular fibers, which are extremely thin except at the beginning of the vagina, where they form a strong sphincter capable of completely closing the entrance; and (6) a distinct cellular layer similar to that recognized by Zschokke in $T$. expansa.

It will be noticed that in the cirrus the circular muscles (4) are surrounded by the longitudinal fibers (5), while in the vagina the reverse is 
the case. This has already been noticed by other authors, Zschokke for instance, in other species (T. expansa).

The vagina runs toward the median field and passes the longitudinal canals on their dorsal side, retaining a nearly uniform diameter for about $0.72^{\mathrm{mm}}$. Then it suddenly enlarges (Plate IIr, Fig. 3) into a short bulb, which is followed by a constricted portion $24 \mu$ long. The latter then empties into the receptaculum seminis, which measures $0.44^{\mathrm{mm}}$ long by $0.096 \mathrm{~mm}$ in diameter and lies on the dorsal side of the ovary. The histology of the receptaculum seminis is entirely different from that of the vagina, there being an inner epithelium with an outer basement membrane. From this point the topography of the cauals can be made out only by a patient study of numerous sections. Our studies on sections in the three directions have led us to the following results (Plate II, Fig. 1 and Plate III, Figs. 1-3):

From the ventral side of the receptaculum seminis, a short distance from its median extremity, a canal takes its origin; this canal runs about $0.132^{\mathrm{mm}}$ in a curved direction, the convexity being ventrad and slightly distal; it is made up of a high interior epithelium (nuclei $3 \mu$ ) with a basement membrane. This canal (oviduct) in which, as we shall see later, the ova come in contact with the spermatozoa (hence "fertilization canal"), leads from the ovary, from which it is separated by a short constriction provided with circular fibers. Thirty two $\mu$ from theorigin of the above-described canal another canal branches off from it dorsally, makes one or more convolutions, and enters the shell gland; in about the center of the latter it divides into two canals, one of which passes straight through the gland in a ventro-distal direction and dilates suddenly into the calyx of the vitellogene gland. This latter canal is the vitello-duct. The other canal runs from the center of the gland dorso-anteriorly, makes several convolutions both in the shell-gland and after it leaves it, then runs to the uterus. The relations of these canals are given in Plate II, Fig. 1, and Plate III, Fig. 1, which are slightly diagrammatic.

Ovary.-The ovary is nearly renal in form, varying slightly according to the contraction of the segment (Plate III, Fig.3). When the segments are expanded, the ovary, shell-gland, and vitellogene gland together form a circular body, but when the segment is more or less contracted, this circular body becomes leugthened in its lateral diameter and shortened in its longitudinal diameter. The ovary in Plate 1II, Fig. 3, measures $1.12^{\mathrm{mm}}$ in its lateral diameter. Coloring liquids differentiate the organ into two distinct parts; a peripheral portion (cortex) which stains very dark and represents the ovarial tubes, and a central portion (calyx) which stains more lightly. All the tubes lead into this central portion from which the oviduet rmos.

The vitellogene gland (Plate 11 , Fig. 1, and Plate II, Figs. 1-3) lies on the posterior (distal) side of the ovary, and is nearly inclosed by the projecting sides of the latter. It measures $0.4^{\mathrm{mm}}$ by $0.24^{\mathrm{mm}}$. In preparations 
this organ appears much darker than the ovary, owing to the fact that the nuclei are of a darker eolor; slightly smaller, and more crowded. The gland is composed of tubes which lead into a common calyx from which the vitello duct takes its origin. While the ovary consists of long, thin tubes, the vitellogene gland is more acinus-like in its character.

shell-gland (Plate II, Fig. 1, and Plate III, Figs. 1-3).-Between the ovary and vitellogene gland, and on the dorsal side of the latter, is sitnated an irregularly shaped organ which is known as the shell-gland. The point where the vitello-duct and oviduct come together (oötyp) forms approximately the center of the gland. The nuclei of the gland $(5 \mu)$ are very similar to those of the ovary, and take on about the same stain.

Uterus.-No connection can be traced between the uteri-anlagen of the two sides of the segment, hence we have originally (ontogenetically) two uteri, corresponding to the two sets of organs.

Excretory cancls. - The ventral longitudinal canal lies laterally of the dorsal canal and slightly ventrally. Its lumen is flattened dorsoventrally $\left(0.2^{\mathrm{mm}}\right.$ by $\left.0.016^{\mathrm{mm}}\right)$ and is extremely irregular; its cuticular lining is very thin. In the posterior portion of each segment it is connected with the corresponding canal of the other side by the transverse canal. The dorsal canal is smaller $\left(0.064^{\mathrm{mm}}\right.$ by $\left.0.008^{\mathrm{mm}}\right)$; the cuticular lining is thicker than that of the ventral canal. Cells are grouped more or less regularly around the dorsal canal, but we can liardly look upon them as forming a distinct epithelium such as Zschokke described in his article on $T$. expansa.

The longitudinal nerves can be distinguished on the lateral side of the lacunes. They cross the genital canals ventrally. From this point to the end of the strobila the segments continue to increase considerably in breadth but very slightly in length. Segments $60^{\mathrm{cm}}$ from the head measured $14^{\mathrm{mm}}$ by $1^{\mathrm{mm}} ; 80^{\mathrm{cm}}$ from the head, $16^{\mathrm{mm}}$ by $1^{\mathrm{mm}} ; 1^{\mathrm{m}}$ from the hearl, $18^{\mathrm{mm}}$ by $1.5^{\mathrm{mm}}$, and $1.25^{\mathrm{mm}}$ from the head, $20^{\mathrm{mm}}$ by $1.75^{\mathrm{mm}}$. In one worm segments $26^{\mathrm{mm}}$ broad were found. The last few segments show a disposition to become slightly longer and narrower.

As the uteri increase in size they make numerous folds which com pletely fill up the median field and extend into the lateral fields, crossing the longitudinal canals dorsally. In the median line the two uteri come together in such a way that it is impossible to distinguish one from the other (Plate II, Fig. 5).

In the meantime the other genital organs atrophy, the testicles being the first to disappear; then the ovary, vitellogene gland, and shell-gland fade; the receptaculum seminis, vagina, and cirrus-pouch persist the longest (Plate II, Fig. 5).

Eggs. - The ova are more or less globular, measuring $63 \mu$ in diameter; by reciprocal pressure they become cuboid. The embryo, which measures $16-18 \mu$ in diameter and is provided with the six eharacteristic hooks $9 \mu$ long, is surrounded by a pyriform apparatus. The bulb 
of the apparatus-i.e., that part directly surrounding the enbryo-measures $20 \%$ in diameter. The horns are $24 \%$ long, appear to be hollow, and end in a disk $15 \mu$ in diameter. When viewed en face the disk is nearly round. In the center are seen the ends of the two horns, each $3 \%$ in diameter, and several-generally four-round nuclear bodies $(3 \mu)$, each containing one or two very refringent dots. These four bodies have every appearance of being true nuclei. When treated with caustic soda the disk frequently assumed the fringed appearance which Curtice figures in Tonia expansa.

There is a thin membrane inside the bulb of the pyriform apparatus, and at a point near the horns this membrane was invaginated toward the embryo (Plate II, Fig. 6). This observation was made upon preserved material, so that it is not impossible that this is to be explained by a contraction of the embryo. The horns are surrounded by a granular fatty substance.

\section{SPECIFIC DIAGNOSIS.}

From the above anatomical description we feel justified in giving the following specific diagnosis:

Moniezia planissima S. \& H., 1892.-Adult strobila 1 to $2^{\mathrm{m}}$ long; yellowish. Head quadrate, $0.4-0.9 \mathrm{~mm}$ broad; suckers at the four corners and directed forwards (diameter $\left.0.25^{\mathrm{mm}}\right)$; dorsal and ventral surfaces usually show a distinct plane running across the suckers and ending about $0.5^{\mathrm{mm}}$ from the antcrior extremity (balsam preparations). Neck broad, $0.6-1.5 \mathrm{~mm}$ long or very narrow and long. First traces of genital organs $7-12^{\mathrm{mm}}$ from the head (sc gment $\left.c a .150\right)$. The segments are at first extremely short; always much broader than long and longer than thick; ripe segments $12-26^{\mathrm{mm}}$ broad by $1-1.75^{\mathrm{mm}}$ long, generally thiu. Interproglottidal glands linear, large and very distinct. Genital pores double, sitnated in the anterior portion of the lateral margin; vagina and cirrus on the same transverse plane-on the right, vagina ventral, cirrus dorsal; left side vagina dorsal, cirrus ventral; genital canals pass dorsally of the longitudinal canals and nerves. Testicles begin about $10^{\mathrm{cm}}$ from the head, at first arranged in two triangles; 400-600 testicles present in ripe segments and generally as numerous in the median line as elsewhere, so that the testicles of each side cannot always be distinctly separated. Uterine folds enter the lateral fields. Eggs $63 \mu$ in diameter; bulb of pyriform apparatus $20 \mu$; horns $24 \mu$; embryo $16-18 \mu$; hooks $9 \mu$. (See also gencrie diagnosis, p. 54).

Type specimen is in the Bureau of Animal Industry; typical specimens will be sent to museums and specialists exchanging with this Bureau.

S. \& H.

(2) Moniezia Benedeni (Moniez, 1879) R. M1., 1891.

[Plate II, Figs. 7, 8.]

Synonymy.-Trenia Benedeni Moniez, 1879; Moniezia Benedeni (M.) R. Bl., 1891; Tania denticulata Neumann, 1892 (fig. of scolex.)

Hosts.-Domestic sheep (Ovis aries), Moniez; eattle (Bos taurus), Moniez, v. Marenzeller.

Geographical distribution.-France (at Lille, Moniez); Austria (at Vienna, v. Marenzeller). 
(1) Moxicz, R. Note sur denx especes nouvelles de Tinnias inermes (Trenia Togti et T. Benedeni); Bulletin scientif. du Department du Nord, 1879, 2 sér., 'T. "2, p. 163.

(2) Raildiwt, A. Éléments de Zoologie Médicale et Agricole, 1886, p. 261, Ténia Benedeni.

(3) Nequxx, L. G. Traité des Maladies parasitaires non-microbiennes des animax domestiqnes, 1st ed., 1888, p. 383, T'pnia Benedeni.

(4) Branchard, R. Sur les Helminthes des Primates anthropoides; Mém. d. 1. Soc. Zool. d. France, 1891, p. 187, fontnote, Moniezia Benedeni.

(5) Monizz, R. Notes sur les Helminthes. vi, 1; Revue Biologique du Nord d. 1. France, 1891 (Extr. pp. 14-16), Moniezia Benedeni.

(6) Buaxchard, R. Notices helminthologiques. 2. sér., 7; Mém. d. 1. Soc. Zool. d. France, 1891, p. 44t, M. Benedeni.

(7) Neumaxs. Traité, etc., 2 ed., 1892, p. 408, T. Bencdeni.-Engl. Transl., p. 418, T. Benedeni.

\section{HISTORICAL REVIEW.}

This species was first described by Moniez (1) as a worm of $4^{\mathrm{m}}$ or more long; head large in proportion to the neck; last segments mons than centimeter broad; longitudinal muscles of one surface developed more than those of the other surface. The parasites were found in the intestine of sheep at Lille, France. The above diagnosis is also given by Railliet (2). Nemmann (3) adds that the head is small, globular, and followed by a filiform portion $4-5^{\mathrm{em}} \mathrm{long}$, in which the segments are visible; the eggs a e 75-80 $\mu$, and show a pyriform apparatus. li. Blanchard (4) thenstates in a footnote that he includes this species in his new genus Moniezia. This publication was immediately followed by one from Moniez (5), in which he accepts Blanchard's classification and adds the following in regard to $M$. Benedeni: "The worm is remarkable on account of the thickness and breadth of the segments; in contraction the latter attain $12 \mathrm{~mm}$ in breadth, $3^{\mathrm{mm}}$ in length, and $2^{\mathrm{mm}}$ or more in thickness; the head is more than $1^{\mathrm{mm}}$ thick; the suckers are strong, prominent, $0.45^{\mathrm{mm}}$ in diameter, and touch each other; same form found in seven individuals; neck $2.5^{\mathrm{mm}}$ long; form of segments the same through entire length; found in cattle as well as sheep. Ova $80-85 \mu$, pyriform apparatus 18 \%, em'jyo 15 ".." In Blanchard's second publication (6) the worm is simply mentioned as belonging to the genus Moniezia. In the second edition of Neumann's work (7) the specific diagnosis is not enlarged, but a figure is given of a head of the worm which the author considers to be T. Benedeni. In response to a request both Moniez and Neumann kindly forwarded specimens which they included in this species. Nemmann's specimen, however, agrees perfectly with Moniezia trigonophora.

Moniez's specimen bears the label "Monton 31 (? indistinct-c. W. s.), 79, i, prep. xx. le 2 gros. frağm.=? du même." As he describerl this parasite in 1879, and as he was asked for his original type, it is assumed that the "79" refers to the year, and that the sample sent is some of his original material; this specimen forms the basis of the 
present description. Moniez's interrogation point on the label signified that he suspected there was more than one species container in the hottle. His suspicions proved to be perfectly correct. for besides the $M$. Benedeni, which was easily recognized from his description of the head, segments of M. expansa and Th. Giardi were found. A tapeworm labeled " $T$. expansa" was received from Dr. von Marenzeller; of Vienna, which agrees with Moniez's type of $M$. Benedeni.

\section{ANATOMY.}

External appearance.-Plate II, Fig. 7, represents a strobila (natural size) which was received from Moniez. The animal is considerably contracted. It will be noticed that the head is quite large, the neck is quite stout, the segments are distinct, thick and much broader than long. Fig. Ta gives two enlarged segments, which show a peculiar checkered appearance of the cuticle; this is common to all the large segments. The strobila is very opaque.

Scolex.-Figs. 8, 8a agree perfectly with Moniez's description (5) of the head. Upon comparing these figures with Nenmann's figure of the scolex of $T$. denticulata $(7, \mathrm{Fig} .183)$, it will be noticed that they are almost identical. Prof. Neumann was kind enough to send 4 strobila of the worm which he looked upon as $T$. Benedeni, and they do not agree with Moniez's type, but, as stated above, with Moniezia triyonophora, so that we are forced to assume that Nemmann's diagnosis of M. Benerleni must be dropped, and that his T. denticulutu (partim) is probably identical with Moniez's M. Benereni.

The hear of Moniez's M. Benedeni, in the possession of the writer, is square, $0.96^{\mathrm{mm}}$ by $0.96^{\mathrm{mm}}$; length $0.48^{\mathrm{mm}}$. Two furrows cross the anterior surface of the head and extend around on the four sides; these furrows are comnected by furrows posterior to the suckers, thus separating the latter from the strobila. 'This arrangenent of furrows gives the head a lobed appearance, each of the 4 lobes representing a large sucker $0.48^{\mathrm{mm}}$ in diameter; the opening of the suckers must of comrse vary with the state of contraction. In Figs. S, Sa the opening is small and circular. The specimen of this species is so contracted that it would be altogether too hazardous to make many statements in regard to its internal anatomy. It can, however, be stated that the interponglottidal glands are extremely indistinct (absent?), without sacs. but appear to be linear, as in M.planissima. There appears to be no reason, however, for uniting these two species, since the heads and strobila are so totally different. Furthermore, Moniez states that the rontracted segments measure $12^{\mathrm{mm}}$ hroad by $3^{\mathrm{mm}}$ long by $2^{\mathrm{mm}}$ thick, which measurements do not agree with those of $M$. planissima. The general topographical rela. tions of the vagina, cirrus, genital (anals, genital glands, longitudinal canals and nerves are the same in this species as in $M$. planissima; the arrangement of the testes, however, was not observed.

Ova.-As stated above, Moniez gives the measurement of the ova as S0-\$5 $\mu$, of the embryo $15 \mu$. 
SPEOHIT: I)IAGNOSIS.

From our present kuowledge of this parasite we may accept the following as a provisional diagnosis:

Moniezia Benedeni (Moniez, 1879) R. B1., 1891.-Strobila attains a length of $4^{\mathrm{m}}$; head $1^{\mathrm{mm}}$ or more large; obtuse, 4-lobed, suckers directed diagonally forwaris. opening circular. Neck present, slightly narrower than the hearl, about 2-2.5mm long. Segments always broader than long; eontracted ripe segments attain the measurement of $12^{\mathrm{mm}}$ broad by $3^{\mathrm{mm}}$ long by $2^{\mathrm{mm}}$ thick. Topography of longitudinal canals, genital eanals, and female glands the same as in M. planissima; testicles? Interproglottidal glands extremely indistinct [absent?], without saes. Ova 80-85 $\mu$ in diameter; embryo $15 \mu$; pyriform apparatus $18 \mu$.

Type specimens are in the possession of Prof. Moniez, Lille, France, and C. W. Stiles, Washington, D. C. One very typical specimen is in the K. K. Museum in Vienua, Austria.

$$
\text { C. W. S. }
$$

\section{(3) Moniezia Neumanni Moniez, $18 \hat{1} 1$.}

[Plate IV, Figs. 1-5.]

Most.--Sheep (Moniez).

Gcographical distribuion.-France (Lille, by Moniez).

\section{I.ITERATURE.}

(1) Moxiez, R. Notes sur les Helminthes, vi, 2; Revne Biologique du Nord de la Franee, 1891, t. iv, 1 page.

HISTORICAL REVIEW.

Moniez gave a very short description of this worm $(1)$, by which it has not been possible to recognize the species. He was, howerer, so kind as to send specimens. He states (1) that the head has the same dimensions as that of $M$. Benedeni, and is more than $1^{\mathrm{mm}}$ in diameter; suckers agree with those of $M$. Benedeni; while the neck is the same length as in $M$. Benedeni but filiform. The strobila is much shorter (1-2 feet); seginents narrower ( $\left.8^{\mathrm{mm}}\right)$, thinner, and $1-1.5^{\mathrm{mm}}$ long; the segments which are about to become detached are 6 by $2^{\mathrm{mm}}$. Ova measure 55-56 $\mu$, embryo 18-21 $\mu$.

\section{ANATOIIY.}

Only contracted segments (Plate IV, Fig. 1) are at hand, and but little in regard to the anatomy of this species can be given.

Scolex.-The head resembles that of $M$. Benedeni in some respects; it is almost square when viewed en fuce (Plate IV, Fig. $2 a$ ), $0.9^{\mathrm{mm}}$ broad; when viewed from the dorsal or ventral surface, the suckers do not appear so distinctly separated from the neck. The openings of the suckers are quite small, situated at the four corners and directed diagonally for ward. The segments are all broader than long.

In several segments measuring $1.2^{\mathrm{mm}}$ wide by $0.56^{\mathrm{mm}}$ long (Plate IV, Figs. 3,4$)$, the genital pore was just on the point of piercing the lat- 
eral margin of the segment in its anterior third. The male and female canals were distinct from each other, but their lumena were very indistinct. The female glands were very small, but could be distinguisheil. A large number of testicles were distributed in the median field, and they were as numerous in the median line as elsewhere. Short linear interproglottidal glands without sacs were present in the median line. In several segments (Plate IV, Fig. 5) measuring $4^{\mathrm{mm}}$ wide by $2.2^{\mathrm{mm}}$ long, the uteri occupied almost the entire segment and were filled with ova. The other organs were atrophied. The genital pore was in the middle or anterior half of the segment. In the segments $6.5^{\mathrm{min}}$ wide wo details were recognized.

\section{SPECIFIC DIAGNOSIS.}

The specific diagnosis of this cestode is very unsatisfactory, owing to our lack of knowledge in regard to important details.

M. Neumanni M., 1891.-Strobila $1 \frac{1}{2}$ to 2 feet long, perhaps longer. Head square, $0.9^{\mathrm{mm}}$ or more broad, suckers powerful, same diameter as in $M$. Benedeni, but not so distinctly lobed as in that species. Neck about $2-2.5^{\mathrm{mm}}$ long, anterior portion filiform, very thin. Segments narrower and thinner than those of $M$. Benedeni; the largest segments measure $8^{\mathrm{mm}}$ broud by $1.5^{\mathrm{mm}} \operatorname{loug}$; the segments at the posterior end somewhat narrower and longer, $6^{\mathrm{mm}}$ by $2^{\mathrm{mm}}$. Topography of lougitudinal cauals, genital canals, and female glands probably the same as in $M$. Benedeni; testicles arranged in a quadrangle. Interproglottidal glands small, linear, without sacs. Ova 55-65 $\mu$; embryo 18-21 $\mu$.

Type specimens are in the possession of Prof. Moniez, Lille, France, Bureau of Animal Industry, and C. W. Stiles, Washington, D. C.

It will be seen from the above that $M$. Neumanni as well as $M$. Benedcni must be subjected to a thorough anatomical study by some one who can obtain the proper material. The powerful contraction of the specimens, and the inability to obtain fresh material, is offered as an apology for not going more into details. See also M. nullicollis (p. 83). C. W. S.

\section{B. Expansa Group. (M. expansa, M.oblongiceps, and $M$. trigonophora.)}

Characterized by the grouping of the interproglottidal glands around b]ind sacs.

(4) Moniezia expansa (R., 1810) R. Bl., 1891.

[Plato V, Figs. 1-3; Plate VI.]

Synonymy.-(After Rudolphi) Tania vasis nutritiis distinctis Bloch, 1782; T. ovina Goeze, 1782; T. ovina Batsch, 1786; T. ovina Schrank, 1788; T. ovina Gmelin, 1789; Halysis ovina Zeder, 1803-(A fter Croplin) T. denticulata Mayor-(After Baird, 1853) Alyselminthus expansus Blainville-(After Stiles) T. expansa, cx parte of Railliet, Neumann, Perroncito, Curtice, and other recent authors.

Hosts. - Sheep (Ovis aries), found by varions anthors; cattlo (Bos taurus), found by varions anthors; Kebu (Bos indicus), found by Porroncito; goats (Capra. hircus); Pyrenian tor or ibex (Capra pyrenaica); roo deer (Capreolus caprea), reported hy Nitzsch; pampas deed (Cariacus campestris), seo Dies.; brocket (Cariacus rufus), see Dies.; Cervus Nambi, see Dies. (collected by Natterer); Gazellu dorras, reported by Bremser, see Dies.; Ovibos moschatus; gemso or chanois (Inpicapra trayns), reported by Bremsor. 
Geographical distribution.-North Ameriea: Chicago, Ill., * Stiles; Washington, D. C., *Curtice, *Hassall, * Stiles; Colcrade, Curtice. South America (Brazil, Natterer). Europe: England, Hassall and others; France, *Railliet, *R. Blawehard, Nemmanu, Moniez, *Stiles, and others; Germany aud Austria, Lenckart, Blocll, Gocze, Nitzsch, *Rndolplii, *'Zsehokke, *Stiles, and many others; Italy, Perroncito and others.

Besides the above, this species is mentioned by numerous other authors in varions parts of the world. In all probability some of the cases are those of true $M$. expanso. while in other cases the data given are too incomplete to determine whether the species found was $M$. expansa or some other form. An examination has been made of the specimens of this species colleeted by those men whose names are starred (*) in the above list. It is very eertain that many of the cases of Tania expansa reported in America are cither $T h$. actinioides, M. planissima, or M. trigonophora. In all probability this is the most widely distributed speeies found in cattle and sheep (See extract from letter by Dr. (Giles, p. 74.)

\section{LITERATURE (1810-1892).}

(1) Rudolpmi. Ent. Hist. nat., ii, 2, 1810, p. 77-79, Trenia expansa.

(2) - Ent. Syn., 1819, p'p. 144, 487.

(3.) Gunct. Lehrbneh der pathologischen Anatomie der Haussängethiere, 1831, i, p. $381, \mathrm{x}, 1-2$.

(4) - Hartwig's Mag. d. Thierheilkumde, iv Jahrg., 2. Heft.

(5) Mayer. Froriep's Neue Notizen i, p. 107 (T. denticulata)

(6) - Analecta f. vergl. Anat. 2. Samml. i, p. 70, figs. 4-5. Review by v. Siebold in Wiegmann's Arch. f. Naturg. 1840, ii, p. 192.

(7) Creplin. Endozoologische Beiträge. 1 Ueber Trenia denticulatu Rud, und Trenia expansa Rud.; Wiegmann's Arch. f. Naturgeschichte, 1842, i, pp. 315-327.

(8) Dujardin. Hist. nat., p. 577.

(9) Diesing. Syst. Helm., i, p. 497.

(10) Verrim, A. E. Parasites of domestic animals; Report of the Connecticut Board of Agriculture for 1870, pp. 65, 100.

(11) Davaine. Traité des Entozoaires, 1877, p. liii, 235.

(12) Moniez. Mémoires sur les Cestodes. Paris, 1881, pp. 15-21, pl. i, figs. 38--58, ii, 1. (Embryology.)

(13) Zürn. Die th. Parasiten, 1882, p. 189-192, taf. iii, figs. 39-41.

(14) Perroncrto. I Parassiti, etc., 1882, p. 239, figs. 99-100.

(15) - Trattato sulle Malattio degli Animali domestici, 1886, pp. 231-232, 233.

(16) McMurricr. Ninth Anumal (1883) Report of the Ontario School of Agriculturo (1884), pp. 174-178, 7 figs. (Zoologieal Report.); F. G. Grixsine, same Report, pp. 200-203. (Veterinary Report). (Rev. by v. Linstow, Arch. f: Naturg., 1888.) [T. expansa=M. trigonophora, vide p. 37.]

(17) Leuckart, R. Bandwiirmer; Koch's Encyelopällie der gesammten Thierlieikŭnde, 1885, p1. 396-399; T. еxpansa, under Dipylidium.

(18) Railliet. Eléments, etc., 1886, p. 257-260, fig. 151 (orig.), fig. 152 (after Neumann), fig. 153, 1-12 (after Moniez).

(19) Neumans. Traite, ete., 1st ed., 1888, p.377-378; figs. 164 (1-12) (after Moniez), 165 (after Railliet), 166 (orig.).

(20) Cuntice, C. Animal Parasites of Sheep, 1890, pp. 113-122, pls. xiv, xv.

(21) Zschoкke. Reehcreh sur la structmre anatomique et histologiqne des Cestodes, 1890, pp. 91-114, taf. ii-iii, tìgs.31-38.

(22) Branchard, R. Sur les Helm. des Anthro. (Footuote); Mém. d. l. Soe. Zool. d. France, 1891, p. 187. (Moniezia expansa.)

(23) Moniez. Notes sur les Helm., vi, 4; Rev. biol du Nord de la France, 1891, 
(21) Buancinald, R. Notices Helm., 22 sór., 6; Mrm d. 1. Soc. Zool de France, $1 \times 91,1) .445$.

(25) Neumans. Traité, ete., ¿nd ed., 18!2. 1. 402. 107; ligs. 184-186.-Wngl. Transt., 1892, pp. 412, 418, 129. (T. expoust.)

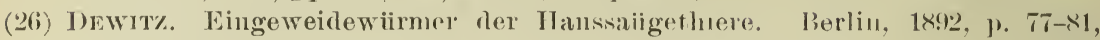
figs. 51-52.

(27) Stmles. Notes sur les Parasites-11: Snr le Truin expansa Rud.: ('ompt. rent. d. 1. Soc. 1. Biol. (Paris) $1 \times 92$, 1). 664.

(28) _- Bemerkungen iiber Parasiten-17: Ueher die topographische Luatomie des Gefaisssystems in der Familie Trniadn'; ('entralblattf. Bakterologie nnd Parasitenkunde, 1893, bd. xiii, p. 457-f(65), figs. 1, 2. (11. еxpansa.)

\section{HISTORICAI, REVIEW.}

It is almost impossible to give a satisfactory historical review of this species, since very few of the diagnoses given by authors can be applied to this worm alone.

Rudolphi (1) starts off his discription of the species with the diagnosis-

"Tania expansa K.-Capite obtuso, collo nullo, articulis auticis brevissimis, reliquis subquadratis, foraminibus margiualibns oppositis."

As synonyms and literature he gives:

Bloch.-Ablandlung von der Erzeugnung der Eigeweidewiirmer und den Mitteln wider dieselben. Berlin, 1782, $4^{\circ} 10$ 'Taf., p. 16, 'Taf. 5, figs. 1-5. Tania rasis nutritiis distinctis.

Goeze.-Versuch einer Naturgeschichte der Eingeweidewiirmer thieriseher Körper. Leipzig, 1782, 44 Taf., p. 360-371, Taf. 28, figs. 1-12. T. ovina.

Batsch.-Naturgeschiehte der Baudwurngattung iiberhaupt und ihrer Arten insbesondere, nach den neuern Beobachtungen in einem systematischen Auszuge. Halle, 1786, p. 182, n. 28, figs. 109, 162. T. orina.

schrank.-Verzeichniss der bisher hinlänglich bekannten Eingeweidewiirmer, nebst einer Abhandlung iiber ihre Anverwandtschaften. Miinchen, 1788, 8 , p. 38, No. $115, T$. ovina.

Gmelin.-Linnés Systema Nature, 1789-1790, p. 3074, No. 55. T. ovina.

Tabl. Encyl. t. 45, figs. 1-12 (ic. (Goeze). T. orina.

Zeder.-Anleitung zur Naturgeschichte der Eingeweidewiirmer. Banberg, 1803, 1. 332, n. 7. Halysis ovina.

Rudolphi then gives a more detailed description of the animal; but that this deseription is insufficient is conclusively proven by the fact that many helminthologists since Rudolphi's time have had difliculty in recognizing the proper T.expansa. Although Rudolphi acknowledges that his species is identical with the species $T$. ovina, he renames it $T$. expansa. There seems to be an impression among certain anthors that the word expansa refers to the breadth of the worm, but this is not the case, for Rudolphi writes: "Ipse maximam ab agni pyloro an cocum usque expansam et villosie allacrentem virli, mole nomen triviale desumsi." Hence Rudolphi referred to the length rather than the breadth in using the term expansa.

As far as the literature and synonyms which Rudolphi gives areconcerned, the following have been foumd:

In the French edition of Bloch's work (1). 35-37, v. 1-5) a worm is described which may or may not be considered identical with lindolphi's 
T. cxpanss. In the former the head is large, and the genital pores, in nearly every segment figured, are exactly in the middle of the margin. The shape of the head is quite similar to that of expansa. In expanse the head is small, the pores generally in front of the middle of the edge. The worm Goeze describes as Trenia oxina could, with a little imagination, be called identical with Rudolphiis expansu.

The articles by Batsch and Schrank are not at hand. Gmelin evidently takes his diagnosis from Bloch's article. The other two books are not to be obtained here.

Rublolphi is probably as far back as it is possible to trace T.expansa with certainty. Having in my possession part of his type material I feel obliged to accept his name expansa in preference to the name ovina, which antedates it. If, however, the original material of Goeze can be examined, and if by that means it can be demonstrated that ocina is really identical with expansa, then I should be in favor of holding to the law of priority and of vindicating Goeze even at this late date. As Rudolphi himself believed his species to be identical with Goeze's ovina, he had no excuse for renaming the species.

In his synopsis Rudolphi (2) adds nothing of any importance in regard to $T$. expunsa.

Gurlt (3) gives a short description of T. expansa, together with two figures. It is probable that this is really T. expansa, although it may possibly belong to some other species. It has not been possible to obtain Gurlt's second article.

Mayer (5-6) speaks of $T$. denticuluta in two articles (see below).

Creplin (7) gives quite a good discussion of the differences between T. expansa and T. denticuluta. His diagnosis for T. expansa reads:

T. capitis parvi oblongi, antice et lateribus rotundati, retrorsum angustati osculis perfecte lateralibus, magnis, tumidis. eollo cum corpore toto plano, subtili, cum capite, se crassiore, continuo, perbrevi, articulis primis hrevissimis, marginibus lateralibus convexinsculis, sequentibus lentissine latescentibus elongatisque et simul marginibus lateralibus rectioribus demumcue fere prorsus rectis, postico rectr, sæpe crispato, semper tumidulo, uti angnlis obtusis, perparum prominentibns, articulis ultimis solis, cum se antecelentibus sensim angustioribus factis, primo quadratis, tum (perpancis, neque semper), alleo longioribus quam latioribus, terminali obtuse finito, osculis genitalibus marginalibns plures pollices a capite monstrari incipientibns, tum ad candau usque, secundis oppositis. (Ova globiformia et angulata.)

Creplin evidently had the true T. exponsa before him, although it is not impossible that he also had some other species. He says that Mayer's (6) denticulata is really exponsa. He thinks also that Carlisle's specimens were expansa.

Dujardin (8) does not add anything new to our knowledge of $T$. expansu. His "large de 5-27 "mm; ventouses dirigées en alvant" seems to indicate that more than one species is inchuded in the diagnosis.

Diesing (9) gives a short Latin diagnosis of the species. 
Verrill (10) evidently has several species included in his diagnosis of T. expansa.*

Davaine's diagnosis (11) is short. He also gives a short account of symptoms and treatment of animals affected by the parasite.

In Moniez's Mémoires sur les Cestodes we find an account of the embryology of tapeworms of the type Tania expansa. His results are as follows:

The ovum of $T$. expansa first appears as a small cell filled with yolk and provided with a body which seems like an excentric nucleus; this latter, lowever, is the true ovum. A vitelline membrane appears after fecundation, and the ovum divides; one of the resulting bodies separates from the vitelline matter and multiplies rapidly, forming the blastodermic cells; the other cell remains inclnded in the vitelline matter and becomes very refringent (polar body). The blastorlermic cells increase in number, forming a morula, while the vitelline matter contains the polar body. The vitelline matter divides into two semilunar bodies which surround the morula, and each of which contains a large vitreous cell. The morula wow gives off in succession two concentric cellular layers; the cells of the onter layer form an envelope for the embryo, then become indistinct and granular. The inner layer of cells then pushes out two horn-like prolongations on one side and forms the so-called pyriform body, inside of which lies the six-hooked embryo (oncosphere). That portion of the ontside cellular layer which is included between the two horus of the pyrifurm apparatus, according to Moniez, forms the prominent circular or radiate body (disk) situated at the end of the horns.

Zürn (13) describes symptoms and gives treatment for animals infested with these tapeworms. His specific diagnosis is short. He goes back to the statement of earlier authors, that $T$. expansa is sometimes $60^{\mathrm{m}}$ long. His figures are very poor. In Figure 39 part of the segments are upside down, i.e., he mistakes the anterior edge for the posterior edge. Perroncito's figures (14) are upside down. His diagnosis seems to apply pretty well to $M$. expansa, although some points in it make one suspicious of $M$. planissima. He states, contrary to Creplin, that the suckers are directed forward. The anterior portion of the strobila is described as colorless or light yellowish, while the posterior portion is a more intense yellow. The segments are said to attain a breadth of $33^{\mathrm{mm}}$.

McMurrich (16) had occasion to investigate a tapeworm epizoötic among sheep in Ontario. He favors a theory that Melophagus ovinus (the sheep tick) acts as intermediate host for the tapeworm. Judging from his description and figures it is probable that the worm he found is the same that the writer found later (see below) in the Blairsville epizoötic (M. trigonophora).

Lenckart (17) gives a very good general description of this parasite,

*In regard to Verrill's report, I might mention that several European authors have concluded that Verrill found in Anerica all the species of parasites which are mentioned in that article. Prof. Verrill, however, laas written to this Burean that such was not the case. As ho does not state in every case in his article which of the parasites mentioned are found in Americin, his report should not be used in making up the geographical distribution of the animals in question.-c. w. s. 
but certain portions of his article lead me to believe that he had both $M$. expansa and M. trigonophora in his possession when he wrote the article. He discusses the question of life history, and suggests that some snail may possibly be the intermediate host.

Railliet (1S) states that the suckers are directed forward, but gives a figme (from Neumann) in which they are directed dorso-and ventrolaterally. An original figure of an adult strobila is given, which seems to be $T$. expansa.

Neumann (19) gives substantially the same as Railliet (18).

Curtice (20) attempted to infect sheep by feeding them eggs of tapeworms. Although his results were not positive, he expresses himself as decidedly in favor of a development without any intermediate host. His experiments cannot be considered as supporting the theory.

Zschokke (21) gives an excellent aceount of the internal organization of T. expansa. There can be no doubt that he had T. expansa before him, for the preparations which he kindly forwarded agreed very well with Rudolphi's type specimen. Zschokke's work will be referred to hereafter in connection with the anatomical description.

R. Blanchard $(22,24)$ placed T. expansa in his new genus Moniezia.

Moniez (23) discusses the difference between $M$. expansa and $M$. denticulata, stating that the segments of expansa are thin, transparent, and yellowish.

Neumann (25) gives a new figure of the head of $T$. expansa. The figure of the head of $T$. expansa given in his first edition is now reproduced as T. Benedeni, but it does not agree with Moniez's original material. That Neumann, as well as Railliet, included M.plunissima in - the species T. expansa is already stated above.

Dewitz (26) mentions Curtice's conclusions in regard to the direct development and copies one of his figures.

Quite recently I obtained some of Rudolphi's specimens and described $(27,28)$ the interproglottidal sacs in them; at the same time attention was called to the fact that $M$. expansa of recent authors contained more than one species, and $M$. planissima was separated from $M$. expansa.

Above have been mentioued nearly all the articles upon $T$. expansa Rud. which it has been possible to find. The species is mentioned in several lists of parasites which are not included in this review. It is further to be expected that there are other articles upon a species so well known (by name), but that they have escaped attention.

\section{ANATOMY.}

After this tedious review of articles published on Tcria expansa, let us pass to a description of that portion of Rudolphi's original specimen in our possession, in order to establish one of the chief points in the diagnosis of this species. 
Prof. Möbius very kindly sent four portions of worms labeled "Tcenia expansa; coll., Rudolphi;" Nos. 1-3 from Ovis aries, No. 4 from Gazella dorcas (Antelope dorcas).

(1) Scolex very much shrunken, nearly sanare when viewed en face, 0.368 by $0.368^{\mathrm{mm}}$. The suckers occupy the four corners, are distinctly raised and directed diagonally towards the front. The openings are slit-form (Plate v, Figs. 1-1b).

(2) A strobila about $10 \mathrm{~mm}$ long by $1.5^{\text {mm }}$ loroad. Poorly preserved. Segments are distinet (Plate v, Fig. 2). Genital organs well developed, but too indistinct to warrant description. Genital pore in anterior half of segment. Interproglottidal sacs almost imperceptible.

(3) Fifteen segments $7 \mathrm{~mm}$ broad by 1-1.3 long. Interproglottidal sacs plainly visible. The material does not warrant any other statements.

(4) (From Gazella dorcas.) Thirty segments; 10 (not $16^{\mathrm{mm}}$ as stated in my Note 14) broad by $1^{\mathrm{mm}}$ long. Somewhat better preserved than 1-3. The segments are filled with eggs; the interproglottidal sacs (Plate v, Fig. 3), are here and there visible.

From the above it will be seen that the species accepted as Moniczia expansa must possess interproglottidal glands grouped around sacs. The literature has been searched very carefully to find mention of these glands, but unless some statement by some author has escaped attention, the glauds were first described in my preliminary note (־6) before the Société de Biologie (Paris).

The question, of course, immediately arises whether these sacs can not be found in other species. As a matter of fact we find these sacs present in several species, which are included in the "Expunsu group." By the aid of the scolex, however, we can determine which one of these is the true $M$. expansa. The scolex of Rudolphi's type agrees very well with the scolex of the worm which Curtice described as Tania expansa; it does not, however, agree with Zscholke's figure, although it resembles one of his preparations. The difference between this figure and Neumann's figure might, perhajs, be explainc ly contraction. At any rate, it is perfectly evident that Curtice's T.exponsu comes the nearest to Rudolphi's type of any specimen in possession of this Burean, and justifies the acceptance of that as the true M.cxpansu.

General appearance.-Specimens of this species show an extreme variation in external appearance, aceording to whether they are alive or fixed by different methods of preservation. These variations, however, are not more than the variations found in specimens of Tomic saginata which have been preserved in different fluids. Older authors state that $T$. expansa grows to more than* 100 feet in length, but

* It is well known that several Ameriean physicins have reported cases of Tonia saginata of man in which the parasites were said to be 50, 100, or oven 210 feot long. Zoölogists have explained these cases by saying that the physicians had taken al number of worms, and, smpposing them to be all from one individual parasite, have given the total length of all the finguents. I have myself followed this explanation in lecturing upon parasites, and believe that this is the true explanation in many, although not in all cases. Only a short time ago I ealled upon a physician who has several bottles in his office containing $T$. saginata, and bearing the labels " 180 fecet. Jong," "18; feet long," "210 feet long," cte. Upon calling into question the supposition 
recent anthors havealleady shown that that shatement is erroneous, and that this enomomoms length was made up omly by placing a number of broken strobila together and assuming that they all belong to one individual tape-worm. It is not infeguent to find worms $4^{\mathrm{m}}$ or even $5^{\mathrm{m}}$ long. The breadth of the worm varies from about $1^{\mathrm{mm}}$ to $16{ }^{m m}$, aroording to the portion (the neck or the posterior end) measnlerl, the age, preservation of the sperimen, and the extent of contraction. The anterior portion is generally whitish, while the posterior end is fiequently of a yellowish or orange color.

s'perimens of worms, agreeing in regard to the shape of the hearl and presence of the blind sacs, vary considerably in form, but it is not thought best to make separate species for these variations, as it is believed that most of them may be accomuted for by contraction.

The scolex varies from $0.368-0.72^{\mathrm{mm}}$; the suckers measure about 0.3 broad by $0.256^{\mathrm{mm}} \operatorname{long}$; the opening is slit-form. The neck is extremely variable, in some cases very thin, in others quite thick (contraction). Segmentation begins from $0.5-2 m m$ back of the head.

Segments $10^{\mathrm{cm}}$ from the head measure $1.5^{\mathrm{mm}}$ wide by $0.24^{\mathrm{mm}}$ loug; about 25 sacs are present; the genital anlage is circular in shape, although, in some cases, slight traces of the barrel of the pistol can be seen.

Segments $15^{\mathrm{cm}}$ back of the head measure $2^{\mathrm{mm}}$ wide by $0.19^{\mathrm{mm}}$ long; about 35 sacs are visible; the genital pores are not yet present; the genital anlage is pistol-shaped, and at the median extremity is already divided into two lobes, the anterior destined to form the male canal, the posterior to form the female canals and glands; the lateral point of the anlage (muzzle of the pistol) is close to the edge of the segment in its anterior third. No testicles are visible.

Segments $20^{\mathrm{cm}}$ from the head (Plate vi, Fig. 3) measure $3.5^{\mathrm{mm}}$ wide by $0.56^{\mathrm{mm}}$ long; about 40 sacs are visible; genital pores not present; the genital canals become more distinct and the ovary, vitellogene and shell-glands can be distinguisherl; numerous testicles are present; they occupy the median line as well as the rest of the median field. $Z$ schokke states that the testicles of $T$. expansu are arranged in two triangles, one each side of the segment, as is the case in $M$. triyonophora (see below). By far the greater number of segiments of $M$. expansa which have been examined do not present this relation, although in a few segments of this species which were collected at Paris,

that these bottles contained only one specimen each, I was assured in the most positive terms that the worm "210 feet long" was entire at the time of measurement. Further conversation, however, elicitud the fact that the worm had not heen measured in the way zö̈logists take measurement, i.e., by placing the parasite upon a table and measuring its length while lying undisturbed, but that the freshly expelled restode was in a stool of warm water; one end of it was lifterl up and the length taken by means of a yard-stick while the parasite was in a perpendicular position. In this way its own weight would, of course, cause an enormous extension, and tho length of " 210 feet" could be easily obtained.-c. w. s.

7114-No. $4-3$ 
the triangular arrangement of the testicles is very distinct. This character appears in the same strobila with a quadrangular arrangement, but as most of the segments show the quadrangular arrangement, it is believed the triangular arrangement in the older segments of this species forms an exception. In Rudolphi's specimens the testicles are not visible.

Segments $30^{\mathrm{cm}}$ from the head measure $5^{\mathrm{mm}}$ broad by $0.4^{\mathrm{mm}}$ long; the genital pore has pierced the anterior third of the margin of the segment; the canals are perfectly distinct; the female glands are all visible; the entire remaining portion of the median field is crowded with testicles $56 \mu$ in diameter. (As the segments were subjected to pressure in making the microscopic preparations, this $56 \mu$ is probably larger than normal.)

The topography of the female glands, canals, vagina, cirrus, nerves, and the longitudinal canals agrees with $M$. trigonophora (see below, $p$. 39-41), so it is useless to repeat the description here. The only difference noticed was that the vagina and cirrus are more exactly on the same transverse plane (Plate vi, Fig. 4). Zschokke states that, as a rule, the vagina is ventral while the cirrus is dorsal, but I have found that the vagina is always ventral on the right side and dorsal on the left, side.

The uteri (Plate VI, Fig. 6) come more plainly into view in segments 80-90 $\mathrm{cm}$ from the head, while the genital glands begin to atrophy. As the segments grow wider the interproglottidal sacs increase in number.

In segments $150^{\mathrm{cm}}$ from the head the uteri are fully developed; they extend into the lateral fields dorsally of the longitudinal eanals. The testicles are no longer visible; but the receptaculum seminis still persists; this organ is generally somewhat longer than in $M$. trigonophora. In the remaining segments the ova come more plainly into view, and finally the segment represents a bag of ova.

Ova.-The eggs measure $50-60 \mu$ in diameter, the bulb of the pyriform apparatus $20 \mu$.

\section{SPECIEIC DIAGNOSIS.}

The specific diagnosis of this form is as follows:

Moniczia expansa (R., 1810) R. B1., 1891.-Strobila attains 4-5m in length; anterior portion usually whitish, posterior portion generally yellowish. Heal $0.36-0.7 m m$ in liameter, obtuse, more or less sfurare, slightly lobed; suckers distinctly raiserl, apertures directed diagonally forwarl. Segments always much broaler than long; end segments attain $16^{\mathrm{mm}}$ in width and are quite thick. Topography of nerres, longitudinal canals, genital canals, and female chlands similar to M. planissima; testicles usually arranged in a ruadrangle, rarely in two triangles except in tho younger segments. Interproglottidal glands localized around blind sacs which open between segments. Ova $50-60 \mu$, bulb of the pyriform apparatus $20 \mu$.

Type specimen is in the Königl. Museum für Naturkunde, Geheimrath P'rof. Dr. Möbiňs, Direktor, Invalidenstrasse, Berlin, Germany. Several segments of the type are in the Burean of $A$ nimal Industry, and in the private collection of $\mathrm{C}$. W. Stiles. Typical specimens will be sent to museums and specialists exchanging with this Bureau.

c. W. S. 
(5) Moniezia oblongiceps sp. n., 1893.

[Plate VII, Figs. 1-4.]

Host.-Conssussp. (S. \&. H.)

(reographical distribution.-Washington, D. C., imported from the Island of Trinidad, South Anerica.

In a post-mortem examination of a Coassus $s p$ ? recently imported for the National Zoölogical Park at Washington, D. C., from South America, two specimens of an adult cestode were found which bear a great resemblance to $M$. expansa, but which differ from it in the form of the head and segments as well as in general appearance.

While for a long time wo were inclined to look upon this as a simple variety of $M$. expansa, we incline at present to the view that the parasite in question represents a distinct species, which, however, is very closely allied to M. expansa of cattle and sheep. If only a few segments of the worm were in our possession we should undoubtedly determine them as identical with that form, but with two well preserver specimens before us it has been decided that they represent a true species.

\section{ANATOMY.}

General appearance.-Two strobilie provided with heads measured respectively $97 \mathrm{~cm}$ and $88 \mathrm{~cm}$ in length. A third strobila, which evidently belonged to one of those provided with in scolex, measured $31^{\mathrm{cm}}$ long. The head is not distinctly separated from the neck; the segments gradually increase in breadth to 50 cm from the head, where they reach the maximum of $9^{m m}$; from this point they diminish in breadth and increase in length so that the end segments measure $6.5 \times 1^{\mathrm{mm}}$. All the segments are quite thin. When fresh the worms have a very light yellowish tinge.

Scolex.-The head measures (preserved) $0.48-0.56^{\mathrm{mm}}$ broad; it is somewhat narrower in its dorso-ventral diameter. The same measurements are preserved for a short distance back of the head, so that the latter is not distinetly separated from the body. While the head of M. expansa viewed en face is more or less square, this head is distinctly oblong. The sucker's (0.19 in diameter) are situated at the four corners. The openings in our specimens are slit-form and directed half forward, half corner-wise. The suckers are not so distinctly raised as in $M$. expansa.

In Fig. $2 b$, Plate vir, the portion back of the head is broader than the head; in another specimen (Plate vIr, Fig. 2), the diameter of the head is preserved for about $2^{\mathrm{mm}}$, then it decreases somewhat for a very short distance, and fually increases again. This variation is due entirely to contraction. 
Segments $10^{\mathrm{cm}}$ from the head measure $5^{\mathrm{mm}}$ wide by $0.16 \mathrm{long}$; ahout $30-36$ sacs are present; no testicles are visible, but the genital anlage has the characteristic pistol shape; the pore is not present; the median end of the anlage is already differentiated into a male and female lobe. In segments measuring $5^{\mathrm{mm}}$ broad by 0.48 long, the pore is just on the point of piercing the margin in its anterior third, the outer cuticle invaginating to meet the genital canals; the cirrus-pouch is not yet formed; the male and female canals are separated in their entire length ; female glands are ail formed but are small. The remaining portion of the median field is occupied by testicles which are found in the median line as well as elsewhere; the interproglottidal sacs have inereased in number in proportion to the width of the segment.

Segments $21^{\mathrm{cm}}$ from the head measure $6^{\mathrm{mm}}$ broad by $0.48^{\mathrm{mm}}$ long; the genital pore is present; all of the organs have increased in size; the testieles have increased in number but are less numerous and smaller than those of 1 . exponso; the genital organs and the longitudinal canals present the same topographical relations found in the other species of Moniezia; copulation has taken place in segments $22^{\mathrm{cm}}$ from the head. The canal inside the cirrus is frequently quite wide. This widening is sometimes seen in other forms also, but it has never been seen so pronounced as in the worm now under consideration. The genital papilla are much more prominent than in any other species examined except $M$. denticulate and $T h$. Giardi.

Segments $30^{\mathrm{cm}}$ from the head measure $6.75^{\mathrm{mm}}$ broad by $0.48^{\mathrm{mm}}$ long. Segments $40^{\mathrm{cm}}$ from the head measure $8^{\mathrm{mm}}$ broad by $0.56^{\mathrm{mm}}$ long; the uteri become more prominent, while the female glands begin to atrophy. Segments $50^{\mathrm{cm}}$ from the hear reach the maximum breath of $9^{\mathrm{mm}}$, while in the next $40^{\mathrm{cm}}$ they decrease in breadth to $6.5^{\mathrm{mm}}$ by $1^{\mathrm{mm}}$ long. In the last segments the female glands and testieles have entirely disappeared.

Eggs.-The ova measure 48-60 $\mu$ in diameter; the bulb of the pyriform organ 16-20 $\mu$; the lorns are very short, $s-10 \mu$.

\section{SPECIFIR' DIAGNOSIS.}

The specific diagnosis of this restode is as follows:

M.oblongiceps sp. 11., 1893. - Strobila attains 97 (probably 120 or more) rentimetersin length; yellowish. Ilead not distinctly separated fom nork; oblong when virwed en face, the brearth $\left(0.48-0.56^{\mathrm{mm}}\right)$ being greater than the thickness; suckers 0.19 in diameter. not prominently raised, situated at the fonr corners; openings slit-form and directed diagonally forwards. Segmentsalways hroader than long; rather thin; maximmm breadth (about 50 $\mathrm{cm}$ from head) 9 ${ }^{m m}$; end segments 6.5 wide by 1 long. Topography of organs same as in $M$. expansa; testicles less momerous and smaller. Interproglottidal glands arranged aromul sacs. Eggs $18-60 \mu$; bulb of pyriform organ 16-20) 11 ; liorus $8-10 \mu$.

Type in the bureau of Animal Ludustry, U. S. Department of Aerirulture. 
(6) Moniezia trigonophora sp. n., 1833.

(I'latis VIII. IX.)

Synonymy. - Ternia rxpansu Curtice, ex parle, sprecimens in the collection of the Burean of Animal Industry. T. axpmasu Me-Murrirh, see p. 30. T. Benedeni Neumimn, ex purle.

Host.-Sheep (Neumam, ('urtire, Mr.Mnrich, Stiles, Hassall).

Ceographical distribution.-North America: U. S. A., Washington, D. C. (Curtice, Stiles, Hassall); Blairsville, Pa. (Ntiles); Canada (MeMurrich). France (Neminns).

\section{LITIERA'TTRE.}

Ma Murrici. See ahove, M. expansa, p. 27.

Suldes. Notes sur les l'arasites-14: Siu le Taniu expansa Rud.; Compt. rend. d. 1. Soc. d. Biol., Paris, 1892, p. 165. (T. Benedeni de Nemuanu.)

Bemerkuugen iiber Parasiten-17: Teber die topographisihe Anatomie des Gefïsssystems in der Familie Truiuda'; ('entrallhatt f. Bakt. u. Par., 1893, bd. Xi11, p. 465 .

\section{IIISTORICAL REVIEW.}

McMurrich (1) mentions a tapeworm epizoïtic in Canada, and diagnoses the parasite as Thuin expansa. From his figures and description, however, we suspect that the worms belong to this new species.*

In September, 18:1, Stiles received instructions from Hon. Edwin Willits, Acting Secretary of A griculture, to proceed to Blairsville, Pa., and study an epizoötic among sheep, which was reported as existing at that place. He found that the sheep were infested with a large number of worms, which were undoubtedly the cause of the trouble. Strongylus contortus was present in the fourth stomach in such numbers that it was almost impossible to see the mucous membrane. Besides this, all of the sheep examined containerl large numbers (10-50) of tapeworms belonging to the species here described. Upon comparing these worms with parasites in the Bureau collection it was found that Curtice had collected a number of the same kind from sheep slanghtered at Washington, D. C., and had determined them as Ternia expansa. As stated above, Neumann's specimen of $M$. Benedeni also agrees with this form.

\section{ANATOMY.}

Gencral appearance.-The worms are of a cream color when fresh. The head is quite small; the strobila attains the length of abont 1.6 meter's. The segments are very sharply defined, in nearly all cases broader than long, althongh some segments were found which were almost square, or even longer than broad. The largest segments are generally $6-\tau^{\text {min }}$ broad by $2^{\mathrm{mm}}$ long.

Scolex.-The heal (Plate vin, Figs. 2-2c) in preserved specimens measures $0.624-0.704^{\mathrm{mm}} \mathrm{broad}$; in a balsam-preparation of the same form

* Throngh the kindness of Prof. MeMurrich I have been able to examine the worm mentioned by him as T. expansa, and have verified the interpretation given above. There is no doubt that the worm is identieal with M. trigonophora.-C. W. S. 
itmeasured $0.768^{\mathrm{mm}}$ broad. The head passesimperceptibly into the neck, which frequently shows a pseudo-segmentation; the true segmentation begins $2^{m i n}$ back of the head, where the worm is 0.48 broarl. The suckers (under pressure) measure 0.272 in diameter. In alcohol specimens the opening is slit-form.

Plate VIII, Fig. 2, represents the head of the smallest worm found. The strobila measured $2.7 \mathrm{~mm}$ long; segmentation is visible, but no genital organs are developed. The head is pear-shaped and measures 0.48 broad ; thesuckers $(0.256$ by 0.2$)$ are directed diagonally forwards-dorso(or ventro-) laterally.

Segments $50^{\mathrm{mm}}$ from the head measure $2^{\mathrm{mm}}$ wide by $0.13 \mathrm{long} ; 10$ 14 interproglottidal sacs are present but indistinct; the genital anlagen are indistinct; no testicles can be seen; $75^{\mathrm{mm}}$ from head, about 18 sacs are visible; no testicles are developed. The genital anlage is represented by a round clump of tissue on each side of every segment.

Segments $10^{\mathrm{cm}}$ (Plate viII, Fig. 3) fiom the head measure $0.4^{\mathrm{mm}}$ long by $4.5^{\mathrm{mm}}$ broad; the genital pores are not present; the genital canals are but little differentiated; the median end-knob of the pistolshaped genital anlage is indistinctly divided into two lobes; the testicles and ovaries are not visible. At the border between every two segments a number (20-27) of blind saes are distinguishable; 150 ${ }^{\mathrm{mm}}$ from head the segments are $3.5^{\mathrm{mm}}$ wirle by $0.4^{\mathrm{mm}}$ long; testicles begin to develop; the genital eanals gradually become more distinet.

In segments $190^{\mathrm{mm}}$ from the head the testiclesare numerous. In segments $200^{\mathrm{mm}}$ from the head, the female anlage becomes quite distinct from the male ducts. It sends a prolongation toward the center of the segment; a portion branches from this toward the posterior edge of the segnent and becomes the anlage of the vitellogene gland; another portionextends toward the anterior edge; its end spreads and forms the anlage of the ovary. Between these two anlagen is seen the anlage of the shell gland. The portion of the male anlage just median of the longitudinal eanals begins to form convolutions, and its end, which is rather thin, can be traced past the female glands a short distance toward the median line of the segment. At about this stage the eanals, which were at first solid, begin to show a lumen.

Segments $30^{\mathrm{cm}}$ (Plate VIII, Fig. 4) from the head measure (balsam preparation) $6^{\mathrm{mm}}$ broad by $0.88 \mathrm{long}$. The interproglotticlal sacs have increased in number (30-36) and size. The genital pores have pierced the cuticle; they are invariably situated in the anterior half of the margin. The cirrus-pouch is well developed; the lateral portion of the vas deferens (vesiculn seminulis) is much convoluted and filled with spermatozoa; the median portion of the vas deferens crosses the ovaries; 150-240 testicles are present in each segment and are arranged in two triangles; the long leg (base) of the triangle is nearly parallel to the posterior edge of the segment, the short leg (perpendicular) parallel with the lateral margin; the hypothenuse runs from the antero-lateral 
portion to the posterior edge near the median line. While the testicles are generally included within these limits, they are frequently found outside the same, so that the triangles must be considered as a convenient but not rigid diagram.

Occasionally testicles were found in the lateral fields. This shows an initial step in the wandering of the testicles from the merlian to the lateral fields, a tendency which reaches its highest development in Th. Giardi. In the female genital organs, the canals present distinet lumens; the vagina is sharply separated from the receptaculum seninis; the three glands are distinctly differentiated from each other, both anatomically and histologically.

Segments $60^{\mathrm{cm}}$ (Plate Ix, Fig. 4) from the head measure (under pressure) $6^{\mathrm{mm}}$ broad by $1^{\mathrm{mm}}$ long. There are $35-37$ interproglottillal sacs to each segment. Copulation has already taken place, as is shown by the presence of spermatozoa in the receptaculum seminis. The various genital organs, with the exception of the uteri, can all be dis. tinguished. The female glands form a circular clump plainly visible to the naked eye on stained specimens. For a careful study of the anatomy, segments $60-80^{\mathrm{cm}}$ from the head are well adapted. The segments are nearly the same width $\left(6-6.5^{\mathrm{mm}}\right)$, but increase gradually in length $\left(1-1.36^{\mathrm{mm}}\right)$. The posterior flap of every segment overlaps the next segment by $0.24^{\mathrm{mm}}$. The arrangement of the testicles in two triangles is general, but testicles are frequently seen in the median line. The arrangement of the female genital glands in a rosette is very striking. In the general topographical relations of the organs there are noticed several important characteristics which agree with $M$. planissima, $i$.e., the genital pores are in the anterior half of the margin; on the right side the vulva is ventral of the cirruspouch, and on the left side the cirrus-pouch is ventral of the vulva; the two organs are, however, not exactly on the same transverse plane, but the vulva is regularly found slightly posterior to the cirrus. The genital canals of each side, in passing to the median field, cross the two longitudinal eanals and nerve dorsally, and the vas deferens passes the ovary dorsally. The relative position of the genital and longitudinal canals, or of the ovary and vas deferens, gives us two excellent points by which we can immediately distinguish the dorsal from the ventral surface.

Male genitalia.-The description of the eirrus-pouch of $M$. planissima applies almost equally well to this species, except that the layer of ciliary projections is not well developed; in fact, in many eases it was impossible to find it. As a general rule, a genital cloaca is present instead of a genital papilla, even when the penis is extended. That, however, is simply a matter of contraction, for, as seen below ( $T h$. Giardi), the development of the papilla in its fullest extent depends upon the absence of the cloaca and vice versa. In the genus Moniezia, however, there is a distinct collar around the genital openings even 
when the genital eloaca is reduced to a minimum. From the base of the cirrus-pouch to the free end of the extenderl penis is $0.32^{m m}$; to the base of the cloaca, 0.16. The median half of the pouch is comsiderably constricted, owing to the presence and contraction of a thick layer of circular muscles. 'The vas deferens branches freely, the branches running to the testicles, which are abont $48 \mu$ in dianeter.

Female genitaliu.-The only practical difference observed between the vulva of this species and that of M. plenissimu is that the former has a position slightly posterior to the center plane of the cirrus-ponch. The vagina is about $0.4^{\operatorname{mm}}$ long; the histology agrees with that of $M$. planissima, except that the muscular layer is slightly more developed. The ciliary layer is somewlat less developed; in fact, in most cases, it has not been possible to recognize it at all.

The receptaculum seminis lies dorsally of the ovary, but generally presents a slightly different aspect from that of $M$. planissima or of $M$. expansa; while in those latter species it was rather long and not very wide, in this species it is much shorter in proportion to its brealth $\left(0.28^{\mathrm{mm}}\right.$ long by 0.144$)$. The wall consists of a simple layer of flat epithelium; in many eases it has been impossible to distinguish any basement membrane, while in several cases it was distinctly visible. As a general rule it may be stated that the receptaculum seminis runs diagonally from before backwards instead of parallel with the anterior edge; this, however, may be subject to variation according to the state of contraction of the segment.

From the ventral side of the receptaculum seminis (Plate vinI, Fig. 5; Plate Ix, Fig. 3) near its median extremity a canal takes its origin and makes a bend ventrally. In this canal the ova come in contact with the spermatozoa (fertilization canal or first portion of the oviduct). The canal can be traced to the calyx of the ovary, with which it is connected by a constricter portion, as in the case of M. planissima.

A short distance from the receptaculum seminis this oviduct gives off a canal dorsally, which, after one or two convolutions, enters the shellgland and runs to its center. Here it meets with the vitello-duct, and from their point of juncture (ootyp) the third portion of the oviduct extends towards the uterus after making several convolutions in and outside of the shell-gland. As it passes towards the anterior portion of the segment it runs directly dorsal of the upper end of the first portions of the oviduct and empties quite suddenly into a cavity elongated laterally, which is the uterus. The exact positions of these canals vary slightly in different segments.

Histology. - The histologieal structure of all these canals agrees very well with that described in M. planissima; $i$. $e$., there is a lining epithelium with basement membrane; the constriction at the upper extremity of the oviduct is surrounded by circular fibers. The ova follow the same comse as those in M. planissima; i. e., from the calyx of the ovat rinm they descend through the constriction to the first portion of the 
oviduct (fertilization canal), come in contact with the spermatozoa, descend throngh the second division of the oviduet to the middle (ooty.p) of the shell-gland, coming in contant with the vitelline matter and ega-shell secretion; they then pass fhrongh the ascending (or third) portion of the oviduct to the uterus.

Excretory cunuls.-The ventral cauals are much larger than the dorsal canals, and are commected at the posterior border of each segment by the transverse canal. As a lule they lie laterally and rentrally of the dorsal canal; rarely a projection of the ventral canal extends somewhat dorsally of the dorsal canal (probaloly a matter of contraction). The lumen of the ventral canals measures $0.36 \mathrm{~s}^{\mathrm{mm}}$ by $0.064^{\mathrm{m} m}$, being longer laterally than dorso-ventrally. It is lined by an extremely thin cuticle, surrounded by parenchyma. The muscle-fibers of the body cluster around it so that it sometimes appears as if they formed a regular layer aromind the lumen.

The dorsal canal seldom measures orer $24 \mu$ by $9 \mu$; its cuticle is thicker than that of the ventral canal. Sudden swellings are seen, such as are described below in Th. Ginirdi.

Interproglottidal glands.-The cells of the interproglottidal glands are grouped, as stated above. Sections through these (Plate Ix, Figs. 1-2) show that numerous large cells are grouped around small blind sacs, which empty between the proglottids. The entire structure is about $0.15^{\mathrm{mm}}$ in diameter. The nuclei of the cells measure $4 \mu$. While the nuclei of these cells in M. planissima generally contained only one nucleolus, two nucleoli are observed to be the rule in this species; the cells converge towards the sac. The wall of the sac has the same histological strusture as the vagina and cirrus, $i$. e., the lumen is surrounded by a layer of ciliary projections $(5 \mu)$, which is followed by a nearly homogenous layer $(3 \mu)$ containing nuclei. It was seen above that the vagina was not an invagination of the outer cuticnlar wall, and the similarity of histology between the vagina and these sacs would naturally lead one to assume that these sacs also are not invaginations in the strictest sense of the term.

Segments $100^{\mathrm{cm}}$ from the head measured, under pressure, $6.5^{\mathrm{mm}}$ broad by $1.12^{\mathrm{mm}} \mathrm{long}$ (median line), 1.28 (on the side). The blind sacs have increased to about 40 in number. In segments $100^{\mathrm{em}}-1.50^{\mathrm{cm}}$ from the head the breadth varies from 5.5 to $6.5^{\mathrm{mm}}$, the length from $1.25-2^{\mathrm{mm}}$. A decided change is brought about in the anatomy of the segment by the change in the genital organs. The testicles, ovaries, vitellogene, and shell-glands gradually atrophy. The two uteri (Plate Ix, Figs. 5, 6) come more plainly into view, one on each side of the segment; they form numerous folds and gradually occupy the lateral and median fields, generally leaving, however, a clear space in the median line. The receptaculum seminis is full of spermatozoa and very prominent.

In connection with the above description it must be mentioned that the point ( $i . e_{\text {., }}$ the distance from the ihead) where the various organs 
begin to develop varies greatly in different specimens. In some cases the interproglottidal sacs appear before the genital organs, in other cases not until after. In several specimens of this species the sacs were very few or even entirely absent (young specimens), while in one specimen they were fused into a regular line very similar to the interproglottidal line of $M$. planissima. This is nudoubtedly to be looked "1pon as a reversion.

In the end segment of one specimen was found a single median but normally developerl set of female genital organs with a single lateral pore. This probably represents a reversion to a former type with single pores.

The mature ova measure $52 \mu-60 \mu$ in diameter; the bulb of the pyriform apparatus is $20 \mu-24 \mu$; the horns $(12 \mu-15 \mu$ long) end in a knob; the hooks of the embryo measure $9 \mu$ long. In this species it was plainly seen that the pyriform body was surrounded by a membrane, a detail which was not so clear in M. planissima.

\section{SPECIFIC DIAGNOSIS.}

The specific diagnosis of this form is as follows:

M. trigonophora sp. n., 1893.-Strobila attains $1.6-2^{\mathrm{m}}$ in length, cream to whitish in color. Head $0.624^{\mathrm{mm}}-0.704^{\mathrm{mm}}$ broad; suckers not distinctly raised, 0.256 by $0.2^{\mathrm{mm}}$, slit form. Neck filiform, $2^{\mathrm{mm}}$ long. Segments generally broader than long, although end segments are occasionally seen which are sqnare or even slightly longer than broad; rarely over $6^{\mathrm{mn}}$ broa $\overline{\mathrm{l}}$ by $2^{\mathrm{mm}}$ long. Topography of lougitudinal cautals, genital canals, and female organs the same as in M. planissima and M. expansa; testicles usually arranged in two triangles; generally absent from the median line of segment. Genital pore never behind the middle of the segment. Ova 52-60 ; bulb of pyriform apparatns 20-24 $\mu$; horns 12-15 $\mu$.

Type in the Burean of Animal Industry, U. S. Department of A griculture. Typical specimens will be sent to museums and specialists exchanging with this Bureau.

S. \& H.

\section{Denticulata Group. (M. denticuluta, and M. alba.)}

Interproglottidal glands ahsent.

(7) Moniezia denticulata (R., 1810) R. B1 1891.

[Plate V, Figs. 4-7.]

Synonymy.-Tonia denticulata Rudolphi, 1810; T. denticolata (R.) Perr., 1882; Moniezia denticulata (R) R. Bl., 1891; (.1lyselminthus denticulatus, Blainville, after Baird, 1853).

Host.-Cattle (Rudolphi).

Geographical distribution.—? ( $\Lambda$ lfort Mnsemm, Rnd.'s specimens.)

\section{LITHRA'TURE.}

(1) Rudolphi. Hist. nat. ii, 2, pl. 79-81.

.(2) - Ent. Syn., p. 145.

(3) Gurı, Lehrbuch der pathologisehen Anatomie der Hansöingethere, 1831, i, p. 381, taf. $\mathrm{x}$, figs. 3-4. 
(4) Craptin. Fndozoologische Beitrïge. 1. Ueber Trenia denticulata Rud. und Tania expansa Rud.; Wiegemanu's Archiv. f. Naturg., 1842, i, pp. 315-327.

(5) DuJardin. Histoire nat., p. 578.

(6) Dicsing. Syst. Helm., i, p. 498.

(7) Banlet. Histoire naturelle des Helıninthes des principanx mammifères domestiques, $1866, \mathrm{pp} .162-163$.

(8) Davainz. Traité, ete., 1877, p. liii.

(9) Zürn. Die tierischen Parasiten, p. 197.

(10) Perroncito. I Parassiti, ete., 1882, p. 240, figs. 101-102.

(11) LecckarT, R. Bandwiirmer; Koch's Encyclop:idie der gesammten Thierheilkunde, 1885, pp. 399-400. T. dentieulata under Inipylidium.

(12) Perroncito. Trattato, etc., 1886, p. 232, fig. 78 (after Gurlt), 80-81. (orig.).

(13) Raildiet. Eiléments, etc., 1886, p. 260.

(14) Neumans. 'Traité, ete., 1888, ı éd., p. 377

(15) R. Bianchard. Sur les Helm. des Anthr. (foot note); Móm. d. 1. Soc. Zool. d. France, 1891, p. 187.

(16) Monicz. Notes sur les Helminthes, vi, 4 ; Rev. Biol. du Nord de la France, 1891, 3 pages.

(17) R. Blanchard. Notices Helmin. 2. sér., 6; Mém. d. 1. Soc. Zool., 1891, p. 444.

(18) Neumann. 'Traité, etc., 2 él., 1892, p. 401, Fig. 183. Engl. Transl., pp. 412, 429; Fig., 183 (M. Benedeni).

(19) Curtice, C. Parasites, etc., 1892.

(20) Stiles. Bemerkungen iiber Parasiten-17: Ueber dio topographische Anatomio des Gefässsystems in der Familie Twnaida; C. f. B. u. P., 1893, bd. xilr, p. 465.

\section{HISTORICAL REVIEW (1810-1892).}

\section{Rudolphi gives as diagnosis of Trenia denticulata:}

Trenia: capite tetragono, collo unllo, articulis brevissimis, foraminibus marginalibus oppositis, lemniscis dentiformibus.

Camper. in Beschäf. d. Berlin. naturf. Freunde, Iv, p. 39.

Gmelin. Syst. Nat., p. 3074, n. 55. Trenia ovina, $\beta$ bovis.

Carlisle. Trans. Soc., Linn. II, tab. 25, Fig. 15-16. T. ovina, bovis.

Rudolphi. Hodœpor vol. I, p. 81; vol. II, p. 39. T. denticulata.

Hab. in Bove. Camperus a bove; Havemannus, Schol:e Vet. Hannov. Director merittissimus, a vitulo (solitariam, capite destitutam); Chabertus a vacca copiose, dejectam observaverunt, hic etiam in vaccæ ventriculo quarto reperit. Specimina mea ex Museo Scholæ Veterinariæ Altorfensis ditissimo.

Deser. Vermes quindecim ad sedecim pollices longi, antice duas ad quinque lineas, postice fere pollicem lati; coloris albidi, vel grisei.

Caput exiguum, tetragonum, latiusculum, osculis quatuor, anticis, subcontiguis, subglobosis, horum apertura exigua, orbiculari. Collum nullum. Articuli aliquot capiti proximi angusti, mox vero latiores, tandem latissimi et subæquales fiunt, ita tamen, ut in uno alterove specimine media vermis pars panllo angustiores et simul longiores objiciat; omnes ceteroquin brevissimi, ut longitudo latissimorum vix lineam, plurimorum ne dimidiam quidem excedat. Margines articnlorum postici crenati sive undulati superficiem insequentium qua partem tegunt; laterales (anterioribus nonnullis, rarius medius quibusdam articulis exceptis) obtusiusculi, foramine utrinque medio, opposito, insignes, e quo denticulus acutus, leviter reflexus et duriusculus (lemniscus) exseritur. Substantia mollis, plus minus crassiuscula, ut crassities interdum lineam adæquet. Ova in substantia media cumulata, ovariis regularibus mihi non vicis.

Of the articles which Rudolphi mentions, Camper has not been consulted. It does not seem that Carlisle's figures could refer to this 
species, and it serms dombtful whother Gimclin's reference is to denticulate.

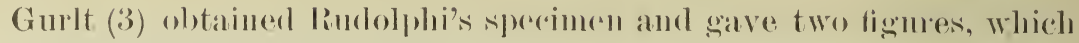
are copied (Plate V, Figs. t, j).

Creplin (4) does not believe that Carlisle's drawings refer to T. dentirmlata. Mayer's drawings (Analecten f. verg. Anat., ‘. Samml., Figs. $4,5)$ he thinks are $T$. expousu, and he is skeptical whether (amper's and Havemann's specimens were T. denticulutu. ('replin himsolf obtained Rudolphi's original specimens, and says in regard to them that there was only one specimen provided with a head; this specimen measured 1-2" long. The hearl was rather four-cornered, and sat like a knob on the end of the anterior cone-shapeal portion; the suckers formed the blunt corners of the head and opened directly in front (Plate V, Fig. 5). The portion inmerliately following the hear was wrinkled, lout it was not clear whether these wrinkles represented true seģments, hence it was doubtful whether a neck was present or not; this portion widened very rapidly. All the segments were very short; the lateral edges were convex; the posterior edge covered the following segment for some distance. The broadest seginents he had were about $\frac{1}{2}^{\prime \prime}$ wide by $1^{\prime \prime \prime}$ long, thick and bloated. Double pores were present. These were situated immediately behind the posterior entye of the next anterior segment, with the exception of "fow cuses where they were nearer the middle; * from the pores the lemuisens (cirrus) protruded; the latter was short, thick, and rather conical, on the end truncate.

Creplin then describes the ova with the pyriform apparatus. As diagnosis of $T$. denticulata he gives the following:

T. capitis tetragoni, parṿi osculis angulos ipsins efficientilus, magnis, antrorsum apertis, collo submullo, articulis ommibns crassis, ecleriter insigniterque latitudine, panlum longitidine crescentibus, anterioribus ideo brevissimis, relicuis onnibus perbrevibus atque aduodum latis, marginibus horum lateralibus convexis, postico protracto et ita sequentis articnli partem anteriorem ciremeirea late tegente, formminibus articulorun latiorem (et lemniscis) marginalilus oppositis secundis. (Articuli postici incogniti. Ova globiformia.)

Dujardin (5), Diesing (6), Davaine (8), and //iim (9) to not add any original observations on $T$. deuticulutr, but give very short diagnoses.

Baillet (7) states that $T$. denticulatu measures 35 to 40 or eren $75^{\mathrm{cm}}$ in length. He gives the measurement of the eggs as $0.09-0.095^{\mathrm{mm}}$, which, as Moniez has alrearly pointed ont, is an error. In his description there is little which aids ns in determining this species.

Perroncito (10) considers identiculate rather as a variety of expunsu. than as a separate species, but it is evident from his figures and dosipiption that he did not have a true denticulatu before him. His fignes seem to mo to resemble exponsa, while a part of his description applies equally well to expormser and planissimu.

The italies are mine. From my drawing of Rudolphi's type (Fig. 6) it is evident that Creplin male his statement on loose segments, and mistook the anterior for the posterior ellge.-C. W.S. 
Lenekart (11) has published by fir the best deseription of this species that has yet appeared (Geplin excepted). It is based upom a specimen in the Leipsic musemm. Lenckint states:

Eine aweite, dem letztern eigenthimliche Art ist dio gleichfalls den IDipylidien zugehörige Truia denticulata Rud. In ihrer Statur der Tomia cxpansa ähnlich, mnterscheidet sio sich aber nicht hloss durch eine mingleich geringere Grösse, sondern schon anf den ersten Bliek, gewisser weiterer Eigenthimlichkeiten einstweilen nicht zu gedenken, durch ein gedrungeneres, derberes und feisteres Aussehen. Ein Exenplar ron $320^{\mathrm{mm}}$ Lünge hat sehon $25^{\mathrm{mm}}$ hinter dem kopfende eine Breite von $4^{\mathrm{mm}}$ nnd in dem 100m langen hinteren Körper eine solehe von $13^{\mathrm{mm}}$. Die Linge der letzten Glieder heträgt $1.5^{\mathrm{mm}}$. IBei grösseren Wiirmern soll die Breite sogar anf $26^{\mathrm{mm}}$ heranwachsen. Der Kopf ist etwas grösser als hei Toniu expansa $(0.7 \mathrm{~mm})$ und mit grösseren Sangniipfen versehen, die ihm eine mehr viereckige Form geben. Auf ihn folgt anch hier zunäichst ein diinnerer Abschnitt, der aber sehr bald schon sich verbreitert und gliedert, and weit rascher als bei Tenia expansa zu den späteren Dimensionen heranwächst. Dabei sind die Glieder áber durchschnittiich kiirzer als bei der letzterı. Die anch hier mit zunehmender Grösse allü̈hlig inmer st:irker hervortretenden nanschettenförmigen fliedrïnder sind gewöhnlich dentlich gewellt und anch iiber die Seitenkanten hinans fortgesetzt, so dass die hinteren Ecken stïrker prominiren und an den letzten und grüssten Gliedern zahnartig nach aussen vorspringeu. Die reifen Eier gleichen denen von Tania expansa, bleiben aber mitsannut denı Embryonalkörper in ihren Dimensionen un Einiges linter denselben zuriick.

Leuckart seems to think that this species is not an uncommon parasite, although, as he has recently written (personal correspondence), this opinion is based upon report more than unon his own observation.

Railliet (13) states that the eggs are cuboid and twice as large as those of $T$. expansa.

Neumanu adds nothing new to our knowledge of this parasite in the first edition of his Traité (14).

R. Blanchard $(15,17)$ places denticulatu in his new genus Moniezia.

Moniez (16) states that denticulute is frequent in sheep killed at Lille. He gives as characteristic of this parasite that the segments are opaque. and thick, and the square head is different in shape from that of expansa. Moniez also cites Baillet's paper, and discusses Creplin's article.

I am at a loss to know how to jurlge this article by my distinguished colleague, for, although his statements are concise, it was found upon comparing specimens of the parasite he determined as $T$. denticulate that they did not agree with Rndolphi's specimens. Hence I am compelled to assume, for the present at least, that Moniez has not examined the true $M$. denticulata.

Neumann (18) lengthens his diagnosis in the second edition of his excellent work, and gives a figure of the scolex of $T$. denticulata. This figure, however, does not agree with Gurlt's figure of Rudolphi's type, but agrees exceedingly well with my drawing of Moniez's original specimen of T. Benedeni. Hence it must be assumed that Nemmann also did not find the true $M$. denticulata.

Neumann quotes Humbold (Berliner thierärztlich. Wochenschrift, 
1892) as stating that he found a specimen 45 meters long in a five.yearold cow. Humbold's statement must, of course, be taken cum grano salis. Curtice (19) is the only author who claims to have found this species in America. His specimens, however, which are now in our Bureau collection, are certainly not $M$. denticulata, although they are too macerated to be definitely determined.

From the above review it must be concluded that scarcely anything is known about $M$. denticulata; that the only authentic specimens of this species which have found their way into print are Rudolphi's specimens which he received from Alfort, and the specimen Leuckart described. We know practically nothing of the microscopic anatomy of $M$. denticulata.

Through the kindness of Geheimrath Karl Möbius, the writer has in his possession a few segments of Rudolphi's original Tania denticulata. These segments are filled with ova; they measure $8^{\mathrm{mm}}$ broad by $1.14^{\mathrm{mm}}$ long. The specimens are so old that it would be unsafe to state much in regard to them, but several points are distinctly visible. In the first place, one is inmediately struck with the position of the cirrus, which pierces the side of the segment (Plate V, Fig. 6) at or very near its posterior border. The cirrus is stout and blunt; the cirrus-pouch is $80 \mu$ long, the proximal portion $(24 \mu)$ being constricted by circular muscles, as observed in other species. The vas deferens conld be traced but a short distance; it could, however, be plainly seen that the genital canals cross over into the median field dorsally of the longitudinal canals. It will be noticed also that the lateral field is very narrow, the ventral canal coming very close to, or even crossing, the proximal portion of the cirrus-pouch. The outer canal can be distinctly seen to occupy a position slightly ventrad of the other canal, and to have a thinner cuticle. Hence the former, by analogy, is the ventral, the latter the dorsal canal. The transverse canals were not visible. The genital glands have all been suppressed by the uteri. The nteri were confined to the median field. The ova are round ( $48 \mu$ in diameter), the embryo measures $12 \mu$ in diameter and is surrounded by a pyriform apparatus. No interproglottidal glands could be discovered.

With the aid of the three figures of this species given in the present paper, it is hoper that some one will be able to determine Moniezia denticulate and give an account of its microscopic anatomy.

\section{SPECIFIC DIAGNOSIS.}

The following may be accepted as a provisional diagnosis of this species:

Moniezia denticulata (R., 1810) R. Bl., 1891.-Strobila 32-40 $\mathrm{cm}$ (to $78^{\mathrm{cm}}$ ) long; head $0.7 \mathrm{~mm}$ broad, with proportionately large suckers directed anteriorly; nock absent or very short, anterior segments very short, increasing rapidly in breadth, but gradually in length as they grow older (Gurlt's figure); $25^{\mathrm{mm}}$ from head they measure $4^{\mathrm{mm}}$ wide; 
segments may attain $13^{\mathrm{nm}}$ to nearly $25^{\mathrm{mm}}$ (R.) in breadth; mature segments quite thick; sogments $8 \mathrm{~mm}$ broad by $1.14 \mathrm{long}$ are filled with ova. Genital pores are in the posterior half of the margin; in segments of Rudolphi's type they are close to the postrior corner of the segment (ef. also Gurlt's figure); penis prominent and blunt; relation of vas deferens (and probably of the vagina) to the longitudinal canals the same as in $M$. planissima; nteri (always?) confined to the median field; eggs $48 \mu$, bulb of pyriform body 12-16 $\mu$, horns 8-16 $\mu$.

Type in the Konigl. Musenm fïr Naturkunde, Berlin. A few segments of the type are in the private collection of C. W. Stiles.

C. W. S.

(8) Moniezia alba (Perroncito, 1879) R. Bl., 1891.

[Plate X.]

Synonymy.-Tania alba Perroncito, 1879; Moniezia alba (Perr.) R. Blanchard, 1891; M. alba var. dubia Moniez, 1891.

Hosts.-Sheep (Perr., Mattozzi, Neumann, Blaise); cattle (Perr., Moniez, Railliet).

Geographical distribution.-Italy ('Turin, by Perroncito; Macerata, prov. Marches, by Mattozzi). France (Lille, by Moniez; Alfort, by Railliet). Algeria (by Blaise, after Neumann).

\section{LITERATURE.}

(1) Perroncito. Di una nuova specie di Tienia (T. alba); Analli della R. Accad. d' Agric. d. Torino, 1879.

(2) —_- Ueber eine neue Bandwurmart (Tania alba); Arch. f. Naturgeschichte, 1879, 45. Jahrg., I, pp. 235-237; taf. xvi, figs. 1-10.

(3) Moniez. Note sur deux espèces nouvelles de tienias inermes, T. Vogti et $T$. Benedeni; Bull. scient. d. Dept. d. Nord (1879) 1830, p. 163.

(4) Perroncito. I parassiti, etc. 1882, pp. 243, 214, figs. 103-105.

(5) Züris. Die tierischen Parasiten. 188:2, p. 197.

(6) Leuckart. Bandwïmer; Koch's Encyclopädie der gesanmten Thierheilkunde, $1885 ;$ T. alba, p. 400.

(7) Railliet. Éléments, etc. 1886, p. 260.

(8) Perroncito. Trattato, etc. 1886, pp. 233-234, figs. 79, 82.

(9) Neumann. Traité, etc. 1. él., 1886, pp. 378, 382.

(10) ——. Observations sur les ténias de moutons; Compt. rencl. d. l. Soc. d' Hist. Nat. d. 'Toulouse, 1891, 18 mars.

(11) Blancharis, R. Sur les Helıninthes des primates anthropoides; Mém. d. l. Soc. Zool. d. France, 1891, p. 187 (foot note).

(12) Monifz. Notes sur les Helminthes, Vi, 5 ; Rev. Biol. d. Nord d. I. France, 1891, IV.

(13) Blancharo, R. Notices Helminthologiques; 2 sér., 7; Mém. d. 1. Soc. Zool. d. France, 1891, p. 444.

(14) Neumann. Traité, etc. 2. éd., 1892, pp. 403-408, Fig. 187; Engl. Transl., pp. $412,418$.

(15) Stules. Notes sur les parasites-14: Sur le Tania expansa Rud; Compt. rend. d. 1. Soc. d. Biol., Paris, 1892, p. 665.

\section{HISTORICAL REVIEIV.}

Perroncito's Italian article (1) is not at my disposal, but he printed an article (2) in Germany about the same time, and that probably contaius substantially the same data. The description reads:

T. candida, multo rarius hinc inde tractu. quodam diluto-flavescente vel flavidoterreo (ochreo) intertincta, sat procera, eximie elastica, metr. 0.60-2.50 longa. Statu 
recrute (mox ex intestino sublata) pallida apparet articulisque s. proglottidilus elongatis, saepe longioribus qunm latis, efformata videtur-qui articuli rleinde contrahuntur magisque bati quam longi fiunt.

Caput subgloboso-quadrangulare, sat distinetum, mun. 1.40-1.15 latum, vix ultra mm. 1 longum, acetabulis (s. osculis suetoriis) pracditum orbicularibus vel subovalibus. extrorsum ae sursum spectantibus, diametro num. 0.356-0.456.

Collum breve, saepe depressione circulari constrictum, mm. 1.5-5.320 longmm, mm. 0.6-0.912 crassum.

Proglottides, quarmu summae num. 0.020-0.038 longae atque valdo angustae sunt, sensim longiores et latiores funt, formanque assequmntur rhomboideam, subcampanulaceam, angulis posticis obtusis, ita prominentibus, nt partim proglottidem subsequentem obtegant corpusque taeniae sat acute scrratum appareat.-Quae c. dem 1 a capite distant, jam ultra $\mathrm{mm}$. 3 longae et c. tertiam 1 m. partem latac smut.- Q que medium taeniae tractum efficient, longitndinem mm.3-3.5, latitudinem 4-5 1 m. praebent atque poris gentitalibus manifestis, in quavis proglottide binis, oppositis, inter tertiam proglottidis partem anteriorem et mediam sitis, instructae sunt. Cirrhus plerumque prominens ac tenuis, conicus vel cylindracens, rectus ant vario incurvus (saepius retrorsum deflexus).--Pars tacniae postica crassior, articnlis brevioribus ac latioribus, circa $1.5 \mathrm{~nm}$. erassis, mun. 2-3.5 nsque ad 4-5, raro 5-6-6.5 longis, mm. 8.5-9.5, raro ultra 10, rarissime 12-14 longis efformata. Proglotticles naturae plerumque contractae cerumtur ac valde breves (2-3 nm. longae), uteris bin is (utrinque singulo) instructae, qui loculis creberrimis, invicem coninnetis, constant, unde, proglottide puncta, ingens ovulorum copia prormmpit.

Ovula candida, cubica (quavis facie quadrata vel rectangulari, angulis rotundatis, lateribus convexis, mm. 0.048-0.052, subiude etiam 0.058 longis), testan praebent duriusculam, chitino efformatam atque guttulis arliposis variae maguitudinis obtectam. Matnra embryonem fovent, qui corpore sphaerico at duobus processibus ancto constat ac plemunque situm diametralem in ovulo tenet (corpus enim embryon is plerumque angulo huic aut illi adiacet, dum processns oppositum ovuli angulum petunt). Corpus, scilicet pars embryonis princeps mainscula, sphaerica et homogenea, ntriculum constituit, cuius media moloscolex inclusns est globosus, protoplasmate homogeneo (dense at pallide gramuloso) nucleisque 2-4-5 efformatus atque unculis longiusculis 6 pracrlitus, quormu ope se in utriculo movet rotatque. Processus snpra dicti ab illo corporis latere, quod centrum oculi spectat (exiguo inter ipsormu exortum spatio interjecto) prodeunt, inter se convergentes ac denique aut decussati ant valde appropinquati : alter glochidliatus, seilicet perlicello constat gracilisnb-conico, sensim attenuato, apice in cuenllum sive appendieulam semilunarem (antrorsum convexam, retrorsum concavan, ad latera utrinque acuminatam ac recnrvam) expanso; alter praecedentis pedicello consinilis et aequilougus, exappendiculatus, illius appendiculum denique attingit (cum ipsa tamen non conflnens).

The author also adds a short differential diagnosis between rlba, expansa, and denticuluta. The ten figures are good so far as outline goes, but details are lacking. 'Tho parasites were found both in cattle and sheep. By comparing this with Perroncito's next article it is noticed that in giving the measurements above "12-14 lomgis efformata," longis is a misprint. 'The brearlth is here referred to.

Moniez (3) states that he has found this species quite common at Lille, France.

Perroncito $(4,8)$ gives sulostantially the same diagnosis in his work on parasites which he gave before (2). Y/ium (5) eopies his diagnosis fom I'erroncito's Italian article. Railliet (7) states that he finds very little difference between this species and T' expansa. Nemmann (10) states in his analytical hable that the segments become longer than broad, 
and do not exceed $10^{\mathrm{mm}}$ in wilth. Blanchard $(11,13)$ places $T$. albo in his new genus Monieziu. Moniez (12) describes a M. ulba var. dubia which, he states, is intermediate between $T$. albu and $T$. expansa.

Prof. Perroncito has kindly sent to me three specimens of his original type material from which he described this species, and Prof. Neumann has sent a specimen of a worm which he diagnosed as $T$. alba. Unfortunately it has been almost impossible to stain Perroncito's specimens satisfactorily, hence it is hazardous to make many definite statements in regard to them.

\section{ANATOMY.}

General appearance.-In regard to the anatomy of the segments, there is no doubt that the topographical relations of the vulva, cirrus, vagina, vas deferens, ovary, shell-gland, vitellogene gland, uterus, longitudinal nerves, dorsal, ventral, and transverse canals are the same as found in the other species of Monieza. The testicles are arranged in a quadrangle. No interproglottidal glands of any description could be distinguished. Had sacs been present they would undonbtedly have been discovered, notwithstanding the poor staining, for the heavy cuticular lining of the sacs always stands out prominently, no matter how macerated the specimens are. As the head and segments are totally different from $M$. expansa, and as no saes are present, it can therefore be stated with certainty that $M$. alba is not identical with $M$. expansa. In some segments of other species, which were even more macerated than Perroncito's three specimens, it has been possible to distinguish linear interproglottidal glands. As they were not seen in Perroncito's specimens, it is assumed that no interproglottidal glands are present in the species $M$. alba.

Ova.-The eggs neasure $61-68 \mu$; the bulb of the pyriform body $20-24 \mu$; the horns 12-20 $\mu$.

Prof. Neumann's specimen is in somewhat better condition than the type specimens, and is evidently identical with Perroneito's Trenic alba. The total length is $105^{\mathrm{cm}}$. The head is too distcrted to warrant description; it does not, however, bear any very great resemblance to the head figured by Neumann as T. alba.

Segments $100^{\mathrm{mm}}$ from the head measure $2^{\mathrm{mm}}$ wide by $0.4 \mathrm{long}$. The median field is filled with testicles, which are as numerous in the median line as elsewhere. The genital canals and female glands have the characteristic pistol-shape, the muzzle of the pistol being situated near the lateral edge of the anterior third of the segment. The anlagen of male and female canals are distinct; the female glands represent a more or less circular clump, which begins to show a slight differentiation into the component parts. There is no sign of interproglottidal glands. The posterior flap, of course, forms a dark line across the segment, but this is not homologous with the line of glands seen in M. plan. issima.

$7114-N o .4-4$ 
Segments $320^{\mathrm{mm}}$ from the head show a very noticeable advance in the development of the sexual organs. The proglottids measure $3^{\text {mm }}$ wirle by 0.8 long. No interploglottidal glands are visible. The posterior flap of each segment extends but slightly over the next following segment.

The genital pore has pierced the segment in the anteriol third of the lateral edge. Cirrus-pouch and vagina are well developed; copulation has already taken place, as is shown by the fact that the receptaculum seminis is full of spermatozoa; the female glands form a large rosette (diameter 0.56) just median of the lateral canals, the vitellogene and shell-glands being almost completely surrounded by the ovarium. 'The numerous testicles occupy the entire remaining part of the median field.

Seginents $55^{\mathrm{cm}}$ from the head measure $4^{\mathrm{mm}}$ wide by $1.44^{\mathrm{mm}}$ long. The uterus now fills the entire.median field and a portion of the lateral fields, suppressing the other genital organs, with the exception of the receptaculum seminis and sexual canals. On these segments it could be proved that on one side the cirrus was ventral and the vagina was dorsal, while on the other side the cirrus was dorsal and vagina ventral. As the longitudinal canals could not be plainly seen, it is impossible to state which side is right and which left, but from analogy with the other forms it may be assumed that the side on which the vagina is ventral is right, the opposite side is left. The vagina is slightly posterior to the cirrus-ponch.

Segurents $80^{\mathrm{cm}}$ from the head measure $4^{\mathrm{mm}}$ wide by $1.76^{\mathrm{mm}}$ long. The uterus occupies almost the entire segment, and is filled with eggs. It could be proven that the uteri cross into the lateral fields only on one side (from analogy, dorsal side) of the large canal, and that the vagina passes on the same side, so that our analogy of ventral and dorsal, right and left sides, made above, is further supported. The dorsal longitudinal canals could not be distinguished. A transverse canal could be distinguished connecting the two ventral canals, thus making our analogy complete, and establishing the fact that we find the - same topographical relations in this species which we find in M.plan. issima. The only point now left undetermined is whether the dorsal canal lies dorsal or ventral of the vagina and vas deferens.

Segments 1 meter from the head measure $5.5^{\mathrm{mm}}$ wide by $3^{\mathrm{mm}}$ long. The proglottids represent sacs filled with the uteri containing eggs; the pores are still plainly visible and a very slight trace of the receptaculum seminis can still be seen.

Eggs. - The ova measure $60-68 \mu$ in diameter, the bulb of the pyriform body $16 \mu$, the horns $8-10 \mu$. Perroneito states that the eggs are cuboid, but that character is of course dependent upon reciprocal pressure, and hence of no diagnostic value. The measurements of the ova given by P'eroncito and Neumann (in his Traite) are $48 \mu-52 \mu$, by Monic\% alonut. $70 \mu$. 
The following may be given as a specific diagnosis of this parasite:

M. alba (Per., 1879) R. B31., '91.-Whitish, 0.60m-2.50m long. Head, sub-quadrangul:u, 1.40-1.15mm broad; suckers slightly lobed, opening small and pointing anteriorly. Neck $1.5-5.3^{\mathrm{mm}}$ long by $0.6-0.9^{\mathrm{mm}}$ broad. Mature segments attain $8-14^{\mathrm{mm}}$ broad by 2-6.5 long by 1.5 thick. Topography of organs same as in M. planissima; testicles arranged in a quarangle; genital pores in anterior half of the segment. Interproglottidal glauds absent. Ova $60-88 \mu$; bulb of pyriform body $16-24 \mu$; horns $8-20 \mu$.

'Type specimens with Prof. Perroncito, Turu, Italy; Bureau of Animal Industry, and private collection of $\mathbf{U}$. W. Stiles, Washington, D. U.

C. W. S.

\section{Moniezia. (Undetermined specimens.)}

In the Bureau collection there are several very peculiar specimens of the genus Moniezia, which at present we are not able to determine specifically. They are all totally different from the forms describer above, and it is believed they will be found to represent new species, at least new varieties, as soon as sufficient material can be obtained upon which to base an opinion.

No. 612, Bureau collection, contains a tapeworm from sheep. The interproglottidal glands are linear, but are very short. 'The head is enormous (Plate XVI, Figs. 5-5a), and totally unlike any head we have ever seen before. It is evidently closely related to $M$. Benedeni and $M$. Neumanni.

Nos. 725 and 732, Bureau collection, contain two heads and numerous loose segments of a worm of the kxpansa group. The segments, in their microscopic anatomy, agree perfectly with $M$. expansa, but the general appearance of the head (Plate XVI, Figs. 4-4b) and segments is totally different from that species. The segments are very distinct, and appear almost like a string of large broad beads. However, a specimen of T. saginata has been found in the Army Medical Museum, the segments of which represent a perfect string of beads, so that we are inclined to doubt the specific value of the peculiar appearance of the segments of these specimens. The parasites came from Virginia sheep.

No. 607, Bureau collection (borrowed from Hassall's private collection), contains a strobila $54.5^{\mathrm{en}}$ long by $2-2.75^{\mathrm{mm}}$ broad (Plate XVI, Figs. 1-2b). Interproglottidal glands could not be distinguished; specimeri is poorly preserved. Genital pores are double; segments become longer than broad. This is the strobila referred to in the discussion of $T$. Vogti. (Of. 84). Segments measure $2.5^{\mathrm{mm}} \operatorname{long}$ by 2 broad, to $1.5 \mathrm{long}$ by 2.75 broad.

C. W. S.

GENERAL SUMMARY OF THE GENUS MONIEZIA.

From the above discussion it will be seen that the species M. planissima, M. Benedeni, M. Neumanni, M. expansa, M. oblongiceps, M. trigonophora, $M$. alba, and $M$. denticulata possess certain character's in com- 
mon which compel us to follow Blanchard in uniting them in one genus. Some of these characters could not be traced in all the species owing to a paucity of material; nevertheless, the following will serve as a general summary of the anatomy of these forms.

The seolex is never provided with hooks. The segments are generally broader than long and longer than thick, althongh the end segments show a tendency to become longer and narrower, in some cases the length becoming equal to or (rarely) even greater than the breadth.

Genital organs.- The first appearance of the genital organs is in seginents near the head, in the form of a round clump of tissue on each side of the segment, situated at the edge of the median field just inside the longitudinal canals. Gradually this genital anlage extends across into the lateral fields on the dorsal side of the lateral canals and nerves, assuming a characteristic pistol shape. It then becomes divided into an anterior (male) portion and a posterior (female) portion, which are at first solid, but in which eanals gradually develop. The cuticle on the edge of the segment then invaginates at a certain point to meet the canals. At this point is situated the genital pore.

Male genitalia.-The anterior canal mentioned above differentiates, at its lateral end, into the cirrus-ponch; the remaining portion (vas deferens) becomes convoluted and runs towards the median line of the segment, passing on the dorsal side of the ovary. The testicles in many (all?) cases are at first arranged in two triangles, one on each side of the segment. This arrangement remains more or less constant throughout life in one species (M. trigonophora), while in (all?) the other species testicles appear in the remaining portion of the median field, thus forming a quadrangle. It is very rare that testicles are found in the lateral fields.

Female genitalia.-The lateral end of the posterior portion of the above-mentioned pistol-shaped genital anlage forms the vagina, the vulva being situated ventrally of the cirrus on the right side of the segment and dorsally of it on the left side. A receptaculum seminis is formed by the portion of the canal inside (median) of the longitudinal canals, while the extreme median lohe-like end differentiates into ovary, vitellogene gland, and shell-gland, together with the canals comnecting the same as described on pages 20,40 . The female glands do not develop quite so early as the male glands, but they persist longer.

A uterus is present on each side of the segment. The two uteri form numerous folds, extending into the lateral ticlds (in all cases?, cf. $M$. den. ticulata), across the dorsal side of the longitudinal canals and nerves, and also toward the median line of the segment. Generally it is impossible to distinguish the uteri from each other in the median line of the older segments, but in M. trigonophora they more frequently remain distinct. As the eggs are developed and the nteri increase in size, the genital glands atrophy, the male organs disappearing before the female organs. Receptaculum seminis, vagina, cirrus-pouch, and the lateral 
portion of the vas deferens persist the longest. One segment has been found with a single median set of female organs and a single lateral pore.

The ova are spherical, but by reciprocal pressure they become cuboid. A pyriform apparatus surrounds the oncosphere.

Excretory organs of the segments.-On each side of the segments are fomd two longitudinal canals rumning fom the head to the posterior extremity, erossing the genital canals on their ventral sicle. The large lateral canal represents the ventral canal; its euticular lining is extremely thin; near the posterior border of each segment the ventral canals of the two sides are connected by a transverse canal possessing a thin euticular lining. T'he other longitudinal canals lie medio-dorsal of the rentral canals, have a thick cnticular lining, and are not connected in the segments by any transverse canals.

Nerves in the segments. - On each side of the strobila there extends a longitudinal nerve from the scolex to the posterior extremity, running lateral of and parallel with the lateral longitudinal canals. It crosses the genital canals ventrally.

Interproglottidal glands.-A number of the species mentioned above possess glaudular structures at the border between every two succeeding segments. The most simple and, as the writer considers, the primitive form of these structures, appears in species of the Planissima group, where the glaudular cells are arranged in a line parallel to the posterior border of the segment. The longitudinal muscles cause a slight grouping of these gland-cells. In members of the Expansa group the gland-cells are encentrated around blind sacs, which open under the flap of the posterior edge. These glands generally appear in segments showing a genital anlage, although the exact distance from the scolex is subject to variation.

R. Blanchard included in this genus the following forms:

Trenia expansa R.; Teuia alba Per.; T. Benedeni M.; T. denticulata R.; M. nullicollis M.; M. Neumanui M.; all of which I also arhnit to the genus.

Blanchard further classes as Monieziu:

Tania festiva R., 1819 (M. festiva R. Bl., 1891), from Macropus gigantens.

Dipylidiun latissimum Riehm, 1881 (M. Goezei (Baird, 1853) R. Bl., 1891), from rabbits.

Dipylidiun Lenckarti Riehn, 1881 (M. Lenekarti (Riehm, 1881) R. B1., (1891), from rabbits.

Tenia marmote Frölich, 1802 (.M. murmote (F.) R. B1., 1891), from Arctomys marmota.

Tania pectinata Goeze, 1782 (Dipylidium pectinatum Riehm, 1881; M. pectinata (G. 1782) R. Bl., 1891), from rabbits.

Moniez includes in the genus Moniczin the species:

Trenia ovilla Riv., 1878 (T. Giardi M., 1879; M. ovilla M. 1891), from sheep.

Thysanosoma actinioides Dies., 1834 (Touia fimbriata, Dies.; M. fimbriata, Moniez, 1891) from sheep.

Blanchard gives as generie diagnosis the following: "Corps lancéolé en avant. Anneaux serrés, beancoup plus larges que longs, avec deux pores sexuels opposés."

As the species Tania marmote, Dipylidium latissimum, D. Lenckarti, 
D. pectinatum, T. Giardi, and Thyssunosoma actiniointes have little in common with the species of Moniesin of eattle and sheep, it is proposed to exchude them from this gen!s.

Anticipating the results obtained below, it will simply be stated here that Thysunosoma actinioides and Th. Giurdi, should be excluded from Moniezia, becáuse in these two species we find in each segment a single uterus with ascon-spore like egg-sacs, instead of two uteri. Further, the genital canals pass between the longitudinal canals. For other particulars see the anatomy of these two forms.

Trenic marmote differs trom Moniezia s. st., (1) in having a single uterus; (2) the dorsal canal lies lateral instead of median of the ventral canal; (3) the cirrus and vagina do not have the same topographical relations that are found in the genus Moniezia.

Dipylidium Leuckarti, according to Riehm,* has but one uterus in each segment, while (Riehm, Plate VI, Fig. 5) what is evidently the dorsal canal agrees with $T$. marmote in lying lateral of the ventral canal.

The uterus of $D$. pectinatum, according to Riehm, agrees with that of D. Lenckarti.

In D. latissimum, according to the same author, the uterus is not "ein einfacher, stellenweise erweiterter Schlanch, der in querer Richtung die Proglottis durchzieht, er erweist sich vielmehr in zwei oder drei solcher Schläuche zerspalten, welche sich vielfach mit einander vereinigen und so Inseln von Parenchym einschliessen." Riehm's figure of the uterus is very unsatisfactory, and resembles the vas deferens more than it does a uterus. Whether he has in this case drawn the uterus or not, the anatomy (to judge from his figure) does not agree with that of Moniezia s. st.

Hence it is proposed to restrict the genus Moniczia, and the following is suggested as a-

\section{GENERIC DIAGNOSIS.}

Moniezia R. Bl., 1891. Char. emend.-Head without hooks; segments generally broader than long and longer than thick, end segments showing a tendency to become longer and narrower. Two full sets of genital organs, with two uteri and two lateral pores in each segment. On the right side the vagina is ventral, cirrus dorsal; on the left side vagina dorsal, cirrus ventral. Dorsal canal lies dorso-median of ventral canal. Genital canals cross the longitudinal canals and norves dorsally. Interproglottidal glands generally present. Calcareous bodies absent from parenchyma. Eggs with well developed pyriform body.

Besides the species mentioned above as belonging to this genus, there are several other forms which may or may not represent new species. This Bureau is not willing to commit itself in regard to the specific determination of specimens Nos. 607, 612, 725, and 732 of its collection, until further material is obtained. We ineline, however, to the belief that at least two of these forms will eventually be proven to represent new species of the genus Moniczia.

For M. nullicollis, see 1). 83.

C. W. S.

\footnotetext{
*Gottfried Riehm, Studien an Cestoden. Inang.-1)iss. Halle, 1881.
} 


\title{
PAR'T II. \\ THYSANOSOMA Dies., 1834.
}

(9) Thysanosoma actinioides Dies., 1834.

\author{
[Plate XI, Figs. 1-8.]
}

Synonymy.-Thysanosoma actinioides Diesing, 1834; Tonia fimbriata Diesing, 1850; Moniezia fimbriata (D.) Moniez, 1891; Th. actinioides Dies. (Reëstablisher, Stiles. 1892.)

Hosts.-Sheep, (Curtice, Hassall, Stiles, et al.) ; Cariacus rufus, (Natterer); C. simplicicornis, (N.); C. Nambi, (N.); C. paludosus (C. dichotomus), (N.).

Geographical distribution.-South America: Brazil, "Registo do Rio Araguay, Cuyaba, Nas Frechas, und Villa Maria" (Natterer). North America: Colorado, Utah, Nebraska (Curtice); New Mexico (Codweiss, Curtice); California, Oregon, Utah (Collins, Curtice); Missouri (Stewart, Curtice); Washington, D. C. (sheep came from Colorado-Hassall and Stiles.)

\section{LITERATURE.}

(1) Diesing. Tropisurus und Thysanosoma, zwey neue Gattungen von Binenwiirmeru aus Brazil; Med. Jahrbuch d. oesterr. Staat. Neue Folge. vii, vide pp. 105-111, taf. III, 1834, Thysanosoma actinioides.

(2) Nordmann. In Lamark, Auim. s. vert., 2 éd., III, 591.

(3) Diesing. Syst. Helm., I, p. 501, 1850, Tania fimbriata.

(4) Zwauzig Arten von Cephalocotyleen. pp. 32-33; Denkschr. d. M.-N. Classe der K. Akad. d. Wiss. Wien, 1856, T. fimbriata.

(5) Kïchenmeister. On Tape and Cystic worms, with an introduction on the origin of the intestiual worms, by C. T. v. Siebold. Trans. by T. H. Huxley, 1857, p. 42.

(6) Faville. Report Veterinary Dept. of the Colorado State Agricultural College, January, 1885, $T$. expansa, p. p.

(7) Cuntice, C. Tapeworm disease of sheep of the Western Plains; Annual Report of the Burean of Animal Industry, U. S. Dept. Agric., 1887-1888 (appeared 1889); pp. 165-184, 2 plates, T. fimbriata.

(8) — Animal Parasites of Sheep, 1890; pp. 89-109, pl. xII (orig.), Pl. xIII (Diesing's figures), T. fimbriata.

(9) Neumans. Traité, 2 éd., 1892, p. 408, figs. 193-194 (after Railliet, unpublisherl), T. fimbriata.-Engl. Transl, p. 419.

(10) Stiles. Bemerkungen iiber Parasiten-17: Ueber die topographische Anatomie des Gefässsystems in der Familie Taniada; Centralblatt f. Bakt. u. Parasitenkunde, bd. XIII, 1893, p. 457-465, figs. 7-8.

\section{HISTORICAL REVIEW.}

Natterer first discovered this parasite in Cervus paludosus in 1823. Only a few segments were found, and these were described by Diesing (1) under the name of Thysanosoma actinioides, for which he was inclined to erect a new order, Crasperlosomata, to be placed between the 
Cestodes and Trematodes. As Diesing misunderstood the nature of the animal, and mistook a single segment for the entire parasite, his description need not be reviewed.

In his Syst. Helm. (3) Diesing, having received complete specimens from Natterer, recognized that the animal was a tapeworm. $\mathrm{He}$ renamed the parasite. Trenia fimbriata and gave as diagnosis:

Caput obtuse tetragonum magnum, acetabulis hemisphericis angularibus anticis. Collum nullum. Corpus antrorsum lanceolatum, articulis cuneatis brevissimis, superiorum margine postico integerrimo, subsequentium crenato, ultimorum utrinque fimbriato. Aperturæ genitalium-. Long. $6^{\prime \prime \prime}-6^{\prime \prime}$; lat. $1-3^{\prime \prime \prime}$.

In his third publication (4) he gives substantially the same diagnosis, adding "articuli maturi soluti 1 "l longi, 2 "l lati, fimbriis lanceolatis."

Siebold's remarks (5) are simply of an historical nature.

The next article at my disposal is by Faville (6), who found tapeworms in sheep in Colorado. The worms were found in the gall-ducts and in the intestines. He includes all the worms under the name Tania expansa, but there can be little doubt that the worms he found in the gall-dncts were Th. actinioides, while the worms he describes as 5 or 6 feet long must belong to some other species.

Curtice (7) had occasion to investigate a Tania fimbriata epizoötic in Colorado in 1886 and 188i. He states that the adult specimens are $15-30^{\mathrm{cm}}$ long by $8^{\mathrm{mm}}$ wide. The broadest portion is about $2^{\mathrm{cm}}$ from the posterior end. Head is $1-1.5^{\mathrm{mm}}$ wide. Genital pores double. The segments attain maturity about the middle of the worm. The male organs develop first, and occupy the entire breadth of the young segments. The uteri develop last. Each uterus is composed of a series of bags, arranged side by sirle in a fringe, which extends along the anterior erlge of the segment. The bags empty into a tube which is connected with the ovaries. The worms are found in the gall-ducts and duodenum. Curtice found one specimen in a lamb 2 months old, but he states that yearlings and 2-year olds are more infested than lambs. The worm requires six to ten months for its development. Curtice's experiments for direct development without change of host were negative. He further diseusses the geographicai distribution and treatment. He also refers to an article by Stewart. The reference is, however, incorrect and it has been impossible to trace the paper. Curtice reprints his observations in his report on the animal parasites of sheep (8).

Neumann (9) evidently takes his diagnosis from Curtice.

Stiles (10) remarks upon the anatomy and reëstablishes the genus Thysunosoma.

Besides the above articles this species is mentioned in several places, but no new facts are given in regard to its anatomy or life history.

specific name.-According to the rules of nomenclature, the specific name given to a fragment of an animal must hold for the entire animal in case the fragment was named first. Hénce nothing remains in this case but to accept the specific name actinioides. In separating this worm, together with $T$. Giardi, from the genera Moniezia and Taenia 
s. st., it is necessary to accept the generic name Thysanosoma instead of creating a new name.

Considerable material is at hand which Curtice collected in 1856; it is, however, very poorly preserved, so that there are a number of points in resard to the structure of the parasite which it lias been foumd necessary to omit.

\section{ANATOMY.}

The scolex.-The head (Plate Xr, Figs 2,2l) measures $1-1.5^{\mathrm{mm}}$ wide; it is very prominent, much broarler (alnost square when viewerl en fuce) than long, so that it looks almost like a crosspiece placed mpon the antr. rior end of the strobila. The four suckers are very powerful, separated from eacl other by deep depressions (in other words, the suckers are prominently raised); each sucker measures about $0.48^{\mathrm{mm}}$ in diameter; the opening is circular or slightly oval, and is directerl half anteriorly at the four corners of the head. The scolex is quite sharply defined from the neck (Plate xI, Figs. 2, 2 $)$, which measures about $0.9^{\mathrm{mm}}$ broad and is very flat. The unsegmented portion is very slort, or almost absent; $0.48^{\mathrm{mm}}$ from the head the segmentation is quite distinct, while at about 1.5 from the head the posterior edge of the segments show short prolongations, which increase gradually in length as the segments grow oller, forming the fimbrice, so characteristic of this species.

Segments $20^{\mathrm{mm}}$ from the head measure $2.4^{\mathrm{mm}}$ wide by $0.24^{\mathrm{mm}}$ long. No internal organs could be distiuguished. No interproglottidal glands are present. The posterior edge of the segments is about $0.25^{\mathrm{mm}}$ broader than the anterior edge; the flapl extends over the anterior edge of the next following segment by $48 \mu$, and is divided into about 70 lobes which form a finge work around the entire segment.

Segments $40^{\mathrm{mm}}$ from the head measure $2.7^{\mathrm{mm}}$ broad by $0.32^{\mathrm{mm}}$ long. A genital anlage becomes visible on rach side of the segment, just nerlian of the longitudinal canals.

Segments $60^{\text {mm }}$ from the head (Plate XI, Fig. 3) measure 3.2mm wide by $0.35^{\mathrm{mm}}$ long; the fringe is somewhat longer in the median line $(0.16)$ than on the edge $(0.032)$.

In segments $80^{\mathrm{mm}}$ from the head the genital anlage has extended into the lateral field towards the posterior half of the erlge. The anlagen of the male and the female canals gradually separate from each other. The fringe on the posterior edge increases in length.

Segments $100^{\mathrm{mm}}$ from the head (Plate xI, Fig 4) ineasure $4.8^{\mathrm{mm}}$ wide by

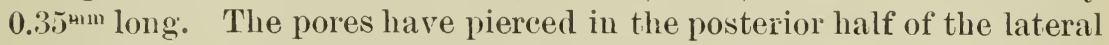
margin. The male and female canals can be distinguisled from each other. Just merlian of the longitudinal canals the vagina ends in a clump) of tissue in which it was impossible to distin guish any differentiation into ovary, etc.; the vas deferens forms a number of convolutions; the posterior portion of the median field of every segment is occupied by the testicles, which generally appear slightly less numerous in the median line than elsewhere. 
Segments $120^{\mathrm{mm}}$ from the hear measure $5.1^{\mathrm{mm}}$ wide by $0.36^{\mathrm{mm}}$ long. The vagina and cimus are very long, thin, and distinct; copulation occurs in these segments. Fig. 7 represents the penis in copulation; the genital pore is closed by contraction; the cirrus is inserted into the vagina.

Unfortunately, the material at hand is so poor that an exact descrip. tion of many interesting points in commection with this species must be onitted for the present, and as Th. actinioides is rarely found in this part of the country, it is doubtful when a complete account of the anatomy can be given. The following points could, however, be established:

The genital canals pass between the longitudinal canals, as in $T \%$. Giardi. The inverted cirrus and the vas deferens are quite convoluted, the cirrus-pouch long; the testicles are confined to the posterior half of the median field. The vagina generally passes on the dorsal side of the proximal portion of the cirrus-ponch, but the position of the vulva conld be distinguished with less certainty; in many (all?) cases it is situated anterior to the cirrus in nearly the same frontal plane. As the vagina passes into the median field (Fig. 6) it rums ventrally across the convolutions of the vas deferens, which tend towards the dorsal surface. The ovaries, etc., lie just merlian of the longitudinal canals, but the topography of the various canals can not be given. Only a single uterus (Fig. 8) is present in each segment. This is like the uterus of Th. Giardi, and so far as observed it develops in the same manner.

- The ventral canals are lateral and run parallel to the nerves; the smaller canals lie somewhat median of the lacunes, and are distinctly dorsal. At the posterior border of each segment there are two small (Fig. 5) but distinct transverse canals; the ventral transverse canal connects the lacunes (ventral longitudinal canals); the dorsal transverse canal connects the dorsal longitudinal canals. In connection with the double transverse canals it is worthy of note that the dorsal canal is not so rudimentary as in the other species described in this paper. From both dorsal and ventral transverse canals a system of extremely minute irregularly branching canals could be traced into the parenchyma of the segment.

Segments $140^{\mathrm{mm}}$ from the head measure $5.1^{\mathrm{mm}}$ wide by $0.4^{\mathrm{mm}}$ long. The fimbrix are about as long as the segment. Segments $170^{\mathrm{mm}}$ fiom the heal measure $5.1^{\mathrm{mm}}$ wide by $0.4^{\mathrm{mm}}$ long. Some of the fimbrice are $0.4^{\mathrm{mn}}$ long.

\section{SPECIFIC DIAGNOSIS.}

The following is given as a specific diagnosis of this form:

Thysanosoma actinioides Dies., 1834.-Strobila 15-30 cm long; head large, nearly square when viewed en face, 1-1.5 $\mathrm{mm}$ broad, placed like a 'T on the nerk. Suckers very large, prominent; openings large, elongated or oval, at the four corners, and directed forward or lialf forward. Nock is exceedingly flat dorso-ventrally, and quite broad. Segmentation hegins almost immediately back of the head. The 
broadest seyments measme $5-8^{m m}$ widl. hy $0.1-0.6^{m m}$ long, and are situated abont gem fron tho posterior end. The posterior segments show al decided tendency to becomes longer and narrower. Matmo segments at tain a thickness of $2.2^{\mathrm{mm}}$. The posterior flap of the segments is hroken "lp inte fimbrie, whieh in the end segments attain the leugth of the segment itself. There are present in each segment two lateral genital pores, two ovaries, two vitellogene glands, but only one uterus. The latter is situated in the anterior portion of the median field, is composed of a sinall canal with numerous blind sacs, and surrounded by a thick fibrous tissue. The genital eanals pass from the median field between the dorsal and ventral canals and dorsally of the nerve. 'The dorsal canals are somewhat smaller than the ventral canals and conneeted by transverse segmental canals. Ova measure? in diameter; horus of pyriform body not developed. (No ova at my disposal).

Typical specimens in the Bureau of Animal Industry. Specimens will be sent to museums and specialists exchanging with this Bureau.

C. W. S.

(10) Thysanosoma Giardi (Riv., 1878) Stiles, 1893.

[Plates vir Fig. 5, xıI, xim.]

Syonymy.-Tania ovilla Rivolta, 1878; Tania Giardi Moniez, 1879; Tania aculeata Perroncito, 1882; Moniezin ovilla (Riv., Mon.) Moniez, 1891; Moniezia orillavar. macilenta Moniez, 1891; Thysanosoma Giardi (Riv., Mon.) Stiles, 1893.

Hosts.-Sheep (Rivolta, Macerta, Moniez, Stiles); cattle (Perroncito, after Riv.).

Geographical distribution.-Italy (Rivolta, Perroncito, Macerta); Germany (R. Lenckart, at Leipsic); France (Moniez, at Lille; Stiles, at Paris). Not reporterl in America up to date.

\section{LITERA'TURE.}

(1) Rivolta. Di una nuova specie di Tienia nella pecora, T. ovilla; Giorn. Anat. Fisiol. e Patolog. degli Animali, Pisa, 1878, pp. 302-308, 3 figs.; (also, in Studi fatti nel Gabin. di Anat. patolog. di Pisa, 1879, p. 79, 3 figs.).

(2) Monız. Sur le Tania Giardi et sur quelques espèces du groups des inermes; Comptes rendus de l'Acad. d. Sc., 26 mai, 1879, pp. 1094-1096.

(3) Perroncito. I Parassiti, etc., Milano, 1882 (1p. 244-246, T. ovilla; pp. 246-247, T. aculeata).

(4) Monicz. Sur quelques types de Cestodes; Comp. rend. d.l'Acad. d. Sc., 6 Mars., 1882, p. 662 (T. Giurdi).

(5) Zürıs. Die tierischen Parasiten, Weimar, 1882 (pp. 198-199, T. orilla).

(6) Railduet. Eléments de Zoologie médicale et agricole, Paris, 1886 (p. 261, T. aculeata; p. 264, T. Giardi).

(7) Perroncrto. Trattato teorico-praticosulle Malattie piu comuni degli Animali domestici, Torino, 1886 (pp. 236-237, T. ovilla; pp. 237-238, T. aculeata).

(8) Neumann. Traité des maladie parasitaires von-microbiennes, Paris, 1888 (p. 383, T. aculeata, T. ovilla, T. Giardi).

(9) Monicz. Les Parasites de l’Homme, Paris, 1889, (pp. 123-124, T. Giardi).

(10) Neumarn. Observations sur les Ténias dı Mouton; Comp. rend. d. 1. Soc. d'Hist. Nat. 凡. Toulorse, 1891, 10 fev., 4 pages (T.ovilla).

(11) Moxizz. Notes sur les Helminthes; Rev. Biolog. du Nord d. 1., France, 1891, T. Iv, Oct. (Moniezia ovilla, M. ovilla var macilenta).

(12) Branchard. Notices helminthologiques, 2 sér. 7; Mém. d. 1. Soc. Zool. d., France, 1891, pp.443-446 (T. ovilla).

(13) Neumans. Traité, ete., 2 éd., 1892, pp. 408-409, 410; figs. 195-196, T. ovilla.English translation by Fleming, London, 1892, pp. 419-421.

(14) - Sur la place dı Tania ovilla Riv. daus la Classification; Comp. rend.d.1. Soc. d'Hist. Nat. d. Toulouse, 2 mars, 1892, 3 pages. 
(15) Stiles. Notes sur les Parasites-13: Sur le Trenia Fiardi; Comp. rend. d. 1. Soc. d. Biol., Paris, 1892, pp. 664-665.

(16) - Bemerkungen iiber Parasiten-17: Ueber die topographische Anatomie des Gefisssytems in der Familie Taniada; C'entralblatt f. Bakt.u. Par., 1893, bd. xI11, p. 457-465, figs. $5-6$.

HISTORICAL REVIEW.

Prof. Rivolta, of Italy, (1) seems to be the first person who noticed that this tapeworm differs from M. expansa, so frequently found in sheep, leer, cattle, etc. In 1874, while working on cestodes, he came across a peculiar form, which he decided did not belong to the species $T$. expansa, and which scarcely agreed with the species $T$. denticulata. He labeled the worm "T. denticulata?". Four years afterward he saw a parasite which Perroncito found in an ox and which he (R.) immediately recognized as identical with the form he had found in 1874 and labeled "T. denticulata?". Rivolta gave a very good description (1) of the worm, identifying most of the anatomical structures; he gave two figures of the parasite, which are easily recognizable, although unfortunately (evidently by a mistake of the editor rather than the author), the segments are turned upside down. For this new species Rivolta proposed the name $T$. ovilla.

Rivolta clearly recognized that the genital pores and ovaries were irregularly alternate; also that the testicles were placed laterally of the longitudinal canals. His description of the uterus is also quite exact. Rivolta's second paper (1879) is not at my disposal.

A year after Rivolta's publication Prof. R. Moniez (2), of Lille, France, described a tapeworm of sheep as a new species, to which he gave the name Tania Girrdi. He distinguisher two ovaries in each segment, as is plainly seen from his statement that "T'wo of them (i. e., currents of spermatozoa) are lost in the neighboring ovary; * the third extends across the segment and fecundates the ovary* of the other side." *

Perroncito $(3,7)$ next mentioned T. ovilla in 1882 and 1886, adding nothing, however, to Rivolta's deseription. He also describer as a new species (3, pp. 246-247) Trenia aculerta, which he considered as "very closely allied to, if not identical with, T. ovilla."

Moniez (4) then mentioned $T$. Ciardi again, but added scarcely anything new to his original description. In his book on the parasites of man (9) he states that the eggs of $T$. Griardi are arranged in bundles of 15-30 each, very similar to the egg bundles of Trenia madugascuriensis.

Railliet $(6)$ considered T. ovilla Riv. and T. aculeata Per. identical, and accepted the specific name $T$. aculeatn, since $T$. ovilla harl previously been used by Gmelin.

Neumann (10) then obtained type specimens of T.ovilla, T. Giardi, and T. aculeata, and decided that all three parasites belonged to one species, 
for which he accepted the name T'ovilla. A point which it is impor. tant to note is that Prof. Neumam states very decidedly that only one genital pore is present in each segment.

Since Neumann's paper appeared Moniez (11) has again written on this parasite. In this last paper he adopts the synonymy given by Nenmann, and again asserts in most positive terms that double genital pores are present, and on this account he places the worm in Blanchard's new genus Moniezia (M. ovilla). ("Je me suis récemment encore assuré de son existence de la façon la plus certaine.") In the same paper Moniez describes as a new variety of sheep-tapeworm under the name Moniezia ovilla var. macilenta, a form in which the egg-shells are small and the strobila is very thin.

R. Blanchard (12) has also recently published a paper in which he speaks of $T$. ovilla, and in which he states very positively that donble genital pores were found in a specimen of $T$. aculeatu fowarded by Perroncito, and of $T$. Giardi forwarded by Moniez (the specimen coming through the hands of Neumann).

At a meeting of the Washington Biological Society (April 16, 1892), the writer presented a paper on "Tonia ovilla and its relation to Blanchard's classification." In that paper, of which only an abstract (15) appeared in print, but which is substantially the same as the present paper, an attempt was made to harmonize the contradictory statements of the authors above reviewed. Since that date Prof. Nemmann has sent me a reprint of a paper (14) presented him before the "Société d'Histoire Naturelle de Toulouse," and also a personal letter in which he reiterates the statements of his former papers.

Prof. Leuckart has also stated (personal correspondence) that a $T$. ovilla, with alternate genital pores, has recently been found in Leipsic, and Prof. Moniez has written (personal correspondence) in regard to the form in which he found the double genital pores.

When two experts like Blanchard and Moniez state that they have found double genital pores in Tania Giardi, their statements must be accepted. Likewise, when Rivolta and Neunann state that they found alternate genital pores in Taniu ovilla, their statements must also be accepted, and we inust try to harmonize the two opposing views. Two alternatives are open to us, $i$. e., we can assume that some of the segments of $T$. Giardi have only one pore while the others have two pores, or that the forms examined represent two species. As will be seen by the text and figures given below, the former alternative is the correct one.*

* Since writing the above review, both Nelumann and I have exanined one of Moniez's slides which appeared to present two pores in one segment. Neumann, Moniez, and I now agree that in this particular slide the section is diagonal and intersects two segments. Thus this preparation does not prove that Moniez saw two pores. However, it seems to me highly probable that he saw double pores in some other specimen.-c. w. s. 
Specific name.-The specific name ovilla must of course be dropled, for Railliet has already pointed out that ovilla was used by Gmelin* to designate another worm. Moniez's specific name Giardi must be accepted, since Neumann has demonstrated that this parasite is identical with T. ovilla Riv., and since the name Giardi antedates aculcutu Per. The parasite certainly can not be united in one genus with such forms as T. saginata, nor can it be placed in the genus Moniczia. As shown below, it bears very close relations to Thysanosoma actinioides, and accordingly these two worms are placed in one genus.

\section{ANATOMY.}

The material studied was collected at one of the Paris abattoirs in the spring of 1891. The results harmonize perfectly with the statements of Rivolta and Neumann, in that as a rule the testicles of $T h$. Giardi are lateral and the genital pores are irregularly alternate. This rule, however, has two very important exceptions, $i$. e., first, segments are not infrequently found in which double genital pores are present (hence the statements of Moniez and Blanchard); secondly, in some segments the testicles are not confined to the lateral spaces, but are also found in the median field, $i$. e., in the space between the lateral canals.

Prof. Rivolta gives the total length of $T$. ovilla as $1.50^{\mathrm{m}}$; Perroncito's specimens of 'T. aculeata measured $2^{\mathrm{m}}$. I have examined a large number of segments, but no complete worm; the longest strobila was $1.20^{\mathrm{m}}$. The torm of the segments varies greatly in different specimens, and according to the state of contraction there is considerable difference in the form of the segments of the same strobila, even when the segments are in the same stage of development. Plate XII, Fig. 1, represents a strobila about $1.20^{\mathrm{m}}$ in length. It will be noticed that most of the segments are broader than long, while the end segments are slightly louger than broad. In the third and fourth portions it will be noticed that the lateral margins are irregular, owing to a slight lengthening of one side and the projection of the genital pore. This is an appearance which is frequent, though not constant. It is confined to the segments which are not full of eggs. Plate xir, Fig. 1a, represents segments in a stage corresponding to the last portion of Plate XII, Fig. 1. All the segments are broader than long, but the last segments show a tendency to grow narrower and longer, the same as in I'late XII, Fig. 1. The segments never attain any very great thickness; the anterior proglottids are quite thin. Plate Xn, Fig. 7, gives a sagittal section of a mature segment. It measured $6^{\mathrm{mm}}$ wide by $1.3^{\mathrm{mm}}$ long by $0.19^{\mathrm{mm}}$ thick. It will be noticed that the posterior erlge of one segment overlaps the anterior edge of the one next following by about $96 \mu$.

* Limmés Systema naturae, p. 3061. "Tania ovilla, 20. T. ovis arielis Goeze, Eiugew, p. 257." 
Among the French tapeworms cxamined not a single head was noticed which could be positively identified as belonging to Th. Giardi. 'Two heads were found in a bottle containing segments of "Th. Giurdi and $M$. expansa, and it is probable that one of these heads belongs to the species now under consideration.

Perroncito $(3$, p. 246) describes the scolex of $T$. aculeata as follows:

Testa depressa, quadrangolare, col iato maggiore di millimetri $0.560-0.589$, col lato minore di millim. 0.250-0.300, lunga millim. 0.500 ; ventose rivolte in fuori, divise da nua depressione, del diametro longitudinale di millim. 0.256-0.261, trasversale di millim. 0.200-0.208. Collo breve rappresentito da un restringimento o collaretto del diametro di millim. 0.536 .

Neumann (13, p. 408) describes the head as "tétragone ayant un peu plus d'un millimetre de largeur, suivie d'un cou assez Iong, de largeur moitié moindre que celle de la tête."

One of the heads mentioned above agrees very nearly with the description, and is probably the head of Th. Giurdi. It was connected witl a strobila $38^{\mathrm{mm}}$ in leugth.

The head. (Plate xir, Fig. 2) is quadrate, stout, $0.506^{\mathrm{mm}}$ broad, $0.33^{\mathrm{mm}}$ long (from the anterior extremity to the constriction of the neck). The suckers are very strong, nearly round, with a diameter of $0.192 m$. The opening of the suckers in my specimen is elongated. The constriction back of the head measures $0.816^{\mathrm{mm}}$. Segmentation, at first extremely indistinct, begins at this point; $1.6^{\mathrm{mm}}$ distal from this point the segmentation is very distinct. The first anlage of the genital organs is noticed about $2.25^{\mathrm{mm}}$ back of the cervical constriction. At first only a small differentiation of tissue is seen in the median line of every segment; this arrangement (Plate xir, Fig. 3) continues for a distance of about $3^{\mathrm{mm}}$, when it is noticed that the anlage of every segment does not lie exactly in the median line, but slightly to the right or left of it. This diversion from the median line continues, so that $17.5^{\mathrm{mm}}$ from the point where it begins the anlagen lie close to the longitudinal (lateral) canals. The oldest segment of this strobila measures $2.5^{\mathrm{mm}}$ broad by $0.8^{\mathrm{mm}}$ long. The genital anlage is very simple, and no traces of testicles can be seen.

Thus it is seen that this head and strobila belong to some cestode with alternate genital pores. If it is not a T. Giurdi, it must be a new species (or T. Vogti???). The other head referred to agrees so closely with the head of $M$. expansa, that it is difficult to believe it can belong to Th. Giardi.

Plate XII, Fig. 4, represents seven young segments, each measuring $3.7^{\mathrm{mm}}$ broad by $0.176^{\mathrm{mm}} \mathrm{long}$. Six of these segments have a single anlage of genital organs, while one of the segments possesses anlagen of two sets of sexual organs, one of which is rather rudimentary. The genital pore lias not yet pierced the cuticle of the margin. From about the middle of the lateral margin of each segment two anrved and nearly parallel portions of tissue, which color darker than the surrounding parenchyma, extend across the longitudinal canal into the median field. The 
anterior string of tissue represents the anlage of the male genital canal, and is thicker in its marginal portion than in its median portion. The posterior (distal) string is the female anlage. The marginal portion is somewhat thinner than the corresponding portion of the male string; the portion which crosses the longitudinal canals is extremcly thin and, upon arriving on the median side of the canals, ends in a small knob, which later differentiates into ovarium, vitellogene glands, etc. In the third segment of the figure, both male and female organs are seen on one side; and on the other side only the male anlage is present.

The segments of Fig. 5, Plate xII, measure (balsam preparation made under pressure) $3.5^{\mathrm{mm}}$ broad by $1^{\mathrm{mm}}$ long (median line); occasionally the edge of the segment upon which the genital pore is situated is slightly longer than the edge of the other side, so that the strobila has a zigzag appearance. The pore is in the middle or back of the middle of the margin.

In segments such as are shown in Fig. 5, Plate xIl, we have the following arrangement of the genital organs. The genital pores are irregularly alternate, sometimes protruding prominently from the edge of the segment in the form of a papilla, at other times nearly on a line with the edge.

Male organs.-The lateral fields are almost entirely ocenpied by the male organs: the anterior portion (of the papilla-lateral field) by the vas deferens* and cirrus-pouch, the posterior portion by the testicles. The cirrus is oecasionally seen protruding from the pore, at other times lying in the cirrus-pouch. The cirrus-pouch $\left(0.32^{\mathrm{mm}} \mathrm{long}\right)$ is more or less bottle-shape, varying somewhat both in size and form in the different segments. Anterior to the cirrus-ponch the vas deferens makes a 11 m. ber of turns, then crosses the lateral eanals into the middle field, extends diagonally across the ovary toward the posterior (distal) portion of the segment, and branches; one branch extends back to the testicles of the pore side, while the other extends toward the opposite side of the segment.

On the pore side about fifty testicles are found ocenpying the posterior portion of the field, and on the opposite side about ninety are found, but they occupy the anterior portion of the field as well as the posterior portion. The testicles are about $0.024^{\mathrm{mm}}$ in diameter; they do not all lie in one plane, but by focusing ean be proven to lie in both the dorsal and ventral portion of the segment.

Female genital organs. - The vulva is situated close to the cimrus; the vagina follows the general curvature of the cirrus-pouch to a point about equidistant fiom the anterior and the posterior margins of the segment, where it curves, crosses between the longitudinal canals diagonally, and enters the median fich. Directly median of the longitudinal canals it dilates slightly into the receptaculum seminis, at the

\footnotetext{
* Neumann incorrectly figures testicles in this portion of tho field.
} 
end of which several organs come together, but in this stage it is scarcely possible to make out their exact relations. It can, however, be seen that a canal (ascending oviduct) extends toward the anterior edge of the segment crossing the ovary and forming a triangular dilatation. The base of this triangle extends parallel to the proximal edge of the segment in both directions towards the longitudinal canals. This, it will be seen later, is the anlage of the uterus. The branched ovary is seen at the point where the vas deferens crosses the oviduct. Posterioi (distal) to the receptaculum seminis and ovary is a differentiation, which will be seen later to be the anlage of the vitellogene gland.

In segments (Plate XII, Fig. 6) measuring $3.4^{\mathrm{mm}}$ broad by 0.8 long, the genital organs have increased in size, and the segments themselves are sharply defined from one another.

Plate VIr, Fig. 5, shows a slightly diagrammatic drawing of the end portion of Fig. 6, Plate XII (microtome section). A small opening on the lateral margin of the segment leads into a sexual cloaca. The size of this sexual cloaca depends upon the position of the cirrus-pouch. If the cirrus-pouch is retracted into the body as in Fig. 6, Plate xII, the cloaca is quite deep, but if the cirrus protudes the cloaca becomes inverted and forms what is called the sexual papilla (see Plate xiI, Fig. 5). Thus the genital cloaca and genital papilla are not coustant organs, but the one (in its fullest development) depends upon the absence of the other. When the papilla is present it is nearly filled up by the cirrus-ponch; when the cloaca is present the pouch begins at the deepest point of the former. The pouch is somewhat pear-shaped; the broader portion is situated towards the anterior margin of the segment; the narrower portion is slightly curved. The pouch of Fig. 6, Plate XII, measures $0.42^{\mathrm{mm}}$ long. From the deepest point of the cloaca (highest point of the papilla) two canals extend towards the interior of the segment.

Male organs.-The anterior caual enters the tip of the pouch, in which it makes a number of irregular turns; the first portion is of nearly uniform size and provided with a lumen of $4 \mu$, which is lined by a rather heavy cuticle. The wall of this portion is about $5 \mu$ thick. 'This is the cirrus or penis which is drawn into the eirrus-pouch. In one case the extruded penis was filiform, $80 \mu$ long. This canal gradually widens until the lunen measures about $25 \mu$; the cuticle and wall become much thinner. The end of the broad canal suddenly dilates into a large vesicula seminalis, with a lumen of $60 \mu$. From the vesicula seminalis the vas deferens, $64 \mu$ in diameter (lumen $8 \mu$ ), extends into the anterior portion of the lateral field; the thick walls contain a tissue which is probably of glandular nature (prostata). The vas deferens decreases in diameter $(32 \mu$, lumen $8 \mu)$ as it extends towards the longitudinal canals; it passes on the dorsal side of the large canal (lacme of some authors) and on the ventral side of the small canal. 7114-No. 4-5 
After passing the longitudinal canals the vas deferens runs across the ovarium, on the dorsal side of the latter. As stated above, it branches; this branching sometimes takes place before it reaches the ovary, but more frequently not until it has passed to the median side of the latter. The epithelial nature of the vas deferens is very evident, and at times an extremely fine cuticle (a basement membrane) could be distinguished. The testicles measure about $80 \mu$ in diameter.

Female organs.-The second canal, which extends from the end of the genital cloaca, represents the vagina. In the genus Moniezia we saw that this vaginal canal bears a constant relation to the cirrus-pouch, for on the right side of the strobila the vaginal opening and canal are constantly on the ventral side of the pouch, while on the left side they are dorsal. There seems to be no such constant topographical arrangement of the vaginal canal in the ease of Th. Giardi, for although in Fig. 5, Plate VII, it is situated somewhat ventrally, it is plainly posterior (distal) in several segments of Fig. 5, Plate XII, and segments have also bees found in which the canal was dorsal. The total thickness of the canal at the beginning is about $28 \mu$; it increases gradually in thickness as it approaches the longitudinal canals, where it measures $40 \mu$.

Zschokke states* that the vagina of $T$. expansa is composed of four layers: 1 (ontside), a simple layer of circular muscular fibers; 2, a simple layer of longitudinal muscular fibers; 3, a homogeneous and brilliant tissue, and 4 (inside), a cellular membrane bearing cilia or hooks which project into the lumen of the vagina, and possibly serve in advancing the sperm cells into the receptaculum seminis.

In Th. Giardi I have been able to distinguish Zschokke's fourth layer very plainly, but have not been able to make ont its cellular nature. The third layer is also very evident in my preparations. The first and second (outside) layers, which the eminent Swiss zoölogist deseribes in his excellent article on $T$. expansa, do not appear in my preparations of Th. Giardi. On the contrary, only one layer appears outside and that is distinctly cellular in its nature. These three layers end abruptly at the receptaculum seminis, which exhibits a distinct inner epithelial layer with a fine outer basement membrane.

Just median of the receptaculum seminis and connected with it is a small bulb in which the vitello-duct and oviduct come together; or, more properly speaking, the first portion of the oviduct extends from the calyx of the ovary toward the receptaculum seminis, on the median side of which it widens to receive the spermatozoa and vitelline matter. The ascending portion of the oviduct (not seen plainly on transverse sections, but very evident on fiontal sections) then extends toward the anterior portion of the segment and empties into the nterus. The vitello-duct lies somewhat dorsally and distally of the descending oviduct, and empties into the bulb just referred to.

* Recherches sur la structure anatomique et histologicuo des Cestodes, p. 108. 
It shows the same histology as the receptaculum seminis, and widens into a calyx around which are grouped the vitellogene cells. The gland in this stage measures about $0.16^{\mathrm{mm}}$ in diameter. The cells of this organ color (in acid carmine) much darker than the cells of the surrounding parenchyma or the epithelial cells of the ducts. The descending oviduct agrees in histology with the vitello-duct, and widens also into a calyx around which the ovarial tubes are grouped.

The ascending oviduct is about $12 \mu$ thick, and consists of a simple epithelium with basement membrane. It passes dorsally of the ovarium, indenting the same, or even passing through its dorsal portion. The part of the oviduct between the ovary and the uterus is surrounded by a cellular tissue (shell-gland?) which colors about the same as the epithelium. The uterus in this stage is made up of a conglomeration of cells, but no lumen can be distinctly seen.

The vascular system drawn in Fig 5, Plate VII, is very instructive. Two longitudinal canals of different thickness and histological structure are seen. The external (ventral) canal presents a lumen of about $80 \mu$, and near the posterior edge of the segment is connected with the corresponding canal of the opposite side by a transverse canal. The latter extends also into the lateral field of the segment. The lumen of the large canals (lacunes of some authors) is bordered by a fine membrane. Zschokke figures and describes a regular epithelium on the outside of this membrane, but in this species it has been impossible to recognize the epithelial nature of the neighboring cells, which seem to be exactly similar to the parenchymatic cells. These lacunes are supposed to be the ventral canals, a view which is supporter in this species by the position of the genital organs-considering the female organs as ventral and the male organs as dorsal. The small longitudinal (dorsal) canals lie dorso-median of the lacunes. The diameter $(24-36 \mu)$ varies at different points, the canal suddenly swelling into bulbs at various distances from each other.

The structure is entirely different from that of the ventral canals. The central portion, which is about $5 \mu$ broad, appears as a lumen when examined under a low power, but when examined under high power appears to be filled with a spongy mass in which no nuclei are present (coagulated contents?). This spongy centrum is bounded on the exterior by a $2 \mu$ enticle which colors very dark. This is followed by a tissue $(3 \mu)$ which colors much more lightly; the exact nature of this tissue is not clear. Next comes a distinct layer of circular fibers, which I am rather inclined to look upon as of muscular nature.

The longitudinal nerves run on the marginal side of the large canals and on the ventral side of the genital canals. In segments $4.25^{\mathrm{mm}}$ broad by $1.0 t^{\mathrm{mm}}$ long the organs are but little changed. The testicles measure $0.064-0.08^{\mathrm{mm}}$ in diameter; the ovary is considerably larger than in the segments described before; the uterus is much thicker and has assumed a wavy appearance. 
In Fig. 8, Plate xIr, where the seginents have reached a breadth of $5^{\mathrm{mm}}$ and a length of $0.72^{\mathrm{mm}}$ (somewhat shorter than the segments described last, a fact to which no importance should be attached, as this can easily be due to contraction) the " waves" of the uterus have advanced so far as to form a number of irregular parallel and connected folds. This peculiar development of the uterus is entirely different from the case presented by tapervorms belonging in the series with Truia solium, T. saginata, ete. In these latter species the uterus develops lateral branches from a median stem, the median uterus not increasing in length disproportionately to the length of the segment. In the case of Th. Giardi the uterus lies parallel to the proximal edge of the segment; the two ends of the uterus are more or less stationary, and the uterus increases in length, totally disproportionately to the width of the segment, instead of sending off branches at riglit angles. The natural physical result of this growth is that the uterus lies in folds, which, in this case, assume a direction nearly parallel to the sicle of the segment. This mode of development of the uterus seems ample ground for separating, generically, worms of this kind from such forms as $T$. solium.

As the segments iucrease still further in size (Plate xıI, Fig. 2) $\left(6^{\mathrm{mm}}\right.$ broad by $1.04^{\mathrm{mm}}$ lor $\left.\mathrm{g}\right)$ the folds of the uterus in growing latger are diverted from their position parallal to the sides and assume irregular positions; the testicles in the meantime become somewhat indistinct. In Fig. 3, Plate xiII (segments $6.5^{\mathrm{mm}}$ broad by $1.0 t^{\mathrm{mm}}$ long) the uterus occupies almost the entire median field, while the testicles are scarcely visible. The other sexual organs gradually atrophy.

Segments with more than one set of orguns.- It has already been stated that Moniez and Blanchard have found segments with double pores, and that they believed that to be the normal condition. In Fig. 4, P'late xII, is pictured a segment with an extra male anlage. A number of segments have also been found in which an extra ovary and vitellogene gland were developed; also six segurents in which double pores were present (Plate xm, Fig. 1). One of these segments belonged to a strobila sent by Prof. Neumann. All the organs were double except the uterus, of which only one could be distinguished. With this the question as to the prosence of single or double pores in Th. Giardi may be considered as definitely settled.

Eggs and egg-capsules.-Former authors have already stated that the eggs of Th. Giardi lie in capsules of tibrous tissue, each capsule containing 10-15 ova. Fig. 4, Plate xum, shows the origin of these packages of eggs. At the top of the figure is seen a canal, which represents a portion of one of the slings of the uterus. Simall blind sacs form on the side of this canal. The blind end of the sac enlarges, but still retains its communication with the utrus-stem through a narow neck. The enlarged end is filled with egass. The entire ntorus with blind sines is surrommded by a fibrous tissue, the layer being especially thick aromo the sacs. 
Fig. 5, Plate xur, represents these sam in a finther stage of development. Several egess are seen at one end, but it is almost impossible to find the neek of the sacs. The egers measme $20-2: 3 \mu$ in diameter, and appear to have but one shell, as Moniez stated. I refiain from discussing the matter of the pyriform body, as no fiesh material is at hand and preserved material is unsatisfactory in this ease. Moniez (11) states:

Il faut considérer la coque chitinense çni revêt immédiatement l'onchosphère conme homologue de l'appareil pyriforme, puiș' elle a la même origine. Le T'unia orilla se trouvant done rattaché anssi anx Anoplowephalines par le plus important des caractères de ce groupe, par l'existence de l'appareil pyriform autour de son embryon.

This is, of course, a point of great importance.

\section{SPECIFIC DIAGNOSIS.}

From the above review of former work on Th. Giardi, and from my own anatomical description, we can accept the following as a diagnosis of this species:

Thysanosoma Giardi (Riv., '78) Stiles, 1892.-Length 1 to 2 meters. Head quadrate, measuring (after Neumann) slightly more than $1 \mathrm{~mm}$; (after Perroncito) 0.56-0.589 (largest diameter), 0.25-0.3 (smallest diameter), 0.5 long; (after Stiles) 0.506 broad by 0.33 long (balsam preparation). Neck (after Nenmann) rather long, half as wide as the head; (after Per.) short, diameter 0.536; (after Stiles) alosent. The most of the segments are broader than long, thin; only the posterior are longer than broad, their breadth being less than that of the segment immediately preceding. Unripe segments present a slightly zigzag appearance, owing to the projection of the side on which the genital pore is situated. Largest segments $5-6.5^{\mathrm{mm}}$ wide by $2-1^{\mathrm{mm}}$ long. Genital pores in the middle or behind the midlle of the segment and generally irregularly alternate, but occasionally segments are found with double pores. Testicles generally confined to the lateral fields, though scattered testes are occasionally found in the median field. Uterus transverse, giving rise to folds, which run more or less parallel to the lateral margin, and possessing ascon-spore-like egg sacs which are surromelet by an extremely thick layer of tissme. Genital canals pass between the dorsal and ventral longitudinal canals and dorsal of nerve. Eggs $20-23 \mu$ in diameter; a number are found together in small sacs of the uterus; horns of pyriform apparatus not developed.

Typical specimens with Burean of Animal Industry and with Messrs. Neumann, Moniez, Rivolta, Lenckart, and Stiles.

THE SYSTEMATIC POSITION OF TANIA GIARDI.

It is evident from the above that this species can not remain in the genus Moniezia, since there is but one uterus present, and the topography of the genital canals, female glands and longitudinal canals is entirely different. It can not enter E. Blanchard's genus Anoplocephala, since the pores are irregularly alternate instead of unilateral. Neumann remarks that it approaches R. Blanchard's genus Bertia. This is true to a certain extent, but it is not deemed wise to place it in that genus until more is known about the position of the testicles, ovaries, etc., in Bertia. The occasional presence of double pores, on the other 
hand, shows that T. Giardi has some relations either with Moniezia or with Thysanosoma, and on account of its great resemblance to $T h$. actinioides in the uterus and in the topographical anatomy it may be provisionally placed in the genus Thysanosoma.

\section{GENERAL CONSIDERATIONS IN REgARD TO TH. GIARDI AND Th.} ACTINIOIDES.

From the foregoing discussion of these two species it will be seen that these forms can notpossibly be mited with the genus Moniezia, since they differ from the species of that genus already studied in a number of important characters, $i$. e., the genital canals pass between the longitudinal canalsinstead of dorsal of both canals; the cirrus-pouch appears to be ventral of the vagina on both sides (Th. actinioides), instead of alternating dorsal right, ventral left; there is but a single uterus present in each segment, and this has an entirely different structure from that of the species of Moniezia lescribed above.

In Th. actinioides the first anlagen of the genital organs appear on both sides of the median field of every segment, while in the anterior portion of what we have above supposed to be Th. Giardi (Plate xII, Fig. 3) the anlage first appears in the median line and then gradually diverges towards the laterai portion of the median field. Notwithstanding these differences the two worms are much more closely allied to each other than to the species of Moniezia above described, and should, for the present at least, be placed in a genus together. The following revised characters for the genus are proposed:

GENERIC DIAGNOSIS.

Thysanosoma Dies., 1834 (reëst. Stiles, 1893). Type species Th. actinioides.-Head unarmed; genital pores double or single; genital canals pass between the dorsal and ventral longitudinal canals and dorsal of nerve; only one uterus present in each segment; this uterus is transverse, waving, and forms blind egg-sacs which are surrounded by a thick layer of tissue; calcareous bodies absent from parenchyma; pyriform body of the eggs not well developed.

Th. actinioides and Giardi agree with each other in the relative position of the genital canals to the longitudinal canals, and in the form of the uterus. They differ from each other chiefly, however, in the position of the testicles and in the transverse canals, of which Th. actinioides has two, Th. Giardi one, to each segment.

Th. actinioides and Th. Giardi agree with Tenia marmota in having but one uterus. They differ from T. marmote chiefly in the relative position of the dorsal longitudinal canals, which in the latter species lie ventral of the genital canals and lateral of the ventral canals, and in the structure of the uterus.

Moniez states that Tania hyracis possesses unilateral pores, and that the eggs are arranged in sacs. We know, however, too little about the exact nature of the sacs in that species as well as about the topographical anatomy of the other organs to determine in what genus the form belongs.

c. W. S. 
(11) * Trenia marmotæe Fröhlich, 180\%.

[Plato VIl, Figs. 6, 7.]

Synonymy.-T. marmota Fröhlich, 1802; Moniezia marmote (F., 1802) R. Bl., 1891.

Host.-Aretomys marmota.

'This species is introdnced in this place for comparison, although it does not ocenr in cattle or sheep, becanse it is an important species to consider in connection with the genera Moniezia and Thysanosoma. Thanks to the kindness of Prof. R. Blanchard, I have been able to examine several speciniens of $T$. marmote.

In the anatomy (Plate VII, Figs. 6, 7) of this species it is important to note that two pores are present in each segment. There are also two ovaries, two vitellogene glands, but only one uterus. This uterus is, however, totally different from that of Thysanosoma, for the branches or egg-sacs are not surrounded by the heavy layer of fibrinous tissue which is so characteristic of that genus. Furthermore, the eggs, according to Blanchard, are exactly like those of Dipylidium latissimum Riehm (T. Goezei Baird-ML.Goezei (B) R. Bl.). In other words, the pyriform apparatus is well developed, which character does not agree with the genus Thysanosoma.

In $T$. marmote the topography of the longitudinal canals differs both from that of Moniezia and that of Thysanosoma, for the dorsal canal lies lateral of the ventral canal, and both of the canals as well as the nerve cross the genital canals ventrally, this latter character agreeing with Moniezia, but differing from Thysanosoma.

These characters seem to furnish sufficient grounds for separating the species Tania marmote from the genus Moniezia, and for not placing it with the genus Thysanosoma. It certainly can not be united with such forms as T. solium, etc., on account of the transverse uterus, double pores, pyriform body, etc. It would also be impossible to place it in the genus Dipylidium, for it differs very greatly from $D$. caninum ( $T$. cucumerina R.), which forms the type of that genus. The differences in the uterus, egg-balls, and rostellum are certainly sufficient grounds for not placing $T$. marmotce in the same genus with $D$. caninum.

Should a new genus be established for this worm, as must undoubtedly be done, the generic diagnosis would read:

Head withont hooks; segments broader than long. Each segment possesses two lateral genital pores; two ovaries and two vitellogene glands in the lateral portion of the median field; one transterse uterus with simple longitudinal branches. Dorsal canal between nerve and ventral canal; genital canals pass dorsally of longitudinal canals and nerve. Calcareous bodies absent from parenchyma. Eggs with well developed pyriform body.

Blanchard has already separated Riehm's species Dipylidium Leuckarti, D. latissimum, and D. pectinatum from the genus Dipylidium, and has placed them in the genus Moniezia, as M. Leuckarti, M. Goezei, and M. pectinata. The writer is in entire accord with Blanchard in the

* Type.?-Typical specimens with Drs. R. Blanchard, of Paris, and C. W. Stiles, of Washington, D. C. 
view that these three forms can not be generically united with 7 . crmimum, but he can not admit them to the genus Moniezin (see 1). 54), since-

(1) There is but one uterus present in the segments of ID. Leuckurti and $D$. pectinatum, while the uterus of I). latissimum, although evidently different from that of $D$. Leuckarti and $D$. pectinatum, is not similar to that of Moniezia;

(2) What is evidently the dorsal canal in D. Leuckurti is figured by Richm (Studien an Cestoden, Taf. VI, 5, ) as lateral of the ventral canal. Riehm's species must be restudied before a positive decision can be made, but it seems very possible that these three forms can be placed in the same genus with $T$. marmote by slightly altering the generic diagnosis given above.

c. W. S. 


\title{
PART III.
}

\section{STILESIA Railliet, 1893}

(12) Stilesia globipunctata (Riv., 1874) Railliet, 1893.

\author{
[Plate XIV.]
}

Synonymy.-T. globipunctata Riv., 1874; T. ovipunctata Riv., 1874; Stilesia globipunctata (Riv.) Rail., 1893.

Hosts.-Sheep (Rivolta, Giles); ? Cattle (See v. Linstow's Compendium.)

Geographical distribution.-Italy (Riv.); India (Giles).

\section{LITERATURE.}

(1) Rivolta. Sopra alcune specie di Tienia della Pecora. Pisa. 1874.

(2) Penroncito. I Parassiti, etc., 1882 (T.globipyuctata, pp. 210, 241; T. ovipunctata, p. 241).

(3) -. Trattato, etc., 1886, p. 235.

(4) Raildiet. Eléments, etc., 1886, pp. 260, 261 (T.globipunctata, T. ovipunctata).

(5) Neumann. Traité, 1888, p. 384.

(6) - Observations, etc.; Compt. rend. d. l. Soc. d'Hist. nat. d. Toulouse, 1891.

(7) —. Traité, 2nd ed., 1892, p.410;-Engl. Transl., p. 420.

(8) Stilfs. Bemerkungen iiber Parasiten-17: Ueber die topographische Anatomie des Gefïsssystems in der Familie Twniada; C. f. B. u. P., 1893, xıI, p. 457.

(9) Rallliet. Éléments, ete., 2nd ed., 1893 (MS).

\section{HISTORICAL REVIEW.}

Rivolta described T. globipunctata and $T$. ovipunctata in the same paper (1) in which he described T. centripunctata. The article is not at hand.

Perroncito $(2,3)$ accepted both species; the following in regard to them is abstracted from his descriptions:

T. globipunctata (globi-punteggiata, Ital.). Head $1 \mathrm{~mm}$ in diameter; suckers directed forwards; neck absent. Length $45-60 \mathrm{~cm}$; color white or greenish yellow, like the contents of the intestines. Ripe segments $2^{\mathrm{mm}}$ wide by $0.15-0.17 \mathrm{long}$. Two spherical uteri are present; they appear as two lateral rows of whitish globules in the middle or posterior third of the strobila. Few eggs are present, oval, $28 \mu$ by $24 \mu$; four hooks visible in the embryo.

T. ovipunctata (ovipunteggiata, Ital.). Head quadrangular, 0.50-0.65 $\mathrm{mm}$ broad; neck absent. Segments in sexual activity present to the naked eye two lateral lines composed of fine points, between which are seen larger bodies which are oval. This punctation is due in part to the vas deferens and in part to the uterus. The segments immediately following the head vary in breadth from $0.33-1^{\mathrm{mm}}$, with a length of 0.03 ; in mature segments the breadth is $1-2.5$, length $0.08-0.12$. Genital organs are on the sides of the segments; eggs oval, $20 \mu$ by $16 \mu$. 
The head of this worm buries itself in the intestinal mucosa and causes a legeneration of a number of the villi. It obstructs the function of the Lieberkiihn glands to a certain extent, maintains an irritation in the cytrgenic substance, giving rise to hyperplasia of the interglandular tissule, and forming nodules or elevations at those portions where the worm has fistened itself. In some places these elevations are confluent, in others isolated. Their size varies from that of a pea to a lentil, and they manifestly proceed from a circumscribed tumefaction of a fold of the mucosa.

Railliet (4) had evidently not examined the worms, but admits the two species. Neumann (5) adds that the testicles of these two species are in the lateral fields. He then (6) obtained a specimen which Rivolta had labeled "ovi-globipunctata." Neumann states that the two species are identical, and that the median line remains transparent. In the second edition of his Traité(7) he holds to the same opinion, and unites the two worms under one diagnosis.

Von Linstow includes these worms in his list of cattle parasites, and following him the writer has done the same, although unable to trace any positive authority for so doing. Later (S) I stated that a new genus would have to be created for this and the following species.

Railliet (9) establishes a new genus Stilesia for T. centripunctata and T. globipunctata. (Personal correspondence; the second edition of Railliet's work is in press.)

Prof. Neumann very kindly sent a number of segments labeled Tania globipunctata which he had received from Prof. Rivolta, and Dr. G. M. Giles, of Sanawar, Punjab, India, has forwarded a number of specimens from India. Dr. Giles writes:

The worms are very social, dozens being found coiled up in a compressed mass in the small intestine. When fresh they are beautifully transparent and delicate. They would escape notice of anyone but a regular helminthologist, although masses half the size of one's fist may occasionally be found. In the fresh state they look so much like a mass of the pultaceous intestinal contents of this part of the intestine that the orlinary reterinarian would be pretty sure to overlook them.

I have found them in every sheep I have examined up here, but the fact that I have sometimes identified the worms as of one species, sometimes as another, makes me suspect that two or more species are present. T. expansa is also common in sheep out here, but not nearly so common as the delicate species. I have never found any tapeworms in the intestines of our oxen, i. e., Bos indicus.

It is upon these two sendings that the following description is based. Unfortunately the material was not preserved for fine microscopic work, so that the description given below is necessarily incomplete in many respects. Nevertheless, data enough are given to show that this worm is entirely different, not only specifically but also generically, from any of those described above.

\section{ANATOMY.}

General appearance.-The strobila in possession of this Bureau vary from $60-150^{\mathrm{mm}}$ in length, but no one strobila is complete. The widest 
segments are $2.5^{\mathrm{mm}}$ broad, while the anterior and posterior segments are much narrower. In a fresh state they were transparent (Giles), and many of them have remained so in alcohol, while others have become more or less opaque. The head on the anterior end is quite prominent and appears like a knob. The anterior portion of the strobila is generally quite regular in outline, but the greater part of the worm is crenate and more or less twisted, thus making it quits difficult to prepare in mounts.

The head (Plate xiv, Figs. 2-2b) measures $0.768-0.9^{\mathrm{mm}}$ in diameter. It is square when viewed en face. 'The suckers are generally quite prominent, and more or less lobed according to the state of contraction. They measure (balsam preparation) $0.336^{\mathrm{mm}}$ in diameter, the muscular wall being $30 \mu$ thick. The opening is round or oval and directed in some cases anteriorly, in others more diagonally. The neck varies greatly according to contraction, in some specimens appearing to be absent; in others it is quite distinct. In one balsam preparation it measured $0.28^{\mathrm{mm}}$ broad directly back of the head, and was $2.2^{\mathrm{mm}}$ long. The longitudinal canals were very distinct, two lying dorsally and two ventrally, and measuring $6 \mu$ in diameter. The ventral canal was $16 \mu$ lateral of the dorsal canals. Segmentation becomes quite distinct at about $2.8^{\mathrm{mm}}$ from the head. The distance between the dorsal and ventral canals gradually widens, and about $8.8^{\mathrm{mn}}$ from the head irregularly alternate pistol-shaped genital anlagen appear, the handle of the pistol being situated in the space between the dorsal and ventral canals of the same side.

Segments $15^{\mathrm{mm}}$ from the head (Plate xIV, Fig. 3) measure $0.56^{\mathrm{mm}}$ broad by $56 \mu$ long. The longitudinal nerves were discovered in the extreme lateral portion of the segments. About $0.12^{\mathrm{mm}}$ from the lateral edge ran the ventral canal, provided with an extremely thin cuticle and having a diameter of $8 \mu ; 40 \mu$ nearer the median line was situated the dorsal canal, with a diameter of $6 \mu$, and with a slightly heavier cuticle than that of the ventral canal. No transverse canals could be discovered connecting the dorsal canals, but near the posterior border of each segment there was a fine transverse canal running between the ventral canals. A short transverse canal also extended from each ventral canal towards the margin of the segment.

Male genital organs.-On each side of every segment are situated, between the ventral canal and the nerve, 4-7 testicles, measuring 8-9 in diameter. The genital pore is not yet present. Irregularly alternate in the anterior portion of the segment there is a clump of tissue $68 \mu$ long by $12-14 \mu$ broad, which afterwards differentiates into the cirruspouch and the vagina.

Female genital organs.-From the posterior portion of this clump of tissue one can trace a thin string of nuclei which runs toward the longitudinal canals, crosses the ventral canal dorsally, and ends in a mass of tissue; the latter is situated between the dorsal and ventral canals, 
and represents the female glands; it is composed of an anterior and a posterior portion.

Segments $27^{\mathrm{mm}}$ from the head (Plate XIV, Figs. 4,5) measure $1.14^{\mathrm{mm}}$ broad by $96 \mu$ long. The nerves liave preserved their extreme lateral position and run ventrally of the genital organs. The ventral canals run about $0.18^{\mathrm{mm}}$ fiom the lateral margin, and have a diameter of $16 \mu$. The dorsal canals rum $0.1^{\mathrm{mm}}$ nearer the median line, and liave a diameter of $7 \mu$. The irregularly alternate genital pores have pierced in the anterior half of the margin. The genital cloaca is ahout $20 \mu$ deep, and from its proximal end two canals extend toward the median line. One of these canals (the vagina) lies dorsal, the other (the cirrus) lies ventral.

Male organs.-The cirrus-pouch is rather pyriform, $56 \mu$ long by $40 \mu$ broad. In its distal portion is situated the inverted cirrus, $50 \mu$ to $60 \mu$ long by $6 \mu$ broad. This colors very intensely, owing to the ciliated layer surrounding its lumen, and is thus quite sharply defined from the portion of the male canal which inmediately follows. This latter makes a turn or two inside of the pouch, and then can be followed across the segment to the testicles of the other side. In its course it runs from the cirrus-pouch anteriorly of the testicles of the pore side, dorsally of the ventral canal and the female glands, ventrally of the dorsal canal, then running through the inedian field it lies anterior and dorsal of the transverse canal. It erosses the dorsal canal ventrally, the ventral canal dorsally, and is finally lost in the testicles. This vas deferens undoubtedly receives the spermatozoa from the testicles on both sides of the segment, although it was impossible to observe any fine branches running to the separate testicles of the pore-side. The testicles have increased considerably in size, and now measure 20-2S $\mu$ in diameter.

Female organs. - The vulva lies on nearly the same frontal plane as the cirrus, or somewhat dorsally. The vagina then curves so as to lie dorsally of the cirrus-pouch. Ciliary projections could be seen for about $20-52 \mu$ from the vulva, then they became indistinct. The vagina then becomes very thin and crosses the ventral canal dorsally. At this portion it is generally somewhat convoluted. Just median of the ventral canal it increases somewhat in diameter, forming the receptaculum seminis. The median end of the latter then branches, forming two canals about $3 \mu$ in diameter. These two canals vary in their relative position. In general, however, one of them lies ventrally of the other, and after rumining a very short distance (about $15 \mu$ ) toward the median line, it turns anteriorly and ends in the ovary. This canal is evidently the oviduct. The other canal extends just beyond the ovary, and is then lost to view. The ovary is a circular body $27-42 \mu$ in diameter.

In these segments one finds the anlagen of two more female organs, $i$. e., the uteri. On the pore-side of the seginent there is a chump of small darkly staining nuclei, situated ventrally of the receptaculum 
seminis and dorsally of the ovary. On the opposite side of the segment there is an anlage, which is exactly similar in appearance to the one just described.

Segments $50^{\mathrm{mm}}$ from the head (Plate XIv, Figs. 4, 5) measure $1.15^{\mathrm{mm}}$ broad by $96 \mu$ long. The ovary has increased in size, now measuring $60 \mu$ in diameter. The anlagen of the uteri have grown but little. For the next $8^{\mathrm{mm}}$ a complete and rapid change (Plate XIV, Fig.6) takes place in the appearance of the female organs. It is noticed that the ovary now begins to grow less distinct, while the uteri come more plainly into view. We should naturally expect to find a canal connecting the descending oviduct aud the uteri. No such canal, however, could be distinguished connecting the ovary with the uterus on the opposite side of the segment. In three different segments the writer thought he could distinguish a canal connecting the oviduct with the uterus on the same side, but was not positive that such was really the case; this point is left to be determined by some one who can procure and fix fresh material. It seems beyond question, however, that the ova descend through the oviduct and become fertilized; then, that a portion of them enter the uterus next to the ovary, while the remainder travel across the segment and enter the uterus of the other side.

In support of this view the following observations are given: The ovary becomes smaller while the uteri become larger; ova have been seen in the oviduct; at first no ova are seen in either uterus, then they appear in the uterus on the pore-sicie, and at the same time a broken line of ova can be seen extending across the segment toward the opposite uterus; the latter is next observed containing ova, while no ova can be distinguished in the median field. I am forced to admit that in no one segment has it been possible to find a continuous line of ova extending across the entire merlian field, but by combining several segments a diagrammatic line of ova may be constructed which extends from ovarium to uterus. An interesting point in connection with this wandering of the ova across the segment is that young ova, especially those found in the median field, have no definite form, a fact which points to their being capable of amøebic motion.

In segments measuring $1.344^{\mathrm{mm}}$ broad by $81 \mu$ long, still another change takes place in the form of the female organs. The uteri of both sides elongate medio-anteriorly, the prolongation having a fibrinous appearance. The oviduct is still faintly visible, and the ovary nearly or quite devoid of eggs can be distinguished on the ventral side of the uterus of the pore-side. At the same time another organ, the function of which I am uuable to explain, appears anterior to the uteri and running transversely. This organ lies ventrally of the vas deferensin those cases where the vas deferens extends so near the anterior margin-and stains quite dark in carmine or hæmatoxylin. The exact bistology of this peculiar organ could not be definitely determined. In 
older segments the portion found in the median field looked very much like a canal.

In segments $1.256^{\mathrm{mm}}$ broad by $0.144 \mathrm{long}$ each uterus is composed of two distinct portions, a posterior-lateral bulb, $96 \mu$ in diameter, containing the eggs which have now segmented and are surrounded by a shell, and an anterior median fibrinous prolongation $0.144^{\mathrm{mm}}$ long by $72 \mu$ broad at the base. The anterior extremity of this prolongation fits into (or connects with ? ?) the curve formed by the organ directly in front of it. This latter has increased considerably in size, and now extends partially around the uterus, crosses the dorsal canal ventrally, and tapers off into a fine point which runs through the median field to meet the corresponding organ of the opposite side. In one or two segments this crossed the dorsal canal dorsally instead of ventrally. The vas deferens which crosses the median field comes very plainly into view.

In segments $2.4^{\mathrm{mm}}$ wide by $0.114^{\mathrm{mm}}$ long the median prolongation of the uterus has partially constricted from the rest, so that we now find three more or less distinct portions of the uterus, $i$. e., the lateral bulb containing eggs and two spherical fibrinous portions. The middle portion lies nearer the ventral surface of the worm than either the lateral or the median portion. In different segments (Plate XIV, Figs. 7-9) these three divisions of the uterus present quite different relations to each other, but the exact order of the changes can not be given, as these variations were seen on loose segments and not on a complete worm. In the segments in which these changes are taking place the vagina is greatly enlarged.

As the segments grow older they become narrower and longer; the uteri assume a more antero-posterior position; the median anterior portion of the same becomes less distinct, and ova appear in the middle portion. The transverse organ in front of the uterus has united with the corresponding organ of the other side; the testicles become indistinct. Some of the oldest segments (Plate xIV, Fig. 9) of Dr. Giles' material measured $0.624^{\mathrm{mm}}$ broad by $0.28^{\mathrm{mm}}$ long.

In many segments one sees at the posterior edge, especially in the lateral fields, a row of small $(4-8 \mu)$ round or oval bodies which stain very dark in hamatoxylin or carmine. These same bodies are occasionally met with in the median field, but their presence is extremely irregular. In general appearance they resemble, to a certain extent, the calcareous bodies found in T. crassicollis, T. solium, etc. In hrematoxylin their margin and center stain very dark, while a lightly staining zone separates the two more darkly staining portions.

Eggs.-The eggs are round or oval, and measure 14-16 $\mu \times 10-14 \mu$. Only one egg-shell could be discovered with certainty, althongh it oceasionally appeared as if two were present; at two opposite poles this egg-shell bears a spinous projection $4-6 \mu$ long. Comparatively few ova $(10-30)$ are found in each uterus. 


\section{SPECIFIC DIAGNOSIS.}

The following is given as a provisional diagnosis of this species:

Stilesia globipunctata (Riv., 1874) Rail., 1893.-Strobila transparent, whitish or greyish yellow, $45-60^{\mathrm{cm}} \mathrm{long}$, very thin and not over $2.5^{\mathrm{mm}}$ broad. Head quadrate $0.5-1^{\mathrm{mm}}$ broad, suekers more or less distinctly lobed, opening round or oval and directed anteriorly or obliquely. Neck present, $2^{\mathrm{mm}}$ or more long (neek absent according to other observers). Developing segments $0.33-2.5^{\mathrm{mm}}$ broad by $0.05-0.17^{\mathrm{mm}}$ long; end segments narrower and longer $(0.6$ by 0.28$)$. Genital pores irregularly alteruate in anterior half of lateral margin. Cirrus-poueh slightly ventral of vagina, dorsal of nerve; vas deferens crosses the ventral eanal dorsally, the dorsal canal ventrally; testicles in both lateral fields. Vulva on about the same frontal plane as cirrus; vagina generally dorsal of eirrus-pouch, erosses the ventral canal dorsally; ovary between the longitudinal canals on pore side of segment; two uteri present in each segment, one on each side and situated clorsally of ovarium; eaeh uterus may be more or less distinctly divided into 3 globular bodies; a transverse organ of undetermined function anterior of uterns. At posterior border of lateral field is found a line of round or oval, refringent, ehromatophil particles, $4-8 \mu$ in diameter. Ova 14-16 $\mu$ by $10-14 \mu$. Single shell with a spinous prolongation $4-6 \mu$ long at two opposite poles.

Type with Prof. Rivolta; typical specimens in Burean of Animal Industry and collection of Stiles, at Washington; in collection of Leidy, at Philadelphia; and with Prof. Neumann, Toulouse, France.

It may be well to call attention to the following points, which should be more definitely determined by some one who is able to obtain perfectly fresh material: (1) What is the nature of the transverse organ anterior to the uterus? (2) Is there a canal extending from the descending oviduct to the uteri, or do the ova gain access to the latter by amœbic motion through the tissue? (3) What is the exact order of the changes in the form of the uterus? (4) What is the function of the canal which joins with the oviduct at the ovarium; do the ova pass through this canal to the median field?

C. W. S.

(13) Stilesia centripunctata (Riv., 1874) Railliet, 1893 MS.

[Plate XV, Figs. 1-6.]

Synonymy.-Tonia centripunctata Rivolta, 1874; T. centripunteggiata (R.) Perr., 1882 (Italian); Stilesia centripunctata (Riv.) Rail., 1893.

Hosts.-Sheep (Riv., Mattozzi);? Cattle (after ? seev. Linstow's Compendinm). Geographical distribution.-Italy (Riv. Mattozzi); Algeria (Neumann's specimens).

\section{LITERATURE.}

(1) Rivolta. Sopra alcune specie di Tenie della Pecora. Pisa. 1874

(2) Perroncito. I Parassiti, etc., 1884, p. 242.

(3) - Trattato, ete., 1886, pp. 235, 236.

(4) RaIlliet. Eléments, etc., 1886, p. 261.

(5) Neumann. Traité, 1 ed., p. 383.

(6) —- Observations sur les Ténias du Monton; Compt. rend. d. 1. Soc. d'Hist. Nat., 1891. 18 Mars.

(7) ——. Traité, 2 ed., 1892, p. 409, fig. 197. Eng. Transl., p. 419.

(8) RaIlliet. Eléments, etc., 2 ed. 1893. 
This parasite was first described by Rivolta (1) in 1874. The original article is not accessible. Perroncito $(2,3)$ says in regard to it that the head (scolex) measures $2^{\mathrm{mm}}$; suckers are large; neck entirely absent; the strobila measures $2.75-2.84^{\mathrm{m}}$ long, wider in the anterior half than in the posterior half; $10^{\mathrm{cm}}$ from the head the proglottids are $2-4^{\mathrm{mm}}$ wide; $50^{\mathrm{cm}}$ from the head they measure $2-3^{\mathrm{mm}}$ wide; $150^{\mathrm{cm}}$ from the head they measure $1.5^{\mathrm{mm}}$ wide; at the end of the strobila they are scarcely $1^{\mathrm{mm}}$ wide. They are quite thick, the thickness increasing as the segments grow older.

The mature specimens (preserved) were yellowish white and almost round. In the center of each segment, beginning at about the middle of the strobila, a round or elliptical and rather prominent spot could be noticed by the naked eye. This prominence increases in the posterior segments, alternates on the two surfaces, and is formed by the female organs in the center of the proglottid. The testicles are situated laterally in each segment; a single genital pore is present in each segment, situated in the middle of the lateral margm. Eggs are spherical with a single shell, diameters $22-24 \mu$ by $21-22 \mu$; six hooks in protoscolex; very few ova present in each proglottid. Rivolta found these worms in the small intestines of a sheep. The mucosa was in part reddish, owing to hyperremia of the villi, and in part pale and grayish on account of the pigmental degeneration of the villi. Dr. Mattozzi noticed only a catarrhal affection in the intestines of sheep infested with this parasite.

Railliet (4) takes his diagnosis from Rivolta or Perroncito, and has evidently not seen the parasite in question.

Neumann (5) does not state that he had examined the worms, but adds to Raillet's diagnosis that the length of the segment is $\frac{1}{10}-\frac{1}{3} \mathrm{~mm}$, increasing with the age of the segments; the testicles are in the lateral portion of the median field; pores irregularly alternate.

Neumann (6) obtained several specimens from Algeria and ards to the foregoing that there are firom 1,800 to 2,200 segments in each strobila. In the second edition of his Traité (7) he gives a figure of the hearl.

v. Linstow in his compendium places this species among the parasites of cattle. Following v. Linstow I also placed it in the Check-list of Animal Parasites of Cattle (Note 8), recently published, although I have been unable to ascertain when, where, or by whom it has been found in cattle.

\section{ANATOMY.}

Through the extreme kindness of Prof. Nemmann I am in possession of a specimen which he obtained from Algeria, and although, as this French savant wrote, the strobila is not well preserved, the anatomy of this species will be described below as fully as the mate- 
rial allows. The seolex of the specimen is too contracted to warrant any statements (cel. I'late xv, Figs. $2-2 b)$.

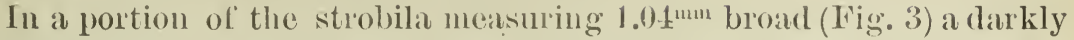
coloring tissue oceupied the median line; on arch side of this was a clear space; lateral from these spaces testicles were visible; then a elear space-a longitudinal enal ; lateral of this there is another longitudinal line (nerve?).

In a portion of the strobila measuring $1.12^{\mathrm{mm}}$ wide (Plate xv, Fig. 4), instead of having a dark tissue on the median line, this portion of the field was clear for a space 0.16 hroad. At the border of this field were found clumps of darkly stained tissue, irregularly alternate. These represent the handle of the pistol-shape genital anlagen; the muzzle of the pistol can be traced almost to the lateral edge of the strobila; further, it could be plainly seen that the testicles were situated not only on the median side of the longitudinal canals, but also dorsally (? or ventrally) and laterally of them.

The testicles measure $64 \mu$ in diameter (preparation was subject to pressure while coloring). In the strobila, measuring $1.24^{\mathrm{mm}}$ broad (Plate $\mathrm{xV}$, Fig. 5), it is noticed that a line of deeply stained tissue runs from the handle of the pistol a short distance torards the opposite side of the segment; the lateral portion of the analage of the canals (muzzle of the pistol) is quite thick and partially differentiated into two parts (male and female). Where the strobila was $1.6^{\mathrm{mm}}$ wide (Plate xv, Fig. 6) the borders betreen the segments could be distinguished; the segments varied from $32 \mu$ long on the side without the pore to $64 \mu$ long on the side with the pore. The median portion $\left(0.24^{\mathrm{mm}}\right.$ broad) of the median field is now entirely oceupied by the dark tissue referred to above as running from the handle of the pistol towards the other side of the strobila (probably uterus).

With the material at hand I do not feel justified in making any further statements.

\section{SPECIFIC DIAGNOSIS.}

The following is given as the diagnosis of this species:

Stilesia centripunctata (Riv., 1ð74) Rail., 1893. (Provisional).-Head large,1.5-2mm broad, suckers large, directed anteriorly (after Neumann), situated at the four corners, directed diagonally forward (after Stiles). Strobila attains nearly $3^{\mathrm{m}}$ in length. Segments $10^{\mathrm{cm}}$ from the head ineasure $2-3 \mathrm{~mm}$ wide, and from this point they grow narrower; posterior segments measure scarcely $1 \mathrm{~mm}$; always much broader than long, and longer on the pore side than on the opposite side. Genital pores irregnlarly alternate. Transverse uterus in the median portion of the median field. Testicles extend from each end of the uterus to the lateral (?) nerve. Eggs splierical with a single shell without pyriform apparatus; diameter $20-24 \mu$ by $21-22 \mu$.

Type with Prof. Rivolta. Typical specimens with L. G. Neumann, Toulouse, Bureau of Animal Industry, and U. W. Stiles, Washington, D. C.

$7114-$ No. $4-6$ 
General Remaris in Regard to Stilesia globipunctata and STILESIA CENTRIPUNCTATA.

From the above description of these two species, although the account of $S$. centripunctata is very incomplete, it is evident that these forms can not be generically united with Moniezia or Thysanosoma. They differ from both of these genera in the form of the uterus and the general arrangement of the genitalia. S. globipunctuta agrees with Thysanosoma in the relation of the genital canals to the nerves and longitudinal canals. This relation could not be definitely determined for S. centripunctata.

A new genus must certainly be established for $T$. globipunctata, and although $T$. centripunctata agrees with this form in comparatively few characters-narrow strobila, short segments, irregularly alternate pores, absence of testicles from median portion of median field, size of egg and single egg-shell-it will probably be better to place the two forms in one genus until T. centripunctuta can be more thoroughly studied. Railliet has also recognized the nevessity of establishing a new genus for these forms, and has proposed the generic name Stilesia (S. globipunctata and S. centripunctata). The following will suffice for a provisional diagnosis until T. centripunctata can be thoroughly studied:

\section{GENERIC DIAGNOSIS.}

Stilesia Railliet, 1893.-Typo species S. globipunctata (Riv.) Railliet, 1893. Head with four suckers but no hooks. Strobila thin and narrow. Genital pores irregularly alternate. Segments broader than loug. 'Two distinct sets of testicles present in each segment, one on each side, but no testicles in the median line. Eggs very small (not over $24 \mu$ ) and with but one shell.

The following points, which may prove to be of generic value, have been established only for S. globipunctata: Genital camals pass dorsally of nerve and ventral canal, but ventrally of dorsal canal. Egg-shell with two conical spinous projectiong at opposite poles.

Habitat.-Intestine of sheep. Development unknown.

C. W. S. 


\section{PAR'T IV. \\ SPECIES INQUIRENDAE.}

(14) Mouiezia nullicollis Moniez, 1891.

[Plate XVI, 3-3b.]

Host.-Sheep (Moniez).

Geographical distribution.-Thus far M. nullicollis has been reported only at Lille, France, by Prof. R. Moniez.

\section{LITERATURE.}

Moniez. Notes sur les Helminthes vi, 3; Revue Biologique du Nord de la France, T. IV, 1891, 1 page.

\section{HISTORICAL REVIEW.}

Prof. Moniez found two specimens of tapeworms at the Lille abattoir in May, 1879. In 1891 he described them as a new species under the name given above, giving, however, only a very short description. He states that the worms were $40^{\mathrm{cm}}$ long. The last segments were perfectly mature and measured, in alcohol, $8^{\mathrm{mm}}$ wide by $1^{\mathrm{mm}}$ long. They were quite thin and all the segments very short. Head cuboid, rounded in front, hemispherical in back, 1.3 in diameter. Suckers 0.65 in diameter. Neck absent, so that the segments begin directly back of the suckers. Ova 55-65 $\mu$; embryo $21 \mu$.

At my request Prof. Moniez forwarded part of his original material. I have only the head (Figs. 3-3b, Plate xvI), and hence can give no statements in regard to the anatomy of the segments.

\section{DIAGNOSIS.}

It is impossible to give a satisfactory diagnosis of this form, hence I can not as yet look upon it as a well-established species.

Type with Prof. Moniez. One scolex in private collection of C. W. Stiles, Washington, D. C.

C. W. S.

(15) * Tænia Vogti Moniez, 1879.

Synonymy.-Tckia Vogti, Moniez, 1879; Anoplocephala Vogti Moniez, 1891.

Host.-Sheep (Moniez).

Geographical distribution.-France (at Lille, Moniez). 


\section{LITER $\Lambda$ TURE.}

(1) Monifz. Note sur denx espèces nouvelles de Trnias inermes (T. Voqti et $T$.

Benedeni); Bulletin scientıfique du Départment du Nord, 1878, II, pp. 163-164.

(2) Railliet. Eléments, etc., 1886, p. 261.

(3) Nuumann. Traité, etc., 1st ed., 1888, p. 383.

(4). - Observations sur les Ténias du Mouton; Comp. rend. d. 1. Soc. d'Hist Nat. d. Toulouse, 1891; 18 Mars.

(5) Monicz. Notes sur les Helminthes; Rev. Biol. du Nord, 1891.

(6) R. Blanchard. Notices helminthologiques, 2 sér.; Mém. d. 1. Soc. Zoöl. d. France, 1891, p. 447, footnote.

(7) Neumans. Traité, 2 ed., p. 408;-Eugl. Transl., p. 419.

\section{HISTORICAL REVIEW.}

Moniez found a strobila $1 \frac{1}{2}$ feet long in a sheep, and gave the following description (1) of it: Head unknown; color of strobila, white; the young segments remain very narrow for a considerable distance of the total length; the largest segments measure $2.5^{\mathrm{mm}}$ broad by $5^{\mathrm{mm}}$ long, and contain eggs with the pyriform body; they are very flat; the muscles are better developed than in the other species, and are not grouped. It is very rare, having been found but once.

Railliet (2) and Neumann (3) take their diagnoses from Moniez. In (4) Neumann adds nothing to the above in his text, but in the analytical table he gives $T$. Vogti as having but a single genital pore to each segment. In Moniez's paper, however, I do not find any authority for this statement.

Moniez (5) then places this species in his analytical table as Anoplocephala Vogti, under the line "Un seul pore genital par anneau." This, however, is too indefinite, as the genus Anoplocephala in Blanchard's classification has unilateral pores.

Blanchard (6) calls attention to this last point, and states that this species is more closely allied to the genus Bertia, since the pores are irregularly alternate. I have been unable to obtain any specimens of this species.

\section{CONCLUSIONS.}

From this review it is seen that the $T$. Vogti is not sufficiently well known to be accepted as a true species. There is considerable doubt as to the genital pores, and our experience with Th. Giardi has taught us that great difference of opinion may arise in such a case, and that the pores alone are no criterion in the generic determination. Furthermore, the fact that the segments are longer than broad is by itself, unassociated with other characters, of little value, for we find that several species sometimes show this character.

In Hassall's collection is found a worm from sheep, the ripe segments of which agree in breadth with Moniez's description of 7 . Vogti, but the genital pores are distiuctly double. In external appearance (Plate 
xvi, Figs 1-2b) the worm is totally different from any tapeworm we have ever seen. While somewhat inclined to believe that this represents a new species-in case it is not identical with $T$. Vogti-I refrain from describing it as such, since the material is so poorly preserved that the internal organs will not stain. I am unable on this account to give any description of the internal anatomy, except that the vas deferens is very much convoluted, and that the uteri are present which resemble those of Moniezia. The time is passed when an authoris justified in describing a tapeworm as a new species unless he can give a recognizable diagnosis.

C. W. S.

(16) *Tænia crucigera Nitzsch and Gicbel, 1866.

Host.-Capreolus caprea.

Geographical distribution.-Germany, found but once, by Nitzsch.

\section{LITERA'TURE.}

Grebel, C. Die im zoölogischen Museum der Universität Halle aufgestellten Eingeveidewürmer nebst Beobachtungen ïber dieselben; Zeitschrift für die gesammten Naturwissenchaften, 1866, xxvıII, p. 259.

Giebel describes a species of tapeworm collected by Nitzsch from Capreolus caprea. His diagnosis and description are as follows:

T. capite obtuso obverse pyramidali tetragona collo brevi; articulis anticis tenuissimis, insequentibus latequadratis, ultimis subquadratis margine laterali arcuatis posteriori vix incumbentibus; vulvis oppositis.

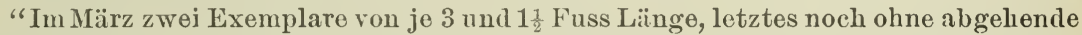
Proglottiden. Der Kopf its umgekehrt kegelförmig oder pyramidal, hinten am breitesten und fast wie abgeschnitten, mit vier hochrandigen mehr nach vorn als zur Seite gerichteten Saugnäpfen, die von vorn gesehen länglich viereckig, gleichsam mit eingediicktem Krenz bezeichnet sind. Der Halstheil hat halbe Kopf breite und etwa die sech bis siebenfache Kopflänge. Von ihm nimmt der Leib ganz allmählig an Breite zu bis zum Ende, wo er vier Linien Breite misst. Die ersten Glieder gleichen bloss sehr feinen Runzeln, werden dann allmälılig länger, anfang $\frac{1}{10}$, dann $\frac{1}{6}-\frac{1}{3}-\frac{1}{2}$ zuletz $\frac{2}{3}$ ihrer Breite lāng, gestreckt viereckig, am hintern Rande nur sehr wenig breiter wie am vordern. Der Seitenrand der vordern Glieder in 3 bis 4 Zoll Entfernung vom Kopfe erscheint durch zwei Hervorragungen gekerbt, deren vordere die Genitalwarze, die hintere die hervorstehende Hinterecke ist. Erste bildet ein selır breites kurzes aufsitzendes Knöpfehen mit dentlicher Oeffunng. Weiter nach hinten wird die Geschlechtspapille schwächer, schon in der Leibesmitte ist sie ganz schwach und in dem letzten Leibesdrittel fehlt sie ganz. Der Hinterrand jedes Gliedes liegt hier nur ganz kurz und unbedentend auf dem Vorderrande des folgendeu auf, bei der nächst verwandten $T$. expansa iibcrragt jener sehr beträchlich. Nahe des Seitenrandes der Glicder macht sich der Seitenkanal als dunkler Streif sehr bemerklich, nur in den ersten Gliedern nicht erkennbar. Die letzten Glieder sind ganz mit grossen reifen Eiern gefüllt, welche käseförmig gestaltet also in den breiten Seiten kreisrund, vom Rande gesehen länglich sind. Die Unterschiede dieser Art von der sehr ähnlichen vorigen sind folgende: Die Saugnäpe stehen sehr nach vorn und sind röhriger; der Halstheil ist deutlicher und länger, die hinteren Glieder in Verhältniss ihrer Briete weit lïnger; der Hinterrand der Glieder wenig oder gar nicht überragend; die Geschlechtspapille knopfförmig, die Eier käseförmig, nicht konisch." 
From the above it is very probable that this species belongs to the genus Moniezia, although, without the internal anatomy, even this point is uncertain.

Dr. Brandes, in reply to a letter, has written that the type specimen has, unfortunately, been lost or destroyed. It would be too uncertain to determine a worm by the above description without the original material at hand, so that it will probably be better to ignore this specific name and diagnosis entirely.

C. W. S.

(17) Tænia capreoli Viborg, 1795 and (18) Tænia capræ Rud., 1810.

These two species should evidently be entirely ignored, for the descriptions given are not sufficient to establish them as true species, and the types can not be found. The following remarks in regard to them are quoted from Rudolphi's Historia Naturalis (pp. 200-201). The forms have been referred to by several authors since Rudolphi's time, but no one seems inclined to arcept the species or able to give any anatomical details in regard to them.

83. Thania Capreolr.-Viborg ind. Mus. Vet. Hafn., p. 238. n. 87. b. Tænia capreoli.

Tania ovina (expansa n.2.) el. viro n. 87. a. dicitur, unde capreoli vermem huic affinem esse, eo magis colligimus, quo major inter expansam (ovis), denticulatum (bovis) et insequentem (eapræ) affinitas observetur.

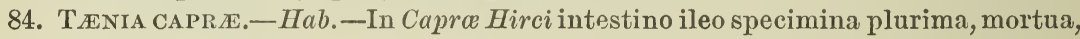
Augisto mense reperi.

Fragmenta tri- vel quinquepollicaria; qnorum alia minora articulis linea angustıoribus, brevissimis, angulis laterlibus acutinsculis, alia majora articulis duas tresve lineas latis, vix lineam longis. Isti crassiusculi marginibus lateralibus crenulatis, foramine utrinque medio sive opposito indistincto, angulis rotundatis, margine postico plicatulo, snbstantia molli, lineis transversis undulatis.

Obs. 1.-Tænia hæe inter expansam n.2. et denticulatam n. 3. quas conferas, media videtur, capite tamen non viso, de eadem jndicare nequeo.

Ob8. 2.-Ne cum Tænia capræa Abilgaardii, caprina Gmelini et Zederi confundas, hæc enm Tænia non est, et sub Polystomatis denticulati nomine supra clescripta sistitur. c. W. s.

(See also addenda, p. 101.) 


\section{PART V.}

\section{LIFE HISTORY.}

Mégnin publisherl an article in which he set forth the theory that certain cestodes conld develop withont a change of host. Moniez and others combatted this idea, while Perroncito suggested that some insect might possibly play the role of intermediate host for forms like Moniezia expansa. MeMurrich was inclined to look upon Melophagus ovinus as the intermediate host of Moniezia expansa. Leuckart has suggested that some snail might act as the intermediate host. Curtice then supported the direct infection theory again.

Hutchinson, in rather an undecided amateurish article, suggests that it is possible that caterpillars, slugs, earthworms, lice, etc., may be intermediate hosts for T. expansa, which he "believes" is the tapeworm found in sheep and ruminants.

For over a year past we have been conducting experiments in this line, but up to date only negative results can be reported. Experiments to infect sheep directly by feeding eggs to them have been totally negative. Experiments to infect Melophagus ovinus, numerous ooprophagous insects, and earthworms, have also been negative. It is the intention to continue these experiments, especially those with earthworms, this coming spring upon a much larger scale, and we hope later to report some positive results. From experiments thus far, however, we feel confident that cattle, etc., can not become infested with tapeworms by swallowing the eggs, as Curtice still contends, and it is believed that some insect, worm, or snail will be found to contain the larval stage.

C. W. S. 


\section{PAR'T VI. \\ CONCLUSIONS.}

In the foregoing stndies the following conclusions have been reached:

(1) Descriptions of cestodes based upon external form alone, unassociated with internal anatomy, are of little value. (See also p. 84,99.)

(2) In the case of those forms of which the original type specimens can be obtained, we may be able to recognize the species, but in most of the cases of which the types have been lost, the determination of the species is extremely uncertain. We shall hence be obliged to ignore a great number of forms which have been described as species

(3) The present genus Tcuia, as generally accepted by authors, contains forms which must be restudied and arranged in several sub-families and a number of genera.

(4) This revision must be based upon internal anatomy.

(5) In the adult cestodes of cattle and sheep three genera Moniczia R. Bl., Thysanosoma Dies., and Stilesia Rail., are recognized.

(6) For a summary of the genus Moniczia, see p.51; for Thysanosoma, p. 70 ; for stilesia, p. 82.

(7) The following table will aid in determining the various species of these genera:

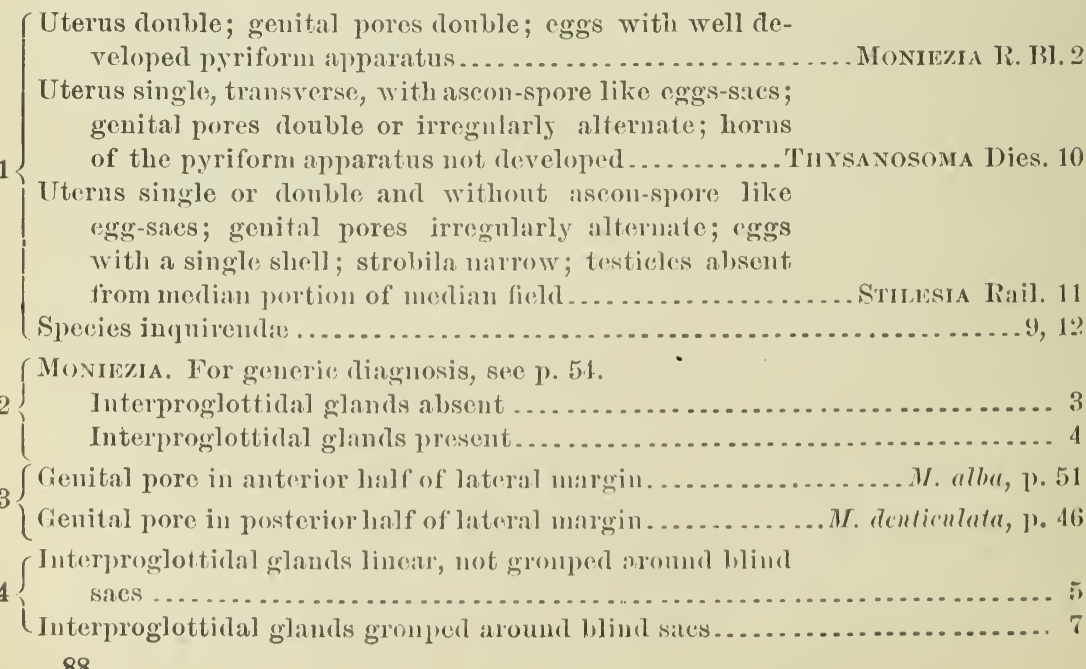


(Interproglottidal glands very prominent and quite long

(broad in reference to the segment); head not very

5 distinetly lobed; opening of suckers slightly elongated; segments attain $20-26^{\mathrm{mm}}$ in breadth............. M. planissima, p. 22

Interproglottidal glands short and not prominent................... 6

Head rery distinctly lobed and sharply separated from neck; opening of sucker rouud; neek nearly as broad

6 as head; segments attain $10-12^{\mathrm{mm}}$ in breadth.

M. Benedeni, p. 25

Lobing of head less distinct; head not so sharply separated from neck; neck filiform; segments attain $8^{\mathrm{mm}}$ in breadth; thinner than foregoing species.............. N. Neumanni, p. 26

(Testicles generally in form of two triangles; space frequently left between the uteri in the median line; head pyriform, almost square when riewed en face;

7 segments attain $6^{\mathrm{mm}}$ in breadth; end segments occasionally as long as broad............................ trigonophora, p. 42

Testicles generally in form of a quadrangle; no space between the fully developed uteri; end segments never as long as broad ......................................... 8

(Head olllong when viewed en face; not lobed; suckers not distinctly raised; entire strobila thin; segments attain $9 \mathrm{~mm}$. in breadth; end segments narrower ........M. oblongiceps, p. 36

Head more or less distinctly lobed; nearly square when

8 viewed en face; suckers raised; openings decidedly elongated; strobila often quite thick; posterior portion frequently of an orange color; segments attain $16 \mathrm{~mm}$., perhaps more, in breadth; end segments show a tendency to break off $1-3$ at a time .................. expansa, p. 34

Neck absent; head large, decidedly lobed; openings of

9 suckers round; segments attain $8 \mathrm{~mm}$. in breadth-sp.

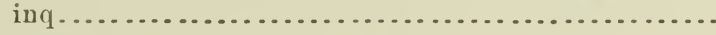

M. mullicollis, p. 83.

Tilysanosoma.-For generic diagnosis, see p. 70

Head very large $(1.5 \mathrm{~mm})$; square; lobed; testieles in median field; posterior flap of segments fimbriate; genital pores donble............................ Th.actinioides, p. 58

Head small; testieles in lateral fields; posterior flap not fimbriate; genital pores irregularly alteruate (rarely double). Th. Giardi, p. 69

Stilesia.-See p. 82.

Median portion of median field oceupied by a transverse nterus; "heart 2 nm. in diameter".................. S. centripunetata, p. 81.

Median portion of median field transparent; two lateral uteri in each segment; "liead less than $1 \mathrm{~mm}$. in diameter"..................................... S.globipunetata, p. 79 .

12 Segments $2 \mathrm{~mm}$. broad by $5 \mathrm{~mm}$. loug, sp. inq........... T. Vogti, p. 83. End segments $8 \mathrm{~mm}$. broad, sp. inq .................. T. erucigera, p. 85 .

(S) For Tania marmotce we shall be compelled to recognize a new genus (p. 71).

(9) Riehm's species Dipylidium Leuckarti, D. pectinatum and D. Zatissimum can not remain in the genus Dipylidium, establisled by Leuckart for Tania cucumerina. It may be found upon further study that they can be placed in the genus with $T$. marmotce. 


\section{PART VII.}

\section{COMPENDIUM OF SPECIES ARRANGED ACCORDING TO THEIR HOSTS.}

I have personally examined those forms marked with an asterisk $\left(^{*}\right)$ under the various hosts, and can hence guarantee that these species occur in the animals cited. The other cases mentioned are compiled. Forms given as $M$. expansa, but not starred, should be reëxamined to establish their specific identity.

The nomenclature of the hosts here adopted is mainly that of Flower and Lydecker's Mammals, Living and Extinct, 1891. In the case of the Cervida, Sir Victor Brooke's Monograph (Proc. Zool. Soc., London, $1878 \mathrm{pp} .883-928)$ of this family has been followed:

Antitope dorcas Pallas. See Gazella dorcas.

Antilope rupicapra Erxl. See RUPICAPra tragus.

Bos indicus. Zebu.

Moniczia expansa (R.) R. Bl., reported as Tunia cxpansa.

Bos taurus L. Domestic cattle.

* Moniezia alba (Per.) R. Bl.

* Moniezia Bencdoni (M.) R. Bl.

* Moniczia denticulata (R.) R. Bl.

* Moniezia cxpansa (R.) R. Bl.

* Moniezia planissima S. \& H.

Thysanosoma Giardi (Riv). S. (See p. 59.) Found by Perroncito; determined by Rivolta.

Stilesia centripunctata. (See p. 79.)

Stilesia globipunctata. (See p. 73.)

Capra hircus L. Goat.

Moniezia expansa (R.) R. Bl., reported as Tania expansa.

Tania capra R. sp. dub.

Capra hispanica Græl. See Capra Pyrenaica.

Capra Pyrenaica Sehinz. (Capra hispanica.) Spanish Ibex.

Moniczia expansa (R.) R. Bl., reported as Tania cxpansa.

Capreolus Caprea. (Cervus capreolus.) Roe or Roe deer.

Moniezia cxpansa (R.) R. Bl., reported as Tania expansa.

Tania crucigera Nitzsch, sp. dub.

Cariacus (Blastocerus) campestris. (Cervus campestris.) Pampas Deer.

Moniczia expansa, reported as Tania expansa.

Cariacus (Blastoctrus) paludosus. (Cervus pa7udosus.)

Thysanosoma actinioides Dies.

Cariacus (Conssus) Nambi. (Corvus nambi.)

Moniczia expansa (R.) R. Bl., reported as Tania expansa.

Thysanosoma actinioides Dies. 
Cariacus (Coassts) rufus. (Cerms rufus.) Brocket.

Moniezia expansa (R.) R. B1., reported as Tania expansa. (See Diesing.)

Thysanosoma actinioides Dies.

Cariacus (Coassus) simplicicornis. (Cervus simpticicornis.)

Thysanosoma actinioides Dies.; found by Natterer.

Cariacus (Coassus) sp.?

* Moniezia oblongiceps S. \& H.

Cervus campestris. See Cariacus (Blastocerus) campestris.

Cervus capreolus. See Capreolus caprea.

Corvus dichotomus. See Cariacus paludosus.

Cervus nambi. See Cariacus Nambi.

Cervus paludosus. See Cariacus paludosus.

Cervus rufus Cuv. Seo Cariacus (CoAssus) rufus.

Cervus simplicicornis. See Cariacus SImplicicorisis.

Gazella dorcas. (Antilope dorcas.) Gazelle.

* Moniezia expansa (R.) R. Bl., one of Rudolphi's original specimens.

Ovibos moschatus (Zimm). Musk ox.

Moniezia expansa (R.) R. Bl.

Ovis ARIES L. Domestic sheep.

* Moniezia alba (P.) R. Bl.

* Moniczia Benedeni (M.) R. Bl.

* Moniezia expansa (R.) R. B1.

* Moniezia Neumanni Moniez.

* Moniezia mullicollis, Moniez, sp. dub.

* Moniezia planissima s. \&. H.

* Moniezia trigonophora S. \& H.

* Thysanosoma actinioides Dies.

* Thysanosoma Giardi (Riv.) S.

*Stilesia centripunctata (Riv.) Rail. .

* Stilesia globipunctata (Riv.) Rail.

RUpicapia tragus Gray. (Antilope rupicapra.) Chamois or Gemso.

Moniezia expansa (R.) R. Bl., reported as Tania expansa. 


\section{PART VIII.}

\section{BIBLIOGRAPHY OF ADULT CESTODES OF CATTLE AND SHEEP.}

We have not been able to find the articles the numbers of which are inclosed in brackets, thus $\Gamma 7$ :

1. D'Arboval, L.H.J. Helminthes; Dictionnaire de Médécine de Chirurgie et d’Hygiéne Vétérinaires, par. A. Zundel. Paris, 1875, pp. 111-159.

(T. expansa, p. $150 ; T$. denticulata, p. 151.)

2. Balletet. Histoire Naturelle des Helminthes des principaux mammifères domestiques. Paris, 1866. (Extr. d. Nouv. Iict. d. Méd., d. Chir. et d'Hvg. vét.)

(T. expansa, p. 161; T. denticulata, p. 161; T. capra, p. 162.)

[3] Batsch, A.J.G.C. Naturgeschichte der Bandwurmgattung uiberhaupt und ihrer Arten insbesondere, nach den neuern Beobachtungen in einem systematischen Auszuge. Halle. 1786.

4. Blanchard, Raphael. Sur les Helminthes des primates anthropoïdes; Mémoires de la Socí́té Zool. de France, 1891.

Vide p. 187, footnote; (Moniezia mentioned as a new genus).

5. - Notices Helminthologiques. (Deuxiéme série.) 7. Cestodes du groupe des Anoplocephalinæ, p. 443; Mémoires de la Société Zoologique de France, 1891.

(pp. 444-446. Generic diagnosis of Moniezia: (1) Moniezia alba, (2) M. Benedeni, (3) M. denticulata, (4) M. expanza, (9) M. Neumanni, (10) M. nullicollis. 'T. ovilla, T. fimbriata.)

[6.] BLoch, M. E. Abhandlung von der Erzengung der Eingeweidewiirmer und den Mitteln wider dieselben. Berlin, 1782. 4\%, 10 taf.

6a. - Traité de la génération des vers des intestius et des Vermifuges. Strasbourg, 1788.

(Tenia vasis nutriciis distinctis, pp. 16-17, v, 1-5.)

[7] Camper. Beschïft. Berlin Naturf. Freunde iv, 1779. 39 T. denticulata.

8. Carlislik, Anthony. On the structure and occurrence of Tienix; Transactions of the Linnaean Society (Lond., 1793), ii, tab. xxv, f. 15, 16.

[T. ovina $=T$. cxpansa, after Rudolphi].

[9] Cha inizt. Traité des Maladies Vermineuse, p. 120, pro parte.

[Tania rubané=T. denticulata, atter Jies.]

10. Coв BoLD, 'T. S. Parasites; a treatise on the entozoa of man and animals. London, 1879. Vide p. 332 .

(Mentions $T$. expansa and $T$. denticuluta; $T$. capre and $T$. fimbriata as doubtful species.)

11. - The Internal Parasites of our Domesticated Animals. I London, 1873.

(T. expansa, pp. 20-22; T. denticnlala, p. 22.)

12. Creptin, F. C. II. Endozoologische Boitrïge:1. Über Tania denticulata Rud. und T. expansa Rud.; Weigemamu's Arehiv fur Naturgeschichte Jarg. viii, 1842. I, pp. 315-327.

13. Curtice, C. 'Tapeworm disease of the sheep of the western plains; Annual Report of the Burean of Animal Iudustry. Washington, J). C., 1887-'88, ply. 167-184, pl. i-ii, $T$. fimbriata. Review by M. Braun in Contralblatt f. Bacteriologie und Parasitenkunde, 1890, viii, p. 732. 
14. - The Animal Parasites of Sheep. Spocial Report of the Bureau of Animal Industry, U. S. Department of $\Lambda$ griculture. Washington, D. C., 1890.

(Tenia alba, 1. 89; T. Benedeni, p. 89) T. centripunctata, p. 89; T. fimbriata, pp. 89-112, pls. xii and xiii; T. Giardi, p. 89; T. ylobipunctata, p. 89; T. ovilla, p. 89 ; T. ovipunctata, p. 89; T. Vogti, p. 89; T. expansa, pp. 113126, pls. xiv and $\mathrm{xv}$.)

15. - Parasites, being a list of those infesting the domesticated animals and man in the United States; Journal of Comparative Medieine and Veterinary Archives, 1892, pp. 223-236.

(T. expansa, pp. 225,227 ; $T$. denticulata, p. 227, is not $T$. denticulata, C. W. S.)

16. Davaine, C. Traité des entozoaires et des maladies vermineuse. Paris, 1877.

(P. liii and p. 235 Tania expansa Rud. and T. denticulata Rud.)

[17] 2. Deutsche Nordpolarfahrt r. 2. Abtheilung, 1874, p. 686.

(Tania expansa.)

18. Dewitz, JoH. Die Eingeweidewiirmer der Haussäugethiere. Berlin, 1892.

(Tania expansa Rud., p. 77-8!, figs. 51 and 52.)

19. Diesing, K. M.-Tropisŭrŭs ŭnd Thysanosoma, zwey neйe Gattŭngen von Binnenw ürmern; Med. Jahrb. d. öst. Staat., n. Folge, vii, 105-111. Tab. iii et anat.

20. - - Systema Helminthum, vol. i. Vindobonæ, 1850.

(T. expansa, p. 497; T. denticulata, p.498; T. fimbriata, p.501; Tania capra, p. 552.)

21. Z Zwanzig Arten von Cephalocotyleen; Denkschrift d. math. naturw. Classe der Kaiserlichen Akademie d. Naturwissenchaften. Wien, 1856.

(T. fimbriata, pp. 32-33, taf. v, 9-15.)

22. Dujardin, F.-Histoire Naturelle des Helminthes. Paris, 1815.

(Tania expansa, p. 577; T. denticulata, p. 578.)

23. Favilie, Geo. C.-Report of the Veterinary Department of the Colorado State Agricultural College, Jan., 1885.

(Tania expansa Rud., ex paı ${ }^{\ddagger} e=$ Thysanosoma actinioides.)

24. Giebel, C.-Die im zoolog. Museum der Universitït Halle aufgestellten Eingeweidewiirmer nebst Beobachtungen iiber dieselben; Zeitsch. f. d. gesammt. Naturw., 1866.

(p. 259, T. expansa; pp. 259-260, T. crucigera; p. 265, T. capra.

25. Guelin.-Linne's Systema Naturæ, p. 3074; No. 55. 1789-1790.

(Tonia ovina $=T$. expansa (after Rud.).

26. Goeze, J. A. E.-Versuch einer Naturgeschichte der Eingeweidew ürmer thierischer Körper. Leipzig, 1782, 44 taf.

(T. ovina, 369 , taf. $\mathrm{xxv}$, iii, 1-12.)

27. GurLT.-Lehrbuch der pathologischen Anatomie der Haussäugethiere, 1831, Bd. I.

(p. 381, pl. x, figs. 1,2, Tania expansa; plate x, figs. 3, 4, Tania denticulata.)

[28] Hertwigs Magazine der Thierheilkunde, iv Jahrg., 2 Heft.

[29] Havemann.-

[30] НuмвоцD.-Berliner thierärztl Wochenschrift, 1889.

(T. denticulata, 45 meters long?!)

31. Hutchinson, J.-On Tapeworms and other Parasites in Sheep and Rabbits; Archiv surg. London, 1891-992, pp. 155-156, 172-175.

[32] KRABBE.-

33. Leukart, R.-Bandwürmer; Koch's Encyklopädie der gesammten Thierheil kunde. Wien und Leipzig, 1885, pp. 361-404.

(T. expansa, pp. 398-399; T. denticulata, pp.399, 400; T. alba, p. 400.)

34.

Die Parasiten des Menschen, 2 Aufl. I, p. 353, 1879-1886. 
35. v. Linstow, O. Compendium der Helminthologie, Hanover, 1878. Nachtrag, 1889.

[36.] Mayer. Froriep's Neue Notizen, i, p. 107.

(Tania denticulat $=T$. expansa, after Creplin.)

[37.] - Analecta für vergl. Anat. 2. Samml. 70, figs. 4,5 (de organ genit.) [T. dcnticulata $=T$. expansa, after Crep. and Dies.]

38. McMurrich, J. P. Zoölogical Report; T. G. Grenside, Veterinary Report, pp. 200-203. Ontario School of Agriculture, 9th Annual Report, 1881 (pp. 174-178, 7 Figs., T. expansa $=M$. trigonophora). (Review by V. Linstow, Archiv fur Naturgeschichte, 1888.)

39. Monicz, R. Note sur deux espèces nouvelles de Tænias inermes. T. Vogti et T. Benedeni; Bulletin Scientifique du Départment du Nord, 1879, sér. ii, T. ii, p. 163-164.

40. - - Sur le Tania Giardi et sur quelques espèces du groupe des inermes; Comptes Rendus de l'Academic des Sciences, lxxxviii, Mai 26, 1879, pp. 1094-1096.

41. - Mémoires sur les Cestodes, Paris, 1881.

('T६nia expansa, pp. 15-20, pl. i, figs. 38-58; pl. ii,'iig. 1. Embryology.)

42. - - Sur quelques types de Cestodes; Comptes Rendus de l'Academie des Sciences, Mar. 6, 1882, p. 662. (T. Giardi).

43. - Les Parasites de l'Homme. Paris, 1889, pp. 123, 124, (T. Giardi).

44. - - Notes sur les Helminthes; Revue Biologique du Nord de la France, Tome iv, 1891 .

(Extrait: V. Moniezia ovilla, pp.11-14; VI. 1. M. Benedeni, pp.14-16; 2. M. Neumanni sp. n., pp. 16-17; 3. M. nullicollis sp. n., p. 17; 4. M. denticulata et M. expansa, pp. 18-21; 5. M. alba var. dubia et M. ovilla var. macilenta, pp. 21-23; vir. Tableau synoptique.pp. 23-24.)

45. Neumann, L. G. Traité des Maladies parasitaires non microbiennes des Animaux domestiques, Paris, 1888.

(Tenia aculeata, p. 383; T. alba, pp. 378, 382; T. Bencdeni, p. $383 ;$ T. capro, p. $389 ;$ T. centripunctata, p. $383 ;$ T. denticulata, p. $377 ;$ T. expansa, pp. 377,382, figs. 164-166; T. Giardi, p. 383; T. globipunctata, p. 384; T. ovillu, p. $383 ;$ T. ovipunctata, p. $384 ;$ T. Vogti, p. 383 .)

46. - Observations sur les Ténias du Mouton; Compt. Rend. do la Société d'Histoire Naturelle de Toulouse. Séance, 10 fév. 1891, 4 pages.

47. - Sur la place du Tenia ovilla Riv. dans la classification; Compt. Rend. de la Société d'Histoire Naturelle de Toulouse. Séance, 2 mars, 1892, 3 pages.

48. - Traité des Maladies parasitaires non-microbienues des animaux domestiques, Paris, 1892, Deuxième édition.

(Tania expansa, pp. 402, 407, 417, Figs. 182, 184-186; T. denticulata, p.401, Fig. 183; T. alba, pp. 403, 408, Fig. 187; T. Benedeni, p. 408, Fig. 192; T. capre, p. $417 ;$ T. centripunctata, 409, Fig. 197; T. fimbriala, p. 408, Figs. 193-194; T. globipunctata, p.410; T. ovilla, p.408, Figs. $195-196 ; T$. ovipunctata, $.410 ; T$. Vogti, p. 408.)

48A. Ncumann's Parasites and Parasitic Diseases of Domesticated Animals. ('Translation of 48 by Fleming.) London, 1892.

[49.] Nordmann.-Lamark Anim. s. vert., 2 ed., iii, 591. Thysanosoma actinioides. [50.] Parona.

51. Perroncito, E. I Parassiti dell' Uomo e degli Animali Utili. Milano, 1882.

(Tania alba, p. 243, figs. 103-105; T. aenleata, p.246; T. contripunclata, p. 242 ; T. expansa, p. 239, figs. 99, 100; T. globipunctata, p. 240; T. ovilla, p. 244; T. ovipunctata, p.241; T. denticulata, p. 240, figs.101-102.)

[52.] - Di una nuova specie di Tienia (T. alba); Aunali della R.Ac.d'Agric. d. Torino, 1879 . 
53. . Ueber eine neve Bandwurmart ( $T$. alba); Archiv fiir Naturgeschichte, 1879, pp. 235-237, taf. xvi, figs. 1-10.

54. - - 'Trattato teorico-pratico sulle malattie piu comuni degli Animali domestici dal punto di vista agricolo, commerciale ed igienico. Afetodi di cura ed appendice sui migliori metodi do disinfezione dei vagoni. Torino, 1886 .

(Tania expansa R., pp. 231-232; T. denticulata R., p. 232, Fig. 78 (after Gurlt), Figs. 80-81 (not denticulata); T. alba P., pp. 233-231, Figs. 79, 82; T. globipunctata, Riv., p. 235 ; T. ovipunctata Riv., p. 235 ; T. contripunctata Riv., pp. 235-236; T. ovilla Riv., pp. 236-237; T. aculeata Riv., pp. 237-238.)

55. Railliet, A. Éléments de Zoologie Médicale et Agricole, Paris, 1886.

(Tania expansa, p. 257, figs. 151-153; T. denticulata, p. 260; T. alba, p. 260; T. globipunctata, pp. 260,261; T. ovipunctata, p. 261; T. centripunctata, p. 261; T. aculeata, p. 261; T. Bonedeni, p. 261; T. Vogti, p. 261; T. capra, p. 262; T. Giardi, p. 264.)

55A. - Second edition, 1893 is in press.

[56.] "Raulis. Traité des maladies oceasionées par les promtes et frequentes variations de l'air, Paris, 1752, p. 444, a tapeworm 26 feet long from a 3 months lamb."

[57.] Rivolta, S. Sopra alcune specie di Tenie della Pecora, Pisa, 1874. (T. centripunctata, T. ovipunctata, T. globipunctata.)

58. - - Di una nuova specie di Trenia nelle pecora (Tania ovilla); Giornale di Anat. Fisiol. et Patol. degli Animali, Pisa, 1878, pp. 302-308, figs. 1-3.

[59.] —- Studi fatti nel Gabin. di Anat. patalog. di Pisa, 1879, p. 79, 3 fig's. T. ovilla.

60. Robertson, W. Tænia in lambs; The Veterinarian, London, 1875, vol. xlviii, p. 80 .

61. Rudolphi, C. A. Entozoorum sive vermium intestinalium Historia naturalis. Amstelædami, 1809, vol. ii, p. 2.

(Tania expansa, pp.77-79; T. denticulata, pp.79-80; Tania capreoli and $T$. capra, pp. 200, 201, [Species dubiæ.])

62. - Entozoorum Synopsis cui accedunt Mantissa duplex et indices. Vindobonnæ 1819.

(Taenia capreoli, p. 144; T. dcnticulata, p. 145; T. expansa, pp.144,487. T. capra p. 170.)

63. Scirrank, F. v.P. Verzeichniss der bisher hinlänglich bekannten Eingeweidewïrmer, nebst einer Abhandlung iiber ihre Anverwandschaften. München, 1788 , p. 80 .

64. Steel, J.H. Diseases of the Ox. London, 1881. (Tania expansa, p. 294.)

65. Stiles, C. W. Notes on Parasites-8: A check list of the animal parasites of cattle; Journal of Comparative Medicine and Veterinary Archives, New York, 1891, p. 346.

(Tania expansa, T. denticulata, T. alba, T. centripunctata, T. globipunctata.)

66. - Notes sur les Parasites-13: Sur le Tania Giardi (Riv.) Moniez; Comp. rend. de la Société de Biologie. Paris, 1892, pp. 664-665.

67. — - Notes sur les Parasites-14: Sur le Trenia expansa Rud.; Compt. Rend. de la Société de Biologie. Paris, 1892, pp. 665-666.

68. - - Bemerkungen über Parasiten-17: Ueber die topographische Anatomie des Gefässystems in der Familie Tæniadæ; Centralblatt für Bakteriologie und Parasitenkunde, 1893, bd. xiii, pp. 457-465, figs. 1-12.

(M. planissima, M. Benedeni, M. Neumanni, M. expansa, M. oblongiceps, $M$. trigonophora, M. denticulata, M. alba, Thysanosoma actinioides, Th. Giardi, Tonia centripunctata, T. globipunctata.)

69. - and Hassall, Albert. A Revision of the Adult Cestodes of Cattle Sheep, and Allied Animals. Bulletin 4, Bureau of Animal Industry, U. S. Department of Agrieulture, Washington, D. C., 1893 (the present paper). Pages 1-134, 16 plates. 
[70.] TABL. ENCYCL. t 45. (Figs. 1-12. (i. e. Goeze). Tania ovina=T. expansa (after Rud.).

71. Verrill, $\Lambda$. E. Parasites of Domestie Animals; Report of the Connecticut Board of $\Lambda$ griculture, 1870.

(Tania cxpansa, pp. 65, 100; T. denticulata, p. 65.)

72. Viltar, Sylney. 'The Veterinarian, Oct., 1886.

[73.] Zener, J. (x. II. Anleitung zur Naturgeschichte der Eingeweidew ürmer. Bamberg, 1803.

(Halysis ovina $=T$. expansa, after Rud.)

74. Zsсnокке, F. Recherches sur la Structure Anatomiqne et Histologique des Cestodes. Genève, 1890. (Truia expansa, pp.93-114, figs. 31-38.)

75. ZürN, F. A. Die thierischeu Parasiten auf und in dem Körper unserer Hauss:iugetiere, Wiemar, 1882.

(Tania expansa, p. 189, taf. iji, figs. 39-41; T. denticulata, p. $197 ;$ T. alba, p. 197; T. ovilla, p. 198.)

Several other references were found but we have been unable to trace the articles.

S. \& H. 


\section{ADDENDA.}

As the manuscript of the preceding pages left the author's hands some months ago, it may be well to add a postseript covering several interesting observations made since that time. During the past six months it has been possible to examine a large number of specimens of adult cestodes of cattle and sheep, and thus to put the analytical table given on pp. 88,89 to a very severe test. The parasites examined came from the several sources enumerated below:

(a) Thirty strobila collected by Dr. Melvin and assistants at the abattoirs of the Union Stock Yards of Chicago. These strobilie were preserved in weak alcohol and did not come into my hands until they were quite macerated. Although disappointed at first because the material was not in better condition, its examination has led to some very important and rather unlooked-for results.

(b) A few segments of Rudolphi's original Tania capra, kindly forwarded by Geheimrath Karl Mïbius.

(c) About 100 strobilæ of Moniezia from cattle and sheep collected by Dr. Hassall at the Washington (D. C.) abattoir, and fixed aceording to the method described on pp. 13,14 .

(d) Several jars of tapeworms from sheep belonging to the Leidy collection, University of Pennsylvania. The specimens are all members of the genus Moniezia, but are very much macerated.

(e) Several strobilæ collected by Dr. Theobald Smith.

(f) About 100 strobilio collecterl by myself in August, from cattle at the abattoirs of Chicago, and preserved according to the method described on p. 13.

The specimens collected by Hassall and myself were very readily determined as belonging to the species M. planissima, M. expansa, and

- M. trigonophora. Several strobila bear a very close resemblance to $M$. Neumanni, but I am not willing at present to state definitely that they belong to that species. A study of the forms not " fixed" before being placed in alcohol emphasized the importance of not relying upon external form in making the specific determinations--a point to which attention has already been drawn in the text-for it is the easiest thing in the world to determine an $M$. expansa as $M$.planissima, or vice versa. If microscopic preparations are made, however, there is not the slightest difficulty in distinguisling the Planissima group from the Expansa group. In many cases, however, it is difficult to distinguish $M$. expansa from $M$. trigonophora by examining only one or two segments, but the species are easily separated by a more careful study.

The specimens of Leidy's collection, although too macerated to be determined specifically, could easily be divided into the Planissima group and the Expansa group. 
The results of the study confirm the position taken in the preceding text, that the furm of the interproglottidal glands furnishes a basis for a natural division into the Planissima group and the Expansa gromp. I am led, however, to doubt the advisability of placing very much stress upon the size of the glands in the I'lanissima group as a criterion in determining the species; in several eases strobila were found which agreed with M.planissima in all respects except that the hear was much larger and the linear glands extended the cntire (or almost the entire) width of the segment. Preferring to be conservative, I lave deemed it best for the present to look upon these specimens as the M. planissima rather than to ereate a new species for them, for in studying parasites, as well as other animals, we must expect to find individual variation. This variation can, for the present, be held to account for the enormous size of the linear glands. The enormous size of the heads in several specimens might possibly be accounted for by individual variation and method of presercation, for I have studied the live heads a number of times, and find that, in their contraction, they can change in size to a consirlerable degree.

An interesting fact was developed in comparing unfixed and fixed material with Perroncito's types of $M$. alba. Specimens of $M$. planissima when fixed with sublimate bear absolutely no resemblance to Perroncito's $M$. alba (the type specimens of $T$. alba were evidently preserved in alcohol without first being treated with sublimate); specimens of M. planissima which have not been fixed in sublimate, but have remained in weak (50) per cent) alcohol for some time, differ from specimens which have been fixed in sublimate to a marked degree. In fact, when I first examined the specimens macroscopically I was entirely at a loss to know to which species they might possibly belong. A microseopie examination of a number of slides, however, left no doubt that they were typical specimens of $M$. planissima, and that any difference in the form of the segments or the genera ag, veupang of the strobilar

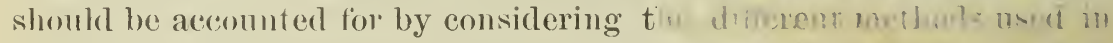
preserving the specimens. Next came an interestmg, whel to my lume? an important observation, i. e., that a few of the more poorly preserved of the alcohol specimens hore a remarkable macoscopic resemblance to Perroncito's type of T. alba. In fact, in the ease of one specimen the resemblance was so striking that when I compared it side by side with one of Perroncito's specimens I was unable to tell which was the Italian and which the Ameriean form without comparing the labels. Having called attention to this remarkable maroscopic resemblance, it is in place to compare the microscopie slides. In no case have I found a single slide, in the many preparations made from three of Perroneito's types, in which there was the slightest trace of interproglottidal glands; on the other hand, I have never failed to find these linear glands in the segments of $M$. planissimu which were treated with sublimate: in the vast majority of cases those nonfixed sperimens of American tape- 
worms which did not belong to the Expansa group have possessed linear interproglottidal glands. Most of these are undonbtedly our $M$. planissima; on the other specimens I prefer to reserve judgment for the present. The same character was present in French specimens sent to no by Railliet and Neumann. Now, euriously enough, seven nonfixed fragments of American forms fail to show any signs of interproglottidal glands. These specinens are poorly preserved, but notbadly macerated; under the microscope they can not be distinguished from $M$. alba. I should not think of uniting them with $M$. planissima, on account of the absence of the linear interproglottidal glands; if such glands were present they would probably have been brought out by staining, for we have frequently found these glands in fragments which were more macerated than those now under discussion. If pressed to a determination of these fragments, I should accordingly label them .M. alba, although rather unwillingly, because they are only fragments and are the only specimens of $M$. alba (?) ever collected in America.

I might add, further, that two of Perroncito's types agree perfectly with each other when examined macroscopically, while the third, as well as Neumann's specimens, would probably not be united with $M$. alba by any worker except after a careful microscopic study.

To recapitulate, in a few words, we may conclude that-

(1) Specimens of a species fixed in sublimate may differ so in macroscopic appearance from specimens of the same species simply preserved in alcohol that scarcely any helminthologist would hesitate to recognize them as belonging to different species.

By using different methods of preservation and different degrees of maceration it has been found possible to bring about such a variation in external form of different specimens of $M$. planissima and $M$. expanse, or of different portions of the strobila of a single specimen that almost any species (if established on external form alone) can be made to order.

(2) It is entirely unsafe to determine a given specimen of Moniezia as $M$. planissima, M. expunsa, or in fact as almost any other species without first examining several stained segments. Segments with well-developed testicles and ovaries are the best for this purpose.

(3) As specimens macerate, the linear interproglottidal glands of $M$. planissima stain less distinctly.

(4) In Perroncito's type of $M$. alba there is no trace of any interproglottidal glands; these specimens were evidently not fixed in sublimate before being placed in alcohol, but they are not badly macerated.

(5) One specimen of an undoubted M. planissima resembles one specimen of Perroncito's type so very closely (macroscopically) that no zoölogist would hesitate to pronounce the two specifically identical, were it not that linear glands are present in the former and absent in the latter. 
(6) The specimen of $M$. alba referred to (5) bears no macroscopic resemblance to Perroncito's other two types, nor to his figures of this species, but on account of the absence of the glands it must be united with $M$. alba.

Upon reading the above, some of my European colleagues will undoubtedly conelude that $M$. planissima and $M$. alba are specifically identical, that the absence of the linear gland is to be accounted for by maceration, and that we have been too hasty in ereating $M$. planissima as a new species. Such a conclusion would, however, be entirely unjustified unless the person based his opinion on a eareful study of the types and a large series of typical specimens. If anyone is able to find linear interproglottidal glands in Perroncito's types and to prove that Perroncito's types contain but one species, then he would be perfectly justified in uniting $M$. planissima and $M$. alba as one species, and no one would be more willing to accept his results than Hassall and myself. I have not been able to find these glands in $M$. alba, but have found them in every specimen of tapeworm of the genus Moniezia except those of the Expansa group, the few segments of $M$. denticulata now at my disposal, in Perroncito's and Neumann's specimens of $M$. alba, and the few fragments mentioned above as M. alba (?).

This Bureau has already sent to a number of zoölogists typical specimens (stained slides and alcohol specimens) of the forms described in this paper, and it can safely be leit to European specialists to decide whether we were justified in creating the species $M$. planissima, and it is needless to add that the Bureau types and preparations are always at the disposal of specialists in helminthology. I feel that we have solved the question of the classification of this genus so far as it applies to the American forms and to the limited amount of European specimens at our disposal. If we have made any errors in judging the European forms they can best be corrected by onr European colleagues. Looking to the possibility of an European revision of this genus in the future I should like to call particular attention to the following points:

(1) Are the scarcely visible linear glands in M. Benedeni, mentioned in this paper, in reality interproglottidal glands or have I here mistaken some of the more intensely stained subenticular cells for glandular cells? This point can be determined only by an examination of properly preserved, freshly collected material. If I have here made an error of interpretation, the species should be placed in the "Denticuĩata group."

(2) Are interproglottidal glands present in freshly collected and properly preserved material of $M$. denticulata?

(3) Can the species Stilesia centripunctata remain in the same genus with S. globipunctata? This certainly can not be so unlews we find more points of resemblance between the two forms than we know at present. 
(4) Any classification of any genus of tapeworms, based upon externial form alone, can not claim the attention of specialists. Anatomy must form the basis of our future revisions.

(5) Individual variation must be taken into consideration in the study of parasites just as weil as in the study of other animals. Slight, or, in many cases, even considerable, difference in the size of the hear, form of the segments, etc., can often be explained as due to different methods of preserving.

Moniezia capre sp. inq.

The segments of Rudolphi's T. caprce, now in my possession, warrant but one statement, $i$.e., that the species is evidently a true Moniezia.

The proglottids are so crowded with ova that one would scarcely expect to distinguish the interproglottidal glands, even if they are present. Experience in comparing the exterual form of specinens has taught me so many lessons that I would not even dare to speculate as to the possible affinities of this specimen with the other species of Moniezia.

Thysanosoma actiniodes.

This species has been found in South Africa (personal conversation with Dr. S. Wiltshire).

C. W. S.

OC'TOBER 28, 1893. 



\section{INDEX TO SPECIFIC NAIr̃ES.}

The nannes in italics are synonyms. In the case of such forms as T. marmota, $T$. pectinata, etc., whose generic position is somewhat uncertain, I have retained them provisionally in the colleetive genus Thuia, pending the establishment of new genera. Private ardviees from Prof. Railliet inform me that he intends to establish several new genera in the second edition of his Traité de Zoologie médicale et agricole (now in press). According to Prof. Railliet's letters he has adopted the MS generic name Audrya for T. marmote and Ctenotenia and Rhopalocephala for Riehm's species. As Railliet's secont edition lias not yet reaehed this comtry, at the time of the final proof-reading and indexing of this bulletin, I an not in a position to state positively whether Railliet has retained in his work the manuscript numes given above, and the reader must, therefore, be referred to Railliet's publication for the generie names to be employer in comneetion with the speeies in question. C. W. s.

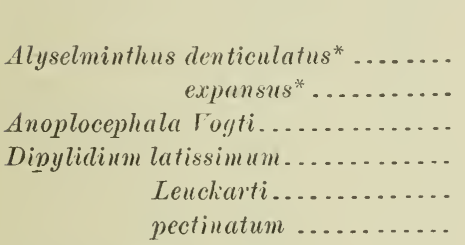

Halysis ovina*.................

Linguatula armilluta ..............

Monieza alba

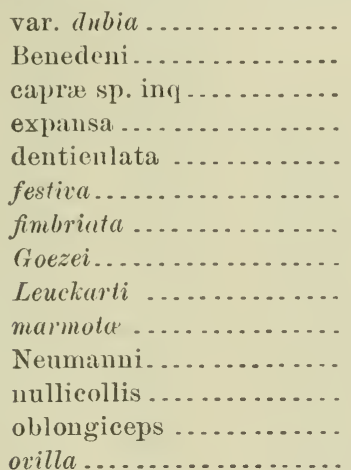

pectinuta.............. 53,71

planissima............. 15,98

trigonophora .......... $\quad 37$

Peutastomum proboscidenm........ 12

Porocephalus anunlosus ......... 12

armillatus ......... 12

constrietus ........ 12

crotali........... 12

megastomns ....... 12

moniliformis....... 12

oxycephalus ........ 12

polyzonus ......... 12

subuliferns ........ 12

Stilesia centripunctata ......... $\quad 79$
42 Stilesia globipmetata .......... Page.

26 Strongylus eontortus ............ $\quad 37$

83 Tania aculeata................ 59

alba................. 47,53

Benedeni .............22, 37, 53

capra................. 86

capraa................ 86

capreoli ................ $\quad 86$

caprina ................ 86

centripunctut $a . . . . . . . . . \quad 79$

centripuntegyiata .......... $\quad 79$

crassicollis ............. 78

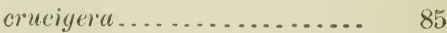

denticolata .............. 42

denticnlata........22, 26,42,53

expansic $\ldots . . . .15,26,37,53,55,74$

festiva ................ 53

fimbriata.............. 53,55

Giardi ................. 53,59

globipunctata............ $\quad 73$

globipunteggiata ........... 73

hyracis ............... $\quad 70$

madagascariensis ........ 60

marinot: .............. 53,71

ovi-globipnnctuta .......... 74

ovilla .................53, 59, 62

ovina ................. 26, 86

ovipunctata ............. 73

ovipnnteggiata........... $\quad 73$

ovis arietis .............. $\quad 62$

pectinata .............. 53

saginata ............... 32

solium................ 78

vasis nutriciis distinctis...... $\quad 26$

Vogti................. 83

Thysonosoma actinioides ......53, 55, 101

Giardi........... 59

* Origiual article not at my disposal. 
Plate I.-Moniezia planissima.

Fig. 1. Adult strobila, natural size, slightly contracted.

Fig. 1a. Ripe segments, uncontracted.

Fig. 2. Head, ventral view, balsam preparation. $\times 17$.

Fig. $2 a$. Head, alcohol specimen. $\times 17$.

Fig. 2b. Head, en face, alcohol specimen. $\times 17$.

Fig. 3. Segments, $10^{\mathrm{min}}$ from the anterior extremity, showing the first appearance of the genital anlagen.

Fig. 4. Segments, $70^{\mathrm{mm}}$ from the anterior extremity, showing the pistol-shape anlagen of the genital canals.

Fig. 5. Sagittal section, $20^{\mathrm{cm}}$ from the anterior extremity, showing the overlapping posterior flap of each segment.

10.4 

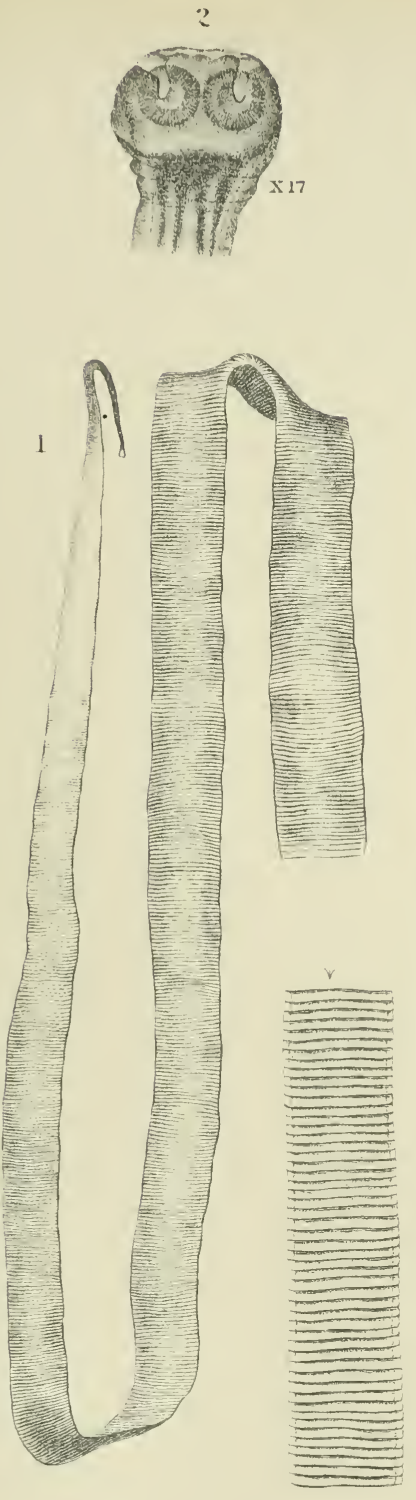
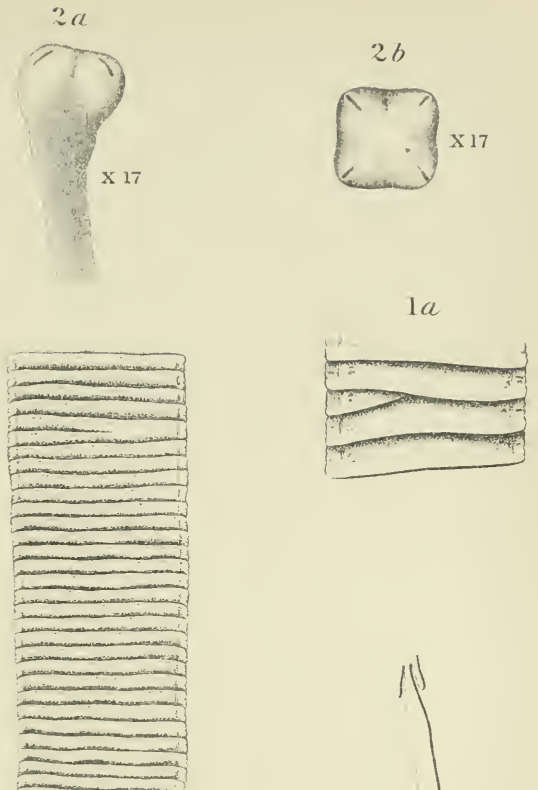

201-
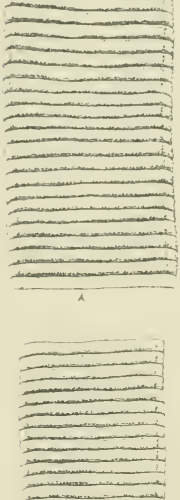

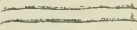
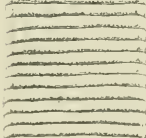

$1 a$

$2 b$
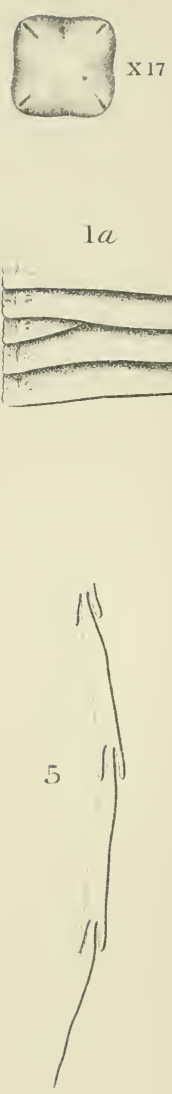

3

tis

4

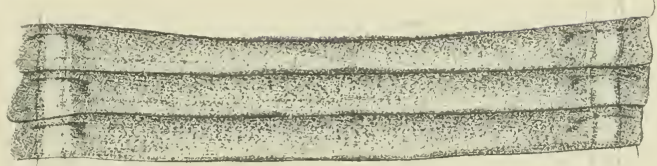




Plate II.-Moniezia planissima and M. Renedeni.

Figs. 1-6 M. planissima.

Fig. 1. Diagram of the female organs, dorsal view : r.s., receptaculum seminis; $o v .$, ovary; s. g., shell-gland; $r . g$., vitellogene gland; $v . d t$., vitello-duct; o. $d_{.,}, o . d^{\prime}$., o. $d^{\prime \prime}$., oviduct.

Fig. 2. Frontal section of the interproglottidal gland.

Fig. 3. Diagrammatic figure of a transverse section; $r$, vagina; $c$., cirrus; $n$., nerve; $v$. c., ventral canal; $d . c$. , dorsal canal.

Fig. 4. Dorsal view of segment, $200 \mathrm{~mm}$ from the anterior extremity; i.g., interproglottidal gland; $t$., testicles; $d$.c., dorsal canal; r. c., ventral canal; n., nerve.

Fig. 5. Ripe segments filled by the uteri.

Fig. 6. Ovum.

Figs. 7, 8. M. Benedeni, drawn from one of Moniez's original specimens.

Fig. 7. Head and strolbila, natmral size.

Fig. 7a. Two segments, drawn to show the peculiar architecture in contraction.

Fig. 8. Scolex, en face. $\times 17$.

Fig. $8 a$. Scolex, ventral view. $\times 17$.

$10 t$ 


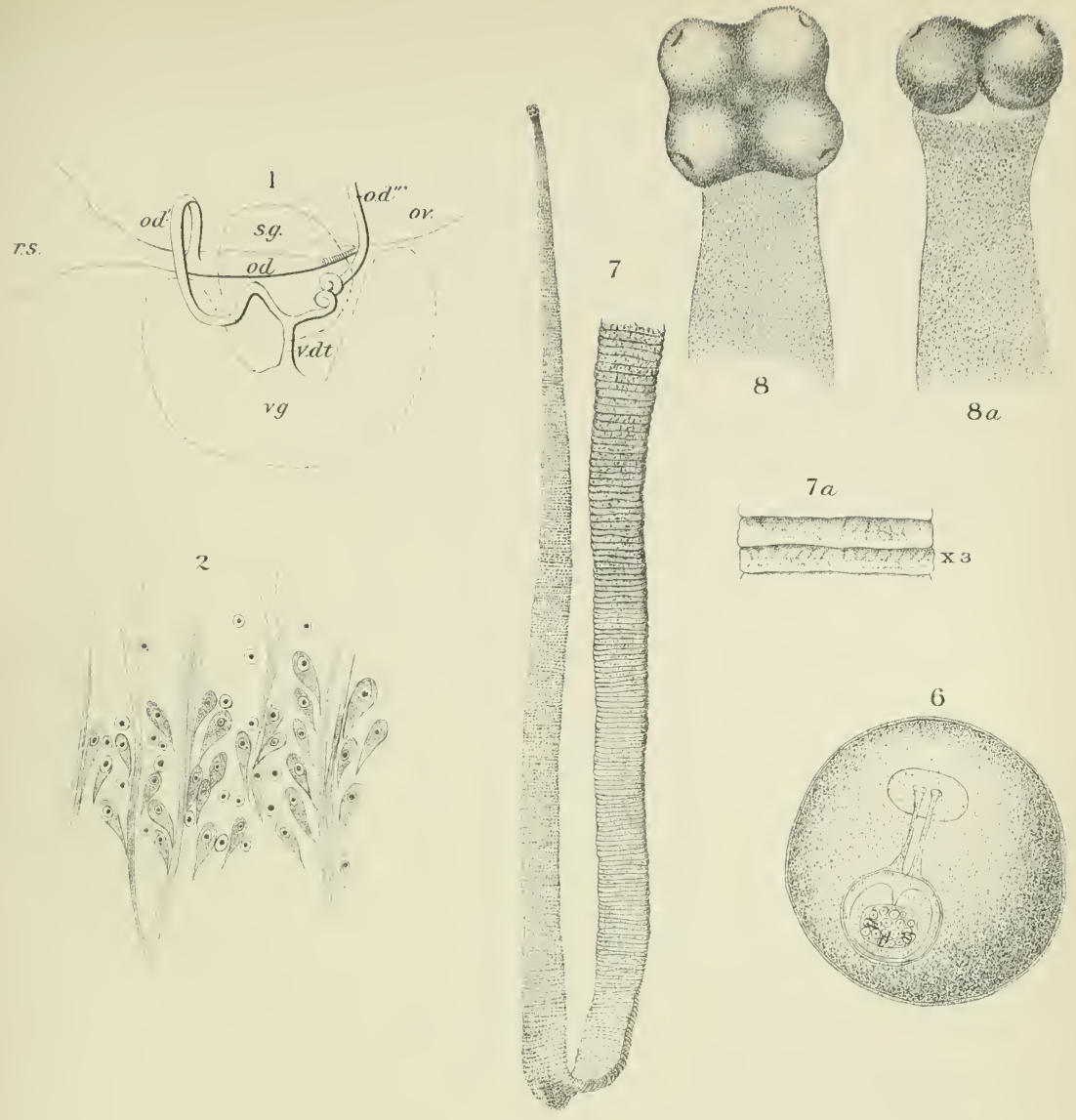

3

Dorsal

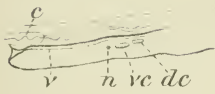

Ventral
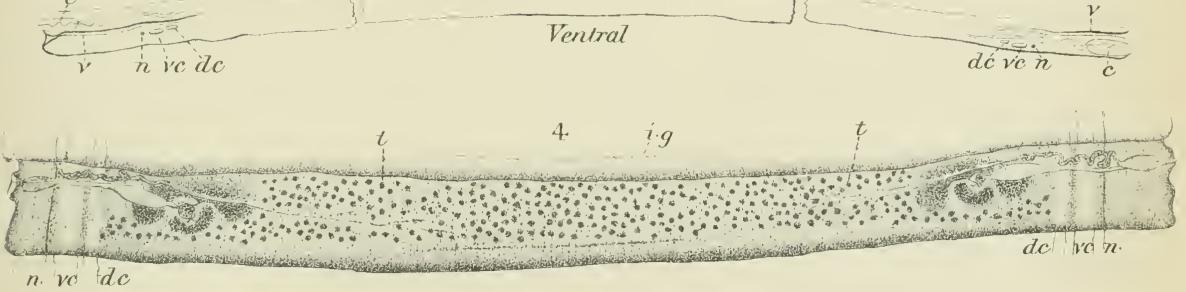

5

Cun

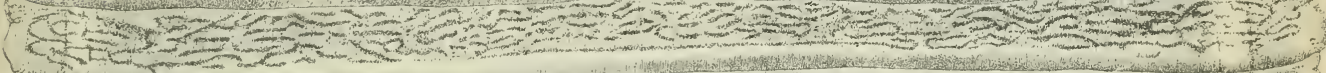

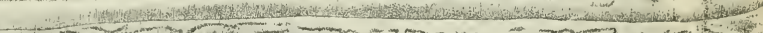

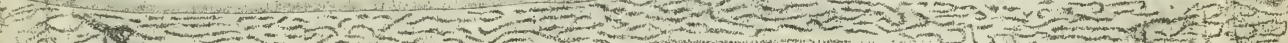

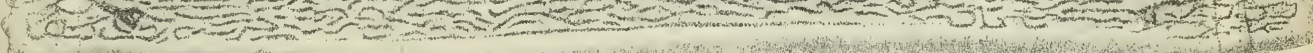

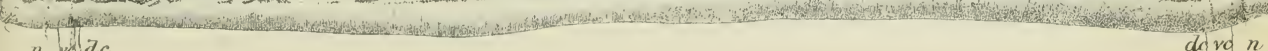




Plate III.-Moniezia planissima.

Fig. 1. Transverse projection of the female canals, diagrammatic.

Fig. 2. Sagittal section of the interproglottidal gland.

Fig. 3. Portion of segment, showing the genital organs.

Fig. 4. Longitudinal section of cirrus and vagina, slightly diagonal.

Fig. 5. Transverse section of vagina.

Fig. 6. Transverse section of cirrus.

Fig. 7. A young egg, diagrammatic.

Fig. 8. Disk of the pyriform body.

108 

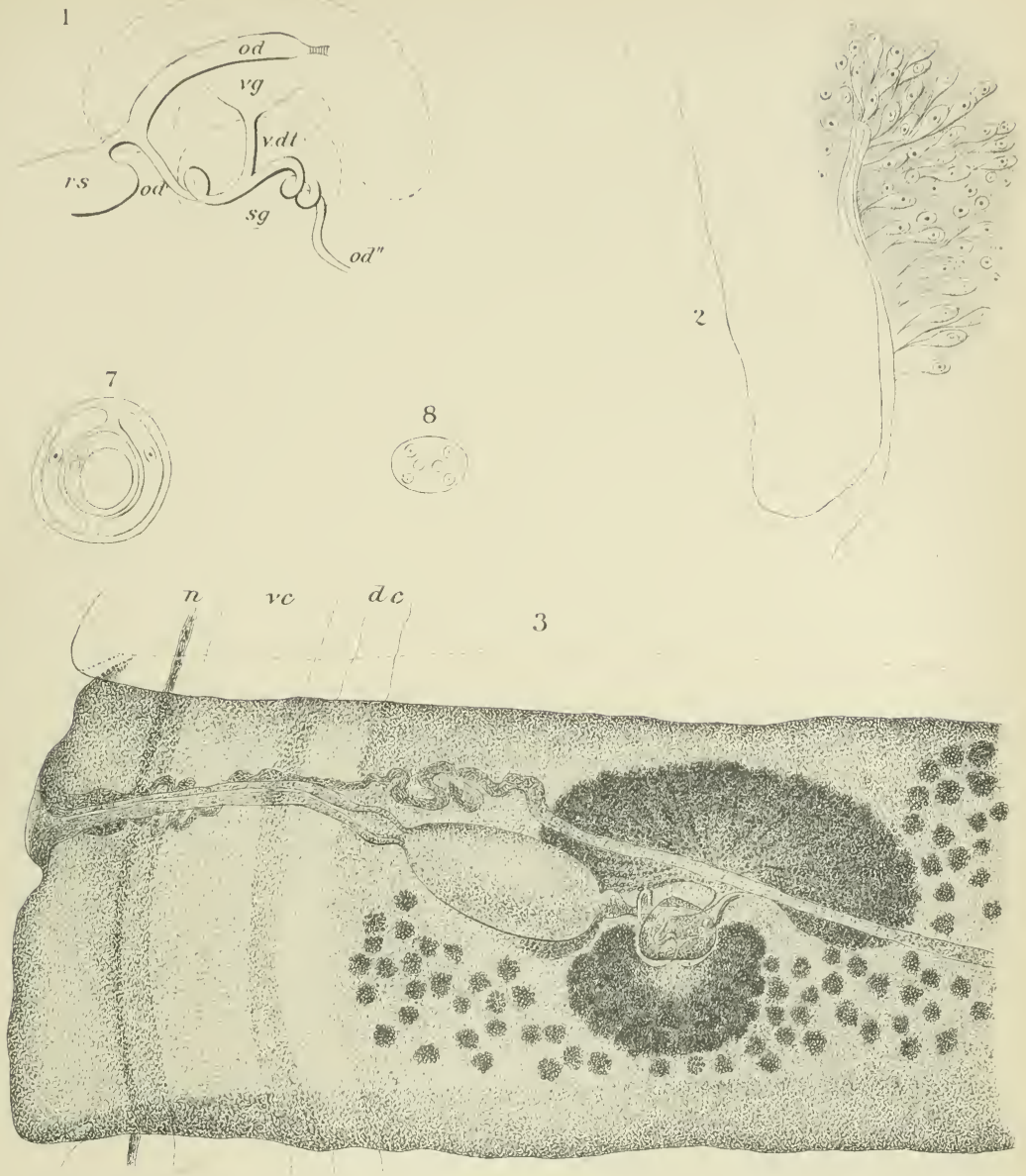

5

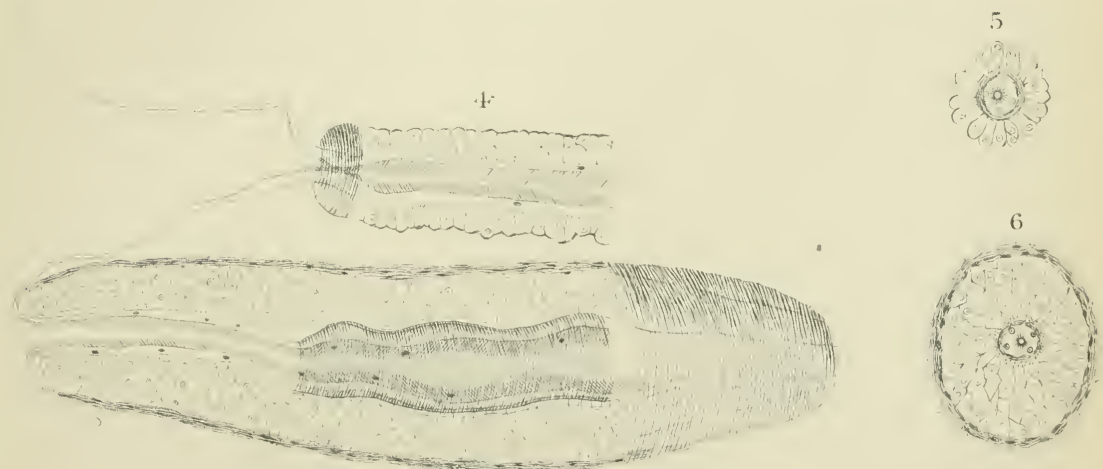




Plate IV.-Moniezia Neumanni (drawn from Moniez's original material).

Fig. 1. Fragments (contracted) natural size.

Fig. 2. Head, ventral view. $\times 17$.

Fig. $2 a$. Head, en face. $\times 17$.

Fig. 3. Segments with testicles and anlagen of the genital eanals. (Ventral view).

Fig. 4. Same, greatly enlarged.

Fig. 5. Segment with fully developed uterus. 


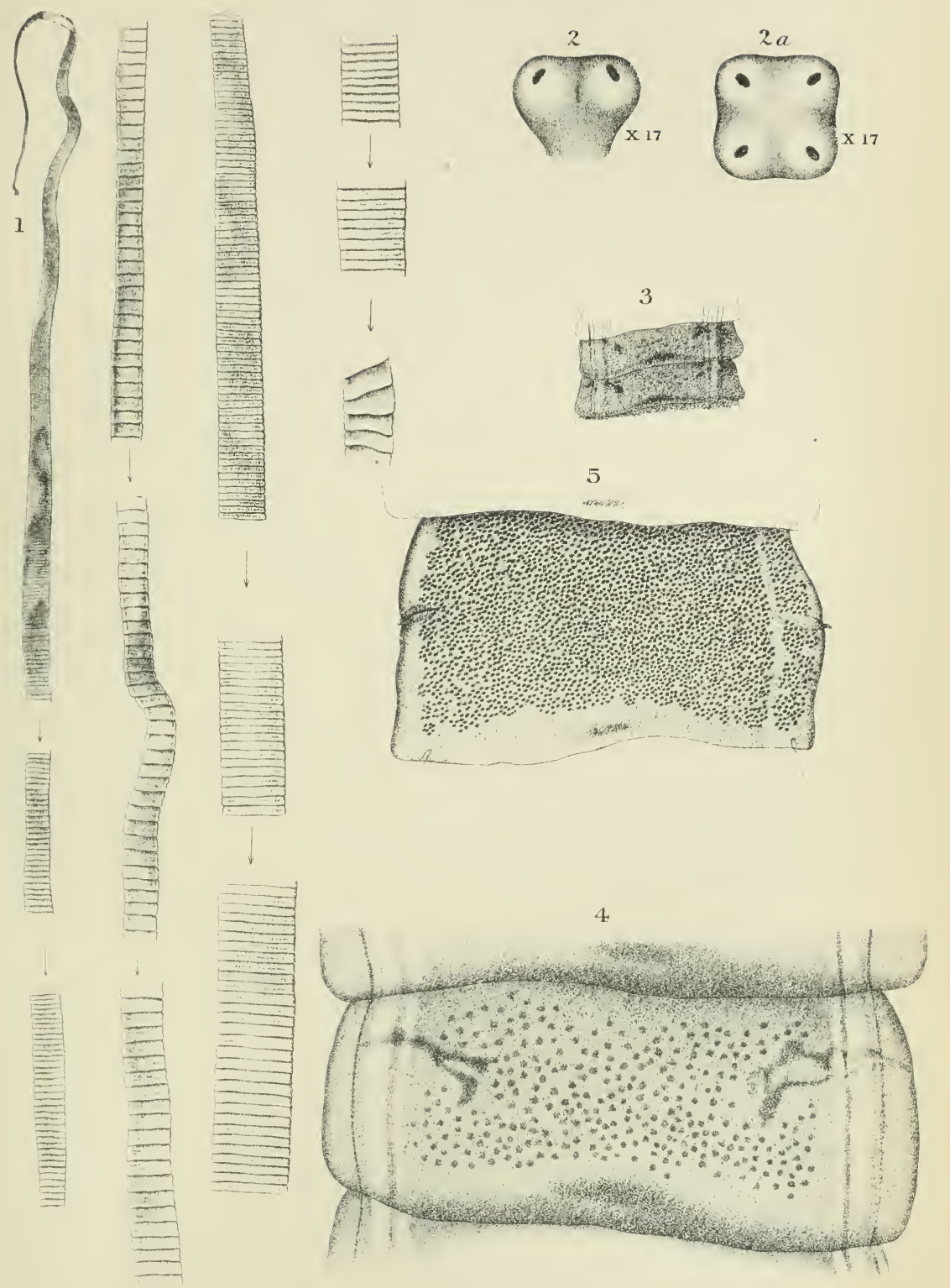




Plate V.-Moniezia expansa and M. denticulata.

Figs. 1-3. M. expansa, drawn from Rudolphi's original material (1810). Orig. Fig. 1. Scolex, ventral view. $\times 17$.

Fig. 1a. The same, en face. $\& 17$.

Fig. 1b. The same, viewed diagonally. $\times 17$.

Fig. 2. Segments in which the uteri were not developed.

Fig. 3. Segments in which eggs were developed.

Figs. 4-6. M. denticulata: 4-5 drawn after Goeze's figures of Ruflolphi original

material; 6 drawn from Rudolphi's original material (orig).
Fig. 4. Strobila with head, natural size.

Fig. 5. Hear.

Fig. 6. Mature segments. 


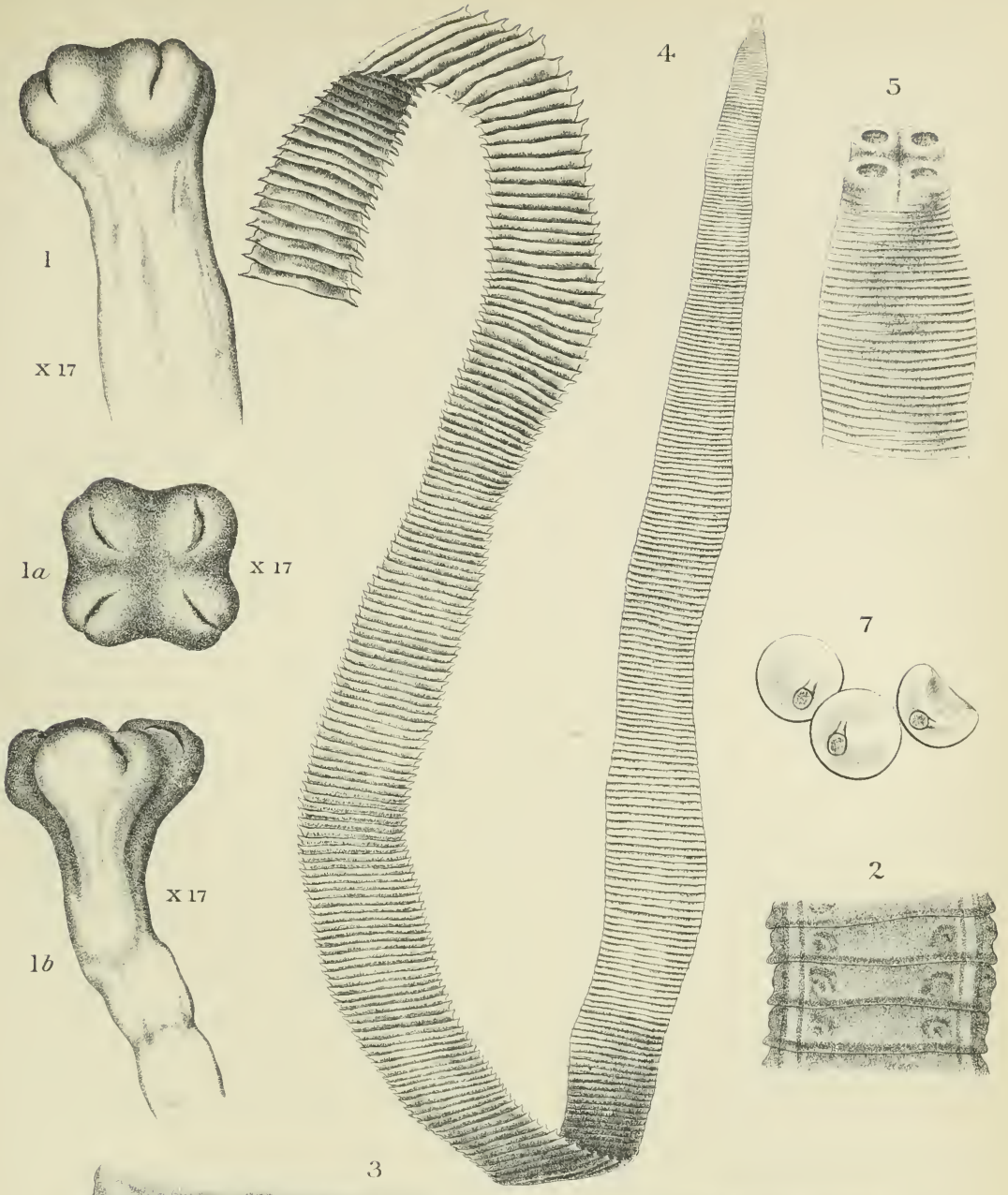

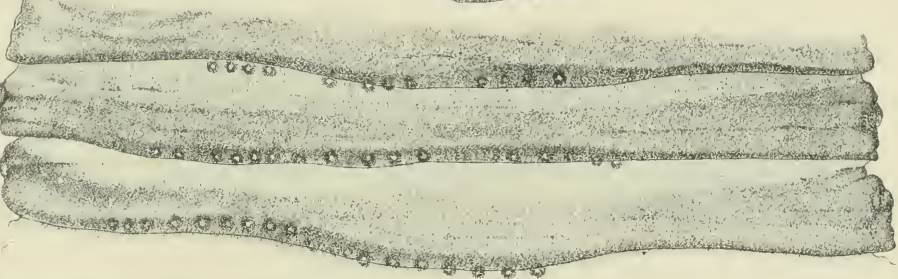

6

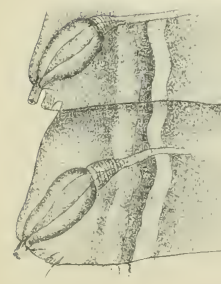

HAINES, DEL 


PIATE VI.-Moniezil expansa.

Fig. 1. Strobila, natural size, collected by Curtice, Washington, D. C. Figs. 1a, 1b. Segments of (urtice's type (Animal Parasites of Sheep). $\quad \times 3$. Fig. 1c. Segments collected at Paris. (Stiles.) $\times 3$.

Figs. 2-2a. Heads collected at Washington, D. C.; very small. $\times 17$.

Fig. 3. Segments $20 \mathrm{~cm}$ from head showing interproglottidal glands, pistul-shape frenital anlagen, testicles. Dorsal view.

Fig. 4. Segments showing complete anatomy except the uteri. Dorsal view.

Fig. 5. Segments from Paris (Stiles' private collection). Ventral view.

lig. 6. Segment showing the uteri. Ventral riew. 


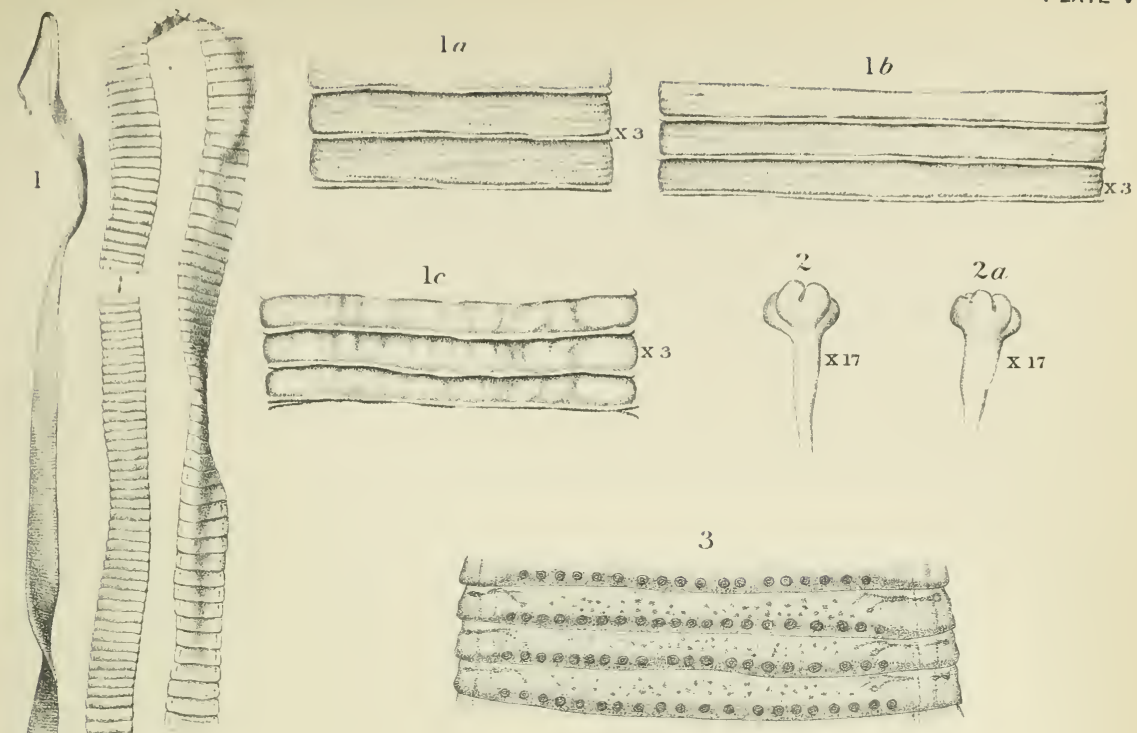

4.

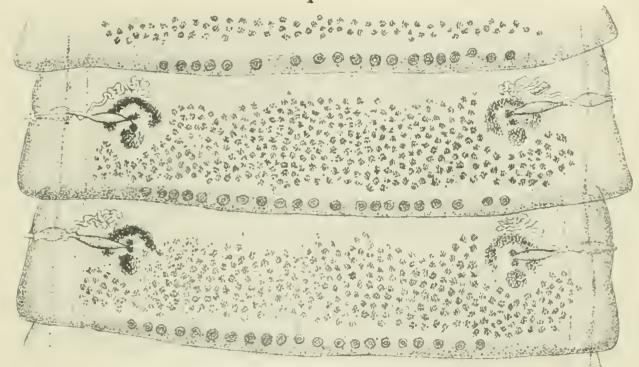

.5

6

How

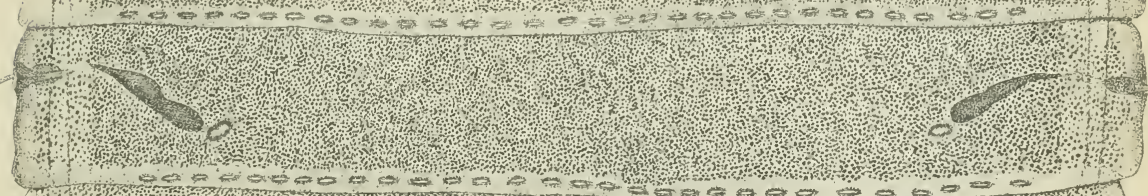
1.1\%का 


PLATE VII.-Moniezia oblongireps-Thysanosoma Giardi-Tania marmota.

Figs. 1-1. M. oblongiceps.

Fig. 1. Strobila, natural size.

Fig. 2. Head, alcohol specimen, ventral view.

Fig. 2a. The same, en face.

Fig. 2b. Hearl, balsam preparation.

Fig. 3. Segments showing genital canals, testicles, interproglottidal glands, etc. Fig. 4. Egg.

Fig. 5. Section of Th. Giardi; g. p., genital pore; $v$. s., vesicula seminalis; $v$. $d$. , convolutions of vas deferens with prostata; $v$. $d$., vas deferens; $v$, vagina; $r . s$, receptaculum seminis; o.d., descending oviduct; o. $d^{\prime}$., ascending oviduct; $u . t$. , uterus; o. $v$, ovarium; v.g., vetellogene gland; $v . c$. , ventral canal; $d . c$. , dorsal canal; $t . c$. , transverse canal; $t$., testes.

Fig. 6-7. Tania marmota.

Fig. 6. Segment showing the anatomy with the exception of the uterus, dorsal view.

Fig. 7. Segments with developed uterus. 
BUL. 4, BUREAU OF ANIMAL INDUSTRY.
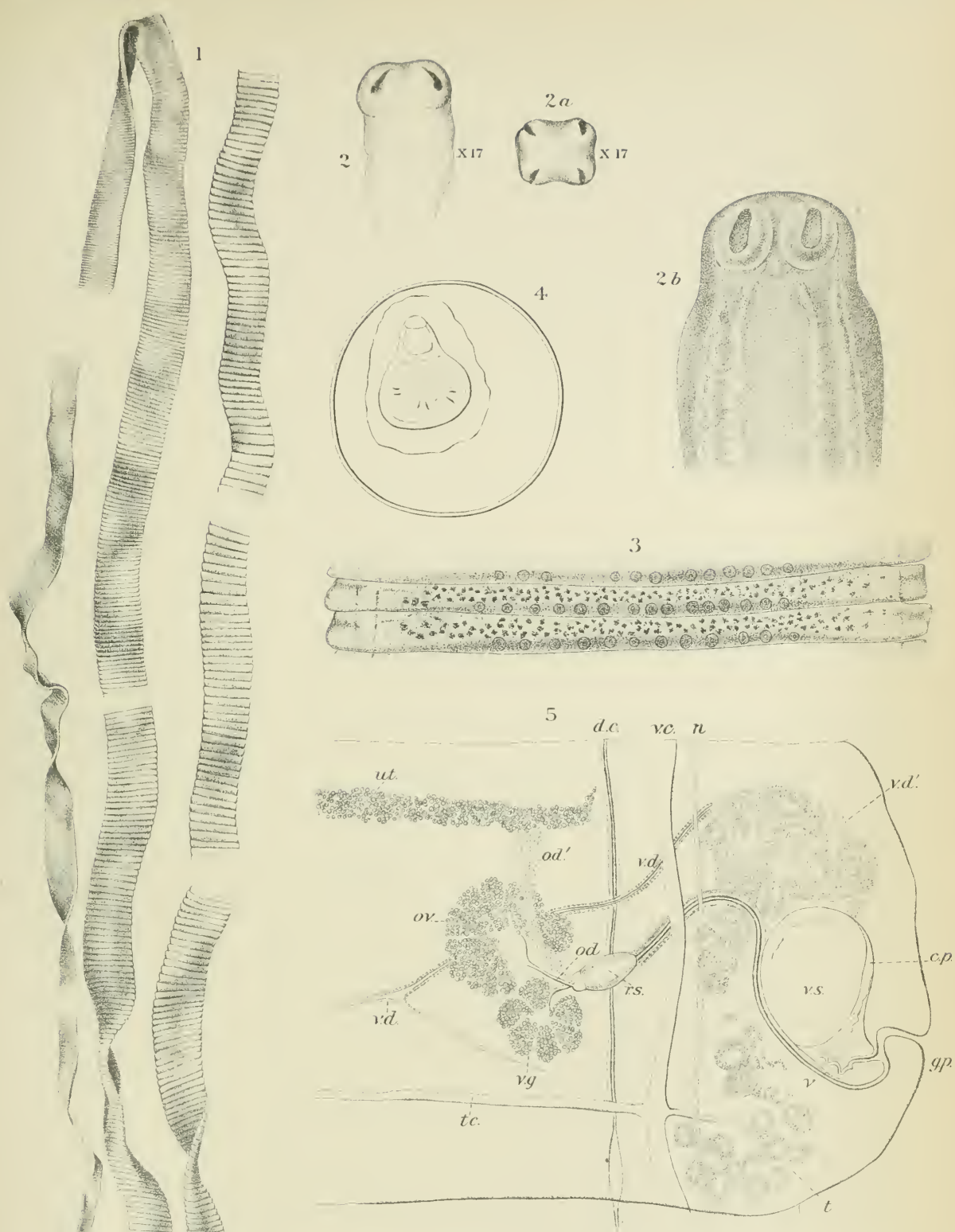

6
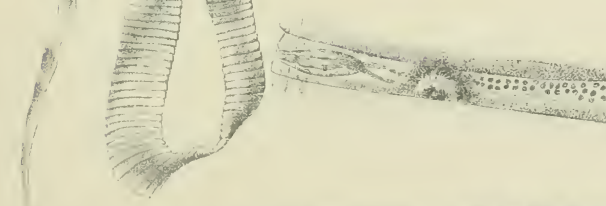

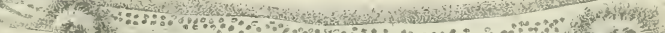
…… 7

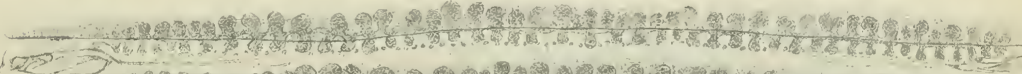

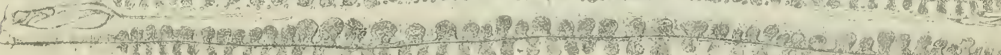




Plate VIII.-Moniezia trigonophora.

Fig. 1. Strobila, natural size.

Fig. 2. Head, balsam preparation. $\times 17$.

Fig. $2 a$. Head, alcohol preparation, ventral view. $\times 17$.

Fig. 2b. The same, en face. $\times 17$.

Fig. 2c. The same, lateral view. $\times 17$.

Fig. 3. Segments $10^{\mathrm{cm}}$ from anterior extremity.

Fig. 4. Segments $30^{\mathrm{cm}}$ from anterior extremity.

Fig. 5. Diagram of female glands and eanals, dorsal view. 
BUL. 4, BUREAU OF ANIMAL INDUSTRY.

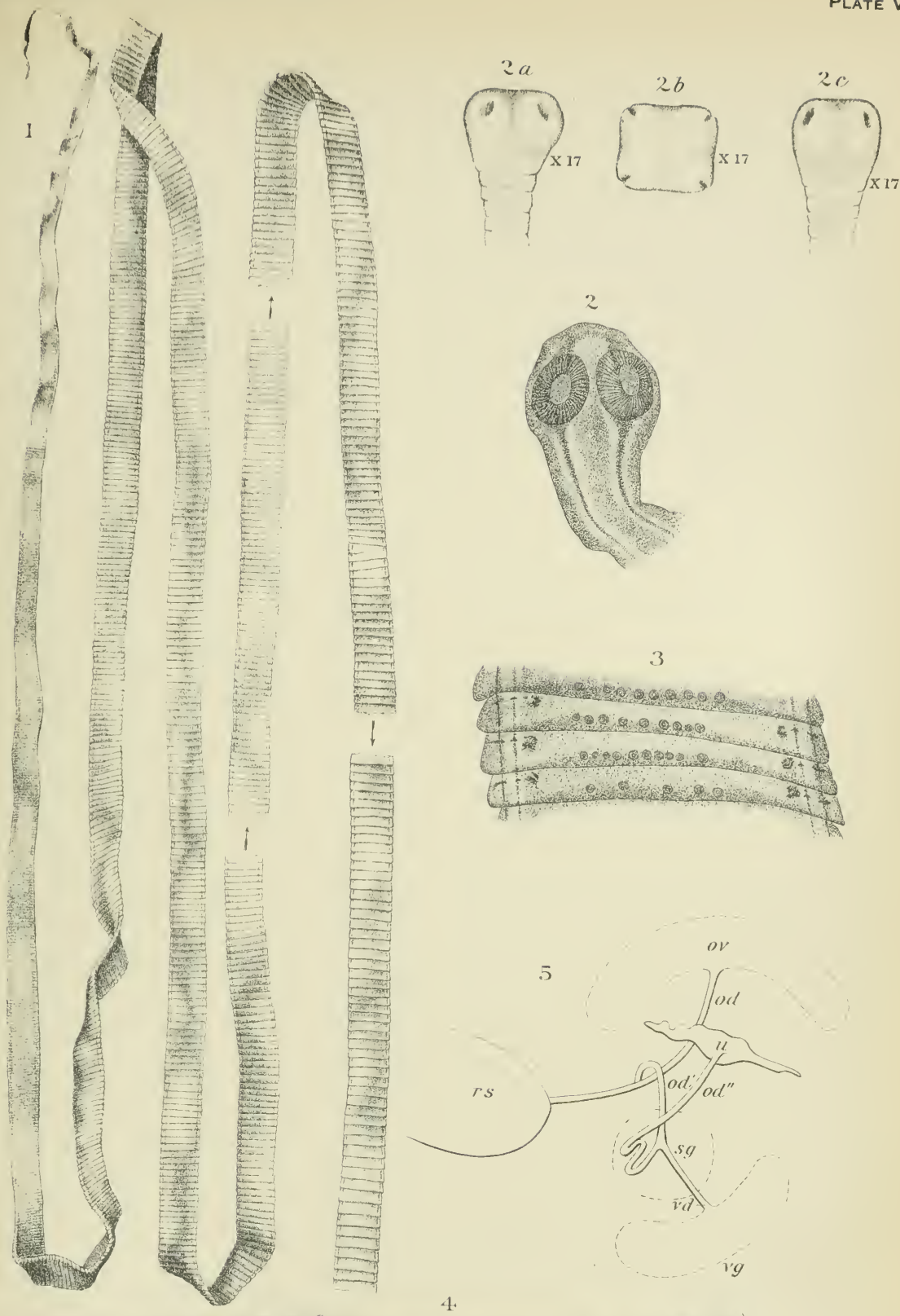

4.

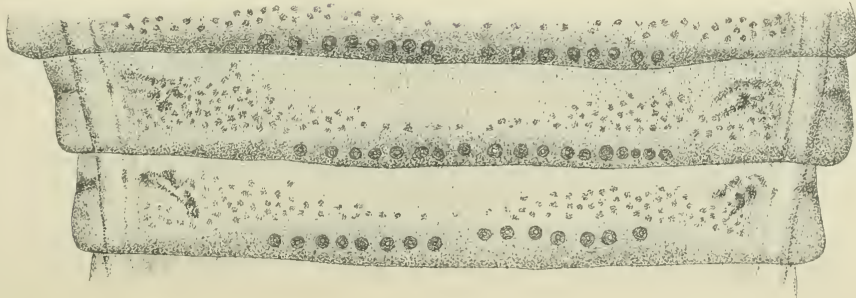




Plate IX.-Moniezia trigonophora.

Fig. 1. Sagittal section of a sac around which the eells of the interproglotticlal gland are grouped.

Fig. 2. The same, frontal section.

Fig. 3. Diagram of the female glands and canals, transverse section.

Fig. 4. Segment $60^{\mathrm{cm}}$ from the anterior extremity, showing all the organs except the uteri, which have not yet developed. Ventral view.

Fig. :. Segments with developing uteri; a space is left vacant in the median line.

Fig. 6. segments with fully developed uteri and eggrs. 

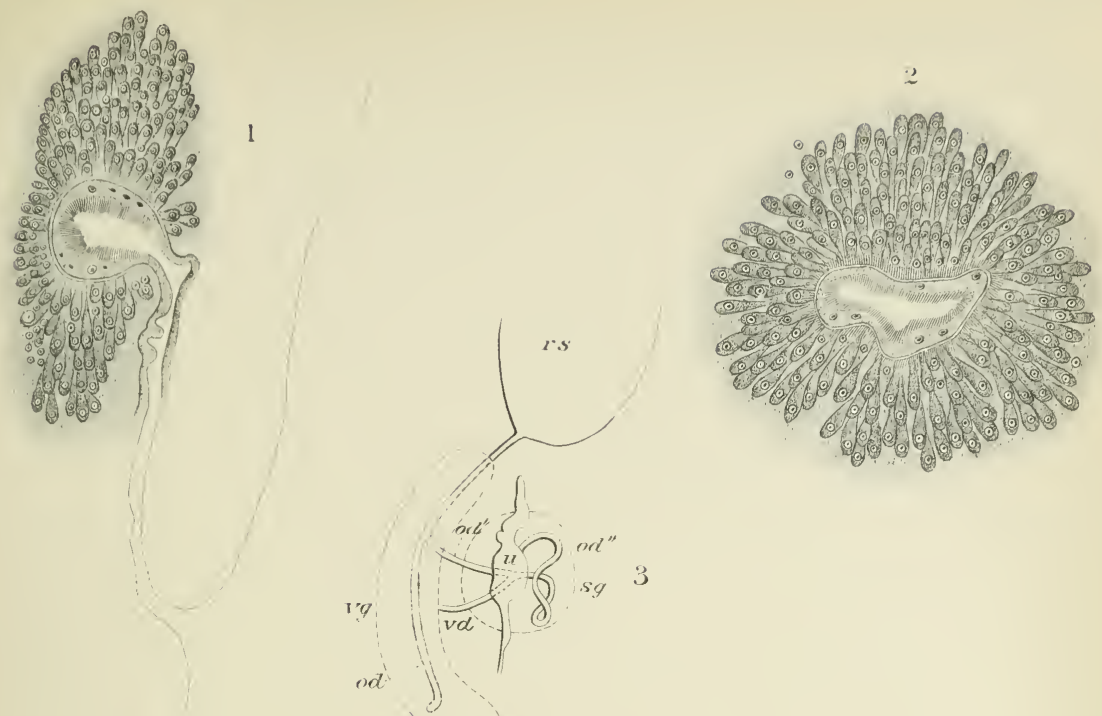

4

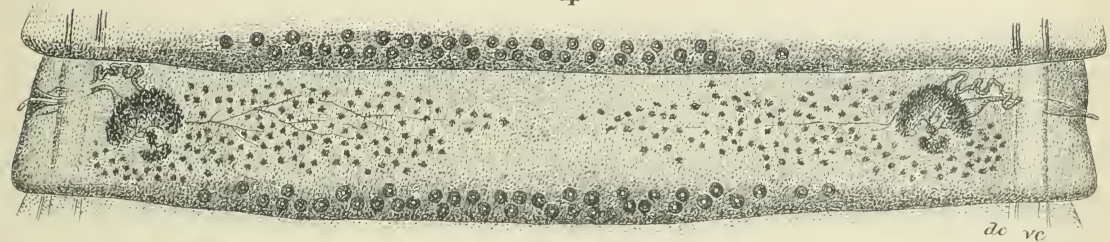

5

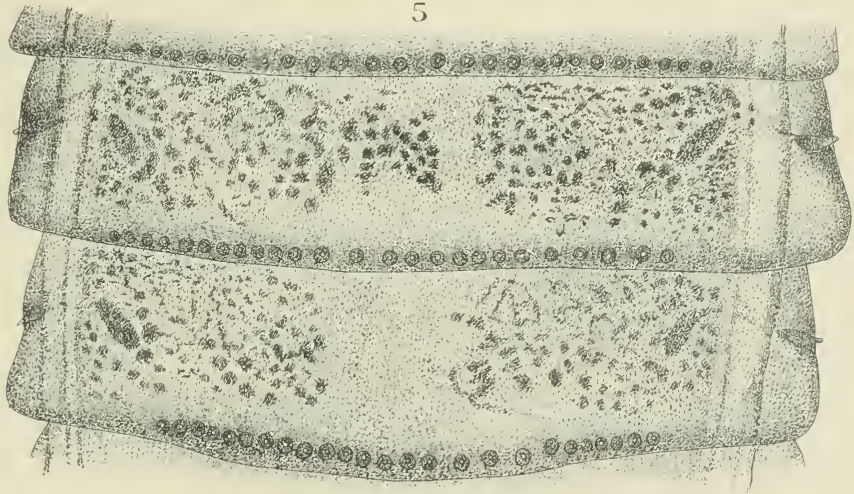

6

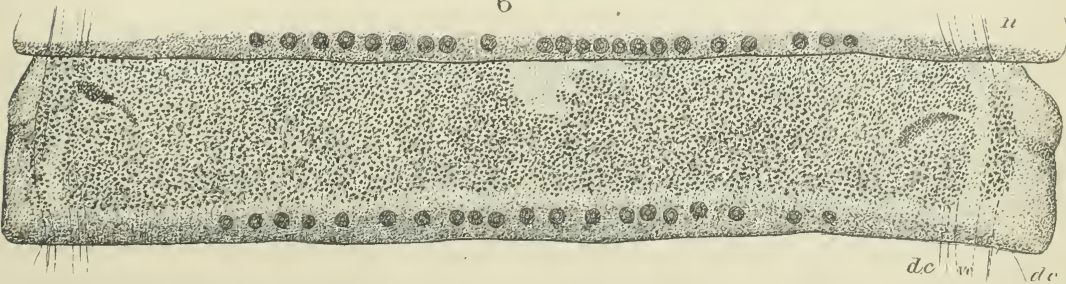

HAINES, DEL. 




\section{Plate X.-Moniezia alba.}

Figs. 1-2b, from Perroneito's original material.

Fig. 1. Strobila, natural size; a, head and anterior portion of one specimen; b-n from a specimen $1^{\mathrm{m}} 40^{\mathrm{cm}}$ long, lacking head; b, anterior portion; $\mathrm{c}, 10^{\mathrm{cm}}$ from anterior end; d $20^{\mathrm{cm}}$, e $30^{\mathrm{cm}}$, f $40^{\mathrm{cm}}, \underline{\mathrm{g}} 50^{\mathrm{cm}}$, h $70^{\mathrm{cm}}$, i $80^{\mathrm{cm}}$, j $90^{\mathrm{cm}}, \mathrm{k} 100^{\mathrm{cm}}, 1110^{\mathrm{cm}}, \mathrm{m} 120^{\mathrm{cm}}$, $\mathrm{n}$ $130 \mathrm{~cm}$, from anterior extremity.

Fig. 2. Head, lateral view. $\times 17$.

Fig. $2 a$. Head, ventral view. $\times 17$.

Fig. 2b. Head, viewed en face. $\times 17$.

Figs. 3-7 from Neumann's specimen.

Fig 3 . Segments $10^{\mathrm{cm}}$ from the hearl, $2^{\mathrm{mm}}$ wide.

Fig. t. Segments $32^{\mathrm{cm}}$ from the head, $3^{\mathrm{mm}}$ wide.

Fig. 5. Segments $55^{\mathrm{cm}}$ from the head, $4^{\mathrm{mm}}$ wille.

Fig. 6. Segments $79^{\mathrm{cm}}$ from the head, $4^{\mathrm{mm}}$ wille.

Fig. 7. Segments $1^{\mathrm{m}}$ from the head, 5.5 wide by $3^{\mathrm{mm}}$ long. 


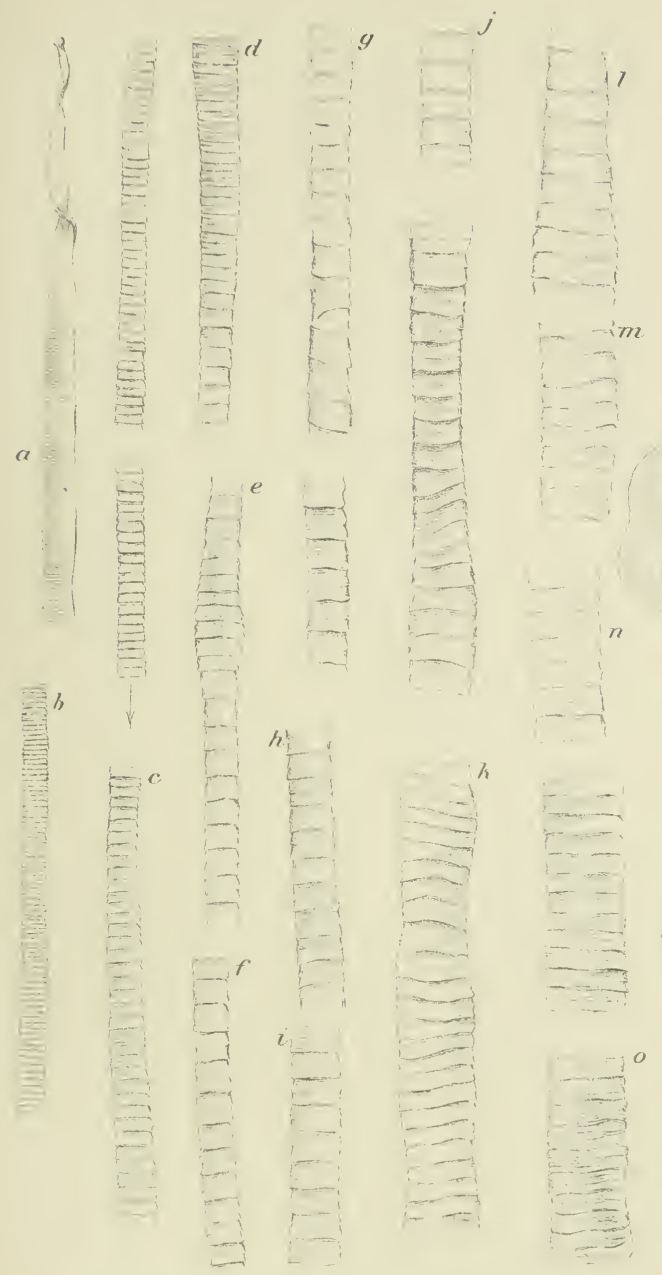

?

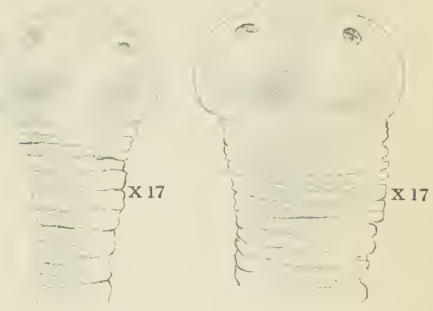

$2 a$

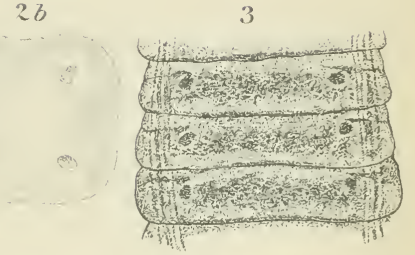

4

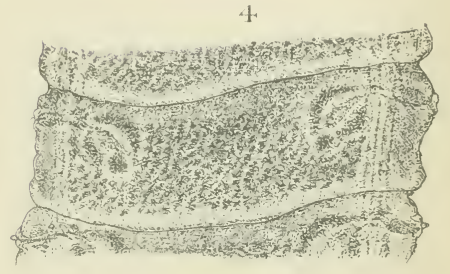

5

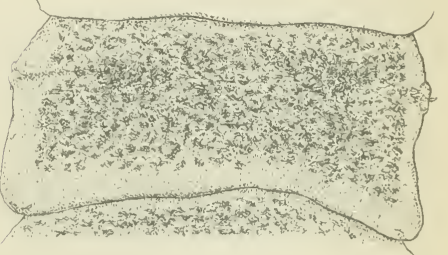

6

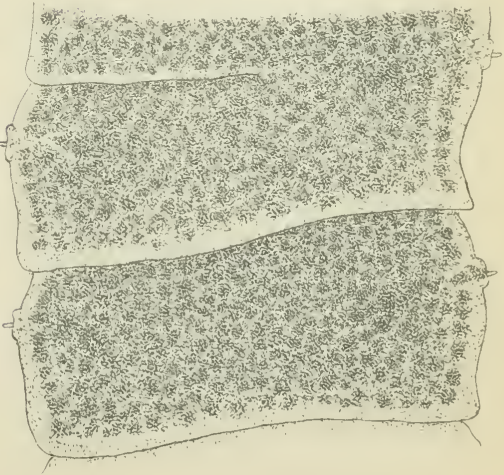




P'LATE XI.-Thysanosoma aclinioides.

Fig. 1. Strobila, natural size.

Fig. 1a. End portion of another strobila.

Fig. 2. Head, rentral view. $\times 17$.

Fig. 2a. The same, lateral view. $\times 17$.

Fig. 2b. The same, en face. $\times 17$.

Fig. 3. Segments $60^{\mathrm{mm}}$ from the hear.

Fig. 4. Segments $100^{\mathrm{mm}}$ from the head, showing testicles and genital canals.

Fig. 5. Sagittal section showing two transverse canals.

Fig. 6. Transverse section showing topography of the genital and longitudinal cantals.

Fig. 7. Cirrus in eopulation.

Fig. 8. Segment with uterus. 

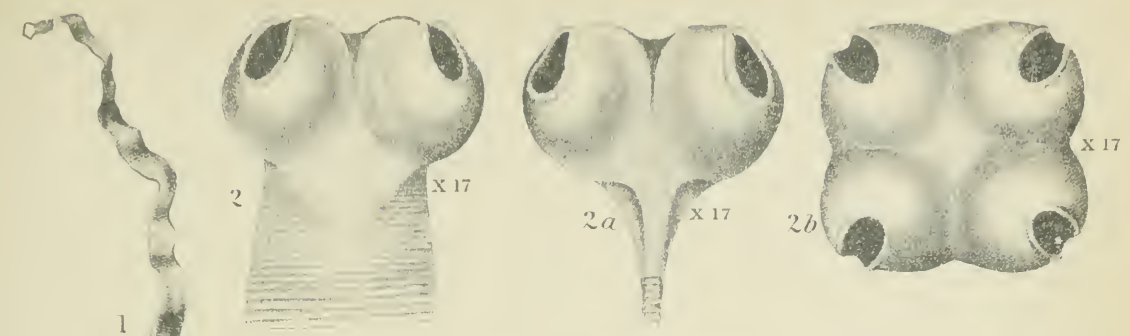

3

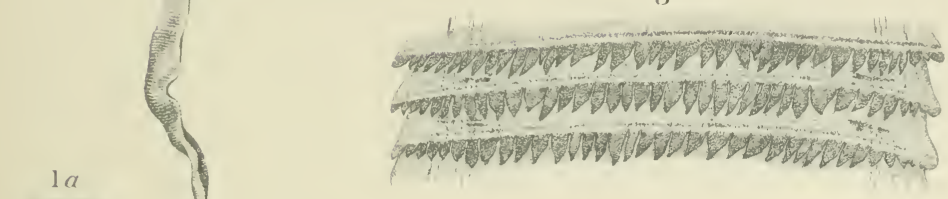

4

2. to 4 *

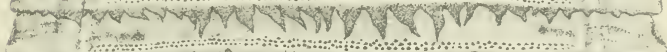
(x)

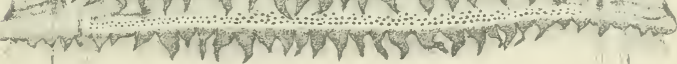

8

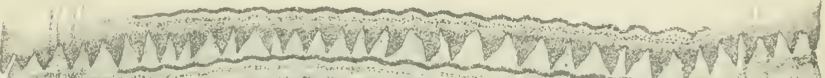
W

5
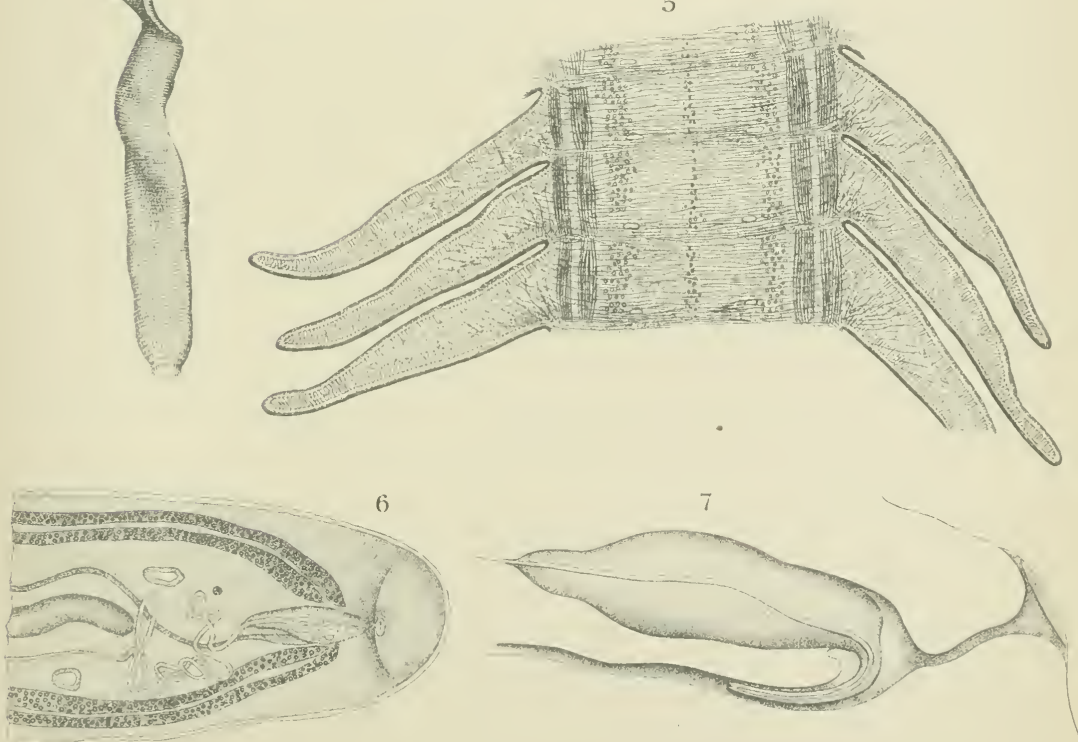

HAINES, DEL. 


Plate XII.-Thysanosoma Giardi.

Fig. 1. Strobila, natural size; head drawn from a separate fragment.

Fig. 1a. Fragment, with larger segments, natural size.

Fig. 2. Head probably of Th. Giardi, balsam preparation. $\times 17$.

Fig. 3. Strobila belonging to head in Fig. 2. The genital anlage is at first in the median line, then diverges towards the right and left in alternate segments.

Fig. 4. Segments with pistol-shaped anlage of the genital canals. One segment has an extra male canal. Dorsal view.

Fig. 5. Six segments with developing genital organs. Dorsal view.

Fig. 6. Genital organs well developed. Dorsal view. See Fig. 5, Plate vir.

Fig. 7. Sagittal section showing the proportion of length to thickness. 'T. c. transverse canal.

Fig. 8. Three segments showing the wavy appearance of the uterus. 
BUL. 4, BUREAU OF ANIMAL INDUSTRY.

Plate XII
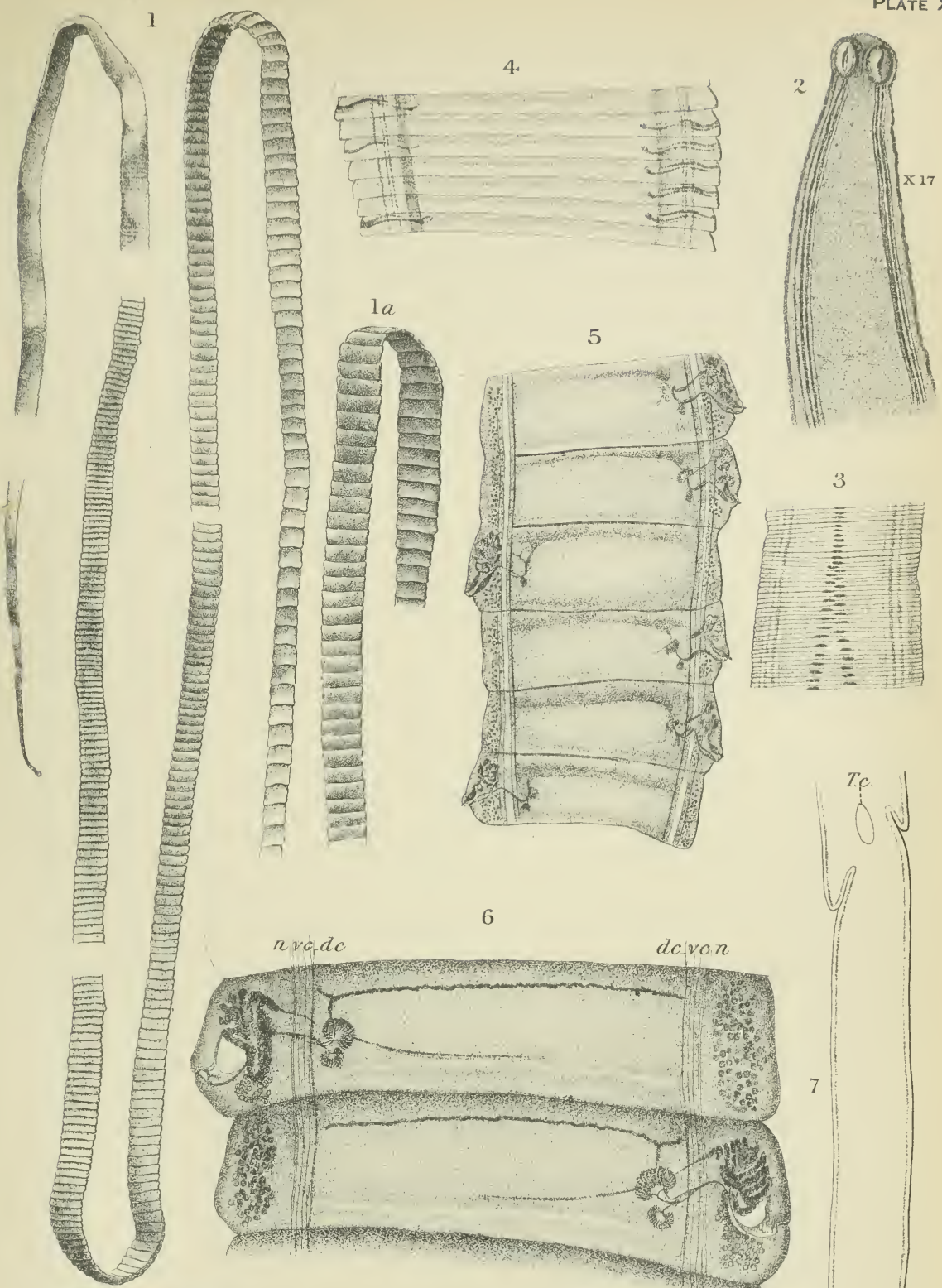

6

$n v a d c$

dc. $v a n$

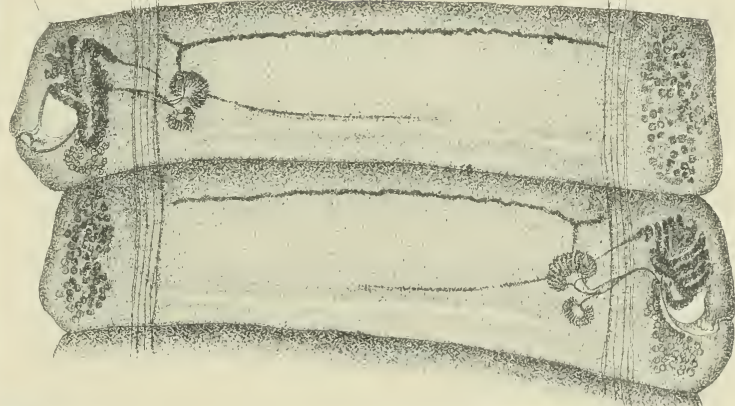

8
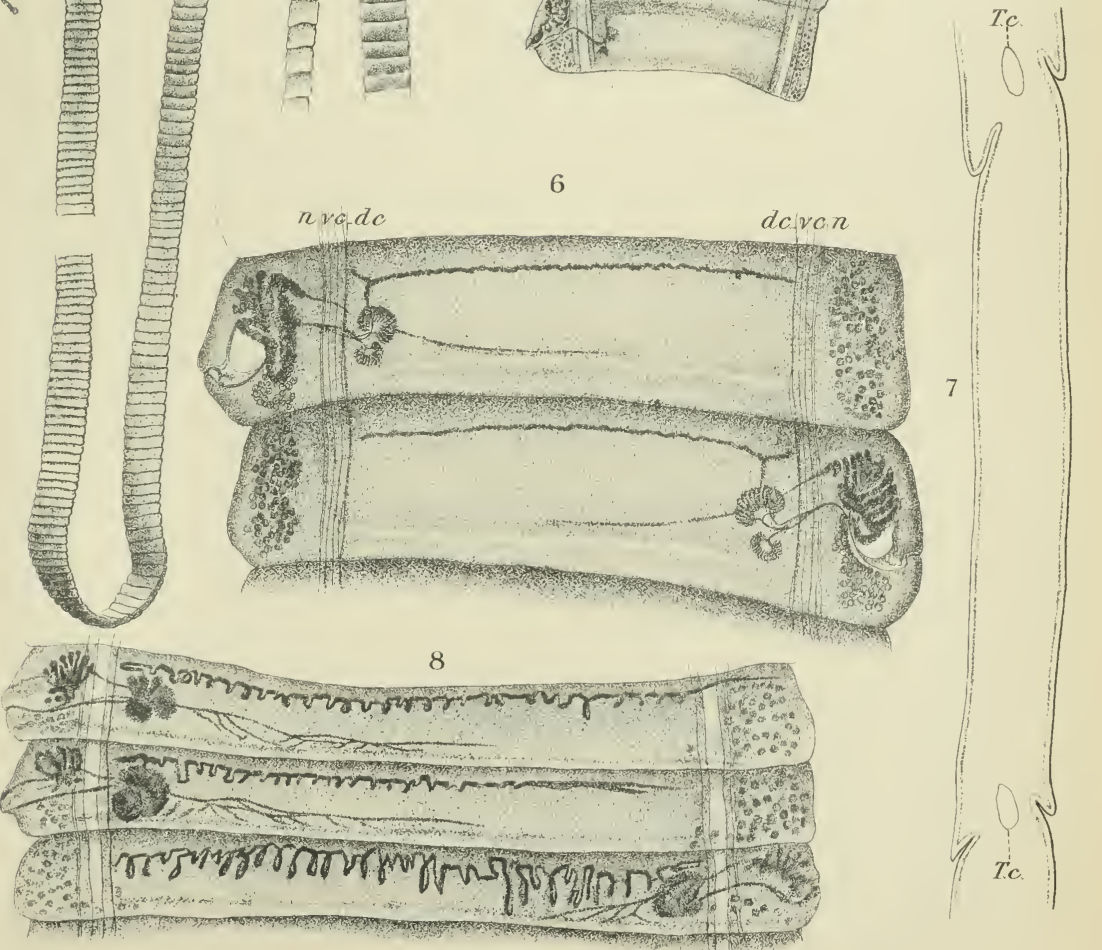


PLATE XIII-Thysanosoma Giardi.

Fig. 1. Three segments, ventral view. The second segment has double genitapores.

Fig. 2. Segments with well-ileveloped nterus. The other genital organs are atrophying. Dorsal view.

Fig. 3. Segment with fully-developed uterus. The other genital organs are almost entirely atrophied.

Fig. 4. Egg-sacs developing.

Fig. 5. Fully developed egg-saes.

128 

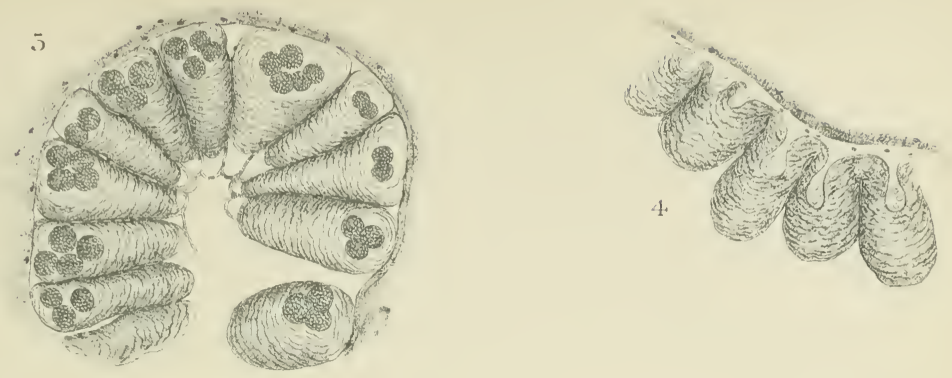

1

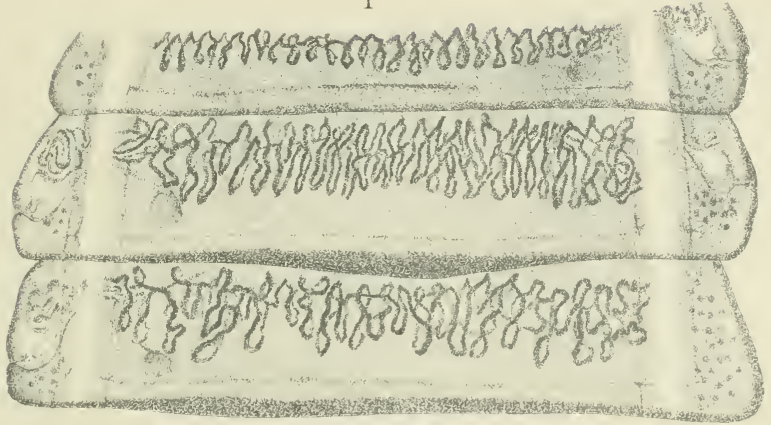

$?$

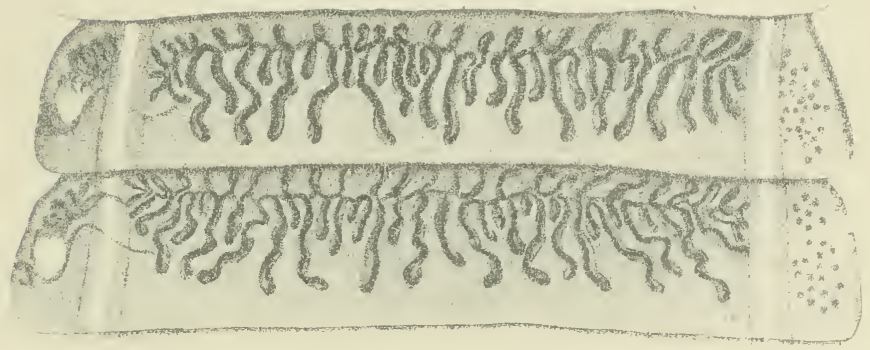

3

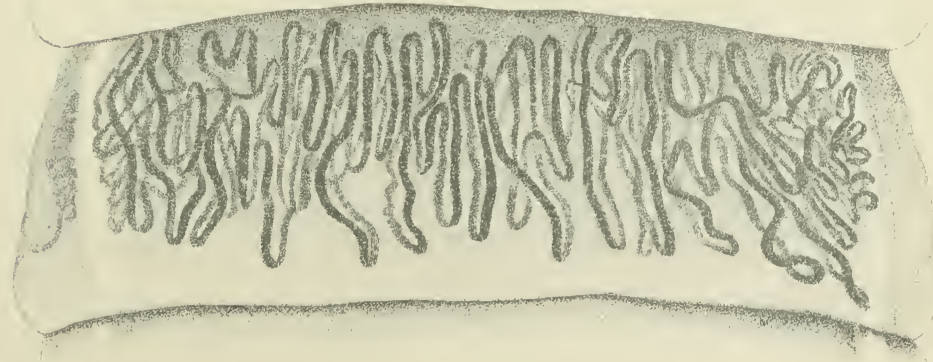



Plate XIV.-Stilesin globipunertata.

Fig. 1. Strobilæ, fragments, natnzìl size.

Fig. 2, 2b. Scolex, viewed ventrally, en face and from the side. $\times 17$.

Fig. 3. Segments $15^{\mathrm{mm}}$ from head, showing nerves, canals and anlagen of genita] organs; dorsal view. About $56 \mu$ long by $0.57^{\mathrm{mm}}$ broad.

Fig. 4. Segments $27-50 \mathrm{~mm}$ from head; the anlagen of the nteri are present; dorsal view. About $96 \mu$ long by $1.14^{\mathrm{mm}}$ broad.

Fig. 5. End of same, greatly enlarged.

Fig. 6. Right end of slightly older segments, showing ova in the median field; dorsal view.

Figs. 7 and 8. Segments showing two stages of the nterns and transverse organ; fig. 7 , ventral view; fig. 8 , dorsal view; in fig. 7 the ovary is still visille. Fig. 7 , about $0.16^{\mathrm{mm}}$ by $1.4^{\mathrm{mm}}$; fig. 8 , about $0.13^{\mathrm{mm}}$ by $2.3^{\mathrm{mm}}$.

Fig. 9. End segments. Abont $0.26^{\mathrm{mm}}$ by $0.64^{\mathrm{mm}}$.

Fig. 10. Egg. Zeiss 娄im. 
BUL. 4, BUREAU OF ANIMAL INDUSTRY.

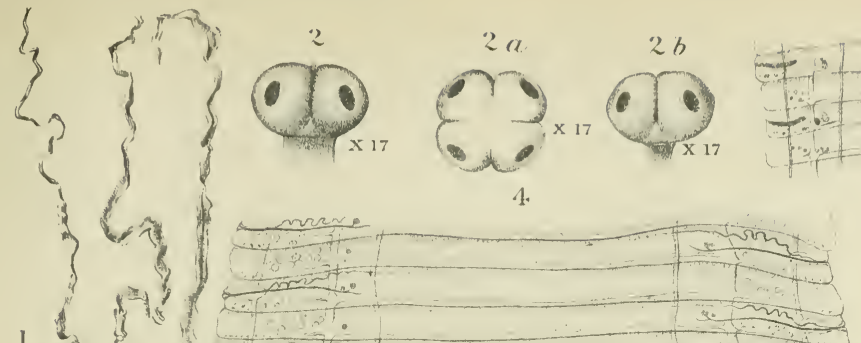

i)

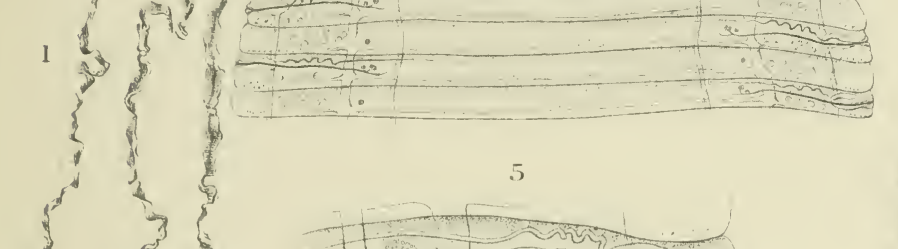

8
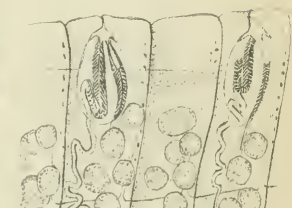

है

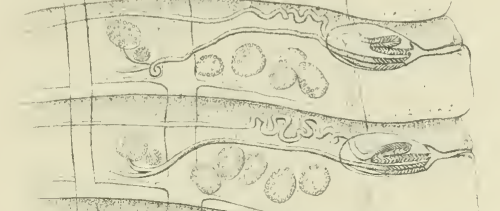

as

3

9
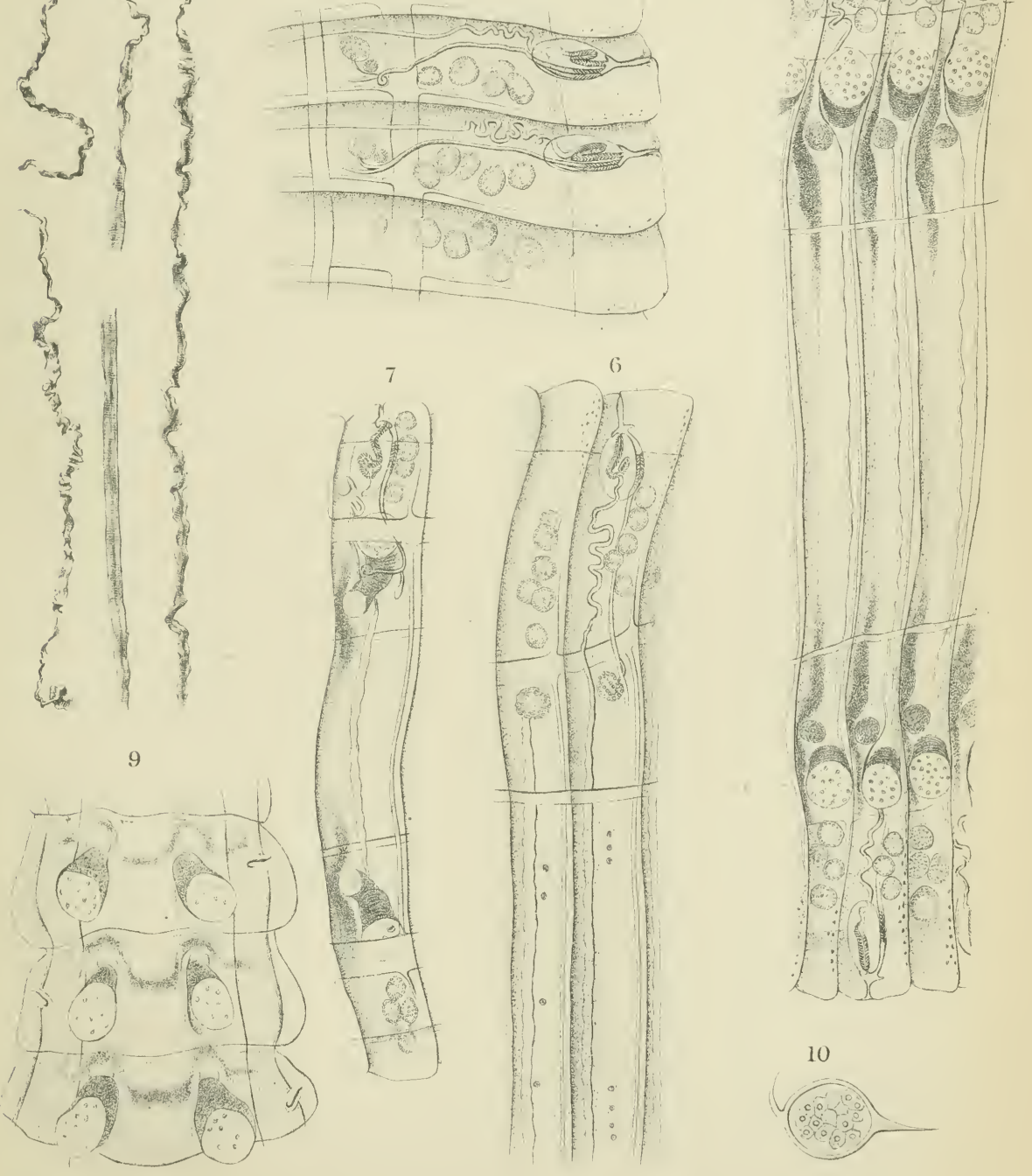

10

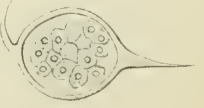




Plate XV.-Stilesia centripunctata,

Fig. 1. Portion of a strobila, natural size.

Fig. 2. Head, ventral view. $\times 17$.

Fig. 2a. Head, lateral view. $\times 17$.

Fig. $2 b$. Head, en face. $\times 17$.

Fig. 2c. Head, after Neumann.

Fig. 3. Segments $1^{\mathrm{mm}}$ broad.

Fig. 4 Segments $1.12^{\mathrm{mm}}$ broad.

Fig. 5. Segments $1.24^{\mathrm{mm}}$ broad.

Fig. 6. Segments $1.6^{\mathrm{mm}}$ broad. 

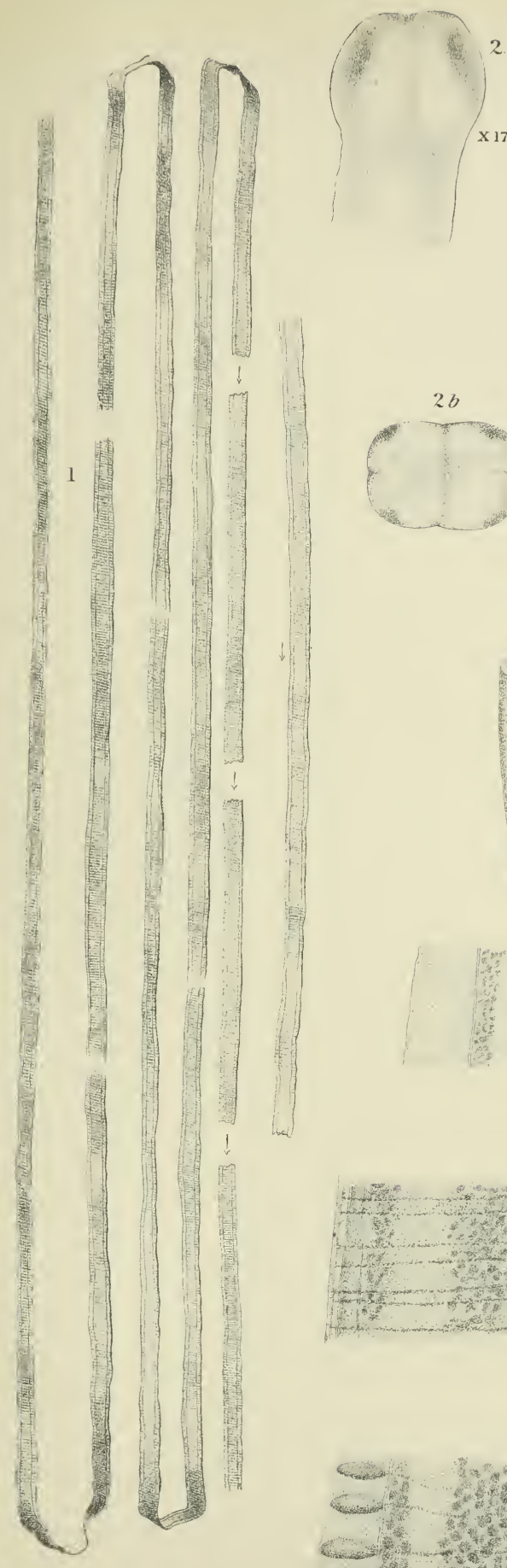

2.

X17

$2 a$

X 17

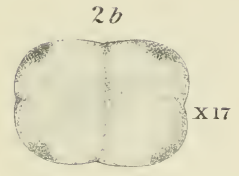

6

(C) $1+10$ $10.1+1$

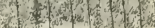
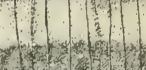

3. 4 r.

437 .

(2) 10
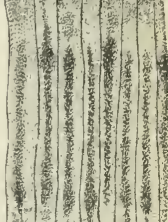

$2.1)$

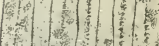

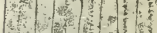

(1) 3 (n)

$2 \cdot x_{0}=$

H.

3.t.

$2 c$
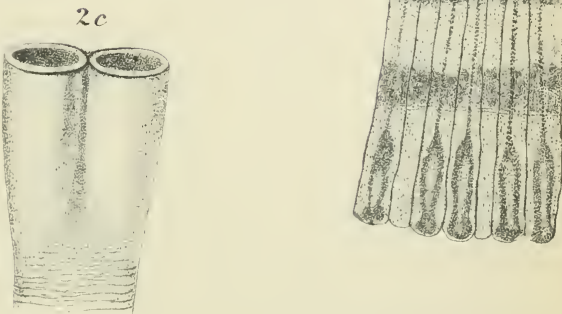

3

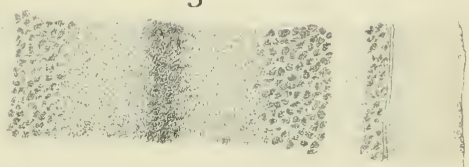

4.

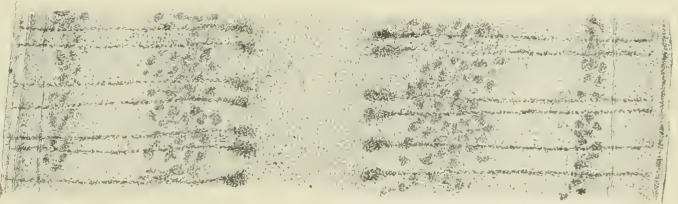

5

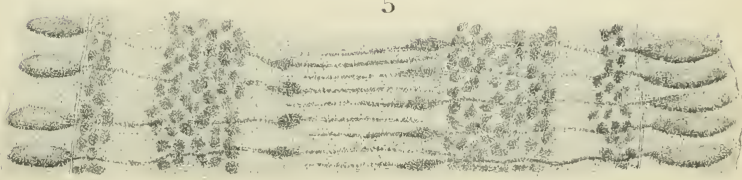

A. HOEN \& 00

STILESIA CENTRIPUNCTATA 


PLATE XVI.-Moniezia, species inquirender.

Figs. 1-2b. Bureau collection, specimen No. 607, borrowed from Hassall's private collection, collected in England.

Fig. 1. Segments, natural size.

Fig. 1a. Segments. $\times 3$.

Fig. 2. Head, viewed en fare, alcohol specimen, much contorted. $\times 17$.

Fig. 2a. The same, ventral view. $\times 17$.

Fig. 2b. The same, lateral view. $\times 17$.

Figs. 3, 3a. Head of Moniez's specimen of $Y$. mullicollis.

Fig. 3. Head, lateral view. $\times 17$.

Fig. $3 a$. The same, ventral view. $\times 17$.

Fig. $3 b$. The same, en face. $\times 17$.

Figs. 4, 4b. Bureau collection, No. 725, Moniezin sp., Expansa group.

Fig. 4. Head, ventral view. $\times \mathbf{1 7}$.

Fig. 4a. The same, lateral view. $\times 17$.

Fig. 4b. The same, en face. $\times 17$.

Figs. 5, כa. Bureau collection, No.612, Moniezia sp., Planissima group.

Fig. 5. Head, ventral view. $\times 17$.

Fig. $5 a$. The same, en face. $\times 17$. 612 .

Fig. 6 and $6 a$. A hearl found in sheep. Possibly the same as B. A. I. collection No. 


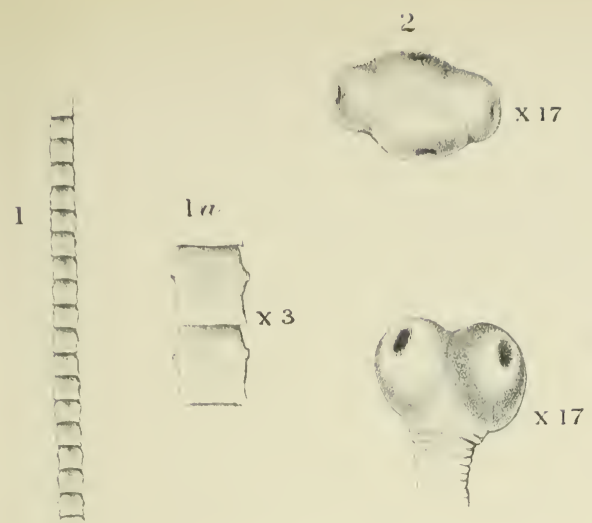

3
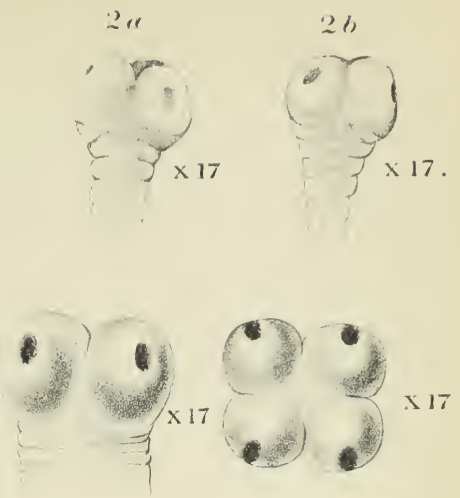

36

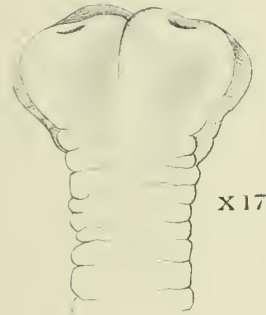

4

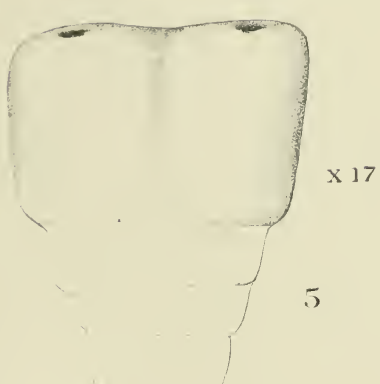

$3 r$
$4 \cdot a$

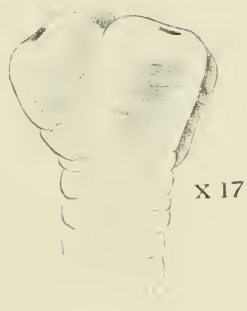

46
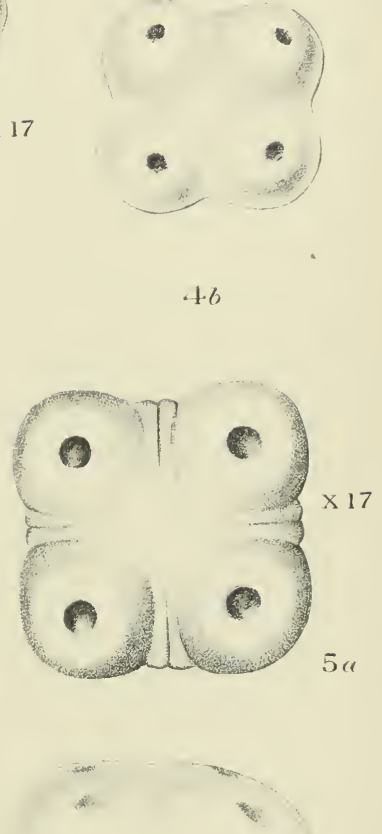

U.S. DEPARTMENT OF AGRICULTURE. BUREAU OF ANIMAL INDUSTRY.

BULLETIN NO. 5.

\section{REPORT}

ON THE

\section{DAIRY INDUSTRY}

OF

\section{DENMARK}

BY

\section{Prof. C. C. GEORGESON.}

PUBLISHED BY AUTHORITY OF THE SECRETARY OF AGRICULTURE.

WASHINGTON:

GOVERNMENT PRINTING OFFICT.

1893. 


\section{ILLUSTRATIONS.}

Fig. 1. Map of Denmark

2. Shallow milk tub....................................... 18

3. Setting milk in shallow iron pans......................... 18

4. Setting milk in ice water................................. 19

5. Cow of the red Danish breed............................. 20

6. Bull of the red Danish breed............................... 20

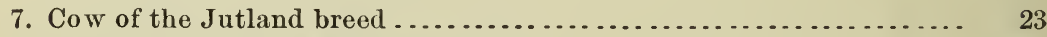

8. The Burmeister and Wains separator ....................... 26

9. Machinery connected with the separator .................... 27

10. The Lawrence cooler...................................... 28

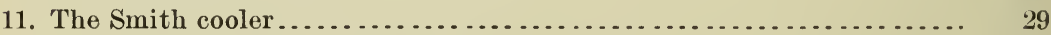

12. Prof. Fjord's control apparatus............................ 30

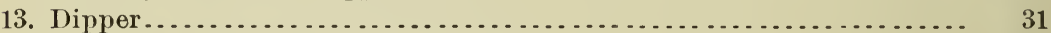

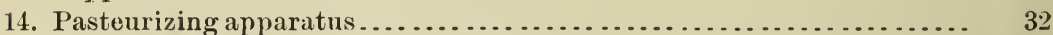

15. Danish churns ................................................... 33

16. Butter trough ............................................... 34

17. Cooling box for butter ...................................... 34

18. Scrubbing brushes ............................................ 35

19. Barrel in which butter is packed for market. ................ 49

20. Side view of cattle barn at Valdemar castle................. 67

21. Ground plan of cattle barn at Valdemar castle $\ldots . \ldots \ldots \ldots \ldots \ldots . .67$

22. Perspective of Madame Nielsen's buildings.................. 73

23. Marlame Nielsen's buildings - plan of basement ................ $\quad 75$

24. A typical coöperative creamery ............................ 109

25. Section of typical cö̈perative creamery ..................... 110

26. Ground plan of typical coöperative creamery .................. 110

27. Arrangement of machinery in creamery ..................... 111

28. Projection of one side of Quist's creamery building ............ 11;

29. Vertical section from end of Quist's creamery building ............ 118

30. Plan of Quist's creamery building ......................... 118

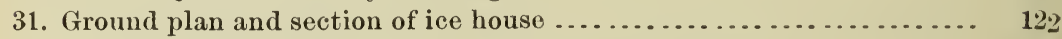

32. Use of ice in storeroom ................................ 124

33. Required shape and marking of oleomargarine packages.......... 131 


\section{TABLE OF CONTENTS.}

Letter of transmittal

Letter of submittal

Introulctory

Facts about Denmark

Situation

Condition of agriculture 10

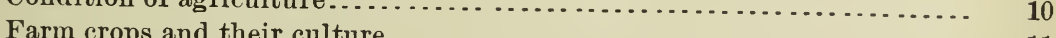

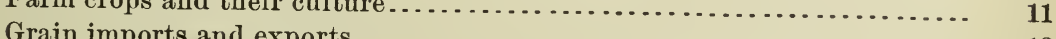

Size of the

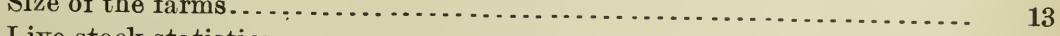

Live-stock statistics..... . . . . . . .

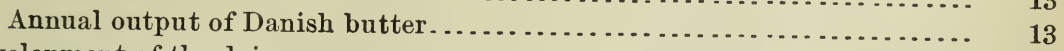

Development of the dairy ..... . . . . .

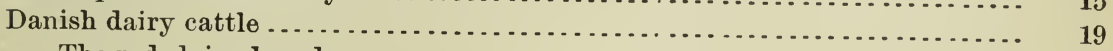

The red dairy breed............................................ 20

The Jutland breed................................................ ${ }_{22}$

Composition of Danish milk..... . . . . . . . . .

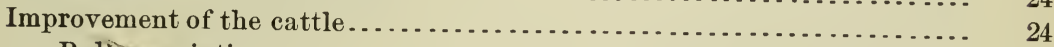

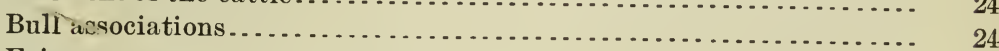

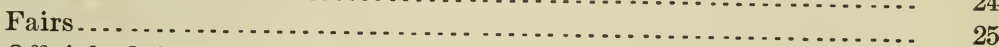

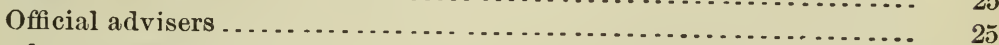

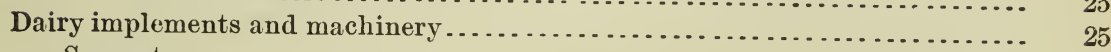

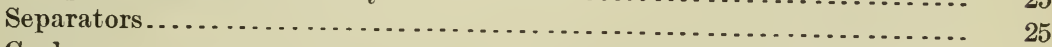

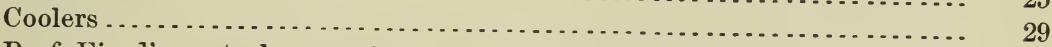

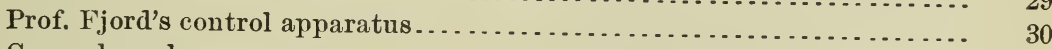

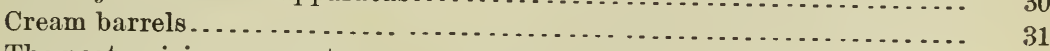

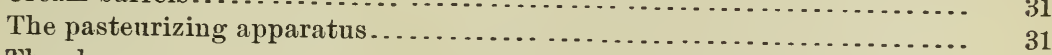

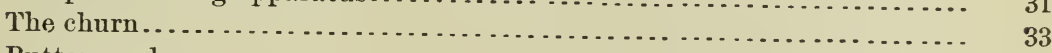

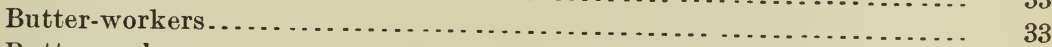

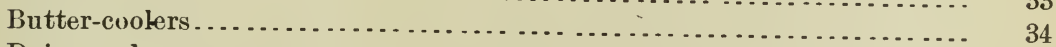

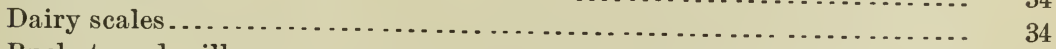

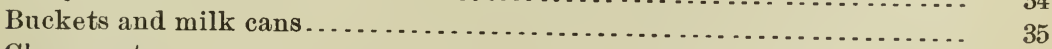

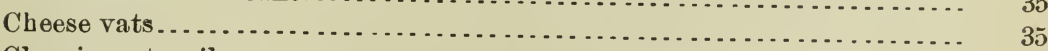

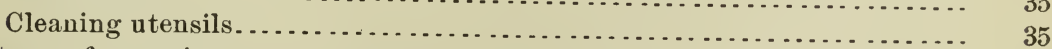

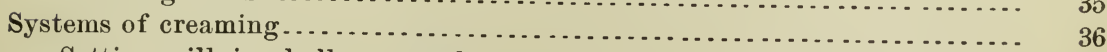

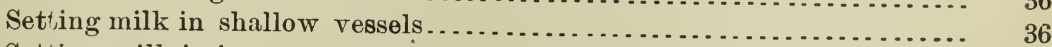

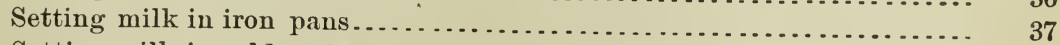

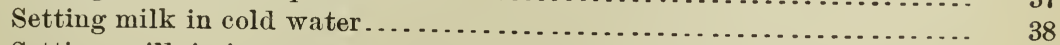

Setting milk in ice water . . . . . . . .

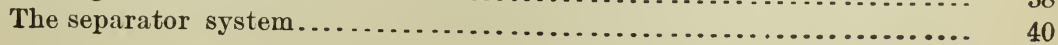

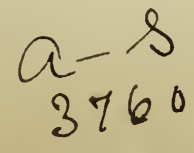


Treatment of the cream. ..... Page

Sterilization of the cream ................................ 40

Methods of fermentation................................... 42

Natural souring ................................... 42

By the use of buttermilk ............................. 42

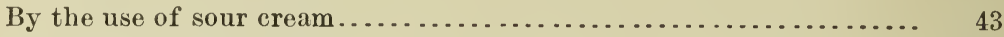

By the use of skim milk.............................. 43

By the use of pure cultures............................ 44

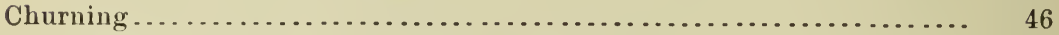

Treatment of the butter................................... 47

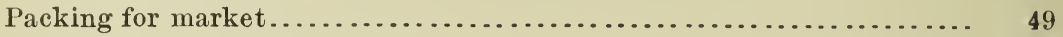

Use of skim milk and buttermilk . . . . . . . . . . . . . . . . . . . . . . . 50

Description of places visited ................................ 50

The Milk-supply Company of Copenhagen..................... 51

Large dairy farms. .................................. 56

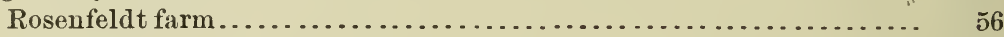

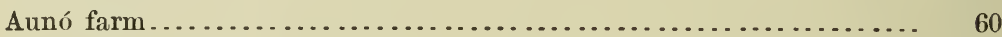

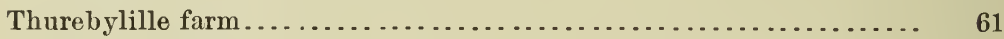

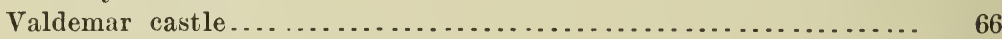

Faareveile farm . . . . . . . . . . . . . . . . . . . . . . . . . . . 69

Julesberg farm .................................... 70

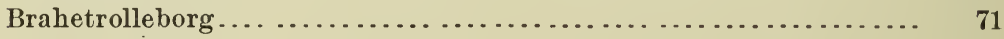

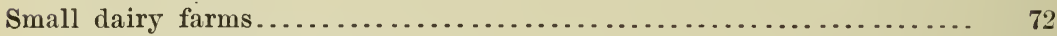

Farm of Madame Nielsen .............................. 73

Farm of P. Pedersen................................... 80

Farm of Hans Hansen.............................. 81

Farm of Christian Rasmussen.......................... 82

Farm of Rasmus Andersen............................. 83

Farm of Andersen Bros .................................. 84

Farm of P. Nielsen ................................ 85

Eskelund dairy farm . . . . . . . . . . . . . . . . . . . . . . . . . 86

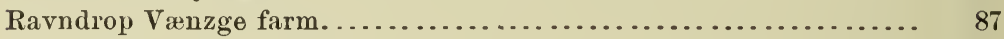

Holev farm ....................................... 89

Hermans Minde farm . . . . . . . . . . . . . . . . . . . . . . . . . . 92

Dalum agricultural school .................................. 94

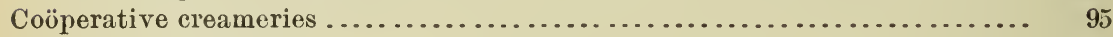

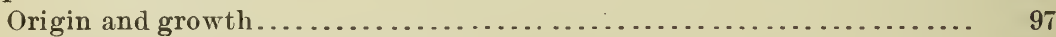

Organization-officers, salaries, etc......................... 99

Kildevald cö̈perative creamery ............................. 100

Constitution of Kildevæld creamery ....................... 102

Practical operations of Kildevæld creamery .................. 105

A typical coöperative creamery . . . . . . . . . . . . . . . . . . . . . . . . 109

Renkolde cö̈perative creamery ............................. 111

Ringe coöperative creamery ................................ 112

Snóde and Stoelse coöperative creamery ... . . . . . . . . . . . . . . . . 113

Nonnebjerg Falles creamery ............................... 115

Dairy bacteriology ... . . . . . . . . . . . . . . . . . . . . . . . . . . . . 119

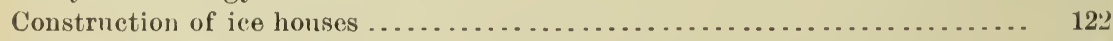

State aid to dairy industry ....................................... 124

Markets for Danish dairy products ... . . . . . . . . . . . . . . . . . . . . . . 127

English imports of Danish butter........................... 127

State aid to foreign markets............................... 12!)

Instructions to Government Agent Faber ... . . . . . . . . . . . . . . 12!

Restrictions on the sale of oleomargarine...................... 130

Agricultural and dairy education ............................... 131 


\section{LETTER OF TRANSMITTAL.}

U. S. Departuent of Agriculture, Bureau of Animal Industry,

Washington, D. C., September 6, 1893.

SIR: I have the honor to transmit herewith for publication the report of Prof. C. C. Georgeson upon his recent investigations of the dairy industry of Denmark.

Very respectfully,

D. E. SALMON, Chief of Bureau of Animal Industry.

Hon. J. Sterling Morton, Secretary of Agriculture. 


\section{LETTER OF SUBMITTAL.}

Kansas State Agricultural College,

Manhattan, Kansas, September 2, 1893.

SIR: I have the honor to submit herewith my report on the dairy industry of Denmark, which, under date of January 7, 1893, I was instructed to investigate under the auspices of your Bureau.

Very respectfully,

Dr. D. E. Salmon,

C. C. Georgeson.

Chief of Bureau of Animal Industry. 


\section{REPORT ON THE DAIRY INDUSTRY OF DENMARK.}

By C. C. Georgeson.

\section{INTRODUCTORY.}

The facts hereinafter detailed were gathered auring my recent visit to Denmark as a special agent of the U. S. Department of Agriculture. I was commissioned by Hon. J. M. Rusk, in January last, for a period of two months, afterwards extended by thirty days, making the time three months in all. I deem it but just to say that the short time at my disposal for the trip and the unfavorable season of the year when it was made-it being midwinter-prevented my investigation from being as thorough as it would have been under more favorable circumstances.

The following quotation from my instructions makes plain the object of my visit:

I desire that your investigations should cover the three phases of the dairy. industry, namely: First, the dairy farm, including the subject of breeds, yield per animal, feeding, general care, and the disposal and handling of the milk; second, the manufacture of dairy products, its methods and appliances; and third, the trade in dairy products, the value of butter and cheese in the home market and at ports of shipment, and other statisties showing the extent and distribution of dairy products anci the various characteristies as to form, color, salting and packing, etc., required to meet the wants of foreign countries. You will proceed at once to Denmark to undertake this work and will complete it in the shortest time possible, returning directly to Washington (via London and Liverpool) to prepare and submit your report.

I arrived in Denmark on the 27th of January and left again on the 6 th of March. The interval between these dates I spent in visiting representative creameries and dairy farms on three of the islands and on the peninsula, and $I$ also made it a point to meet as many as possible of the men, both scientific and practical, who are identified with the development of the dairy industry. Among those to whom I am indebted for valuable information I would especially mention Prof. T. R. Segelcke and Prof. V. Storch, the former of the Royal Agricultural College at Copenhagen, and the latter of the Experimental Laboratory, same place; Konsulent B. Bóggild, Secretary of the Royal Agricultural 
Society, also of Copenhagen; Konsulent J. X. Dall, of Fredericia; Konsulent A. Apple, of Aarhus, and Mr. E. A. Quist, of Skanderborg.

I am also indebted to a large number of dairy managers and farmers, some of whom will be mentioned hereafter, for information in regard to their individual methods in the dairy and on the farm.

I was everywhere courteously received. Although my presence on such a mission gave rise to a natural and freely expressed suspicion that perhaps the United States intended to compete with Denmark for favors in the English dairy market, this suspicion did not cause any secretiveness or unwillingness to give information. My many questions, which often concerned private business affairs, were in every instance answered cheerfully and satisfactorily. This much, by way of acknowledgment, is due to the many who, by their unstinted readiness to give information, met me more than half way and enabled me to collect the facts here presented.

The agricultural, and especially the dairy, literature of Denmark has been another rich source of information. I have perused no small number of publications of this character, first among which should be mentioned a work on the dairy by Bernhard Bóggild, entitled "Mrlkeribruget i Danmark." The others are mostly pamphlets and reports issued by agricultural societies, and statistical works published by the Danish Government. Among the most important I may name the bulletins of the agricultural experiment station at Copenhagen, the reports of the official "Konsulenter" (advisers) in matters pertaining to dairy and stock-breeding, and publications by the Royal Danish Agricultural Society.

\section{FACTS ABOUT DENMARK.}

\section{SITUATION.}

A glance at the accompanying map (Fig. 1) shows that Denmark is almost entirely surrounded by water. It lies far to the north, being between $54^{\circ} 30^{\prime}$ and $50^{\circ} 30^{\prime}$ north latitude and between $8 \circ$ and $12^{\circ} 45^{\prime}$. east longitude. The climate belonging to this latitude is, however, materially modified by the large body of water surrounding the country, so that the extremes of temperature are really not so great there as they are on the adjoining continent, farther south. The map also shows the situation of the leading cities and the lines of railroad. The heavy double line across the neck of the peninsula is the German frontier. On the west coast, just above this line, will be noticed the port of Esbjerg. This port has sprung into importance during the last few years, and grown in population with phenomenal rapidity, solely because the establishment of a steanship line fiom this port to England has made it the port of shipment for nearly all the Danish butter going to that country. 
AREA, SOLLS, ANI) CLIMA'TE.

The area of Denmark is $14, \overline{0} 53$ English square miles, which is less than one-tenth the size of the State of California or about half the size of the State of Maine. The population, in round numbers, is $2,000,000$ people, giving 137 persons to the square mile. The topographical features are not striking; there are no mountains at all in the country and the hills are not high. On the islands the land is generally undulating, with here and there a ridge of hills which range somewhat higher than the average. On the peninsula occur several long

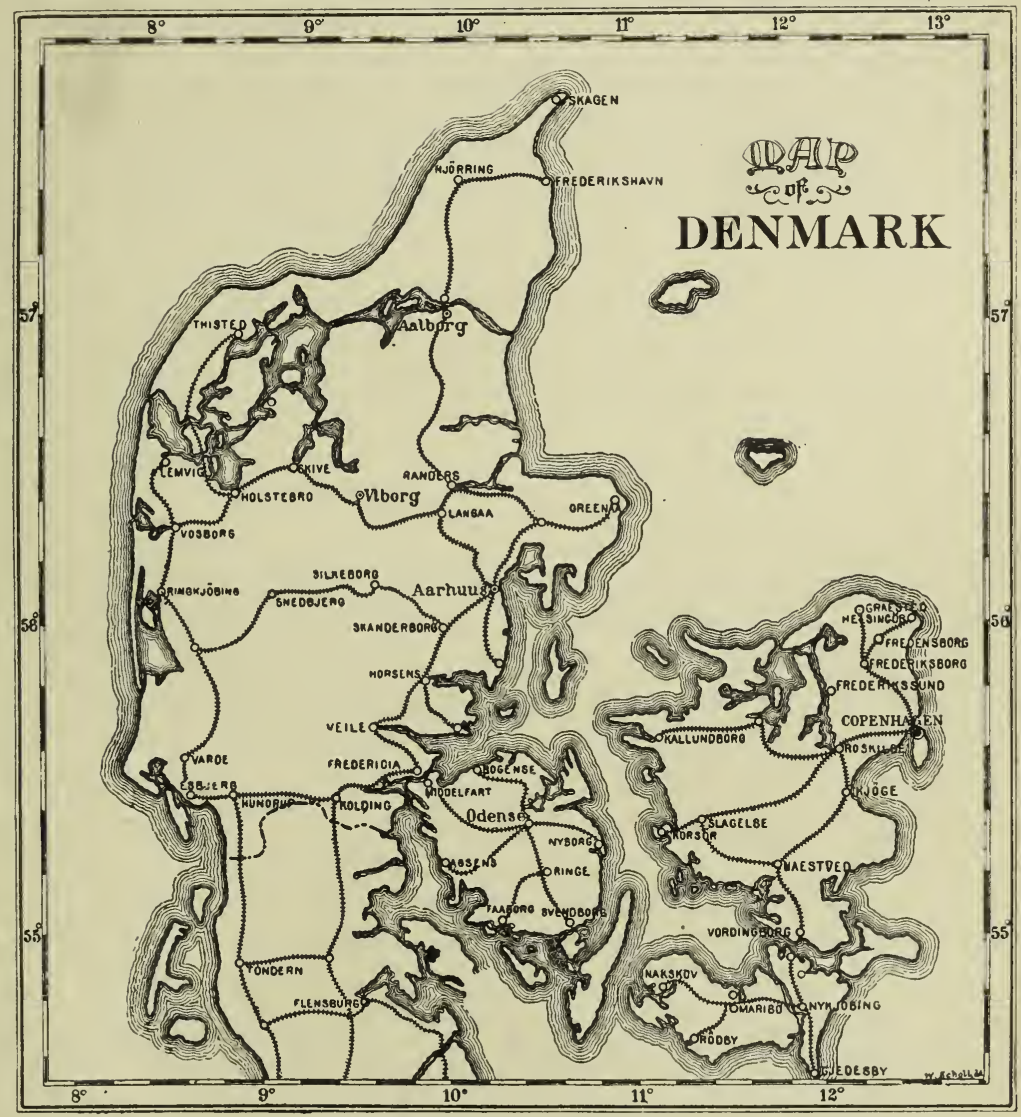

Fig. 1.-Map of Denmark.

stretches of level ground, portions of which consist chiefly of drift sand and are overgrown with heather. These tracts are, of course, unsuited for agriculture. The soil is varied in character. In many places it is clayey and in others it is very sandy. This variation is due chiefly to glacial action, which is everywhere evident. The clay soil is, as a rule, fertile but difficult to work; the sandy soil, on the other hand, requires constant addition of fertilizers in order to produce paying crops. The 
climate is moist, although the rainfall is not excessive; but there are frequent storms of light misty rains which last sometimes for days, and fog and mist are very frequent, especially during the winter. The snowfall is variable, but, as a rule, the snow lies on the ground so as to furnish sleigling for two or three months each winter, and in some years much longer.

\section{TEMPERATURE AND RAINFALL.}

The average temperature for the three winter months ranges from $31.1^{\circ}$ to $34.7^{\circ} \mathrm{F}$; for the spring months, from $40.1^{\circ}$ to $43.7^{\circ}$; for the summer inonths, from $57.2^{\circ}$ to $61.7^{\circ}$; and for the fall months from $44.6^{\circ}$ to $49.1^{\circ}$, the average for the whole year being from $43.7^{\circ}$ to $47.3^{\circ}$.

The rainfall averages about as follows: For the winter months, 5 inches; for the spring months, 4 inches; for the summer months, 6.8 inches; and for the fall months, 8 inches. This seems to be a light rainfall, but it should be borne in mind that in that far northern latitude evaporation does not take place so rapidly as it does in nearly all parts of the United States, and hence a greater proportion of the rain can go to the direct benefit of the crops. Complete failure of crops on account of drought never occurs, and there is rarely a deficiency in the crops from lack of rainfall.

\section{CONDITION OF AGRICULTURE.}

Agriculture is prosperous, but its present condition has been reached only through a gradual improvement which began a century ago. At that time the peasants, the cultivators of the soil, owned but a very small proportion of the land they cultivated; it was nearly all in the hands of landlords. Since then the latter have gradually sold to the peasants a large proportion of the land, and now somewhat more than 50 per cent of the area under cultivation is owned by peasant farmers, and of the remaining land the greater portion is worked by them under a system of life tenancy by which the farm will sometimes remain in the same family for several generations, the contract being renewed by the son on the death of his father. A farmer, for example, desires to rent a farm which is tenantless. He agrees to pay so much in annual rent, in addition to which a certain sum is always paid down at the time the bargain is made. He begins to work the place, and contimnes to work it ummolested as long as he lives and his wife after him, if he dies before she does, but if she marries again the old contract is abro. gated and a new one is made with the incoming farmer. This pian is the most satisfactory arrangement that can be made, next to ownership) of the lands. The farmer feels that he is secme in reaping whatever benefits may accrue from his inprovements. He can drain his land, or fence it, sub-soil it, and manure it without the fear that his rent will 
be increased or that he will be turned off and some one else will take the place.

A limited number of farms are rented from year to year, according as the parties concerned can agree. There are in the country quite a number of large farms ranging from 500 acres to 1,500 or upwards. These are, as a rule, owned by the families of the old-time landlords who in former days owned all of the surrounding country. They seldom work these large farms themselves, but rent them to tenants, who usually pay as high a rent as the farms warrant, leaving but a small margin for the farmer. He tries to widen this marginal profit by improved methods of culture and economic handling and sale of his crops. In these cases the lease usually lasts for a series of years, the number varying with the system of rotation in rogue, it being so arranged that the farmer can take one of each of the crops in the rotation from each field. Thus in an eight-year rotation the farm will be divided into eight ficlds and the lease run for eight years at so much per year.

\section{FARM CROPS AND THEIR C'LTURE.}

To get a correct idea of the relation which dairying bears to general farming it will be desirable to look briefly into the character of the farm crops, and the relative proportion in which they are grown.

The following tables have been deduced from "Danmarks Statistik," fourth series, Litra C, No. 7, and show how the soil of the whole kingdom was in use on the 16th of July, 1888. What changes have taken place in the cropping since that time have been in the direction of enlarged areas in fodder crops. I have reduced the figures to acres in order to make the statements more intelligible to American readers:

Acres seeded in Denmark in 1888.

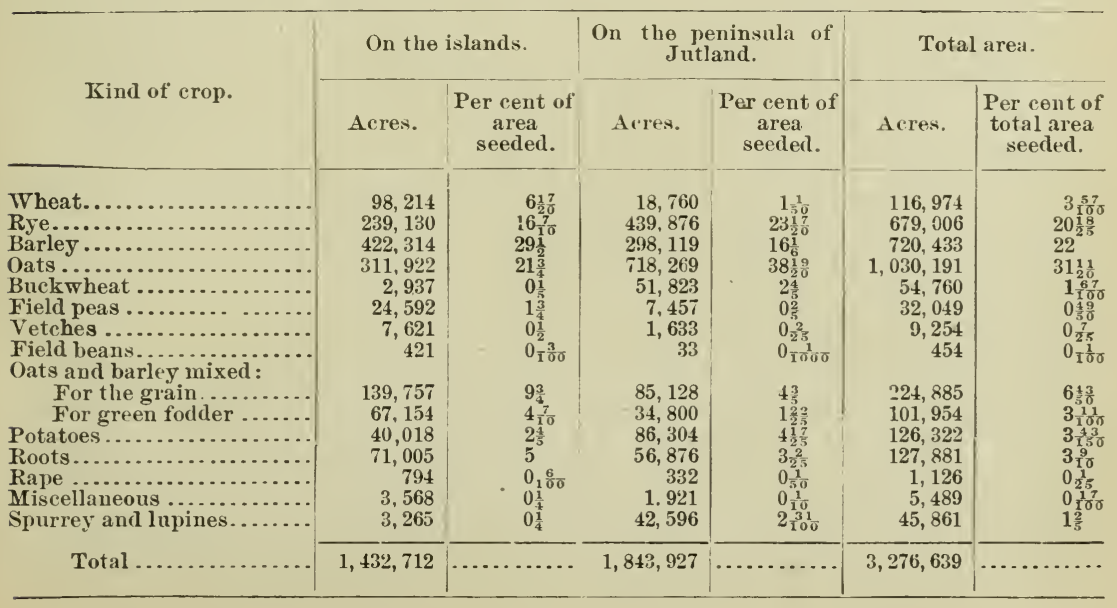


Area in grass in 1888.

\begin{tabular}{|c|c|c|c|c|c|c|}
\hline & \multicolumn{2}{|c|}{ On the islands. } & \multicolumn{2}{|c|}{ On the peninsula. } & \multicolumn{2}{|c|}{ Total area. } \\
\hline & Acres. & $\begin{array}{l}\text { Per cent } \\
\text { of grass } \\
\text { land. }\end{array}$ & Acres. & $\begin{array}{c}\text { Per cent } \\
\text { of grass } \\
\text { land. }\end{array}$ & Acres. & $\begin{array}{l}\text { Per cent } \\
\text { of total. }\end{array}$ \\
\hline 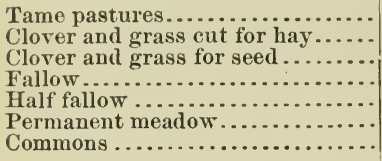 & $\begin{array}{r}395,749 \\
296,152 \\
4,632 \\
224,721 \\
84,572 \\
120,610 \\
49,108\end{array}$ & $\begin{array}{r}33 \frac{2}{12} \\
25_{5}^{1} \\
0 \frac{2}{5} \\
19 \frac{1}{9} \\
72 \\
10 \frac{1}{4} \\
4 \frac{1}{6}\end{array}$ & $\begin{array}{r}1.380,149 \\
143,829 \\
2,985 \\
249,527 \\
66,372 \\
433,892 \\
114,868\end{array}$ & $\begin{array}{l}57 \frac{1}{10} \\
6 \frac{1}{100} \\
0 \frac{3}{25} \\
10 \frac{11}{25} \\
2 \frac{4}{5} \\
18 \frac{1}{7} \\
4 \frac{4}{5}\end{array}$ & $\begin{array}{r}1,775,898 \\
439,981 \\
7,617 \\
474,248 \\
150,944 \\
554,502 \\
163,976\end{array}$ & $\begin{array}{r}48 \frac{7}{10} \\
12 \frac{3}{50} \\
0 \frac{2}{5} \\
13 \\
4 \frac{1}{7} \\
15 \frac{1}{5} \\
6 \frac{7}{10}\end{array}$ \\
\hline Total $\ldots . . . \ldots \ldots \ldots \ldots$ & $1,175,544$ & $\ldots$ & $2,391,622$ & $\cdots \cdots \cdot$ & $3,567,: 66$ & \\
\hline
\end{tabular}

Area in miscellaneous uses in 1888.

\begin{tabular}{|c|c|c|c|c|c|c|}
\hline 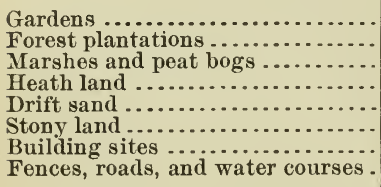 & $\begin{array}{r}39,148 \\
300,520 \\
500,116 \\
16,228 \\
2,860 \\
12,924 \\
32,174 \\
65,161\end{array}$ & $\begin{array}{l}4 \frac{1}{25} \\
31_{110}^{\frac{1}{100}} \\
51_{5}^{3} \\
1 \frac{1}{25} \\
0 \frac{3}{10} \\
1 \frac{1}{3} \\
3 \frac{1}{3} \\
6 \frac{1}{25}\end{array}$ & $\begin{array}{r}29,221 \\
247,771 \\
242,264 \\
768,707 \\
95,399 \\
43,357 \\
32,764 \\
79,166\end{array}$ & $\begin{array}{l}1 \frac{9}{10} \\
16 \frac{1}{10} \\
15 \frac{3}{4} \\
50 \\
6 \frac{1}{5} \\
8 \frac{1}{50} \\
2 \frac{1}{8} \\
5 \frac{3}{20}\end{array}$ & $\begin{array}{r}68,369 \\
747,961 \\
292,380 \\
784,935 \\
98,259 \\
56,281 \\
64,938 \\
144,327\end{array}$ & $\begin{array}{l}3 \frac{1}{56} \\
33 \frac{1}{8} \\
12 \frac{19}{20} \\
34 \frac{77}{100} \\
4 \frac{7}{20} \\
2 \frac{1}{2} \\
2 \frac{2}{25} \\
6 \frac{2}{5}\end{array}$ \\
\hline Total.. & 969,131 & & $1,538,649$ & & $2,257,450$ & \\
\hline
\end{tabular}

Referring to these tables it will be noticed that a very large proportion of these crops is designed for feed.

\section{GRAIN IMPORTS AND EXPORTS.}

As a matter of fact Denmark does not produce breadstuffs enough for the consumption of her own people. The statistics of imports and exports issued by the Government for the year 1891 show that the following quantities of grain were imported over and above the export of the same articles:

Bushels.

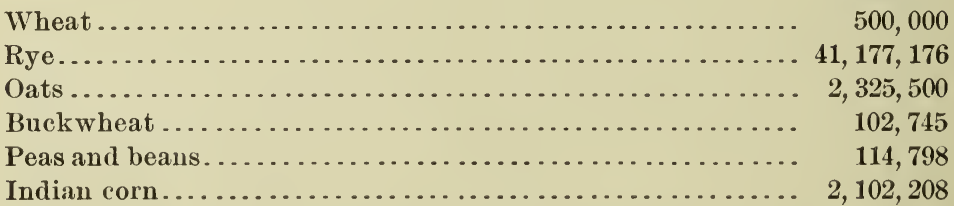

The figures have been summarized and reduced to bushels for ease of comprehension by American readers. The last item, Indian corn, is used chiefly for feed for live stock; as yet only a small amount of corn is used as food for the people.

This shows that a considerable quantity of grain is imported for home consumption and indirectly confirms the prominence which is given to the dairy. Barley is the only grain the export of which exceeds the import. The export of barley amounts to about 1,500,000 bushels; these figures, it should be noted, are not for any single year, but they represent the average of the years 1887, 1888, 1889, 1890, and 1891. 
Graded according to their size, the farms of Denmark may be put into three classes, which we may designate respectively as large, medium, and small farms. The average size of the farms of each of these classes is difficult to ascertain because the statisties do not give the area of the land belonging to each class. They are classified upon the basis of taxation according to the quality of the land. The present basis, which is called "hartkorn," was established in 1844, when all the lands were revalued. But knowing the total area and the number of farms, it is easy enough to ascertain the average size of the Danish farms. Thus, in 1888 , there were in the country 1,954 large farms. The size of these will range between 500 and 1,500 acres. There were 71,778 mediumsized farms, the areas of which range from 50 to 500 acres, and there were 150,260 small farms ranging in size from a small patch up to 50 acres. Now the total area of the country, excluding forests and waste land, and taking only what is under actual culture and in grass, is $6,843,805$ acres, which makes the average size of the farm 30.55 acres.

\section{LIVE-STOCK STATISTICS.}

The latest available statistics I have on the subject are for 1888. In this year there were in Denmark 1,459,527 head of cattle, 375,533 head of horses, 1,225,196 head of sheep, and 770,785 head of swine. This gives 1.67 horses, 6.51 head of cattle, 3.44 head of swine, and 5.47 head of sheep for the average farm of 30.55 acres. It should be borne in mind that in estimating the average much land has been counted in which, for various reasons, does not support any live stock at all, such as gardens, and it also includes all of the very poor land and land fit only for pasture, and poor at that. In fact, it includes land of every description which has any agricultural value. At this same rate a 160. acre farm should support 8.7 head of horses, 34 head of cattle, 28.6 head of sheep, and 18 head of swine.

\section{ANNUAL OUTPUT OF DANISH BUTTER.}

The output of dairy products during recent years from this little country has been astonishingly large. Statistics published by the Government show that during 1891 Denmark exported 91,455,262 Danish pounds of butter, which amounts to $100,600,788$ pounds avoirdupois. This is, however, inclusive of what was imported into the country and exported again. A considerable amount of foreign butter is imported and consumed there, presumably in order to save for export the more valuable Danish product. The total import of butter for 1891 amounted to $24,277,557$ pounds avoirdupois. If we deduct the total imports from the total exports we get a surplus of $76,323,231$ pounds avoirdupois as the product of the Danish creameries exported in 1891; but it appears from the statistics that of the total import of butter, 
17,870,456 Danish pounds were destined for home consumption. Subtracting this from the total imports we have left 4,200,051 Danish pounds $(4,620,056$ pounds avoirdupois) of the import which was again exported. Now, if we subtract this small amount from the total export we shall get the actual amount of Danish-made butter which was put upon the foreign market in 1891, and this, it will be seen, amounts to $95,980,732$ pounds avoirdupois. There are no statistics to throw light on the amount of butter which is annually consumed in the country, but it must be considerable. Bread and butter form an important part of the diet of the people. All through the spring and summer, from March to November, it is customary among the farmer's and working classes to eat a lunch of bread and butter and cold meat or cheese in the middle of the forenoon, and another in the middle of the afternoon. And at the three regular meals of breakfast, dinner, and supper, bread and butter are, as a rule, also eaten freely. And this aside from what is used in cooking. In two cases in which I obtained data on this point I found that the family and help, in each case averaging about nine persons throughout the year, consumed annually at home about 660 pounds of butter. This would be an allowance of about 70 pounds to each person for the year, which seems high; but I believe it would not be too high an estimate if we should put the home consumption at 1.1 pounds per capita per week, or 55 pounds per year. The population may be reckoned at 2,000,000 in round numbers, though it is somewhat in excess of this (the census of 1890 puts the population at $2,172,205)$. The annual home consumption of butter would therefore amount to $110,000,000$ pounds avoirdupois. On this estimate, as a basis, it is possible to approximate the total annual output of butter from the Danish dairies by a simple process of addition and subtraction. But first we should note that the consumption of oleomargarine is considerable, which, of course, goes to set free at least an equal amount of butter for export. The amount of oleomargarine imported in 1891 was $1,931,461$ pounds, and the amount of same material produced at home $13,339,984$ pounds; total, $15,271,445$ pounds. The amount exported of this material was very slight-in round numbers, 500,000 pounds. 'This leaves a total of $16,248,589$ pounds avoirdupois. The account stands as follows in pounds avoirdupois:

Pounds avoirdupois.

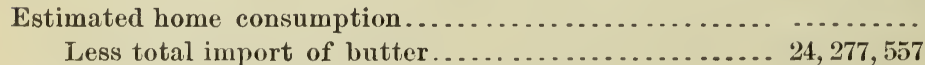

Less total consumption of oleomargarine......... 16, 248, 589

$40,526,146$

Danish-made butter consumed at home................. $69,473,854$

Total export of butter........................... 100, 600, 788

Total annual production.......................... 170, 074, 642

This is done in a country less than one-tenth the size of the State of California and but little more than one-sixth the size of the State of 
Kansas. It is an interesting study and in line with the purposes of this report to consider how such results have been attained. An output so large would not be possible if it were not for a conjunction of favorable conditions, which makes Demmark a natural dairy country.

\section{DEVELOPMENT OF THE DAIRY.}

The growth of the dairy has been slow, and it is only recently that it has reached the present high standard. Some ninety or one hundred years ago the dairy was of little consequence except on the large farms; the smaller farms did well if they could keep the family in butter. It was not unfrequently the case that a farmer kept more horses than cattle, and the cattle received but poor treatment. They frequently had to seek their food from early spring until the snow fell, without any grain whatever, and when stabled in winter they were kept alive on straw and a little hay, the object being simply to winter them until they could again be turned to grass in the spring. Under these conditions they gave, of course, but little milk.

On the large farms the agriculture was, as a rule, farther advanced. The owners were well-to-do people of education who kept abreast of the times, and they generally employed competent superintendents for their farms. Their live stock was, therefore, of a better quality than that owned by the small farmers; still, there was but little interest shown in the dairy. Steer-feeding, on the other hand, was an important industry, and every farm raised and fattened for market a number of steers commensurate with the size of the farm. The Duchy of Holstein, which at that time belonged to Denmark, was the region where the dairy first developed, and somewhat later the island of Funen followed the same example. It was in these two districts that butter was first made for export and for the supply of the larger towns. The butter from Holstein held the first place and realized the best price in the market. Twelve cents a pound was at that time considered a fair price for butter.

The priests hold their parishes by the appointment of the Government, and to each country church a farm is attached for the support of the priest. These gentlemen were often the leaders not only in spiritual matters, but also in improved farming, and they, as a class, did their best to interest the farmers in the dairy and in the improvement of their live stock. Not a few of them have written instructive works on the subject. The political situation, in the beginning of this century, was unfavorable to the development of industries of any kind, but, as the troubles subsided, farming gradually improved, and it became more and more customary to employ skilled dairy women on the larger farms. These dairy people came from Holstein, and the methods they introduced were those practiced in their native districts. They had discovered that it was advantageous to keep the milk in a 
low, even temperature, and for this purpose cellars were built under the dairy-houses, and the milk was strained in shallow wooden tubs or buckets only 4 or 5 inches deep, and these were placed upon the cold stone floor for the cream to rise. However, the whole treatment of the milk left much to be desired, and it was only during the summer, while the pastures were good, that there was a surplus of butter produced. The cows calved in the spring, and nearly all of them were dry during the greater part of the winter.

The Royal Agricultural Society was organized in 1769, with the object of advancing agriculture by every possible means. In the year 1837 this society undertook to provide instruction in dairying for the young and promising daughters of farmers. It was exclusively a practical course and covered two years, which time they spent on one and sometimes on two or even three of the largest farms in the country where dairying was recognized as an important branch of the farming. They participated in all kinds of work in the dairy and thus learned the business from thorough, practical experience. Those who had thus qualified themselves had no difficulty in obtaining positions on similar farms, as head dairy maids, where they had complete charge of the milk and butter and cheese-making. Cheese-making, however, never became an important branch of industry. It was only now and then that any attempts were made to make sweet-milk cheese, and as to skim-milk cheese it had no sale outside of the country; this branch of the dairy was naturally confined to the manufacture of cheese for home consumption. Butter, on the other hand, gradually grew in importance. That which was produced on the larger farms was sent to Hamburg and Keil for export to England, and the poorer grades were sent to Norway.

In the beginning of the '50's many of the smaller farmers had begun to take an interest in the dairy, and the merchants who handled the products began to send their butter directly to England instead of sending it by way of Hamburg or Keil, as formerly. In 1854 the Royal Agricultural Society undertook, for the first time, to provide practical instruction for young men in the dairy business, as they had already provided for the young women. The object was, however, mainly to train herdsmen and feeders, who with this united the trade of a cooper, and made the tubs and buckets and other wooden utensils required in the dairies, particularly the barrels in which the butter was transported to market. This departure proved successful, and even to the present day the same class of employees is kept on the larger farms. In 1858 the Royal Agricultural School, at Copenhagen, was started as a branch of the already existing veterinary school, and thus gradually the number of skilled helpers on the farm was increased.

This gradual advancement received further impetus by the example set by a few of the most enlightened landowners. These were, as a rule, large property-holders who had been well educated. Etatsraad 
A. Valentiner and Gehjmekonferensraad E. Tesdorpf are examples of this class of leaders. By the improvements these and others like them made upon their farms, they set worthy examples for others to follow. Much of the advancement was also due to the direct teaching of a few specialists, who, through their published articles, their lectures, and their investigations of scientific questions called attention to improved systems in the treatment of the dairy cattle and of the milk. The most prominent of the latter class is Prof. Thos. R. Segelcke, who has spent upwards of thirty years in continued work for the improvement of the dairy. He was first employed by the agricultural society as a public teacher in dairy inatters, in which position he traveled all over the country, studied the various methods in use, both at home and abroad, and devised many and important improvements. In 1874 he was transferred to the agricultural school at Copenhagen as professor of Dairy Science, which important post he still fills. He had at first to encounter many difficulties in introducing the improvements he proposed. The knowledge of dairying, when he began work, was entirely empirical. The milk was kept cool because it was found by practice that it would thus remain sweet longer. The temperature of the cream in the churn was determined by feeling it with the finger, and salt was added to the butter not by weight but by guess. Prof. Segelcke began at once to alter these empirical methods by pointing out the reasous for the practices followed, and explaining how better results might be reached by following more exact methods. Having received a scientific training as chemist, he soon discovered that exact methods were necessary to produce uniform and satisfactory results.

He thus introduced the practice of weighing the milk that was delivered to the dairy, weighing the cream that was churned, and using the thermometer instead of trial by finger; and he particularly called attention to the advantage of keeping accounts with the cows, crediting them with the amount of milk furnished and charging them with the amount of feed and labor necessary to its production. He soon devised several forms of account which he published and which gradually came into general use, at least in the larger dairies. In traveling from place to place in the pursuit of this work he came into personal contact with all the leading farmers of the country, to whom he pointed out the methods by which they could improve their system, and he thusexercised a paramount influence on the development of the industry. Since his connection with the agricultural school he has, of course, been more confined and less able to take an active leadership in the practical dairy, but his influence has nevertheless been felt through upwards of a thousand pupils who have taken instruction under him at this school.

The system of straining the milk into shallow wooden tubs (Fig. 2), which originated in Holstein and gradually became adopted all over the country, after a time gave way to better methods, although on the $8538-$ No. $5-2$ 
smaller farms this plan is yet followed to some extent. These tubs are about 2 feet in diameter and $\dot{x}$ or 5 inches deep. They were sometimes painted both inside and out, and usually held together by two iron

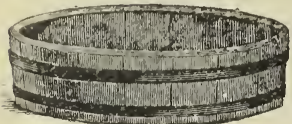
hoops. They were, in some instances, replaced by glass vessels which had obvious advantages in point of cleanliness, but they were too easily broken, and it was therefore too costly to maintain the Fia. 2.-Shallow milk tub. necessary number in the dairy. Next, large shallow iron pans were introduced as vessels in which to set the milk. These pans were 6 to 8 feet loug, from 2 to 3 feet wide, and about 4 inches deep. Fig. 3 gives a good illustration of these pans as they appear when in use. One end was made flaring, so that by tipping the pan the milk could easily run out, and they were frequently balanced on pivots in the center so that they could be easily tilted. This system of shallow setting was supposed to facilitate the rising of the cream,

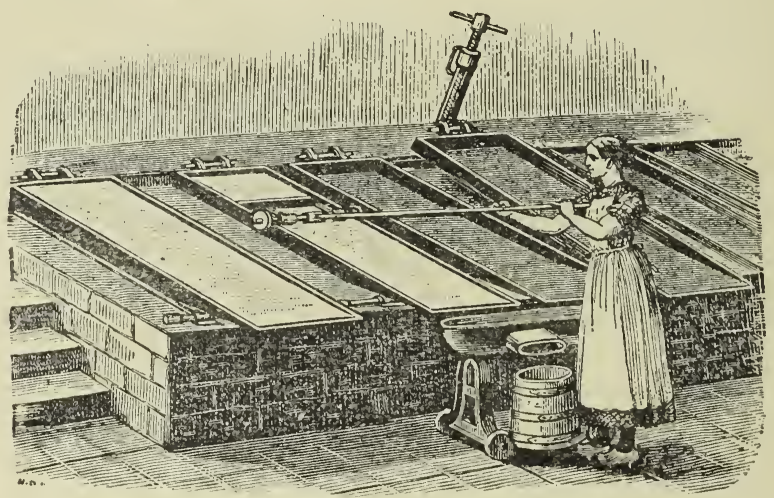

FIG. 3.-Setting milk in shallow iron pans.

since the fat globules thus had but a short distance to traverse to reach the surface. These pans were skimmed by drawing the edge of a board slowly from one end to the other, thus pushing the layer of creau before it. This system is yet occasionally met with, although it is considered antiquated and unsuited to the most economical production of butter.

The next step in advance was the introduction of water in which to cool the milk. This, however, was not always practicable, as cold water of the necessary low temperature was, in places, difficult to obtain; but, the dairymai being convinced that a low temperature was essential to the best results, the plan of using ice instead of water was an easy step to take. Setting in ice water becane common. For this purpose cement basins were built in the old milk cellars, and the milk was set in deep cylindrical cans which were sunk into the basins. 'This is still the method followed where the separator has not done away entirely with the setting of milk. Instead of cement basins, which were rather costly, ordinary large wooden tubs were often used to hold the ice water, as shown in Fig. 4. 
It was in the beginning of the '70's that the idea of the separator began to take hold of inventors; but not until 1878 was the first Danish patent granted for a separator, and in the same year a machine was constructed, and exhibited in Copenhagen, which could discharge both cream and skim milk simultaneously with the introduction of whole milk, in a continuous strean. In 1879 it was improved and given the form which in the main it still has, and two years later, in 1881, the patent was bought by a firm who still manufacture it in yearly increasing quantities. The construction of separators had occupied other inventors. In Germany a firm had constructed a machine as early as 1877, but it could not run continuously nor could it remove the cream itself. It was necessary to stop and skim it and refill it with fresh milk.

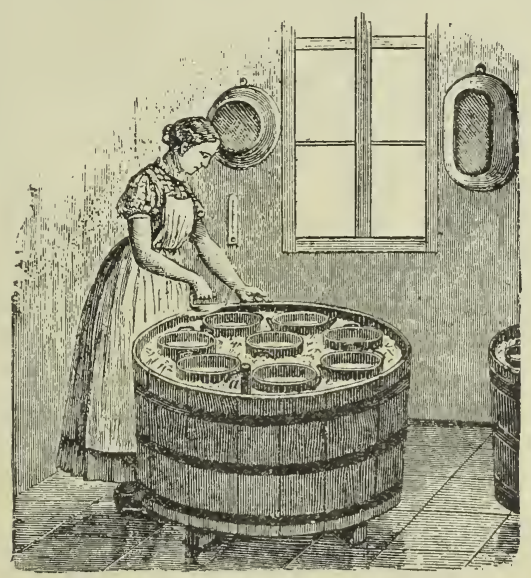

Fig. 4.-Setting milk in ice water.

In 1879 another German firm constructed a machine which retained the cream and let the skim milk run out, and it was thus necessary to stop and empty it every time a given quantity of eream had accumulated. In the same year, 1879, the Swedish scientist, De Laval, perfected his first well-known separator, which was at once put in use in some of the eading Swedish dairies. Separators are now in general use all over the country. The "butter extractor" which, at one time, promised to revolutionize dairying, has never gained any foothold in Denmark.

\section{DANISH DAIRY CATTLE.}

There are, in Denmark, two distinct breeds of cattle besides a large number of nondescript animals which can not be referred to any breed. One of these breeds is found chiefly on the islands, and is known as the "Red Danish Cattle," the other is in the peninsula and is called the "Jutland Breed." Both breeds, or races, have been much improved during the last twenty or twenty-five years, but neither of them has, as yet, reached the highest limit of perfection. 
THE RED DAIRY BREED.

The origin of this breed is not readily traced, but it doubtless takes its origin from a blending of the Angler breed of the Duchy of Holstein, which has been imported quite largely into the country, with the native cattle. There are other foreign races which, possibly, contribute, though in a slight degree, to the make-up of this breed. Thus,

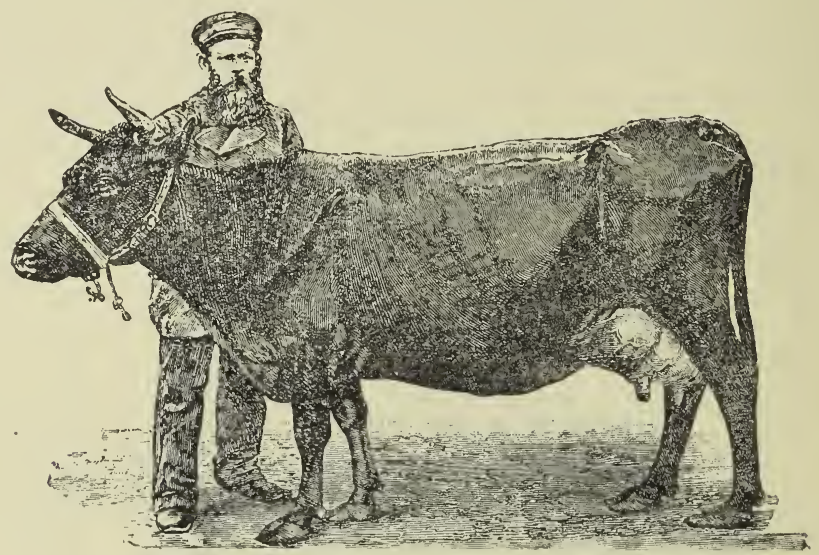

FIG. 5.-Cow of the red Danish dairy breed.

Shorthorns have been imported from England, Ayrshires from Scotland, and some few animals have been brought from Switzerlancl. But the Angler breed has been the most important, and the present red breed also closely resembles the Angler cattle. As the name indicates, they are of a red color, the head is of medium size, horns small

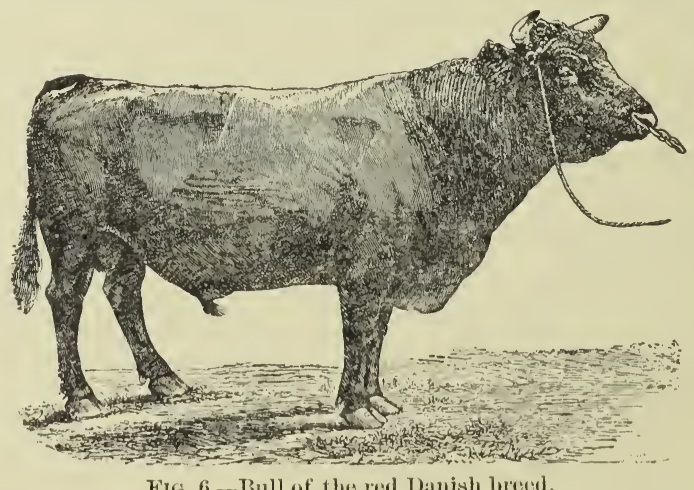

Fig. 6.-Bull of the red I)anish bred.

and curved inward, seldom symmetrical, neck light and short, rather depressed, shoulders fine, chest light, chine prominent, hips broad, bag large, milk veins very prominent, legs short and stout, weight about 1,000 to 1,100 pounds. The whole make-up of the cow is that of the ideal dairy animal. She is gentle and easily handled. Figs. 5 and 6 show, respectively, a cow and a bull which are representative animals 
of this breed. It is only during the last forty years that the farmers have awakened to the necessity of improving their dairy cattle. Pre. vious to that time the dairy, as we have seen, was a subordinate branch of the agriculture of the country. At that time the farmers thought more of developing a beef breed than of rearing a race which should be superior at the pail; nor has the development been simultaneous in all parts of the islands. The start in improving cattle for the dairy was made by a few far-seeing men, and as their aims and objects became known, others have followed their example; but, even to-day, by no means all of the cattle on the islands can be dignified by a classification in this breed. Owing, moreover, to the division of the country into islands, which of necessity puts a barrier to the fiee intermixture of the cattle, we may find different types of the breed on the different islands. The Island of Funen contains the best specimens of the breed, and several herds which I there visited are unquestionably of high merit.

There are many examples of the gradual development of the milking qualities of the cattle simultaneously with the fixing of breed characteristics. Mr. Goldschmidt, in his treatise on The Development of the Cattle Interest in Denmark, has compiled statisties from several farms, which shows this development even in a brief period of time. Thus, in 1868, the average yield of butter per cow, on twenty-six farms, was 112.7 Danish pounds. In 1869 the average produce from these farms was 132.5 pounds, per cow. In 1871 the average yield from forty farms was 140.1 pounds, and in 1872 it was 146.4 pounds. Many other similar instances could readily be produced. The following table of milk yields in pounds avoirdupois from several farms and a large number of cows will, in a like manner, show a gradual increase in production:

Average yearly milk yield per cow in the herds of five farms named.

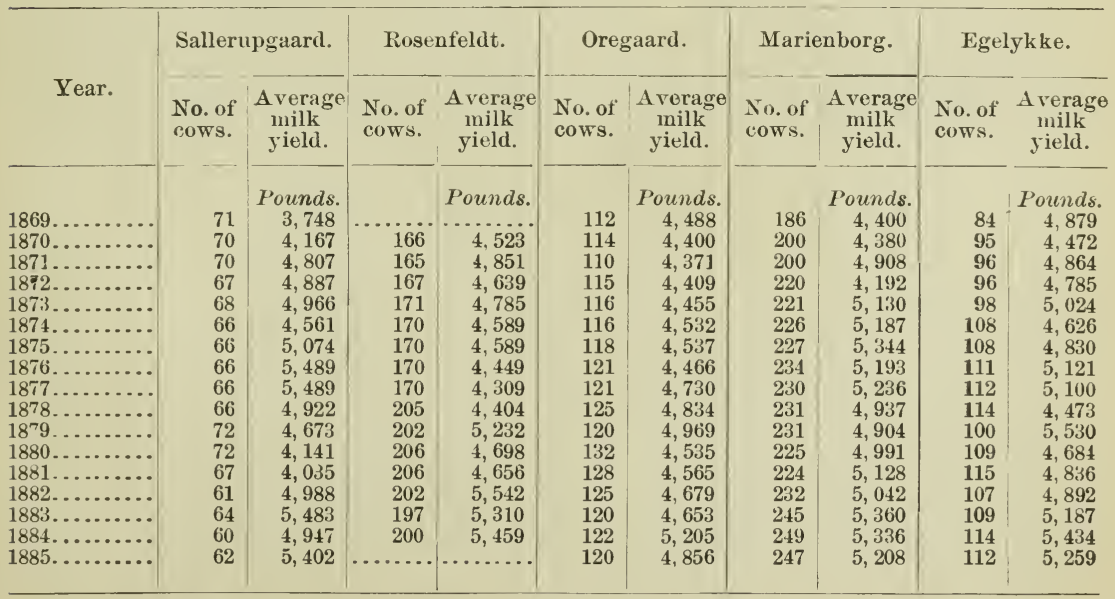

These yields are from common cattle of all ages and conditions. The table represents about the average of the Danish unimproved dairy 
cattle as we find them to-day on the ordinary farms. The improved red cattle are decidedly better, as shown in the following: On the farm named "Naesbyholm," on which in 1882 there were 89 cows, one milked between 11,000 and 12,000 pounds yearly, one between 10,000 and 11,000 pounds, two between 8,800 and 9,000 pounds, six een reached 8,000 pounds, sixteen between 6,600 and 7,000 pounds, thirty between 5,500 and 6,000 pounds, and eighteen between 4,400 and 5,000 pounds avoirdupois. A farm on the island of Funen, which has for many years been noted for its excellent specimens of the red Danish dairy cattle, has given the following average per cow of five years old or more:

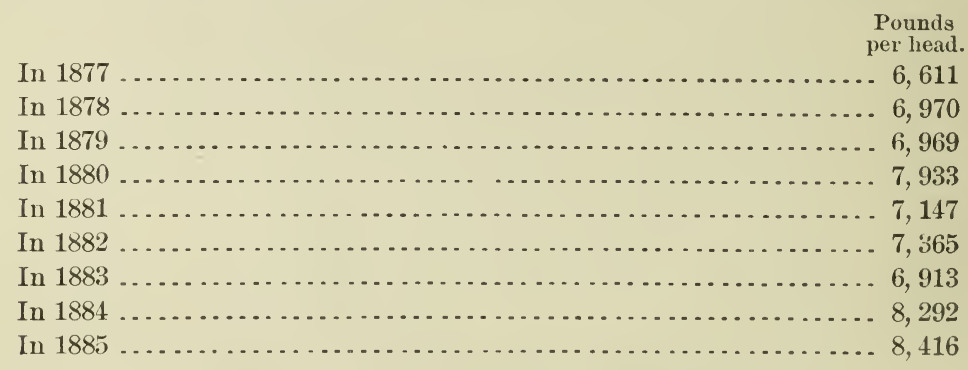

I have given the yields in aroirdupois. Some few individual cows of the breed will exceed these figures and rise to upwards of 13,000 pounds per year, but it would scarcely be fair to judge the breed as a whole by a few of the choicest specimens. Good average cows of the breed, with good care, may be expected to yield from 8,000 to 9,000 pounds avoirdupois yearly.

\section{THE JUTLAND BREED.}

The characteristic cattle in the peninsula of Jutland, which go by the above name, are black and white in color and the dairy qualities are not quite so well developed as those of the red cattle of the islands. They have been handled more as a beef breed, and, until comparatively recently, the fattening of steers was considered of more importance and more remunerative than the dairy business. The cattle had, therefore, for a long series of years been developed for beef rather than for milk. This is, however, now ehanged, and dairying is there, as elsewhere, the leading branch of farming. The origin of this race is unknown, as this class of eattle has been there from time immemorial. It may be said to be native to the soil and it has been only slightly modified by the introduction of foreign breeds. The color is not altogether uniform, but a large majority of them are black and white, much resembling the Holstein-Friesian breed. The head is usually black and the legs and tail white. In shape they are somewhat heavier in front than the red cattle, the dairy type being not quite so fully developed as in the latter, and the steers fatten readily and attain a good weight. 'The cows are a little smaller than those of the red cattle, especially in those regions where the soil is poor and the feed not abundant. They are distin. 
guished for hardiness and thrive well on moderate feed. The milk yield is also somewhat less than that given for the red cattle. On many farms the average yield of all ages will not much exceed 3,500 pounds per year and on other farms the average reaches 4,500 pounds. This is for common, unimproved stock. In some exceptional cases. where special attention has been given to the selection of the herd, the average will go as high as 6,500 pounds, for cows in good condition. One of the best cows of the breed, which is shown in the accompanying illustration (Fig. 7) has, in the years 1886-'89 inclusive, given respective] y 9,273 pounds, $10,488,10,719$, and 9,905 pounds avoirdupois; but this is an exceptional animal. On the farm where she belongs the average for the same years was $6,176,6,799,7,317$, and 7,334 pounds per cow. One of the best herds of this breed is found on the farm named Borupgaard, in central Jutland. This herd has been so improved during the last twenty years that it is now one of the leading herds of that breed in

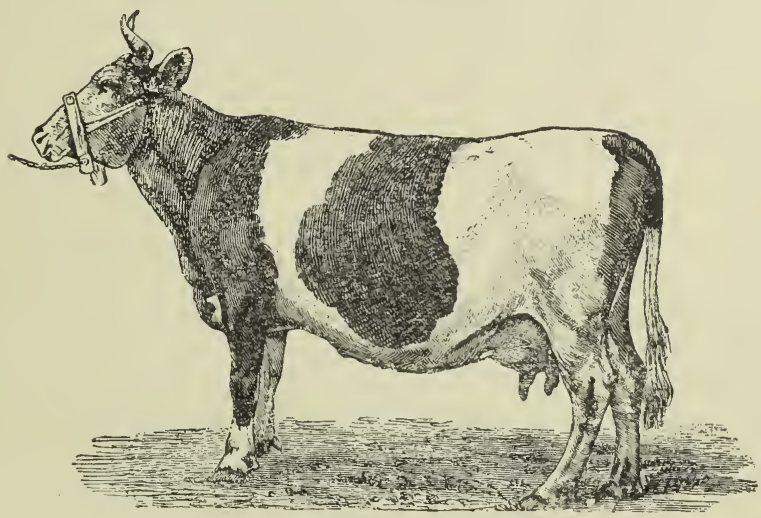

Fig. 7.-Cow of the Jutland breed.

the country. The improvement has been made by selecting the best cattle which could be found, regardless of cost, and breeding from these only. The milk yields of this herd, from $18 \div 0$ to 1890 , were as follows :

\begin{tabular}{|c|c|c|c|}
\hline Year. & $\begin{array}{c}\text { Number } \\
\text { of cows. }\end{array}$ & $\begin{array}{c}\text { Average } \\
\text { yield. }\end{array}$ & $\begin{array}{c}\text { Number of } \\
\text { cows yielding } \\
\text { over 6,600 } \\
\text { pouncls. }\end{array}$ \\
\hline & & Pounds. & \\
\hline & & 5,995 & 36 \\
$1880 \ldots$ & 124 & 5,1 \\
$1881 \ldots$ & 127 & 5,830 & 31 \\
$1882 \ldots$ & 124 & 5,720 & 32 \\
$1883 \ldots$ & 122 & 5,912 & 34 \\
$1884 \ldots$ & 127 & 5.830 & 30 \\
$1885 \ldots$ & 140 & 5,720 & 32 \\
$1886 \ldots$ & 145 & 5,610 & 29 \\
$1887 \ldots$ & 142 & 5,610 & 38 \\
$1888 \ldots$ & 145 & 6,116 & 52 \\
$1889 \ldots$ & 138 & 5.610 & 35 \\
$1890 \ldots$ & 142 & 6,380 & 43 \\
\hline
\end{tabular}

This average includes from 15 to 25 cows each year which were not at their best either by reason of having lost their calves or by reason of being barren. 
In regard to the quality of the milk of the two breeds, the experiments of Prof. Fjord, in which the two breeds were kept on the same farm and treated in the same manner, indicated that if the Jutland breed gave somewhat less milk than the red breed, the per cent of fat was also a trifle higher, so that for the production of butter there was practically no difference between them, so far as this one experiment would indicate. The average composition of 43 samples of Danish milk, according to Prof. V. Storch, is as follows:

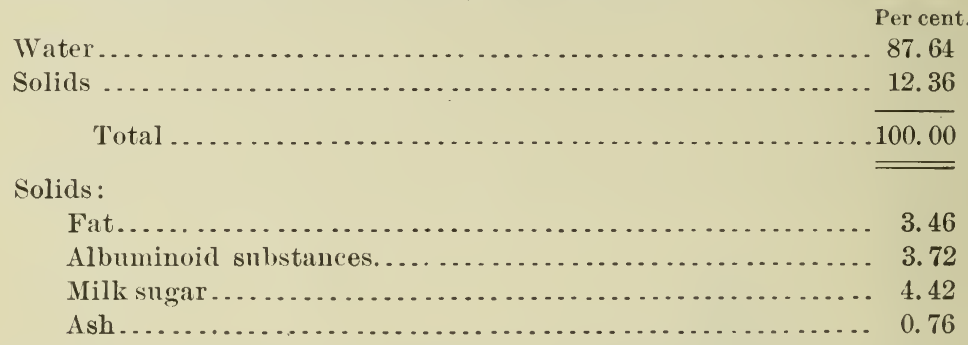

This is, doubtless, a fair average of the composition of Danish milk generally.

\section{IMPROVEMENT OF THE CAT'TLE.}

As has been stated, the best specimens of these respective breeds are in the hands of a few breeders who have labored skillfully and perseveringly during the last two decades in order to improve their herds; but the general desire to improve has now spread all over the country and there is scarcely a farmer, be his farm large or small, who is not striving with might and main to improve his herd. 'This is shown in many ways. It has given rise to numerous associations anong which, perhaps, the so-called "Bull associations" are the most important.

Bull associations._-These associations are made up of the farmers in a given neighborhood who unite and purchase a superior bull which they use in their respective herds. The purchase price is borne by the association and his keep is paid for out of the service fees. In many instances these associations are aided by small gifts from local farmers' clubs and from a state fund set apart for that purpose. I give an example which will suffice to illustrate all cases, and this is one of several which fell under ny own observation. Such an association bull was stationed on a farm in the town of Ringe on the island of Funen. The association that owned him consisted of twenty-nine members, all farmers in the neighborhood. They had selected one hundred of their best cows and he was put to none but those chosen; inferior cows were not eligible. Ife was a good-sized, handsome animal of the red breed and weighed about 1,900 pounds avoirdupois. He cost abont $\$ 162$. The service fee paid by members was about 70 cents, but this fee fluctuates with the cost of his keep, as the members are charged only the actual cost. He was 
recorded in the herdbook for that breed, was 5 years old and had taken several prizes. The keep of this bull amounterl last year to about \$68, which sum was paid to the farmer who had him in charge, and one-third of this was covered by state aid.

Fairs.-The numerous district, county, and association fairs at which animals are exhibited and compete with each other for prizes have a wholesome influence on the improvement of the dairy cattle. Here the best types are shown, their good qualities are made prominent by the prizes they win, and purchases and transfers commonly also take place at these fairs. These institutions are helped, or maintained, under the auspices of the Royal Agricultural Society, which I shall have occasion to mention later. The fairs are a potent force in the improvement of the Danish dairy cattle.

Officiul advisers.-Another important aid, especially for the inexperienced breeders, is a class of officials who have become quite numerous. They are called "Kousulenter," from the word to consult, which, in a free translation, may be rendered "advisers." The state maintains three such officials in cattle-breeding, and three more for horses and sheep, each of whom is assigned to a given district. Many of the leading local societies have, in like manner, "Konsulenter" or "advisers" of their own. Altogether there are between twenty and thirty "advisers" of the latter class in the country at present. Their business is to answer questions and give advice on all kuotty points which the farmer himself does not feel competent to decide upon. They are supposed to be expert in all questions pertaining to cattlebreeding. They are familiar with the history and breeding of all the herds and even individual animals in their respective districts, and, not infrequently, they are also veterinarians. It will be readily seen how they can render important service to the plain farmer in, for instance, the selection of a breeding bull, or in almost any proposed line of improvement. In addition to this they lecture at the gatherings of farmers in their respective districts, thus serving as public teachers, and in a hundred ways are of assistance in the improvement of the dairy stock. Under such methods it is apparent that rapid strides are being made in the development, not only of the dairy breeds, but of the dairy industry as a whole.

\section{DAIRY IMPLEMENTS AND MACHINERY.}

\section{SEPARATORS.}

First among the long list of machinery and utensils used in the Danish dairy should be placed the separator. There are several patterns in use, but the one most commonly used is the machine shown in the accompanying illustration (Fig. 8). It is there known as the Burmeister \& Waine separator, after the manufacturers. The machine, which was invented by a Dane, P. Nielsen, was first used successfully 
in Copenhagen in 1878. It lhas since been improved and altered in many ways, but retains the essential original features. It can raise the skim milk and cream through its discharge pipes to a height of 8 feet, thereby enabling them to run off to other parts of the creamery where the reservoirs may be located. It is the only separator which can do this. Fig. $9 c$ shows this arrangement. It is manufactured in several sizes. The larger forms revolve with a speed of 2,800 revolutions a minute and the smaller form, which is designated as " $B$ " by the manufacturers, revolves with a speed of 4,000 revolutions per minute.

Another separator, which I believe to be gaining favor in Denmark, is the so-called "Alpha" separator, invented by De Laval. I saw it in use in several places, and the superintendents invariably spoke highly of it. This form is peculiar in that it has a series of inverted tin pans in the drum, between which the milk must pass and which, it is claimed, facilitate the more complete separation of the cream. It is claimed that it can skim 1,750 pounds avoirdupois per hour, at a rate of speed

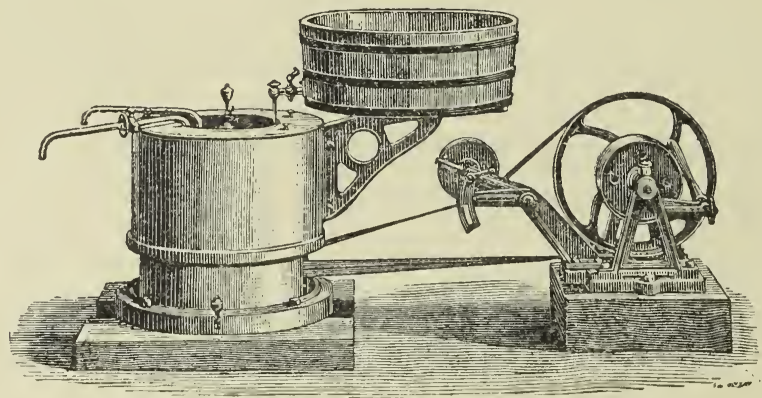

FIG. 8.-The Burmeister \& Waine separator.

of 5,600 revolutions per minute, with the milk at a temperature of $75^{\circ}$ to $80^{\circ} \mathrm{F}$. The still larger form known as "Alpha 2 " can skim, it is claimed; 3,500 pounds per hour at a speed of 5,600 revolutions per minute.

A third separator, which is used to a considerable extent, is also of Danish origin. It was invented and first brought into use in 1885 by M. Peterson and J. Nielson. They sold it, however, soon after to the present manufacturers, Koefoed \& Hauberg, by whose name it is known. It is claimed for it that it skims the milk clean and works with comparatively light power. There are still one or two others in use of German origin, but only the three here named can be said to play any important part in the dairy business, and in point of numbers in use of each kind they rank about in the order here given. Each of these patterns can also be had in smaller machines for hand power, and these "Baby" separators are coming more and more into use in the smaller dairies. 
Burmeister \& Waine's hand separator is manufactured in two sizes, costing, respectively, about $\$ 68$ and $\$ 95$, and with respective capacities of 250 and 350 pounds of milk per hour. The Koefoed \& Hauberg hand separator is also manufactured in two sizes, costing, respectively, about $\$ 154$ and $\$ 117$ and with respective capacities of 500 pounds and 400 pounds of milk per hour.

De Laval's hand separators are likewise in two forms, the "Baby" costing about $\$ 62$, with a capacity of 120 pounds per hour, and the "Alpha

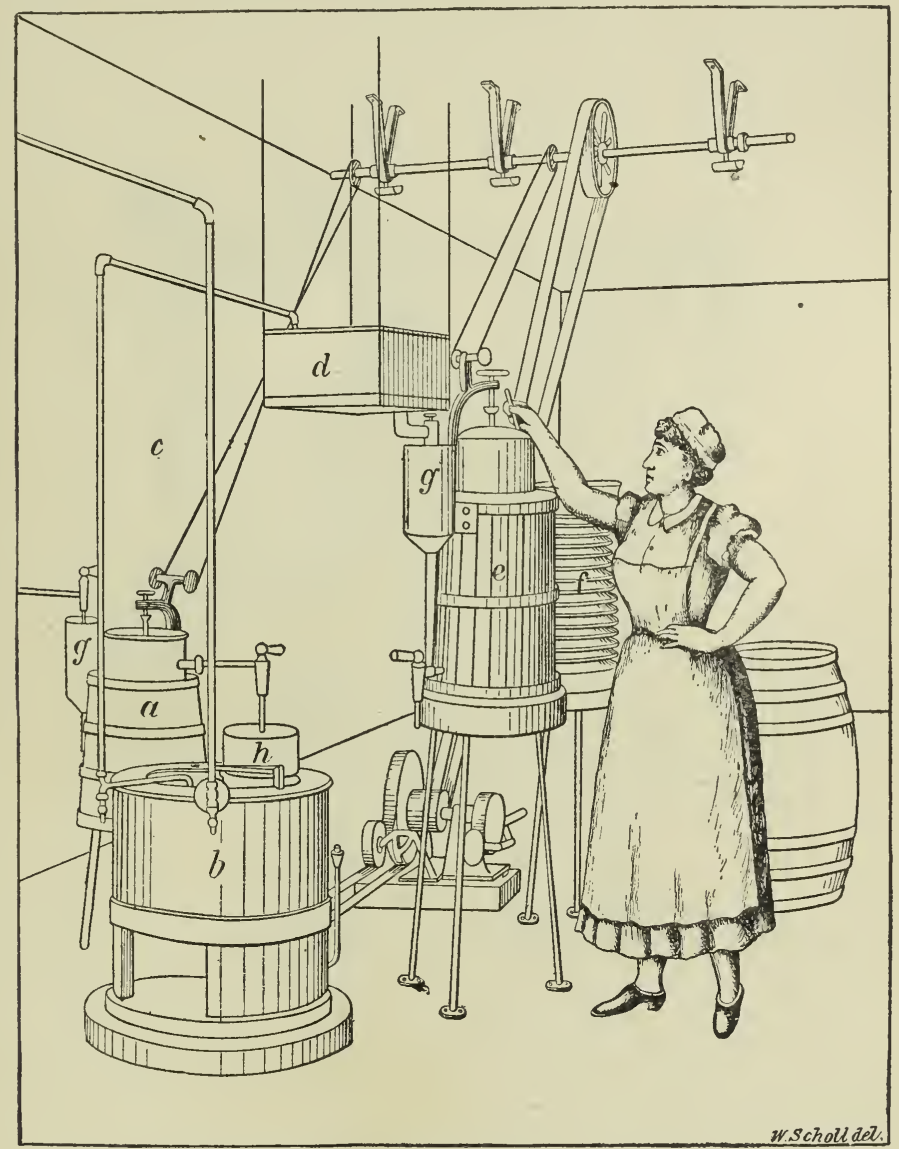

FIG. 9.-Machinery connected with the separator.

baby" which skims 250 pounds per hour. I did not learn the price at which it was sold.

We will next notice a few pieces of machinery directly connected with the separator. The receiving basin, into which the milk is poured after it has been received at the creamery and weighed, is sometimes a large wooden vessel, either square or circular in form, and in other places it is made of heavy tin. It is always placed on a platform so high above the floor where the separators stand that the milk cau run by gravity into 
them. It is a well-known fact that the milk is skimmed cleanest when warmed to a temperature upwards of $75 \circ \mathrm{F}$. The milk does not lave this temperature when received from the dairy, nor can it be attained unless it is heated, and for this purpose a piece of machinery has been invented, through which the milk rums in a continuous stream on its way to the separator, and in passing is heated by steam to the required temperature. This machine is known as the "forewarmer" (see Fig. 9 a). It is a simple cylindrical barrel, nade of tin or copper, which is "jacketed;" that is, placed inside another and a little larger vessel, so that a space is left between the two, and this space is made steam-tight. The steam is introduced into this space, and it thus varms

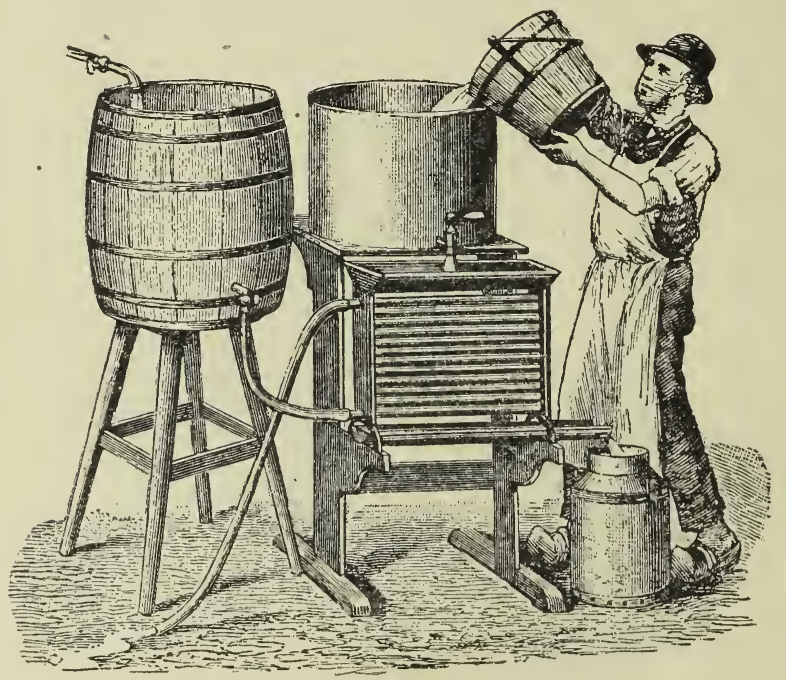

FIG. 10.-The Lawrence cooler.

the milk contained in the inner vessel. To warm it evenly an agitator is introduced, which revolves slowly, so as to bring all the milk into equal contact with the heated sides of the vessel. The milk enters the forewarmer at the bottom and runs out at the top. An opening in the discharge pipe permits of inserting the bulb of a thermometer so that the temperature can always be noted.

There are several devices which regulate the inflow of milk into the separator, or other vessels, so that the stream is constant from beginning to end, although there may be a variation in pressure. One of these is known as "Prof. Fjord's regulating fummel," having been invented by him. Another arrangement is to put a "swimmer" in the fumnel. A "swimmer" is a cylinder with conical ends, into each of which is put a piece of wire, which thus forms the axis of the cylinder. One end, with its wire, projects in to the discharge pipe of the funnel, and the other end, with its wire, projects into the discharge cock of the basin, or reservoir. Now, if the milk runs too fast, this "swimmer" rises up against 
the end of the discharge cock, partially closing it and thus regulating the flow. The "swimmer" is used in the funnels shown at gg, Fig. 9, and "Fjord's regulating funnel" at $h$. The cream from the separator is run into the suspended vessel $d$, from which it runs through the sterilizer $e$ to the cooler $f$. The cream may run from the separator either directly to a cooling apparatus, or, as noted above, through a sterilizer to the cooler. We will note the latter first.

\section{COOLERS.}

Three forms of coolers were noticed, and all three cool the milk or cream upon the same principle. A stream of cold water enters the apparatus from below and rumning through a "worm," or a piece of thin zigzag pipe to the top of the apparatus, it is discharged, the end of the pipe being directed downwards and discharging the water below. The milk or cream is poured into a hopper on top of the apparatus and runs slowly in a thin sheet down over the corrugated sides,

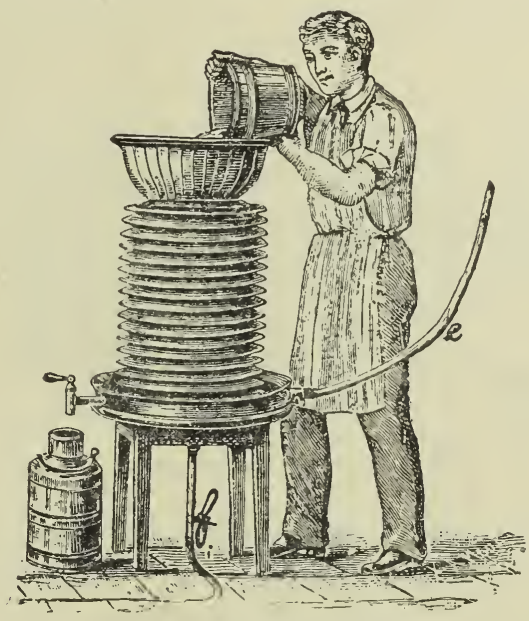

Fig. 11.-The Smith cooler.

and coming thus into direct contact with the cool metal it is cooled to within 4 or 5 degrees of the temperature of the water used. The water may be supplied from a tank or large barrel placed so high that it will run through the cooler. Fig. 10 is a good illustration of the "Lawrence cooler," which I described in my preliminary report. The barrel should be filled with broken ice and a jet of water turned on to keep up the supply. From the barrel it runs into the bottom of the cooler, and following the zigzag pipe it runs out at the top and is discharged through a piece of hose. The substance to be cooled is poured into the small reservoir, whence it flows into the hopper, and then flows over the cooler. The Smith cooler (Fig. 11) is cylindrical in shape instead of flat. The water enters through the hose $e$ and is discharged at $f$. 
In some cases I found that the water running from the cooler was again raised to the reservoir by a pump worked by the engine that supplied the power for the establishment. This effected a saving of ice. and the water was cooler than it could be if drawn from some outside source. These coolers are made in many sizes. The largest is 3 feet, high in the body, and is claimed to cool 5,000 pounds of milk per hour, from $180^{\circ} \mathrm{F}$. to about $3 \circ \mathrm{F}$. above the temperature of the water used.

PROF. FJORD'S CONTROL APPARATUS.

This invention, which is illustrated in Fig. 12, is in universal use all over the country. It has, by successive steps, been improved and enlarged until it now has the form herewith represented. The Danes think a great deal of this piece of testing machinery, and are firm in the belief that there is nothing equal to it. The inventor is the highest authority in dairy matters that they recognize, and it is impossible that a thing he recommends could be inferior to anything else. I told them of our Babcock milk tester, but my representations met with no favor. The fact that ehemicals were to be used in the Babcock test,
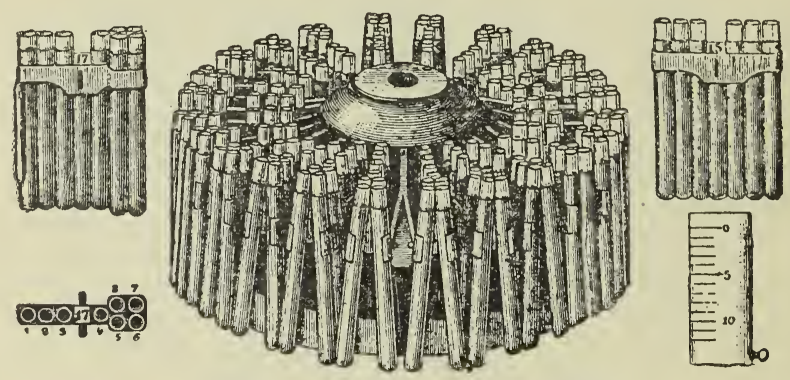

FIG. 12.-Prof. Fjord's control apparatus.

was considered an insuperable obstacle which might work dire disaster in the hands of common dairymen who have had no training in chemistry. Fjord's milk tester' presented no such difficulty, and to them represented the acme of simplicity and accuracy. As shown in the illustration, it is a nest of glass tubes built up of twenty-four little frames which are suspended from as many arms radiating from a central hub. Each of the small frames holds eight tubes, the whole containing one hundred and ninety-two tubes, and being capable of making 192 tests at once. A small frame is shown both inside view and end view in the illustration. 'The tubes are about 6 inches long, and half an inch in diameter. It is essential that they should be of the same diameter throughout. In shape they are like the ordinary test tubes, except that the month is straight, not flaring. There are no seales or marks on these tubes, but the places they occupy in the frame are numbered fiom one to eight, and the frames are numbered from one to twenty-four. It will thus be seen that in testing a sample of milk it is only necessary to keep a 
record of the place it occupies in the nest. To fill the tubes they use a little dipper, represented in Fig. 13, made of tin. It has a little projecting arm on one side which is put into the tube after it is filled, and thus forces out a quantity of milk equal to the space it occupies. By this means it is possible to get exactly the same amount of milk in each tube. The test is on the centrifugal principle.

The Burmeister \& Waine separator has a very large bowl and it is made to attach to an axis which stands perpendicular in the center of the bowl. The hub of the nest is put over this axis and when the bowl is set in motion the nest of samples turns with it, the frames being swung out : o that the tubes occupy a horizontal position with the opening pointing inward. The cream in each sample is forced to the mouth of the tube, and by continuing to run the machine for forty-five minutes, which is the usual time, the cream forms a perfectly solid mass which fits like a cork over the milk. Now, by means of the little scales shown in the lower right-hand corner of theillustration (Fig.12), this layer of cream is measured and recorded for each sample. Then, by reference to a table, constructed by Prof. Fjord to be used in this connection, the operator reais off the per cent of fat contained in each sample of milk. I was told by more than one chemist, who had compared the results of this milk-tester with chemical analysis of

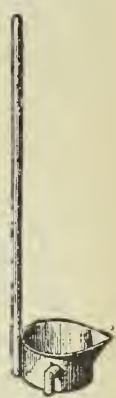

Frg. 13. Dipper. the milk, that when carefully handled it would work with perfect accuracy. It is not likely to meet with favor in this country; but I describe it because of the importance it has in Danish creameries.

\section{CREAM BARRELS.}

The vessels in which the cream is set to ferment may next be mentioned. These were, in nearly every case that came under my observation, simple wooden barrels, although I learned that in some places were used large tin cans of a size suited to the size of the dairy. Sometimes only one or two were needed, while at other places I saw three or more in use. They were made of oak wood and each was supplied with a lid. A double cream barrel I learned was in use in some creameries. It consisted of a tin can fitted inside a wooden tub or barrel. The object aimed at was to maintain a uniform temperature in the cream by guarding against the effect of external fluctuations.

\section{THE PASTEURIZING APPARATUS.}

I described briefly this piece of dairying machinery in my preliminary report, and here present an illustration with a more detailed description (see Fig. 14). The form used in Denmark was invented by Prof. Fjord. He introduced an agitator to prevent the burning of the milk or cream. It is made of a copper cylinder covered with tin (Fig. 14ua). 
This is fitted steam-tight into a vessel of somewhat larger diameter, $b b$, made either of copper or galvanized iron, and then covered on the outside with wood to retard cooling. The steam is introduced through the opening at $g$ into the space between the two vessels and passes out through the pipe $d d$. The milk or cream enters through the pipe $c$ into the bottom of the apparatus and it is lischarged through the pipe $e$, which has a pocket on the upper side into which a thermometer can be inserted, thus enabling the operator to control the temperature, which he does by letting in more or less steam. The agitator $f$ is a simple frame of wood or metal. It is turned at a speed of about 150 revolutions per minute by a belt connected with the shafting. This apparatus is in common use in Danish dairies. It is intended to kill

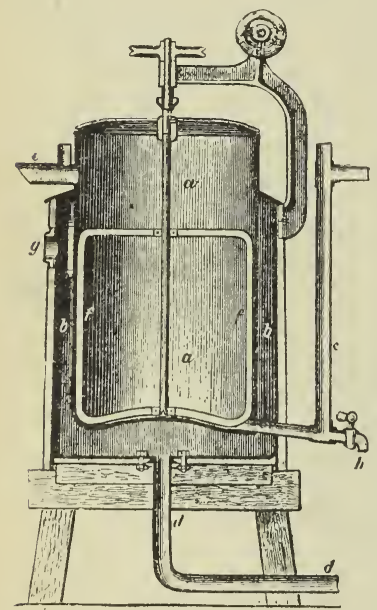
the microbes, or at least the greater part of them, and thus prevent or retard their propagation, and it is used for sweet milk, for cream, or for skim milk, as the case may be. In some instances the sweet milk is sterilized instead of being merely warmed by the "forewarmer" before it runs into the separator. In other clairies I found that the cream was sterilized and again cooled at once before it was set into the cream barrels to await fermentation. And in nearly all cases where the sweet milk was not sterilized before it was separated I found that the skim milk was sterilized as it left the separator. This was especially the case in coöperative dairies, where nearly all the skim FIG.14.-Pasteurizing apparatus. milk was taken back to the producers. Thus treated it would keep better, and in feeding it to calves it was claimed that all danger of possible infection with tuberculosis was avoided.

The temperature to which the milk or cream was heated I found to vary somewhat, but it was usually somewhere between $160^{\circ}$ and $180^{\circ}$ F. It should be noted that in order to maintain this temperature constantly the steam should be admitted directly from the boiler. Waste steam, it has been found, does not answer the purpose. It may be noted in this connection that when the sweet milk is sterilized by being heated, say to $170^{\circ} \mathrm{F}$., the separator can skim it much cleaner than when it runs through at a lower temperature; but, on the other hand, sterilization of either sweet milk or cream slightly diminishes the amount of butter. It increases the amomnt of butter fat retained in the buttermilk and it diminishes the amount of water in the butter. 
THE CHURN.

The next important piece of dairy apparatus is the churn. Of this I found but one pattern and, I believe, only one size. Where one chum Was not sufficient to do the work, several were used. The pattern used is represented in the accompanying illustration (Fig. 15). It is a large truncated barrel made of oak wood and suspended, as shown in the illus. tration, by pivots near the center of gravity, resting in upright posts. This arrangement facilitates the removal of butter and buttermilk and the cleaning of the churn, in that it can readily be tilted to any angle. The cream is churued by a revolving agitator made of wood, shown in the lower left-hand corner of the cut. Sometimes cleats are nailed on the inside of the churn to the number of three or four, which, by break-

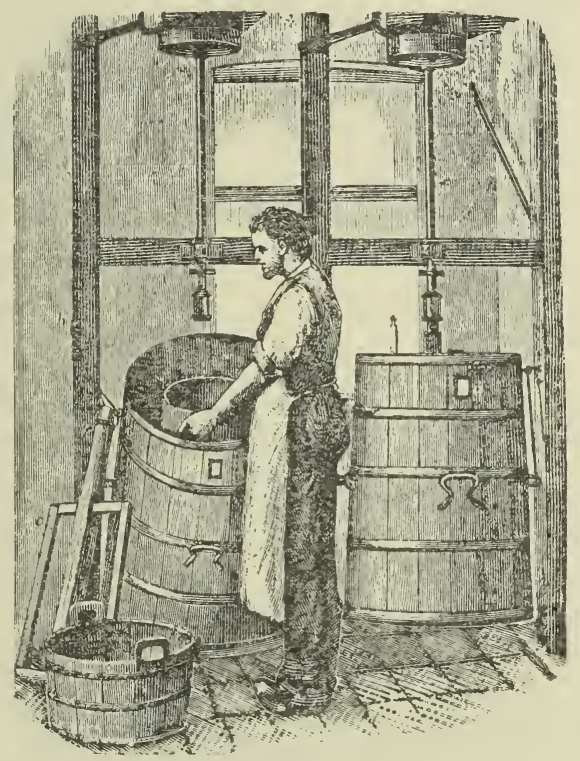

FIG. 15.-Danish churns.

ing the rotary motion of the cream, assist in the agitation. In the majority of instances the power used is steam, but I saw also the churn worked by a horse, through the medium of a sweep. The illustration shows the butter-maker in the act of removing the butter with a sieve which drains off the buttermilk.

\section{BUTTER-WORKERS.}

The butter-workers used are of the same patterns which we find in this country; in fact, I believe they are of American origin. In former times, before the introduction of mechanical butter-workers, the butter was worked by hand altogether, in a trough, as shown in Fig. 16. It is simply a log of hard wood, usually beech wood, which is scooped out and made smooth. It is so placed that it inclines very slightly to one 
end, and thus the buttermilk as it is pressed out will run into a vessel placed underneath, through a hole in the trough. It is only in a few places, and where the dairying is conducted on a small scale, that the butter is worked by hand. The old-fashioned butter trough will soon be classed among the obsolete articles.

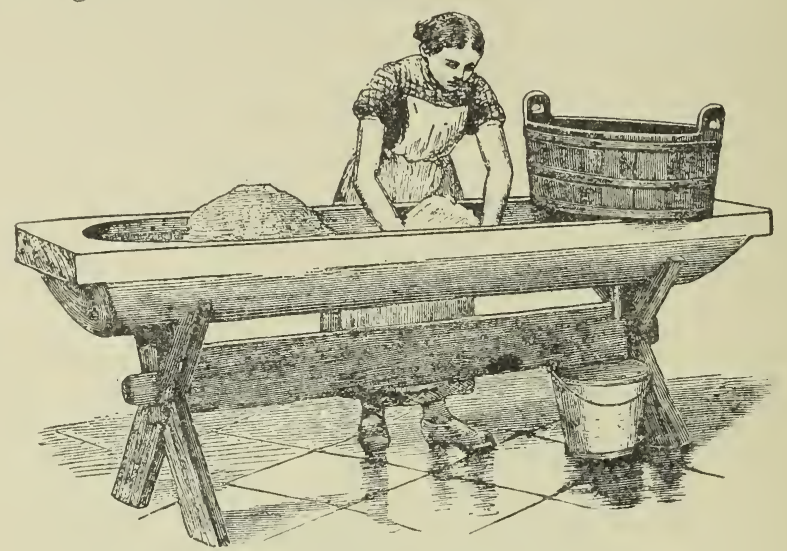

Fig. 16.-Butter tromgh.

BUTTER-COOLERS.

Butter-coolers are found in every dairy. The cooler is a simple box made either of wood or, in some cases, of zinc, in which the butter is kept to cool after it is removed from the butter-worker, and it remains there until it is time to give it the next working. The box is provided with one or more cleats inside, according to its depth, and slats are laid across the box on these cleats on which the butter is put, as shown in Fig. 17. In the illustration, $a$ represents the cleat, $c$ the slats, and $s$ the butter. The box is covered with a lid on which is put a layer

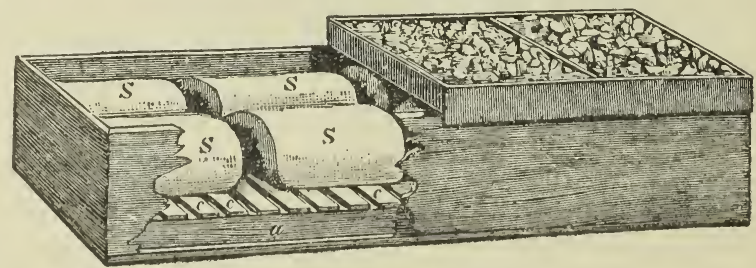

FIG. 17,-Cooling box for butter.

of broken ice, and the ice water from the melting of this ice runs down into the bottom of the box. To facilitate the cooling, the butter is rolled into an arch, as shown in the illustration. In some of the larger dairies which I visited these boxes admitted of placing two or three layers of butter to cool at once.

DAIRY SCALES.

Every dairy is supplied with several scales of varying sizes. The decimal weight is used everywhere. Instead of moving the weight 
along on the lever arm, as we usually do, there are two platforms, one on which to place the object to be weighed and on the other the weights of different sizes. In many dairies they use a weight on the receiving platform for the milk, which has a basin holding from 50 to 75 gallons. The milk is poured into this and when a certain weight is reached it is checked off and the basin is tipped so that it empties its contents into the large milk vat.

\section{BUCKET'S AND MILK CANS.}

Of these many styles are used. They are generally made of heavy tin and they arc not infrequently enormous in size. The cans in which the milk is transported are of two general forms, square and round, and the mouth is so large that it admits a hand and arm readily. Frequently the weight of the can is stamped upon it, or it is stamped upon a brass plate and soldered to the can, thus obviating the weighing of the can.

\section{CHEESE VATS.}

These are of two general forms, the oblong box and the cylindrical vat. In the latter the milk is warmed only through the bottom, which is double and made of copper, admitting steam to be injected botween it and the real bottom of the rat. The square form is of the type in common use in this country. The cheese presses are also found in several patterns, none of which are superior to those in use in this country. The horizontal press is known as the "American" cheese press, and has been imported from this country.
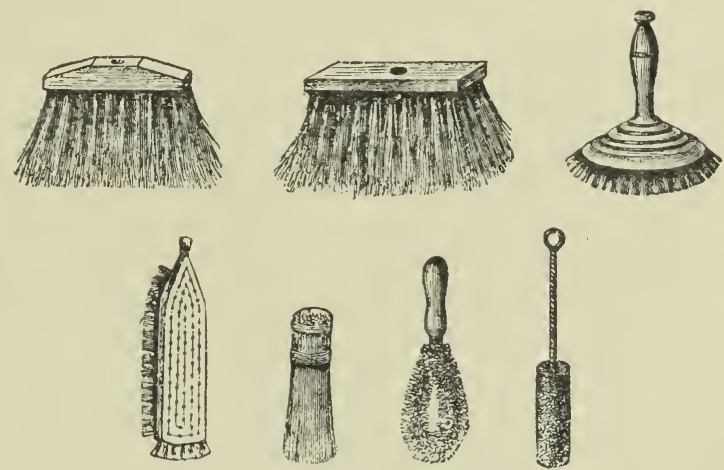

FIG. 18.-Scrubbing brushes.

('LEANING UTENSILS.

Of these there is a large variety of scrubbers and brushes in use, some of which are represented in Fig. 18. Strainers, thermometers, measuring glasses, etc., are of numerous patterns, but I did not notice any that were improvements on those in use here. 


\section{SYSTEMS OF CREAMING.}

The system now in use is, as has already been inclicated, the separator system, but previous to its introduction there were, as noted under the heading of the development of the dairy, several transitions from one improved methorl to another, and all of these can yet be seen represented on Danish farms. I will, therefore, briefly describe them, inasmuch as conditions in this country may be such that a farmer now and then can use one or the other of them to advantage.

\section{I.-SETTING MILK IN SHALLOW VESSELS.}

It was early recognized that it was necessary to keep the milk in a uniformly cool temperature in order that the cream might rise in a reasonable time and the milk keep sweet, and to produce this condition it became customary to keep the milk in cellars or basements. These were not, as the word might imply, dark places deep in the ground, but they were usually 4 or 5 feet deeper than the surface of the ground, winclows and ventilators to supply light and fresh air being put in; and to-day, with the improved separator system, we find these basements still in use, not because they are a recognized necessity, but because they remain in the buillings as remmants of a former system. In the new creamelies, which are now being constructed, cellars are frequently wanting. The old method of setting the milk in shallow vessels where it stood from twenty-four to forty-eight hours before it was skimmerl required an even, cool temperature, and the cellar was, in that day, the nearest approach to that condition. The milk was strainer into shallow wooden tubs and these placed by companies and platoons in rows upon the floor, a narrow alley way separating one milking from another.

This system of setting the milk required an unusually large cellar. It required from 12 to 20 square feet of space for each cow in the dairy. 'This had the advantage that there was always a large volume of air in the room and that by careful ventilation the air could be kept sweet and fresh. It was also necessary that these cellars be thoroughly drained, so that all moisture which collected in the course of cleaning could be easily led off. The floor was usually made of brick set on eilge; later on it was occasionally made of cement, and in exceptional cases it was marle of hewn stone. The ressel most commonly in use, in which the milk was set, is slıwn in Fig. 2. The cream was skimmerl off when it had stook, as stated, from twenty-four to forty-eight hours. In summer the skimming usually took place in twenty-four hours and in winter in thirty-six to forty-eight hours. This shallow tub was made of oak wood 2 feet in rliameter and 5 inches high insirle. The quantity of milk set in the tub liffered with the different seasons, fiom $1 \frac{1}{2}$ inches to about $3 \frac{1}{2}$ inches reep. The chief objection to this system is that too much of the eream remains in the milk. In summer when it is neces- 
sary to skim the milk in twenty-four hours, frequently 1 per cent or more of the fat will remain and, of course, become lost to the buttermaker. The cream is skimmerl off by means of a large flat spoon made sometimes of wood but usually of tin, circular in shape, some 6 to 10 inches in diameter and but little dished. The dairy maid would, with a dexterous move with this spoon, first loosen the cream from the sides of the tub and then gather it in rapidly in two or three spoonfuls and collect it in the "ream bucket which she earried with her. The most difficult part of the management of this system is to know when to skim the milk, and to so ventilate the cellar that the cream and the butter receive no bad taste or smell. To get the best quality of butter the milk is skimmerl while it is sweet. What butter fat is left in the milk is not to be consilered as a total loss inasmuch as a large part of the skim milk is made into cheese, and what is thus lost in quantity of butter is gainerl in quality of cheese.

\section{II.-SETTING MILK IN IRON PANS.}

The leng, shallow, iron pans which I have already mentioned (see Fig. 3), and which are yet seen in many dairies, were brought into use for the first time in 1843 by Hr. V. Destinon in Holstein. They are of several sizes, but perhaps the most common size is 7 feet long by 2 feet broad and 4 inches deep, having at one end a handle by which it can be raised and the other end flaring for the outlet of the milk and cream. Sometimes they are placed upon the edges of a brick basin, which is filled with cold water, the water, if possible, being made to circulate or run through the basin and reach to near the edge of the pan. The water is supposed to aid the cream in rising more rapidly and also in keeping the milk at a uniform temperature, but it has this disadvantage, that it keeps the air in the milk room very moist and more difficult to hold sweet and fresh, and in summer, when fresh air is most needed, it favors the formation of molds, which is decidedly undesirable. For these reasons water is not often introduced, nowadays, andinstead of the basins the pans are most frequently placed on low stools or blocks of wood. These pans are somewhat easier to handle than the wooden tubs which, in a large dairy, are very numerous and of necessity have to be carried about from the milk cellar to the wash room, where they are boiled out and then cleaned in cold water: they are next placed in pyramids on racks out of doors to air and dry. The iron pans ean be scrubbed down in scalding water without being required to be aërated and sun-dried in order to keep them sweet.

On the smaller farms, where the dairy consists of only a few cows, the milk has very generally been set either in earthenware dishes or in enameled iron pans. These are still in common use on small farms, and they are placed either on the floor or on shelves in the milk room. The chief objection to all of these forms of setting the milk lies usually in 
the premises. If it is possible to have a dry cellar on the north side of the house, so situated that no bad odors from outside can get into it with any direction of the wind and which permits free ventilation, this system of setting milk, especially when the skim milk is user for cheese, is not a bad one. But in too many cases it is difficult to meet these necessary conditions, and whenever the cellar is objectionable it is impossible to make a first-class article of butter.

\section{III.-SETTING MILK IN COLD WATER.}

This system, which was used in some dairies for a number of years, was borrowed from America, and it was even sometimes called the "Orange County System," after Orange County, N. I., where it was common. The plan consisted, as in this country, of setting the milk in deep cans in cold water-spring water, if it could be obtained; well water raised by a pump, if nothing better could be had. However, it never came into general use, although the results were, on the whole, satisfactory where the water supply was sufficient. Some of the leading farmers adopted the plan, but after some years it was modified by the use of ice.

\section{IV.-SETTING MILK IN ICE WATER.}

The use of ice was the outgrowth of the cold-water method. A few trials proved conclusively the great advantage of cooling the milk rapidly and maintaining it at the lowest possible temperature by the use of ice. But the great difficulty was to preserve a sufficient quantity of ice for this use in a large dairy. This question led to Prof. Fjord's experiments in the construction of ice houses, which I shall mention later on. Early in the '70's the practice of using ice to cool the milk became general wherever it was practicable to obtain and store the ice. The method was to use the old water basins constructed some eight or ten years before, when the water system came into repute. The conditions were favorable to the use of ice; that is, the aim had been all along in the construction of milk cellars to reduce the temperature to the minimum in warm weather, so that the ice lasted longer than it would have done had the milk room been entirely above ground and no provision made for the use of cold water. On the smaller farms the ice basin frequently consisted of large wooden tubs of the proper depth, into which the cans were sunk (see Fig. 4). I found this method in use yet on many of the smaller places where the milk was not sold soon after milking to some neighboring creamery, or where the separator had not been introduced. Prof. Fjord, who has done so much for the development of the Danish dairy, experimented largely in order to ascertain the relative values of setting milk in cold water and in ice water. By cold water we are to understand such water as can be obtained fom springs and wells, usually in that country ranging in 
temperature between $50^{\circ}$ and $60^{\circ}$ Fahrenheit. He proved conclusively that the milk ought to be cooled as soon as possible to a temperature as near freezing as could be obtained, by the use of ice water in sum. mer; and he proved further that the depth of the milk in the can had but little influence on the rapidity of the rising of the cream, provided it was kept at a low temperature.

The following table, by Prof. Fjord, shows the advantage of rapid cooling to a low temperature compared with the use of cold, still water:

\begin{tabular}{|c|c|c|c|c|c|c|}
\hline & \multicolumn{3}{|c|}{$\begin{array}{l}\text { Cream from milkskimmed after } \\
\text { standing ten hours. }\end{array}$} & \multicolumn{3}{|c|}{$\begin{array}{l}\text { Cream from milk skimmed after } \\
\text { standing thirty-four hours. }\end{array}$} \\
\hline & \multirow{2}{*}{$\begin{array}{c}\text { In ice } \\
\text { cooled at } \\
35^{\circ} \text { to } 36^{\circ} \mathrm{F} .\end{array}$} & \multicolumn{2}{|c|}{ In still water at- } & \multirow{2}{*}{$\begin{array}{c}\text { In ice } \\
\text { cooled at } \\
35^{\circ} \text { io } 36^{\circ} \mathrm{F}\end{array}$} & \multicolumn{2}{|c|}{ In still water at- } \\
\hline & & $39^{\circ}$ to $40^{\circ} \mathrm{F}$ & $50^{\circ} \mathrm{F}$ & & $39 \circ$ to 40 $\mathrm{F}$. & $50^{\circ} \mathrm{F}$. \\
\hline
\end{tabular}

From this it will be seen that a great loss is sustained by setting the milk even at so low a temperature as $500^{\circ}$, compared with a temperature but little above freezing. The next table shows the loss in per cent of butter, which is sustained by skimming the milk after standing ten hours, compared with thirty-four hours' standing, both by the use of ice and the use of water at different temperatures.

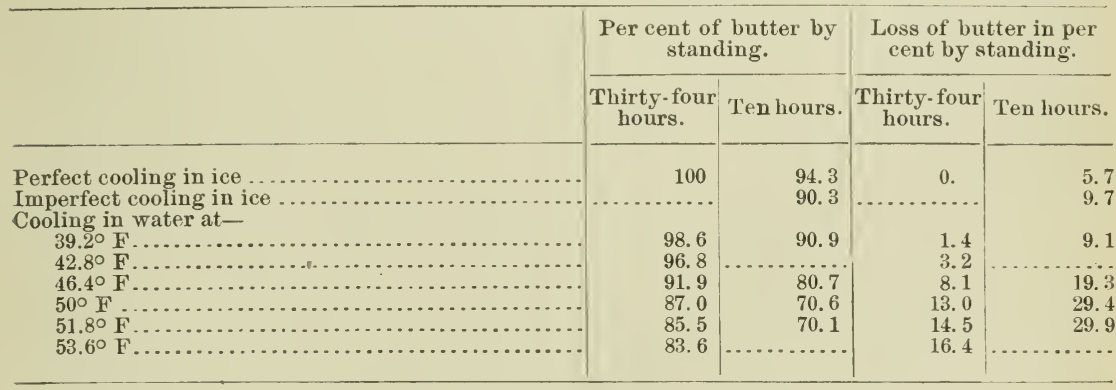

These results were obtained by Prof. Fjord in his experiments on the subject. The table is copied from "Mælkeribruget i Danmark," by B. Bóggild.

Prof. Fjord also constructed tables showing the amount of ice required to cool 100 pounds of milk under different conditions, during the several months of the year. The experiment, however, was based upon the average temperature of each month in Denmark. As the conditions in America vary greatly in different places, all of which will have a higher average temperature in summer than there is in Denmark, these tables will not have much practical value for us. By the use of good ice houses and by careful, economical use of the ice, the safest guide to follow will be to put up as much ice as the weight of the milk which is to be handled throughout the year, pound for pound. 


\section{V.-THE SEPARATOR SYS'TEM.}

As already stated, the separator is now in general nse. It will not bo neressary to detail the method of operation followerl, inasmuch as it is known to every creamery man in this comntry. I will note only a point or two which are not generally practiced here. I have already called attention to the Burmeister \& Waine separator, by means of which the cream and milk can be transported through pipes to their respective reservoirs directly from the separator (see Fig. 9). In the coïperative creameries it is now a common practice to sterilize the skim milk directly after it leaves the separator, and wherever this make of the separator is used the skim milk is carried to the sterilizer, often at an elevation of 6 or more feet above the separator, without the intervention of pump or any other force than that given to it by the drum in the separator. The cream is in like manner carrierl to the cream barrel, if so desired. This is a saving of labor which is not possible with any other separator. The one objection which I found urged against this method of transmitting milk and cream was that it frothed too much. The skim milk is sterilized at a temperature very near the boiling point when it is not intended to use it for cheese. The object is twofoldfirst, to increase its kecping qualities so that it can be carried back to the farms without souring, and, second, it is sterilizer in order to kill possible germs of contagion, such as tuberculosis. The milk coming in from so many places there is always a strong presumption that some of the cows may be tuberculous, although they may not show it by examination; and the milk being mixed, other dairy farmers with sound cattle object to using the milk in thrir households and for their calves and pigs muless it is thus sterilized.

\section{TREATMENT OF THE CREAM.}

Sweet-cream butter has so far not eome into demand in Demmark either for home consumption or for export, and there is lout little, if any, of that article made. The treatment of the subject is therefore confined to the methods practiced for the production of butter from somr cream. There are two reasons for somring the crean aside from the one that sweet-cream butter finds no sale: First, it is found that sour crean yields more butter than sweet cream, and, second, soming or fermenting the cream is, in accordance with the present standard, thought to increase the aroma and good taste of the hutter. Experiments have proverl that the difference in yield of butter by the two systems is about 4 per cent, or very nearly equal to the difference of one pound of milk for each pound of hutter.

\section{S'TERILIZATION OF THE CREAM.}

The first essential condition to success in butter-making is to have a good quality of cream. It must not be affected by bad orlors, nor by bitter, salt, or other offensive taste. These faults may be due to the 
cows, in which case their mili should be withdrawn from the ereamery. Investigations have proved. however, that in the vast majority of instances faulty cream is due to bacteria, many forms of which, both useful and injurious, have been discovered. To counteract their influence it has been the practice in many Danish dairies, during the last three or four year's, either to sterilize the cream immediately after it is separated or the milk before separation takes place. How this could be done was first pointed out by, the great Frenchman, Pasteur. He showed that by heating the liquid containing these bacteria up to $75 \circ$ or $80 \circ \mathrm{C}$., the greater part of them would be killed, and after him the method is frequently called pasteurization. Prof. Fjord was the inventor of the sterilization apparatus, which has already been described and which is in common use; but it is not absolutely necessary to use this machine. The same result can be reached by the use of boiling water, whether this water is heated by steam or in a kettle over the fire. The latter method I saw used in several places with success. In that case the cream is put into tall cylindrical milk cans, which have first been thoroughly cleaned by washing in mild lye, or by the addition of soda to the wash water, or with water containing lime in solution, and thereafter cleaned by a jet of steam, or, if steam cannot be had, scalding water.

The cream cans are sunk to the rim in the hot water, and this is kept near the boiling point either by the constant addition of boiling water or, what is more convenient, by heating it with a jet of steam through a pipe. The cream in the cans is constantly stirred, so that it may warm equally through the mass, care being taken that the paddle used to stir with has been thoroughly cleaned. It is, in most cases, made of galvanized iron. The dairymaid holds in one hand her thermometer immersed in the cream, while with the other she keeps up the stirring, watehing the rise of the temperature all the while, and when it reaches $80^{\circ}$ C. $\left(176^{\circ} \mathrm{F}.\right)$, which temperature should not be exceeded, she lifts the can out and at once immerses it in ice water; where, by continual stirring again, it is cooler as rapidly as possible till it reaches about $35^{\circ}$ or $36^{\circ} \mathrm{F}$. It can then remain standing in the ice until such time as it is found convenient to begin the fermentation. The sterilizing apparatus differs from this as to result only in that it does the work more rapidly, with greater certainty, and with less labor on behalf of the dairy people. As alrearly described, the cream runs into the bottom of the apparatus from a reservoir, which may be filled directly from the separator, and after being heated by the steam while it is stirred by the agitator in the machine it ruus out again at the top in a continuous stream, and the cream is then either cooled by immediately running over one of the coolers described or it may be placed in cans and these be set in ice water. The object of it all is to free the cream from bacteria of all kinds, and then, by the addition of a previously prepared ferment which is known to be pure, to ferment the cream to such a degree that it shall be capable of producing the best quality of butter. 
(1) Natural souring.-Treating of these in the progressive order in which they have been brought into use, I shall first mention the souring of the cream without attempting to influence the result by artificial means. This is the old-fashioned way. It is still used now and then on small farms, theowners of which have not kept pace with the times. Sterilizing is unknown to this method. When skimmed from the milk cream is atonce poured into the cream barrel, which is kept in some corner of the milk room, or, in the colder seasons of the year, may be alongside the stove in the kitchen, or in any warm room where it will be liable to sour quickly. This method, as stated, is now used only in small places; but I mention it here because with careful manipulation the results may be entirely satisfactory, provided that the milk room is clean and sweet, that the milk is from sound cows, that the stable is kept clean and airy, and that there are no contaminating influences outside, such as from stagnant water or manure heaps and the like, which can gain access to the dairy room through the air. This might be styled self-souring, or natural souring. I would not have it understood that all Danish dairies sterilize their cream or milk, for this is far from being the case; they do so only when they find it necessary in order to produce butter of first quality. I visited several places where the conditions were so favorable, the premises kept so clean, that it was not necessary to sterilize the cream at all, but they, nevertheless, fermented the cream by one of the following methods.

(2) By the use of buttermilk.-In this case a portion of the buttermilk is taken from the churn immediately after the butter is removed and added to the cream barrel. The quantity used will differ much according to the quantity of cream, the sourness of the buttermilk, and the season of the year, and also with the length of time that the buttermaker clesires to give to the fermentation. It is a matter which each butter-maker decides upon for himself according to his experience. But I can say that it does not usually exceed 10 per cent of the cream nor less than 5 per cent. The objections to the use of buttermilk are, that if there is any defect in the churning from which it is taken it will, of course, transmit the same defect to the cream, and the undesirable qualities are thus perpetuated. It is also somewhat more difficult to keep the fermentation under complete control, in that the buttermilk is liable to vary more in acidity than any other form of ferment. If it is found by experience that the buttermilk does not produce the right flavor in the butter, from whatever cause, it is a common practice to resort to a neighboring dairy for the buttermilk; and experience has proved that sometimes this expedient will entirely overcome the injurious influences which affect the eream and butter, and that when this is done once or twice the buttermilk from the home dairy can again be used for some time with entire satisfaction. Before the ferment, of whatever kind, is adderl the cream should be raised to a temperature 
which varies between $70^{\circ}$ and $95^{\circ} \mathrm{F}$. This is done either by ruming it through a forewarmer-the apparatus described as being used to heat the milk before it runs into the separator (see Fig. 9)-or by simply immersing the cans of cream in warm water and gently stirring until it reaches the desired temperature. Still another method of warming it is to insert a cylindrical can of small diameter. filled with boiling water into the cream can or cream barrel and allowing this to stand until the cream reaches the desired temperature, it being gently stirred in the meantime. In any case the cream should be warmed to the degree mentioned before the buttermilk or other ferment is added. In the cool season of the year precautions are taken to prevent the falling of the temperature too low in the cream barrel until the fermentation is complete. These precantions consist either in keeping the room warm by a stove or steam pipe or in having the cream barrel stand in a box or large barrel prepared for the purpose, so that it can be packed all around with hay, and a similar covering, either a quilt or hay mattress, is put over the lid of the cream barrel. The proper degree of fermentation is usually reached in the course of eighteen to twenty hours. The butter-maker starts the fermentation at such a time that the cream will be ready to churn at the most convenient hour the following day. I found, in most cases, that the fermentation was started at about noon; the cream would then be ready to churn by 6 o'clock the next morning. During this interval it was gently stirred two or three times and the progress of the fermentation watched by the changes in appearance, in taste, and in smell.

(3) By the use of sour cream.-Dour cream is occasionally used as a starter. A portion of the cream churned to-day is thus set aside in the morning, and at noon added to the cream that is to be churned to-morrow. Practical butter-makers admit, however, that sour cream is even less desirable than buttermilk, for the reason that, owing to the presence of the fat and off taste which it might have, it is not so readily detected as in the use of buttermilk, and it, in like manner, perpetuates whatever faults there may be from one churning to the next. In all other respects the preparation of the cream for churning is the same as already described.

(4) By the use of skim milk.-In the use of both buttermilk and sour cream as a ferment there is a continual perpetuation of the same fermenting elements from week to week, and month to month, as long as continued. It is found that, in many cases, injurious bacteria creep in, and, after a time, the ferment degenerates and fails to produce the good quality of butter that it did at first. This is, in a measure, obviated by the production of fresh ferment every week or two, as the case may require. Such ferment is usually made from half-skimmed milk, that is, milk which has stood from ten to twelve hours and then been skimmed. It is this milk which, by being heated to about $100^{\circ} \mathrm{F}$, and then allowed to stand at a temperature of about $70^{\circ} \mathrm{F}$. for from 
twenty-four to forty hours, according to the season, will (levelop) the ferment, which can be used for souring the cream The inilk used for this purpose should be from a nearly fresh cow. Milk from cows about to go dry does not answer the purpose. Good judgment is reouired on the part of the butter-maker to obtain the right degree of acidity in this ferment. He examines it frequently, smells it, tastes it, and notices its consistency. It should have a certain degree of thickness, and show the formation of small granules, and should have a clear, sharp taste. If it has a bad smell or taste, or if it does not thicken properly, it should be rejected. As soon as it reaches the proper degree of consistency and sourness the fermentation is stopped, by immersing the can containing it into cold water, where it can remain for a few hours, if necessary, before it is added to the cream. The cream is prepared to receive this ferment by being warmed to a degree varying with the season, from $75^{\circ}$ to $84^{\circ} \mathrm{F}$., or even more. The top of the ferment in the can should then be skimmed off and discarded, as it may be contaminated with bacteria from the air, and from 3 to 5 per cent of the volume of the cream is added to the cream barrel from the remainder. The rream barrel is now covered and kept at a temperature of about $75^{\circ} \mathrm{F}$. until it is ready to churn on the following day. The length of time that it stands varies with the season and the temperature in the cream room, from eighteen to twenty-four hours. This, however, is not the invariable method followed.

Sometimes cream is adder to the skim milk before the fermentation begins; at other times the milk is not skimmed at all. The result, however, is the same. A new ferment is obtained, which, if the proper care has been exerciserl in its production, is capable of souring the cream in such a manner that it will produce a first quality of butter. However, this expedient does not always work satisfactorily. If the dairy building or the surroundings are impregnated with injurious bacteria these are sure to infest this new ferment also, and it fails in a short time.

(5) By the use of pure cultures.-When all things else fail to produce the desired quality of butter, resort is harl to the use of an artificial ferment, the so-called "pure cultures."

The Danish dairy experts have been at work for several years on the isolation and culture of those bacteria which have been found to be the active agents in the fermentation of the cream and, some two or three years ago, success was so far attainel that these artificial cultures were offered on the market for use in the creameries; and at the time of my visit last winter there were three laboratories in which these ferments were cultivated and sold. The methorls followerl in the production of these cultures are serrets belonging to the respective establishments, which will not be divmlger. However, it would appear that any good bacteriologist who had studied the question carefully could reach the same result. The bacteria used were obtained from the finest qualities of butter. They have been isolated and experiments havo 
revealed the mutrients needed for their growth and the temperatures at which they can most readily be propagaterl. I do not think that any of them claim to know all there is to be known on the subject. They steadily discover that improvements ean be made by slight alterations in the methods and that new bacteria heretofore unknown are added to the list. It is certain that the different species of bacteria produce notably different results in the taste and aroma of the cream and butter. Mr. L. A. Quist, of Skanderborg, uses only two species, very distinct in appearance and in the quality they impart to the butter. They are cultivated separately and mixed just before they are sent out to the customers. Blauenfeldt \& Trede, on the other hand, use several kinds in their ferment, which may or may not include those used by Mr. Quist. The results of the use of these pure cultures have been so eminently satisfactory in practice that I found them in very common use. A new starter of these cultures is not needed every day or even every week; and as they are sold reasonably cheap, the expense connected with their use is but slight. When used according to directions sent with them they insure the production of a first quality of butter, which is of greater consideration than the expense their purchase involves. The three laboratories I have mentioned prescribe different methods of procedure in their use, which proves that there is no hard and fast rule that must be followed in order to obtain the desired results. That is, there is a possibility of the extension of this science far beyond our present knowledge of the subject. These artificial cultures are used more particularly in dairies which seem to be infected with injurious bacteria and, to attain the best results, the cream should be sterilized before the ferment is added. The pure culture is added to a small portion of sterilized milk or cream, and then set aside at a given temperature until it has attained the proper growth. It is then further propagated in a still larger quantity of milk or cream and, when a sufficient quantity has been obtained, it is added to the cream in the cream barrel, where it accomplishes the desired fermentation in from eighteen to twenty hours. At the time of my visit none of the laboratories had succeeded in devising means to perpetuate the ferment outside the laboratory for any leugth of time. It was, therefore, a difficult matter to obtain pure cultures for transmission to America. The preparation required for their transportation during two or three days would not answer the purpose when the journey was extended to as many weeks. I am convinced, however, that it will not be long before this difficulty will be overeome.

There are a few points in the treatment of the cream concerning which no definite directions ean be given. One of these is the per cent of ferment, whatever kind is used, which should be added to produce the best results. This depends upon so many things that no definite rule can be given. It depends upon the sourness of the ferment when it is added, npon the length of time it is desired to have the 
cream stand before it is churned, and upon the temperature which it is practicable to maintain; and in the adjustment of these to one another the butter-maker must follow his own judgment and experience. It is a well-known fact that the fermentation proceeds more rapidly in warm weather than in cold weather, and in summer the process, therefore, usually takes a shorter time than it does in winter. It is essential that the cream should stand in a room with fresh air. In damp and musty air the cream will be sure to lack the aroma and pleasant taste which are so highly prized. It is a maxim in Danish dairying that to obtain the finest quality of butter, which shall keep well, the fermentation must be strong, and that it should not be stopped until the cream has become thick and shows a peculiar granular appearance and at the same time develops the pleasant aroma belonging to good butter. When this point is reached the cream should be churned.

\section{CHURNING.}

I saw but one pattern of the churn, and this has already been described. Years ago, before the dairy had reached its present perfection of methods, the old-fashioned dash churn was common in the small dairies. It then required the united aid of the entire household to keep it going at the requisite speed until butter "came." On the large farms the ehurn with the revolving dasher has always been the favorite, and is the one still in use. The churn is scalded inside with boiling water and immediately afterwards rinsed in fresh cold water before the cream is poured in. The cream should be of the tem. perature which experience has proved to be the best for the season of the year. This temperature may vary from $50 \circ$ to $70 \circ \mathrm{F}$. Thus, milk from cows that are about to go dry requires to be clumed at a higher temperature than that from fresh eows. The usual temperature at which to churn is between $55^{\circ}$ and $600 \mathrm{~F}$. The quantity of cream should be known in order that the right quantity of butter color can be added. This quantity differs with the demands of the markets, the quality of the color used, and the feed given the eows; so that no rule whatever ean be laid down, and there is no uniformity in the Danish practice in this particular. The English market, where practically all the surplus butter is sent, demands that it shall be of a rather light straw color, and so well do the producers meet this demand, under the varying conditions, that it is only the expert eye that can detert differences in the color of the butter from different parts of the country when collected in the warehouses of the wholesale dealers. I saw this practically illustrated in London.

When all conditions are right the length of time taken in churning varies from thirty to forty minutes, but the time, too, is dependent upon several conditions. It depends upon the speed at which the dasher is revolved, upon the construction of the dasher, and upon the condition 
of the cream, both as to consistency and temperature. The kind of feed given the eattle also has an influence upon it. The higher the temperature, the shorter the time required; but all butter-maker's with whom I came in contact were agreed that it is better to chum at a too low than at a too high temperature, as the grain of the butter will, in the latter case, be spoiled. 'There is, however, danger of getting the temperature too low, especially towards fall when the cows begin to go dry and the weather gets cooler. It is then often the case that the cream will froth and the butter refuse to "come." This is obviated by raising the temperature above the normal. In obstinate cases of this kind it is a common practice to stop the churn and let the cream stand until the following day when it is warmed some $\tilde{5}^{\circ}$ or $6^{\circ}$ higher than usual and again churned, when the difficulty is generally overcome. The temperature of the cream is watched from time to time as the churning proceeds, and for this purpose there is a hole in the lid through which a thermometer can be dipped into the churn. Usually the agitation of the cream causes the temperature to rise some $3 \circ$ to $5^{\circ}$ in the course of thirty minutes. This is as it ought to be, but if the rise is much greater than that it is an indication that the cream was not at the proper temperature when the churn was started. The formation of the first grarules of butter is carefully watched for, and for this purpose there is a short piece of wood or stick with a deep furrow on one side put through a hole in the lid, in which it fits closely. This is removed from time to time to see the size of the granules as they collect in the groove. It is of the utmost importance that the churn be stopped at the right time. If continued longer than necessary the grain of the butter is injured. It is the usual practice, when the granules are about as large as small peas, to stop the churn and to add a small quantity of cold water which is thrown upon the lid and down the sides of the churn, after which the dasher is again started at a slow motion until the butter is sufficiently gathered.

\section{TREATMENT OF THE BUTTER}

The first thing the butter-maker does after the churn is stopped is to wash down the sides of the churn with fresh cold water. In some places boiled water which has again been reduced to normal temperature is used for this purpose. This is to avoid contaminating the butter with bacteria which the water may contain. Perfectly pure well or spring water is, however, used without first being boiled. The churn is invariably stopped while the butter is still in small granules or pellets. The butter is next removed from the churn, which is done as follows: The churn is tipped, as shown in Fig. 15, and with a sieve the butter is gathered as it floats in the buttermilk and transferred to a tub by the side, allowing the buttermilk to drain through the sieve as much as it will. Usually a fine hair sieve is used. Finally the 
buttermilk is run through a strainer in order to collect the last floating particles of butter. In some places I noticed that it was the practice to dip each sieve full of butter into a tub of clean, cool water, and let this drain through the sieve, thereby washing out a portion of the buttermilk. This, however, is not a common practice. Formerly the butter was sometimes washed in the churn before it was removed, the buttermilk first having been clrained off', but this, too, is now obsolete. The Danes believe that butter so washed absorbs too much water, and is less liable to keep.

The next operation consists in pressing the buttermilk out of the butter. In former times this was always done in the butter trough shown in Fig. 16, and by the hands only, or simply by the use of a large wooden paddle made for the purpose. Now, in comparatively few dairies is the butter touched with the hands, and in that case a lump weighing perhaps 8 or 10 pounds is taken up with both hands and put against the sides of the trough, pressed gently with the palms of the hands five or six times, the ends of the flattened mass being turned in after each pressure. In most places the butter-worker is used and is permitted to run under the valve three or four times. When the hands are used, the greatest care is taken that they are clean and cool. They are washed frequently and thoroughly and they are always dipped in cold water before the butter is touched. This is essential, not only to cool the hands, but to prevent the butter from sticking to the fingers, and the same precaution is taken with all the utensils with which the butter comes in contact. Everything is scrubbed first in boiling water to sterilize the implements, and next in clean cool water.

As soon as the buttermilk has been remover the butter is weighed in order to calculate the amount of salt required, and the salt is worked in at this time, always on the butter-worker. Sometimes it is put in at one working, and in other places it is preferred to add it in two workings. The amount of salt used I found was not uniform. It was adapted to the taste of the market where it was expected to be sold, but it varies between 4 and 5 per cent of the weight of the butter. The salt is not weighed, but measured in a large glass with a scale graduated to grams on the side, 5 grams being equal to 1 per cent. Usually the butter is sold, as I shall explain later, to butter dealers, who handle it either on commission or they buy it outright on their own acconnt. These dealers are supposed to know the wants of the market, and it is customary to accept their instructions in regard to the amount of salt and color to be added to the butter. The salt is worked into the butter with the least possible amount of handling, and it is then laid aside for some time before the next working takes place. In summer it is put in the butter-coolers already described (sce Fig. 17), which are a sort of ice box. In winter it may simply be latid in large rolls in the butter trough or on a table provided for the purpose. It 
lies here for at least two hours in the cold season, and when the weather is warm it may lie for eight or ten hours, or even until in the cool of the following morning, before it receives the final working. The object is, not only to cool the butter, and thus allow it to become firmer, but also to allow the salt to dissolve and to penetrate the whole mass. When it has attained the proper degree of firmmess it is again put under the butter-worker, and the last buttermilk and a considerable portion of the brine formed from the salt is worked out. How inuch working it can stand differs much in individual cases. Care is taken, however, that it is not the least bit overworked so as to become greasy and sticky. This working may be repeated a couple of times, or it may be packed for shipment at once; practice is not uniform on this point. It is common, however, to give it one more working an hour or two later. The main point is not to work it until it has become lecidedly firm, and then work it only to the extent it can bear without injuring the grain, and yet remove as much as possible of the brine which has formed from the dissolving salt. It is worthy of notice that although 4 per cent of salt may be added, with proper working about half of this is removed in the form of brine.

\section{PACKING FOR MARKET.}

The Danish butter is invariably packed in barrels. These may vary in size according to the demands of the market, but they are always of the same pattern. One of these barrels and the wooden mallet used to pack the butter firmly in it are represented in the accompanying illustration (Fig. 19). The most usual size is one which will contain an English hundredweight (112 pounds), but this size is not uniform. These barrels are usually manufactured on the farm where they are to be used, the "feed-master" combining with this important office the trade of a cooper. While the cows are eating he makes the butter barrels. These barrels are usually made of beech wood. The European beech wood is white, fine grained and hard,

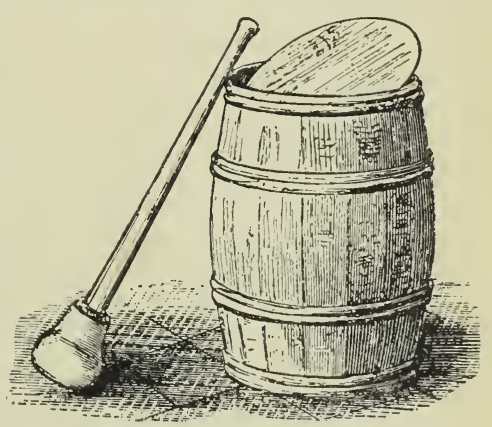

FIG. 19.-Barrel in which butter is packed for market. and of course it is thoroughly seasoned. The staves are held together by wooden hoops and the barrel is marle perfectly smooth inside. The day before it is to be filled with butter it is first thoroughly scrubbed in scalding water and next filled with clean cold water, which remains standing in it until a short time before it is wanted. It is next rubbed with salt all over the inside, letting as much as possible adhere to the sides. The bottom and sides are next lined with paraffin paper to prevent the butter coming into contact with the wood. Then the butter is packed in lumps of 10 
and 12 pounds, and by the use of the mallet it is pressed firmly down in order to prevent any openings in it. This process is continued until the barrel is full. It is filled slightly higher than will permit the putting on of the lid, and with a sharp, flat spoon the excess is cut off to just the point required to admit the lid. Now a sheet of paraffin paper is put over it and this is covered with a thin layer of fine salt. The lid is then put on, the hoops tightened, and the barrel is ready for market. During the time it remains in the creamery it is placed in a cool storeroom, usually adjacent to the ice house, until enough has been collected for shipment to the dealer. It is customary in most dairies to ship their export butter to the agent about once a week.

\section{USE OF SKIM MILK AND BUTTERMILK.}

Much of the skim milk is used for cheese. Considerable is also used in the household, and what is not used for these purposes is fed to calves and pigs. It is customary in feeding calves to gradually sub. stitute skim milk for whole milk, after the first two weeks. As the older cows drop out of the herd, or cease to be profitable, their places are taken by young stock raised on the place. It is customary, also, to keep a number of swine, which are fed, for the most part, on the offal from the dairy. In the coöperative dairies the farmers who furnish the milk take back the skim milk, this being, in most cases, a part of the contract, and in some cases the buttermilk is in like manner returned to the producer at a moderate valuation. These products are then used in the same manner as on the larger farms. Near the towns there is always a good market for skim milk among the poor people, who can buy it at a low rate. It will thus be seen that nothing is wasted, everything is utilized, and it is to be noted that the skim milk is returned to the producer while it is sweet. In no case did I find the stinking skim-milk reservoir which seems to be a fixture in American creameries, and from which our farmers help themselves. The skim milk is also fed to colts with good results.

\section{DESCRIPTION OF PLACES VISITED.}

In what follows I shall attempt to give some idea of the methods followed on Danish dairy farms, both in the dairy and in the production of crops. Of course many details lave been overlooked, but it is hoped that enough has been gathered to give the reader a fairly correct notion of the system manifest in the work, and the care given to details. I was compellerl, for lack of time, to confine myself to the salient points in farming methods. Had more time been at my disposal it would have been possible to have gone more into details, but I could usually spend only one day at a place, and this, too, at a season when there was practically no farm work going on. 
I took early occasion to visit the city milk-supply station of Copenhagen, which I shall briefly describe. I am aware that it has already been partially described in our agricultural press, but it is an institution of such extraordinary interest, owing to the methods there adopted in handling milk, that it will bear further mention, and should serve as the worthy example for our many large cities to follow. It is in the hands and under the exclusive control of a private organization. Neither the Government nor city has anything whatever to do with it. It should also be explained that it does not by any means supply all the milk consumed in Copenhagen. Just what part it supplies of the total amount consumed in the city I am unable to state, but it is considerably less than one-fourth. The Milk-supply Company of Copenhagen is simply an organization which has taken upon itself to supply its patrons with absolutely pure and wholesome milk at a slightly advanced price above what milk can be bought for from the numerous other sources of supply. The company began business some fifteen years ago on a small scale, but for the month of December, 1879 , the daily sales averaged 9,733 Danish pounds (10,728 pounds avoirdupois), and for December, 1892, 36,194 Danish pounds (39,896 pounds avoirdupois), and this at the season when milk is scarcest. The merit of the system consists, first, in the strict rules which have been laid down concerning the quality of the milk; second, in the painstaking cleanliness which obtains in the handling of the milk; and, third, in the unflinching enforcement of the rules mentioned. Each dairy farmer of whom the company buys the milk must agree to conform to the following requirements in every particular:

(1) The feerl must be such that it does not affect the taste or character of the milk injuriously. The use of distillery slop ancl like substances for feed is absolutely prohibited, and the use of all feed that has been injured or is not well preserved. The use of turnips, kohlrabi, rutabagas, and the leaves of all kinds of root crops is prohibited. Carrots and mangels may be used to the extent of half a bushel per day for each cow, but only when the grain feed given amounts to 7 pounds per day. Cows which supply milk for the use of children must not be fed mangels and carrots beyond the extent of 1 peck per day. Oil cake (rape-seed cakes) may be fed to the extent of but $1 \frac{1}{2}$ pounds per day, and this only in connection with at least 5 pounds of grain feed. Cows supplying milk for the use of children must not be fed oil cake of any kind. For other cows the grain mixture used shall receive the company's approval before delivery of milk can begin.

(2) In the summer time the cows must not be fed in the barn under any conditions. They must be pastured on clover and grass. Vetches must not be used. When necessary, arrangements may be made with the company for the use of grain or green grain crops during the summer.

(3) The cows must be clipped on the udder, tail, and hind quarters in the fall before they are put in the barn.

(4) The time of calving of cows in the herd must be distributed as evenly as possible through the year, so that the amount of milk delivered, especially during September and October, shall not be less than two-thirds of the greatest amount delivered in any month. 
(5) Fresl milk up to twelve days after ealving must not be delivered, nor will the company receive milk from cows which give less than 6 pounds per day.

(6) The utmost eleanliness must be observed in milking, and the milk must be strained throngh a metal strainer covered with a clean woolen cloth.

(7) There must be at the disposal of the dairy at least 30 pounds of ice for every 100 pounds of milk produced on the farm.

(8) Every dairy must be supplied with a Lawrence milk-cooler. This may be rented from the company if desired.

(9) As soon as it is drawn from the cow, the milk must be cooled by the use of ice water on the milk cooler, and this at all seasons of the year. This cooling should reduce the temperature of the milk to at least $4^{\circ}$ Réaumur $\left(41^{\circ} \mathrm{F}\right.$.) before it is shipped.

(10). The milk must be delivered at the railway station once or twice daily, as the company may desire, either as sweet milk or as half-skimmed milk and cream. It must not be sent from the dairy farm sooner than wecessary to make the train, and in summer the delivery wagon must be covered so as to shade the cans.

(11) The company will supply the cans used for transportation, and they will be cleaned before they are shipped to the dairy farm.

(12) The cans must be rinsed in cold water immediately on their arrival at the dairy. They must be kept in an airy place, protected from all dirt, with the lids removed and opening downward, but so that the air has free access to the interior, until they are used.

(13) The can must under no circumstances be used for anything else than the transportation of milk.

(14) The dairy farmer must agree to answer all questions which the company may put to him concerning the milk.

(15) The dairy farmer must permit one of the company's veterinarians to examine his cattle whenever he chooses, and must carry ont the directions which the latter may give him. He must also agree to furnish transportation for the veterinarian to and from the railroad station.

(16) Cows which the veterinarian finds have tubcrculosis must be removed from the herd at once and disposed of as soon as possible.

(17) Cows which are taken with any suspicious disease must be removed from the herd at once and the company informed of the fact, and, if necessary, the delivery of milk may be stopped until the veterinarian has had opportunity to examine the case. But in such cases the company will pay for the milk at the same rate as though it were delivered.

(18) If any contagions disease occurs among the persons who live on the farm, or at the homes of the laborers who work on the farm, it shall be the duty of the dairyman to inform the company of the fact at once. The milk will, in such cases, be paid for at the usual rate.

(19) This contract may be terminated either by the company or the dairyman on the first day of any year, but with at least six months' notice.

(20) Should the milk be found to be of such an inferior quality as to be unfit for sale, the company rescrves the right to stop its delivery without remuneration.

(21) If the sale of milk in Copenhagen should be stopped by reason of an epidemic or other non-preventable cause, the delivery must be stopped for a shorter or longer period without remuneration.

This very strict code is observed to the letter. At present the company receives milk from 42 dairy farms, representing in round numbers 4,600 milch cows. To watch the health of these cows the company employs three skilled veterinarians, who spend all their timo in traveling from farm to farm in order to examine periodically each individual in the herds, and also to see that the rules as regards feeding, 
etc., are observed. When an animal is found to be suffering from any sort of disease, it is withdrawn at once. And should a disease occur suddenly the dairyman is encouraged in withdrawing of his own accord the animals so affected, and is paid the price of the milk which they give until one of the company's veterinarians can pass judgment on the case.

In addition to this the company employs a staff of trained clairymaids who travel from farm to farm, call unexpectedly, examine the surrounding conditions with special reference to cleanliness and care in milking, watch the feeding, the cooling of the milk, etc., and then promptly report the facts they obtain to the company on blanks furnished them for the purpose. On some of the larger farms such dairymaids are permanently located, the company paying the dairymen for their board. At present there are four such dairywomen located on as many farms. But the inspection does not end here. At the receiving station in Copenhagen a sample of the milk from each farm is taken inmediately on its arrival and subjected to the Babcock test, a large machine of this pattern having been procured for that purpose a short time ago, and once or twice a month similar samples are subjected to chemical analysis. Moreover, when the sample is taken an experienced dairywoman stands by, who tastes and smells of the milk from each farm, and who quickly notes whether it falls below the high standard that is required.

I spent a night and the greater part of two days at the company's receiving and distributing station in order to watch its operations. Including the drivers of delivery wagons and the boys who assist them by carrying the milk from the wagon to the house, the number of employees is about 250 . The milk is shipped to the city in the evening. The trains begin to arrive about 9 p. m., and continue until after midnight. The milk is at once hauled to this receiving station or dairy, where it is weighed, sampled, tasted, and the cans deposited in large vats of ice water, where they remain till 4 a. m., when the delivery wagons begin to make ready.

The cans are of tin, made very massive, and hold about 100 pounds each. Two forms are used, one round, the other square. The round cans are used for the unskimmed milk and the square cans for the halfskimmed milk. Otherwise they are shaped like a jug, with contracted neck, into which a lid fits very closely. In the interior of the cans for unskimmed milk is an arrangement intended to equalize the distribution of the cream with the milk. It will be seen that when a can of sweet milk stands on a delivery wagon several hours the upper stratum of milk must be richer in cream than the lower stratum, and that if the milk is drawn from the bottom the first customer will practically get skim-milk and the last cream. To prevent such injustice, the tube, or pipe, in which the discharge valve is placed is continned clear to the top of the can on the inside, and a row of small holes the whole length 
of this tube admits the milk. Now, when the valve is opened, the milk is drawn neither from the bottom nor top of the can, exclusively, but through these sinall holes in the tube-from the whole side of the can from bottom to top, or as high as the milk may reach, equally. The weight of each can is stamped upon a brass plate which is soldered to the top of the can. This greatly facilitates weighing. Before leaving the farm each can is sealed with a cord and a piece of lead stamped with a punch, in the same manner as our railroad freight cars are sealed. This precludes all possibility of tampering with the contents in transit, and a label bearing the name of the farm in large print is also pasted on each can.

The company supplies its customers with three grades of milk, namely, whole milk, half skim milk, and milk for children, and with two grades of cream, designated respectively as first and second grade. The skim milk received, and for which there is a large demand for use in cooking, is in most cases the morning's milk which has stood ten to twelve hours before shipment. The cream that may have risen is then skimmed off and shipped in separate cans. The children's milk is intended as food for infants. For this supply special cows are picked by the company's veterinarians, and these are fed somewhat differently, or at least with greater care than the others, and their milk is kept separate. This milk is filtered and bottled and the bottles sealed immediately on arrival, and not allowed to stand in the eans till morning, as is the other milk. The ordinary whole and skim milk is also filtered before it goes to the customer, but this is done at $4 \mathrm{a} . \mathrm{m}$., just before it is loaded into the delivery wagons.

The filtering machines are of special interest. There are several of them, and they vary somewhat in size, but otherwise the construction is the same. Imagine a large bowl of enameled iron with a capacity of about 40 gallons, and this raised on supports about 5 feet from the floor. There is a hole in the bottom through which the milk enters, and near the rim are two discharge pipes, one on each side of the bowl. Now, the filtering material consists of three layers of gravel and six thicknesses of fine muslin stretched over a ring that fits closely inside the bowl and is placed above the upper layer of gravel. The gravel is of three grades of fineness. The lower is about the size of buekshot, the middle layer finer still, and the upper layer as fine as small pinheads. Each layer rests mpon a tin plate, perforated with many fine holes, and which fits closely to the sides of the bowl. Each layer is about 2 inches thick, and there is a space of an inch from the top of one layer to the tin plate which supports the next layer above. The milk is ponred into a large reservoir which stands somewhat higher than the filter. A brass pipe leads from the bottom of this reservoir to the bottom of the filter bowl. The pressure thus attained forces the milk through the suceesive layers of gravel and the six thicknesses of eloth, and when it rises to the discharge pipes it runs off throngh these into the 
vessels placed for its reception. Such treatment throughout assures the customer that it is absolutely pure, clean, and wholesome.

Probably no other eity in the world is blessed with so thorough a system of control as regards the quality of the milk. And be it noter that it is not done under compulsion of law, but as a piece of business enterprise in private hands. The constitution of the company forbids a greater dividend than 5 per cent, and the price to the consumer is regulated on this basis. It further prohibits the two principal directors from having any financial interest in the company which might tempt them to work for greater profits. The prices paid to the producer for the milk are as follows: From the 1st of April to the 1st of September 20 öre* per "Kande" ( 2 kilograms $=4,409$ pounds avoirdupois), which is very nearly $\$ 1.25$ per hundred pounds avoirdupois. From September 1 to December 16,26 öre per 2 kilograms, or $\$ 1.61$ per 100 pounds, and from December 16 to April 1, 22 öre per 2 kilograms, or $\$ 1.36$ per 100 pounds. This is for the ordinary sweet milk. Milk for children costs rather more and skim milk correspondingly less. The company retails this milk from its wagons in the street at the following rates: Milk for children at 10 öre per half kilogram, ordinary milk at 8 öre per half kilogram, and skim milk and buttermilk at 4 öre per half kilogram, which is approximately $5 \frac{1}{2}$ cents per quart for children's milk, $4 \frac{1}{2}$ cents per quart for sweet milk, and $2 \frac{1}{4}$ cents per quart for skim-milk and buttermilk. The cream is sold at 27 cents per quart for first quality and $16 \frac{1}{2}$ cents per quart for second quality. The cream which is not sold is made into butter, and in like manner the whole milk which is returned from the wagons is creamed and the skim milk resulting sold to poor people at half price.

Pay of employees-Their method of treating their employees is interesting, to say the least, and I think it contains a lesson not uuworthy the attention of employers of this country. The company has in its employ, altogether, some 250 persons. Most of these are drivers of milk wagons and drivers' assistants, boys who carry the milk from the wagon to the house; but there are also a number of skilled dairy people, clerks, etc. The pay of all is graded to the character of the work; but it is the policy of the company to retain faithful servants as long as possible, and this they do by liberal treatment. The pay of a clerk, for instance, is about $\$ 43$ a month; but on New Year's day he gets a present of about $\$ 54$ besides; and each clerk who can show an increase in his bank account of not less than about $\$ 2.70$ per month gets about $\$ 1.35$ more deposited to his credit in the bank. In the same manner each driver or other working man who can show a monthly increase of about 54 cents in his bank account gets about 54 cents more deposited to his credit in the bank. This money remains at compound inter.

\footnotetext{
* The unit of Danish money is the crown (krone), which contains 100 öre. One dollar is at present worth 3 crowns and 65 öre, and one cent is consequently equal to 3.65 öre.
} 
est for a certain number of years, and by the time it becomes available it amounts to a substantial sum, which may help the owner to a home or enable him to open a business. The regular wages of a driver is. from about 70 to 80 cents a day, but in addition to this he gets a commission on the milk sold of one-half öre for each Danish pound, the commission being the same for both sweet and skim milk, and at the end of the year he gets a commission of 1 ore for each pound of butter he has taken orders for during the year. By these means the company not only gets efficient servants but it retains them, and through its encouragements to work and to save it has a beneficial influence on all its employees. The credit for this model plan of supplying a large city with milk belongs to Grosserer Busk, of Copenhagen, more than to any other one man. He has been the leading spirit of the company fiom its inception and organization to the present day.

\section{LARGE DAIRY FARMS.}

ROSENFELDT FARM.

The first large dairy farm I had opportunity to visit is named Rosenfeldt, which is situated near the city of Vordinborg. It is the property of a Danish nobleman, Kammerherre Oxholm, who lives on the place. The farm contains 1,300 acres. The actual number of cows on the place was 276, which number was, however, to be increased to upwards of 300 by the addition of heifer's which were soon to come in. Of the above number of cows 223 were in milk at the time of my visit, and they produced, on an average, 3,747 pounds of milk per day. This gives but an average of 17 pounds per day per cow, or about 2 gallons. If this seems a small output it should be remembered that it was in midwinter, when many of them were about to go dry, and that the native Danish dlairy cow is but a small animal. The average weight would not much exceed 900 pounds per head. When these things are taken into consideration the milk yield was not small. These cattle were fed as follows: The first feed was given them at 4 o'clock in the morning and consisted of oat straw or barley straw. They got a liberal amomut, and what was left was used for bedding. At 7 a. m. they were fed their grain, which averaged about 7 pounds per head per day, divided into two feeds, and consisted of 3 pounds barley and oats mixed and cruslied, 2 pounds oil cake, usually half rapeseed cake and half palm cake or sumflower cake (of rapeseed cake they never fed more than $1 \frac{1}{2}$ pounds), and 2 pounds bran. At $8: 30$ a. m. they got 20 pounds sugar-beet refise each. The beets are grown on the farm and delivered to a neighboring sugar factory, but the refuse pulp is hanled back for feed. (O) if the prlp is missing mangels are fed instead. Next they are watered in the stable, the water being turned right into the mangers, and alter this they get 10 pounds each of hay from the meadow. The hay is of mixed grasses, containing also some clover. This completes the feeding for 
the forenoon. At $1 \mathrm{p}$. $\mathrm{m}$. the feeding begins again with the same feeds in the same quantities, beginning first with the grain, then roots, water, hay, and straw, keeping them busy eating all the afternoon, the straw being fed at 7 p. m., and this time wheat straw.

The milking begins at 4 o'clock in the morning and at 4 o'clock in the afternoon. It is all done by women, each of whom milks 20 cows, and they do it in from two to two and one-half hours. The churning temperature is $55^{\circ} \mathrm{F}$., and butter comes in about thirty minutes.

The particular ferment which was used in this dairy received the following treatment: It is sold in bottles holding about a pint, all of which is used at one time. It it not added directly to the cream, but is first propagated in skim-milk. For this purpose the milk from a fresh cow should, if possible, be selected. The milk is set in ice water for twelve hours and then skimmed by hand. It is next sterilized by being heated to $180^{\circ} \mathrm{F}$., at which temperature it should be kept for two hours. It is then cooled to $82^{\circ} \mathrm{F}$. and the bottle of ferment is added, and this temperature should, as far as practicable, be maintained during the next twenty or twenty-four hours while the ferment is growing, which takes about that time. At the close of this process another batch of skim milk is sterilized as before. It is next cooled to $50^{\circ} \mathrm{F}$., then again warmed to $82 \circ \mathrm{F}$., and 10 per cent of its weight is added to it from the ferment made the previous day. This stands again another twenty-four hours at the same temperature as nearly as may be, and then it is ready to be added to the cream, for which purpose the cream should be at $70^{\circ} \mathrm{F}$.

A herdsman styled "feed-master" has charge of the feeding. The milk is, of course, weighed as fast it arrives at the dairy, but twice a month there is a "trial milking," at which the milk is weighed from each cow, which enables them to get an approximately correct idea of the yield of each cow. The cows do not get out of the barn all winter. When tied up in the fall they remain there till they are put in pasture the following spring, which usually occurs the latter part of May. This, I find, is the practice on nearly all dairy farms.

The morning milk is run through the separator as fast as it arrives in the dairy from the barn, and the evening milk stands in the rat and is separated also in the morning.

The cream runs from the separator into the "pasteurizing" or sterilizing apparatus, where it is heated to $16 \tau^{\circ} \mathrm{F}$., after which it is at once cooled on the cooling apparatus to $44^{\circ} \mathrm{F}$. This heating and cooling is completed by about halt past 7 in the morning, and from that hour mntil $10 \mathrm{a} . \mathrm{m}$. it stands in ice water. It is then heated again, but this time only to $70^{\circ} \mathrm{F}$., at which temperature the prepared cream ferment is added, and it now stands for about twenty hours, or until 6 a. m. the next morning, while the ferment does its work, and then it is churned. The amount of ferment added to the cream will in a measure depend upon the season, the cows, and other conditions. In the dairy to which 
this refers it was found that of this impregnated milk 4 to 5 per cent of the weight of the cream should be added at this season to have the proper effect. The ferment is perpetuated in sterilized skim milk, which is prepared fresh every day, and what is made one day is used the next day both to ferment the cream and to start a new lot in fresh skim milk. On Mr. Oxholn's dairy farm it is found necessary to get a fresh lot of ferment from the laboratory about once in six weeks.

I have briefly described the process followed on this farm because the butter made there stands in high repute. It frequently takes prizes at the national butter exhibits, and I was told that it brought usually about $\$ 1.62$ per 100 pounds above the top market quotation. It is all exported to England. Nearly all the work in this dairy is done by women, and the chief dairymaid was certainly a competent person. Most of the skim milk was made into cheese of good quality. It is possible to make good cheese from skim milk, but the success lies in the knowing how, and I believe the time is coming when good skim-milk cheese will find a market in America.

At Rosenfeldt it required at this season 29 pounds of milk to make a pound of butter. The cream loses some of its butter by being sterilized as it was here.

About 1,000 pounds of skim milk was made into cheese daily, and the remaining skim milk was sterilized and fed to the calves and pigs. The cheese milk was warmed to $70^{\circ} \mathrm{F}$. When it reached this temperature 10 per cent of fiesh buttermilk was added-that is, 100 pounds to the 1,000 pounds of milk-and immediately afterwards rennet of a given strength was added. In this case it required seventeen one-hundredths of a pound of rennet for the amount of milk used. After the rennet had been stirred in, the milk was allowed to stand perfectly still until it had coagulated, which happened, usually, in about thirty minutes. The chief dairynaid informed me that if it coagulated in less than twenty minutes the cheese would be tough, and if it took over forty minutes in winter, the milk got too cool. The time when the desired degree of coagulation was reached was determined by inserting the finger and notieing how readily the cheese matter would break when the finger was gently lifted up through it from bolow. A certain brittleness was a sign that the process liad proceeded far enough. The "urd was then cut both ways with wire cutters and allowed to stand five minutes undisturbed, but at the end of this time it was stirred gently and continuously from twenty to thirty minutes. 'This stirring separated the curd from the whey. The whey was then drawn off and the curd was worked with the hands and pressed in the bottom of the cheese vat until all the whey that could be separated by this process was removed.

Four per cent of salt was next added to the curd and thoronghly mixed through it with the hands. The cheese emps were then filled and the cheeses were put under light pressure for twenty hours, being turned 
three times during that period. On removal from the press they were put into a large tub of strong brine, so strong that the cheese would swim on the surface, and allowed to remain there from twelve to fourteen hours. On removal from the brine they were placed upon shelves in the cheese room, where a temperature is maintained of from $56^{\circ}$ to $60^{\circ} \mathrm{F}$. They remained there fourteen days, during which time they were daily turned and wiped on both sides with a cloth. After two weeks of this treatment they were transferred to another room where the temperature was kept lower than in the cheese room, and there kept until sold. During the first few weeks in this room they were washed every other day in strong brine, the object being to make the crust soft and thin. They were usually sold when about three months old and brought in the home market 19 ore per Danish pound, which is equal to about 5 cents per pound avoirdupois.

All the dairy work was in the direct charge of the chief dairymaid, who had half a dozen assistants, most of whom were pupils. The farm was managed by a superintendent, a scientifically educated gentleman and a graduate from the Royal Agricultural School of Copenhagen. He had several assistants. As stated, the farm consisted of about 1,300 acres, and it required 18 teams ( 36 horses) and 8 yoke of oxen to work it. The rotation observed at that time was as follows: First year, fallow, this being manured for the next year's crop; second year, rye or wheat; third, barley; fourth, sugar beets; fifth, barley; sixth, oats; seventh and eighth, clover and grass. But the superintendent informed me that it was contemplated to change the rotation to this: First year, rye or wheat; second year, one-third in potatoes, one-third in beans, and one-third in peas; third year, barley; fourth year, sugar beets; fifth, barley; sixth, oats; seventh and eighth, clover and grass. All of the land is fall-plowed and the manure is applied for the wheat and rye. Occasionally, also, artificial manure for the sugar beets.

On all of these large farms we find young mer. who are there to learn the business of farming. They may remain one or two years, according to contract, and some of them receive a small wage when they consent to eat at the tables with the hired men and in other respects "hoe their row" alongside the hired men. Others are there without any pay, and still others pay for the privilege of being there.

On this farm there were two classes of farm pupils, one class receiving 100 erowns a year in wages (about $\$ 2.7$ ) and the other class receiving no pay. All were required to work alike, however, and they were expected to work as steadily and hard as was required of the hired men, the only difference in their privileges being that they got their meals in a separate room. The hired men who worked on the farm received 200 crowns a year $(\$ 54)$ and their board, and day laborers received from 36 to 37 cents per day, boarding themselves. 
ALNO FARM.

This farm also belongs to Kammerherre Oxholm, the owner of Rosenfeldt, already described, but it was tenanted by Mr. Stolpe, who had rented the farm for a series of years. The farm is some 700 acres in extent and was worked on a system of mixed husbandry, with special attention to dairying. At the time of my visit there were 260 cows on the farm, 50 of which were dry. An average of 3,000 pounds of milk per day was obtained from the 210 cows giving milk, and in addition to this 1,000 pounds per day were bought from neighboring farmers. The total amount of milk was, therefore, 4,000 pounds per day. The dairy work was in charge of a man, but the milking, cleaning of utensils, etc., was done by women. The feeding of the cattle was in charge of a so-called feed-master, who is also the cooper and makes the butter barrels. The cows were fed as follows: The first feed was given them at $4 \mathrm{a} . \mathrm{m}$., and consisted of either oat straw or barley straw. The milking was done while this was being eaten. At 7 they were watered, after which they received a small feed of chaff. When this was eaten they got their grain, consisting of 1 pound of bran, 3 pounds of barley and oats ground together, and 1 pound of rape-seed cake. Half an hour later they each got 10 pounds of mangels and next a feed of hay. This completed the feeding for the forenoon. At $1 \mathrm{p} . \mathrm{m}$. they were again watered and then followed in succession feels of chaff, grain (the same as above), mangels, hay, aud straw, the last feed being given about 6 in the evening. Milking was done about twelve hours apart, beginning at 4 in the morning and again at 4 o'clock in the evening. All the cattle remained in the barn during the entire winter, the water also being given them in the mangers. The cattle were of the red Danish dairy breed, with some crosses of the Angler breed. The evening milk was put in tall, tin milk cans, which were set in cold water over night. In the morning these were skimmed by liand and the milk, which had thus stood only twelve hours, was run through a separator, together with the morning's milk. The milk ran through a forewarmer, a machine already described, which raiser it to $95^{\circ} \mathrm{F}$., before it ran into the separator. The cream, as it came from the separator, ran into large milk cans which were at once immersed in ice water and cooled under constant stirring to about $35^{\circ} \mathrm{F}$., and here it remained until it was again warmed for the reception of the ferment and set to sour in the cream barrels. They occasionally used artificial cream ferments and occasionally they marle their own ferment from half-skimmed milk. The method followed on this place for the production of the latter was as follows: The evening milk from a nearly fiesh cow was set by itself in ice water over night and in the morning was skimmed. The skim milk was warmed to $170^{\circ} \mathrm{F}$., and then cooled to between 720 and 750 F., at which temperature it remained for twenty-four hours, when it was found to be sour. Of this sour milk "5 per cent of the weight of the 
cream was added to the cream which had been separated in the morning and cooled off' in ice water, as already stated. The cream was warmed to $70^{\circ} \mathrm{F}$., before the sour milk was added, and it was, as far as possible, kept at this temperature until 6 the following morning, being gently stirred two or three times in the ineantime.

During the winter the churning temperature was at $62^{\circ} \mathrm{F}$, and the cream rose to $66^{\circ} \mathrm{F}$. during the process of churning. In the summer, I was informed, they churned at $50 \circ \mathrm{F}$., or a little above, according to the weather, and butter always came in from thirty to thirty-five minutes.

The erops on the farm consisted chiefly of barley, oats, rye, and a little wheat, and besides these, mangels for the cattle, some potatoes, grasses and clover. Of the grains only so much was sold as could be spared from the amount necessary to keep the live stock. The tenant, Mr. Stolpe, was an able farmer, and in spite of the fact that the rent of the farm came to some $\$ 1,890$ per year, he managed to pay all the expenses and still put something in the bank, the dairy being the main source of income.

\section{THUREHYLILE FARM.}

This farm can also be classed among the large estates. It contains an area of a little over 700 acres, of which some 60 acres are in permanent meadow, and the rest under culture. The farm was owned by a landlord who had large estates in that part of the country, but it was worked by a company who used it as a stock farm largely, their objects being not only to pay expenses, but also to carry out certain experiments in breeding, in order to improve the breed of dairy cattle. Superintendent Tuxon, the able manager of the place, was a man of established reputation as a farm manager.

While they also had a dairy there, it was not the chief feature, but was simply coördinate with breeding of stock and the production of grain crops. They kept 150 head of cows on the place. These remained tied in the stable all winter. I did not see this herd, for the reason that there was a "foot-and-mouth-disease" scare in that part of the country and no stranger was admitted to the barn; but Superintendent Tuxon informed me that the cattle were all of the red Danish breed and that they were all superior animals. Their management was of interest because it varied somewhat from the common custom and may be said to stand out as the best example of the management of dairy cattle to be found in the country. The cows were classified in the barn as follows: All those which were in full flow of inilk; all those which had passed the period of full flow but still gave milk; all dry cows; and, lastly, all barren cows, each class standing by itself in a different part of the same barn. The feeding of these varied, and this was the main reason for their classification. Cows in full flow of milk got their first feed at 4 o'clock in the morning, consisting of 6 pounds of hay, 
and they were milked while eating this, between 4 and 6 a. $m$. At 6.30 a. m. each cow received 4.1 pounds of grain, consisting of $1 \frac{1}{2}$ pounds of oil-meal (which was made up of one-half pound rape cake, one-half pound sunflower cake, and one-half pound palm cake), 2 pounds barley and oats mixed and ground together, and 1 pound bran. Immediately after this, each cow was given 15 pounds of whole mangels, and next they got a feed of barley straw.

Water stood before them at.all times, in a little basin between each two cows, so that both drank out of the same basin. By a self-regulating valve in the supply pipe the water stood at the same height in these basius at all times. This very handy arrangement cost twelve crowns, or a trifle more than $\$ 3$ per head to put up. At 1 p. $\mathrm{m}$. the afternoon feeding began, which was simply a repetition of that of the forenoon.

All other cows in the barn, not in full milk, got hay, straw, and mangels as these, but they were fed different amounts of grain, according to their condition. The strictest attention was paid to cleanliness. The barn was cleaned and swept twice daily, and all the cattle were bedderl twice daily. Superintendent Tuxon believed that not only the barn should be kept clean, but that it paid to keep the skins of the animals scrupulously clean. To this end he employed a man who spent his entire time currying the cattle; he went over the entire herd with currycomb and brush twice daily. One man did all of the cleaning of the stable, and another curried the cattle, while a third attended to the feeding. The temperature of the barn was kept, as nearly as possible, at $60^{\circ} \mathrm{F}$. for the cows and $55^{\circ} \mathrm{F}$. for the calves. No artificial heating apparatus was used, but the barn was a substantial stone structure with perfect ventilation and provision against drafts, so that it was possible to maintain this temperature even in very cold weather. None of the cows were let out of their stalls during the entire winter. The summer management was equally exacting. The cattle were put on grass about the 15th of May and remained ont of doors until about the 10th of October. During the greater part of this time they were tethered in the field, each cow being secured to a stick driven in the ground, and allowed the freedom afforded by a 20 -foot rope. The whole herd was thus staked out in the pasture, beginning on one side and gradually inoving over to the other side. This is, doubtless, the most economical way of utilizing the pasture. Each cow was given some 3 or 4 feet of fresh pasture at each change; the grass was thus eaten up clean, and nothing was wasted by being tramped down or dirtied by the herd. This method of pastming is in very common use on the smaller farms and also on many of the larger ones, as in this instance. The entire herd was changed five times a day at equal intervals, all the work being done by one man. The watering was attended to by another man, who, with a horse and cart, hauled the water to tubs placed between each two cows. These tubs were moved forward 
once in three or four days, as occasion required. All the cows giving milk were blanketed with light cotton blankets during the summer to keep the flies off, and, in addition to the pasture, they were fed a little grain daily, for the most part bran, and not to exceed 3 pounds per cow per day.

The management of the calves is also worthy of note. As soon as the calf is dropped, it is put in a small pen by itself, and within a couple of days it is injected with tuberculine to test whether or not it is affected with tuberculosis. If there is a reaction from this injection, shown by a rising temperature, then it is slaughtered at once, as this is a sure sign that it is affected; but if there is no reaction, it is spared. Then the healthy calves are put together by twos until they are 2 months old. Then four are put together in the same pen, and when the weather begins to get milder in spring they have access to a little open run in the yard. In May, when the cows are let out, all the calves are again injected with tuberculine to learn whether any of them have been infected during the winter. All which sbow a rise of temperature by this test are killed at once, and those which are healthy are allowed to run together without restriction as to number.

The object is to raise a herd which shall be entirely free from tuberculosis. It is admitted that at present a large percentage of the cattle in the country are affected by this disease. It is impracticable to kill all of the affected cows at once, as the loss would be too great, but by this method of weeding out infected calves the country will eventually be clear of this curse, and the object of the company, under whose auspices these experiments are carried out, is to ascertain what means can best be adopted so as to be at once effective and attended with the least loṣs. As to the value of tuberculine, Prof. Koch's great discovery, I was assured that in no instance had the slightest sign of the disease been found in animals which did not show a rise of temperature as a result of the injection, and, on the other hand, the germs of disease had, in every instance, been found in animals where this rise in tem. perature took place.

During the first summer the calves run together in a pasture to themselves. In the fall, when they are taken in, they are put, fifteen together, in a large pen and allowed to go loose. The following summer they are tethered in the same manner as the cows. The feeding of the calves is done with equal care and system. Each calf gets 300 pounds of whole milk and 2,000 pounds of skim milk up to the age of 5 months, and after that age they get no milk. It is divided about as follows: Until three weeks old all are fed the whole milk, the quantity being proportioned to the age of the calf. At 3 weeks old a little skim milk is added to the whole milk and the quantity of skim milk is then gradually increased during the next two or three weeks until, at 6 weeks old, the whole milk is entirely withdrawn. The maximum amount which is allowed in one day is $\mathbf{1 6}$ pounds of whole milk 
or 20 pounds of skim milk. All the milk is boiled and then cooled to a temperature at which the ealves can drink it.

The grain feed begins at 2 months of age. They then get a small quantity of a mixture consisting of equal parts of bran and linseed meal, and this is gradually increased up to $1 \frac{1}{2}$ to 2 pounds per day, each, and in addition to this they always have hay before them. When the skim milk is withdrawn they are given mangels instead until they are put on pasture, and during the entire summer, while on pasture, each gets $1 \frac{1}{2}$ to 2 pounds of grain daily. During the following winter each gets 40 pounds of mangels, 10 pounds of hay, and pounds of grain (bran and linseed cake) daily.

The heifers are bred when 15 months old and hence drop their first calves at 2 years old. During the second summer they are together as stated, but without any grain. The calves are dropped from September to February. The following is the average weight of the cattle at the ages given: Calves average the first of May about 330 pounds; the first of November, about 5.5 pounds; at $1 \frac{1}{2}$ years, about 700 pounds; at 2 years, about 825 pounds, and at 3 years, about 924 pounds. The heifers get 4 pounds of grain daily, in the fall, from the time they are put in the barn until they calve, and after calving they are treated in the same manner as cows. All cows in the herd which are not in calf are tried by a teaser every day, and if they show signs of heat, are bred to one of the fine bulls. The herd remains tied on pasture until about the middle of September, when they are turned loose on the stubble fields, these having been cleared of grain by that time.

At the time of my visit, in the beginning of February, the cows gave about 2,200 pounds of milk per day. Of this about 100 pounds was required for the young calves and the rest was set and skimmed. In this dairy no separator was used. It was run on the old plan of setting the milk in ice water and in the flat iron pans already described. The evening milk was set in the deep milk cans in ice water and the morning milk in the shallow iron pans. The milk stood from twenty-four to thirty-six hours before it was skimmed and then it was skimmed twice. The first skimming took place in the morning, when the morning milk har stood twenty-four hours and the evening milk twelve hours, and the second skimming in the evening when the evening milk had stood twenty-four hours and the morning milk thirty-six hours. In summer the iron pans were not used, all the milk being set in ice water. The cream was sterilized, not by means of a sterilizing apparatus, but by being heated to $170^{\circ} \mathrm{F}$. in hot water. The water stood in a vat and was heated by steam formed in a little boiler set up for the purpose of providing hot water and steam to aid in cleaning the utensils, but not for the production of power. The cream, which had been put in deep cans, was heated by inserting the cans in this vat of hot water, the contents of each can being gently agitated all the while. When the thermometer indicated that the desired temperature was 
reached, the cream can was at once removed and immersed in a basin of ice water, where it was cooled to about 450 $\mathrm{F}$. The dairy was in charge of an able dairywoman, and all the help in the dairy consisted of pupils who worked for their board, and neither gave nor received remuneration. Artificial cream ferment from the laboratory of Blanenfeldt \& Tvede was used. The cream was warmed to $70^{\circ} \mathrm{F}$. before the addition of the ferment, and at this temperature was set in the cream barrels, where it remained for twenty-eight hours in winter, falling in that time to about $60^{\circ} \mathrm{F}$, at which temperature it was churned.

The churn was worked by horse power and the butter came in the same time as in the other dairies I visited where steam power was used. It required, at that time of year, 29 to 30 pounds of milk to produce a pound of butter. The butter was worked three times, in each case by nand; first, for the removal of the buttermilk, immediately after which 5 per cent of salt was added; then it was allowed to harden and the salt to melt for one hour, when it got the second working, the object being, this time, to mix the salt thoroughly; the third working took place two hours after the second.

A fine quality of skim-nilk cheese was made at this dairy. The process was, briefly, as follows: The milk was warmed to $95^{\circ} \mathrm{F}$. and at this temperature was added 8 per cent of buttermilk and the necessary quantity of rennet, according to its strength. If color was used it was also added then. It then stood untouched for twenty minutes while the rennet did its work. The curd was next cut and then stirred for fifty-five minutes. If the temperature had fallen during that time, it was warmed up to $96^{\circ} \mathrm{F}$. by admitting steam under the vat. Half the whey was then drawn oft. It was next stirred for fifteen minutes, when the remaining whey was drawn off and the curd was worked by hand. 'One-fourth of a pound of salt was then added for every 100 pounds of milk. The curd was then put into forms and given half pressure. At the end of an hour it was turned and replaced under the press, and two hours later it was turned again. Full pressure was then applied and it stood undisturbed until evening, when it was turned once more and a fresh cloth wrapped about it and it was again put under the press. Next morning it was taken from the cheese cup and put in strong brine, where it remained for two days; it was then put in the cheese room where a temperature of $52 \circ$ to $55^{\circ} \mathrm{F}$. was maintained, and there all the cheeses were daily turned and rubbed with salt or strong brine once a week. This continued for three months.

The rotation followed on this farm was as follows: First year, fallow; second year, rye; third year, barley; fourth year, one-fourth roots and three-fourths annual grass; fifth year, oats; sixth year, clover; and seventh year, one-half mixed barley and oats, and the other half in grass from the previous year's seeding. On a small portion of the farm a four-years' rotation was followed, being first year, rye; second year, roots; third year, barley; and fourth year, grass. The grass was $8538-N o .5-5$ 
sown with the oats and barley, respectively, in rotation. The yield of hay was about two tons to the acre. For the annual grass they used a mixture of oat grass, English rai grass, yellow medick, and sometimes a little bromus arvenses.

For the two years' grass crop in the seven-year rotation they used red clover, orchard grass, English rai grass, and meadow fescue. The average yield of rye was 50 bushels to the acre; of barley, 45 bushels; of oats, 60 bushels to the acre; and the yield of roots went as high as 24 tons to the acre. I append a statement of the receipts from the dairy, which Superintendent Tuxon kindly permitted me to draw from his books.

Statement of the products of Thurebylille Dairy and their values for the years named.

[Year reckoned from May 1 to April 30; weights in pounds avoirdupois.]

\begin{tabular}{|c|c|c|c|c|c|c|}
\hline & $1886-' 87$. & $1887-' 88$. & $1888-89$. & $1889-’ 90$. & $1890-91$. & $1891-' 92$. \\
\hline Number of cows giving milk............. & 135 & 129 & 127 & 123 & 127 & \\
\hline Yield of milk ...... pounds a voirdupois. & 726,752 & 771,810 & 746,447 & 738.133 & 766,286 & 772,275 \\
\hline A verage yield of milk per cow ..... do... & 5,383 & 5,982 & 5,877 & 6,000 & 6,033 & 5,940 \\
\hline A verage yield of butter per cow....do... & 183.5 & 207.6 & 204.4 & 198.9 & 196. 2 & 203.2 \\
\hline $\begin{array}{l}\text { Butter sold per cow (inclusive of house- } \\
\text { hold consumption) pounds avoirdupois. }\end{array}$ & 182.1 & 205.9 & 203.3 & 191.9 & 189.7 & 199.8 \\
\hline $\begin{array}{l}\text { Butter value of sweetmilk used on farm } \\
\text { per cow........... pounds avoirdupois.. }\end{array}$ & 6.53 & 4.8 & 5.8 & 8.1 & 15 & 5 \\
\hline Produced green cheese per cow.....do... & $\begin{array}{l}0.03 \\
233.9\end{array}$ & $\begin{array}{r}4.8 \\
289.7\end{array}$ & 253.1 & $\begin{array}{r}8.1 \\
232.8\end{array}$ & $\begin{array}{c}15 \\
246.7\end{array}$ & 266.9 \\
\hline Produced ripe cheese per cow ...... dlo... & 195.5 & 245 & 212.6 & 208 & 220.1 & 238.1 \\
\hline Live werght of swine sold per cow..do... & 185. 7 & 214.5 & 245.7 & 240.6 & 231 & 236.1 \\
\hline $\begin{array}{l}\text { Shrink in butter................ per cent.. } \\
\text { Milk required to make } 1 \text { pound butter }\end{array}$ & .8 & .8 & .4 & 3.1 & 3.4 & 1.9 \\
\hline Skiw........................... pounds. & 28.5 & 28.2 & 28 & 29.5 & 29. 2 & 28.9 \\
\hline cheese ..................... pounds... & 13.5 & 13.4 & 13. 3 & 13.4 & 13.1 & 13.2 \\
\hline $\begin{array}{l}\text { Shrink in eheese } \\
\text { Price of butter per pound a voirdupois: }\end{array}$ & 16. 4 & 12.2 & $16^{\circ}$ & 10.7 & 10.8 & 10.8 \\
\hline In summer ....................... cents. & 21.6 & 22.8 & 21.5 & 22.9 & 21.6 & 22.3 \\
\hline In winter.......................... & 25.02 & 25 & 25 & 25.02 & 27 & 27 \\
\hline $\begin{array}{l}\text { Price per pound aroirdupois of skim- } \\
\text { milk cheese..................... cents.. }\end{array}$ & 3.15 & 4. 72 & 4.7 & 3. 97 & 5.2 & 5. 62 \\
\hline $\begin{array}{l}\text { Price per pound of swine (live weight } \\
\text { avoirdupois).......................... }\end{array}$ & 6. 27 & 5. 88 & 6.41 & 6.97 & 7. 38 & 7.63 \\
\hline Receipts from each pound of skim milk: & 2 & 31 & 29 & 26 & 35 & 1.00 \\
\hline 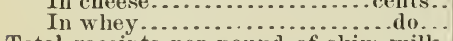 & .098 & .31 & .29 & .20 & $\begin{array}{l}.35 \\
.19\end{array}$ & $\begin{array}{l}.42 \\
.198\end{array}$ \\
\hline Total receipts per pound of skim milk & 298 & 42 & 4 & 41 & .54 & 618 \\
\hline Receipts from each pound sweet milk & & & & & & \\
\hline Gross receipts per cow. & $\begin{array}{r}1.08 \\
\$ 58.14\end{array}$ & $\begin{array}{r}1.19 \\
\$ 71.51\end{array}$ & $\begin{array}{ll}1.2 \\
\$ 71.54\end{array}$ & $\begin{array}{r}1.15 \\
\$ 69.51\end{array}$ & $\begin{array}{c}1.3 \\
\$ 77.89\end{array}$ & $\begin{array}{r}1.36 \\
\$ 81.21\end{array}$ \\
\hline
\end{tabular}

VALUEMAR CASTLE.

This old eastle is situated on the small island called Tosinge, which lies close to the Isle of Funen. The farm consisted of 630 acres. On this area were kept, at the time of my visit, 120 dairy cows, about half of which were in milk and the other half dry. They were stabled in an excellent barn, the plan of which may be seen in the accompanying illustration (Figs. 20 and 21), which I give as a sample of the barns that are generally found on the large farms.

The barn was built of briek with stone foundation, and was one story high, with a high roof. It. was entirely fireproof. The ceiling was of brick laid on iron arches, and the only openings from the inside were 
those necessary to admit hay and bedding, which were stored under the roof. At intervals there were brick flues for ventilators, which ran to the crest of the roof, and the roof itself was of tiles. There was no wood in the interior of the structure except the door and wiudow frames. There were no partitions between the stalls, and all the posts were of inoll.

Fig. 21 shows a plan of the barn; $a$ represents the rows of stalls, and $b$ the feed alley between the stalls. The floor of this alley was raised a foot and a half above the floor of the stalls. At both ends of

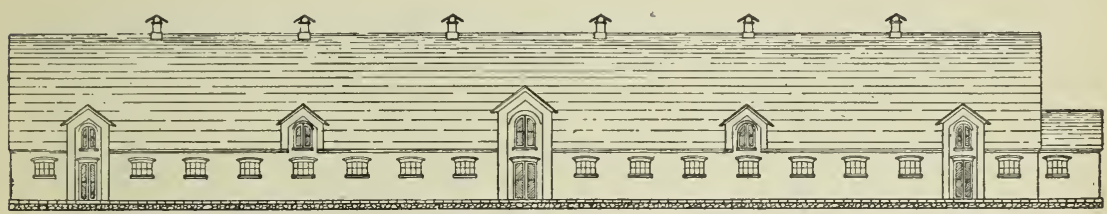

FIG. 20.-Side view of cattle barn at Valdemar castle.

the barn were louse boxes marked $c$, inclosed with an iron fence. These were, for the most part, used for calves; $m$ represents the mangers, which were made of curved tiles laid in cement so that they were perfectly water-tight, and the cattle were watered in these mangers twice daily. At one end of the barn were three feed-bins indicated by $f$, and in the middle of the barn was a short wing which contained the herdsman's room $h$, and a large room for mangels, $n$. The floor of the barn, except the feed alley, was a pavement of squared stones nicely

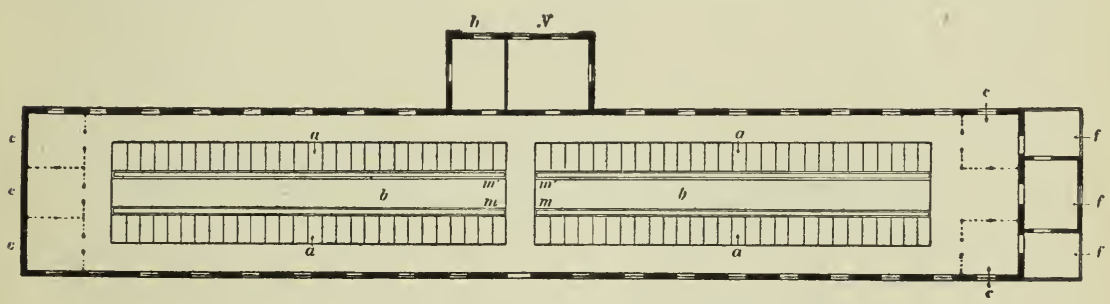

Fig. 21.-Ground plan of cattle barn at Valdemar castle.

fitted together, and provision was made for the drainage of the liquid manure into large cisterns. I did not learn what the cost of this barn was, but it must have been considerable. On the other hand, it was of such a substantial nature that it will last for centuries.

The cows were fed as follows: At 5 a. m. they were fed straw, and the milking took place while this was being eaten; at $7 \mathrm{a} . \mathrm{m}$. each cow got 10 pounds of mangels, and immediately after 5 pounds of grain, consisting of the following mixture: One pound of cocoanut cake, 2 pounds of sunflower cake, 2 pounds of bran, 3 pounds of oats, and 2 pounds of barley, the two latter being ground together. After this was eaten they were watered. Next they got a feed of straw or of chaff. At $12 \mathrm{~m}$. they got a small feed of hay. At $1 \mathrm{p}$. m. the afternoon feeding began with 10 pounds of roots, then half of the above mix- 
ture of grain, and then water. Next straw, and again a feed of straw at $7 \mathrm{p} . \mathrm{m}$. The cows were fine specimens of the red Danish breed. The average yield of milk per cow for the year was 4,950 pounds avoirdupois, including barren cows and heifers. Some of the cows were large milkers, one of them yielding 68 pounds per day.

The milk is handled as follows, according to the statement of the chief dairymaid:

The evening milk is set in tall 50-pound cans, and these are sunk in ice water. These cans are hand skimmed in the morning, and the skim milk is run through the separator, which still produces 4 pounds of cream to 100 pounds of milk. When the milk has been skimmed in this way all the cream is sterilized by being heated to $167^{\circ}$ $\mathrm{F}$. This is accomplished by immersing the cream cans in hot water, the sterilizing apparatus being out of order. It is next cooled to about $40^{\circ} \mathrm{F}$, at which temperature it remains for a couple of hours. It is then heated again to $78^{\circ} \mathrm{F}$., and at this temperature the ferment is added. The ferment in this case consists of sour cream saved from the morning's churning, 20 per cent of this sour cream being added. It then stands in the cream barrel, in a protected place, for eighteen hours, or from noon one day to 6 a. m. the next, when it is churned.

The dairymaid held that this cream ferment had given her better results than any other methods she had tried. In summer she set the cream to sour at only $60^{\circ} \mathrm{F}$, but she then added a greater quantity of sour cream, and the same results were obtained. She churned the cream in winter at from $59^{\circ}$ to $62{ }^{\circ} \mathrm{F}$. and washed the utensils with clean water at $56^{\circ} \mathrm{F}$. The butter came, usually, in twenty-five minutes. The revolving dasher in the churn made 140 revolutions per minute. In summer the churning temperature was $52^{\circ} \mathrm{F}$. The butter she made was of first quality, and always brought about $\$ 1.35$ per 100 pounds over the highest quotation at the exchange in Copenhagen. She added $4 \frac{1}{2}$ per cent of salt in winter and 6 per cent in summer, and she assured me that the butter would keep for half a year.

She took the following precautions in packing butter: First, the barrel was soaked for twenty-four hours in cold water and next it was filled with brine. which remained in it for twenty-four hours. Then the inside was thoroughly rubbed with salt. In the meantime paraffin paper was prepared by soaking in brine for several hours, and lastly in saltpeter for a half hour, and the barrel was then lined with this; finally a half inch of salt was put on the bottom of the barrel. The butter was then firmly packed in, the top covered with a sheet of paraffin paper, on top of this half an inch of salt, and then the head was put on. The barrels thus packed were kept in a cool room adjoining the ice house until they were shipped, and each barrel was rolled about for some time once a week in order to prevent the brine from settling too much in one place.

The skim milk was sterilized before it was fed to the calves, and cheese was made from what was left after feeding the calves and taking out what was required for the household. It required 22 pounds of milk to make a pound of butter in summer and fall and 25 pounds in winter. 
This farm is situated on the island of Langeland (Longland). I paid a flying visit to it because, in my travels about the country, I had heard the dairy products from this place highly spoken of, and I even heard of the butterfrom this farm while among the butter dealers in London. My time, however, was so limited that I could only interview the dairy manager, but the establishment owed its reputation chiefly to his skill. He was a young man by the name of Neilsen Holm, who had studied dairying in the best establishments of the country. The following notes were taken from his statements:

There were 250 cows on the farm, and of these 200 were in milk at the time of my visit. The stalls were cleaned in the morning from 3 to $4: 30$ a. m., and immediately after this the cows were milked. The first feed was given at 7 a. m. and consisted of half a bushel of whole mangels. When these were eaten they were fed grain consisting of 2 pounds of barley and oats ground together, 2 pounds of bran, and 1 pound of rape-seed cake; they were next watered at about 8 a. m., the water being run into the manger in front of them. After the water they got 4 pounds of hay per head, and when this was eaten a liberal feed of barley straw. They were then bedded down, the doors were closed and the stable kept as quiet as possible for the remainder of the forenoon. At $1 \mathrm{p} . \mathrm{m}$. the feeding began again with another half bushel of whole mangels and then 5 pounds of grain of the same mixture as above ; next water, hay, and straw in succession as in the forenoon. The stable was again cleaned from 3 to $4: 30 \mathrm{p}$. $\mathrm{m}$. and the milking took place immediately after ; finally a feed of straw was given about 7 in the evening.

The herd, as a whole, was first class. Many of the cows gave as much as 6,600 pounds avoirdupois of milk per year. He selected calves intended to be raised to fill places in the herd only from the best cows. Good cows were boughtwherever they could be found. The cows were tested by the so-called "trial-milkings," which occurred twice a month. It consists in weighing the milk from each cow separately, at both milkings, on fixed dates, and from this the yield is estimated. The cows were not named but numbered, each being known by her number only. Special attention was also given to the breeding of bulls on this farm, and the few bull calves which, in point of pedigree and individual merits, were considered good enough to raise, sold for high prices. The milk was run through a De Laval "Alpha" separator as soon as it reached the dairy, at a temperature of $86^{\circ} \mathrm{F}$., and the cream was at once put in ice water and cooled to $50^{\circ} \mathrm{F}$. in the winter, and in summer to $45^{\circ} \mathrm{F}$, and was kept at this until time to sour it. It was then warmed to $77^{\circ} \mathrm{F}$. and at $10 \mathrm{a} . \mathrm{m}$. put in the cream barrel at this temperature and 5 per cent of ferment added.

The ferment was made thus: Healthy cows were selected which had been in milk about six weeks. This milk was put in ice water as 
soon as it arrived at the dairy and cooled to $40^{\circ} \mathrm{F}$. Here it remained five hours, and whatever crean rose in that time was skimmed off. The skim milk was then warmed to $100^{\circ} \mathrm{F}$. and immediately put aside in large earthenware jars, and these, in winter, kept in a box lined with a hay mattress to keep out the cold. Put away thus at $11 \mathrm{a} . \mathrm{m}$. it remained until the next morning at 8 a. m., when the jars are opened and the upper 2 inches skimmed off to avoid contamination from injurious bacteria which might have found lodging on the surface. The milk, which had become sour, was then thoroughly mixed by being violently stirred and then cooled while exposed to fresh air to $50^{\circ}$ or $54^{\circ} \mathrm{F}$., and finally, at $10 \mathrm{a} . \mathrm{m}$., it was added to the cream in the cream barrels. Occasionally buttermilk was used as a ferment, but, as a rule, this skim milk ferment was prepared and used daily. After the ferment was added the cream stood from 10 a. $m$. until 5 a. m. the next morning, but was stirred four times during the day. At 5 a. m. it was transferred to tall cans which were sunk in ice water until the cream showed a temperature of $12 \circ \mathrm{R}$. (59 F.), at which temperature it was churned in winter. From the 200 cows in milk 330 pounds of cream was obtained daily. The churn was run by steam, the dasler making 140 revolutions per minute, and butter came in from 30 to 35 minutes. The butter received three workings on the machine; after the first working it was weighed and 4 per cent of salt was added; onehalf hour later it was worked again, and two to two and a half hours later it received the final working. It was then packed in barrels in the same manner as has already been described. It took 26 pounds of milk to make a pound of butter. The butter was sold by contract to one dealer for the whole year, the agreement being to pay about $\$ 26$ per 100 pounds of butter; but for the ensuing year's contract I was informed the price would be raised to about $\$ 30$ per 100 pounds. The butter was strictly a first-quality article.

JULESBERG FARM.

I made a brief visit to a large farm of that name situated a short distance from the city of Nyborg. There were 130 cows on the place, but, in addition to the milk fron these, milk was bought from some of the smaller farms in the neighborhood, so that the total amount han dled was nearly 5,000 pounds daily. The creamery was of the improved type, equipped with all the machinery denanded by the most advanced methods. 'This milk produced about 195 pounds of butter daily. All the cream was pasteurized before it was fermented. The ferment used was usmally produced on the place, from skim milk, in the manner already described, but they also used pure cultures when it was thought advantageous to do so. The dairy was in charge of a woman, who informed me that she churned at $570 \mathrm{~F}$. luring the winter. and that the cream was set to sour at from $68^{\circ}$ to $720 \mathrm{~F}$., according to the weather, and she used $4{ }^{1} \cdot$ per cent salt to the butter. 
The cows on the farm were fed as follows: They were milked between 4 and $6.30 \mathrm{a} . \mathrm{m}$. This done they got their first feed, which consister of $t$ pounds of a mixture made up of one-half part each of rape-seed cake and cotton-seed cake, and one part each of bran and barley and oats ground together. After this they got a feed of hay, then water, and next straw. This completed the feeding for the forenoon. At 1 p. m. it began again with grain, as above, then 15 pounds of sliced mangels, then two feeds of straw in succession. Of the 130 cows on the place 92 of all ages were in milk, and these gave 1,850 pounds of milk daily, and the best cow gave at that time 48 pounds of milk daily.

In summer the cows were tethered in the pasture. The milking was done by women, each of whom milked 20 cows in two and one-half hours. I found on this farm some 25 or 30 head of steers which were being fattened. They were fed entirely on oil cake and mangels. Each steer got 5 pounds of rape-seed cake daily and all the mangels he would eat, but no water, the mangels supplying water enough for the needs of the system. This mode of fattening is so unusual that I can not forbear to mention it here. The feed-master assured me that they gained about 3 pounds daily, per head, under this treatment. They were of the ordinary dairy breed of the country, and were tied up in the stable, never being let out.

BRAHETROLLEBORG.

This is the country seat of Count Reventlow. The home farm of the estate comprised about 660 acres, about 100 acres of which were in permanent meadow and the rest under culture. The herd consisted of 161 cows and 3 bulls, and 22 horses were used to work the farm. The cattle got their first feed at 4 o'clock in the morning and while they ate this the milking took place. The second feed was given at $6: 30$ in the morning, cousisting of three pounds of barley and oats ground together, 2 pounds of bran, 1 pound of rape-seed cake and one pound of palm cake. They were next watered in the mangers, after which they got a feed of chaff, and when this was eaten they had a feed of oat or barley straw, which completer the feeding for the forenoon. At 1 p. m. they were again given grain, water, chaff, and hay in succession, and finally a feed of straw in the evening. The milking began at 4 a. m., the stable being cleaned before milking. The bulls had each 4 pounds of grain per day and straw and hay, as the cows. No roots were fed upon this place. At the time of my visit 82 cows were in milk, which yielded abont 1,450 pounds milk per day. They still used the old system of setting the milk in cold water, where it stood twenty-four hours before it was skimmed. The eream was then warmed to $68^{\circ} \mathrm{F}$. and soured by the use of buttermilk from last churning. Pure culture cream ferment was used when necessary. The cream stood 24 hours after the buttermilk was added. They also occasionally made fresh ferment from skim milk in the manner already described 
elsewhere. From the amount of milk given they obtained 57 pounds of butter daily. The butter was worked three times, four per cent of salt being added at the first working. The price realized from the sale of the butter was somewhat less than that of some of the other places I have noted.

Cheese was made from the skim milk by the following process: The milk was warmed to $95^{\circ} \mathrm{F}$. in winter and $92^{\circ}$ in summer, and prepared rennet added when the temperature was reached. It then stood undisturbed for three-quarters of an hour, when the curd was cut and slowly stirred for twenty minutes, until the whey became clear. It then rested for teu minutes, after which it was again stirred a little and when the whey was perfectly clear it was drawn off and the curd pressed with the hands in the cheese vat, to work off the whey. Salt and caraway seed were then added. This completed, it was transferred to the cheese cups and put under the press, the pressure being light at first. At the end of an hour the cheese was turned in the cups and replaced under the press, where it remained until next morning, but it was turned twice more in the meantime. On being removed from the press the cheeses were put in strong brine, where they remained for twenty-four hours, and they were then put in the cheese room, where the temperature was kept at about $54^{\circ} \mathrm{F}$. Here they were turned daily and each time wiped with a dry cloth, this process being continued until they were thoroughly dry. They were kept for at least two months and sometimes much longer, according to the market. 'The dairy was in charge of a superintendent, to whom I am indebted for these facts. He stated, further, that he made a pound of green cheese from 11 pounds of milk, and that his cheese shrank 15 per cent in weight in the process of drying.

On this farm were 20 head of high-grade shorthorn heifers, 3 and 4 years old, being fattened for market. They were in extra fine condition and estimated to average about 1,600 pounds in weight. They were tied up in the barn, as the dairy cows, and were never let out, being ferl and watered in the stalls. They had been on good pasture without grain during the summer and when put in the stable in the fall they were fed hay and straw, turnips and kohlrabi, all they would eat. but no grain. This treatment was continued for one month, then 2 pounds of linseed cake was added to the ration. During the next month this was increased to 6 pounds per head, and in addition some of the largest got 2 pounds of barley and oats.

\section{SMALL DAIRY FARMS.}

In the following account I shall briefly describe the processes, both in the dairy and in the cultivation of the soil, which are followed on the small Danish farms. Possibly they may furnish hints of greater usefulness to the majority of farmers in the United States than do those of the large farms. There are but few farms in this country where one 
hundred or even fifty dairy cows are kept, and fewer still where the dairy is fully equipped with all the modern machinery. Here the farm dairy is on a small scale, and as to material and practical methods of work, it therefore comes nearer the small Danish farms.

FARM OF MADAME NIELSEN.

One of the first of this class of farms which it was my privilege to visit is situated an hour's ride on the railway from Copenhagen and belongs to Madame Nielsen, who named it Havarthigaard. This lady is known ontside of Denmark for her dairy products. For nearly thirty-five years she has been a close student of dairying; both at home and abroad, having made several trips to various European countries

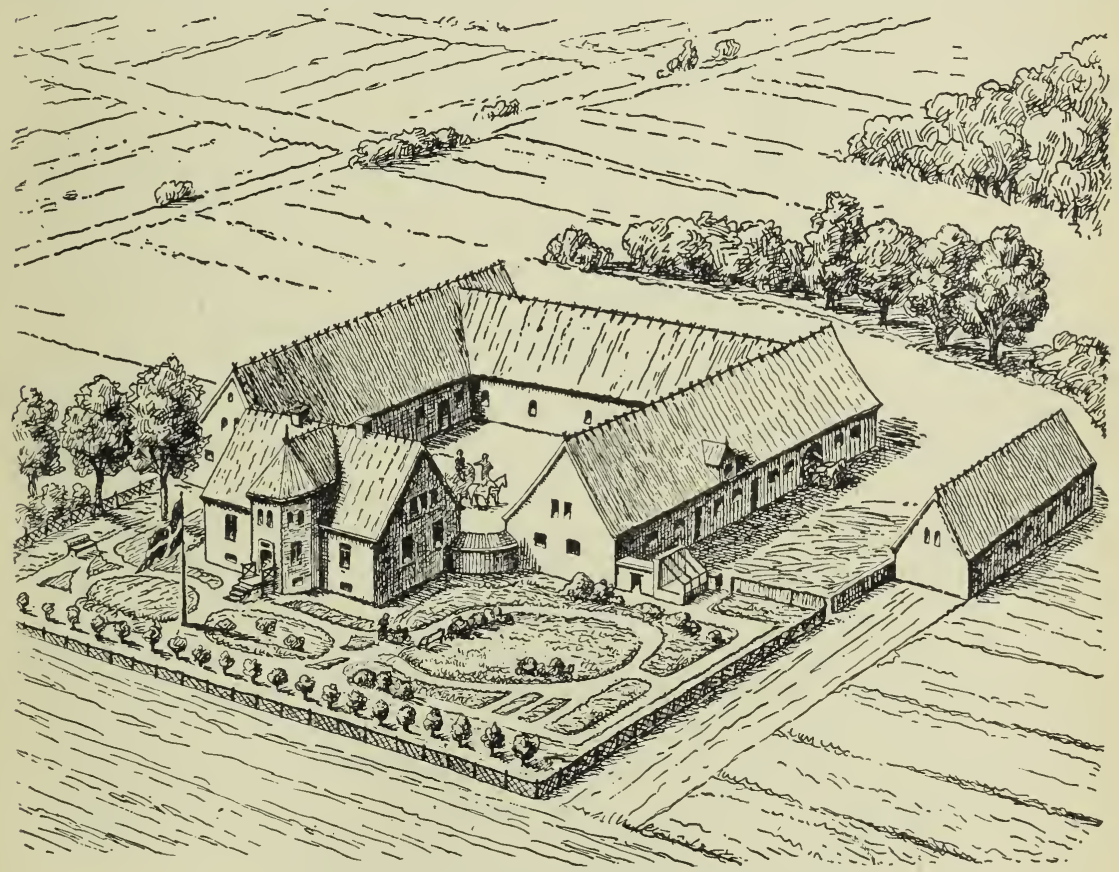

FIG. 22.-Perspective of Madame Nielsen's buildings.

in order to study their methods and obtain points which she could apply to her own practice. She has thus visited England, Holland, Switzerland, Norway, and France at various times. She has metwith signal success. She supplies the butter for the table of the King and Queen, a few pounds being sent to the palace by express every morning from her house. In cheese-making she is equally successful. Her cheese sells for many times the price obtained for ordinary cheese. She even sends cheese to the Emperor of Russia, who learned to appreciate her dairy products on his visits to Copenhagen. He usually carries home a supply when returning from one of these visits, and consignments are occasionally sent to the palare in St. Petersburg. 
Most of her produce, both butter and cheese, is sold to special highclass customers, and the surplus not sold in this manner is disposed of in a little shop in the exhibition building in Copenhagen, where she herself waits upon customers every afternoon. She is famous not only as a practical dairywoman but as a teacher of darying as well. She informed me that she had instructed no fewer than 1,000 pupils in her dairy methods. It is quite the thing for young persons of both sexes, who have completed their course in dairying elsewhere, to finish up with a few weeks or a few months with Madame Nielsen, and many of the chief dairywomen that I met elsewhere had, at some time or other, been at her establishment. She charges a good round fee of them, usually about $\$ 27$, whether they stay for a long or short time, and while there, by the terms of the agreement, they do the work in the dairy of whatever kind. At the time of my visit, in January, there were four young ladies studying dairying, and I was informed that by the first of March two more were to come.

The farm on which she has developed this famous little dairy is $\mathbf{1 6 6}$ acres in extent. The farm management is in the hands of her husband and son-in-law, who, with his wife, lives with them; but the management of the dairy cows and of the dairy is all under her own supervision. I obtained an introduction to this lady through Prof. Segelcke, and by special arrangement it was agreed that I should visit her place, stay over night, so as to be on hand early in the morning, when I might have the privilege of learning what I could of what was going on about me.

At the time of my visit she had 19 cows giving milk, the total number in the herd being between 25 and 30 cows. The milking began at $4: 30$ in the morning and was done by the pupils. The cows were not fed until $6: 30$, when they got a feed of 3 pounds of grain, consisting of a mixture of bran, barley, and oats ground together in equal quantities, and three-fourtlis of a pound of rape-seed cake, which was soaked in water for twenty-four hours pieviously and mixed with the grain. In half to three fourths of an hour each cow had three-fourths of a bushel of mangels. At $8 \mathrm{a}$. m. they received a big feed of barley straw or oat straw, the surplus not eaten being used for bedding. At 11 a. m. they were watered in the manger, after which they had a feed of hay, all they could eat. This finished the feeding for the forenoon. They were not ferl again until 5 p. m., when they harl the same amount of grain as above and after that all the straw they could eat and later water, but no mangels or hay was given them in the afternoon.

In the beginning of May the cows are put on pasture for two or three weeks, but as soon as the grass gets tall enough to eut they are again stabled and stall-fed on green fodder until after harvest, when they are again let out for five or six weeks. They are not tethered, as is the usual custom, but run loose when they are out. The rows were of the red Danish breed, and there were several excellent animals 
among them. Ten or twelve heifer calves are raised every year, those not wanted to take the place of older cows being sold.

The calves are fed during the first two weeks of their lives on whole milk; but at the end of this time skim milk is mixed with the whole milk, gradually increasing the skim milk until they get no whole milk at all, and when about ten weeks old the skim milk is partly substituted by whey and bran. This, with hay and straw in winter and grass in the summer, forms the diet of the calres. The following winter they get roots in addition to hay and straw and a little grain. The heifers are bred when fifteen months old so as to drop the first calf when two years of age. 'Twice a month there is a trial milking, when the milk from each cow is weighed separately, but for the rest of the time all the milk is weighed together.

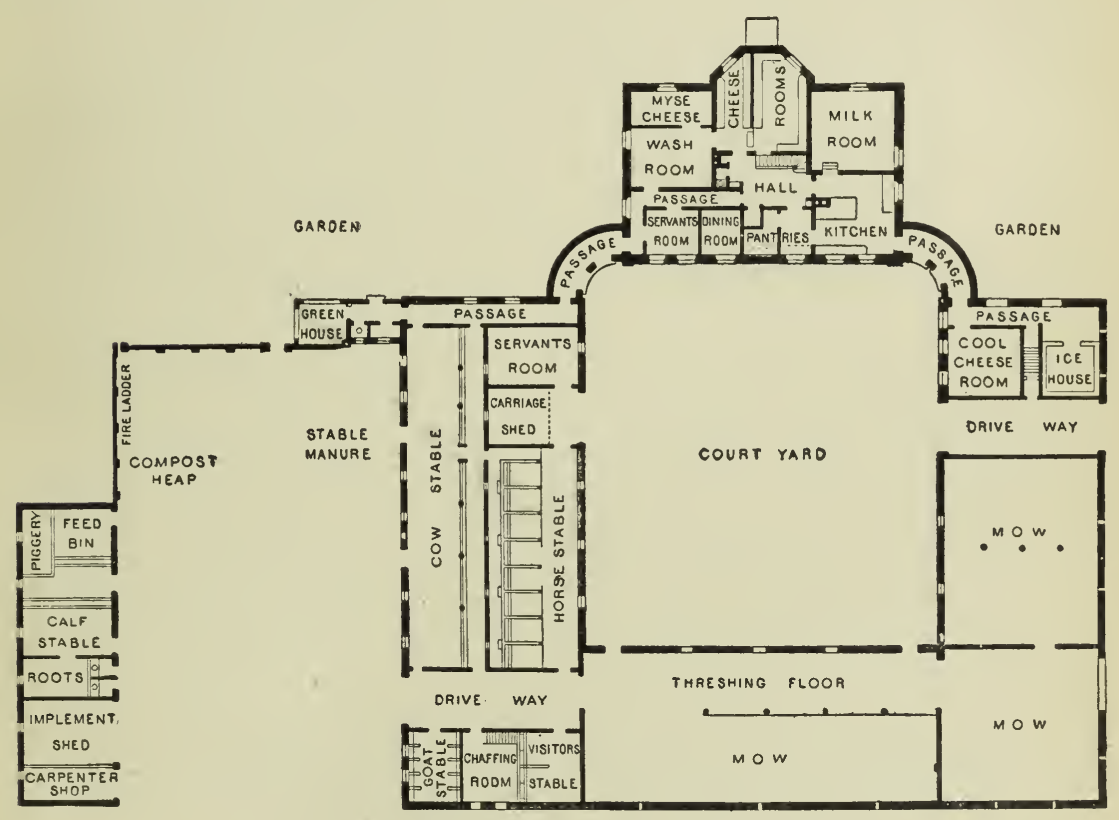

FIG. 23.-Madame'Nielsen's buildings-plan of basement.

Nadame Nielsen's dairy, I found, was small and by no means of a style which is unattainable by American farmers. It was all in the basement (Fig. 23) under the dwelling house. The milk was set in tall cylindrical cans, and these sunk in ice water, there being several basins constructed of brick laid in cement for tine purpose of holding this ice water. The kitchen adjoined the milk-room, and most of the work in buttter and cheese making was done in the kitchen. The milk stood twenty-four hours before it was skimmed. As only about 300 pounds of milk were handled per day during the winter, no separator was needed. nor was any sterilizing apparatus used; all the work was done in the good old-fashioned way and with the best results. The utmost cleanliness 
was apparent everywhere. All the utensils were scoured and scrubbed until they fairly shone. The floors were clean. Care was used in the ventilation to let in all the fresh air possible and still maintain the desired temperature, and pure water and ice were used in abundance. The cream, when skimmed from the milk, was put in an earthenware jar scrupulonsly clean. She used in souring the cream a ferment that she makes from skim milk. She used milk from a cow nearly fresh. This stood twelve hours and was then skimmed. It was then warmed to $100^{\circ}$ or $105^{\circ} \mathrm{F}$., and set away in a place where the temperature could be kept moderately uniform, where it remained twenty-four hours. At the end of this time it developed the proper degree of sourness, the temperature in the winter, in the meantime, having fallen to $68^{\circ}$ or $70^{\circ} \mathrm{F}$. Some 5 per cent of this prepared ferment was added to the cream about the middle of the forenoon, the cream being first warmed to $75^{\circ} \mathrm{F}$. The cream jar was packed in a box lined with a hay mattress, in order to prevent cooling too rapidly. In the course of the day the cream was stirred several times and in the evening it began to thicken. It was then removed from the hay box and kept at a somewhat cooler temperature over night, and at 5 o'clock the following morning the churn was started. The churning temperature varies greatly with the season and the condition. It may range from $55^{\circ}$ to $75^{\circ} \mathrm{F}$.

When I was there the cream was warmed to $61^{\circ} \mathrm{F}$. The churning was done by hand by the side of the kitchen stove in a little churn of the iisual pattern of the country, the revolving dasher being turned by a crank and the power supplied by the unuscular arms of one of the young lady pupils. On the sign of formation of butter the churning proceeded more slowly, and when the granules were as large as pin heads the churn was washed down with water at about $50^{\circ} \mathrm{F}$. Madame Nielsen herself takes the butter out of the churn and works it in an oldfashioned wooden butter trough, but she never tonches it with her hands. It was worked three times at brief intervals. At the second working, the buttermilk having been removed at the first working, 4 per cent of salt was added, and immediately after the third working it was packed in china crocks for transmission by express to the several special customers.

The method of souring the cream here described was not her invariable practice, however. Sometimes she would warm the cream in an enameled kettle to $84^{\circ} \mathrm{F}$., after which she would add 5 per cent of buttermilk from the last churning. This would stand for three or four hours in the cream jar until the temperature of the cream had fallen to $66^{\circ}$ or $68^{\circ} \mathrm{F}$., when it was transferred to the hay-lined box to maintain this temperature as nearly as possible, and it remained there until bedtime, when it had begun to thicken. If the box should then afford too much warmth the jar was taken ont and set on the floor or in water, according to circumstances, until morning. She attached special importance to the following points :

(1) That the churning temperature was properly adjusted to the season 
and the quality of the cream, as this might be affected by the feed of the cows, or the number of fresh cows in the herd, and other varying conditions; (2) that the fermentation of the cream began at a relatively high degree of warmth; (3) that the temperature of the cream was gradually reduced during the time that the ferment was acting; and (4) that the cream thus treated should stand for some time (over night) at the temperature of about $50^{\circ} \mathrm{F}$. before it was churned. In the morning the cream was warmed to the proper temperature for churning by setting the cream jar in warm water. By this method she claimed to be able always to produce the finest quality of butter. She had not, as yet, found it necessary to use the pure cultures in the fermentation of her cream.

Simultaneously with the churning, preparations were made for the making of cheese. She made three kinds of cheese while I was there, sweet-milk cheese, Camembert cheese, and Myse cheese, and occasionally she made some half dozen other kinds of cheese, in all of which she was very expert. The sweet-milk cheese was begun first. As soon as the milk was received from the barn and it had been weighed and strained, it was warmed to $88^{\circ} \mathrm{F}$. by setting the cans of milk in kettles of warm water on the stove. To a hundred pounds of sweet milk she added 64 pounds of skim milk, so it was not strictly a full-cream cheese. When the milk had reached the temperature of $88^{\circ} \mathrm{F}$. the rennet was added.

She used rennet at the rate of two one-hundredths of a pound of the fluid extract to 100 pounds of milk. A little buttermilk was added along with the rennet. It was then allowed to stand undisturbed for twenty-five minutes, at the end of which time it had coagulated, when it was cut with a curd knife. After this it was gently stirred to clear the whey, and if the temperature had fallen during the interval it was warmed a little during the process of stirring. When the curd had settled to the bottom the whey was drawn off and the lump of curd quartered and the outside turned into the middle of the vat in order, as she stated, to drain it equally and make it of the same consistency throughout; otherwise it would have been firmer in the middle. When the whey had drained off, the curd was broken into large pieces by hand and these were packed in the cheese cup with layers of caraway seed. No salt was added, as it gets enough of that substance during the process of curing.

The form of the cheese cup should be mentioned. It could be enlarged by the addition of rings, and as the cheese was compressed and required less room these rings were removed. When the cup was filled it was placed under the cheese press, but only a very light pressure was applied. It was put in press at 9 a. $\mathrm{m}$. usually, and at $9: 30$ the cheese was turned and pricked through and through with a prong. It was again turned at 11 and again soon after noon, at 4 p.m., and at 9 p.m., but remained in the press until the following morning. It was then removed 
from the cup, rubbed with salt and putin a wooden bowl with a rounded bottom, where it stood for some time in order that the sharp edges might be removed and the cheese take a rounded shape. It was rubbed with salt and turned in this bowl three times a day for four days; then it was put in the cheese room and rubbed with salt once a day. This was continued for six weeks; it was then changed to another room of a slightly cooler temperature and rubbed with brine once every other day, which process was continued until the cheese was sold. She attached great importance to this frequent rubbing with salt. It not only makes the rind soft and practically prevents the formation of any rind whatever, but she called my attention to the fact that her cheese was covered with a fatty layer, which she said was drawn out by the salt, and which effectually prevented the access of air to the interior of the cheese. It is a well-known fact that the best cheese is cured in the absence of air in the interior. She made, at that time of the year, only one cheese of 16 pounds' weight per day. After about three months' curing, or longer, the cheese was sold, and it brought about 25 cents per pound, when the ordinary skim-milk cheese brought but from 4 to 5 cents per pound.

Camembert cheese is a kind of specially rich cheese which she makes from whole sweet milk every morning, as soon as the milk is drawn from the cows, weighed, and strained. It was my privilege to assist in making this cheese. The process was as follows: The fresh milk, warm from the cow, was warmed to $100 \circ \mathrm{F}$. At this temperature she added one one-humdredth pound of rennet for each 10 pounds of milk, after which it was gently stirred, and then allowed to stand for four and one-lıalf hours, when it had coagulated to the proper degree. The cheese vat used was a large earthen jar, which was kept wrapped up and placed near the stove so as to maintain the same temperature of $100^{\circ} \mathrm{F}$. until coagulation was completed. The curd was then cut into small squares, but not worked or pressed in the least. When cut sufficiently fine, curd and whey together were dipped out and put in small tin rings or molds with holes in the sides, but with neither bottom nor top. Each form, or mold, consisted of two rings, one placed on top of the other, and when thus arranged they were about 6 inches high and measured 5 inches in diameter. They rested on a strainer placed on top of a vessel, and over the strainer was laid a little mat made of clean, stiff, rye straws, single straws being tied parallel to each other. This was to drain off the whey, and the straw mats facilitated the turning of the cheese. When the cups were full they were left undisturbed for eight hours in a warm place near the stove. By the end of that time the cheese had settled so much that the upper ring or half of the mold was removed and the cheese was turned, but left in the cup over night. Next morning they were turned again and salted on one side. Three or four hours later they were again turned and salted on the other side. No pressure was applied at any time. During all this time the temperature did not fall below 
$78^{\circ} \mathrm{F}$. After the second salting it hat acquired sufficient solidity to be removed from the mold. It was then placed in a room with the temperature from $55^{\circ}$ to $60^{\circ} \mathrm{F}$., where it remained for two or three weeks, when it was placed in the cheese room with a somewhat higher temperature. It was ready for sale when two months old, and sold at 1 crown, or 27 cents, apiece and weighed three-fourths of a pound. She made about fourteen cheeses daily of this kind, from 80 pounds of milk, the total weight being 10 pounds of cheese. Some cream was sometimes added to the milk before it was curdled, if she had the cream to spare.

Myse cheese is made of the solid matter left in the whey after the water has been evaporated. It consists chiefly of milk-sugar, with most of the ash elements of the milk, and the albumen and fat not removed in cheese-making. It originated in Norway, where they use the milk of goats chiefly, which is said to produce the finest quality. But it can be made from any kind of whey, sweet milk, or skim milk, and, as a matter of fact, is so made. It therefore varies greatly in composition. Mr. Bòggild makes, on the authority of a Norwegian chemist named Werenskjold, the statement, that Norwegian Myse cheese will vary in composition as follows: Water, from 24 to 38 per cent; ash, from $5 \frac{1}{2}$ to $6 \frac{1}{4}$ per cent; fat, from 1 to 10 per cent; lactic acid, from $\frac{1}{10}$ to $\frac{1}{2}$ per cent; albuminoids, from $6 \frac{1}{2}$ to 9 per cent, and other substances, consisting chiefly of milk sugar, from 46 to 61 per cent.

Madame Nielsen allowed nothing to go to waste. Every morning the whey, which was obtained from her sweet milk and her Camembert cheese, was made into Myse cheese and, to give it the genuine flavor, she kept half a dozen goats, whose milk also contributed to it. As soon as all the whey was collected it was divided between half a dozen enameled pots, each holding several gallons, and these were placed over the cook stove and here the whey was evaporated, being constantly kept at the boiling point while it was continuously and violently stirred. This kept all her pupils busy for a couple of hours. As the whey evaporated, so that the contents in the pot became less, it was poured into an adjoining pot until there was only one kettle over the fire. As the mass thickened the stirring increased, and finally it became so thick that it could no longer be prevented from burning, It was then put into a trough, where it was pounded and beaten with wooden clubs like potato mashers, with all the might the young ladies possessed and, when made perfectly homogeneous, it was tamped closely into a square wooden mold and put under pressure. It forms a greenish-yellow, sweetish mass, rather agreeable to the taste and very nutritious. No rennet is added, nor any flavoring whatever. It is ready to eat as soon as it comes out of the mold, but it will also keep for a couple of months. It should be kept in a covered vessel to prevent it from drying up. This cheese sold at the same price as the sweet-milk 
cheese. During the summer season she made also Chester cheese, Gorkonsola, Edam cheese, and other kinds.

There is a lesson to be learned from this remarkable woman. With no advantages in education or resources above those possessed by the vast majority of her sisters, she has, by devoting herself to the household arts with energy and skill, attained not only an international reputation, but has accumulated a comfortable little fortune. Fig. 22 shows a perspective of her house and barns, and Fig. 23 a plan of the same, in which the dairy rooms are pointed out.

FARM OF P. PEDERSEN.

I will next briefly describe a small place which I visited in the village of Sóllinge, on the island of Funen. The place is owned by a school teacher, Mr. P. Pedersen, who teaches school daily in addition to overseeing the work on his little farm. The property consists of 7 acres, which he described to me as a soil of ordinary quality with a clay subsoil. He has owned it since 1876. About one acre was occupied with buildings and garden; the garden was closely planted with apples, pears, cherries, and between these gooseberries, currants, and raspberries. The remaining 6 acres were divided into five lots, onefifth being in perpetual meadow. The other four parts are cultivated as follows, in a four-year rotation: The first year, one-third of one of these quarters was in rye or wheat, one-third in some soiling crop, chiefly a mixture of vetches and oats, and one-third was given up to the production of seed of turnips, mangels, and cabbage. A second quarter of the small farm was given up to roots for the cattle, potatoes, and green peas, the latter being raised for market. Of the third quarter, two-thirds was sown in barley or a mixture of barley and oats, which was used for feed, and one-third was given up to a variety of vegetables. The last quarter was two-thirds in grass and one-third in vegetables. This rotation was followed constantly; thus, the quarter with rye and soiling crops one year was the next year planted with roots, potatoes, and green peas; the third year with barley and vegetables, and the fourth year with grass and vegetables. High manuring and thorough culture make it possible to keep four high-class dairy cows and one heifer on this very small farm.

Every particle of manure was saved. The liquid manure was collected in a cistern and applied to the grass land, to the fruit trees, and plats devoted to seed growing, and the solid manure was applied to the root crops, the soiling crops, and to the vegetables. Besides the cows he kept two brood sows and yearly fattened 8 or 10 head of swine, all from the products of this little place. But with all this live stock, which produced considerable manure, he informed me that he still bought yearly 200 pounds of kainit, 400 pounds of Thomas slag, and 200 pounds of Chile saltpeter, which were applied to the grain erops. The dary cows were fed grain both summer and winter. The winter ration 
consisted of a mixture of oil cake, bran, and barley and oats ground together. The quantity given each varied with the amount of milk she produced, but the feed was not excessively heavy, usually from 8 to 10 pounds of this mixture perday, in addition to which each cow would get 40 pounds of roots, 4 pounds of hay, and 8 pounds of straw. During the summer the cows were, for the mostpart, fed in the stablealso, but were tethered on good pasture an average of two hours daily, and in addition each cow received about 4 pounds of grain daily.

I was interested in this very intensivw system of agriculture and procured detailed accounts of receipts and expenditures. During the year 1892 the five head of cattle mentioned consumed 10,788 pounds of grain feed of the mixture above mentioned, valued at 608 crowns (\$166), and they also consumed roots and hay valued at 292 crowns (\$64). Total expense for feed, $\$ 230$, all of which, except the oil cake, was produced upon the place. The cows gave in return 30,438 pounds avoirdupois of milk, which, at the price received for milk at the coöperative dairy, was worth 1,073 crowns ( $\$ 294)$. This left but $\$ 64$ profit, but he placed a very high estimate on the feed, and the milk was valued at only factory prices. As a matter of fact, more was made from it by making it into butter and cheese at home, and the sale of a heifer brought 165 crowns $(\$ 45)$. The swine ate 8,845 pounds grain, together with the whey and skim milk not otherwise used, and offal from the garden, on which he put a total value of 745 crowns (\$204), which seems a high valuation. The receipts were 902 crowns (\$247), from seven fat swine and twentyone sucking pigs, but not including two or three head slaughtered for home consumption. I did not receive details of the fruit, vegetables, and seed sold, which must have been no inconsiderable item. These articles would have brought still better prices if the place had been located near the city, where they could have been sold to the best advantage. But, as it is, it is a fine example of the possibilities of a small place when properly attended to.

FARM OF HANS HANSEN.

I next visited another farmer in the same neighborhood, Hans Hansen, of Sóllinge. He owus a farm of 93 acres and he had, at the time of my visit, 32 cows, 16 heifers, and 3 bulls. His cattle were of the best strain of the red Danish dairy cattle. He was, in fact, a breeder of fine dairy stock as well as a farmer. Several of his cows gave between 50 and 60 pounds of milk daily, and he got in the neighborhood of $\$ 100$ apiece for promising heifers. The average milk yield of his cows had been up to 8,800 pounds per cow, young and old, yearly; but during the last two years his herd had been troubled with abortion, which had somewhat reduced the milk yield. Still, during the year 1892, his 32 cows produced 226,317 pounds of milk, avoirdupois, which was sold to the coöperative creamery, besides what was used in the household, conceruing which I did not get details. This brings the 8538-No. 5-6 
average to 7,072 pounds per cow for the milk sold. His cattle are fed in the following mamner: During the winter, first feed was given at $6: 30$ a. $\mathrm{m}$., and consisted of 6 pounds of hay, and a little later threefourths of a bushel of mangels. He used oil cake to a considerable extent, each cow getting about 6 pounds per day when in full flow, which was divided into two feeds, each feed being mixed with an equal weight of ground oats. His oil cake consists of equal parts of cottonseed cake and sunflower cake, rape-seed cake and hemp-seed cake. After 3 pounds of this oil cake and 3 pounds of oats had been eaten the cows were watered, and then followed a feed of barley or oat straw, which finished the feeding for the forenoon. The afternoon feeding began at $3: 30$, this time with the roots, then grain, as above, water, and straw for the night, no hay being given in the afternoon. The cattle remained in the barn all winter without being let out once; the manger was built water-tight and the water turned into it twice a day, and what was not drunk was again drained off.

His barns were models of solidity and convenience. They were built of brick, vith floors of squared stone and stalls and all auxiliaries of the most substantial nature. The drainage of the stables was well nigh perfect. Nothing was allowed to go to waste. The urine was collected in large cisterns, from which it was pumped out and hauled to the grass lands during the spring and summer, and the solid manure was kept under shelter. In summer the whole herd was tethered in the pasture, or stall-fed with soiling erops if the pasture was at all short. $\mathrm{He}$ practiced a seven-year rotation, as follows: First year, oats, half of which is sown to grass; second year, this half remains in grass and the other half is sown in soiling crops, which is either a mixture of vetches and oats or field peas and oats; third year, it is in rye; fourth year, three-fourths is in roots and one-fourth in barley; fifth year, oats sown with clover and grass seed; sixth year, meadow, and seventh year, pas. ture, which ends the rotation, the pasture being again broken up for oats. The manure is applied chiefly to the grass land and to the roots. The owner is now past middle age, but still active and energetic. By careful attention to his farm he has accumulated a fortume which euables him to present each of his several children with a confortable farm, while he and his wife will live in comfort during their coming old age.

FARM OF CHRISTIAN RASMUSSEN.

I next visited several small farms on the island of Langeland, some of which I shall describe. In the village of Clansebólle I had a talk with a farmer named Christian Rasmussen. He owns 32 acres of land, on which he kept two horses, nine cows, several head of young cattle, besides lialf a dozen hogs, and a lot of poultry. His cows were of a good ordinary quality, but made no pretentions to be pure-bred stock. The nine cows averaged 6,600 pounds avoirdupois of milk per year. 
The products of his dairy netted him in 1892 about $\$ 55$ per cow, not counting the labor; this is very nearly equal to $\$ 500$ from the cows alone. He sold pigs for about $\$ 54$, and wheat for about $\$ 200$. This makes a total of nearly $\$ 750$ as the receipts from this little farm. He followed a six-year rotation: First year, fallow; second year, wheat; third year, barley; fourth year, oats which were sown to grass; fifth and sixth years, pasturage and hay. This rotation is not so well arranged as it might be. As is the case on all other successful small farms, nothing is allowed to go to waste. Every particle of manure is saved and applied to the land. The solid manure is for the most part put on the fallow ground, as this is to be sown in wheat. The liquid manure is applied to the pasture in the spring.

FARM OF RASMUS ANDERSEN.

Rasmus Andersen, in the hamlet of Tressebólle, is one of the fore most farmers in that region, who also gives much attention to dairying. His farm is not so heavily stocked as is the case on some others. He owns 107 acres and this he works with five large horses, and he keeps from 20 to 25 milch cows, besides young stock and swine. He sells the milk to a neighboring coöperative dairy, except what is used in the household. During 1892 he sold milk for 4,489 crowns $(\$ 1,230)$ from 21 cows, being an average income of $\$ 58.50$ per cow.

The cattle were of the red Danish breed and were superior animals. During the winter they were cared for as follows: At 6 a. m. they got a feed of wheat straw, and the milking took place while they ate this. About 7 o'clock they were watered, aud immediately afterward they had a feed of chaffed barley or oat straw, which was moistened and mixed with the grain feed. The grain consisted of one-half pound of rape-seed cake, one-half pound of sunflower cake, 1 pound of bran, and 2 pounds of mixed barley and oats ground together. This was the ordinary grain feed which they had twice a day, but extra heavy milkers received a little more. When this was eaten they had a feed of hay, all they would eat up clean, and this was followed by a feed of straw, which completed the feeding for the forenoon. In the afternoon the same routine was repeated of straw, water, grain feed, or chaff, hay and straw, the milking taking place at $6 \mathrm{p} . \mathrm{m}$. The cattle remained in the barn all winter, not being let out once. They were watered in the manger, which was built of tiling and water-tight. In the summer the cattle were lariated in the pasture and received no grain except when the pastures were short; but pains were taken to provide first-class clover pasture.

Mr. Andersen followed an eight-year rotation: First year, bare fallow, which was heavily manured and which received four plowings during the summer and was sown in wheat in the fall. I was struck with the heavy seeding which he practiced; he never sowed less than two bushels of wheat per acre and preferred broad casting to drilling; 
second year, wheat; third year, barley, which was sown with clover and mixed grasses. In the fourth year this was pastured, and in the fifth year cut for hay and was then manured and plowed up in the fall; and the sixth year sown in soiling crops, consisting of oats and peas mixed, or oats and vetches. This was fed in the stable when the pastures were short. The ground was plowed as fast as cleared; and the seventh year it was sown in barley and a portion in carrots, which was followed the eighth year by oats.

Mr. Andersen was highly impressed with the value of carrots for both cattle and horses, and he was planting increasing areas in this crop and intended, as soon as practicable, to raise enough to give the cattle at least one feed daily all winter. He recommended especially a yellow variety called "Long Green Head." The cattle eat the tops, as well as the roots. He raised them in rows one and one-half feet apart.

FARM OF ANDERSEN BROS.

I next visited a farm near the village of Leibólle, owned by two brothers named Andersen, who worked it in company. Their farm consisted of 66 acres of land on which they kept three good horses, or sometimes four, sixteen cows, some six or eight head of young stock, besides some swine and a few sheep. Their cows were of the red Danish breed and were considered superior animals, several of them milking between 40 and 50 pounds each per day. Their milk was sold to a neighboring coöperative dairy at fixę prices. The receipts from these sixteen cows during the year 1891 were as follows, by months. I am able to give this as they kindly permitted me to look into their account books:

January, 265.96 crowns; February, 310.91 crowns; March, 398.84 crowns; April, 380.88 crowns; May, 346.38 crowns; June, 292.15 crowns; July, 273.64 crowns; August, 250.44 crowns; September, 239.00 crowns; October, 169.93 crowns; November, 226.99 crowns; December, 328.06 crowns; total, $3,483.20$ crowns; to which should be added 864.88 crowns, as their pro rata share in the profits of the creamery for the year. Grand total, $4,348.08$ crowns $(\$ 1,191)$ as the receipts from sixteen cows in one year. The cows were fed as foliows: At 6 a. m. a feed of straw, which they ate while the milking proceeded. Next a feed of moistened chaff made from barley or oat straw mixed with the grain feed. The grain feed consisted of 2 pounds of sunflower cake and 2 pounds of bran. When this was eaten they were watered and after their water they had a feed of hay. In the afternoon about 3 o'clock each cow got a feed of 50 pounds of roots, mangels, or earrots, while they lasted; at 4 p. m., again moistened chaff and grain. This time the grain consisted of 1 pound of rape-seed cake and 3 pounds of equal parts of barley and oats ground together, then they were watered. Next followed a feed of hay, and lastly, about 6 p. m., a feed of straw. The cattle stand in the stable all winter. In 
Sv:mmer they were tethered on good pasture until harvest, except for a period during the last of June and beginning of July, when they were again stabled and fed on soiling crops. After harvest they run loose in the stubble fields. They get 3 pounds of bran per hearl daily during the summer. All the manure produced upon the place was saved with great care. The urine from all the stock was collected in cisterus and applied to the grass lands in the spring. Their system of rotation was as follows: First year, fallow, which was plowed several times and manured, and in the fall sown to wheat. They seeded also very heavily, using seed wheat at the rate of $2 \frac{1}{2}$ bushels per acre. The yield of wheat one year with another had for several years averaged 55 bushels to the acre. Second year, wheat; third year, barley, which was sown at the same rate and yielded about 50 bushels to the acre; fourth year, mangels and potatoes and a soiling crop consisting of oats and vetches grown together, the three occupying nearly equal areas; fifth year, oats, which were sown at the rate of 4 bushels to the acre and yielded, on an average, upwards of 80 bushels to the acre. The oats were sown with grass and clover. Sixth year, grass, used for pasture and hay; and seventh, grass, used for pasture and hay, which ended the rotation, the pasture being broken in the fall of the seventh year and followed by fallow. The two young men who owned an $l$ worked this farm had received more than an average education and were fine types of the best class of small farmers.

FARM OF P. NIELSEN.

On leaving the island I met a prominent breeder at an inn and had an interesting talk with him in regard to his business; but I did not see his place or any of his stock, it being too much out of my way to go there. His name was P. Nielsen, and he lived in the village of Torpe, Langeland. He informed me that he owned 69 acres of land, on which he kept 14 cows and about 6 head of young stock, the number he usually reared annually. His cows were of the red Danish breed, and several of the best ones gave each between 10,000 and 11,000 pounds of milk per year. All of his stock were recorded in the herdbook and he sold his heifers at high prices. He managed hiscows somewhat differently from what is customary there, in that he fell them oftener. Each cow in full milk got 6 pounds of mixed barley and oats grouud together, 2 pounds of bran, and 2 pounds of sunflower cake daily, this being divided into three feeds. The first feed was given at 5 a. m., consisting of straw; next they had one-third of the grain mentioned, then three-fourths of a bushel of roots, then a small feed of hay and again a feed of straw, and this course was repeated twice more during the day. Mr. Nielsen followed a seven-year rotation: First year, fallow, which was manured and sown to wheat in the fall; second year, wheat; third year, oats, laid out with clover and grass; fourth year, pasture; fifth year, hay; sixth year, half roots and half barley and oats 
mixed, which is grown for feed; seventh year, barley. He stated that his yields were about as follows: Wheat, 50 bushels to the acre; oats, 70 to 75 bushels; barley, about 50 bushels. Mr. Nielsen was superintendent of one of the many "bull associations" which are common in the country and of which I have already written. His association was a stock company of farmers, who bought bulls of superior quality, to be used on the cattle in their herds. These bulls were boarded out at various places, always, however, with some member of the company. Mr. Nielsen's association owned seven such bulls. The service fee to members was 2 crowns, or about 55 cents, and to non-members it was about $\$ 1.35$.

ESKELUND DAIRY FARM.

This farm, on the island of Funeu, was cultivated by a tenant farmer, who was absent at the time of my visit, and the following facts in regard to the place were obtained from the foreman: The farm is 240 acres in extent, soil of only fair average quality. They kept 12 work horses, 3 brood mares, 50 head of dairy cows, 2 stock bulls, a varying number of young cattle, which, however, amounted to as many during the year as there were cows. The cattle were all highly-bred, herdbook stock, and all the calves were saved. Besides this they had from 50 to 60 head of swine and the usual complement of poultry of all kinds. This herd is known all over the country, it being one of the most highly-improved herds of the red Danish cattle. It has been bred with care for about fifty years, and the herd has acquired a uniformity in color and build which is not always found in highly-bred stock. All the cattle were solid red and of an average weight of hetween 1,000 and 1,100 pounds. They were stabled in a fine brick baru, where they remained all winter, not being let out for water. Their feed during the winter season was as follows: at 4 a. $m$. they got a feed of barley straw, which they ate while the milking was in progress. At 6:30 they were watered, after which they got a feed of grain, consisting of 2 pounds of barley and oats mixer and ground, 1 pound of bran, one-half pound of palm-nut cake, one-half pound of rape cake, and one-half pound of sunflower cake. After this came a feed of roots. At 8 a. m. a small feed of hay was given and at 8:30 a. m. straw. At $11: 30$ a. m. they were again watered. The afternoon feeding began at $1: 30 \mathrm{p}$. $\mathrm{m}$. with a feed of straw and then the rest in the order as given above, with the exception of water. The management of the calves is of interest. Up to 2 weeks old they were fed on milk from their dams, each calf getting his own mother's milk, but about the tenth day they begin to mix in skim milk, the quantity being daily increased until in a few days the sweet milk is entirely supplanted, when they continue to get warm skim milk three times daily. But no one calf is ever allowed more than 24 pounds in a day, and that much only when about 2 months old. Soon after the skin-milk feeding begins a little linseed-oil meal is mixed with it and later 
ground oats, with hay and roots constantly before them. When between 5 and 6 months old the skim milk is gradually replaced with whey, which they get for a few weeks. The calves are pastured in summer and receive, in addition, some oil meal and ground barley and oats mixed. The heifers are bred to calve at 2 years old, and they calve at all seasons in order to have a moderately regular supply of milk. It is no small amount of work to feed some 30 or 40 head of calves in the above manner, each one having his own special "bill of fare." I was told that all the young stock sold readily at high prices. Bulls often brought 700 crowns $(\$ 190)$ apiece. The tenant farmer, Mr. R. Christiansen, is one of the most enlightened men in the neighborhood. He exhibited his cattle at all the leading shows and always carried off prizes. In 1378 he was awarded a silver medal at the Paris Exposition for a herd he had sent there. The cropping of the farm was an eight-year rotation, beginning the first year with fallow, which was manured. It was followed the second year with wheat and rye, and the third year, barley. The fourth year it was again manured for soiling crops, consisting of oats and vetches mixed, or oats and peas and mangels; fifth year, mixed grain, barley and oats used for feed, and with this laid out to clover and grass; sixth year, hay; seventh, pasture, the cattle being tethered in the field; and eighth year, oats, which completed the rotation.

RAVNDROP VANZGE FARM.

In the same neighborhood I visited another farm owned by Mr. P. Nielsen. This farm consisted of 84 acres of only medium quality soil, it being rather light, but it sustained 4 good horses, 40 head of cattle of all ages, and a small herd of swine. The cattle were all of the red Danish breed, purely bred and recorded stock, and the herd contained some of the best individuals of that breed. It was in many respects a model place. The barn was fireproof, built of brick with iron posts and well lighted and ventilated, and the floor was made of cement, perfect drainage being provided. At the time of my visit he had 20 cows, 7 yearling heifers, 3 bulls, and 12 calves. Mr. Nielsen practiced in-and-in breeding closely and so far with the best results. The cattle were of the usual size of that breed, between 1,000 and 1,100 pounds avoirdupois in weight. They were fed as follows: The first feed was given at $7 \mathrm{a} . \mathrm{m}$., which was a grain feed and consisted of one-half pound of rape-seed cake, one-half pound of palm-nut cake, 1 pound of sunflower cake, one-half pound of hemp-seed cake, three-fourths of a pound of cotton-seed cake, one-half pound of bran, and 1 pound of ground barley and oats. This was for the best cows, however, those not in full milk not getting so much. When this was eaten they had a feed of straw; third, about 25 pounds of mangels per head; fourth, a small feed of hay; fifth, water, and sixth, a feed of straw, which finished the feeding for the forenoon. At $3 \mathrm{p}$. $\mathrm{m}$. the afternoon feed- 
ing began exactly in the same order as above, the last feed being straw, which was given at $5 \mathrm{p} . \mathrm{m}$. They are watered in the stable, which is customary everywhere, and are not turned out all winter. They are milked three times a day both winter and summer. The first milking begins at $6 \mathrm{a} . \mathrm{m}$. in the winter and at $4 \mathrm{a} . \mathrm{m}$. in the summer. During the summer the cows which are in milk are let out two hours each day on good pasture; but for the rest of the time they are in barns where they are fed equal parts of palm-nut cake, sunflower cake, and bran to the amount of 4 or 5 pounds a day in two feeds. Besides this they get green clover, or green oats and vetches growu together, and some little hay. All the grain is fed dry. Heifers and cows not in milk are tethered in the pasture most of the time. In the beginning of the ' 60 's Mr. Nielsen's father procured a couple of fine heifers, which became the foundation of this very interesting herd, and during the last fifteen years the herd has been bred in-and-in closely.

A few figures in regard to the feed and produce of this herd may be of interest.

Average consumption of feed and yield of milk per cow, including heifers and barren cows.

\begin{tabular}{|c|c|c|c|c|c|}
\hline Year. & $\begin{array}{c}\text { Grain and } \\
\text { oil cake. }\end{array}$ & Roots. & Hay. & $\begin{array}{c}\text { Green } \\
\text { fodder. }\end{array}$ & $\begin{array}{l}\text { Yield of } \\
\text { milk. }\end{array}$ \\
\hline 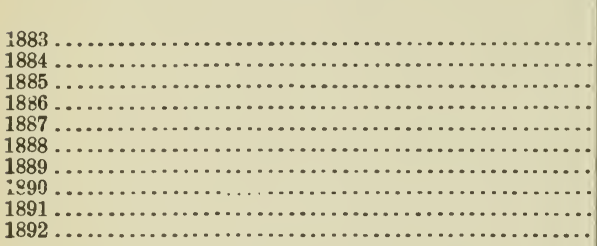 & \begin{tabular}{r|} 
Pounds. \\
3,033 \\
2,910 \\
2,904 \\
2,910 \\
2,922 \\
3,148 \\
2,447 \\
3,195 \\
2,663 \\
2,787
\end{tabular} & $\begin{array}{r}\text { Pounds. } \\
4,620 \\
3,938 \\
4,103 \\
5,170 \\
11,110 \\
11,770 \\
10,560 \\
10,569 \\
9,534 \\
12,079\end{array}$ & \begin{tabular}{r} 
Pounds. \\
\hdashline. \\
607 \\
968 \\
484 \\
404 \\
610 \\
830 \\
1,479 \\
1,513
\end{tabular} & $\begin{array}{l}\text { Pounds. } \\
\cdots \\
12,914 \\
11,704 \\
12,056 \\
11,528 \\
17,875 \\
12,205 \\
11,616 \\
12,848\end{array}$ & $\begin{array}{r}\text { Pounds. } \\
7,870 \\
7,694 \\
7,887 \\
8,083 \\
8,082 \\
8,516 \\
8,373 \\
9.253 \\
8,322 \\
8,795\end{array}$ \\
\hline
\end{tabular}

Yields of some of the individual cows in the herd._-"Tolly" gave as a two-year old, 8,195 pounds of milk avoirdupois; at 3 years, 9,473 pounds ; at 4 years, 9,744 pounds; at 5 years, 11,327 pounds; at 6 years, 10,080 pounds; at 7 years, 11,982 pounds; at 8 years, 13,043 pounds; at 9 years, 13,171 pounds. The latter figure is her yield in 1892. "Habele" gave, as six-year old, in 1892, 12,056 pounds of milk. "Anmaette" at 9 years oli in 1892, 12,219 pounds. "Purtse" at 8 years old in 1892, 11,044 pounds. These are some of the very best yields of the breed.

Mr. Nielsen practiced a six year rotation, as follows: First year, rye; second year, barley, for which he uses a light dressing of Chile saltpeter; third year, mangels; fourth year, oats, which is laid out with clover and grass; fifth year, hay; and sixth year, pasture until July, when it is manured and broken for rye, being plowed three times before seeding in the latter part of September. All the manure produced upon the place is carefully saved. 
HOLEV FARM.

In the village of Holev, some miles from the city of Odense, is a farm belonging to Jens Hansen's widow, which is one of the many interesting smaller farms in the neighborhood. It is superintended by her son, a young man who has the advantage of a good erlucation, but the credit for the present condition belongs to the young man's father, who died only two years ago. The farm contains 125 acres, 19 of which are taken up by building sites, garden, orchard, farmyard, and a large wood lot. The remaining 106 acres are under culture. On this they had, at the time of my visit, 7 work horses, 2 colts, 26 cows, 8 heifers, 6 bulls, and 12 calves, besides 19 head of hogs, several of which were brood sows. The cattle were all of the red Danish breed and recorded in the herdbook. Jens Hansen had been a noted breeder ever since 1838, but the present herd has been bred since 1852, when he purchased a cow which proved to be of superior value and from which most of the herd has sprung, though some ten years later he procured three other excellent cows, whose progeny are in the herd. The young stock sells at good prices. A choice heifer, or bull, frequently brings as much as $\$ 270$. I saw a three-year-old bull there for which I was told they had been offered about $\$ 378$, but refused the offer. The cattle are fed in the following manner: The first feed consists of straw and is given at $5 \mathrm{a} . \mathrm{m}$. when they are milked, and afterwards the stable is cleaned. Next, they are watered at 7:30 a. m. and immediately after they get a grain feed consisting of one-half pound of rape-seed cake, one-half pound of palm-nut cake, one-half pound of sunflower cake, one-half pound of cocoanut cake, $1 \frac{1}{2}$ pounds of mixed barley and oats ground, and 1 pound of bran. At 8:30 a. m. they get a small feed of hay. At 9 they get about 25 pounds of mangels and at 10 a feed of straw. The stable is then closed and kept as quiet as possible until 12, when they are again milked. After another season of rest the feeding begins again at $2: 30 \mathrm{p} . \mathrm{m}$. and consists of the same material in the same order, with the exception that they get no hay, but instead a feed of straw. In the evening they are milked at $7: 30$.

In summer the herd is let out some time during the first half of May, but is taken in at night, if the season is cold and rainy. They are tethered on the pasture until after harvest, when they are turned loose in the stubble field. During hot weather and when the flies are bad they are taken in during the day and let out at night, and in the fall again they are taken in at night and are out only in the daytime. If the grass gives out in the latter part of June, as is often the case, then they are put in the stable and fed on soiling crops until the pastures improve. During the entire summer each cow gets from 4 to 6 pounds of grain daily, half of which is oil cake and the other half barley and oats mixed. As in former cases, already mentioned, the calves receive special care. Each calf is kept in a pen by itself and fed on its mother's milk until 2 weeks old. The whole milk is then gradually supplanted 
by skim milk, and in the course of eight to ten days they get nothing but skim milk. The quantity allowed them is also carefully graded. The first day they get but $1 \frac{1}{2}$ pounds of milk in three feeds. When a week old a thrifty calf is allowed $1 \frac{1}{2}$ pounds three times a day, and when 2 weeks old 4 pounds each time, three times daily. In addition to this, they get crushed oats and linseed cake, the latter stirred in the milk as soon as they will eat it, and a little cut roots and hay constantly kept before them. When a month old they get 8 pounds of skim milk at each feed, three times daily, the milk being warmed to the temperature of the body. They never get more milk than this at any age, but the grain is gradually increased to 2 pounds daily, besides what roots and hay they will eat. They get no water at all while they are fed milk. Fall calves, which have gone through this course of treatment during the winter, are put on pasture the follow. ing summer, but spring calves are kept in the barn all summer, and in addition to the above treatment get a little cut grass daily. The grain continues, however, all summer for those on pasture. The following winter the yearlings get 3 pounds grain daily each, and about 30 pounds of roots. The heifers are bred when 15 months old, and after they calve the grain is increased gradually, as they can take it, up to the normal feed for cows. The cows have a disposition to milk perpetually, but they are dried up for six weeks, during which time each gets 6 pounds grain daily and 40 pounds roots, if her dry season happens to be in the winter. Their service bulls were fed from 4 to 6 pounds of oats daily, but no oil cake or any other kind of grain; in addition to this they got 25 pounds of mangels and all the hay and st raw they would eat.

The milk was creamed and churned at home. There was a convenient ice dairy in the basement of the dwelling house where the milk was set in the usual tall tin cans and skimmed by hand. There was no separator on the place. The following: are the outputs of milk and butter for each month during the year 1892, in pounds avoirdupois. The milk yield does not include the sweet milk fed to the calves, nor the new milk from fresh cows:

\begin{tabular}{|c|c|c|c|c|}
\hline Month. & $\begin{array}{l}\text { Milk } \\
\text { yield. }\end{array}$ & $\begin{array}{c}\text { Used in } \\
\text { household }\end{array}$ & $\begin{array}{l}\text { Milk creamed } \\
\text { and churned. }\end{array}$ & $\begin{array}{l}\text { Butter } \\
\text { marle. }\end{array}$ \\
\hline anury & Pounds. & Pounds. & $\begin{array}{l}\text { Pounds. } \\
14,070\end{array}$ & Pounds. \\
\hline ebr & $\begin{array}{l}14,617 \\
14,617\end{array}$ & 440 & 14,177 & 528 \\
\hline Marc & 18, & 440 & 17,578 & 698 \\
\hline Apri & 20 & 440 & 20,464 & 782 \\
\hline May & 19,644 & 440 & 19,204 & 737 \\
\hline Jun & 19,504 & 440 & 19,064 & 667 \\
\hline July & 17,360 & 440 & 16,922 & 602 \\
\hline Aug & 13,129 & 440 & 12,689 & 471 \\
\hline Sep & 10,511 & 440 & 10,071 & 387 \\
\hline Octol & 11,089 & 440 & 10,649 & 427 \\
\hline & 12,042 & 440 & 11,602 & 4.48 \\
\hline December.. & 10,945 & 440 & 10,505 & 389 \\
\hline
\end{tabular}


I was kindly permitted to draw these figures from their account book. The processes followed in the butter-making were the same as those already repeatedly described. The butter sold for the same prices as were realized by the large dairies. Cheese was made of the skim milk, but only for home use, though a small surplus had been sold during the year to the value of about $\$ 54$.

The ground was cultivated in a seven-year rotation. First year, oats, for which the pasture had been broken the previous fall; second year, roots, chiefly mangels, but also şome carrots and turnips, the first two being for the milch cows and the last for the young stock. This crop is manured; third, two-thirds of the field in barley and one-third sown in Italian rye grass and yellow clover; fourth, the two-thirds which was in barley was manured and sown with soiling crops, vetches, and oats mixed; fifth year, the one-third which was in grass during the third and fourth years of the rotation is broken and sown in barley, which is cut for hay in June, and the two-thirds which was in soiling crops lies fallow and is plowed two or three times, and the whole field sown in wheat or rye in the middle of September. Sixth, this rye field is fed off and half of it sown in red clover and grass and the remaining half sown with barley and oats mixed, which is laid out with grass; seventh year, all is in grass.

The milk yield, not of the best cows, but of the whole herd including heifers, barren cows, and those that aborted, has averaged the following amounts per head for the years mentioned, in pounds avoirdupois: In $1886,7,453$; in $1887,8,525$; in $1888,8,074$; in $1889,6,921$; in $1890,7,067$; in $1891,7,619$; in $1892,8,145$. This, of course, gives no indication of the yield of the best cows, which runs up to 10,000 and 11,000 pounds each.

To show the possibilities of such a dairy on a 100-acre farm I drew from their books the sales and prices realized during 1892:

\begin{tabular}{|c|c|}
\hline Products sold. & Prices. \\
\hline 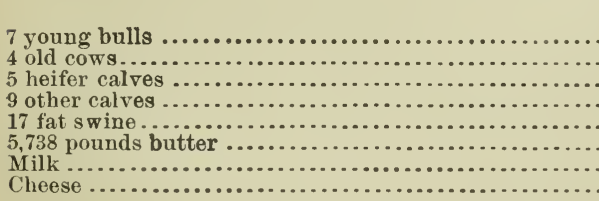 & \begin{tabular}{|r} 
Crowns. \\
3,150 \\
740 \\
1,065 \\
410 \\
1,339 \\
5,049 \\
24 \\
200
\end{tabular} \\
\hline 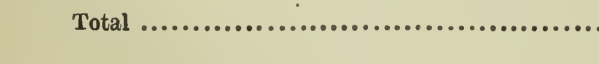 & $\begin{array}{r}11,977 \\
(\$ 3,282)\end{array}$ \\
\hline
\end{tabular}

Cost of bran, grain, oil cake and all the feed bought for the herd 3,027 crowns (\$829). The rest of the feed was produced upon the place. The herd is exhibited in all the leading shows in Denmark and sometimes in foreign countries. In 1879 representatives from this herd took first premium in London, England. Since 1859 the herd has twice 
taken sweepstakes, in competition with the whole country, and taken sixty-six other prizes, of which thirty-eight were first prizes.

HERMANS MINDE FARM.

This property is owned by a Mr. Hermansen, and is situated on the peninsula of Jutland, a few miles from the city of Fredericia. It is a beautiful place, with a large, rather imposing brick dwelling house, substantial brick barn, and supplied with all modern conveniences. The cow stable was especially large and airy, built of iron and brick, so as to be entirely fireproof, with a good floor, and well drained. The property consisted of 150 acres, which was worked with 6 horses, and there were at the time of my visit 36 head of dairy cows, 13 head of young stock, a few sheep, and some 30 swine. The cattle were treated in the following way: They were watered the first thing in the morning, at 5 a. $\mathrm{m}$; then they were given a feed of grain consisting of onehalf pound of rape-seed cake, 2 pounds of mixed barley and oats, ground, and 2 pounds of bran per head; after this they got a feed of straw, then a small feed of hay, then a feed of straw again, when they rested until $1 \mathrm{p} . \mathrm{m}$. At this time they were again watered and then given the same routine of feeds as in the forenoon. Some roots were raised, but seldom enough to last through the winter. No beeves were fattened except such cows as did not prove profitable for the dairy. The herd remained in the stable all winter, and they were also st ibled every night during the summer, when they were fed soiling crops and hay, the object being to save the manure. The cattle were of a rather mixed character, none of them pure bred, though most of them belonged to the red dairy breed. The average milk yield per cow approached 6,000 pounds avoirdupois. The dairy department was in charge of a grown daughter, who kindly gave me details concerning her management.

The dairy room was located in the basement, at one end of the dwelling house, and was very conveniently arranged. It was an ice dairy, a capacious ice house near by being filled every year. Instead of the cement basins to hold the ice water, large tubs were used. These have some advantages over the brick and cement basins. They are very much cheaper, in the first place, and they are more readily cleaned and can be moved to the most eonvenient point in the dairy. These tubs were a few inches taller than the milk cans and each would hold nine or ten cans (see Fig. 4). A deep, cool well supplied all the water wanted, of the purest kind. It was raised to a tank on top of the house by means of a pump worked by horse power and from this tank pipes supplied water wherever needed. At the time of my visit 28 cows were giving milk, but many of them were nearly dry. This number supplied 500 pounds of milk daily. As soon as it was received from the barn, and was strained and weighed, it was transferred to the eans, which were sunk in the ice basins, where it remained thirty-six hou's; but the milk was skimmed three times during that time, at the expiration of 
twelve hours, twenty-four hours, and thirty-six hours. The cream was kept in ice witer until enough was collected for a churning; then it was warmed to a temperature of $70^{\circ}$ to $74^{\circ} \mathrm{F}$., when half a gallon of buttermilk from last churning was added. It then stood for twenty-four hours packed in a hay mattress, the object being to keep it as nearly as possible at about $60^{\circ} \mathrm{F}$. The process of souring was watched in the meantime and if too rapid the temperature was reduced. She churned, at that time of year, at $54^{\circ} \mathrm{F}$. The churn was of the pattern already described; in fact, I saw no other styles anywhere. She churned by horse power, and the butter always came in about thirty minutes. The butter was removed from the buttermilk with a sieve and worked by hand. It received three workings. At the iirst one, the buttermilk was pressed out. Soon after this 4 per cent of salt was added and worked in a little. It was then put in a cooling box and about an hour later it received a final working, when it was packed in butter barrels of the customary form. It required about 27 pounds of milk to make a pound of butter. In the fall, when many of the cows were about to go dry, the milk was more difficult to manipulate; the cream did not rise so readily, nor did it churn so well. She then set the milk in shallow iron pans, of the style already described, the pans being used also for the reason that they save ice. The ice supply usually runs short at that time of the year. She had not found it necessary to use pure cream cultures. The butter was first class and usually brought about $\$ 1.08$ per 100 pounds over the top quotation of butter by the exchange in Copenhagen.

All the skim milk which was not used in the household, or for pigs and calves, was made into cheese. Her process was as follows: The cheese vat was a round tub which could hold about 400 pounds. The milk was warmed to $86^{\circ} \mathrm{F}$., when she added 12 per cent of buttermilk. Then she added four one-hundredths of a pound of rennet extract to 350 pounds of milk. It was then allowed to stand thirty minutes to coagulate, when the curd was stripped and the whole mass stirred for fifteen to twenty minutes until the whey became clear and the small grains of curd would break when pressed. The whey was then drawn oft and the curd put at once in the cheese cup. Her practice differed at this point from most other cheese-makers in that she did not permit the curd to be worked before it was put under the press. This, she maintained, would make the cheese tough; nor should it be allowed to cool before it was put under the press. The pressure applied is very light at first, and in half an hour the cheese is taken out and turned and again placed in the press, this being repeaterl four times during the first day. The following morning it is taken out and weighed and put in strong brine, where it remains for two days. It is next placed on the shelves in the cheese room, where it is turned and wiped with a dry cloth every other day. She got 23 öre per Danish pound, for her skim-milk cheese, which is about equal to 6 cents per pound avoirdupois. 
An eight-year rotation was followed on this farm. First year, oats, the ground being broken from grass the previous fall. The oats were sown very thick, at the rate of nearly 6 bushels to the acre, and the yield reached nearly 100 bushels per acre; second year, fallow and manured with some 14 to 16 tons of stable manure per acre in May or June, and the fallow was plowed five times during the summer; third year, wheat or rye, sown on the fallow the previous fall. About 3 bushels of seed wheat per acre were used and the usual yield was from 40 to 45 bushels per acre; fourth year, barley, the barley ground being manured after harvest before the fall plowing; fifth year, barley and oats mixed, this being grown for feed, and the land was laid out with clover and grass; sixth, pasture or hay; seventh year, pasture or hay; and eighth year, pasture or hay, the grassland being broken and manured late in the summer of the eighth year. The help on the farm consisted, first, of a herdsman, who tended the stock and did odd chores about the place; second, two men and a boy of some 17 years. These were kept the year round and got their board and lodging and the customary wages (about $\$ 60$ a year). In the house were kept three hired girls. The butter consumed in the household averaged 660 pounds avoirdupois annually, and about 6,000 pounds was yearly sold for export at an average price of nearly 27 cents a pound. Fat swine were sold to the value of about $\$ 540$ annually, besides what was made into bacon for home consumption. The sale of skim-milk cheese amounted to about $\$ 162$ yearly, and what grain was sold would balance the feed bought.

DALUM AGRICULTURAL SCHOOL.

In conclusion I will add a few notes from the farm of Dalum Agrieultural School, situated near the city of Odense. This is a private school owned by J. Peterson, who was president and head teacher. The foundation of it is a small farm of 80 acres, in connection with which the proprietor has erected buildings and engaged teachers for the instruction, in agriculture, of abont 100 pupils. I shall speak more fully of the school under another heading, and will at present note only what I learned concerning their farm practice. Owing to the absence of the proprietor, who is himself also professor of agrienlture, and takes charge of the management of the farm, I conld not obtain as detailed an account of the place as I desired. As stated, the farm consisterl of 80 acres, on which were kept, at the time of my visit, 26 cows, 1 bull, and 3 heifers, all of the red Danish dairy breed, and superior individuals. These cattle were treated as follows: They were milked at 5 a.m.; at 7 a. m. they got a grain feed consisting of threequarters of a pound of rape-seed eake, 1 pound of sunflower seed cake, three-quarters of a pound of cotton-seed cake, 1 pound of hemp-seed cake, and $1 \frac{1}{2}$ pounds of bran, and immediately after 25 pounds of sliced mangels. When this was eaten they were watered and they then got 
4 pounds of hay, which completed the feeding for the forenoon. They were again milked at 12, and about 3 o'clock the above feeding was repeated, with the exception that they received straw instead of hay. They were milked the third time at $8 \mathrm{p.} \mathrm{m}$. The average annual yield per cow, of all ages, reached 7,700 pounds avoirdupois. After the milk had been strained and weighed in the dairy the noon and evening milkings were put in ice water until the following morning, when it, together with the morning's milk, was run through a small separator, the milk being first warmed to $90^{\circ} \mathrm{F}$. The cream was at once cooled off and kept in ice water until the ferment was added. They used the pure ferment produced by Mr. Quist, of Skanderborg. When enough for churning had been gathered, it was warmed to $66^{\circ}$ F., and the ferment was added. At the time of my visit the churning temperature was $57^{\circ} \mathrm{F}$. The dairy is not considered part of the school, except during three months of the summer, when there is a special dairy class in attendance. The rotation followed on the farm was as follows: First year, barley, which was laid out with clover and grass; second year, part of this was pastured and part was cut for hay, the cattle being tethered on the pasture; third year, oats; fourth year part of it was in soiling crops, which consisted of mixed barley and oats, oats and vetches, and oats and peas, and part was sown in buckwheat and yellow mustard, which were used as pasture. These were sown at different times, and what remained after pasturing was plowed under for green manure. This is termed a "cultivated fallow," to which manure is applied in the fall; fifth year, wheat or rye; sixth year, barley; and seventh year, mangels and sugar beets, which they use for feed. All the manure from the stables is saved with the greatest care. Drains from cow stable, pig-pens and horse stables led to a large cistern where all the liquid was collected, and the solid manure was kept under a shed. The liquid was partly applied to the grass land and partly sprinkled on the solid nanure. A portion of the farm, comprising some 5 acres, was in permanent meadow and to this the liquid manure had been liberally applied for a long time, and it was found that when thus applied it, after a time, lost its beneficial influence unless lime and phosphoric acid were also added.

\section{COÖPERATIVE CREAMERIES.}

I have so far confined myself to the description of private dairies, large and small. I shall now consider the organization and work of the large number of coöperative creameries throughout the country. It is chiefly these that benefit the sinall farmers. They are patronized by men who, as a rule, keep but a limited number of cows, say from one to fifteen or twenty head, though occasionally the milk from much larger farms is also worked up in these creameries. It is from this class of creameries that the bulk of the export butter comes, and it is 
really these that fix the standing of Danish butter in foreign countries. There are two classes of creameries, which are distiuguished rom each other only in ownership, but which are alike in methods of operation and all other characteristics, and they can therefore be treated together uder the above heading. The first class is the coöperative creamery p oper, which is owned in company by all those who deliver milk there. The second class is the kind so largely represented in America in which the creamery plant is owned by one man, or at most by a few individuals, who buy the milk from the farmers of the neighborhood. The former are called "Andels" creameries, which is, properly translated, coïperative creameries. The second class is called "Fælles" creameries, and the name denotes delivery of milk by several individuals to the same creamery. In the former class the producers of milk have a personal interest. Their profits depend upon the management of the institution. In the second class they have no direct interestit is merely a place where they sell their milk at so much per hundredweight, and the owner takes all the financial responsibility. The latter class of creameries has eonstantly decreased in number, while the former class has constantly increased. When it was found that coöperative creameries could be conducted without disagreement among those interested, and on an economical basis which would yield the best attainable returns, everybody wanted to join them. The Fælles creamery was doomed to go. None were willing to grant profits to the creamery owner if they could just as well share them. The creamery owners, ou the other hand, became obliged to pay so much for their milk, if they wanted any, that a profit was practically impossible, and it has not unfrequently happened that when the farmers did not get the prices they thought they ought to have, they have built a coöperative creamery to which they have sent their milk and left the other creamery owner out in the cold.

I have nowhere been able to obtain definite statistics in regard to the number of coöperative creameries in the country, but I have seen various estimates placing them at from 1,000 to 1,500 , and $I$ am inclined to believe that the latter figure is very nearly correct. The number of Fælles creameries, on the other hand, does not probably exceed 400 .

All these structures are alike in their essential points, and one traveling in the country soon learns to recognize them afar off. There are the two-story brick buildings with short wings for engine-houses, and the imposing brick chimneys, which are invariably present. They are usually located near the highways, at some central point of the district contributing milk, and the arrangement of detail, which I shall describe more fully hereafter, is essentially the same in all of them. They differ, however, in capacity from about 300 cows up to 1,500 or even more. The Frelles creamery has been in operation there for many years, but it is only during the last ten years that the coopperativo 
creameries have sprung into existence. The history of their beginning and later growth is exceedingly interesting.

\section{ORIGIN AND GROW'TH.}

They had their origin in the desire on the part of the small farmer to make the most possible out of his dairy. He had the example of the larger farms before him, where the dairy had always been an important and well-paying branch. How would it be possible for him to get relatively as much out of his few cows as the large farmers got out of their cows? The latter found it economical to place the dairy in the hands of expert help who could make first-class butter, which would realize the top market price; but the wives of the small farmers were by no means expert dairywomen. Their butter could not compare in quality with that produced upon the larger farms, nor was there any hope of so improving their practice that any considerable number could be counted on to make first-class butter. The only way open was for the farmers in the district, who were all equally interested, to unite, build a creamery, and deliver their milk there for creaming and churning; and this was what they finally did. But it resulted in this through some intermediate steps. The first of these was taken during the winter of 1881-'82. A few farmers in a certain part of the peninsula, after cousultation, conceived the idea of engaging an expert at a stated salary per year, who should go from farm to farm and give instruction in dairy methods, and act as joint adviser and agent for them all, which position was offered to a Mr. S. Andersen, an educated man in the neighborhood. He saw, however, the insuperable obstacles to this course. Even though each farmer might provide the necessary utensils and the proper accommodations for the dairy, still their wives and daughters, his prospective pupils, would not all be equally apt learners, and those who found that the enterprise did not bring the desired results would soon withdraw from the arrangement. So for a time it amounted to nothing. But Mr. Andersen made them the proposition that if they would furnish him with a proper creamery and allow him the necessary help he would receive and churn their milk under his personal supervision and for a stated yearly salary. After many meetings it was finally decided to adopt this plan. But it was found that a sufficient number of farmers to represent 400 cows could not be obtained, and Mr. Andersen refused to begin operations with a less number. Three hundred cows were subscribed at once, and there were one or two hundred more in the neighborhood, but their owners did not think well of this new and untried coöperative scheme. They were willing to sell their milk, but did not care to risk anything on the proposed plan. This obstacle was finally overcome by Mr. Andersen, who offered to buy their milk on his own account, and on this basis, partly "Fælles," the first coöperative creamery in Denmark was started in 1882.

It was a task of no little difficulty. There was no similar institu$8538-N_{0} .5-7$ 
tion from which even a hint could be taken in regard to the best method of organization, or the drafting of the laws and regulations, which it became necessary to have; but it was fortunate, very fortunate for the future of the enterprise, that those to whom this task of the first organization was assigned were able, far-seeing men. The constitution which was finally adopted proved to be so satisfactory that it has been more or less literally copied in the organization of all later coöperative creameries. This first plant, including building, machinery, and all dairy utensils, cost 8,000 crowns $(\$ 2,160)$. The enterprise proved to be a success from the start, and those farmers who at first hesitated to join the organization, but preferred to sell their milk, one after another joined the company, and by the end of the second year all who delivered milk there were members.

The coöperative creameries have benefited the Danish farmers in more ways than one. They have been educators in addition to being the means of augmenting their incomes. The common interest which they had in the creamery brought the farmers together in frequent consultation, at which the brighter and more enterprising minds among them influenced the easier-going ones to adopt better methods. It compelled those who had not been in the habit of keeping accounts to study the art of bookkeeping in order to assure themselves that they received pay for all the milk they delivered.

The constitution usually requires a minimum amount of oil cake to be fed daily to each cow, and this, of course, had to be bought. This raised the question of cost of feed and the return in milk from the feed given, questions which were to be closely studied. And now every Danish farmer interested in a coöperative creamery is able to tell to the fraction of a cent what his feed has cost him and what his returus in milk have been. Under the Frelles creamery system the farmers sold their milk directly to the creamery man. They were not particularly interested in the quality of the milk. No practical test had at that time been devised by which the amount of fat in the milk could be ascertained with ease and certainty. Under the coöperative system they soon recognized that the milk from some herds was worth more for butter than from others, and, since butter was the product aimed at, they all conceded the equity of paying for the milk in accordance with the per cent of fat it contained. At this juncture Prof: Fjord came to their assistance by inventing his "control" apparatus, which has already been described (Fig. 12) in the foregoing pages, and his invention is now in use in every creamery in the land. Indirectly this testing apparatus led to the improvement of the cattle. When one farmer found that his neighbor received more money for his milk than he, it was natural to inquire into the cause, which, in most cases, was traced to the cow herself. This, again, led to the organization of numerous societies for the improvement of the cattle, one form of which is represented by the "bull associations" already mentioned; and thus 
one ruestion suggested another until the present standard has been reacherl. But they will by no means stop here. The same forces are still at work. Improvement in every line of the dairy interest is the ambition of the whole country. It is this wholesome development of the coöperative creameries which has increased the Danish exports of butter to the present astonishing figures, the amount having almost doubled during the last half-dozen years.

\section{ORGANIZATION-OFFICERS, SALARIES, ETC.}

When an organization is to be effected the farmers of the neighborhood meet together, and, having agreed upon the general plan, they elect a board of directors from their number. There is considerable difference, numerically, in the composition of this board in different organizations. Thus, the number of directors varies from three to fifteen. This board is charged with the duty of conducting the business of the creamery according to the requirements of the constitution which the organization adopts. A chairman, a secretary, and a treasurer are elected from among the board of directors. In some cases a small salary is paid to each, while in other cases they give their services. The board of directors hire a competent dairyman to take charge of the creamery. He, in all cases that came under my observation, had rooms in the building. Sometimes he is paid a salary only, at other times he is paid both salary and commission, this latter plan being the most common. He gets his commission from the surplus which the butter brings over the highest quotation on the exchange in Copenhagen, and he thus becomes financially interested in producing the finest quality possible. He also has certain allowances of milk and butter, and is furnished fuel, light, etc.

The salary of such a man will vary from $\$ 270$ to $\$ 405$ a year, in addition to his commission; or from $\$ 432$ to $\$ 1,080$ a year when he works without a commission. The board of directors hold him responsible for the operation of the creamery. According to the terms of the agreement he is either allowed a given sum with which to hire his helpers, or, as happens in many cases, he supplies the helpers himself and pays them out of his own pocket. In any case he is the only responsible person. When he pays for the help it is very generally the case that he gets young men and women who desire to learn the business and who work for a small salary in order to get the benefit of his instruction and become familiar with the details of the business.

The capital with which the plant is erected is, in nearly all cases, money borrowed at 4 per cent interest. The terms of the agreement usually require that a given amount shall be paid off each year for a period running from tell to twenty years, and each member of the organization becomes personally responsible for a share of the capital thus borrowed, in proportion to the number of cows that he has entered. In the meetings of the organization the members vote also 
by the number of cows represented by each, or, in some cases, by the square root of the number of cows represented by each. When new members wish to join a body already organized, they must obtain their places by paying a certain amount of cash into the treasury of the association. The value of the partnership is usually based upon the amount of milk delivered and the profits made during the year pre. cerling.

The methods of procedure in these creameries are so nearly alike that only a few examples are necessary to convey an idea of them.

\section{KILDEV ALD COÖPERATIVE CREAMERY.}

This is a typical creamery, on the island of Zealand, some 12 or 14 miles from the citý of Hilleród. I investigated this institution thoroughly, and am under obligations both to the president of the board of directors, Mr. M. F. J. Grónbeck, and to the superintendent in charge of the creamery, Mr. L. H. Larsen, who, with unreserved kindness, answered all my questions fully.

The building was a two-story brick, much like the pattern shown in Figs. 24, 25, and 26 (pp.109, 110), although these illustrations are not identical with the building in question. The lower floor was devoted to the dairy alone. It was level with the ground, and was divided into only two rooms, the larger one of which contained the separator and the churn, and there also the milk was received. In the smaller room the butter was worked, cooled, and stored until shipment. A small "L" projecting back from the main wing contained the boiler and engine with storage room for coal, and back of this was an ice house. The necessary water was supplied by a good well, from which it was pumped by the engine into a suitable reservoir. The upper story was set apart for the superintendent and his family and also contained rooms for the helpers.

President Grónbeck kindly furnished me with the following facts concerning the plant: The association was organized in 1888, with 69 members, representing 400 cows; but, as they anticipated that others would join, they decided to erect a creamery with a capacity for 1,000 cows.

At first it was agreed that each member should pay into the treasury a sum of 27 cents per cow owned by him. This was raised to 54 cents, and later to $\$ 1.35$ per cow. The following is a statement of capital and expenses of the plant:

Capital.

Assessiment of members at $\$ 1.35$ per cow............... \$675.00

Loan from bank............................. 8,610.00

Total ............................ 
Lixpenses.

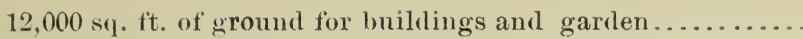

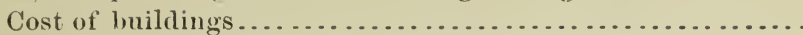

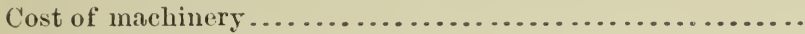

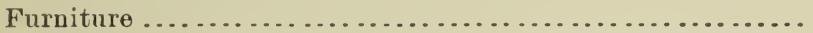

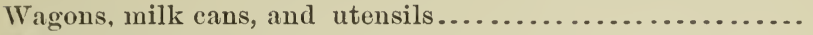

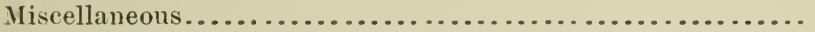

$\$ 400.92$

$3,995.40$

$3,148.82$

434. 09

$1,052.85$

133.71

$9,165.79$

Later improvements

$1,080.00$

Total

$10,245.79$

The later improvements have been made from the profits of the business. The buildings have been constructed in the most substantial manner, and the machinery is the best obtainable. The business is in the hands of a board of directors, who elect their own chairman, secretary, and treasurer, and their actions are binding on the association.

The creamery began business the first of November, 1888, with 69 members, representing 400 cows, and in February, 1893, there were 177 members, representing 1,400 cows. The superintendent of the cream. ery was hired by the board of directors, and he had direct charge of the work in the creamery. His salary consisted in 6 per cent of the value of the butter made from the "overskud" (surplus). This term "overskud," which may be translated "surplus," is, when used in this sense, a little difficult to understand. They estimate that it requires 28 pounds of milk to make a pound of butter. Now if the creamery superintendent can make a pound of butter from 25 or 26 pounds of milk, the difference between that and the estimated 28 pounds is called the "surplus," and his wages consisted of 6 per cent of this and 20 per cent of the price which his butter realized more than the highest quotation made by the exchange in Copenhagen each respective week; in addition, he had furnished quarters, fuel, light, milk, butter, garden, ete., but out of this he paid for his hired help. The members of the company were paid for their milk once in four weeks, according to the per cent of fat in the milk, calculated by the system invented by Prof. Fjord. They got the price of a pound of butter for every 28 pounds of milk of average per cent fat, the highest quotation of the exchange each week fixing the price of butter; and if there was still a surplus in the treasury it was divided among the members in the form of a dividend twice yearly. In this creamery there had so far always been a considerable dividend distributed in this manner. The members were bound to take back their proportion of the skim milk and the buttermilk, and for this they paid the creamery 1 öre per pound, a trifle more than one-fourth of a cent.

The creamery provided the cans, fetched the milk from each farm, and brought each man back his proportion of the skim milk and the buttermilk; but the teams used were hired and paid for at the rate of 
19 öre per 100 pounds of milk hauled. The skim milk was sterilized before it was returned, by being heated to a temperature $190 \circ \mathrm{F}$. as fast as it ran from the separator. These were the fundamental principles on which the creamery was run, all of which I learned from the president of the company.

CONSTITUTION OF KILDEVALD CREAMERY.

To give a still clearer idea of the workings of a coöperative creamery I insert the following translation of the constitution of Kildevald Creamery :

First. The object of the association is to secure to its members the best possible returns from their dairy cows. The milk shall be separated in common and from the cream butter shall be made and marketed. The members shall take back their proportion of the skim milk and butternilk and shall pay for the same at about their actual value.

Second. Any one can become a member of this association by paying 1 crown per cow which he proposes to enter; provided, however, that not fewer than twothirds of the votes cast are in his favor. But all who join after the creamery begins business shall pay 5 crowns per cow into the association's treasury. Each member shall on all occasions be entitled to vote as follows :

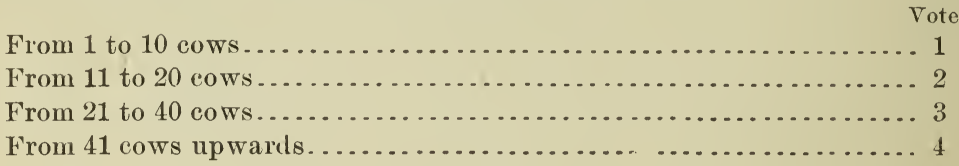

Third. The association shall elect from their number a board of directors consisting of five members, and this board shall elect its own chairman, secretary, and treasurer, which offices must not be united in the same person. The board of directors shall represent the association in all business affairs, and their action shall be binding on the association. No member shall refuse to hold office when duly elected. The board of directors shall hold office five years, and the term of one of the original board shall expire each year during the first term, as determined by lot. Those who have once held office may decline reëlection if they choose. The board of directors shall receive no pay, but they may employ a bookkeeper, at a salary not to exceed 40 öre per cow, and all necessary expenses incurred for the association shall be refunded from the treasury.

Fourth. An annual meeting of all the members shall be held each year in February, at which meeting the board of directors shall give an account of the business done during the past year and present plans for the business during the current year. At these meetings all differences that may arise between the board and members, or between the members themselves, shall be settled, and the moeting shall appoint an assessor and two auditors, the latter to hold office for two years, and one of those first appointed shall resign at the end of ono year, as determined by lot. Amendments to the constitution shall ręuiro a two-thirds majority, but all other questions, not otherwise specified, are to be determined by a simple majority of the votes cast. Extraordinary meetings may be held at the call of the board, or npon the written request to the board of not less than one-third of the members. The board shall then give at least ten days' notice of the meeting to each momber, who shall also be notified of the purposes of the meeting.

Fifth. The board shall contract a loan as large as necessary for the erection of the contemplated plant. For this loan each momber shall be responsible, in proportion to the number of cows he enters, until all is paid. 
Sixth. If a nember sells or transfers his property, his share in the ereamery shali pass to the new owner, if the latter will accept the privileges and responsibilities it confers; if not, the association shall pay the outgoing member only one-half of his share. No member can withdraw from the association before the debt is paid, except by removal from the neighborhood. Members desiring to withdraw after the debt is paid shall give at least three months' written notice of such withdrawal to the board of directors, and they shall be entitled to only one-half of their share from the association. Members who are expelled br the annual meeting shall lose their shares.

Seventh. The board of directors shall hire a superintendent for the creamery, who shall keep account with the members and shall be responsible for the work in the creamery. The superintendent shall hire, pay, and board all his help. He shall have at least one assistant who is a competent butter-maker, and at least one other assistant who is competent to take charge of the machinery (engineer). The superintendent of the croamery shall be paid quarterly, and shall be furnished quarters with garden, fuel and light, and the creamery products necessary for his household, and at the end of the year, when accounts are balanced, he shall be paid a premium for every hundred pounds butter sold at a satisfactory price.

Eighth. The milk shall be paid for in accordance with its content of fat, which shall be determined by the superintendent of the creamery. Milk of the average per cent of fat shall be paid for at the rate of the highest quotation for a pound of butter for each 28 pounds of milk.

Ninth. Until the debt of the creamery is paid the members shall be bound to receive their respective portions of skim milk and buttermilk, for which theyshall pay about one-third of a cent per pound. After the debt is paid the price of this milk can be fixed at each annual meeting.

Tenth. The rates fixed in paragraphs 8 and 9 presuppose that there will be a surplus in the treasury over and above what is paid for the milk. This surplus shall be employed to cover current expenses, to pay interest, as well as the principal of the debt, as it falls due, or it may be divided among the members.

Eleventh. When the debt is paid, the president of the board, the assessor, and superintendent of the ereamery shall assess the property of the company, which shall be assigned to the members in proportion to the amount of milk each has delivered since the business began. Thereafter the yearly profits shall be divided so that each member shall receive, first, 5 per cent of the value of his share, and if there is still a surplus in the treasury it shall be divided among the members in proportion to the amount of milk each has delivered during the past year.

Twelfth. The association shall provide and maintain the necessary number of milk cans and shall transport the milk to and from each farm; however, members who deliver less than 100 pounds of milk daily shall bring their milk to the nearest point on the highway where the transport wagons pass, and shall receive the skim milk in the same place. Members located off the main road shall keep the roads to their respective farms open and passable, and the milk cans shall be ready for the driver when he calls at the time named the day before.

Thirteenth. It shall be the duty of each member to keep his milk cans serupulously clean, to see that strict cleanliness is observed in milking, and to strain the milk at once into the milk cans and place these in cold water, which shall be changed if necessary. Milk from sick cows must not be sent to the creamery, and milk from fresh cows must not be sent until four days after calving. It shall be the duty of the superintendent of the creamery to see that the milk is sweet and good. If the cans are not properly cleaned, or if the milk is objectionable, he shall give written notice thereof to the members interested, and if the fault is not corrected at once he shall refuse to receive the milk. If this happens a second time such member shall be fined two crowns for each cow in his herd. The members are forbidden to make butter or cheese for sale, and also to sell sweet milk to other 
ereamerics; but they are allowed to retain what they desire for use in the household aud to give away milk to poor people who do not keep cows. If desired, the members have the right to get their butter from the creamery at the same price that it will then loring on the market.

Fourteenth. It shall be the duty of the members to feed their cows in such a manner that the milk shall not be affected disadvantageously or the quality of the butter deteriorated. Cabbage, turnip tops, and kohlrabi tops must not be fed. Members shall notify the superintendent of the creamery of ehanges in feed, and keep him informed concerning the feeds used, so that he may give speeial attention to the milk. If the feed affeets the quality of the milk or the butter, it shall be the duty of the superintendent to give written notice thereof to the member in question, and if the fault continnes he shall refuse to reeeive the milk. The president of the board and the superintendent may, when they agree, forbid the use of any feed which they have reason to think is detrimental to the creamery. Members who refuse to be goverued by their decision, or who do not live up to the regulation of this paragraph, shall be fined from two to ten erowns per cow, and pay for the injury they have caused the creamery as estimated by the president of the board, the superintendent of the creamery, and the assessor.

Fifteenth. To enable the members to obtain good feed stuffs at the lowest rate, the board, or a eommittee appointed for the purpose, may purchase such feerls at wholesale for the benefit of the members. It shall further be the duty of the members to buy rape-seed cakes enough for each cow to have at least one pound daily all the year through, and it shall be the duty of the board of directors to aid the members in feeding their cattle in an intelligent and economical manner, and,for example, by the purchase of seed, aid in the culture and use of plants which have a desirable inflnenee on the quality of the butter.

Sixtenth. It shall be the right and duty of the board of directors and the supertendent of the creamery to inspeet the premises of each member as often as they see fit, to note the quality of their fodder, pastures, cleanliness in stables and milk room, ete., and each member shall give all the information they may desire on these subjects, and if it can be proverl that a member lias withheld information wanted, or given them wrong information in regard to these matters, he shall be fined from two to ten erowns per eow and pay for the injury he may have caused the creamery, aeeording to the estimates made by the president, superintendent, and the assessor.

Serenteenth. If any eontagious disease appears either among the people or the cattle, on a member's farm, it shall be his duty to stop the delivery of milk at once until the disease has disappeared and proper disinfection taken plaee, and in case such disease appears at the ereamery, the superintendent shall eause the sick person to be moved away and the place to be disinfected. To prevent possible transportation of disease in the milk, the skim milk shall be sterilized at the creamery before it is returned to the members. Non-complianee with the provisions of this paragraph shall be punished by fine in an amount not to exceed 100 erowns.

Eighteenth. It shall be the duty of the board to oversee the management of the superintendent and to see that the unarhinery and ntensils are at all times in satisfietory coudition, and particularly shall it he the dnty of the board to give the whole creamery a thorough overhanling in regard to repairs every spring so that it may be in good condition for the warm season. At the elose of the yearly account an inventory of the property owned by the assoeiation shall be taken jointly by the president of the board, the superintendent of the creamery, and the assessor, so that the members may always have a correct idea of the value of their common property.

Nineteenth. It shall be the duty of the board of directors to see that the eash on hand is employer to the best advantage of their association. The members shall be paid every four weeks for the amount of milk they have delivered, less the value of 
the products they have received from the creamery. Division of the profits shall take place yearly after the ammul meeting of the association.

Twentieth. In all cases when points arise not covered by this constitution the board of directors shall use their best judgment in the interest of the association, and, if necessary, bring the matter up at the next annual meeting, which alone is einpowered to make alterations in or additions to these regulations.

Twenty-first. The board of directors shall have authority to buy milk for the creamery fiom farmers who are not members of the association.

Twenty-second. This constitution shall go into effect on aud after the 27th of May, 1888.

I would call especial attention to those paragraphs in this constitution which are intended to enforce cleanliness in the handling of the milk, and which aim to compel the farmer to feed his cattle in such a manner that the milk shall not be objectionable. It is worthy of note that the board of directors are empowered to impose fines severe enough to deter any slovenly or careless person from breaking the rules. The fact that none can withdraw from the association until the debt is paid, except by removal from the neighborhood, unless he is willing to lose half his vested interest in the concern, is a feature which could probably not be put in force in this country.

All the essential features in this constitution are found in the constitutions of all the coöperative creameries, and although this represents but one of the many hundreds of associations which there are in the country, it is, nevertheless, a type of them all.

PRACTICAL OPERATIONS OF KILDEVALD CREAMERY.

The superintendent of Kildevæld ereamery, Mr. L. H. Larsen, kindly gave me the following details concerning the operations of the creamery:

Although there were 1,400 cows entered by the members of the association, the creamery was receiving milk from only about 1,300 of them at the time of my visit, and from this number they obtain in round numbers 18,000 pounds of milk daily. A large majority of the members had only from 1 to 10 cows. A few had from 20 to 30 cows, but there were only two larger farms which contributed to the creamery, on one of which there were 60 cows and on the other 80 . It will thus be seen that it was especially the small farmer, with only a few acres of land, who profited by the coopperative system. At that time of the year (February) it required 27 pounds of milk to make a pound of butter. The milk from all these patrons was tested twice each week, but the patrons did not know from what deliveries the samples were taken. They were paid in proportion to the per cent of fat contained in the milk. For this analysis Fjord's control apparatus was used, by means of whirch 192 samples could be tested at once. (See Fig. 12.) This apparatus can be used only in connection with the Burmeister \& Waine separator, this machine having a larger bowl than the others. The bowl 
is filled with water heated to a temperature of $133 \circ \mathrm{F}$., the tubes of milk being warmed by it to this temperature. The separator is theu run at a low speed, 1,500 revolutions per minute, for three-quarters of an hour. The cream has then formed a hard, perfectly solid mass in the opening of the tube. The thickness of this mass of cream is measured by means of a scale applied to the glass, and by reference to a table constructed by Prof. Fjord the per cent of fat ean be told with accuracy.

All the patrous milk their cows three times a day, and the noon and the evening milk is kept in cold water, or ice water at the farms, until the following morning, when the transport wagon arrives and takes it, together with that morning's milk. As already stated, the creamery owns the cans and transport wagons, but the teams and drivers are hired and remunerated at from 5 to 7 cents per 100 pounds of milk hauled, according to the length of the route. All milk is tested upon its arrival at the creamery. If something is found wrong with it, and the superintendent is disposed to think that it is caused by one or more cows in the herd, he sends a case of small bottles to the farm and each cow's milk is sampled, and upon the return of these samples to the creamery the fault can frequently be traced to some individual animal, and her milk is then excluded until the fault is remedied. The milk furnished by each patron is of course weighed and the weight entered in the record.

From the receiving vat the milk runs through the "forewarmer," where it is heated to $84^{\circ} \mathrm{F}$. in winter and $80^{\circ} \mathrm{F}$. in summer before it runs through the separator. There were four of the Burmeister \& Waine separators in use. From the separator the cream is discharged into tall cans which are sunk in ice water, one after another, as they are filled. The skim milk is raised by the separators themselves to a tank on the same level as the receiving platform, from which it runs through a pasteurizing apparatus, where it is heated to $190 \circ \mathrm{F}$. From this it is discharged into a large tank from which the amount due each patron is weighed out and sent back. The price, as already mentioned, was one öre per Danish pound. There was no difficulty in getting the patrons to take the milk at this price and some were glad to get more than their share. This milk reaches the farms perfectly fresh and sweet. It is used largely for the manufacture of skim-milk cheese and to feed to calves and pigs. The buttermilk is in like manner returned to the patrons at the price given for skim milk.

When all the cream had been separated and cooled, about noon, the ferment was started. To this end the cream was first warmed to $640 \mathrm{~F}$. In this creamery they used the somewhat troublesome method of warming it by setting the eans in hot water and constantly stirring their contents and testing with the thermometer until the desired temperature was reached. In most other places they used a pasteurizing 
apparatus for this purpose. When the proper temperature was reached the prepared ferment was added, to the amount of from 6 to 8 per cent of the weight of the cream, and it then stood in the cream barrels for 18 hours, from noon until 6 the following morning, when the churning began." Mr. Larsen used pure culture ferment for his cream. He propagated this ferment from day to day in skim milk, and he found it necessary to buy new cultures only once in about two months. For propagating this ferment he used 200 pounds of fresh milk daily. This was secured from special cows which had calved only four or five weeks. He used the morning's milk. It was set in cold water immediately on arrival and skimmed by hand. After skimming it was pasteurized at $176^{\circ} \mathrm{F}$. and then kept in warm water of the same temperature for 15 minutes, after which it was cooled to $82^{\circ} \mathrm{F}$., when a portion of the ferment made in the same manner the previous day was added. This ferment was added about $2 \mathrm{p} . \mathrm{m}$. The skim milk containing the ferment was then warmed to $88^{\circ} \mathrm{F}$. by setting the cans in warm water, and by means of a steam pipe connected with the watertank it was kept at the temperature until evening, when it was thick. It was then taken out of the warm water and set in cold water of about $50^{\circ} \mathrm{F}$., where it remained until the next noon, when it was ready to be added to the cream. He considered this the best method. He deprecated the use of buttermilk as a starter, and would never use it unless compelled to do so.

The churns are started at between 5 and 6 a. $\mathrm{m}$. At that season he churned at a temperature of $57^{\circ} \mathrm{F}$., but in summer he frequently went as low as $52^{\circ} \mathrm{F}$. The churning temperature varied with the character of the butter and with the weather. He judged of the required temperature by the character of the butter. The creamery was furnisked with four churns of the pattern already described. The dasher in each made 130 revolutions per minute, at which speed it required forty-five minutes to churn. When the butter began to form in small granules the size of pin heads, the churns were stopped and the sides and lids were washed down with water at a temperature of about $50{ }^{\circ} \mathrm{F}$. Each cliurn contained from 400 to 450 pounds of cream, which filled it about three-quarters full. The butter was dipped out of the churn with a sieve or strainer and immediately worked a little on the butter worker to remove most of the buttermilk. It was then weighed and four and a half per cent of salt added, which was worked in lightly. The butter was next made into crescent-shaped rolls and placed in ice boxes made for that purpose (see Fig. 16). Here it stood for two hours, after which it was worked for the last time and at once packed in barrels holding about 110 pounds each. The butter was sent off every Monday. It was sold to a dealer in Copenhagen, who paid about $\$ 1.00$ per 100 pounds above the highest quotation on the exchange for the preceding week. The cream in this dairy was not pasteurized 
before it was churned. All unfavorablo conditions were so well under control that this was not found necessary. The leading pieces of machinery and apparatus in this creamery were as follows:

(1) An 8-horse power engine.

(2) A 10-horse power boiler.

(3) Four "B. \& W." separators, each with a capacity of 4,000 pounds per hour when run at a speed of 2,700 revolutions per minute.

(4) Four churns of the usual pattern.

(5) One "forewarming" apparatus for sweet milk.

(6) Two Pasteurizing machines for the skim milk.

(7) Three large milk vats, one each for sweet milk, skim milk, and buttermilk, besides several smaller ones used as occasion demanded.

(8) Eight cream barrels.

(9) One large butter-worker.

(10) Several ice boxes in which to cool butter.

(11) Three large water-tanks for cold and warm water.

(12) Forty cream cans.

(13) Six hundred cans used in the transportation of milk. They varied in capacity from 40 to 100 pounds.

Besides these, there was a large reservoir on top of the building which was always kept full of water, pumped by steam from a good well, and with this tank there were connections to all parts of the creamery where cold water was needed. In like manner a hot-water tank furnished hot water to all parts of the creamery, and there was a miscellaneous collection of smaller utensils not necessary to specify.

The working force of the creamery consisted of, first, the superintendent ; second, an engineer, who had charge not only of the engine and boiler but also of the separators ; third, a weighmaster, who weighed all the milk that came in or left the creamery and assisted with other things ; fourth, a butter-maker ; fifth, an assistant who had no special duties; sixth, two pupils, a young woman and a young man, who worked, however, the full time as did the others. All of these people received their pay, board and lodging from the superintendent of the creamery. The pupils got their board and a very small wage besides, for which they worked as much as would be required of a hired person. The other four assistants were paid according to their value, the total amount paid to all being about $\$ 300$ per year, besides board and lodging.

The accompanying summary of the business of the creamery for the years 1889, 1890 and 1891, which I obtained permission of Mr. Larsen to transcribe from his books, will give a clear idea of the profit to the patrons as well as the business of the creamery in a more condensed form than any deseription could give. 


\begin{tabular}{|c|c|c|c|}
\hline General statistics. & $1888-' 89$. & $1889-90$. & $1890-' 91$. \\
\hline 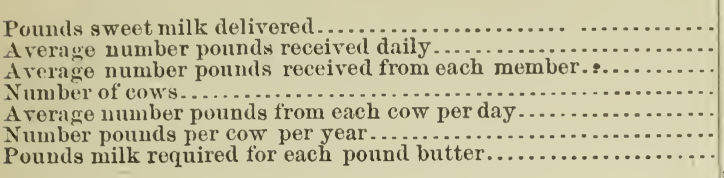 & $\begin{array}{r}4,614,831 \\
12,643 \\
134 \\
945 \\
13.37 \\
4,883.40 \\
29.31\end{array}$ & $\begin{array}{r}5,712,081 \\
15,649 \\
173 \\
1,115 \\
13.53 \\
4,939.50 \\
30.12\end{array}$ & $\begin{array}{r}6,395,909 \\
17,523 \\
179 \\
1,240 \\
14.22 \\
5,157.90 \\
29.39\end{array}$ \\
\hline 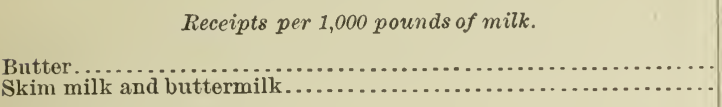 & $\begin{array}{r}\$ 9.93 \\
2.57\end{array}$ & $\begin{array}{r}\$ 9.33 \\
2.57\end{array}$ & $\begin{array}{r}\$ 9.91 \\
2.60\end{array}$ \\
\hline Total..... & 12.54 & 11.90 & 12.51 \\
\hline Expenses per 1,000 pounds of milk. & & & \\
\hline 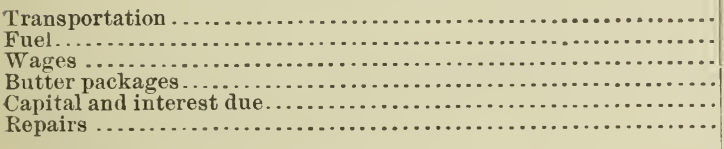 & $\begin{array}{r}\$ 0.29 \\
.18 \\
.22 \\
.18 \\
.10 \\
.14\end{array}$ & $\begin{array}{r}\$ 0.35 \\
.15 \\
.20 \\
.14 \\
.17 \\
.21\end{array}$ & $\begin{array}{r}\$ 0.37 \\
.15 \\
.22 \\
.16 \\
.16 \\
.05\end{array}$ \\
\hline 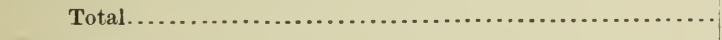 & 1.11 & 1.22 & 1. 11 \\
\hline 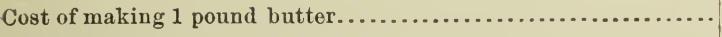 & 0.027 & 0.03 & 0.027 \\
\hline 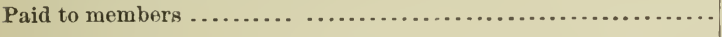 & 10.37 & 9.69 & 10.34 \\
\hline
\end{tabular}

\section{A TYPICAL COÖPERATIVE CREAMERY.}

I append here illustrations of a coöperative creamery calculated to handle the milk from about 700 cows. I did not find time to visit this creamery, but the illustrations presented, with their explanations, will serve to convey an idea of the construction and equipment of the typi-

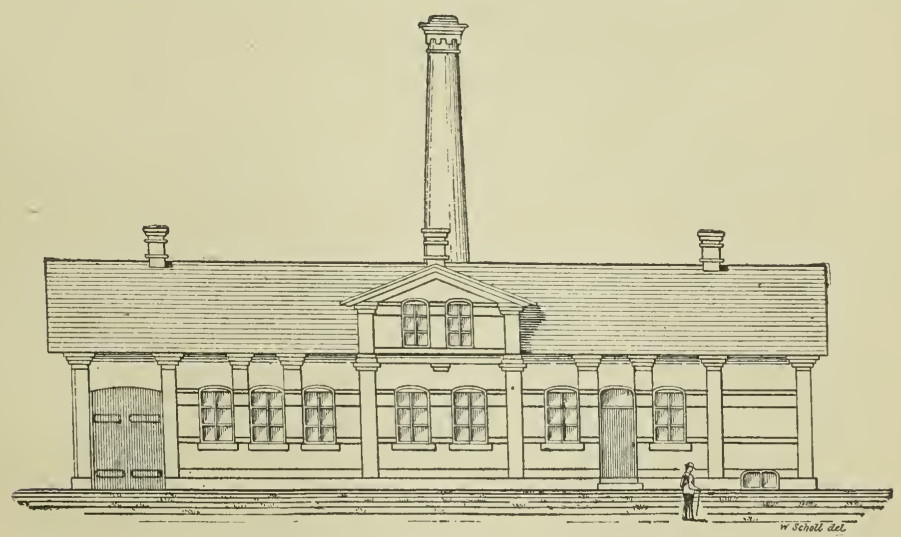

EIG. 24.-A typical coöperative creamery.

cal coöperative creamery. Fig. 24 gives a front view of the building. On the left is seen the driveway in which the milk is delivered. There are rooms above for the accommodation of the superintendent and help. Fig. 25 shows a section of the same building with some of the appara- 
tus in place. Fig. 26 shows a ground plan of the same. $A$ is a stable, $B$ coal room, $C$ driveway, $D$ the main dairy room, $E$ butter room, $F$ cheese room with cellar below, $G$ office, and $I I$ a raised platform on which

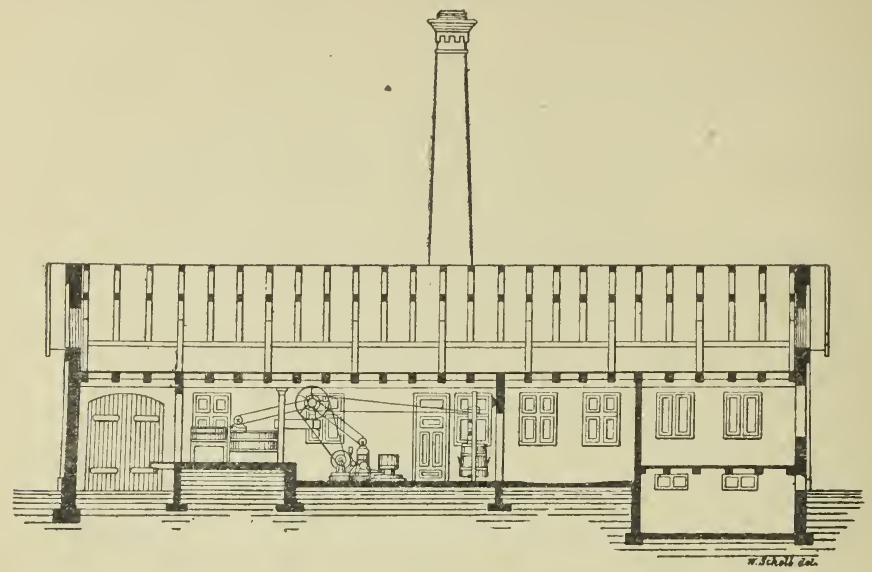

FIG. 25.-Sectiou of typical coöperative creamery.

the milk is received and weighed. The machinery is designated as follows: $a_{1}$ and $a_{2}$ two separators, $b$ a forewarmer, $c_{1}$ and $c_{2}$ represent two churns, $d$ cheese vat, $e$ vat for sweet milk, $f$ vat for skim milk, $g$ tank for hot water, $h$ butter worker, and $s$ sterilizing applaratus.

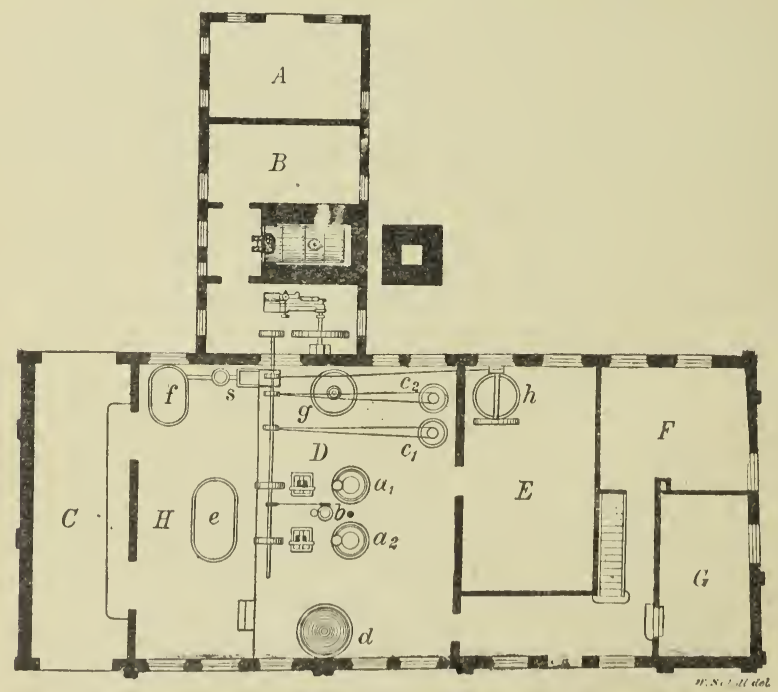

FIG. 26.-Ground plan of typical coöperative creamery.

I will here add an illustration (Fig.27) which has been prepared by $\mathrm{H}$. C. Petersen \& Co., manufacturers of creamery goods, Copenhagen. It shows the arrangement of the machinery in a well-equipped dairy, and although it is an idealistic arrangement it corresponds with the arrangement generally found in the eoöperative ereameries. It is best studied 
by following the milk and cream through the various machines. The milk is carried from the delivery wagon $A$ to the weigh box $C$, into which it is emptied. From this it runs into the vat for sweet milk $D$, and through the pipe $d$ into the forewarmer $E$, and from this into the separator $F$. The skim milk rises of itself through the pipe $L$ and through the trough $l$ into a small suspended vat $M$. This vat is found necessary in order to give the froth time to settle. From this it runs into the pasteurizing apparatus $N$ and from here over the cooler $O$ into the skim milk vat $P$. From this it is drawn into the cans $R$ and weighed on the scale $S$ and then disposed of to customers. If cheese is made, the skim milk runs directly into the cheese vat from the sepa-

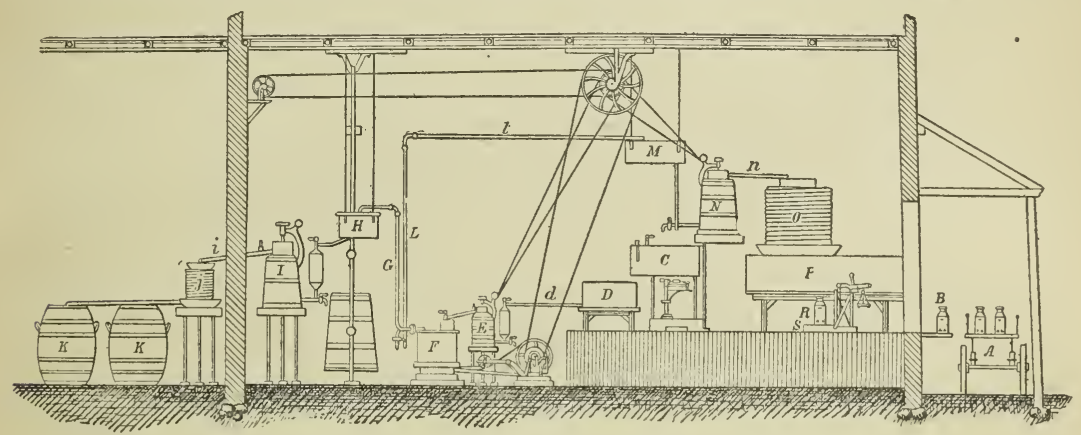

FIG. 27.-A rraugement of machinery in creamery.

rator. The cream leaves the separator through the pipe $G$ into the small suspended vat $H$. From this it runs through the pasteurizing apparatus $I$ over the cooler $j$ and through the trough $j$ into the cream barrels at $K$. The whole is arranged on the gravity plan, so that the milk or cream can run of itself to any place it may be wanted.

\section{RENKOLDE COÖPERATIVE CREAMERY.}

I mentioned this creamery briefly in my preliminary report. It is organized exactly on the plan just described and run on the same methods. The plant consisted of a large, substantial brick building with rooms for the superintendent and help. The ground and building with ice house cost about $\$ 5,400$ and the machinery $\$ 2,835$ and later additions and utensils and improvements cost $\$ 405$, making a total of $\$ 8,640$. All this money was borrowed from a bank at 4 per cent interest, to be paid off in sixteen years, at the rate of $\$ 540$ per year. It had been running only a few years, but had so far been entirely successful. The patrons were paid the maximum quotation per pound of butter for 28 pounds of milk, 28 pounds being the standard assumed as necessary to make a pound of butter. It is, however, only in a few instances that it requires that much milk. The superintendent was paid a certain per cent of the profits, as is usually the case, and he hired his help, which consisted of two men and two women. During the past 
year the surplus, after paying the patrons at the rate stated above for milk delivered, was sufficient not only to pay all running expenses, but to pay the interest, the $\$ 540$ falling due on the capital invested, and still give each patron a dividend of about 17 cents for each 100 pounds of milk delivered. The superintendent informed me that he, at that time of year, only used :5 pounds of milk to a pound of butter, and that in summer he used 29.5 pounds. They received, at that time, milk from about 1,100 cows daily, from which they made on an average 350 pounds of butter per day. The arrangement of the building was the same in pattern as the one already described, with only slight differences. Near the center of the building was an arched gateway for the wagons to drive through, and on one side of this was the creamery and on the other the rooms for the superintendent and his help. The delivery and weight platform was raised to the height of a wagon box, but the other portions of the building were on a level with the ground. There was first a large room, in which the machinery was placed. Beyond this was a butter room, where the butter was worked and packed. Next to this a storage room, which stood in immediate connection with the ice house. The engine was placed in a small "L" projecting from the main building. The creamery was equipped with an eight horse-power engine, three separators, and two churns, with the necessary smaller utensils, water tanks, etc. The skim milk and buttermilk were taken back by the patrons who paid three-fourths of an öre per pound.

RINGE COÖPERATIVE CREAMERY.

On the island of Funen I visited a representative coöperative dairy in the village of Ringe, where Superintendent L. Rasmussen kindly gave me the following details in regard to the business: The building was of brick, with cement floors and excellent drainage arrangements. The association was organized five years ago, and then represented 500 cows. Each nember paid $\$ 2.70$ per cow entered by him, which made a total capital of $\$ 1,350$. The cost of the plant was $\$ 6,588$. The balance of $\$ 5,292$ was borrowed at 4 per cent interest, with notes for $\$ 540$ falling due each year until all was paid. Each member was retponsible for the debt in proportion to the number of cows entered. At she time of my visit there were 214 members, representing 700 cows. They were paid, as in other eases, the maximum price of a pound of butter for 28 pounds of milk. The organization differed from those already described in that the farmers were not compelled to take back the skim milk and buttermilk, but those who wanted it got what they. required at about one-fourth of a cent per pound. The skim milk not sold was made into cheese at the creamery. They received at the time of my visit about 10,000 pounds of milk daily, in round numbers. The milk was raised to a temperature of $8(; \circ \mathrm{F}$. by running through a "forewarmer" before it ran into the separator. The cream was cooled with 
ice water as soon as separated, and about noon the prejared ferment was added, and by 6 a. 11 . the following day it was ready to churn. It was churned at $56^{\circ} \mathrm{F}$, and butter usually came in thirty-five minutes. The superintendent worked the butter the first time by hand, after which it was laid away to cool for two hours; it was then worked on the butter-worker, 4 per cent of salt added, and immediately. packed in barrels for shipment. It was kept in cold storage while in the creamery and sold once a week. Mr. Rasmussen prepared his own ferment from hand-skimmed milk in exactly the same manner as described for the Kildevæld creamery. The skim milk was sterilized at $185^{\circ} \mathrm{F}$. From the sterilizing apparatus it ran into a large vat, from which the orders from the farmers were filled, and what remained was made into skim-milk cheese, of which they made about 170 pounds daily. It required 16 pounds of milk to make a pound of cheese.

The milk was warmed and the rennet added in the manner already described. The cheese remained in the press for twenty-four hours. It was then put in strong brine for twenty-four hours and next put in the cheese-room, where it was daily wiped and turned for a month, and when the rind began to harden it was washed daily with brine. Their cheese sold, wholesale, for $4 \frac{1}{2}$ cents per pound avoirdupois. Patrons of the creamery had the privilege of buying cheese and butter at a trifle lower price than that asked in the market. The establishment was equipped with a seven horse-power boiler, a five horse power engine, a DeLaval "Alpha" separator, with the necessary line of tanks, cream barrels, buckets, etc., and also a line of cheese-making utensils. The superintendent in this creamery received about $\$ 272$ per year as salary, with house, fuel, light, and dairy products, and 25 per cent of the receipts for butter above the top quotation by the exchange in Copenhagen. Out of this he paid and boarded his help, of which there were four persons: first, a chief dairywoman who got $\$ 67.50$ per year and board; second, a young man who got $\$ 59.40$ a year and board; third, a young man who got $\$ 33.75$ per year and board, and, lastly, a dairy pupil, a young woman, who got $\$ 16.20$ per year and board.

\section{SNÓDE AND STOELSE COÖPERATIVE CREAMERY.}

The superintendent of this creamery, Mr. P. Andersen, kindly furnished me with the following information: Eight thousand one hundred dollars was invested in the plant. Of this sum the building site cost $\$ 378$, the bnilding $\$ 2,970$, machinery $\$ 2,970$, and furniture and all other dairy utensils, with some other further improvements, some $\$ 1,782$. All of this was borrowed money, on which interest was paid at the rate of 4 per cent per annum. Five thousand four hundred dollars were to be paid back in ten years, at the rate of $\$ \tilde{540}$ a year, and the remainder was to stand indefinitely. The association contained only 123 members, who had entered 650 cows. At that time $8538-N o .5-8$ 
the receipt of milk amounted to only 6,000 pounds daily, but in summer time this was more than doubled. From this was made an average of 210 pounds of butter daily, which in summer arose to 550 pounds. The members were paid, as in other places, the highest price per pound of butter for 28 pounds of milk; but they possessed a good class of cows, and it required only 26.6 pounds of milk to make a pound of butter. Ijord's control apparatus was used to test the milk, and all the milk was tested at least twice a week. It was not compulsory on the members to take back the skim milk and buttermilk, but it was sold to those who wanted it at about one-fourth of a cent per pound for buttermilk and one-third of a cent per pound for skim milk. All the skim milk not disposed of in this manner was made into cheese. That little creamery produced aunually 121,000 pounds of cheese, or at an average rate of about 330 pounds daily. The cream was not sterilized because it was not found necessary. The strictest precautious to maintain absolute cleanliness were taken at every step, and they never had any cause to sterilize the cream. In winter the churning temperature was at about $60^{\circ} \mathrm{F}$.; in summer it was somewhat lower. The churn was of the usual pattern, the dasher making 150 revolutions per minute. When granules began to show in the churn it was washed down with a few quarts of water of the same temperature as the cream. The butter was worked as soon as taken out of the churn for the removal of the buttermilk, and 4 per cent of salt added at once. One hour later it received a second working. It was then placed in a cooling-box, and in winter a third working was given it in two hours from the last, but in summer it remained in the cooler until the next morning, when it received the final working and was packed. Superintendent Andersen did not find it necessary to use any butter color. In fact, there are many places where none is used, and only little is added where color is used at all.

There being so much cheese manufactured, I obtained details in regard to the method followed. The skim milk was warmed to $85^{\circ} \mathrm{F}$. and 14 per cent buttermilk was added and stirred violently and rènuet added. It then stood for thirty minutes, when the curd wis cut and stirred slowly, and at the same time the temperature was raised to $90^{\circ} \mathrm{F}$. by injecting steam under the vat. When the whey was clear it was drawn off, and three-tenths of a pound of salt was added to the curd for every 100 pounds of milk, and worked in by hand. It was next put in the cheese cups and a light pressure applied. At the end of an hour it was taken out and turned and replaced under the press. Ten hours later it was turned again, and the following morning it was taken outand put in brine, where it remained for twenty-four hours. From the brine it was put into the cheese room, which was so arranged that it could be heated at pleasure by the waste steam from the engine. It was first placed near the steam pipes where there was a temperature of about $77^{\circ} \mathrm{F}$. in win. ter; here it remained four days, being daily turued and wiped. After 
that it was gradually moved into a cooler temperature, remaining, however, in the cheese room for three months. If stored after that it was kept at a temperature of $55^{\circ} \mathrm{F}$. The price received varied considerably with the market, the maximum being abont $6 \frac{1}{4}$ cents per pound avoirdupois. The whey was sold to farmers at about one-twelfth of a cent per pound.

\section{NONNEBJERG FALLES CREAMERY.}

This creamery is, in several respects, the most interesting commercial creamery I visited. It is located at the city of Skanderborg, and is owned and operated by a noted bacteriologist, Mr. E. A. Quist, who, in his laboratory connected with the creamery, prepares the pure cultures which $I$ have already had occasion to mention. By the courtesy of Mr. Quist I was permitted to look into the details of his business. The plant consisted of a large and very substantial brick building (Fig. 28), the cost of which is immaterial, since it also contained a laboratory and other compartments not essential to a creamery. Mr. Quist, bought the milk from about 1,500 cows, but at the time of my visit

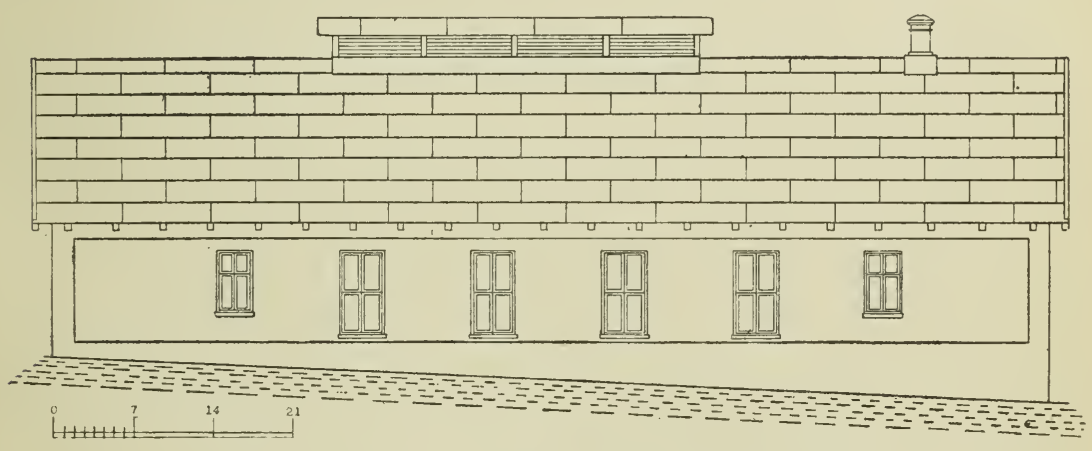

FIG. 28.-Projection of one side of Quist's creamery building.

the supply amounted to only 10,000 pounds daily. He had built and equipped this creamery chiefly to afford him opportunities for testing his pure cultures, and as he was compelled to pay a high price for the milk the profit was not great. However, the equipment of the creamery and the management of the product reached the highest point of modern skill attainable under the conditions there presented.

The sweet milk was sterilized as soon as received, at a temperature of $1670 \mathrm{~F}$. It ran directly from the sterilizing apparatus into the separators. Mr. Quist had tested the effect of separating at different temperatures, and he found that the milk was skimmed cleaner when run through the separator at about $167^{\circ} \mathrm{F}$. than it was when separated at any lower temperature. He would under no circumstances separate at a lower temperature than $95^{\circ} \mathrm{F}$. He found that at $167 \circ \mathrm{F}$. there remained fifteen one-hundredths of 1 per cent of fat in the milk; at $95^{\circ}$ F. there remained nineteen one-hundredths of 1 per cent, but at $77^{\circ} \mathrm{F}$. 
analysis showed that there remained twenty-two one-huudredths of 1 per cent of fat in the milk; and he maintained that, aside from the fact that the milk was sterilized, it was economy to raise it to that temperature in order to skim it cleaner. He used two Burmeister \& Waine separators, as he maintained that they had advantages over others. They can raise the skim milk and cream to a height of 8 feet through pipes which can be directed to any part of the creamery, and much work in handling milk and cream is thus saved. Again, the latest pattern of this separator, which I saw in use there, was provided with a lid which could be closed perfectly air-tight. There is, however, a suction of air into the bowl when the machine is running; this air was admitted through a tube filled with sterilized cotton, which thus, as it were, strained the air before it came into contact with the milk and cream. Both milk and cream were run from the separators directly to coolers. In this case Smith's round cooler was used, which lowered the tempera. ture to $46^{\circ} \mathrm{F}$. These coolers were provided with a cap, or sort of lirl made of tin, which fitted closely, and was designed to exclude the air as much as possible while the cream was trickling slowly down the sides. The notion that the coolers served any good purpose by aërating the milk is a mistaken one. It would be much better if the air were excluded, as the milk or cream becomes impreguated with bacteria from the air when thus exposed in a thin sheet over the sides of the cooler. The sole object in cooling the milk is to thus reduce the temperature so that the bacteria will cease to grow, and not, as is sometimes advocated, to expose the milk to the air, which has no effect on the creaming quality of the milk. When all the milk for the day had been separated Mr. Quist warmed a portion of the cream to $160{ }^{\circ} \mathrm{F}$., and this was mixed with the bulk of the cream in order to raise it all to a temperature of $68^{\circ} \mathrm{F}$., and at this temperature he added 12 per cent of his pure culture. Cream thus prepared stood from 1 p. m. till $7: 30$ a. m. the next day, when it was ready to churn. The pure culture was used every day if the milk was not sterilized, as was sometimes the case; but when the milk was sterilized daily he used pure culture only once a week and buttermilk the rest of the time. When the milk was sterilized it was found that the cream required nearly twice the amount of pure culture in order to cause the proper degree of fermentation in the desired time than was needed when not sterilized. Sterilized cream, on the other hand, did not require such close watching. If kept at a given temperature it would always attain a given degree of acidity in the allotted time, and the souring could also be permitted to go further without injurious effects.

The churns were of the usual pattern of the country. The churning temperature varied with the season. At the time of my visit it was $570 \mathrm{~F}$., but in summer it frequently went as low as $520 \mathrm{~F}$. The butter was dipped out of the churn with a sieve, placed on the butter-worlier, the buttermilk pressed out, and immediately afterward it was weighed 
and is per cent of salt added, thus giving it two workings at once. The third working took place three or four hours later in the winter, the butter being kept in a cooling box in the meantime; but in the summer it was not worked the last time until the next morning, being kept in the cooler all the time. Immediately after the last working it was packed in barrels of the usual size and placed in the storeroom to await shipment. Mr. Quist was particular to stop the churn at the right moment, not only for the sake of the consistency of the butter, but he maintained that if it was churned too long, or churned at too warm a temperature, the butter would contain a greater per cent of water than otherwise, and this affected its quality. The skim milk from this creamery was, for the most part, sold to people in the city, and that not disposed of in this manner was fed to swine.

I note the following points in regard to this creamery: (1) It was a solid brick structure which might last for centuries; (2) the ventilation was perfect; air could be admitted from all sides and the warm air could be let out by means of the ventilator in the roof; (3) it was constructed on the "gravity" plan; milk and cream could run directly to any desired place without having to be transported in buckets, thus saving a large amount of labor; (4) the floors were of natural Trinidad asphalt, and aside from being durable they could be kept scrupulously clean, and the drainage arrangements were perfect. I could not help comparing this to the wooden floors in our American creameries full of cracks and crevices, in which bacteria breed to perfection and through which not unfrequently bad odors arise from the water-soaked ground below.

The machinery and utensils had cost the owner the sum of $\$ 3,240$. I enumerate the following:

(1) An eight horse-power boiler.

(2) A six horse-power engine.

(3) Two "B \& W" separators, each with a capacity of 2,800 Danish pounds per hour.

(4) A receiving weight, on which rested a balanced weighing can, with a capacity of several hundred pounds. When the desired amount of milk had been weighed a light touch tilted this can so that the milk was discharged into-

(5) The receiving tank. A large, square can of heavy tin.

(6) A "forewarmer" or sterilizing apparatus, where the milk was heated to the desired temperature.

(7) The separators, which raise the cream through brass pipes to a little tin pan suspended from above, from which it runs through-

(8) Smith's cooler, which, in turn discharges into barrels.

(9) The skim milk is in like manner raised to a small tank above, directly from the separators, from which it runs to the delivery tank or through the sterilizer, as may be desired.

(10) A pump forced the water used in the coolers in a circle from a reservoir, filled with ice above the cooler, through the cooler and back again to the reservoir. This arrangement made it possible to save a good deal of ice, since the same ice water was used over and over again.

(11) The same pump was also used to convey buttermilk from the churns through a hose to a tank in the delivery room, where it was weighed ont to customers. 
(12) Two Smith's coolers, which have been described elsewhere.

(13) Two cream barrels, double, a tin can being fitted in an oaken casing.

(14) Two churns, also of oak wood.

(15) A large revolving butter-worker, of the American pattern, but which was highest in the center, so that the fluid as it was pressed ont ran to the circumference.

(16) An oak-wood butter trough, serving more especially as a table.

(17) Two butter-coolers, of the pattern described and illustrated under Dairy Implements.

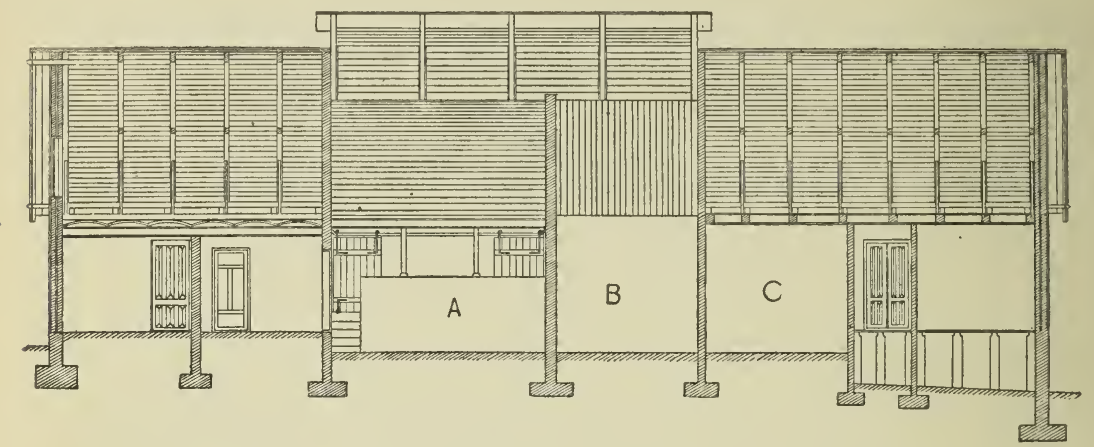

FIG. 29.-Vertical section from end of Quist's creamery building.

(18) A butter weight, on which the butter is weighed after the first working, in order to ascertain the required amount of salt.

(19) In a separate room cement basins for ice water in which to cool milk when this is desirable.

(20) A large cheese vat with copper bottom.

(21) Cheese presses.

(22) In wash room a hot-water tank, from which the necessary amount of hot water could be had at all times, when the engine was running.

(23) In the same place a steam jet, with arrangement for sterilizing all milk cans and other utensils.

I give herewith further illustrations of Mr. Quist's creamery. Fig. 29 shows a vertical section, from end to end, of the ereamery. $A$ is the

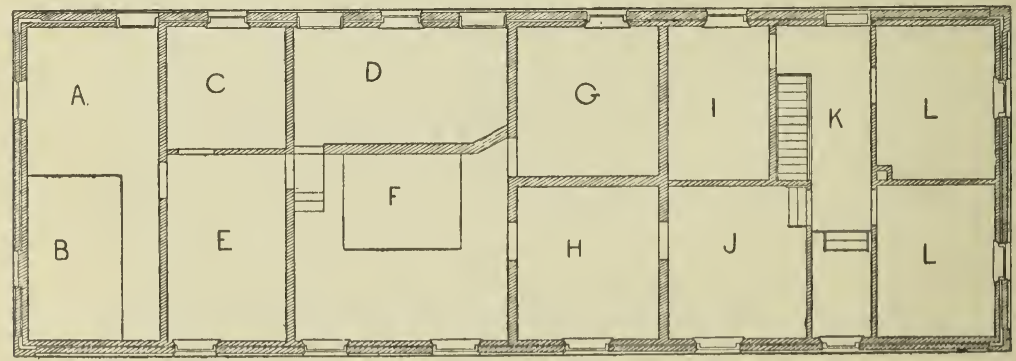

Frg. 30.-Plan of Quist's creamery building.

raised platform on which the milk is received and weighed and from which it runs down to the separator, $B$ is the churning room, and $C$ the room in which the butter is worked. The ventilator over the work room is a noticeable feature. This ventilator could be closed or opened at pleasure. Fig. 30 is the plan of the building. A is the coal room, $B$ the boiler room, (C the cheese room. This room would be warmed by 
steam from the boiler. $E$ the engine room, $D$ the platform on which were the milk vats, $F$ the separating room below this platform, $G$ the wash room, $H$ the churning room, $I$ office, $J$ the room in which the butter was worked and cooled, $\dot{K}$ hall, and $L L$ laboratories. There were rooms above the laboratories for the accommodation of the help.

To these descriptions I could add much more material of the same nature, but I trust that the methods described in the foregoing cases will enable creamery and dairy men to get a reasonably clear idea of the practice in the Danish dairies.

\section{DAIRY BACTERIOLOGY.}

It is only between two and three years since the use of pure cultures of bacteria were introduced into the creameries of Denmark, as a means of improving the butter, but the results bave been so uniformly successful that they are now used in all dairies whenever there is necessity for them. The honor of the introduction of this improvement in creamery methods does not belong to any one man, though, perhaps Prof. V. Storch, director of the experimental laboratory at Copenhagen, deserves the "lion's share" of the credit. He has been at work on the problem for some six or eight years, and from time to time has published the results of his researches. Other bacteriologists took up the same line of work, and the result was that pure cultures of the beneficial bacteria were put upon the market by the three different laboratories at about the same time. Prof. Storch had already then isolated and cultivated several forms of these bacteria, but he has not put any of them on the market.

As the several investigators worked independently of each other, each can be credited with an original discovery, especially since the bacteria employed are not the same in all cases. From the investigations by Prof. Storch, Prof. Fjord, and others, it soon became evident that the quaiity of the butter depended, at least in a large degree, on the presence or absence of certain minute organisms. It was found that in faulty butter certain forms were present which, when isolated and cultivated, produced the characteristics which were objectionable. In like manner it was found that in high-class butter certain other forms were present, which would, in like manner, produce the characteristic aroma and flavor when cultivated by themselves. This led to the natural recognition of two general classes of bacteria, one of which was injurious to the interests of the dairy, and the other one beneficial. When this fact had been settled, the practical question before the investigators was how it would be possible to repress one class and encourage the other. Prof. Fjord had, in the meantime, perfected his pasteurizing apparatus, which has been figured and described elsewhere. This was invented chiefly with a view to improve the keeping qualities of skim milk so that it might reach the patrons and other con- 
sumers in a fresh and sweet condition. This was accomplished by heating it to a temperature of upwards of $150^{\circ} \mathrm{F}$. It was found that this temperature destroyed enough of the bacteria which caused the milk to change to make it possible to keep it sweet for from twelve to twenty-four hours longer than when it is not thus heated. The next step was to apply the same treatment to the cream, or to the sweet milk before it was separated, with the same results. Heated to a temperature of between $150^{\circ}$ and $160^{\circ} \mathrm{F}$., or upwards, it was found that the most active forms of the bacteria were killed, and that by again cooling the cream to about $75^{\circ}$ or $80^{\circ} \mathrm{F}$., at which bacterial life becomes active, the cream could be inoculated by any desirable form of bacteria if these could be obtained from pure cultures, and that the forms with which the cream was thus impregnated, meeting with no opposition from other torms, would develop rapidly in enormous numbers and give their peculiar characteristics to the butter. This is exactly what is now done in practice. The cream is not always pasteurized because, if there are no injurious bacteria present, at least in such numbers as to cause a deterioration of the product, there is no necessity for attempting to kill them, and the addition in sufficient quantity of a pure cul. ture would at once give the latter the upper hand, and their peculiarities would become prominent. A rather more thorough sterilization would be effected if the milk were raised to the boiling point; but a temperature of much over $160^{\circ} \mathrm{F}$. gives the characteristic boiled taste to both milk and cream, which is objectionable and must be avoided. It is found in practice that this temperature destroys nearly all the organisins in active growth. The spores will survive this temperature, but it takes them longer to develop and by the time they become ready the pure culture has the mastery.

As a higher degree of heat than that required for their normal development is destructive to the bacteria, so in like manner a reduction of the temperature retards their growth. It does not destroy them, if not below the freezing point, but it stops their development and renders them inactive. It is for this reason that pains should always be taken to reduce the temperature of the milk, by means of ice water, to a point as near the freezing point as practicable. Heat and cold, relatively speaking, are therefore effective means in controlling bacterial life in the creamery.

There were at the time of my visit three laboratories in which pure cultures were propagated and sold to the dairies. One of these was the laboratory of Christian Hansen, the famous butter color and rennet mannfacturer, whose products are well known in this country. Another was a firm of chemists named Blauenfeldt \& Tvede. Both of these laboratories were in Copenhagen. $\Lambda$ third laboratory belonged to Mr. E. A. Quist, of Skanderborg, whom I have already had occasion to mention in the description of his creamery. These dealers in the pure cultures keep their methods of propagation secret. The isolation of the germs 
can, of course, be accomplished by any bacteriologist; but they claim that it is only through a long series of experiments that they have hit upon a composition for the nutritive fluid in which to grow them to best advantage. It also requires certain forms of apparatus to which they may lay claim as inventors, and these features they do not propose to divulge, as it would probably increase competition to a point beyond profitable production. Moreover, I was informed that each form of these minute organisms had to be treated in a manner peculiar to itself, and as the several growers did not deal in the same bacterial forms, their methods of treatment in the laboratory were not alike. How many forms of the beneficial bacteria are in existence is at present unknown; but it is known that they are numerous. A dozen or more forms have already been discovered. Blanenfeldt \& Tvede informed me that their culture contained many forms, but how many was not stated.

Mr. Quist, on the other hand, uses only two forms, which I had opportunity to study through the microscope. There was a decided difference in the smell and taste of the preparations offered by the two firms. Blauenfeldt \& Tvede's cultures had a sharp, sour taste and smell. Mr. Quist's cultures were less acid and possessed a peculiar nutty aroma, such as we find in the best samples of butter. I have had opportunity to see the effect of Mr. Quist's culture in this country. I bought samples of him which he shipped to the Department of Agriculture. A portion of these samples was forwarded to me, and I tried them in the Belle Springs creamery, owned by Mr. J. E. Nissley, the president of the Kansas Dairymen's Association.

The creain was not sterilized before the cultures were added, but they had a decided influence on the butter. Both Mr. Nissley and his butter-maker pronounced the quality superior to any they had made formerly, and they at once became converts to the process. I forwarded samples of the pure cultures to Prof. H. C. Wallace, who has charge of the dairying in the Iowa State Agricultural College, but I have at this writing not heard with what results they were used. In Denmark, where the cultures will be in transit at most only a couple of days before they reach their destination on the dairy farms, they are put up in a preparation of skim milk; but in shipping to this country, where they may be in transit for many weeks, they must be specially prepared in order to retain their activity the necessary length of time. The samples shipped by Mr. Quist were sent in three mediums; one a preparaticn of milk sugar; another in sterilized cotton, and the third in a nutritive fluid of his own invention. At the Belle Springs ereamery we used only the first and the last of these. Although they had been in transit from the 10th of March until the 5th of June, they appeared to be perfectly fresh and ready for business. The cotton preparation I did not try. Should any of our creamery men decide to try Mr. Quist's cultures, they can be had without fear of deterioration on the journey. It is, however, to be supposed that our bacteriologists will soon take 
this matter in hand and give us pure cultures within easy reach of the creameries in this country. The American branch of Christian Hansen's laboratory prepares and sells the pure cultures, which had their origin in his laboratory at Copenhagen.

\section{CONSTRUCTION OF ICE HOUSES.}

Ice being used so largely in the Danish dairies, they have given considerable attention to the construction of ice houses. Formerly it was customary to store the ice in a pit in the ground, this pit being dug on a hill or rising ground, so that a drain could be laid from the bottom of it to carry the water off readily. These pits were walled up with brick or granite and covered with thatch. But it was found that this construction was neither convenient-nor economical, and it has long since been abandoned. Their ice houses are now constructed above ground, and, whenever practicable, in direct connection with the ereamery. Although ice is not a scarce article in that country, it has been found more economical to build a substantial ice house with double walls
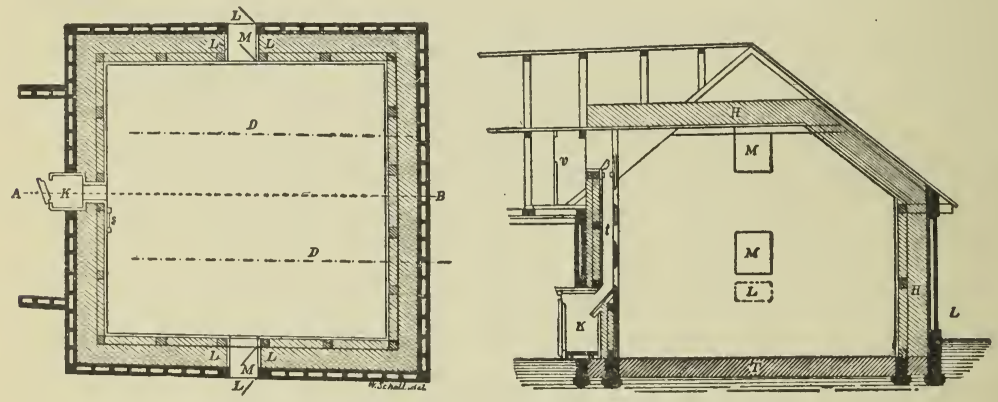

FIG. 31.-Ground plan and section of ice house.

than to put the ice in a flimsy structure with thin walls, through which the changes of temperature are readily felt.

Fig. 31 shows the plan and section of an ice house of modern construction, built in connection with a creamery. Both the outside and inside walls are, in this case, of wood, but they are also frequently built of brick. The plan shows that the outside studding is boarded up on both sides. On the outside, ordinary weather-boarding is used, but on the inside it should be made as tight as possible. Inside of this shell, and 2 feet from it all around, is another set of studding which is also boarded up on both sides, the boards being fitted nicely together. The 2-foot space between these two walls is filled with some non-heatconducting material, chafted straw or hay being the most common. Any material thus used will, in the course of two or three years, have gathered moisture so that it will begin to mold, or, if it is of a nature which prevents its molding, it will nevertheless be moist, and in this condition it has partly lost its non-conducting properties and it onght to be renewed. It is therefore essential to have small door's at con- 
venient places in the outside shell, near the ground, through which the packing can be removed, and others near the top through which it can be renewed. The space is large enough to admit a person to tramp it together.

The ice house here represented opeus into the creamery and, in this case, no special anteroom is therefore needed; but if the entrance is from the outside an anteroom is essential in order that the warm air may be excluded from the ice house on entering. In the section of this ice house the letters $H H$ represent the chaff which is packed in between the double walls. $M M$ are doors through which the ice house is to be filled and $L L$ are doors used for the renewal of the chaff. The letter $T$ represents a layer of peat on which the ice rests. It will be noticed that the layer of chaff extends over the ice as well as up the sides of the building. The ice is removed through the shute $t$ and falls into the box $K$, from which it is removed as wanted during the day. Entrance to this ice house is gained at $V$, under the ceiling, where there also is a window to admit some light. In the plan the letters $L$ and $I I$ represent the openings, the same as in the section. $K$ is the box into which the ice is thrown, $D D$ are drains which carry off the water as the ice melts and the line $A B$ represents the point at which the section is drawn.

In this connection it is interesting to note an experiment carried out by Prof. Fjord to ascertain the efficiency of the substances named as packing materials to be used between the walls of ice houses. The results are based upon the amount of water obtained from melting ice kept in an ice box, placed in a warm room, and the double walls of which were successively tilled with the materials named. Chaffed straw is taken as the standard and represents 100. The figures are as follows :

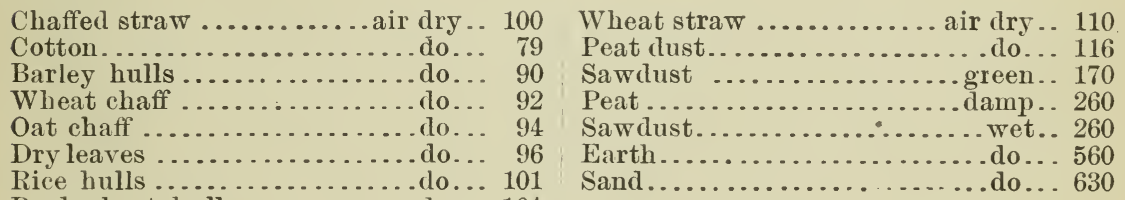

Buckwheat hulls ............ dlo... 104

The proper use of ice in storerooms is also an important point. In connection with the experimental laboratory in Copenhagen is an exhibition building for dairy products. It is in this building that the frequent competitive exhibitions of butter from dairies all over the country are held. It is essential that the butter should be kept at a low temperature, and to this end Prof. Fjord constructed an ice house in connection with it which is so arranged that the air which enters the strreroom must pass over the ice in the ice house. There are ventilators placed near the bottom of the wall which separates the ice house from the storeroom, and the cold air from the ice house flows through these into the storeroom. The principle is illustrated in Fig. 32, the 
arrows showing the course of the air currents. The cold air comes out from the ice directly under the butter packages, and as it becomes warmer it rises and again enters the ice house, or large ice box, as the case may be, at the top. It is a principle which it may be advantageous for our creameries to put into practice.

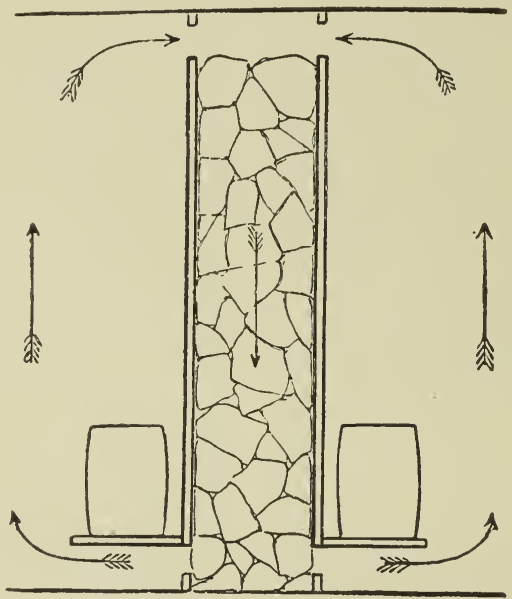

FIG. 32.-Use of ice in storeroom.

\section{STATE AID TO THE DAIRY INDUSTRY.}

As I have shown in the early portion of this report, dairying is the chief industry in Denmark, and it would manifestly be to the interest of the state to aid in its development in every possible way. The persons with whom I conversed on the subject were, as a rule, of the opinion that the state did not do its full duty in this respect. However, considering that it is a small country, under heavy expenses in maintaining the Government, and with but limited resources, the state has given no inconsiderable aid in this direction. It does not expend large sums in any one direction. Its policy is, on the other hand, to encourage the people by small money donations, and, by furnishing them expert assistants, to solve for themselves the problems as they appear. The experimental laboratory at Copenhagen should perhaps be named first. It is maintained wholly by the state in the interest of the indus. tries among which dairying, of course, takes the leading position. It was in the eapacity of director of this laboratory that Prof. Fjord and his many able assistants made the long series of experiments and investigations into the various phases of the dairy industry which have been so fruitful in beneficial results. Yet these investigations were made without any great cost to the state. As a rule, the large farmers throughout the country. who were interested in the results, lent their herds to these experiments with unstinted hand, free of charge. Under these conditions Prof. Fijord was able to earry on ex- 
periments at several-frequently at as many as half a dozen-farms at once. He furnished the expert assistants, who had direct charge of the experiments aud supervised the work, while the farmer, who owned the place where the work was carried oil, as a rule paid for the labor.

The Government aids indirectly by offering premiums for the best dairy cattle at the shows. Such premiums are not, however, restricted to the dairy interest, but have in view the. improvement of domestic animals of all kinds. It encourages the improvement of the dairy breeds by aiding the "bull associations," to which I have already had occasion to refer. For this purpose $\$ 13,500$ are at present divided yearly among the associations in various parts of the country. Thus, the state will pay one-third of the cost of keeping these bulls, within the limits of the sum named, provided the association conforms to specified rules. And, in order to be entitled to this aid the bull must be at least two years old, he must not be put to more than 100 cows, and he must be of approved quality and pure breed.

The state gives direct aid to a limited number of young men and women who desire to become experts in the dairy business. They will thus be sent to some approved agricultural school for a time, and later to some practical dairy of more or less renown, where they can obtain the necessary information and experience. Twenty-two pupils received state aid of this character in 1892 at a cost of only about $\$ 1,620$.

The state aids indirectly in the advancement of the dairy industry through the Royal Agricultural Society, which is supported in part by the state. This society has been of untold benefit to Danish agriculture. It was organized in 1769, and has ever since labored by the most effective means within its reach to improve agriculture and the condition of the farmer. Its membership has, as a rule, at all times been drawn from the leading men of the country, and these have contributed freely both of their substance and work to further the object for which the society was organized. Their most effective means are, perhaps, the education in agriculture of promising young men who, in turn, become leaders in the industry and influential factors in its improvement. This has been the main line of work for many years and is still continued. The society also maintains at least one expert adviser in dairying, whose counsel is available to any one who stands in need of advice. This post is at present filled by Mr. Bernhard Bóggild, who is not only a scientist but an expert in all dairy matters aud an authority everywhere recognized. He is author of the book on dairying I have already mentioned, which is, perhaps, the best work on the subject in print. The society also aids in the improvement of the dairy cattle by supplementing the Government in giving prizes at shows and in aiding in the purchase of good bulls to be used by the bull associations. It aids by giving publicity to all useful information in the form of pamphlets and reports, which otherwise would not reach any considerable number of the farmers. The Royal Agricultural 
Society of Demmark must thus be considered as affording important aid to the dairy industry, in which work it is assisted and encouraged by the state.

The state aids the dairy by maintaining three dairy experts as advisers to the people. Each one of these is assigned a definite territory. They are all under the direction of the minister of the interior. In all there are nine such advisers for the benefit of agriculture, but three of them have their work confined to the dairy. These gentlemen may be called by any creamery or dairy man in the district to assist in discovering faults in the butter, to give their advice in regard to methods of treatment of the cattle, and of the milk and butter, and, in short, to render every assistance in their power in aiding the dairy industry. For this service the adviser is paid by the state; but the person calling him pays a certain proportion of the traveling expenses and maintains him with board and lodging while at his place. I can perhaps give no better idea of the nature of this work than by quoting a portion of the report of one of them to the minister of the interior for the year 1891. This report is by Stats-Konsulent (State Adviser) J. Nissen-Dall, who is located in the city of Fredericia, and whose activity is confined to a portion of the southern peninsula and several of the smaller islands. He says:

I have during the time covered by this report been called upon to give assistance in the dairies of $\mathrm{my}$ district one hundred and forty-five times. I have given twenty-seven lectures on dairy matters. I have, by invitation, attended eight meetings of the farmers, and $I$ have acted as judge of dairy exhibits nineteen times, most of the exhibitions being held at the experimental laboratory. I attended an exhibition of cheese at the same laboratory, and I attended three exhibitions of butter under the auspices of the dairymen's association. In conjunction with Konsulenterne (advisers) Bóggild, Segelcke, and Buhl-all dairy advisers-I have visited three dairies on Funen to witness experiments in pasteurizing milk and cream. Further, I have, with pecuniary assistance from the Royal Danish Agricultural Society, visited a number of dairies where the pasteurizing of cream was being introduced, and I also made investigations in the neighborhood of Vejle. Toward the close of July, 1891, I was present at the final examination of the pupils in the Ribe Dairy school. In connection with the exhibition of butter, to be held in Góteborg (Sweden), I visited seven dairies in my đistrict during May and June. I attended the exhibition in Góteborg, from the 31 to the 9th of August. Besides all of this I have answered a large number of questions by mail. The majority of my lectures have been held in the interest of coöperative creameries, where my subjec has been The Production and Treatment of the Milk. I lectured once before the members of the Fodder Stuff Association, and once at an exhibition of cheese in Ringe.

This will give a fair idea of the rature of the work assigned to these alvisers, and the results prove that the plan is thoroughly practical. 'This business of advising in dairy matters has become a profession in Denmark. Besides the four official advisers, one maintained by the agricultural society and three by the state, very many of the dairy associations, of which there are many thoughout the country, maintain advisers of their own, and whenever a fault appears in the dairy or creamery which the man in charge is unable to remedy an adviser 
is called in to investigate the cause and remedy the fault, just as a farmer here will call a veterinarian when his horse or cow gets sick, or a doctor when a member of his family gets sick. In dairies where hundreds of pounds of butter are made daily and where a deterioration in quality is sure to be followed by a decrease in price, it is of the ntmost importance to remedy a defect as soon as it appears, and this is the business of the adviser.

\section{MARKETS FOR DANISH DAIRY PRODUCTS.}

Statistics published by the Government for the year 1891 show that butter was exported from Denmark to the following countries during that year, to the amounts named:

\begin{tabular}{|c|c|c|}
\hline & $\begin{array}{l}\text { Danish } \\
\text { pounds. }\end{array}$ & $\begin{array}{c}\text { Pounds } \\
\text { avoirdupois. }\end{array}$ \\
\hline Faroe Islands. & 17. 543 & 19,297 \\
\hline Iceland ................... & 15,293 & 16,822 \\
\hline Greenland $\ldots \ldots \ldots \ldots \ldots$ & 13,618 & 14,980 \\
\hline Danish Possessions in West Ind1 & 1,314 & 1,445 \\
\hline 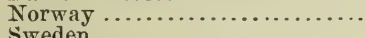 & 202,730 & 223,003 \\
\hline Sweden. & 482,950 & 531,245 \\
\hline Russia........ & 623 & 685 \\
\hline To Hamburg ......... & 203,425 & 223.767 \\
\hline Lubeck ........................ & 348,350 & 383,185 \\
\hline Slesrig-Holstein and Lauenburg & 860,474 & 946,521 \\
\hline To the rest of Germany ......... & $\begin{array}{l}10,240 \\
080,171\end{array}$ & 11,264 \\
\hline To England .............. & $88,959,471$ & $97,855,418$ \\
\hline Holland & 155,953 & 171,548 \\
\hline Belgium & 52,726 & 57,998 \\
\hline France. & 1,211 & 1,332 \\
\hline Portugal & 2,803 & 3,083 \\
\hline Spain.... & 18,952 & 20,847 \\
\hline Itals $\ldots . . .$. & 94,526 & 103,978 \\
\hline United States ............ & 5,500 & 6,050 \\
\hline To miscellaneous places.. & 7,560 & 8,316 \\
\hline Total & $91,455,262$ & $100,602.784$ \\
\hline
\end{tabular}

\section{ENGLISH IMPORTS OF DANISH BUTTER.}

England, it will be noticed, is the only country which consumes Danish butter in any considerable quantity, and this is the market which they cultivate above all others. I stopped in London a few days in order to investigate this subject on the spot, and spent considerable time among the importers of Danish butter. What I learned in this regard I stated briefly in my preliminary report, and I can not do better now than to repeat what I then said, which was as follows:

All the testimony I gathered agrees in this, that the Dauish butter brings the top price in the English market with one exception, and that exception is made in favor of the comparatively small amount of fresh unsalted butter shipped to London from northern France. It caters to the taste of a small but select class of consumers, and it reaches their tables inside of twelve hours from the churn. This butter, I was told, brings from one to three or four pence more per pound than the Danish butter. I tasted this butter, and to me it lacked flavor and the pleasant briskness peculiar to a fine grade of salted butter, but there is no accounting for tastes. There is a market for such butter in London, and the dairymen from northern France supply it. The Danes have tried to supply it, but their product can not reach England in less than three days, and this length of time was fatal to the rich, creamy flavor which the market demanded, and the attempt was given up. 
I found also that the Danish butter finds its best market in the manufacturing towns, and that these consume relatively much more of it than the capital. This would indicate that it goes especially to the middle class and well-to-do artisans.

I inquired particularly about the manner of shipment and whether any attempt was made to keep it in a uniform temperature during transit, and I found that so far the temperature had not been controlled. It had been tried and proved that nothing was gained by it. The conditions are such that it must be handled several times, and often many times, between the dairy and its destination. It must be transferred from wagon to railway, and from railway to boat, several times, following the route of transportation from island to island to the port of shipment, and during these transfers it is often exposed for hours to a burning heat or chilling cold, which would so far affect it that no attempt to equalize the temperature where it could be controlled could remedy the damage it might already have received, while it stood in the sun on the wharf or railroad station. It is true only a very small per cent is injured from these causes, but the risk still exists. Transportation which shall be absolutely safe to the texture and flavor of the Danish butter is still an unsolved problem. Nearly all Danish butter is packed and shipped to England in small barrels, of which there are two sizes, holding, respectively, 56 and 112 pounds. This sort of package is easily handled and has become customary. The Australian and New Zealand butter is usually shipped in square boxes, holding almost 36 pounds. The French butter above mentioned is done up in 2-pound rolls and forwarded in baskets containing usually 24 pounds. To my surprise I found in several of the warehouses considerable quantities of Russian butter. Russia began to ship butter to England five or six years ago, and the amount is growing year by year. It is put up in barrels in imitation of the Danish butter.

The Danish butter maintains its high standing chiefly through its uniformity. I sampled numerous packages from various parts of the country and found them very nearly all alike. It is all first class; it all has the same bright straw color, and the same degree of saltness, and it varies but very slightly in aroma, flavor, and texture. Mr. Jacobsen told me that he had endeavored to secure consignments of a cheaper grade of butter, as he thought he had a market for it, but word came back that it could not be found; no inferior butter was offered for export. This uniformity in quality inspires both dealer and consumer with confidence. They know they can buy the Danish butter and be sure of getting a good article without necessitating their tasting it before they purchase. Not so, I was told, with other countries. While they might forward some very fine butter, it was never safe to depend upon it.

As to the prices which wholesale dealers in London realize on Danish butter, they rule low from an American point of view. There are fluctuations, of course, but it sells generally from $14 d$ to $18 d$ a pound, the latter figure being considered high.

To give an idea of the growth of the Danish export butter to England in recent years, I quote the following figures from the "Annual statement of the tracle of the United Kingdom," which I procured. It is an official and entirely reliable publication:

English imports of butter from Denmark.

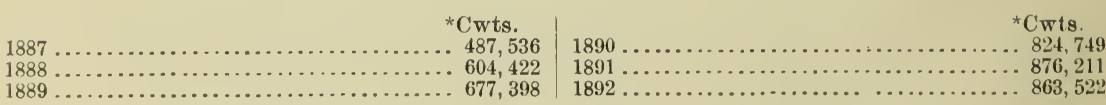

ralue of same in pounds sterling.

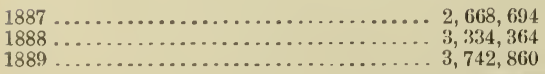

${ }^{*}$ Cwts. $=112$ pounds avoirdupois. 
I can not comment on these figures now, but they speak for themselves. At the same time the import of butter from the following countries has also increased: Sweden, Russia, Belgium, France, United States, New South Wales, New Zealand, and Canada. The greatest increase has been made by New Zealand, but still the largest import therefrom was in 1891, and amounted to only $28,647 \mathrm{cwts}$. The imports of butter from Norway, Germany, and Holland have materially decreased in this time.

\section{STATE AID TO FOREIGN MARKETS.}

To aid in the sale of Danish agricultural products in the English market, the Government detailed an official in 1888 to be permanently located in England, whose duties were to aid by all means in his power in the sale of the Danish dairy products. The office is filled by an accomplished scientist, Mr. H. Faber, who has, according to the published records, done most excellent work in the line of his appointment. To give a more complete idea of the nature of his mission I translate the following instructions to him from the minister of the interior, as published in a report from the interior department for the years 1889 and 1890, entitled "Report of the Landókonomiske Konsulentvirksomhed:"

\section{INSTRUCTIONS TO GOVERNMENT AGENT FABER.}

(1) It will be your duty to support the good name and sale of Danish agricultural products, and particularly the dairy products on the English markets, by all the means at your disposal; and in so far as you find these moans unsatisfactory, to make propositions for new and effective aids.

(2) You must direct your attention to the conditions governing trade and transportation of Danish agricultural products, and particularly the dairy products, both before they reach the English market and after they arrive there.

(3) It will be your duty to meet and correct through the press, and chiefly through the English press, all false and injurious statements concerning the Danish agriculture, and particularly our dairy industry and its products, and take the lead in setting a proper valuation on these.

(4) Your attention must be directed to frauds, both in and outside of England, which can injure the good name of Danish butter-as, for example, the misuse of Danish names, adulteration of Danish butter with oleomargarine, and the like-and make proposals for counteracting such frauds. For that purpose you must be supplied with the necessary apparatus, chemical and microscopical, to enable you to discover such adulterations.

(5) It will be your duty to keep your attention directed to the markets and ascertain if other and profitable markets can be opened for our agricultural and particularly our dairy products.

(6) When occasion demands it, and at least once a year, you must report through the Royal Danish Agricultural Society on the English market for our agricultural and especially for our dairy products, with such observations as you may see fit to make on the subject, particularly concerning the demands of the consumers, the faults and wants in our products, which should be corrected, and concerning such alterations in trade methods as may seem desirable.

(7) It will be your duty, as far as possible, to answer all inquiries directed to you, whether from Danish institutions or from men in private life concerning agriculture and the dairy. In like manner you ought to answer similar inquiries from trades-

8538-No. 5- 9 
men and consumers of Danish agricultural and dairy products in England, in so far as you may thereby serve Danish interests.

(8) You must neither directly nor indirectly allow yourself to become financially interested in the sale of Danish agricultural products or in other trade affairs.

In compliance with the above instructions, Mr. Faber took hold of his work with a vim. We learn from reports to his Government that he set about to correct false impressions concerning Danish dairying, through the press, exposed dealers who pretended to sell Danish butter, but in reality handled inferior articles from other countries. He even goes so far as to prosecute under English law those whom he can prove adulterate Danish butter or in other ways injure the trade in the genuine article. Comments are unnecessary. He renders signal service to the country in his appointed field of labor, and doubtless the increasing demand for Danish butter in England is due largely to his efforts.

\section{RESTRICTIONS ON THE SALE OF OLEOMARGARINE.}

In Denmark, as in the United States, the unrestricted sale of oleomargarine has proved to be injurious to the agricultural interests and especially to the dairy interests. To prevent this, strict laws have been enacted which regulate the manufacture and sale of this article. To quote the law, or even the chief points of it in this report, would perhaps be improper; but I will briefly note some of the leading features of the traffic and its restrictions. The law makes a distinction between "margarine" and "oleomargarine." It appears from statistics for the year, from the 1st of April, 1891, to the 1st of March, 1892, that there were in all sixteen factories where "margarine" was made and one where "oleomargarine" was made, which together produced 13,339,984 Danish pounds, for the period covered, and that there were in all 7,991 places in the country on the 31st of March, 1892, where this material was sold. This included both retail and wholesale houses. Among the chief points in the regulations concerning its sale are to be noted-

(1) The oleomargarine shall be colored light yellow. And as a standard of comparison color tables have been adopted in which different shades are numbered, the law providing that the color must not be darker than No. 9 of that scale, which is a very light straw yellow.

(2) That oleomargarine shall always be put in tubs or barrels, which must differ in shape from the ordinary packages in which butter is kept. The form adonted is an oval tub or barrel, in which the major axis shall be at least one and a half times longer than the niinor axis, and the term "Margarine" shall be marked upon the ends and sides in very large letters. The accompanying illustration (Fig. 32) shows the form of the receptacle and the marking. This is not confined to large packages. All smaller vessels nsed to hold oleomargarine in the retail stores shall be of the same form and distinctly marked in the same manner, and all wrapping material used when purchases are wrapped up for customers shall be marked iu the same manner; and, further, the per cent of butter in the mixture, if any, shall likewise be marked on the packages. 
(3) All who deal in oleomargarine in any form shall procuro a license from the Government before they can begin business.

(4) Three oleomargarine inspectors are appointed by the Government, each of whom has a definite district. These inspectors are charged with the duty of sceing that dealers and mauufacturers comply with the law. They have authority to enter any establishment where butter is manufactured and sold and where oleomargarine is manufactured and sold, to examine the books and look into the details of the business, and they are required to take samples frequently, which are forwarded to a laboratory for analysis.

(5) The law prohibits the manufacture of butter and oleomargarine on the same premises.

These are only a few of the leading points, which, however, may serve to give an idea of the thoroughness of the control under which it is kept.

The penalties for violation of the law are severe and punishment is sure and swift. This law was first enacted in 1885, when, however, it was found to be too lenient. Later amendments have strengthened it

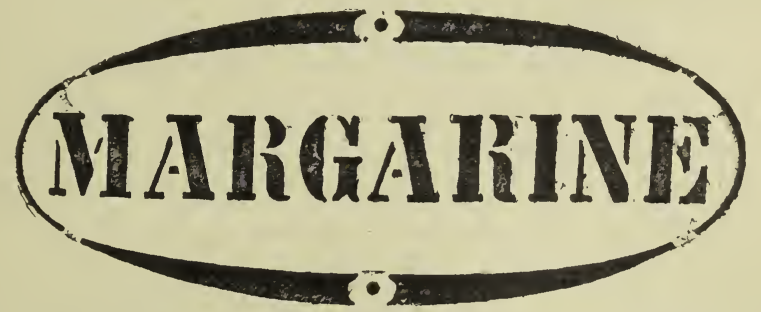

FIG. 33.-Required shape and narking of oieomargarıne package (reduced one-lıalf.)

on several points, and evasions of the law are now of comparatively rare occurrence, which is shown by the following statistics: From the 1st of May, 1888, to 31st of March, 1889, there were 176 convictions for violations of the law; from the 1st of April, 1889, to the 31st of March, 1890, there were $\$ 1$ convictions; and from the 1st of April, 1890, to the 31 st of March, 1891, there were but 36 convictions. The dairymen generally are satisfied with the workings of the law.

\section{AGRICULTURAL AND DAIRY EDUCATION.}

The two lines of education are so intimately connected that it is difficult to separate them from each other, and I therefore treat them together. There are a large number of agricultural schools and high schools in the country, nearly all of which give more are less instruc. tion in dairy matters. I shall in the following account give a brief description of these schools, as far as I could ascertain the facts during the short time at my disposal. The institution which ranks above all others in the country is the "Royal Veterinary and Agricultural College" at Copenhagen. 'This is an old institution, having been in exist. ence more than one hundred years. It was first begun as a veterinary school, and later branches in agriculture were added to it, but the veterinary branch of the institution continues to be an important oue. 
A law dated April 12, 1892, concerning the institution, of which I have obtained a copy, requires in its first paragraph the number of teachers in each branch to be as follows:

(1) In veterinary science, 3 ; (2) in agriculture, including dairying, 3 ; (3) in stock-breeding, 2 ; (4) in forestry, 2 ; (5) in surveying, 2; (6) in botany, 2 ; (7) in economic horticulture, 1 ; (8). in anatomy, 1 ; (9) in animal physiology, $1 ;(10)$ in chemistry, including agricultural chemistry, 2 ; (11) in physics, 1 ; (12) in zoölogy, 1.

This is exclusive of assistants and lecturers, for the employment of which the same law makes an appropriation of about $\$ 8,834.50$ annually. The same law puts the salary of a professor at $\$ 864$ a year, with an addition of $\$ 162$ for every five years he remains in the college. This is a relatively much higher salary than is paid to the same class of teachers in this country. The same law appropriates $\$ 233,550$ for the enlargement of the institution in nearly all of its branches, said amount to be expended in two years. In a report of the institution for 1891, which I obtained, I find the number of students given as 372 . Provision is made for both long and short courses, so that it is difficult to say just what the length of the course is, but it does not exceed four years. The instruction is not free, but certain of the students are assisted by the state. Such assistance is invariably given to the best students. The dairy instruction is in charge of Prof. Segelcke, to whom I have already had occasion to refer. The teaching at the school is entirely theoretical, as far as the dairy instruction is concerned, but before a diploma is granted the student is required to spend a given time, which varies with his accomplishments, in a good dairy where the practical information can be fully supplied. The dairying is given as a part of the course in agriculture. I visited the institution and saw some of the students at work. The instruction is most thorough and confined strictly to agriculture and the related sciences. There is no literary instruction whatever given in the institution. This students are required to have before they enter.

to this institution comes a class of agricultural schools, some teri number, all of which are private institutions. These, however, recelve state aid in proportion to the number of students they have, the larger schools receiving each $\$ 810$ annually. At all of these schools the students pay for their instruction. In most of them the course is two years in length and confined strictly to agriculture and the related sciences. Most of them give only theoretical instruction in dairying, the practice being obtained from some first-class dairy. I will briefly mention some of these schools.

(1) Dalum Agricultural School, per Odense.-This is the largest of that class of institutions. It had at the time of my visit to it 107 pupils, young men of the average age of 23 years, and from all parts of the country. The course was two years in length, thoroughly practical in its nature, and included as much of the sciences related to agriculture 
as the time would permit. Dairy instruction was given during the summer months. I have already had occasion to mention the farm connected with this institution.

(2) The Classenske Agricultural School, Noesgaard, on the Island of Falster.-They have here but a limited number of pupils. The instruction is confined strictly to agriculture and dairying.

(3) Lyngby Agricultural School, per Lyngby.-There is an experiment station connected with this school maintained by the state.

(4) Thune Agricultural School.-This gives theoretical instruction only, and is designed to accommodate students who can spend only a short time in school. It has two courses, une of nine months and one of six months. It has 58 pupils.

(5) Ladelund Agricultural School, per Brórub.-This institution has some 70 students and it gives theoretical instruction in dairying, as well as in agriculture.

(6) Malling Agricultural School.-There are 40 students at this school. It is a well-equipped institution of high standing.

(7) Yding Agricultural School, per Skanderborg.

(8) Morsó Agricultural School.

(9) Odense Agricultural School.

(10) Lyby Agricultural School.

All of these are technical agricultural schools. But aside from them there are a large number of ordinary high schools which give agricultural instruction. It is estimated that there are about 100 of this class of institutions, many of which are most excellent. They are not classed with the agricultural schools because their main instruction is literary in its character. But, as stated, nearly all give some instruction in agriculture and dairying, and in the aggregate their influence is felt as a force in agricultural education.

Next to the schools comes the instruction received in the dairies. There are some 2,000 dairies in the country which receive pupils, and it is here that the majority of dairymen are educated. The instruction is of the most practical nature, the pupils doing any work which the business requires. As I have already had occasion to remark, these pupils usually receive their board and lodging and a very small salary besides, though in a few cases they pay for the privilege of working in the dairy. In some cases the dairy superintendent lectures to the pupils and thus, in a measure, supplies the lack of theoretical instruction, but this is the exception and not the rule. 






an

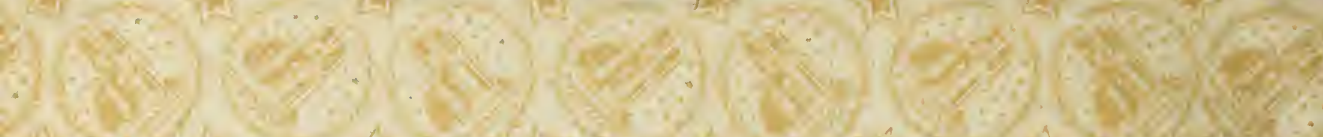

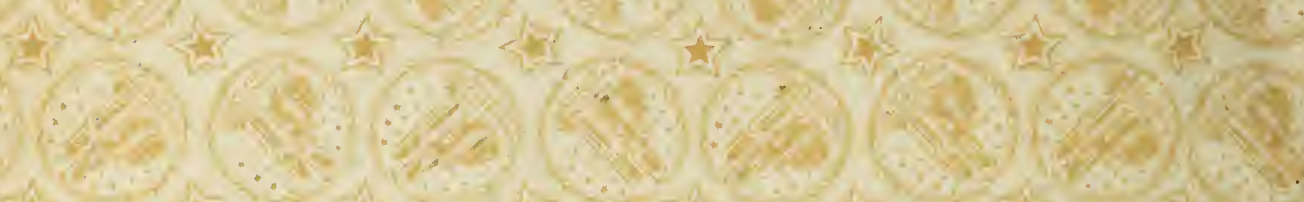

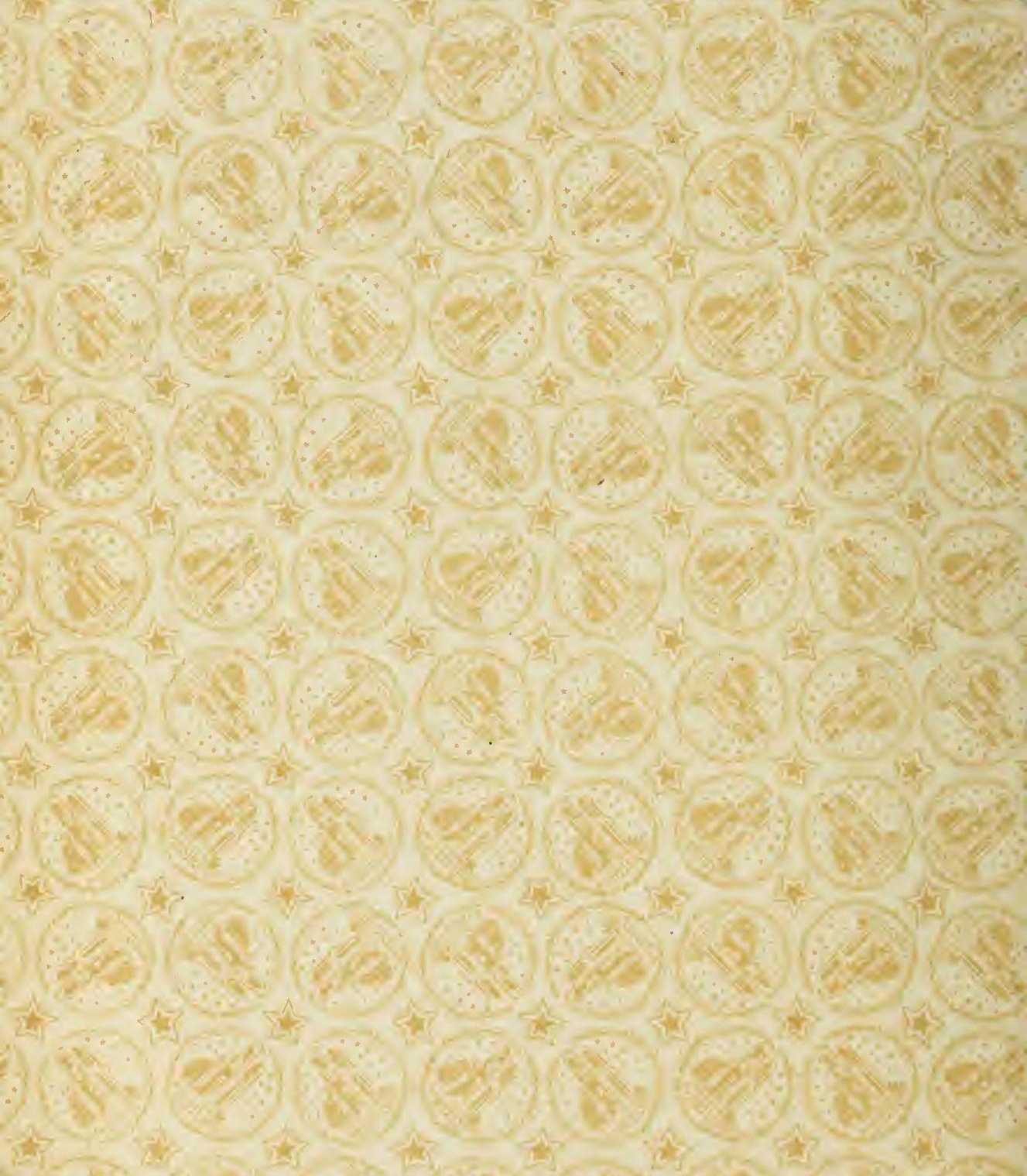



